\title{
Asymmetric transfer hydrogenation of $\alpha$-ketoamides; highly enantioselective formation of malic acid diamides and $\alpha$ - hydroxyamides
}

Shweta K. Gediya, Vijyesh K. Vyas, Guy J. Clarkson and Martin Wills*

Department of Chemistry, The University of Warwick, Coventry, CV4 7AL, UK.

Corresponding author email: m.wills@warwick.ac.uk

Supporting information.

\section{Contents:}

General experimental details $\quad$ S2

Section on oxoacetate preparations $\quad$ S3

Section on the synthesis of $\alpha$-keto amide derivatives $\quad$ S51

Section on reductions to alcohols $\quad \mathbf{S 1 9 4}$

Section on Dynamic Kinetic Resolution (DKR). $\quad$ S405

Section on applications $\quad \mathbf{S 4 3 4}$

X-ray data $\quad \mathbf{S 5 0 4}$ 


\section{General experimental details.}

Reagents and solvents were used as purchased and without further purification. Reactions were carried out under a nitrogen atmosphere unless otherwise specified. Reactions at elevated temperature were maintained by thermostatically controlled oil-baths or aluminium heating blocks. A temperature of $0{ }^{\circ} \mathrm{C}$ refers to an ice slush bath, $-78{ }^{\circ} \mathrm{C}$ to a dry ice acetone bath. NMR spectra were recorded on a Bruker AV (250 MHz), Bruker DPX (300 or $400 \mathrm{MHz})$ or Bruker DRX (500 MHz). Chemical shifts are rounded to the nearest $0.01 \mathrm{ppm}$ for ${ }^{1} \mathrm{H}$ spectra and the nearest $0.1 \mathrm{ppm}$ for ${ }^{13} \mathrm{C}$ spectra and are referenced to the solvent chemical shift. Coupling constants are rounded to the nearest $0.1 \mathrm{~Hz}$. Mass spectra were recorded on an Esquire 2000 and high-resolution mass spectra were recorded on a Bruker Micro ToF or MaXis. IR spectra were recorded on a PerkinElmer spectrum100 and peaks are reported in wavenumbers. Optical rotations were measured on an Optical Activity Ltd. AA1000 Polarimeter and are reported in $\operatorname{deg} \mathrm{dm}^{-1} \mathrm{~cm}^{3} \mathrm{~g}^{-1}$. The chiral GC measurements were performed using a Hewlett-Packard 1050 instrument linked to a PC running DataApex Clarity software. HPLC measurements were performed out using a Hewlett Packard 1050 Series with a quaternary pump, autosampler and variable wavelength detector linked to a PC running DataApex Clarity software. Melting points were determined on a Stuart scientific melting point apparatus and are uncorrected. Flash column chromatography was performed using silica gel of 230-400 mesh size. Thin layer chromatography was carried out on aluminium backed silica gel 60(F254) plates, visualised using 254nm UV light, potassium permanganate or cerium ammonium molybdate (CAM). Column chromatography was performed either by gradient elution (reported as a range, eg EtOAc/Petroleum ether (2-12\%), or by isocratic elution 


\section{Section on oxoacetate preparations.}

\section{General procedure A for acetylation or oxoacetate formation.}

To a stirred solution of amine in dichloromethane was added triethylamine and the mixture was cooled to $0^{\circ} \mathrm{C}$. This was followed by dropwise addition of acetyl chloride or methyl 2chloro-2-oxoacetate (fumes observed) and the resulting reaction mixture was stirred at room temperature for $24 \mathrm{~h}$. Once the reaction was complete (as assessed by TLC), the mixture was quenched with water and extracted with dichloromethane. The combined organic layer was washed with $2 \mathrm{~N} \mathrm{HCl}$ solution, water and finally with brine solution. The organic layer was dried over $\mathrm{MgSO}_{4}$, filtered and filtrate was concentrated under reduced pressure to afford acetylated derivatives which were used in the next step without further purification.

\section{1-(Piperidin-1-yl)ethan-1-one.}<smiles>CC(=O)N1CCCCC1</smiles>

This compound has been reported and was prepared following general procedure A using piperidine (2.00 g, $2.32 \mathrm{~mL}, 23.5 \mathrm{mmol}, 1.0 \mathrm{eq})$, acetyl chloride (1.84 g, $1.68 \mathrm{~mL}, 23.5 \mathrm{mmol}$, $1.0 \mathrm{eq})$ and triethylamine $(4.74 \mathrm{~g}, 6.54 \mathrm{~mL}, 47.0 \mathrm{mmol}, 2.0 \mathrm{eq})$ in dichloromethane $(40 \mathrm{~mL})$ to give the product as a clear yellow liquid $(2.83 \mathrm{~g}, 22.3 \mathrm{mmol}, 95 \%)$.

${ }^{1} \mathrm{H}$ NMR (400 MHz, $\left.\mathrm{CDCl}_{3}\right) \delta$ 3.48-3.45 (2H, m, $\left.\mathrm{CH}_{2}\right), 3.33-3.29\left(2 \mathrm{H}, \mathrm{m}, \mathrm{CH}_{2}\right), 2.00(3 \mathrm{H}, \mathrm{s}$, $\left.\mathrm{COCH}_{3}\right), 1.60-1.45\left(6 \mathrm{H}, \mathrm{m}, \mathrm{CH}_{2}\right)$;

${ }^{13} \mathrm{C} \mathrm{NMR}\left(101 \mathrm{MHz}, \mathrm{CDCl}_{3}\right) \delta 168.8,47.4,42.5,26.4,25.5,24.4,21.4$.

The data matches the reported data.

a) Talukdar, R. IrIII-Catalyzed direct syntheses of amides and esters using nitriles as acid equivalents: a photochemical pathway, New J. Chem. 2020, 44, 5303-5308. b) Ghosh, S.; Purkait, A.; Jana, C. K. Environmentally benign decarboxylative N-, O-, and S-acetylations and acylations, Green Chem. 2020, 22, 8721-8727. 
1-(Piperidin-1-yl)ethan-1-one.

${ }^{1} \mathrm{H} \mathrm{NMR}\left(400 \mathrm{MHz}, \mathrm{CDCl}_{3}\right)$.

Sep14-2020

PROTON.w CDCl3 /opt/topspin3.5pl2 SKG 31

$\stackrel{\substack{n \\ i}}{i}$

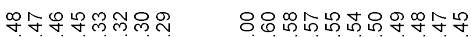

mmmmm<smiles>CC(=O)N1CCCCC1</smiles>

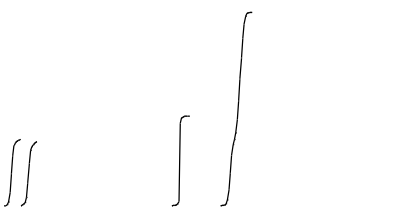

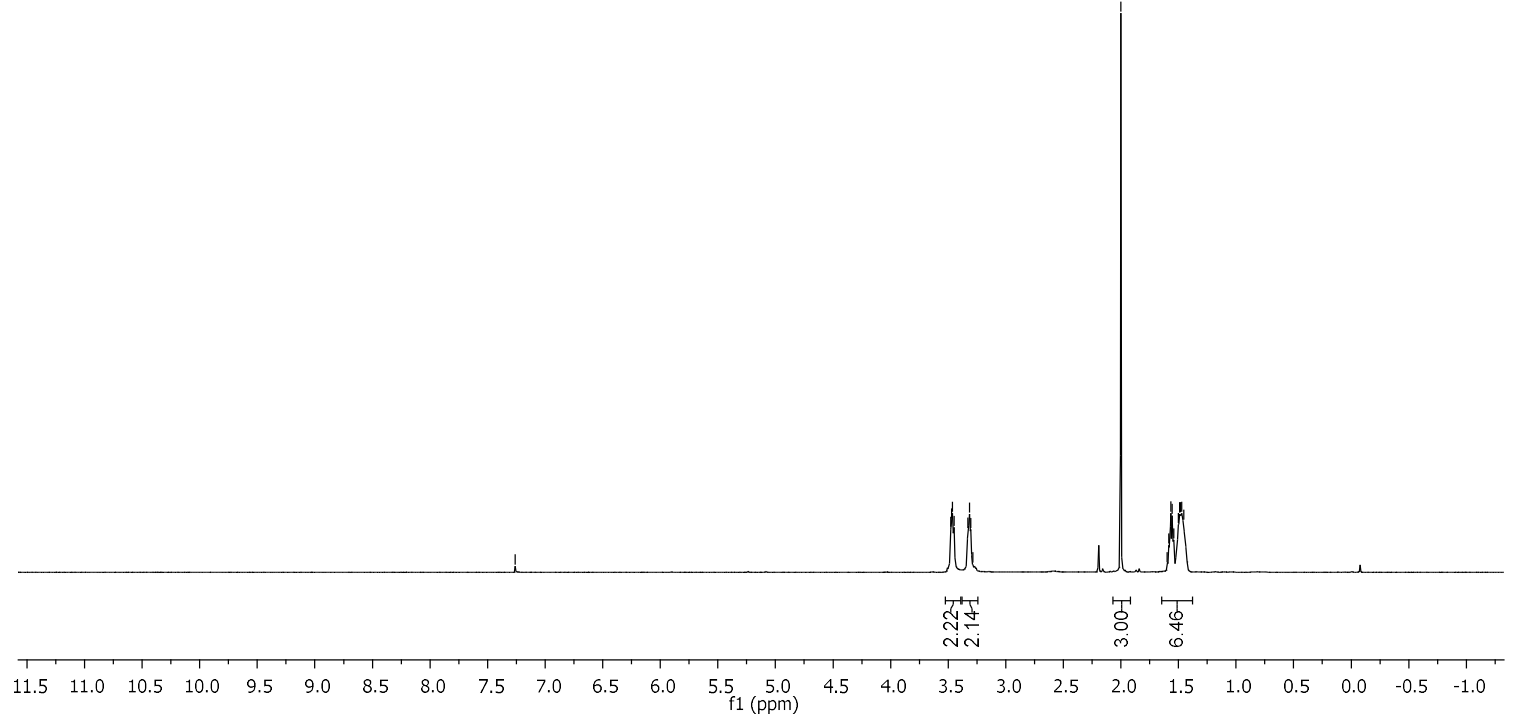

${ }^{13} \mathrm{C}$ NMR (101 MHz, $\left.\mathrm{CDCl}_{3}\right)$.

Apr12-2021

C13APTIong.w CDCI3

\begin{tabular}{l}
9 \\
$\infty$ \\
$\infty$ \\
$@$ \\
\hline 1
\end{tabular}

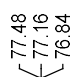

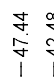

焉等寺学

떳ㅊ

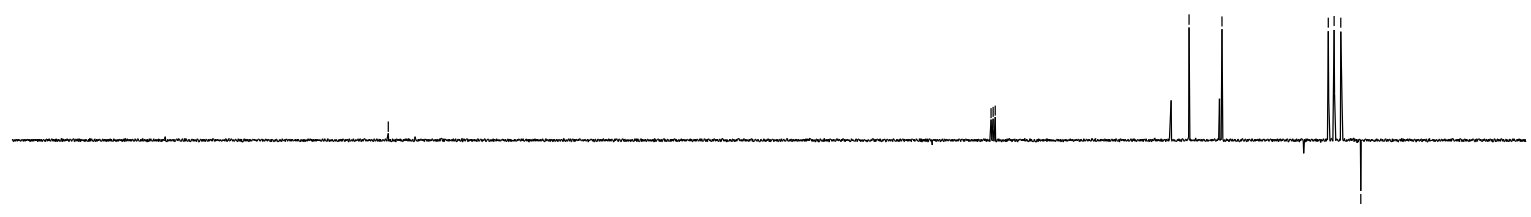




\section{$N$-methyl- $N$-phenylacetamide.}

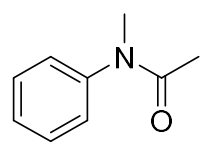

This compound has been reported and was prepared following general procedure $\mathbf{A}$ using $\mathrm{N}$ methylaniline $(3.00 \mathrm{~g}, 28.0 \mathrm{mmol}, 1.0 \mathrm{eq})$, acetyl chloride $(2.64 \mathrm{~g}, 2.40 \mathrm{~mL}, 33.6 \mathrm{mmol}, 1.2$ eq) and triethylamine $(5.66 \mathrm{~g}, 7.77 \mathrm{~mL}, 56.0 \mathrm{mmol}, 2.0 \mathrm{eq})$ in dichloromethane $(40 \mathrm{~mL})$ to give the product as a pale-yellow solid (2.89 g, $19.4 \mathrm{mmol}, 69 \%)$.

${ }^{1} \mathrm{H}$ NMR (400 MHz, $\left.\mathrm{CDCl}_{3}\right) \delta$ 7.44-7.30 (3H, m, ArH), $7.19(2 \mathrm{H}, \mathrm{d}, J=7.5 \mathrm{~Hz}, \mathrm{ArH}), 3.27$ $\left(3 \mathrm{H}, \mathrm{s}, \mathrm{N}-\mathrm{CH}_{3}\right), 1.87\left(3 \mathrm{H}, \mathrm{s}, \mathrm{COCH}_{3}\right)$;

${ }^{13} \mathrm{C}$ NMR $\left(101 \mathrm{MHz}, \mathrm{CDCl}_{3}\right) \delta 170.6,144.7,129.8,127.8,127.1,37.2,22.4$.

The data matches the reported data.

Andrew J. Smith, A. J.; Young, A.; Rohrbach, S.; O'Connor, E. F.; Allison, M.; Wang, H.-S.; Poole, D. L.; Tuttle, T.; Murphy, J. A. Electron-Transfer and Hydride-Transfer Pathways in the Stoltz-Grubbs Reducing System (KOtBu/Et $\left.{ }_{3} \mathrm{SiH}\right)$, Angew. Chem. Int. Ed. 2017, 56, $13747-13751$. 
$N$-Methyl- $N$-phenylacetamide.

${ }^{1} \mathrm{H}$ NMR (400 MHz, $\mathrm{CDCl}_{3}$ ).
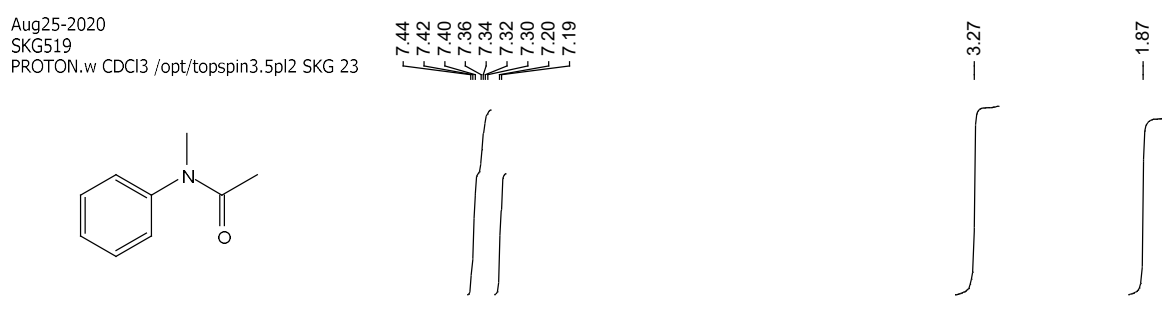

$\stackrel{8}{i}$

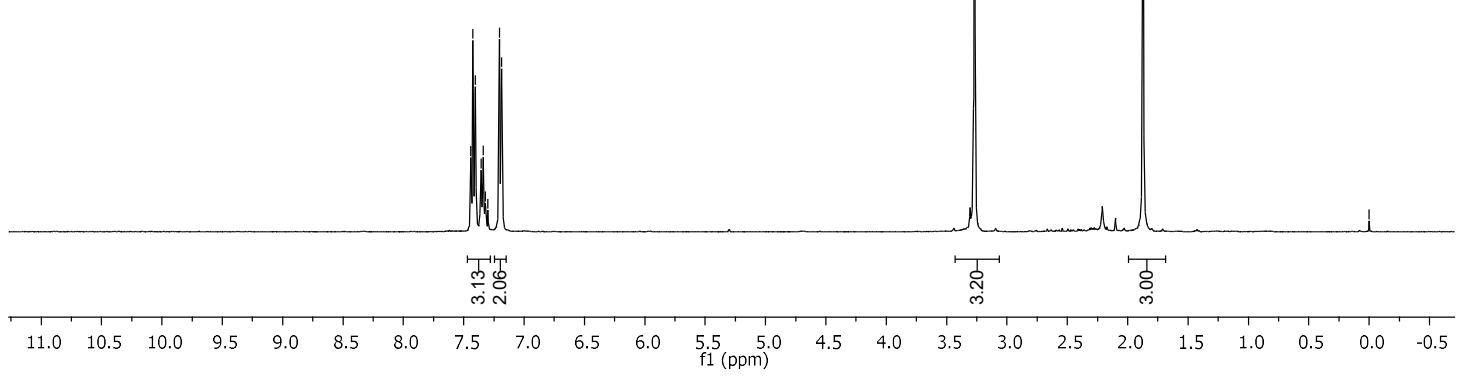

${ }^{13} \mathrm{C}$ NMR (101 MHz, $\mathrm{CDCl}_{3}$ ).

Aug25-2020
SKG519

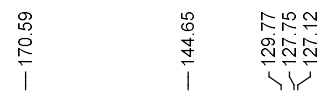

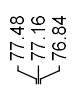
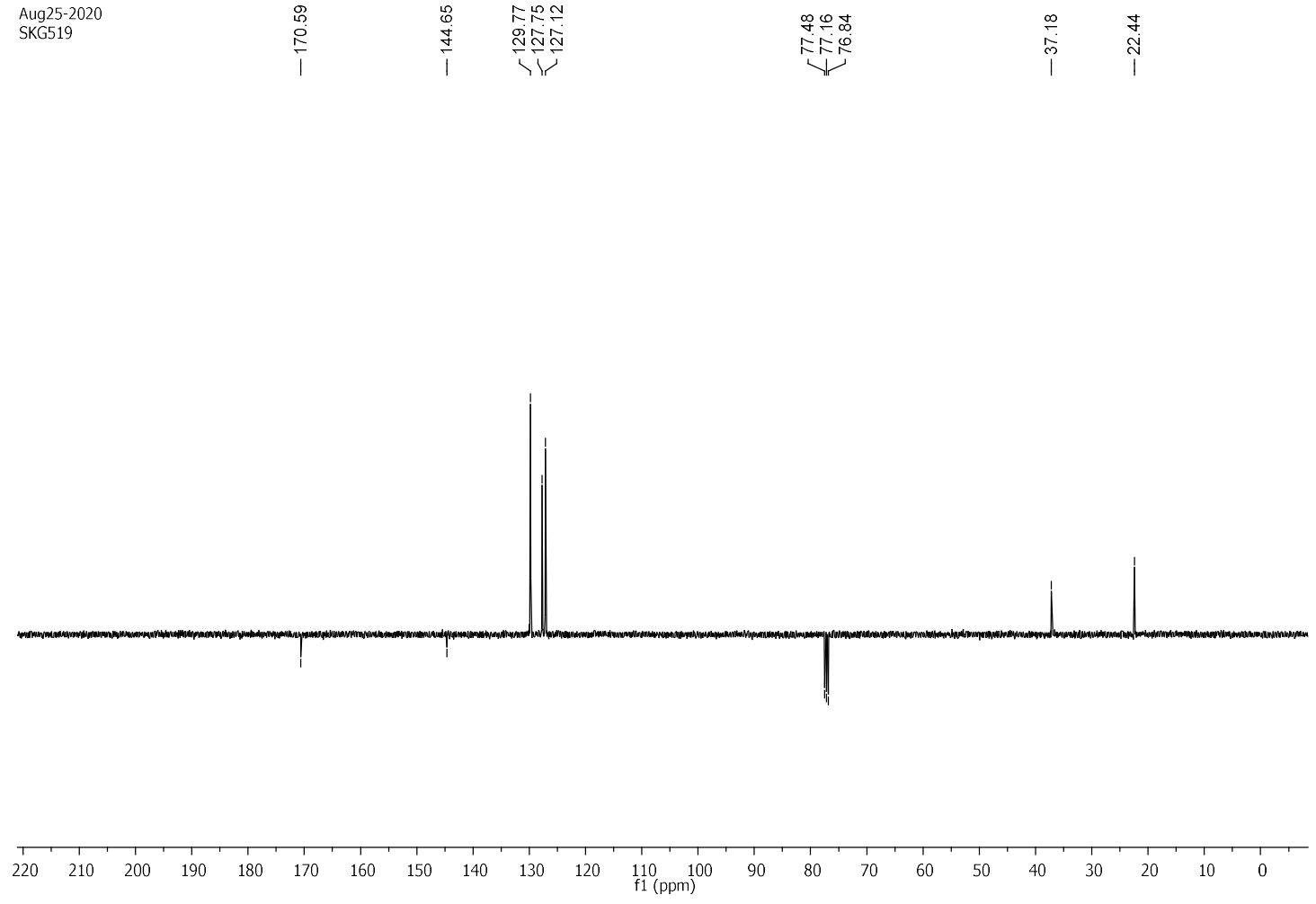

S6 


\section{$N$-Cyclohexyl- $N$-methylacetamide.}

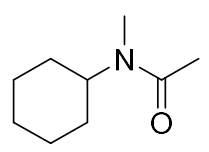

This compound has been reported and was prepared following general procedure A using $\mathrm{N}$ methylcyclohexanamine (1.00 g, $8.85 \mathrm{mmol}, 1.0 \mathrm{eq})$, acetyl chloride (1.38 g, $1.26 \mathrm{~mL}, 17.7$ mmol, $2.0 \mathrm{eq})$ and triethylamine $(1.07 \mathrm{~g}, 1.45 \mathrm{~mL}, 10.6 \mathrm{mmol}, 1.2 \mathrm{eq})$ in dichloromethane (40 $\mathrm{mL})$ to give the product as a clear liquid $(1.21 \mathrm{~g}, 7.74 \mathrm{mmol}, 88 \%)$. Two rotamers are visible in the NMR spectra.

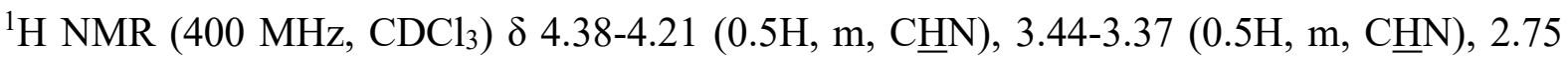
$\left(1.5 \mathrm{H}, \mathrm{s}, \mathrm{NCH}_{3}\right), 2.71\left(1.5 \mathrm{H}, \mathrm{s}, \mathrm{NCH}_{3}\right), 2.03\left(1.5 \mathrm{H}, \mathrm{s}, \mathrm{COCH}_{3}\right), 1.99\left(1.5 \mathrm{H}, \mathrm{s}, \mathrm{COCH}_{3}\right), 1.79-$ $0.93\left(10 \mathrm{H}, \mathrm{m}, \mathrm{CH}_{2}\right)(2$ rotamers ca. 1:1);

${ }^{13} \mathrm{C}$ NMR $\left(101 \mathrm{MHz}, \mathrm{CDCl}_{3}\right) \delta 170.2,170.1,57.8,51.2,30.8,30.2,29.8,26.9,25.7,25.5$, 25.2, 22.4, 21.5.

The data matches the reported data.

Henry, C.; Bolien, D.; Ibanescu, B.; Bloodworth, S.; Harrowven, D. C.; Zhang, X.; Craven, A.; Sneddon, H. F.; Whitby, R. J. Generation and Trapping of Ketenes in Flow, Eur. J. Org. Chem. 2015, 1491-1499. 


\section{$N$-Cyclohexyl- $N$-methylacetamide.}

${ }^{1} \mathrm{H}$ NMR $\left(400 \mathrm{MHz}, \mathrm{CDCl}_{3}\right)$.

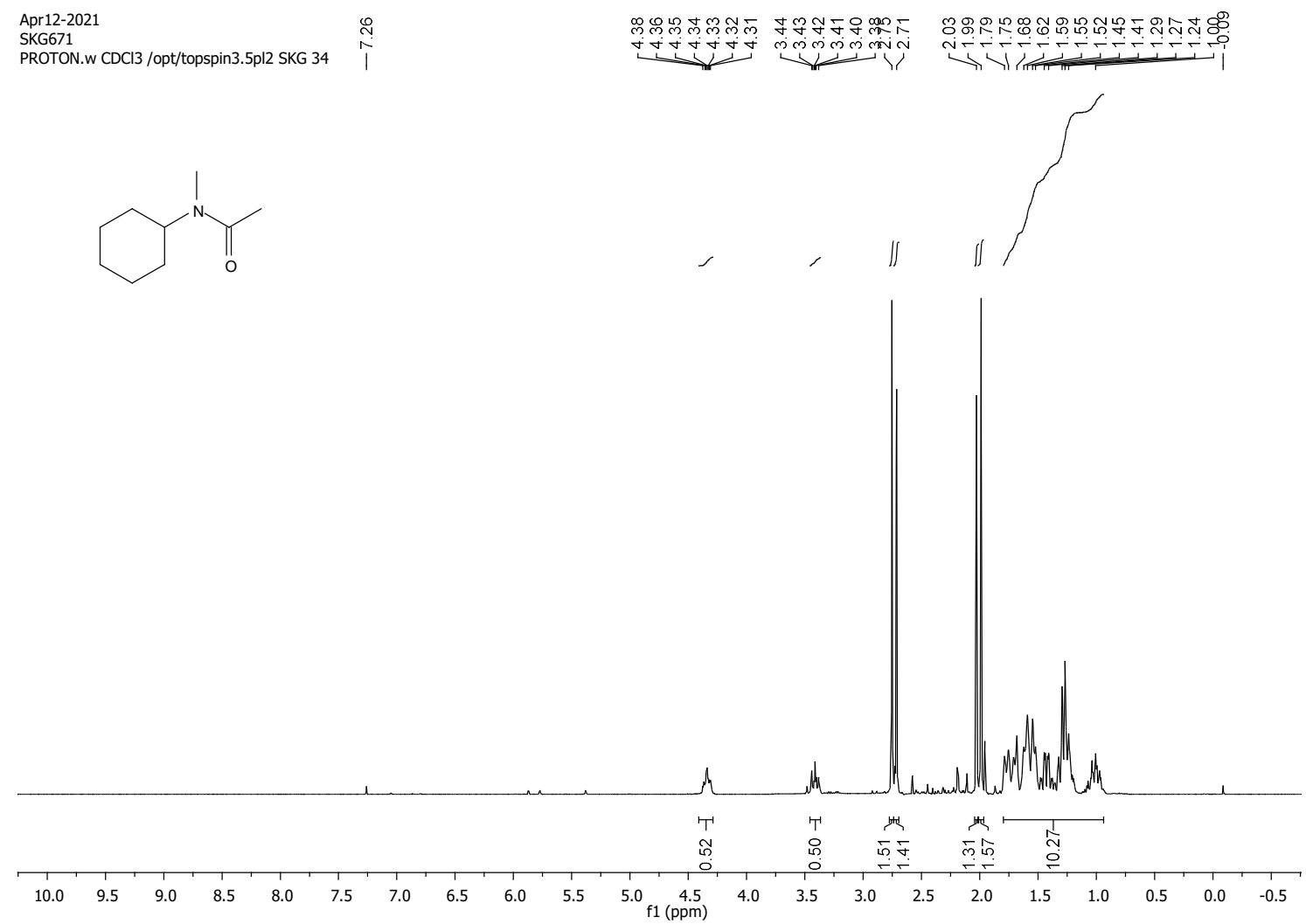

${ }^{13} \mathrm{C}$ NMR (101 MHz, $\left.\mathrm{CDCl}_{3}\right)$.

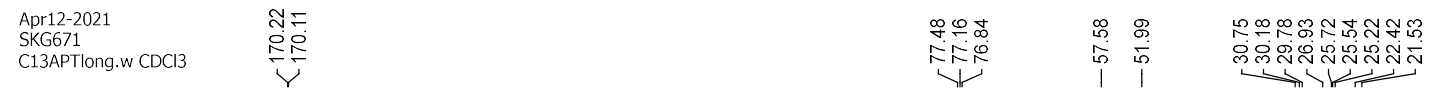

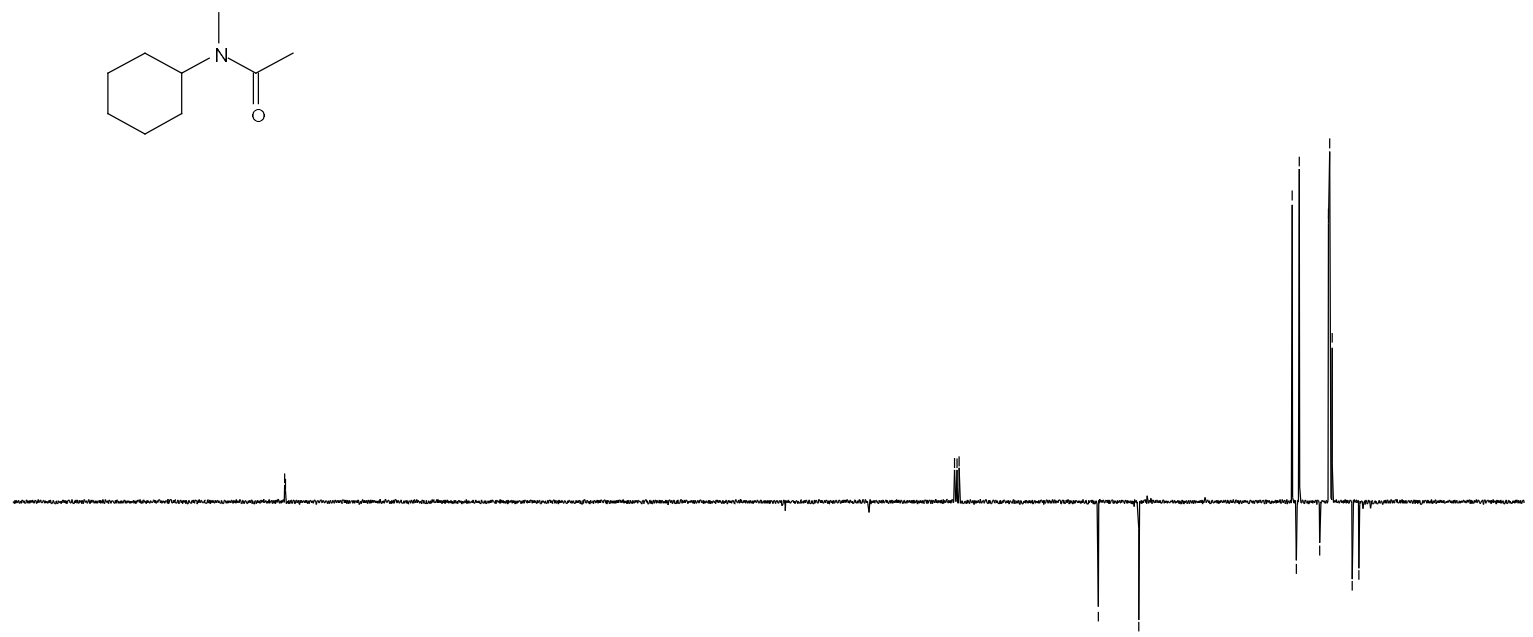

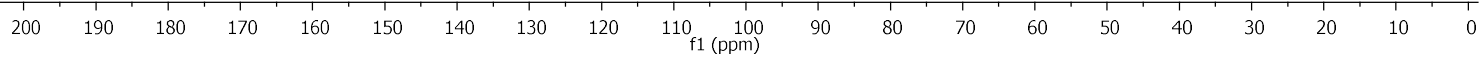




\section{$N$-Allyl- $N$-methylacetamide.}<smiles>C=CCN(C)C(C)=O</smiles>

This compound is reported and was prepared following the general procedure A using $\mathrm{N}$ methylcyclohexanamine $(0.500 \mathrm{~g}, 7.04 \mathrm{mmol}, 1.0 \mathrm{eq})$, acetyl chloride $(0.658 \mathrm{~g}, 0.59 \mathrm{~mL}, 8.44$ mmol, $1.2 \mathrm{eq})$ and triethylamine $(1.42 \mathrm{~g}, 1.95 \mathrm{~mL}, 14.1 \mathrm{mmol}, 2.0 \mathrm{eq})$ in dichloromethane (10 $\mathrm{mL})$ to give the product as a clear liquid $(0.430 \mathrm{~g}, 3.80 \mathrm{mmol}, 54 \%)$.

${ }^{1} \mathrm{H}$ NMR (400 MHz, $\mathrm{CDCl}_{3}$ ) (Two rotamers, ca 1:1) $\delta 5.83-5.70\left(1 \mathrm{H}, \mathrm{m}, \mathrm{CH}_{2}=\mathrm{C}_{\underline{H C H}}\right.$ ), 5.24 -5.12 (2H, m, $\left.\underline{\mathrm{CH}}_{2}=\mathrm{CHCH}_{2}\right), 4.00-3.97\left(1 \mathrm{H}, \mathrm{m}, \mathrm{CH}_{2}=\mathrm{CHC}_{2}\right), 3.92-3.89(1 \mathrm{H}, \mathrm{m}$, $\left.\mathrm{CH}_{2}=\mathrm{CHCH}_{2}\right), 2.95\left(1.5 \mathrm{H}, \mathrm{m}, \mathrm{NCH}_{3}\right), 2.92\left(1.5 \mathrm{H}, \mathrm{m}, \mathrm{NCH}_{3}\right), 2.11\left(1.5 \mathrm{H}, \mathrm{m}, \mathrm{COCH}_{3}\right), 2.08$ $\left(1.5 \mathrm{H}, \mathrm{m}, \mathrm{COCH}_{3}\right)$;

${ }^{13} \mathrm{C}$ NMR $\left(101 \mathrm{MHz}, \mathrm{CDCl}_{3}\right) \delta 170.9,170.5,133.1,132.4,117.2,116.6,53.1,49.8,35.5$, $33.5,21.8,21.2$ (2 rotamers ca. 1:1).

The data matches the reported data.

a) Ohmura, N.; Nakamura, A.; Hamasaki, A.; Tokunaga, M. Hydrolytic Deallylation of NAllyl Amides Catalyzed by $\mathrm{Pd}^{\mathrm{II}}$ Complexes. Eur. J. Org. Chem. 2008, 5042-5045. B) Della, E. W.; Knill, A. M. A Theoretical and Experimental Investigation of the Kinetics of Ring Closure of the 3-Methyl-3-azahex-5-enyl Radical. Aust. J. Chem. 1995, 48, 2047-2051. 


\section{$N$-Allyl- $N$-methylacetamide.}

${ }^{1} \mathrm{H}$ NMR (400 MHz, $\left.\mathrm{CDCl}_{3}\right)$.

Nov26-2020

SKG672

BD

PROTON.w CDCl3 /opt/topspin3.5pl2 SKG 50 on

นูก

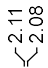

8
$i$
$i$<smiles>C=CCN(C)C(C)=O</smiles><smiles>C=CCCCC</smiles>

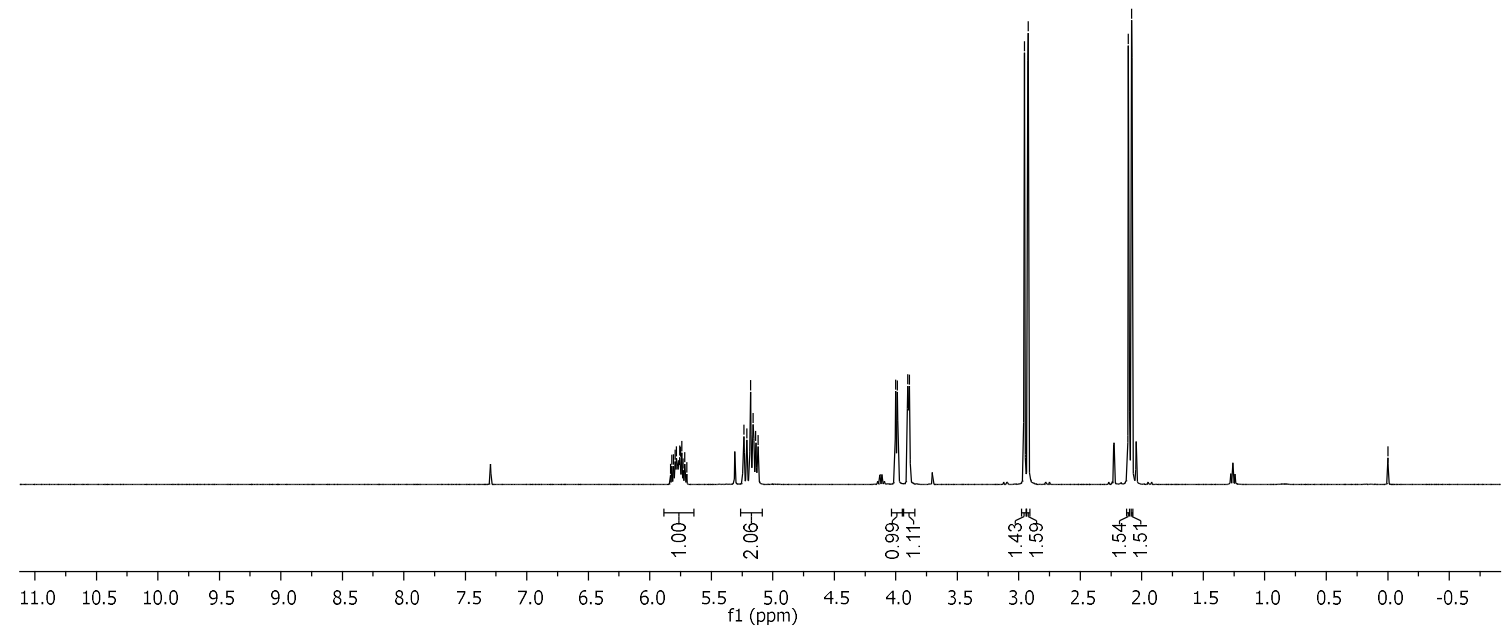

${ }^{13} \mathrm{C}$ NMR (101 MHz, $\left.\mathrm{CDCl}_{3}\right)$.
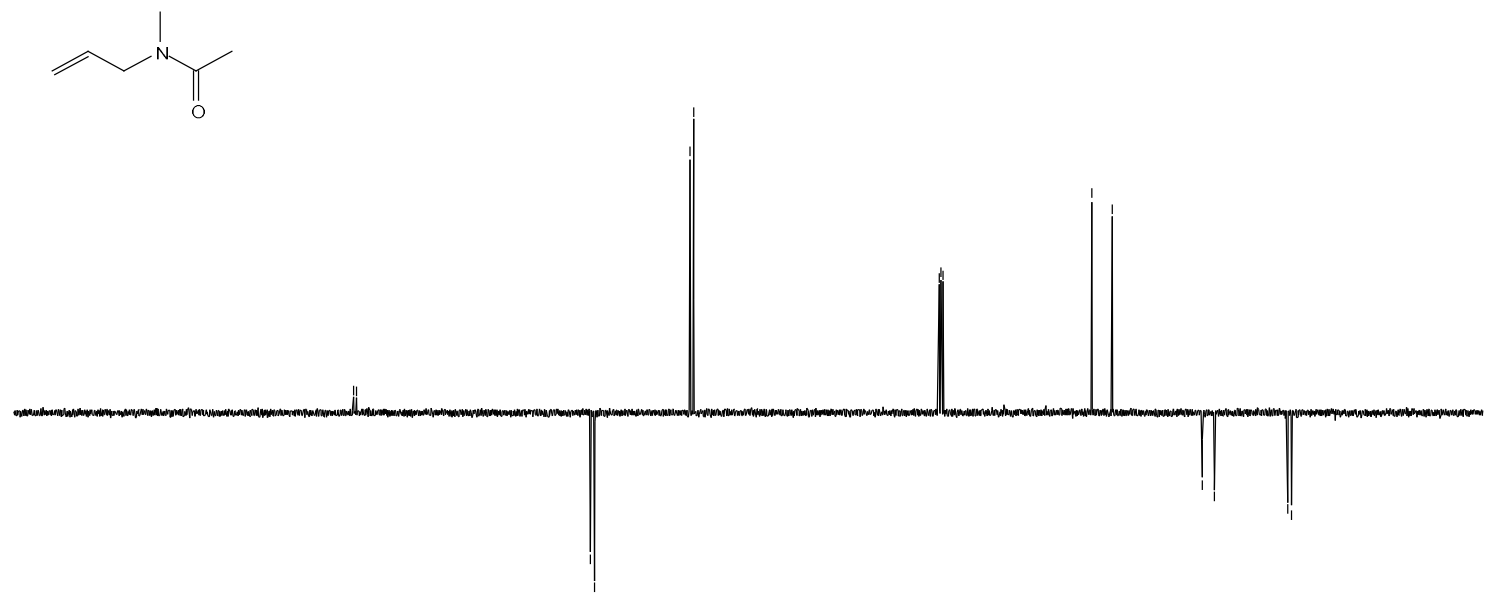

$\begin{array}{llllllllllllllllllllllllll}220 & 210 & 200 & 190 & 180 & 170 & 160 & 150 & 140 & 130 & 120 & 110 & 100 & 90 & 80 & 70 & 60 & 50 & 40 & 30 & 20 & 10 & 0\end{array}$ 
Methyl 2-oxo-2-(phenylamino) acetate.<smiles>COC(=O)C(=O)Nc1ccccc1</smiles>

This compound has been reported and was prepared following the general procedure A using methyl 2-chloro-2-oxoacetate $(2.00 \mathrm{~g}, 1.50 \mathrm{~mL}, 16.4 \mathrm{mmol}, 1.05 \mathrm{eq})$, aniline (1.46 g, 15.6 mmol, $1.0 \mathrm{eq})$ and triethylamine $(1.89 \mathrm{~g}, 2.60 \mathrm{~mL}, 18.7 \mathrm{mmol}, 1.2 \mathrm{eq})$ in dichloromethane (40 $\mathrm{mL})$ to give the product as a white solid $(2.52 \mathrm{~g}, 14.1 \mathrm{mmol}, 90 \%)$.

TLC: $\mathrm{R}_{\mathrm{f}}$ ca 0.3 (8:2, Hexane: EtOAc), strong UV active;

${ }^{1} \mathrm{H}$ NMR $\left(500 \mathrm{MHz}, \mathrm{CDCl}_{3}\right) \delta 8.97(1 \mathrm{H}, \mathrm{s}, \mathrm{CONH}), 7.65(2 \mathrm{H}, \mathrm{d}, J=8.0 \mathrm{~Hz}, \mathrm{ArH}), 7.38-7.35$ (2H, m, ArH), 7.20-7.17 (1H, m, ArH), $3.93\left(3 \mathrm{H}, \mathrm{s}, \mathrm{OCH}_{3}\right)$;

${ }^{13} \mathrm{C}$ NMR $\left(126 \mathrm{MHz}, \mathrm{CDCl}_{3}\right) \delta 161.5,153.7,136.3,129.2,125.6,119.9,54.0$;

$m / z(\mathrm{ESI}) 202.0[(\mathrm{M}+\mathrm{Na})+, 60 \%]$.

The data matches the reported data.

1. Adib, M.; Seyed, R. P.; Gohari, J. A.; Shahsavaric, F. Metal-Free Oxidative $\mathrm{C}=\mathrm{C}$ Bond Cleavage of Electron-Deficient Enamines Promoted by tert-Butyl Hydroperoxide. Synlett 2017, 1481-1485.

2. Zhang, Z.; Gao, X.; Yu, H.; Zhang, G.; Liu, J. Copper-Catalysed (Diacetoxyiodo)benzene-Promoted Aerobic Esterification Reaction: Synthesis of Oxamates from Acetoacetamides. Adv. Synth. Catal. 2018, 360, 3406-3411. 
Methyl 2-oxo-2-(phenylamino) acetate.

${ }^{1} \mathrm{H}$ NMR (500 MHz, $\mathrm{CDCl}_{3}$ ).
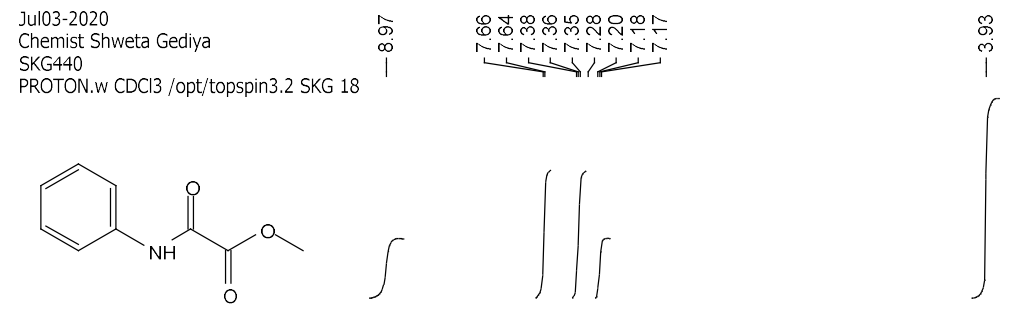

8
0
$i$

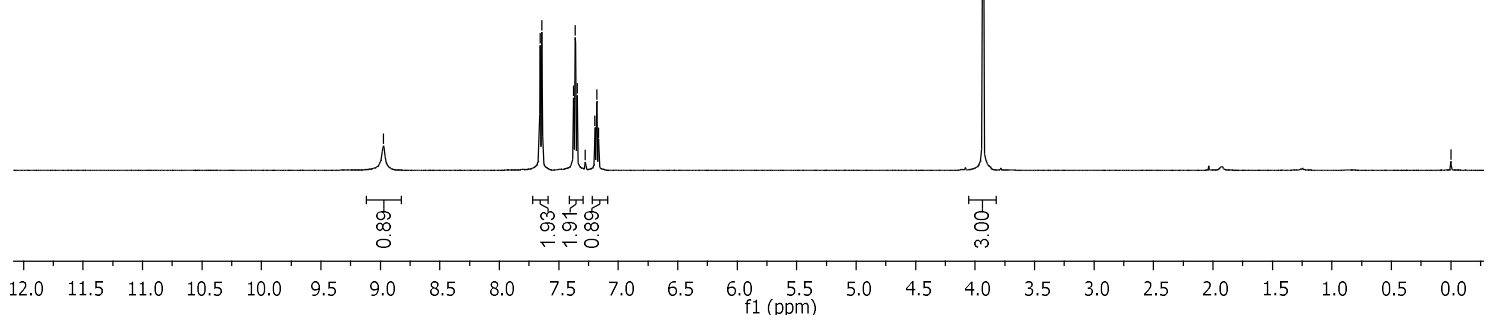

${ }^{13} \mathrm{C}$ NMR (126 MHz, $\mathrm{CDCl}_{3}$ ).

Jul03-2020

Chemist Shweta Gediya

C13APT.w CDCl3 /opt/topspin3.2 SKG 18

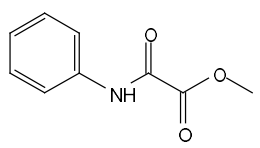

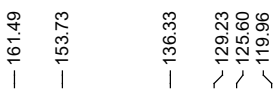

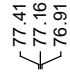
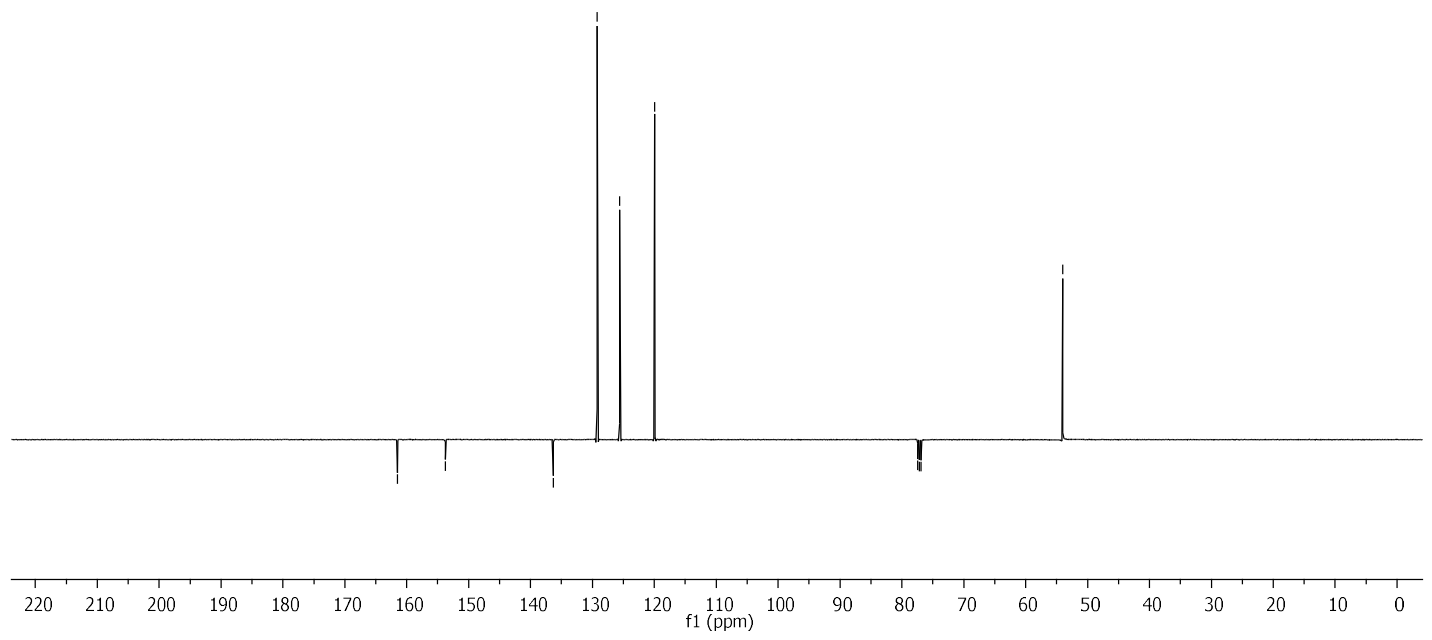

S12 
Methyl 2-oxo-2-(piperidin-1-yl) acetate.<smiles>COC(=O)C(=O)N1CCCCC1</smiles>

This compound is reported and was prepared following the general procedure A using methyl 2-chloro-2-oxoacetate (2.00 g, $1.50 \mathrm{~mL}, 16.4 \mathrm{mmol}, 1.05 \mathrm{eq})$, piperidine (1.33 g, $15.6 \mathrm{mmol}$, $1.0 \mathrm{eq})$ and triethylamine $(1.89 \mathrm{~g}, 2.60 \mathrm{~mL}, 18.7 \mathrm{mmol}, 1.2 \mathrm{eq})$ in dichloromethane $(40 \mathrm{~mL})$ to give the product as a colourless liquid $(2.63 \mathrm{~g}, 15.4 \mathrm{mmol}, 99 \%)$.

TLC: $\mathrm{R}_{\mathrm{f}}$ ca 0.4 (7:3, Hexane: EtOAc), strong UV active;

${ }^{1} \mathrm{H}$ NMR $\left(400 \mathrm{MHz}, \mathrm{CDCl}_{3}\right) \delta 3.83\left(3 \mathrm{H}, \mathrm{s}, \mathrm{OCH}_{3}\right), 3.54-3.51\left(2 \mathrm{H}, \mathrm{m}, \mathrm{CH}_{2}\right), 3.31-3.28(2 \mathrm{H}$, $\left.\mathrm{m}, \mathrm{CH}_{2}\right), 1.69-1.55\left(6 \mathrm{H}, \mathrm{m}, \mathrm{CH}_{2}\right)$;

${ }^{13} \mathrm{C}$ NMR (101 MHz, $\left.\mathrm{CDCl}_{3}\right) \delta 163.5,160.1,52.5,47.3,42.3,26.3,25.2,24.4$;

$m / z(\mathrm{ESI}) 194.1\left[(\mathrm{M}+\mathrm{Na})^{+}, 10 \%\right]$. The data matches the reported data.

Kolekar, Y. A.; Bhanage, B. M.; Pd/C-catalyzed synthesis of oxamates by oxidative cross double carbonylation of alcohols and tertiary amines through $\mathrm{C}-\mathrm{N}$ bond cleavage, New J. Chem. 2019, 43, 18072-18078. 
Methyl 2-oxo-2-(piperidin-1-yl) acetate.

${ }^{1} \mathrm{H} \mathrm{NMR}\left(400 \mathrm{MHz}, \mathrm{CDCl}_{3}\right)$.

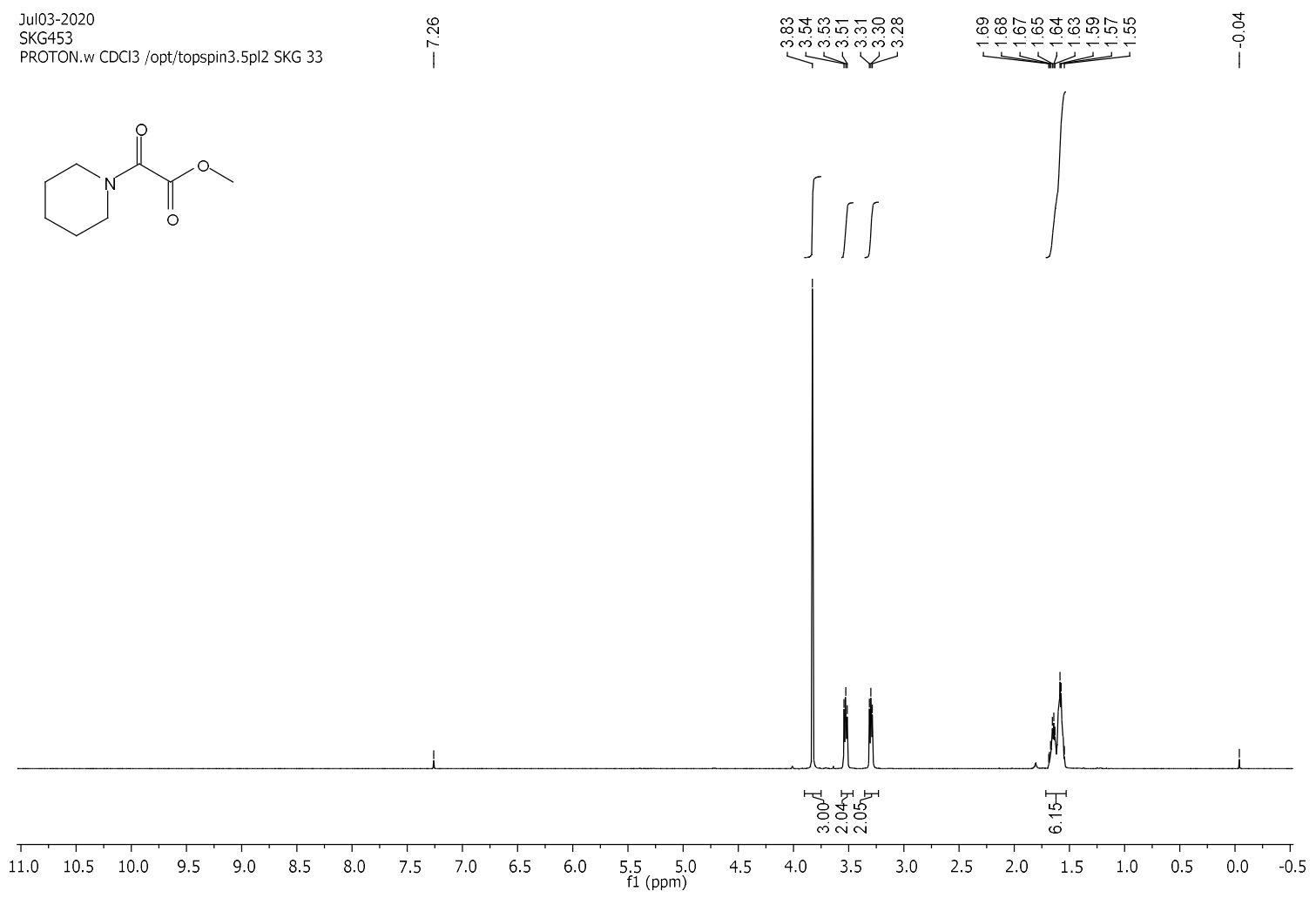

${ }^{13} \mathrm{C}$ NMR (101 MHz, $\left.\mathrm{CDCl}_{3}\right)$.

Jul03-2020
SKG453

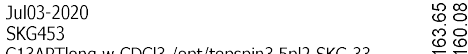

C13APTlong.w CDCl3 /opt/topspin3.5pl2 SKG 33<smiles>C=C(OC)C(=O)N1CCCCC1</smiles>

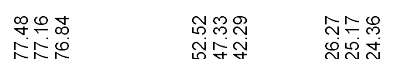

年少尔

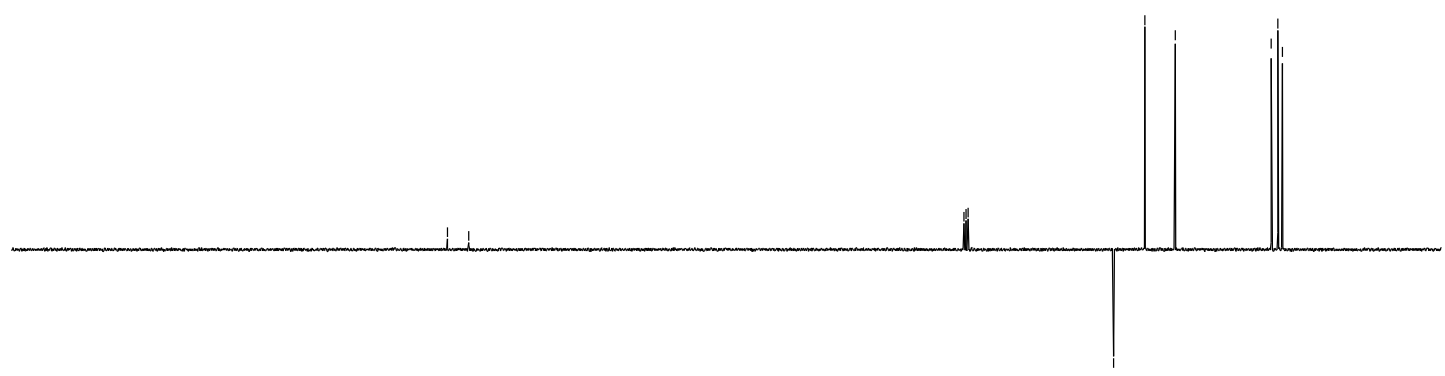

$\begin{array}{lllllllllllllllllllllllllllllllllllll}230 & 220 & 210 & 200 & 190 & 180 & 170 & 160 & 150 & 140 & 130 & 120 & 110 & 100 & 90 & 80 & 70 & 60 & 50 & 40 & 30 & 20 & 10 & 0\end{array}$ 
Methyl 2-(cyclohexylamino)-2-oxoacetate.<smiles>COC(=O)C(=O)NC1CCCCC1</smiles>

This compound is reported and was prepared by following the general procedure A using methyl 2-chloro-2-oxoacetate (2.00 g, $1.50 \mathrm{~mL}, 16.4 \mathrm{mmol}, 1.05 \mathrm{eq})$, cyclohexanamine (1.54 $\mathrm{g}, 15.6 \mathrm{mmol}, 1.0 \mathrm{eq})$ and triethylamine $(1.89 \mathrm{~g}, 2.60 \mathrm{~mL}, 18.7 \mathrm{mmol}, 1.2 \mathrm{eq})$ in dichloromethane $(40 \mathrm{~mL})$ to give the product as a colourless liquid (1.89 $\mathrm{g}, 10.2 \mathrm{mmol}, 65 \%)$. TLC: $R_{\mathrm{f}}$ ca 0.2 (7:3, Hexane: EtOAc), strong UV active;

${ }^{1} \mathrm{H}$ NMR (400 MHz, $\left.\mathrm{CDCl}_{3}\right) \delta 6.97(1 \mathrm{H}, \mathrm{s}, \mathrm{CONH}), 3.87\left(3 \mathrm{H}, \mathrm{s},-\mathrm{OCH}_{3}\right), 3.83-3.73(1 \mathrm{H}, \mathrm{m}$, CㅌNH), 1.94-1.90 (2H, m, $\left.\mathrm{CH}_{2}\right), 1.74-1.59\left(3 \mathrm{H}, \mathrm{m}, \mathrm{CH}_{2}\right), 1.42-1.31\left(2 \mathrm{H}, \mathrm{m}, \mathrm{CH}_{2}\right), 1.26$ $1.12\left(3 \mathrm{H}, \mathrm{m}, \mathrm{CH}_{2}\right)$;

${ }^{13} \mathrm{C}$ NMR $\left(101 \mathrm{MHz}, \mathrm{CDCl}_{3}\right) \delta 161.6,155.4,53.7,48.9,32.7,25.4,24.7$;

$m / z(\mathrm{ESI}) 208.1\left[(\mathrm{M}+\mathrm{Na})^{+}, 100 \%\right]$.

The data matches the reported data.

Nair,V.; Sheeba, V. A Facile CAN-Mediated Transformation of Acetoacetamides to Oxamates, J. Org. Chem. 1999, 64, 6898-6900. 
Methyl 2-(cyclohexylamino)-2-oxoacetate.

${ }^{1} \mathrm{H}$ NMR (400 MHz, $\left.\mathrm{CDCl}_{3}\right)$.

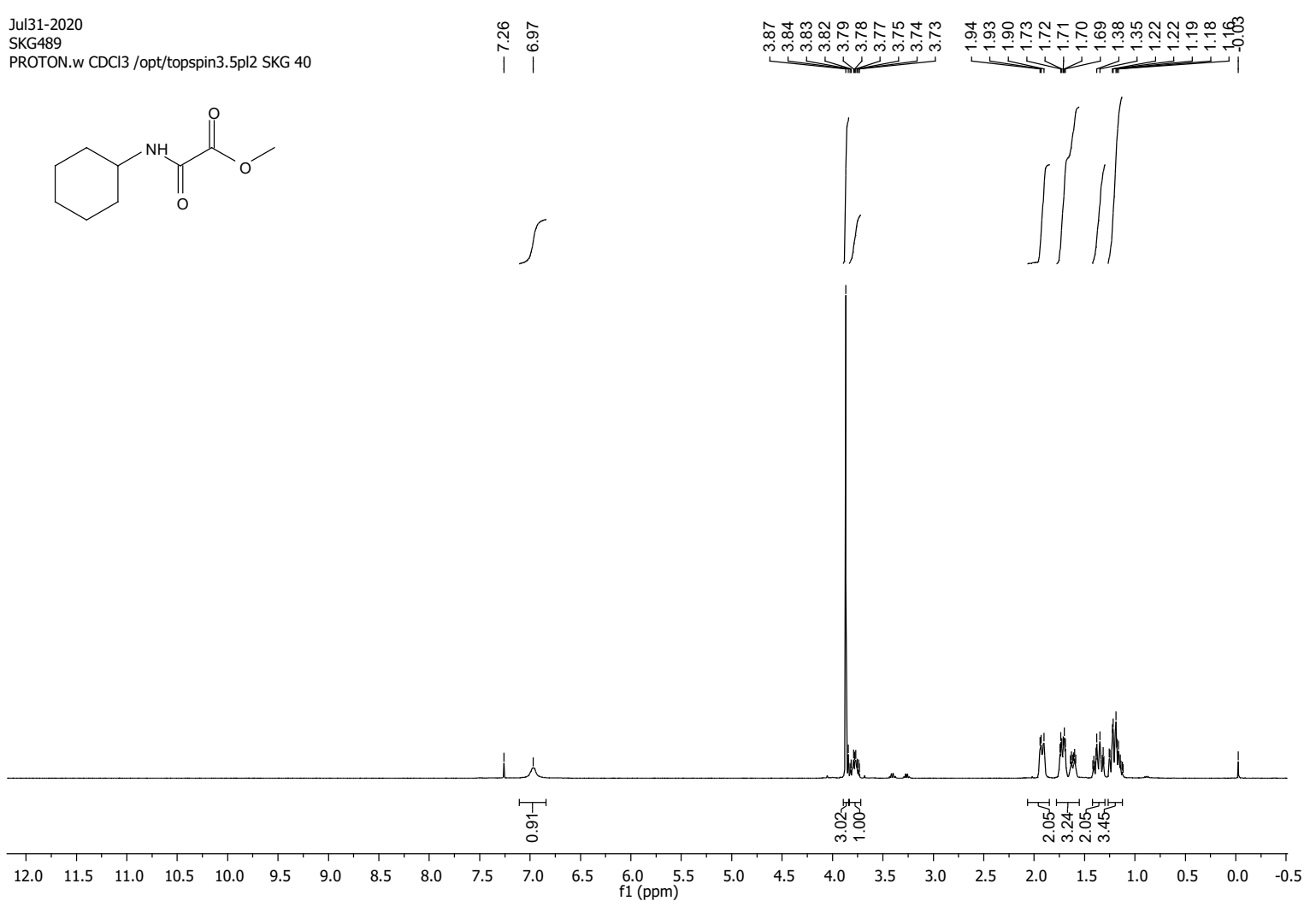

${ }^{13} \mathrm{C}$ NMR (101 MHz, $\left.\mathrm{CDCl}_{3}\right)$.

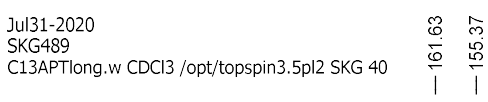

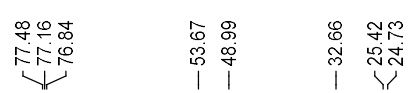
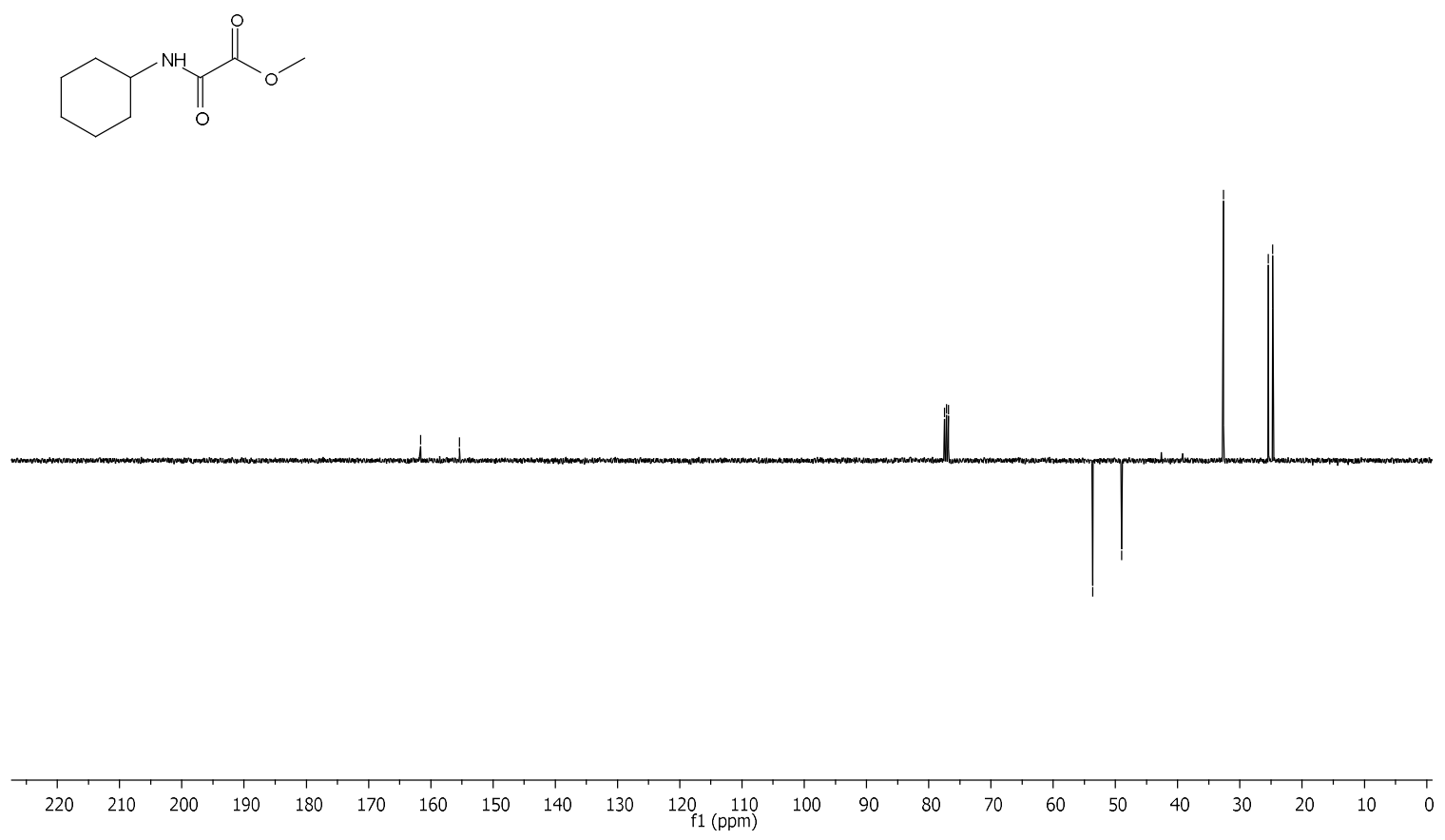

S16 
Methyl 2-(methylamino)-2-oxoacetate.<smiles>CNC(=O)C(=O)OC</smiles>

This compound has been reported and was prepared by following the general procedure $\mathbf{A}$ using methyl 2-chloro-2-oxoacetate $(2.00 \mathrm{~g}, 1.50 \mathrm{~mL}, 16.4 \mathrm{mmol}, 1.0 \mathrm{eq})$, methylamine (33\% in ethanol) (0.688 g, $32.8 \mathrm{mmol}, 2.0 \mathrm{eq})$ and triethylamine $(1.89 \mathrm{~g}, 2.60 \mathrm{~mL}, 18.7 \mathrm{mmol}, 1.15$ eq) in dichloromethane $(40 \mathrm{~mL})$ to give the product as a white solid $(0.870 \mathrm{~g}, 7.43 \mathrm{mmol}$, $45 \%)$.

TLC: $\mathrm{R}_{\mathrm{f}}$ ca 0.2 (7:3, Hexane: EtOAc), strong UV active;

${ }^{1} \mathrm{H}$ NMR $\left(400 \mathrm{MHz}, \mathrm{CDCl}_{3}\right) \delta 7.16(1 \mathrm{H}, \mathrm{s}, \mathrm{CONH}), 3.88\left(3 \mathrm{H}, \mathrm{s},-\mathrm{OCH}_{3}\right), 2.92(3 \mathrm{H}, \mathrm{d}, J=5.1$ $\left.\mathrm{Hz},-\mathrm{NHCH}_{3}\right)$;

${ }^{13} \mathrm{C}$ NMR (101 MHz, $\left.\mathrm{CDCl}_{3}\right) \delta 161.3,157.1,53.7,26.6$;

$m / z(\mathrm{ESI}) 140.1\left[(\mathrm{M}+\mathrm{Na})^{+}, 40 \%\right], 257.2\left[(2 \mathrm{M}+\mathrm{Na})^{+}, 100 \%\right]$.

The data matches the reported data.

Zhang, Z.; Gao, X.; Yu, H.; Zhang, G.; Liu, J. Copper-Catalysed (Diacetoxyiodo)benzenePromoted Aerobic Esterification Reaction: Synthesis of Oxamates from Acetoacetamides, Adv. Synth. Catal. 2018, 360, 3406-3411. 
Methyl 2-(methylamino)-2-oxoacetate.

${ }^{1} \mathrm{H}$ NMR $\left(400 \mathrm{MHz}, \mathrm{CDCl}_{3}\right)$.

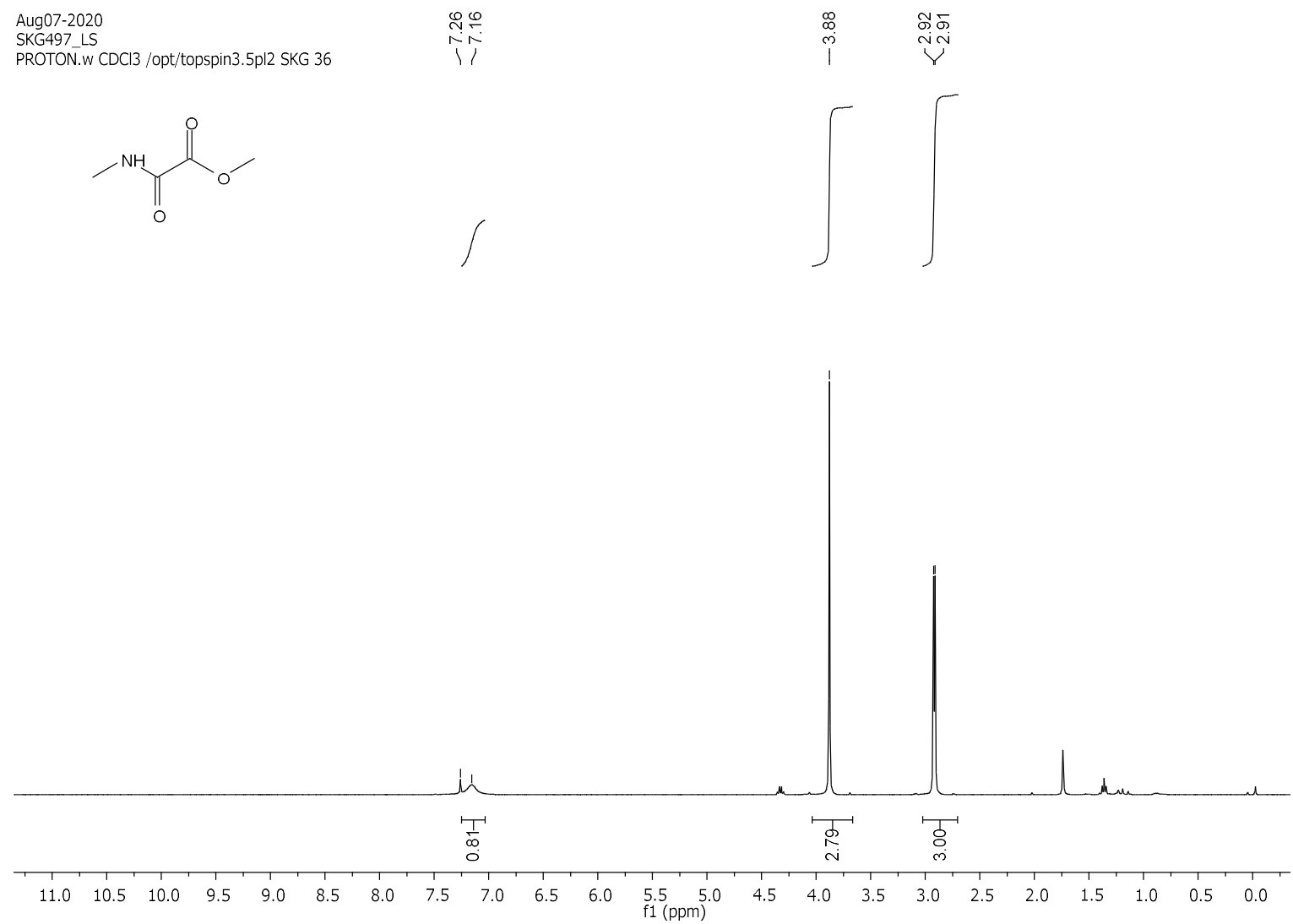

${ }^{13} \mathrm{C}$ NMR (101 MHz, $\left.\mathrm{CDCl}_{3}\right)$.

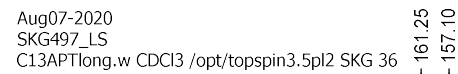

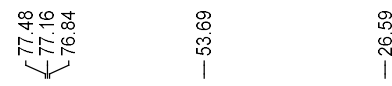<smiles>CNC(=O)C(=O)OC</smiles>

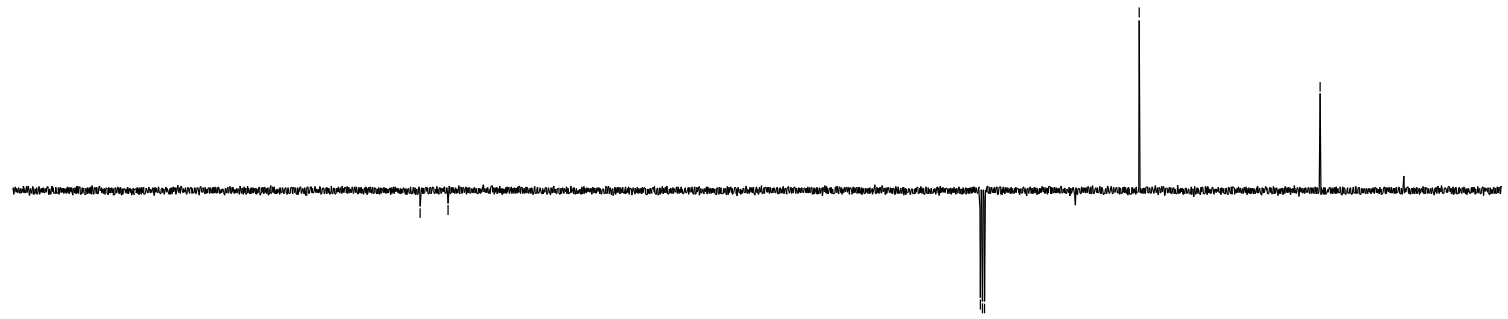

\begin{tabular}{lllllllllllllllllllllllllllll}
\hline 220 & 210 & 200 & 190 & 180 & 170 & 160 & 150 & 140 & 130 & 120 & 110 & 100 & 90 & 80 & 70 & 60 & 50 & 40 & 30 & 20 & 10 & 0
\end{tabular} 
Methyl 2-morpholino-2-oxoacetate.<smiles>COC(=O)C(=O)N1CCOCC1</smiles>

This compound has been reported and was prepared by following the general procedure $\mathbf{A}$ using methyl 2-chloro-2-oxoacetate $(2.00 \mathrm{~g}, 1.50 \mathrm{~mL}, 16.4 \mathrm{mmol}, 1.05 \mathrm{eq})$, morpholine (1.36 $\mathrm{g}, 15.6 \mathrm{mmol}, 1.0 \mathrm{eq})$ and triethylamine $(1.89 \mathrm{~g}, 2.60 \mathrm{~mL}, 18.7 \mathrm{mmol}, 1.2 \mathrm{eq})$ in dichloromethane $(40 \mathrm{~mL})$ to give the product as a white solid $(2.10 \mathrm{~g}, 12.1 \mathrm{mmol}, 78 \%)$.

TLC: $\mathrm{R}_{\mathrm{f}}$ ca 0.5 (4:6, Hexane: EtOAc), strong UV active;

${ }^{1} \mathrm{H}$ NMR (400 MHz, $\left.\mathrm{CDCl}_{3}\right) \delta 3.79\left(3 \mathrm{H}, \mathrm{s},-\mathrm{OCH}_{3}\right), 3.65-3.61\left(4 \mathrm{H}, \mathrm{m}, \mathrm{CH}_{2}\right), 3.57-3.36(2 \mathrm{H}$, $\left.\mathrm{m}, \mathrm{CH}_{2}\right), 3.39-3.37\left(2 \mathrm{H}, \mathrm{m}, \mathrm{CH}_{2}\right)$;

${ }^{13} \mathrm{C}$ NMR $\left(101 \mathrm{MHz}, \mathrm{CDCl}_{3}\right) \delta 162.6,159.8,66.6,66.3,52.7,46.4,41.7$;

$m / z(\mathrm{ESI}) 196.1\left[(\mathrm{M}+\mathrm{Na})^{+}, 20 \%\right], 369.2\left[(2 \mathrm{M}+\mathrm{Na})^{+}, 100 \%\right]$.

The data matches the reported data.

Kolekar, Y. V.; Bhanage, B. M. Pd/C-catalyzed synthesis of oxamates by oxidative cross double carbonylation of alcohols and tertiary amines through $\mathrm{C}-\mathrm{N}$ bond cleavage, New $J$. Chem. 2019, 43, 18072-18078. 
Methyl 2-morpholino-2-oxoacetate.

${ }^{1} \mathrm{H}$ NMR (400 MHz, $\left.\mathrm{CDCl}_{3}\right)$.

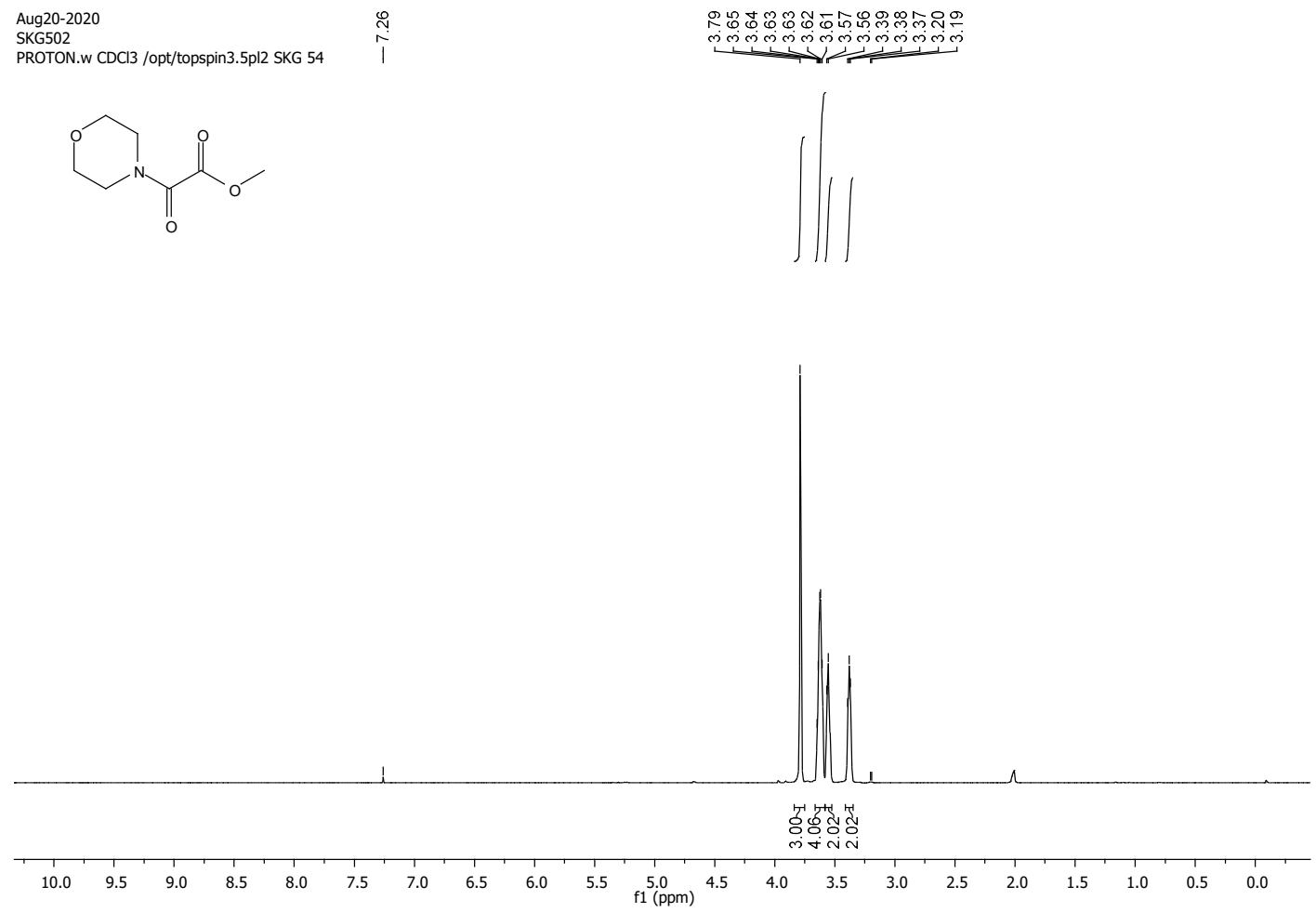

${ }^{13} \mathrm{C}$ NMR (101 MHz, $\left.\mathrm{CDCl}_{3}\right)$.

Aug11-2020

SKG502

C13APTlong.w CDCl3

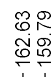

11 $\underbrace{\text { VI }}$

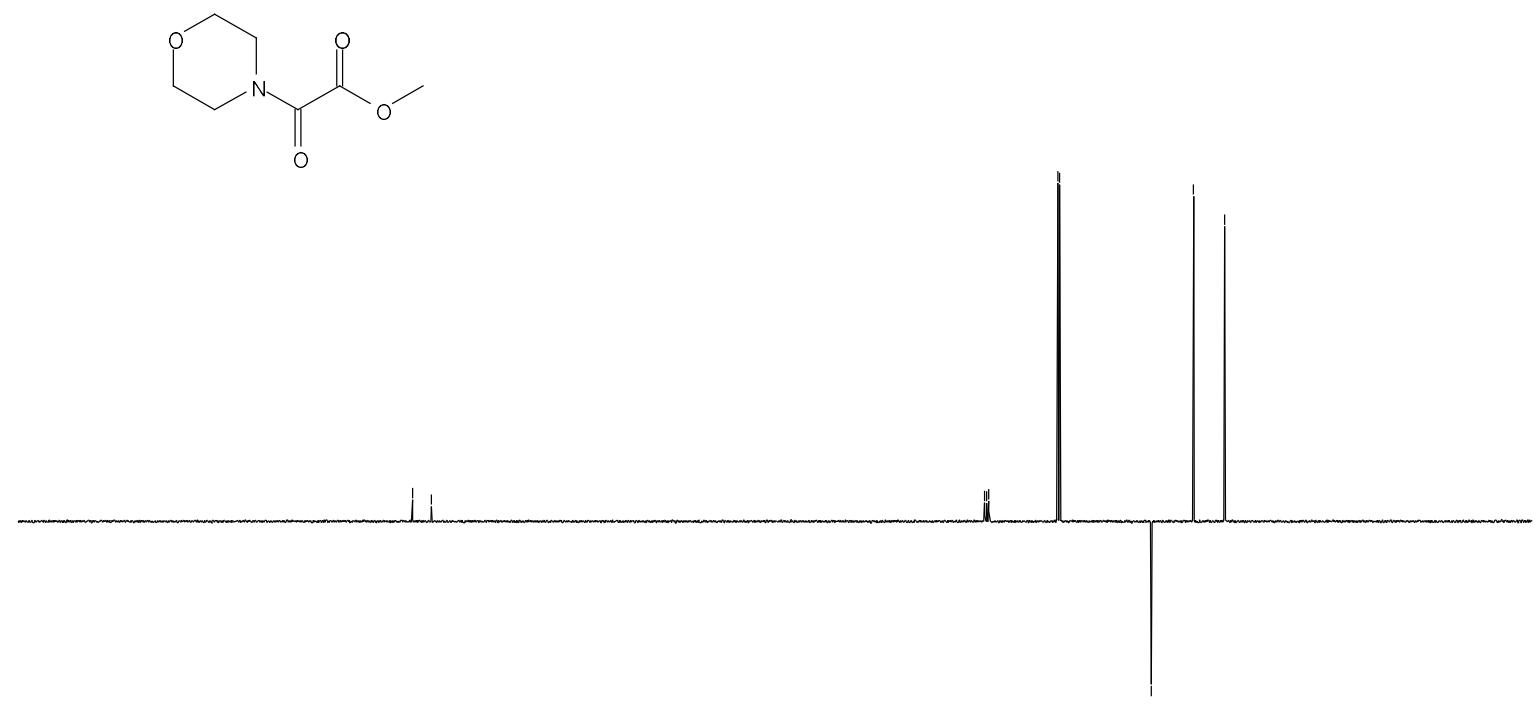

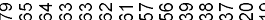

$\begin{array}{llllllllllllllllllllllllllll} & 1 \\ 220 & 210 & 200 & 190 & 180 & 170 & 160 & 150 & 140 & 130 & 120 & 110 & 100 & 90 & 80 & 70 & 60 & 50 & 40 & 30 & 20 & 10 & 0\end{array}$ 
Methyl 2-(methyl(phenyl)amino)-2-oxoacetate.<smiles>COC(=O)C(=O)N(C)c1ccccc1</smiles>

This compound is known however it has not been characterised previously. This compound was prepared by following the general procedure A using methyl 2-chloro-2-oxoacetate (2.00 g, $1.50 \mathrm{~mL}, 16.4 \mathrm{mmol}, 1.05 \mathrm{eq})$, N-methyl aniline (1.65 g, $15.6 \mathrm{mmol}, 1.0 \mathrm{eq})$ and triethylamine $(1.89 \mathrm{~g}, 2.60 \mathrm{~mL}, 18.7 \mathrm{mmol}, 12 \mathrm{eq})$ in dichloromethane $(40 \mathrm{~mL})$ to give the product as a clear liquid (2.48 g, $12.9 \mathrm{mmol}, 83 \%)$.

TLC: $\mathrm{R}_{\mathrm{f}}$ ca 0.3 (8:2, Hexane: EtOAc), strong UV active;

HRMS (ESI) m/z: [M+Na] $]^{+}$Calcd for $\mathrm{C}_{10} \mathrm{H}_{11} \mathrm{NO}_{3} \mathrm{Na} 216.0631$; Found 216.0638 (error -3.1 ppm);

${ }^{1} \mathrm{H}$ NMR (400 MHz, $\left.\mathrm{CDCl}_{3}\right) \delta 7.42-7.33$ (3H, m, ArH), $7.23(2 \mathrm{H}, \mathrm{d}, J=7.4 \mathrm{~Hz}, \mathrm{ArH}), 3.56$ $\left(3 \mathrm{H}, \mathrm{s}, \mathrm{OCH}_{3}\right), 3.36\left(3 \mathrm{H}, \mathrm{s}, \mathrm{NCH}_{3}\right)$;

${ }^{13} \mathrm{C}$ NMR $\left(101 \mathrm{MHz}, \mathrm{CDCl}_{3}\right) \delta 163.1,161.7,141.5,129.7,128.5,126.3,52.3,36.3$;

$m / z(\mathrm{ESI}) 216.0\left[(\mathrm{M}+\mathrm{Na})^{+}, 100 \%\right]$.

1) Bai, Q.-F.; Jin, C.; He, J.-Y.; Feng, G. Carbamoyl Radicals via Photoredox Decarboxylation of Oxamic Acids in Aqueous Media: Access to 3,4-Dihydroquinolin-2(1H)ones, Org. Lett. 2018 20, 2172-2175.

2) Fier, P. S.; Maloney, K. M. Reagent Design and Ligand Evolution for the Development of a Mild Copper-Catalyzed Hydroxylation Reaction, Org. Lett. 2017, 19, 3033-3036. 
Methyl 2-(methyl(phenyl)amino)-2-oxoacetate.

${ }^{1} \mathrm{H}$ NMR (400 MHz, $\left.\mathrm{CDCl}_{3}\right)$.

Aug20-2020

SKG506a

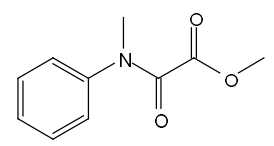

ᄀำ

rivininis

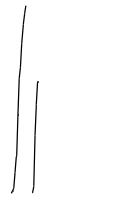

il

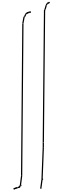

8
$i$
$i$

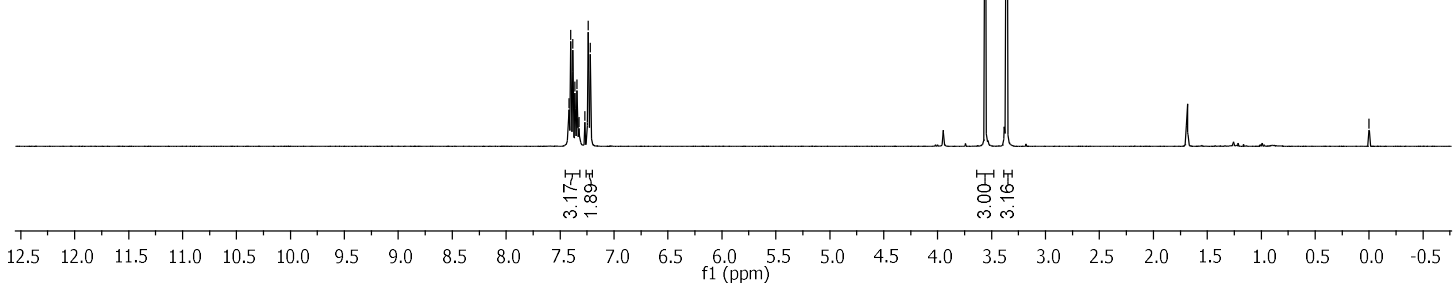

${ }^{13} \mathrm{C}$ NMR $\left(101 \mathrm{MHz}, \mathrm{CDCl}_{3}\right)$.

Aug14-2020

SKG506a

C13APTlong.w $\mathrm{CDCl} 3$

두우
ஸ் $\frac{1}{1}$
11

$\frac{\mathfrak{d}}{\mathfrak{d}}$

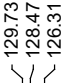

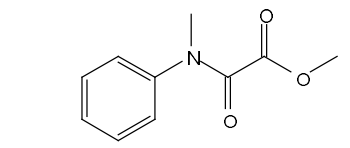

年

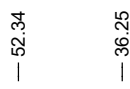

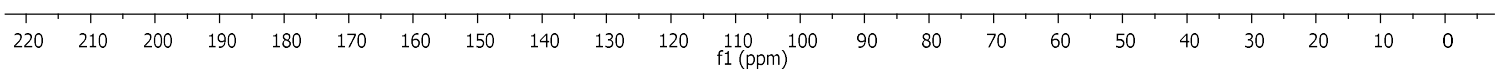

S22 
Methyl 2-(cyclopropylamino)-2-oxoacetate.<smiles>COC(=O)C(=O)NC1CC1</smiles>

This compound has been reported and was prepared by following the general procedure $\mathbf{A}$ using methyl 2-chloro-2-oxoacetate $(2.00 \mathrm{~g}, 1.50 \mathrm{~mL}, 16.4 \mathrm{mmol}, 1.05 \mathrm{eq})$, cyclopropanamine $(0.880 \mathrm{~g}, 15.6 \mathrm{mmol}, 1.0 \mathrm{eq})$ and triethylamine $(1.89 \mathrm{~g}, 2.60 \mathrm{~mL}, 18.7$ mmol, $1.2 \mathrm{eq})$ in dichloromethane $(40 \mathrm{~mL})$ to give the product as a white solid $(1.32 \mathrm{~g}, 9.23$ mmol, 59\%).

TLC: $\mathrm{R}_{\mathrm{f}}$ ca 0.3 (6:4, Hexane: EtOAc), strong UV active;

${ }^{1} \mathrm{H}$ NMR (400 MHz, DMSO-d $d_{6} \delta 8.94(1 \mathrm{H}, \mathrm{s}, \mathrm{CONH}), 3.75\left(3 \mathrm{H}, \mathrm{s}, \mathrm{OCH}_{3}\right), 2.77-2.70(1 \mathrm{H}$, $\mathrm{m}, \mathrm{CH}), 0.68-0.55\left(4 \mathrm{H}, \mathrm{m}, \mathrm{CH}_{2}\right)$;

${ }^{13} \mathrm{C}$ NMR (101 MHz, DMSO-d6) $\delta 161.1,158.0,52.7,22.8,5.47$;

$m / z(\mathrm{ESI}) 166.0\left[(\mathrm{M}+\mathrm{Na})^{+}, 80 \%\right], 309.2\left[(2 \mathrm{M}+\mathrm{Na})^{+}, 100 \%\right]$.

The data matches the reported data.

De Francesco, R; Donnici, L.; Guidotti, L.; Iannacone, M.; Di Fabio, R.; Summa, V.; Prandi, A.; Randazzo, P.; Ivanova Bencheva, L.; De Matteo, M.; Ferrante, L.; Gornati, D.; Grillo, A. Oxalamido-substituted tricyclic inhibitors of hepatitis B virus. WO2020/234483, 2020, A1. 
Methyl 2-(cyclopropylamino)-2-oxoacetate.

${ }^{1} \mathrm{H}$ NMR (400 MHz, DMSO-d 6 ).

Apr12-2021
SKG527
PROTON.w DMSO /opt/topspin3.5pl2 SKG 35 I

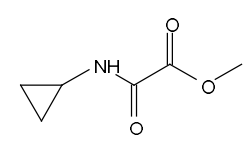

$\int$
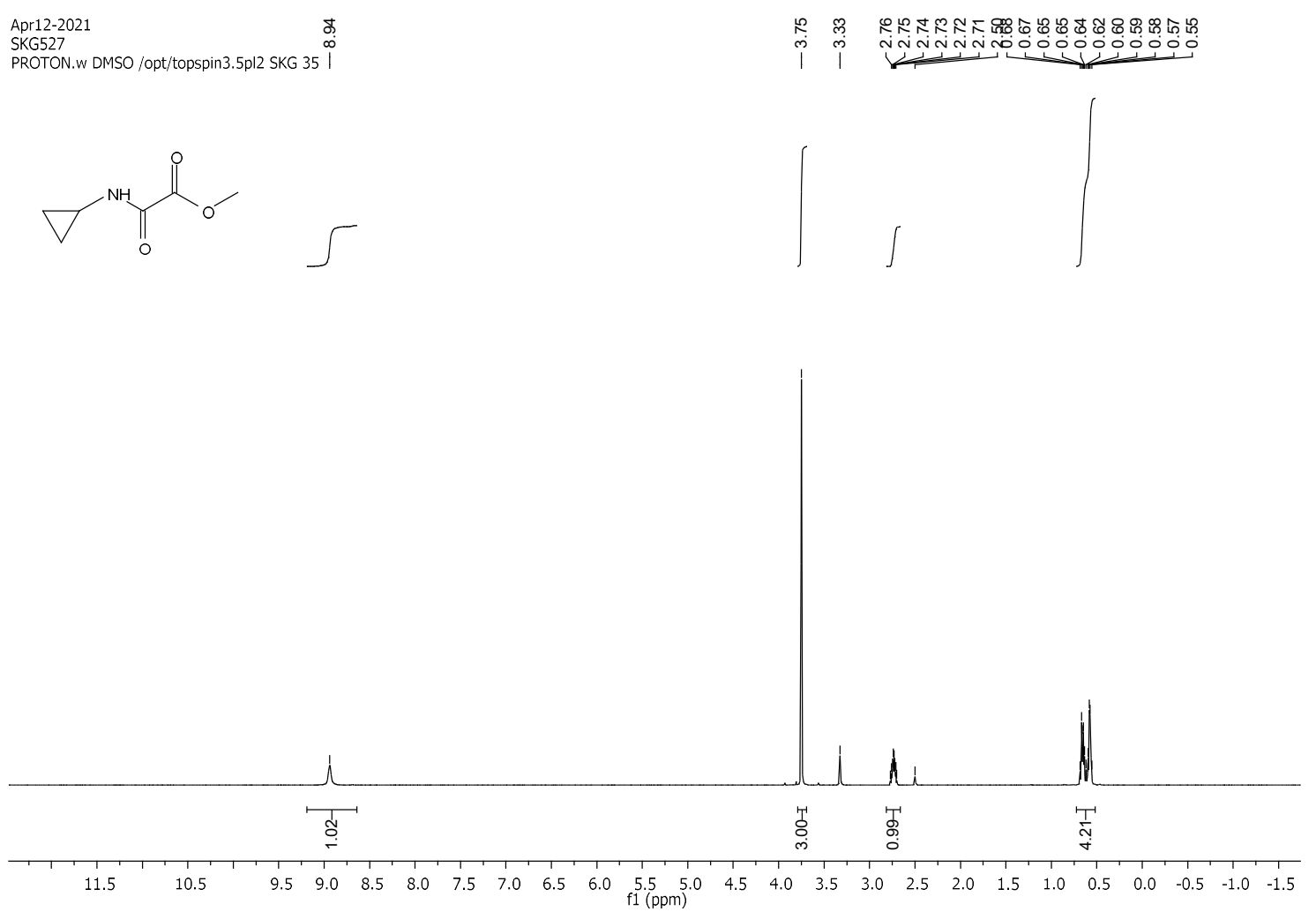

${ }^{13} \mathrm{C}$ NMR (101 MHz, DMSO-d 6 ).

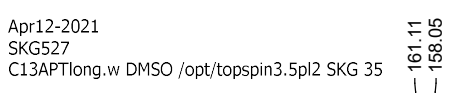

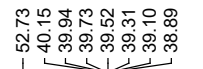

先
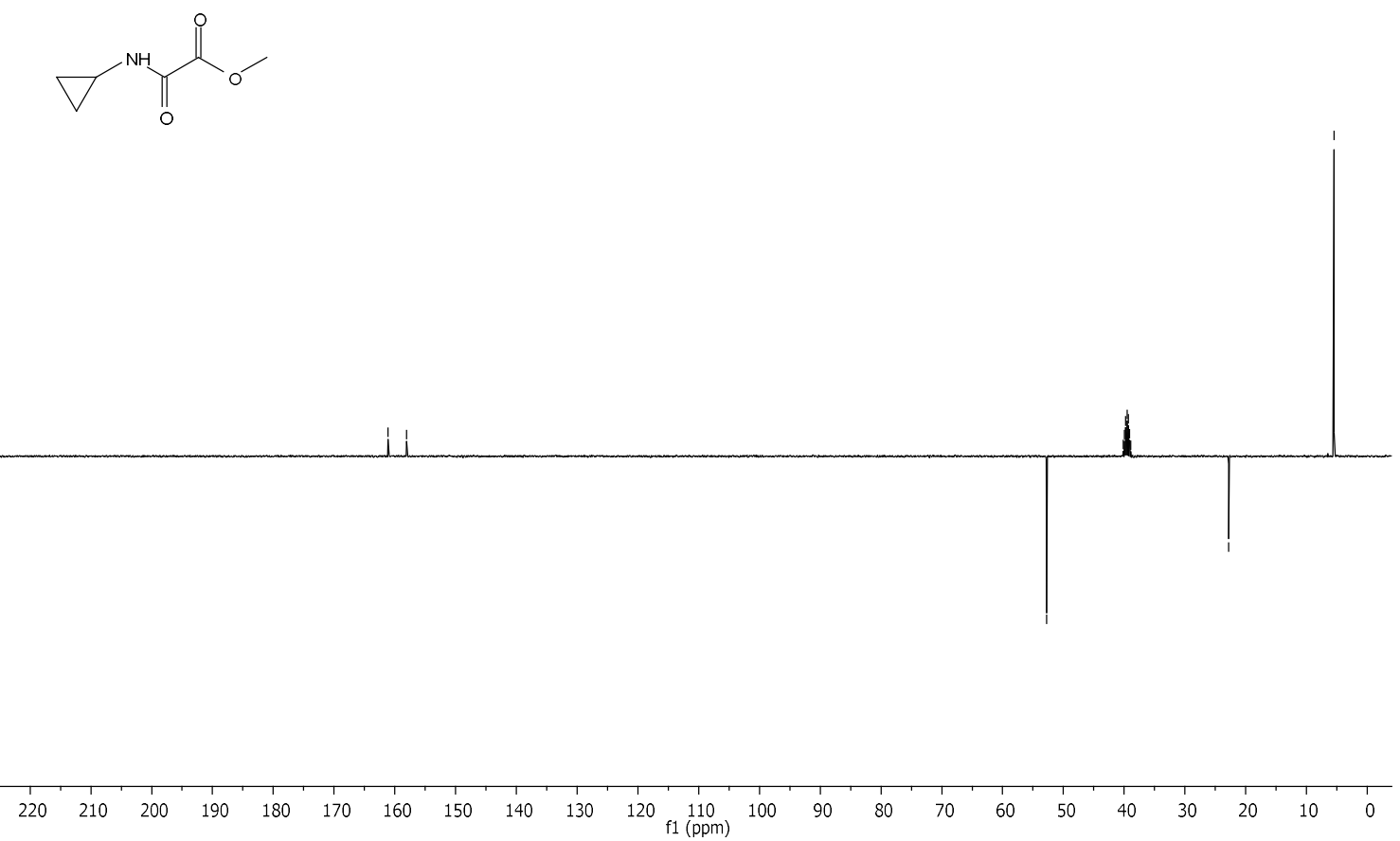

S24 


\section{Methyl 2-((4-chlorophenyl)amino)-2-oxoacetate.}<smiles>COC(=O)C(=O)Nc1ccc(Cl)cc1</smiles>

This compound has been reported and was prepared by following the general procedure $\mathbf{A}$ using methyl 2-chloro-2-oxoacetate (1.00 g, $0.752 \mathrm{~mL}, 8.13 \mathrm{mmol}, 1.05 \mathrm{eq})$, 4-chloro aniline $(0.988 \mathrm{~g}, 7.72 \mathrm{mmol}, 1.0 \mathrm{eq})$ and triethylamine $(0.946 \mathrm{~g}, 1.30 \mathrm{~mL}, 9.35 \mathrm{mmol}, 1.2 \mathrm{eq})$ in dichloromethane $(20 \mathrm{~mL})$ to give the product as an off white solid $(1.52 \mathrm{~g}, 7.13 \mathrm{mmol}, 92 \%)$. TLC: $\mathrm{R}_{\mathrm{f}}$ ca 0.3 (8:2, Hexane: EtOAc), strong UV active;

${ }^{1} \mathrm{H}$ NMR $\left(400 \mathrm{MHz}, \mathrm{CDCl}_{3}\right) \delta 8.87(1 \mathrm{H}, \mathrm{s}, \mathrm{CONH}), 7.60(2 \mathrm{H}, \mathrm{d}, J=8.8 \mathrm{~Hz}, \mathrm{ArH}), 7.35(2 \mathrm{H}$, d, $J=8.8 \mathrm{~Hz}, \mathrm{ArH}), 3.98\left(3 \mathrm{H}, \mathrm{s}, \mathrm{OCH}_{3}\right)$;

${ }^{13} \mathrm{C}$ NMR (101 MHz, $\left.\mathrm{CDCl}_{3}\right) \delta 161.4,153.7,134.9,130.9,129.5,121.2,54.3$;

$m / z(\mathrm{ESI}) 449.2\left[\left(2 \mathrm{M}\left({ }^{35} \mathrm{Cl}\right)+\mathrm{Na}\right)^{+}, 100 \%\right], 451.2\left[\left(2 \mathrm{M}\left({ }^{37} \mathrm{Cl}\right)+\mathrm{Na}\right)^{+}, 60 \%\right]$.

The data matches the reported data.

Zhang, Z.; Gao, X.; Yu, H.; Zhang, G.; Liu, J. Copper-Catalysed (Diacetoxyiodo)benzenePromoted Aerobic Esterification Reaction: Synthesis of Oxamates from Acetoacetamides, Adv. Synth. Catal. 2018, 360, 3406-3411. 
Methyl 2-((4-chlorophenyl)amino)-2-oxoacetate.

${ }^{1} \mathrm{H}$ NMR $\left(400 \mathrm{MHz}, \mathrm{CDCl}_{3}\right)$.

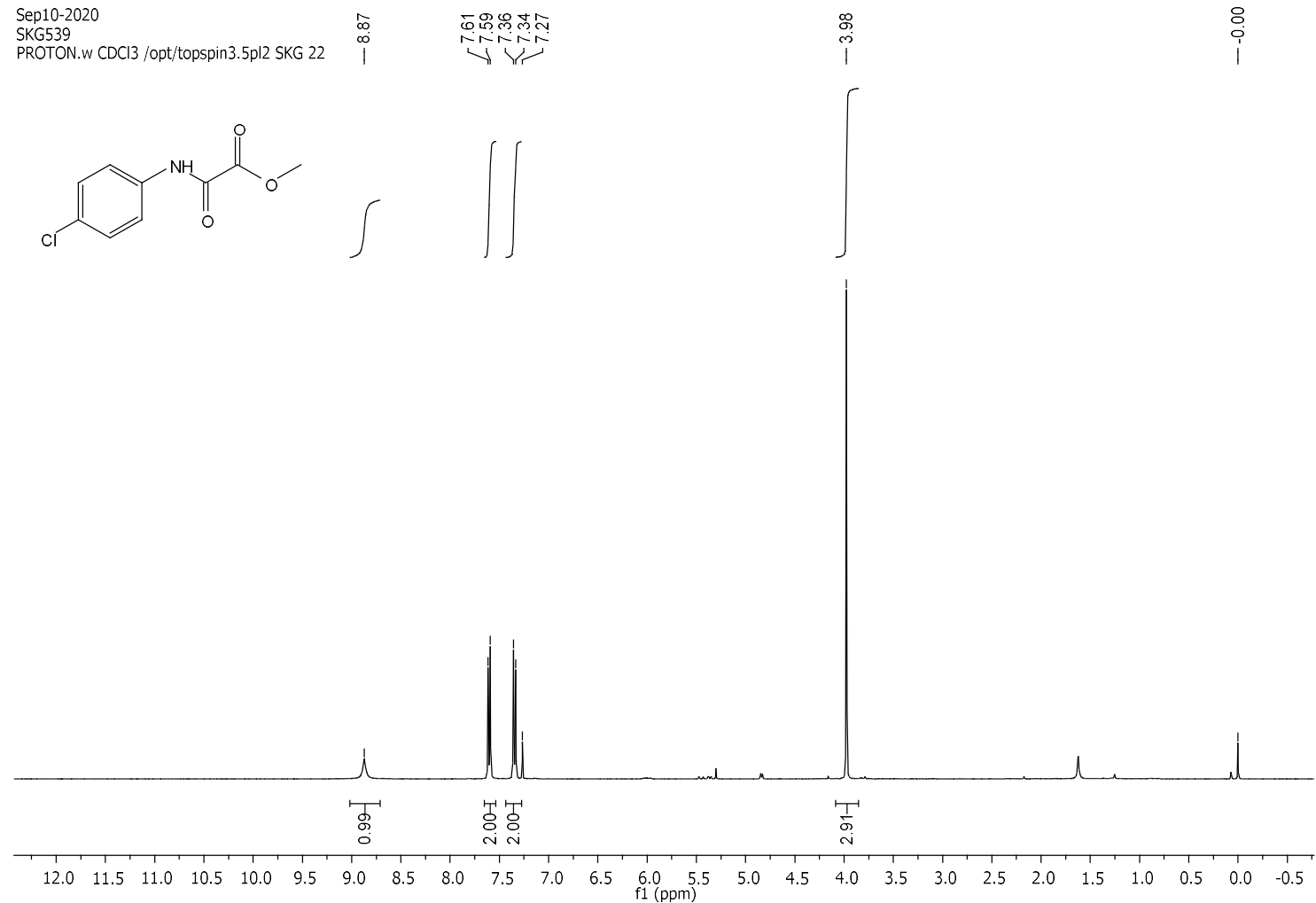

${ }^{13} \mathrm{C}$ NMR (101 MHz, $\left.\mathrm{CDCl}_{3}\right)$.
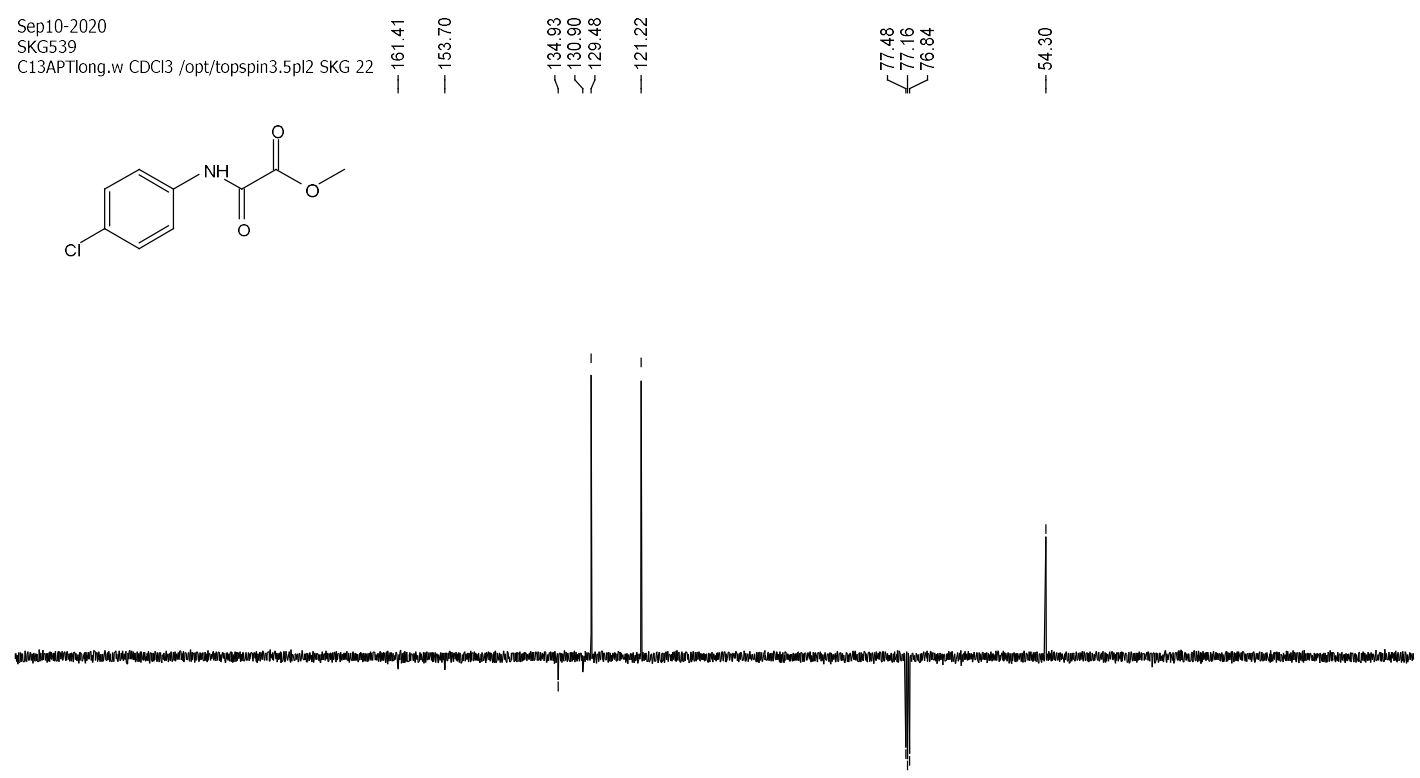

$\begin{array}{llllllllllllllllllllllll} & 220 & 210 & 200 & 190 & 180 & 170 & 160 & 150 & 140 & 130 & 120 & 110 & 100 & 90 & 80 & 70 & 60 & 50 & 40 & 30 & 20 & 10 & 0\end{array}$ 
Methyl 2-(methoxy(methyl)amino)-2-oxoacetate.<smiles>COC(=O)C(=O)N(C)OC</smiles>

This compound is reported and was prepared by following the general procedure A using methyl 2-chloro-2-oxoacetate $(2.00 \mathrm{~g}, \quad 1.50 \mathrm{~mL}, 16.4 \mathrm{mmol}, 1.0 \quad$ eq $), \quad \mathrm{N}, \mathrm{O}-$ dimethylhydroxylamine hydrochloride $(2.24 \mathrm{~g}, 23.2 \mathrm{mmol}, 1.5 \mathrm{eq})$ and triethylamine $(3.28 \mathrm{~g}$, $4.51 \mathrm{~mL}, 32.5 \mathrm{mmol}, 2.0 \mathrm{eq})$ in dichloromethane $(40 \mathrm{~mL})$ to give the product as a clear liquid (2.31 g, $15.7 \mathrm{mmol}, 97 \%)$.

TLC: $\mathrm{R}_{\mathrm{f}}$ ca 0.3 (8:2, Hexane: EtOAc), strong UV active;

${ }^{1} \mathrm{H}$ NMR (400 MHz, $\left.\mathrm{CDCl}_{3}\right) \delta 3.83\left(3 \mathrm{H}, \mathrm{s}, \mathrm{OCH}_{3}\right), 3.70\left(3 \mathrm{H}, \mathrm{s}, \mathrm{OCH}_{3}\right), 3.18\left(3 \mathrm{H}, \mathrm{s},-\mathrm{NCH}_{3}\right)$;

${ }^{13} \mathrm{C} \mathrm{NMR}\left(101 \mathrm{MHz}, \mathrm{CDCl}_{3}\right) \delta 162.8,161.8,62.3,52.6,31.4$;

$m / z(\mathrm{ESI}) 170.0\left[(\mathrm{M}+\mathrm{Na})^{+}, 100 \%\right], 317.2\left[(2 \mathrm{M}+\mathrm{Na})^{+}, 70 \%\right]$.

The data matches the reported data.

Honda, K; Ohkura, S.; Hayashi, Y.; Kawauchi, S.; Mikami, K., Cationic Chiral Pd-Catalyzed "Acetylenic" Diels-Alder Reaction: Computational Analysis of Reversal in Enantioselectivity. Chem - Asian J. 2018, 13, 2842 - 2846. 
Methyl 2-(methoxy(methyl)amino)-2-oxoacetate.

${ }^{1} \mathrm{H}$ NMR (400 MHz, $\left.\mathrm{CDCl}_{3}\right)$.

Sep15-2020
SKG545

PROTON.w CDCl3/opt/topspin3.5pl2 SKG 4<smiles>COC(=O)C(=O)N(C)OC</smiles>

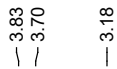

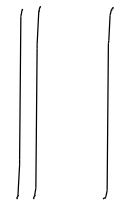

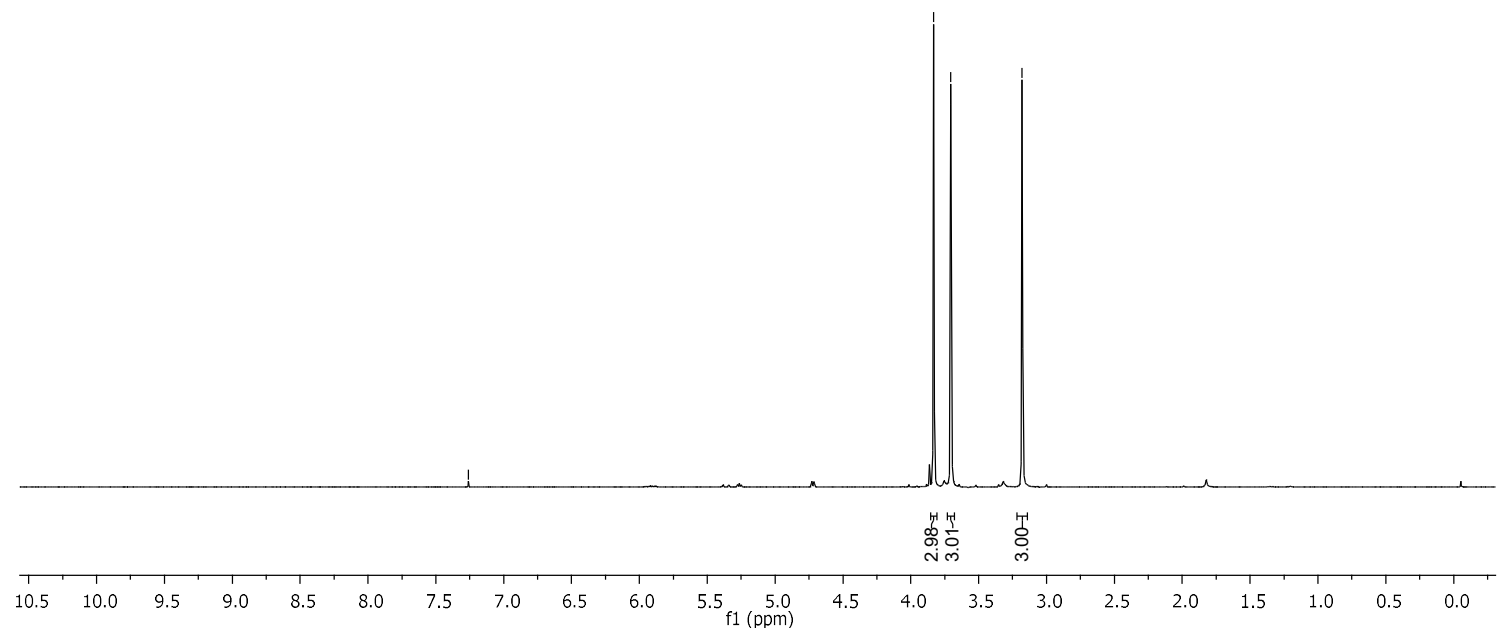

${ }^{13} \mathrm{C}$ NMR $\left(101 \mathrm{MHz}, \mathrm{CDCl}_{3}\right)$.

\section{Sep $15-2020$
SKG545}

SKG545

C13APTlong.w CDCl3

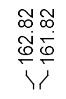<smiles>COC(=O)C(=O)N(C)OC</smiles>

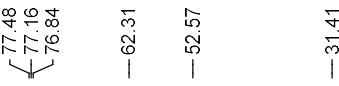

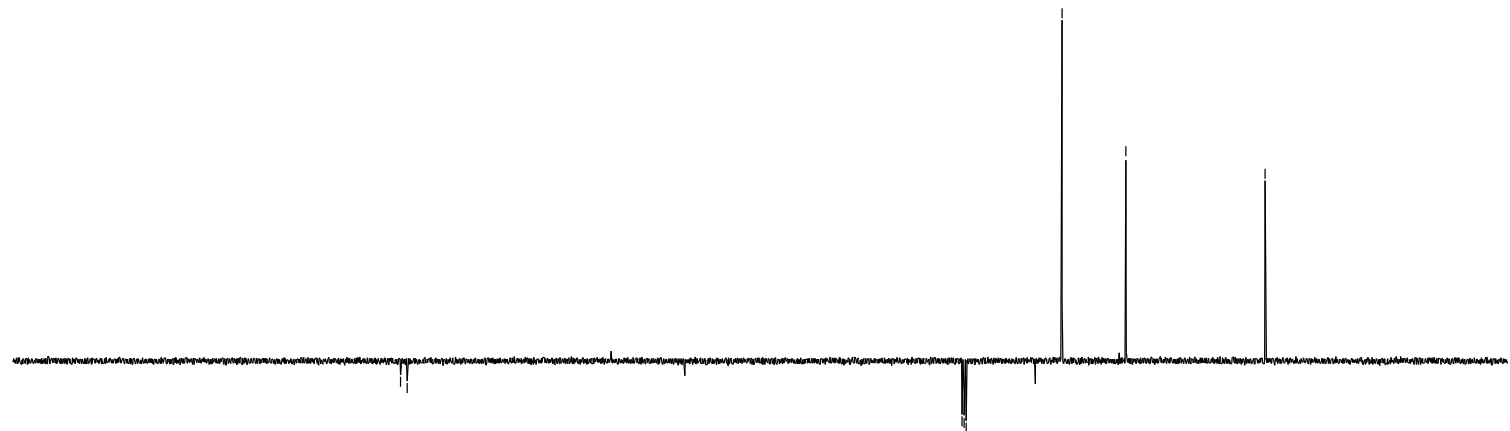

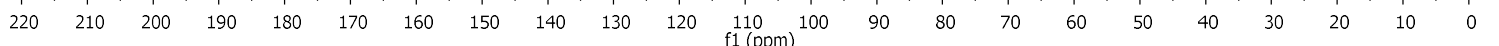




\section{Synthesis of Methyl 2-(phenylamino)-2-thioxoacetate.}

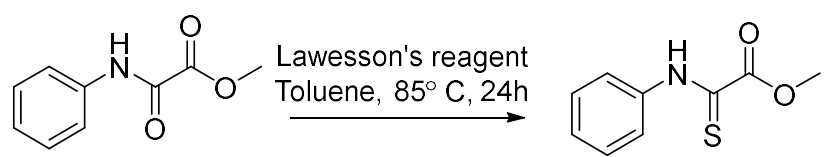

This compound is known however it has not been characterised previously. To a solution of methyl 2-oxo-2-(phenylamino) acetate $(0.500 \mathrm{~g}, 2.79 \mathrm{mmol}, 1.0 \mathrm{eq})$ in toluene $(20 \mathrm{~mL})$ was added Lawesson's reagent $(0.564 \mathrm{~g}, 1.39 \mathrm{mmol}, 0.50 \mathrm{eq})$ and the resulting reaction mixture was stirred at $85{ }^{\circ} \mathrm{C}$ for $24 \mathrm{~h}$. Once the reaction was complete (as assessed by TLC), the reaction mixture was quenched with saturated aqueous $\mathrm{NaHCO}_{3}$ solution $(25 \mathrm{~mL})$ and the resulting suspension was filtered. The obtained filtrate was further extracted with ethyl acetate $(3 \times 20 \mathrm{~mL})$. The combined organic layers were washed with brine $(50 \mathrm{~mL})$. The organic layer was dried over $\mathrm{MgSO}_{4}$, filtered and the filtrate was concentrated under reduced pressure to generate crude product which was further purified by column chromatography (5$10 \%$ EtOAc in petroleum ether) to afford the product as an orange solid $(0.452 \mathrm{~g}, 2.32 \mathrm{mmol}$, $83 \%)$.

TLC: $\mathrm{R}_{\mathrm{f}}$ ca 0.4 (7:3, hexane: EtOAc), UV-active and strong $\mathrm{KMnO}_{4}$;

MP: $84-86{ }^{0} \mathrm{C}$;

HRMS (ESI) m/z: $[\mathrm{M}+\mathrm{Na}]^{+}$Calcd for $\mathrm{C}_{9} \mathrm{H}_{9} \mathrm{NO}_{2} \mathrm{SNa} 218.0246$; Found 218.0251 (error -2.3 ppm);

$\mathrm{U}_{\max } 3261,1705,1524,1434,1287,1066,794,737,683,502 \mathrm{~cm}^{-1}$;

${ }^{1} \mathrm{H}$ NMR (400 MHz, $\left.\mathrm{CDCl}_{3}\right) \delta 10.57(1 \mathrm{H}, \mathrm{s}, \mathrm{NH}), 7.98(2 \mathrm{H}, \mathrm{d}, J=7.9 \mathrm{~Hz}, \mathrm{ArH}), 7.46-7.42$ (2H, m, ArH), $7.31(1 \mathrm{H}, \mathrm{t}, J=7.4 \mathrm{~Hz}, \mathrm{ArH}), 4.00\left(3 \mathrm{H}, \mathrm{s}, \mathrm{OCH}_{3}\right)$;

${ }^{13} \mathrm{C}$ NMR (101 MHz, $\left.\mathrm{CDCl}_{3}\right)$ 178.8, 160.3, 137.8, 129.3, 127.6, 122.1, 55.2;

$m / z(\mathrm{ESI}) 218.1\left[(\mathrm{M}+\mathrm{Na})^{+}, 100 \%\right]$. 
Methyl 2-(phenylamino)-2-thioxoacetate.

${ }^{1} \mathrm{H}$ NMR (400 MHz, $\left.\mathrm{CDCl}_{3}\right)$.

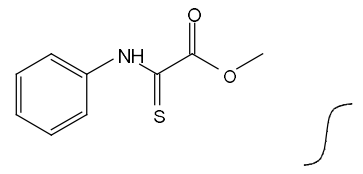

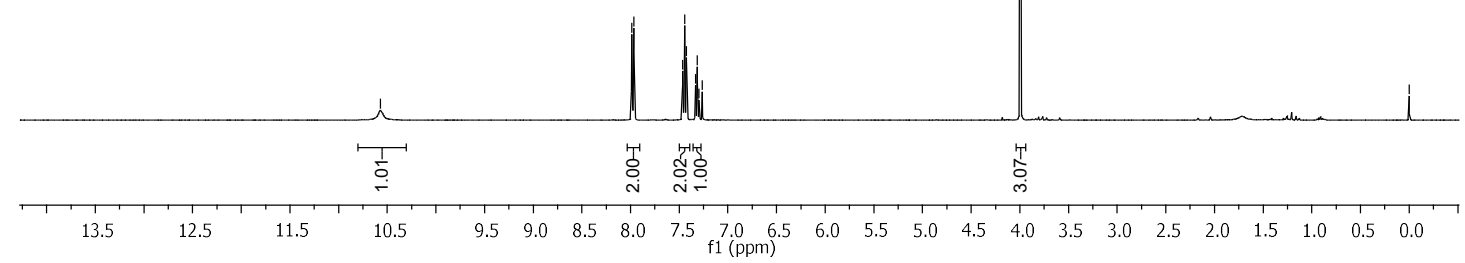

${ }^{13} \mathrm{C}$ NMR (101 MHz, $\left.\mathrm{CDCl}_{3}\right)$.

Oct02-2020
SKG572 US

SKG572 US
C13APTlong.w $\mathrm{CDCl} 3$

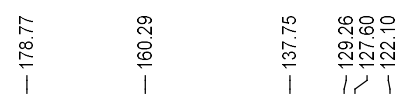<smiles>COC(=O)C(=O)Nc1ccccc1</smiles>

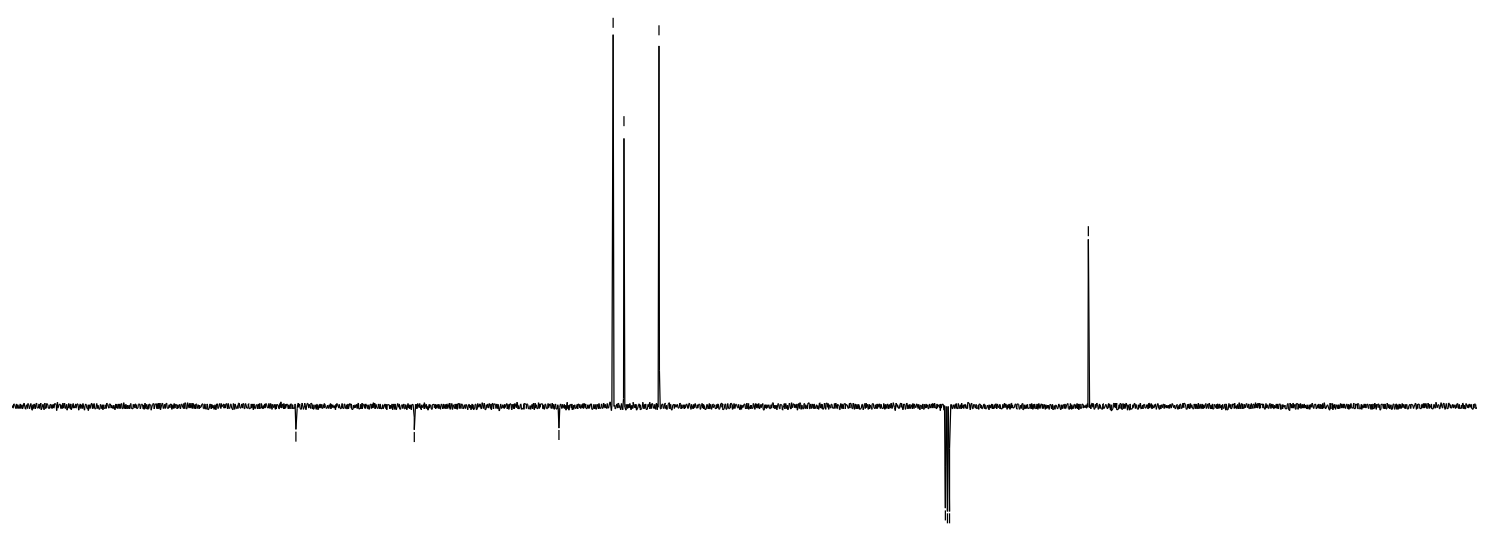

\begin{tabular}{llllllllllllllllllllllllll}
\hline & 220 & 210 & 200 & 190 & 180 & 170 & 160 & 150 & 140 & 130 & 120 & 110 & 100 & 90 & 80 & 70 & 60 & 50 & 40 & 30 & 20 & 10 & 0
\end{tabular} 
Methyl 2-((2,6-diisopropylphenyl)amino)-2-oxoacetate.<smiles>COC(=O)C(=O)Nc1c(C(C)C)cccc1C(C)C</smiles>

This compound has been reported and was prepared by following the general procedure $\mathbf{A}$ using methyl 2-chloro-2-oxoacetate $(2.00 \mathrm{~g}, 1.50 \mathrm{~mL}, 16.4 \mathrm{mmol}, 1.05 \mathrm{eq}), 2,6$ diisopropylaniline $(2.76 \mathrm{~g}, 15.6 \mathrm{mmol}, 1.0 \mathrm{eq})$ and triethylamine $(1.89 \mathrm{~g}, 2.60 \mathrm{~mL}, 18.7$ mmol, $1.2 \mathrm{eq})$ in dichloromethane $(40 \mathrm{~mL})$ to give the product as a white solid $(3.25 \mathrm{~g}, 12.6$ mmol, 81\%).

TLC: $\mathrm{R}_{\mathrm{f}}$ ca 0.3 (7:3, Hexane: EtOAc), strong UV active;

${ }^{1} \mathrm{H}$ NMR (400 MHz, $\left.\mathrm{CDCl}_{3}\right) \delta 8.38(1 \mathrm{H}, \mathrm{s}, \mathrm{CONH}), 7.34(1 \mathrm{H}, \mathrm{t}, J=7.7 \mathrm{~Hz}, \mathrm{ArH}), 7.21(2 \mathrm{H}$, d, $J=7.7 \mathrm{~Hz}, \mathrm{ArH}), 3.98\left(3 \mathrm{H}, \mathrm{s}, \mathrm{OCH}_{3}\right), 3.06-2.95(2 \mathrm{H}, \mathrm{m}, \mathrm{CH}$ of isopropyl group), 1.20 $\left(12 \mathrm{H}, \mathrm{d}, J=6.9 \mathrm{~Hz}, \mathrm{CH}_{3}\right.$ of isopropyl group);

${ }^{13} \mathrm{C} \mathrm{NMR}\left(101 \mathrm{MHz}, \mathrm{CDCl}_{3}\right) \delta 161.5,155.8,145.9,129.3,129.2,123.8,54.2,29.0,23.7$; $m / z(\mathrm{ESI}) 286.1\left[(\mathrm{M}+\mathrm{Na})^{+}, 100 \%\right]$.

The data matches the reported data.

Chinn, A. J.; Hwang, J.; Kim, B.; Parish, C. A.; Krska, S. W.; Miller, S. J. Application of High-Throughput Competition Experiments in the Development of Aspartate-Directed SiteSelective Modification of Tyrosine Residues in Peptides. J. Org. Chem. 2020, 85, 9424-9433. 
Methyl 2-((2,6-diisopropylphenyl)amino)-2-oxoacetate.

${ }^{1} \mathrm{H} \mathrm{NMR}\left(400 \mathrm{MHz}, \mathrm{CDCl}_{3}\right)$.

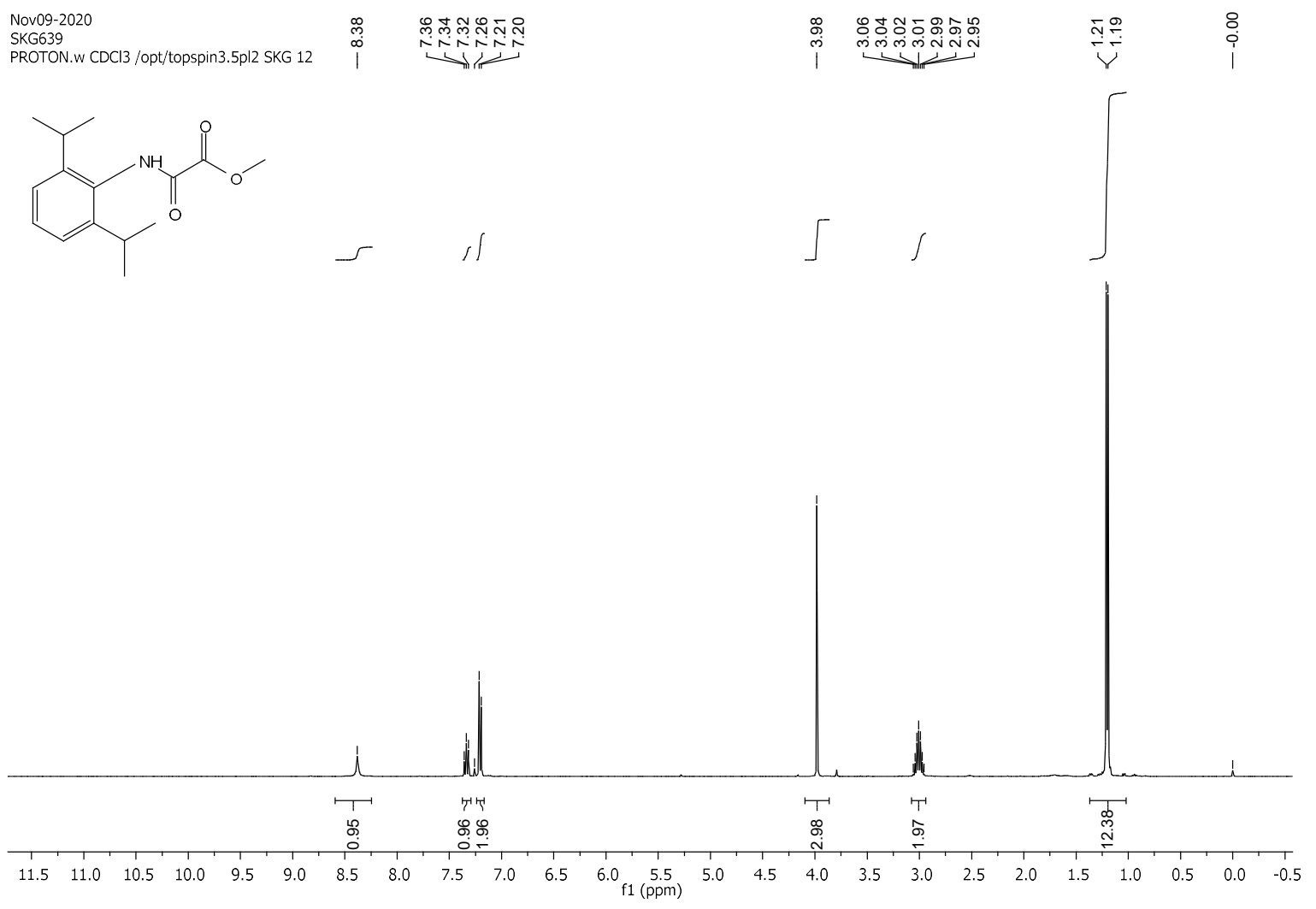

${ }^{13} \mathrm{C}$ NMR (101 MHz, $\left.\mathrm{CDCl}_{3}\right)$.

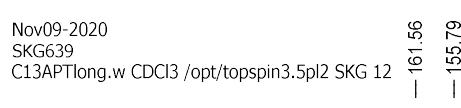

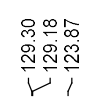

$\sqrt{1}$

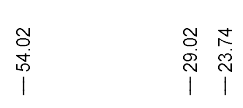
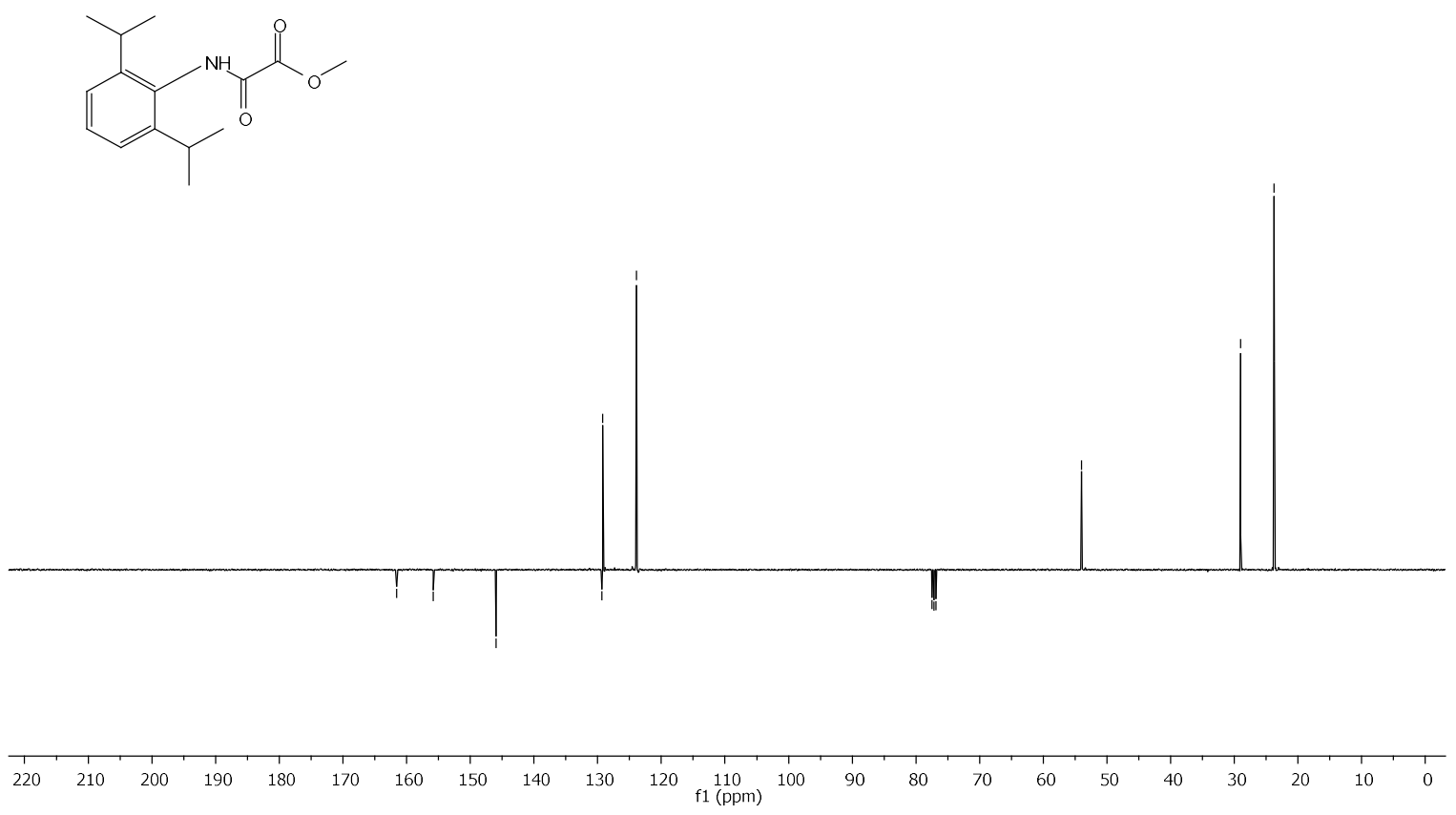

S32 
Methyl 2-(diphenylamino)-2-oxoacetate.<smiles>COC(=O)C(=O)N(c1ccccc1)c1ccccc1</smiles>

This compound has been reported and was prepared by following the general procedure $\mathbf{A}$ using methyl 2-chloro-2-oxoacetate $(1.00 \mathrm{~g}, 0.752 \mathrm{~mL}, 8.13 \mathrm{mmol}, 1.05 \mathrm{eq})$, diphenylamine $(1.30 \mathrm{~g}, 7.72 \mathrm{mmol}, 1.0 \mathrm{eq})$ and triethylamine $(0.946 \mathrm{~g}, 1.30 \mathrm{~mL}, 9.35 \mathrm{mmol}, 1.2 \mathrm{eq})$ in dichloromethane $(20 \mathrm{~mL})$ to give the product as a white solid $(1.83 \mathrm{~g}, 7.17 \mathrm{mmol}, 93 \%)$.

TLC: $\mathrm{R}_{\mathrm{f}}$ ca 0.3 (7:3, Hexane: EtOAc), strong UV active;

${ }^{1} \mathrm{H}$ NMR (400 MHz, $\left.\mathrm{CDCl}_{3}\right) \delta$ 7.40-7.24 (10H, m, ArH), $3.60\left(3 \mathrm{H}, \mathrm{s}, \mathrm{OCH}_{3}\right)$;

${ }^{13} \mathrm{C} \mathrm{NMR}\left(101 \mathrm{MHz}, \mathrm{CDCl}_{3}\right) \delta 163.1,161.2,140.7,140.2,129.8,129.3,128.7,127.9,127.2$, 125.6, 52.5;

$m / z(\mathrm{ESI}) 278.1\left[(\mathrm{M}+\mathrm{Na})^{+}, 100 \%\right]$.

The data matches the reported data.

Chinn, A. J.; Hwang, J.; Kim, B.; Parish, C. A.; Krska, S. W.; Miller, S. J. Application of High-Throughput Competition Experiments in the Development of Aspartate-Directed SiteSelective Modification of Tyrosine Residues in Peptides. J. Org. Chem. 2020, 85, 9424-9433. 
Methyl 2-(diphenylamino)-2-oxoacetate.

${ }^{1} \mathrm{H}$ NMR (400 MHz, $\mathrm{CDCl}_{3}$ ).

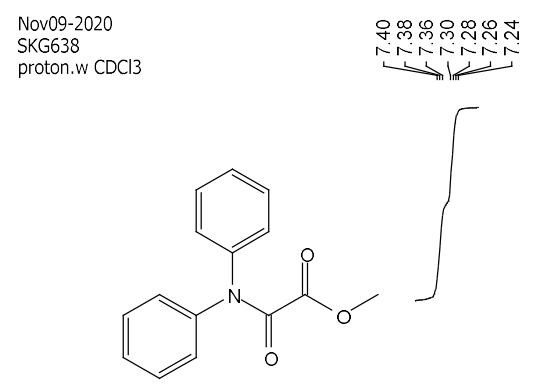

$\underset{8}{8}$

$\stackrel{8}{i}$

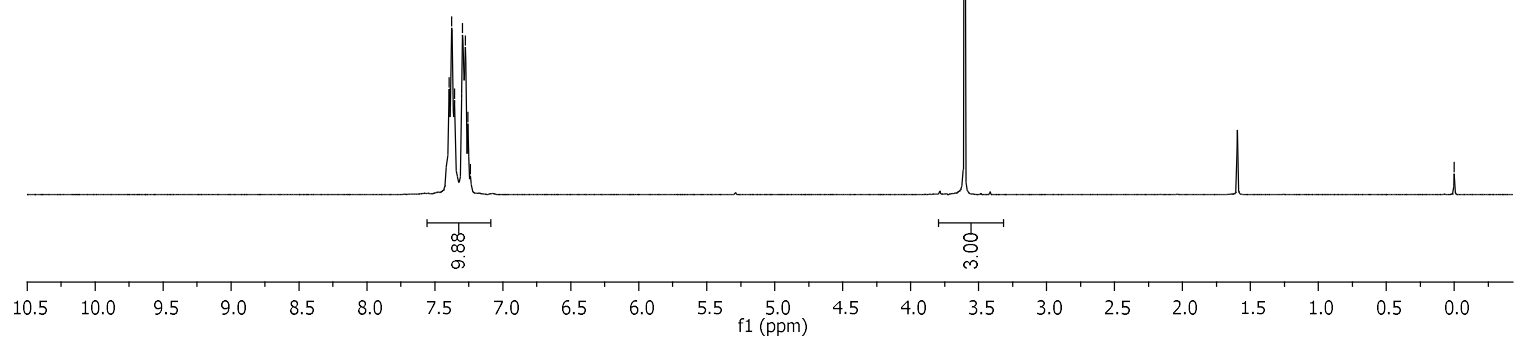

${ }^{13} \mathrm{C}$ NMR (101 MHz, $\left.\mathrm{CDCl}_{3}\right)$.

Nov09-2020

SKG638

C13APTlong.w $\mathrm{CDCl} 3$

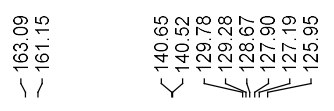

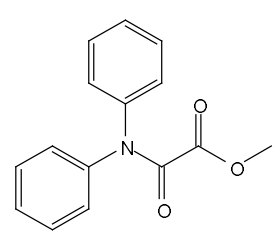

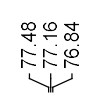

กัก
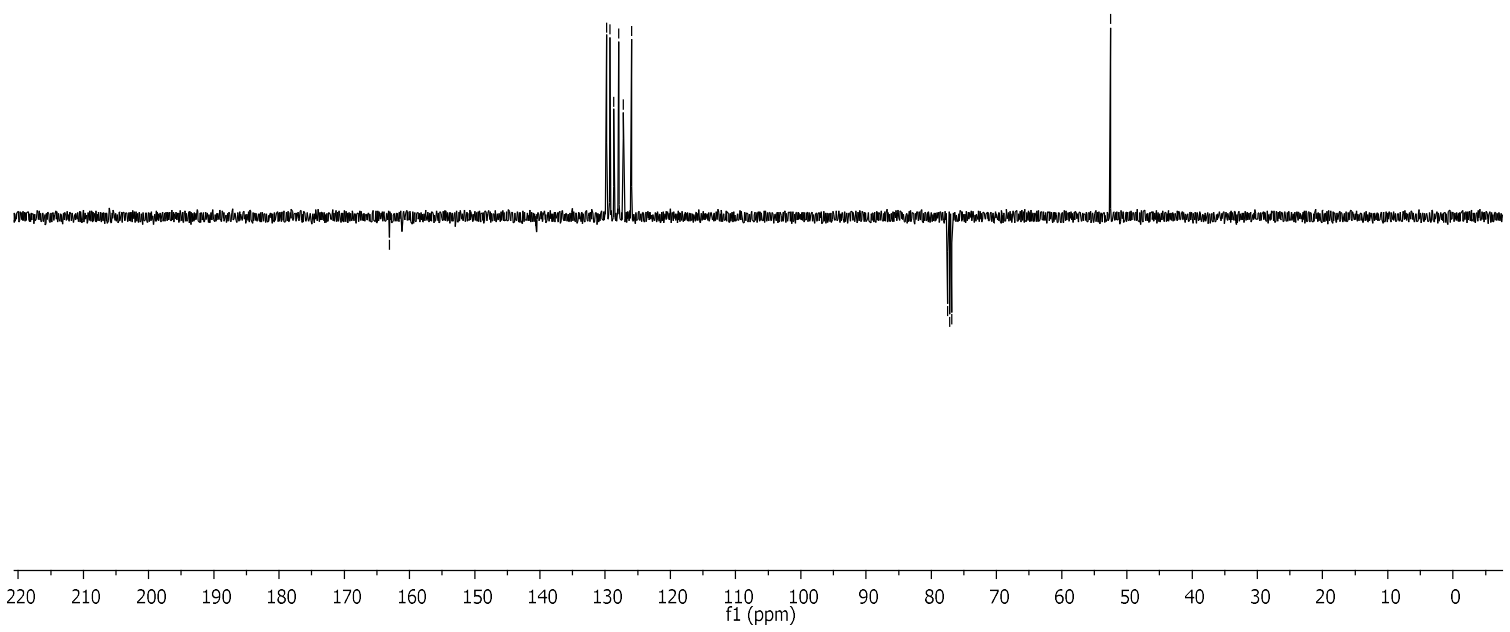

S34 
Methyl 2-(allyl(methyl)amino)-2-oxoacetate.<smiles>C=CCN(C)C(=O)C(=O)OC</smiles>

This compound is known however it has not been characterised previously. This compound was prepared by following the general procedure A using methyl 2-chloro-2-oxoacetate (1.00 g, $0.752 \mathrm{~mL}, 8.13 \mathrm{mmol}, 1.05 \mathrm{eq})$, N-methylprop-2-en-1-amine (0.548 g, $7.72 \mathrm{mmol}, 1.0 \mathrm{eq})$ and triethylamine $(0.946 \mathrm{~g}, 1.30 \mathrm{~mL}, 9.35 \mathrm{mmol}, 1.2 \mathrm{eq})$ in dichloromethane $(20 \mathrm{~mL})$ to give the product as a clear oil $(1.15 \mathrm{~g}, 7.29 \mathrm{mmol}, 94 \%)$.

TLC: $\mathrm{R}_{\mathrm{f}}$ ca 0.4 (7:3, Hexane: EtOAc), strong UV active;

HRMS (ESI) m/z: [M+Na] $]^{+}$Calcd for $\mathrm{C}_{7} \mathrm{H}_{11} \mathrm{NO}_{3} \mathrm{Na} 180.0631$; Found 180.0630 (error 0.6 ppm);

$U_{\max } 1738,1654,1409,1239,1203,1088,986,822 \mathrm{~cm}^{-1}$;

${ }^{1} \mathrm{H}$ NMR (400 MHz, $\mathrm{CDCl}_{3}$ ) (2 rotamers ca. 1:1) $\delta$ 5.83-5.72 $\left(1 \mathrm{H}, \mathrm{m}, \mathrm{CH}_{2}=\mathrm{C}_{\mathbf{H C H}}\right.$ ), 5.31$5.22\left(2 \mathrm{H}, \mathrm{m}, \mathrm{C}_{2}=\mathrm{CHCH}_{2}\right), 4.03-4.00\left(1 \mathrm{H}, \mathrm{m}, \mathrm{CH}_{2}=\mathrm{CHCH}_{2}\right) \quad 3.90-3.86 \quad(4 \mathrm{H}, \mathrm{m}$, $\left.\mathrm{CH}_{2}=\mathrm{CHCH}_{2}+\mathrm{OCH}_{3}\right), 2.97\left(1.5 \mathrm{H}, \mathrm{s},-\mathrm{NCH}_{3}\right), 2.95\left(1.5 \mathrm{H}, \mathrm{s}, \mathrm{NCH}_{3}\right)$;

${ }^{13} \mathrm{C}$ NMR $\left(101 \mathrm{MHz}, \mathrm{CDCl}_{3}\right) \delta 163.3,163.2,161.7,161.4,132.0 .131 .2,119.1,118.7,52.7$, 52.6, 49.1, 34.7, 31.2;

$m / z(\mathrm{ESI}) 180.0\left[(\mathrm{M}+\mathrm{Na})^{+}, 100 \%\right], 337.1\left[(2 \mathrm{M}+\mathrm{Na})^{+}, 80 \%\right]$. 
Methyl 2-(allyl(methyl)amino)-2-oxoacetate.

${ }^{1} \mathrm{H}$ NMR (400 MHz, $\mathrm{CDCl}_{3}$ ).

Nov19-2020

Chemist Shweta Gediya

PROTON.w CDCl3 /opt/topspin3.2 SKG 32<smiles>C=CCN(C)C(=O)C(=O)OC</smiles>

$\stackrel{\text { న }}{\sim}$

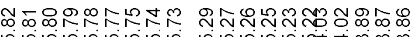
W

ปิ่

$\stackrel{8}{i}$

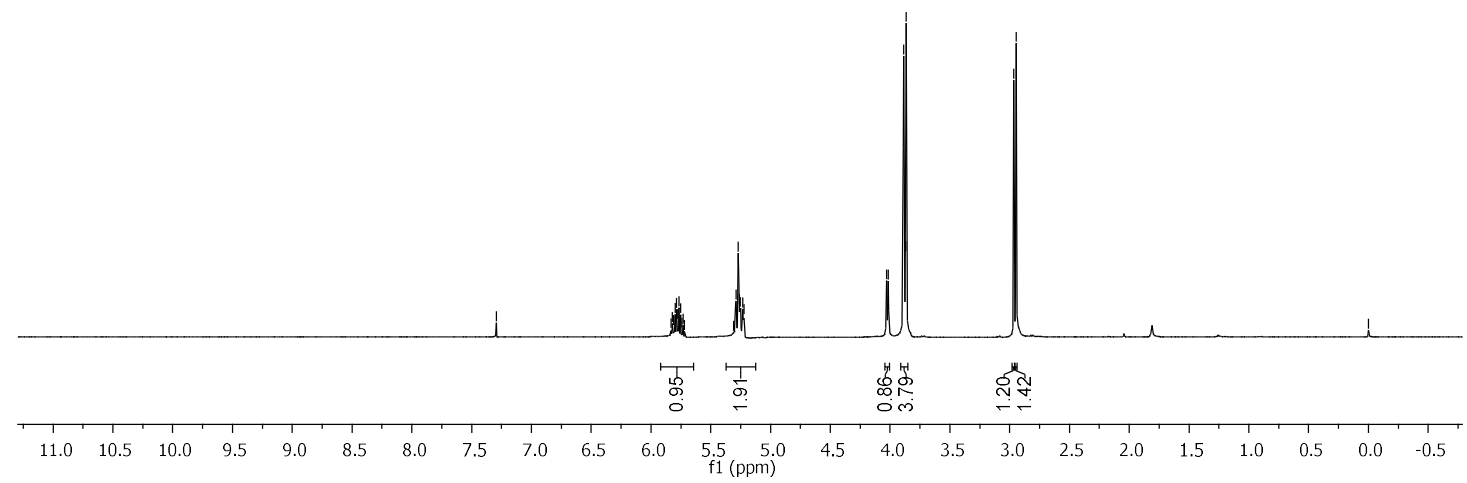

${ }^{13} \mathrm{C}$ NMR (101 MHz, $\left.\mathrm{CDCl}_{3}\right)$.

\begin{tabular}{|c|c|c|c|c|c|}
\hline $\begin{array}{l}\text { Nov19-2020 } \\
\text { Chemist Shweta Gediya } \\
\text { SKG646 } \\
\text { C13APT.w CDCl3 /opt/topspin 3.2 SKG } 32\end{array}$ & 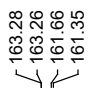 & 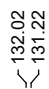 & 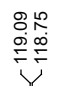 & 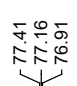 & 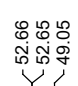 \\
\hline
\end{tabular}
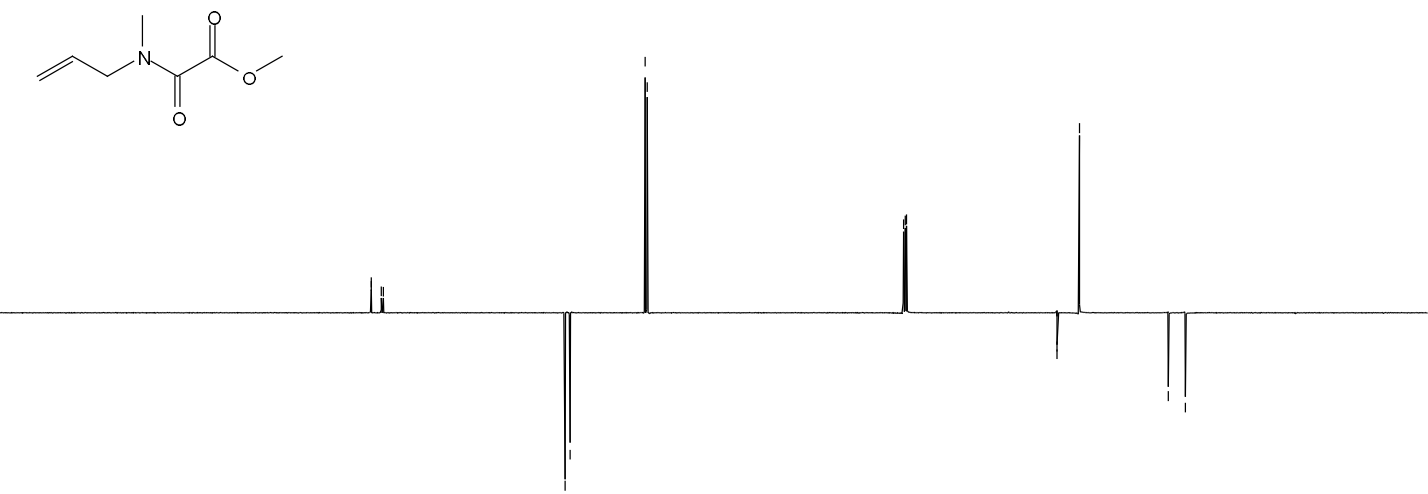

\begin{tabular}{lllllllllllllllllllllllllll}
\hline 220 & 210 & 200 & 190 & 180 & 170 & 160 & 150 & 140 & 130 & 120 & 110 & 100 & 90 & 80 & 70 & 60 & 50 & 40 & 30 & 20 & 10 & 0
\end{tabular} 
Methyl 2-(methyl(prop-2-yn-1-yl) amino)-2-oxoacetate.<smiles>C=CCN(C)C(=O)C(=O)OC</smiles>

This compound is known however it has not been characterised previously. This compound was prepared by following the general procedure A using methyl 2-chloro-2-oxoacetate (0.500 g, $0.376 \mathrm{~mL}, 4.07 \mathrm{mmol}, 1.05 \mathrm{eq})$, N-methylprop-2-yn-1-amine (0.266 g, 3.86 mmol, $1.0 \mathrm{eq})$ and triethylamine $(0.452 \mathrm{~g}, 0.62 \mathrm{~mL}, 4.47 \mathrm{mmol}, 1.2 \mathrm{eq})$ in dichloromethane $(10 \mathrm{~mL})$ to give the product as a dark brown oil $(0.350 \mathrm{~g}, 2.25 \mathrm{mmol}, 58 \%)$.

TLC: $\mathrm{R}_{\mathrm{f}}$ ca 0.3 (7:3, Hexane: EtOAc), strong UV active;

HRMS (ESI) m/z: $[\mathrm{M}+\mathrm{Na}]^{+}$Calcd for $\mathrm{C}_{7} \mathrm{H}_{9} \mathrm{NO}_{3} \mathrm{Na}$ 178.0475; Found 178.0474 (error 0.5 ppm);

$U_{\max } 3273,2956,1736,1653,1204,1100,686 \mathrm{~cm}^{-1}$;

${ }^{1} \mathrm{H}$ NMR $\left(500 \mathrm{MHz}, \mathrm{CDCl}_{3}\right)(2$ rotamers ca $3: 2) \delta 4.25\left(1.2 \mathrm{H}, \mathrm{d}, J=2.5 \mathrm{~Hz}, \mathrm{CH}_{2}\right), 4.15$ $\left(0.8 \mathrm{H}, \mathrm{d}, J=2.4 \mathrm{~Hz}, \mathrm{CH}_{2}\right), 3.90\left(1.8 \mathrm{H}, \mathrm{s}, \mathrm{OCH}_{3}\right), 3.88\left(1.2 \mathrm{H}, \mathrm{s}, \mathrm{OCH}_{3}\right), 3.10(1.8 \mathrm{H}, \mathrm{s}$, $\left.\mathrm{NCH}_{3}\right), 3.07\left(1.2 \mathrm{H}, \mathrm{s}, \mathrm{NCH}_{3}\right), 2.37(0.4 \mathrm{H}, \mathrm{t}, J=2.4 \mathrm{~Hz}, \mathrm{CH}), 2.29(0.6 \mathrm{H}, \mathrm{t}, J=2.5 \mathrm{~Hz}, \mathrm{CH})$;

${ }^{13} \mathrm{C} \mathrm{NMR}\left(126 \mathrm{MHz}, \mathrm{CDCl}_{3}\right) \delta 162.7,162.6,161.0,160.9,73.8,73.2,53.0,52.9,39.8,35.8$, $34.5,34.1,32.4$

$m / z(\mathrm{ESI}) 178.0\left[(\mathrm{M}+\mathrm{Na})^{+}, 100 \%\right]$. 
Methyl 2-(methyl(prop-2-yn-1-yl) amino)-2-oxoacetate.

${ }^{1} \mathrm{H}$ NMR (500 MHz, $\mathrm{CDCl}_{3}$ ).
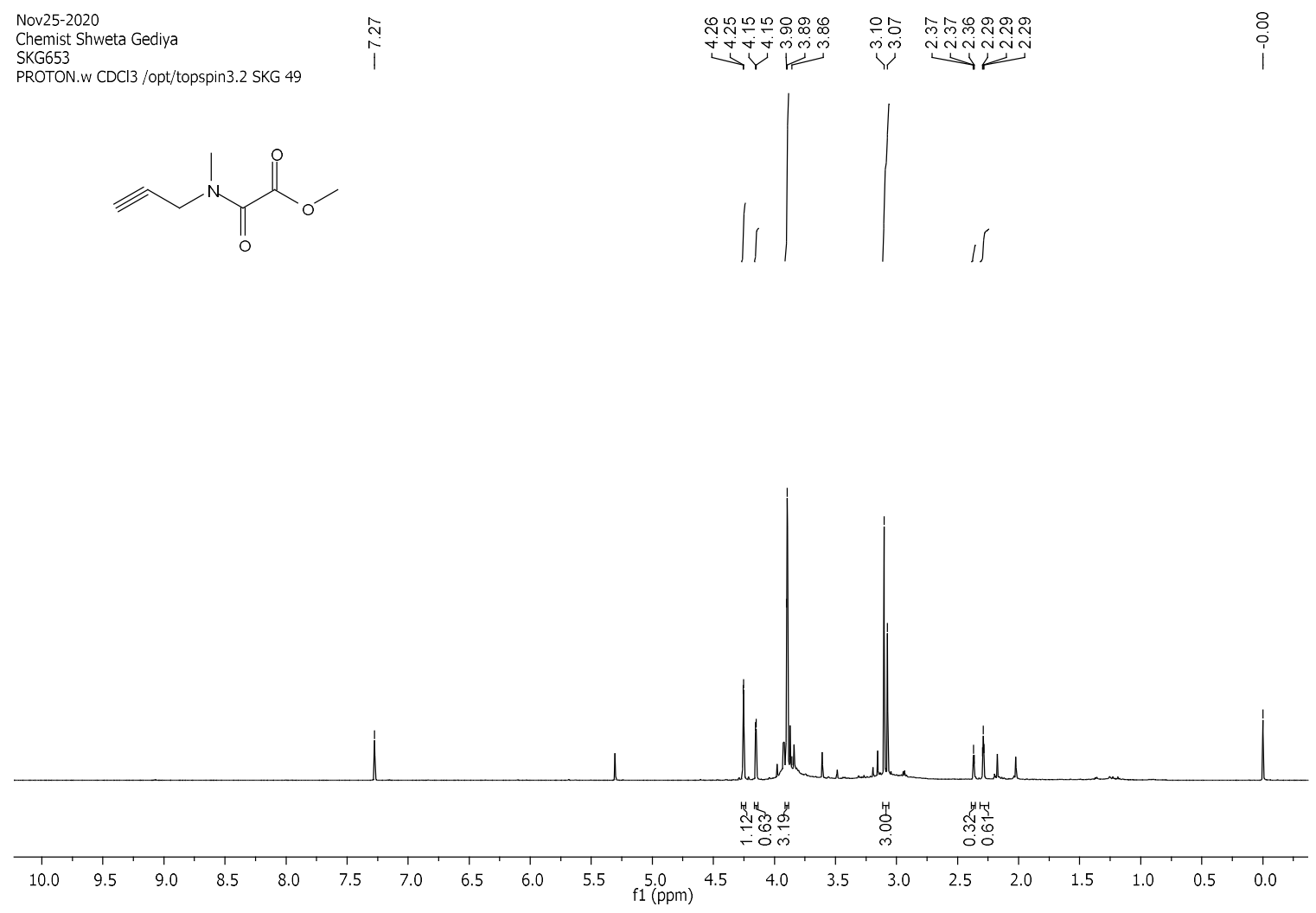

${ }^{13} \mathrm{C}$ NMR (126 MHz, $\left.\mathrm{CDCl}_{3}\right)$.

Nov25-2020

Chemist Shweta Gediya

C13APT.w CDCl3 /opt/topspin3.2 SKG 49

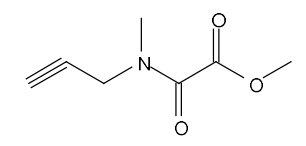

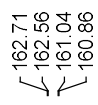

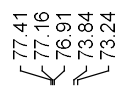

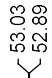

8
0
1

(1)

\begin{tabular}{rlllllllllllllllllllllllllllllllllll}
\hline & 230 & 220 & 210 & 200 & 190 & 180 & 170 & 160 & 150 & 140 & 130 & 120 & 110 & 100 & 90 & 80 & 70 & 60 & 50 & 40 & 30 & 20 & 10 & 0
\end{tabular}

S38 
Methyl 2-(cyclohexyl(methyl)amino)-2-oxoacetate.<smiles>COC(=O)C(=O)N(C)C1CCCCC1</smiles>

This compound is known however it has not been characterised previously. This compound was prepared by following the general procedure A using methyl 2-chloro-2-oxoacetate (1.00 g, $0.752 \mathrm{~mL}, 8.13 \mathrm{mmol}, 1.05 \mathrm{eq})$, N-methylcyclohexanamine (0.873 g, $7.72 \mathrm{mmol}, 1.0 \mathrm{eq})$ and triethylamine $(0.946 \mathrm{~g}, 1.30 \mathrm{~mL}, 9.35 \mathrm{mmol}, 1.2 \mathrm{eq})$ in dichloromethane $(20 \mathrm{~mL})$ to give the product as a yellow liquid $(1.25 \mathrm{~g}, 6.28 \mathrm{mmol}, 81 \%)$.

TLC: $\mathrm{R}_{\mathrm{f}}$ ca 0.4 (7:3, Hexane: EtOAc), strong UV active;

HRMS (ESI) m/z: $[\mathrm{M}+\mathrm{Na}]^{+}$Calcd for $\mathrm{C}_{10} \mathrm{H}_{17} \mathrm{NO}_{3} \mathrm{Na} 222.1101$; Found 222.1109 (error 3.6 ppm);

$U_{\max } 2932,1738,1647,1450,1412,1210,1142,1084 \mathrm{~cm}^{-1}$;

${ }^{1} \mathrm{H} \mathrm{NMR}\left(400 \mathrm{MHz}, \mathrm{CDCl}_{3}\right)$ (2 rotamers ca. 2:3) $\delta$ 4.33-4.26 $(0.4 \mathrm{H}, \mathrm{m}, \mathrm{CH}$ of cyclohexyl), $3.87\left(3 \mathrm{H}, \mathrm{s}, \mathrm{OCH}_{3}\right), 3.32-3.24\left(0.6 \mathrm{H}, \mathrm{m}, \mathrm{CH}\right.$ of cyclohexyl), $2.86\left(1.8 \mathrm{H}, \mathrm{s}, \mathrm{NCH}_{3}\right), 2.85$ $\left(1.2 \mathrm{H}, \mathrm{s}, \mathrm{NCH}_{3}\right), 1.92-1.03\left(10 \mathrm{H}, \mathrm{m}, \mathrm{CH}_{2}\right)$;

${ }^{13} \mathrm{C} \mathrm{NMR}\left(101 \mathrm{MHz}, \mathrm{CDCl}_{3}\right) \delta 163.9,163.7,161.8,161.4,58.1,52.5,52.4,30.6,29.9,29.3$, 26.4, 25.5, 25.4, 25.3, 25.1;

$m / z(\mathrm{ESI}) 222.1\left[(\mathrm{M}+\mathrm{Na})^{+}, 65 \%\right], 421.2\left[(2 \mathrm{M}+\mathrm{Na})^{+}, 100 \%\right]$. 
Methyl 2-(cyclohexyl(methyl)amino)-2-oxoacetate.

${ }^{1} \mathrm{H}$ NMR (400 MHz, $\left.\mathrm{CDCl}_{3}\right)$.

Dec01-2020

SKG663
PROTON.w CDCl3 /opt/topspin3.5pl2 SKG 35

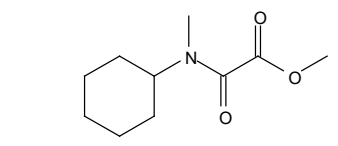

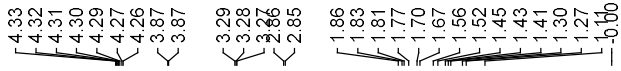

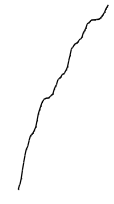

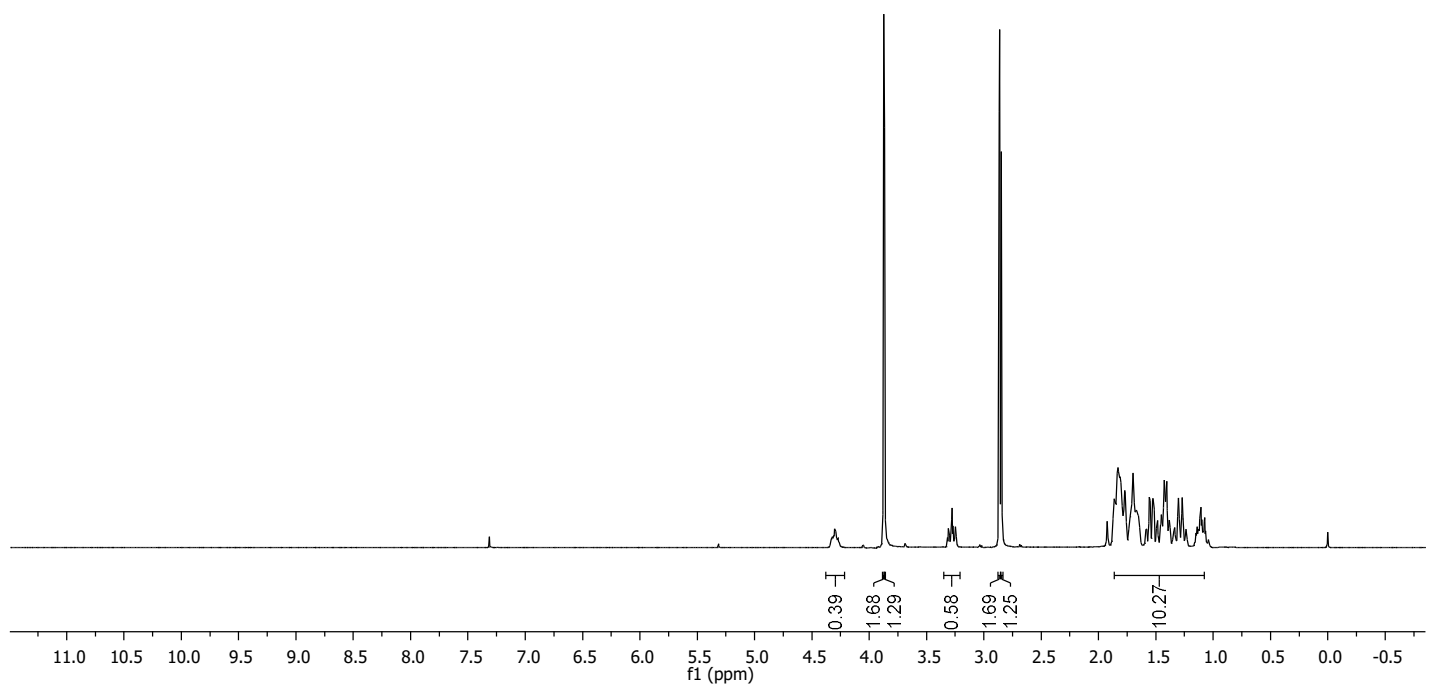

${ }^{13} \mathrm{C}$ NMR (101 MHz, $\left.\mathrm{CDCl}_{3}\right)$.

Dec01-2020

SKG663

C13APTlong.w CDCl3

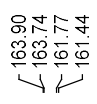

哭最

$\overbrace{\substack{0 \\ 0}}^{\infty}$

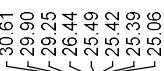
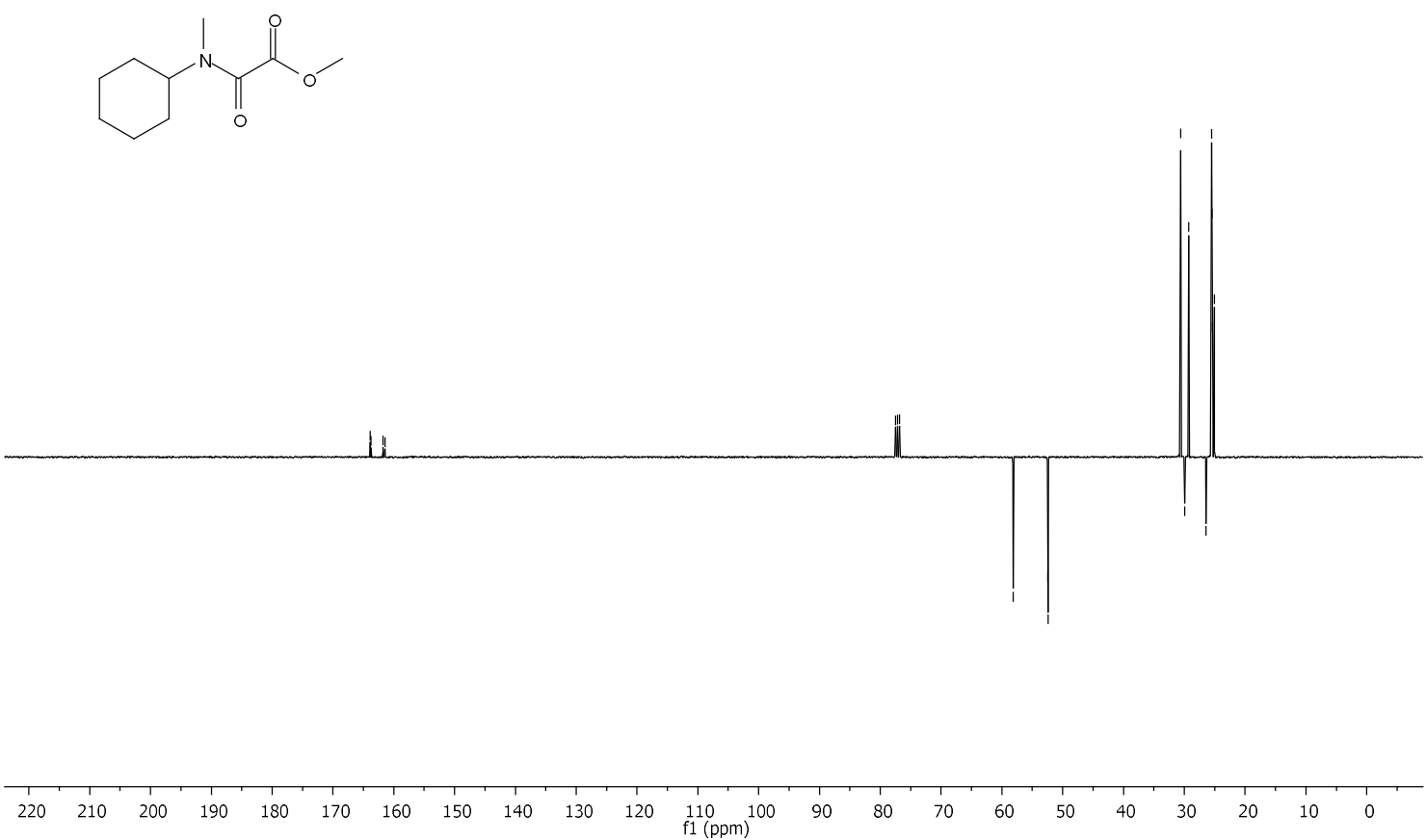

S40 


\section{Methyl 2-((2-chlorophenyl)amino)-2-oxoacetate.}<smiles>COC(=O)C(=O)Nc1ccccc1Cl</smiles>

This compound is reported and was prepared by following the general procedure A using methyl 2-chloro-2-oxoacetate (1.00 g, $0.752 \mathrm{~mL}, 8.13 \mathrm{mmol}, 1.05 \mathrm{eq}), 2$-chloro aniline $(0.988 \mathrm{~g}, 7.72 \mathrm{mmol}, 1.0 \mathrm{eq})$ and triethylamine $(0.946 \mathrm{~g}, 1.30 \mathrm{~mL}, 9.35 \mathrm{mmol}, 1.2 \mathrm{eq})$ in dichloromethane $(20 \mathrm{~mL})$ to give the product as a white solid $(1.25 \mathrm{~g}, 5.86 \mathrm{mmol}, 76 \%)$.

TLC: $\mathrm{R}_{\mathrm{f}}$ ca 0.3 (8:2, Hexane: EtOAc), strong UV active;

${ }^{1} \mathrm{H}$ NMR $\left(400 \mathrm{MHz}, \mathrm{CDCl}_{3}\right) \delta 9.46(1 \mathrm{H}, \mathrm{s}, \mathrm{CONH}), 8.44(1 \mathrm{H}, \mathrm{d}, J=8.3 \mathrm{~Hz}, \mathrm{ArH}), 7.42(1 \mathrm{H}$, $\mathrm{d}, J=8.0 \mathrm{~Hz}, \mathrm{ArH}), 7.33(1 \mathrm{H}, \mathrm{t}, J=7.8 \mathrm{~Hz}, \operatorname{ArH}), 7.13(1 \mathrm{H}, \mathrm{t}, J=7.8 \mathrm{~Hz}, \operatorname{ArH}), 4.00(3 \mathrm{H}, \mathrm{s}$, $\left.\mathrm{OCH}_{3}\right)$;

${ }^{13} \mathrm{C}$ NMR $\left(101 \mathrm{MHz}, \mathrm{CDCl}_{3}\right) \delta 161.1,153.7,133.3,129.4,128.0,126.1,123.6,121.4,54.3$;

$m / z(\mathrm{ESI}) 236.0\left[\left(\mathrm{M}\left({ }^{35} \mathrm{Cl}\right)+\mathrm{Na}\right)^{+}, 100 \%\right], 238.0\left[\left(\mathrm{M}\left({ }^{37} \mathrm{Cl}\right)+\mathrm{Na}\right)^{+}, 40 \%\right]$.

The data matches the reported data.

Zhang, Z.; Gao, X.; Yu, H.; Zhang, G.; Liu, J. Copper-Catalysed (Diacetoxyiodo)benzenePromoted Aerobic Esterification Reaction: Synthesis of Oxamates from Acetoacetamides. Adv. Synth. Catal. 2018, 360, 3406-3411. 
Methyl 2-((2-chlorophenyl)amino)-2-oxoacetate.

${ }^{1} \mathrm{H}$ NMR (400 MHz, $\left.\mathrm{CDCl}_{3}\right)$.

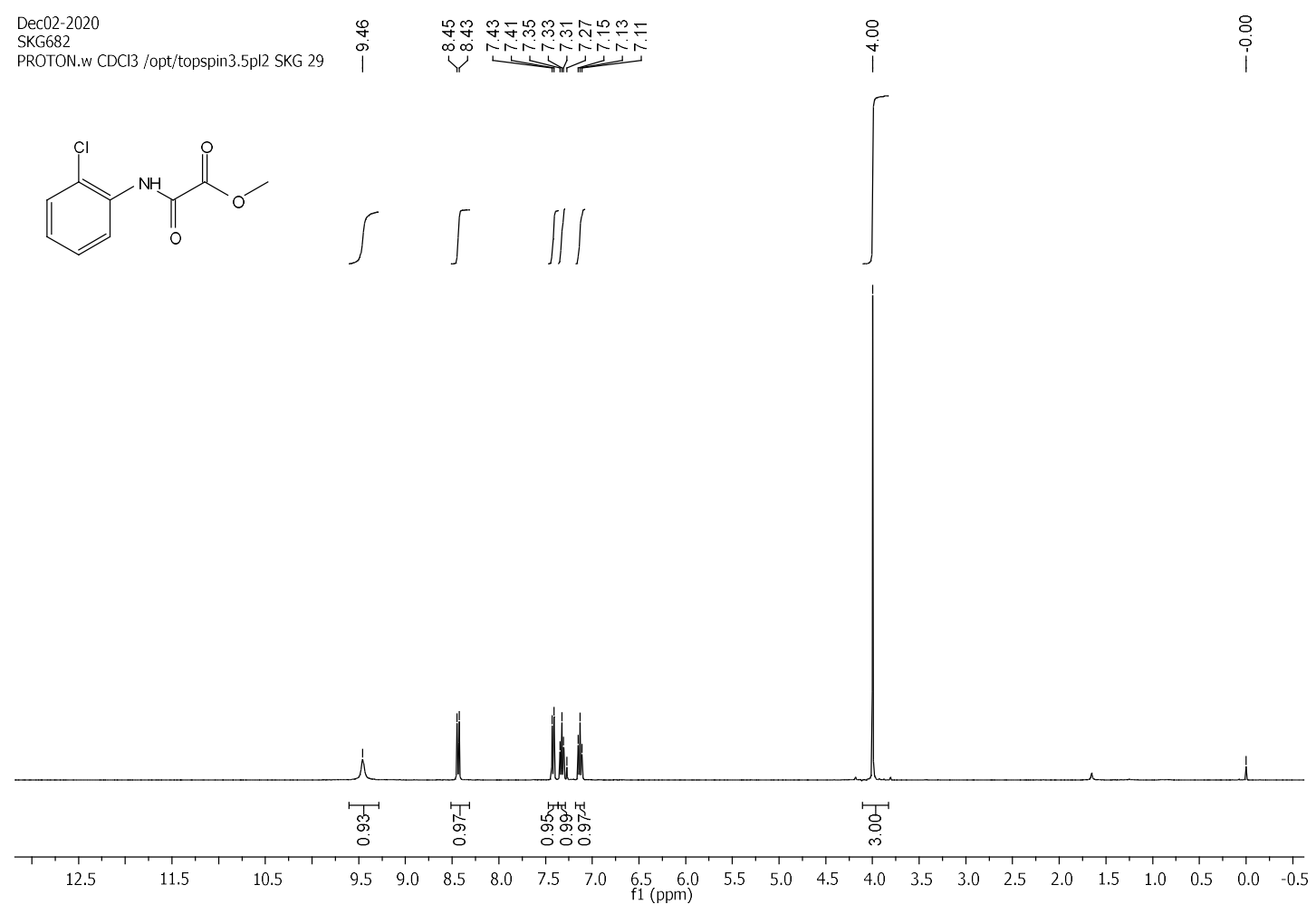

${ }^{13} \mathrm{C}$ NMR (101 MHz, $\left.\mathrm{CDCl}_{3}\right)$.

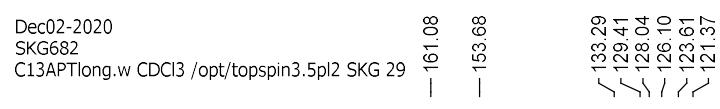

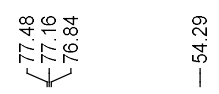
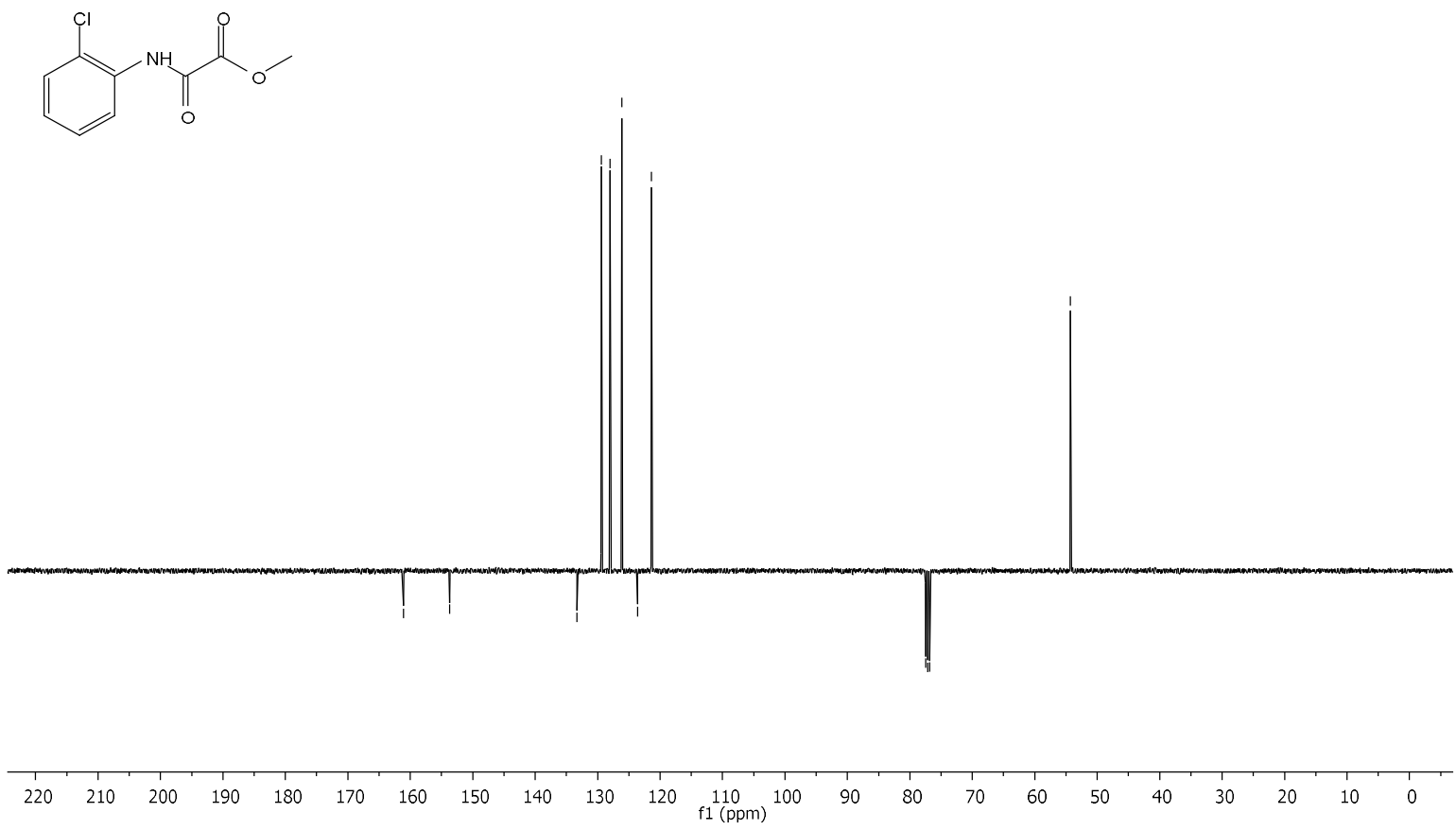

S42 
Methyl 2-(dimethylamino)-2-oxoacetate.<smiles>COC(=O)C(=O)N(C)C</smiles>

This compound has been reported and fully characterized. This was prepared following the general procedure A using methyl 2-chloro-2-oxoacetate $(1.00 \mathrm{~g}, 0.752 \mathrm{~mL}, 8.13 \mathrm{mmol}, 1.0$ eq), dimethylamine hydrochloride $(0.994 \mathrm{~g}, 12.2 \mathrm{mmol}, 1.5 \mathrm{eq})$ and triethylamine (2.05 $\mathrm{g}$, $2.81 \mathrm{~mL}, 20.3 \mathrm{mmol}, 2.5 \mathrm{eq})$ in dichloromethane $(20 \mathrm{~mL})$ to give the product as a clear liquid $(0.967 \mathrm{~g}, 7.38 \mathrm{mmol}, 91 \%)$.

TLC: $\mathrm{R}_{\mathrm{f}}$ ca 0.3 (8:2, Hexane: EtOAc), strong UV active;

${ }^{1} \mathrm{H}$ NMR (400 MHz, $\left.\mathrm{CDCl}_{3}\right) \delta 3.83\left(3 \mathrm{H}, \mathrm{s}, \mathrm{OCH}_{3}\right), 2.99\left(3 \mathrm{H}, \mathrm{s}, \mathrm{NCH}_{3}\right), 2.95\left(3 \mathrm{H}, \mathrm{s}, \mathrm{NCH}_{3}\right)$;

${ }^{13} \mathrm{C} \mathrm{NMR}\left(101 \mathrm{MHz}, \mathrm{CDCl}_{3}\right) \delta 163.3,161.5,52.6,37.2,34.2$;

$m / z(\mathrm{ESI}) 154.0\left[(\mathrm{M}+\mathrm{Na})^{+}, 80 \%\right], 285.1\left[(2 \mathrm{M}+\mathrm{Na})^{+}, 100 \%\right]$.

The data matches the reported data.

Duguet, N.; Petit, S. M.; Marchand, P.; Harrison-Marchand, A.; Maddaluno, J.; Heterocyclic Lithium Amides as Chiral Ligands for an Enantioselective Hydroxyalkylation with n-BuLi, $J$. Org. Chem. 2008, 73, 5397-5409. 
Methyl 2-(dimethylamino)-2-oxoacetate.

${ }^{1} \mathrm{H}$ NMR $\left(400 \mathrm{MHz}, \mathrm{CDCl}_{3}\right)$.

May13-2021

PROTON.w CDCl3 /opt/topspin3.5pl2 SKG 22<smiles>COC(=O)C(=O)N(C)C</smiles>

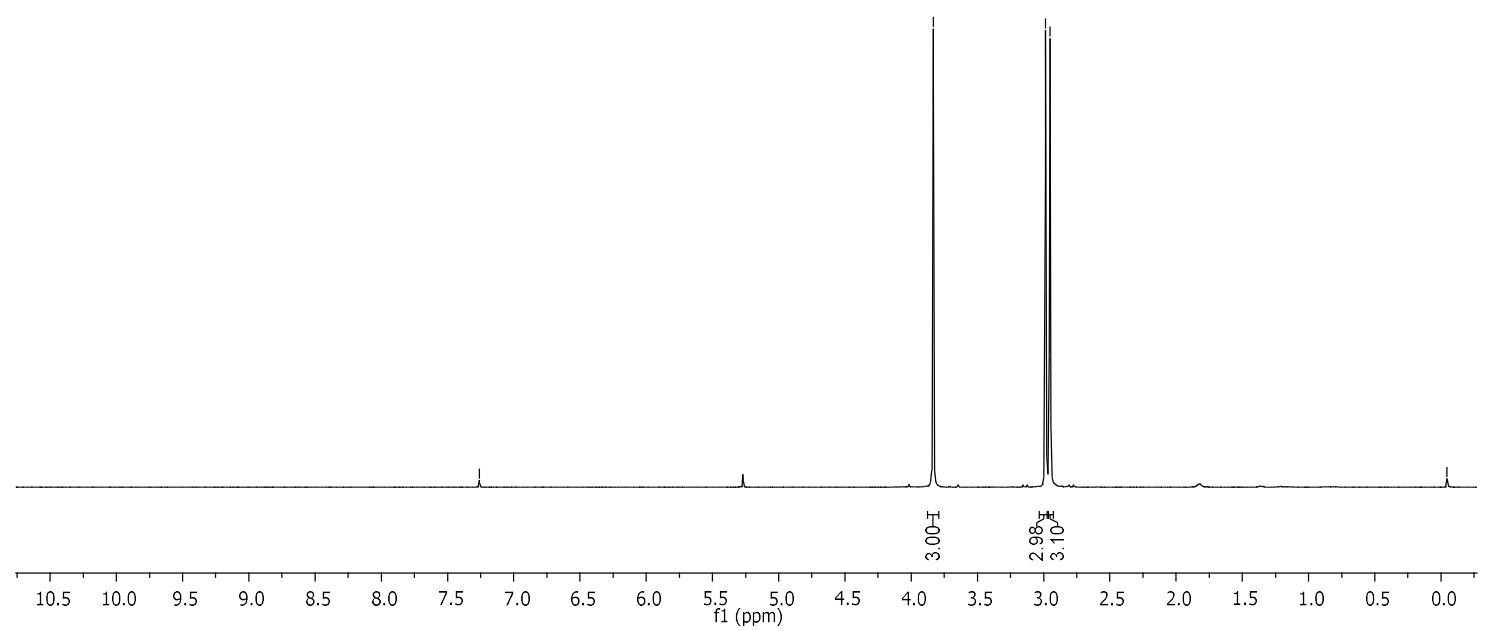

${ }^{13} \mathrm{C} \mathrm{NMR}\left(101 \mathrm{MHz}, \mathrm{CDCl}_{3}\right)$.

May13-2021
SKG869
C13APTlong.w CDCl3<smiles>COC(=O)C(=O)N(C)C</smiles>

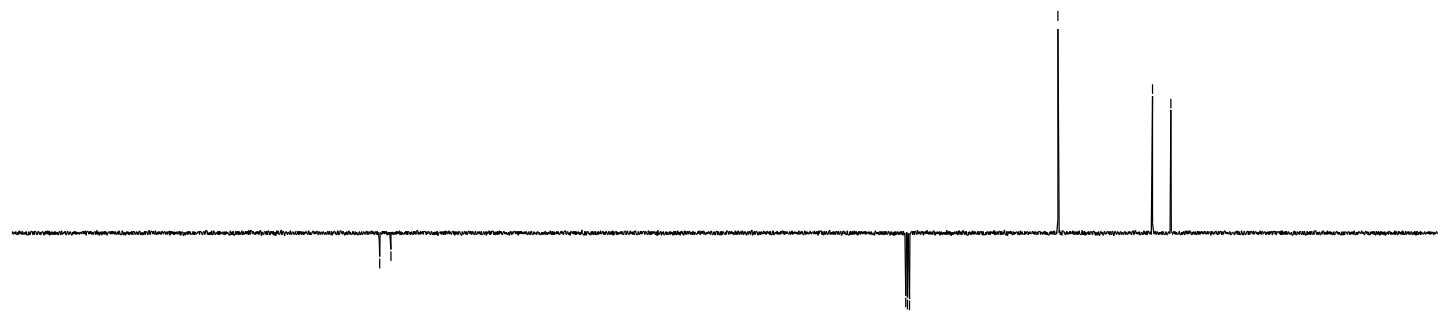

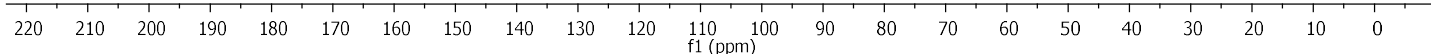


Methyl 2-oxo-2-(propylamino) acetate.<smiles>CCCNC(=O)C(=O)OC</smiles>

This compound is reported however it has not been characterized previously. This compound was prepared by following the general procedure A using methyl 2-chloro-2-oxoacetate (2.00 g, $1.50 \mathrm{~mL}, 16.4 \mathrm{mmol}, 1.05 \mathrm{eq})$, n-propyl amine (0.920 g, $15.6 \mathrm{mmol}, 1.0 \mathrm{eq})$ and triethylamine $(1.89 \mathrm{~g}, 2.60 \mathrm{~mL}, 18.7 \mathrm{mmol}, 1.15 \mathrm{eq})$ in dichloromethane (40 mL) to give the product as a clear liquid $(1.75 \mathrm{~g}, 12.1 \mathrm{mmol}, 78 \%)$.

TLC: $\mathrm{R}_{\mathrm{f}}$ ca 0.3 (5:5, Hexane: EtOAc), strong UV active;

HRMS (ESI) m/z: [M+Na] $]^{+}$Calcd for $\mathrm{C}_{6} \mathrm{H}_{11} \mathrm{NO}_{3} \mathrm{Na}$ 168.0631; Found 168.0632 (error -0.4 ppm);

$U_{\max } 3308,2963,1739,1680,1529,1437,1209,965 \mathrm{~cm}^{-1}$;

${ }^{1} \mathrm{H}$ NMR (400 MHz, $\left.\mathrm{CDCl}_{3}\right) \delta 7.21\left(1 \mathrm{H}\right.$, br. s, NH), $3.90\left(3 \mathrm{H}, \mathrm{s},-\mathrm{OCH}_{3}\right), 3.35-3.30(2 \mathrm{H}, \mathrm{m}$, $\left.\mathrm{CH}_{2}\right), 1.65-1.56\left(2 \mathrm{H}, \mathrm{m}, \mathrm{CH}_{2} \mathrm{CH}_{3}\right), 0.96\left(3 \mathrm{H}, \mathrm{t}, J=7.4 \mathrm{~Hz}, \mathrm{CH}_{2} \mathrm{C}_{3}\right)$;

${ }^{13} \mathrm{C}$ NMR $\left(101 \mathrm{MHz}, \mathrm{CDCl}_{3}\right) \delta 161.4,156.4,53.6,41.6,22.5,11.3$;

$m / z(\mathrm{ESI}) 168.1\left[(\mathrm{M}+\mathrm{Na})^{+}, 60 \%\right], 313.1\left[(2 \mathrm{M}+\mathrm{Na})^{+}, 100 \%\right]$.

Minisci, F.; Fontana, F.; Coppa, F.; Yan, Y. M. Reactivity of Carbamoyl Radicals. A New, General, Convenient Free-Radical Synthesis of Isocyanates from Monoamides of Oxalic Acic, J. Org. Chem. 1995, 60, 5430-5433. 
Methyl 2-oxo-2-(propylamino) acetate.

${ }^{1} \mathrm{H}$ NMR (400 MHz, $\mathrm{CDCl}_{3}$ ).

Feb03-2021

topt/topspin3.5p12 SKG 60

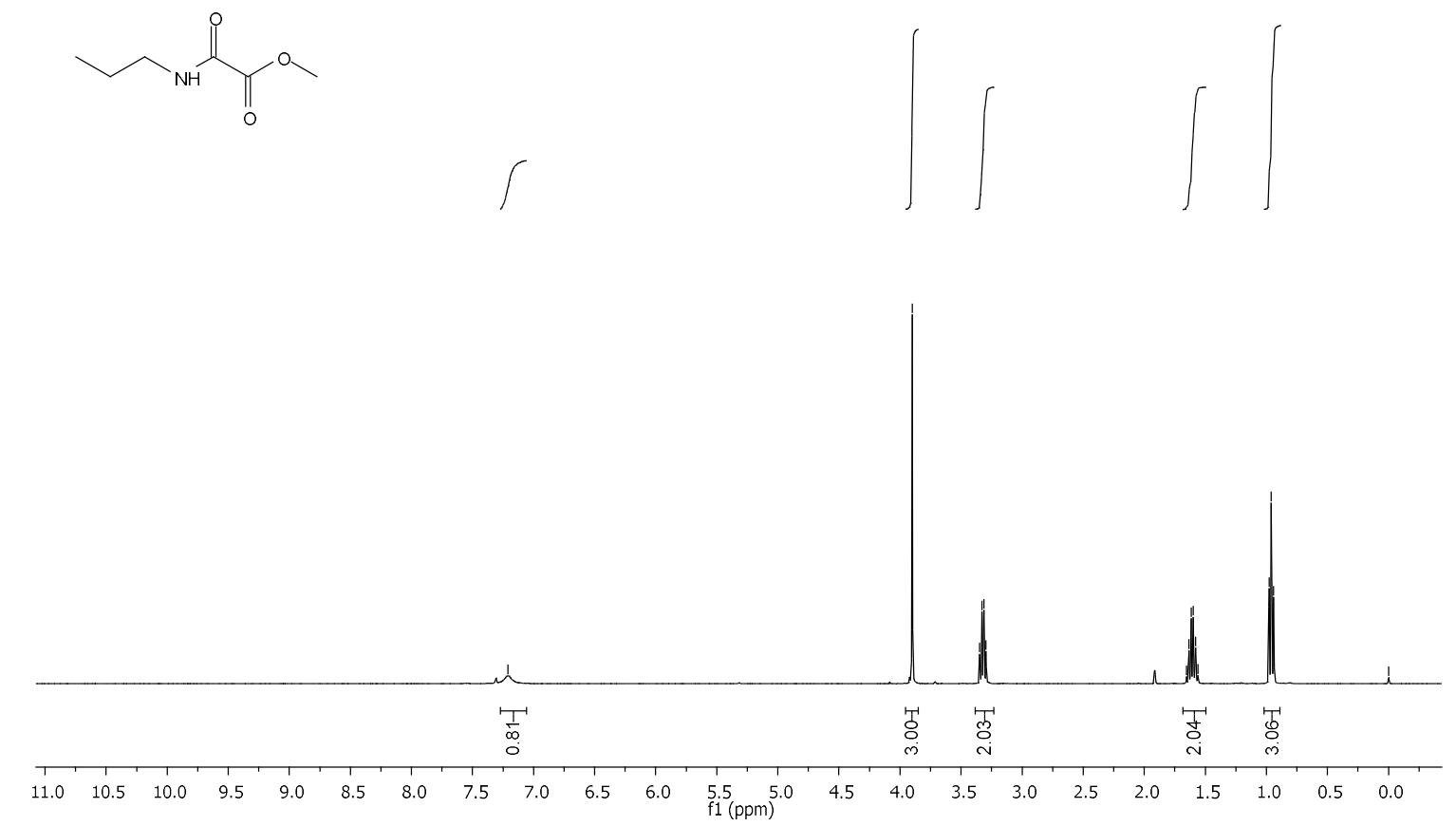

았 ल요

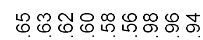
$-1--000$

${ }^{13} \mathrm{C}$ NMR (101 MHz, $\left.\mathrm{CDCl}_{3}\right)$.

Feb03-2021
SKG776 run

C13APTlong.w.CDCl3

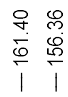<smiles>CCCNC(=O)C(=O)OC</smiles>

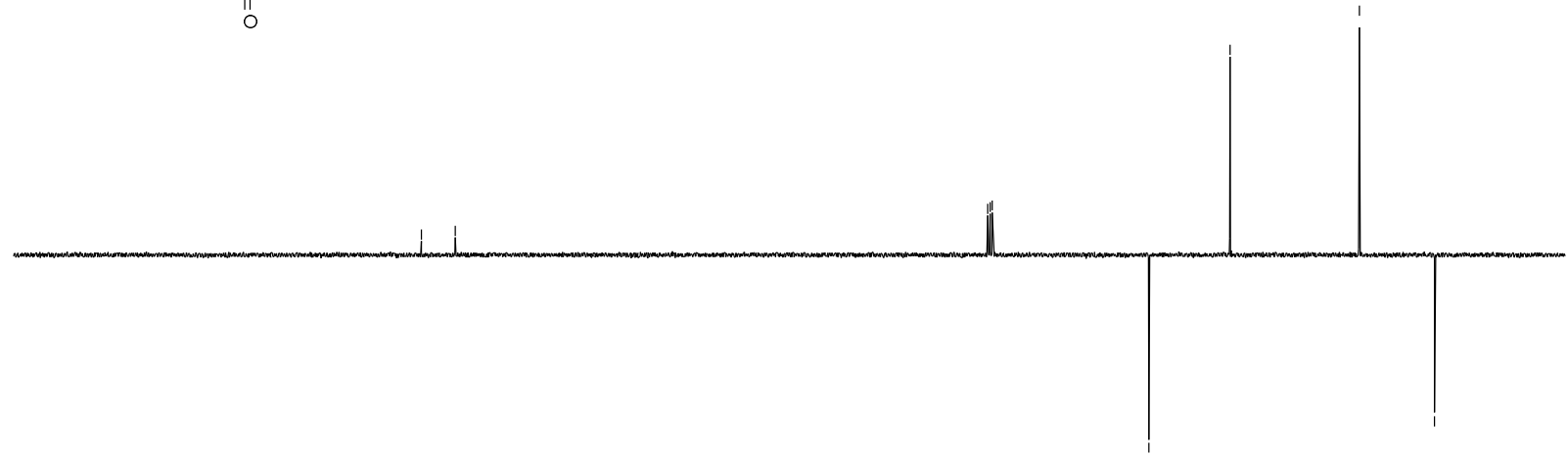

$\begin{array}{llllllllllllllllllllllllllll}220 & 210 & 200 & 190 & 180 & 170 & 160 & 150 & 140 & 130 & 120 & \frac{110}{\mathrm{f} 1(\mathrm{ppm})} & 100 & 90 & 80 & 70 & 60 & 50 & 40 & 30 & 20 & 10 & 0 & \end{array}$ 
COSY.

SKG776 run2
COSY.w CDCl3 /opt/topspin3.5pl2 SKG 60

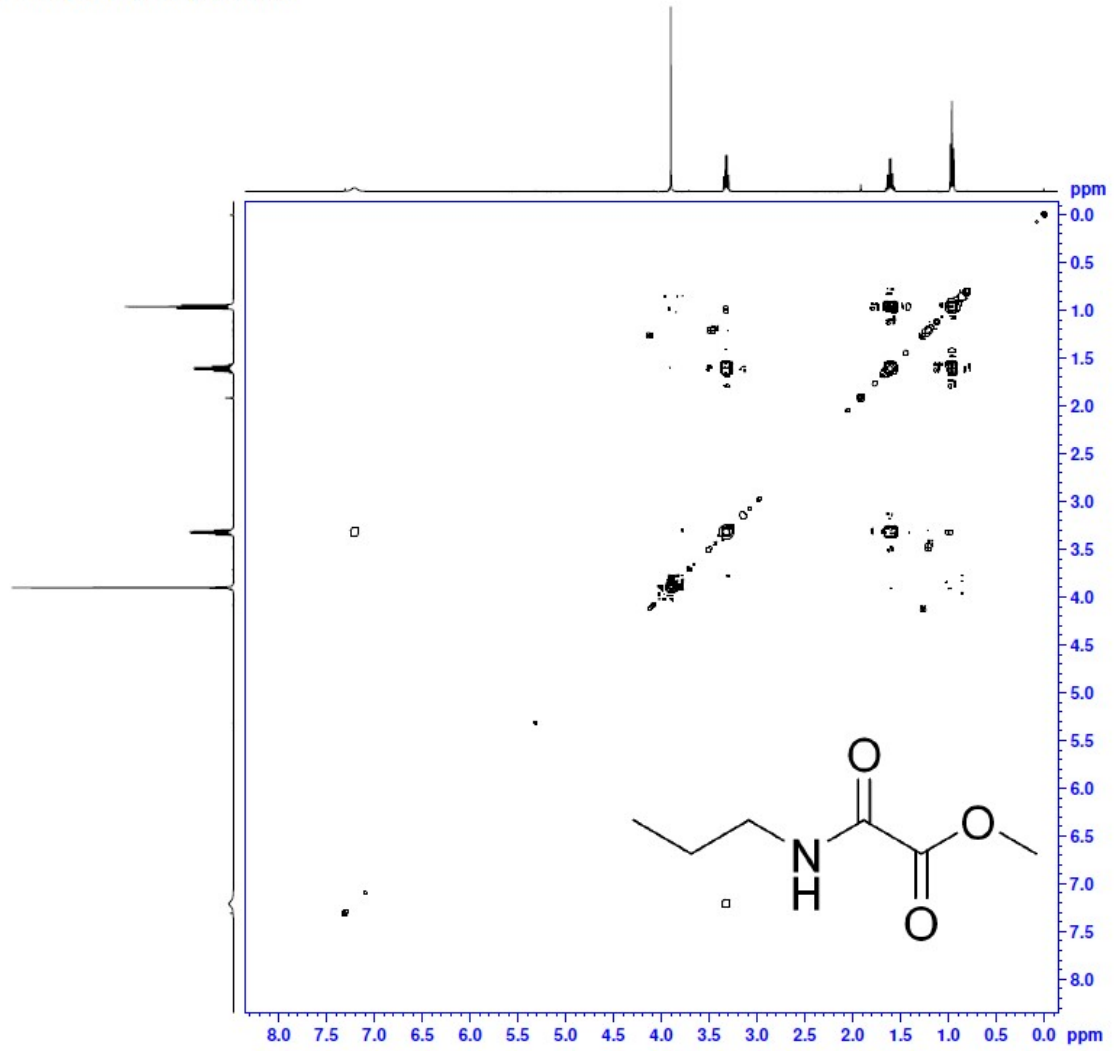


Methyl 2-(benzyl(methyl)amino)-2-oxoacetate.<smiles>COC(=O)C(=O)N(C)Cc1ccccc1</smiles>

This compound has been reported; however, it has not been fully characterized previously. It was prepared following the general procedure A using methyl 2-chloro-2-oxoacetate $(2.00 \mathrm{~g}$, $1.50 \mathrm{~mL}, 16.4 \mathrm{mmol}, 1.05 \mathrm{eq}), N$-methyl-1-phenylmethanamine (1.88 g, $15.6 \mathrm{mmol}, 1.0 \mathrm{eq})$ and triethylamine $(1.89 \mathrm{~g}, 2.60 \mathrm{~mL}, 18.7 \mathrm{mmol}, 1.2 \mathrm{eq})$ in dichloromethane $(40 \mathrm{~mL})$ to give the product as a clear liquid $(1.56 \mathrm{~g}, 7.53 \mathrm{mmol}, 48 \%)$.

TLC: $\mathrm{R}_{\mathrm{f}}$ ca 0.4 (7:3, Hexane: EtOAc), strong UV active;

HRMS (ESI) m/z: $[\mathrm{M}+\mathrm{Na}]^{+}$Calcd for $\mathrm{C}_{11} \mathrm{H}_{13} \mathrm{NO}_{3} \mathrm{Na} 230.0788$; Found 230.0784 (error 1.6 ppm);

$U_{\max } 2955,1737,1651,1453,1408,1237,1200,1097,700 \mathrm{~cm}^{-1}$;

${ }^{1} \mathrm{H}$ NMR (400 MHz, $\mathrm{CDCl}_{3}$ ) $\delta$ 7.39-7.31 (3H, m, ArH), 7.29-7.26 (2H, m, ArH), 4.60 (1H, s, $\mathrm{C}_{2} \mathrm{Ph}$ Rotamer 1), 4.44 (1H, s, $\mathrm{CH}_{2} \mathrm{Ph}$ Rotamer 2), 3.88 (1.5H, s, $\mathrm{OCH}_{3}$ Rotamer 1), 3.86 (1.5H, s, $\mathrm{OCH}_{3}$ Rotamer 2), 2.90 (1.5H, s, $\mathrm{NCH}_{3}$ Rotamer 1), 2.86 (1.5H, s, $\mathrm{NCH}_{3}$ Rotamer 2);

${ }^{13} \mathrm{C} \mathrm{NMR}\left(101 \mathrm{MHz}, \mathrm{CDCl}_{3}\right) \delta 163.5,163.3,161.8,161.7,135.4,134.9,128.9,128.8,128.3$, 128.2, 127.9, 127.7, 53.7, 52.7, 52.6, 49.9, 34.6, 31.6;

$m / z(\mathrm{ESI}) 230.0[(\mathrm{M}+\mathrm{Na})+, 100 \%]$. 
Methyl 2-(benzyl(methyl)amino)-2-oxoacetate.

${ }^{1} \mathrm{H}$ NMR (400 MHz, $\left.\mathrm{CDCl}_{3}\right)$.

Apr20-2021
SKG894

PROTON.w CDCl3 /opt/topspin3.5pl2 SKG 10

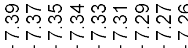

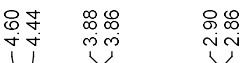

8
$i$
$i$<smiles>COC(=O)C(=O)N(C)Cc1ccccc1</smiles><smiles>C=CCCC</smiles>

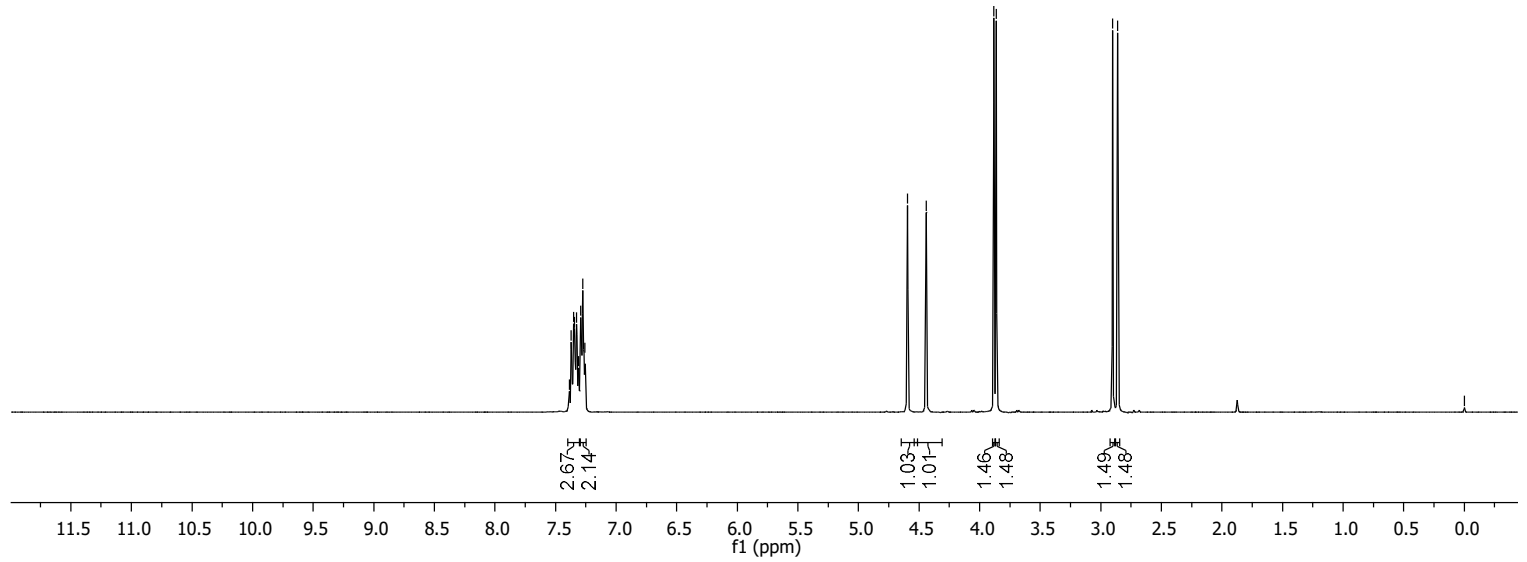

${ }^{13} \mathrm{C}$ NMR (101 MHz, $\left.\mathrm{CDCl}_{3}\right)$.

Apr20-2021

C13APTlong.w CDCl3 /opt/topspin3.5p12 SKG 10

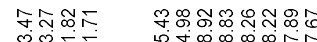

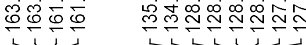

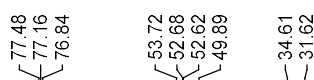

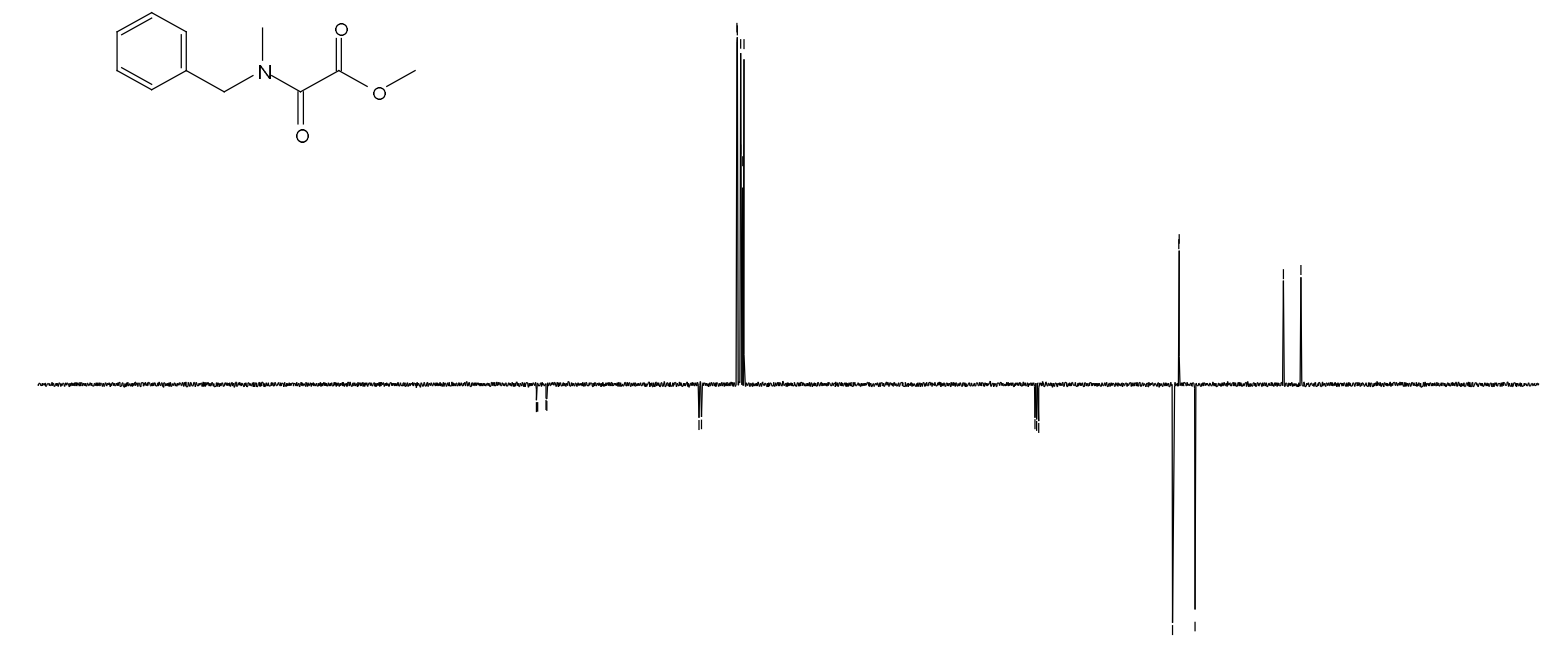

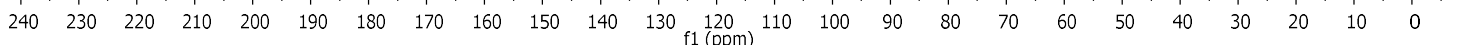


COSY.

SKG894

COSY.w $\mathrm{CDCl}$ /opt/topspin3.5pl2 SKG 10

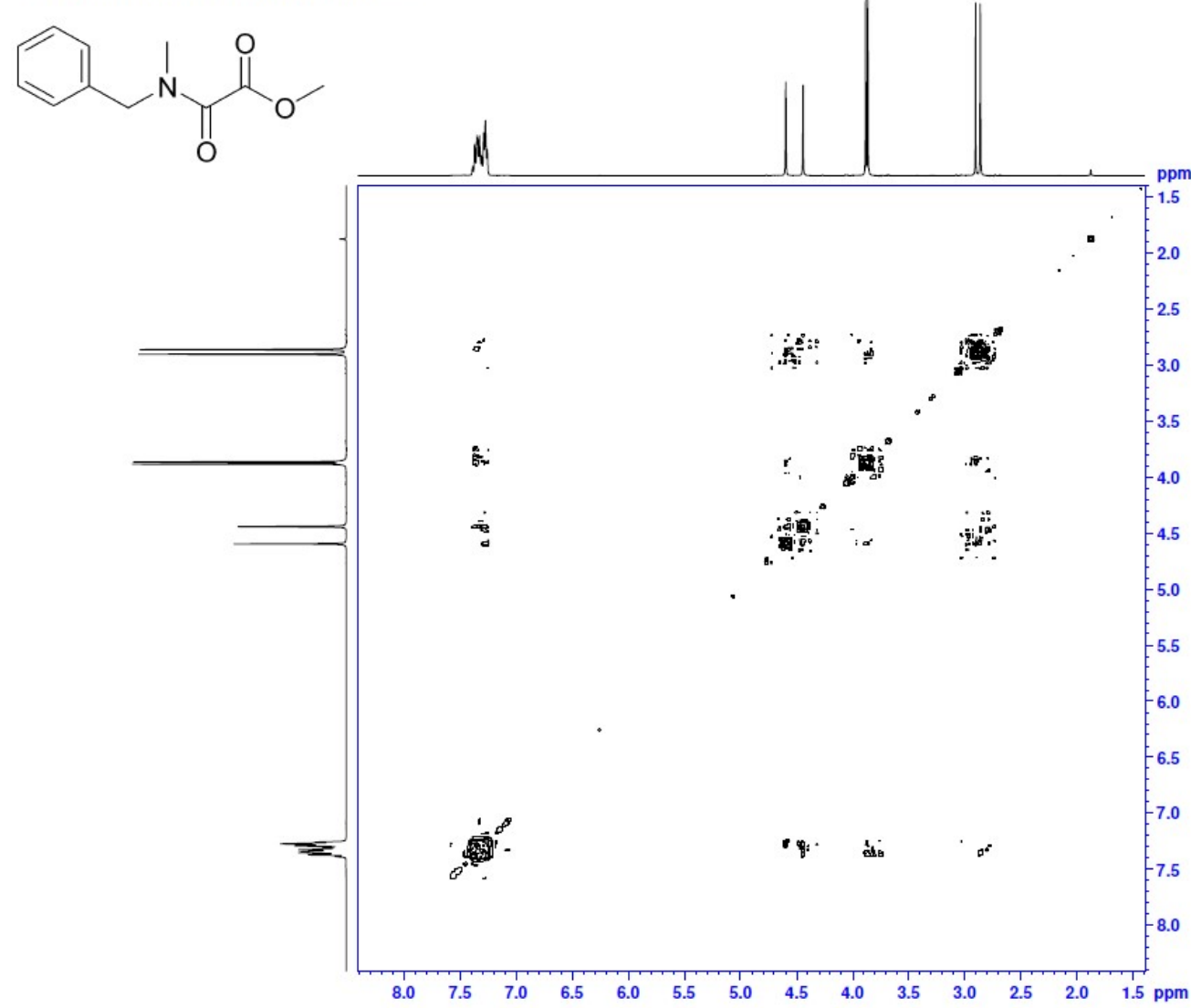




\section{Section on the synthesis of $\alpha$-keto amide derivatives.}

\section{General procedure B for synthesis of $\alpha$-keto amide derivatives.}

To a stirred solution of LDA in anhydrous THF was added a solution of the substituted acetamide in anhydrous THF dropwise, at $-78^{\circ} \mathrm{C}$ and the reaction mixture was stirred at the same temperature for 20 minutes. A solution of the acetate in anhydrous THF was added dropwise and the resulting reaction mixture was stirred at the same temperature for 20 minutes followed by stirring at room temperature for $1 \mathrm{~h}$. Once the reaction was complete (as assessed by TLC), the reaction mixture was quenched with saturated ammonium chloride solution and the aqueous layer was extracted with ethyl acetate $(3 \times 30 \mathrm{~mL})$. The combined organic layers were washed with brine $(30 \mathrm{~mL})$ and dried over $\mathrm{MgSO}_{4}$. After filtration, the organic layer was concentrated under reduced pressure to generate the crude product which was further purified my flash chromatography on silica gel to afford the $\alpha$-keto amide derivatives. 
$N^{1}, N^{4}$-dimethyl-2-oxo- $N^{1}, N^{4}$-diphenylsuccinamide (precursor of 5).<smiles>CN(C(=O)CC(=O)C(=O)N(C)c1ccccc1)c1ccccc1</smiles>

This compound is novel and was prepared following general procedure B using methyl 2(methyl(phenyl)amino)-2-oxoacetate $(0.500 \mathrm{~g}, 2.59 \mathrm{mmol}, 1.0 \mathrm{eq})$ in anhydrous THF $(8 \mathrm{~mL})$, $N$-methyl- $N$-phenylacetamide $(0.386 \mathrm{~g}, 2.59 \mathrm{mmol}, 1.0 \mathrm{eq})$ in anhydrous THF $(8 \mathrm{~mL})$ and LDA (1M solution in THF) $(3.36 \mathrm{~mL}, 3.36 \mathrm{mmol}, 1.5 \mathrm{eq})$ to give crude material which was further purified by column chromatography (10-30\% EtOAc in petroleum ether) to afford the product as a yellow liquid $(0.525 \mathrm{~g}, 1.69 \mathrm{mmol}, 65 \%)$. The title compound was obtained as a keto/enol tautomer $(3: 1)$ as per ${ }^{1} \mathrm{H}$ NMR.

TLC: $\mathrm{R}_{\mathrm{f}}$ ca 0.3 (7:3, hexane: EtOAc), UV-active and strong $\mathrm{KMnO}_{4}$;

HRMS (ESI) m/z: [M+Na] $]^{+}$Calcd for $\mathrm{C}_{18} \mathrm{H}_{18} \mathrm{~N}_{2} \mathrm{O}_{3} \mathrm{Na} 333.1210$; Found 333.1214 (error -1.2 ppm);

$U_{\max } 3540,2972,2922,1734,1639,1592,1490,1383,1212,1093,1072,779,697 \mathrm{~cm}^{-1}$;

${ }^{1} \mathrm{H}$ NMR (500 MHz, $\mathrm{CDCl}_{3}$ ) (keto/enol, 3:1) $\delta 13.99$ (0.25H, br.s., OH of enol form), 7.44 $7.26(9 \mathrm{H}, \mathrm{m}, \mathrm{ArH}), 7.10-6.99(1 \mathrm{H}, \mathrm{m}, \mathrm{ArH}), 5.04(0.25 \mathrm{H}, \mathrm{s}=\mathrm{C} \underline{\mathrm{H}}$ of enol form), 3.64-3.24 $\left(7.5 \mathrm{H}, \mathrm{m}, \mathrm{NCH}_{3}+\mathrm{CH}_{2}\right.$ of keto form);

${ }^{13} \mathrm{C}$ NMR (126 MHz, $\left.\mathrm{CDCl}_{3}\right)$ (keto/enol, 3:1) $\delta 192.8(\mathrm{C}=\mathrm{O}), 171.1(\mathrm{C}=\mathrm{O}$ amide), 167.3 $(\mathrm{C}=\mathrm{O}$ amide $), 164.7,164.5,143.5,142.6,142.7,131.1,130.1,129.9,129.5,129.4,128.6$, $128.5,128.1,127.7,127.3,127.2,126.5,125.9,125.8,93.4(\mathrm{C}=\underline{\mathrm{C}} \mathrm{H}$, enol form $), 45.9\left(\mathrm{CH}_{2}\right.$ keto form), 38.7, 37.5, 37.3, 36.6;

$m / z(\mathrm{ESI}) 333.1\left[(\mathrm{M}+\mathrm{Na})^{+}, 100 \%\right]$. 
$N^{1}, N^{4}$-Dimethyl-2-oxo- $N^{1}, N^{4}$-diphenylsuccinamide.

${ }^{1} \mathrm{H}$ NMR (500 MHz, $\left.\mathrm{CDCl}_{3}\right)$.

Dec17-2020 I
SKG626<smiles>COC(=O)CC(=O)N(C)c1ccccc1</smiles>

enol form
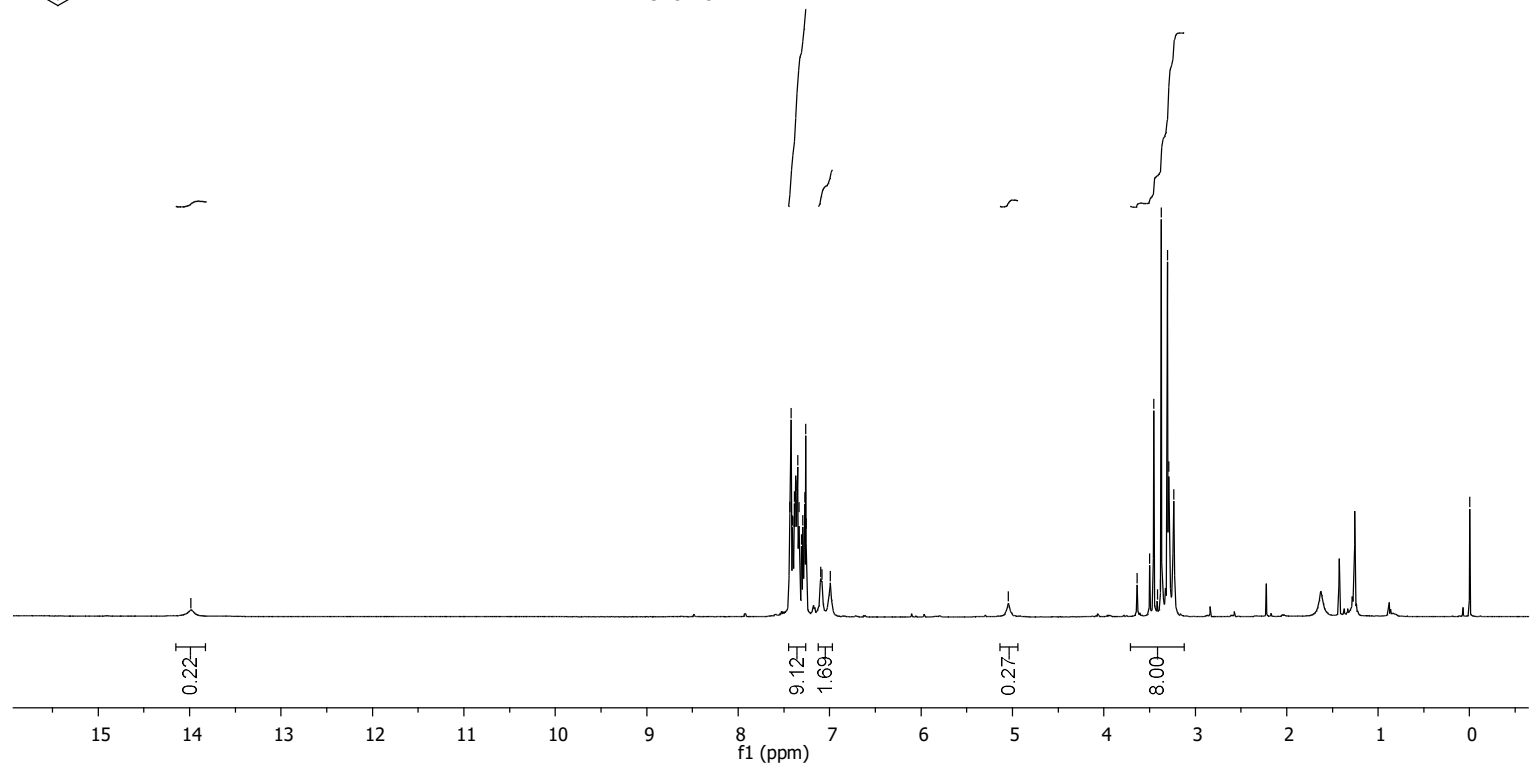

${ }^{13} \mathrm{C}$ NMR (126 MHz, $\left.\mathrm{CDCl}_{3}\right)$.

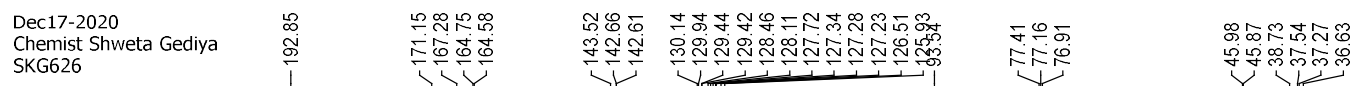<smiles>COC(=O)C(=O)N(C)c1ccccc1</smiles>

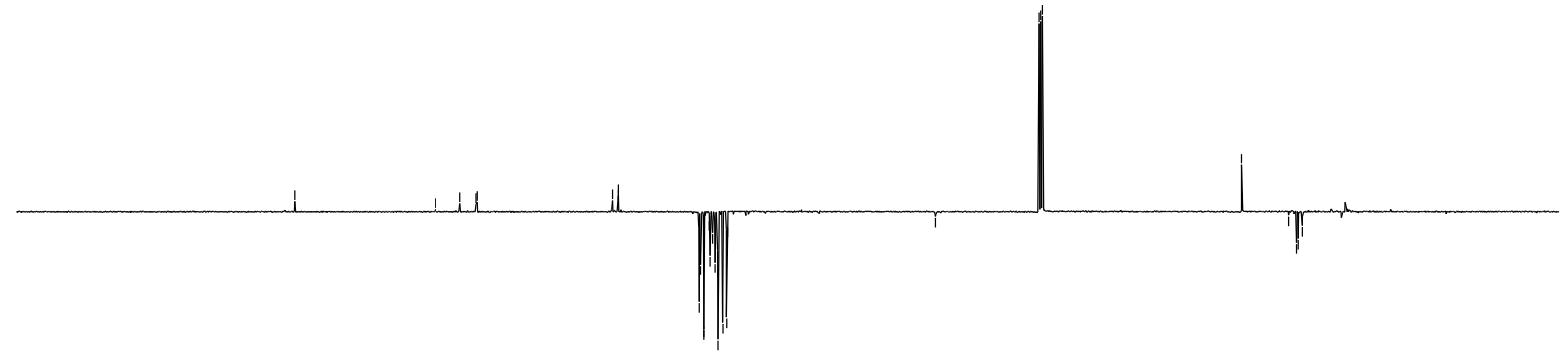

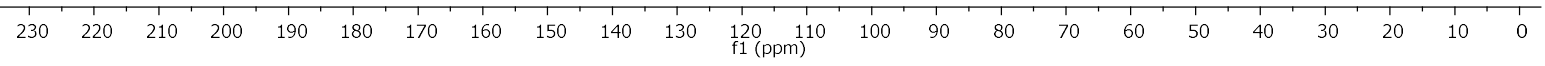


COSY.

Chemist Shweta Gediya

COSY.w CDCl3/opt/topspin3.2 SKG 53

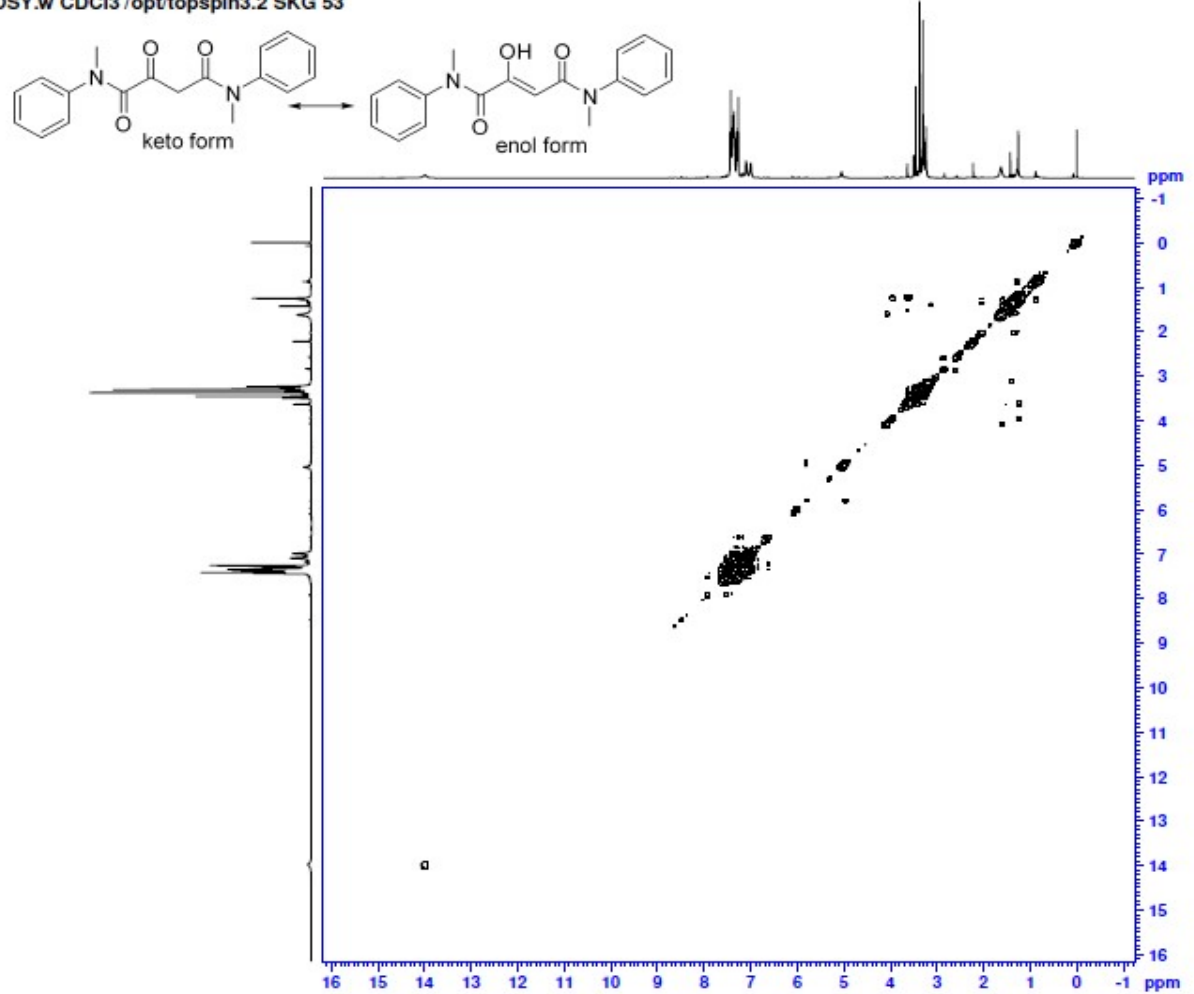

HSQC.

Chemist Shweta Gediya

HSGC.w $\mathrm{CDCl}$ /opt/topspin3.2 SKG 53

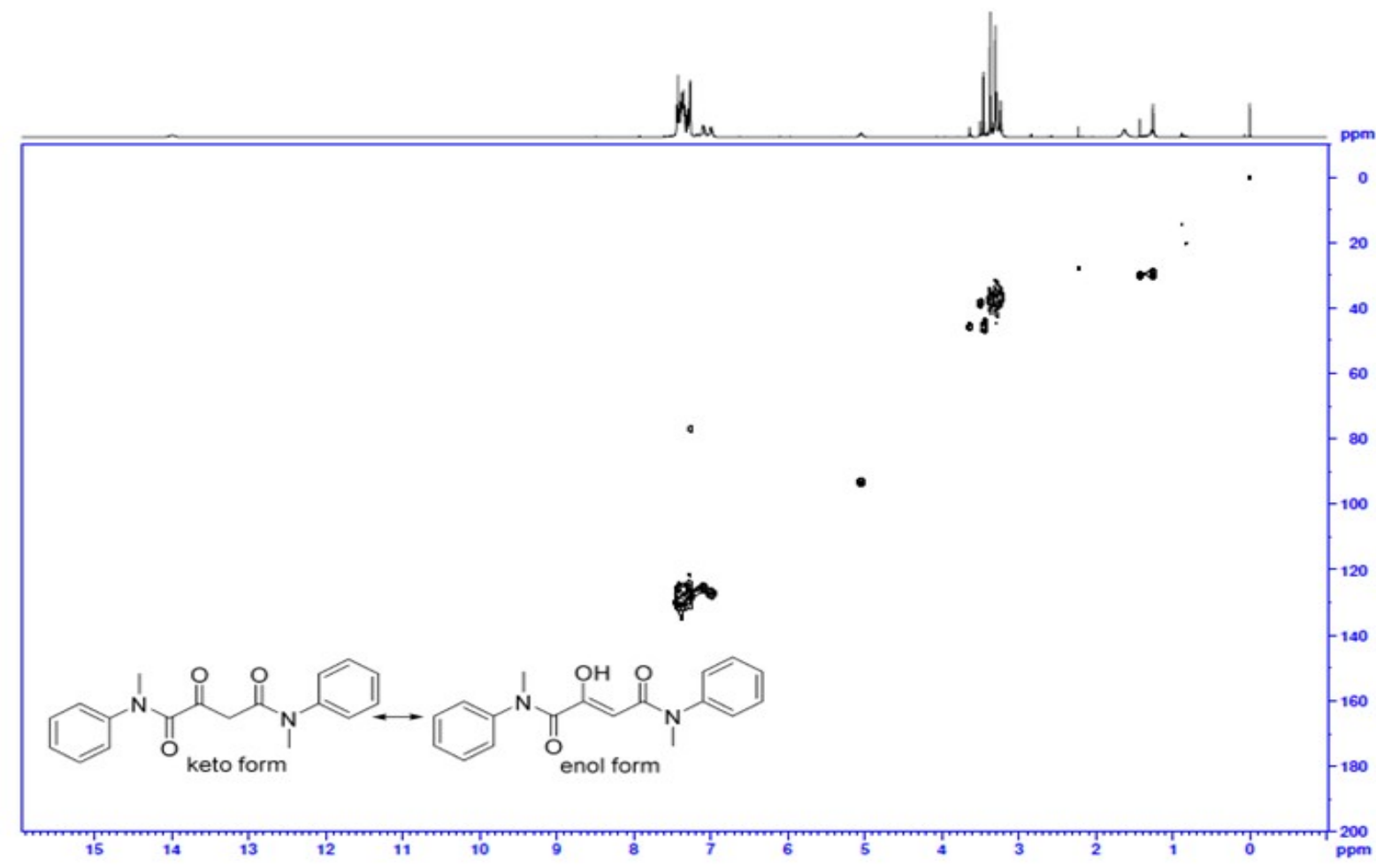


$N^{1}$-Benzyl- $N^{1}, N^{4}$-dimethyl-2-oxo- $N^{4}$-phenylsuccinamide (precursor of 6).<smiles>CN(Cc1ccccc1)C(=O)CC(=O)CC(=O)N(C)c1ccccc1</smiles>

This compound is novel and was prepared following general procedure B using methyl 2(benzyl(methyl)amino)-2-oxoacetate $(0.517 \mathrm{~g}, 2.50 \mathrm{mmol}, 1.0 \mathrm{eq})$ in anhydrous THF $(8 \mathrm{~mL})$, $N$-methyl- $N$-phenylacetamide $(0.447 \mathrm{~g}, 2.99 \mathrm{mmol}, 1.2 \mathrm{eq})$ in anhydrous THF $(8 \mathrm{~mL})$ and LDA (1M solution in THF) $(3.48 \mathrm{~mL}, 3.48 \mathrm{mmol}, 1.4 \mathrm{eq})$ to give crude material which was further purified by column chromatography (30-40\% EtOAc in petroleum ether) to afford te product as a clear liquid $(0.715 \mathrm{~g}, 2.20 \mathrm{mmol}, 88 \%)$. The title compound was obtained as a keto/enol tautomer $(1: 1)$ and rotamers as per ${ }^{1} \mathrm{H}$ NMR.

TLC: $\mathrm{R}_{\mathrm{f}}$ ca 0.2 (7:3, hexane: EtOAc), UV-active and strong $\mathrm{KMnO}_{4}$;

HRMS (ESI) m/z: [M+Na] ${ }^{+}$Calcd for $\mathrm{C}_{19} \mathrm{H}_{20} \mathrm{~N}_{2} \mathrm{O}_{3} \mathrm{Na}$ 347.1366; Found 347.1360 (error 1.7 ppm);

$U_{\max } 3061,2931,1720,1638,1592,1494,1383,1120,1070,696 \mathrm{~cm}^{-1}$;

${ }^{1} \mathrm{H}$ NMR $\left(400 \mathrm{MHz}, \mathrm{CDCl}_{3}\right)$ (keto/enol $(1: 1)+$ rotamers) $\delta 14.70(0.5 \mathrm{H}$, br.s., $\mathrm{OH}$ of enol form), 7.46-7.14 (10H, m, ArH), 5.29-5.28 $(0.5 \mathrm{H}, \mathrm{m},=\mathrm{CH}$ of enol form), 4.62-4.60 (1.5H, m, $\mathrm{CH}_{2} \mathrm{Ph}$ - major), 4.53 (0.5H, m, $\mathrm{CH}_{2} \mathrm{Ph}$ - minor) 3.65-3.63 (1H, m $\mathrm{CH}_{2}$ of keto form), 3.33$3.26\left(3 \mathrm{H}, \mathrm{m}, \mathrm{NCH}_{3}\right), 3.05\left(1 \mathrm{H}, \mathrm{s}, \mathrm{NCH}_{3}\right), 2.98\left(0.5 \mathrm{H}, \mathrm{s}, \mathrm{NCH}_{3}\right), 2.85\left(1 \mathrm{H}, \mathrm{s}, \mathrm{NCH}_{3}\right), 2.80$ $\left(0.5 \mathrm{H}, \mathrm{s},-\mathrm{NCH}_{3}\right)$;

${ }^{13} \mathrm{C}$ NMR $\left(101 \mathrm{MHz}, \mathrm{CDCl}_{3}\right)$ (keto/enol $(1: 1)+$ rotamers $) \delta 194.1,194.0(\mathrm{C}=\mathrm{O}$, keto form), $167.6,167.5,166.4,166.1,143.2,142.5,136.2,135.9,135.8,130.1,129.9,129.8,128.7$, $128.6,128.5,128.2,128.1,128.0,127.8,127.7,127.6,127.5,127.2,127.1,92.2(\mathrm{C}=\underline{\mathrm{C}} \mathrm{H}$, enol form), 54.0, 53.6, 50.9, 50.8, 45.9, 45.8, 37.0, 36.9, 36.7, 36.6, 35.5, 35.0, 32.7, 32.3;

$\mathrm{m} / z(\mathrm{ESI}) 347.1\left[(\mathrm{M}+\mathrm{Na})^{+}, 100 \%\right]$. 
$N^{1}$-Benzyl- $N^{1}, N^{4}$-dimethyl-2-oxo- $N^{4}$-phenylsuccinamide.

${ }^{1} \mathrm{H}$ NMR (400 MHz, $\left.\mathrm{CDCl}_{3}\right)$.

Mar31-2021

PROTON.w CDCl3 /opt/topspin3.5pl2 SKG 24
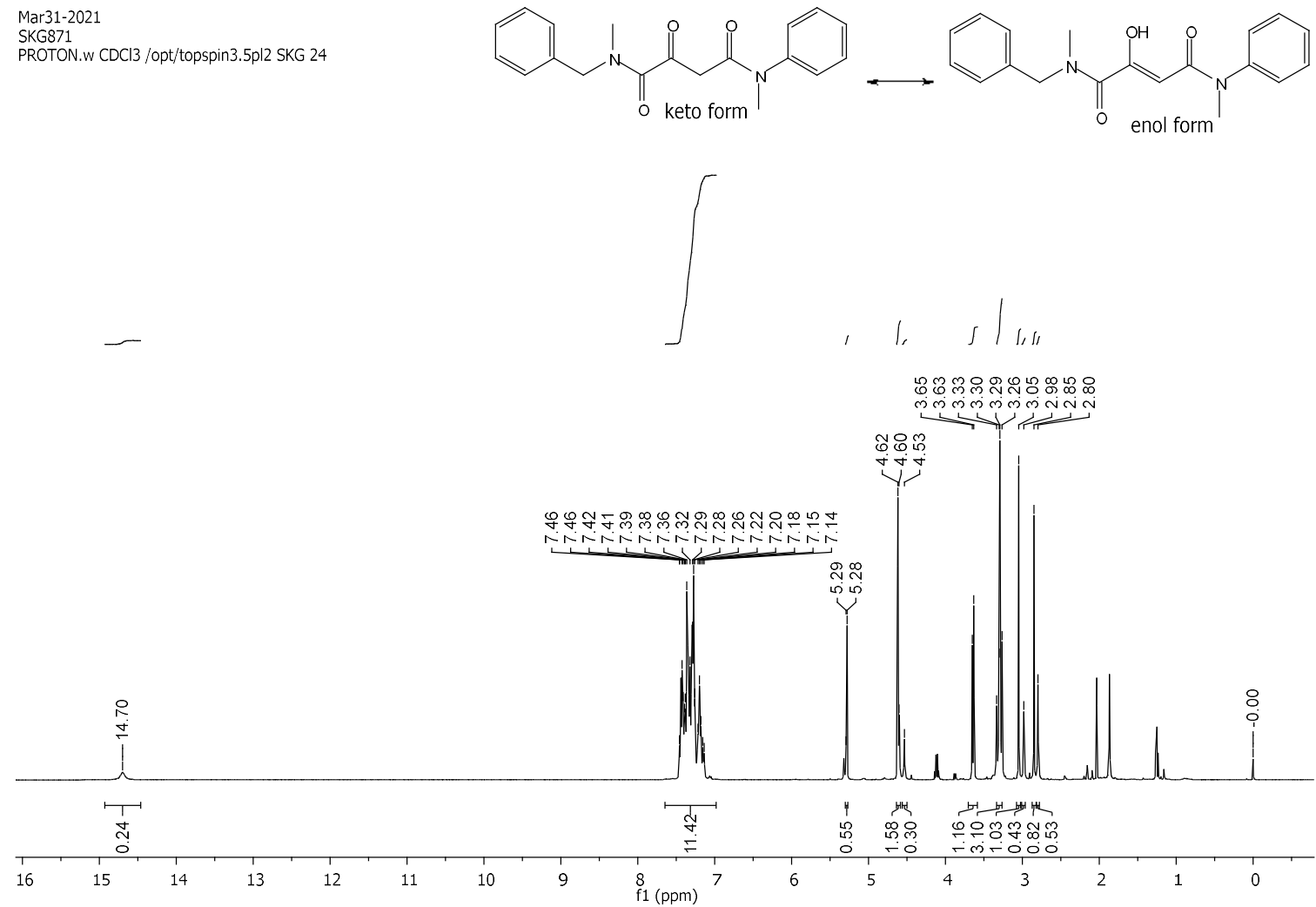

${ }^{13} \mathrm{C}$ NMR (101 MHz, $\left.\mathrm{CDCl}_{3}\right)$.

Mar31-2021
SKG871

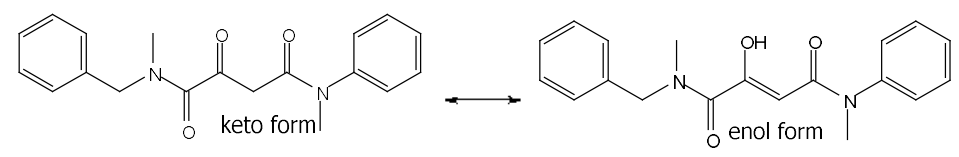

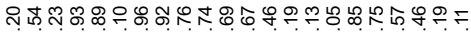

守守

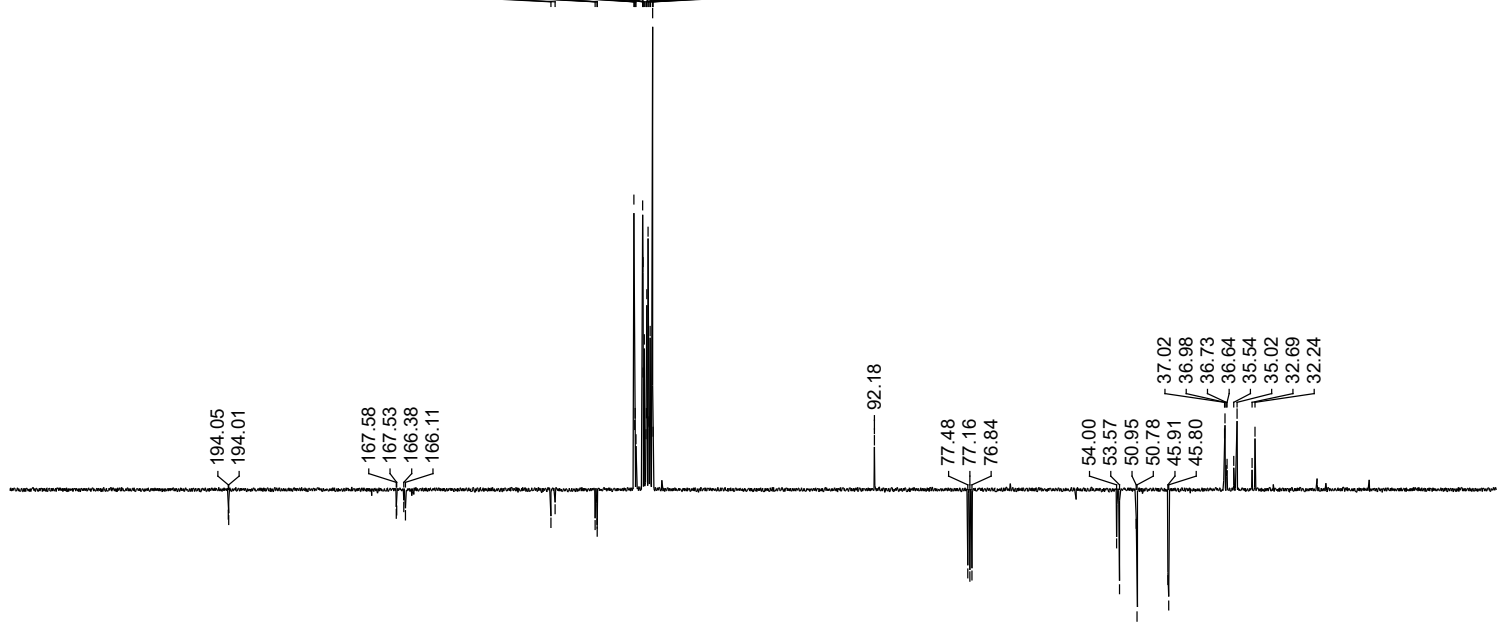

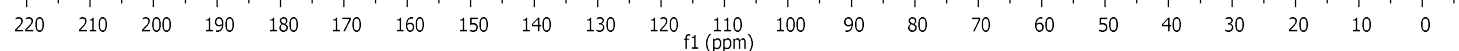


COSY.

SKG871

COSY.w CDCl3 /opt/topspin3.5pl2 SKG 24

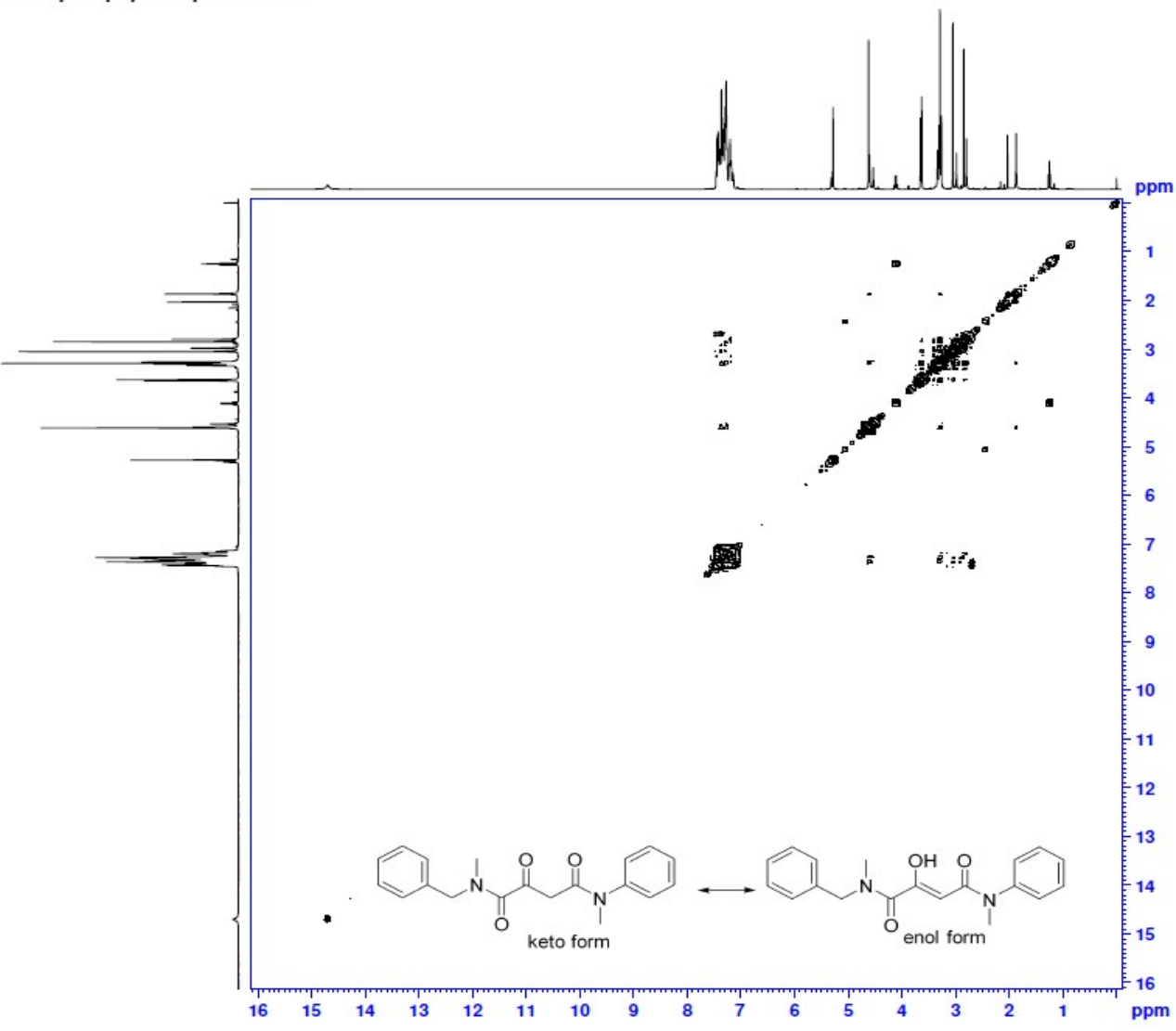

HSQC.

SKG871
HSOC.w CDCI3 /opt/topspin3.5p12 SKG 24

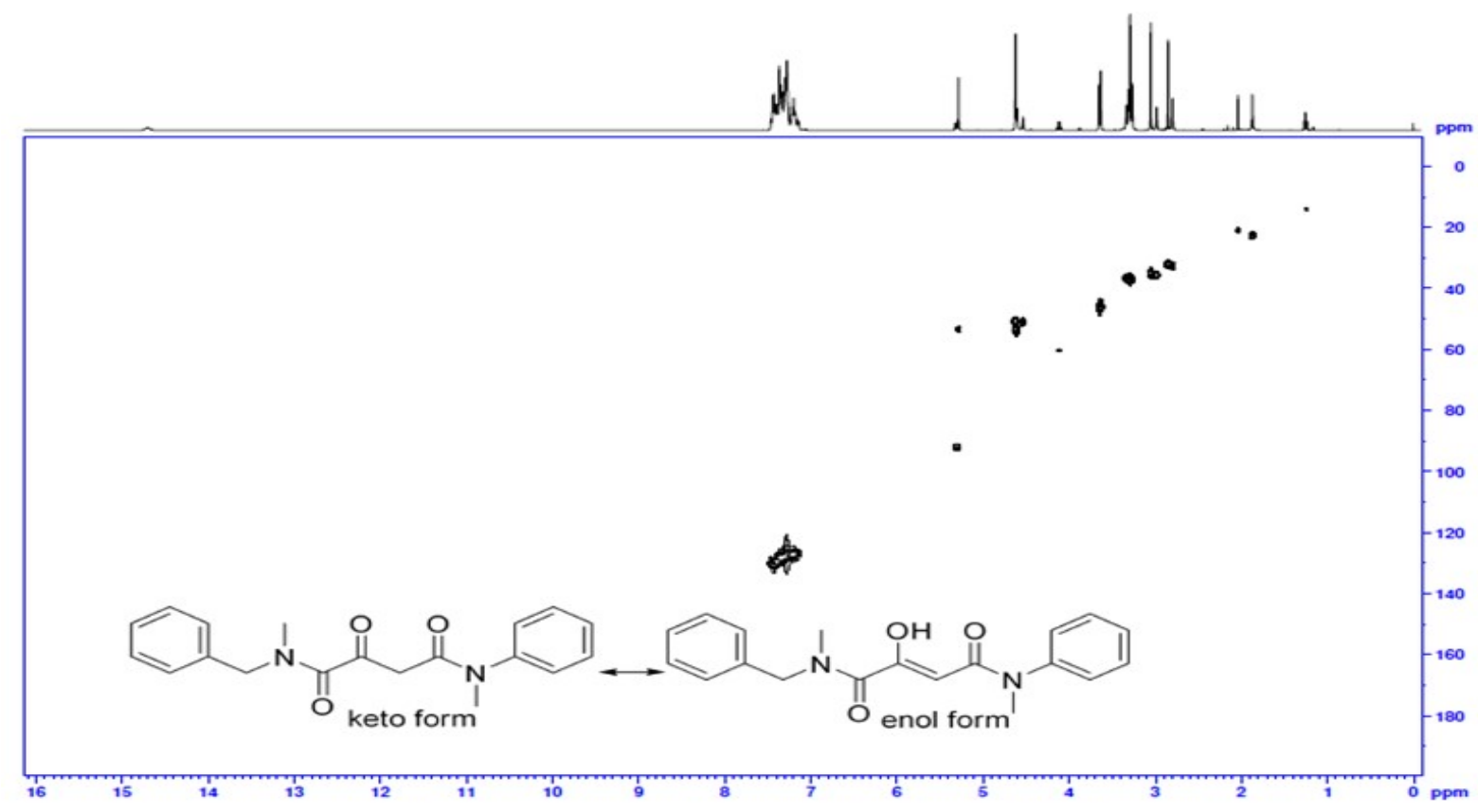


$N^{1}, N^{4}$-Dibenzyl- $N^{1}, N^{4}$-dimethyl-2-oxosuccinamide (precursor of 7 ).<smiles>CN(Cc1ccccc1)C(=O)CC(=O)C(=O)N(C)Cc1ccccc1</smiles>

This compound is novel and was prepared following general procedure B using methyl 2(benzyl(methyl)amino)-2-oxoacetate $(0.517 \mathrm{~g}, 2.50 \mathrm{mmol}, 1.0 \mathrm{eq})$ in anhydrous THF $(8 \mathrm{~mL})$, $N$-benzyl- $N$-methylacetamide (0.488 g, $2.99 \mathrm{mmol}, 1.0 \mathrm{eq})$ in anhydrous THF ( $8 \mathrm{~mL})$ and LDA (1M solution in THF) $(3.48 \mathrm{~mL}, 3.48 \mathrm{mmol}, 1.4 \mathrm{eq})$ to give crude material which was further purified by column chromatography $(60-70 \%$ EtOAc in petroleum ether) to afford the product as a pale yellow liquid $(0.530 \mathrm{~g}, 1.56 \mathrm{mmol}, 63 \%)$. The title compound was obtained as a keto/enol tautomer (5:1) and rotamers as per ${ }^{1} \mathrm{H}$ NMR.

TLC: $\mathrm{R}_{\mathrm{f}}$ ca 0.3 (3:7, hexane: EtOAc), UV-active and strong $\mathrm{KMnO}_{4}$;

HRMS (ESI) m/z: [M+Na] ${ }^{+}$Calcd for $\mathrm{C}_{20} \mathrm{H}_{22} \mathrm{~N}_{2} \mathrm{O}_{3} \mathrm{Na}$ 361.1523; Found 361.1517 (error 1.5 ppm);

$U_{\max } 3029,2929,1721,1633,1601,1484,1451,1401,1070,696 \mathrm{~cm}^{-1}$;

${ }^{1} \mathrm{H}$ NMR $\left(500 \mathrm{MHz}, \mathrm{CDCl}_{3}\right)($ keto/enol $(5: 1)+$ rotamers) $\delta 15.35$ (0.16, br.s., $\mathrm{OH}$ of enol form), 7.41-7.19 (10H, m, ArH), 5.83-5.82 (0.16H m, =CH of enol form), 4.66-4.51 (4H, m, $\left.\mathrm{CH}_{2} \mathrm{Ph}\right), 3.99-3.97\left(1.68 \mathrm{H}, \mathrm{m}, \mathrm{CH}_{2}\right.$ of keto form), 3.09-2.85 (6H, m, $\left.\mathrm{NCH}_{3}\right)$;

${ }^{13} \mathrm{C} \mathrm{NMR}\left(126 \mathrm{MHz}, \mathrm{CDCl}_{3}\right)$ (keto/enol (5:1; + rotamers) $\delta$ 193.8, 193.7, $193.6(\mathrm{C}=\mathrm{O}$, keto), $172.0,171.9,168.3,168.2,168.1,167.9,166.6,166.3,136.5,136.4,136.1,136.0,135.9$, $135.6,129.2$, 128.9, 128.8, 128.3, 128.2, 128.1, 128.0, 127.9, 127.8, 127.7, 127.6, 126.7, 126.6, 90.4, $90.3(\mathrm{C}=\underline{\mathrm{CH}}$, enol), 54.2, 54.1, 54.0, 53.8, 53.7, 51.3, 50.9, 50.6, 50.5, 45.0, 44.9, $44.7,44.6,35.7,35.3,35.2,34.9,34.8,33.6,33.5,33.2,32.5,32.4$;

$m / z(\mathrm{ESI}) 361.1\left[(\mathrm{M}+\mathrm{Na})^{+}, 100 \%\right]$. 
$N^{1}$-Benzyl- $N^{1}, N^{4}$-dimethyl-2-oxo- $N^{4}$-phenylsuccinamide.

${ }^{1} \mathrm{H}$ NMR $\left(500 \mathrm{MHz}, \mathrm{CDCl}_{3}\right)$.

May05-2021

Chemist Shweta Gediy

PROTON.w CDCl3 /opt/topspin3.2 SKG 32
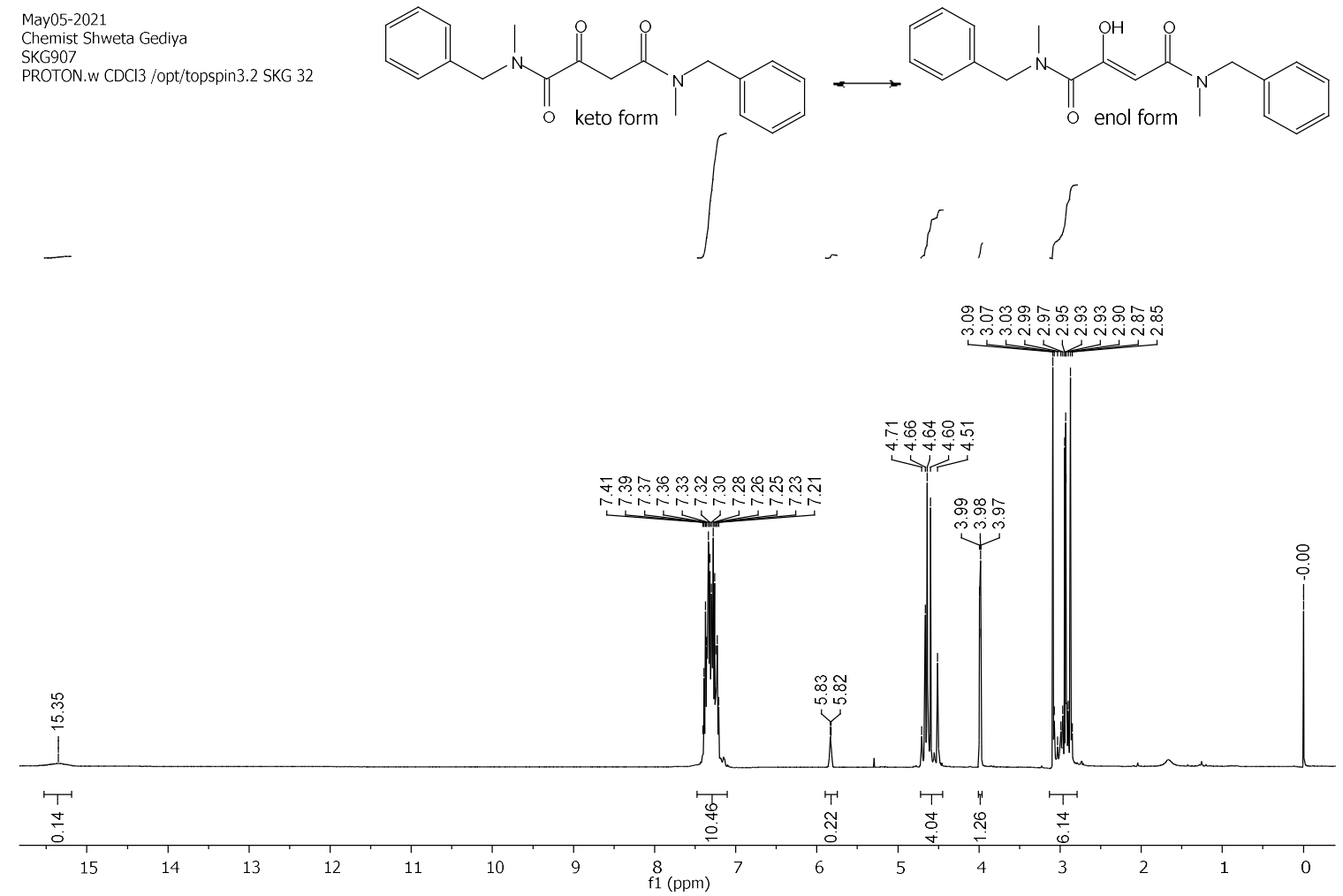

${ }^{13} \mathrm{C} \mathrm{NMR}\left(126 \mathrm{MHz}, \mathrm{CDCl}_{3}\right)$.

May05-2021 SKG907

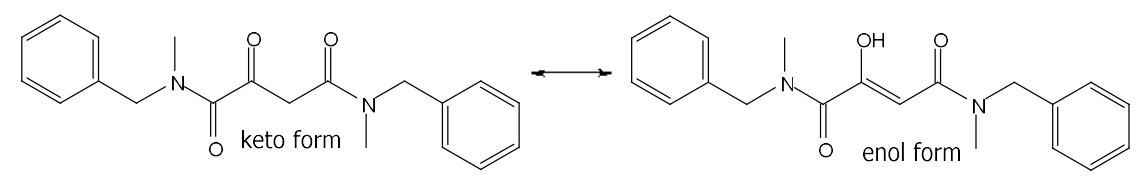

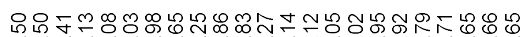

임

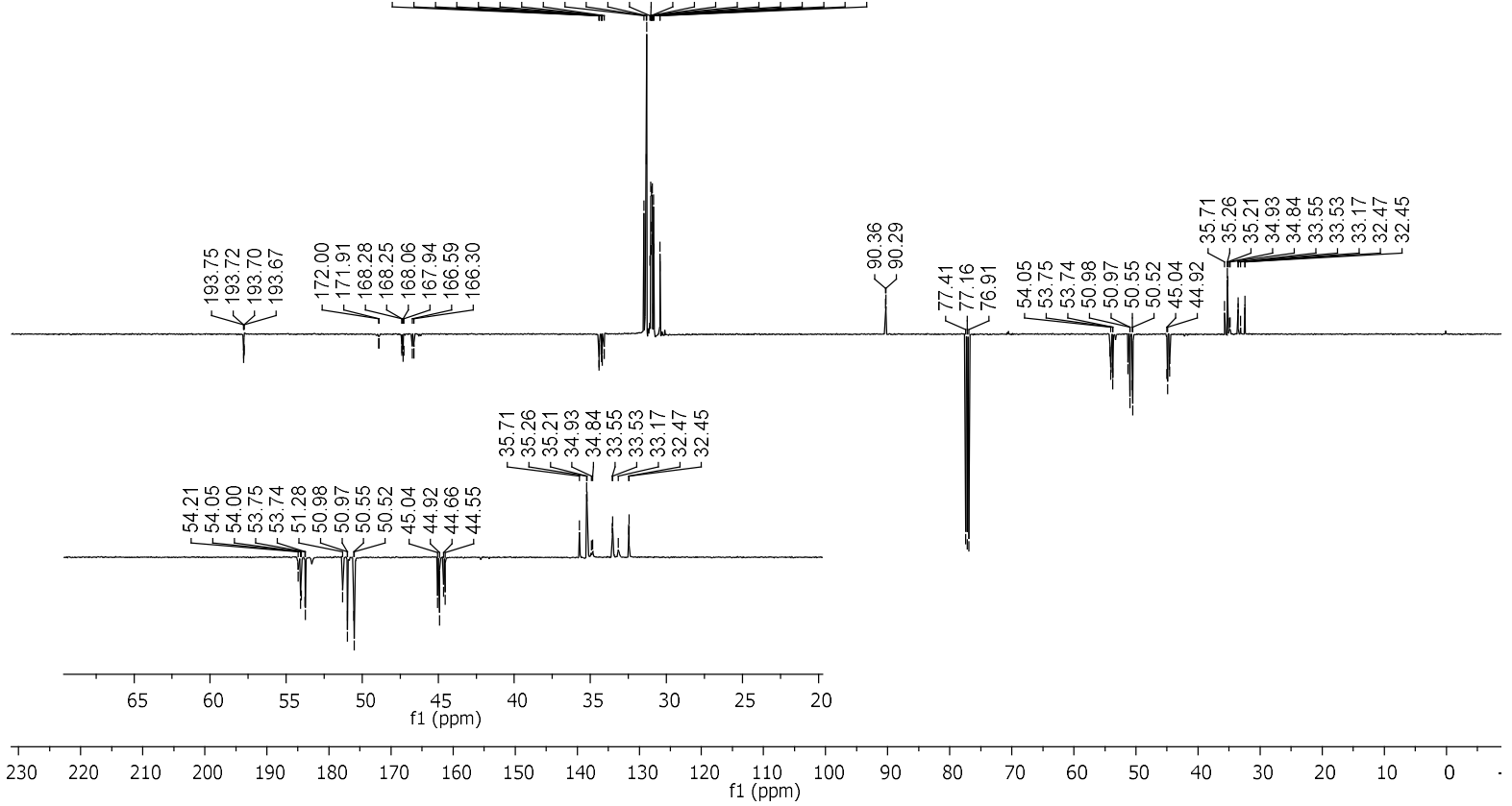


COSY.

Chemist Shweta Gediya

COSY.w CDCl3 /opt/topspin3.2 SKG 32

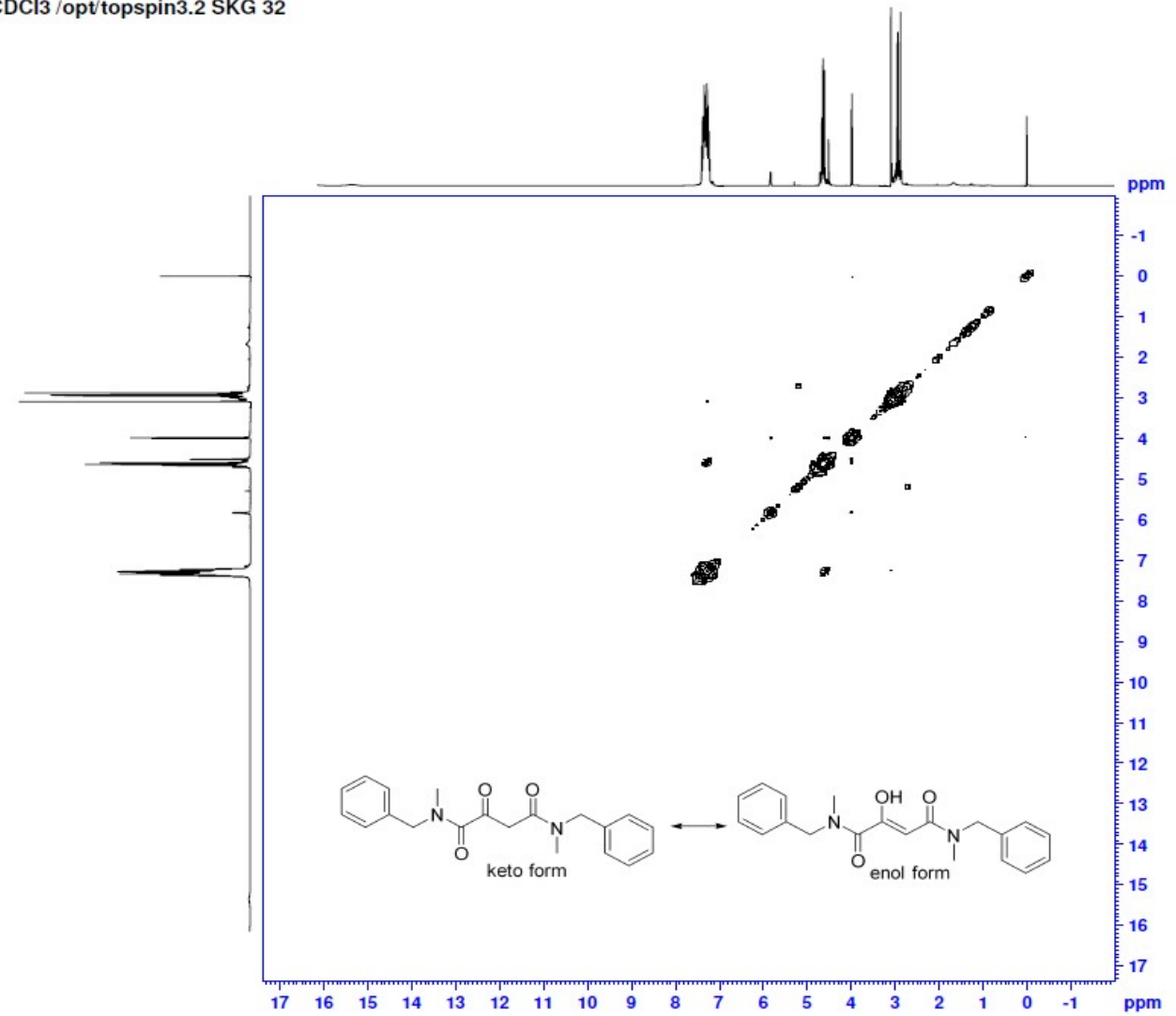

HSQC.

HSOC.w CDCl3 /opt/topspin3.2 SKG 32

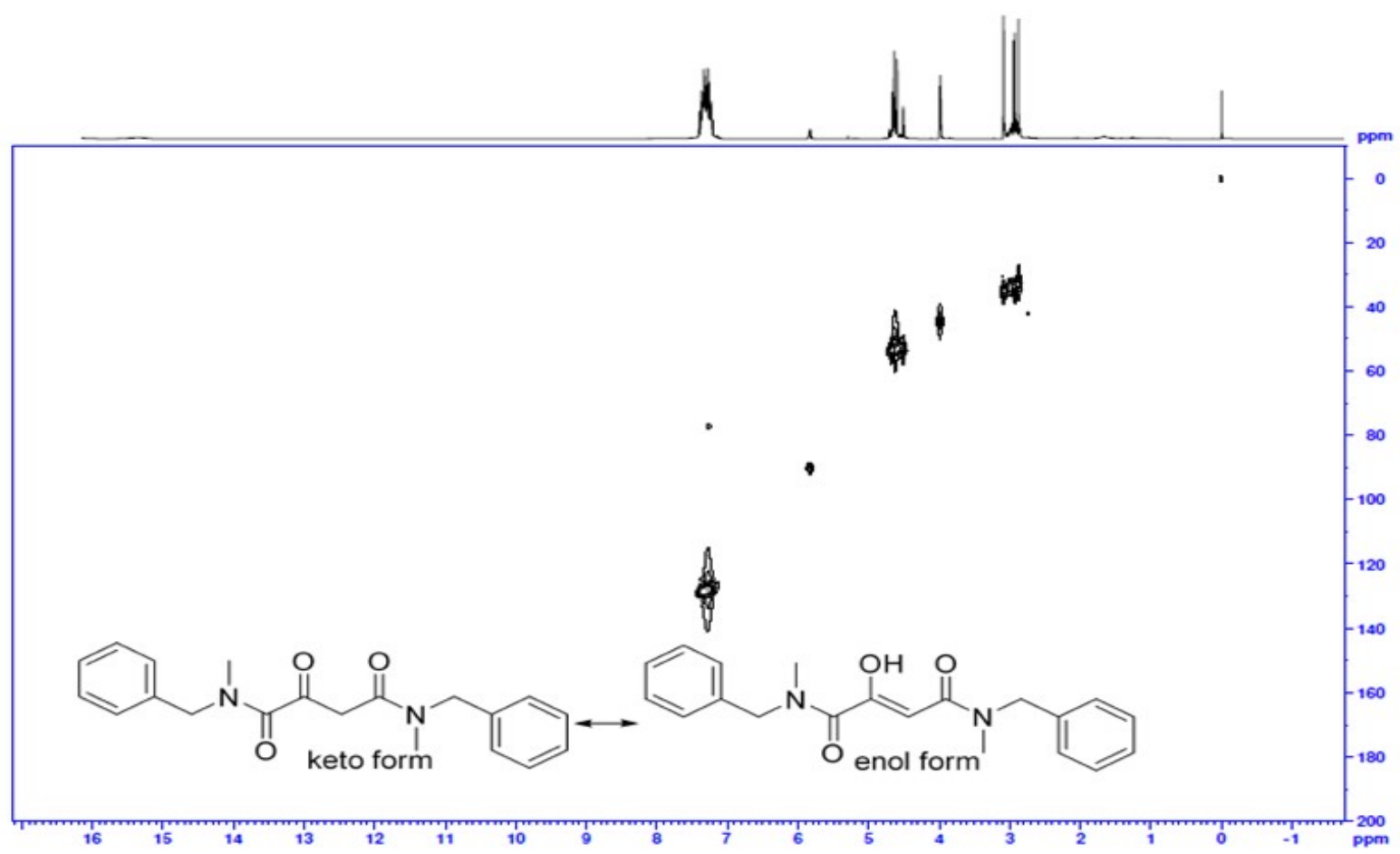


$N^{1}, N^{1}, N^{4}$-Trimethyl-2-oxo- $N^{4}$-phenylsuccinamide (precursor of 8).<smiles>CN(C)C(=O)CC(=O)N(C)c1ccccc1</smiles>

This compound is novel and was prepared following general procedure B using methyl 2(dimethylamino)-2-oxoacetate $(0.500 \mathrm{~g}, 3.81 \mathrm{mmol}, 1.0 \mathrm{eq})$ in anhydrous THF $(8 \mathrm{~mL}), \mathrm{N}$ methyl- $N$-phenylacetamide $(0.568 \mathrm{~g}, 3.81 \mathrm{mmol}, 1.0 \mathrm{eq})$ in anhydrous THF $(8 \mathrm{~mL})$ and LDA (1M solution in THF) $(4.57 \mathrm{~mL}, 4.57 \mathrm{mmol}, 1.2 \mathrm{eq})$ to give crude material which was further purified by column chromatography (50-60\% EtOAc in petroleum ether) to afford as a clear liquid $(0.521 \mathrm{~g}, 2.10 \mathrm{mmol}, 55 \%)$. The title compound was obtained as a keto/enol tautomer (3:1) and rotamers as per $\left.{ }^{1} \mathrm{H} \mathrm{NMR}\right)$.

TLC: $\mathrm{R}_{\mathrm{f}}$ ca 0.3 (6:4, hexane: EtOAc), UV-active and strong $\mathrm{KMnO}_{4}$;

HRMS (ESI) m/z: [M+Na] $]^{+}$Calcd for $\mathrm{C}_{13} \mathrm{H}_{16} \mathrm{~N}_{2} \mathrm{O}_{3} \mathrm{Na} 271.1053$; Found 271.1047 (error 2.1 ppm);

$U_{\max } 3548,2933,1720,1639,1590,1494,1385,1120,1074,771,698,561 \mathrm{~cm}^{-1}$;

${ }^{1} \mathrm{H}$ NMR $\left(500 \mathrm{MHz}, \mathrm{CDCl}_{3}\right)($ keto/enol $(3: 1)+$ rotamers) $\delta 14.60(0.25 \mathrm{H}$, br.s., OH of enol form), 7.46-7.33 (3H, m, ArH), 7.28-7.20 (2H, m, ArH), $5.25(0.25 \mathrm{H}, \mathrm{s},=\mathrm{C} \underline{\mathrm{H}}$, of enol form), $3.59\left(1.5 \mathrm{H}, \mathrm{s}, \mathrm{CH}_{2}\right.$ of keto form), $3.33\left(0.75 \mathrm{H}, \mathrm{s},-\mathrm{NCH}_{3}, \operatorname{minor} 1\right), 3.27\left(2.25 \mathrm{H}, \mathrm{s},-\mathrm{NCH}_{3}\right.$, major 1), $3.13\left(2.25 \mathrm{H}, \mathrm{s},-\mathrm{NCH}_{3}\right.$, major 2), $3.08\left(0.75 \mathrm{H}, \mathrm{s},-\mathrm{NCH}_{3}\right.$, minor 2), $3.00(2.25 \mathrm{H}$, s, $\mathrm{NCH}_{3}$, major 3), $2.92\left(0.75 \mathrm{H}, \mathrm{s},-\mathrm{NCH}_{3}\right.$, minor 3);

${ }^{13} \mathrm{C}$ NMR (126 MHz, $\left.\mathrm{CDCl}_{3}\right)$ (keto/enol (3:2) + rotamers) $\delta 194.2(\mathrm{C}=\mathrm{O}$, keto form), 171.6, $167.7,166.2,165.9,164.7,143.2,142.6,130.2,129.9,128.5,128.2,127.3,127.2,92.1$ $\left(\mathrm{C}=\underline{\mathrm{CH}}\right.$, enol form), $45.2\left(\mathrm{CH}_{2}\right.$ of keto form $), 38.0,37.4,37.0,36.7,35.3,35.2$; $m / z(\mathrm{ESI}) 271.1\left[(\mathrm{M}+\mathrm{Na})^{+}, 100 \%\right]$. 
$N^{1}, N^{1}, N^{4}$-Trimethyl-2-oxo- $\mathrm{N}^{4}$-phenylsuccinamide.

${ }^{1} \mathrm{H}$ NMR $\left(500 \mathrm{MHz}, \mathrm{CDCl}_{3}\right)$.

Apr20-2021
SKG896

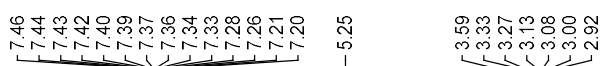

8
$i$
$i$<smiles>COCN(C)c1cccc(I)c1</smiles>

$$
11
$$
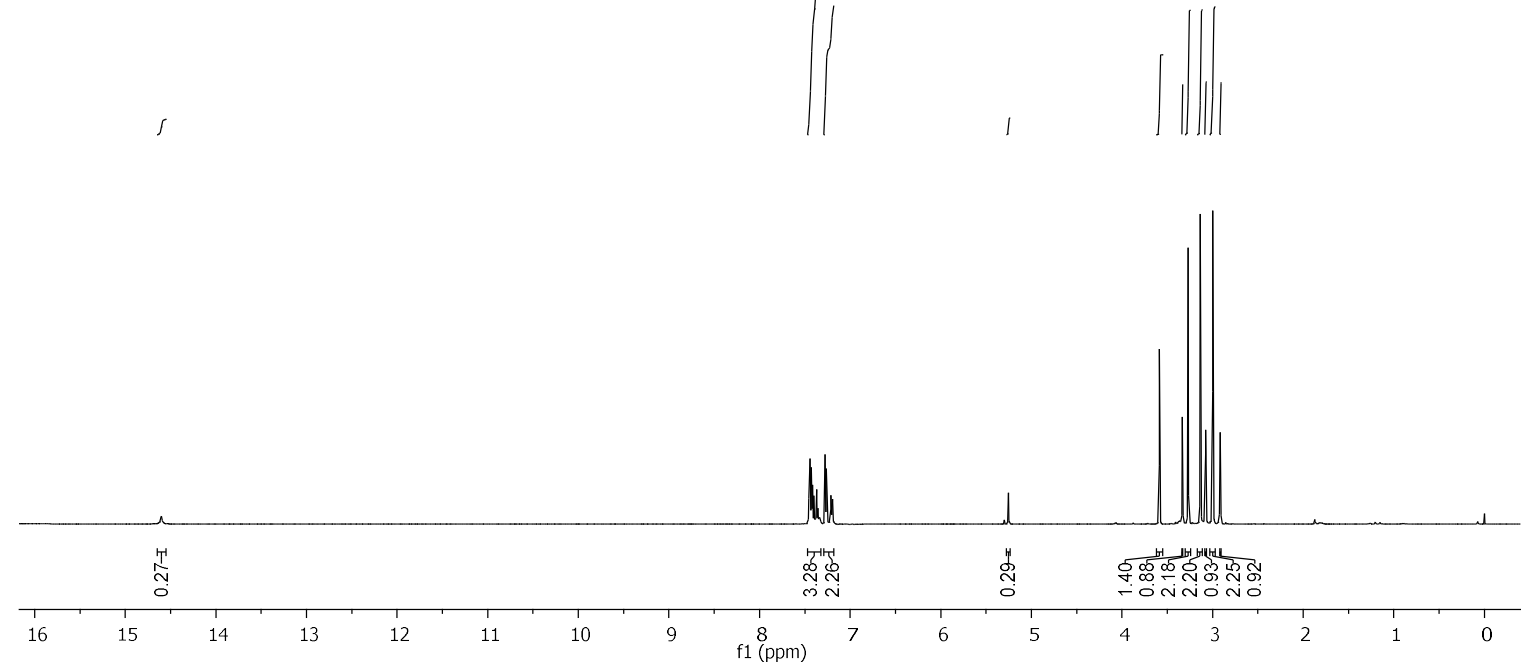

${ }^{13} \mathrm{C} \mathrm{NMR}\left(126 \mathrm{MHz}, \mathrm{CDCl}_{3}\right)$.

\begin{tabular}{|c|c|c|c|c|c|c|}
\hline $\begin{array}{l}\text { Apr20-2021 } \\
\text { SKG896 }\end{array}$ & 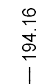 & 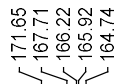 & 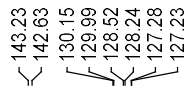 & $\sim$ & 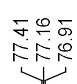 & 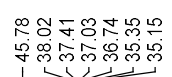 \\
\hline
\end{tabular}<smiles>CCOCN(C)C(=O)/C(O)=C/C(=O)N(C)c1ccccc1</smiles>

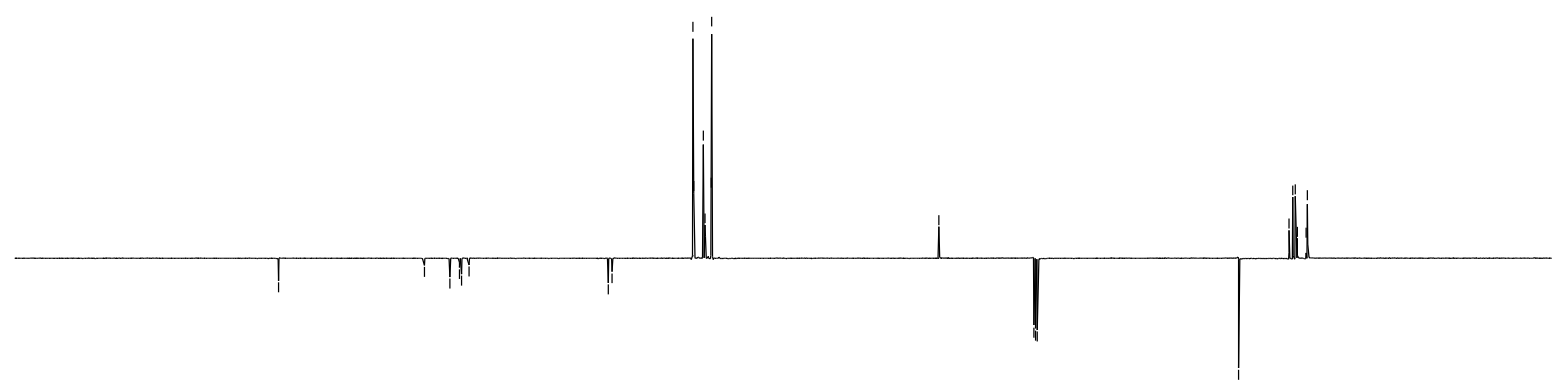

$\begin{array}{lllllllllllllllllllllllllllllllllllll}230 & 220 & 210 & 200 & 190 & 180 & 170 & 160 & 150 & 140 & 130 & 120 & 110 & 100 & 90 & 80 & 70 & 60 & 50 & 40 & 30 & 20 & 10 & 0\end{array}$ 
COSY

Chemist Shweta Gediya

COSY.w CDCl3 /opt/topspin3.2 SKG 58

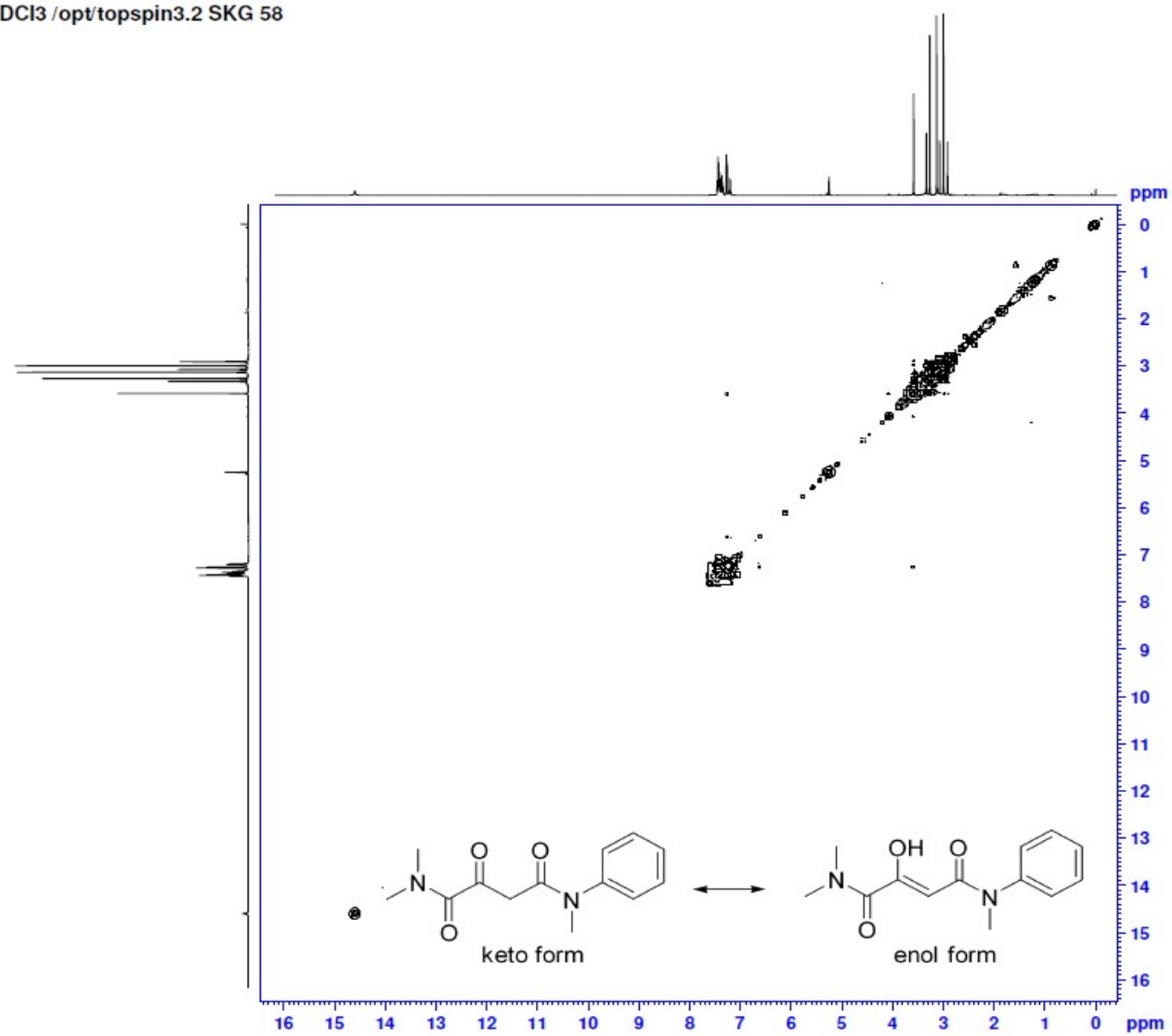

HSQC.

\section{Chemist Shwota Gediya}

HSOC.w CDCl3 /opt/topspin3.2 SKG 58

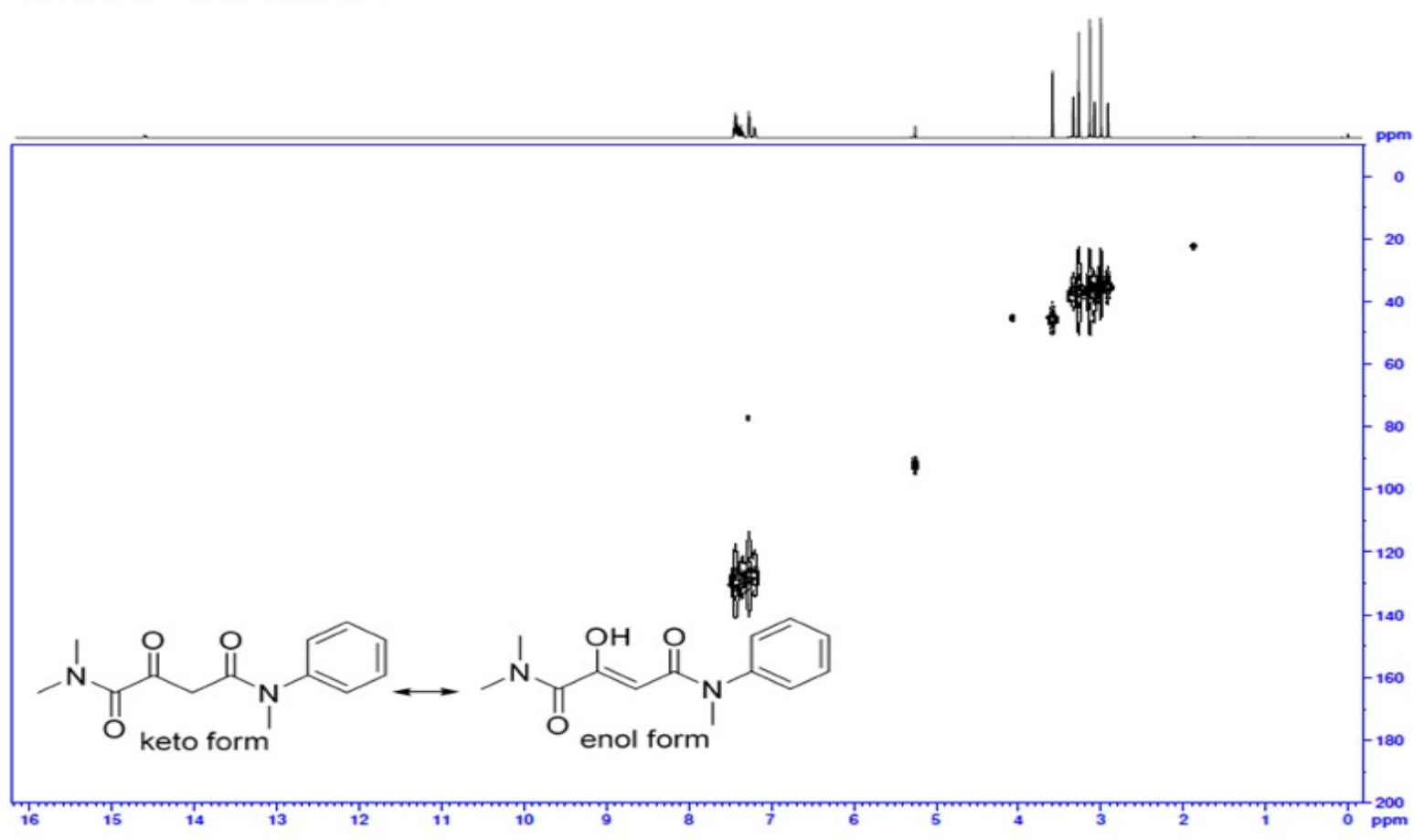




\section{$N^{1}$-Cyclohexyl- $N^{1}, N^{4}$-dimethyl-2-oxo- $N^{4}$-phenylsuccinamide (precursor of 9).}<smiles>CN(C(=O)CC(=O)C(=O)N(C)C1CCCCC1)c1ccccc1</smiles>

This compound is novel and was prepared following the general procedure B using methyl 2(cyclohexyl(methyl)amino)-2-oxoacetate $(0.300 \mathrm{~g}, 1.50 \mathrm{mmol}, 1.0 \mathrm{eq})$ in anhydrous THF (4 $\mathrm{mL}), N$-methyl- $N$-phenylacetamide $(0.317 \mathrm{~g}, 1.65 \mathrm{mmol}, 1.1 \mathrm{eq})$ in anhydrous THF $(4 \mathrm{~mL})$, and LDA (1M solution in THF) $(1.95 \mathrm{~mL}, 1.95 \mathrm{mmol}, 1.3 \mathrm{eq})$ to give crude material which was further purified by column chromatography (30\% EtOAc in petroleum ether) to afford the product as a clear liquid $(0.210 \mathrm{~g}, 0.664 \mathrm{mmol}, 44 \%)$. The title compound was obtained as a keto/enol tautomer (7:3) and rotamers as per ${ }^{1} \mathrm{H}$ NMR.

TLC: $\mathrm{R}_{\mathrm{f}}$ ca 0.3 (7:3, hexane: EtOAc), UV-active and strong $\mathrm{KMnO}_{4}$;

HRMS (ESI) m/z: [M+Na] ${ }^{+}$Calcd for $\mathrm{C}_{18} \mathrm{H}_{24} \mathrm{~N}_{2} \mathrm{O}_{3} \mathrm{Na}$ 339.1679; Found 339.1675 (error 1.2 ppm);

$U_{\max } 2930,2856,1718,1629,1591,1382,1331,1119,1065,698 \mathrm{~cm}^{-1}$;

${ }^{1} \mathrm{H}$ NMR $\left(500 \mathrm{MHz}, \mathrm{CDCl}_{3}\right)($ keto/enol $(7: 3)+$ rotamers $) \delta 14.59(0.1 \mathrm{H}, \mathrm{s}, \mathrm{OH}$ enol, minor rotamer 1), $14.43(0.2 \mathrm{H}, \mathrm{s}, \mathrm{OH}$ enol, major rotamer 2$), 7.45-7.34(3 \mathrm{H}, \mathrm{m}, \mathrm{ArH}), 7.28-7.19$ $(2 \mathrm{H}, \mathrm{m}, \mathrm{ArH}), 5.19(0.1 \mathrm{H}, \mathrm{s},=\mathrm{CH}$ of enol form, minor rotamer 1$), 5.13(0.2 \mathrm{H}, \mathrm{s},=\mathrm{CH}$ of enol form, major rotamer 2), 4.29-4.23 $(0.36 \mathrm{H}, \mathrm{m}, \mathrm{CH}$ of cyclohexylamine), 3.80-3.58 $(0.41 \mathrm{H}, \mathrm{m}$, $\mathrm{CH}$ of cyclohexylamine) $3.58\left(1.4 \mathrm{H} \mathrm{s}, \mathrm{CH}_{2}\right.$ of keto form $), 3.34\left(1 \mathrm{H}, \mathrm{s}, \mathrm{NCH}_{3}-\right.$ minor rotamer 1), $3.27\left(2 \mathrm{H}, \mathrm{s}, \mathrm{NCH}_{3}\right.$ - major rotamer 2), $2.96\left(1 \mathrm{H}, \mathrm{s}, \mathrm{NCH}_{3}\right.$ - major rotamer 2), $2.89(0.5 \mathrm{H}$, $\mathrm{s}, \mathrm{NCH}_{3}$ - minor rotamer 1), $2.86\left(1 \mathrm{H}, \mathrm{s}, \mathrm{NCH}_{3}-\right.$ major rotamer 2$), 2.77\left(0.5 \mathrm{H}, \mathrm{s}, \mathrm{NCH}_{3}-\right.$ minor rotamer 1), 1.91-1.02 (10H, $\mathrm{m}, \mathrm{CH}_{2}$ of cyclohexylamine);

${ }^{13} \mathrm{C}$ NMR $\left(126 \mathrm{MHz}, \mathrm{CDCl}_{3}\right)(($ keto/enol $(7: 3)+$ rotamers $)) \delta 194.6(\mathrm{C}=\mathrm{O}$, keto form $), 194.6$ $(\mathrm{C}=\mathrm{O}$, keto form), 171.8, 171.7, 167.6, 167.3, 167.2, 166.9, 166.4, 165.8, 164.9, 164.5, 143.5, $143.4,142.8,142.7,130.2,130.0,128.5,128.4,128.3,127.4,127.3,91.6$ ( $\mathrm{C}=\underline{\mathrm{C}} H$, enol form), $91.3\left(\mathrm{C}=\underline{\mathrm{CH}}\right.$, enol form), 57.9, 57.1, 53.2, 53.1, $46.2\left(\mathrm{CH}_{2}\right.$ keto form $), 45.9\left(\mathrm{CH}_{2}\right.$ keto form), $37.2,37.1,36.8,36.7,30.9,30.6,29.9,29.5,29.3,27.3,27.1,25.7,25.6,25.5,25.4$; $m / z(\mathrm{ESI}) 339.2\left[(\mathrm{M}+\mathrm{Na})^{+}, 100 \%\right]$. 


\section{$N^{1}$-Cyclohexyl- $N^{1}, N^{4}$-dimethyl-2-oxo- $N^{4}$-phenylsuccinamide.}

${ }^{1} \mathrm{H}$ NMR $\left(500 \mathrm{MHz}, \mathrm{CDCl}_{3}\right)$.

SKG667
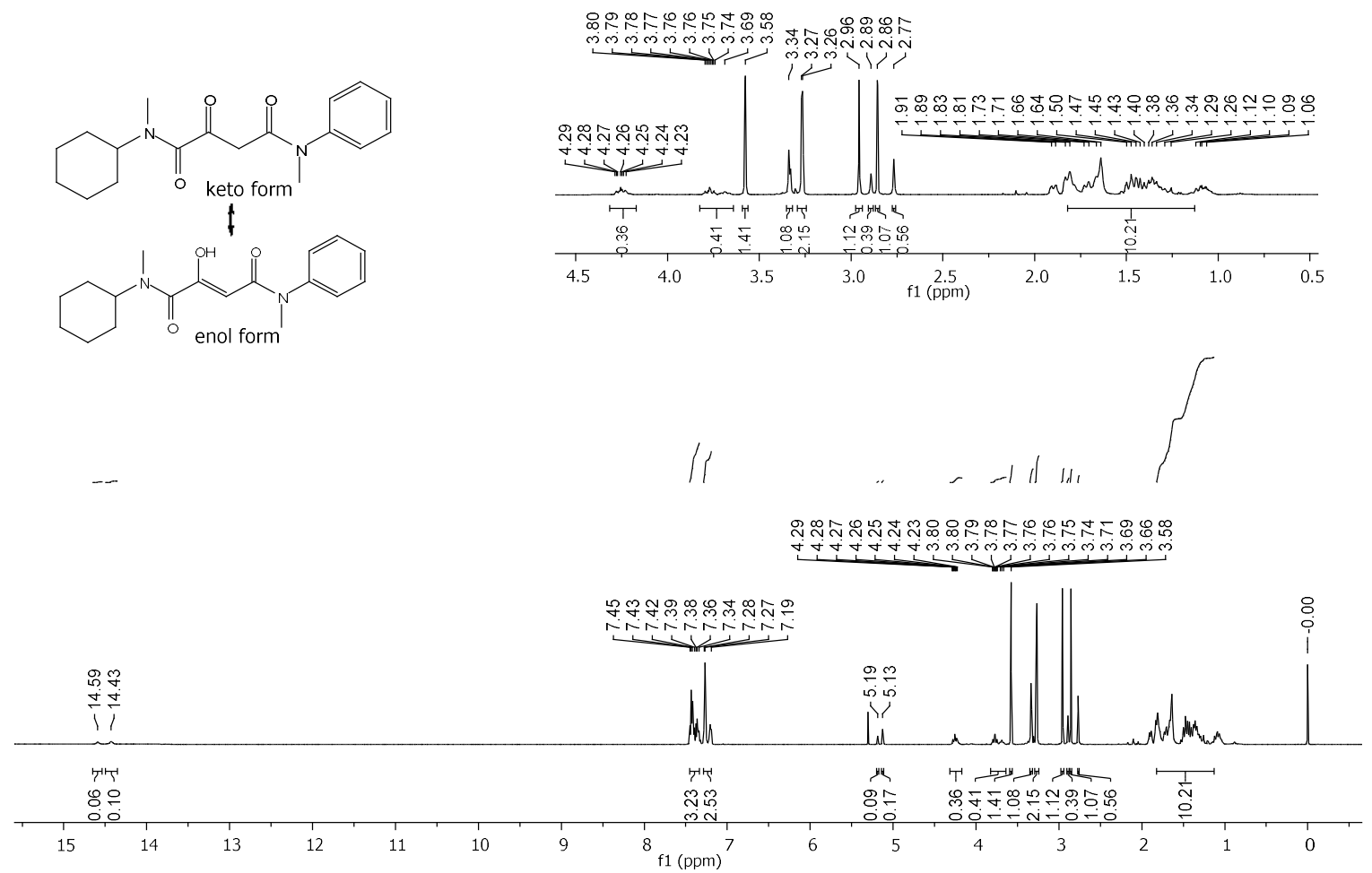

${ }^{13} \mathrm{C}$ NMR (126 MHz, $\left.\mathrm{CDCl}_{3}\right)$.

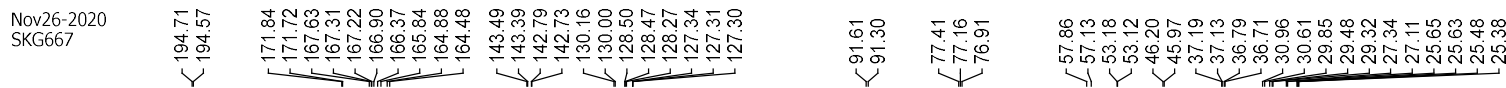<smiles>COCN(C(=O)CC(=O)C(=O)N(C)C1CCCCC1)c1cccc(C)c1</smiles>

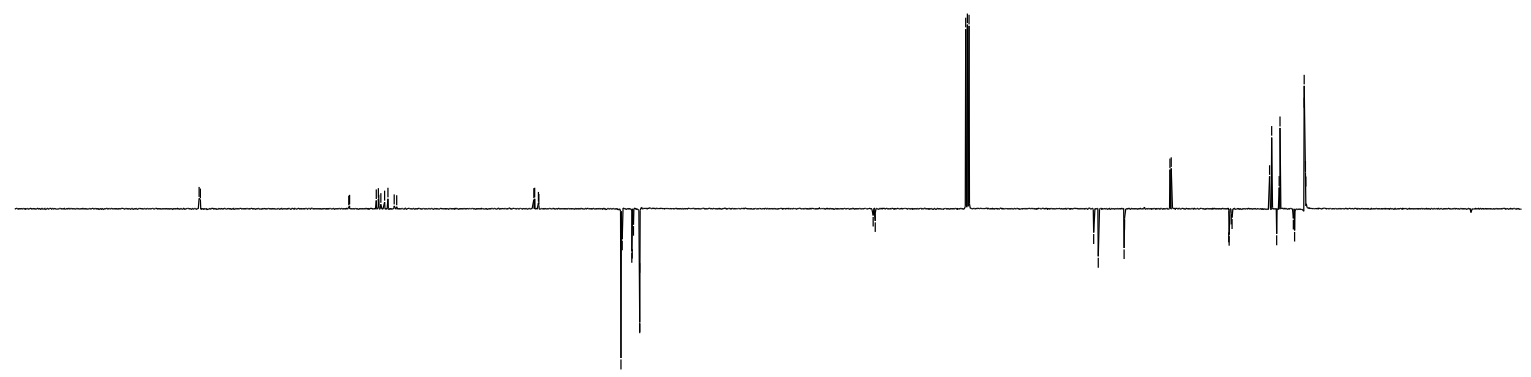

$\begin{array}{lllllllllllllllllllllllll}1 & 220 & 210 & 200 & 190 & 180 & 170 & 160 & 150 & 140 & 130 & 120 & 110 & 100 & 90 & 80 & 70 & 60 & 50 & 40 & 30 & 20 & 10 & 0\end{array}$ 
COSY.

Chemist Shweta Gediya

COSY.w CDCl3 /opt/topspin3.2 SKG 53

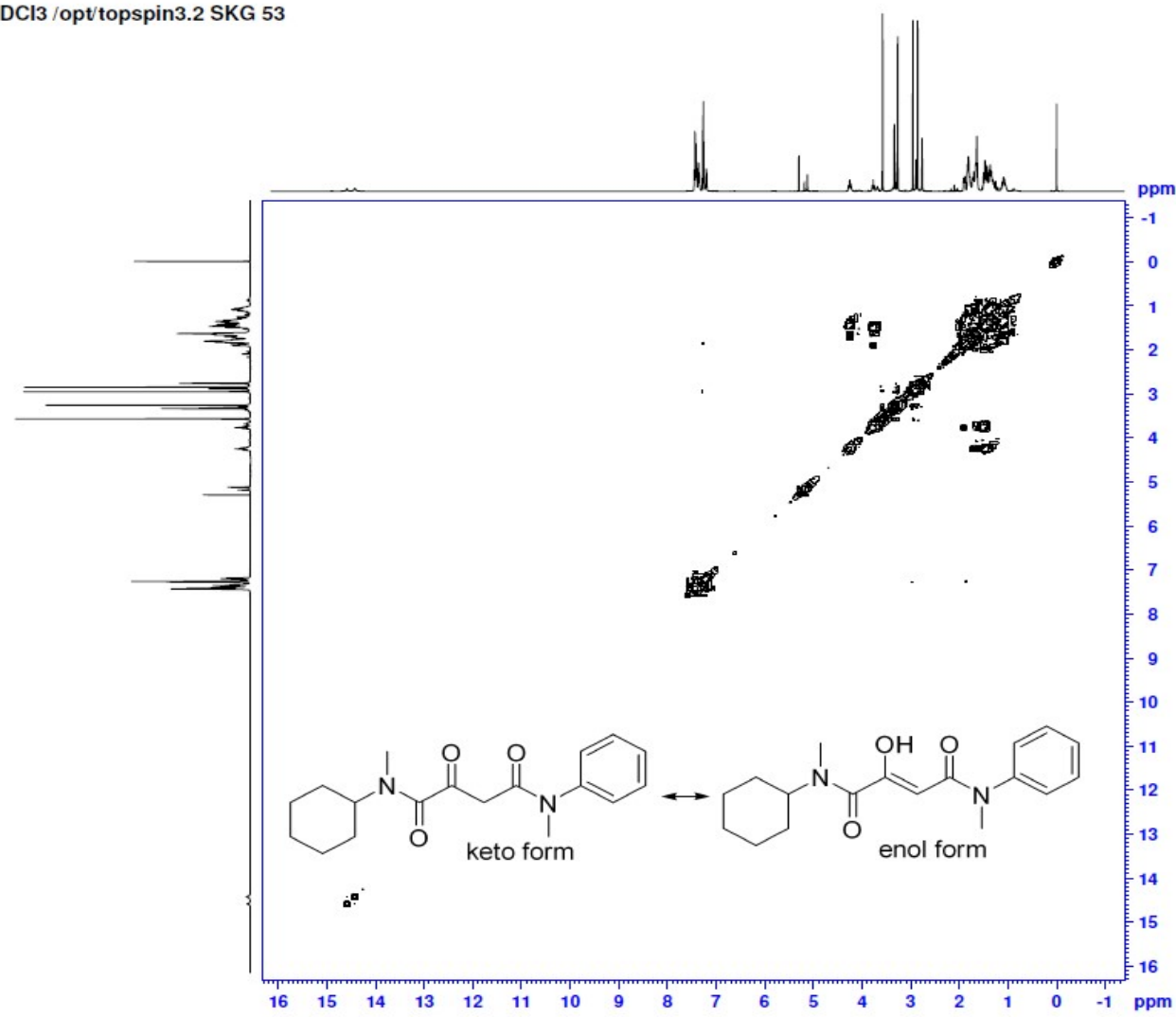

HSQC.

\section{Chemist Shweta Gediya}

HSQC.w CDCl3 /opt/topspin3.2 SKG 53

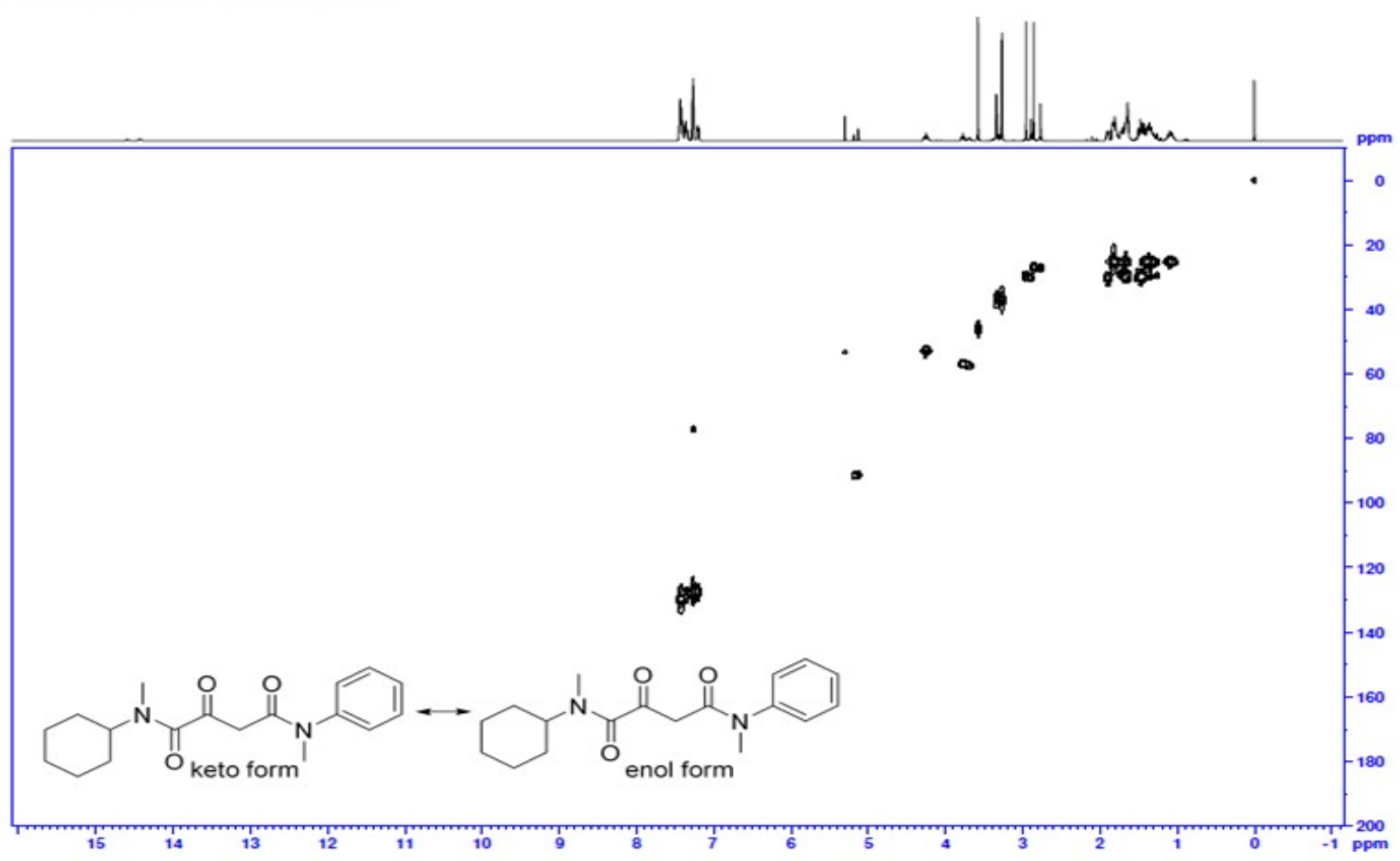


$N^{1}, N^{4}$-Dicyclohexyl- $N^{1}, N^{4}$-dimethyl-2-oxosuccinamide (precursor of 10 ).<smiles>CN(C(=O)CC(=O)C(=O)N(C)C1CCCCC1)C1CCCCC1</smiles>

This compound is novel and was prepared following general procedure B using methyl 2(cyclohexyl(methyl)amino)-2-oxoacetate $(0.300 \mathrm{~g}, 1.50 \mathrm{mmol}, 1.0 \mathrm{eq})$ in anhydrous THF (4 $\mathrm{mL}), N$-cyclohexyl- $N$-methylacetamide $(0.255 \mathrm{~g}, 1.65 \mathrm{mmol}, 1.1 \mathrm{eq})$ in anhydrous THF (4 $\mathrm{mL})$, and LDA (1M solution in THF) (1.95 mL, $1.95 \mathrm{mmol}, 1.3 \mathrm{eq})$ to give crude material which was further purified by column chromatography (50\% EtOAc in petroleum ether) to afford the product as a yellow oil $(0.235 \mathrm{~g}, 0.729 \mathrm{mmol}, 49 \%)$. The title compound was obtained as a keto/enol tautomer $(2: 1)$ and rotamers as per ${ }^{1} \mathrm{H}$ NMR.

TLC: $\mathrm{R}_{\mathrm{f}}$ ca 0.4 (4:6, hexane: EtOAc), UV-active and strong $\mathrm{KMnO}_{4}$;

HRMS (ESI) m/z: [M+Na] $]^{+}$Calcd for $\mathrm{C}_{18} \mathrm{H}_{30} \mathrm{~N}_{2} \mathrm{O}_{3} \mathrm{Na} 345.2149$; Found 345.2137 (error 3.3 ppm);

$U_{\max } 2927,2854,1718,1628,1446,1406,1322,1088,1064,893 \mathrm{~cm}^{-1}$;

${ }^{1} \mathrm{H}$ NMR $\left(500 \mathrm{MHz}, \mathrm{CDCl}_{3}\right)$ (keto/enol $(2: 1)+$ rotamers) $\delta 15.84(0.33 \mathrm{H}$, br.s., OH of enol form), 5.66-5.54 $(0.33 \mathrm{H}, \mathrm{m},=\mathrm{CH}$ of enol form $), 4.40-4.20(1 \mathrm{H}, \mathrm{m}, \mathrm{NCH}$ of cyclohexyl group), 3.86-3.81 (2H, m, $\mathrm{CH}_{2}$ of keto form + $\mathrm{NCH}$ of cyclohexyl group), 3.39- 3.36 (0.33, m, $\mathrm{NCH}$ of cyclohexyl group), $2.95\left(1 \mathrm{H}, \mathrm{s}, \mathrm{NCH}_{3}\right.$, rotamer 1), $2.93\left(0.5 \mathrm{H}, \mathrm{NCH}_{3}\right.$ rotamer 2), 2.83-2.75 (4.5H, m, $-\mathrm{NCH}_{3}$, rotamer 1 and 2), 1.86-1.05 (20H, m, $\mathrm{CH}_{2}$ of cyclohexyl group); ${ }^{13} \mathrm{C}$ NMR $\left(126 \mathrm{MHz}, \mathrm{CDCl}_{3}\right)(\mathrm{keto} / \mathrm{enol}(2: 1)+$ rotamers $) \delta 194.5(\mathrm{C}=\mathrm{O}$ keto $), 194.4(\mathrm{C}=\mathrm{O}$ keto), 171.6, 171.4, 168.8, 168.6, 167.2, 167.1, 166.7, 166.6, 166.5, 166.4, 165.9, 165.8, 90.0 $(\mathrm{C}=\underline{\mathrm{C}} \mathrm{H}$ enol form), 89.8, $89.5(\mathrm{C}=\underline{\mathrm{CH}}$, enol form), 89.4, 57.7, 57.6, 57.5, 57.0, 56.9, 53.2, 53.1, 52.2, 52.1, 46.1, 45.5, 45.3, 44.7, 30.9, 30.8, 30.6, 30.5, 30.0, 29.9, 29.8, 29.5, 29.3, $27.0,26.9,25.7,25.6,25.5,25.4,25.3,25.2$;

$m / z(\mathrm{ESI}) 345.2\left[(\mathrm{M}+\mathrm{Na})^{+}, 100 \%\right]$. 
$N^{1}, N^{4}$-Dicyclohexyl- $N^{1}, N^{4}$-dimethyl-2-oxosuccinamide.

${ }^{1} \mathrm{H}$ NMR $\left(500 \mathrm{MHz}, \mathrm{CDCl}_{3}\right)$.

Nov30-2020

Chemist Shweta Gediya

PROTON.w CDCl3 /opt/topspin3.2 SKG 60
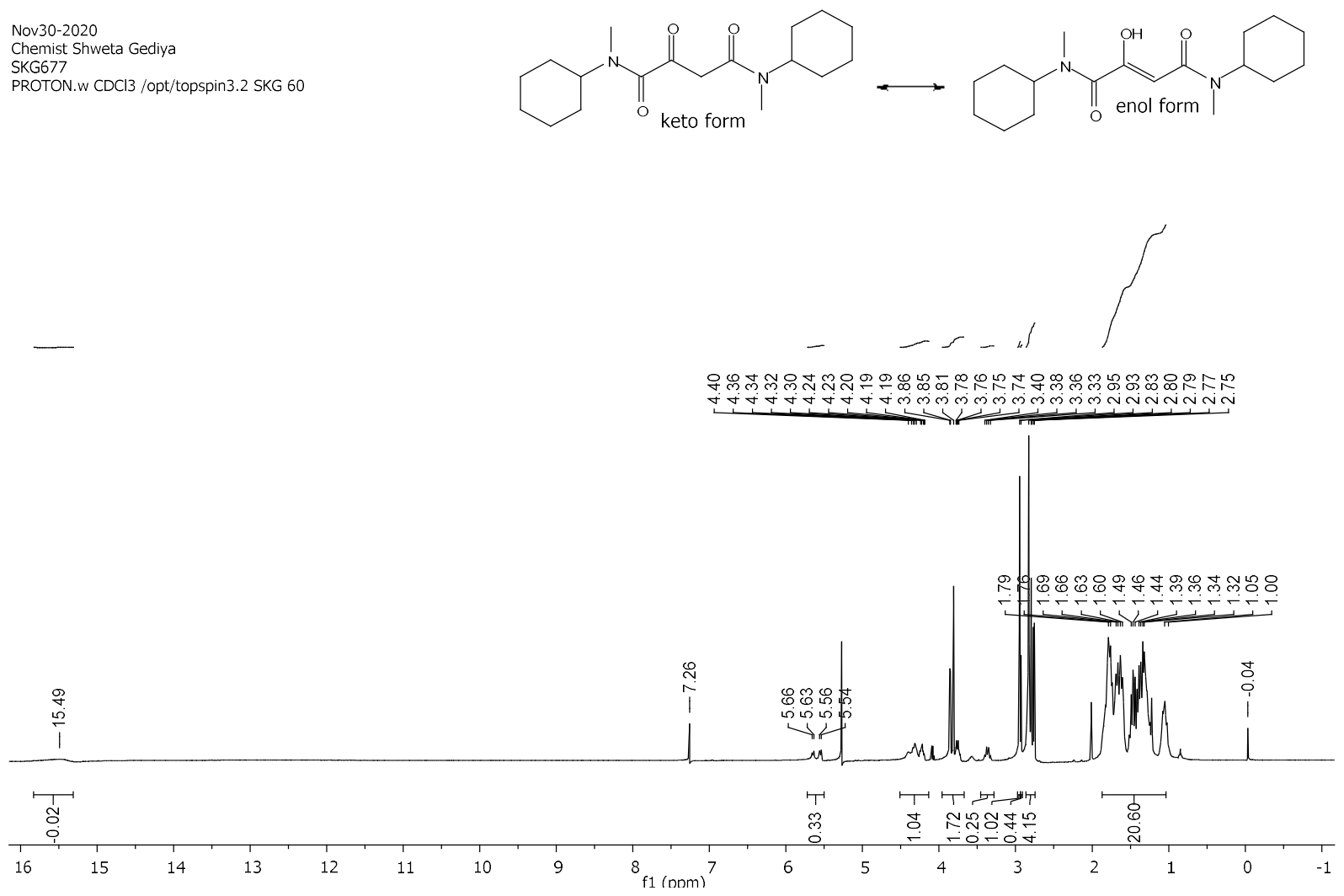

${ }^{13} \mathrm{C}$ NMR (126 MHz, $\left.\mathrm{CDCl}_{3}\right)$.

Nov30-2020

SKG677

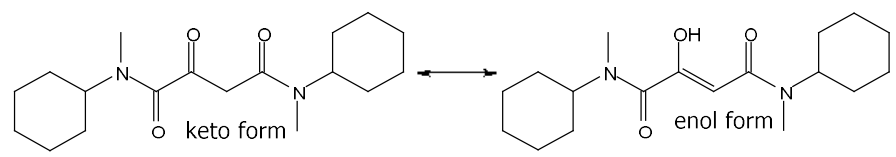

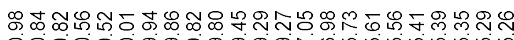

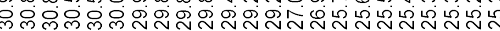
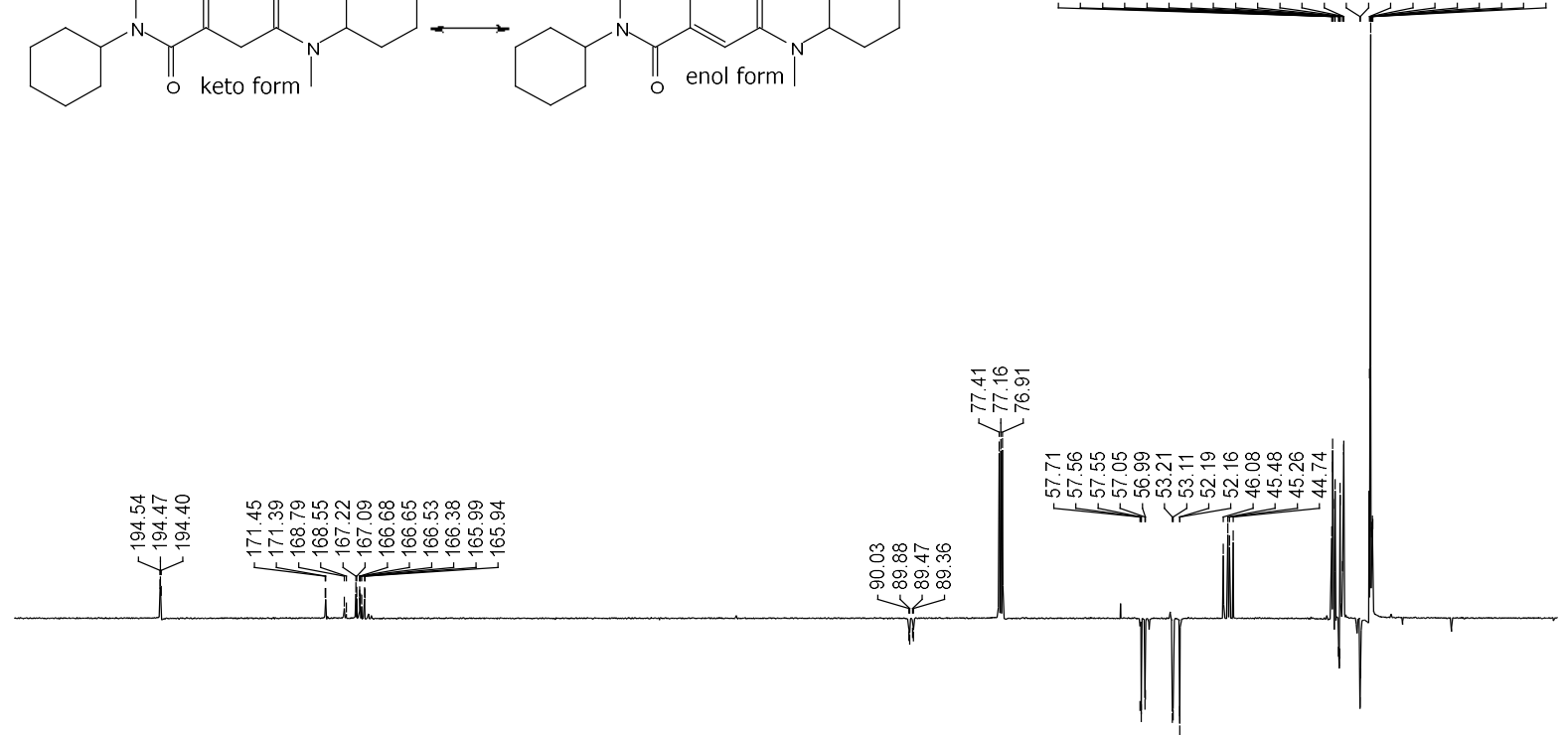

$\begin{array}{llllllllllllllllllllllll}210 & 200 & 190 & 180 & 170 & 160 & 150 & 140 & 130 & 120 & 110 & 100 & 90 & 80 & 70 & 60 & 50 & 40 & 30 & 20 & 10 & 0\end{array}$ 
COSY.

Chemist Shweta Gediya

COSY.w $\mathrm{CDCl}$ /opt/topspin3.2 SKG 60

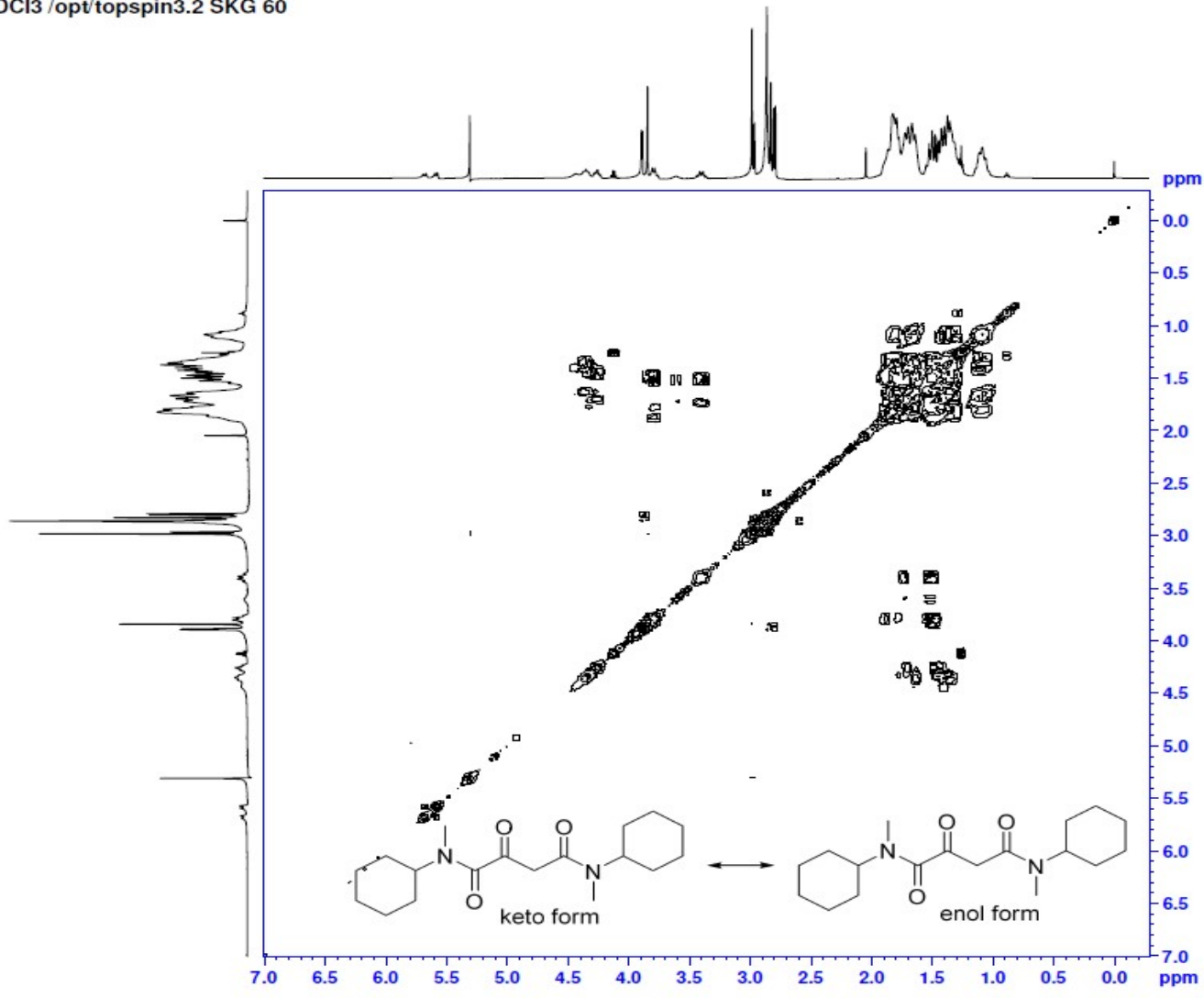

HSQC.

Chemist Shweta Cediya

HSOC.w CDCl3 /opt/topspin3.2 SKG 60

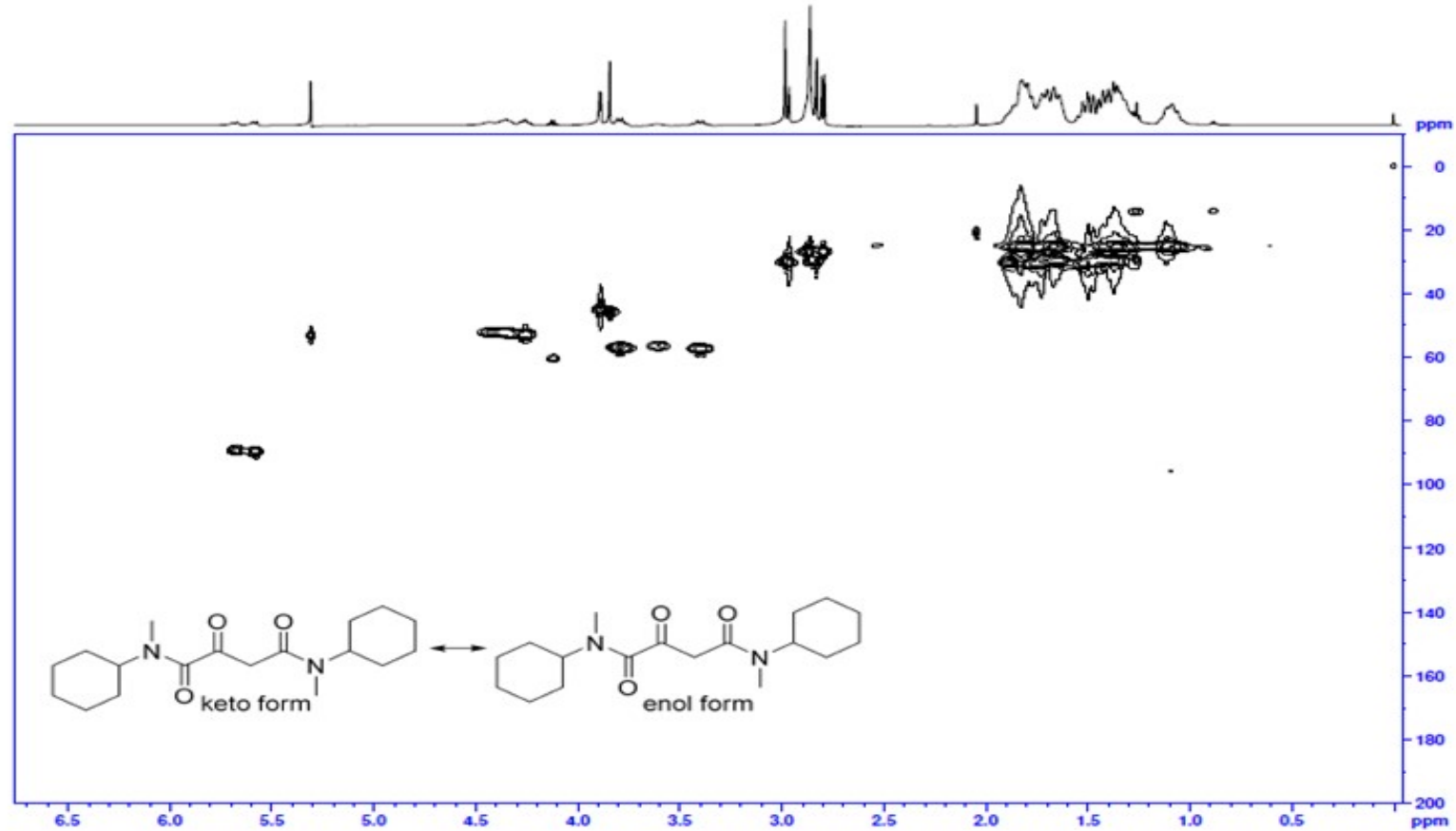


$N^{1}$-Allyl- $N^{1}, N^{4}$-dimethyl-2-oxo- $N^{4}$-phenylsuccinamide (precursor of 11).<smiles>C=CCN(C)C(=O)C(=O)CC(=O)N(C)c1ccccc1</smiles>

This compound is novel and was prepared following general procedure B using methyl 2(allyl(methyl)amino)-2-oxoacetate $(0.400 \mathrm{~g}, 2.54 \mathrm{mmol}, 1.0 \mathrm{eq})$ in anhydrous THF $(5 \mathrm{~mL})$, $N$-methyl- $N$-phenylacetamide $(0.417 \mathrm{~g}, 2.80 \mathrm{mmol}, 1.1 \mathrm{eq})$ in anhydrous THF $(5 \mathrm{~mL})$ and LDA (1M solution in THF) $(3.30 \mathrm{~mL}, 3.30 \mathrm{mmol}, 1.3 \mathrm{eq})$ to give crude material which was further purified by column chromatography $(20-50 \%$ EtOAc in petroleum ether) to afford the product as a yellow liquid $(0.429 \mathrm{~g}, 1.56 \mathrm{mmol}, 61 \%)$. The title compound was obtained as a keto/enol tautomer $(1: 1)$ and rotamers as per ${ }^{1} \mathrm{H}$ NMR.

TLC: $\mathrm{R}_{\mathrm{f}}$ ca 0.3 (5:5, hexane: EtOAc), UV-active and strong $\mathrm{KMnO}_{4}$;

HRMS (ESI) m/z: [M+Na] ${ }^{+}$Calcd for $\mathrm{C}_{15} \mathrm{H}_{18} \mathrm{~N}_{2} \mathrm{O}_{3} \mathrm{Na}$ 297.1209; Found 297.1209 (error 0.1 ppm);

$U_{\max } 2972,2929,1720,1636,1590,1494,1336,1293,1119,1067,925,698 \mathrm{~cm}^{-1}$;

${ }^{1} \mathrm{H}$ NMR $\left(500 \mathrm{MHz}, \mathrm{CDCl}_{3}\right)$ (keto/enol $(1: 1)+$ rotamers $) \delta 14.63-14.56(0.5 \mathrm{H}, \mathrm{m}, \mathrm{OH}$ of enol form), 7.46-7.33 (3H, m, ArH), 7.28-7.26 (1H, m, ArH), 7.21-7.18 (1H, m, ArH), 5.94 $5.67\left(1 \mathrm{H}, \mathrm{m}, \mathrm{CH}_{2}=\mathrm{C}_{\underline{H C H}}\right)$, 5.28-5.12 $\left(2.5 \mathrm{H}, \mathrm{m}, \mathrm{C}_{2}=\mathrm{CHCH}_{2}+=\mathrm{CH}\right.$ of enol form), 4.02$3.94\left(2 \mathrm{H}, \mathrm{m}, \mathrm{CH}_{2}=\mathrm{CHCH}_{2}\right), 3.60-3.59\left(1 \mathrm{H}, \mathrm{s}, \mathrm{CH}_{2}\right.$ of keto form $), 3.33\left(1 \mathrm{H}, \mathrm{s}, \mathrm{NCH}_{3}\right.$-minor, rotamer 1), $3.27\left(2 \mathrm{H}, \mathrm{s}, \mathrm{NCH}_{3}\right.$-major, rotamer 2), 3.08 (1H, s, $\mathrm{NCH}_{3}$ - major, rotamer 2), 3.02 $\left(0.5 \mathrm{H}, \mathrm{s}, \mathrm{NCH}_{3}\right.$ - minor, rotamer 1), $2.93\left(1 \mathrm{H}, \mathrm{s}, \mathrm{NCH}_{3}\right.$ - major, rotamer 2), $2.85(0.5 \mathrm{H}, \mathrm{s}$, $\mathrm{NCH}_{3}$ - minor, rotamer 1);

${ }^{13} \mathrm{C}$ NMR (126 MHz, $\left.\mathrm{CDCl}_{3}\right) \delta$ (keto/enol, (1:1) + rotamers) $194.1(\mathrm{C}=\mathrm{O}$, keto form), 194.0 $(\mathrm{C}=\mathrm{O}$ keto form $), 171.7,171.6,167.7,167.6,166.2,166.1,165.9,165.8,164.9,164.5,143.2$, $142.6,133.2$, 132.0, 131.5, 130.1, 130.0, 129.9, 138.5, 128.3, 127.3, 127.2, 118.7, 118.2, 118.1, 117.9, 92.2( $\mathrm{C}=\underline{\mathrm{C}} \mathrm{H}$ enol form $), 92.0(\mathrm{C}=\underline{\mathrm{C}} \mathrm{H}$ enol form $), 53.2,52.8,50.2,49.9,45.9$, 45.8;

$m / z(\mathrm{ESI}) 297.2\left[(\mathrm{M}+\mathrm{Na})^{+}, 100 \%\right]$. 
$N^{1}$-Allyl- $N^{1}, N^{4}$-dimethyl-2-oxo- $N^{4}$-phenylsuccinamide.

${ }^{1} \mathrm{H}$ NMR $\left(500 \mathrm{MHz}, \mathrm{CDCl}_{3}\right)$.

SKG647

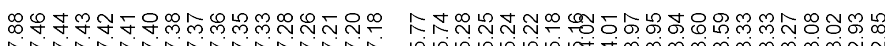

Й

$\stackrel{8}{0}$
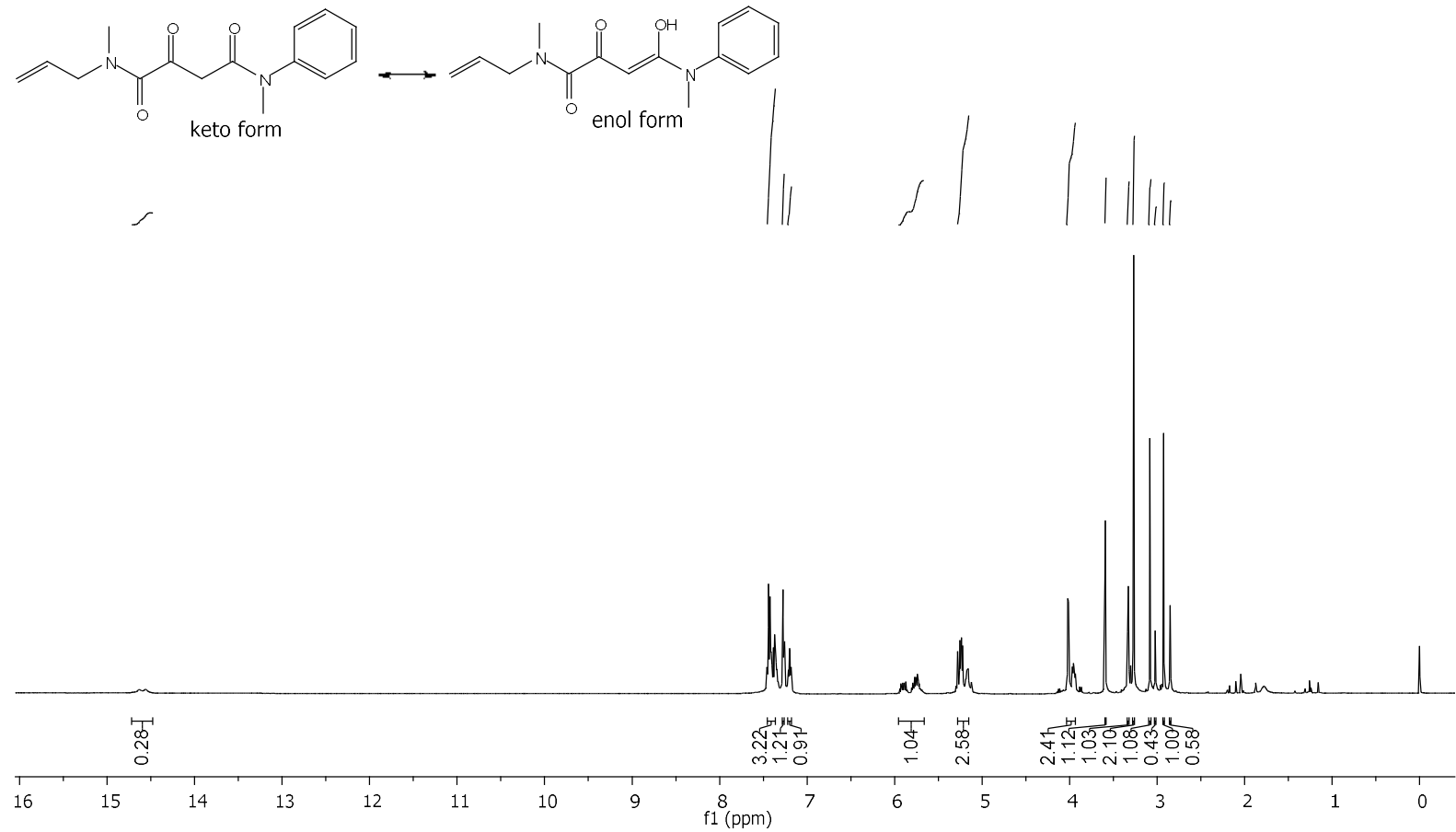

${ }^{13} \mathrm{C}$ NMR (126 MHz, $\left.\mathrm{CDCl}_{3}\right)$.

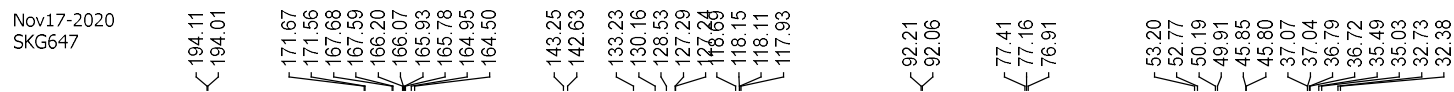

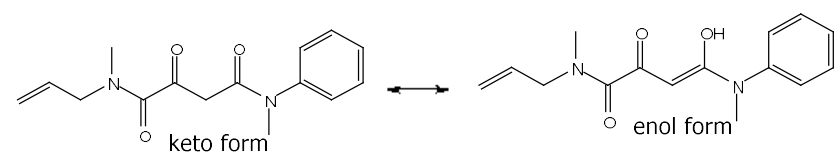

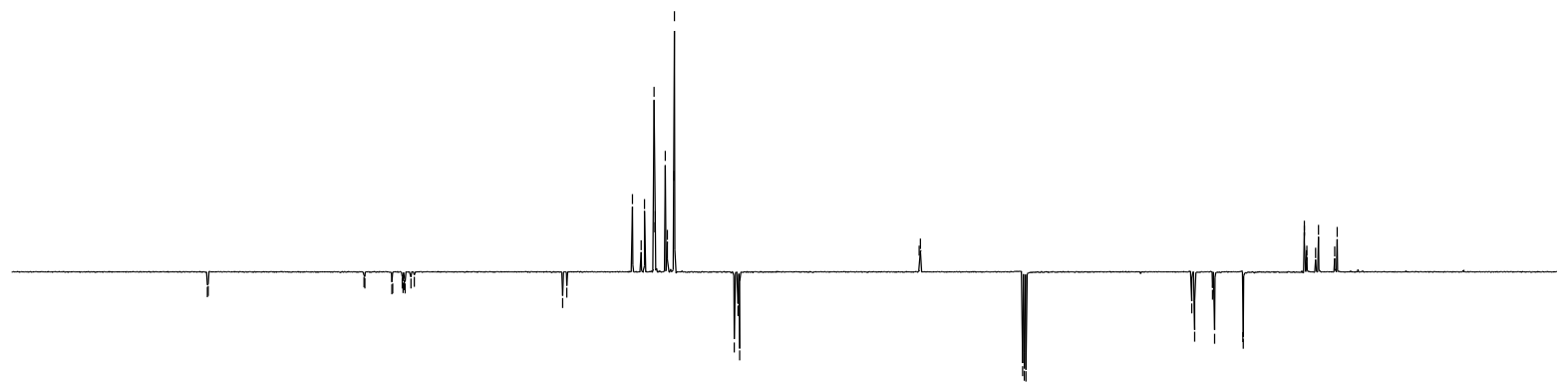

$\begin{array}{lllllllllllllllllllllllllll}120 & 210 & 200 & 190 & 180 & 170 & 160 & 150 & 140 & 130 & 120 & 110 & 100 & 90 & 80 & 70 & 60 & 50 & 40 & 30 & 20 & 10 & 10\end{array}$ 
COSY.

Chemist Shweta Gediya

COSY.w $\mathrm{CDCl}$ /opt/topspin3.2 SKG 12

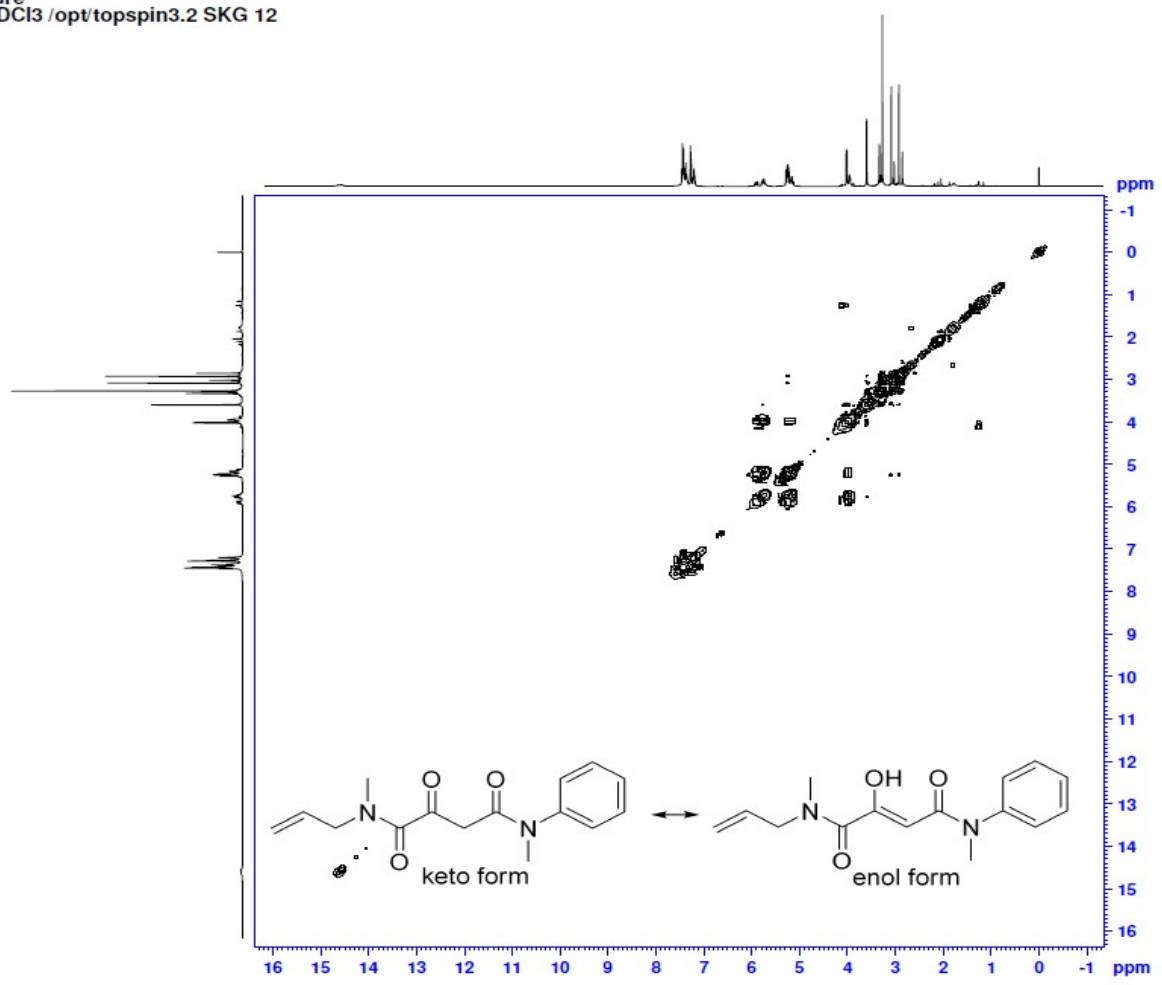

HSQC.

Chemist Shweta Gediya

HSQC.w CDCl3 /opt/topspin3.2 SKG 12

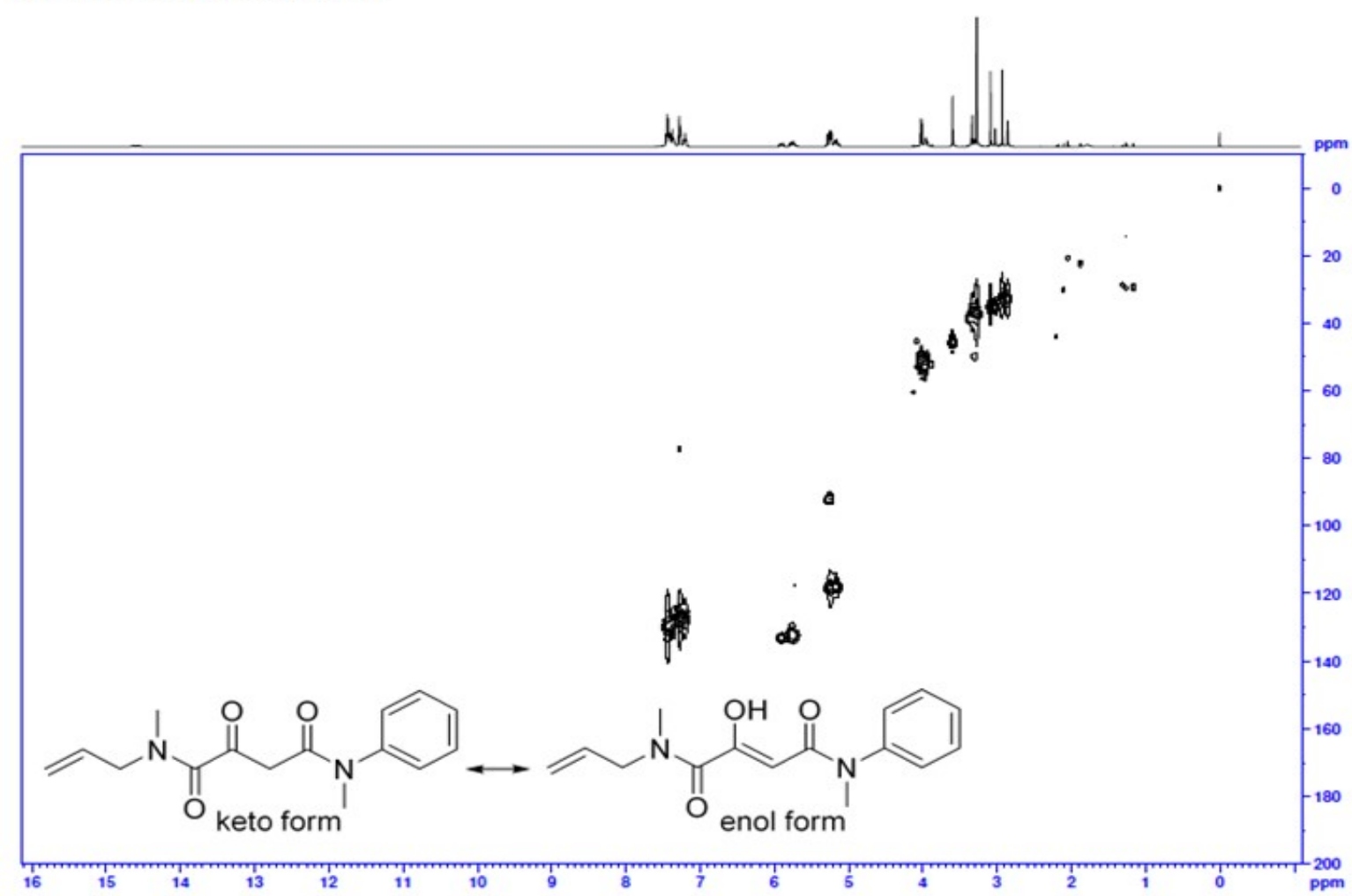


$N^{1}, N^{4}$-Dimethyl-2-oxo- $N^{4}$-phenyl- $N^{1}$-(prop-2-yn-1-yl)succinimide (precursor of 12).<smiles>C#CCN(C)C(=O)C(=O)CC(=O)N(C)c1ccccc1</smiles>

This compound is novel and was prepared following general procedure B using methyl 2(methyl(prop-2-yn-1-yl)amino)-2-oxoacetate (0.300 g, $1.93 \mathrm{mmol}, 1.0 \mathrm{eq})$ in anhydrous THF (4 mL), $N$-methyl- $N$-phenylacetamide $(0.317 \mathrm{~g}, 2.12 \mathrm{mmol}, 1.1 \mathrm{eq})$ in anhydrous THF (4 $\mathrm{mL})$, and LDA (1M solution in THF) $(2.50 \mathrm{~mL}, 2.50 \mathrm{mmol}, 1.3 \mathrm{eq})$ to give crude material which was further purified by column chromatography (50\% EtOAc in petroleum ether) to afford the product as a clear liquid $(0.261 \mathrm{~g}, 0.959 \mathrm{mmol}, 50 \%)$. The title compound was obtained as a keto/enol tautomer $(1: 1)$ and as rotamers as per ${ }^{1} \mathrm{H}$ NMR.

TLC: $\mathrm{R}_{\mathrm{f}}$ ca 0.3 (4:6, hexane: EtOAc), UV-active and strong $\mathrm{KMnO}_{4}$;

HRMS (ESI) m/z: [M+Na] $]^{+}$Calcd for $\mathrm{C}_{15} \mathrm{H}_{16} \mathrm{~N}_{2} \mathrm{O}_{3} \mathrm{Na}$ 295.1053; Found 295.1051 (error 0.9 ppm);

$U_{\max } 3285,3251,2929,1721,1640,1590,1494,1384,1070,772,697 \mathrm{~cm}^{-1}$;

${ }^{1} \mathrm{H}$ NMR $\left(500 \mathrm{MHz}, \mathrm{CDCl}_{3}\right)$ (keto/enol $(1: 1)+$ rotamers) $\delta$ 14.71-14.66 $(0.5 \mathrm{H}, \mathrm{m}, \mathrm{OH}$ of enol form), 7.44-7.19 (5H, m, ArH), 5.35-5.33 $(0.5 \mathrm{H}, \mathrm{m},=\mathrm{C} \underline{\mathrm{H}}$, of enol form), 4.27-4.16 (2H, m, $\mathrm{CH} \equiv \mathrm{CC}_{2}$ ) 3.60-3.59 (1H, m, $\mathrm{CH}_{2}$ of keto form), 3.34 (1H, s, $\mathrm{NCH}_{3}$ - major, rotamer 1), 3.30 $\left(0.5 \mathrm{H}, \mathrm{s}, \mathrm{NCH}_{3}\right.$ - minor, rotamer 2$), 3.27\left(2 \mathrm{H}, \mathrm{s}, \mathrm{NCH}_{3}\right.$ - major, rotamer 1), $3.20\left(1 \mathrm{H}, \mathrm{s}, \mathrm{NCH}_{3}\right.$ - minor, rotamer 2), $3.16\left(0.5 \mathrm{H}, \mathrm{s}, \mathrm{NCH}_{3}\right.$ - minor, rotamer 2$), 3.07\left(0.5 \mathrm{H}, \mathrm{s}, \mathrm{NCH}_{3}\right.$ - minor, rotamer 2), $2.98\left(0.5 \mathrm{H}, \mathrm{s}, \mathrm{NCH}_{3}-\right.$ minor, rotamer 2$), 2.35-2.17\left(1 \mathrm{H}, \mathrm{m}, \mathrm{C} \underline{\mathrm{H}} \equiv \mathrm{CCH}_{2}\right)$;

${ }^{13} \mathrm{C}$ NMR $\left(126 \mathrm{MHz}, \mathrm{CDCl}_{3}\right) \delta$ (keto/enol $(1: 1)+$ rotamers) $193.6(\mathrm{C}=\mathrm{O}$, keto form), 193.5 $(\mathrm{C}=\mathrm{O}$ keto form $), 171.6,171.5,170.7,167.7,167.6,165.5,165.4,165.3,164.9,164.4,143.2$, 143.1, $142.5130 .2,130.0,129.8,128.7,128.6,127.4,127.3,92.7$ ( $\mathrm{C}=\underline{\mathrm{C}} \mathrm{H}$, enol form), 73.1, $72.8,72.5,45.8,45.7,39.5,37.1,36.8,36.7,36.5,35.2,34.7$;

$m / z(\mathrm{ESI}) 297.2\left[(\mathrm{M}+\mathrm{Na})^{+}, 100 \%\right]$. 
$N^{1}, N^{4}$-Dimethyl-2-oxo- $N^{4}$-phenyl- $N^{1}$-(prop-2-yn-1-yl)succinamide.

${ }^{1} \mathrm{H}$ NMR $\left(500 \mathrm{MHz}, \mathrm{CDCl}_{3}\right)$.

Nov25-2020

Chemist Shweta Gediya

SKG655

PROTON.w CDCl3 /opt/topspin3.2 SKG 50
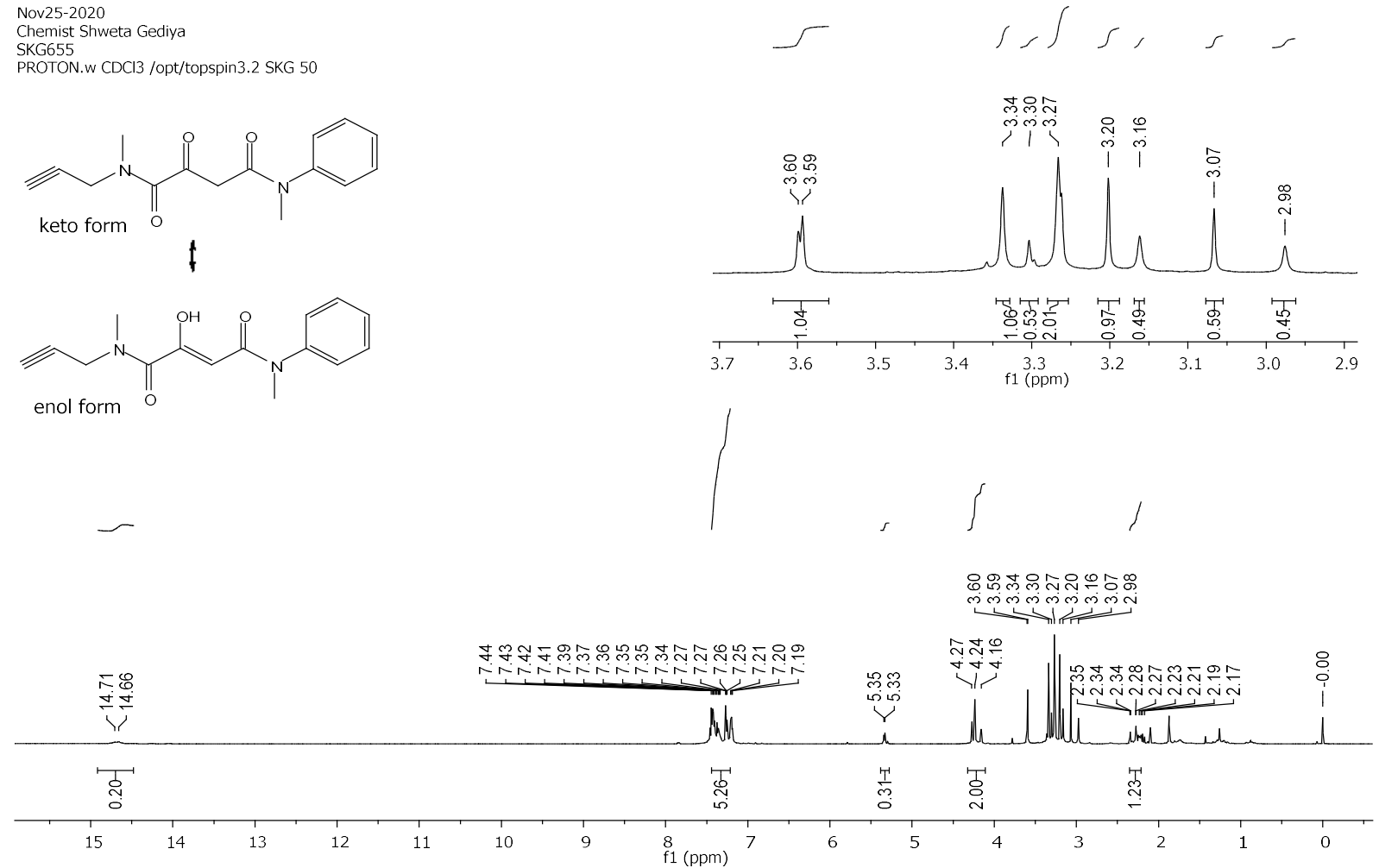

${ }^{13} \mathrm{C}$ NMR (126 MHz, $\left.\mathrm{CDCl}_{3}\right)$.

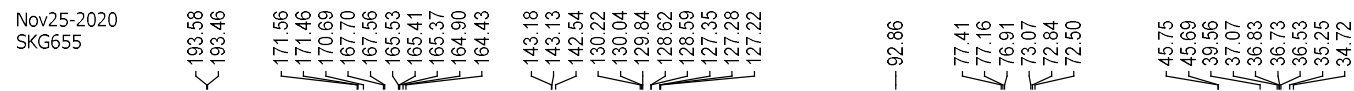<smiles>C#CCN(C)C(=O)C(=O)CC(=O)N(C)c1cccc(CC#CCN(C)C(=O)/C(O)=C/C(=O)N(C)c2ccccc2)c1</smiles>

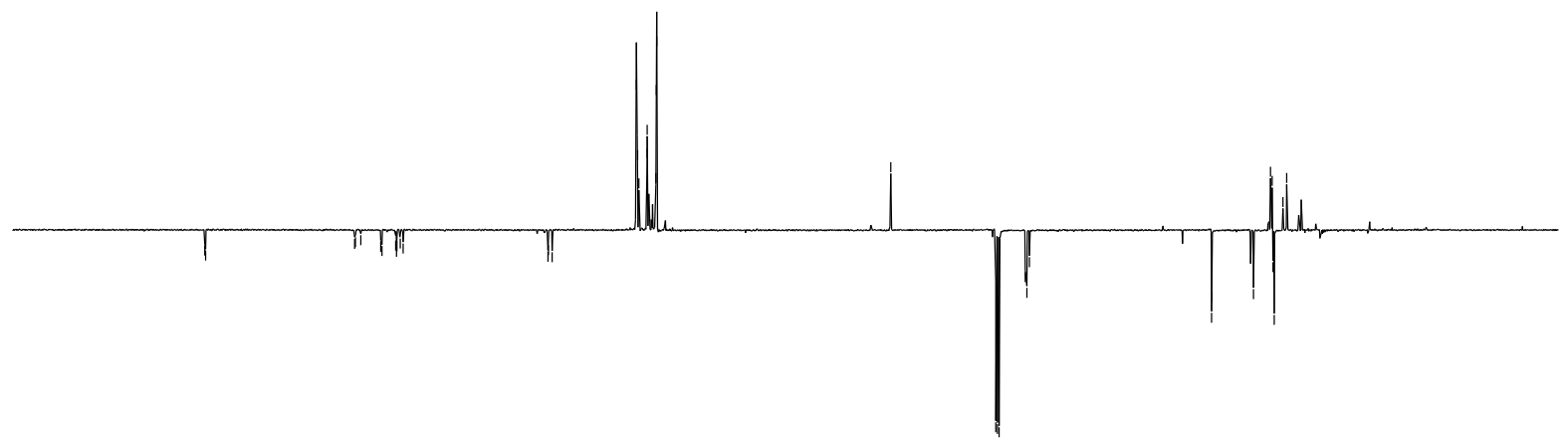

$\begin{array}{llllllllllllllllllllllllll}220 & 210 & 200 & 190 & 180 & 170 & 160 & 150 & 140 & 130 & 120 & \begin{array}{l}110 \\ \mathrm{f} 1(\mathrm{ppm})\end{array} & 100 & 90 & 80 & 70 & 60 & 50 & 40 & 30 & 20 & 10 & 0\end{array}$ 
COSY.

Chemist Shweta Gediya

COSY.w CDCl3 /opt/topspin3.2 SKG 50

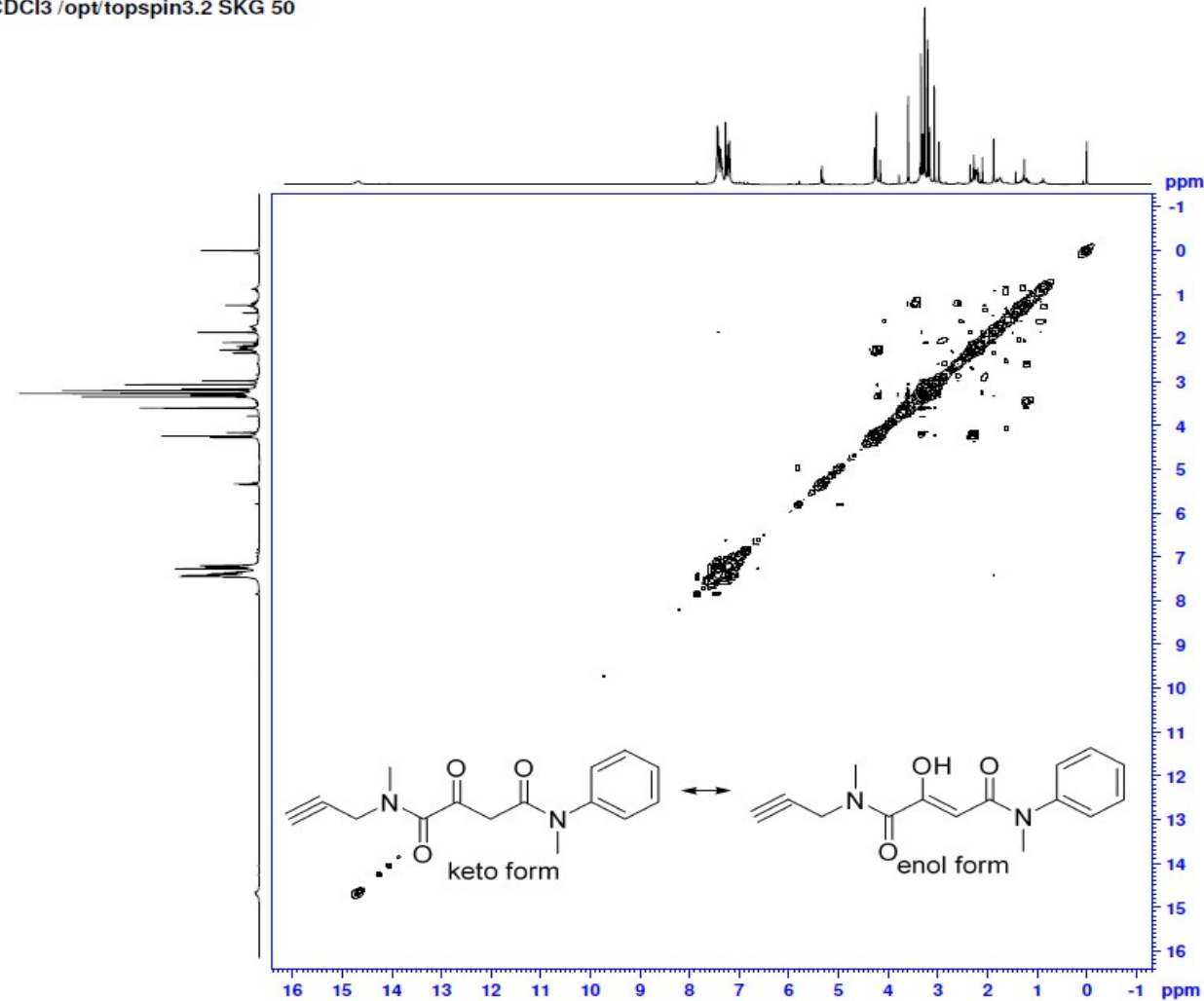

HSQC.

Chemist Shweta Gediya

HSG6.w CDCI3 /opttopspin 3.2 SKG 50

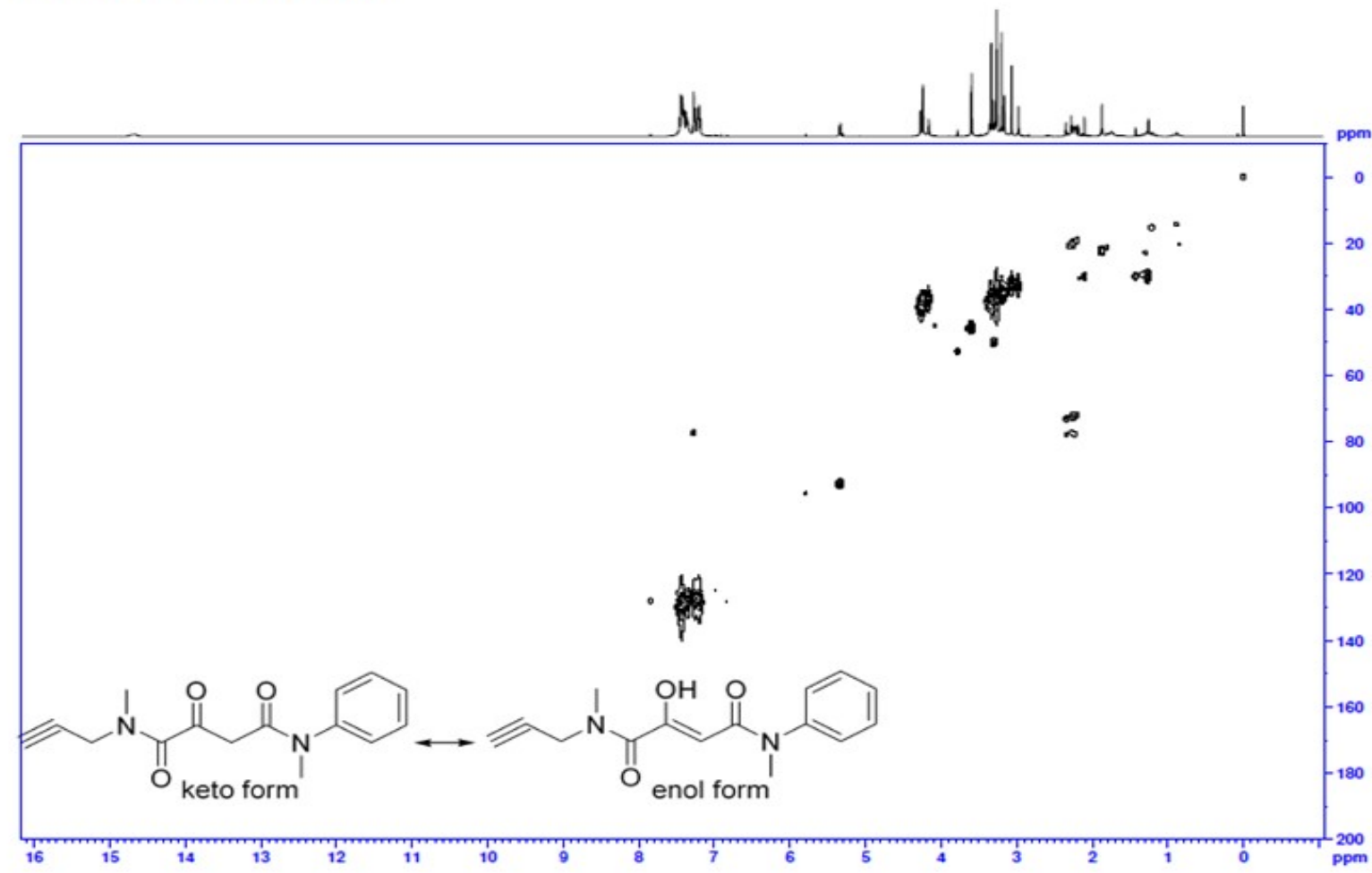


$N^{1}, N^{4}$-Diallyl- $N^{1}, N^{4}$-dimethyl-2-oxosuccinamide (precursor of 13).<smiles>C=CCN(C)C(=O)CC(=O)C(=O)N(C)CC=C</smiles>

This compound is novel and was prepared following general procedure B using methyl 2(allyl(methyl)amino)-2-oxoacetate $(0.300 \mathrm{~g}, 1.91 \mathrm{mmol}, 1.0 \mathrm{eq})$ in anhydrous THF $(4 \mathrm{~mL})$, $N$-allyl- $N$-methylacetamide $(0.237 \mathrm{~g}, 2.10 \mathrm{mmol}, 1.1 \mathrm{eq})$ in anhydrous THF (4 mL), and LDA (1M solution in THF) $(2.48 \mathrm{~mL}, 2.48 \mathrm{mmol}, 1.3 \mathrm{eq})$ to give crude material which was further purified by column chromatography (70\% EtOAc in petroleum ether) to afford the product as a yellow liquid $(0.250 \mathrm{~g}, 1.05 \mathrm{mmol}, 55 \%)$. The title compound was obtained as a keto/enol tautomer (3:1) and rotamers as per ${ }^{1} \mathrm{H}$ NMR.

TLC: $\mathrm{R}_{\mathrm{f}}$ ca 0.5 (1:9, hexane: EtOAc), UV-active and strong $\mathrm{KMnO}_{4}$;

HRMS (ESI) m/z: [M+Na] ${ }^{+}$Calcd for $\mathrm{C}_{12} \mathrm{H}_{18} \mathrm{~N}_{2} \mathrm{O}_{3} \mathrm{Na} 261.1210$; Found 261.1214 (error -1.8 ppm);

$U_{\max } 3606,3082,2929,1721,1631,1481,1400,1360,1258,1066,923 \mathrm{~cm}^{-1}$;

${ }^{1} \mathrm{H}$ NMR $\left(500 \mathrm{MHz}, \mathrm{CDCl}_{3}\right)$ (keto/enol, $(3: 1)+$ rotamers) $\delta 15.20-15.11(0.25 \mathrm{H}, \mathrm{OH}$ of enol form), 5.94-5.64 (2.25H, m, $\mathrm{CH}_{2}=\mathrm{CHCH}_{2}+=\mathrm{CH}$ of enol form), 5.28-5.14 (4H, m, $\left.\mathrm{C}_{2}=\mathrm{CHCH}_{2}\right), 4.02-3.85\left(5.5 \mathrm{H}, \mathrm{m}, \mathrm{CH}_{2}=\mathrm{CHCH}_{2}+\mathrm{CH}_{2}\right.$ of keto form), 3.08-3.07 (1.5H, m, $\mathrm{NCH}_{3}$, rotamer 1), $2.97\left(1 \mathrm{H}, \mathrm{m},-\mathrm{NCH}_{3}\right.$, rotamer 2), 2.93-2.90 (3.5H, m, $-\mathrm{NCH}_{3}$, rotamer 1+ rotamer 2);

${ }^{13} \mathrm{C}$ NMR (126 MHz, $\left.\mathrm{CDCl}_{3}\right)$ (keto/enol, $(3: 1)+$ rotamers) $193.8(\mathrm{C}=\mathrm{O}$ keto $), 193.7(\mathrm{C}=\mathrm{O}$ keto), 193.6, 168.0, 167.9, 167.6, 167.5, 166.2, 165.9, 165.2, 164.8, 133.5, 133.3, 133.2, 132.3, 132.2, 132.1, 131.7, 131.6, 131.5, 118.6, 118.1, 117.9, 117.6, 90.2 (=ㄷH enol), 53.3, 52.8, 52.7, 49.9, 49.8, 44.9, 44.8, 44.3, 44.2, 35.6, 35.2, 35.1, 35.0, 33.3, 33.0, 32.4;

$m / z(\mathrm{ESI}) 261.1\left[(\mathrm{M}+\mathrm{Na})^{+}, 100 \%\right]$. 
$N^{1}, N^{4}$-Diallyl- $N^{1}, N^{4}$-dimethyl-2-oxosuccinamide.

${ }^{1} \mathrm{H}$ NMR $\left(500 \mathrm{MHz}, \mathrm{CDCl}_{3}\right)$.

Dec01-2020

Chemist Shweta Gediya

PROTON.w CDCl3 /opt/topspin3.2 SKG 4
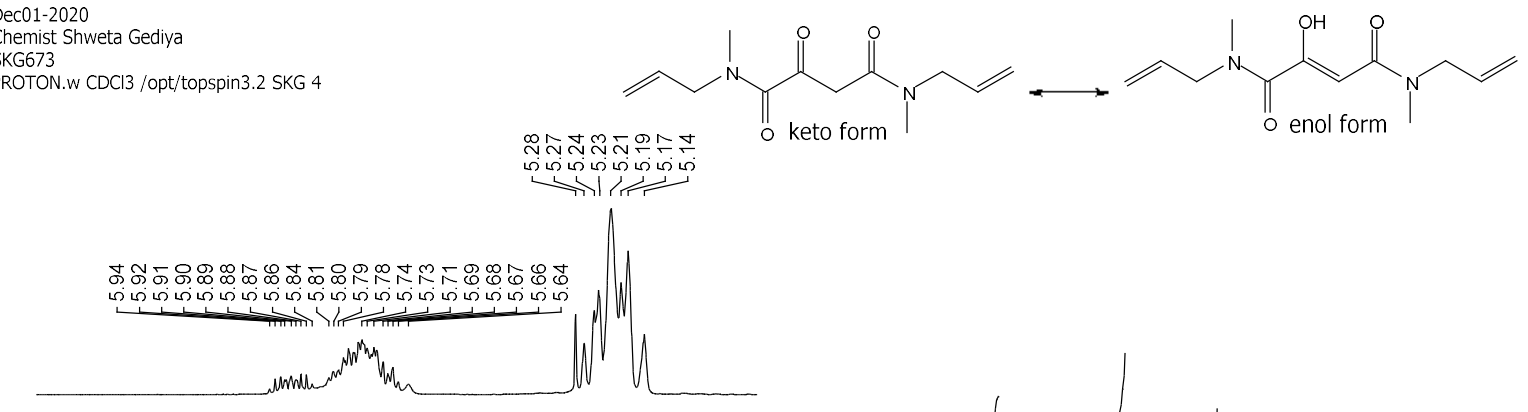

$\begin{array}{lllllllllllllllllll}6.4 & 6.3 & 6.2 & 6.1 & 6.0 & 5.9 & 5.8 & \begin{array}{c}5.7 \\ \mathrm{f}(\mathrm{ppm})\end{array} & 5.5 & 5.4 & 5.3 & 5.2 & 5.1 & 5.0\end{array}$
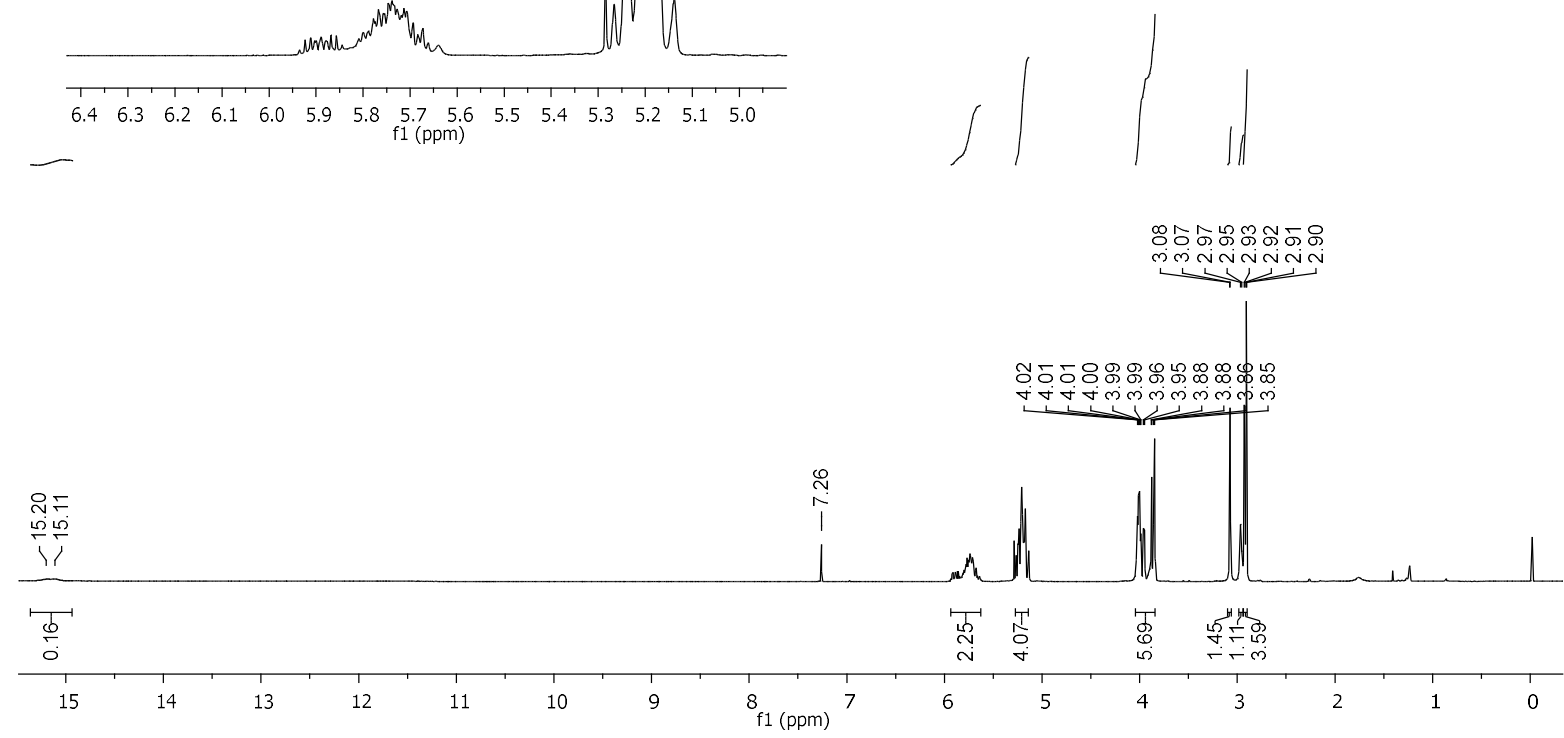

${ }^{13} \mathrm{C}$ NMR (126 MHz, $\left.\mathrm{CDCl}_{3}\right)$.

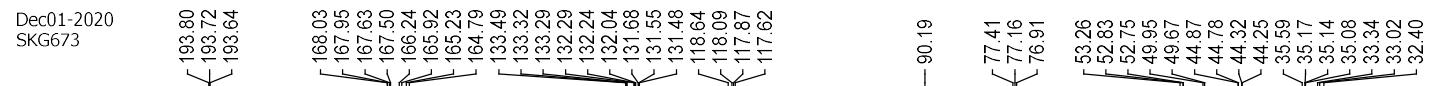<smiles>C=CCN(C)C(=O)/C=C(\C)C(=O)N(C)CC=CC#CCN(CO)C(=O)CC(=O)C(=O)N(C)CC=C</smiles>

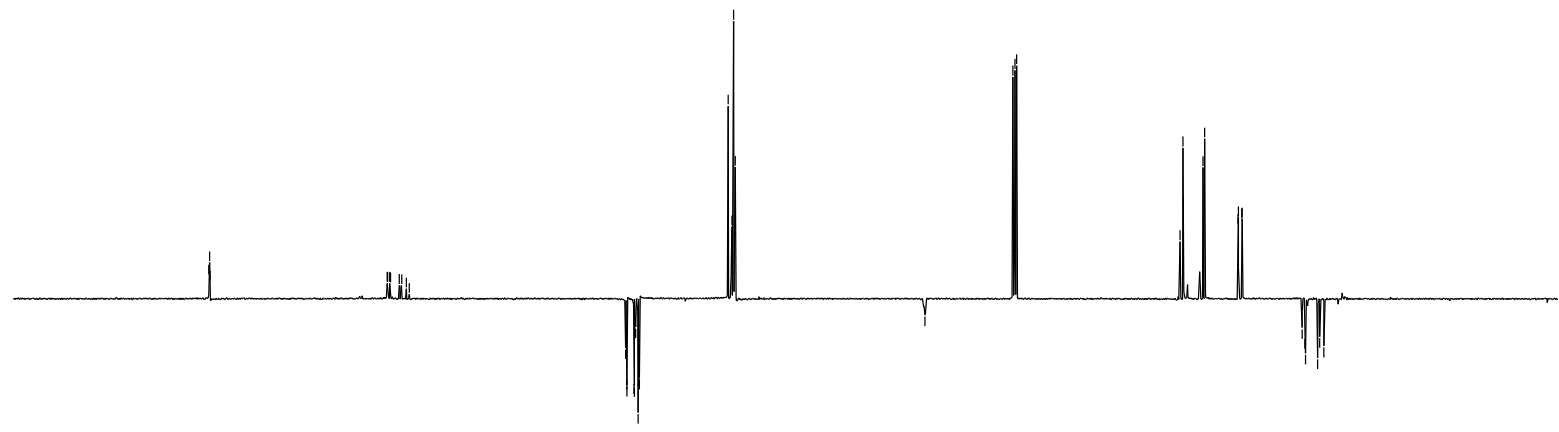

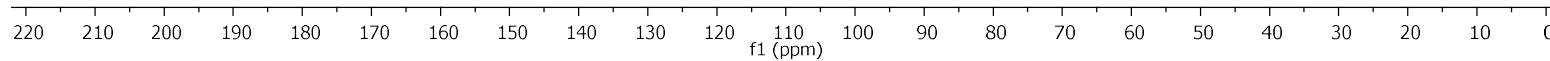


COSY.

Chemist Shweta Gediya

COSY.w CDCl3/opt/topspin3.2 SKG 4

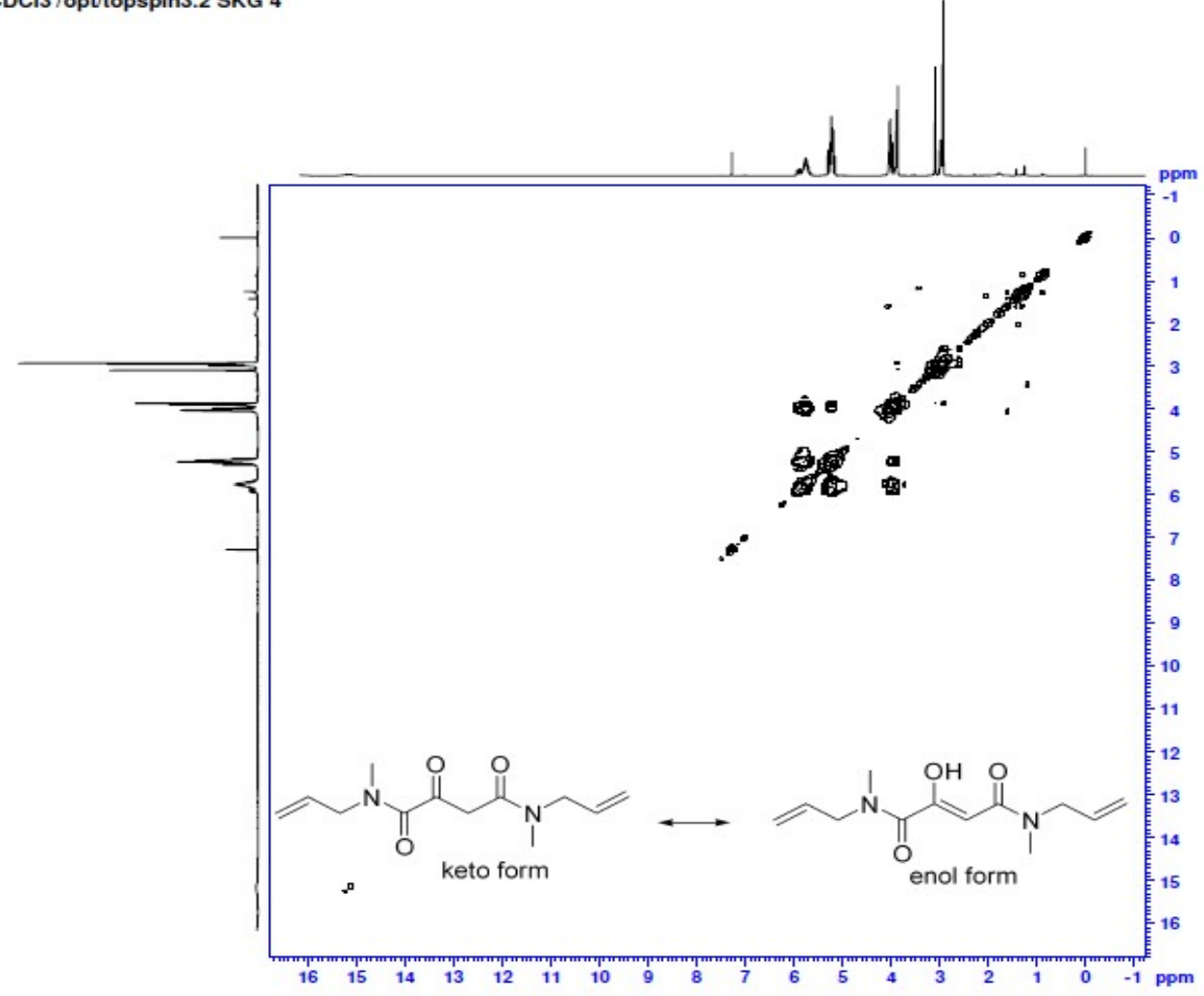

HSQC.

Chemist Shwota Gediya

HSOC.w CDCl3 /opt/topspin3.2 SKG 4

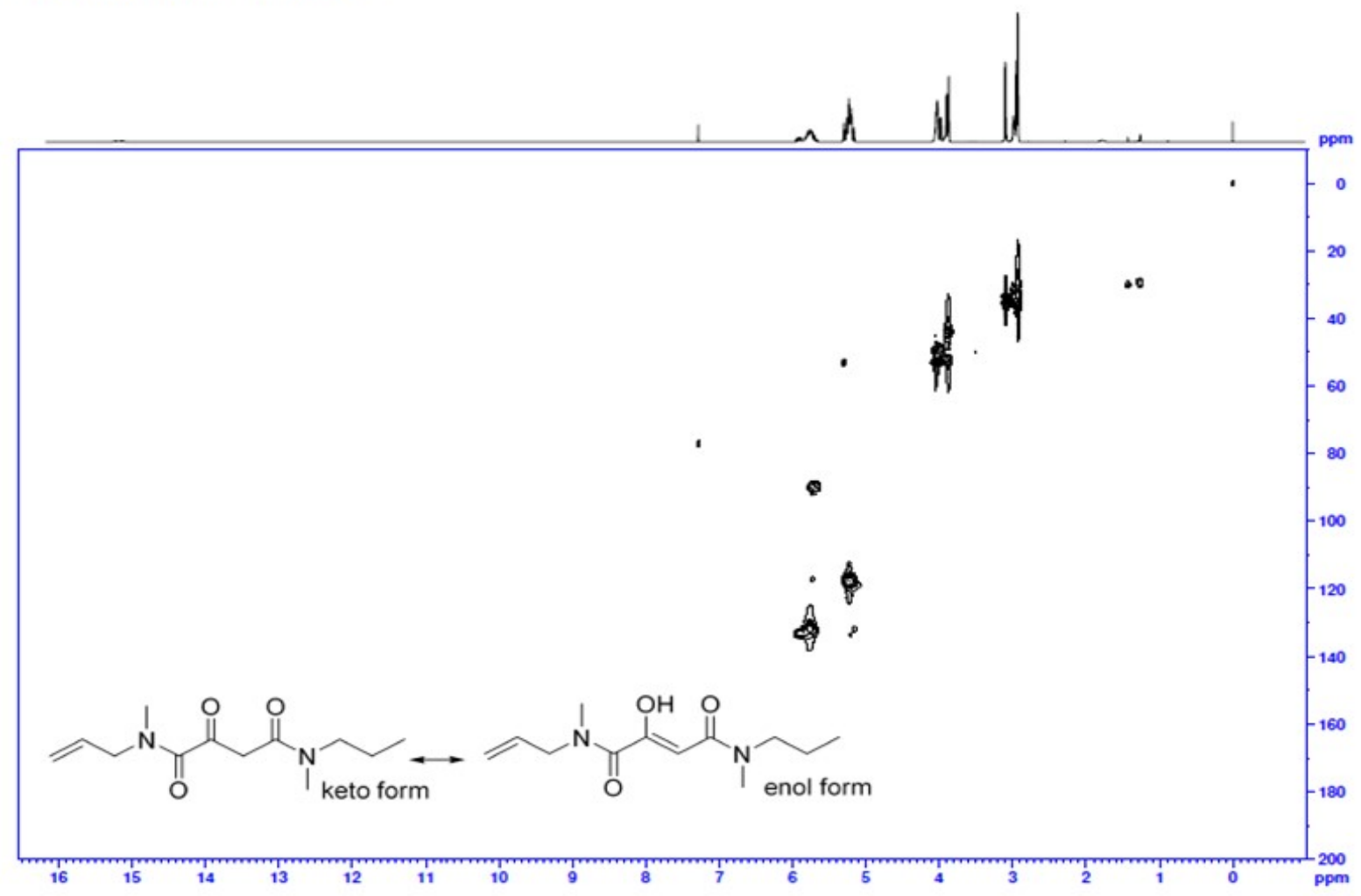


Methyl-2-oxo- $N^{1}, N^{1}, N^{4}$-triphenylsuccinamide (precursor of 14).<smiles>CN(C(=O)CC(=O)C(=O)N(c1ccccc1)c1ccccc1)c1ccccc1</smiles>

This compound is novel and was prepared following general procedure B using methyl 2(diphenylamino)-2-oxoacetate $(0.500 \mathrm{~g}, 1.96 \mathrm{mmol}, 1.0 \mathrm{eq})$ in anhydrous THF $(8 \mathrm{~mL}), \mathrm{N}$ methyl- $N$-phenylacetamide $(0.321 \mathrm{~g}, 2.15 \mathrm{mmol}, 1.1 \mathrm{eq})$ in anhydrous THF $(8 \mathrm{~mL})$ and LDA (1M solution in THF) (2.54 mL, $2.54 \mathrm{mmol}, 1.3 \mathrm{eq})$ to give crude material which was further purified by column chromatography $(10-30 \%$ EtOAc in petroleum ether) to afford the product as a white solid $(0.458 \mathrm{~g}, 1.17 \mathrm{mmol}, 63 \%)$. The title compound was obtained as a keto/enol tautomers $(2: 3)$ and rotamers as per ${ }^{1} \mathrm{H}$ NMR.

TLC: $\mathrm{R}_{\mathrm{f}}$ ca 0.3 (8:2, hexane: EtOAc), UV-active and strong $\mathrm{KMnO}_{4}$;

MP: $130-132{ }^{\circ} \mathrm{C}$;

HRMS (ESI) m/z: $[\mathrm{M}+\mathrm{Na}]^{+}$Calcd for $\mathrm{C}_{23} \mathrm{H}_{20} \mathrm{~N}_{2} \mathrm{O}_{3} \mathrm{Na}$ 395.1366; Found 395.1368 (error -0.5 ppm);

$U_{\max } 3061,2940,1661,1636,1589,1491,1387,1227,1120,696,553 \mathrm{~cm}^{-1}$;

${ }^{1} \mathrm{H}$ NMR (500 MHz, $\left.\mathrm{CDCl}_{3}\right)$ (keto/enol, (2:3) +rotamers) $\delta 13.80$ (0.6H, br.s., $\mathrm{OH}$ enol), 7.53-7.05 (15H, m, ArH), $5.56(0.2 \mathrm{H}, \mathrm{s},=\mathrm{CH}$ of enol form 1$), 5.27(0.4 \mathrm{H}, \mathrm{s},=\mathrm{CH}$ of enol form 2), $3.49\left(0.8 \mathrm{H}, \mathrm{s}, \mathrm{CH}_{2}\right.$ of keto form), $3.29\left(1.8 \mathrm{H}, \mathrm{s},-\mathrm{NCH}_{3}\right), 3.26\left(1.2 \mathrm{H}, \mathrm{s},-\mathrm{NCH}_{3}\right)$;

${ }^{13} \mathrm{C}$ NMR (126 MHz, $\left.\mathrm{CDCl}_{3}\right) \delta$ (keto/enol, (2:3) +rotamers) 193.1 (C=O, keto), 171.5, 171.1, $167.2,165.1,164.6,164.5,143.5,142.9,142.6,142.5,141.7,130.1,129.9,129.4,1129.3$, $129.2,128.4,128.2,127.9,127.8,127.3,127.2,127.1,127.0,126.8,126.2,94.1(\mathrm{C}=\underline{\mathrm{C}} \mathrm{H}$, enol form), $94.0\left(\mathrm{C}=\underline{\mathrm{CH}}\right.$, enol form), $45.8\left(\mathrm{CH}_{2}\right.$ keto form $), 37.2,36.6$.

$m / z(\mathrm{ESI}) 395.2\left[(\mathrm{M}+\mathrm{Na})^{+}, 100 \%\right]$. 
$N^{4}$-Methyl-2-oxo- $N^{1}, N^{1}, N^{4}$-triphenylsuccinamide.

${ }^{1} \mathrm{H}$ NMR (500 MHz, $\mathrm{CDCl}_{3}$ ).

Nov $17-2020$

Chemist Shweta Gediya
SKG640a

PROTON.w CDCl3 /opt/topspin3.2 SKG 59
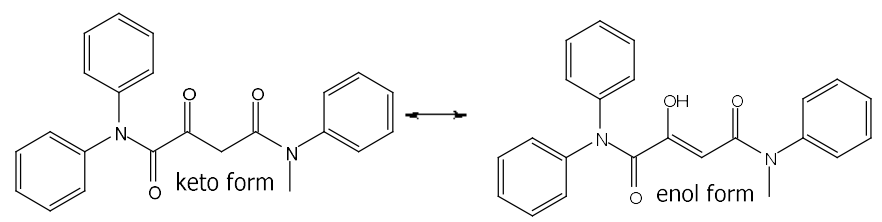

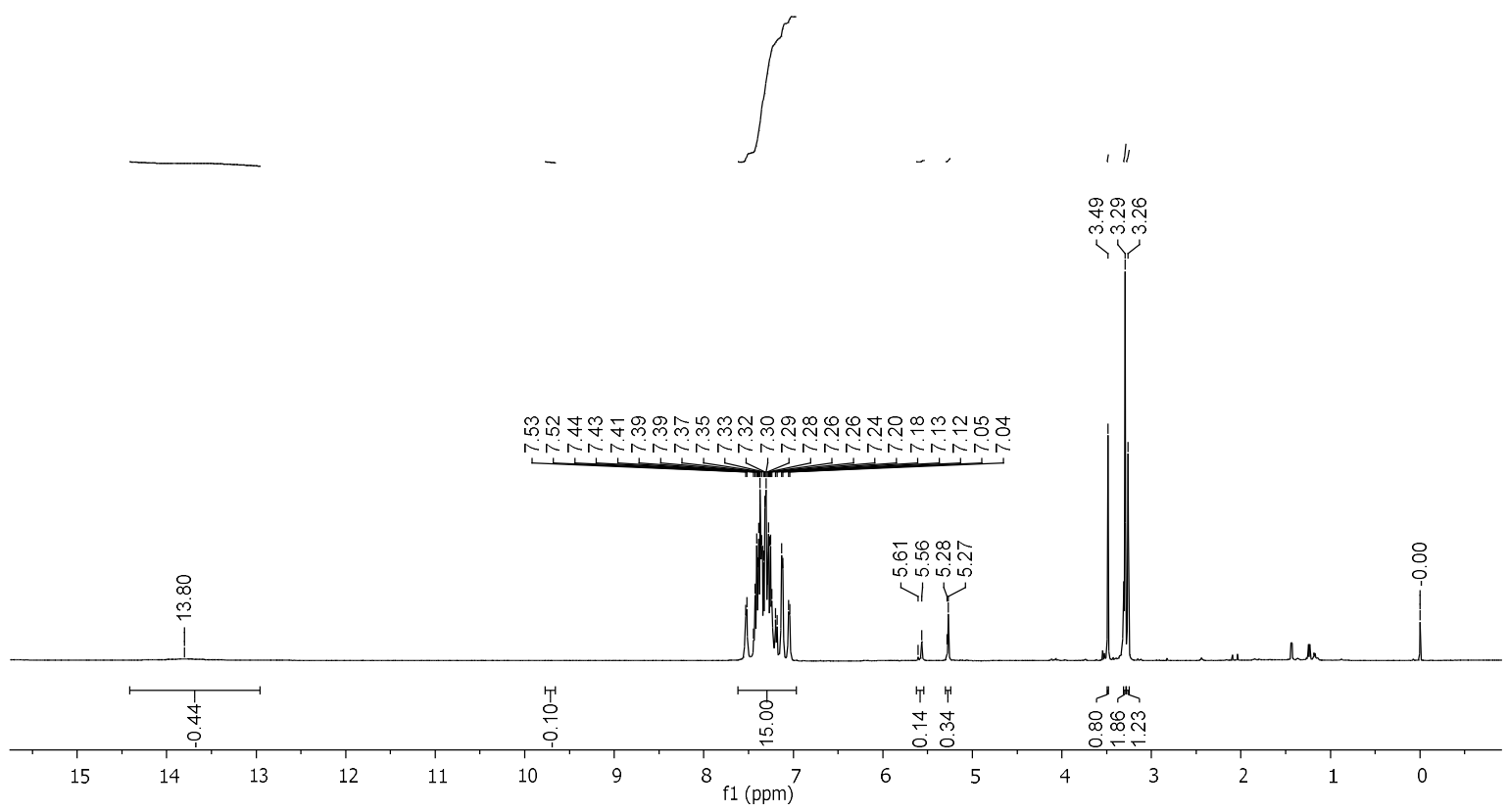

${ }^{13} \mathrm{C}$ NMR $\left(126 \mathrm{MHz}, \mathrm{CDCl}_{3}\right)$.

Nov17-2020

SKG640
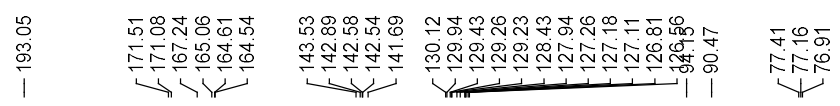

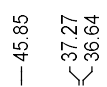
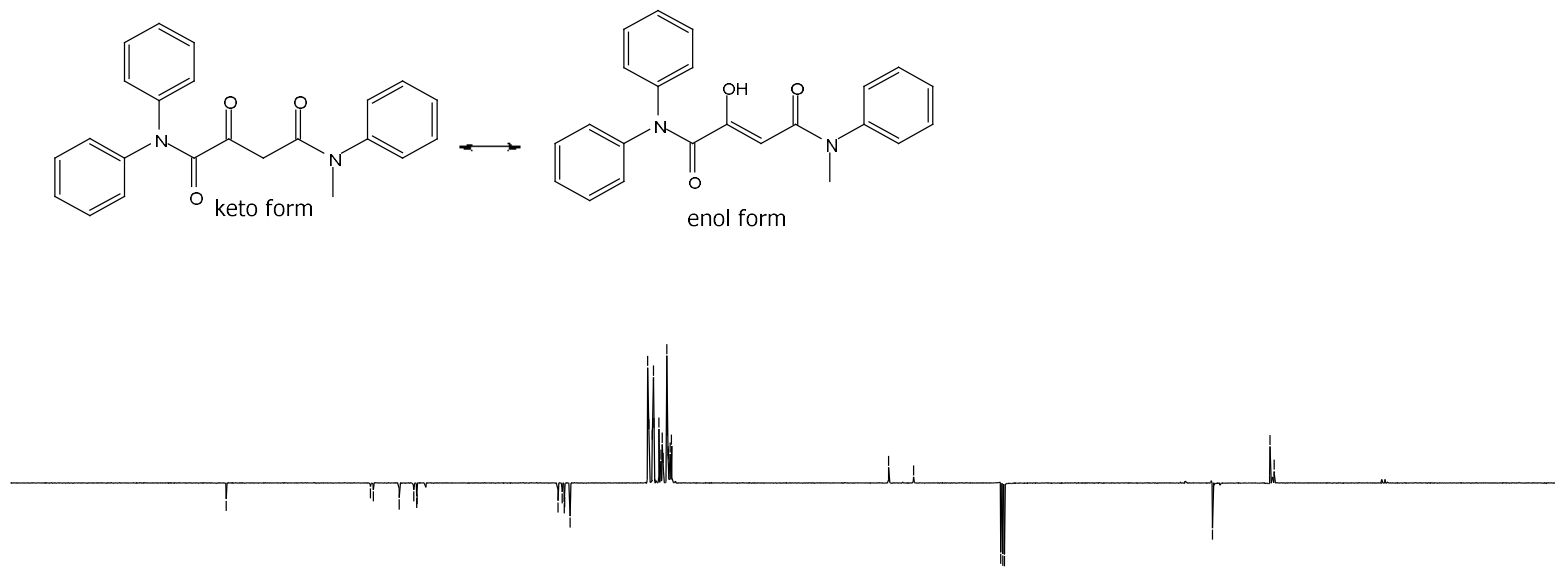

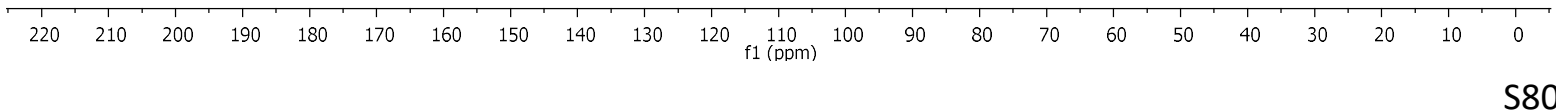


COSY.

Chemist Shweta Gediya

SKG640a

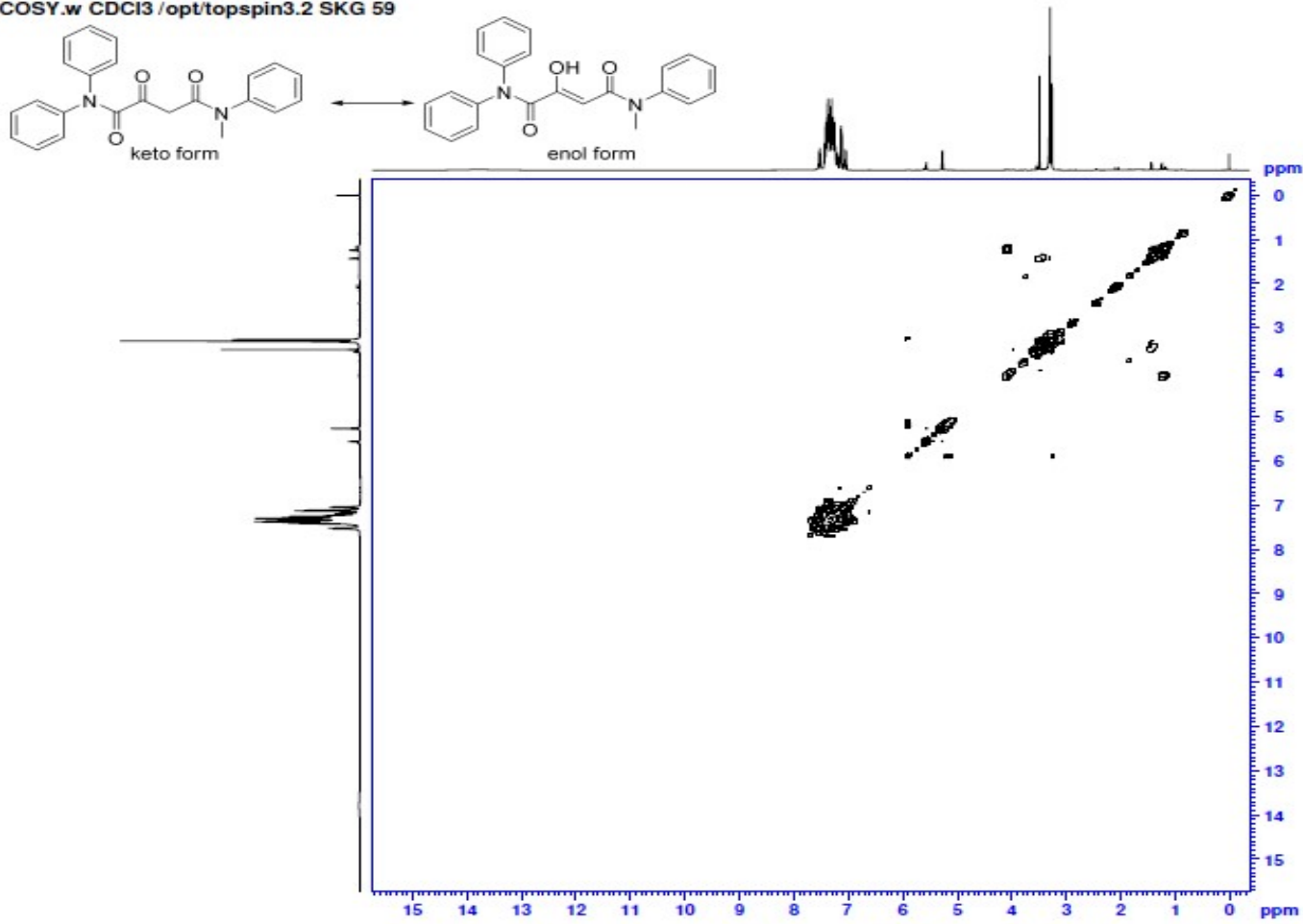

HSQC.

Chemist Shweta Gediya

HSOC.w CDC13 /opt/topspin3.2 SKG 59

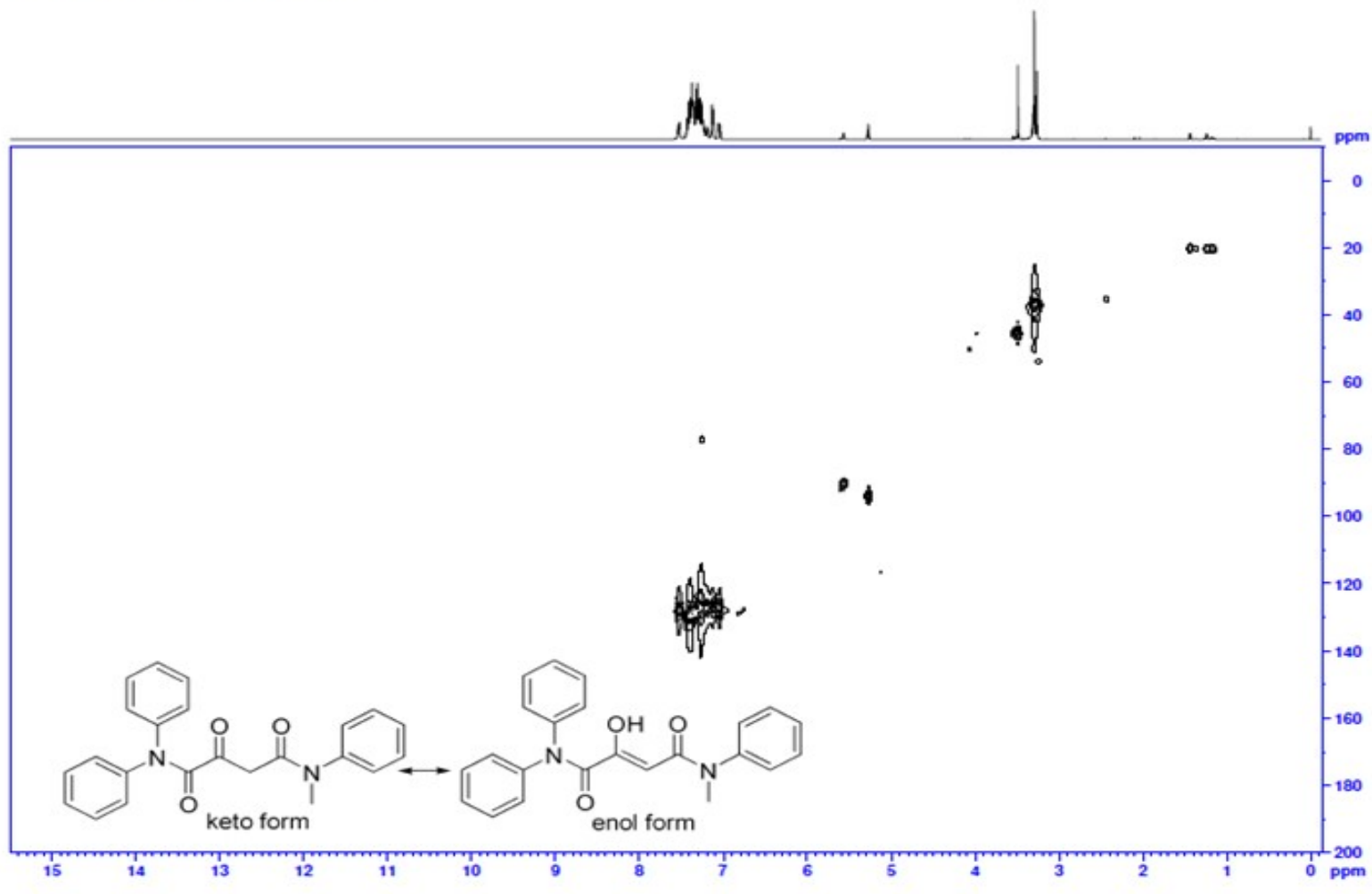




\section{$N^{4}$-Methyl-2-oxo- $N^{1}, N^{4}$-diphenylsuccinamide (precursor of 15)}<smiles>CN(C(=O)CC(=O)C(=O)Nc1ccccc1)c1ccccc1</smiles>

This compound is novel and was prepared following the general procedure B using methyl 2oxo-2-(phenylamino)acetate $(0.480 \mathrm{~g}, 2.68 \mathrm{mmol}, 1.0 \mathrm{eq})$ in anhydrous THF $(5 \mathrm{~mL})$, and $\mathrm{N}$ methyl- $N$-phenylacetamide $(0.400 \mathrm{~g}, 2.68 \mathrm{mmol}, 1.0 \mathrm{eq})$ in anhydrous THF $(5 \mathrm{~mL})$, and LDA (1M solution in THF) (3.48 mL, $3.48 \mathrm{mmol}, 1.3 \mathrm{eq})$ to give crude material which was further purified by column chromatography $(30-50 \%$ EtOAc in petroleum ether) to afford the product as a light pink solid $(0.310 \mathrm{~g}, 1.04 \mathrm{mmol}, 39 \%)$. The title compound was obtained as a keto/enol tautomer (1:9) as per ${ }^{1} \mathrm{H}$ NMR.

TLC: $\mathrm{R}_{\mathrm{f}}$ ca 0.3 (8:2, hexane: EtOAc), UV-active and strong $\mathrm{KMnO}_{4}$;

MP: $128-129.5^{\circ} \mathrm{C}$;

HRMS (ESI) m/z: [M+Na] $]^{+}$Calcd for $\mathrm{C}_{17} \mathrm{H}_{16} \mathrm{~N}_{2} \mathrm{O}_{3} \mathrm{Na}$ 319.1053; Found 319.1041 (error 3.9 ppm);

$U_{\max } 3370,1685,1631,1585,1530,1440,1316,1264,1206,1199,689 \mathrm{~cm}^{-1}$;

${ }^{1} \mathrm{H}$ NMR (400 MHz, $\mathrm{CDCl}_{3}$ ) (keto/enol, 1:9) $\delta 14.91$ (0.9H br.s., OH of enol form), 9.01 $(0.1 \mathrm{H}$, br.s., CONH of keto form), $8.63(0.9 \mathrm{H}$, br.s, $\mathrm{CONH}$ of enol form), 7.59 (2H, d, $J=$ 8.1 Hz, ArH), 7.46 -7.42 (2H, m, ArH), 7.39-7.30 (3H, m, ArH), 7.26-7.21 (2H, m, ArH), 7.16-7.10 $(1 \mathrm{H}, \mathrm{m}, \mathrm{ArH}), 5.96(0.9 \mathrm{H}, \mathrm{s},=\mathrm{CH}$, of enol form $), 3.75\left(0.2 \mathrm{H}\right.$, s, keto $\left.\mathrm{CH}_{2}\right), 3.37$ $\left(2.7 \mathrm{H}, \mathrm{s}, \mathrm{NCH}_{3}\right), 3.31\left(0.3 \mathrm{H}, \mathrm{s}, \mathrm{NCH}_{3}\right)$;

${ }^{13} \mathrm{C}$ NMR (101 MHz, $\left.\mathrm{CDCl}_{3}\right)$ (keto/enol, 1:9) $\delta 171.4(\underline{\mathrm{C}}=\mathrm{CH}$, enol), 160.1 ( $\mathrm{C}=\mathrm{O}$ amide), 159.5 ( $\mathrm{C}=\mathrm{O}$ amide), 142.4, 137.1, 130.2, 129.3, 129.2, 128.5, 127.5, 127.2, 125.5, 124.9, 120.1, 119.9, $92.7\left(\mathrm{C}=\underline{\mathrm{C}} \mathrm{H}\right.$, enol), 49.8, 47.1, $43.3\left(\underline{\mathrm{CH}_{2}}\right.$, keto form), 37.1; $m / z(\mathrm{ESI}) 319.1\left[(\mathrm{M}+\mathrm{Na})^{+}, 100 \%\right]$. 
$N^{4}$-Methyl-2-oxo- $N^{1}, N^{4}$-diphenylsuccinamide.

${ }^{1} \mathrm{H}$ NMR (400 MHz, $\mathrm{CDCl}_{3}$ ).

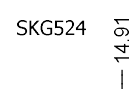

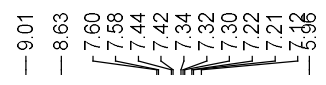

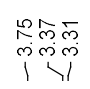

8
0
$i$
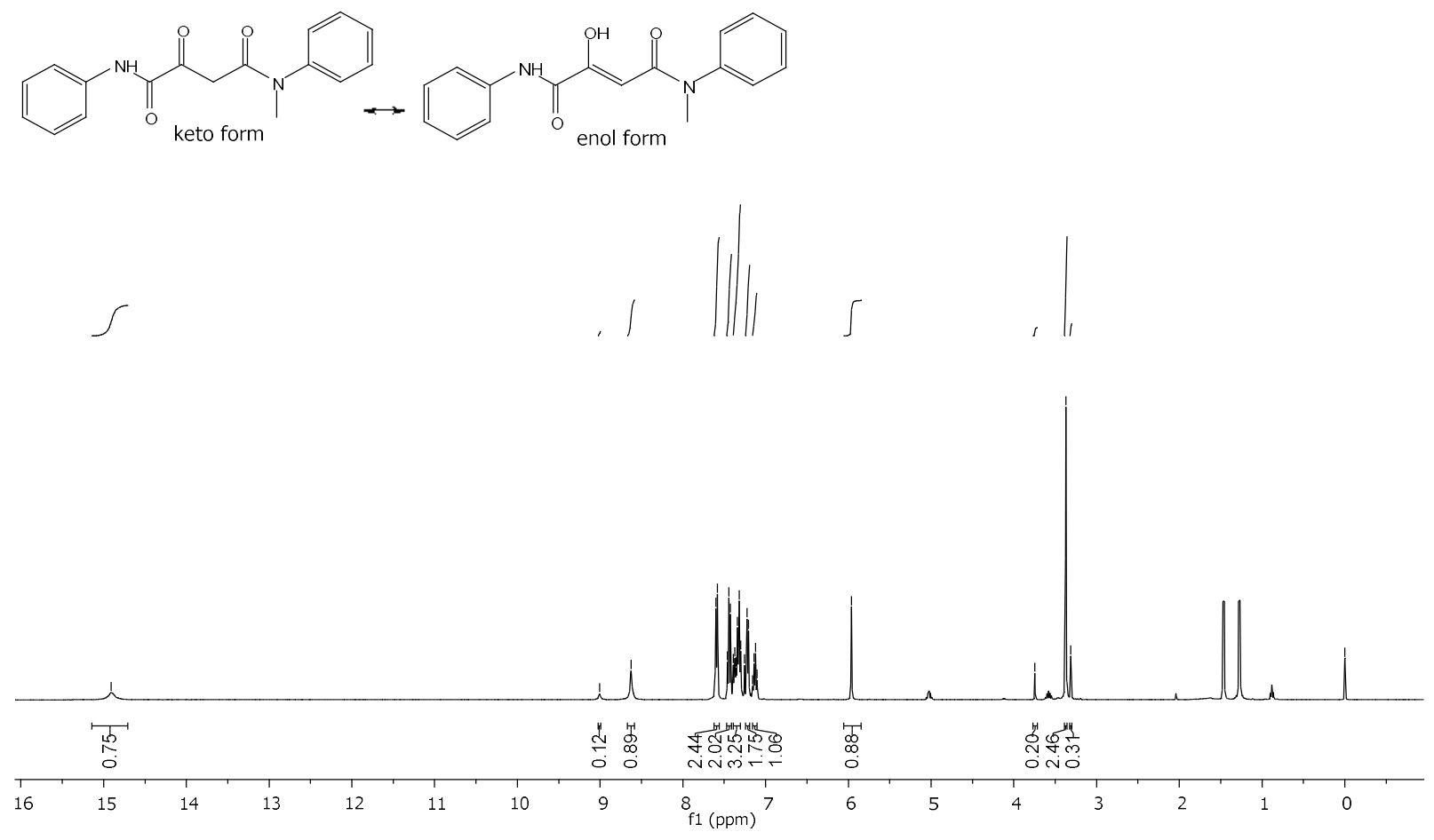

${ }^{13} \mathrm{C}$ NMR (101 MHz, $\left.\mathrm{CDCl}_{3}\right)$.
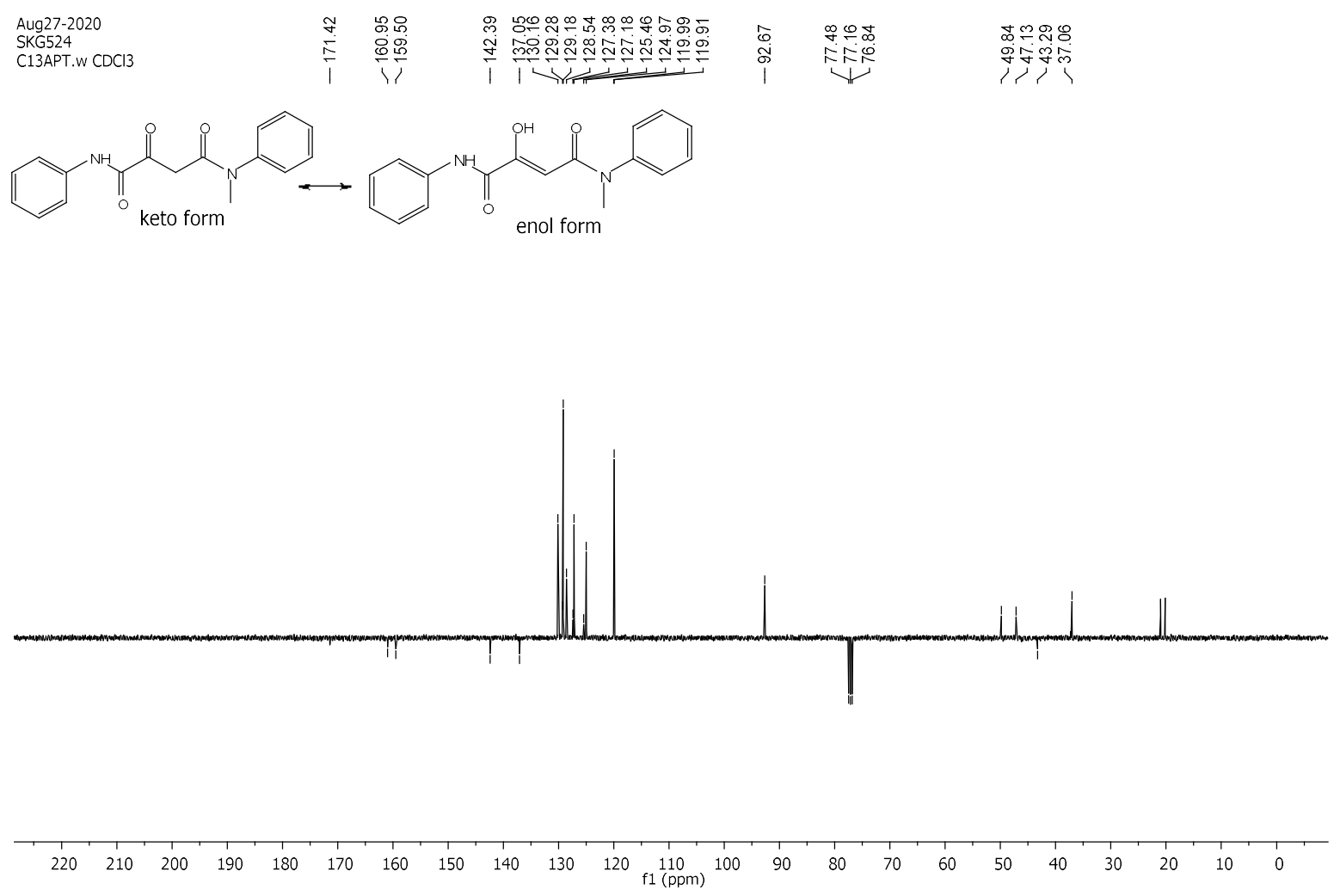

S83 
COSY.

SKG524

COS w $\mathrm{CDCl} /$ opt/topspin3.5pl2 SKG 8

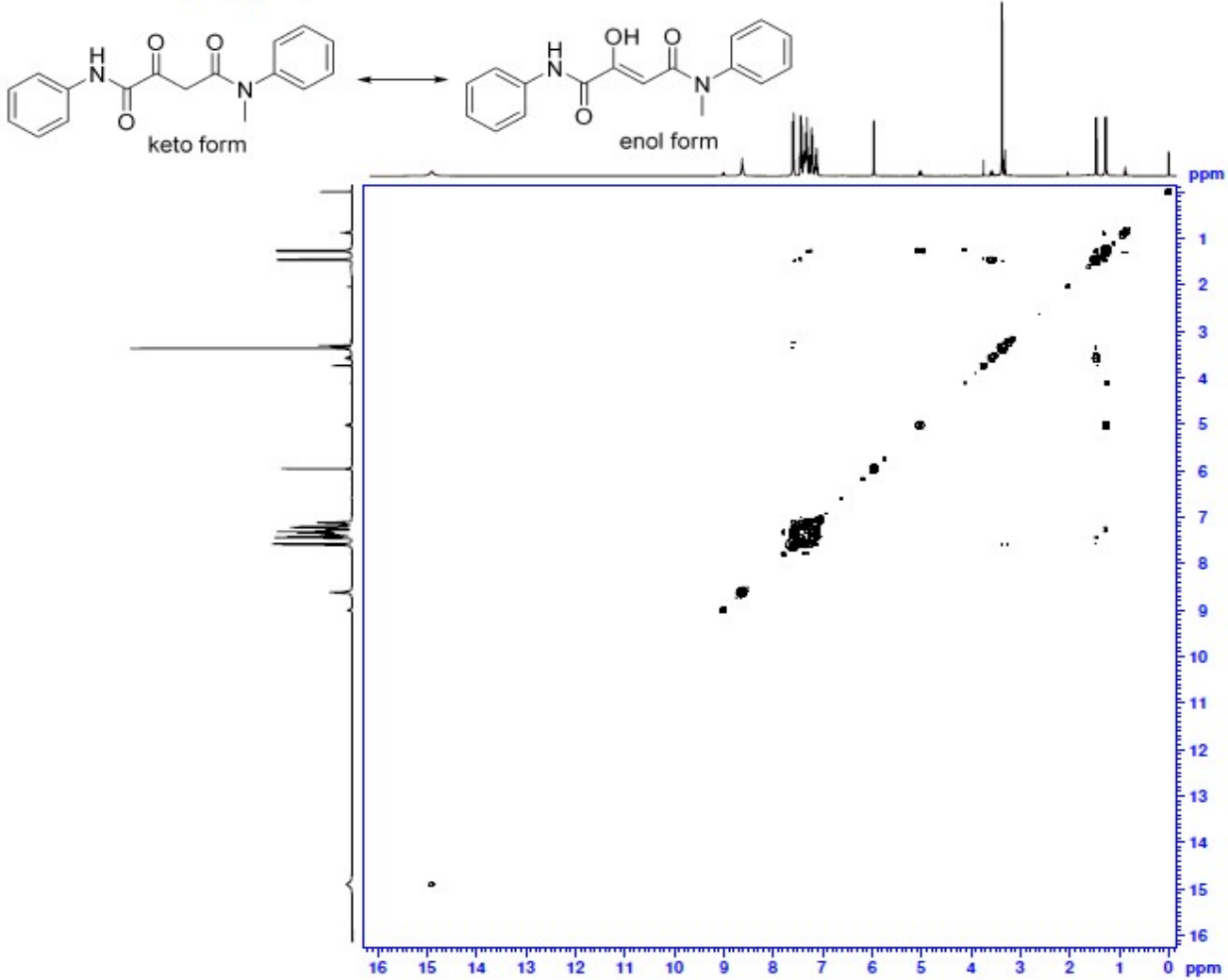

HSQC.

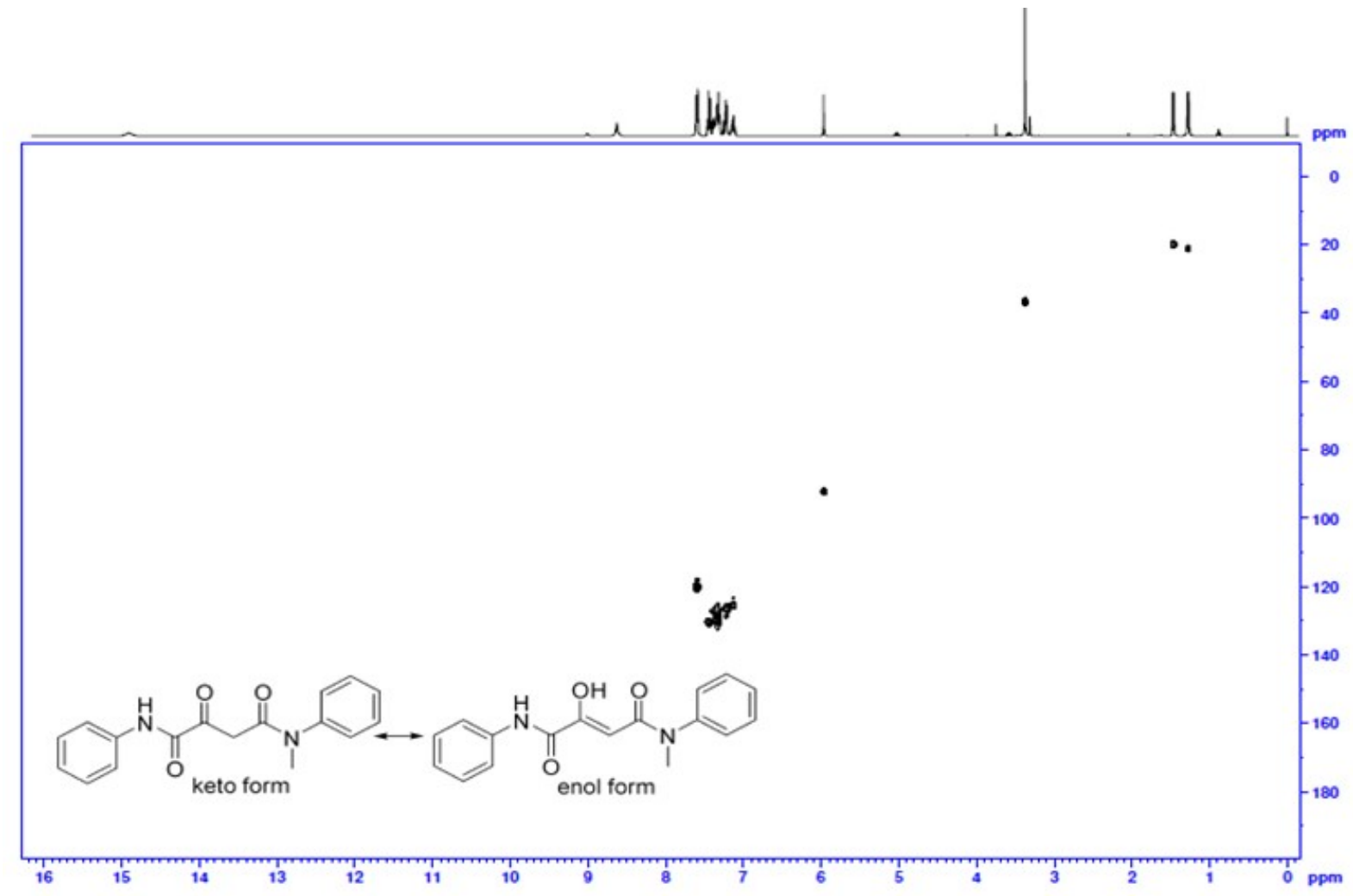


$\mathrm{N}$-Methyl-3-oxo- $\mathrm{N}$-phenyl-4-(phenylamino)-4-thioxobutanamide (precursor of 16).<smiles>CN(C(=O)CC(=O)C(=S)Nc1ccccc1)c1ccccc1</smiles>

This compound is novel and was prepared following general procedure B using methyl 2(phenylamino)-2-thioxoacetate $(0.400 \mathrm{~g}, 2.05 \mathrm{mmol}, 1.0 \mathrm{eq})$ in anhydrous THF $(8 \mathrm{~mL}), \mathrm{N}$ methyl- $N$-phenylacetamide $(0.336 \mathrm{~g}, 2.25 \mathrm{mmol}, 1.1 \mathrm{eq})$ in anhydrous THF $(8 \mathrm{~mL})$ and LDA (1M solution in THF) $(3.07 \mathrm{~mL}, 3.07 \mathrm{mmol}, 1.5 \mathrm{eq})$ to give crude material which was further purified by column chromatography $(10-30 \%$ EtOAc in petroleum ether) to afford the product as a yellow solid $(0.401 \mathrm{~g}, 1.28 \mathrm{mmol}, 63 \%)$. The title compound was obtained as a keto/enol tautomer (40:60) as per from ${ }^{1} \mathrm{H}$ NMR.

TLC: $\mathrm{R}_{\mathrm{f}}$ ca 0.3 (6:4, hexane: EtOAc), UV-active and strong $\mathrm{KMnO}_{4}$;

MP: $102-104^{\circ} \mathrm{C}$;

HRMS (ESI) m/z: [M+Na] ${ }^{+}$Calcd for $\mathrm{C}_{17} \mathrm{H}_{16} \mathrm{~N}_{2} \mathrm{O}_{2} \mathrm{SNa} 335.0825$; Found 335.0811 (error 4.0 ppm);

$U_{\max } 3298,1610,1585,1528,1439,1369,1323,1291,1024,811,762,683 \mathrm{~cm}^{-1}$;

${ }^{1} \mathrm{H}$ NMR (400 MHz, $\mathrm{CDCl}_{3}$ ) (keto/enol, 40:60) $\delta 15.76$ (0.6H s, br.s., OH of enol form), 10.39 (0.4H s, CONH of keto form), $10.29(0.6 \mathrm{H}, \mathrm{s}, \mathrm{CONH}$ of enol from), 7.96-7.86 (2H, m, ArH), $7.48-7.24(8 \mathrm{H}, \mathrm{m}, \mathrm{ArH}), 6.37\left(0.6 \mathrm{H}, \mathrm{s},=\mathrm{C} \underline{\mathrm{H}}\right.$, of enol form), $4.05\left(0.8 \mathrm{H}, \mathrm{s}, \mathrm{CH}_{2}\right.$ of keto form), $3.39\left(1.8 \mathrm{H}, \mathrm{s}, \mathrm{NCH}_{3}, 3.30\left(1.2 \mathrm{H}, \mathrm{s}, \mathrm{NCH}_{3}\right)\right.$;

${ }^{13} \mathrm{C}$ NMR (101 MHz, $\left.\mathrm{CDCl}_{3}\right)$ (keto/enol, 40:60) $\delta 188.5$ (C=O keto), $185.6(\mathrm{C}=\mathrm{O}$ amide), $183.9,171.3(\underline{\mathrm{C}}=\mathrm{CH}$, enol), 167.3, 163.8, 143.5, 142.4, 138.5, 137.6, 130.4, 130.1, 129.2, $129.1,128.5,128.4,127.4,127.3,127.1,122.9,121.8,94.0(\mathrm{C}=\underline{\mathrm{CH}}$, enol form $), 43.7\left(\mathrm{CH}_{2}\right.$, keto form), 37.4, 37.2;

$m / z(\mathrm{ESI}) 335.1\left[(\mathrm{M}+\mathrm{Na})^{+}, 100 \%\right]$. 
$\mathrm{N}$-Methyl-3-oxo- $\mathrm{N}$-phenyl-4-(phenylamino)-4-thioxobutanamide.

${ }^{1} \mathrm{H}$ NMR $\left(400 \mathrm{MHz}, \mathrm{CDCl}_{3}\right)$.

\section{Oct05-2020}

PROTON.w CDCl3 /opt/topspin3.5pl2 SKG 27
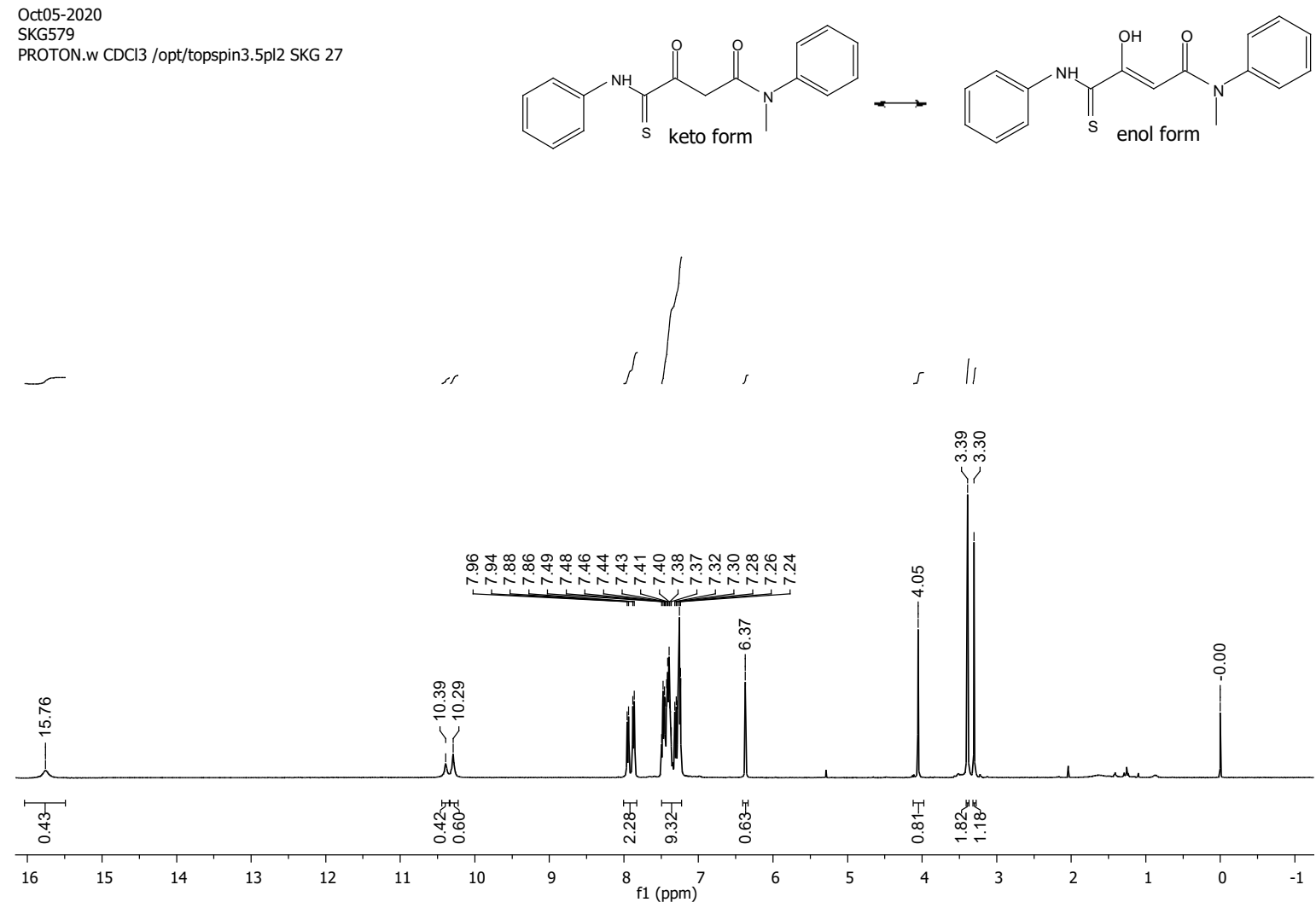

${ }^{13} \mathrm{C} \mathrm{NMR}\left(101 \mathrm{MHz}, \mathrm{CDCl}_{3}\right)$.<smiles>COC(=O)C(=S)C(=O)CC(=O)N(C)c1cccc(C)c1</smiles><smiles>CN(C(=O)/C=C(/O)C(=S)Nc1ccccc1)c1ccccc1</smiles>

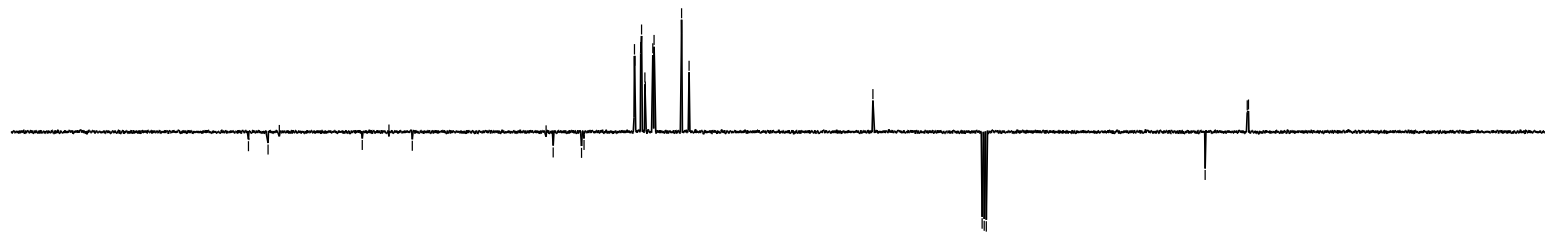


COSY.

SKG579

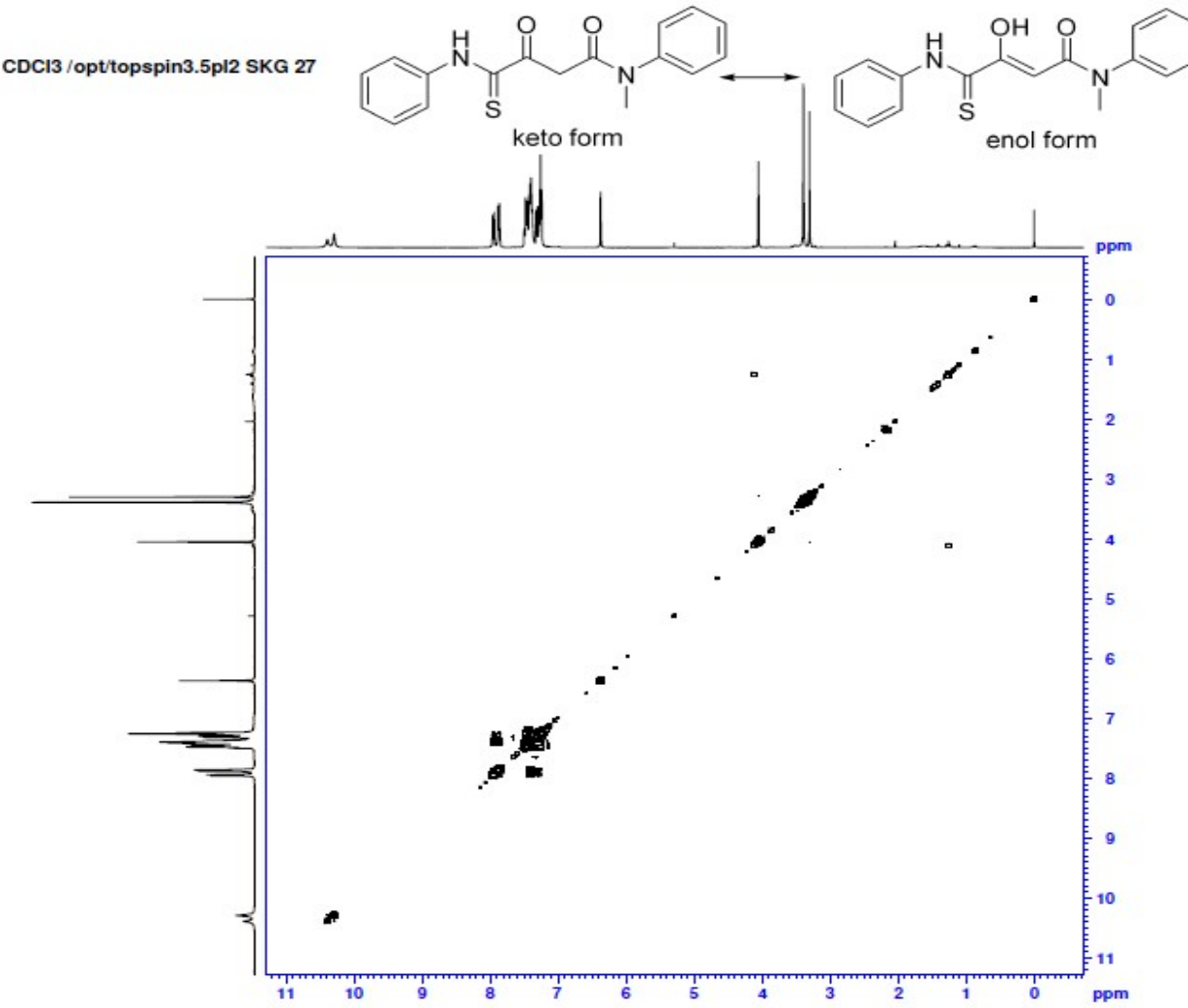

HSQC.

SKG579
HSQC.w CDCI3 /opt/topspin3.5p12 SKG 27

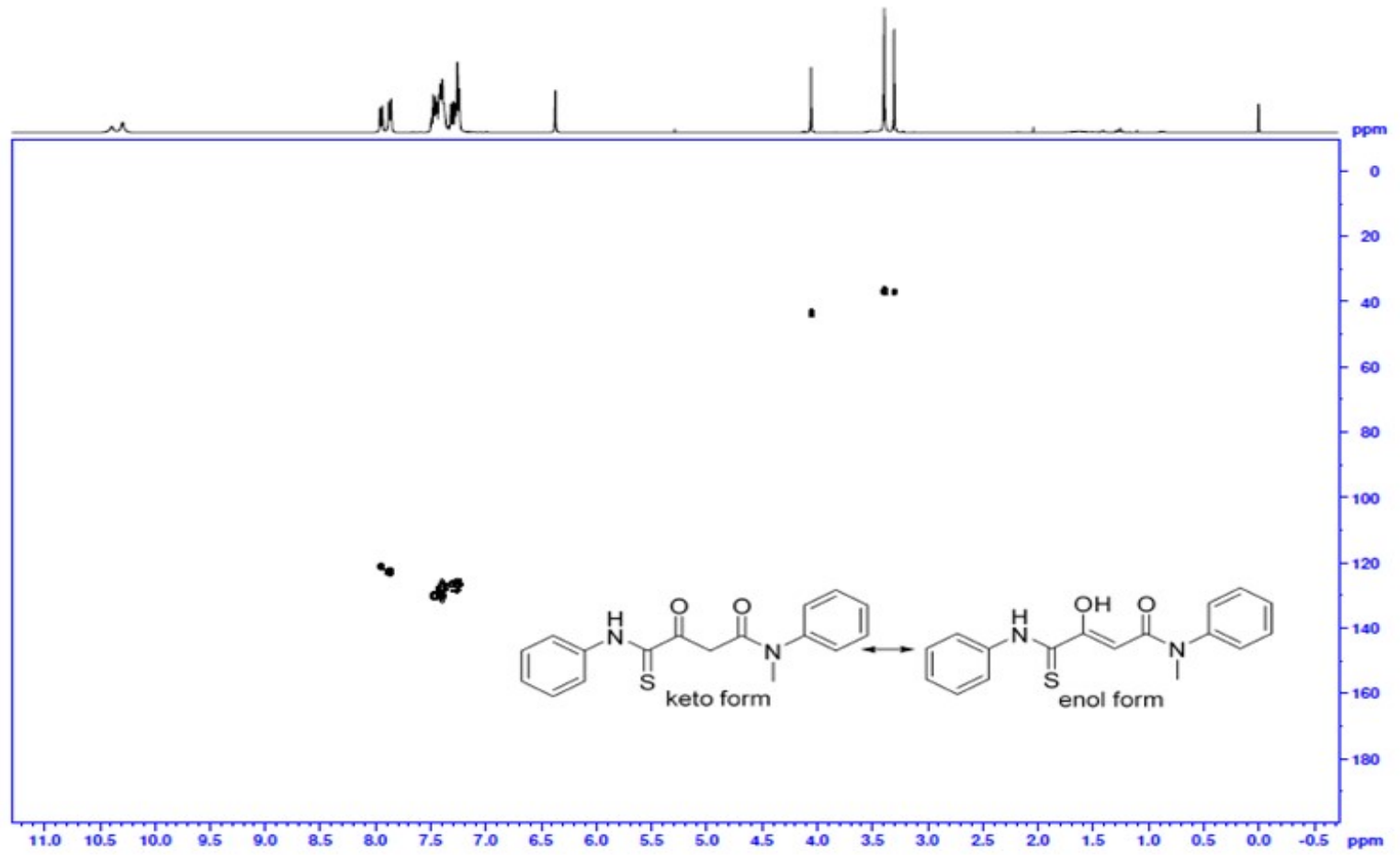




\section{$N^{1}, N^{5}$-Dimethyl-2-oxo- $N^{1}, N^{5}$-diphenylpentanediamide (precursor of 17).}<smiles>CN(C(=O)CCC(=O)C(=O)N(C)c1ccccc1)c1ccccc1</smiles>

This compound is novel. In $25 \mathrm{~mL}$ RBF was dissolved 2-oxopentanedioic acid ( $0.500 \mathrm{~g}, 3.42$ mmol, $1.0 \mathrm{eq})$ in water $(5 \mathrm{~mL})$ and the solution was cooled to $0^{\circ} \mathrm{C}$. In a separate flask, $N$ methylaniline $(0.660 \mathrm{~g}, 6.16 \mathrm{mmol}, 1.8 \mathrm{eq})$ was mixed with water $(3 \mathrm{~mL})$ and $2 \mathrm{M}$ hydrochloric acid was added to adjust the $\mathrm{pH}$ to around 3-4 and was added to above reaction mixture followed by addition of aqueous 1-ethyl-3-(3-dimethylaminopropyl) carbodiimide hydrochloride (EDC.HCl) (1.96 g, $10.3 \mathrm{mmol}, 3.0 \mathrm{eq})$ solution in water $(3 \mathrm{~mL})$ and it was further stirred at room temperature for $2 \mathrm{~h}$. The reaction mixture was quenched with water and extracted with ethyl acetate $(3 \times 20 \mathrm{~mL})$. The combined organic layer was washed with dilute cold $\mathrm{HCl}(25 \mathrm{~mL})$, saturated aqueous $\mathrm{NaHCO}_{3}$ solution $(25 \mathrm{~mL})$ followed by washing with brine $(50 \mathrm{~mL})$. The organic layer was dried over $\mathrm{MgSO}_{4}$, filtered and the filtrate was concentrated under reduced pressure to yield the product as a yellow semisolid material (0.422 g, $1.03 \mathrm{mmol}, 38 \%)$.

TLC: $\mathrm{R}_{\mathrm{f}}$ ca 0.3 (6:4, hexane: EtOAc), UV-active and strong $\mathrm{KMnO}_{4}$; HRMS (ESI) m/z: [M+Na] ${ }^{+}$Calcd for $\mathrm{C}_{19} \mathrm{H}_{20} \mathrm{~N}_{2} \mathrm{O}_{3} \mathrm{Na} 347.1366$; Found 347.1365 (error 0.3 ppm);

$U_{\max } 3061,2926,1714,1643,1593,1493,1386,1120,1090,768,696 \mathrm{~cm}^{-1}$;

${ }^{1} \mathrm{H}$ NMR (400 MHz, $\left.\mathrm{CDCl}_{3}\right) \delta$ 7.45-7.27 (7H, m, ArH), 7.18-7.12 (3H, m, ArH), 3.47 (0.75H, $\mathrm{s},-\mathrm{NCH}_{3}$, minor rotamer 1), $3.34\left(2.25 \mathrm{H}, \mathrm{s},-\mathrm{NCH}_{3}\right.$, major rotamer 1) $3.28\left(0.75 \mathrm{H}, \mathrm{s},-\mathrm{NCH}_{3}\right.$, minor rotamer 2), $3.20\left(2.25 \mathrm{H}, \mathrm{s},-\mathrm{NCH}_{3}\right.$, major rotamer 2$), 3.04\left(0.5 \mathrm{H}\right.$, br.s, $\mathrm{CH}_{2}-\mathrm{minor}$ peak), $2.95\left(1.5 \mathrm{H}, \mathrm{t}, \mathrm{J}=6.6 \mathrm{~Hz}, \mathrm{CH}_{2}\right.$ major peak), 2.50 (0.5H, br.s, $\mathrm{CH}_{2}$-minor peak), 2.20 $\left(1.5 \mathrm{H}, \mathrm{t}, \mathrm{J}=6.6 \mathrm{~Hz}, \mathrm{CH}_{2}\right.$ major peak);

${ }^{13} \mathrm{C}$ NMR (101 MHz, $\left.\mathrm{CDCl}_{3}\right) \delta 199.2,170.9,166.8,143.8,141.8,129.9,129.7,129.5,129.3$, $128.1,127.4,126.4,125.6,37.5,36.8,35.8,27.6$;

$m / z(\mathrm{ESI}) 347.1\left[(\mathrm{M}+\mathrm{Na})^{+}, 100 \%\right]$. 
$N^{1}, N^{5}$-Dimethyl-2-oxo- $N^{1}, N^{5}$-diphenylpentanediamide.

${ }^{1} \mathrm{H}$ NMR $\left(400 \mathrm{MHz}, \mathrm{CDCl}_{3}\right)$.

Feb05-2021
SKG716

PROTON.w CDCl3 /opt/topspin3.5pl2 SKG 29<smiles>CN(C(=O)CCC(=O)C(=O)N(C)c1ccccc1)c1ccccc1</smiles>

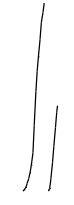

$\|_{d, 1}, \int$

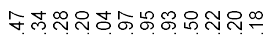

mmmminninan

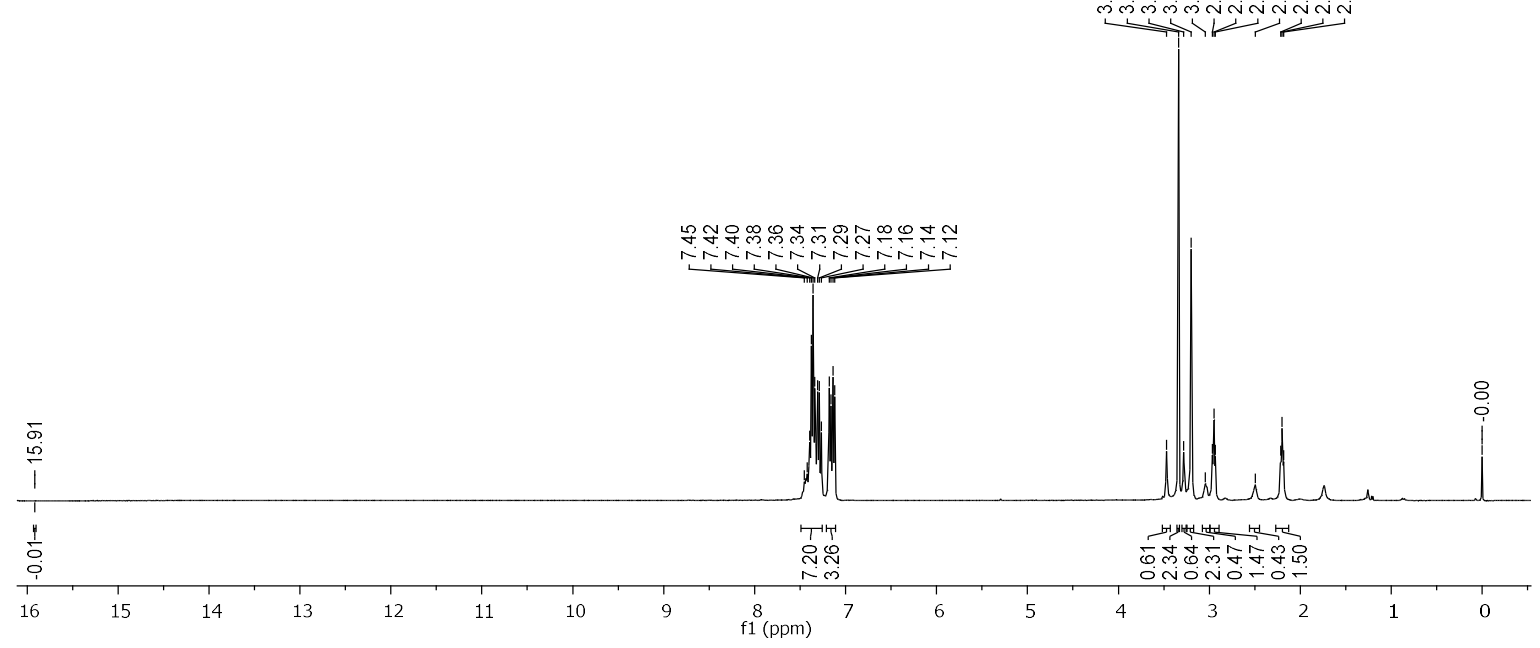

${ }^{13} \mathrm{C}$ NMR (101 MHz, $\left.\mathrm{CDCl}_{3}\right)$.

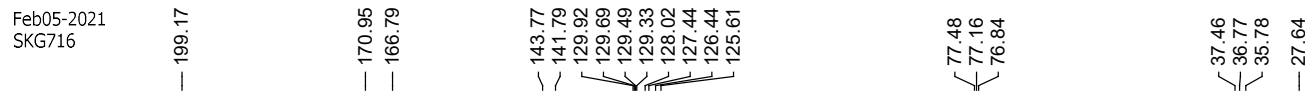<smiles>CN(C(=O)CCC(=O)C(=O)N(C)c1ccccc1)c1ccccc1</smiles>

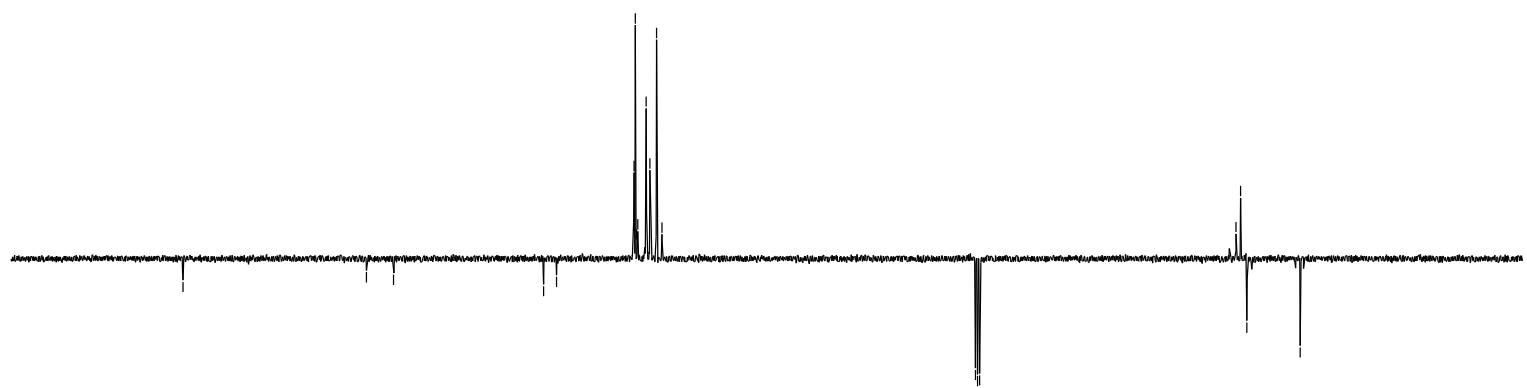

$\begin{array}{lllllllllllllllllllllll}220 & 210 & 200 & 190 & 180 & 170 & 160 & 150 & 140 & 130 & 120 & \begin{array}{l}1110 \\ \mathrm{f} 1(\mathrm{ppm})\end{array} & 100 & 90 & 80 & 70 & 60 & 50 & 40 & 30 & 20 & 10 & 0\end{array}$ 
COSY.

SKG716
COSY.w CDCl3/opt/topspin3.5pl2 SKG 29

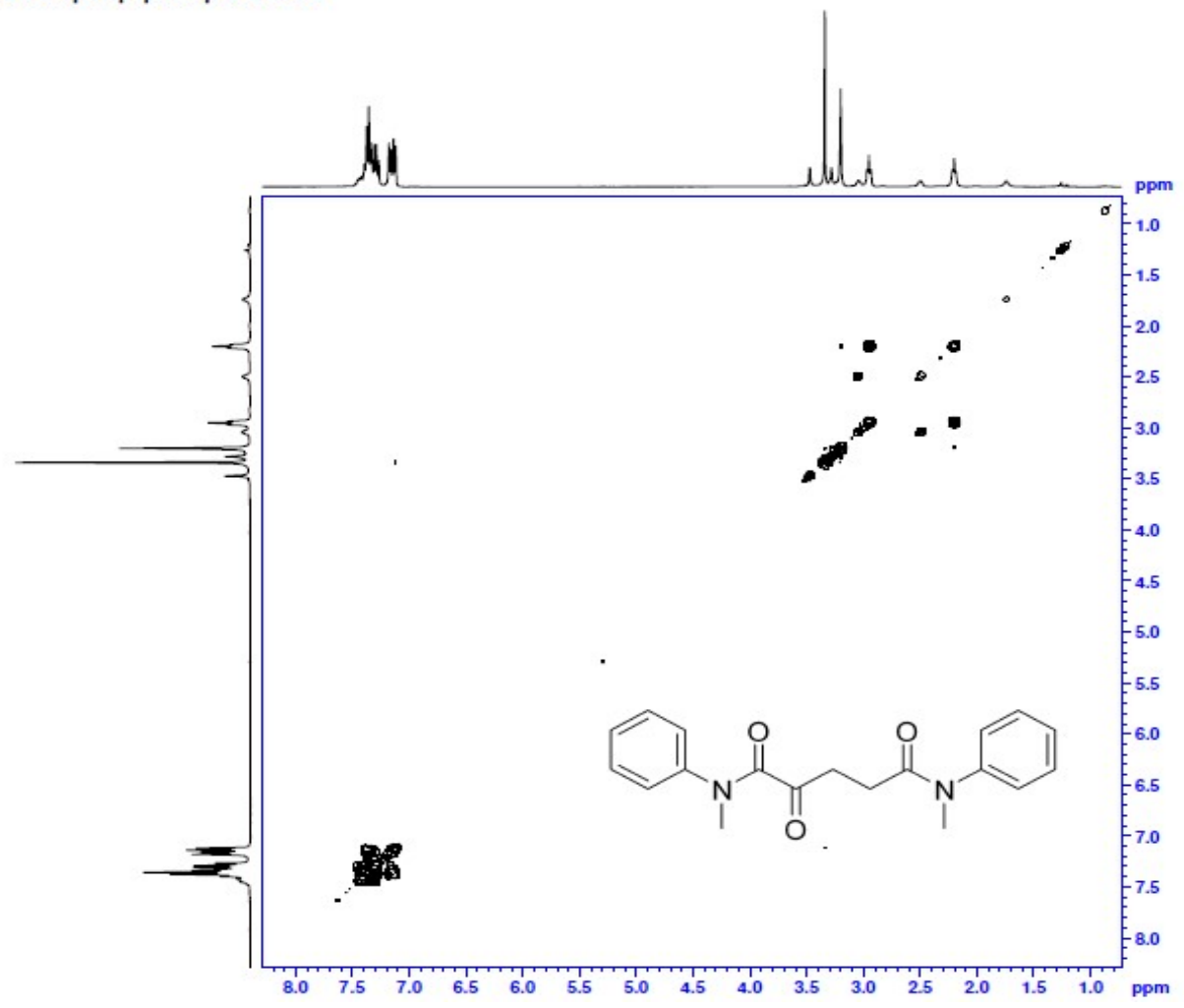

HSQC.

SKG716
HSQC.w $\mathrm{CDCl}$ /opt topspin3.5pl2 SKG 29

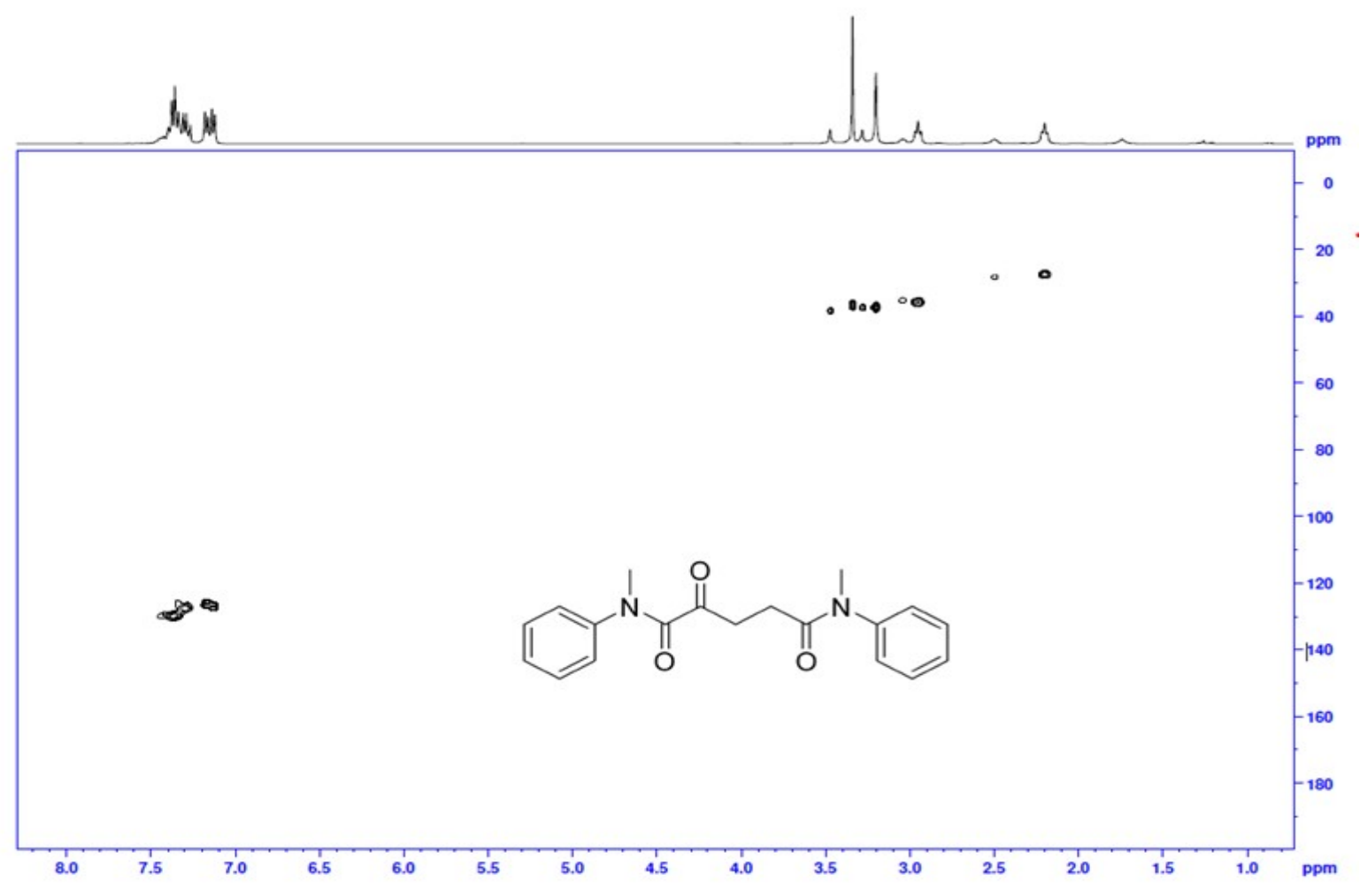

S90 
2-Oxo- $N^{1}, N^{4}$-diphenylsuccinamide (precursor of 18).<smiles>CC(C)(Cl)CC(=O)C(=O)Nc1ccccc1NC(=O)CC(=O)CC(=O)O</smiles>

In $25 \mathrm{ml}$ RBF was dissolved 2-oxosuccinic acid (0.250 g, $1.89 \mathrm{mmol}, 1.0 \mathrm{eq})$ in water (3 mL) and the mixture was cooled to $0{ }^{\circ} \mathrm{C}$. In a separate flask, aniline $(0.316 \mathrm{~g}, 3.40 \mathrm{mmol}, 1.8 \mathrm{eq})$ was mixed with water $(1 \mathrm{~mL})$ and $2 \mathrm{M}$ hydrochloric acid was added to adjust the $\mathrm{pH}$ to around 3-4. The aqueous acidic solution of aniline was added to the acid solution followed by addition of aqueous 1-ethyl-3-(3-dimethylaminopropyl) carbodiimide hydrochloride (EDC.HCl) (1.08 g, $5.67 \mathrm{mmol}, 3.0 \mathrm{eq})$ solution in water (3 mL). Upon addition of EDC.HCl, the reaction mixture became a yellow suspension and it was further stirred at room temperature for $2 \mathrm{~h}$. the reaction mixture was poured into ice cold water $(50 \mathrm{~mL})$ and the solid material was filtered, washed with dilute cold $\mathrm{HCl}$ to remove traces of aniline, then with dilute $\mathrm{NaHCO}_{3}$ and finally with water and then dried under vacuum to afford the product as a yellow solid ( $0.322 \mathrm{~g}, 1.14 \mathrm{mmol}, 60 \%)$ which was used in the next step without further purification. The title compound was obtained as a keto/enol tautomer (40:60) as per ${ }^{1} \mathrm{H}$ NMR.

TLC: $\mathrm{R}_{\mathrm{f}}$ ca 0.5 (7:3, hexane: EtOAc), UV-active and strong $\mathrm{KMnO}_{4}$;

MP: $208-210^{\circ} \mathrm{C}$;

HRMS (ESI) m/z: $[\mathrm{M}+\mathrm{Na}]^{+}$Calcd for $\mathrm{C}_{16} \mathrm{H}_{14} \mathrm{~N}_{2} \mathrm{O}_{3} \mathrm{Na}$ 305.0897; Found 305.0906 (error -3.0 ppm);

$U_{\max } 3304,1642,1594,1533,1442,1303,1252,685 \mathrm{~cm}^{-1}$;

${ }^{1} \mathrm{H}$ NMR (400 MHz, DMSO- $d_{6}$ ) ( keto/enol, 40:60) $\delta 14.06$ (0.6H s, OH of enol form), 10.59$10.57(1 \mathrm{H}, \mathrm{m}, \mathrm{CONH}$, keto + enol form), 10.29 (0.6H s, CONH of enol), $10.23(0.4 \mathrm{H}, \mathrm{s}$, CONH of keto form), 7.83-7.80 (2H, m, ArH), 7.64-7.56 (2H, m, ArH), 7.39-7.27 (4H, m, $\mathrm{ArH}), 7.15-7.02(2 \mathrm{H}, \mathrm{m}, \mathrm{ArH}), 6.30(0.6 \mathrm{H}, \mathrm{s},=\mathrm{C} \underline{\mathrm{H}}$ of enol form $), 4.01\left(0.8 \mathrm{H} \mathrm{s}, \mathrm{CH}_{2}\right.$ of keto form);

${ }^{13} \mathrm{C}$ NMR (101 MHz, DMSO- $\left.d_{6}\right)$ (keto/enol, 40:60) $\delta 193.2(\mathrm{C}=\mathrm{O}), 169.5,165.1,161.1$, $159.6,158.9,138.9,137.9,137.8,137.6,128.9$, 128.8, 128.7, 128.6, 128.5, 124.5, 124.4, $124.3,124.2,123.4,120.8,120.4,120.3,119.9,119.8,119.3,118.9,95.8$ ( $\mathrm{C}=\underline{\mathrm{C}} \mathrm{H}$, enol form), 46.1 ( $\mathrm{CH}_{2}$ keto form); $m / z(\mathrm{ESI}) 305.1\left[(\mathrm{M}+\mathrm{Na})^{+}, 100 \%\right]$. 
2-Oxo- $N^{1}, N^{4}$-diphenylsuccinamide.

${ }^{1} \mathrm{H}$ NMR (400 MHz, DMSO- $\left.d_{6}\right)$.

Nov02-2020

SKG627
PROTON.w DMSO
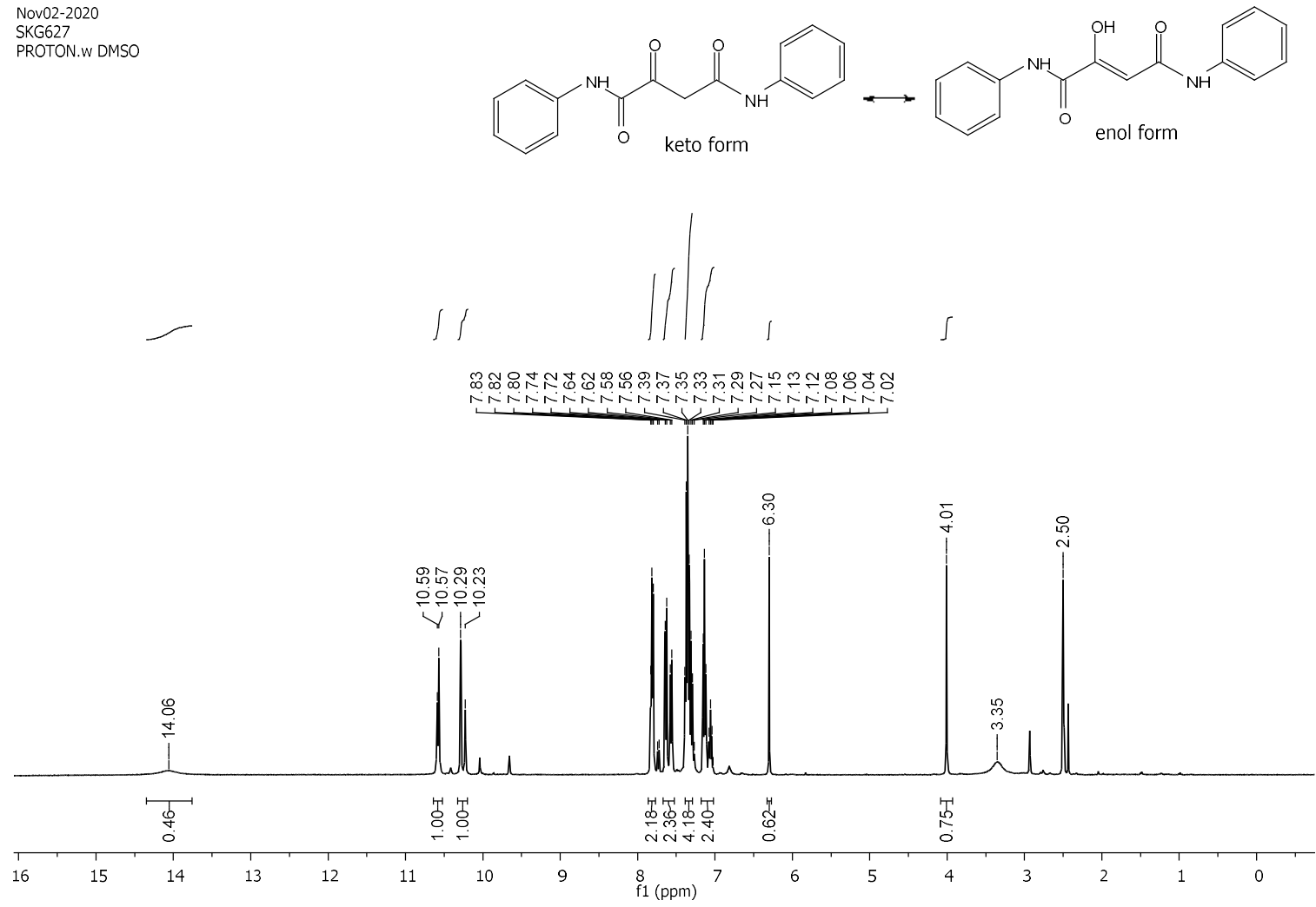

${ }^{13} \mathrm{C}$ NMR (101 MHz, DMSO- $\left.d_{6}\right)$.
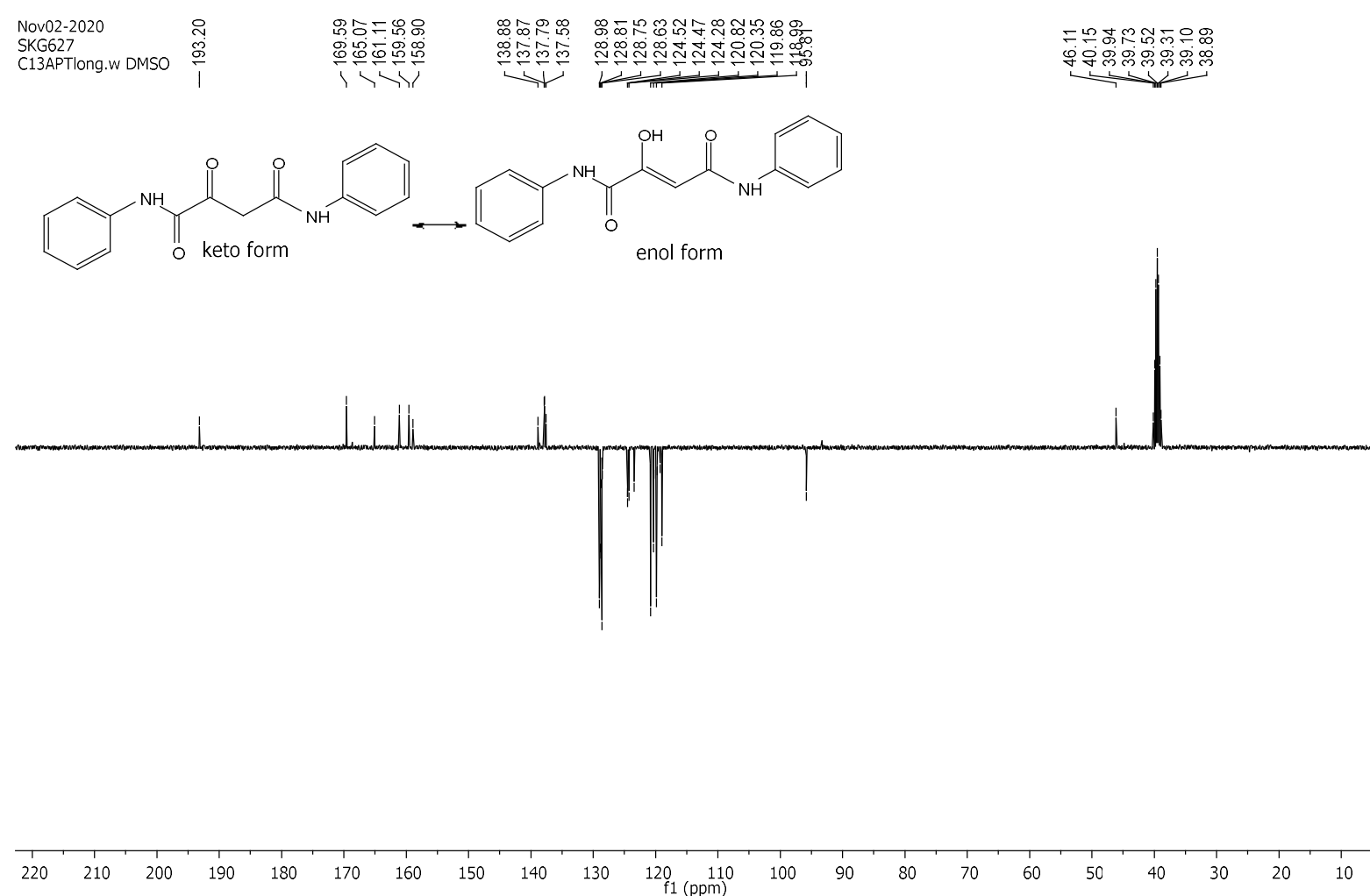
COSY.

SKG627

DMSO/opt/topspin3.5pl2 SKG 56

ketoform

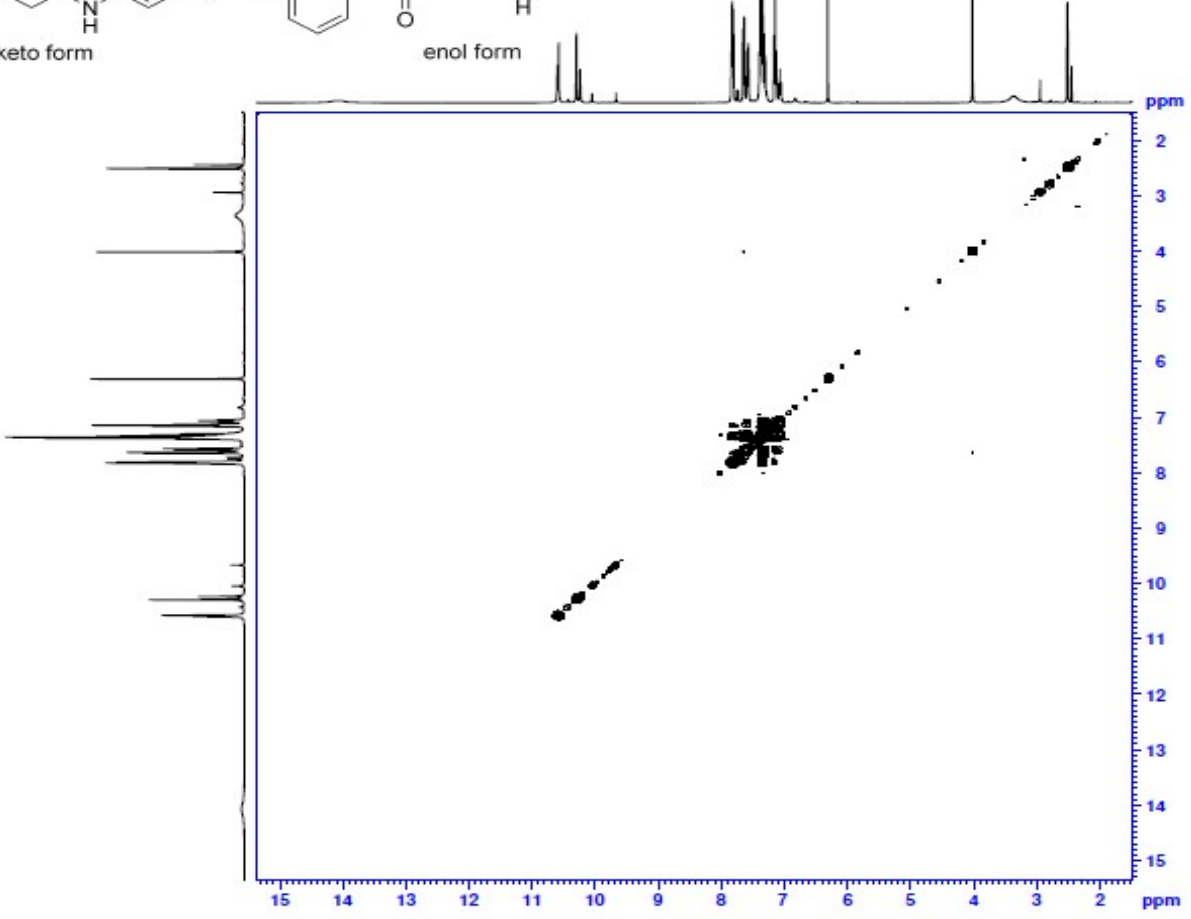

HSQC.

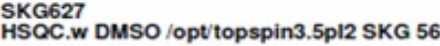

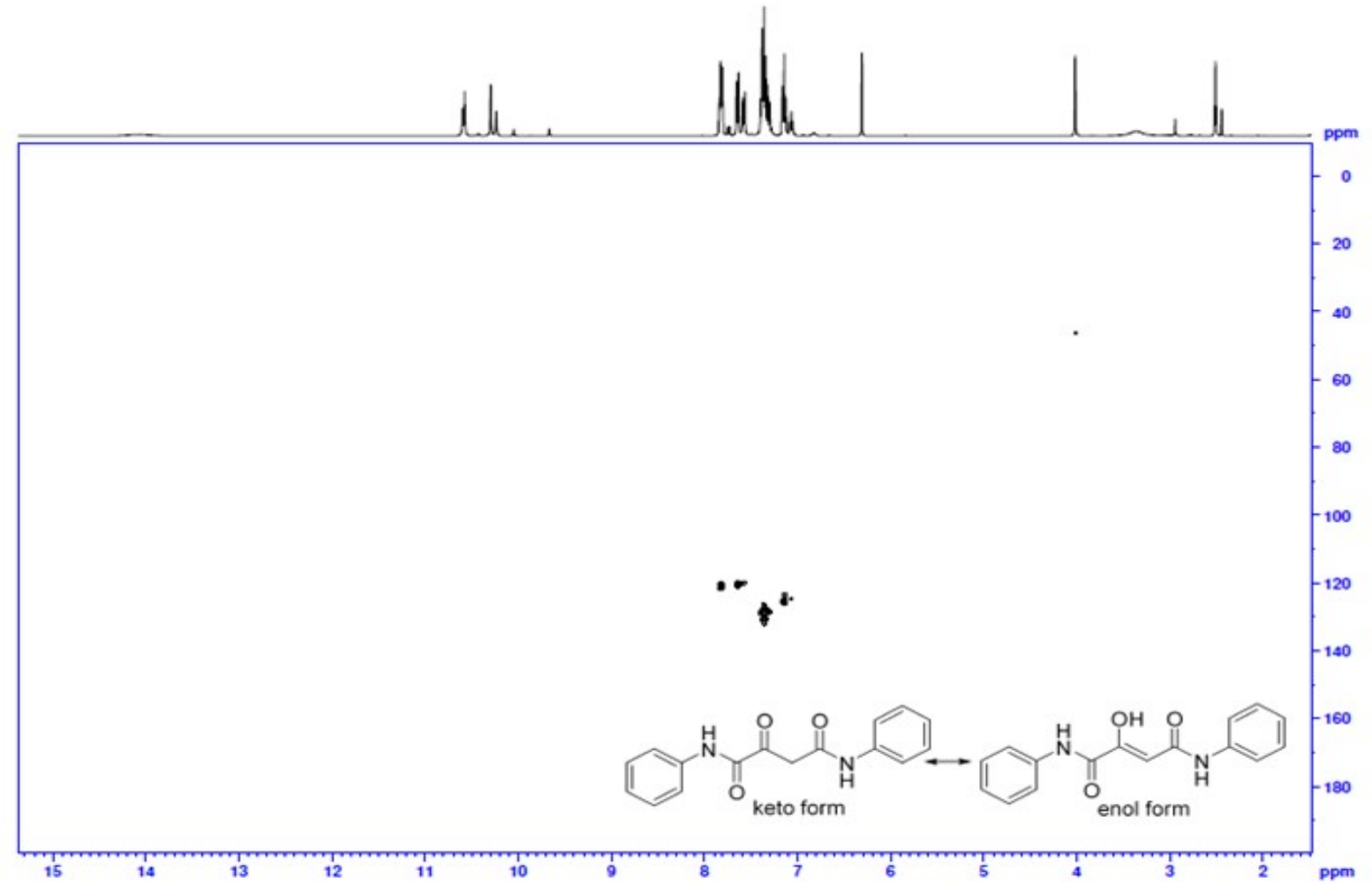


Methyl 4-(methyl(phenyl)amino)-2,4-dioxobutanoate (precursor of 19).<smiles>COC(=O)CC(=O)N(C)c1ccccc1</smiles>

This compound is novel and was prepared following general procedure B using methyl 2(methoxy(methyl)amino)-2-oxoacetate $(0.175 \mathrm{~g}, 1.34 \mathrm{mmol}, 1.0 \mathrm{eq})$ in anhydrous THF (5 $\mathrm{mL}), N$-methyl- $N$-phenylacetamide $(0.200 \mathrm{~g}, 1.34 \mathrm{mmol}, 1.0 \mathrm{eq})$ in anhydrous THF $(5 \mathrm{~mL})$, and LDA (1M solution in THF) $(1.74 \mathrm{~mL}, 1.74 \mathrm{mmol}, 1.3 \mathrm{eq})$ to give crude material which was further purified by column chromatography (10-30\% EtOAc in petroleum ether) to afford the product as a white solid $(0.163 \mathrm{~g}, 0.694 \mathrm{mmol}, 52 \%)$. The title compound was obtained as an enol form as per ${ }^{1} \mathrm{H}$ NMR.

TLC: $\mathrm{R}_{\mathrm{f}}$ ca 0.5 (7:3, hexane: EtOAc), UV-active and strong $\mathrm{KMnO}_{4}$;

MP: $86.5-88.3^{\circ} \mathrm{C}$;

HRMS (ESI) m/z: $[\mathrm{M}+\mathrm{Na}]^{+}$Calcd for $\mathrm{C}_{12} \mathrm{H}_{13} \mathrm{NO}_{4} \mathrm{Na} 258.0737$; Found 258.0735 (error 0.6 ppm);

$U_{\max } 2958,1737,1633,1588,1431,1351,1210,1116,770,698 \mathrm{~cm}^{-1}$;

${ }^{1} \mathrm{H}$ NMR (400 MHz, $\left.\mathrm{CDCl}_{3}\right) \delta 14.05$ (1H, br.s., OH of enol form), 7.60-7.39 (3H, m, ArH), 7.22-7.19 (2H, m, ArH), $5.79(1 \mathrm{H}, \mathrm{s},=\mathrm{C} \underline{\mathrm{H}}$, of enol form $), 3.78\left(3 \mathrm{H}, \mathrm{s}, \mathrm{COOCH}_{3}\right), 3.36(3 \mathrm{H}, \mathrm{s}$, $\left.-\mathrm{NCH}_{3}\right)$;

${ }^{13} \mathrm{C}$ NMR (101 MHz, $\left.\mathrm{CDCl}_{3}\right) \delta 170.8(\underline{\mathrm{C}}=\mathrm{CH}$, enol form), $163.2(\mathrm{C}=\mathrm{O}$ amide $), 158.3(\mathrm{C}=\mathrm{O}$ amide), 142.4, 130.1, 129.9, 128.5, 127.2, 95.8 ( $\mathrm{C}=\underline{\mathrm{CH}}$, enol form), 52.9, 37.1; $m / z(\mathrm{ESI}) 258.0\left[(\mathrm{M}+\mathrm{Na})^{+}, 100 \%\right]$. 
Methyl 4-(methyl(phenyl)amino)-2,4-dioxobutanoate.

${ }^{1} \mathrm{H}$ NMR $\left(400 \mathrm{MHz}, \mathrm{CDCl}_{3}\right)$.

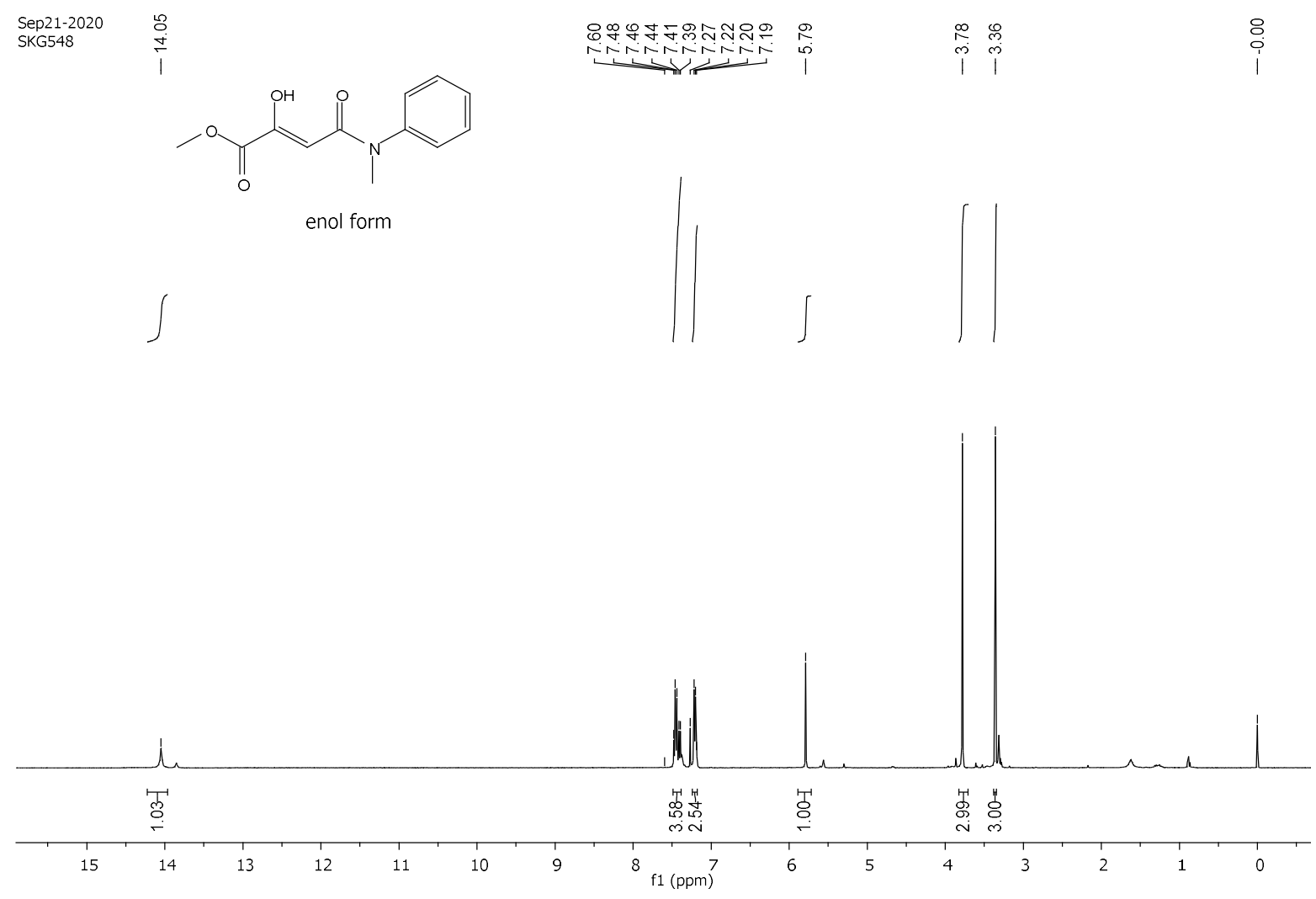

${ }^{13} \mathrm{C}$ NMR (101 MHz, $\left.\mathrm{CDCl}_{3}\right)$.

\begin{tabular}{|c|c|c|c|c|c|c|c|}
\hline $\begin{array}{l}\text { Sep21-2020 } \\
\text { SKG548 } \\
\text { C13APTlong.w CDCI3 }\end{array}$ & $\begin{array}{l}\stackrel{0}{1} \\
\stackrel{2}{P}\end{array}$ & 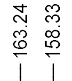 & $\frac{\text { J }}{\text { 先 }}$ & 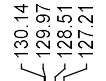 & $\begin{array}{c}1 \\
2 \\
2 \\
0 \\
1 \\
1\end{array}$ & 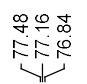 & @ \\
\hline
\end{tabular}
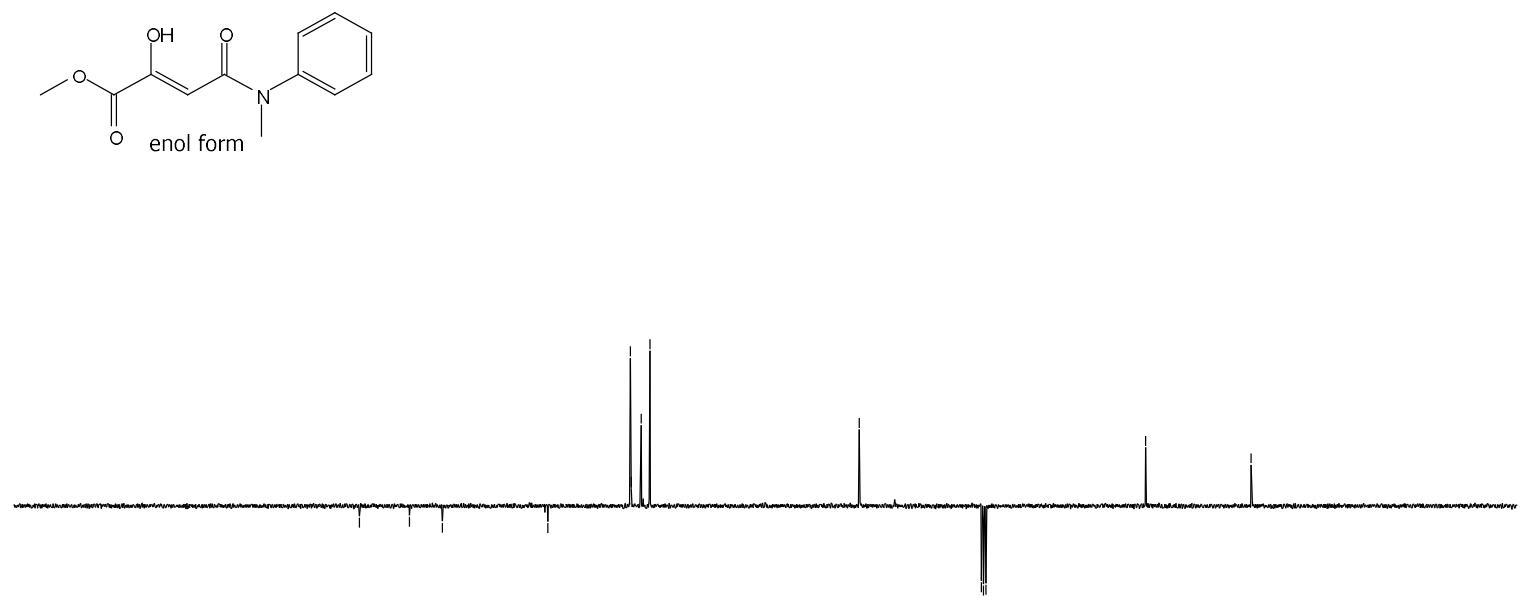

$\begin{array}{llllllllllllllllllllllll}220 & 210 & 200 & 190 & 180 & 170 & 160 & 150 & 140 & 130 & 120 & \underset{\mathrm{f} 1}{110}(\mathrm{ppm}) & 100 & 90 & 80 & 70 & 60 & 50 & 40 & 30 & 20 & 10 & 0\end{array}$ 
COSY.

SKG548 US

COSY.w CDCl3/opt/topspin3.5pl2 SKG 17

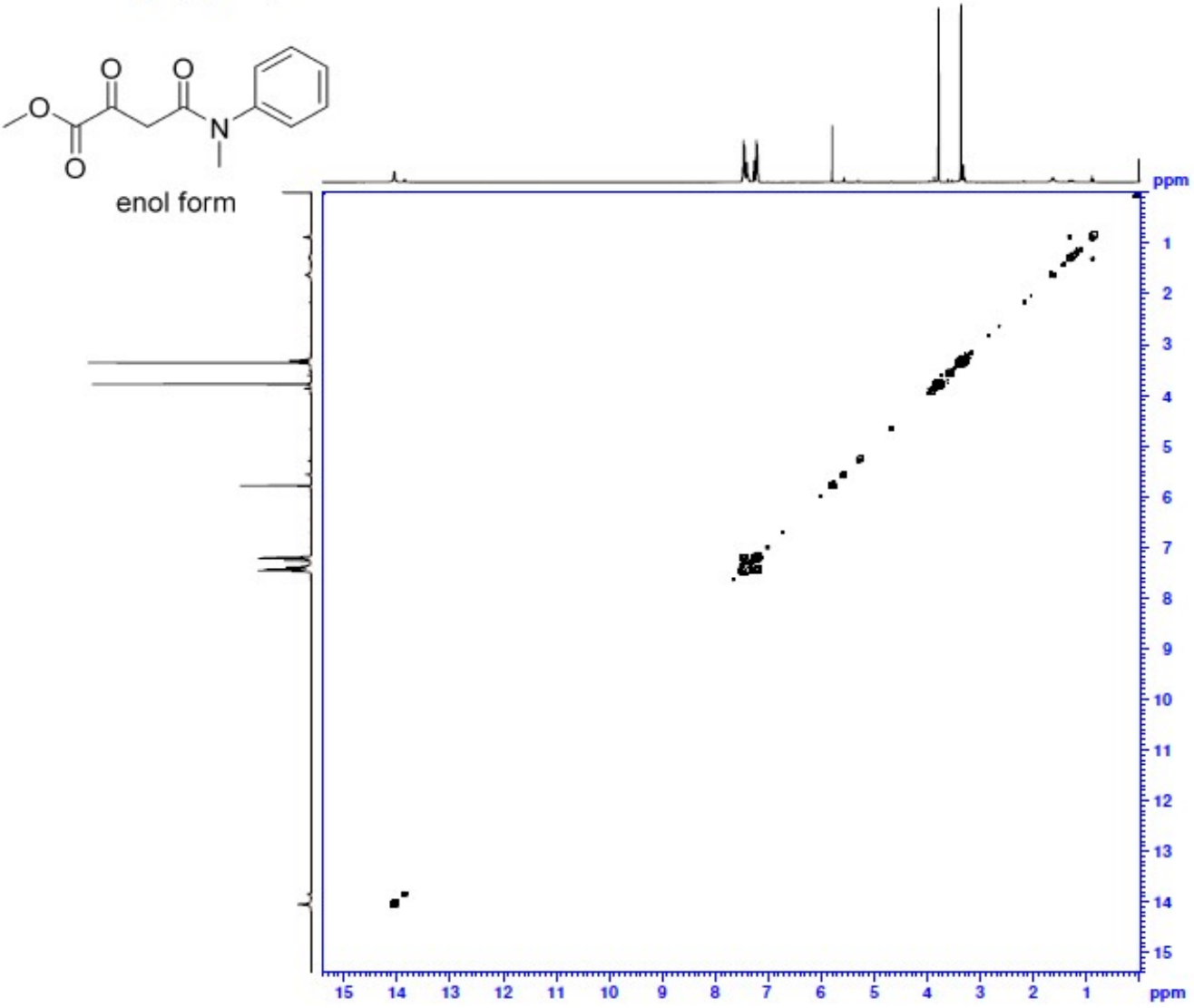

HSQC.

SKG548 US
HSQC.w CDCl3/opt/topspin3.5pl2 SKG 17

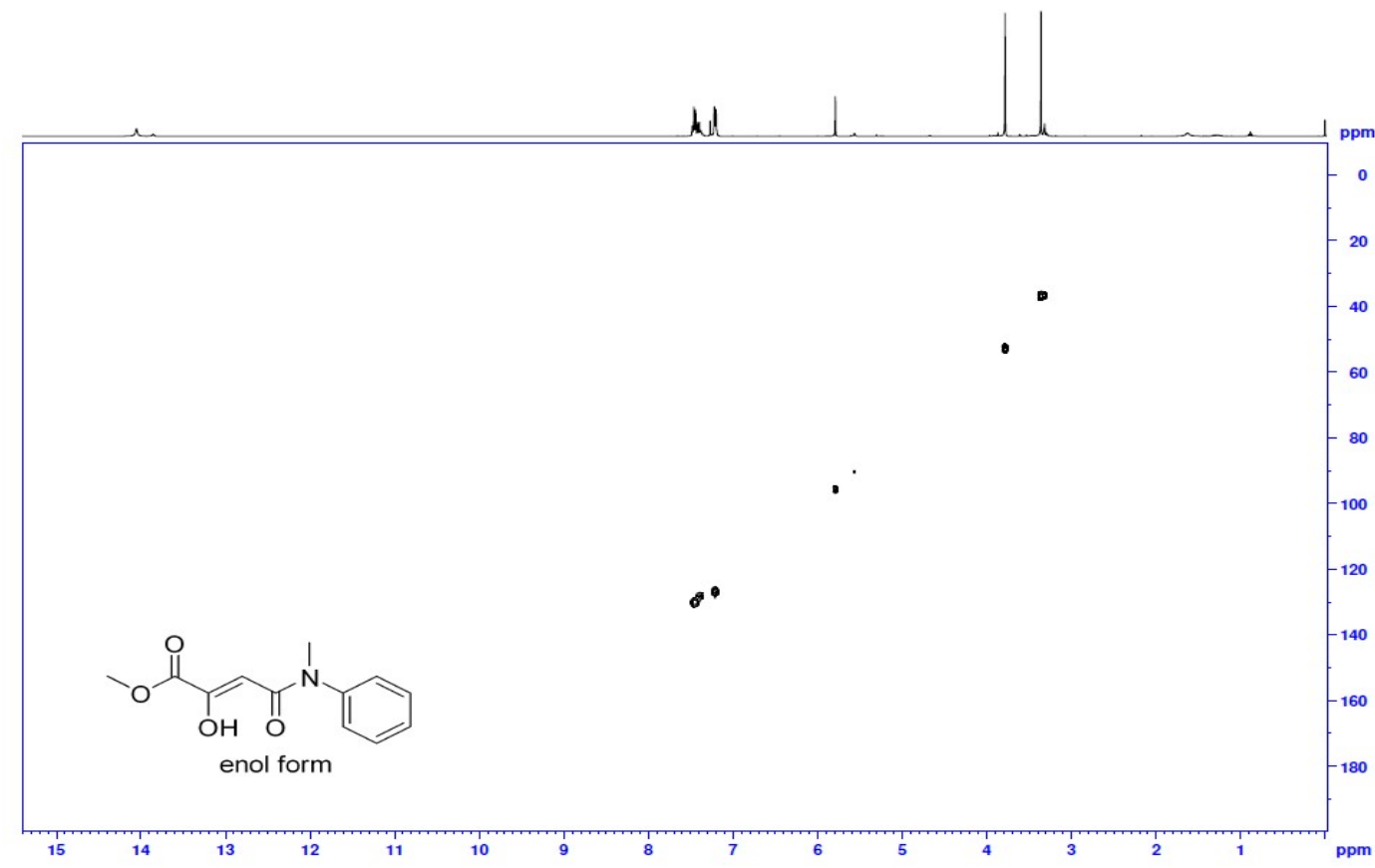


Ethyl 4-(methyl(phenyl)amino)-2,4-dioxobutanoate (precursor of 20).<smiles>CCOC(=O)C(=O)CC(=O)N(C)c1ccccc1</smiles>

This compound is novel and was prepared following general procedure B using diethyl oxalate $(0.245 \mathrm{~g}, 1.67 \mathrm{mmol}, 1.0 \mathrm{eq})$, in anhydrous THF (5 mL) $N$-methyl- $N$-phenylacetamide $(0.250 \mathrm{~g}, 1.67 \mathrm{mmol}, 1.0 \mathrm{eq})$ in anhydrous THF ( $5 \mathrm{~mL})$, and LDA (1M solution in THF) (2.17 $\mathrm{mL}, 2.17 \mathrm{mmol}, 1.3 \mathrm{eq})$ to give crude material which was further purified by column chromatography (10-30\% EtOAc in petroleum ether) to afford the product as a white solid $(0.256 \mathrm{~g}, 1.03 \mathrm{mmol}, 62 \%)$. The title compound was obtained as an enol form as per ${ }^{1} \mathrm{H}$ NMR.

TLC: $\mathrm{R}_{\mathrm{f}}$ ca 0.5 (6:4, hexane: EtOAc), UV-active and strong $\mathrm{KMnO}_{4}$;

MP: $87-89^{\circ} \mathrm{C}$;

HRMS (ESI) m/z: [M+Na] $]^{+}$Calcd for $\mathrm{C}_{13} \mathrm{H}_{15} \mathrm{NO}_{4} \mathrm{Na} 272.089$; Found 272.0891 (error 0.7 ppm);

$U_{\max } 3061,2988,1728,1631,1588,1472,1348,1250,1134,769,695 \mathrm{~cm}^{-1}$;

${ }^{1} \mathrm{H}$ NMR (400 MHz, $\left.\mathrm{CDCl}_{3}\right) \delta 14.03(1 \mathrm{H}, \mathrm{s}$, br.s., OH of enol form) 7.47-7.39 (3H, m, ArH), 7.21-7.20 (2H, m, ArH), $5.79(1 \mathrm{H}, \mathrm{s},=\mathrm{C} \underline{\mathrm{H}}), 4.24\left(2 \mathrm{H}, \mathrm{q}, J=7.1 \mathrm{~Hz}, \underline{\mathrm{C}}_{2} \mathrm{CH}_{3}\right), 3.36(3 \mathrm{H}, \mathrm{s},-$ $\left.\mathrm{NCH}_{3}\right), 1.28\left(3 \mathrm{H}, \mathrm{t}, J=7.1 \mathrm{~Hz}, \mathrm{CH}_{2} \underline{\mathrm{C}}_{3}\right)$;

${ }^{13} \mathrm{C}$ NMR (101 MHz, $\left.\mathrm{CDCl}_{3}\right) \delta 170.8(\underline{\mathrm{C}}=\mathrm{CH}$, enol form), $162.3(\mathrm{C}=\mathrm{O}$ amide), $158.7(\mathrm{C}=\mathrm{O}$ amide), 142.3, 130.2, 130.1, 128.5, 127.2, 95.6 ( $\mathrm{C}=\underline{\mathrm{C}} \mathrm{H}$, enol form), 62.3, 37.1, 14.1; $m / z(\mathrm{ESI}) 272.2\left[(\mathrm{M}+\mathrm{Na})^{+}, 100 \%\right]$. 
Ethyl 4-(methyl(phenyl)amino)-2,4-dioxobutanoate.

${ }^{1} \mathrm{H}$ NMR $\left(400 \mathrm{MHz}, \mathrm{CDCl}_{3}\right)$.

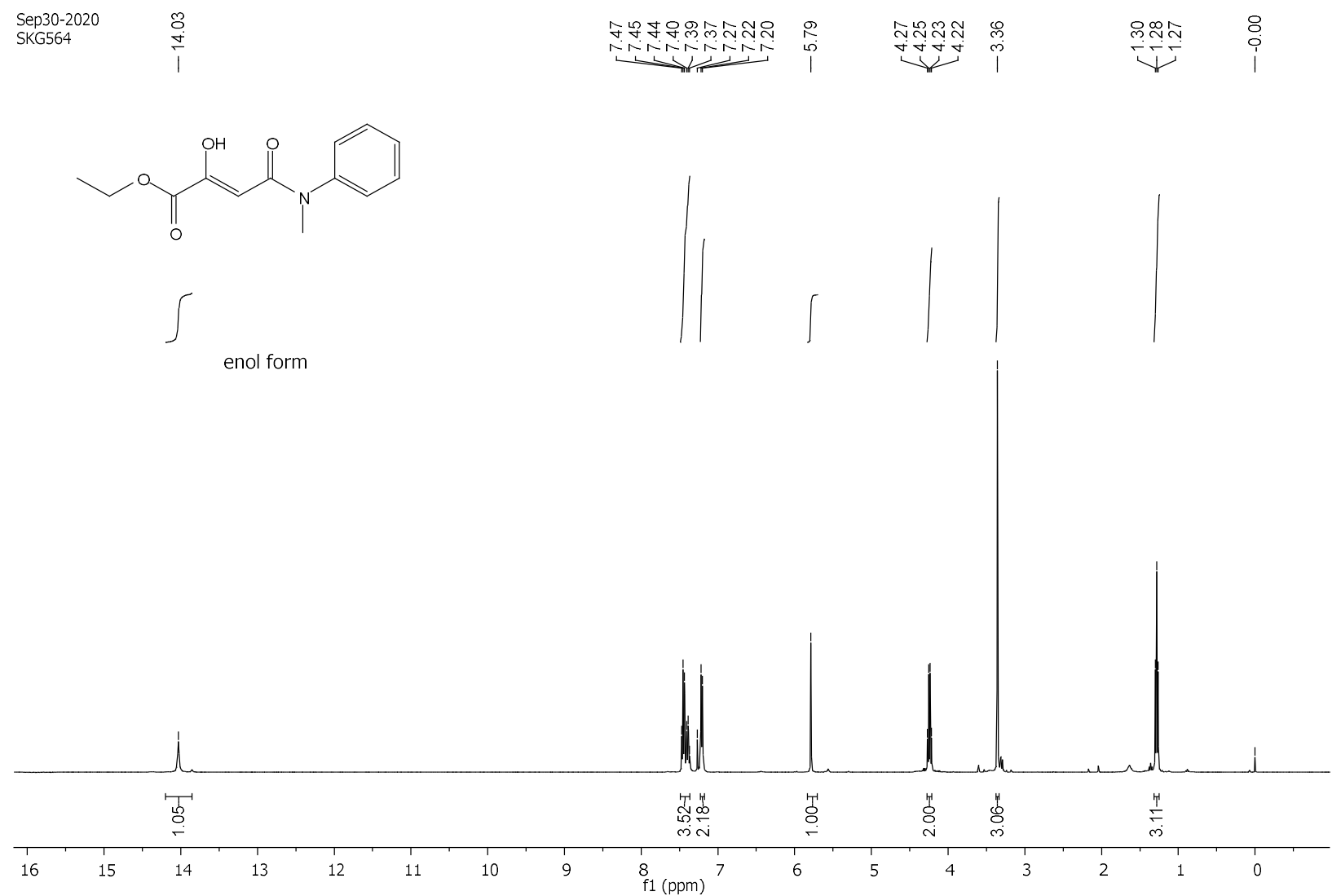

${ }^{13} \mathrm{C}$ NMR (101 MHz, $\left.\mathrm{CDCl}_{3}\right)$.

Sep30-2020
SKG564

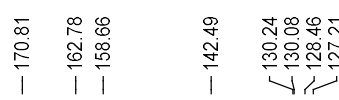

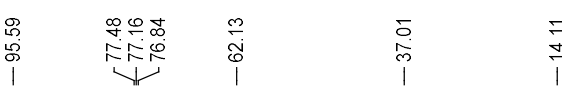

enol form

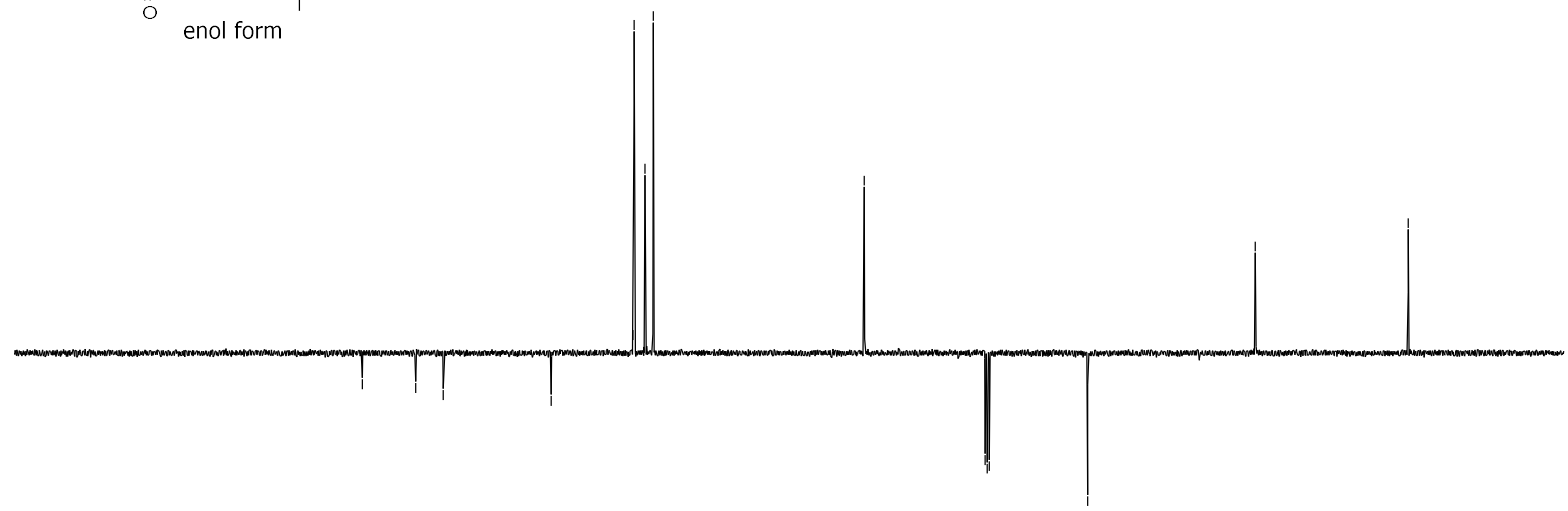

\begin{tabular}{llllllllllllllllllllllllll}
\hline 220 & 210 & 200 & 190 & 180 & 170 & 160 & 150 & 140 & 130 & 120 & $\frac{110}{f 1}(\mathrm{ppm})$ & 100 & 90 & 80 & 70 & 60 & 50 & 40 & 30 & 20 & 10 & 0 & 10
\end{tabular} 
COSY.

SKG564 US pure
COSY.w CDCl3/opt/topspin3.5pl2 SKG 54

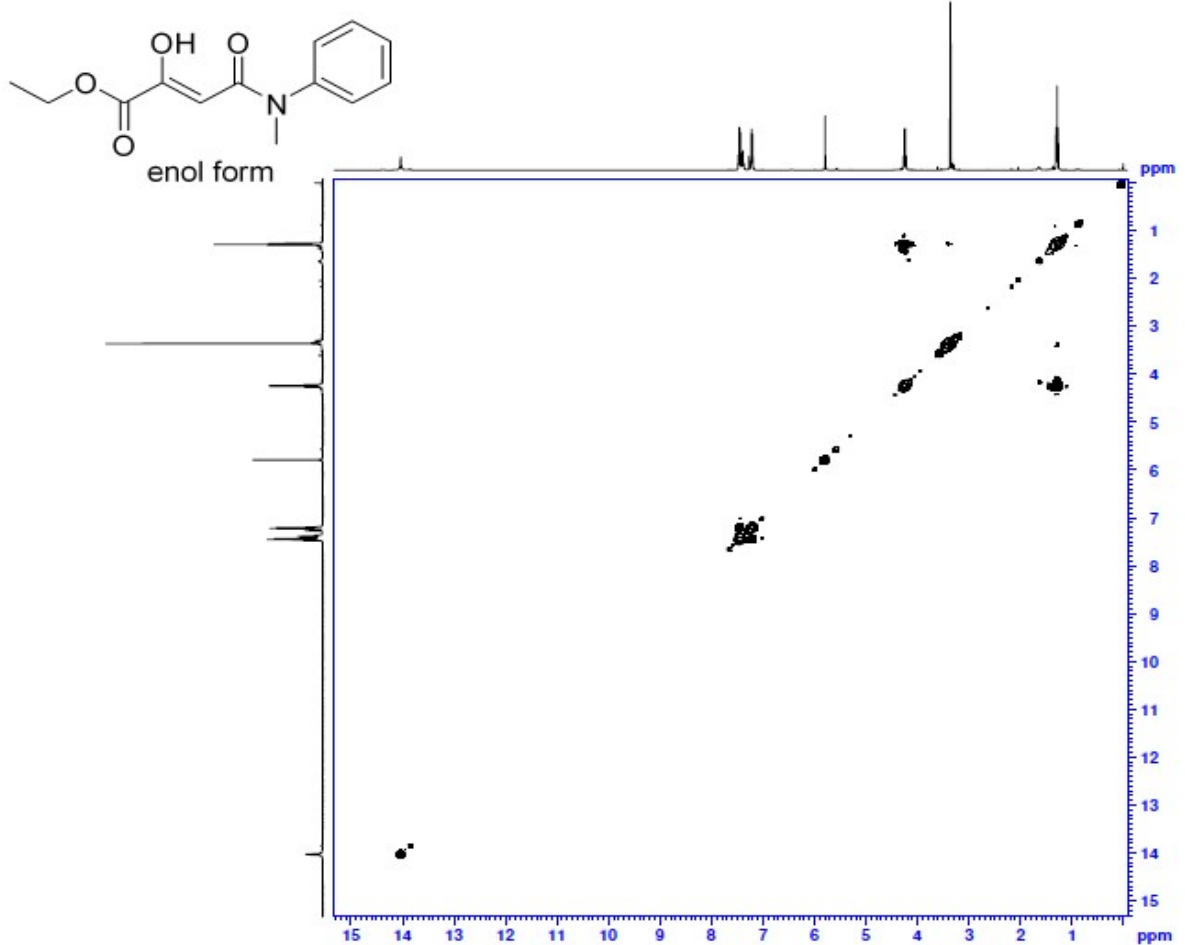

HSQC.

SKG564 US pure
HSOC.w CDCl3 /opt/topspin3.5pl2 SKG 54

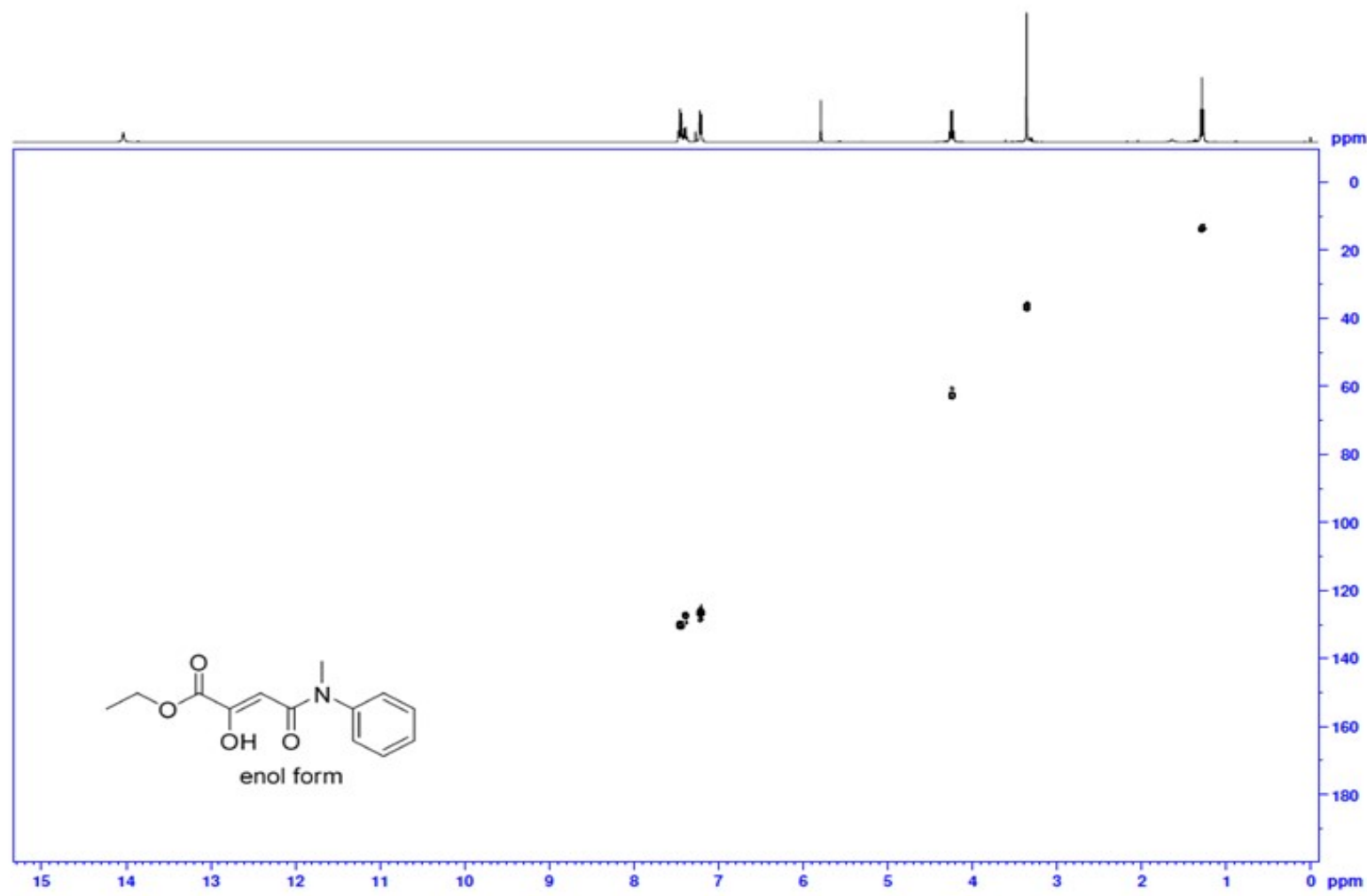


Methyl-2,4-dioxo-N-phenyl-4-(piperidin-1-yl)butanamide (precursor of 21).<smiles>CN(C(=O)C(=O)CC(=O)N1CCCCC1)c1ccccc1</smiles>

This compound is novel and was prepared following the general procedure B using methyl 2(methyl(phenyl)amino)-2-oxoacetate $(0.700 \mathrm{~g}, 3.93 \mathrm{mmol}, 1.0 \mathrm{eq})$ in anhydrous THF (10 $\mathrm{mL}), 1$-(piperidin-1-yl)ethan-1-one $(0.500 \mathrm{~g}, 3.93 \mathrm{mmol}, 1.0 \mathrm{eq})$ in anhydrous THF $(8 \mathrm{~mL})$ and LDA (1M solution in THF) $(5.11 \mathrm{~mL}, 5.11 \mathrm{mmol}, 1.3 \mathrm{eq})$ to give crude material which was further purified by column chromatography (50-100\% EtOAc in petroleum ether) to afford the product as a yellow liquid $(0.632 \mathrm{~g}, 2.19 \mathrm{mmol}, 56 \%)$. The title compound was obtained as keto/enol tautomer $(3: 1)$ and rotamers as per ${ }^{1} \mathrm{H}$ NMR.

TLC: $\mathrm{R}_{\mathrm{f}}$ ca 0.4 (7:3, hexane: EtOAc), UV-active and strong $\mathrm{KMnO}_{4}$; HRMS (ESI) m/z: [M+H] $]^{+}$Calcd for $\mathrm{C}_{16} \mathrm{H}_{21} \mathrm{~N}_{2} \mathrm{O}_{3}$ 289.1547; Found 289.1544 (error 0.8 ppm); $U_{\max } 2935,2857,1721,1625,1591,1494,1442,1383,1250,1224,1016,765,695 \mathrm{~cm}^{-1}$; ${ }^{1} \mathrm{H}$ NMR (500 MHz, $\mathrm{CDCl}_{3}$ ) (keto/enol, 3:1) $\delta 14.73$ (0.25H, br. s, OH of enol form), 7.41$7.20(5 \mathrm{H}, \mathrm{m}, \mathrm{ArH}), 5.75(0.25 \mathrm{H}, \mathrm{s},=\mathrm{C} \underline{\mathrm{H}}$, of enol form $), 3.92\left(0.25 \mathrm{H}, \mathrm{s}, \mathrm{CH}_{2}\right.$ of keto form, rotamer 1), $3.77\left(1.25 \mathrm{H}, \mathrm{s}, \mathrm{CH}_{2}\right.$ of keto form, rotamer 2$), 3.55-3.26\left(7 \mathrm{H}, \mathrm{m}, \mathrm{NCH}_{2}\right.$ of piperidine $\left.+-\mathrm{NCH}_{3}\right), 1.64-1.54\left(6 \mathrm{H}, \mathrm{m}, \mathrm{CH}_{2}\right.$ of piperidine);

${ }^{13} \mathrm{C}$ NMR (126 MHz, $\mathrm{CDCl}_{3}$ ) (keto/enol, 3:1) $\delta 194.2$ (C=O ketone), 193.1 (C=O ketone), $169.7,166.2$, 166.0, 165.1, 165.0, 164.9, 142.5, 142.3, 129.5, 129.4, 129.3, 127.8, 127.3, 127.2, 126.6, 125.9, 125.8, $90.9(\mathrm{C}=\underline{\mathrm{CH}}$, enol), 47.4, 47.3, 45.2, 44.4, 42.8, 42.6, 38.8, 37.6, 26.3, 25.5, 25.4, 24.5, 24.4, 24.3;

$m / z(\mathrm{ESI}) 289.2\left[(\mathrm{M}+\mathrm{H})^{+}, 100 \%\right]$. 
$N$-Methyl-2,4-dioxo- $N$-phenyl-4-(piperidin-1-yl)butanamide.

${ }^{1} \mathrm{H}$ NMR $\left(500 \mathrm{MHz}, \mathrm{CDCl}_{3}\right)$.

Apr29-2021

Chemist Shweta Gediya

PROTON.w CDCl3 /opt/topspin3.2 SKG 26
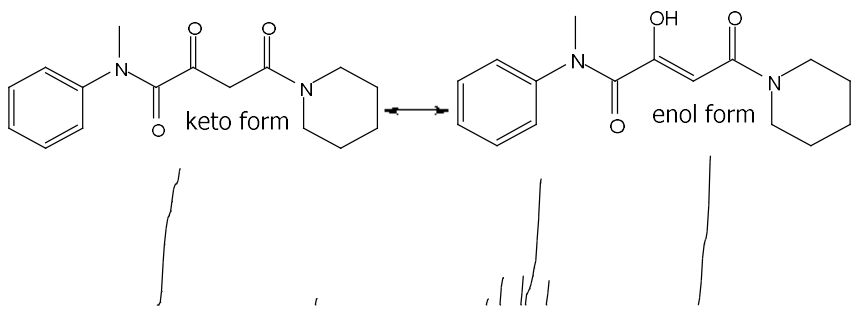

111

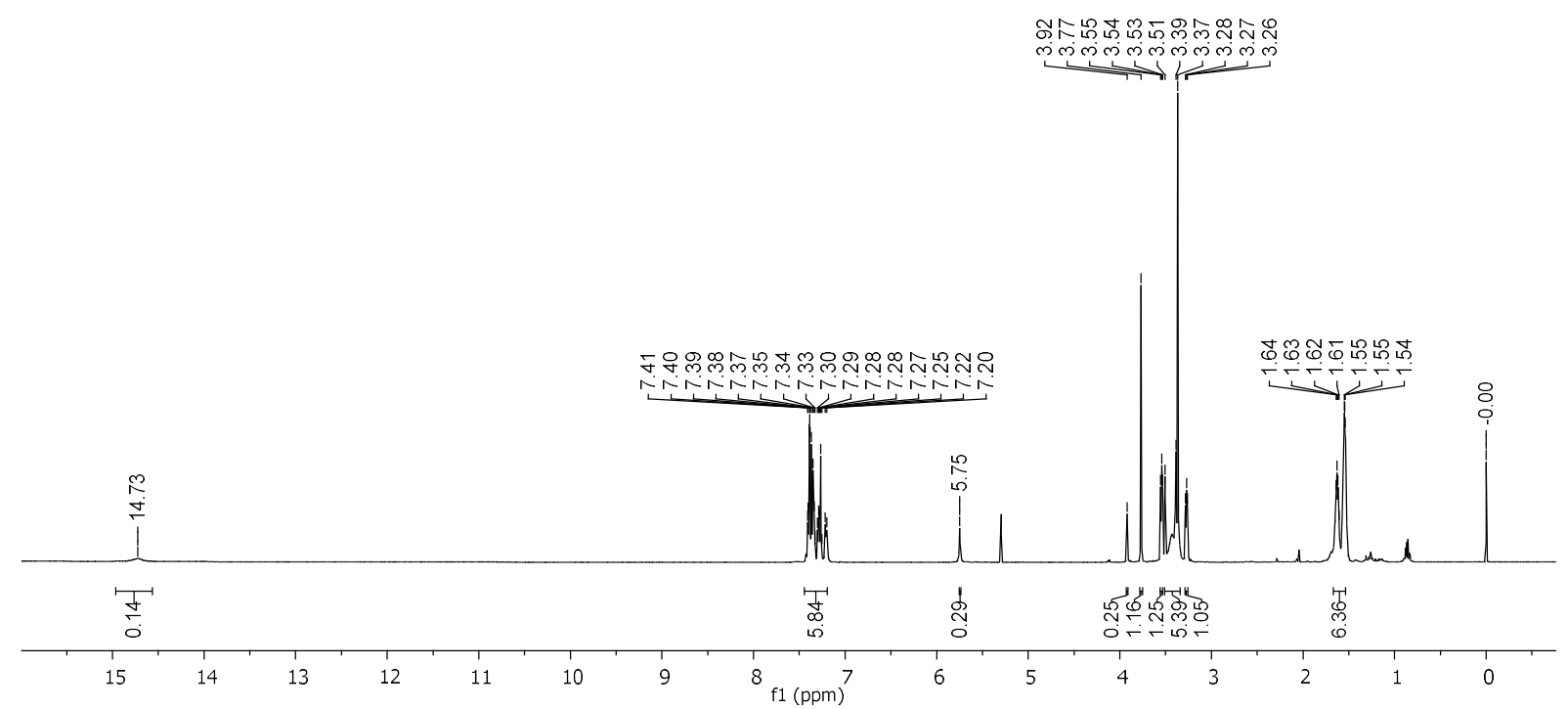

${ }^{13} \mathrm{C} \mathrm{NMR}\left(126 \mathrm{MHz}, \mathrm{CDCl}_{3}\right)$

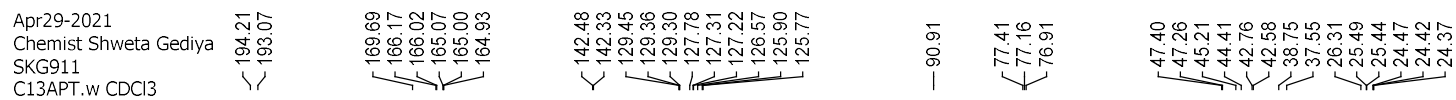

(1)

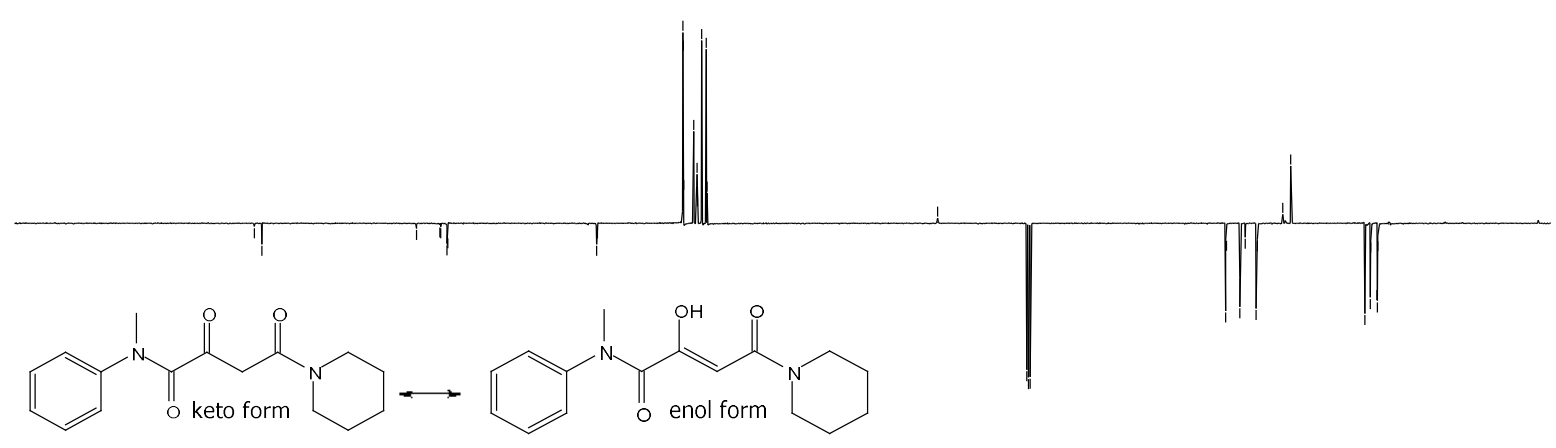

\begin{tabular}{rlllllllllllllllllllllll}
\hline 230 & 220 & 210 & 200 & 190 & 180 & 170 & 160 & 150 & 140 & 130 & $\underset{\mathrm{f} 1}{120}(\mathrm{pmm})$ & 100 & 90 & 80 & 70 & 60 & 50 & 40 & 30 & 20 & 10 & 0
\end{tabular} 
COSY.

Chemist Shweta Gediya

COSY 1 run2 2 (opt topspin3.2 SKG 26

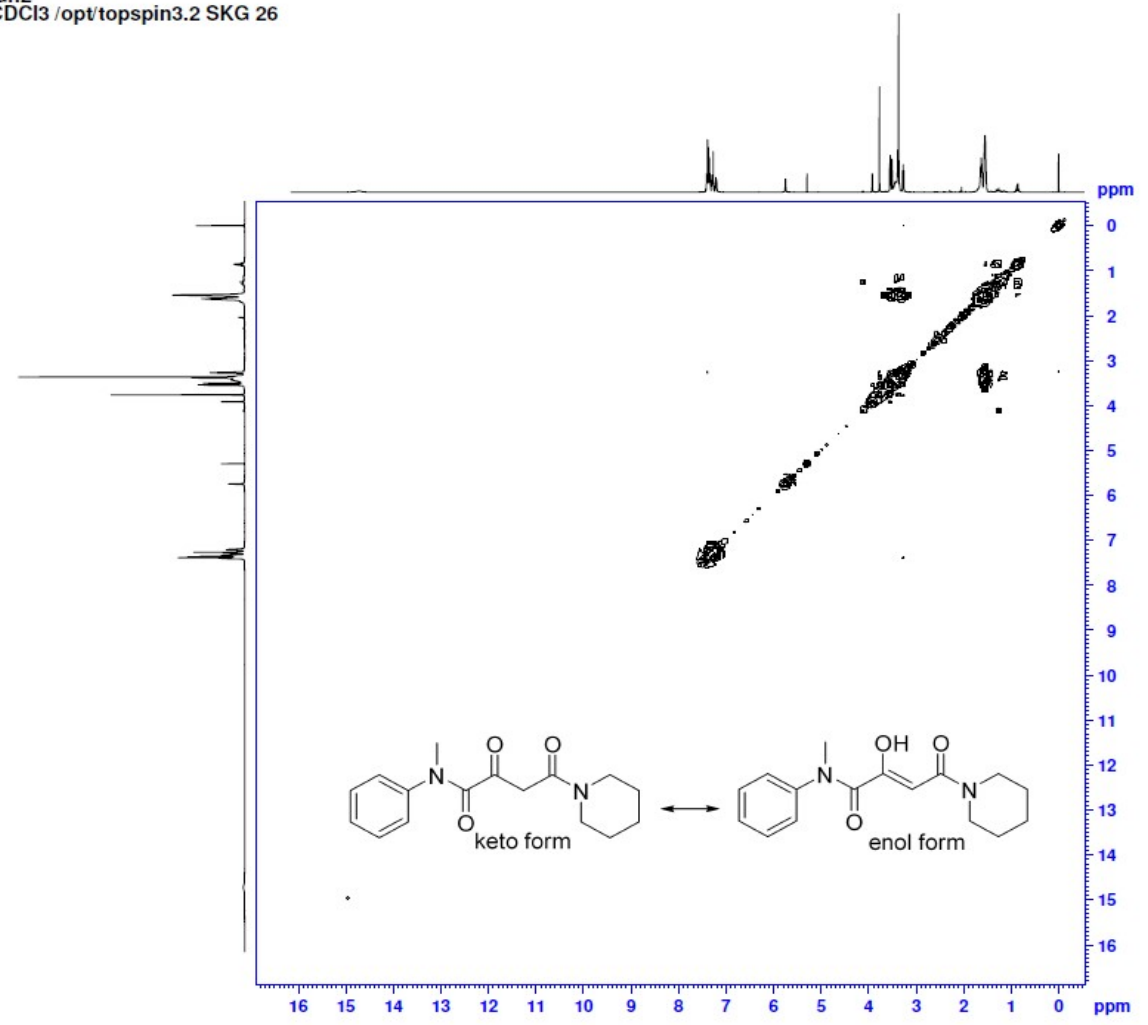

HSQC.

Chemist Shweta Gediya

HSGC.w $\mathrm{CDCl}_{3}$ /opt/topspin 3.2 SKG 26

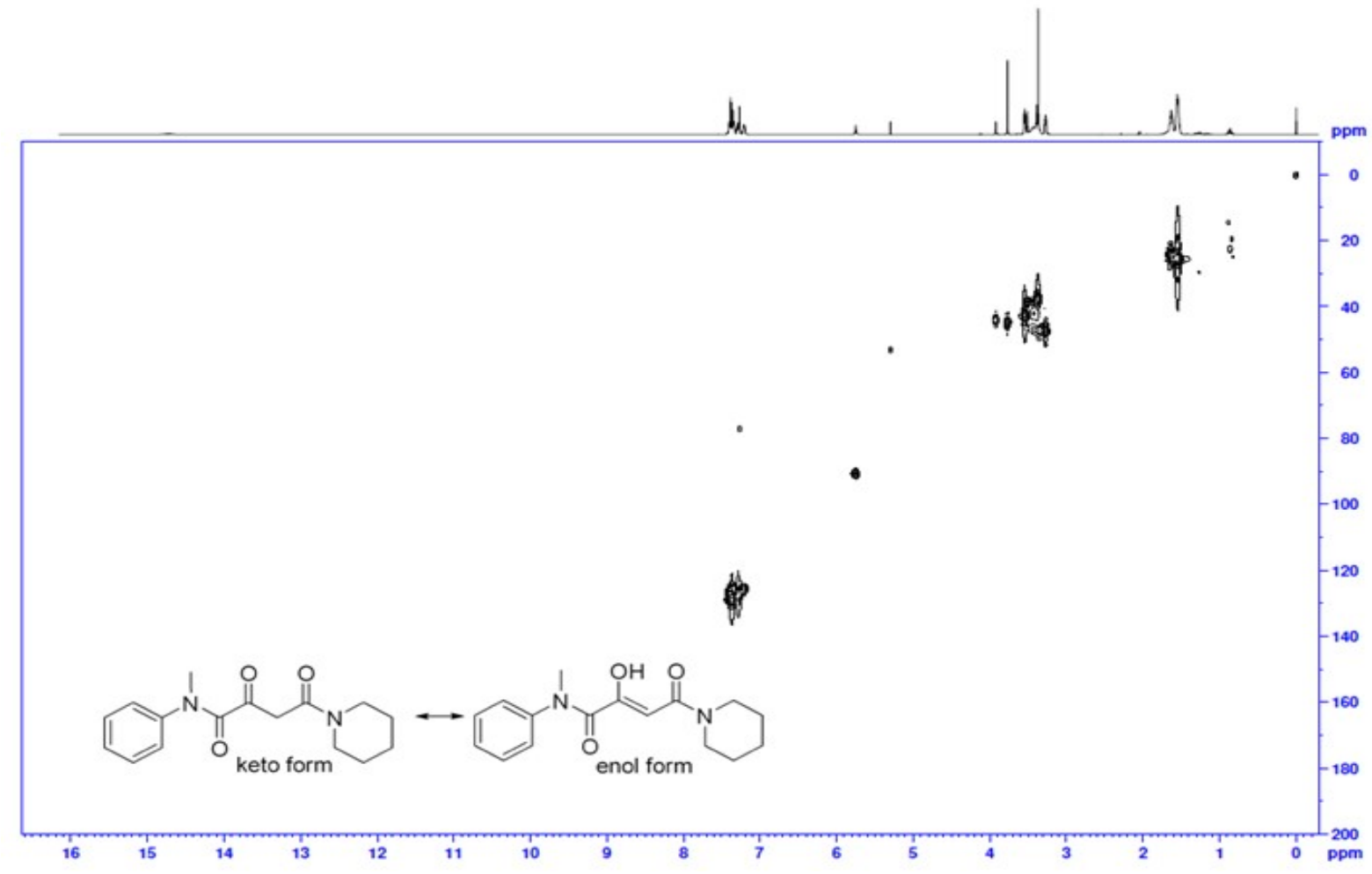




\section{1,4-Di(piperidin-1-yl)butane-1,2,4-trione (precursor of 22).}<smiles>O=C(CC(=O)N1CCCCC1)C(=O)N1CCCCC1</smiles>

This compound is novel and was prepared following general procedure B using methyl 2oxo-2-(piperidin-1-yl)acetate (0.673 g, $3.93 \mathrm{mmol}, 1.0 \mathrm{eq})$ in anhydrous THF (10 mL) and 1(piperidin-1-yl)ethan-1-one (0.500 g, $3.93 \mathrm{mmol}, 1.0 \mathrm{eq})$ in anhydrous THF (10 mL) and LDA (1M solution in THF) $(5.11 \mathrm{~mL}, 5.11 \mathrm{mmol}, 1.3 \mathrm{eq})$ to give crude material which was further purified by column chromatography (50-100\% EtOAc in petroleum ether) to afford the product as a white solid $(0.710 \mathrm{~g}, 2.67 \mathrm{mmol}, 68 \%)$. The title compound was obtained as a keto/enol tautomer (3:1) as per ${ }^{1} \mathrm{H}$ NMR.

TLC: $\mathrm{R}_{\mathrm{f}}$ ca 0.4 (4:6, hexane: EtOAc), UV-active and strong $\mathrm{KMnO}_{4}$; MP: $70-71{ }^{\circ} \mathrm{C}$;

HRMS (ESI) m/z: [M+Na] $]^{+}$Calcd for $\mathrm{C}_{14} \mathrm{H}_{22} \mathrm{~N}_{2} \mathrm{O}_{3} \mathrm{Na}$ 289.1523; Found 289.1513 (error 3.2 ppm);

$U_{\max } 2983,2855,1701,1619,1437,1406,1226,1125,1099,1008,851,615 \mathrm{~cm}^{-1}$;

${ }^{1} \mathrm{H}$ NMR (500 MHz, $\mathrm{CDCl}_{3}$ ) (keto/enol, 3:1) $\delta 15.33(0.25 \mathrm{H}, \mathrm{s}, \mathrm{OH}$ of enol form), 5.67 $(0.25 \mathrm{H}, \mathrm{s},=\mathrm{C} \underline{\mathrm{H}}$, of enol form $), 3.85\left(1.5 \mathrm{H}, \mathrm{s}\right.$, keto $\mathrm{CH}_{2}$ of keto form $), 3.57-3.49(6 \mathrm{H}, \mathrm{m}$, $\mathrm{NCH}_{2}$ of piperidine), 3.34-3.31 (2H, m, $\mathrm{NCH}_{2}$ of piperidine) $1.62-1.53\left(12 \mathrm{H}, \mathrm{m}, \mathrm{CH}_{2}\right.$ of piperidine);

${ }^{13} \mathrm{C}$ NMR (126 MHz, $\mathrm{CDCl}_{3}$ ) (keto/enol, 3:1) $\delta 194.3$ (C=O keto form), 170.1, 168.3, 165.7, 164.9, 163.9, $89.4(\mathrm{C}=\underline{\mathrm{C}} \mathrm{H}$, enol), 47.8, 47.3, 47.2, 44.7, 43.3, 43.0, 42.6, 26.7, 26.3, 26.1, 25.6, 25.5, 24.6, 24.5, 24.4;

$m / z(\mathrm{ESI}) 289.2\left[(\mathrm{M}+\mathrm{Na})^{+}, 100 \%\right]$. 


\section{1,4-Di(piperidin-1-yl)butane-1,2,4-trione.}

${ }^{1} \mathrm{H}$ NMR (500 MHz, $\left.\mathrm{CDCl}_{3}\right)$.

\section{Jul21-2020}

Chemist Shweta Gediya

PROTON.w CDCl3 /opt/topspin3.2 SKG 60
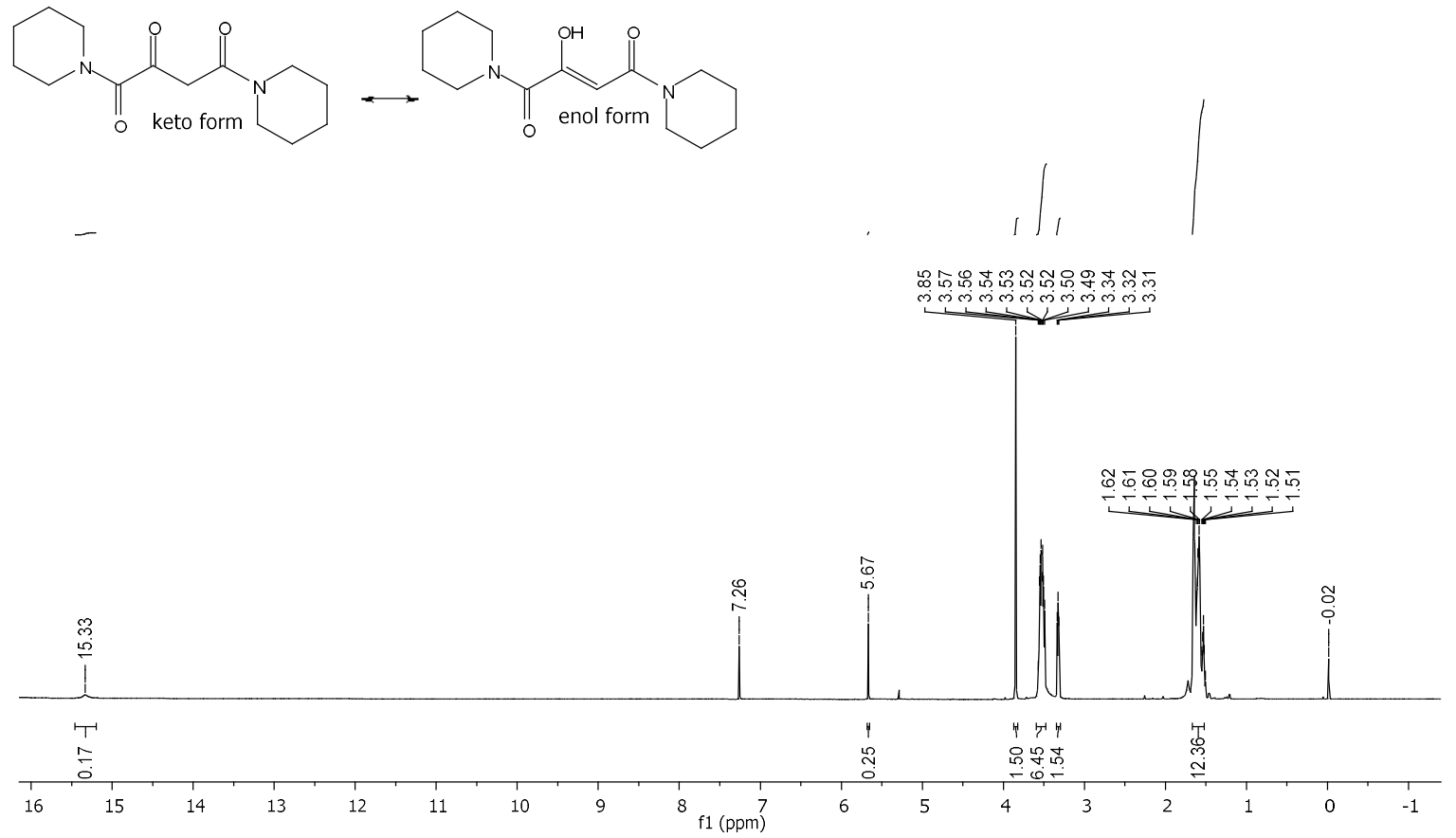

${ }^{13} \mathrm{C} \mathrm{NMR}\left(126 \mathrm{MHz}, \mathrm{CDCl}_{3}\right)$.

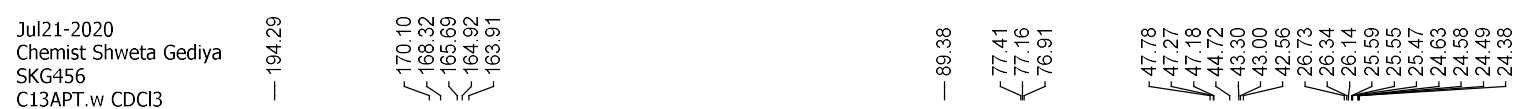

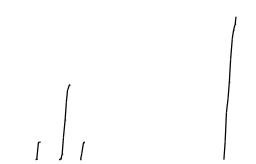

11

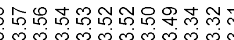
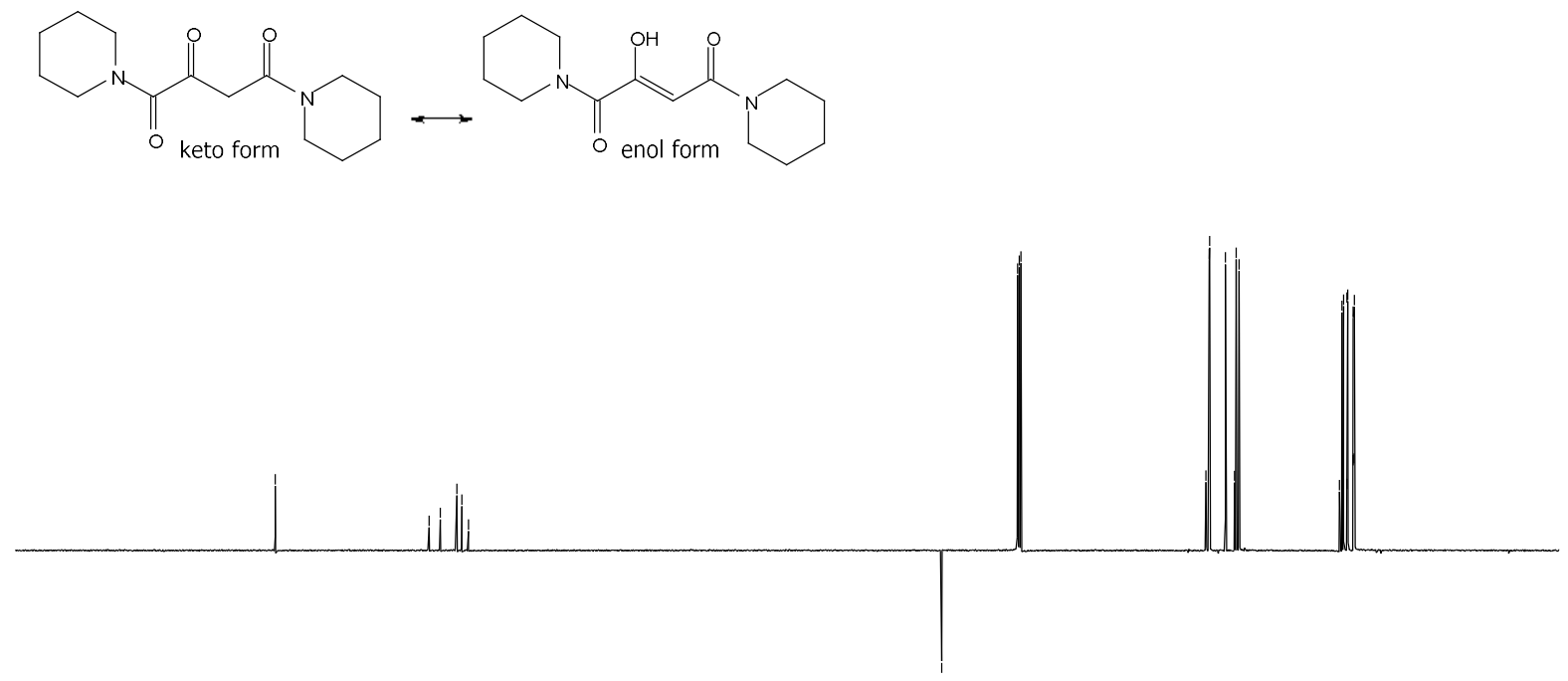

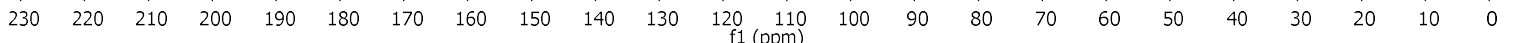


COSY.

Chemist Shweta Gediya

SKG456

COSY.w CDCl3 /opt/topspin3.2 SKG 60

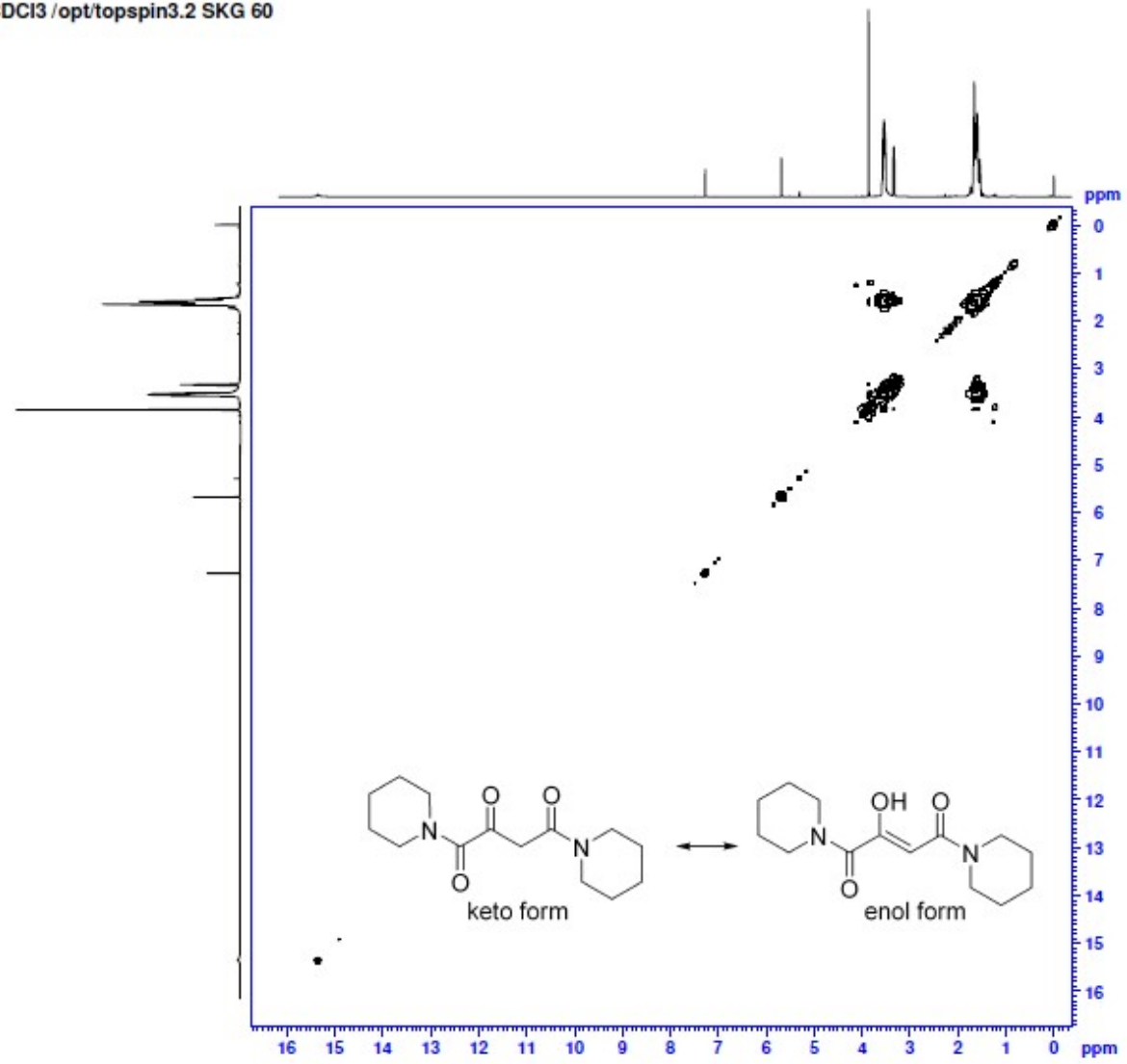

HSQC.

Chemist Shweta Gediya

SKG456
HSQC.w CDCl3 /opt/topspin3.2 SKG 60

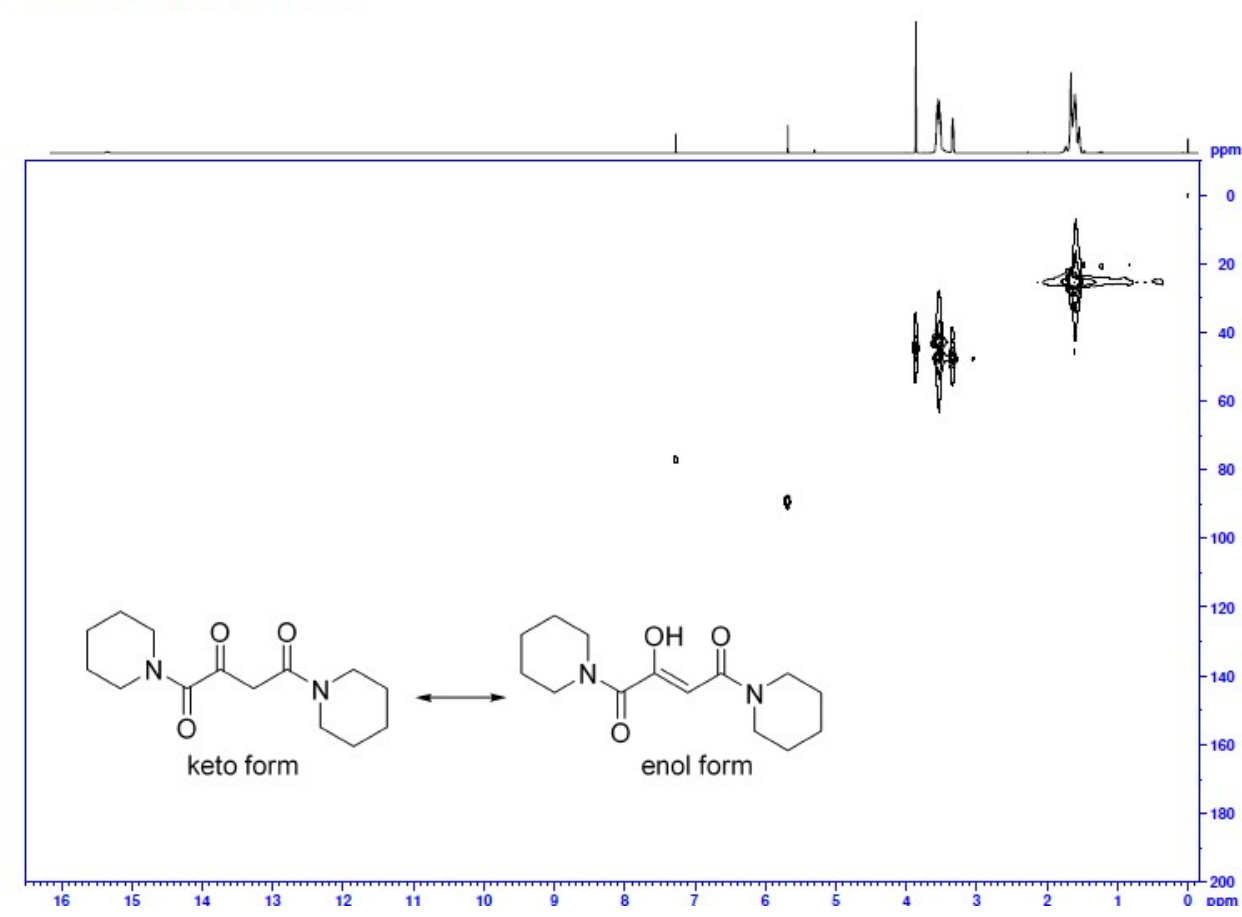


1-Morpholino-4-(piperidin-1-yl)butane-1,2,4-trione (precursor of 23).<smiles>O=C(CC(=O)N1CCCCC1)C(=O)N1CCOCC1</smiles>

This compound is novel and was prepared following the general procedure B using methyl 2morpholino-2-oxoacetate $(0.678 \mathrm{~g}, 3.93 \mathrm{mmol}, 1.0 \mathrm{eq})$ in anhydrous THF (10 mL), 1(piperidin-1-yl)ethan-1-one (0.500 g, $3.93 \mathrm{mmol}, 1.0 \mathrm{eq})$ in anhydrous THF (10 mL), and LDA (1M solution in THF) $(5.11 \mathrm{~mL}, 5.11 \mathrm{mmol}, 1.3 \mathrm{eq})$ to give crude material which was further purified by column chromatography (50-100\% EtOAc in petroleum ether) to afford the product as a brown solid $(0.630 \mathrm{~g}, 2.35 \mathrm{mmol}, 60 \%)$. The title compound was obtained as keto/enol tautomer (5:2) as per $\left.{ }^{1} \mathrm{H} \mathrm{NMR}\right)$.

TLC: $\mathrm{R}_{\mathrm{f}}$ ca 0.5 (2:8, hexane: EtOAc), UV-active and strong $\mathrm{KMnO}_{4}$;

MP: $58-60{ }^{\circ} \mathrm{C}$;

HRMS (ESI) m/z: $[\mathrm{M}+\mathrm{H}]^{+}$Calcd for $\mathrm{C}_{13} \mathrm{H}_{21} \mathrm{~N}_{2} \mathrm{O}_{4} 269.1496$; Found 269.1502 (error -2.4 ppm);

$U_{\max } 2938,2857,1708,1618,1551,1468,1438,1270,1246,1107,1019,623 \mathrm{~cm}^{-1}$;

${ }^{1} \mathrm{H}$ NMR (400 MHz, $\mathrm{CDCl}_{3}$ ) (keto/enol, 5:2) $\delta 15.49$ (0.28H, br. s, OH of enol form), 5.78 $\left(0.28 \mathrm{H}, \mathrm{s},=\mathrm{C} \underline{\mathrm{H}}\right.$ of enol form), $3.83\left(1.44 \mathrm{H}, \mathrm{s}, \mathrm{CH}_{2}\right.$ of keto form $), 3.74-3.60\left(8 \mathrm{H}, \mathrm{m}, \mathrm{CH}_{2}\right.$ of morpholine), 3.50- 3.29 (4H, m, $\mathrm{NCH}_{2}$ of piperidine), $1.67-1.52\left(6 \mathrm{H}, \mathrm{m}, \mathrm{CH}_{2}\right.$ of piperidine); ${ }^{13} \mathrm{C}$ NMR (101 MHz, $\mathrm{CDCl}_{3}$ ) (keto/enol, 5:2) $\delta 193.7$ (C=O ketone), 169.9 ( $\underline{\mathrm{C}}=\mathrm{CH}$, enol), 167.1 $(\mathrm{C}=\mathrm{O}$ amide), $165.9(\mathrm{C}=\mathrm{O}$ amide $), 164.9(\mathrm{C}=\mathrm{O}$ amide $), 163.9$ ( $\mathrm{C}=\mathrm{O}$ amide), 90.4 $(\mathrm{C}=\underline{\mathrm{C}} \mathrm{H}$ enol), 67.2, 66.9, 66.8, 66.5, 47.1, 46.6, 44.2, 42.8, 42.5, 42.3, 26.3, 25.4, 24.4, 24.3; $m / z(\mathrm{ESI}) 269.2\left[(\mathrm{M}+\mathrm{H})^{+}, 100 \%\right]$. 
1-Morpholino-4-(piperidin-1-yl)butane-1,2,4-trione.

${ }^{1} \mathrm{H}$ NMR (400 MHz, $\left.\mathrm{CDCl}_{3}\right)$.

Sep $11-2020$

SKG504

PROTON.w CDCl3 /opt/topspin3.5pl2 SKG 58
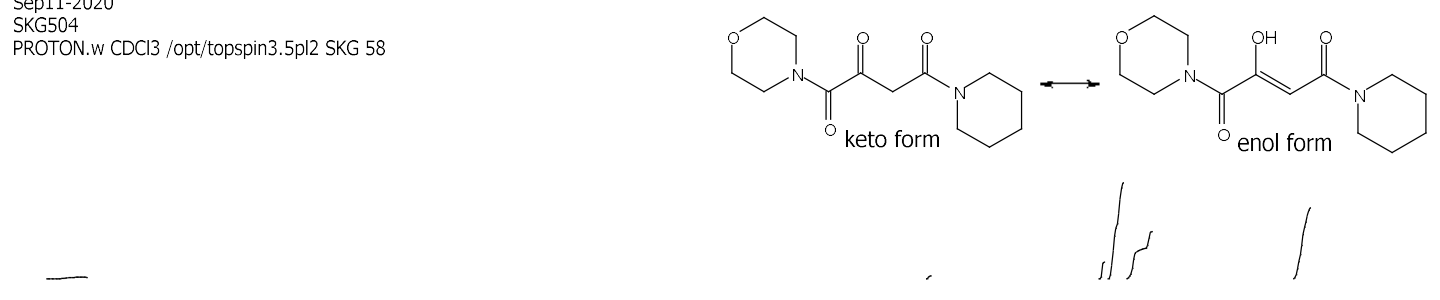

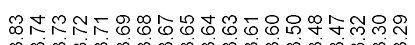
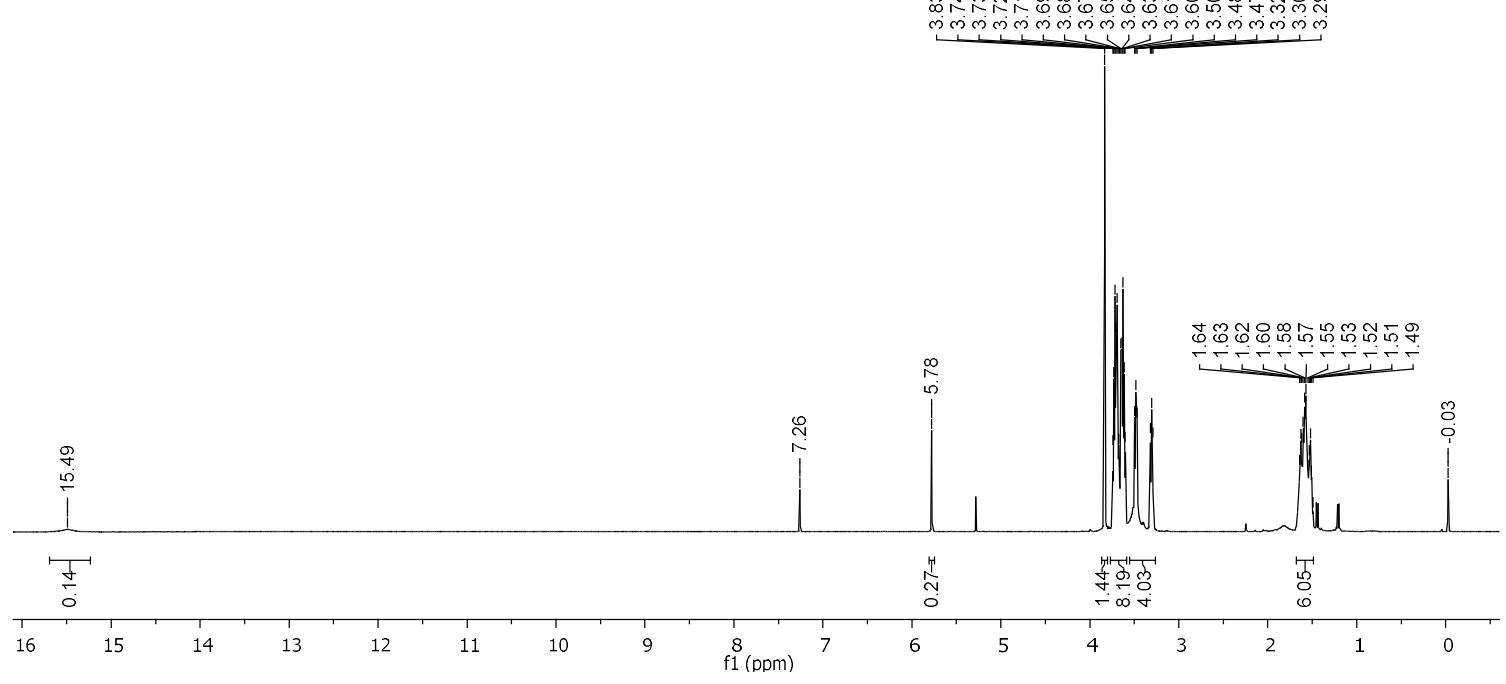

${ }^{13} \mathrm{C}$ NMR (101 MHz, $\left.\mathrm{CDCl}_{3}\right)$.

Sep11-2020

SKG504

C13APT.w $\mathrm{CDCl} 3$

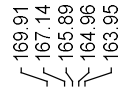

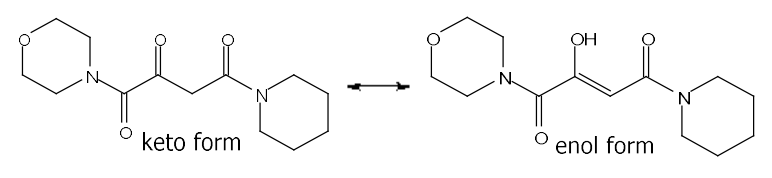

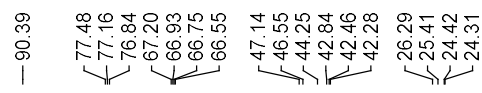
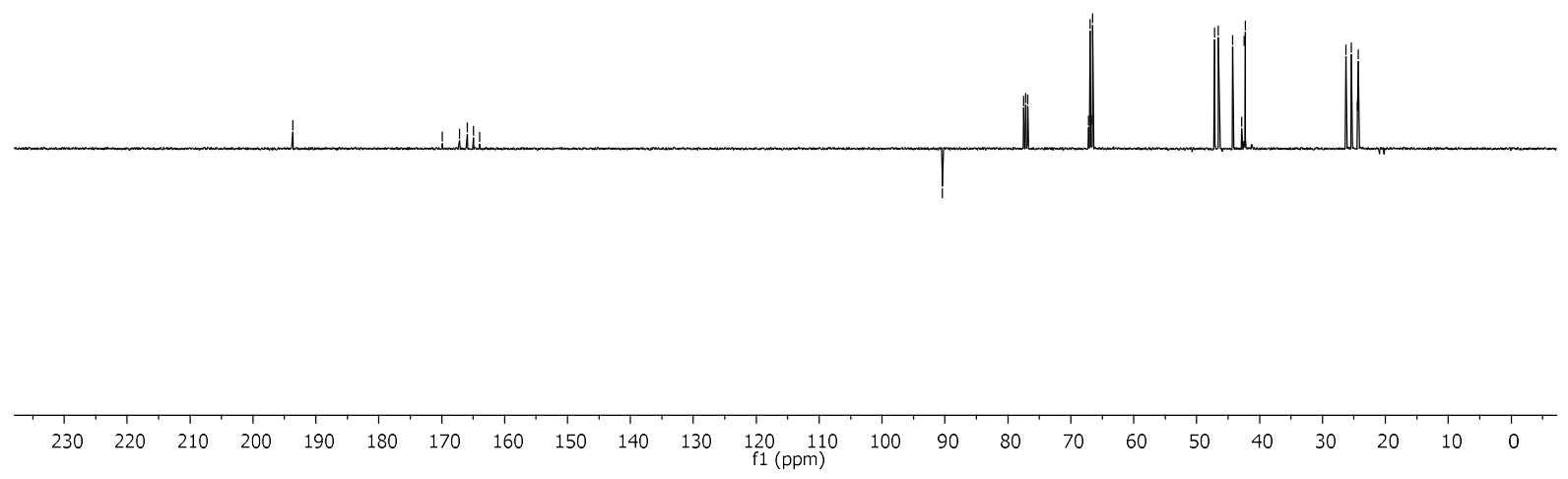

S107 
COSY.

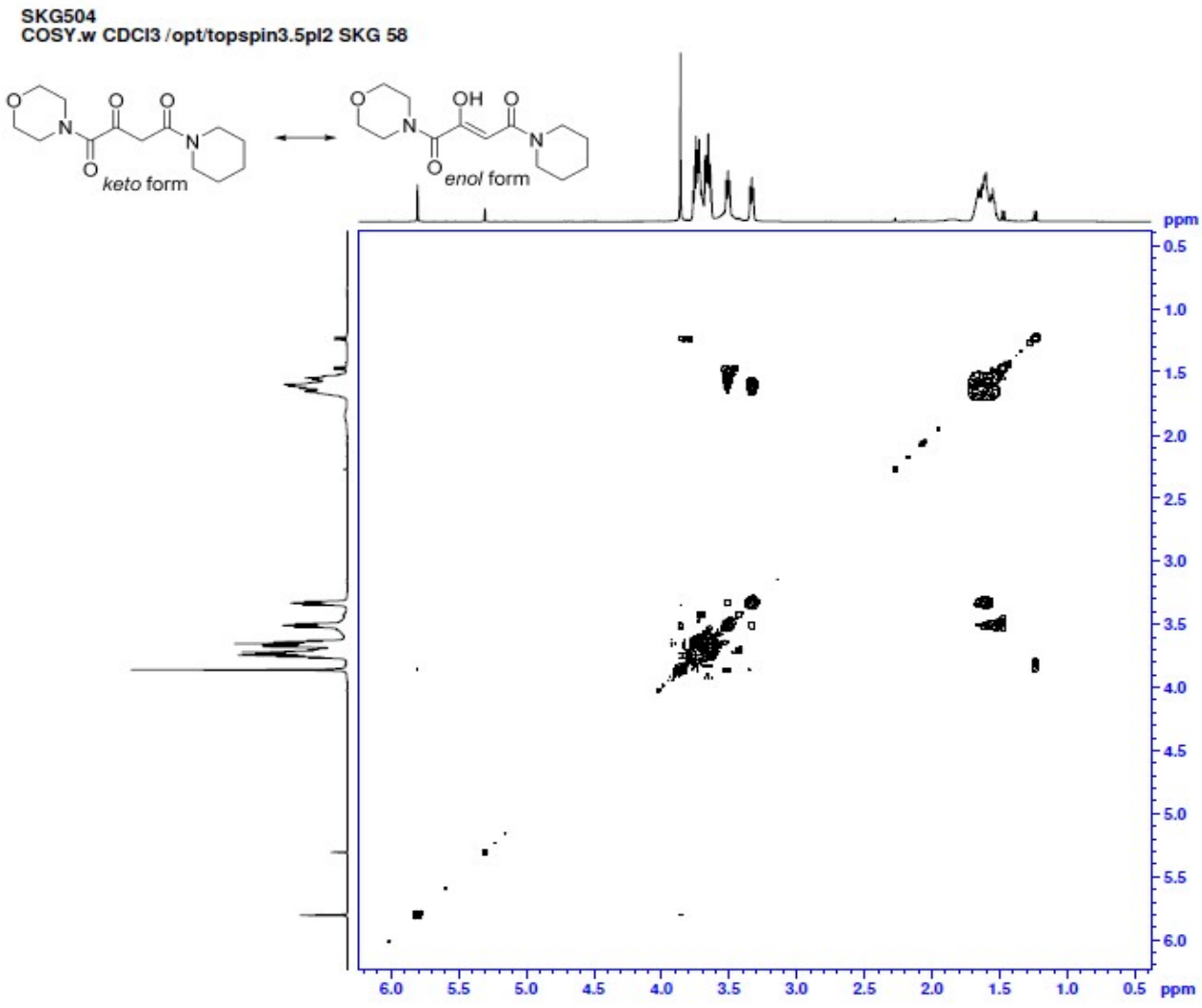

HSQC.

SKG504

HSOC.w CDCl3 /opt/topspin3.5pl2 SKG 58

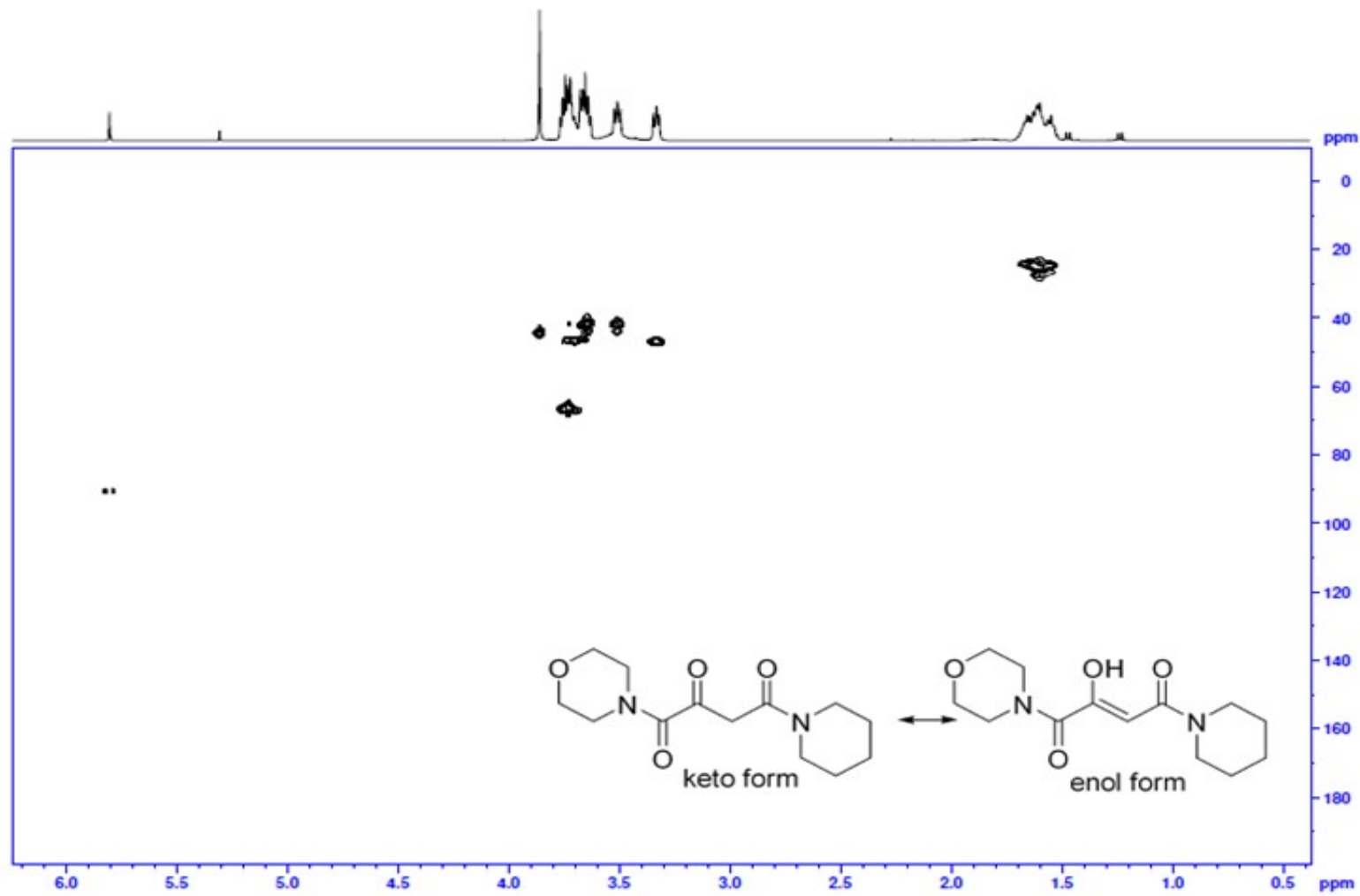




\section{2,4-Dioxo- $N$-phenyl-4-(piperidin-1-yl)butanamide (precursor of 24).}<smiles>O=C(CC(=O)N1CCCCC1)C(=O)Nc1ccccc1</smiles>

This compound is novel and was prepared following general procedure B using methyl 2oxo-2-(phenylamino)acetate $(0.493 \mathrm{~g}, 2.75 \mathrm{mmol}, 1.0 \mathrm{eq})$ in anhydrous THF $(8 \mathrm{~mL})$, 1(piperidin-1-yl)ethan-1-one $(0.350 \mathrm{~g}, 2.75 \mathrm{mmol}, 1.1 \mathrm{eq})$ in anhydrous THF $(8 \mathrm{~mL})$, and LDA (1M solution in THF) $(3.57 \mathrm{~mL}, 3.57 \mathrm{mmol}, 1.3 \mathrm{eq})$ to give crude material which was further purified by column chromatography (30\% EtOAc in petroleum ether) to afford the product as a white solid $(0.463 \mathrm{~g}, 1.68 \mathrm{mmol}, 62 \%)$. The title compound was obtained as keto/enol tautomer (5:95) as per ${ }^{1} \mathrm{H}$ NMR.

TLC: $\mathrm{R}_{\mathrm{f}}$ ca 0.4 (8:2, hexane: EtOAc), UV-active and strong $\mathrm{KMnO}_{4}$;

MP: $147-149^{\circ} \mathrm{C}$;

HRMS (ESI) m/z: $[\mathrm{M}+\mathrm{Na}]^{+}$Calcd for $\mathrm{C}_{15} \mathrm{H}_{18} \mathrm{~N}_{2} \mathrm{O}_{3} \mathrm{Na}$ 297.1210; Found 297.1207 (error 0.9 ppm);

$U_{\max } 3674,3327,2297,1667,1593,1529,1483,1441,1247,1076,755 \mathrm{~cm}^{-1}$;

${ }^{1} \mathrm{H}$ NMR (500 MHz, $\mathrm{CDCl}_{3}$ ) (keto/enol, 5:95) $\delta 15.79(0.95 \mathrm{H}$, br.s, $\mathrm{OH}$ of enol form), 8.72 $(1 \mathrm{H}, \mathrm{s}, \mathrm{CONH}), 7.66(2 \mathrm{H}, \mathrm{d}, J=7.7 \mathrm{~Hz}, \mathrm{ArH}), 7.36(2 \mathrm{H}, \mathrm{t}, J=7.7 \mathrm{~Hz}, \mathrm{ArH}), 7.16(1 \mathrm{H}, \mathrm{t}, J=$ $7.7 \mathrm{~Hz}, \mathrm{ArH}), 6.43$ (0.95 s, =C프, enol of enol form), 4.01 (0.10 s, $\mathrm{CH}_{2}$ of keto form), 3.59 $3.55\left(4 \mathrm{H}, \mathrm{m}, \mathrm{CH}_{2}\right.$ of piperidine), 1.72-1.60 (6H, m, $\mathrm{CH}_{2}$ of piperidine);

${ }^{13} \mathrm{C}$ NMR (126 MHz, $\mathrm{CDCl}_{3}$ ) (keto/enol, 5:95) $\delta 193.4$ (C=O keto form), 169.8, 165.1, 162.8, $160.1,157.6,137.1,136.4,129.3,129.2,125.4,125.0,120.0,119.9,89.6$ (C=ㅡH, enol), 47.5, 42.8, 41.7, 26.4, 25.4, 24.5;

$m / z(\mathrm{ESI}) 297.2\left[(\mathrm{M}+\mathrm{Na})^{+}, 100 \%\right]$. 
2,4-Dioxo- $N$-phenyl-4-(piperidin-1-yl)butanamide.

${ }^{1} \mathrm{H} \mathrm{NMR}\left(500 \mathrm{MHz}, \mathrm{CDCl}_{3}\right)$.

Jul03-2020

Chemist Shweta Gediya

SKG447

PROTON.w CDCl3 /opt/topspin3.2 SKG 19

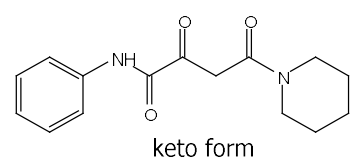

$\rightleftarrows$
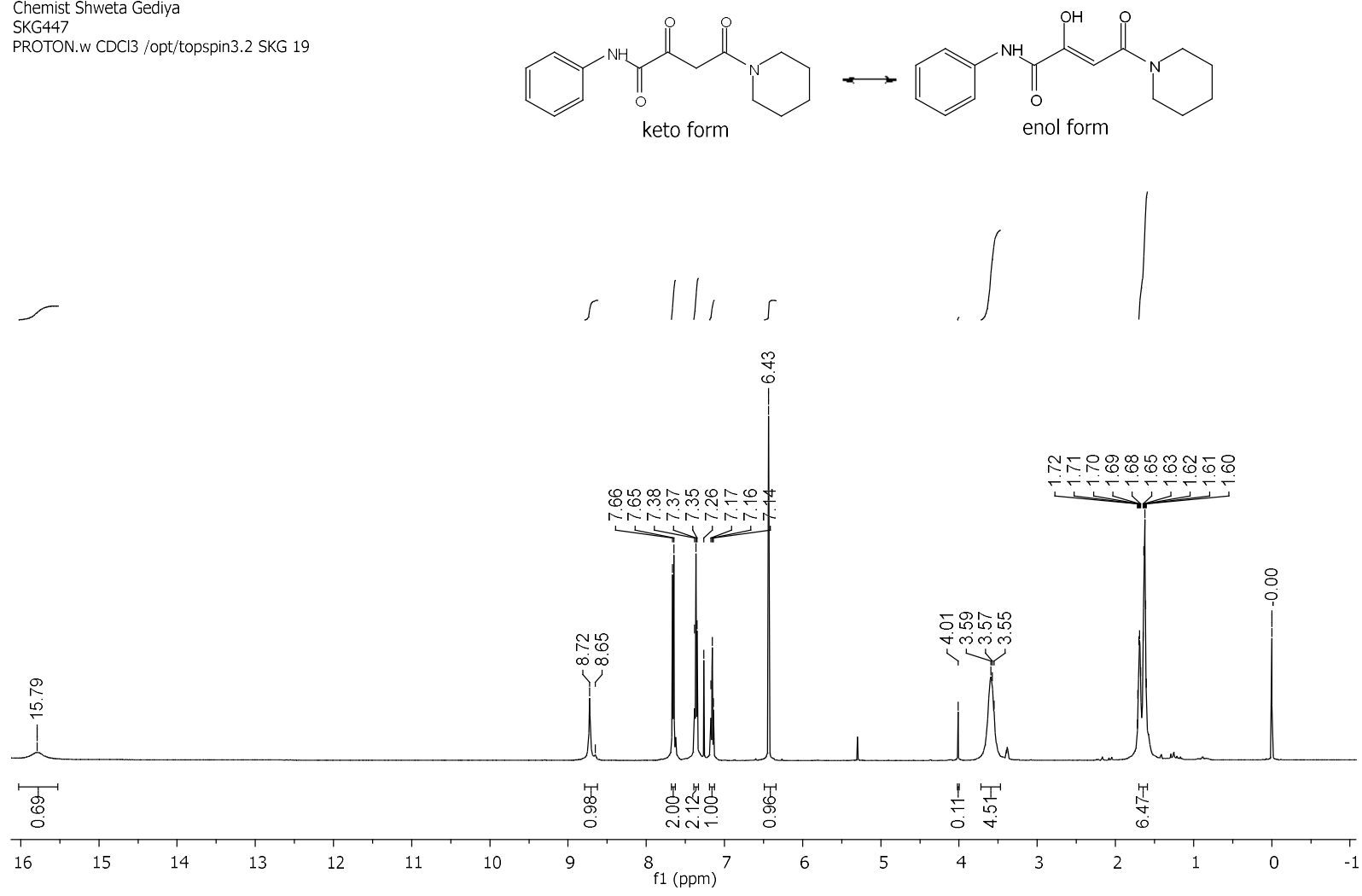

${ }^{13} \mathrm{C}$ NMR (126 MHz, $\left.\mathrm{CDCl}_{3}\right)$.

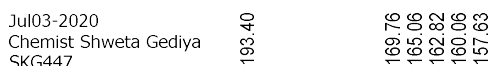

C13APT.w CDCl3

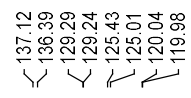

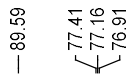

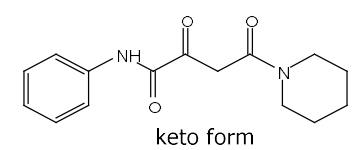

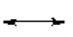

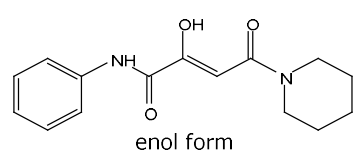

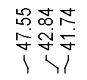

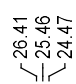

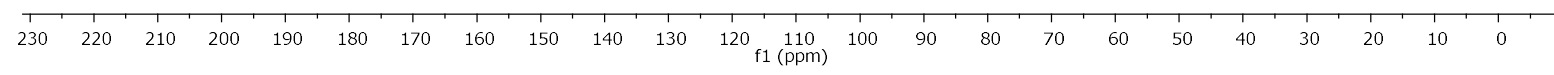

S110 
COSY.

Chemist Shweta Gediya

COSY.w CDCI3/opt/topspin3.2 SKG 19
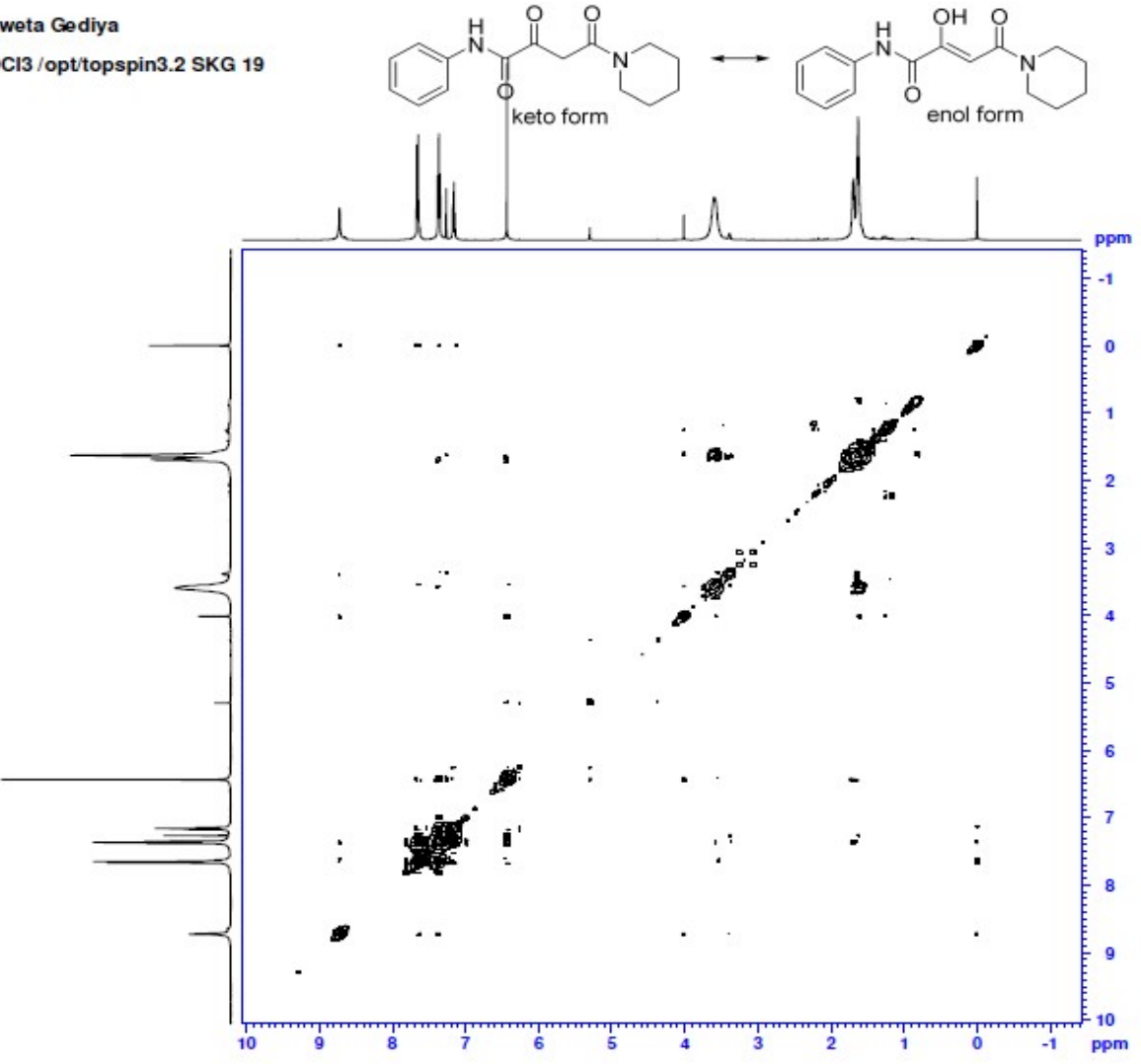

HSQC.

HSOC.w CDCI3 /opt/topspin3.2 SKG 19

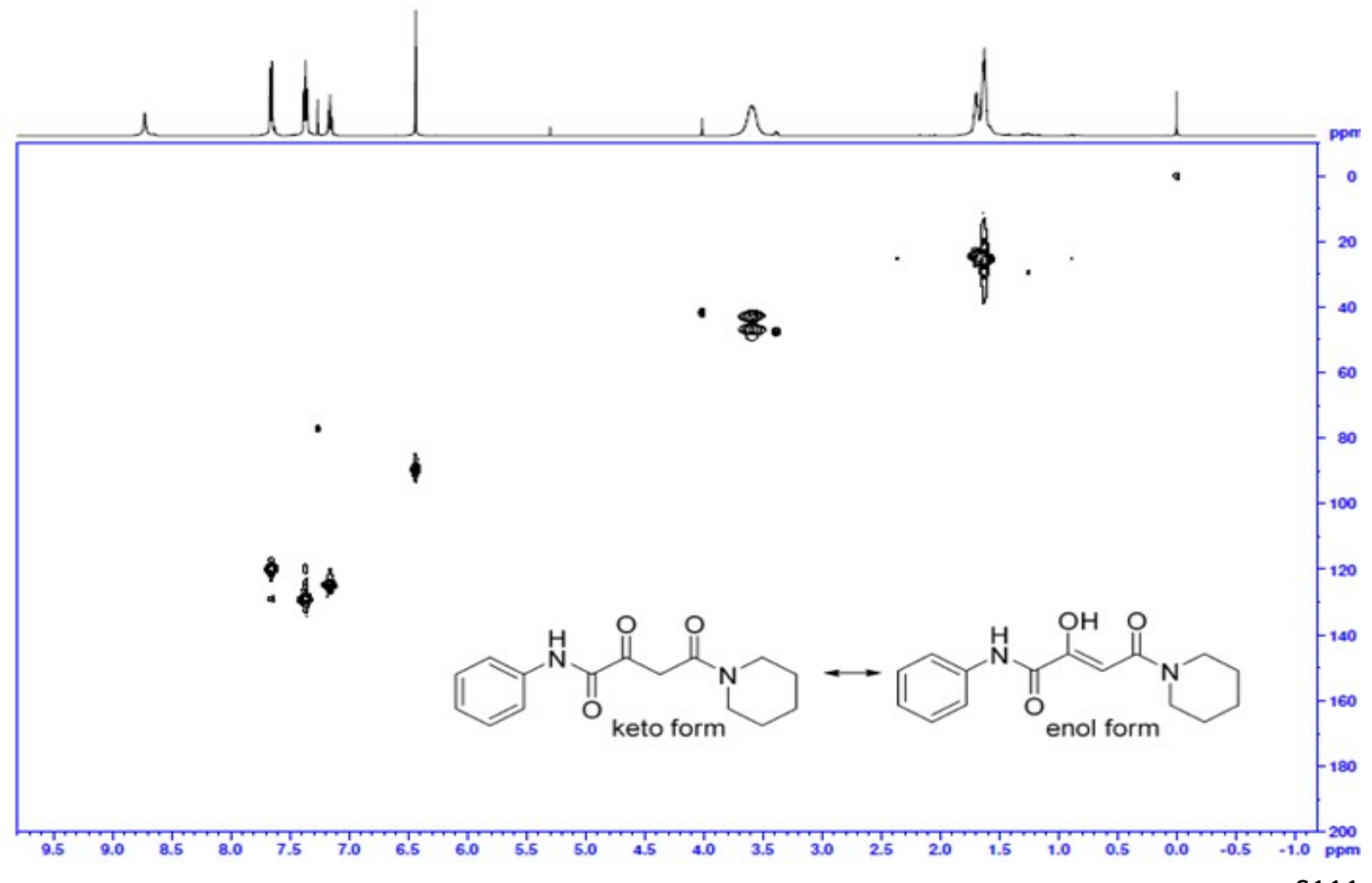


$\mathrm{N}$-(4-Chlorophenyl)-2,4-dioxo-4-(piperidin-1-yl)butanamide (precursor of 25).<smiles>O=C(CC(=O)N1CCCCC1)C(=O)Nc1ccc(Cl)cc1</smiles>

This compound is novel and was prepared following general procedure B using methyl 2-((4chlorophenyl)amino)-2-oxoacetate $(0.668 \mathrm{~g}, 3.14 \mathrm{mmol}, 1.0 \mathrm{eq})$ in anhydrous THF (5 mL), 1(piperidin-1-yl)ethan-1-one (0.400 g, $3.14 \mathrm{mmol}, 1.0 \mathrm{eq})$ in anhydrous THF (5 mL), and LDA (1M solution in THF) $(4.08 \mathrm{~mL}, 4.08 \mathrm{mmol}, 1.3 \mathrm{eq})$ to give crude material which was further purified by column chromatography (10-30\% EtOAc in petroleum ether) to afford the prodict as a white solid $(0.546 \mathrm{~g}, 1.77 \mathrm{mmol}, 56 \%)$. The title compound was obtained as a keto/enol tautomer (1:9) as per ${ }^{1} \mathrm{H}$ NMR.

TLC: $\mathrm{R}_{\mathrm{f}}$ ca 0.5 (7:3, hexane: EtOAc), UV-active and strong $\mathrm{KMnO}_{4}$;

MP: $143-145^{\circ} \mathrm{C}$;

HRMS (ESI) m/z: $[\mathrm{M}+\mathrm{Na}]^{+}$Calcd for $\mathrm{C}_{15} \mathrm{H}_{17}{ }^{35} \mathrm{ClN}_{2} \mathrm{O}_{3} \mathrm{Na} 331.0820$; Found 331.0810 (error 2.9 ppm); $\cup_{\max } 3360,2939,2852,1683,1630,1589,1528,1470,1397,1223,1210,814,656$ $\mathrm{cm}^{-1}$;

${ }^{1} \mathrm{H}$ NMR (400 MHz, $\mathrm{CDCl}_{3}$ ) (keto/enol, 1:9) $\delta 15.85$ (0.9H, br.s., $\mathrm{OH}$ of enol form), 8.74 $(0.9 \mathrm{H}, \mathrm{s}, \mathrm{CONH}$ of enol form $), 8.68(0.1 \mathrm{H}, \mathrm{m}, \mathrm{CONH}$ of keto form $), 7.62(2 \mathrm{H}, \mathrm{d}, J=8.8 \mathrm{~Hz}$, $\mathrm{ArH}), 7.32(2 \mathrm{H}, \mathrm{d}, J=8.8 \mathrm{~Hz}, \mathrm{ArH}), 6.41(0.9 \mathrm{H}, \mathrm{s},=\mathrm{C} \underline{\mathrm{H}}$ of enol form $), 3.99\left(0.2 \mathrm{H}, \mathrm{CH}_{2}\right.$ of keto form), $3.59-3.37$ (4H, m, $\mathrm{NCH}_{2}$ of piperidine), 1.73-1.52 (6H, $\mathrm{m}, \mathrm{CH}_{2}$ of piperidine);

${ }^{13} \mathrm{C}$ NMR (101 MHz, $\left.\mathrm{CDCl}_{3}\right)$ (keto/enol, 1:9) $\delta 169.7(\underline{\mathrm{C}}=\mathrm{CH}$, enol form), $162.6(\mathrm{C}=\mathrm{O}$ amide), 160.2 ( $\mathrm{C}=\mathrm{O}$ amide), 135.7, 129.9, 129.3, 121.3, 121.2, 89.6 ( $\mathrm{C}=\underline{\mathrm{CH}}$, enol form), 47.5 $\left(\mathrm{CH}_{2}\right.$ of keto form), 42.3, 41.6, 26.4, 25.4, 24.4 ( $\mathrm{C}=\mathrm{O}$ not observed, typically ca $\left.\delta 193\right)$; $m / z(\mathrm{ESI}) 331.4\left[\left(\mathrm{M}\left({ }^{35} \mathrm{Cl}\right)+\mathrm{Na}\right)^{+}, 100 \%\right]$. 
$\mathrm{N}$-(4-Chlorophenyl)-2,4-dioxo-4-(piperidin-1-yl)butanamide.

${ }^{1} \mathrm{H}$ NMR (400 MHz, $\left.\mathrm{CDCl}_{3}\right)$.

Sep15-2020

SKG543

PROTON.w CDCl3 /opt/topspin3.5pl2 SKG 3
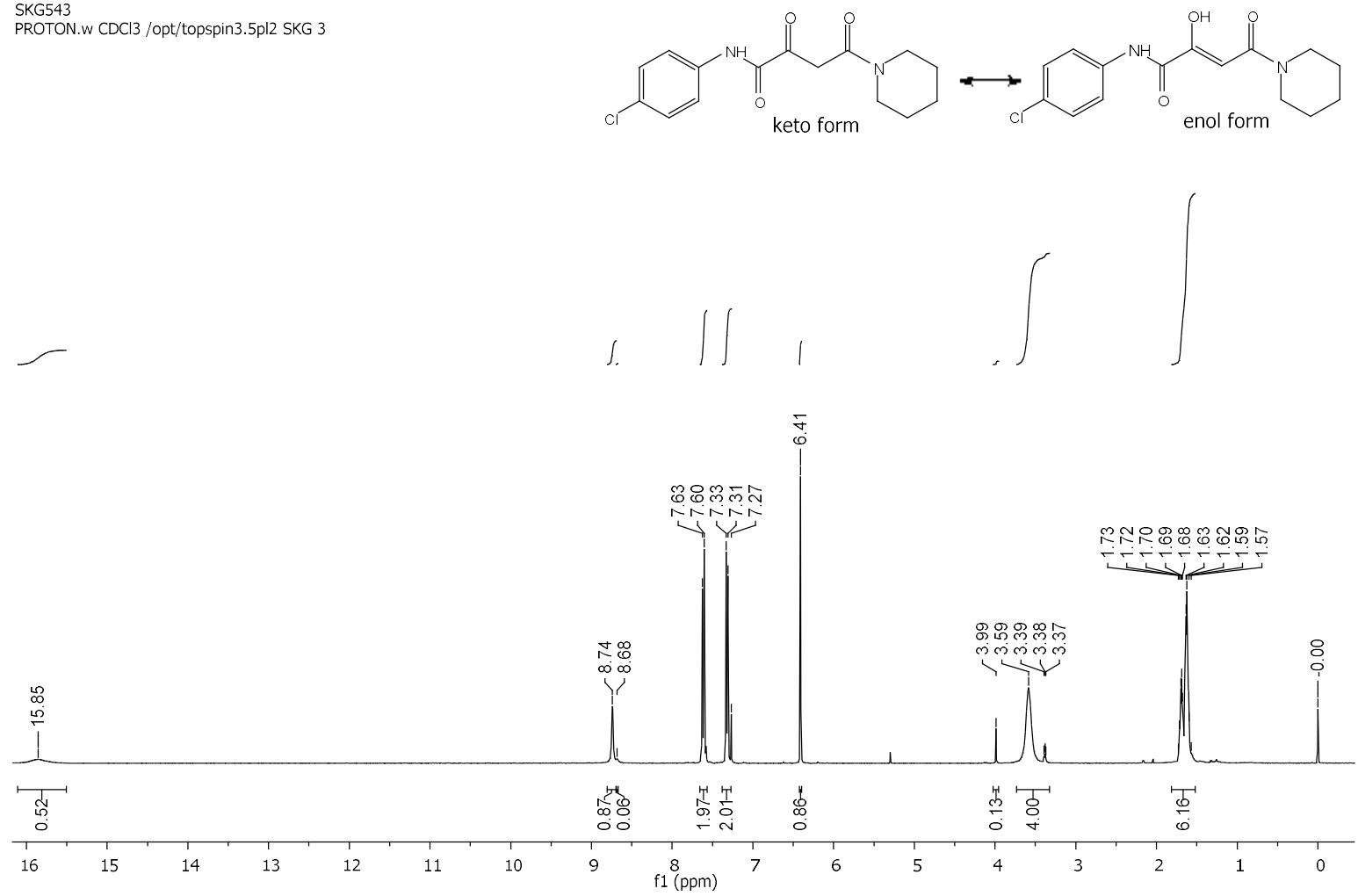

${ }^{13} \mathrm{C}$ NMR $\left(101 \mathrm{MHz}, \mathrm{CDCl}_{3}\right)$.

Sep15-2020

SKG543

C13APTlong.w $\mathrm{CDCl} 3$

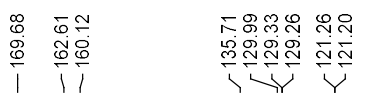

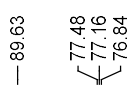

醊新

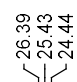
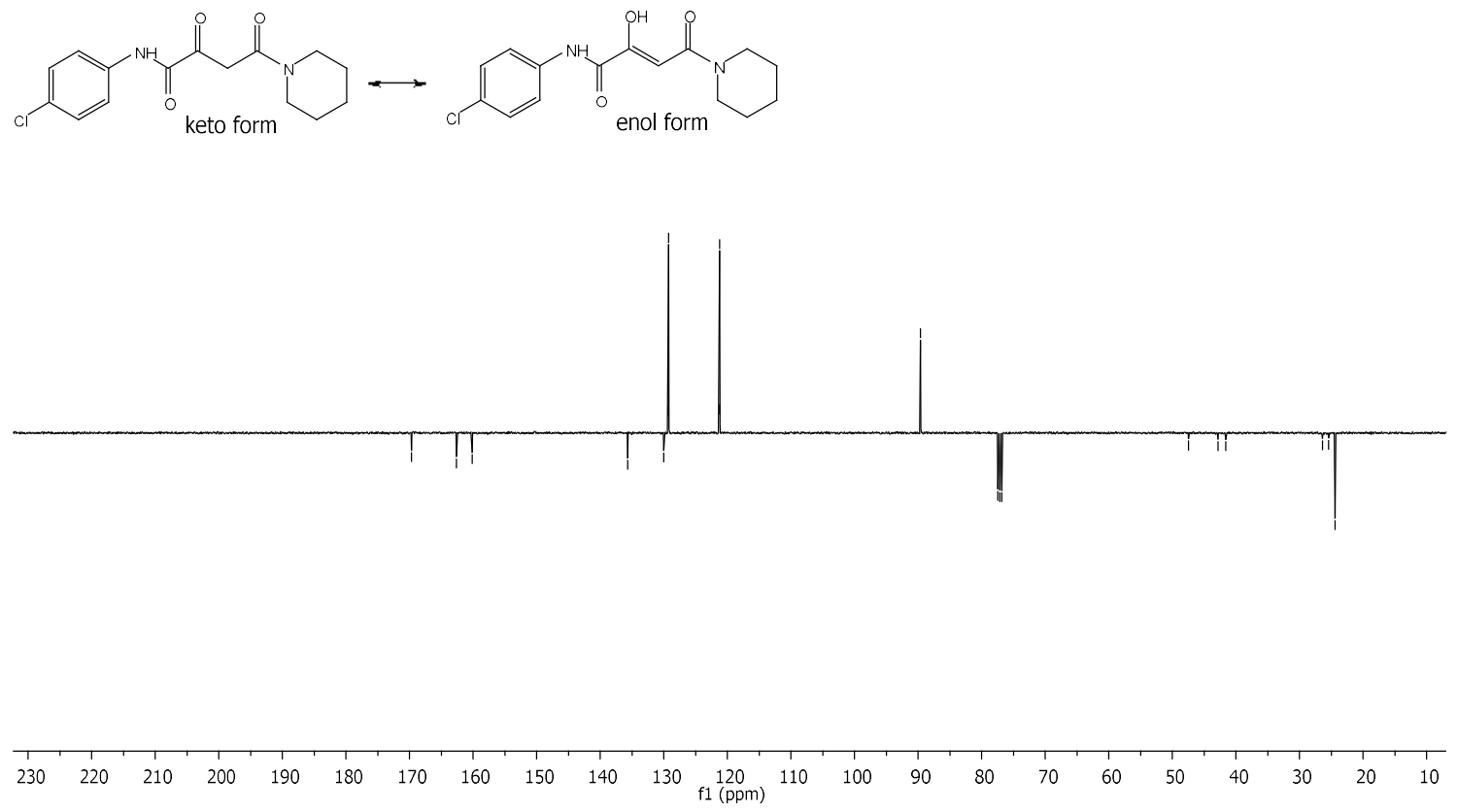

S113 
COSY.

SKG543
COSY.w

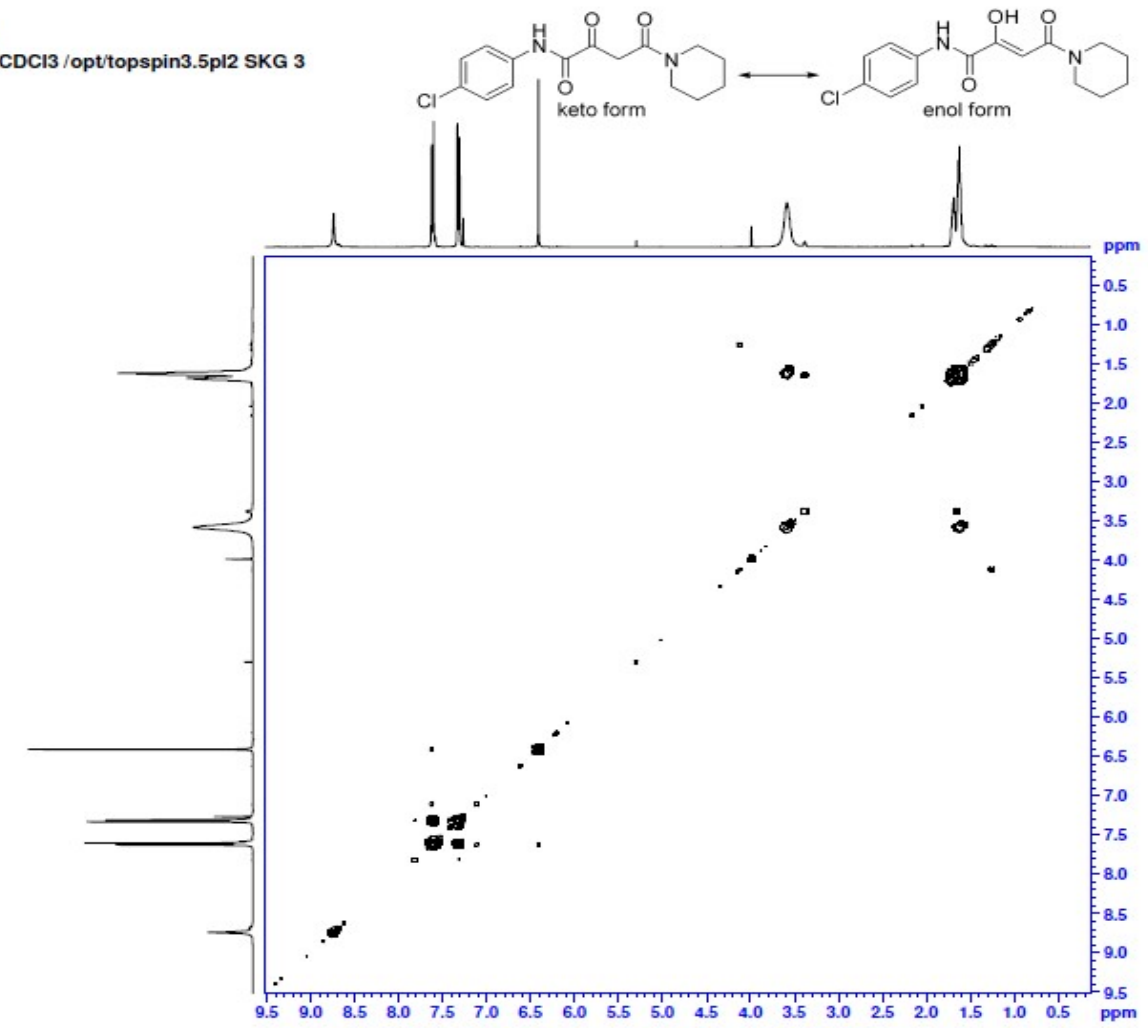

HSQC.

SKG543
HSOC.w CDCl3 /opt/topspin3.5pl2 SKG 3

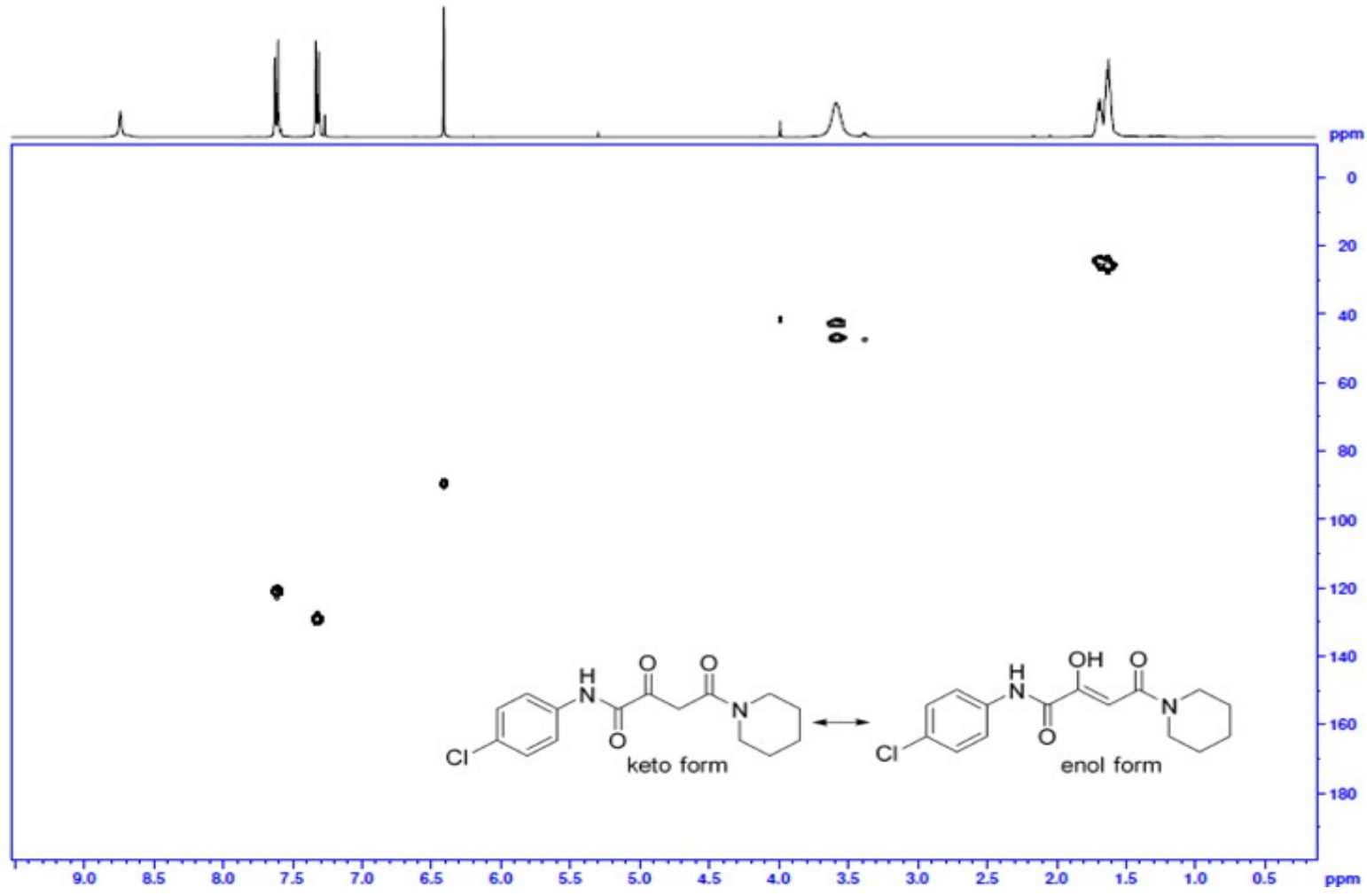


$\mathrm{N}$-(2-Chlorophenyl)-2,4-dioxo-4-(piperidin-1-yl)butanamide (precursor of 26).<smiles>O=C(CC(=O)N1CCCCC1)C(=O)Nc1ccccc1Cl</smiles>

This compound is novel and was prepared following general procedure B using methyl 2-((2chlorophenyl)amino)-2-oxoacetate $(0.400 \mathrm{~g}, 1.87 \mathrm{mmol}, 1.0 \mathrm{eq})$ in anhydrous THF (5 mL), 1(piperidin-1-yl)ethan-1-one (0.356 g, $2.80 \mathrm{mmol}, 1.5 \mathrm{eq})$ in anhydrous THF $(5 \mathrm{~mL})$, and LDA (1M solution in THF) $(3.18 \mathrm{~mL}, 3.18 \mathrm{mmol}, 1.7 \mathrm{eq})$ to give crude material which was further purified by column chromatography ( $50 \%$ EtOAc in petroleum ether) to afford the product as a clear liquid $(0.350 \mathrm{~g}, 1.13 \mathrm{mmol}, 60 \%)$. The title compound was obtained as a keto/enol tautomer (3:7) as per ${ }^{1} \mathrm{H}$ NMR.

TLC: $\mathrm{R}_{\mathrm{f}}$ ca 0.4 (7:3, hexane: EtOAc), UV-active and strong $\mathrm{KMnO}_{4}$;

HRMS (ESI) m/z: [M+Na] ${ }^{+}$Calcd for $\mathrm{C}_{15} \mathrm{H}_{17}{ }^{35} \mathrm{ClN}_{2} \mathrm{O}_{3} \mathrm{Na} 331.0820$; Found 331.0816 (error $1.3 \mathrm{ppm})$;

$U_{\max } 3364,2937,2860,1730,1699,1592,1581,1528,1439,1299,1169,1019,742 \mathrm{~cm}^{-1}$;

${ }^{1} \mathrm{H}$ NMR (500 MHz, $\mathrm{CDCl}_{3}$ ) (keto/enol, 3:7) $\delta 15.77$ (0.7H, m, br.s., OH enol), 9.46-9.29 (1H, m, CONH), 8.50-8.43 (1H, m, ArH), 7.43-7.07 (3H, m, ArH), 6.43 (0.7H, s, =CH enol), 4.02-4.00 (0.6H m, $\mathrm{CH}_{2}$ of keto form), $3.60\left(4 \mathrm{H}, \mathrm{s}, \mathrm{NCH}_{2}\right.$ of piperidine), 1.70-1.61 (6H, m, $\mathrm{CH}_{2}$ of piperidine);

${ }^{13} \mathrm{C}$ NMR (126 MHz, $\mathrm{CDCl}_{3}$ ) (keto/enol, 3:7) $\delta 192.7$ (C=O keto), 169.6, 162.6, 161.1, 160.2, $153.7,134.1,133.3,129.4,129.3,128.1,127.8,126.1,125.3,123.6,121.4,121.4,89.8,47.5$, 42.8, 41.7, 29.8, 26.4, 25.5, 24.5;

$m / z(\mathrm{ESI}) 331.1\left(\left[\mathrm{M}\left({ }^{35} \mathrm{Cl}\right)+\mathrm{Na}\right]+, 100 \%\right)$ and $333.1\left(\left[\mathrm{M}\left({ }^{37} \mathrm{Cl}\right)+\mathrm{Na}\right]+, 40 \%\right)$. 
$\mathrm{N}$-(2-Chlorophenyl)-2,4-dioxo-4-(piperidin-1-yl)butanamide.

${ }^{1} \mathrm{H}$ NMR (500 MHz, $\left.\mathrm{CDCl}_{3}\right)$.

May24-2021

Chemist Shweta Gediya

SKG685
PROTON.w CDCl3 /opt/topspin3.2 SKG 15
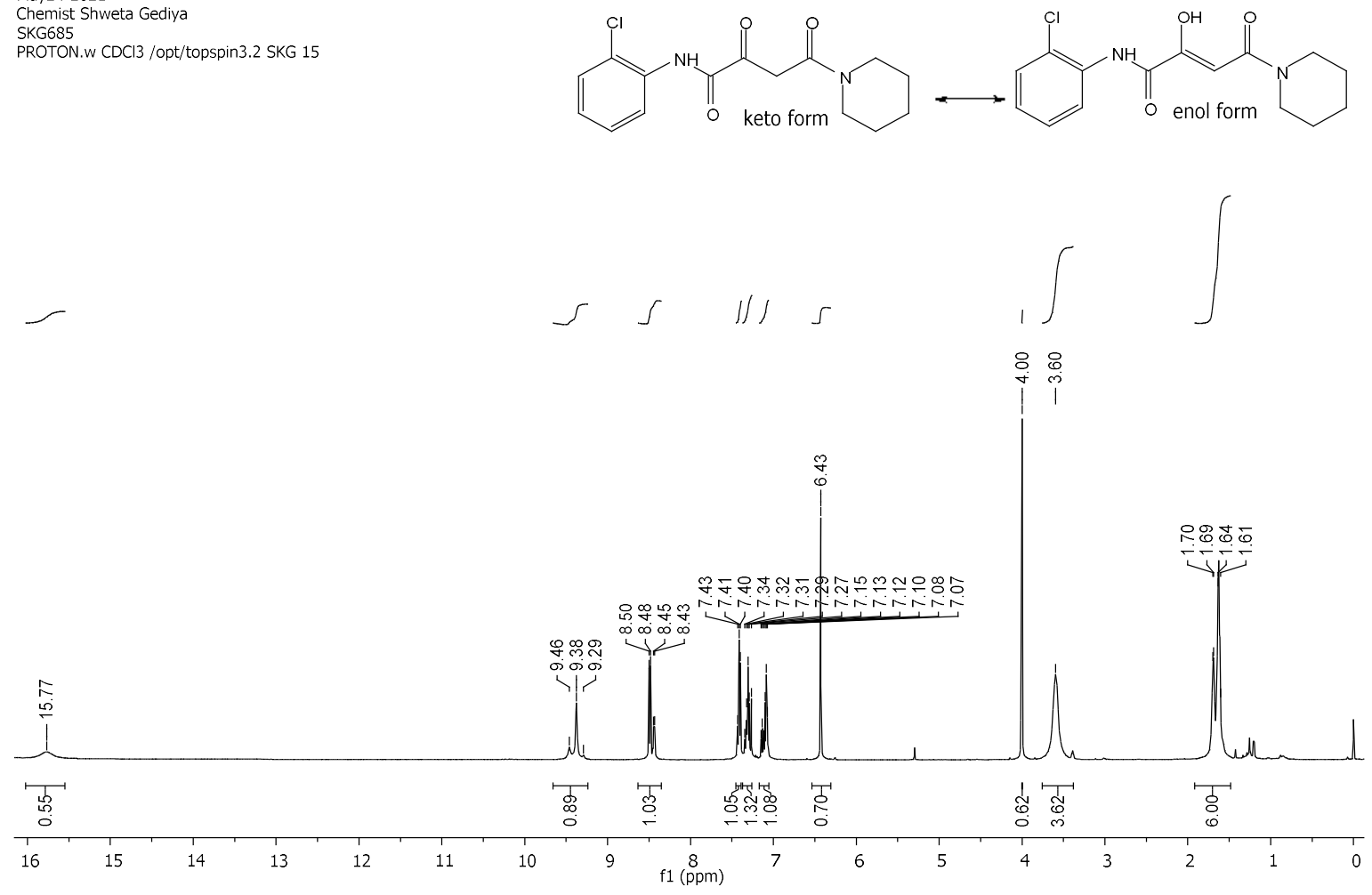

${ }^{13} \mathrm{C}$ NMR $\left(126 \mathrm{MHz}, \mathrm{CDCl}_{3}\right)$.

May24-2021
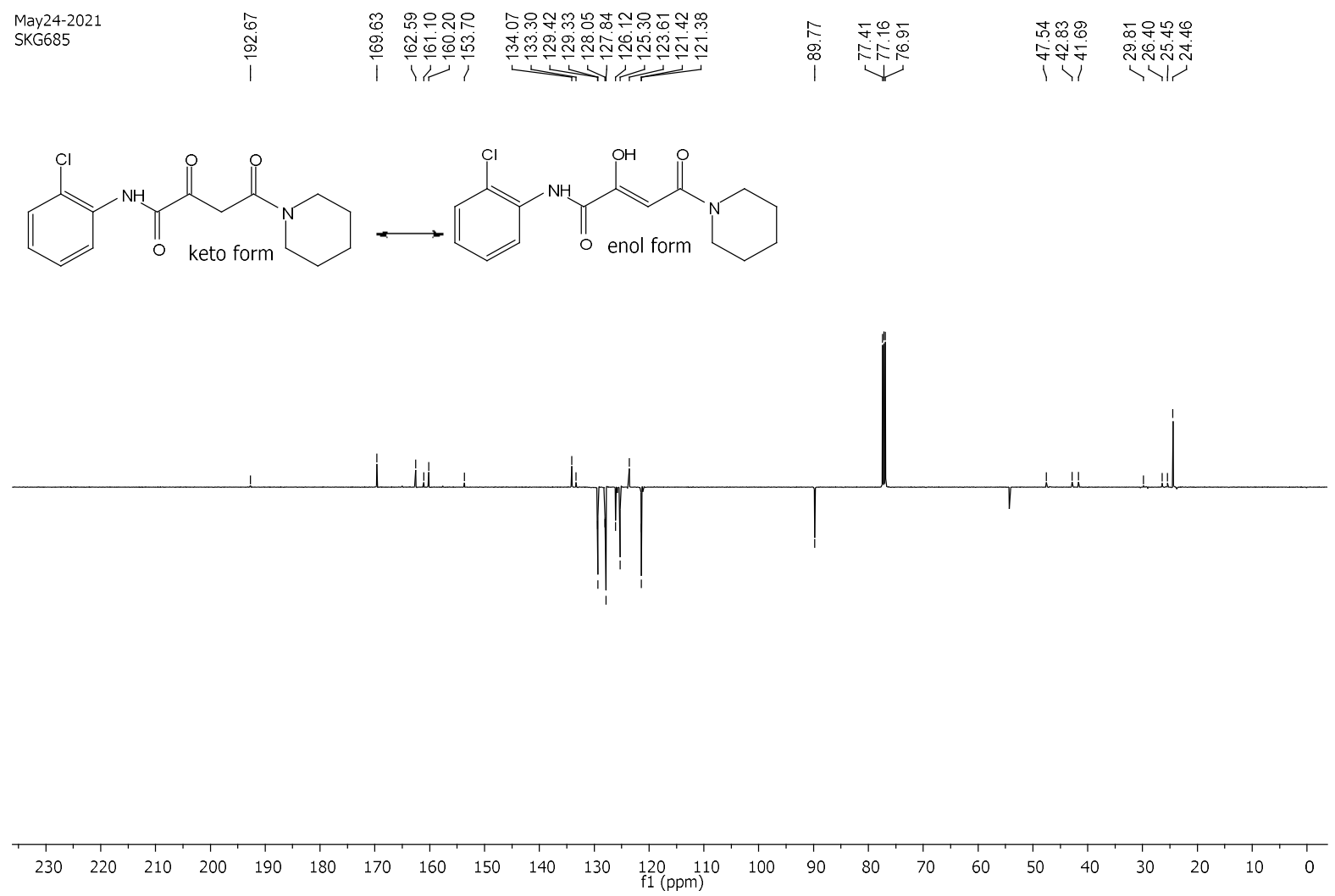

S116 
COSY.

Chemist Shweta Gediya

SKG685
COSY.w CDCl3 /opt/topspin3.2 SKG 15

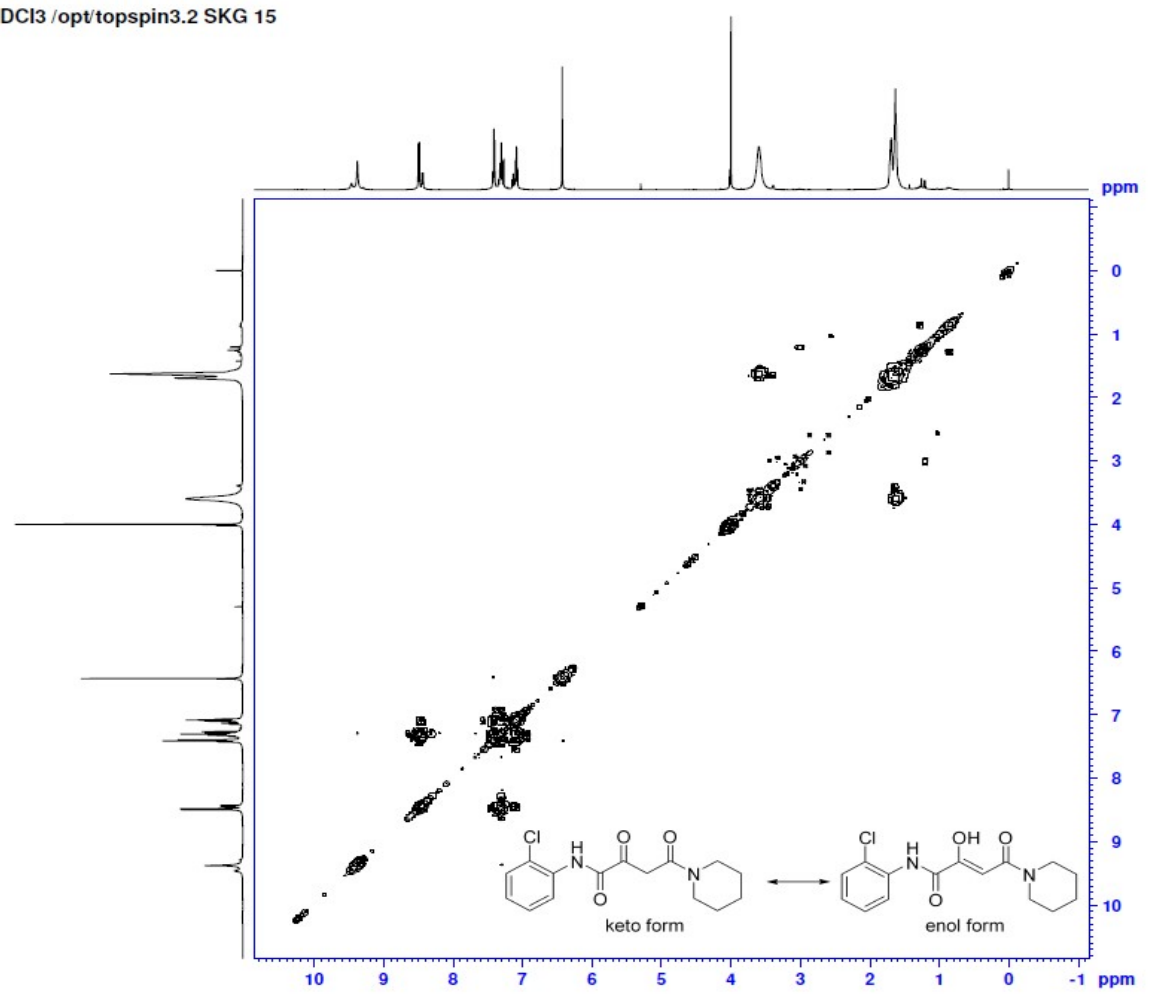

HSQC.

Chemist Shweta Gediya

SKG685
HSQC.w CDCI3 /opt/topspin3.2 SKG 15

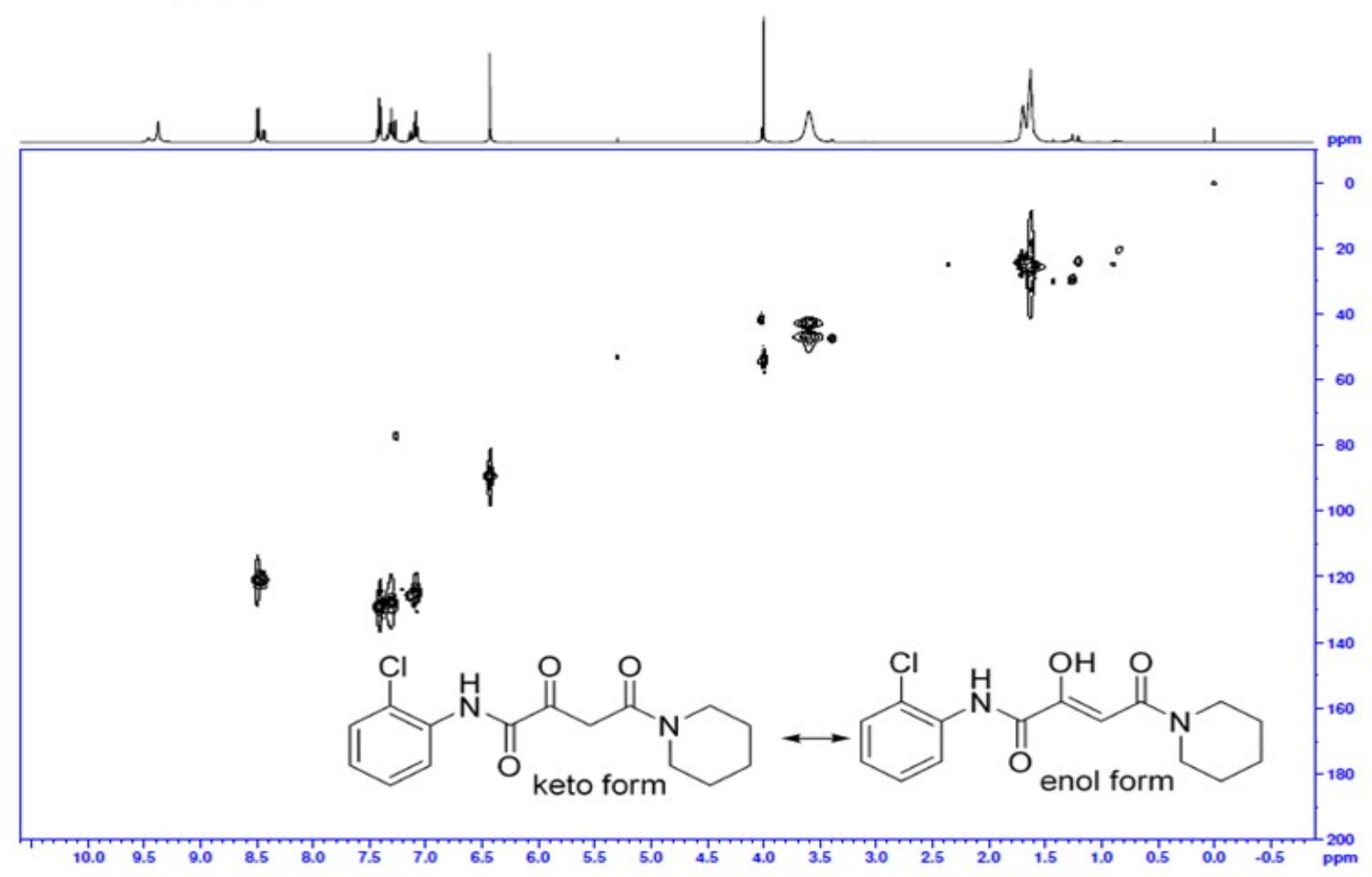

S117 
N-(2,6-Diisopropylphenyl)-2,4-dioxo-4-(piperidin-1-yl)butanamide (precursor of 27).<smiles>CC(C)c1cccc(C(C)C)c1NC(=O)C(=O)CC(=O)N1CCCCC1</smiles>

This compound is novel and was prepared following general procedure B using methyl 2((2,6-diisopropylphenyl)amino)-2-oxoacetate $(0.500 \mathrm{~g}, 1.90 \mathrm{mmol}, 1.0 \mathrm{eq})$ in anhydrous THF $(8 \mathrm{~mL}), 1$-(piperidin-1-yl)ethan-1-one $(0.482 \mathrm{~g}, 3.80 \mathrm{mmol}, 2.0 \mathrm{eq})$ in anhydrous THF $(8 \mathrm{~mL})$ and LDA (1M solution in THF) $(4.37 \mathrm{~mL}, 4.37 \mathrm{mmol}, 2.3 \mathrm{eq})$ to give crude material which was further purified by column chromatography (10-30\% EtOAc in petroleum ether) to afford the product as a white solid $(0.378 \mathrm{~g}, 1.06 \mathrm{mmol}, 56 \%)$. The title compound was obtained as a keto/enol tautomer (1:9) as per ${ }^{1} \mathrm{H}$ NMR.

TLC: $\mathrm{R}_{\mathrm{f}}$ ca 0.5 (7:3, hexane: EtOAc), UV-active and strong $\mathrm{KMnO}_{4}$;

MP: $163-164.7^{\circ} \mathrm{C}$;

HRMS (ESI) m/z: [M+Na] $]^{+}$Calcd for $\mathrm{C}_{21} \mathrm{H}_{30} \mathrm{~N}_{2} \mathrm{O}_{3} \mathrm{Na} 381.2149$; Found 381.2142 (error 1.7 ppm);

$U_{\max } 3355,2965,2865,1681,1584,1457,1377,1251,1227,1020,800,547 \mathrm{~cm}^{-1}$;

${ }^{1} \mathrm{H}$ NMR (500 MHz, $\mathrm{CDCl}_{3}$ ) (keto/enol, 1:9) $\delta 15.70$ (0.9H, br.s., OH of enol form), 8.23 $(0.9 \mathrm{H}, \mathrm{s}, \mathrm{CONH}$ of enol form $), 7.33-7.30(1 \mathrm{H}, \mathrm{m}, \mathrm{ArH}) 7.21-7.18(2.1 \mathrm{H}, \mathrm{m}, \mathrm{ArH}+\mathrm{CONH}$ of enol form), $6.43(0.9 \mathrm{H}, \mathrm{s},=\mathrm{C} \underline{\mathrm{H}}$ of enol form $), 4.01-4.00\left(0.2 \mathrm{H}, \mathrm{m}, \mathrm{CH}_{2}\right.$ of keto form), 3.63$3.55\left(4 \mathrm{H}, \mathrm{m}, \mathrm{NCH}_{2}\right.$ of piperidine), 3.09-3.01 $(2 \mathrm{H}, \mathrm{m}, \mathrm{CH}$ of isopropyl), 1.72-1.60 $(6 \mathrm{H}, \mathrm{m}$, $\mathrm{CH}_{2}$ of piperidine), $1.20\left(12 \mathrm{H}, \mathrm{d}, J=6.9 \mathrm{~Hz}, \mathrm{CH}_{3}\right.$ of isopropyl group);

${ }^{13} \mathrm{C}$ NMR (126 MHz, $\mathrm{CDCl}_{3}$ ) (keto/enol, 1:9) $\delta 169.8(\underline{\mathrm{C}}=\mathrm{CH}$, enol form), 162.6, 161.7, $146.3,146.2,130.3,128.7,123.7,89.9(\mathrm{C}=\underline{\mathrm{C} H}$, enol form), 29.0, 24.5, 23.7;

$m / z(\mathrm{ESI}) 381.2\left[(\mathrm{M}+\mathrm{Na})^{+}, 100 \%\right]$. 
N-(2,6-Diisopropylphenyl)-2,4-dioxo-4-(piperidin-1-yl)butanamide.

${ }^{1} \mathrm{H}$ NMR $\left(500 \mathrm{MHz}, \mathrm{CDCl}_{3}\right)$.

Nov17-2020
Chemist Shweta Gediya

SKG 643

PROTON.w CDCl3 /opt/topspin3.2 SKG 58
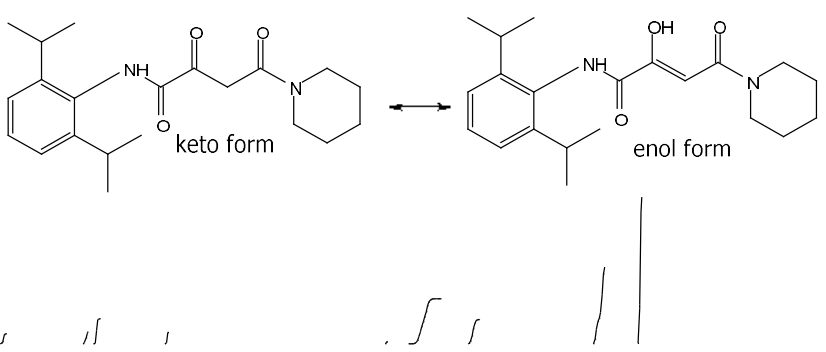

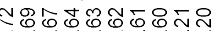

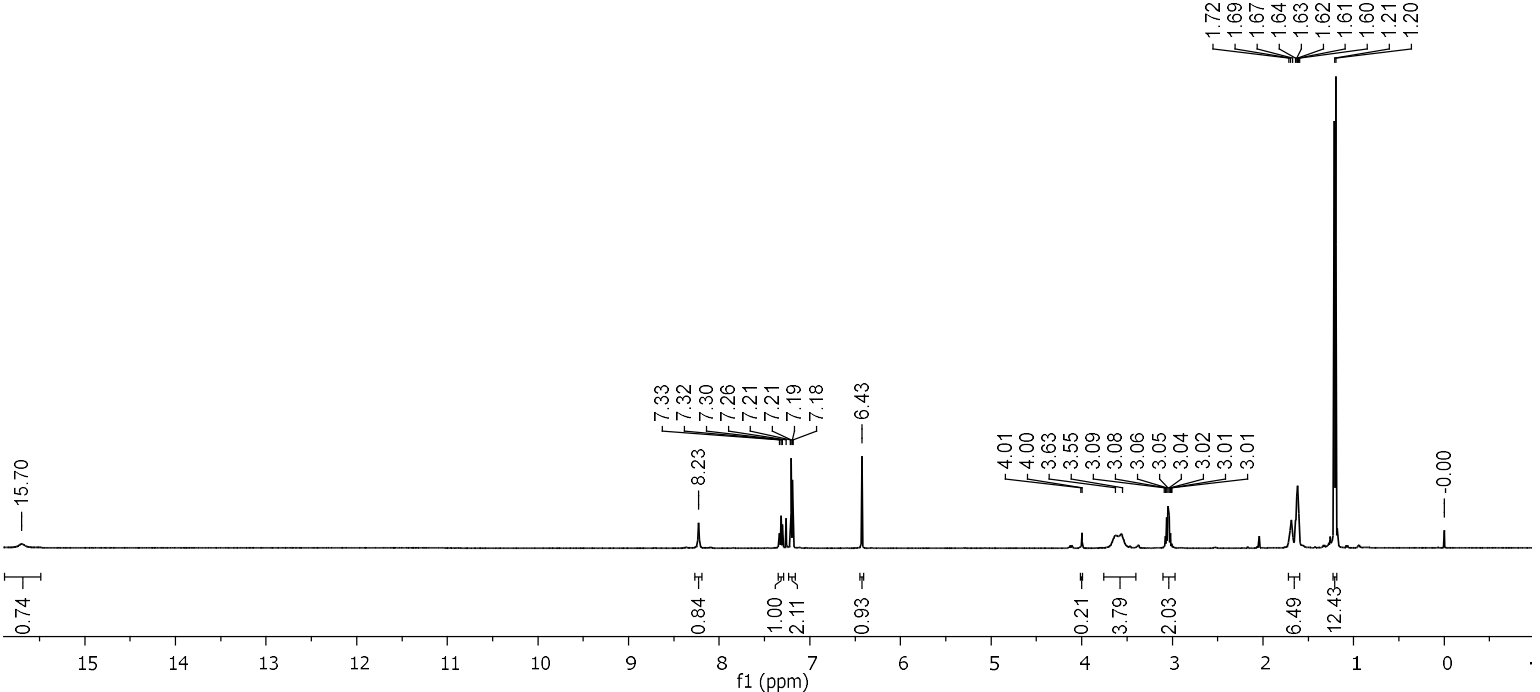

${ }^{13} \mathrm{C}$ NMR $\left(126 \mathrm{MHz}, \mathrm{CDCl}_{3}\right)$.

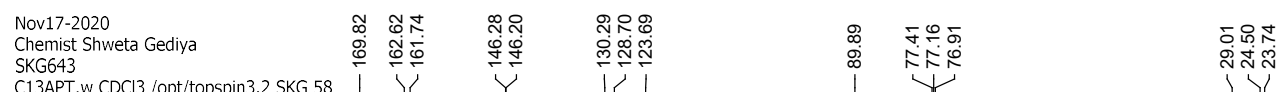

SKG643<smiles>C=C(O)C(=O)Nc1c(C(C)C)cccc1C(C)C</smiles>

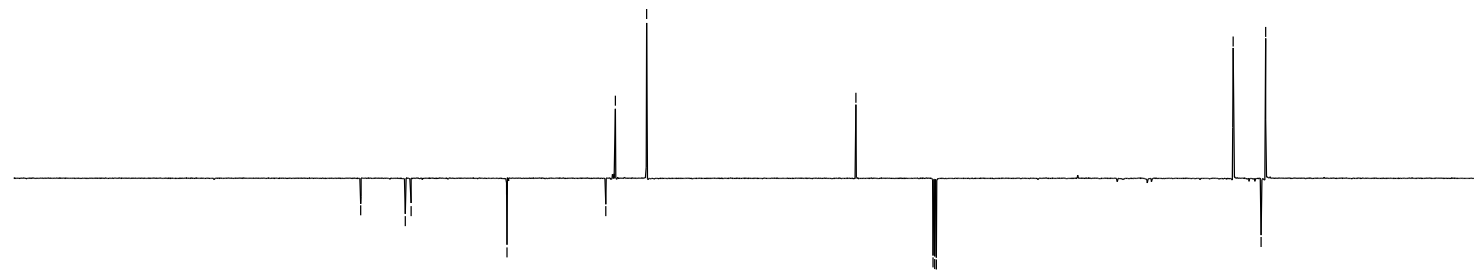

$\begin{array}{lllllllllllllllllllllll}220 & 210 & 200 & 190 & 180 & 170 & 160 & 150 & 140 & 130 & 120 & 110 & 100 & 90 & 80 & 70 & 60 & 50 & 40 & 30 & 20 & 10 & 0\end{array}$ 
COSY.

Chemist Shweta Gediya

COSY.w CDCl3/opt/topspin3.2 SKG 58
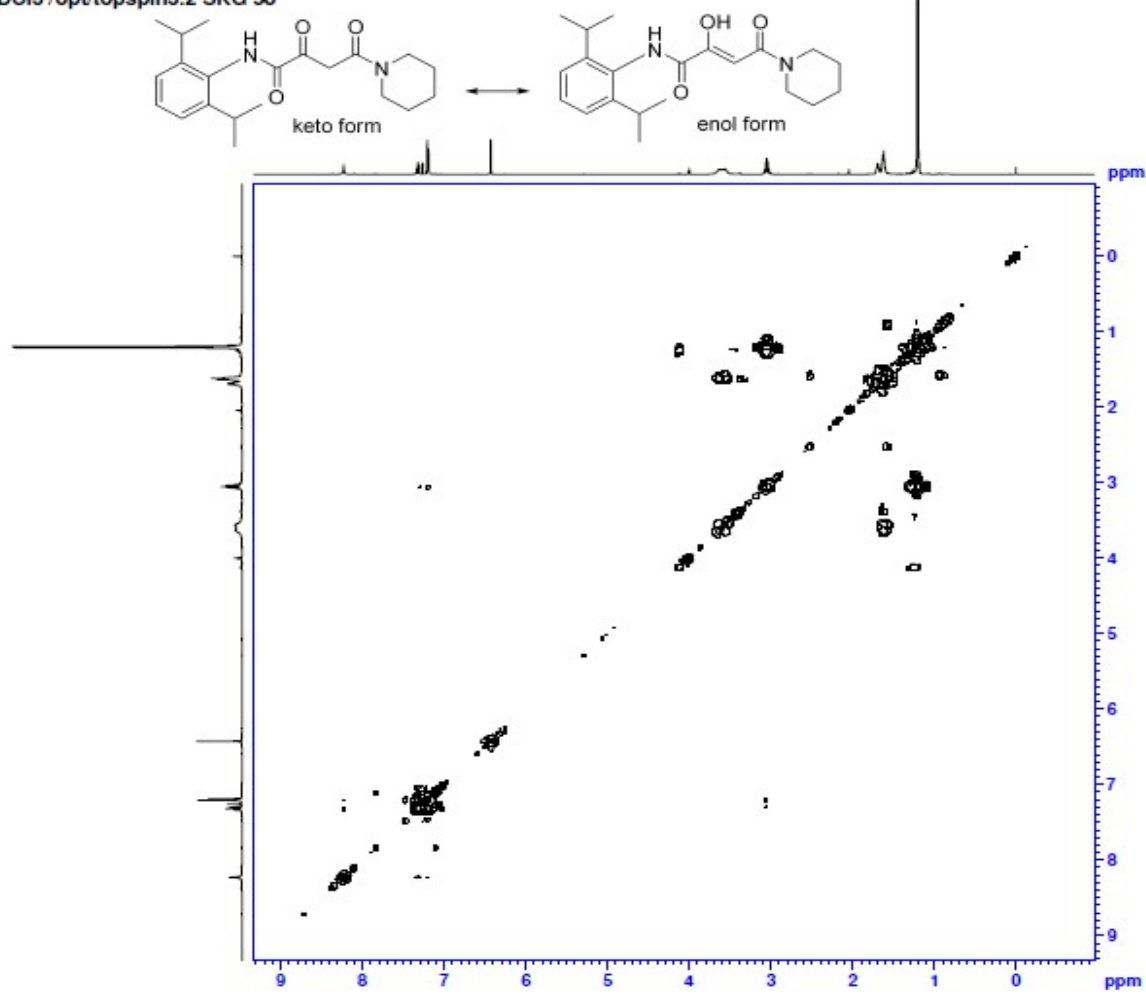

HSQC.

Chemist Shwota Gediya

HSOC.w CDCI3 /opt/topspin3.2 SKG 58

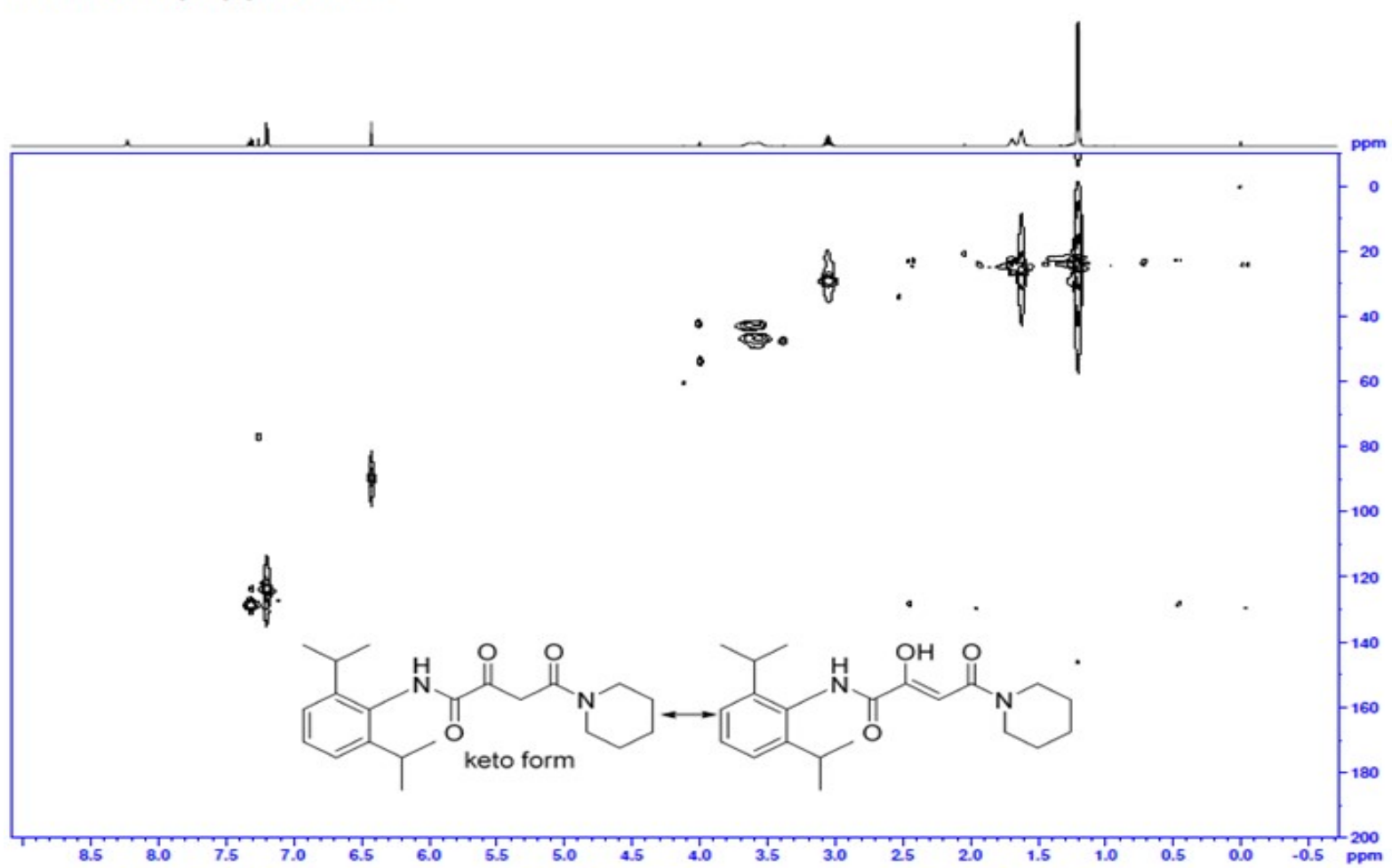


N-Cyclohexyl-2,4-dioxo-4-(piperidin-1-yl)butanamide (precursor of 28).<smiles>O=C(CC(=O)N1CCCCC1)C(=O)NC1CCCCC1</smiles>

This compound is novel and was prepared following general procedure B using methyl 2(cyclohexylamino)-2-oxoacetate $(0.400 \mathrm{~g}, 2.16 \mathrm{mmol}, 1.0 \mathrm{eq})$ in anhydrous THF $(5 \mathrm{~mL}), 1$ (piperidin-1-yl)ethan-1-one $(0.275 \mathrm{~g}, 2.16 \mathrm{mmol}, 1.0 \mathrm{eq})$ in anhydrous THF (8 mL) and LDA (1M solution in THF, $2.81 \mathrm{~mL}, 2.81 \mathrm{mmol}, 1.3 \mathrm{eq}$ ) to give crude material which was further purified by column chromatography $(40-50 \%$ EtOAc in petroleum ether) to afford the product as an off white solid $(0.389 \mathrm{~g}, 1.39 \mathrm{mmol}, 64 \%)$. The title compound was obtained as a keto/enol tautomer (1:9) as per ${ }^{1} \mathrm{H}$ NMR.

TLC: $\mathrm{R}_{\mathrm{f}}$ ca 0.4 (5:5, hexane: EtOAc), UV-active and strong $\mathrm{KMnO}_{4}$;

MP: $108-110^{\circ} \mathrm{C}$;

HRMS (ESI) m/z: [M+Na] $]^{+}$Calcd for $\mathrm{C}_{15} \mathrm{H}_{24} \mathrm{~N}_{2} \mathrm{O}_{3} \mathrm{Na}$ 303.1679; Found 303.1671 (error 2.7 ppm);

$U_{\max } 3329,2928,2852,1656,1627,1587,1520,1478,1445,1246,1224,1140,1019,744$ $\mathrm{cm}^{-1}$;

${ }^{1} \mathrm{H}$ NMR (500 MHz, $\mathrm{CDCl}_{3}$ ) (keto/enol, 1:9) $\delta 15.29$ (0.9H, s, OH of enol form), 6.85-6.83 $(1 \mathrm{H}, \mathrm{m}, \mathrm{CONH}), 6.31(0.9 \mathrm{H}, \mathrm{s},=\mathrm{CH}$, of enol form $), 4.59-4.28\left(0.4 \mathrm{H}, \mathrm{m}, \mathrm{NCH}_{2}\right.$ of piperidine, keto form), $3.93\left(0.2 \mathrm{H}, \mathrm{s}, \mathrm{CH}_{2}\right.$ of keto form), 3.87-3.80 (0.9H, m, $\mathrm{C} \underline{H} \mathrm{H}$, enol form), 3.563.55 (3.6H, br. m, $\mathrm{NCH}_{2}$ of piperidine, enol form), 3.36-3.33 (0.1H, m, $\underline{\mathrm{HNH}}$, keto form), 2.43-1.93 (3H, m, $\mathrm{CH}_{2}$ of cyclohexyl /piperidine), 1.75-1.58 $\left(9 \mathrm{H}, \mathrm{m}, \mathrm{CH}_{2}\right.$ of cyclohexyl /piperidine), 1.42-1.35 (2H, m, $\mathrm{CH}_{2}$ of cyclohexyl/piperidine), 1.26-1.16 $\left(2 \mathrm{H}, \mathrm{m}, \mathrm{CH}_{2}\right.$ of cyclohexyl/piperidine);

${ }^{13} \mathrm{C}$ NMR (126 MHz, $\mathrm{CDCl}_{3}$ ) (keto/enol, 1:9) $\delta 193.6$ (C=O keto form), 169.9, 162.8, 161.2, 159.1, 89.3 ( $\mathrm{C}=\underline{\mathrm{C}} \mathrm{H}$, enol), 48.7, 48.4, 47.6, 47.4, 47.0, 46.4, 45.8, 42.9, 42.2, 32.9, 32.7, 26.4, $25.6,25.5,24.8,24.5,24.4$;

$m / z(\mathrm{ESI}) 303.2\left[(\mathrm{M}+\mathrm{Na})^{+}, 100 \%\right]$. 
$\mathrm{N}$-Cyclohexyl-2,4-dioxo-4-(piperidin-1-yl)butanamide.

${ }^{1} \mathrm{H}$ NMR (500 MHz, $\left.\mathrm{CDCl}_{3}\right)$.

Aug07-2020

Chemist Shweta Gediya

SKG490

PROTON.w CDCl3 /opt/topspin3.2 SKG 52<smiles>O=C(CC(=O)N1CCCCC1)C(=O)NC1CCCCC1C(=O)NC1CCCCC1</smiles><smiles>C#CCCCCCC</smiles>

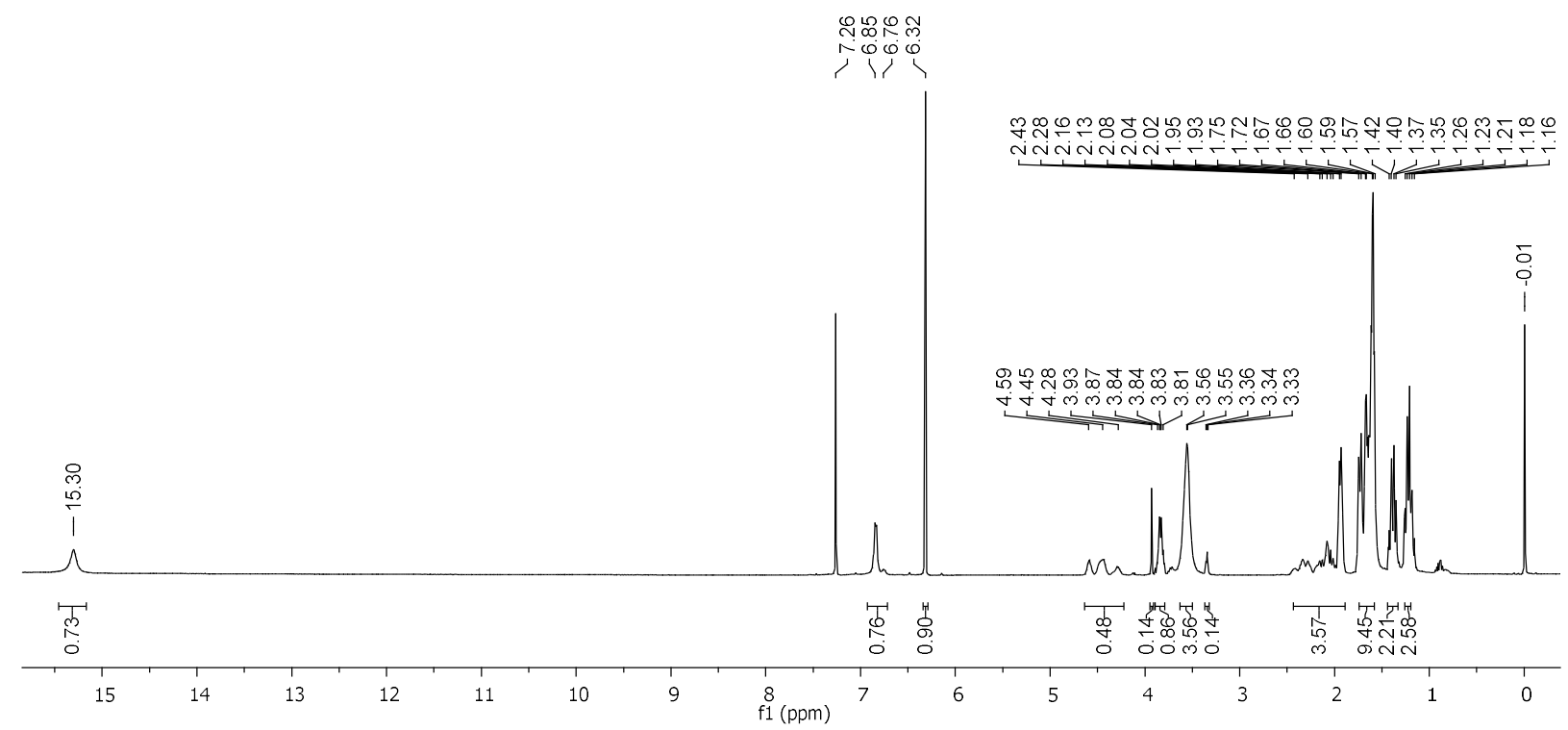

${ }^{13} \mathrm{C}$ NMR (126 MHz, $\left.\mathrm{CDCl}_{3}\right)$.

\begin{tabular}{|c|c|c|c|c|}
\hline $\begin{array}{l}\text { Aug07-2020 } \\
\text { Chemist Shweta Gediya } \\
\text { SKG490 }\end{array}$ & 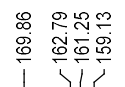 & $\underset{\substack{\infty \\
\infty \\
\infty \\
\infty}}{\infty}$ & 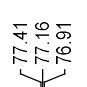 & 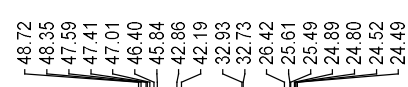 \\
\hline
\end{tabular}<smiles>O=C(NC1CCCCC1)C(=O)NC1CCCC(C(=O)N2CCCCC2)C1</smiles>

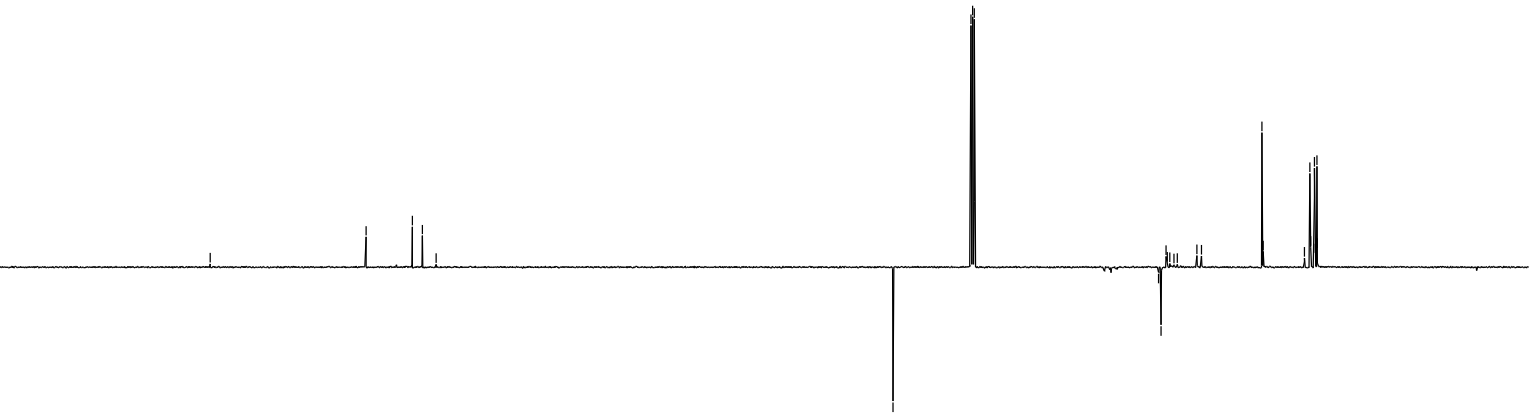

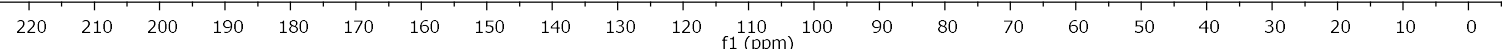


COSY.

Chemist Shweta Gediya

COSY.w CDCl3 /opt/topspin3.2 SKG 52

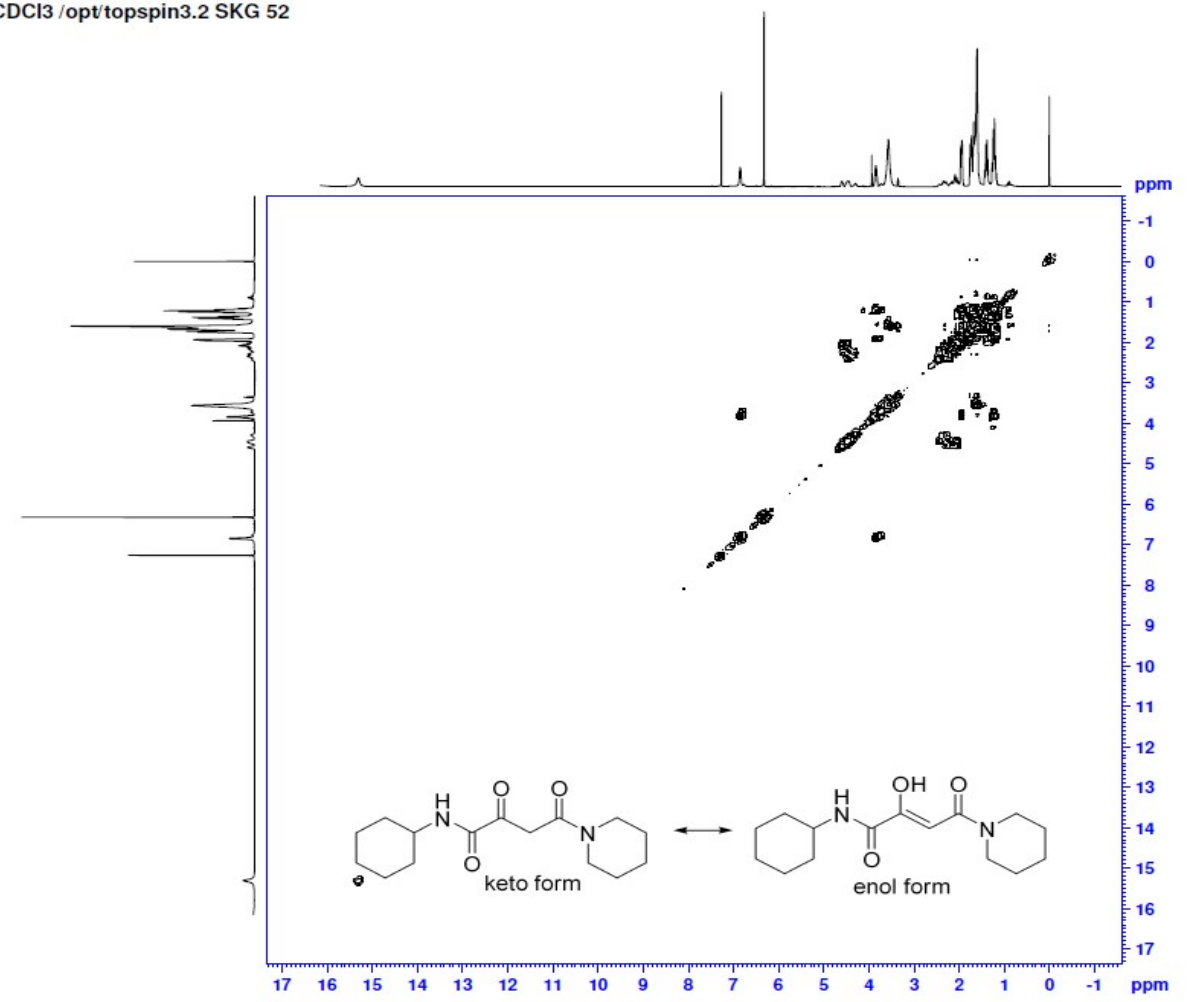

HSQC.

Chemist Shweta Gediya

HSQC.w $\mathrm{CDCl} 3 /$ opt/topspin3.2 SKG 52

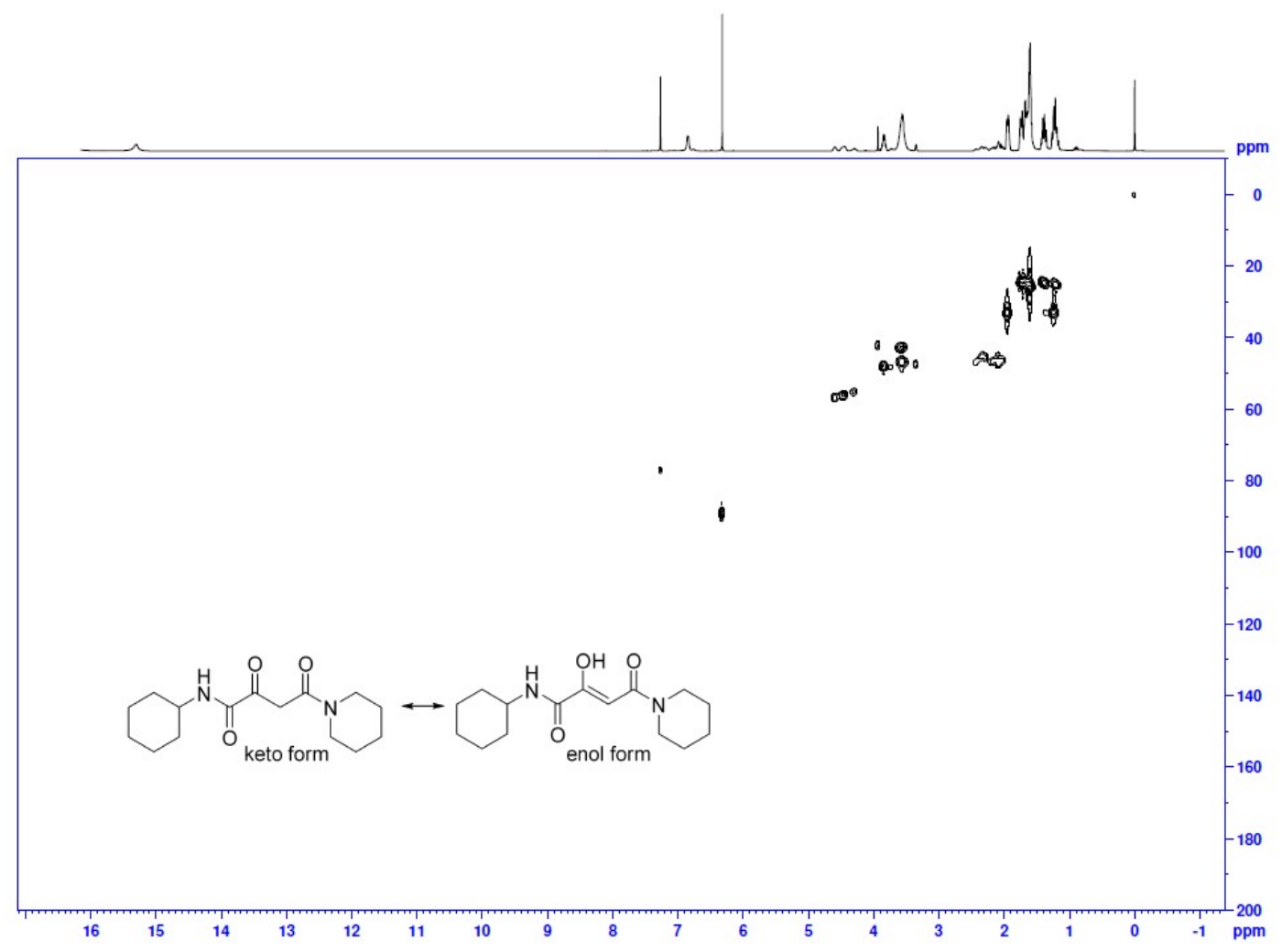


N-Methyl-2,4-dioxo-4-(piperidin-1-yl)butanamide (precursor of 29).<smiles>CNC(=O)C(=O)CC(=O)N1CCCCC1</smiles>

This compound is novel and was prepared following general procedure B using methyl 2(methylamino)-2-oxoacetate(0.673 g, $3.93 \mathrm{mmol}, 1.0 \mathrm{eq})$ in anhydrous THF (10 mL) , 1(piperidin-1-yl)ethan-1-one $(0.500 \mathrm{~g}, 3.93 \mathrm{mmol}, 1.1 \mathrm{eq})$ in anhydrous THF (10 mL) and LDA (1M solution in THF) $(5.11 \mathrm{~mL}, 5.11 \mathrm{mmol}, 1.3 \mathrm{eq})$ to give crude material which was further purified by column chromatography $(50-100 \%$ EtOAc in petroleum ether) to afford the product as a brown solid $(0.562 \mathrm{~g}, 2.65 \mathrm{mmol}, 67 \%)$. The title compound was obtained as keto/enol tautomer (1:9) as per ${ }^{1} \mathrm{H}$ NMR.

TLC: $\mathrm{R}_{\mathrm{f}}$ ca 0.4 (4:6, hexane: EtOAc), UV-active and strong $\mathrm{KMnO}_{4}$;

MP: $85-86.7^{\circ} \mathrm{C}$;

HRMS (ESI) m/z: [M+Na] $]^{+}$Calcd for $\mathrm{C}_{10} \mathrm{H}_{16} \mathrm{~N}_{2} \mathrm{O}_{3} \mathrm{Na}$ 235.1053; Found 235.1052 (error 0.5 ppm);

$U_{\max } 3382,2938,2851,1678,1615,1517,1446,1392,1270,1246,1150,1107,828,772 \mathrm{~cm}^{-}$ 1 ;

${ }^{1} \mathrm{H}$ NMR (400 MHz, $\mathrm{CDCl}_{3}$ ) (keto/enol, 1:9) $\delta 15.25$ (0.9H, br. s, OH of enol form), 7.01 (1H, s, CONH), $6.33\left(0.9 \mathrm{H}, \mathrm{s},=\mathrm{CH}\right.$, of enol form), 3.93 (0.2H s, $\mathrm{CH}_{2}$ of keto form), 3.56-3.34 $\left(4 \mathrm{H}, \mathrm{s}, \mathrm{NCH}_{2}\right.$ of piperidine), 2.94-2.89 $\left(3 \mathrm{H}, \mathrm{m},-\mathrm{NHCH}_{3}\right), 1.68-1.57\left(6 \mathrm{H}, \mathrm{m}, \mathrm{CH}_{2}\right.$ of piperidine);

${ }^{13} \mathrm{C}$ NMR $\left(101 \mathrm{MHz}, \mathrm{CDCl}_{3}\right)($ keto + enol) $\delta 169.7(\underline{\mathrm{C}}=\mathrm{CH}$, enol), $162.9(\mathrm{C}=\mathrm{O}$ amide $), 162.4$ $(\mathrm{C}=\mathrm{O}$ amide), 89.3 ( $\mathrm{C}=\underline{\mathrm{C}} \mathrm{H}$ enol), 47.5, 42.8, 42.2, 26.3, 26.1 , 25.4, 24.5;

$m / z(\mathrm{ESI}) 253.2\left[(\mathrm{M}+\mathrm{Na})^{+}, 100 \%\right]$. 
N-Methyl-2,4-dioxo-4-(piperidin-1-yl)butanamide.

${ }^{1} \mathrm{H}$ NMR (400 MHz, $\left.\mathrm{CDCl}_{3}\right)$.

Aug28-2020

PROTON.w CDCl3 /opt/topspin3.5p12 SKG 11<smiles>CNC(=O)C(=O)CC(=O)N1CCCCC1C(=O)NCCOC(=O)/C(O)=C/C(=O)N1CCCCC1</smiles>

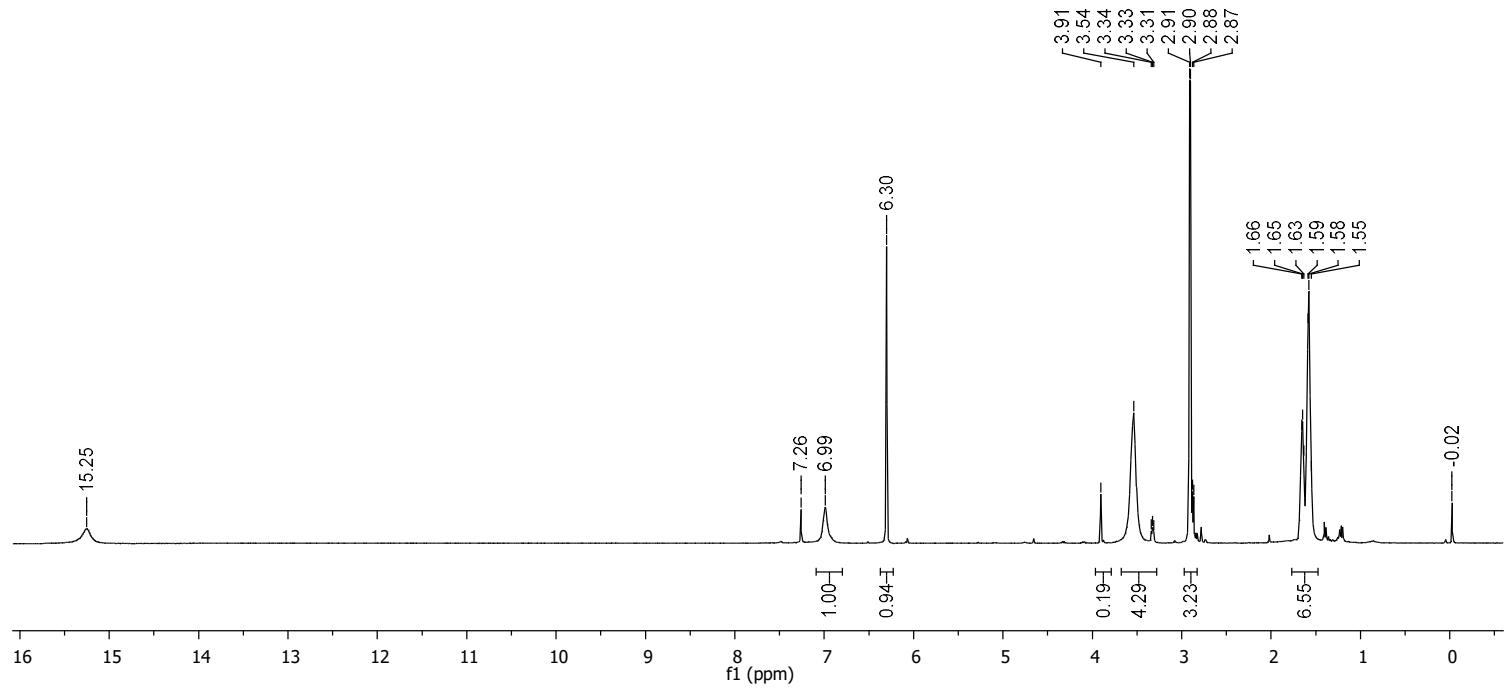

${ }^{13} \mathrm{C}$ NMR (101 MHz, $\left.\mathrm{CDCl}_{3}\right)$.

Aug28-2020

SKG509

C13APT.w CDCI3

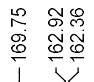

$\stackrel{\substack{1 \\ \infty}}{\infty} \quad \infty$

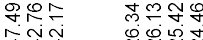

뉸ำ
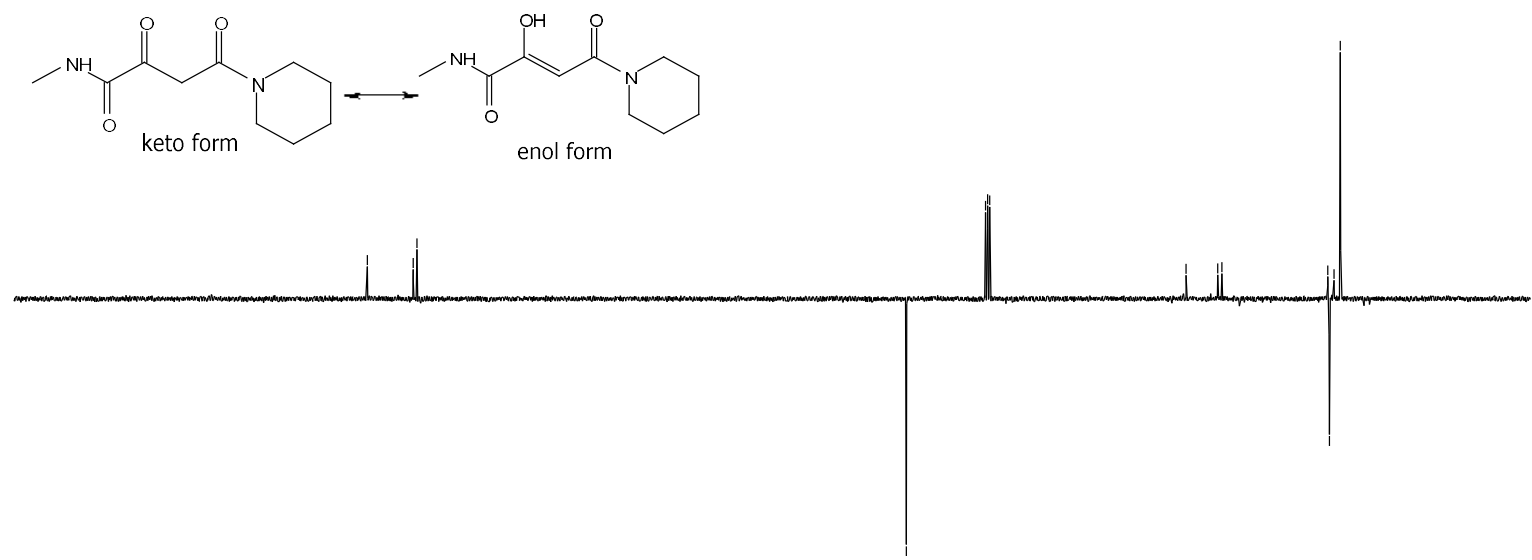

$\begin{array}{llllllllllllllllllllllll}220 & 210 & 200 & 190 & 180 & 170 & 160 & 150 & 140 & 130 & 120 & 110 & 100 & 90 & 80 & 70 & 60 & 50 & 40 & 30 & 20 & 10 & 0\end{array}$ 
COSY.

SKG509

COSY.w CDCl3/opt/topspin3.5pl2 SKG 11

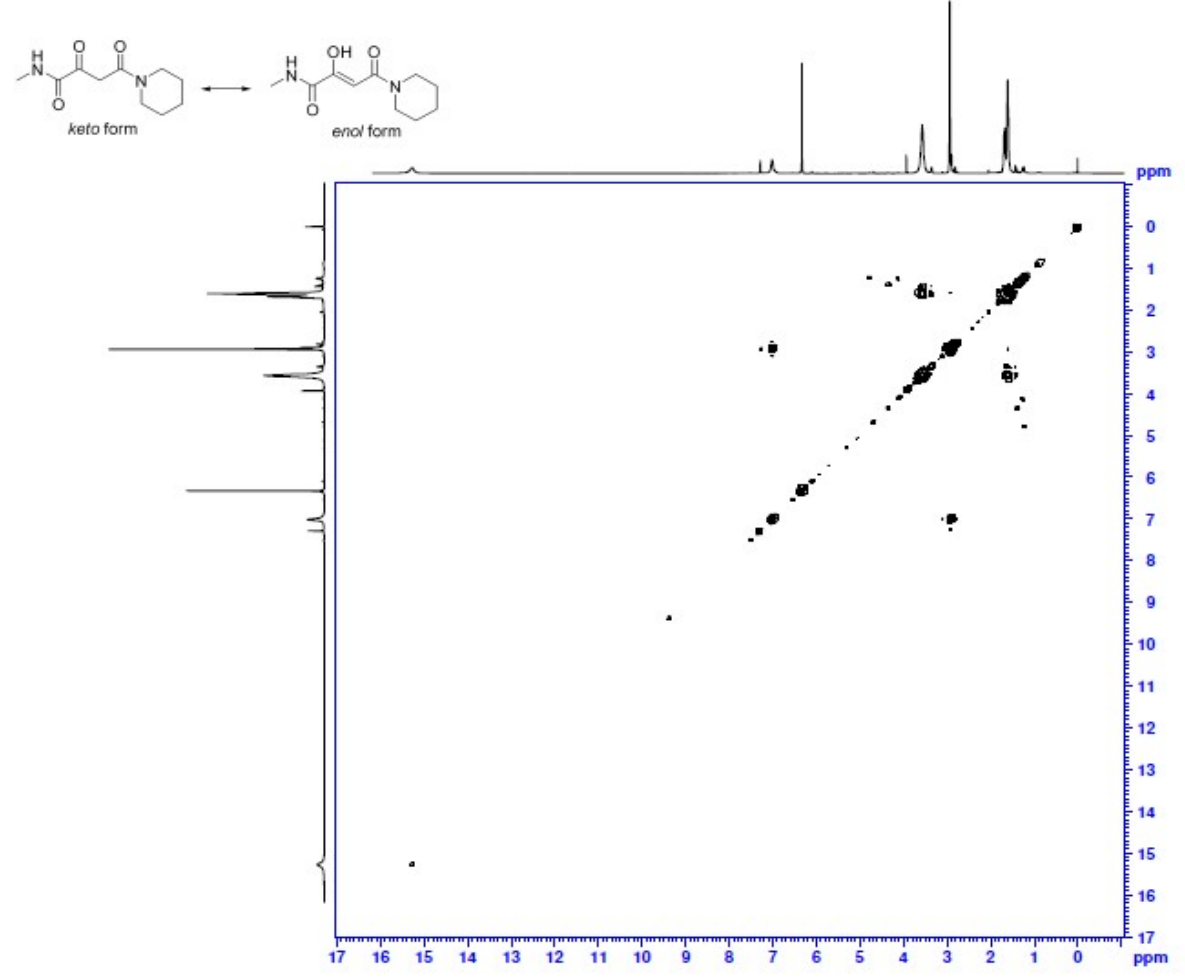

HSQC.

\section{SKG509}

.w. CDCl3 /opt/topspin3.5pl2 SKG 11

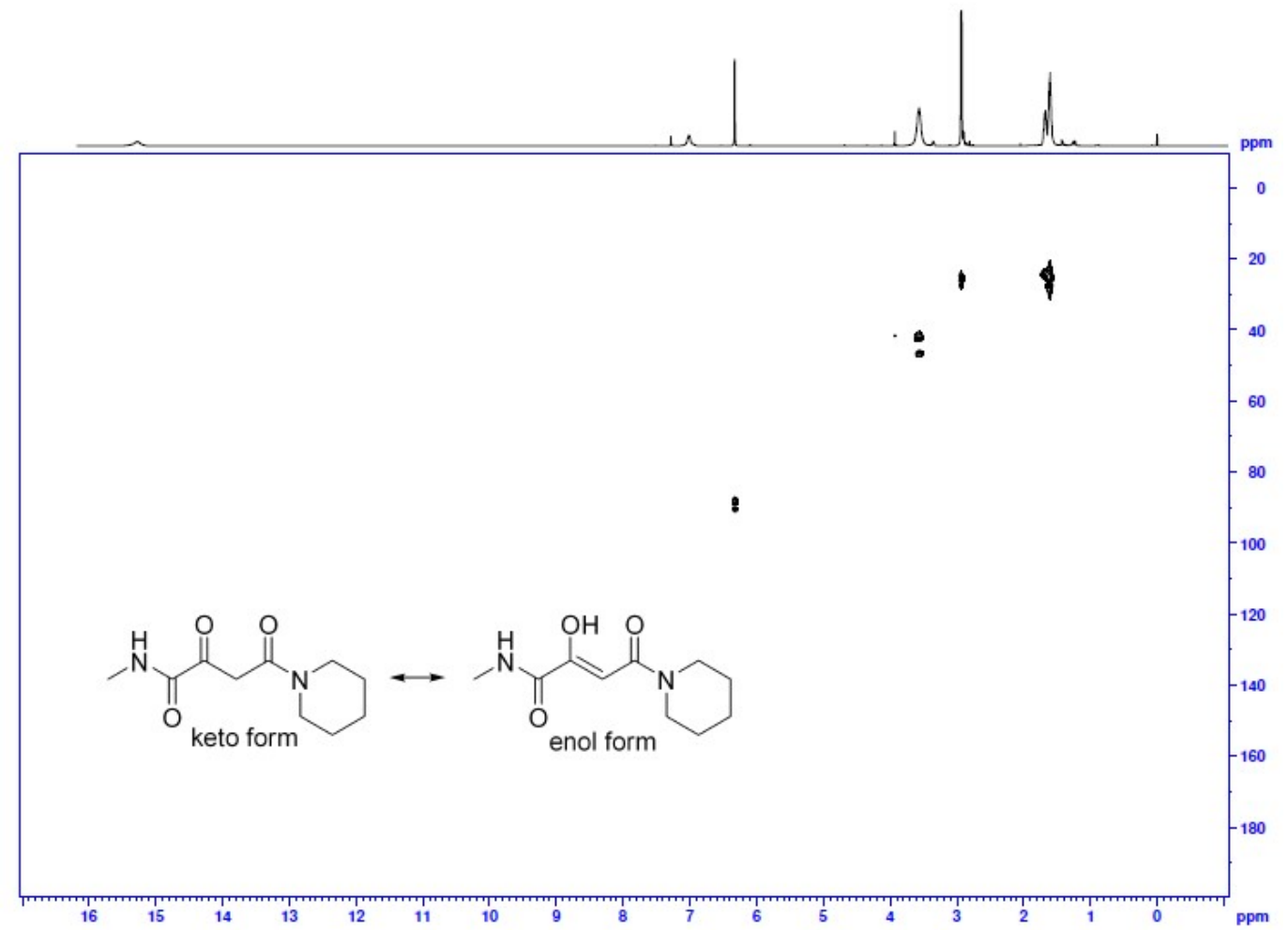


N-Cyclopropyl-2,4-dioxo-4-(piperidin-1-yl)butanamide (precursor of 30).<smiles>O=C(CC(=O)N1CCCCC1)C(=O)NC1CC1</smiles>

This compound is novel and was prepared following the general procedure B using methyl 2(cyclopropylamino)-2-oxoacetate $(0.450 \mathrm{~g}, 3.14 \mathrm{mmol}, 1.0 \mathrm{eq})$ in anhydrous THF $(5 \mathrm{~mL}), 1$ (piperidin-1-yl)ethan-1-one (0.400 g, $3.14 \mathrm{mmol}, 1.0 \mathrm{eq})$ in anhydrous THF (8 mL) and LDA (1M solution in THF) $(4.08 \mathrm{~mL}, 4.08 \mathrm{mmol}, 1.3 \mathrm{eq})$ to give crude material which was further purified by column chromatography $(30-50 \%$ EtOAc in petroleum ether) to afford the product as a white solid $(0.326 \mathrm{~g}, 1.36 \mathrm{mmol}, 44 \%)$. The title compound was obtained as a keto/enol tautomer (1:9) as per ${ }^{1} \mathrm{H}$ NMR.

TLC: $\mathrm{R}_{\mathrm{f}}$ ca 0.5 (5:5, hexane: EtOAc), UV-active and strong $\mathrm{KMnO}_{4}$;

MP: $98-100{ }^{\circ} \mathrm{C}$;

HRMS (ESI) m/z: [M+Na] ${ }^{+}$Calcd for $\mathrm{C}_{12} \mathrm{H}_{18} \mathrm{~N}_{2} \mathrm{O}_{3} \mathrm{Na}$ 261.1210; Found 261.1206 (error 1.6 ppm);

$U_{\max } 3294,2956,2857,1667,1627,1529,1479,1376,1251,1230,1131,704 \mathrm{~cm}^{-1}$;

${ }^{1} \mathrm{H}$ NMR (400 MHz, $\mathrm{CDCl}_{3}$ ) ( keto/enol, 1:9) $\delta 15.28$ (0.9H s, br.s., OH of enol form), 7.45 (0.1H br.s., CONH of keto form), $6.98(0.9 \mathrm{H}, \mathrm{s}, \mathrm{CONH}$ of enol form $), 6.30(0.9 \mathrm{H}, \mathrm{s},=\mathrm{CH}$, of enol form), $3.90\left(0.2 \mathrm{H}, \mathrm{s}, \mathrm{CH}_{2}\right.$ of keto form), 3.54-3.32 (4H, m, $\mathrm{NCH}_{2}$ of piperidine), 2.87$2.741\left(1 \mathrm{H}, \mathrm{m}, \mathrm{CH}\right.$ of cyclopropyl), 1.71-1.57 $\left(6 \mathrm{H}, \mathrm{m}, \mathrm{CH}_{2}\right.$ of piperidine), $0.87-0.82(2 \mathrm{H}, \mathrm{m}$, $\mathrm{CH}_{2}$ of cyclopropyl), $0.64-0.56\left(2 \mathrm{H}, \mathrm{m}, \mathrm{CH}_{2}\right.$ of cyclopropyl);

${ }^{13} \mathrm{C}$ NMR (101 MHz, $\left.\mathrm{CDCl}_{3}\right)$ (keto/enol, 1:9) $\delta 169.7(\underline{\mathrm{C}}=\mathrm{CH}$, enol $\mathrm{C}=\mathrm{O}$ ?), $163.6(\mathrm{C}=\mathrm{O}$ amide), $162.3\left(\mathrm{C}=\mathrm{O}\right.$ amide), $89.2\left(\mathrm{C}=\underline{\mathrm{CH}}\right.$, enol), $47.5\left(\mathrm{CH}_{2}\right.$ of keto form $), 42.3,41.2,26.3$, 25.4, 24.5, 22.3, 6.6, 6.5. 6.4;

$m / z(\mathrm{ESI}) 261.1\left[(\mathrm{M}+\mathrm{Na})^{+}, 100 \%\right]$. 
N-Cyclopropyl-2,4-dioxo-4-(piperidin-1-yl)butanamide.

${ }^{1} \mathrm{H}$ NMR (400 MHz, $\left.\mathrm{CDCl}_{3}\right)$.

Sep $11-2020$
SKG534

SKG534
PROTON.w CDCl3 /opt/topspin3.5pl2 SKG 60
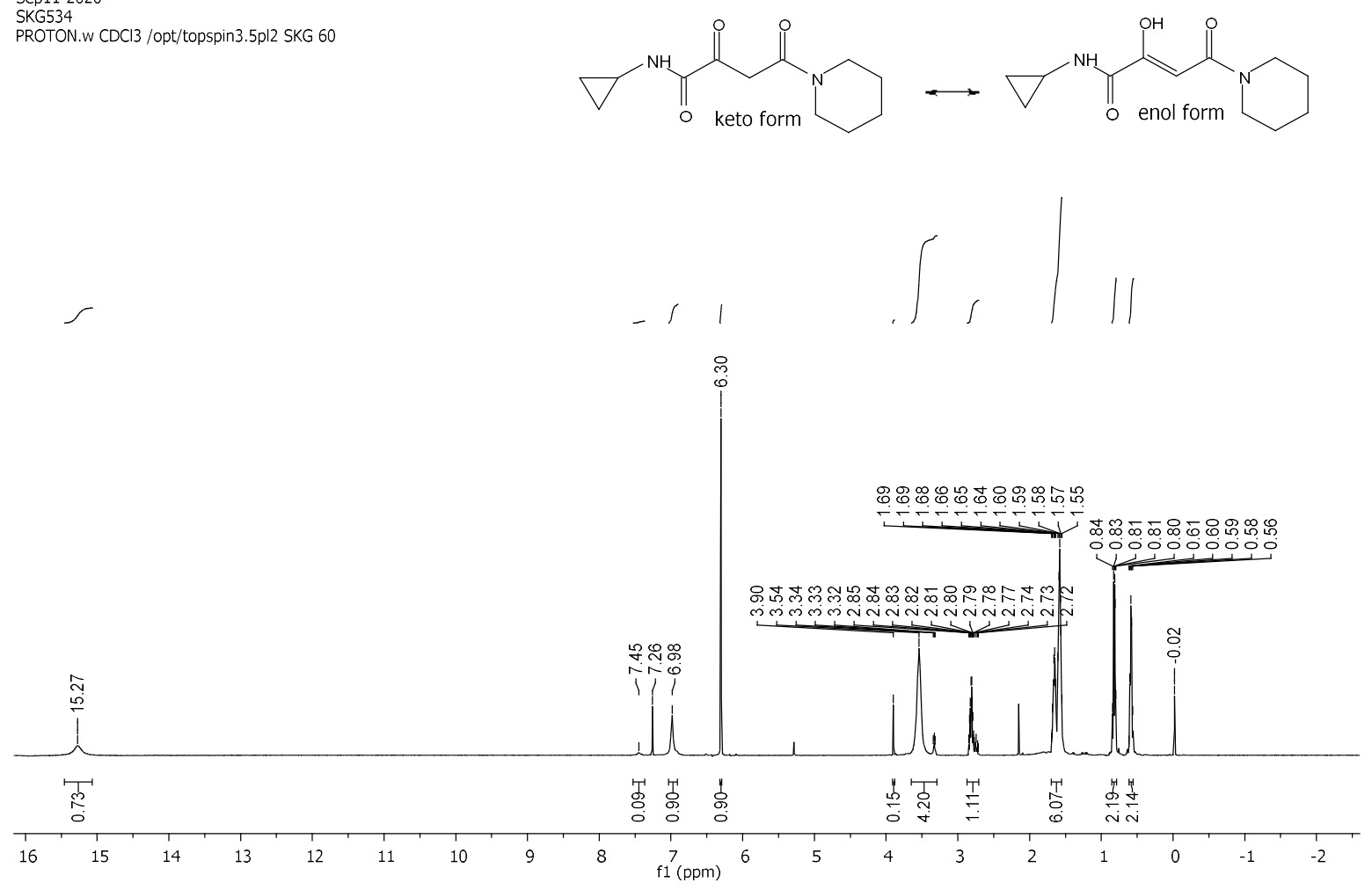

${ }^{13} \mathrm{C}$ NMR (101 MHz, $\left.\mathrm{CDCl}_{3}\right)$.

Sep $11-2020$
SKG534

C13APT.w $\mathrm{CDCl} 3$

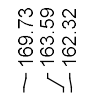

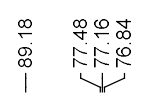

䠉是

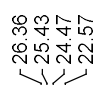

踾
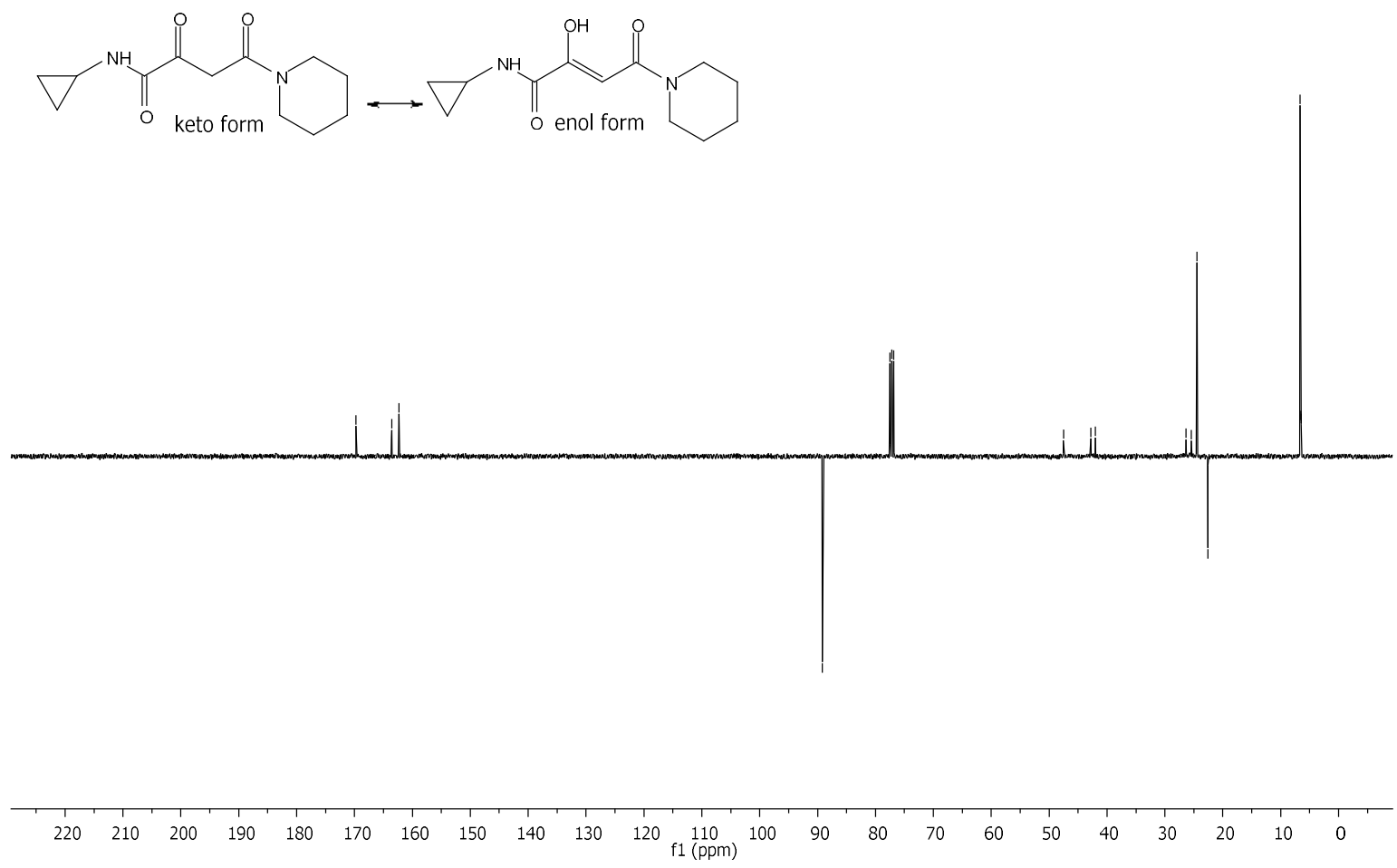

S128 
COSY.

SKG534

COSY.w CDCl3/opt/topspin3.5pl2 SKG 60

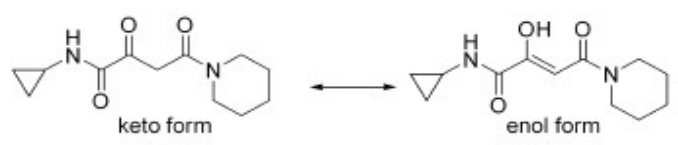

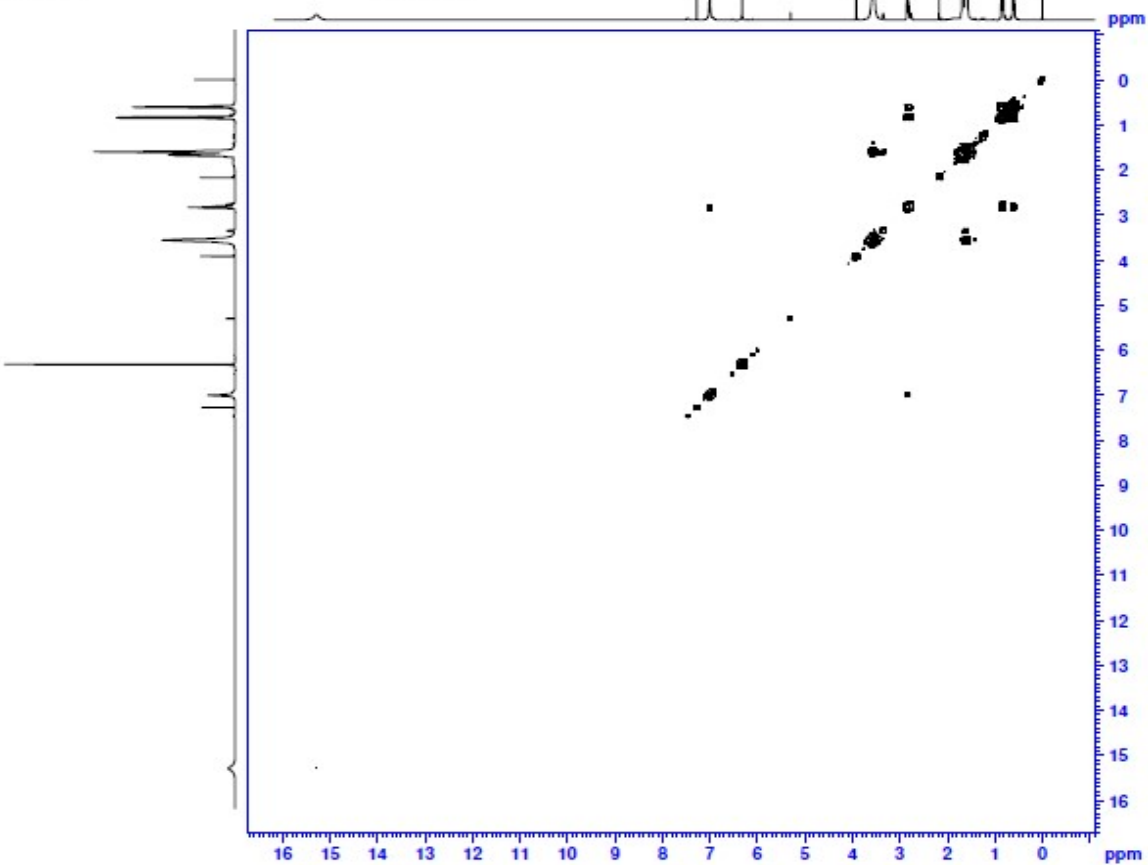

HSQC.

SKG534

HSQC.w CDCl3 /opt/topspin3.5pl2 SKG 60

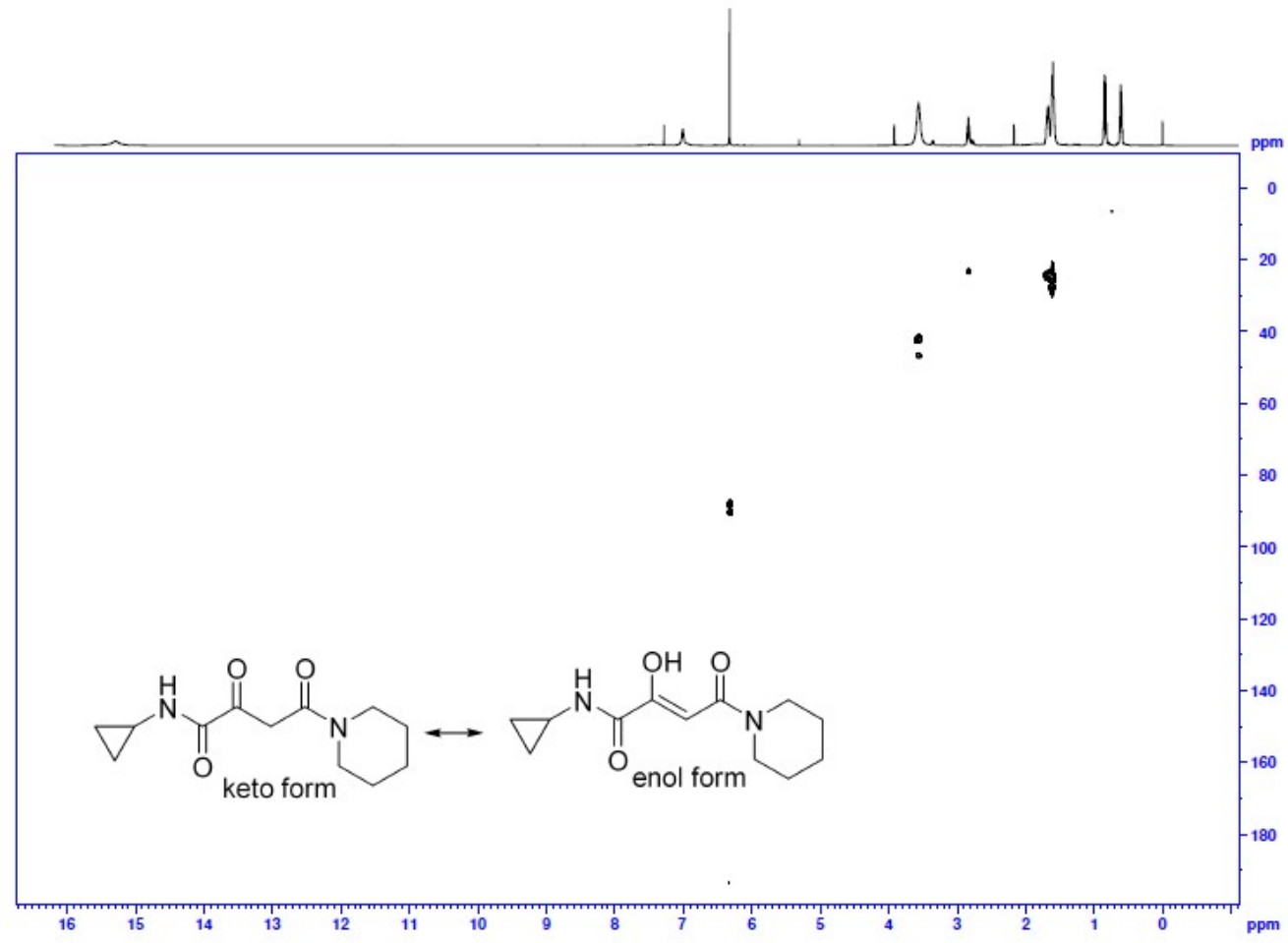


Synthesis of 2, 5-dioxo- $N$-phenyl-5-(piperidin-1-yl)pentanamide (precursor of 31).

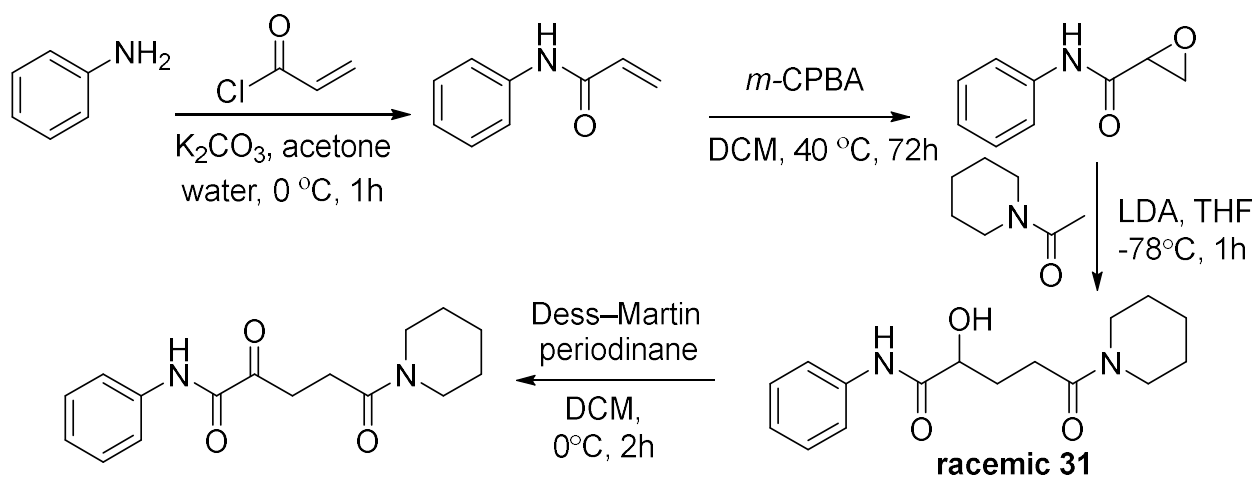

$N$-Phenylacrylamide.<smiles>C=CC(=O)Nc1ccccc1</smiles>

This compound is reported and has been fully characterised previously.

To a suspension of potassium carbonate (14.8 g, $107.6 \mathrm{mmol}, 2.0 \mathrm{eq})$ in distilled water (25 $\mathrm{mL})$ and acetone $(100 \mathrm{~mL})$ was added acryloyl chloride $(9.73 \mathrm{~g}, 107.6 \mathrm{mmol}, 2.0 \mathrm{eq})$ at $0{ }^{\circ} \mathrm{C}$ under a nitrogen atmosphere, followed by dropwise addition of aniline $(5.0 \mathrm{~g}, 53.8 \mathrm{mmol}, 1.0$ eq) and the resulting reaction mixture was stirred for $1 \mathrm{~h}$ at $0{ }^{\circ} \mathrm{C}$. Once the reaction was complete (as assessed by TLC), the reaction mixture was filtered, and the filtrate was concentrated under reduced pressure. The solid residues were suspended in water and filtered, washed with n-pentane and dried to afford the product as white solid $(7.52 \mathrm{~g}, 51.1 \mathrm{mmol}$, $95 \%)$.

TLC: $\mathrm{R}_{\mathrm{f}}$ ca 0.3 (8:2, hexane: EtOAc), UV-active and strong $\mathrm{KMnO}_{4}$;

HRMS (ESI) m/z: $[\mathrm{M}+\mathrm{Na}]^{+}$Calcd for $\mathrm{C}_{9} \mathrm{H}_{9} \mathrm{NONa}$ 170.0576; Found 170.0575 (error 1.0 ppm);

${ }^{1} \mathrm{H}$ NMR $\left(400 \mathrm{MHz}, \mathrm{CDCl}_{3}\right) \delta 7.94(1 \mathrm{H}$, br.s, CONH), $7.59(2 \mathrm{H}, \mathrm{d}, J=7.8 \mathrm{~Hz}, \mathrm{ArH}), 7.32-$ $7.30(2 \mathrm{H}, \mathrm{m}, \mathrm{ArH}), 7.11(1 \mathrm{H}, \mathrm{t}, J=7.3 \mathrm{~Hz}, \mathrm{ArH}), 6.41\left(1 \mathrm{H}, \mathrm{d}, J=16.8 \mathrm{~Hz}, \mathrm{CH}=\mathrm{CH}_{2}\right), 6.29$ $\left(1 \mathrm{H}, \mathrm{dd}, J=16.8,10.0 \mathrm{~Hz}, \mathrm{CH}=\mathrm{C}_{2}\right), 5.72\left(1 \mathrm{H}, \mathrm{d}, J=10.0 \mathrm{~Hz}, \mathrm{CH}=\mathrm{C}_{2}\right)$;

${ }^{13} \mathrm{C}$ NMR $\left(101 \mathrm{MHz}, \mathrm{CDCl}_{3}\right) \delta 164.0,137.9,131.4,129.1,127.8,124.6,120.3$; $m / z(\mathrm{ESI}) 170.2\left[(\mathrm{M}+\mathrm{Na})^{+}, 100 \%\right]$.

Chanthamath, S.; Takaki, S.; Shibatomi, K.; Iwasa, S. Highly Stereoselective Cyclopropanation of $\alpha, \beta$-Unsaturated Carbonyl Compounds with Methyl (Diazoacetoxy)acetate Catalyzed by a Chiral Ruthenium(II) Complex, Angew. Chem. Int. Ed. 2013, 52, 5818 -5821. 


\section{$\mathrm{N}$-Phenylacrylamide.}

${ }^{1} \mathrm{H}$ NMR (400 MHz, $\mathrm{CDCl}_{3}$ ).
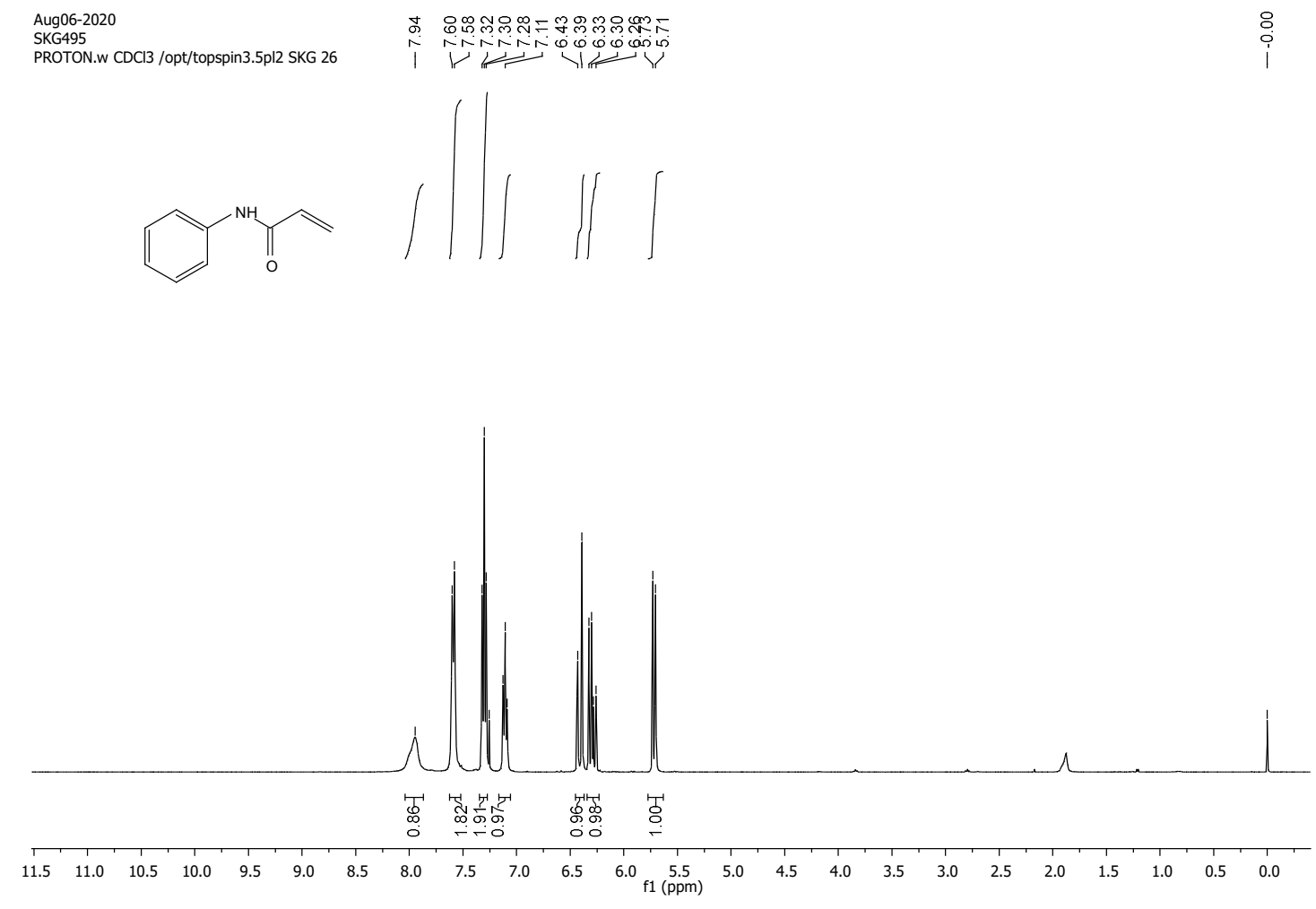

${ }^{13} \mathrm{C}$ NMR (101 MHz, $\left.\mathrm{CDCl}_{3}\right)$.

Aug06-2020
SKG 495

SKG495
C13APT.w CDCl3

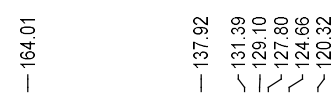<smiles>C=CC(=O)Nc1ccccc1</smiles> 


\section{$N$-Phenyloxirane-2-carboxamide.}<smiles>O=C(Nc1ccccc1)C1CO1</smiles>

This compound is known and but has not been fully characterised previously.

To a solution of $N$-phenylacrylamide $(5.00 \mathrm{~g}, 34.0 \mathrm{mmol}, 1.0 \mathrm{eq})$ in dichloromethane (100 $\mathrm{mL}$ ) was added $m$-chloroperoxybenzoic acid ( $m$-CPBA) $(29.2 \mathrm{~g}, 170 \mathrm{mmol}, 5.0 \mathrm{eq})$ and the resulting reaction mixture was stirred at $40{ }^{\circ} \mathrm{C}$ for $72 \mathrm{~h}$. After $72 \mathrm{~h}$, the reaction was cooled and quenched with saturated sodium bicarbonate solution to give a dark brown solution which was further stirred at rt overnight. The aqueous solution was extracted with DCM (3 x $50 \mathrm{~mL}$ ) and the combined extracts were dried over $\mathrm{MgSO}_{4}$, filtered and filtrate was concentrated to generate crude material which was further purified by column chromatography (20\% EtOAc in petroleum ether) to afford the product as a brown solid (2.52 g, $15.5 \mathrm{mmol}, 46 \%)$.

TLC: $\mathrm{R}_{\mathrm{f}}$ ca 0.2 (7:3, hexane: EtOAc), UV-active and strong $\mathrm{KMnO}_{4}$;

HRMS (ESI) m/z: $[\mathrm{M}+\mathrm{Na}]^{+}$Calcd for $\mathrm{C}_{9} \mathrm{H}_{9} \mathrm{NO}_{2} \mathrm{Na}$ 186.0525; Found 186.0524 (error 0.6 ppm);

${ }^{1} \mathrm{H}$ NMR (400 MHz, $\left.\mathrm{CDCl}_{3}\right) \delta 7.86(1 \mathrm{H}$, br. s, CONH), $7.52(2 \mathrm{H}, \mathrm{d}, J=8.0 \mathrm{~Hz}, \mathrm{ArH}), 7.34-$ $7.31(2 \mathrm{H}, \mathrm{m}, \mathrm{ArH}), 7.13(1 \mathrm{H}, \mathrm{t}, J=7.4 \mathrm{~Hz}, \mathrm{ArH}), 3.57-3.55(1 \mathrm{H}, \mathrm{m}, \mathrm{CH}), 3.08(1 \mathrm{H}, \mathrm{t}, J=5.0$ $\left.\mathrm{Hz}, \mathrm{CH}_{2}\right), 2.91-2.89\left(1 \mathrm{H}, \mathrm{m}, \mathrm{CH}_{2}\right)$;

${ }^{13} \mathrm{C} \mathrm{NMR}\left(101 \mathrm{MHz}, \mathrm{CDCl}_{3}\right) \delta 166.6,136.8,129.2,124.9,119.9,50.0,47.7$;

$m / z(\mathrm{ESI}) 186.0\left[(\mathrm{M}+\mathrm{Na})^{+}, 100 \%\right]$. 


\section{$\mathrm{N}$-Phenyloxirane-2-carboxamide.}

${ }^{1} \mathrm{H}$ NMR (400 MHz, $\mathrm{CDCl}_{3}$ ).

Sep04-2020
SKG521
PROTON.w CDCl3 /opt/topspin3.5pl2 SKG 10

โิ

लूलmmm
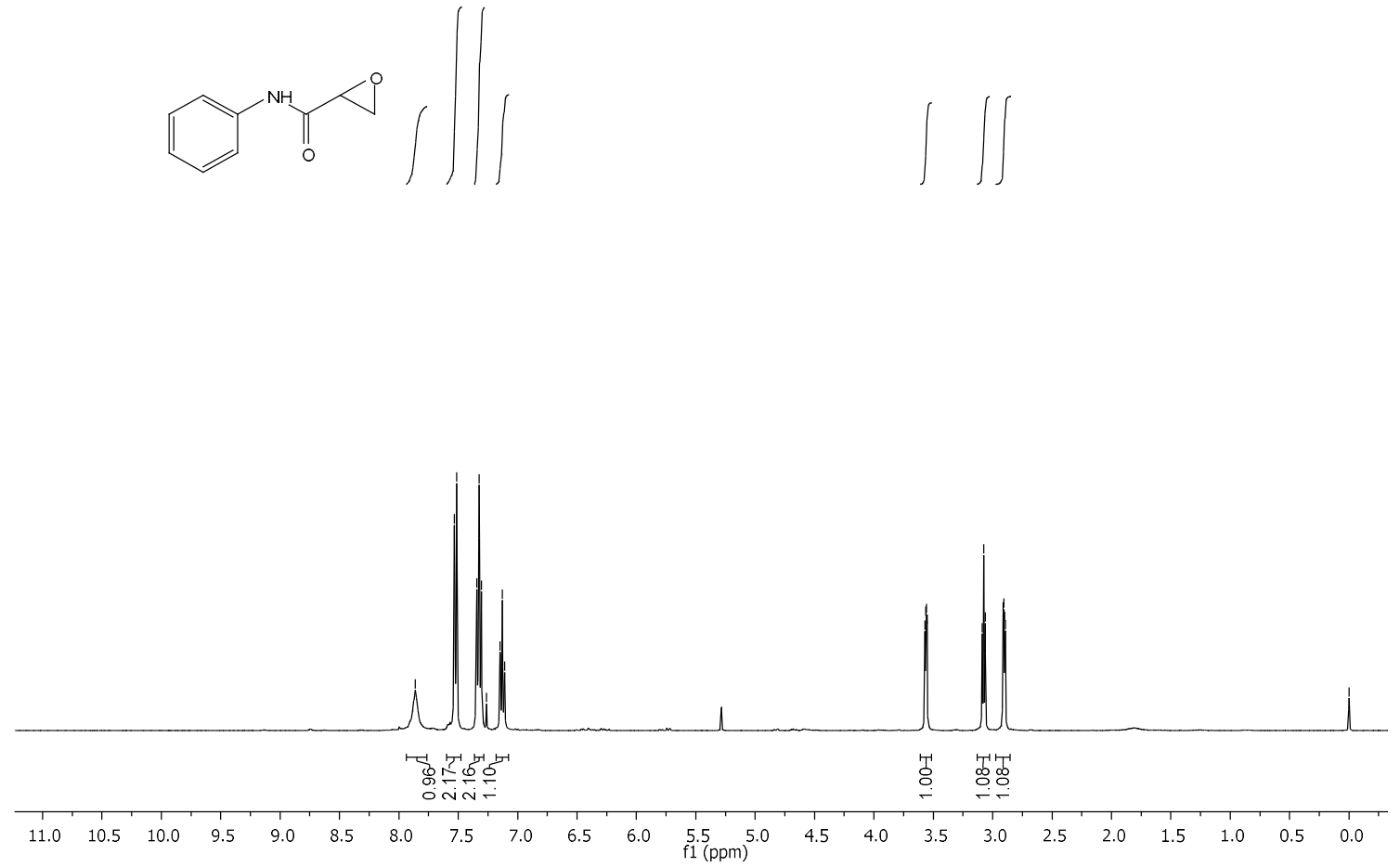

${ }^{13} \mathrm{C}$ NMR $\left(101 \mathrm{MHz}, \mathrm{CDCl}_{3}\right)$.

Sep04-2020

C13APT.w CDCl3

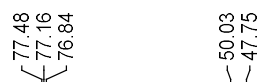

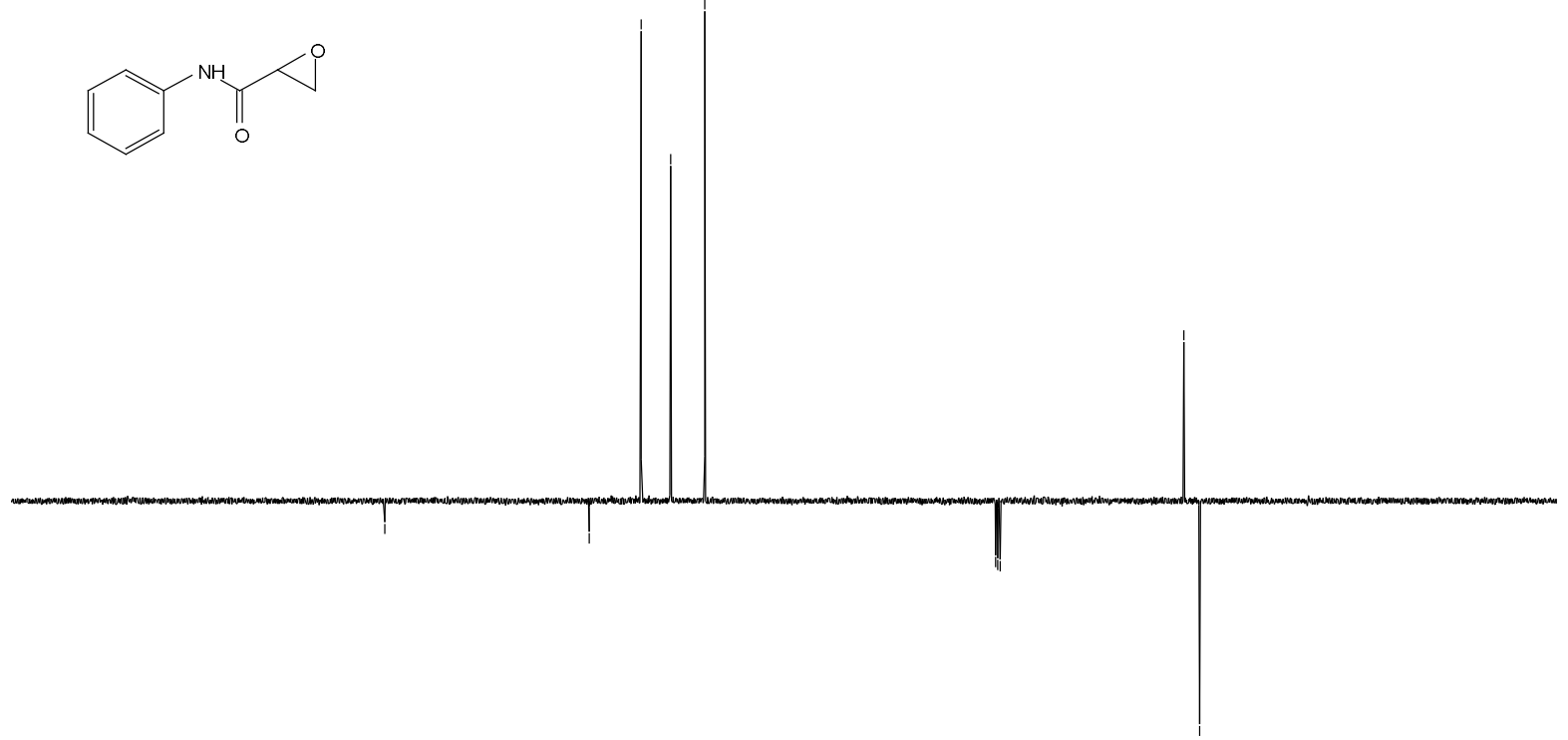

\begin{tabular}{lllllllllllllllllllllllll}
\hline 220 & 210 & 200 & 190 & 180 & 170 & 160 & 150 & 140 & 130 & 120 & 110 & 100 & 90 & 80 & 70 & 60 & 50 & 40 & 30 & 20 & 10 & 0
\end{tabular} 
COSY.

SKG521

COSY.w CDCl3/opt/topspin3.5pl2 SKG 10

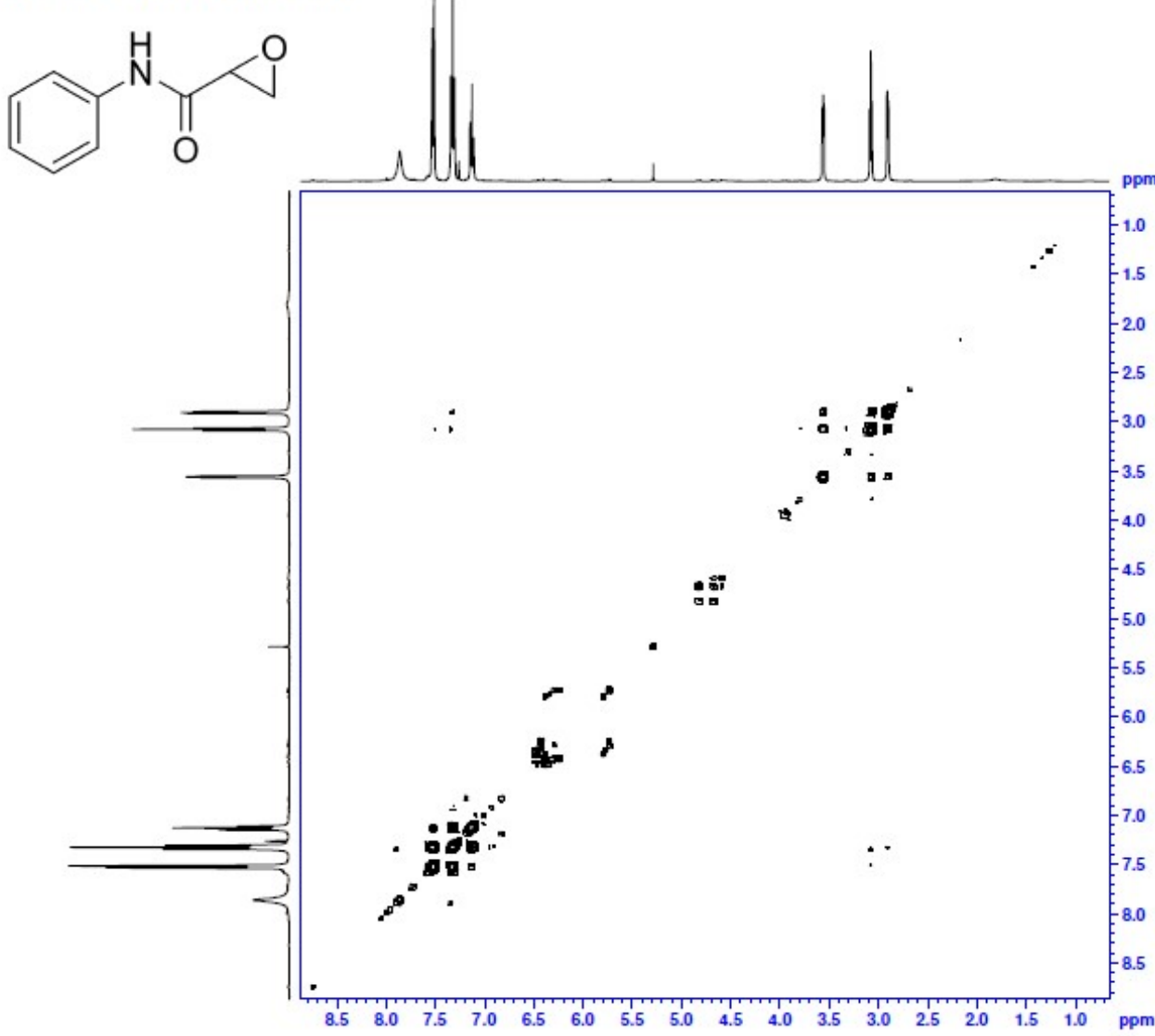


2-Hydroxy-5-oxo- $N$-phenyl-5-(piperidin-1-yl)pentanamide (racemic).<smiles>O=C(Nc1ccccc1)C(O)CCC(=O)N1CCCCC1</smiles>

This compound is novel and was prepared following the general procedure B using $\mathrm{N}$ phenyloxirane-2-carboxamide $(0.300 \mathrm{~g}, 1.84 \mathrm{mmol}, 1.0 \mathrm{eq})$ in anhydrous THF (8 mL), 1(piperidin-1-yl)ethan-1-one (0.279 g, $2.20 \mathrm{mmol}, 1.2 \mathrm{eq})$ in anhydrous THF (8 mL), and LDA (1M solution in THF) $(2.57 \mathrm{~mL}, 2.57 \mathrm{mmol}, 1.4 \mathrm{eq})$ to give crude material which was further purified by column chromatography $(50-100 \%$ EtOAc in petroleum ether) to afford the product as a white $(0.210 \mathrm{~g}, 0.724 \mathrm{mmol}, 39 \%)$. All the physical and spectral data correspond to that reported for in detail in the section on the synthesis of alcohols. 


\section{2,5-Dioxo- $N$-phenyl-5-(piperidin-1-yl)pentanamide (precursor of 31).}<smiles>O=C(CCC(=O)N1CCCCC1)C(=O)Nc1ccccc1</smiles>

This compound is novel. To a solution of 2-hydroxy-5-oxo- $N$-phenyl-5-(piperidin-1yl)pentanamide $(0.160 \mathrm{~g}, 0.551 \mathrm{mmol}, 1.0 \mathrm{eq})$ in dichloromethane $(10 \mathrm{~mL})$ was added DessMartin periodinane $(0.373 \mathrm{~g}, 0.881 \mathrm{mmol}, 1.6 \mathrm{eq})$ at $0{ }^{\circ} \mathrm{C}$ under a nitrogen atmosphere and the resulting reaction mixture was stirred at $0{ }^{\circ} \mathrm{C}$ for 15 minutes, then at room temperature for $2 \mathrm{~h}$. Once the reaction was complete (as assessed by TLC), the mixture was quenched with saturated sodium bicarbonate solution and extracted with DCM $(3 \times 10 \mathrm{~mL})$ and combined extracts were dried over $\mathrm{MgSO}_{4}$, filtered and the filtrate was concentrated under reduced pressure to generate crude which was further purified by column chromatography $(50-60 \%$ EtOAc in petroleum ether) to afford the product as a white solid $(0.112 \mathrm{~g}, 0.388 \mathrm{mmol}, 71 \%)$. TLC: $\mathrm{R}_{\mathrm{f}}$ ca 0.5 (1:9, hexane: EtOAc), UV-active and strong $\mathrm{KMnO}_{4}$; MP: $141-143^{\circ} \mathrm{C}$;

HRMS (ESI) m/z: [M+Na] $]^{+}$Calcd for $\mathrm{C}_{16} \mathrm{H}_{20} \mathrm{~N}_{2} \mathrm{O}_{3} \mathrm{Na} 311.1366$; Found 311.1364 (error 0.8 ppm);

$U_{\max } 3662,3238,2986,1720,1682,1619,1600,1440,1251,1231,1038,1071,749,668 \mathrm{~cm}^{-}$ 1 ;

${ }^{1} \mathrm{H}$ NMR (400 MHz, $\left.\mathrm{CDCl}_{3}\right) \delta 8.75$ (1H, br.s, CONH), $7.64(2 \mathrm{H}, \mathrm{d}, J=7.8 \mathrm{~Hz}, \mathrm{ArH}), 7.35$ $(2 \mathrm{H}, \mathrm{t}, J=7.8 \mathrm{~Hz}, \mathrm{ArH}), 7.15(1 \mathrm{H}, \mathrm{t}, J=7.8 \mathrm{~Hz}, \mathrm{ArH}), 3.53-3.50\left(2 \mathrm{H}, \mathrm{m}, \mathrm{NCH}_{2}\right.$ of piperidine), $3.45-3.42\left(2 \mathrm{H}, \mathrm{m}, \mathrm{NCH}_{2}\right.$ of piperidine $), 3.25-3.22\left(2 \mathrm{H}, \mathrm{m}, \mathrm{CH}_{2}\right), 2.82-2.79(2 \mathrm{H}$, $\left.\mathrm{m}, \mathrm{CH}_{2}\right), 1.70-1.50\left(6 \mathrm{H}, \mathrm{m}, \mathrm{CH}_{2}\right.$ of piperidine);

${ }^{13} \mathrm{C}$ NMR $\left(101 \mathrm{MHz}, \mathrm{CDCl}_{3}\right) \delta 198.6,169.2,158.0,136.7,129.3,125.1,119.9,46.7,43.2$, 31.5, 28.5, 26.5, 25.6, 24.6; $m / z(\mathrm{ESI}) 311.2\left[(\mathrm{M}+\mathrm{Na})^{+}, 100 \%\right]$. 


\section{2,5-Dioxo- $N$-phenyl-5-(piperidin-1-yl)pentanamide.}

${ }^{1} \mathrm{H}$ NMR $\left(400 \mathrm{MHz}, \mathrm{CDCl}_{3}\right)$.
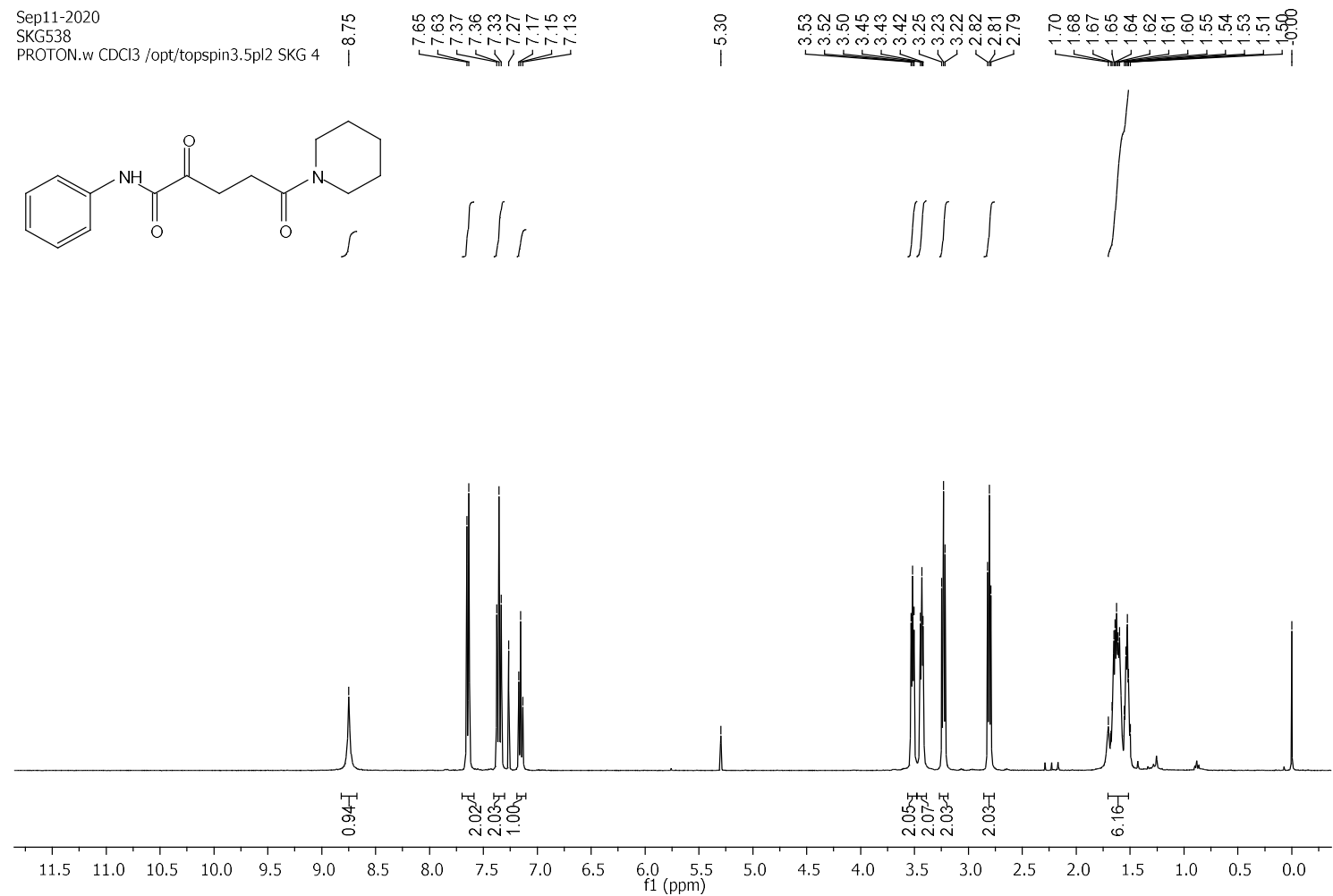

${ }^{13} \mathrm{C}$ NMR $\left(101 \mathrm{MHz}, \mathrm{CDCl}_{3}\right)$.

\begin{tabular}{|c|c|c|c|c|c|c|c|}
\hline $\begin{array}{l}\text { Sep11-2020 } \\
\text { SKG538 } \\
\text { C13APT.w CDCl3 }\end{array}$ & 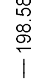 & $\frac{\infty}{5}$ & 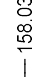 & $\begin{array}{l}0 \\
0 \\
\stackrel{0}{2} \\
\stackrel{2}{1}\end{array}$ & 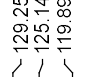 & 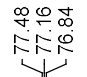 & 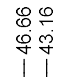 \\
\hline
\end{tabular}

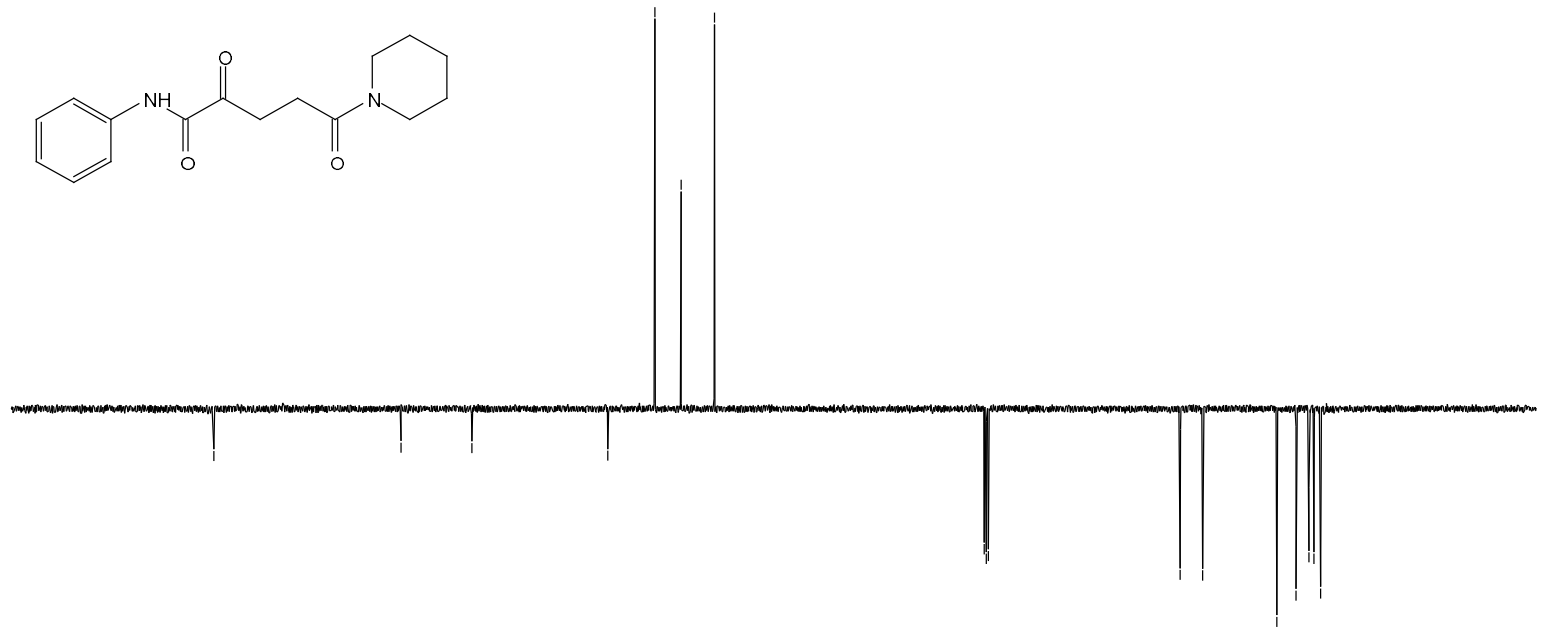

$\begin{array}{lllllllllllllllllllllllllllllllll}230 & 220 & 210 & 200 & 190 & 180 & 170 & 160 & 150 & 140 & 130 & 120 & 110 & 100 & 90 & 80 & 70 & 60 & 50 & 40 & 30 & 20 & 10 & 0\end{array}$ 
COSY.

SKG538

COSY.w CDCl3/opt/topspin3.5pl2 SKG 4

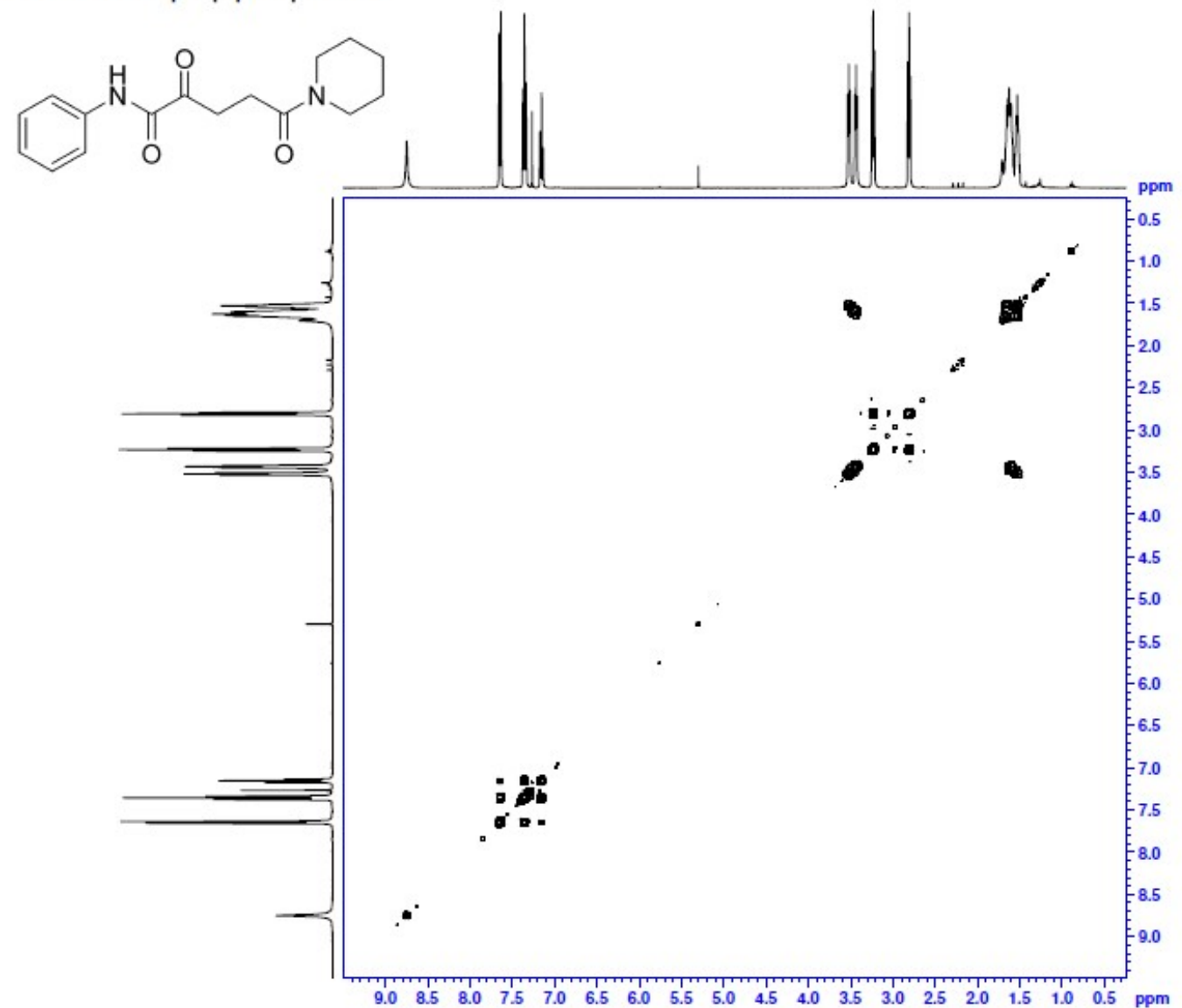


Ethyl 2,4-dioxo-4-(piperidin-1-yl)butanoate (precursor of 32).<smiles>CCOC(=O)C(=O)CC(=O)N1CCCCC1</smiles>

This compound is novel and was prepared following general procedure B using diethyl oxalate $(0.400 \mathrm{~g}, 2.73 \mathrm{mmol}, 1.0 \mathrm{eq})$ in anhydrous THF $(5 \mathrm{~mL}), 1$-(piperidin-1-yl)ethan-1-one (0.382 g, $3.01 \mathrm{mmol}, 1.1 \mathrm{eq})$ in anhydrous THF (5 mL) and LDA (1M solution in THF) (3.54 $\mathrm{mL}, 3.54 \mathrm{mmol}, 1.3 \mathrm{eq})$ in THF $(10 \mathrm{~mL}$ split) to give crude material which was further purified by column chromatography $(30-50 \%$ EtOAc in petroleum ether) to afford the product as a clear oil $(0.321 \mathrm{~g}, 1.41 \mathrm{mmol}, 51 \%)$. The title compound was obtained as an enol form as per ${ }^{1} \mathrm{H}$ NMR.

TLC: $\mathrm{R}_{\mathrm{f}}$ ca 0.4 (7:3, hexane: EtOAc), UV-active and strong $\mathrm{KMnO}_{4}$;

HRMS (ESI) m/z: [M+Na] ${ }^{+}$Calcd for $\mathrm{C}_{11} \mathrm{H}_{17} \mathrm{NO}_{4} \mathrm{Na} 250.1050$; Found 250.1058 (error -3.4 ppm);

$U_{\max } 2938,2858,1727,1617,1599,1585,1484,1377,1244,1226,1128,1014,766 \mathrm{~cm}^{-1}$;

${ }^{1} \mathrm{H}$ NMR (500 MHz, $\mathrm{CDCl}_{3}$ ) (enol form) $\delta 14.72(1 \mathrm{H}$, br.s., $\mathrm{OH}$ of enol form), $6.25(1 \mathrm{H}, \mathrm{d}, J$ $=3.0 \mathrm{~Hz},=\mathrm{C} \underline{\mathrm{H}}$, of enol form), 4.33-4.28 (2H, m, $\left.\mathrm{CH}_{2} \mathrm{CH}_{3}\right), 3.52$ (4H, br.s., $\mathrm{NCH}_{2}$ of piperidine), 1.65-1.57 (6H, m, $\mathrm{CH}_{2}$ of piperidine), $1.35-1.31\left(3 \mathrm{H}, \mathrm{m}, \mathrm{CH}_{2} \mathrm{C}_{3}\right)$;

${ }^{13} \mathrm{C}$ NMR (126 MHz, $\left.\mathrm{CDCl}_{3}\right) \delta 169.1(\mathrm{C}=\mathrm{CH}$, enol), 163.1, 159.8, 92.9 (=CH enol), 62.1, $47.3,42.6,26.3,25.4,24.4,14.2$;

$m / z(\mathrm{ESI}) 250.1\left[(\mathrm{M}+\mathrm{Na})^{+}, 100 \%\right]$. 
Ethyl 2,4-dioxo-4-(piperidin-1-yl)butanoate.

${ }^{1} \mathrm{H}$ NMR (500 MHz, $\left.\mathrm{CDCl}_{3}\right)$.

Dec17-2020

Chemist Shweta Gediya

PROTON.w CDCl3 /opt/topspin3.2 SKG 54<smiles>CCOC(=O)/C(O)=C/C(=O)N1CCCCC1</smiles>
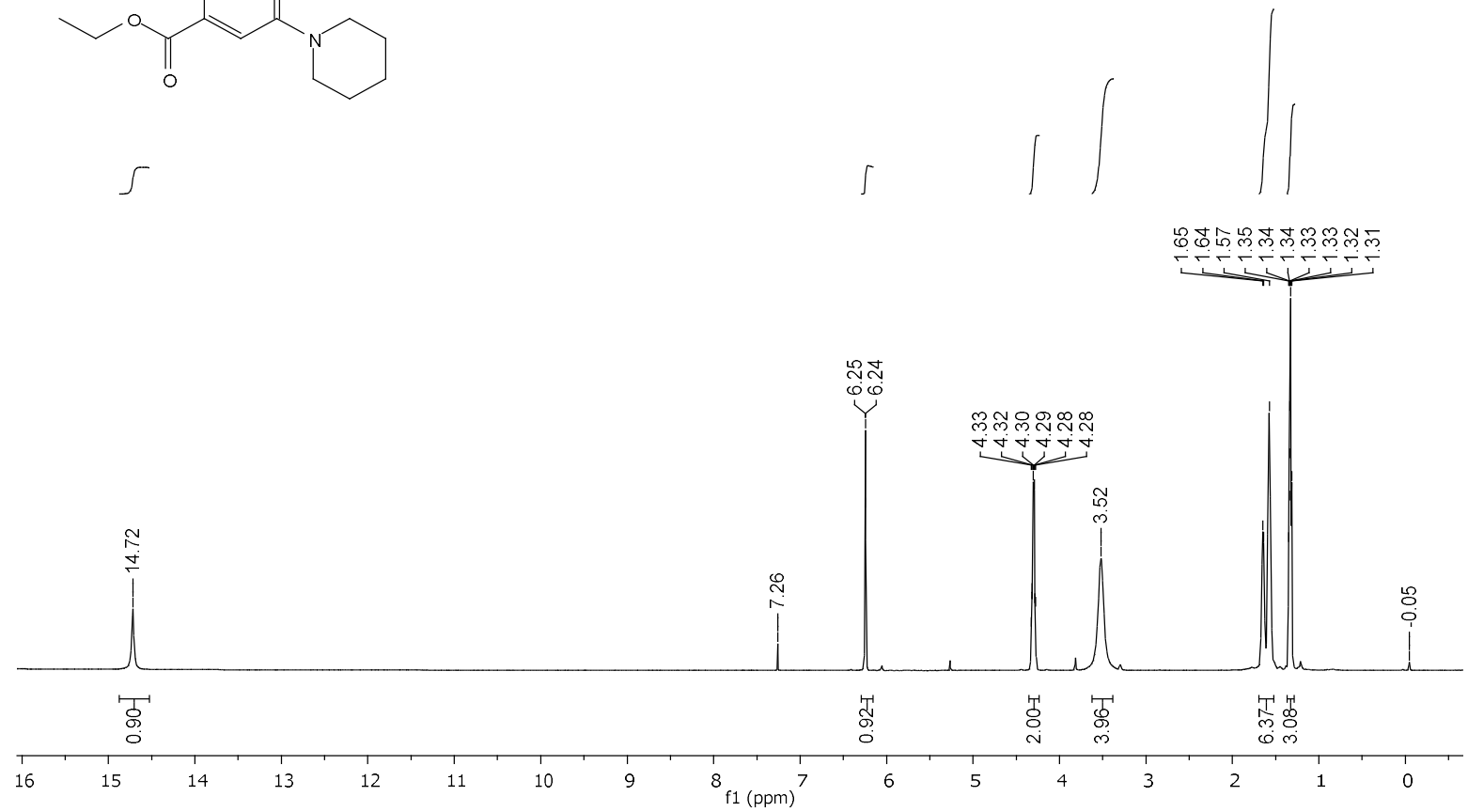

${ }^{13} \mathrm{C}$ NMR (126 MHz, $\left.\mathrm{CDCl}_{3}\right)$.

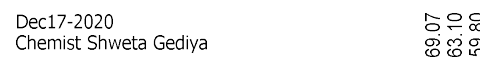

Chemist Shweta Gediyc

C13APT.w CDCl3 /opt/topspin3.2 SKG 54<smiles>CCOC(=O)/C(Cl)=C/C(=O)N1CCCCC1</smiles>

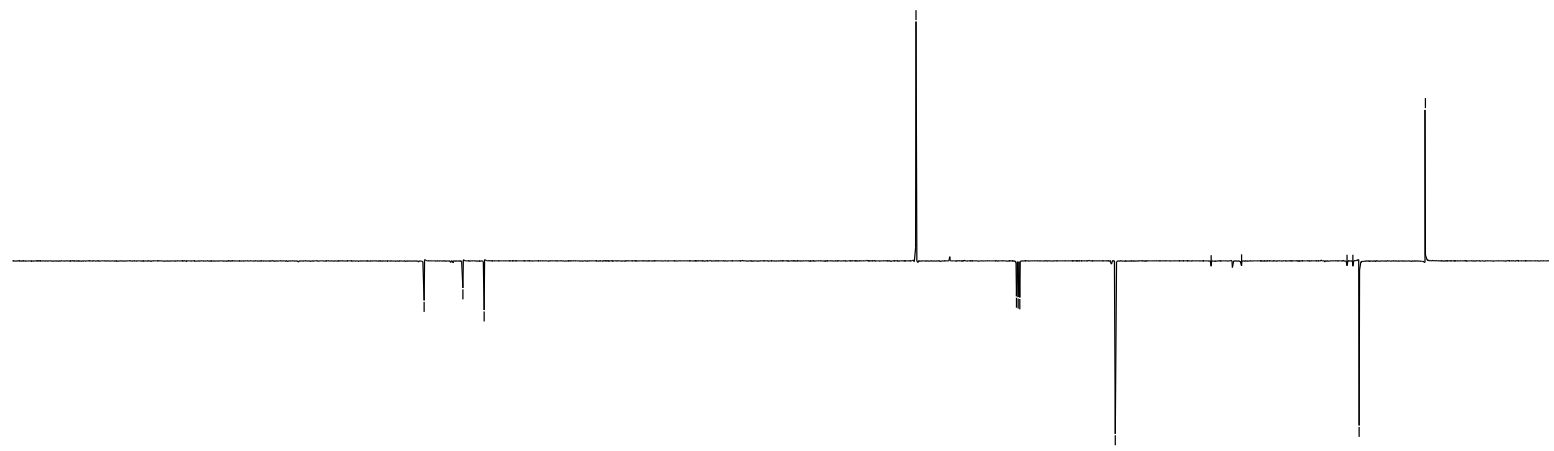

$\begin{array}{lllllllllllllllllllllllll}230 & 220 & 210 & 200 & 190 & 180 & 170 & 160 & 150 & 140 & 130 & 120 & 110 & 100 & 90 & 80 & 70 & 60 & 50 & 40 & 30 & 20 & 10 & 0\end{array}$ 
COSY.

\section{Chemist Shweta Gediya}

COSY.w CDCl3/opt/topspin3.2 SKG 54

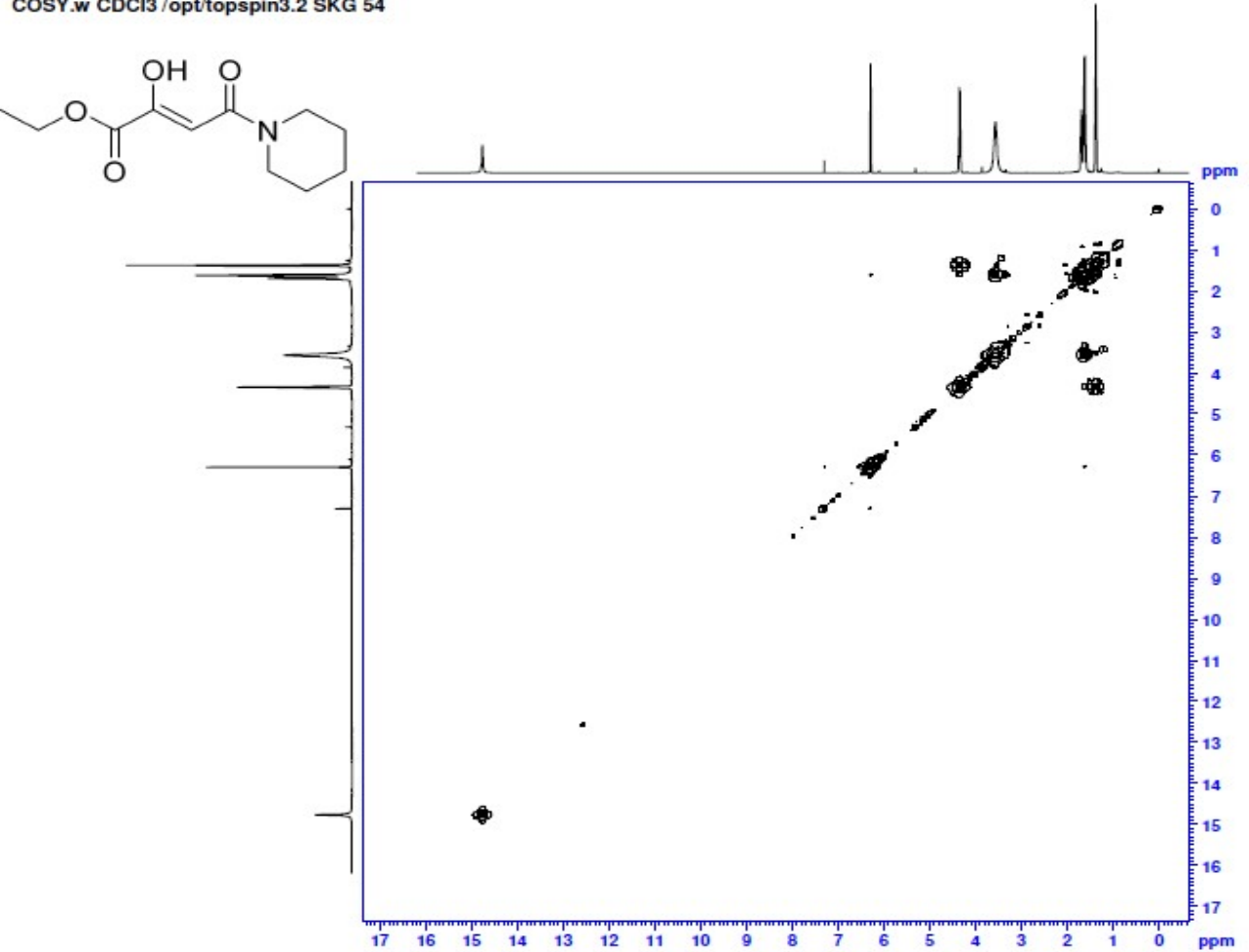

HSQC.

Chemist Shweta Gediya

HSQC.w CDCl3 /opt/topspin3.2 SKG 54

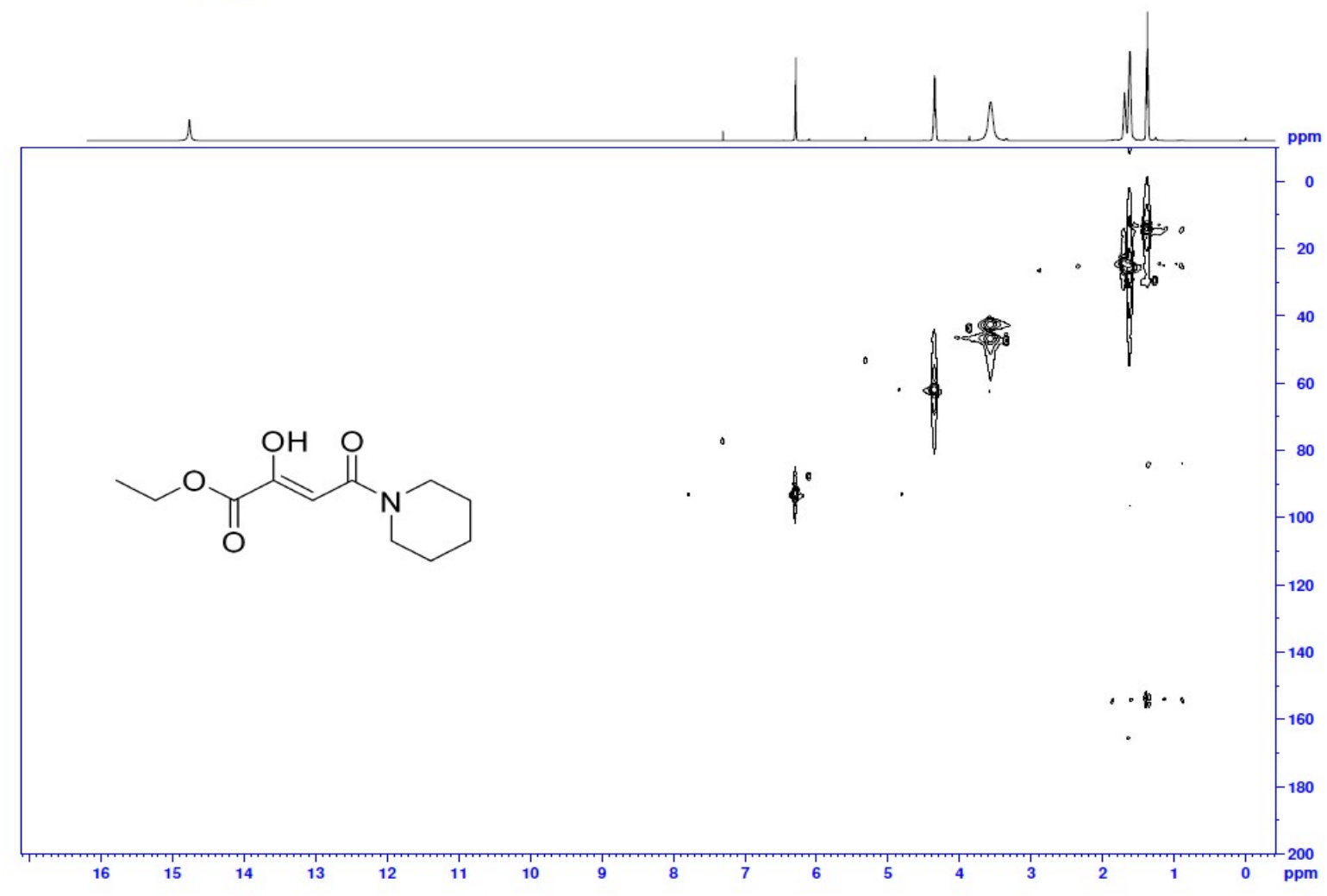


Ethyl 4-((2,6-diisopropylphenyl)amino)-3,4-dioxobutanoate (precursor of 33).<smiles>CCOC(=O)CC(=O)Nc1c(C(C)C)cccc1C(C)C</smiles>

This compound is novel and was prepared following general procedure B using methyl 2((2,6-diisopropylphenyl)amino)-2-oxoacetate $(0.250 \mathrm{~g}, 0.951 \mathrm{mmol}, 1.0 \mathrm{eq})$ in anhydrous THF (6 mL), ethyl acetate $(0.200 \mathrm{~g}, 2.28 \mathrm{mmol}, 2.0 \mathrm{eq})$ in anhydrous THF (2 mL) and LDA (1M solution in THF) $(2.85 \mathrm{~mL}, 2.85 \mathrm{mmol}, 3.0 \mathrm{eq})$ to give crude material which was further purified by column chromatography (5-10\% EtOAc in petroleum ether) to afford the product as a white solid $(0.150 \mathrm{~g}, 0.471 \mathrm{mmol}, 50 \%)$. The title compound was obtained as keto/enol tautomer (1:1) as per ${ }^{1} \mathrm{H}$ NMR.

TLC: $\mathrm{R}_{\mathrm{f}}$ ca 0.5 (8:2, hexane: EtOAc), UV-active and strong $\mathrm{KMnO}_{4}$;

MP: $125-128^{\circ} \mathrm{C}$;

HRMS (ESI) m/z: $[\mathrm{M}+\mathrm{Na}]^{+}$Calcd for $\mathrm{C}_{18} \mathrm{H}_{25} \mathrm{NO}_{4} \mathrm{Na}$ 342.1676; Found 342.1666 (error 2.8 ppm);

$U_{\max } 3261,2962,1752,1727,1644,1511,1327,1209,1137,1038,738 \mathrm{~cm}^{-1}$;

${ }^{1} \mathrm{H}$ NMR (500 MHz, $\mathrm{CDCl}_{3}$ ) (keto/enol, 1:1) $\delta 12.16(0.5 \mathrm{H}, \mathrm{s}$, br.s, $\mathrm{OH}$ of enol form), 8.07 (0.5H, s, CONH), 7.98 (0.5H, s, CONH), 7.25-7.19 (1H, m, ArH), 7.14-7.12 (2H, m, ArH), $6.13(0.5 \mathrm{H}, \mathrm{s},=\mathrm{C} \underline{\mathrm{H}}$ of enol form $), 4.23\left(1 \mathrm{H}, \mathrm{q}, J=7.0 \mathrm{~Hz}, \mathrm{OCH}_{2} \mathrm{CH}_{3}\right), 4.16(1 \mathrm{H}, \mathrm{q}, J=7.0$ $\left.\mathrm{Hz}, \mathrm{OCH}_{2} \mathrm{CH}_{3}\right), 3.91$ (1H, s, $\mathrm{CH}_{2}$ of keto form), 3.00-2.86 (2H, m, $\mathrm{CH}$ of isopropyl group), $1.27\left(1.5 \mathrm{H}, \mathrm{t}, J=7.1 \mathrm{~Hz}, \mathrm{OCH}_{2} \underline{\mathrm{CH}}_{3}\right), 1.22\left(1.5 \mathrm{H}, \mathrm{t}, J=7.1 \mathrm{~Hz}, \mathrm{OCH}_{2} \underline{\mathrm{CH}}_{3}\right), 1.14-1.11(12 \mathrm{H}$, $\mathrm{m}, \mathrm{CH}_{3}$ of isopropyl group);

${ }^{13} \mathrm{C}$ NMR (126 MHz, $\mathrm{CDCl}_{3}$ ) (keto/enol, 1:1) $\delta 192.3$ (C=O keto), 172.7, 166.8, 161.6, 160.2, 158.8, 146.2, 146.0, 129.9, 129.3, 129.1, 128.9, 123.9, 123.8, 94.7 ( $\mathrm{C}=\underline{\mathrm{C}} \mathrm{H}$, enol), 61.7, 61.4, $43.2\left(\underline{\mathrm{CH}}_{2}\right.$, keto), 29.1, 28.9, 23.8, 23.7, 14.3, 14.2;

$m / z(\mathrm{ESI}) 342.2\left[(\mathrm{M}+\mathrm{Na})^{+}, 100 \%\right]$. 
Ethyl 4-((2,6-diisopropylphenyl)amino)-3,4-dioxobutanoate.

${ }^{1} \mathrm{H}$ NMR (500 MHz, $\left.\mathrm{CDCl}_{3}\right)$.

Jan11-2021

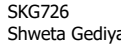

C13APTlong.w.CDCl3

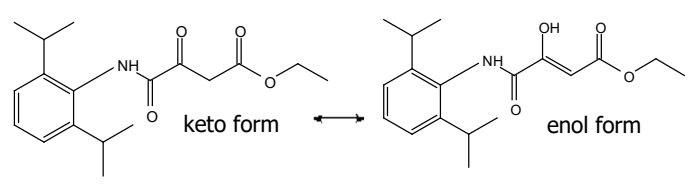

se $d \int^{2}$

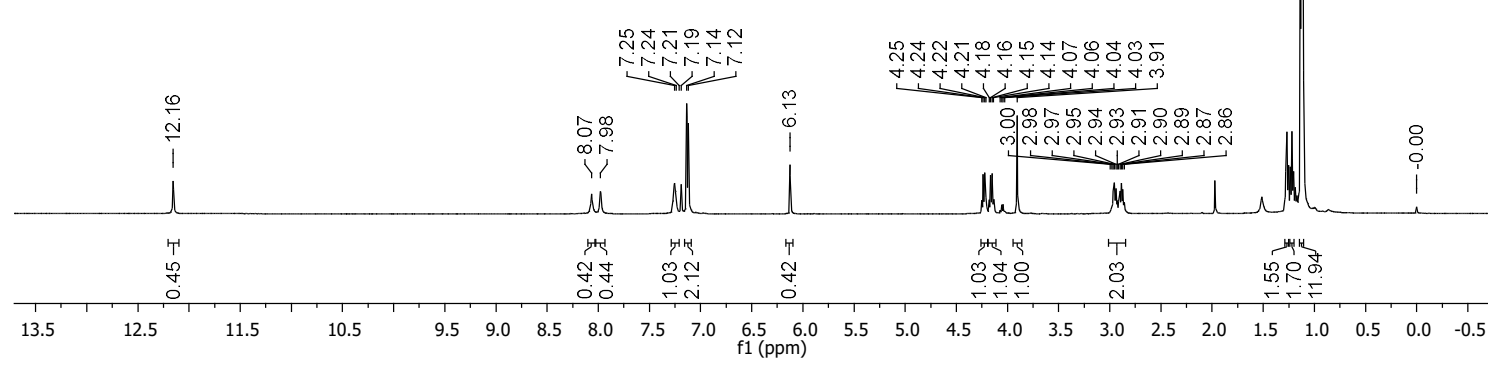

${ }^{13} \mathrm{C}$ NMR (126 MHz, $\left.\mathrm{CDCl}_{3}\right)$.
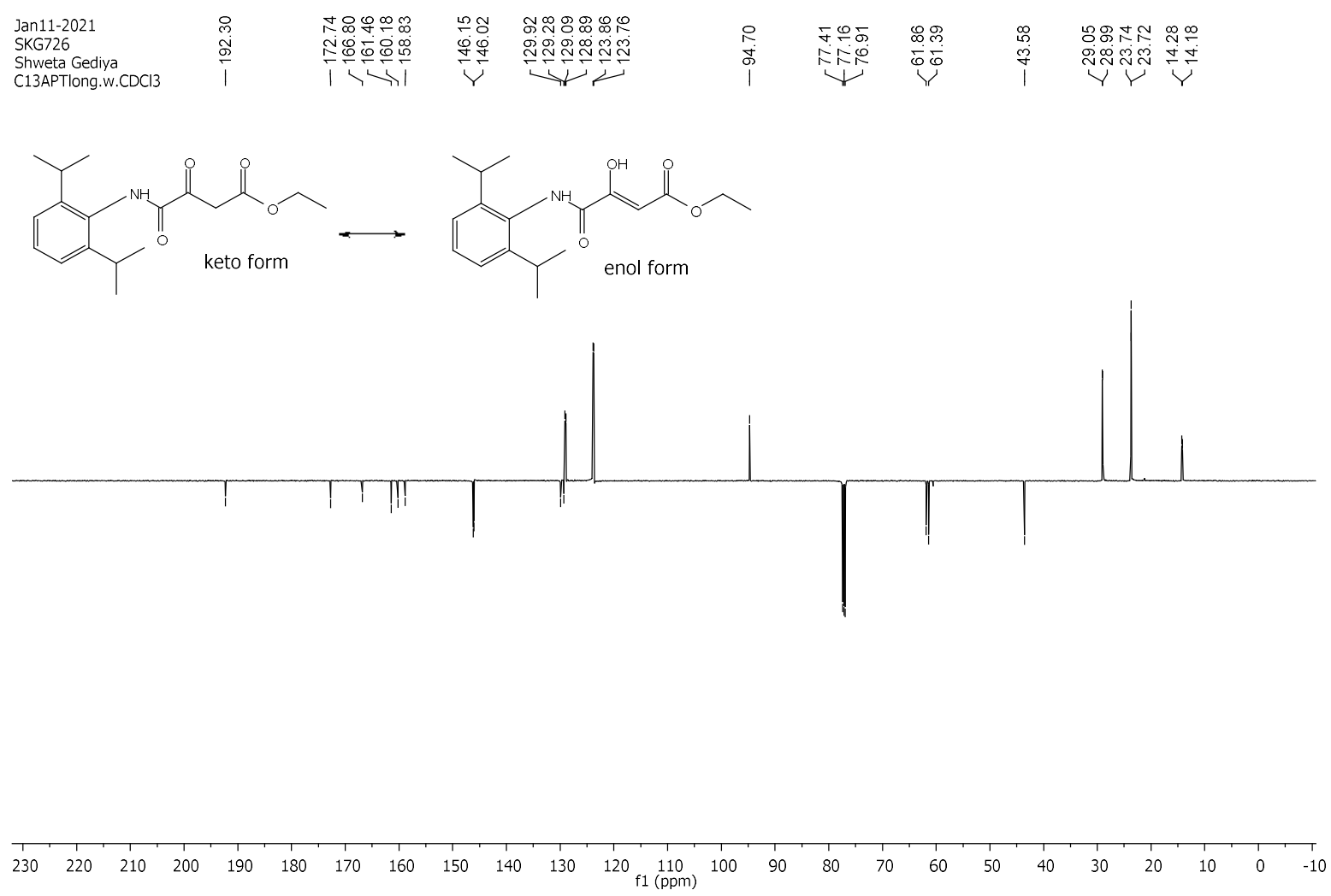

S143 
COSY.

Chemist Shweta Gediya

SKG726
COSY.w CDCl3/opt/topspin3.2 SKG 15

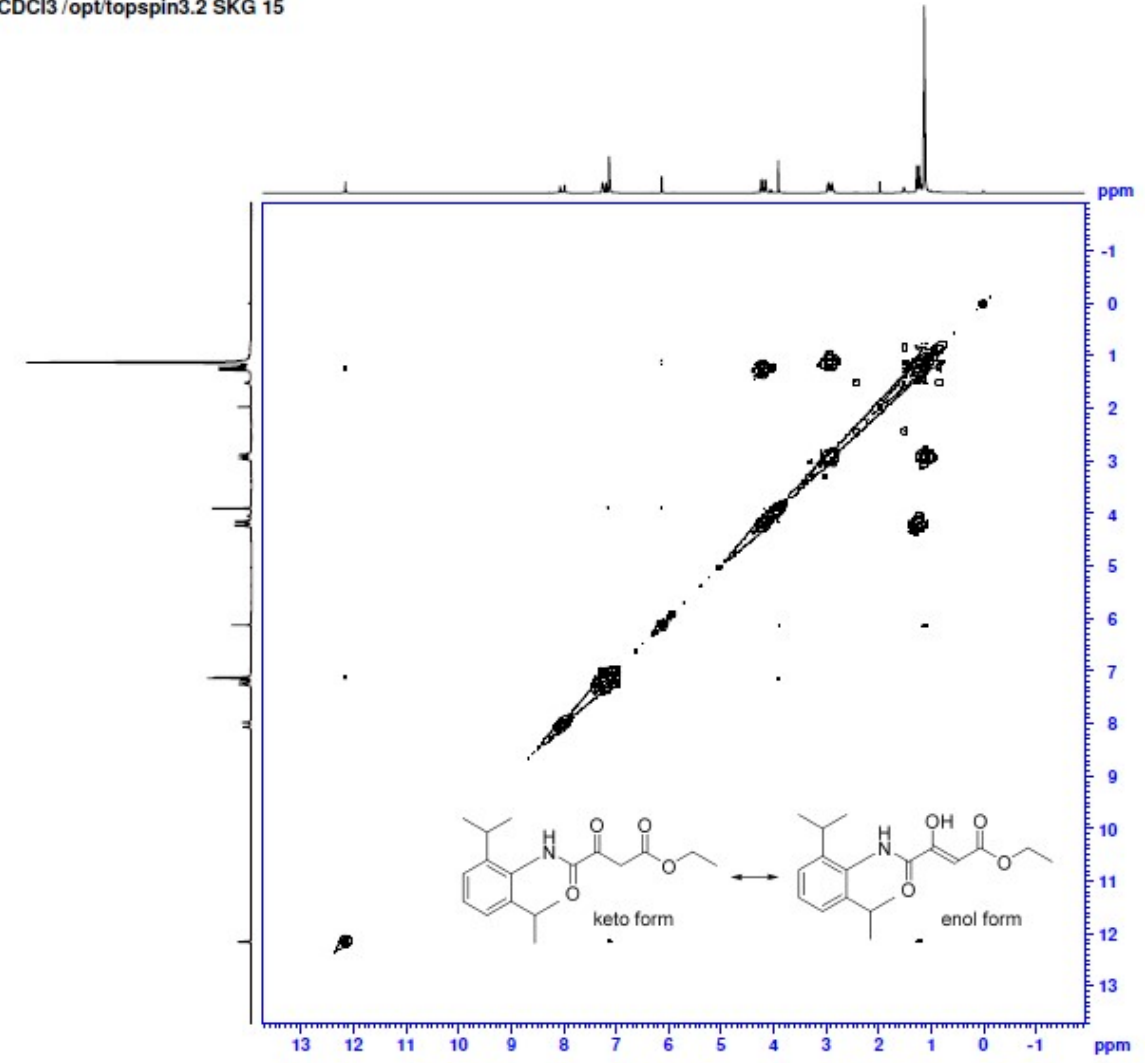

HSQC.

Chemist Shweta Gediya

HSOC.w CDCl3 /opttopspin3.2 SKG 15

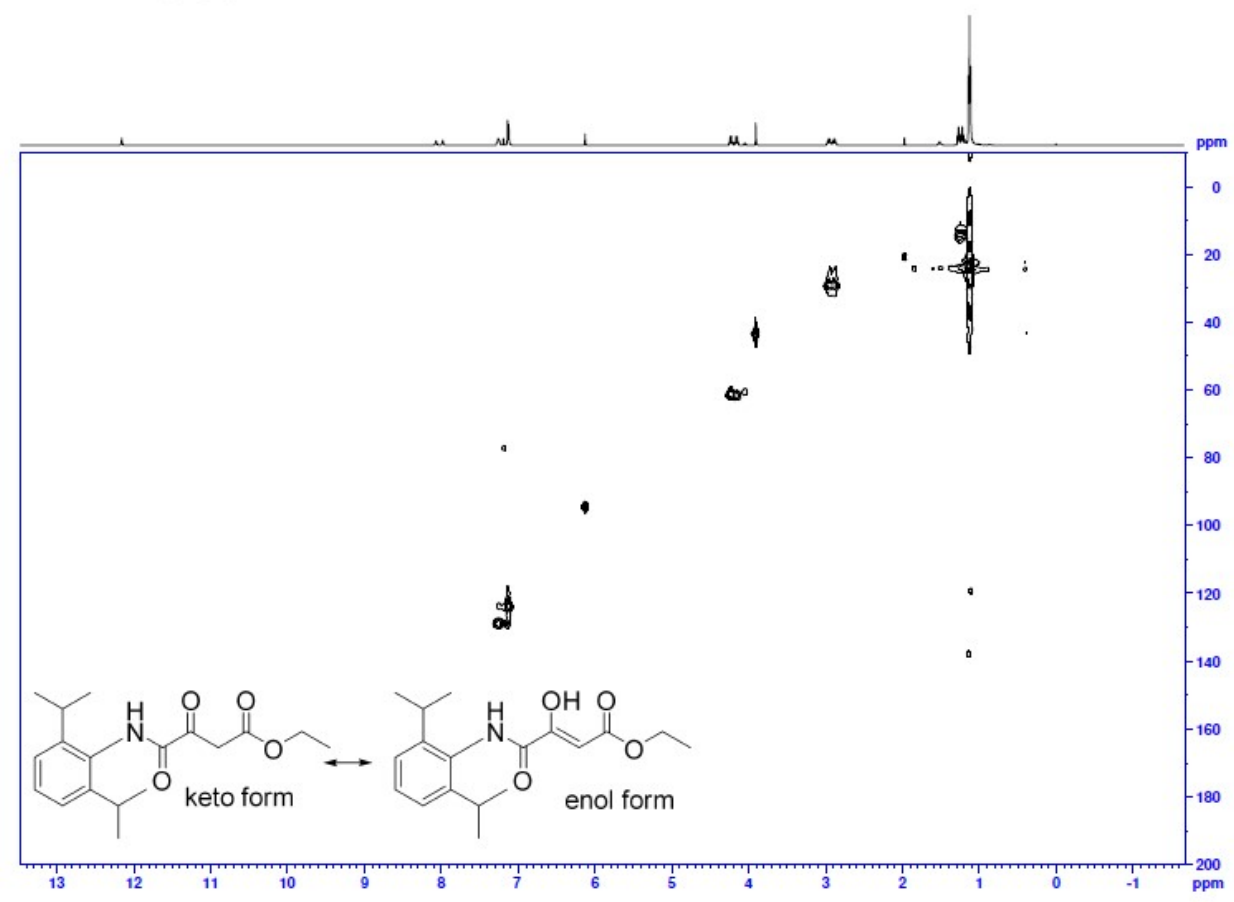


Synthesis of ethyl 5-((2,6-diisopropylphenyl)amino)-4,5-dioxopentanoate (precursor of 34).

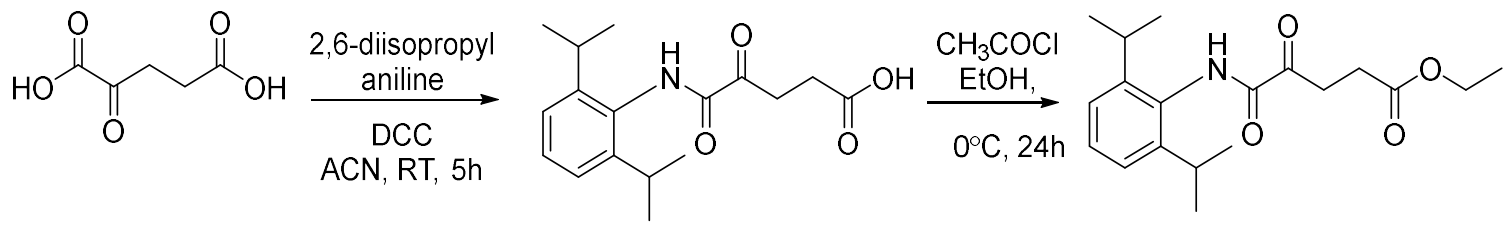

5-((2,6-Diisopropylphenyl)amino)-4,5-dioxopentanoic acid.<smiles>CC(C)c1cccc(C(C)C)c1NC(=O)C(=O)CCC(=O)O</smiles>

This compound is novel and was prepared using following procedure. In a $100 \mathrm{~mL}$ RBF was dissolved 2-oxopentanedioic acid $(0.730 \mathrm{~g}, 5.00 \mathrm{mmol}, 1.0 \mathrm{eq})$ in acetonitrile $(10 \mathrm{~mL})$ followed by addition of N,N'-dicyclohexylcarbodiimide (DCC) (1.03 g, $5.00 \mathrm{mmol}, 1.0 \mathrm{eq})$. Upon addition of DCC, the reaction mixture became pink coloured, and it was stirred at room temperature for 10 minutes. 2,6-Diisopropylaniline $(0.885 \mathrm{~g}, 5.00 \mathrm{mmol}, 1.0 \mathrm{eq})$ was added and colour changed to dark grey and the reaction was stirred at room temperature for $5 \mathrm{~h}$. The dark blue coloured reaction mixture was filtered off and filtrate was diluted with ethyl acetate $(30 \mathrm{~mL})$ and washed with saturated aqueous $\mathrm{NaHCO}_{3}$ solution $(2 \times 10 \mathrm{~mL})$ and the aqueous layer was collected. The aqueous layer was acidified with $2 \mathrm{~N} \mathrm{HCl}$ to adjust $\mathrm{pH} \sim 3$ and it was extracted with ethyl acetate $(3 \times 20 \mathrm{~mL})$. The combined organic layer was washed with water $(20 \mathrm{~mL})$ followed by washing with brine $(50 \mathrm{~mL})$. The organic layer was dried over $\mathrm{MgSO}_{4}$, filtered and the filtrate was concentrated to give crude material which was further purified by column chromatography $(2.5-5 \% \mathrm{MeOH}$ in DCM) to afford the product as a yellow solid (0.352 g, $1.15 \mathrm{mmol}, 23 \%)$.

TLC: $\mathrm{R}_{\mathrm{f}}$ ca 0.3 (9.5:0.5, $\left.\mathrm{CHCl}_{3}: \mathrm{MeOH}\right), \mathrm{UV}$-active and strong $\mathrm{KMnO}_{4}$;

MP: $127-130{ }^{\circ} \mathrm{C}$;

HRMS (ESI) m/z: $[\mathrm{M}+\mathrm{Na}]^{+}$Calcd for $\mathrm{C}_{17} \mathrm{H}_{23} \mathrm{NO}_{4} \mathrm{Na}$ 328.1519; Found 328.1519 (error 0.1 ppm);

$U_{\max } 3254,2968,2928,1713,1674,1508,1361,1254,1079,917 \mathrm{~cm}^{-1}$;

${ }^{1} \mathrm{H}$ NMR (400 MHz, $\left.\mathrm{CDCl}_{3}\right) \delta 9.43(1 \mathrm{H}$, br.s, $\mathrm{COOH}), 8.21(1 \mathrm{H}, \mathrm{s}, \mathrm{CONH}), 7.33(1 \mathrm{H}, \mathrm{t}, J=$ $7.8 \mathrm{~Hz}, \mathrm{ArH}), 7.20(2 \mathrm{H}, \mathrm{d}, J=7.8 \mathrm{~Hz}, \mathrm{ArH}), 3.32\left(2 \mathrm{H}, \mathrm{t}, J=6.4 \mathrm{~Hz}, \mathrm{CH}_{2}\right), 2.99-2.88(2 \mathrm{H}, \mathrm{m}$, 
$\mathrm{CH}$ of isopropyl group), $2.79\left(2 \mathrm{H}, \mathrm{t}, J=6.4 \mathrm{~Hz}, \mathrm{CH}_{2}\right), 1.18\left(12 \mathrm{H}, J=6.8 \mathrm{~Hz}, \mathrm{CH}_{3}\right.$ of isopropyl group);

${ }^{13} \mathrm{C}$ NMR $\left(101 \mathrm{MHz}, \mathrm{CDCl}_{3}\right) \delta 197.7,178.0,159.3,146.0,129.4,129.0,123.8,31.9,29.0$, 27.9, 23.7;

$m / z(\mathrm{ESI}) 328.1\left[(\mathrm{M}+\mathrm{Na})^{+}, 100 \%\right]$. 
5-((2,6-Diisopropylphenyl)amino)-4,5-dioxopentanoic acid.

${ }^{1} \mathrm{H}$ NMR $\left(400 \mathrm{MHz}, \mathrm{CDCl}_{3}\right)$.
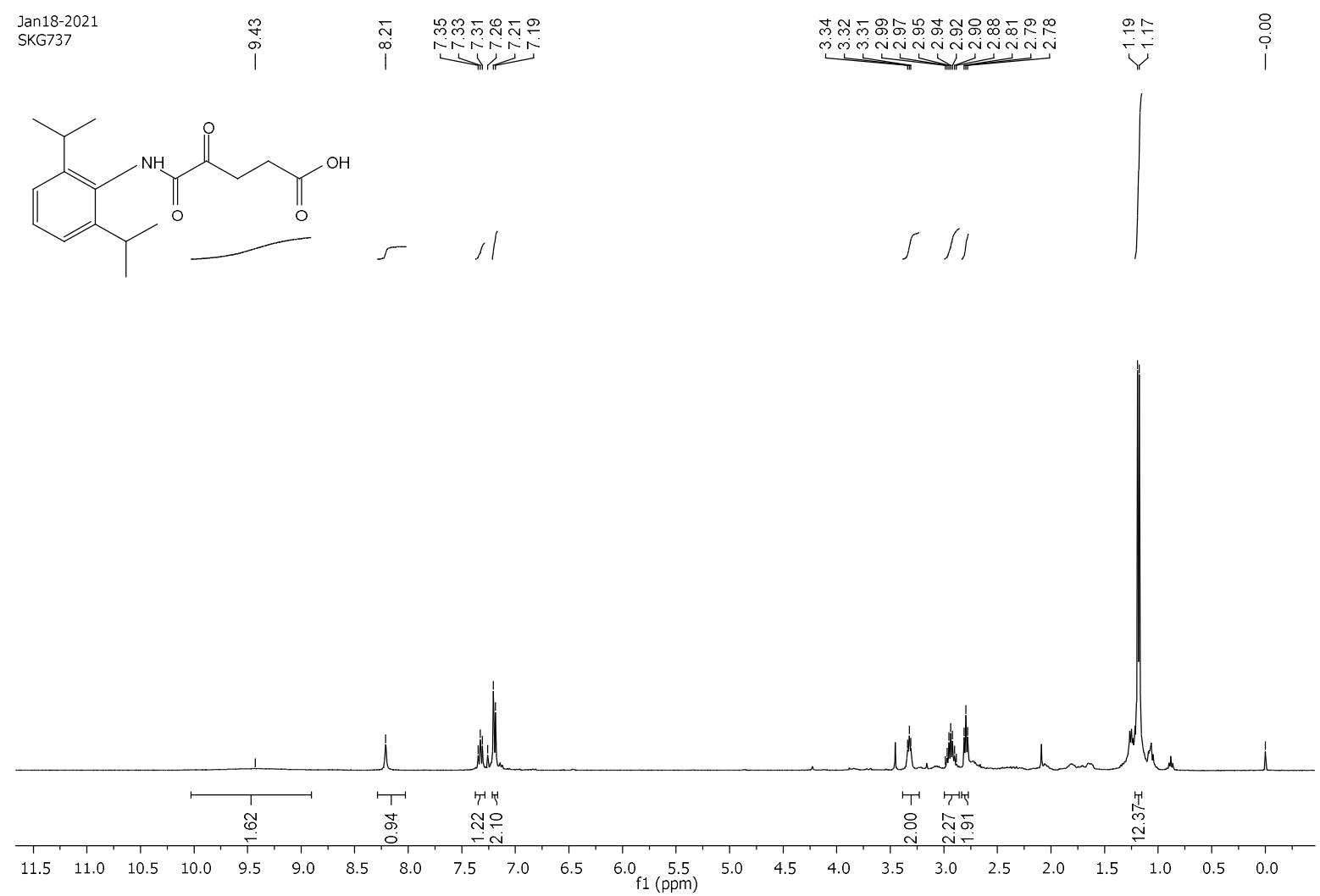

${ }^{13} \mathrm{C}$ NMR (101 MHz, $\left.\mathrm{CDCl}_{3}\right)$

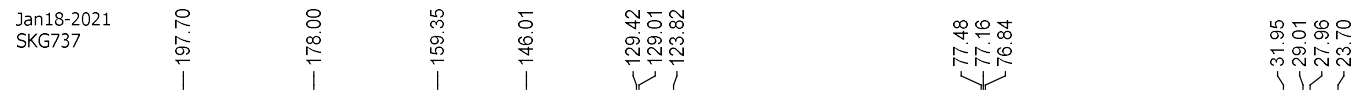<smiles>CC(C)c1cccc(C(C)C)c1NC(=O)C(=O)CCC(=O)O</smiles>
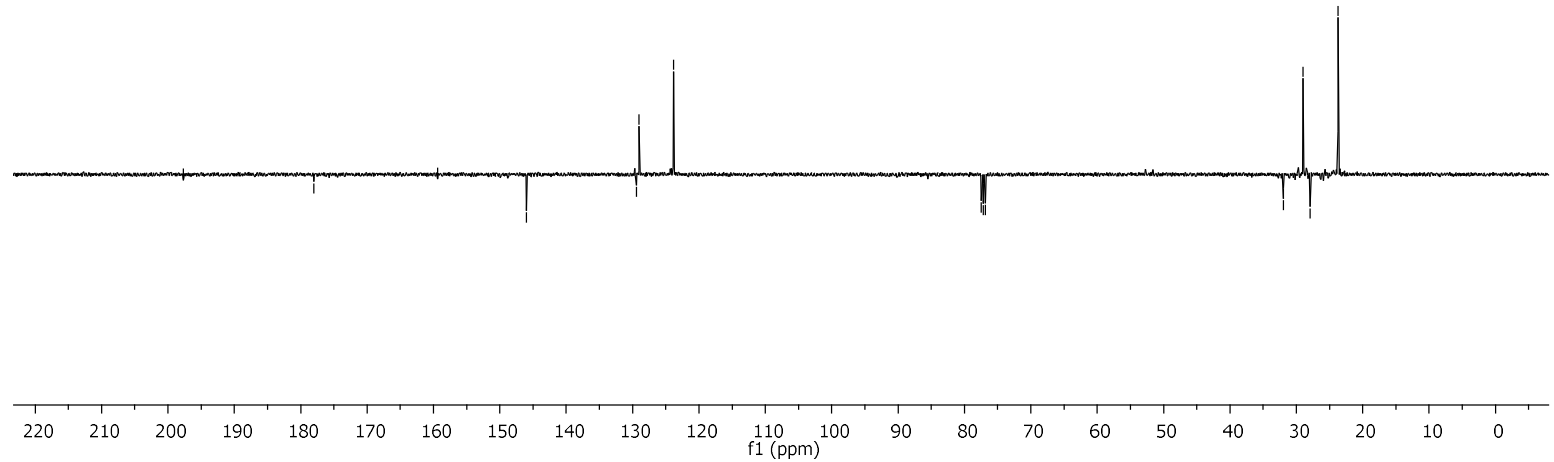
COSY.

SKG737

COSY.w CDCl3 /opt/topspin3.5pl2 SKG 46

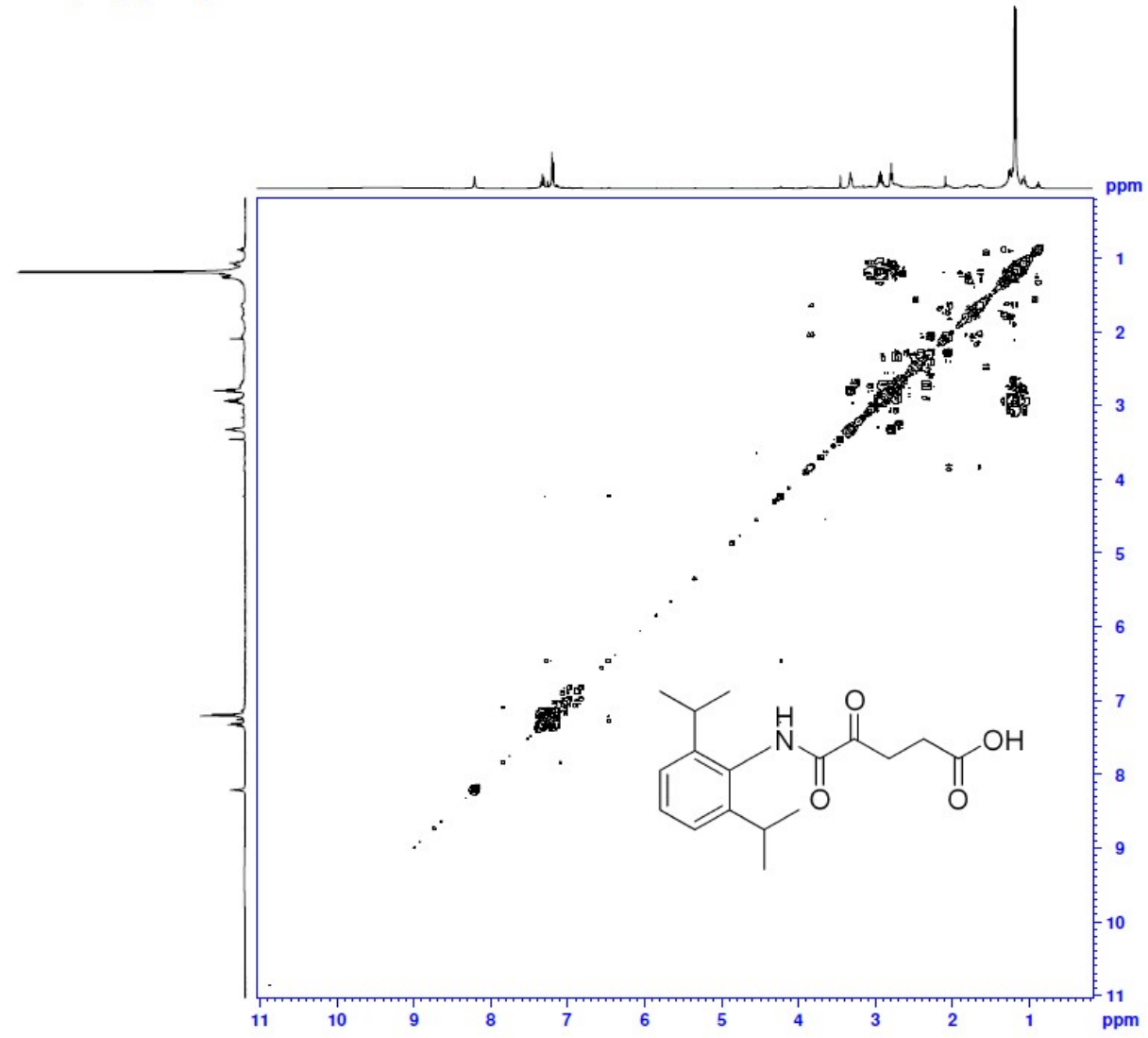


Ethyl 5-((2,6-diisopropylphenyl)amino)-4,5-dioxopentanoate.<smiles>CCOC(=O)CCC(=O)C(=O)Nc1c(C)cccc1C(C)C</smiles>

This compound is novel and was prepared using the following procedure; In a $50 \mathrm{~mL}$ RBF was dissolved 5-((2,6-diisopropylphenyl)amino)-4,5-dioxopentanoic acid (0.305 g, 1.00 mmol, $1.0 \mathrm{eq})$ in ethanol $(10 \mathrm{~mL})$ and the solution was cooled to $0{ }^{\circ} \mathrm{C}$, followed by dropwise addition of acetyl chloride $(0.117 \mathrm{~g}, 1.50 \mathrm{mmol}, 1.5 \mathrm{eq}$.$) . The resulting reaction mixture was$ stirred at room temperature for $24 \mathrm{~h}$. Once the reaction was complete, the reaction mixture was diluted with water $(30 \mathrm{~mL})$ and extracted with ethyl acetate $(2 \times 15 \mathrm{~mL})$. The combined organic layer was washed with saturated aqueous $\mathrm{NaHCO}_{3}$ solution $(2 \times 10 \mathrm{~mL})$, water $(20$ $\mathrm{mL})$ and brine $(50 \mathrm{~mL})$. The organic layer was dried over $\mathrm{MgSO}_{4}$, filtered and filtrate was concentrated to give crude material which was further purified by column chromatography (30-50\% EtOAc in petroleum ether) to afford the product as a yellow oil $(0.173 \mathrm{~g}, 0.519$ mmol, 52\%).

TLC: $\mathrm{R}_{\mathrm{f}}$ ca 0.4 (5:5, hexane: EtOAc), UV-active and strong $\mathrm{KMnO}_{4}$;

HRMS (ESI) m/z: $[\mathrm{M}+\mathrm{Na}]^{+}$Calcd for $\mathrm{C}_{19} \mathrm{H}_{27} \mathrm{NO}_{4} \mathrm{Na}$ 356.1832; Found 356.1831 (error 0.4 ppm);

$U_{\max } 3244,2961,1736,1672,1505,1336,1194,1036,917,743 \mathrm{~cm}^{-1}$;

${ }^{1} \mathrm{H}$ NMR $\left(500 \mathrm{MHz}, \mathrm{CDCl}_{3}\right) \delta 8.19(1 \mathrm{H}, \mathrm{s}, \mathrm{CONH}), 7.32(1 \mathrm{H}, \mathrm{t}, J=7.8 \mathrm{~Hz}, \mathrm{ArH}), 7.20(2 \mathrm{H}$, d, $J=7.8 \mathrm{~Hz}, \mathrm{ArH}), 4.18\left(2 \mathrm{H}, \mathrm{q}, J=7.1 \mathrm{~Hz}, \mathrm{CH}_{2} \mathrm{CH}_{3}\right), 3.33\left(2 \mathrm{H}, \mathrm{t}, J=6.5 \mathrm{~Hz}, \mathrm{CH}_{2}\right), 2.98-$ $2.93\left(2 \mathrm{H}, \mathrm{m}, \mathrm{CH}\right.$ of isopropyl group), $2.73\left(2 \mathrm{H}, \mathrm{t}, J=6.5 \mathrm{~Hz}, \mathrm{CH}_{2}\right), 1.30-1.27(3 \mathrm{H}, \mathrm{m}$, $\left.\mathrm{CH}_{2} \underline{\mathrm{C}}_{3}\right), 1.19\left(12 \mathrm{H}, \mathrm{d}, J=6.9 \mathrm{~Hz}, \mathrm{CH}_{3}\right.$ of isopropyl group);

${ }^{13} \mathrm{C}$ NMR $\left(126 \mathrm{MHz}, \mathrm{CDCl}_{3}\right) \delta 197.7,172.3,168.4,159.3,146.0,129.5,128.9,123.8,60.9$, $32.4,28.9,28.1,23.7,14.3$;

$m / z(\mathrm{ESI}) 356.2\left[(\mathrm{M}+\mathrm{Na})^{+}, 100 \%\right]$. 
Ethyl 5-((2,6-diisopropylphenyl)amino)-4,5-dioxopentanoate.

${ }^{1} \mathrm{H}$ NMR $\left(500 \mathrm{MHz}, \mathrm{CDCl}_{3}\right)$.

Jan21-2021

Chemist Shweta Gediya

PROTON.w CDCl3 /opt/topspin3.2 SKG 43

i

$\underbrace{(1}_{1}$

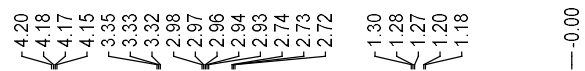

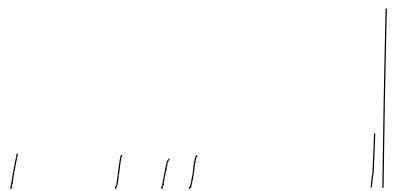

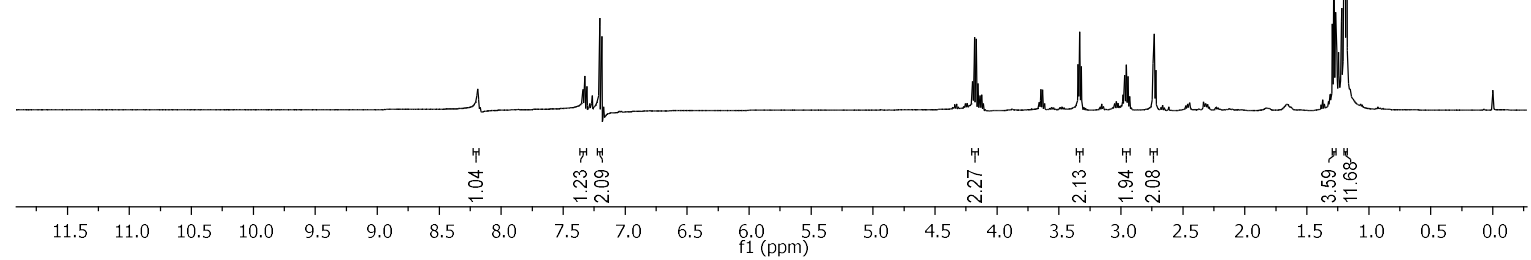

${ }^{13} \mathrm{C}$ NMR $\left(126 \mathrm{MHz}, \mathrm{CDCl}_{3}\right)$.

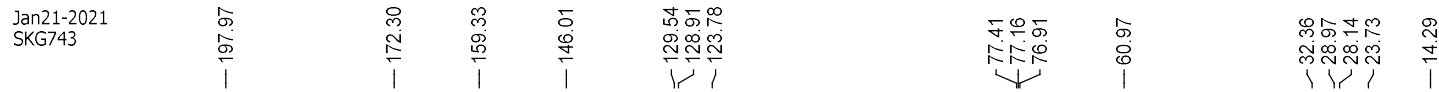<smiles>CCOC(=O)CCC(=O)C(=O)Nc1c(C(C)C)cccc1C(C)C</smiles>

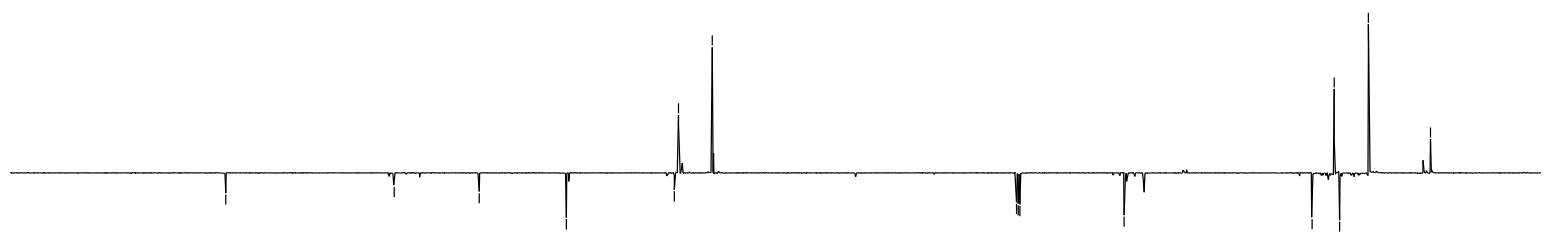

$\begin{array}{lllllllllllllllllllllllllllllllllllll}230 & 220 & 210 & 200 & 190 & 180 & 170 & 160 & 150 & 140 & 130 & 120 & 110 & 100 & 90 & 80 & 70 & 60 & 50 & 40 & 30 & 20 & 10 & 0\end{array}$ 
COSY.

Chemist Shweta Gediya

COSY.w CDCl3 /opt/topspin3.2 SKG 43

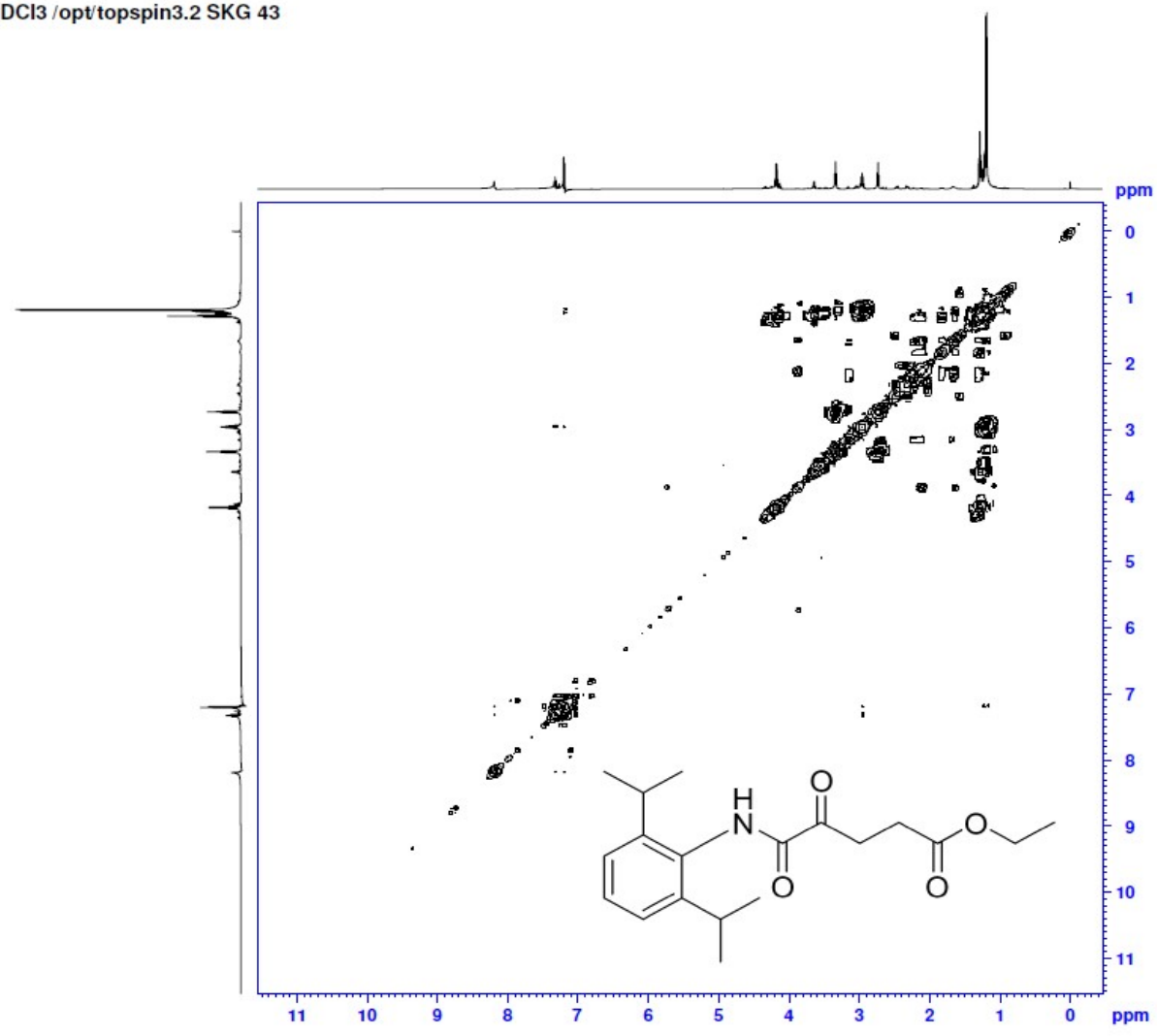


$N$-(2,6-Diisopropylphenyl)-2-oxohexanamide (precursor of 35).<smiles>CCCCC(=O)Nc1c(C(C)C)cccc1C(C)C</smiles>

This compound is known and was synthesised following similar procedure used for synthesis of $N$-methoxy- $N$-methyl-2-oxohexanamide using magnesium turnings $(0.200 \mathrm{~g}, 8.23 \mathrm{mmol}$, 1.1 eq.), n-butyl bromide (0.90 mL, $1.14 \mathrm{mg}, 8.3 \mathrm{mmol}, 1.1 \mathrm{eq})$, methyl 2-((2,6diisopropylphenyl)amino)-2-oxoacetate $(1.89 \mathrm{~g}, 7.21 \mathrm{mmol}, 1.0 \mathrm{eq})$ and THF $(8 \mathrm{~mL})$ to generate crude material which was further purified by column chromatography $(10 \%$ EtOAc in petroleum ether) to afford the product as a yellow solid (1.21 g, $4.18 \mathrm{mmol}, 58 \%)$.

TLC: $\mathrm{R}_{\mathrm{f}}$ ca 0.4 (9:1, hexane: EtOAc), UV-active and strong $\mathrm{KMnO}_{4}$;

MP: $110-112^{\circ} \mathrm{C}$;

HRMS (ESI) m/z: $[\mathrm{M}+\mathrm{Na}]^{+}$Calcd for $\mathrm{C}_{18} \mathrm{H}_{27} \mathrm{NO}_{2} \mathrm{Na}$ 312.1934; Found 312.1928 (error 1.8 ppm);

${ }^{1} \mathrm{H}$ NMR (500 MHz, $\left.\mathrm{CDCl}_{3}\right) \delta 8.22(1 \mathrm{H}, \mathrm{s}, \mathrm{CONH})$, 7.34-7.31 (1H, m, ArH), 7.20-7.19 (2H, $\mathrm{m}, \mathrm{ArH}), 3.03\left(2 \mathrm{H}, \mathrm{t}, J=7.4 \mathrm{~Hz}, \mathrm{COCH}_{2} \mathrm{CH}_{2}\right), 2.97-2.91(2 \mathrm{H}, \mathrm{m}, \mathrm{CH}$ of isopropyl group), 1.71-1.65 (2H, m, $\left.\mathrm{COCH}_{2} \mathrm{C}_{2}\right), 1.44-1.42\left(2 \mathrm{H}, \mathrm{m}, \mathrm{C}_{2} \mathrm{CH}_{3}\right), 1.19\left(12 \mathrm{H}, \mathrm{d}, J=6.9 \mathrm{~Hz}, \mathrm{CH}_{3}\right.$ of isopropyl group), $0.96\left(3 \mathrm{H}, \mathrm{t}, J=7.4 \mathrm{~Hz}, \mathrm{CH}_{2} \mathrm{CH}_{3}\right)$;

${ }^{13} \mathrm{C} \mathrm{NMR}\left(126 \mathrm{MHz}, \mathrm{CDCl}_{3}\right) \delta 199.9,159.6,145.9,129.8,128.9,123.8,36.8,29.0,25.6$, 23.7, 22.4, 13.9;

$m / z(\mathrm{ESI}) 312.2\left[(\mathrm{M}+\mathrm{Na})^{+}, 100 \%\right]$. 
$\mathrm{N}$-(2,6-Diisopropylphenyl)-2-oxohexanamide.

${ }^{1} \mathrm{H}$ NMR (500 MHz, $\left.\mathrm{CDCl}_{3}\right)$.

Nov23-2020
Chemist Shweta Gediya
SKG659
PROTON.w CDC13 /opt/topspin3.2 SKG 40 I

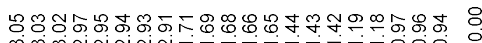

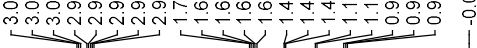

$\underbrace{(1}_{1}$

11

$\iint$
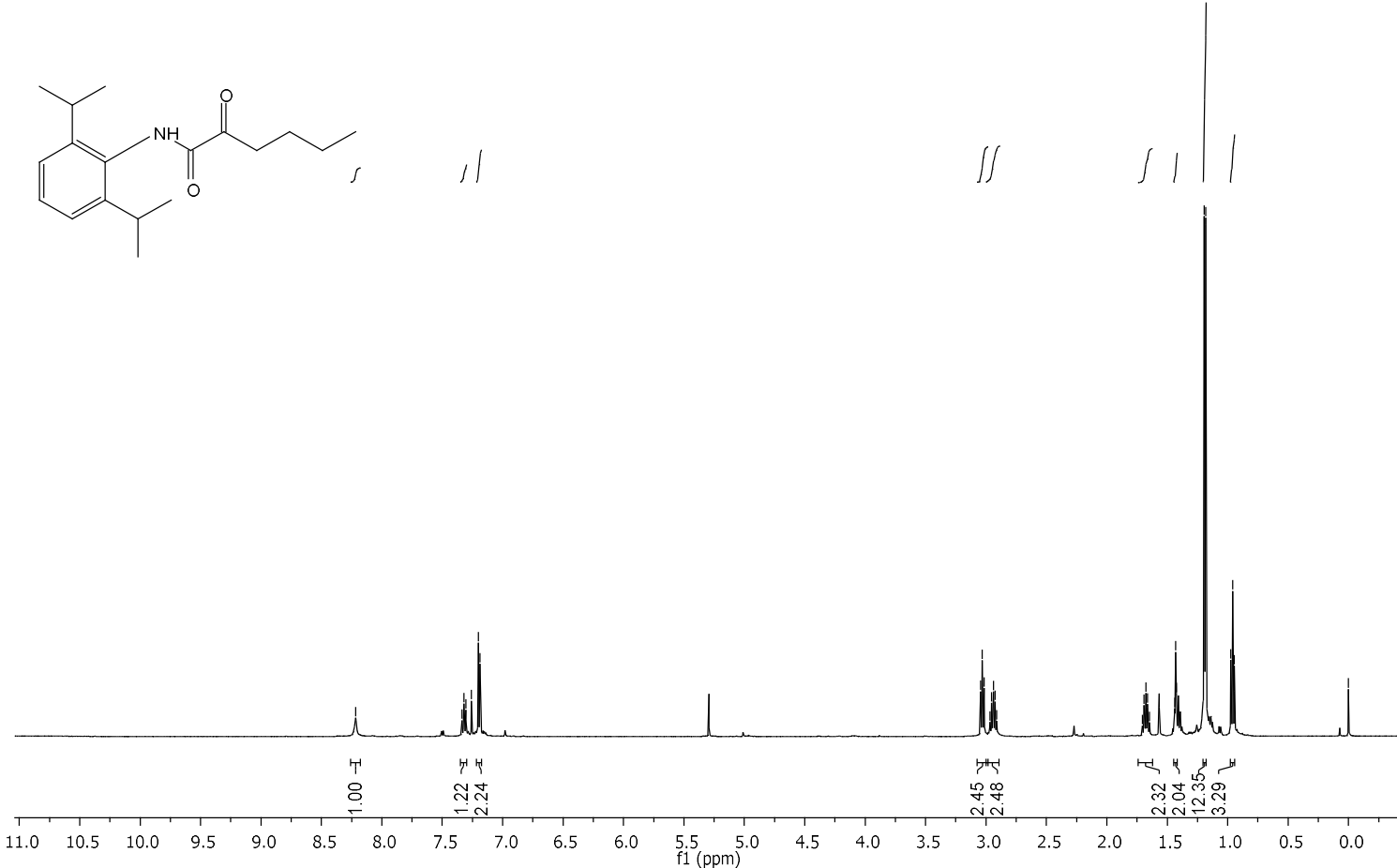

$\left.{ }^{13} \mathrm{C} \mathrm{NMR} \mathrm{(126} \mathrm{MHz,} \mathrm{CDCl}_{3}\right)$.

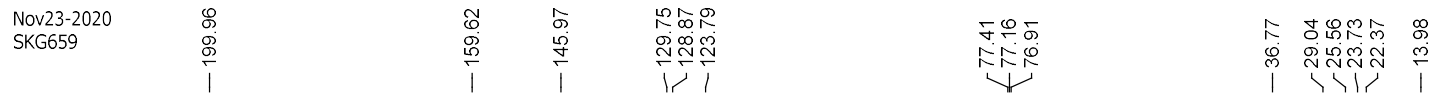<smiles>CCCCC(=O)C(=O)Nc1c(C(C)C)cccc1C(C)C</smiles>

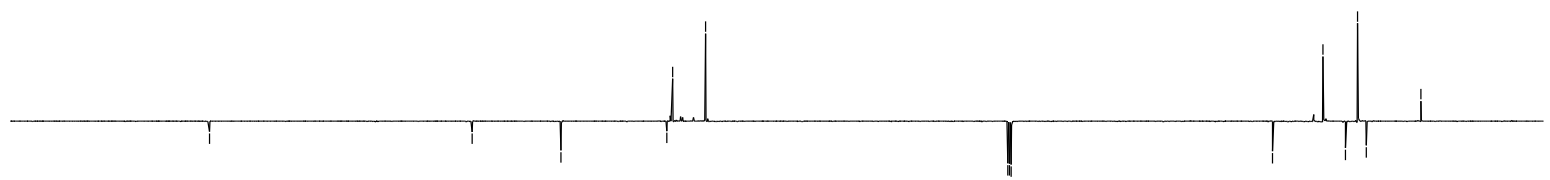

$\begin{array}{rllllllllllllllllllllllllllllllllllllllll}230 & 220 & 210 & 200 & 190 & 180 & 170 & 160 & 150 & 140 & 130 & 120 & 110 & 100 & 90 & 80 & 70 & 60 & 50 & 40 & 30 & 20 & 10 & 0\end{array}$ 
COSY.

Chemist Shweta Gediya

COSY.m CDCI3/opttopspin3 2 SKG 40

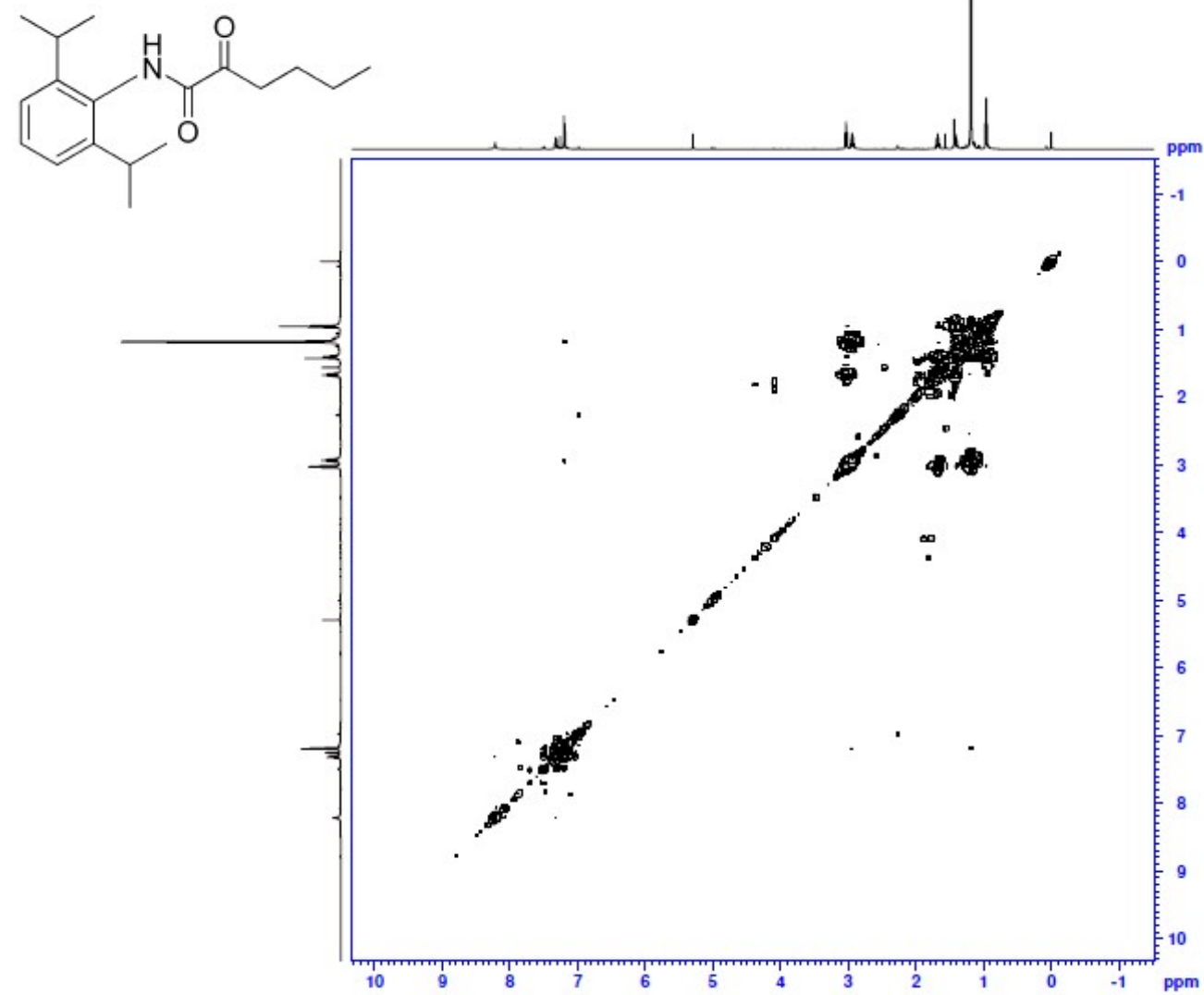


N-(2,6-Diisopropylphenyl)-2-oxo-3-phenylpropanamide (precursor of 36).<smiles>COC(=O)C1=C(C)OC(C)c2cccc(C(C)C)c2N1</smiles>

This compound is novel and was prepared by following the procedure for the previous compound using methyl 2-((2,6-diisopropylphenyl)amino)-2-oxoacetate $(0.538 \mathrm{~g}, 2.00 \mathrm{mmol}$, $1.0 \mathrm{eq})$ in THF (10 mL) and benzyl magnesium bromide (2M in THF) (1.0 mL, $2.00 \mathrm{mmol}$, $1.0 \mathrm{eq})$ to give crude material which was further purified by column chromatography $(5-10 \%$ EtOAc in petroleum ether) to afford the product as a white solid $(0.436 \mathrm{~g}, 1.34 \mathrm{mmol}, 67 \%)$.

TLC: $\mathrm{R}_{\mathrm{f}}$ ca 0.5 (9:1, hexane: EtOAc), UV-active and strong $\mathrm{KMnO}_{4}$;

MP: $105-107^{\circ} \mathrm{C}$;

HRMS (ESI) m/z: $[\mathrm{M}+\mathrm{Na}]^{+}$Calcd for $\mathrm{C}_{21} \mathrm{H}_{25} \mathrm{NO}_{2} \mathrm{Na}$ 346.1777; Found 346.1771 (error 2.0 ppm);

$U_{\max } 3274,2959,1716,1666,1511,1205,1087,925,742,698 \mathrm{~cm}^{-1}$;

${ }^{1} \mathrm{H}$ NMR (500 MHz, $\left.\mathrm{CDCl}_{3}\right) \delta 8.12(1 \mathrm{H}, \mathrm{s}, \mathrm{CONH}), 7.37-7.26(6 \mathrm{H}, \mathrm{m}, \mathrm{ArH}), 7.17(2 \mathrm{H}, \mathrm{d}, J=$ 7.7 Hz, ArH), $4.31\left(2 \mathrm{H}, \mathrm{s}, \mathrm{CH}_{2} \mathrm{Ph}\right), 2.87-2.79(2 \mathrm{H}, \mathrm{m}, \mathrm{CH}$ of isopropyl group), $1.13(12 \mathrm{H}, \mathrm{d}$, $J=6.9 \mathrm{~Hz}, \mathrm{CH}_{3}$ of isopropyl group);

${ }^{13} \mathrm{C} \mathrm{NMR}\left(126 \mathrm{MHz}, \mathrm{CDCl}_{3}\right) \delta 196.5,159.7,145.9,132.4,130.1,129.6,128.9,128.8,127.5$, 123.8, 43.5, 28.9, 23.7;

$m / z(\mathrm{ESI}) 346.2\left[(\mathrm{M}+\mathrm{Na})^{+}, 100 \%\right]$. 
N-(2,6-Diisopropylphenyl)-2-oxo-3-phenylpropanamide.

${ }^{1} \mathrm{H}$ NMR $\left(500 \mathrm{MHz}, \mathrm{CDCl}_{3}\right)$.

Jan08-2021

Chemist Shweta Gediy

PROTON.w CDCl3 /opt/topspin3.2 SKG 13

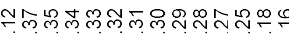

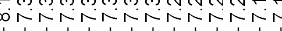

1<smiles>CC(C)c1cccc(C(C)C)c1NC(=O)C(=O)Cc1ccccc1</smiles>

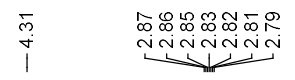

$\begin{array}{ll}\frac{m}{2}= & 8 \\ & 0\end{array}$

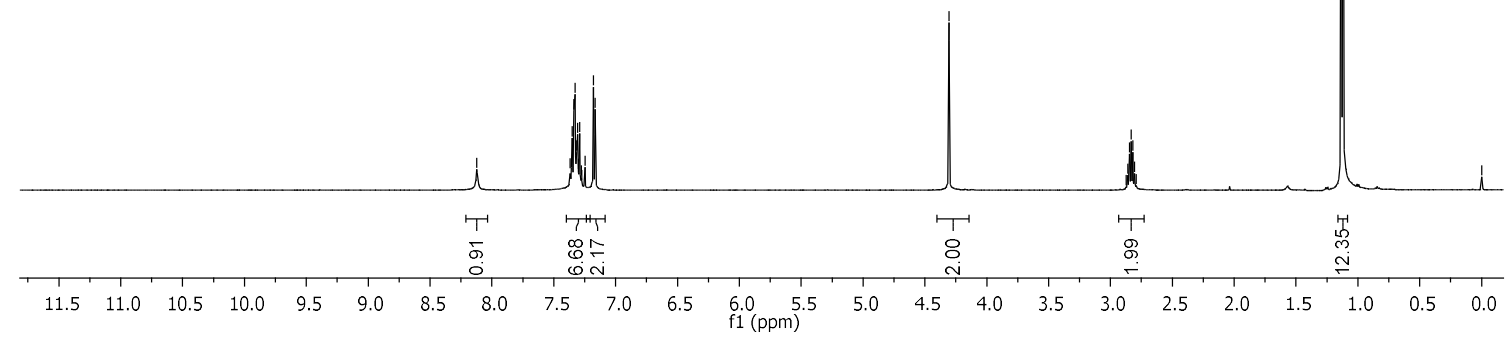

${ }^{13} \mathrm{C}$ NMR (126 MHz, $\left.\mathrm{CDCl}_{3}\right)$.

Jan08-2021
SKG721

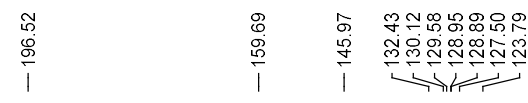

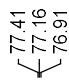

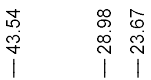<smiles>CC(C)c1cccc(C(C)C)c1NC(=O)C(=O)Cc1ccccc1</smiles>

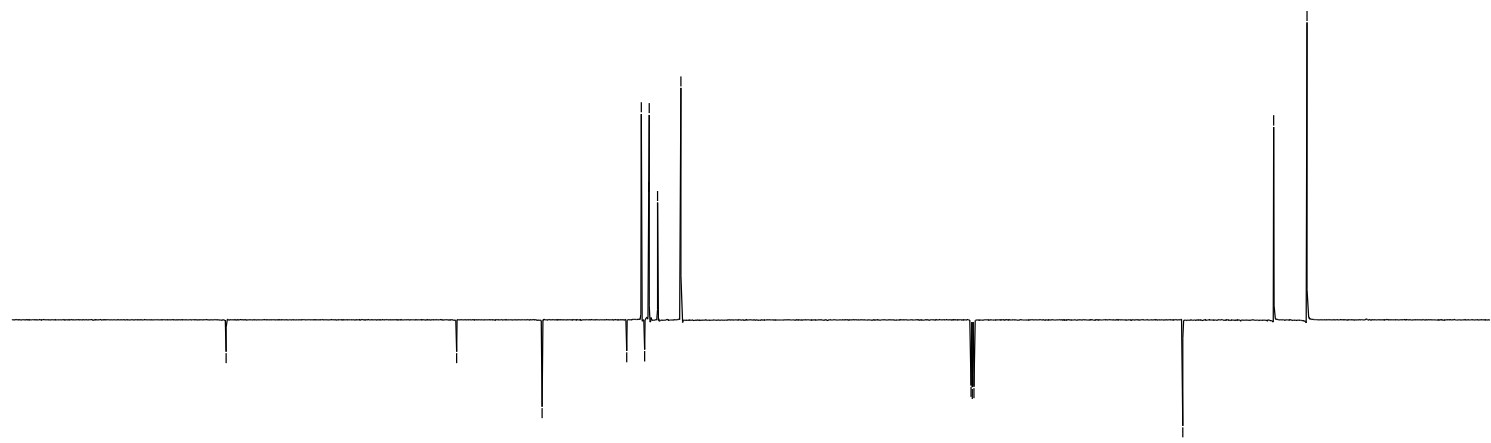

$\begin{array}{rlllllllllllllllllllllllllllllll}230 & 220 & 210 & 200 & 190 & 180 & 170 & 160 & 150 & 140 & 130 & 120 & 110 & 100 & 90 & 80 & 70 & 60 & 50 & 40 & 30 & 20 & 10 & 0\end{array}$ 
COSY.

Chemist Shweta Gediya

COSY.w CDCl3 /opt/topspin3.2 SKG 13

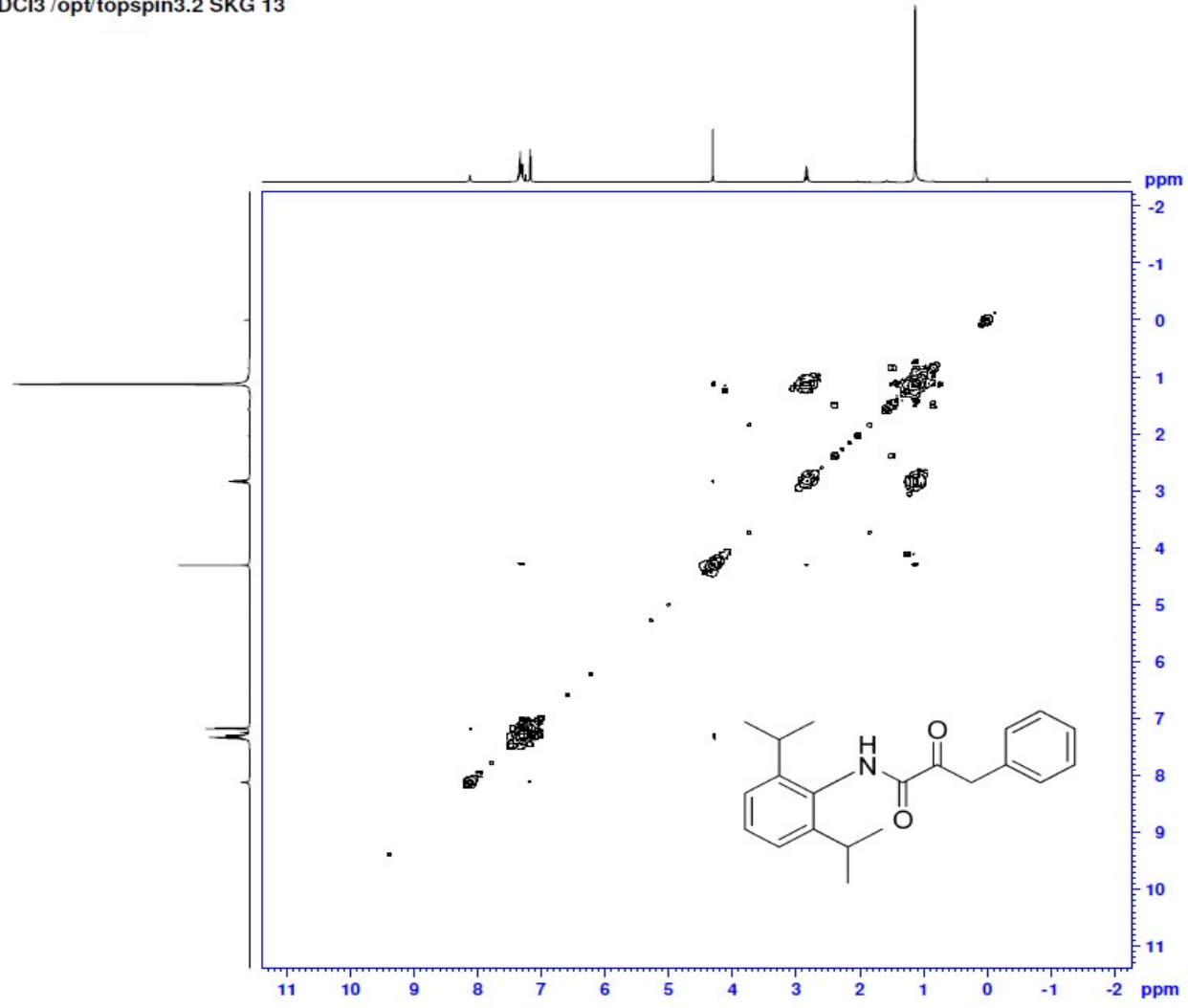


$N$-(2,6-Diisopropylphenyl)-2-oxo-2-phenylacetamide (precursor of 37).<smiles>COC(=O)C1Nc2c(C(C)C)cccc2C(C)O1</smiles>

This compound is novel.

To a $50 \mathrm{~mL}$ RBF was charged methyl 2-((2,6-diisopropylphenyl)amino)-2-oxoacetate (0.300 $\mathrm{g}, 1.14 \mathrm{mmol}, 1.0 \mathrm{eq})$ in THF $(10 \mathrm{~mL})$ and the mixture was cooled to $-78^{\circ} \mathrm{C}$. To this was added phenyl magnesium bromide (3M in diethyl ether) $(0.40 \mathrm{~mL}, 1.22 \mathrm{mmol}, 1.1 \mathrm{eq})$ and the resulting reaction mixture was stirred at $-78^{\circ} \mathrm{C}$ for $2 \mathrm{~h}$, followed by a fast quenching of the reaction at $-78^{\circ} \mathrm{C}$ with $2 \mathrm{~N}$ hydrochloric acid aqueous solution $(10 \mathrm{~mL})$. The reaction was warmed to room temperature and DCM $(20 \mathrm{~mL})$ was added, and the organic layer was separated. The aqueous layer was extracted with DCM $(2 \times 20 \mathrm{~mL})$. The combined organic layers were washed with brine $(60 \mathrm{~mL})$ and dried over $\mathrm{MgSO}_{4}$. The organic layer was concentrated under reduced pressure to generate crude material which was further purified by column chromatography $(10 \%$ EtOAc in petroleum ether) to afford the product as a yellow solid (0.152 g, $0.492 \mathrm{mmol}, 43 \%$ ).

TLC: $\mathrm{R}_{\mathrm{f}}$ ca 0.5 (8:2, hexane: EtOAc), UV-active and strong $\mathrm{KMnO}_{4}$;

MP: $142-143.8^{\circ} \mathrm{C}$;

HRMS (ESI) m/z: $[\mathrm{M}+\mathrm{Na}]^{+}$Calcd for $\mathrm{C}_{20} \mathrm{H}_{23} \mathrm{NO}_{2} \mathrm{Na} 332.1621$; Found 332.1615 (error 1.7 ppm);

$\mathrm{U}_{\max } 3268,2958,2865,1655,1596,1507,1446,1270,1214,1174,736 \mathrm{~cm}^{-1}$;

${ }^{1} \mathrm{H}$ NMR $\left(500 \mathrm{MHz}, \mathrm{CDCl}_{3}\right) \delta$ 8.42-8.40 (2H, m, ArH), $8.35(1 \mathrm{H}, \mathrm{s}, \mathrm{CONH}), 7.66(1 \mathrm{H}, \mathrm{t}, J=$ $7.4 \mathrm{~Hz}, \operatorname{ArH}), 7.54-7.50$ (2H, m, ArH), $7.35(1 \mathrm{H}, \mathrm{t}, J=7.7 \mathrm{~Hz}, \operatorname{ArH}), 7.26-7.23$ (2H, m, $\mathrm{ArH}), 3.13-3.05$ (2H, m, CH of isopropyl group), $1.23\left(12 \mathrm{H}, \mathrm{d}, J=6.9 \mathrm{~Hz}, \mathrm{CH}_{3}\right.$ of isopropyl group);

${ }^{13} \mathrm{C} \mathrm{NMR}\left(126 \mathrm{MHz}, \mathrm{CDCl}_{3}\right) \delta 187.9,161.3,146.2,134.9,133.6,131.6,129.8,129.0,128.8$, 123.9, 29.1, 23.8;

$m / z(\mathrm{ESI}) 332.2\left[(\mathrm{M}+\mathrm{Na})^{+}, 100 \%\right]$. 
$N$-(2,6-Diisopropylphenyl)-2-oxo-2-phenylacetamide.

${ }^{1} \mathrm{H}$ NMR (500 MHz, $\left.\mathrm{CDCl}_{3}\right)$.

Dec07-2020
Chemist Shweta Gediya
SKG690

PROTON.w CDCl3 /opt/topspin3.2 SKG 23

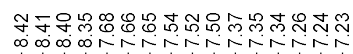

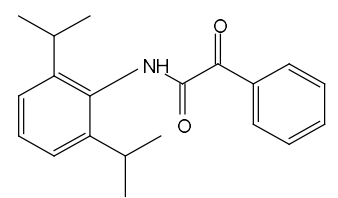

Is 5 d d

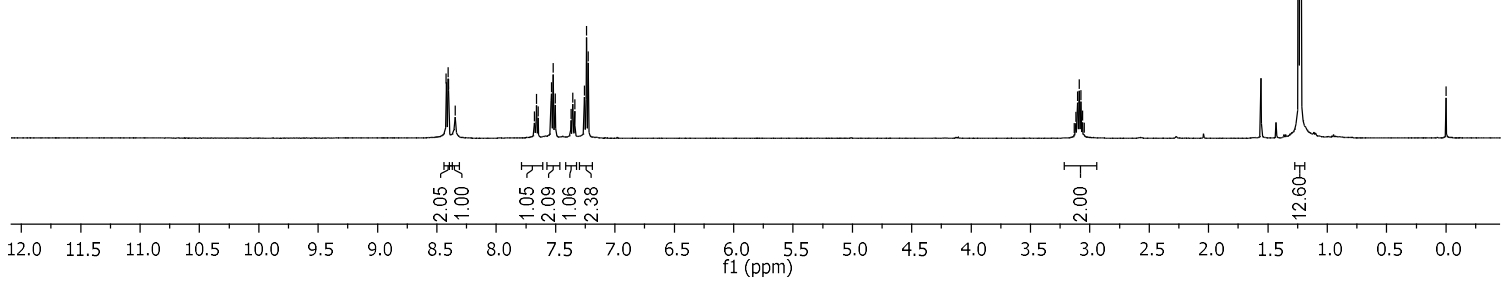

${ }^{13} \mathrm{C}$ NMR $\left(126 \mathrm{MHz}, \mathrm{CDCl}_{3}\right)$.

Dec07-2020
SKG690

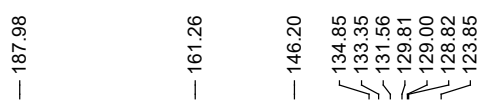

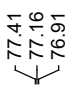

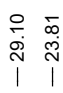
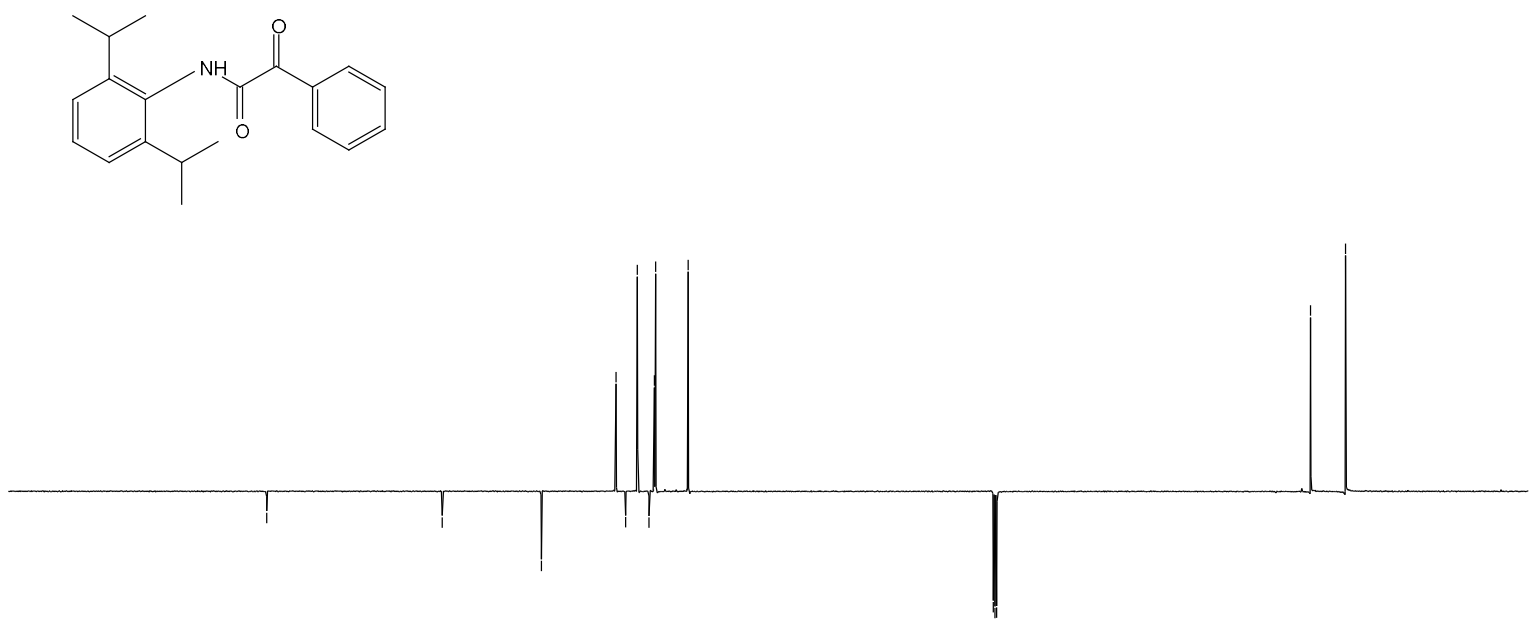

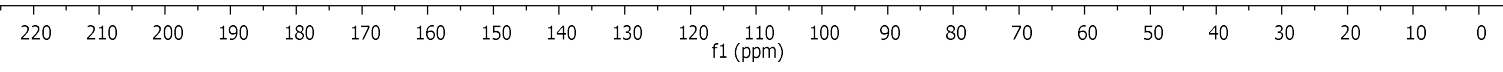

S159 
COSY.

Chemist Shweta Gediya SKG690
COSY.w CDCl3 /optitopspin3.2 SKG 23
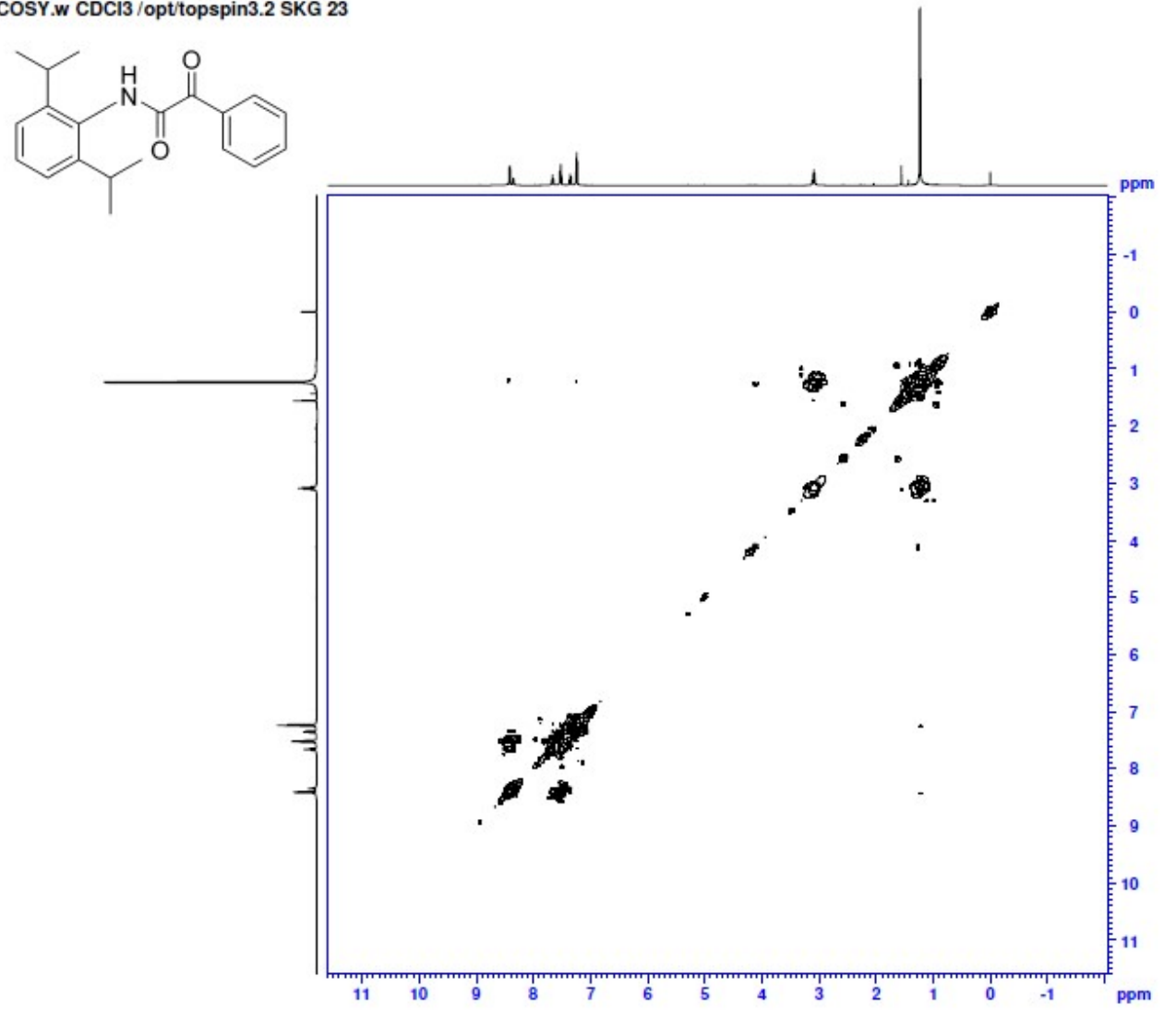

S160 
Ethyl 4-(methyl(phenyl)amino)-3,4-dioxobutanoate (precursor of 38).<smiles>CCOC(=O)CC(=O)C(=O)N(C)c1ccccc1</smiles>

This compound is novel and was prepared following general procedure B using methyl 2(methyl(phenyl)amino)-2-oxoacetate $(1.00 \mathrm{~g}, 5.18 \mathrm{mmol}, 1.0 \mathrm{eq})$ in anhydrous THF $(13 \mathrm{~mL})$, ethyl acetate (1.09 g, $12.4 \mathrm{mmol}, 2.4 \mathrm{eq})$ in anhydrous THF (7 mL) and LDA (1M solution in THF) (13.5 mL, $13.5 \mathrm{mmol}, 2.6 \mathrm{eq})$ to give crude material which was further purified by column chromatography (20-30\% EtOAc in petroleum ether) to afford the product as a yellow liquid $(0.773 \mathrm{~g}, 3.10 \mathrm{mmol}, 60 \%)$. The title compound was obtained as keto/enol tautomer (9:1) as per ${ }^{1} \mathrm{H}$ NMR.

TLC: $\mathrm{R}_{\mathrm{f}}$ ca 0.3 (7:3, hexane: EtOAc), UV-active and strong $\mathrm{KMnO}_{4}$;

HRMS (ESI) m/z: $[\mathrm{M}+\mathrm{Na}]^{+}$Calcd for $\mathrm{C}_{13} \mathrm{H}_{15} \mathrm{NO}_{4} \mathrm{Na} 272.0893$; Found 272.0885 (error 3.0 ppm);

$U_{\max } 2982,1718,1649,1594,1495,1026,730,667 \mathrm{~cm}^{-1}$;

${ }^{1} \mathrm{H}$ NMR $\left(500 \mathrm{MHz}, \mathrm{CDCl}_{3}\right.$ ) (keto/enol, 9:1) $\delta$ 7.43-7.19 (5H, m, ArH), $5.42(0.1 \mathrm{H}, \mathrm{s},=\mathrm{C} \underline{\mathrm{H}}$ of enol), 4.26-4.11 (2H, m, $\left.\underline{\mathrm{OCH}}_{2} \mathrm{CH}_{3}\right), 3.66\left(1.8 \mathrm{H}, \mathrm{m}, \mathrm{CH}_{2}\right.$ of keto form), $3.45(0.3 \mathrm{H}, \mathrm{s}$, $\left.\mathrm{NCH}_{3}\right), 3.37-3.38\left(2.7 \mathrm{H}, \mathrm{m},-\mathrm{NCH}_{3}\right), 1.27\left(3 \mathrm{H}, \mathrm{t}, J=7.1 \mathrm{~Hz}, \mathrm{OCH}_{2} \underline{\mathrm{CH}_{3}}\right), \mathrm{OH}$ of enol form not observed;

${ }^{13} \mathrm{C}$ NMR (126 MHz, $\left.\mathrm{CDCl}_{3}\right)$ (keto/enol, 9:1) $\delta$ 191.5(C=O keto), 167.1, 164.7, 142.1, 129.6, $128.1,126.5,61.6,45.9,37.4,14.2$;

$m / z(\mathrm{ESI}) 272.1\left[(\mathrm{M}+\mathrm{Na})^{+}, 100 \%\right]$. 
Ethyl 4-(methyl(phenyl)amino)-3,4-dioxobutanoate.

${ }^{1} \mathrm{H}$ NMR $\left(500 \mathrm{MHz}, \mathrm{CDCl}_{3}\right)$.

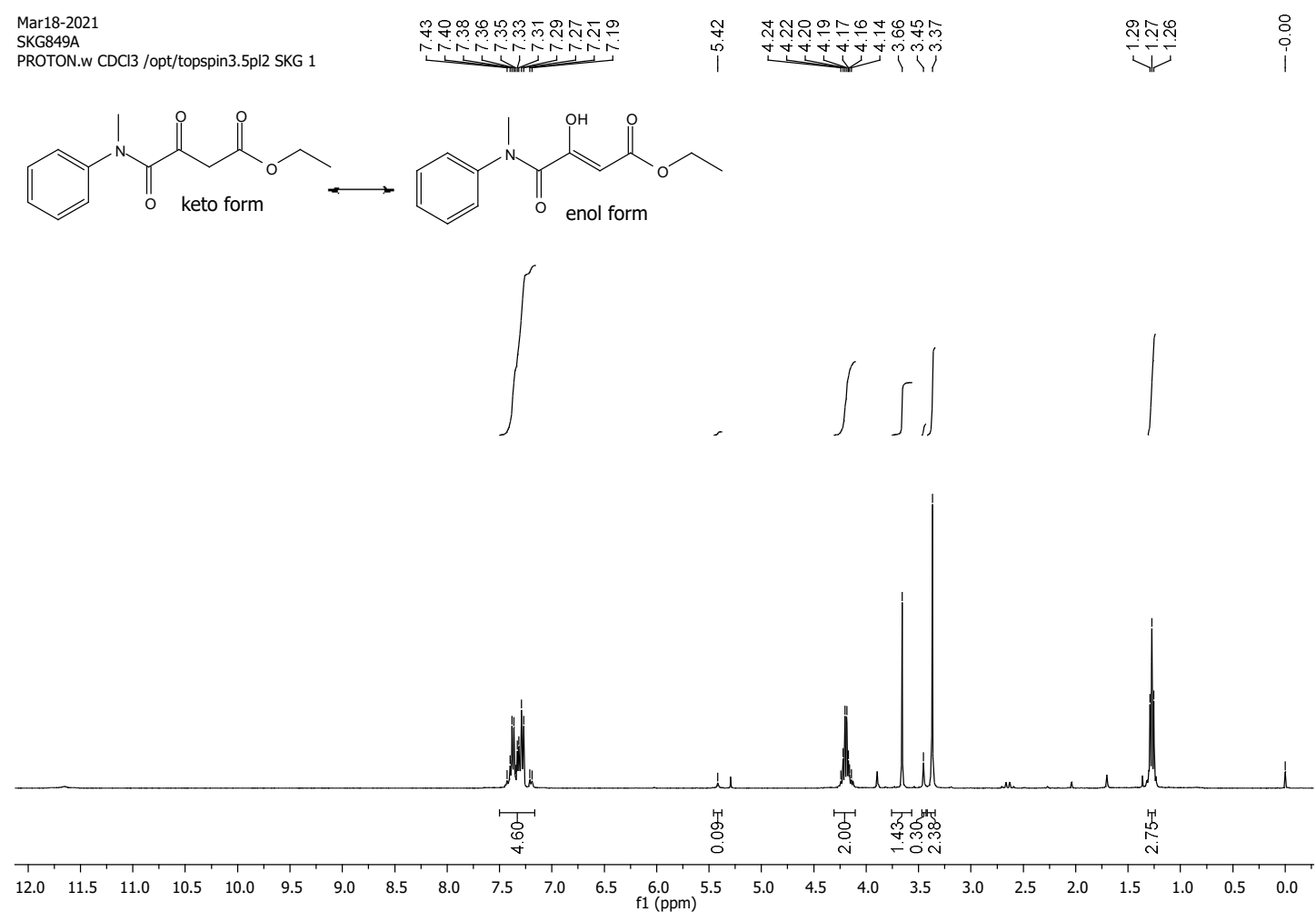

${ }^{13} \mathrm{C}$ NMR $\left(126 \mathrm{MHz}, \mathrm{CDCl}_{3}\right)$.

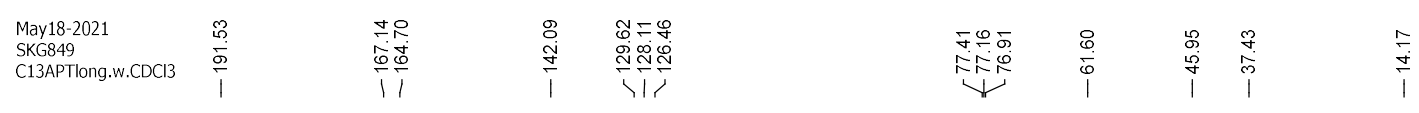
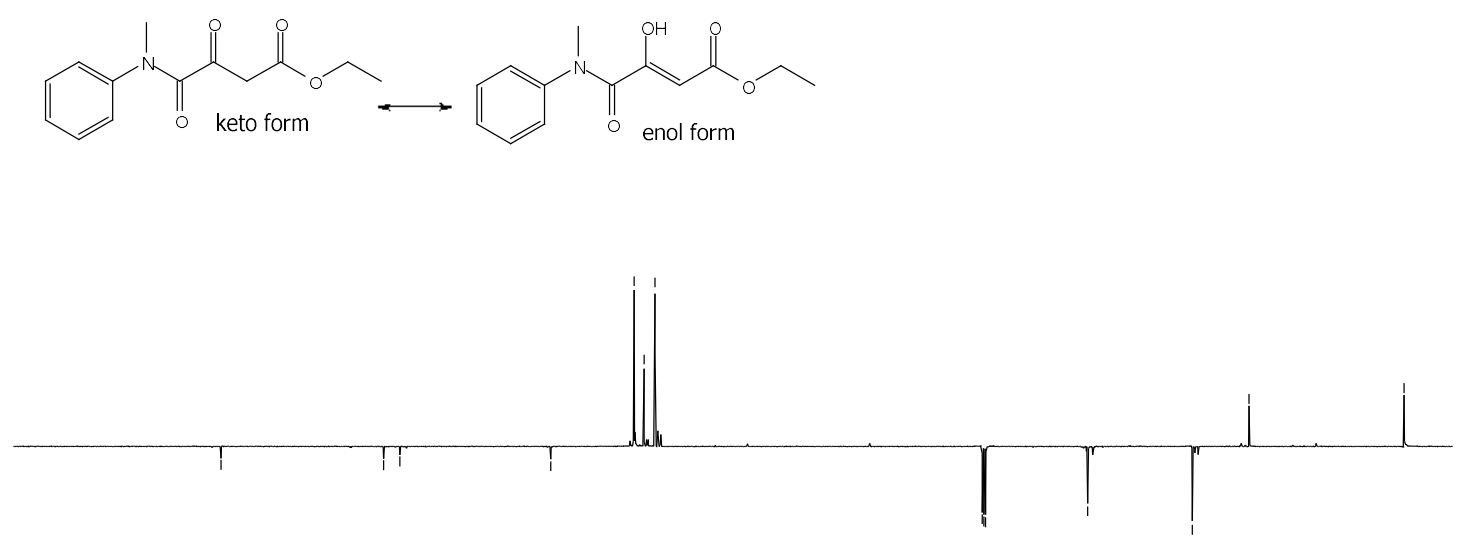

\begin{tabular}{llllllllllllllllllllll}
\hline 220 & 210 & 200 & 190 & 180 & 170 & 160 & 150 & 140 & 130 & 120 & 110 & 100 & 90 & 80 & 70 & 60 & 50 & 40 & 30 & 20 & 10
\end{tabular} 
COSY.

Chemist Shweta Gediya
SKG8 49 COSY.w CDCl3/opt/topspin 3.2 SKG 4

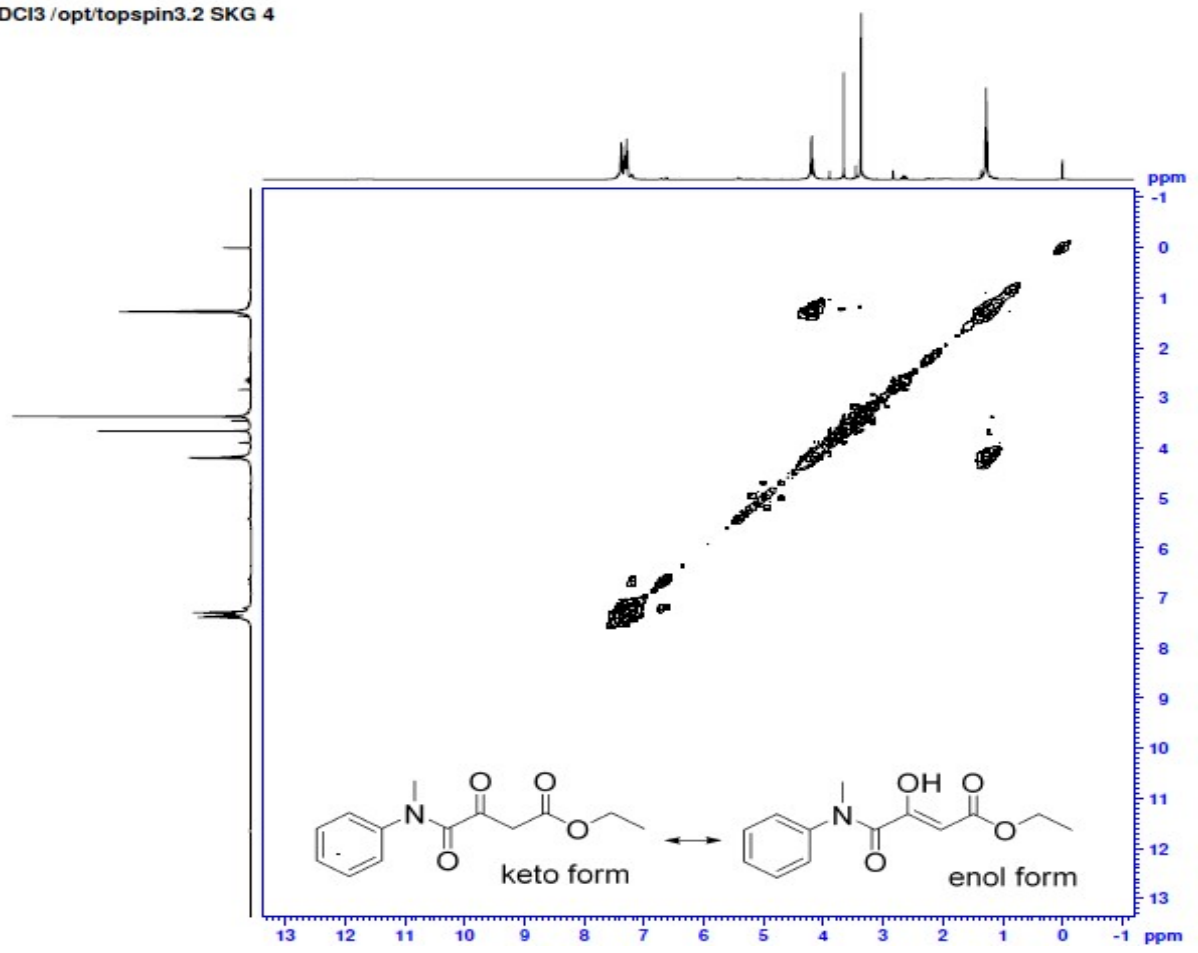

HSQC.

SKG849A
HSQC.w CDCl3 /opt/topspin3.5pl2 SKG 1

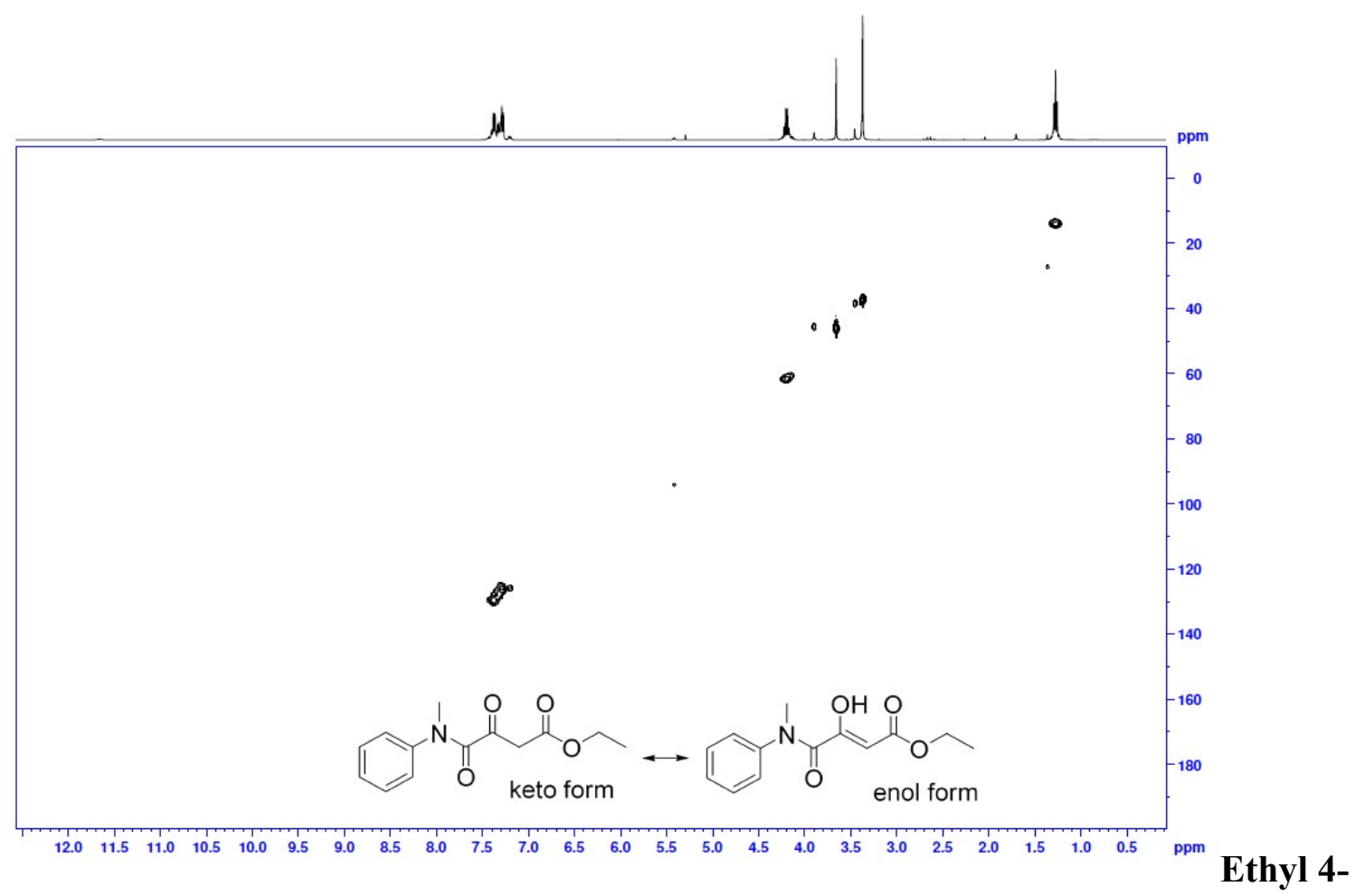

S163 
(Benzyl(methyl)amino)-3,4-dioxobutanoate (precursor to 39).<smiles>CCOC(=O)CC(=O)C(=O)N(C)Cc1ccccc1</smiles>

This compound is novel and was prepared following general procedure B using methyl 2(benzyl(methyl)amino)-2-oxoacetate $(2.07 \mathrm{~g}, 10.0 \mathrm{mmol}, 1.0 \mathrm{eq})$ and ethyl acetate $(2.11 \mathrm{~g}, 24$ mmol, $2.4 \mathrm{eq})$ and LDA (1M solution in THF) $(26.0 \mathrm{~mL}, 2.60 \mathrm{mmol}, 2.6 \mathrm{eq})$ in THF $(30 \mathrm{~mL})$ to give crude material which was further purified by column chromatography $(30-50 \%$ EtOAc in petroleum ether) to afford the product as a yellow oil (1.75 g, $6.61 \mathrm{mmol}, 66 \%)$. The title compound was obtained as keto/enol tautomer (95:5) as per ${ }^{1} \mathrm{H}$ NMR and rotamers. The color changed from yellow to black upon standing at room temperature.

TLC: $\mathrm{R}_{\mathrm{f}}$ ca 0.4 (7:3, hexane: EtOAc), UV-active and strong $\mathrm{KMnO}_{4}$; HRMS (ESI) m/z: [M+Na] $]^{+}$Calcd for $\mathrm{C}_{14} \mathrm{H}_{17} \mathrm{NO}_{4} \mathrm{Na} 286.1050$; Found 286.1050 (error -0.1 ppm);

$U_{\max } 2981,1719,1640,1452,1256,1180,1025,698 \mathrm{~cm}^{-1}$;

${ }^{1} \mathrm{H}$ NMR (400 MHz, $\left.\mathrm{CDCl}_{3}\right)$ (keto/enol $(95: 5)+$ rotamers) $\delta$ 7.38-7.26 (5H, m, ArH), 5.52 $(0.05 \mathrm{H}, \mathrm{s},=\mathrm{C} \underline{\mathrm{H}}$ enol, Rotamer 1 and 2$), 4.61\left(1.3 \mathrm{H}, \mathrm{s}, \mathrm{CH}_{2} \mathrm{Ph}\right.$ major rotamer $), 4.58(0.7 \mathrm{H}, \mathrm{s}$, $\mathrm{CH}_{2} \mathrm{Ph}$, minor rotamer), 4.26-4.13 (2H, m, $\mathrm{CH}_{2}$ of ester group), 3.87-3.86 (1.9H, m, $\mathrm{CH}_{2}$ of keto form, Rotamer 1 and 2), $3.00\left(1.6 \mathrm{H}, \mathrm{s},-\mathrm{NCH}_{3}\right.$ major rotamer), $2.88\left(1.4 \mathrm{H}, \mathrm{s},-\mathrm{NCH}_{3}\right.$ minor rotamer), 1.31-1.22 (3H, $\mathrm{m}, \mathrm{CH}_{3}$ of ester group);

${ }^{13} \mathrm{C}$ NMR $\left(101 \mathrm{MHz}, \mathrm{CDCl}_{3}\right)($ keto/enol (95:5) + rotamers) $\delta 192.3(\mathrm{C}=\mathrm{O}$ keto), 171.9, 167.3, 165.6, 165.4, 135.7, 135.6, 128.8, 128.1, 128.0, 127.9, 127.8, 93.6, 93.3 (C= $\underline{\mathrm{CH}}$, enol), 61.6, $61.4,53.4,50.9,45.8,45.7$, , 34.8, 32.5, 14.2, 14.1, 14.0; $m / z(\mathrm{ESI}) 266.1\left[(\mathrm{M}+\mathrm{Na})^{+}, 100 \%\right]$. 
Ethyl 4-(benzyl(methyl)amino)-3,4-dioxobutanoate.

${ }^{1} \mathrm{H}$ NMR (400 MHz, $\mathrm{CDCl}_{3}$ ).

Apr16-2021

PROTON.w CDCl3 /opt/topspin3.5pl2 SKG 20

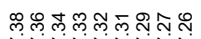

䓍

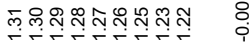

(2)

$\Gamma$
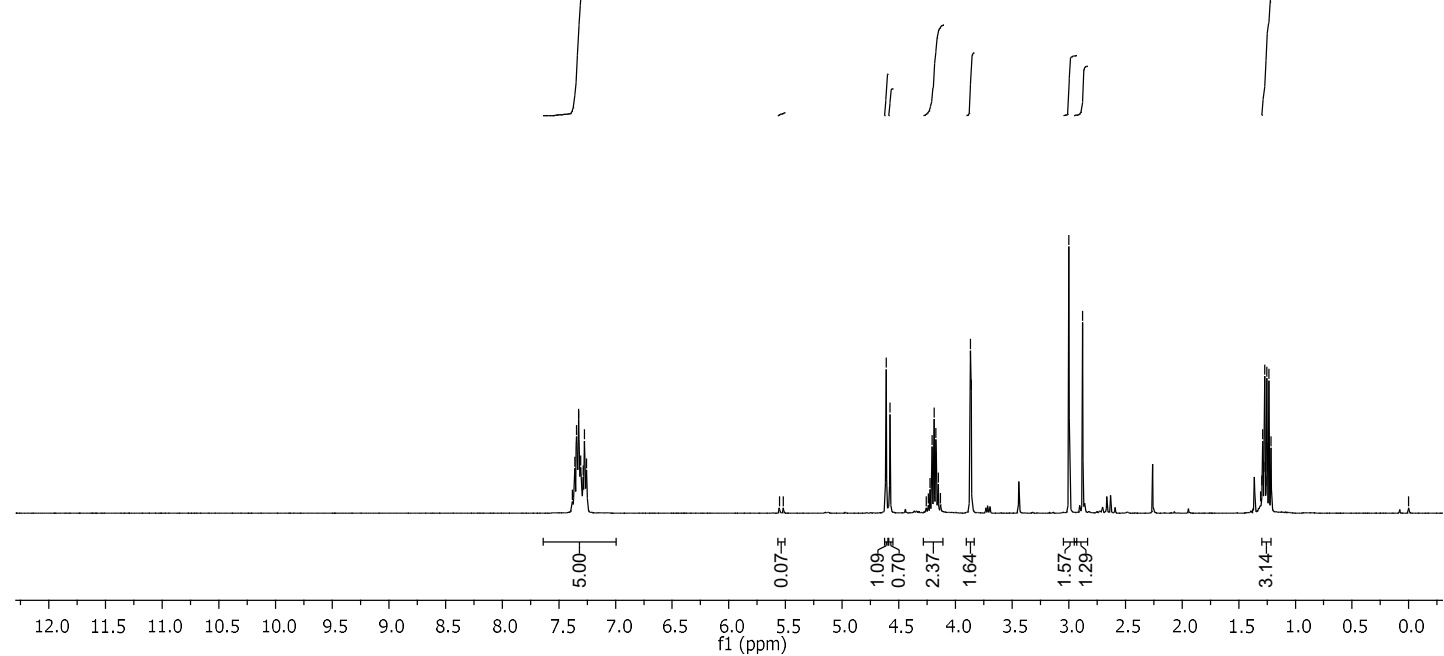

${ }^{13} \mathrm{C}$ NMR (101 MHz, $\left.\mathrm{CDCl}_{3}\right)$.

\begin{tabular}{|c|c|c|c|c|c|c|c|}
\hline $\begin{array}{l}\text { Apr16-2021 } \\
\text { SKG888 }\end{array}$ & 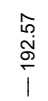 & 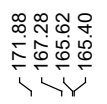 & 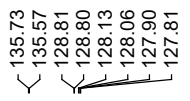 & 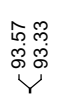 & 年 & 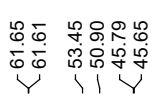 & 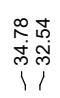 \\
\hline
\end{tabular}
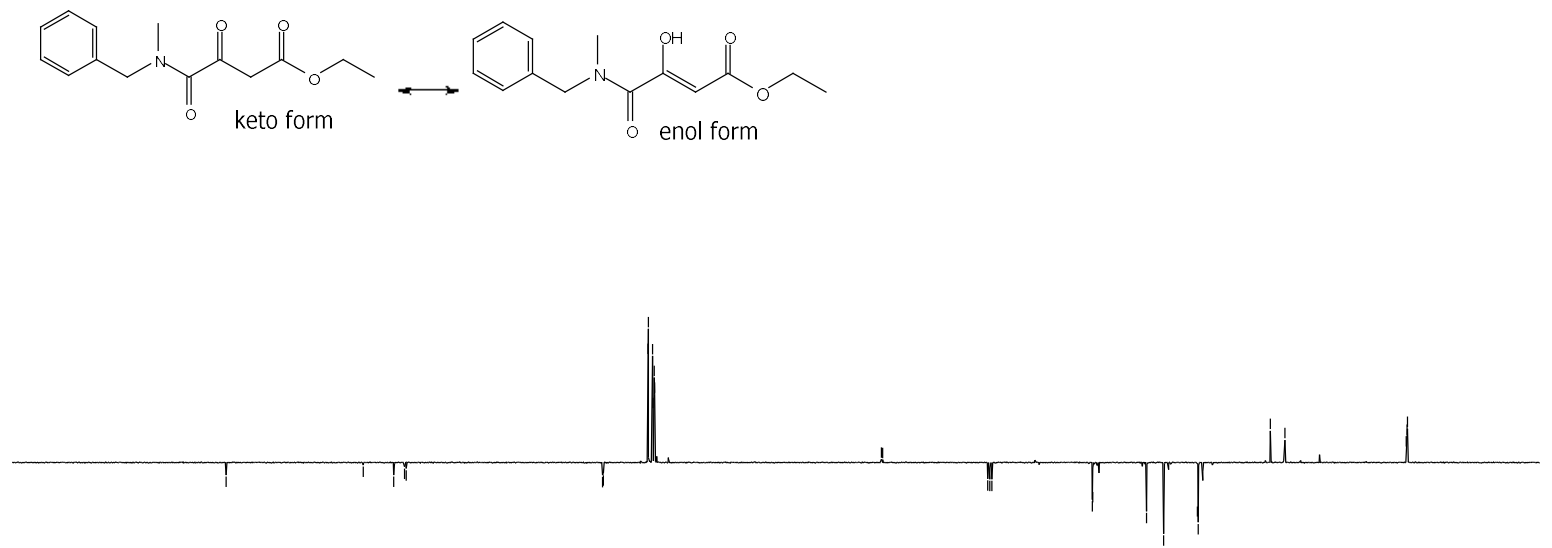

$\begin{array}{lllllllllllllllllllllll}220 & 210 & 200 & 190 & 180 & 170 & 160 & 150 & 140 & 130 & 120 & \left.\begin{array}{l}110 \\ \mathrm{f} 1(\mathrm{ppm})\end{array}\right) & 100 & 90 & 80 & 70 & 60 & 50 & 40 & 30 & 20 & 10 & 0\end{array}$ 
COSY.

SKG888
COSY.w CDCl3 /opt/topspin3.5pl2 SKG 20

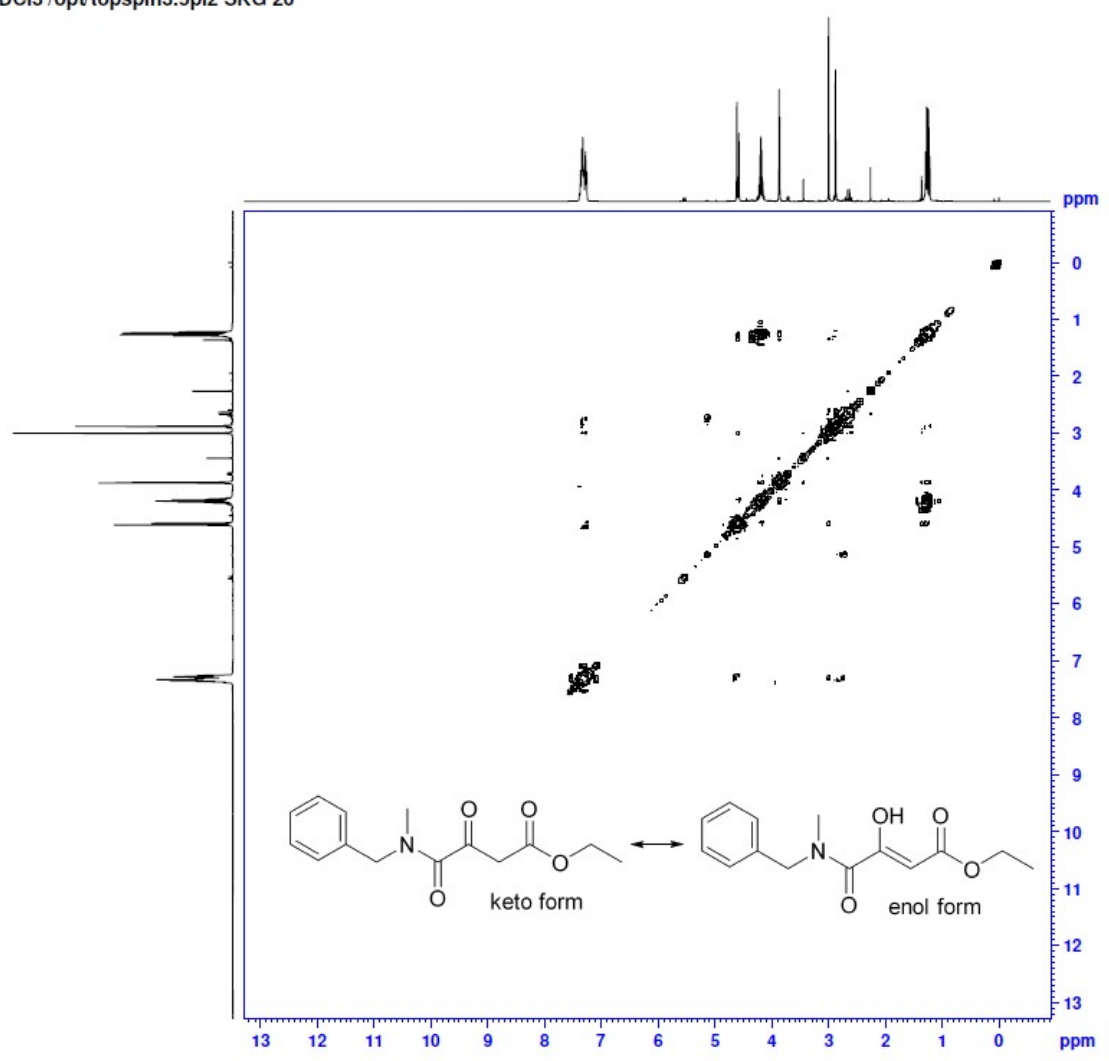

HSQC.

SKG888
HSQC.w CDCl3 /opt/topspin3.5pl2 SKG 20

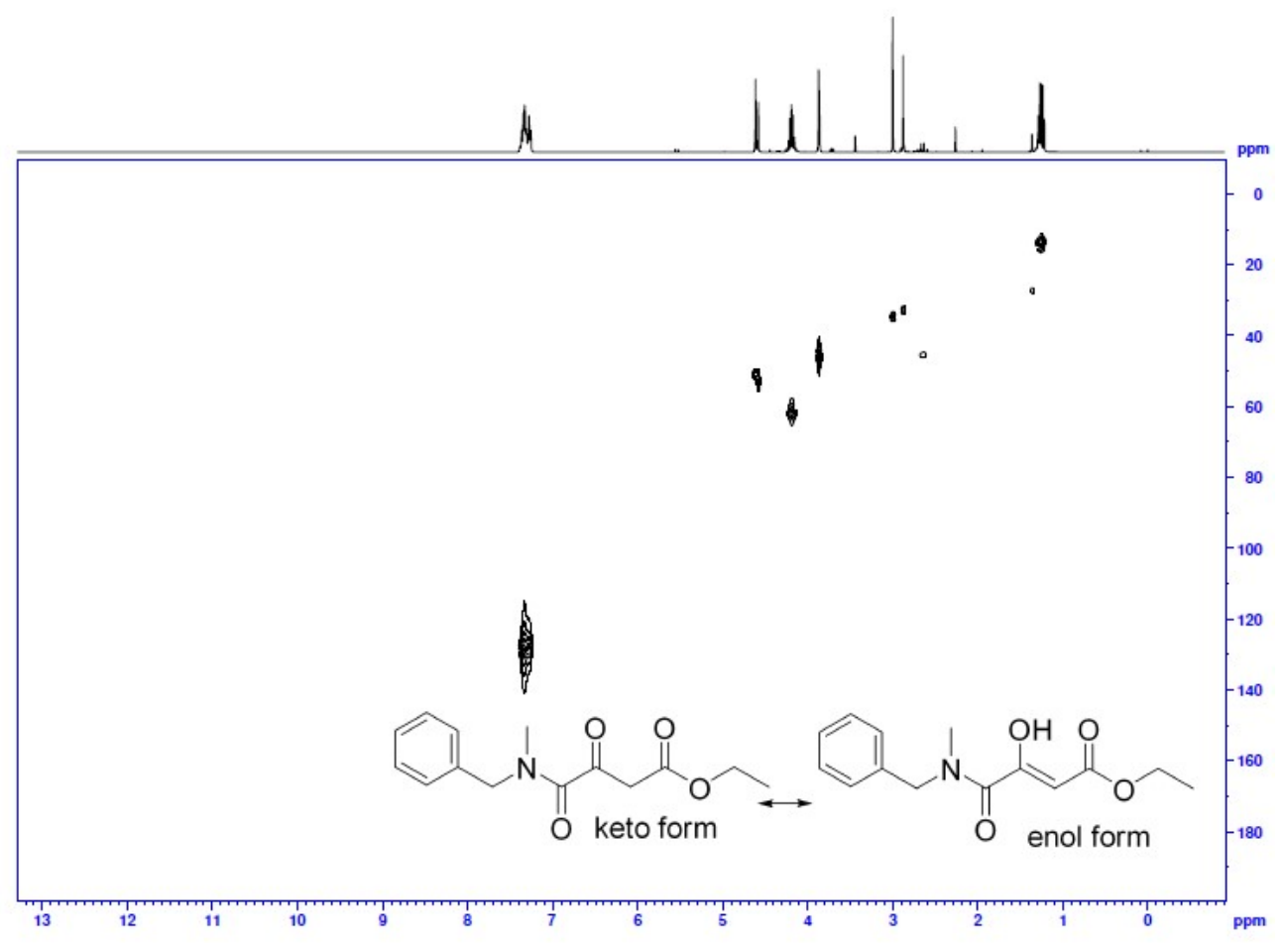

S166 
Ethyl 3,4-dioxo-4-(piperidin-1-yl)butanoate (precursor of 40).<smiles>CCOC(=O)CC(=O)C(=O)N1CCCCC1</smiles>

This compound is novel and was prepared following general procedure B using methyl 2oxo-2-(piperidin-1-yl)acetate (0.850 g, $4.97 \mathrm{mmol}, 1.0 \mathrm{eq})$ in anhydrous THF (13 mL), ethyl acetate (1.05 g, $11.9 \mathrm{mmol}, 2.4 \mathrm{eq})$ in anhydrous THF (7 mL) and LDA (1M solution in THF) (14.9 mL, $14.9 \mathrm{mmol}, 3.0 \mathrm{eq})$ to give crude material which was further purified by column chromatography (30-40\% EtOAc in petroleum ether) to afford the product as a yellow oil $(0.689 \mathrm{~g}, 3.03 \mathrm{mmol}, 61 \%)$. The title compound was obtained as a keto form with trace (ca $5 \%)$ of enol tautomer as per ${ }^{1} \mathrm{H}$ NMR.

TLC: $\mathrm{R}_{\mathrm{f}}$ ca 0.3 (6:4, hexane: EtOAc), UV-active and strong $\mathrm{KMnO}_{4}$;

HRMS (ESI) m/z: $[\mathrm{M}+\mathrm{Na}]^{+}$Calcd for $\mathrm{C}_{11} \mathrm{H}_{17} \mathrm{NO}_{4} \mathrm{Na} 250.1050$; Found 250.1046 (error 1.6 ppm);

$U_{\max } 2936,2858,1731,1633,1603,1444,1176,1024 \mathrm{~cm}^{-1}$;

${ }^{1} \mathrm{H}$ NMR (400 MHz, $\mathrm{CDCl}_{3}$ ) (predominantly keto form (ca 5\%) enol form trace) $\delta 4.20-4.14$ ( $\left.2 \mathrm{H}, \mathrm{m}, \mathrm{OCH}_{2} \mathrm{CH}_{3}\right), 3.78$ (2H, s, $\mathrm{CH}_{2}$ of keto form), 3.55-3.53 (2H, m, $\mathrm{CH}_{2}$ of piperidine), 3.46-3.44 (2H, m, $\mathrm{CH}_{2}$ of piperidine), 1.64-1.60 (6H, m, $\mathrm{CH}_{2}$ of piperidine), 1.27-1.23 (3H, $\left.\mathrm{m}, \mathrm{OCH}_{2} \mathrm{CH}_{3}\right)$;

${ }^{13} \mathrm{C}$ NMR (101 MHz, $\mathrm{CDCl}_{3}$ ) (predominantly keto form (ca 5\%) enol form trace) $\delta 192.9$, $(\mathrm{C}=\mathrm{O}$, keto), 167.3, 164.1, $92.7(\mathrm{C}=\underline{\mathrm{C}} \mathrm{H}, \mathrm{enol}), 61.6,47.0,45.8,43.0,26.2,25.5,24.5,14.2$; $m / z(\mathrm{ESI}) 250.1\left[(\mathrm{M}+\mathrm{Na})^{+}, 100 \%\right]$. 
Ethyl 3,4-dioxo-4-(piperidin-1-yl)butanoate.

${ }^{1} \mathrm{H}$ NMR (400 MHz, $\left.\mathrm{CDCl}_{3}\right)$.

Mar12-2021

SKG837
PROTON.w CDCl3 /opt/topspin3.5pl2 SKG 34

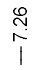
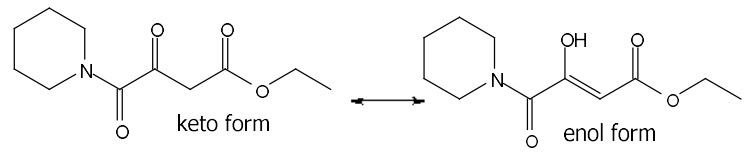

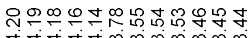
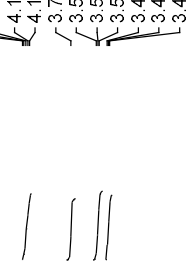

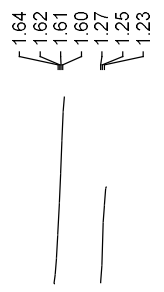

Iọ

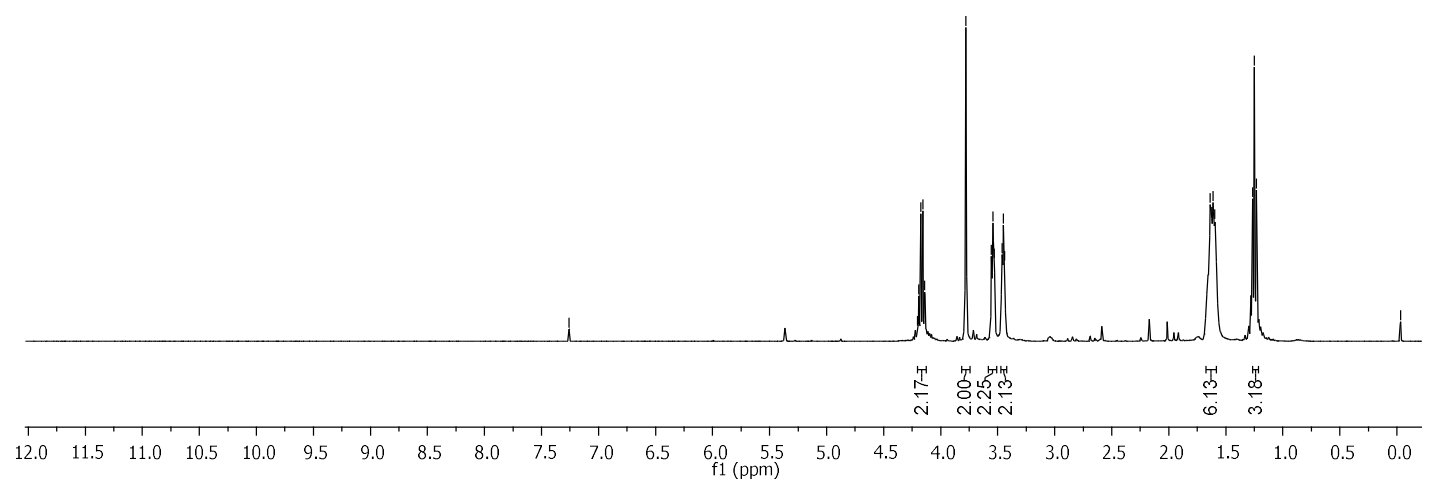

${ }^{13} \mathrm{C}$ NMR (101 MHz, $\left.\mathrm{CDCl}_{3}\right)$.

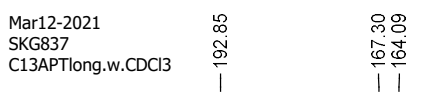

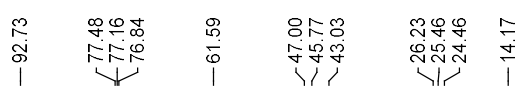
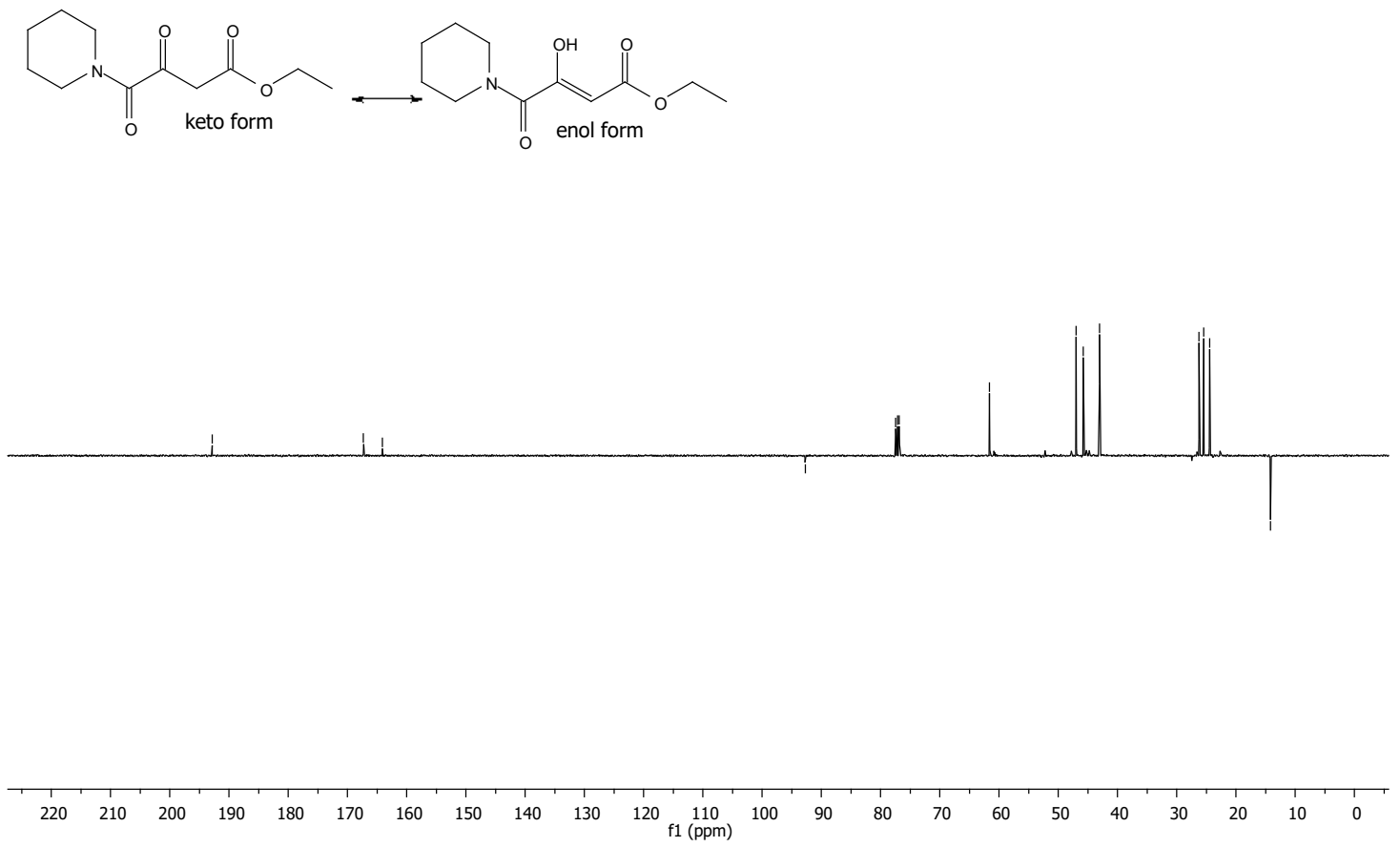

S168 
COSY.

SKG837

COSY.w CDCl3 /opt/topspin3.5pl2 SKG 34

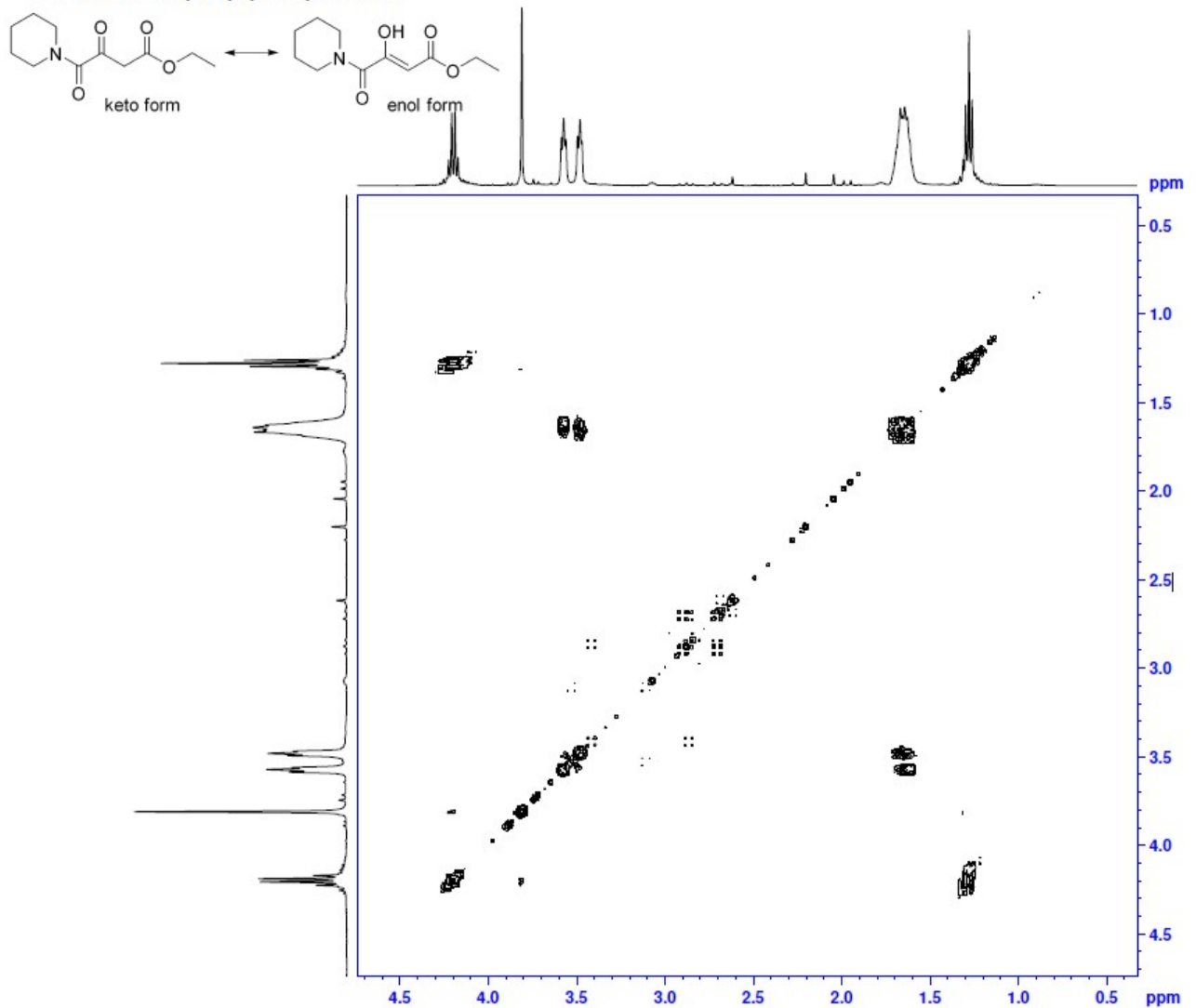

HSQC.

SKG837
HSQC.w CDCI3 /opt/topspin3.5pl2 SKG 34

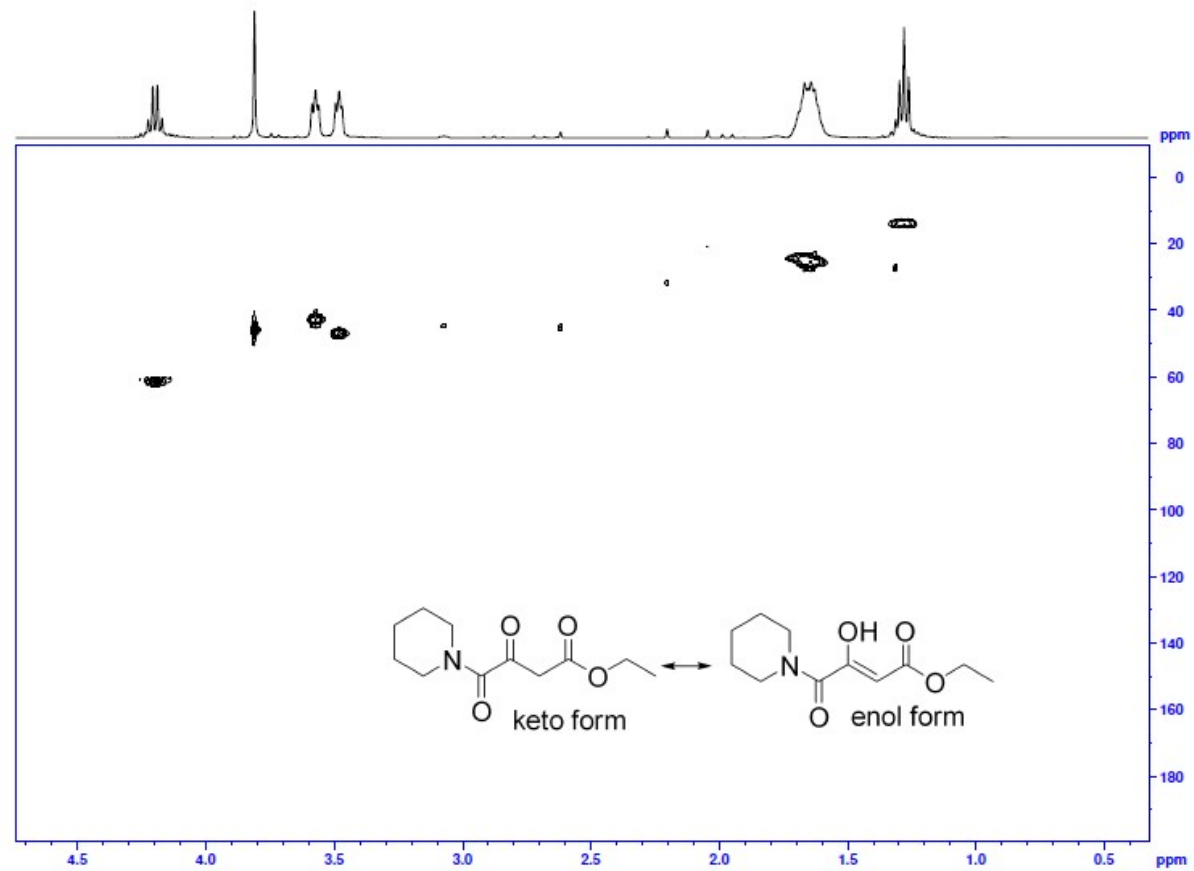


Ethyl 4-morpholino-3,4-dioxobutanoate (precursor of 41).<smiles>CCOC(=O)CC(=O)C(=O)N1CCOCC1</smiles>

This compound is novel and was prepared following general procedure B using methyl 2morpholino-2-oxoacetate (1.01 g, $5.77 \mathrm{mmol}, 1.0 \mathrm{eq})$ in anhydrous THF (13 mL), ethyl acetate (1.20 g, $13.8 \mathrm{mmol}, 2.4 \mathrm{eq})$ in anhydrous THF (7 mL) and LDA (1 M solution in THF) (17.1 mL, $17.1 \mathrm{mmol}, 3.0 \mathrm{eq})$ to give crude material which was further purified by column chromatography (30-40\% EtOAc in petroleum ether) to afford the product as a clear oil $(0.570 \mathrm{~g}, 2.48 \mathrm{mmol}, 44 \%)$. The title compound was obtained as keto/enol tautomer (9:1) as per ${ }^{1} \mathrm{H}$ NMR.

TLC: $\mathrm{R}_{\mathrm{f}}$ ca 0.4 (6:4, hexane: EtOAc), UV-active and strong $\mathrm{KMnO}_{4}$;

HRMS (ESI) m/z: [M+Na] $]^{+}$Calcd for $\mathrm{C}_{10} \mathrm{H}_{15} \mathrm{NO}_{5} \mathrm{Na} 252.0842$; Found 252.0843 (error -0.3 ppm);

$U_{\max } 2977,2908,1719,1639,1441,1179,1108,1019,580 \mathrm{~cm}^{-1}$;

${ }^{1} \mathrm{H}$ NMR (500 MHz, $\mathrm{CDCl}_{3}$ ) (keto/enol, 9:1) $\delta 12.12$ (0.1H, br.s., OH of enol form), 5.49 $\left(0.1 \mathrm{H}, \mathrm{m},=\mathrm{CH}\right.$ enol), 4.25-4.16 $\left(2 \mathrm{H}, \mathrm{m}, \mathrm{C}_{2} \mathrm{CH}_{3}\right), 3.81$ (1.8H, s, $\mathrm{CH}_{2}$ of keto form), 3.73$3.70\left(4 \mathrm{H}, \mathrm{m}, \mathrm{CH}_{2}\right.$ of morpholine), 3.64-3.62 (2H, m, $\mathrm{CH}_{2}$ of morpholine), 3.60-3.58 (2H, m, $\mathrm{CH}_{2}$ of morpholine), 1.31-1.25 (3H, m, $\left.\mathrm{CH}_{2} \underline{\mathrm{C}}_{3}\right)$;

${ }^{13} \mathrm{C}$ NMR (126 MHz, $\mathrm{CDCl}_{3}$ ) (keto/enol, 9:1) $\delta 192.4$ (C=O, keto), 172.4, 167.5, 166.1, 163.9, 94.1 ( $\mathrm{C}=\underline{\mathrm{C}} \mathrm{H}, \mathrm{enol}), 67.1,66.9,66.7,66.6,61.7,61.1,47.1,46.4,45.5,42.7,42.4,14.3$, 14.2;

$m / z(\mathrm{ESI}) 252.1\left[(\mathrm{M}+\mathrm{Na})^{+}, 100 \%\right]$. 
Ethyl 4-morpholino-3,4-dioxobutanoate.

${ }^{1} \mathrm{H}$ NMR (500 MHz, $\mathrm{CDCl}_{3}$ ).

Mar25-2021
SKG862

Shweta Gediya

Shweta Gediya
PROTON.w.CDCl3
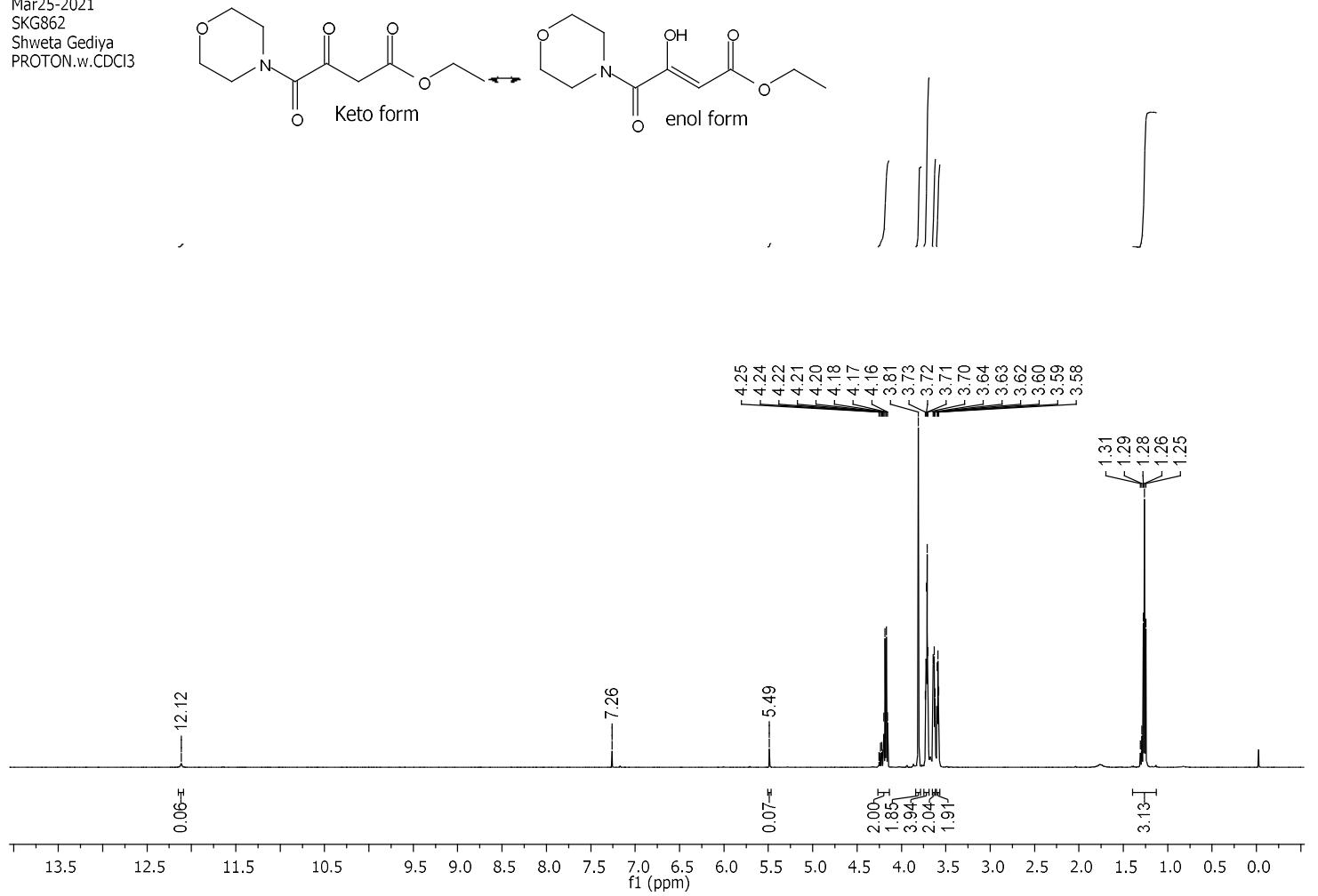

${ }^{13} \mathrm{C}$ NMR $\left(126 \mathrm{MHz}, \mathrm{CDCl}_{3}\right)$.

Mar25-2021

Shweta Gedy

Shweta Gediya<smiles>CCOC(=O)/C=C(\O)C(=O)N1CCOCC1</smiles>

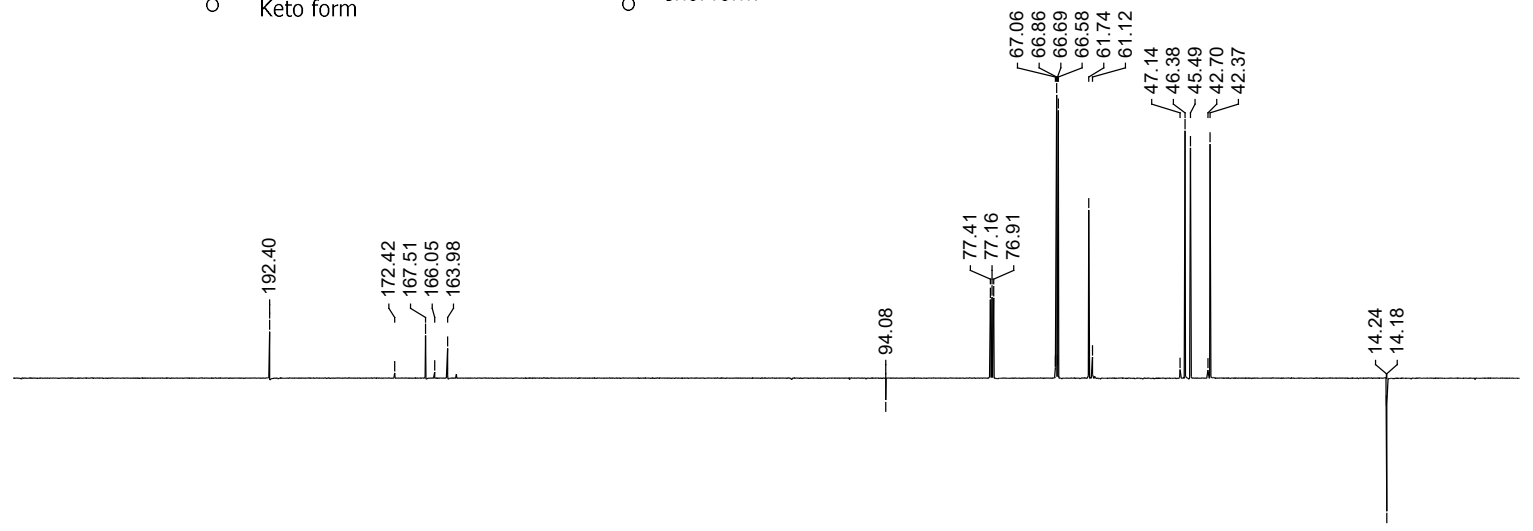

$\begin{array}{lllllllllllllllllllllllllllllllllll}230 & 220 & 210 & 200 & 190 & 180 & 170 & 160 & 150 & 140 & 130 & 120 & 110 & 100 & 90 & 80 & 70 & 60 & 50 & 40 & 30 & 20 & 10 & 0\end{array}$ 
COSY.

Chemist Shweta Gediya

COSY.w CDCl3 /opt/topspin3.2 SKG 27

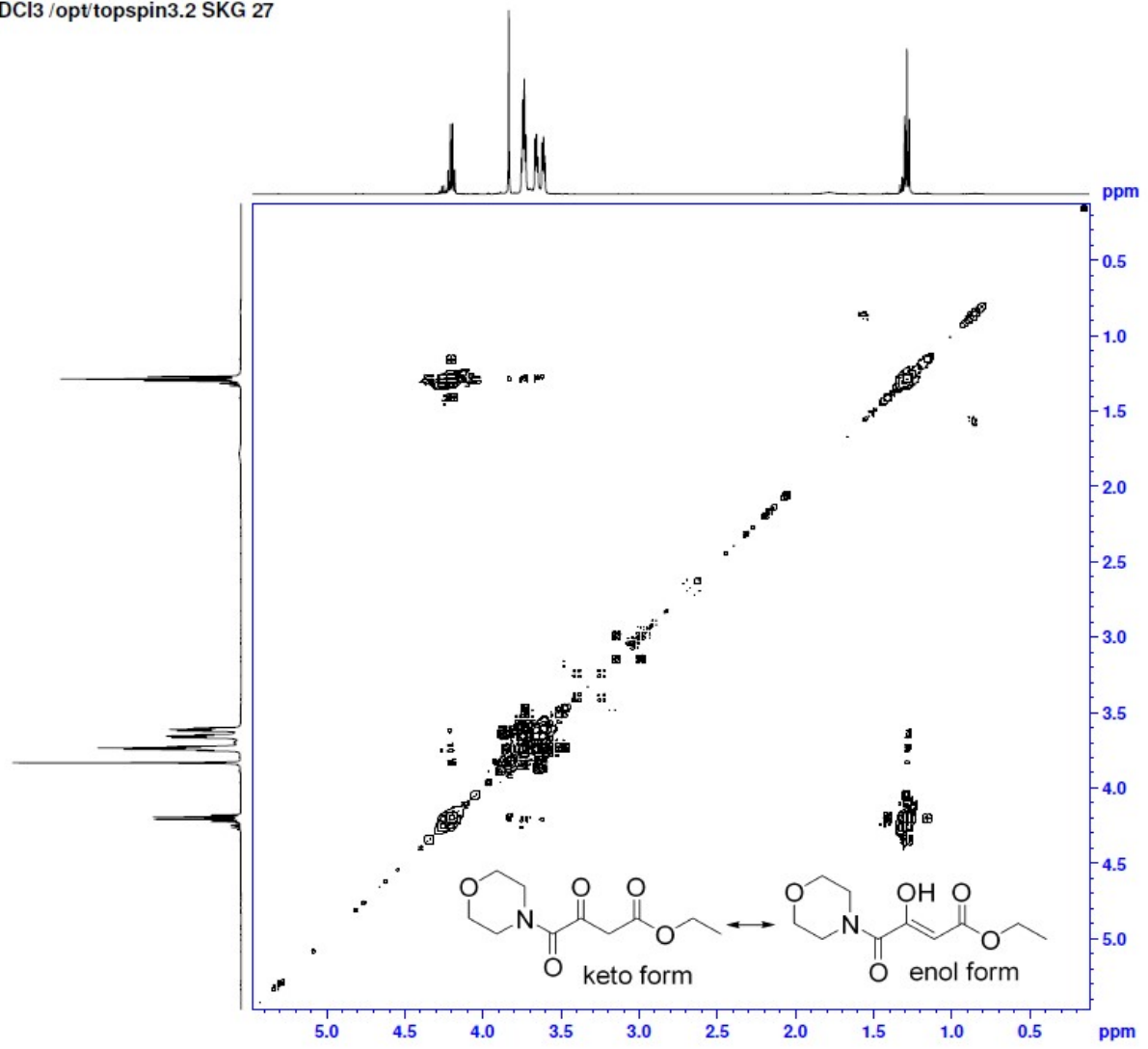

HSQC.

HSQC.w CDCl3/opt/topspin3.2 SKG 27

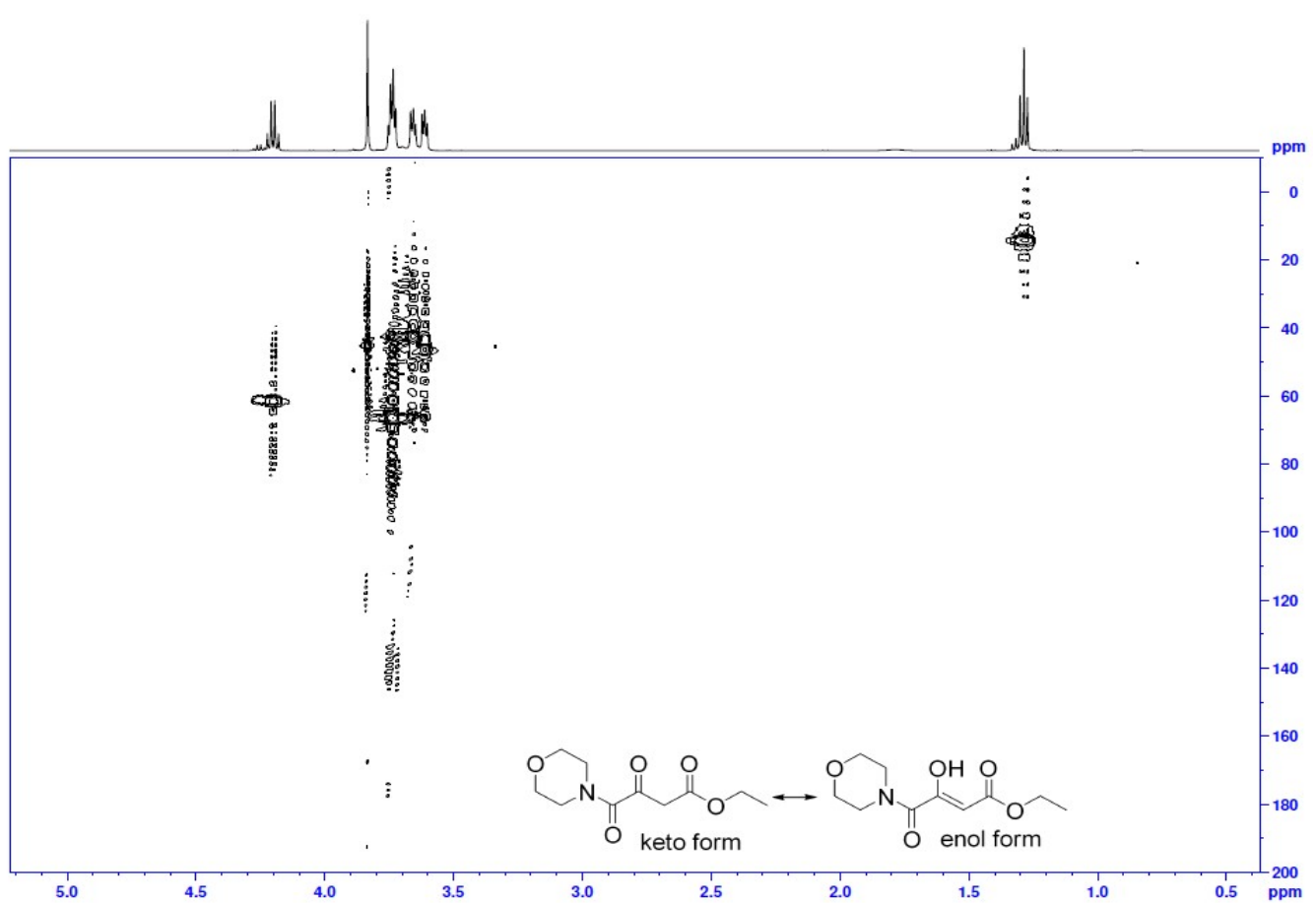


Ethyl 3,4-dioxo-4-(propylamino)butanoate (precursor of 42).<smiles>CCCNC(=O)C(=O)CC(=O)OCC</smiles>

This compound is novel and was prepared following general procedure B using methyl 2((2,6-diisopropylphenyl)amino)-2-oxoacetate $(0.549 \mathrm{~g}, 3.78 \mathrm{mmol}, 1.0 \mathrm{eq})$ in anhydrous THF (6 mL), ethyl acetate $(0.798 \mathrm{~g}, 9.07 \mathrm{mmol}, 2.4 \mathrm{eq})$ in anhydrous THF (4 mL) and LDA (1M solution in THF) $(11.3 \mathrm{~mL}, 11.3 \mathrm{mmol}, 3 \mathrm{eq})$ to give crude material which was further purified by column chromatography (5-10\% EtOAc in petroleum ether) to afford the product as a white solid $(0.201 \mathrm{~g}, 1.16 \mathrm{mmol}, 31 \%)$. The title compound was obtained as keto/enol tautomer (3:2) as per ${ }^{1} \mathrm{H}$ NMR.

TLC: $\mathrm{R}_{\mathrm{f}}$ ca 0.5 (5:5, hexane: EtOAc), UV-active and strong $\mathrm{KMnO}_{4}$;

HRMS (ESI) m/z: [M+Na] ${ }^{+}$Calcd for $\mathrm{C}_{9} \mathrm{H}_{15} \mathrm{NO}_{4} \mathrm{Na}$ 224.0893; Found 224.0886 (error 3.4 ppm);

$U_{\max } 3324,2967,1735,1714,1678,1659,1533,1371,1198,1157,1024 \mathrm{~cm}^{-1}$;

${ }^{1} \mathrm{H}$ NMR (400 MHz, $\mathrm{CDCl}_{3}$ ) (keto/enol, 3:2) $\delta 11.99$ (0.4H, s, OH enol), 6.91-6.86 (1H, br. m, NHCO), $6.06\left(0.4 \mathrm{H}, \mathrm{s},=\mathrm{CH}\right.$ enol), $4.28-4.17\left(2 \mathrm{H}, \mathrm{m}, \mathrm{C}_{2} \mathrm{CH}_{3}\right.$ of ester), $3.87(1.2 \mathrm{H}, \mathrm{s}$, $\mathrm{CH}_{2}$ keto), 3.35-3.24 (2H, m, $\left.\mathrm{CH}_{2}\right), 1.63-1.53\left(2 \mathrm{H}, \mathrm{m}, \mathrm{C}_{2} \mathrm{CH}_{3}\right.$ of propyl), 1.31 (1.2H, t, $J=$ $7.1 \mathrm{~Hz}, \mathrm{CH}_{2} \underline{\mathrm{CH}}_{3}$ of ester), $1.26\left(1.8 \mathrm{H}, \mathrm{t}, J=7.1 \mathrm{~Hz}, \mathrm{CH}_{2} \underline{\mathrm{C}}_{3}\right.$ of ester), 0.96-0.91 (3H, m, $\mathrm{CH}_{2} \underline{\mathrm{C}}_{3}$ of propyl);

${ }^{13} \mathrm{C}$ NMR (101 MHz, $\left.\mathrm{CDCl}_{3}\right)$ (keto/enol, 3:2) $\delta$ 192.1(C=O keto), 172.7, 166.9, 161.8, 160.7, 159.4, 93.8 (=ㄷH enol), 61.7, 61.3, 43.5, 41.3, 41.2, 22.7, 22.6, 14.2, 14.1, 11.5, 11.4; $m / z(\mathrm{ESI}) 224.1\left[(\mathrm{M}+\mathrm{Na})^{+}, 100 \%\right]$. 
Ethyl 3, 4-dioxo-4-(propylamino) butanoate.

${ }^{1} \mathrm{H}$ NMR (400 MHz, $\left.\mathrm{CDCl}_{3}\right)$.

Feb09-2021

SKG785 batch 1

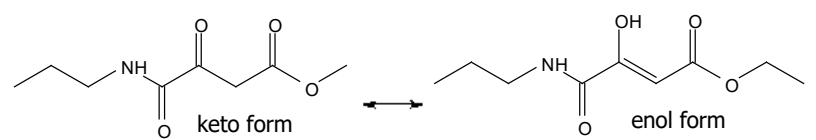

s
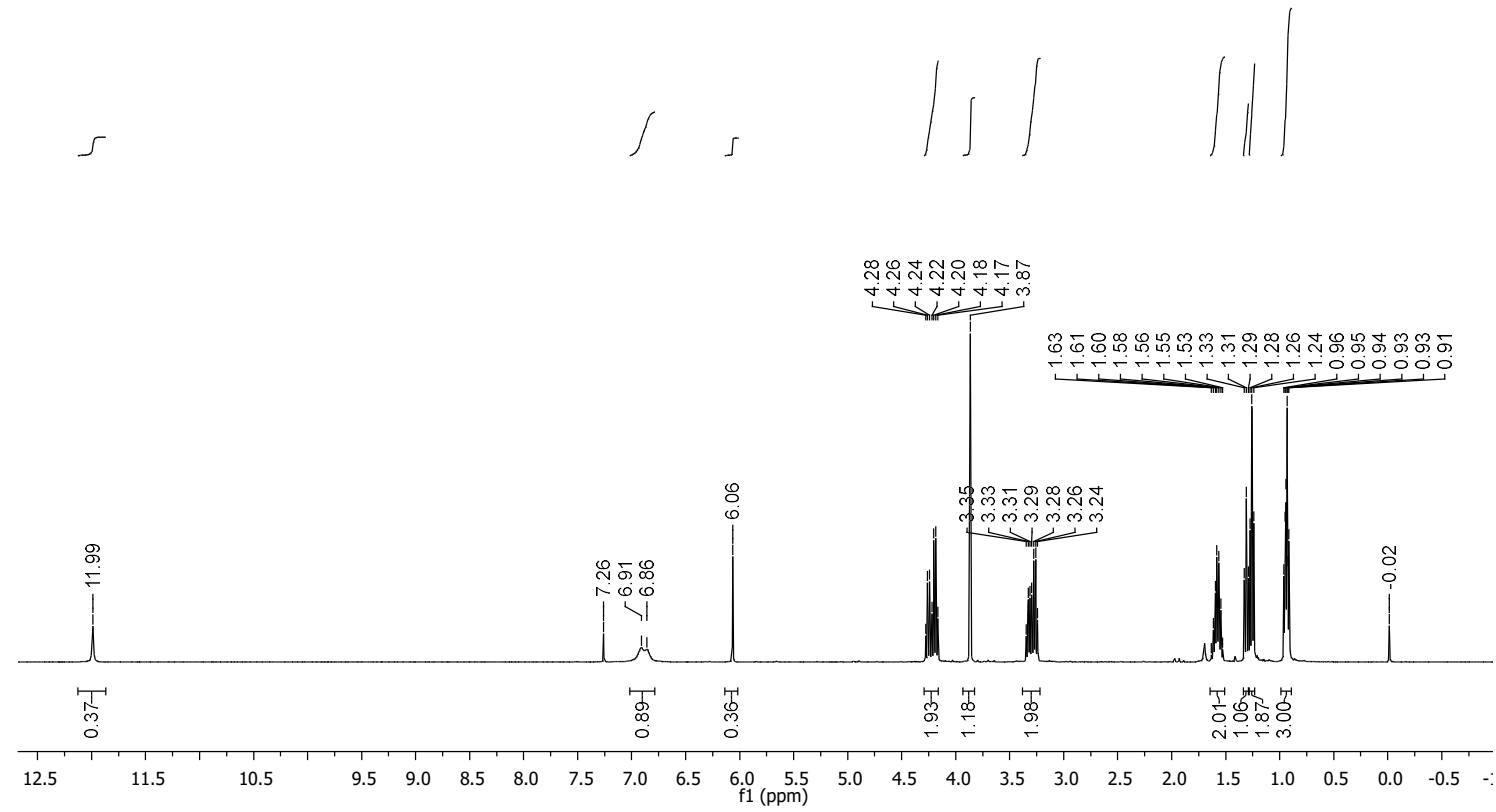

${ }^{13} \mathrm{C}$ NMR (101 MHz, $\left.\mathrm{CDCl}_{3}\right)$.

SKG785 batch 1

C13APTlong.w.CDCl3
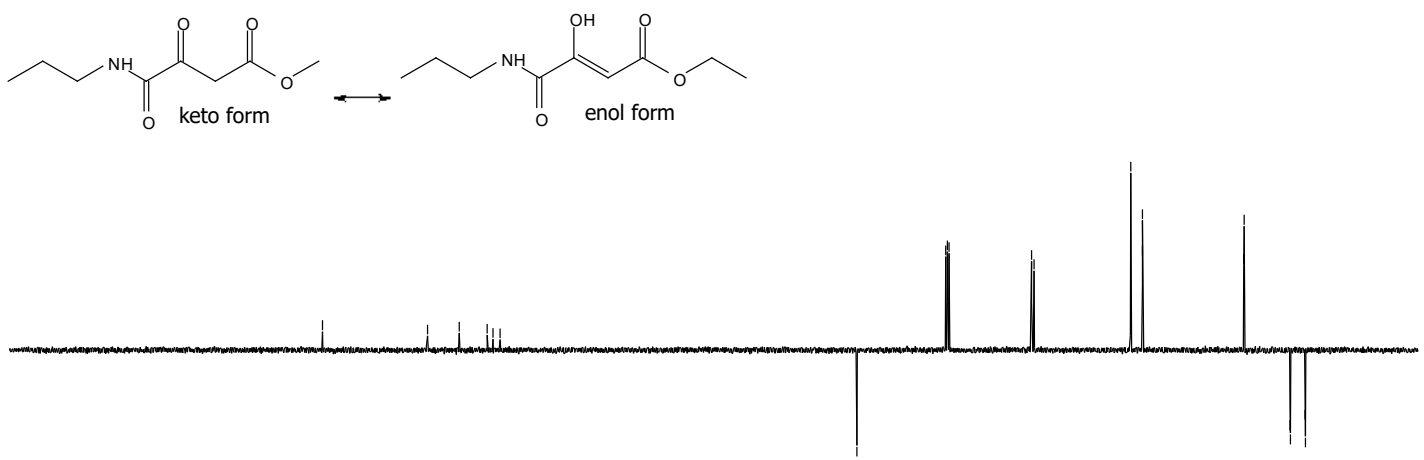

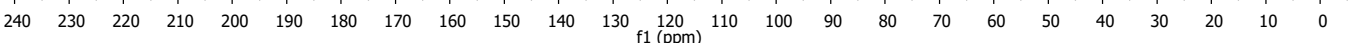


COSY.

SKG785 batch 1

COSY.w CDCl3 /opt/topspin3.5pl2 SKG 34

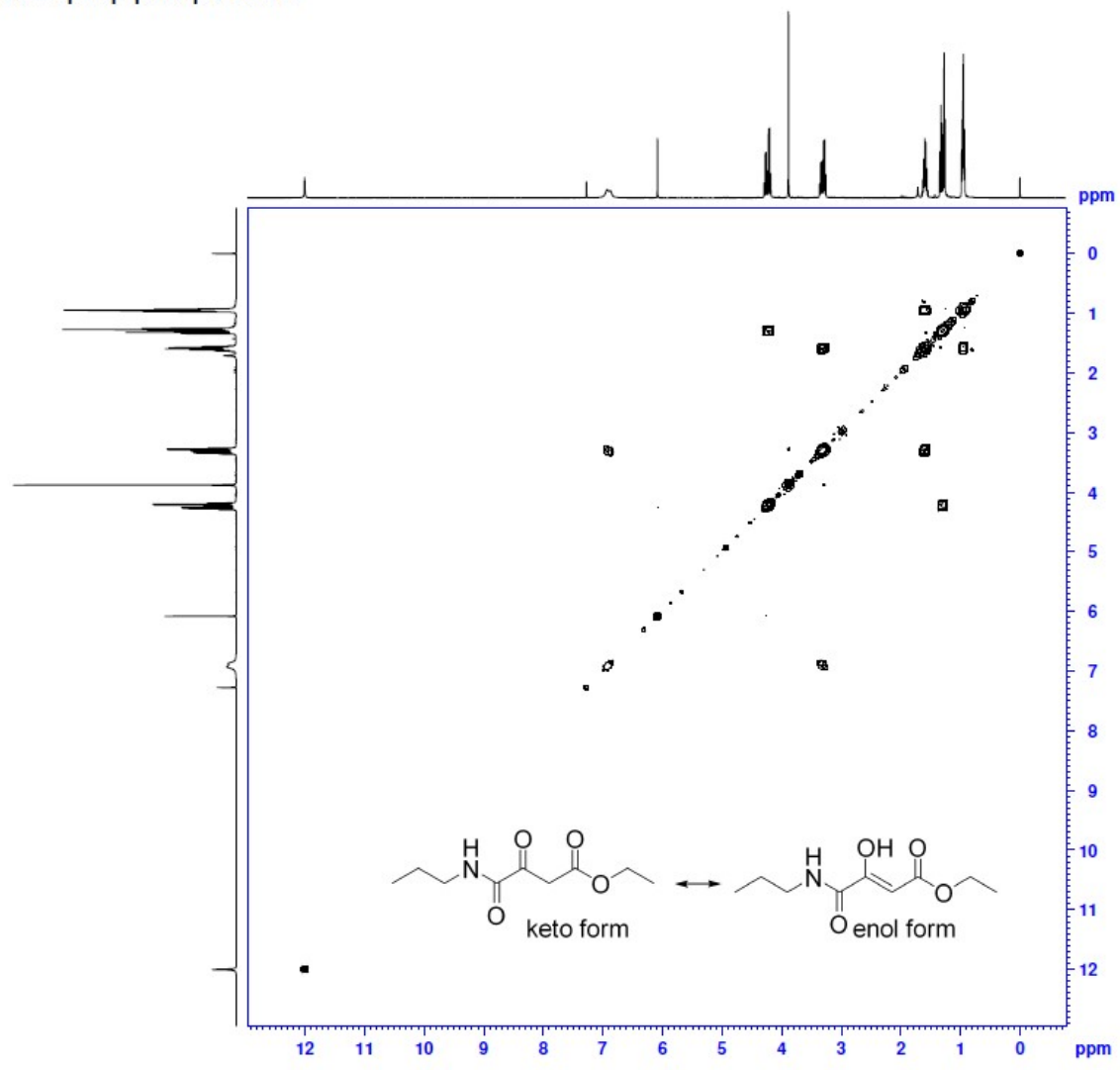

HSQC.

SKG785 batch 1
HSQC.w CDCl3 $/$ opt/topspin 3.5pl2 SKG 34

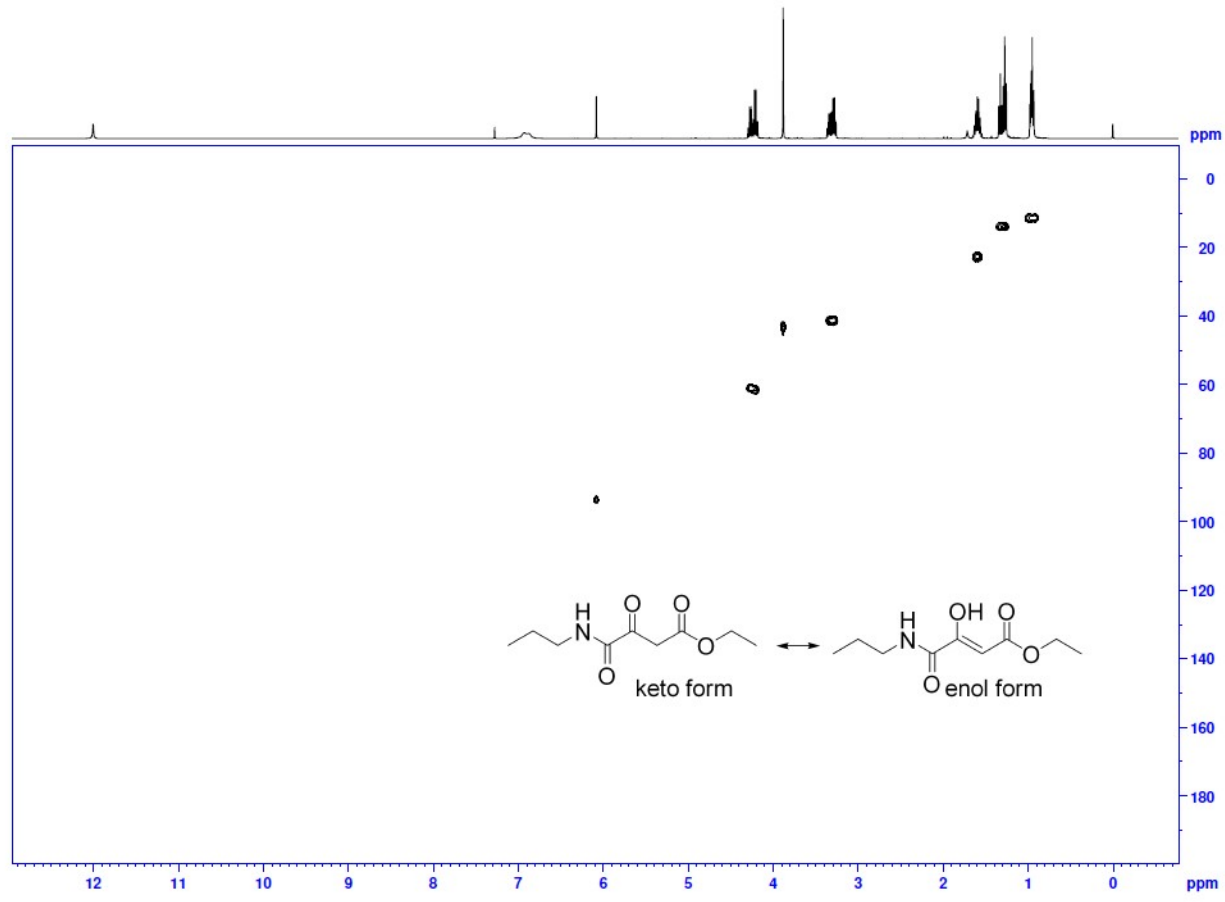


2-Oxo- $N$-phenylhexanamide (precursor of 43).<smiles>CCCCC(=O)C(=O)Nc1ccccc1</smiles>

This compound is reported and has been characterised fully.

To a $25 \mathrm{ml}$ oven dried one neck RBF was charged methyl 2-oxo-2-(phenylamino) acetate (0.179 g, $1.00 \mathrm{mmol}, 1.0 \mathrm{eq})$ and dissolved in anhydrous THF (5 mL) under nitrogen atmosphere. The solution was cooled to $-78^{\circ} \mathrm{C}$, followed by dropwise addition of $\mathrm{n}$-BuLi (1 $\mathrm{M}$ in THF) (1.0 mL, $1.00 \mathrm{mmol}, 1.0 \mathrm{eq})$ and the resulting reaction mixture was stirred at same temperature for $2 \mathrm{~h}$. The reaction was quenched with saturated ammonium chloride solution $(10 \mathrm{~mL})$ and DCM $(5 \mathrm{~mL})$ was added, and the organic layer was separated. The aqueous layer was extracted with DCM $(2 \times 5 \mathrm{~mL})$. The combined organic layers were washed with brine $(30 \mathrm{~mL})$ and dried over MgSO4. The organic layer was concentrated under reduced pressure to generate crude material which was further purified by column chromatography $(10 \%$ EtOAc in petroleum ether) to afford the product as a white solid $(0.095 \mathrm{~g}, 0.463 \mathrm{mmol}, 46 \%)$. TLC: $\mathrm{R}_{\mathrm{f}}$ ca 0.6 (8:2, hexane: EtOAc), UV-active and strong $\mathrm{KMnO}_{4}$; HRMS (ESI) m/z: [M+Na] ${ }^{+}$Calcd for $\mathrm{C}_{12} \mathrm{H}_{15} \mathrm{NO}_{2} \mathrm{Na} 228.1010$; Found 228.0995 (error -2.0 ppm);

${ }^{1} \mathrm{H}$ NMR (400 MHz, $\left.\mathrm{CDCl}_{3}\right) \delta 8.75(1 \mathrm{H}, \mathrm{s}, \mathrm{CONH}), 7.64(2 \mathrm{H}, \mathrm{d}, J=7.8 \mathrm{~Hz}, \mathrm{ArH}), 7.39-7.25$ $(2 \mathrm{H}, \mathrm{m}, \mathrm{ArH}), 7.17(1 \mathrm{H}, \mathrm{t}, J=7.4 \mathrm{~Hz}, \mathrm{ArH}), 3.01\left(2 \mathrm{H}, \mathrm{t}, J=7.4 \mathrm{~Hz}, \mathrm{COC}_{2} \mathrm{CH}_{2}\right), 1.69-1.62$ $\left(2 \mathrm{H}, \mathrm{m}, \mathrm{COCH}_{2} \mathrm{CH}_{2}\right), 1.44-1.35\left(2 \mathrm{H}, \mathrm{m}, \mathrm{C}_{2} \mathrm{CH}_{3}\right), 0.95\left(3 \mathrm{H}, \mathrm{t}, J=7.3 \mathrm{~Hz}, \mathrm{CH}_{3}\right)$;

${ }^{13} \mathrm{C}$ NMR $\left(101 \mathrm{MHz}, \mathrm{CDCl}_{3}\right) \delta 199.6(\mathrm{C}=\mathrm{O}$ ketone $), 157.7(\mathrm{C}=\mathrm{O}$ amide $), 136.5,129.4,125.4$, $119.9,36.2,25.6,25.4,13.9$;

$m / z(\mathrm{ESI}) 228.1\left[(\mathrm{M}+\mathrm{Na})^{+}, 100 \%\right]$.

Huang, H.; He, G.; Xiaolin Zhu, X.; Jin, X.; Qiu S,; Zhu, H. Iodine-Mediated Oxidation of Ynamides: A Facile Access to N-Monosubstituted $\alpha$-Ketoamides and $\alpha$-Ketoimides, Eur. $J$. Org. Chem. 2014, 7174-7183.

Sagadevan, A.; Ragupathi, A.; Lin, C.-C.; Hwu, J. R.; Hwang, K. C. Visible-light initiated copper(i)-catalysed oxidative $\mathrm{C}-\mathrm{N}$ coupling of anilines with terminal alkynes: one-step synthesis of $\alpha$-ketoamides, Green Chem. 2015, 17, 1113-1119. 
2-Oxo- $N$-phenylhexanamide.

${ }^{1} \mathrm{H}$ NMR (400 MHz, $\mathrm{CDCl}_{3}$ ).
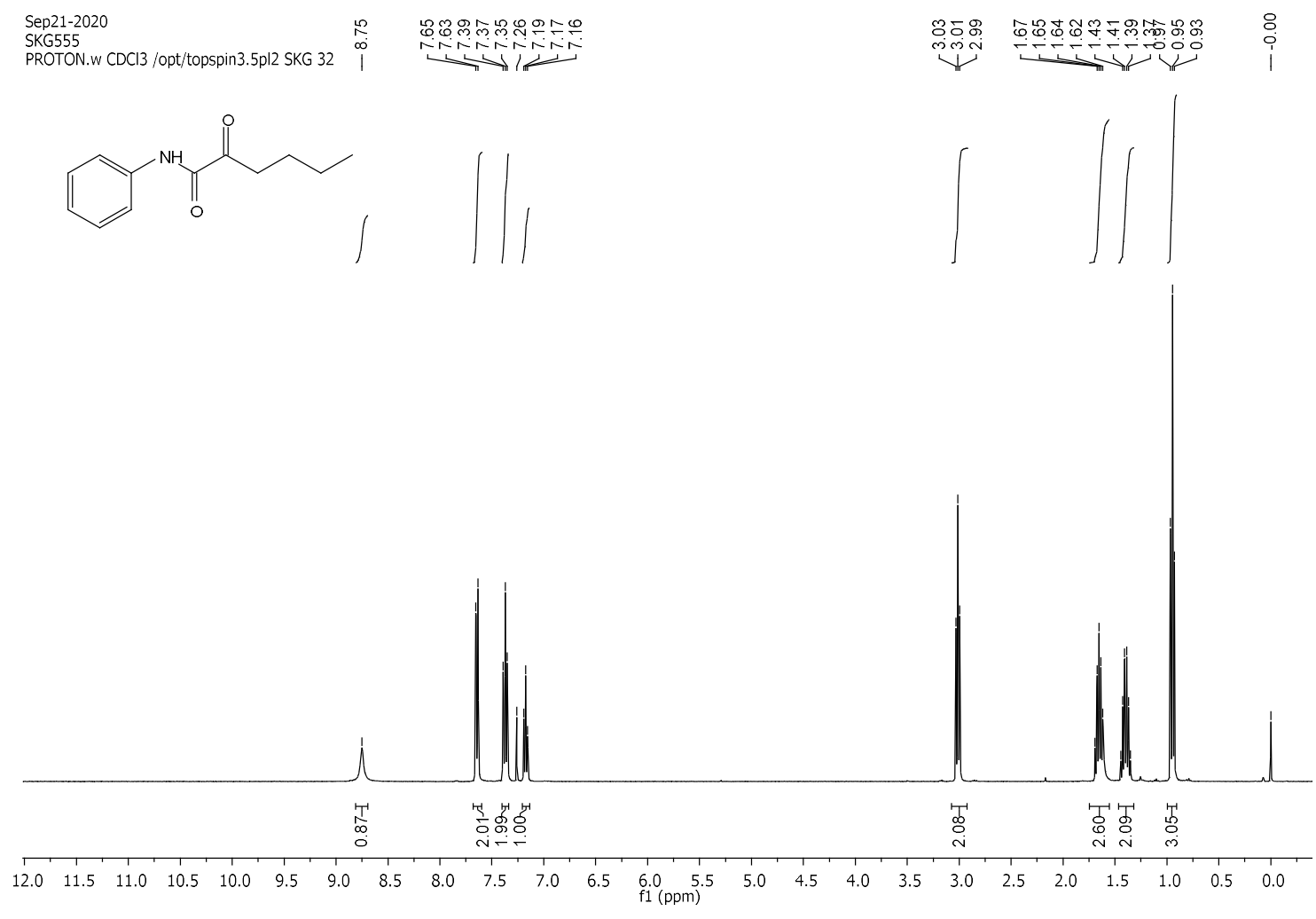

${ }^{13} \mathrm{C}$ NMR (101 MHz, $\left.\mathrm{CDCl}_{3}\right)$.

Sep21-2020

SKG555

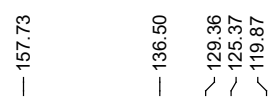

䠝

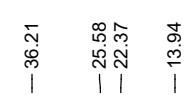

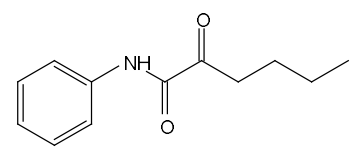
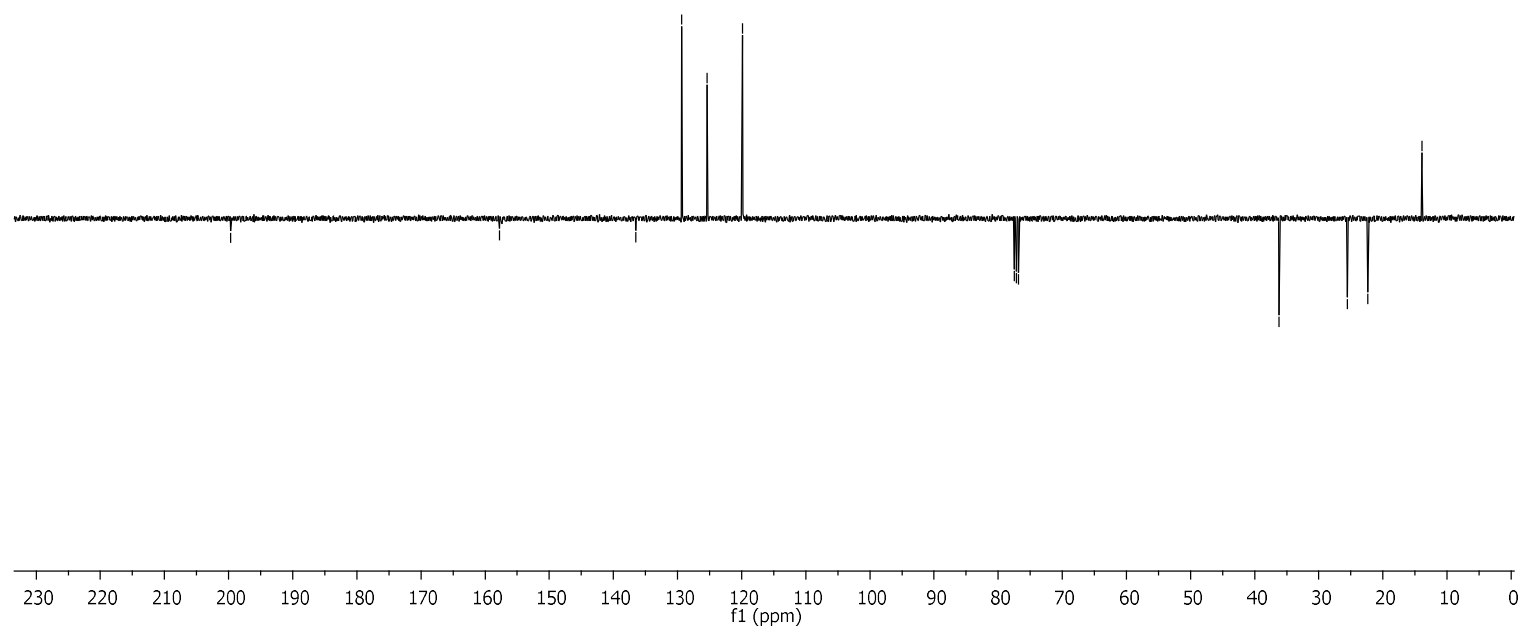

S177 
COSY.

SKG555

COSY.w CDCl3 /opt/topspin3.5pl2 SKG 32

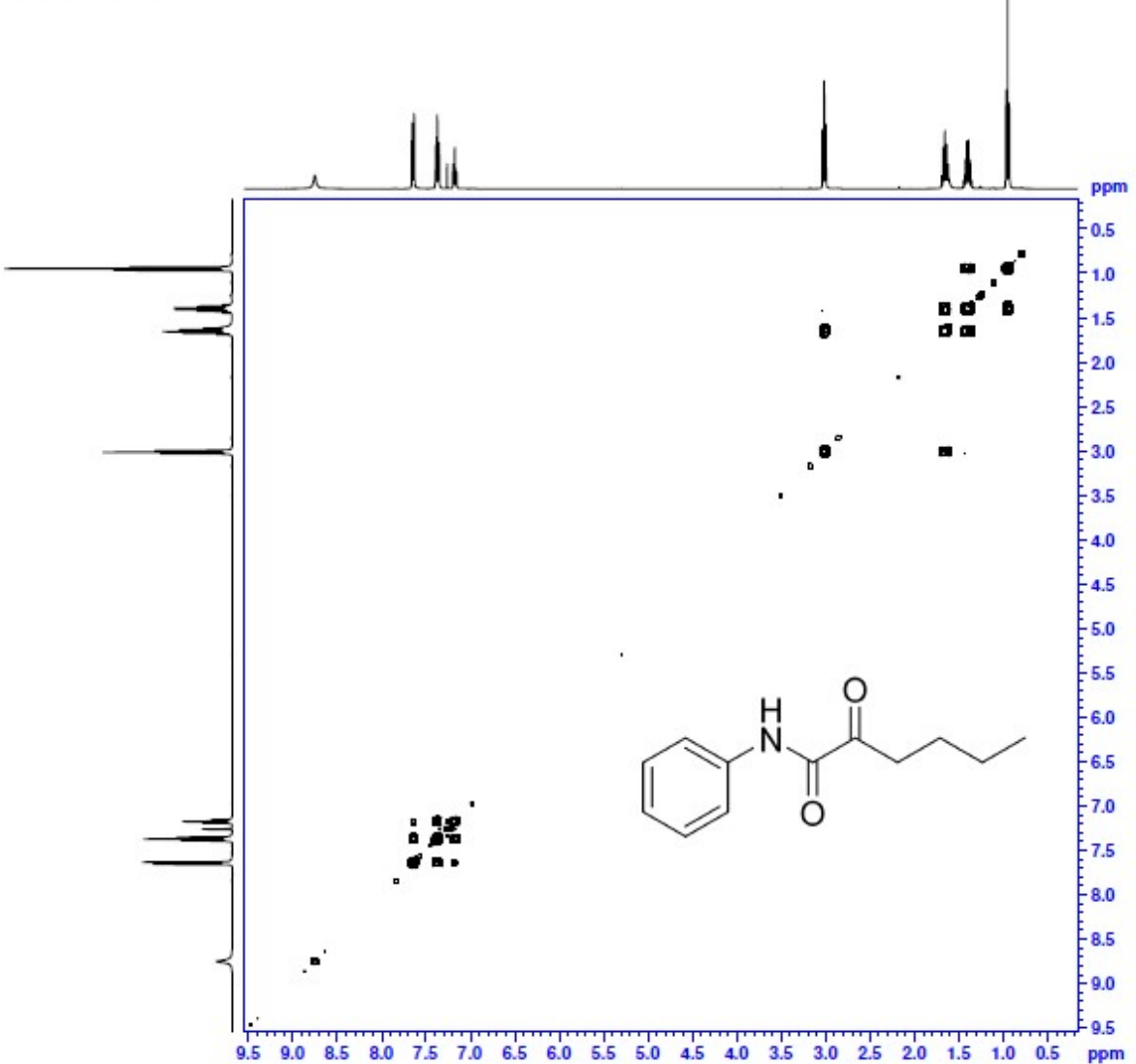


$\mathrm{N}$-Ethyl-2-oxohexanamide (precursor of 44).<smiles>CCCCC(=O)C(=O)NCC</smiles>

This compound is known but has not been characterised fully.

To a solution of ethyl 2-oxohexanoate $(0.150 \mathrm{~g}, 0.936 \mathrm{mmol}, 1.0 \mathrm{eq})$ in dichloromethane (2 $\mathrm{mL}$ ) was added Aliquat $336(4 \mathrm{mg})$ and $68 \%$ aqueous ethylamine $(0.042 \mathrm{~g}, 0.936 \mathrm{mmol}, 1.0$ eq) and the resulting reaction mixture was stirred at room temperature for $72 \mathrm{~h}$. After the completion of the reaction, the reaction mixture was dried over $\mathrm{MgSO}_{4}$, filtered, and concentrated under reduced pressure to generate crude material which was further purified by column chromatography (30\% EtOAc in petroleum ether) to afford the product as a clear oil $(0.0823 \mathrm{~g}, 0.522 \mathrm{mmol}, 56 \%)$.

TLC: $\mathrm{R}_{\mathrm{f}}$ ca 0.5 (8:2, hexane: EtOAc), UV-active and strong $\mathrm{KMnO}_{4}$;

HRMS (ESI) m/z: $[\mathrm{M}+\mathrm{Na}]^{+}$Calcd for $\mathrm{C}_{8} \mathrm{H}_{15} \mathrm{NO}_{2} \mathrm{Na}$ 180.0995; Found 180.0999 (error -2.5 ppm);

${ }^{1} \mathrm{H}$ NMR $\left(400 \mathrm{MHz}, \mathrm{CDCl}_{3}\right) \delta 6.96(1 \mathrm{H}, \mathrm{s}, \mathrm{CONH}), 3.35-3.38\left(2 \mathrm{H}, \mathrm{m}, \mathrm{CH}_{2} \mathrm{CH}_{3}\right.$ of ethyl group), 2.89 (2H, t $\left.J=7.4 \mathrm{~Hz}, \mathrm{COC}_{2} \mathrm{CH}_{2}\right), 1.60-1.52\left(2 \mathrm{H}, \mathrm{m}, \mathrm{COCH}_{2} \underline{\mathrm{CH}}_{2}\right), 1.40-1.28(2 \mathrm{H}$, m, $\underline{\mathrm{C}}_{2} \mathrm{CH}_{3}$ of butyl group), $1.16\left(3 \mathrm{H}, \mathrm{t}, J=7.3 \mathrm{~Hz}, \mathrm{CH}_{2} \underline{\mathrm{CH}}_{3}\right.$ of ethyl group), 0.89 (3H, t, $J=$ $7.3 \mathrm{~Hz}, \mathrm{CH}_{2} \underline{\mathrm{C}}_{3}$ of butyl group);

${ }^{13} \mathrm{C}$ NMR (101 MHz, $\left.\mathrm{CDCl}_{3}\right) \delta 199.6(\mathrm{C}=\mathrm{O}$, ketone), 160.2, ( $\mathrm{C}=\mathrm{O}$ amide), 36.5, 34.3, 25.4, $22.3,14.5,13.9$;

$m / z(\mathrm{ESI}) 180.1\left[(\mathrm{M}+\mathrm{Na})^{+}, 100 \%\right]$.

Seyferth, D.; Hui, R. C. High yield acyl anion trapping reactions: direct nucleophilic acylation of isocyanates and isothiocyanates., Tetrahedron Lett. 1984, 25, 5251-5254. 
Ethyl 2-oxohexanoate.

${ }^{1} \mathrm{H}$ NMR (400 MHz, $\left.\mathrm{CDCl}_{3}\right)$.

Nov02-2020
SKG615

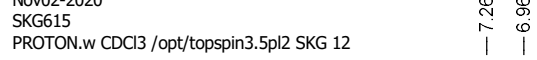

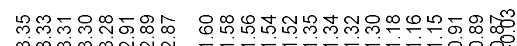<smiles>CCCCC(=O)C(=O)NCC</smiles>
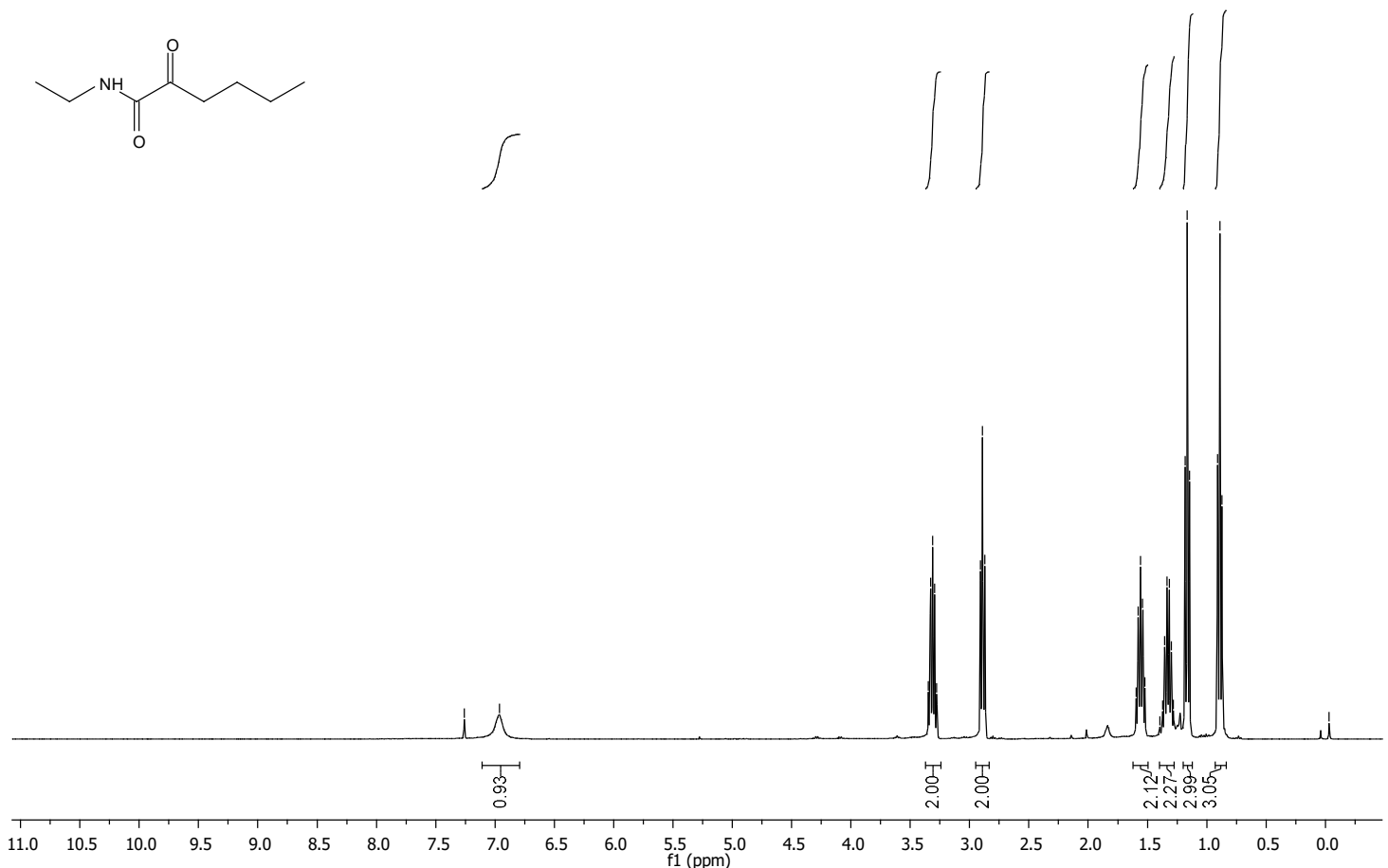

${ }^{13} \mathrm{C}$ NMR $\left(101 \mathrm{MHz}, \mathrm{CDCl}_{3}\right)$.

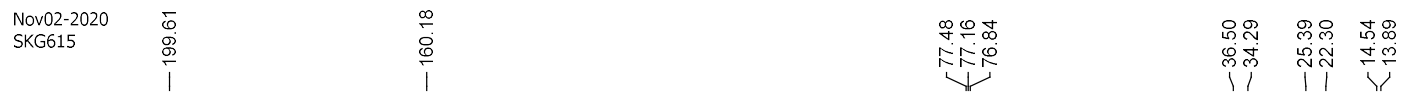<smiles>CCCCC(=O)C(=O)NCC</smiles>

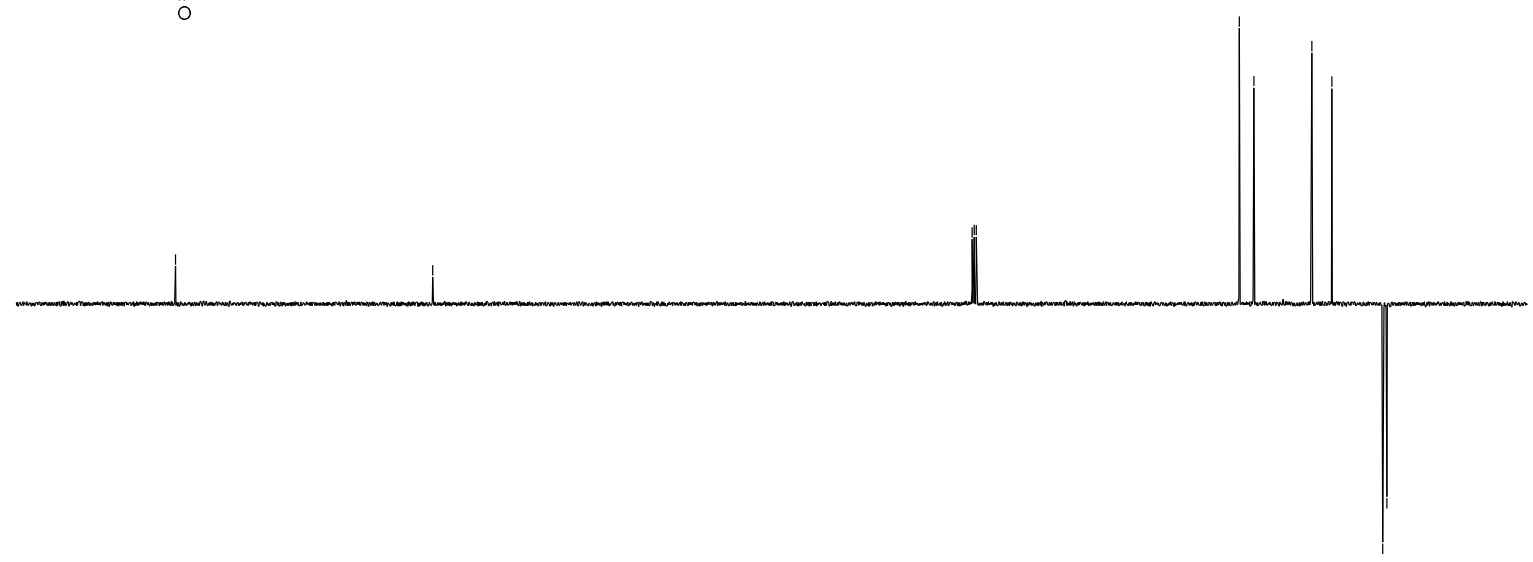

$\begin{array}{llllllllllllllllllllllllllllll}220 & 210 & 200 & 190 & 180 & 170 & 160 & 150 & 140 & 130 & 120 & 110 & 100 & 90 & 80 & 70 & 60 & 50 & 40 & 30 & 20 & 10 & 0\end{array}$ 
COSY.

SKG615

COSY.w CDCl3/opt/topspin3.5pl2 SKG 12

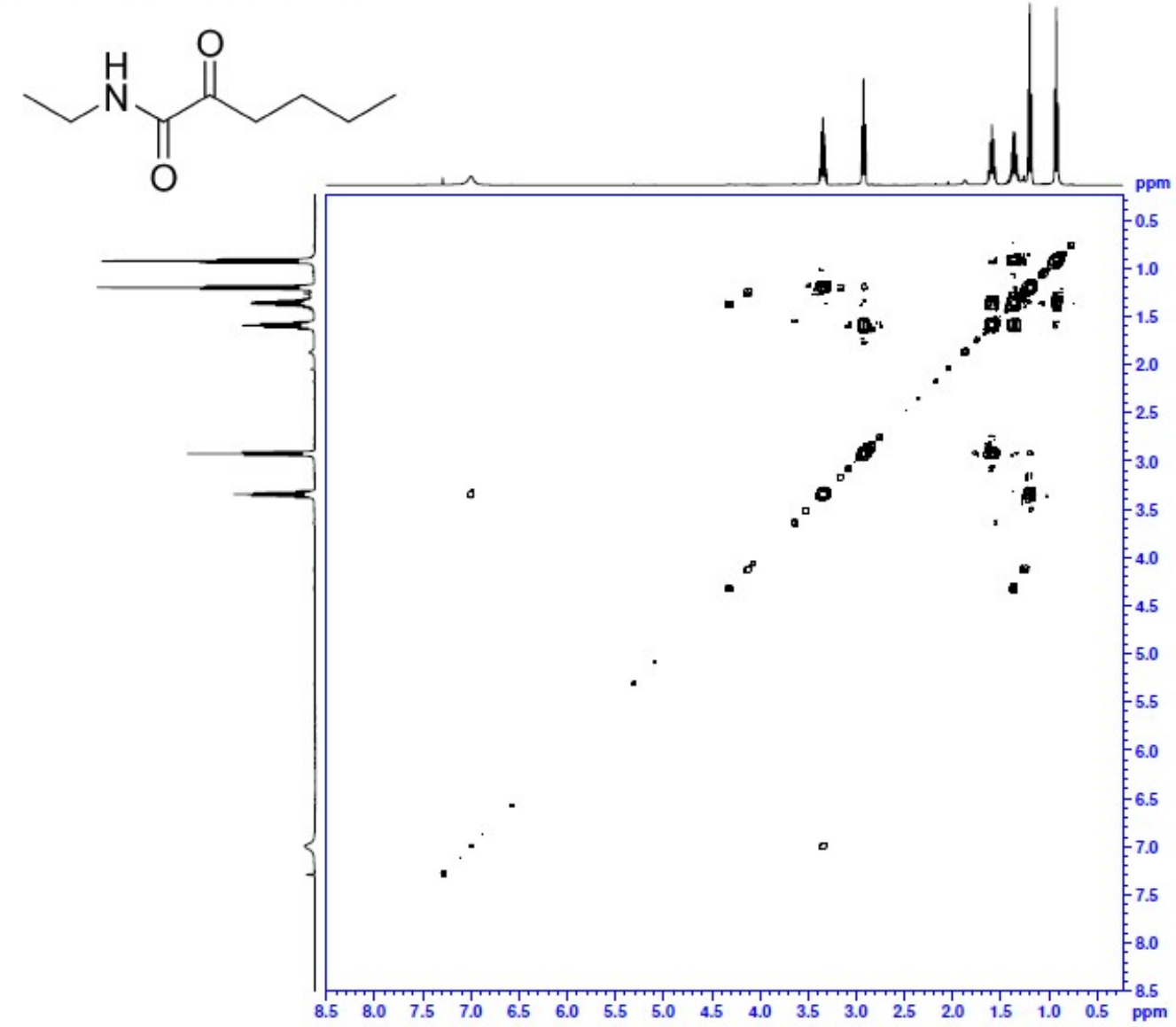


Synthesis of $N$-methoxy- $N$-methyl-2-oxohexanamide (precursor of 45).

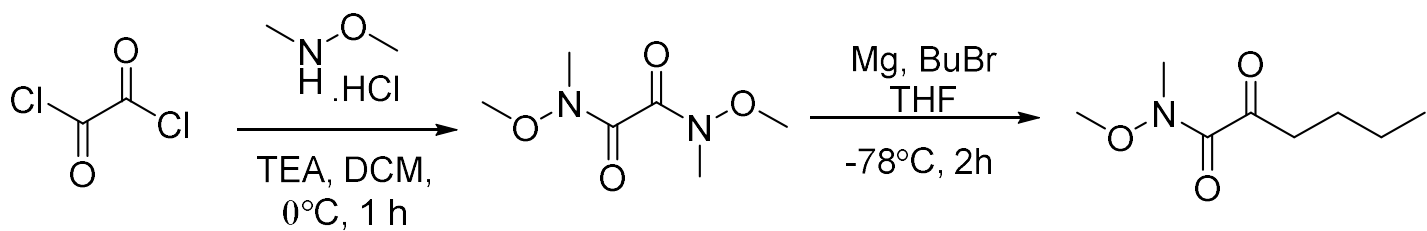

$N^{1}, N^{2}$-Dimethoxy- $N^{1}, N^{2}$-dimethyloxalamide.<smiles>CON(C)C(=O)C(=O)N(C)OC</smiles>

This compound is reported and has been characterised fully.

A solution of $\mathrm{N}, \mathrm{O}$-dimethyl hydroxylamine hydrochloride $(2.31 \mathrm{~g}, 23.7 \mathrm{mmol}, 2.0 \mathrm{eq})$ in dichloromethane $(23 \mathrm{~mL})$ was cooled to $0{ }^{\circ} \mathrm{C}$ and oxalyl chloride $(1.00 \mathrm{~mL}, 11.8 \mathrm{mmol}, 1.0$ eq) was added all at once at the same temperature. A solution of triethylamine $(6.6 \mathrm{~mL}, 47.8$ mmol, $2.0 \mathrm{eq})$ in dichloromethane $(18 \mathrm{~mL})$ was added dropwise over $30 \mathrm{~min}$ and the resulting suspension was stirred at $0{ }^{\circ} \mathrm{C}$ for $1 \mathrm{~h}$. The reaction was quenched with water $(100 \mathrm{~mL})$ and the aqueous layer was extracted with dichloromethane $(2 \times 50 \mathrm{~mL})$. The combined organic layers were washed with brine $(30 \mathrm{~mL})$ and dried over $\mathrm{MgSO}_{4}$. The organic layer was concentrated under reduced pressure up to $20 \mathrm{~mL}$, and cyclohexane $(30 \mathrm{~mL})$ was added dropwise to the obtained white solid. The resulting white suspension was stirred for $30 \mathrm{~min}$ at room temperature, filtered, washed with cyclohexane $(20 \mathrm{~mL})$, and finally dried under high vacuum to afford the product as a white solid (1.81 $\mathrm{g}, 10.3 \mathrm{mmol}, 87 \%)$.

${ }^{1} \mathrm{H}$ NMR (400 MHz, DMSO-d $) \delta 3.66\left(3 \mathrm{H}, \mathrm{s},-\mathrm{OCH}_{3}\right), 3.16\left(3 \mathrm{H}, \mathrm{s},-\mathrm{NCH}_{3}\right)$;

${ }^{13} \mathrm{C}$ NMR (101 MHz, DMSO- $\left.d_{6}\right) \delta 164.1,61.7,30.8$

Guercio, G.; Bacchi, S.; Goodyear, M.; Carangio, A.; Tinazzi, F.; Curti, S. Synthesis of the NK1 Receptor Antagonist GW597599. Part 1: Development of a Scalable Route to a Key Chirally Pure Arylpiperazine. Org. Process Res. Dev. 2008, 12, 6, 1188-1194.

Sibi, M. P.; Marvin, M.; Sharma, R.; alpha Keto Amides and 1,2-Diketones from $N, N^{\prime}-$ Dimethoxy- $N, N^{\prime}$-dimethylethanediamide. A Synthetic and Mechanistic Investigation. J. Org. Chem. 1995, 60, 5016-5023. 
$N^{1}, N^{2}$-Dimethoxy- $N^{1}, N^{2}$-dimethyloxalamide.

${ }^{1} \mathrm{H}$ NMR (400 MHz, DMSO-d6).

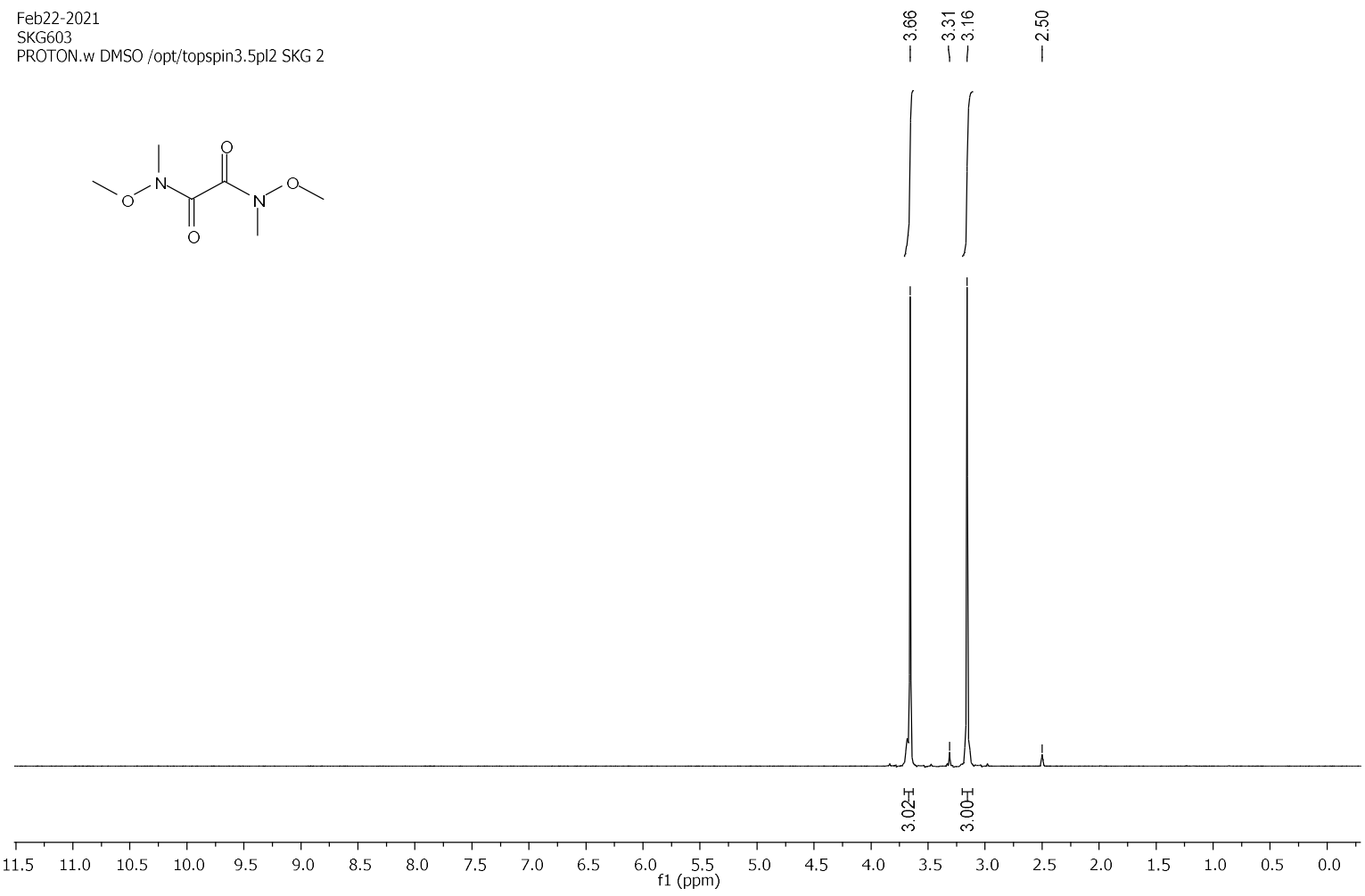

${ }^{13} \mathrm{C}$ NMR (101 MHz, DMSO- $\left.d_{6}\right)$.

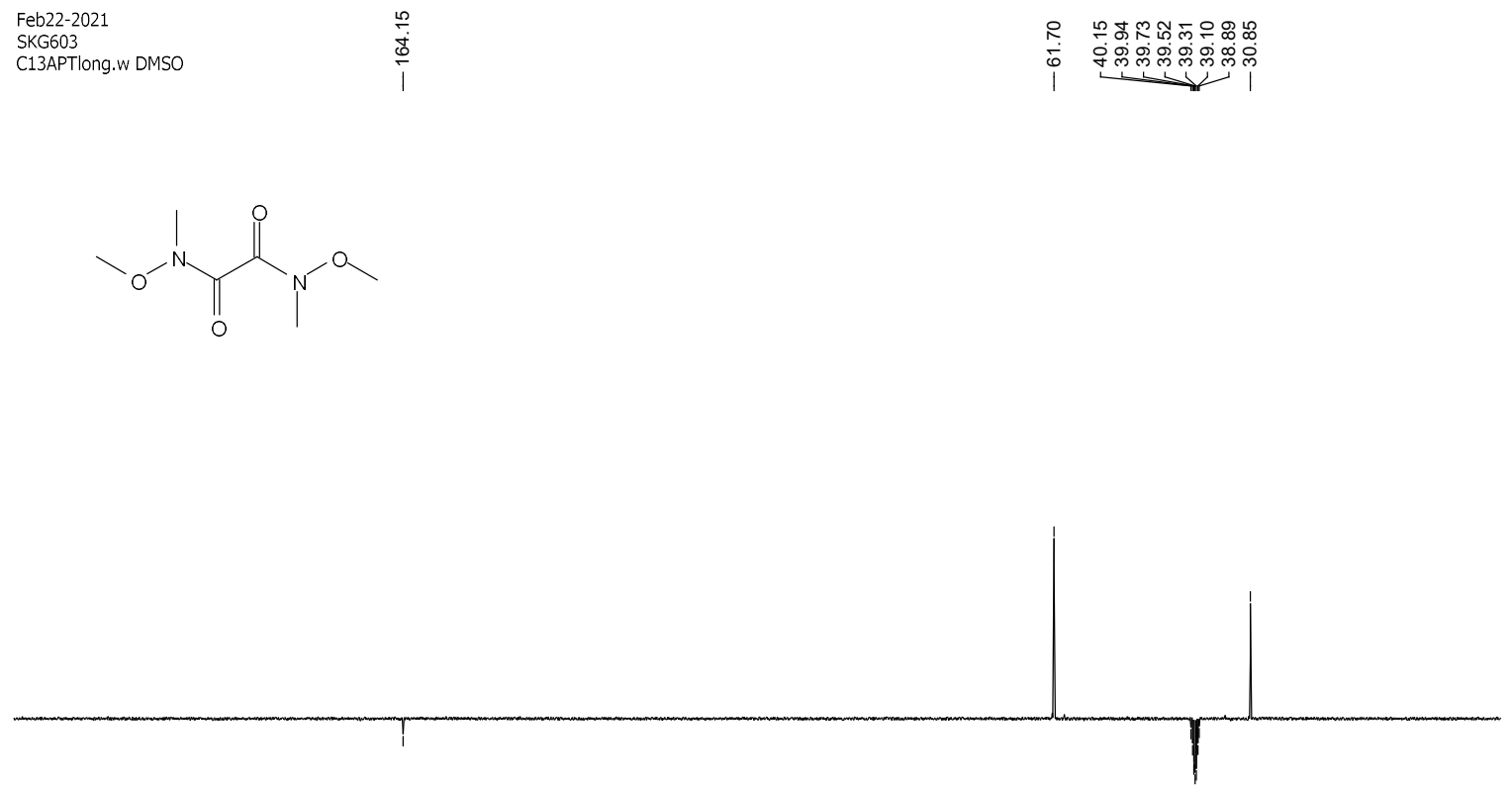

$\begin{array}{lllllllllllllllllllllllllll}220 & 210 & 200 & 190 & 180 & 170 & 160 & 150 & 140 & 130 & 120 & \begin{array}{l}110 \\ \mathrm{f1}(\mathrm{ppm})\end{array} & 100 & 90 & 80 & 70 & 60 & 50 & 40 & 30 & 20 & 10 & 0\end{array}$ 


\section{$N$-Methoxy- $N$-methyl-2-oxohexanamide.}<smiles>CCCCC(=O)C(=O)N(C)OC</smiles>

This compound is reported and has been characterised fully.

In a $25 \mathrm{ml}$ two neck, oven dried RBF was placed activated and dried magnesium turnings $(\mathrm{Mg})(0.200 \mathrm{~g}, 8.00 \mathrm{mmol})$ and a catalytic amount of iodine (a few crystals) and the solution was degassed with vacuum and nitrogen. In another flask, a solution of $n$-butyl bromide in THF $(0.9 \mathrm{~mL}, 8.00 \mathrm{mmol}, 1.1 \mathrm{eq})$ was prepared and $2 \mathrm{~mL}$ of this halide solution was transferred to the flask containing mixture of $\mathrm{Mg}$ and iodine and the mixture was vigorously stirred. After 5 minutes, the colour started to change from orange to grey solution, confirming the activation of the reagent. At this point, the remaining alkyl-halide solution was added slowly dropwise to the round-bottom flask and the resulting reaction mixture was heated at $40^{0} \mathrm{C}$ for complete activation. In another $100 \mathrm{~mL}$ oven dried one neck RBF was charged $N^{l}$, $N 2$-dimethoxy- $N^{1}, N 2$-dimethyloxalamide (1.26 g, $\left.7.21 \mathrm{mmol}, 1.0 \mathrm{eq}\right)$ in THF (3 mL) and cooled to $-78^{\circ} \mathrm{C}$, then the Grignard solution was added to this dropwise. Once the addition was complete, the resulting reaction mixture was stirred at $-78^{\circ} \mathrm{C}$ for $2 \mathrm{~h}$, followed by a fast quenching of the reaction at $-78^{\circ} \mathrm{C}$ with $3 \mathrm{~N}$ hydrochloric acid aqueous solution $(20 \mathrm{~mL})$. The reaction was warmed to room temperature and DCM $(20 \mathrm{~mL})$ was added, and the organic layer was separated. The aqueous layer was extracted with DCM $(2 \times 20 \mathrm{~mL})$. The combined organic layers were washed with brine $(60 \mathrm{~mL})$ and dried over $\mathrm{MgSO}_{4}$. The organic layer was concentrated under reduced pressure to generate crude material which was further purified by column chromatography (10\% EtOAc in petroleum ether) to afford the product as a clear oil (0.653 g, $3.77 \mathrm{mmol}, 52 \%)$.

TLC: $\mathrm{R}_{\mathrm{f}}$ ca 0.5 (7:3, hexane: EtOAc), UV-active and strong $\mathrm{KMnO}_{4}$;

HRMS (ESI) m/z: $[\mathrm{M}+\mathrm{Na}]^{+}$Calcd for $\mathrm{C}_{8} \mathrm{H}_{15} \mathrm{NO}_{3} \mathrm{Na}$ 196.0944; Found 196.0946 (error -1.1 ppm);

${ }^{1} \mathrm{H}$ NMR $\left(400 \mathrm{MHz}, \mathrm{CDCl}_{3}\right) \delta 3.67\left(3 \mathrm{H}, \mathrm{s},-\mathrm{OCH}_{3}\right), 3.20\left(3 \mathrm{H}, \mathrm{s},-\mathrm{NCH}_{3}\right), 2.67(2 \mathrm{H}, \mathrm{t}, J=7.4$ $\left.\mathrm{Hz}, \mathrm{COC}_{2} \mathrm{CH}_{2}\right), 1.66-1.58\left(2 \mathrm{H}, \mathrm{m}, \mathrm{COCH}_{2} \underline{\mathrm{C}}_{2}\right), 1.40-1.31\left(2 \mathrm{H}, \mathrm{m}, \mathrm{C}_{2} \mathrm{CH}_{3}\right), 0.91(3 \mathrm{H}, \mathrm{t}, J$ $\left.=7.3 \mathrm{~Hz}, \mathrm{CH}_{3}\right)$;

${ }^{13} \mathrm{C}$ NMR $\left(101 \mathrm{MHz}, \mathrm{CDCl}_{3}\right) \delta 200.9(\mathrm{C}=\mathrm{O}$ ketone), $168.6(\mathrm{C}=\mathrm{O}$ amide $), 62.4,39.4,31.2$, 24.7, 22.3, 13.9; $m / z(\mathrm{ESI}) 196.1\left[(\mathrm{M}+\mathrm{Na})^{+}, 100 \%\right]$. 
Sibi, M. P.; Marvin, M.; Sharma, R.; alpha Keto Amides and 1,2-Diketones from N,N'Dimethoxy- $N, N^{\prime}$-dimethylethanediamide. A Synthetic and Mechanistic Investigation. J. Org. Chem. 1995, 60, 5016-5023.

3. 
$N$-Methoxy- $N$-methyl-2-oxohexanamide.

${ }^{1} \mathrm{H}$ NMR $\left(400 \mathrm{MHz}, \mathrm{CDCl}_{3}\right)$.

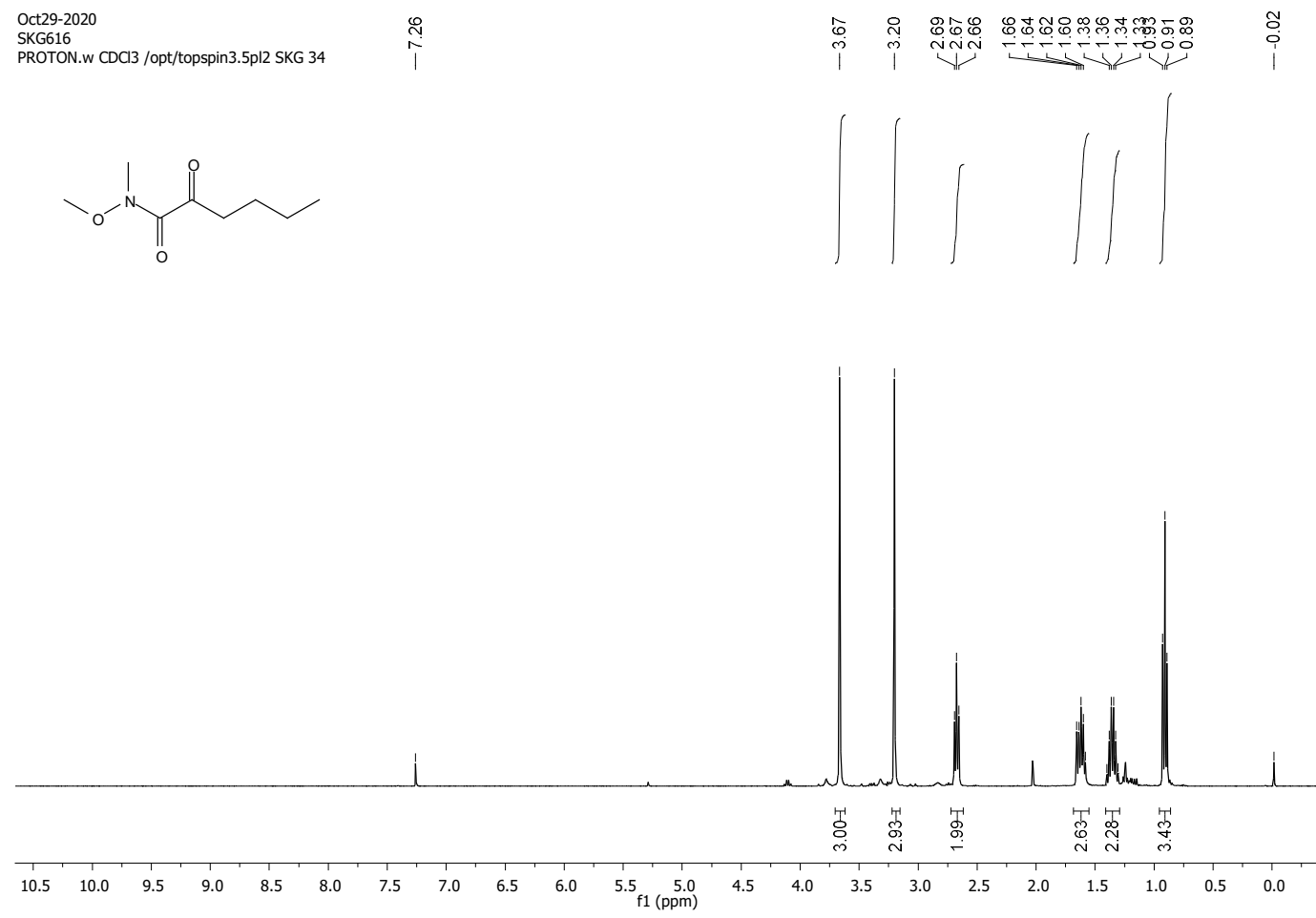

${ }^{13} \mathrm{C}$ NMR (101 MHz, $\left.\mathrm{CDCl}_{3}\right)$.

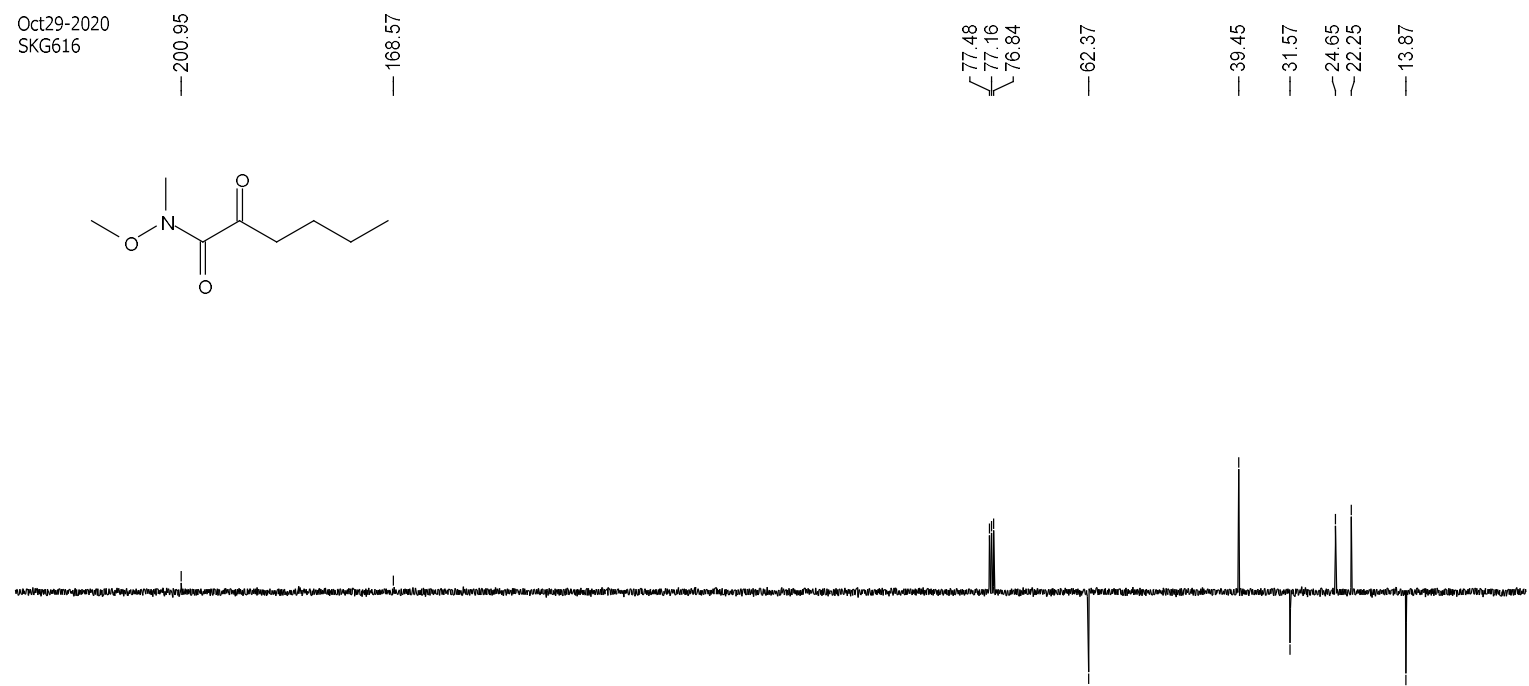

$\begin{array}{lllllllllllllllllllllllllll}220 & 210 & 200 & 190 & 180 & 170 & 160 & 150 & 140 & 130 & 120 & \begin{array}{ll}110 \\ \mathrm{f} 1(\mathrm{ppm})\end{array} & 100 & 90 & 80 & 70 & 60 & 50 & 40 & 30 & 20 & 10 & 0\end{array}$ 
COSY.

SKG616

COSY.w CDCl3/opt/topspin3.5pl2 SKG 34

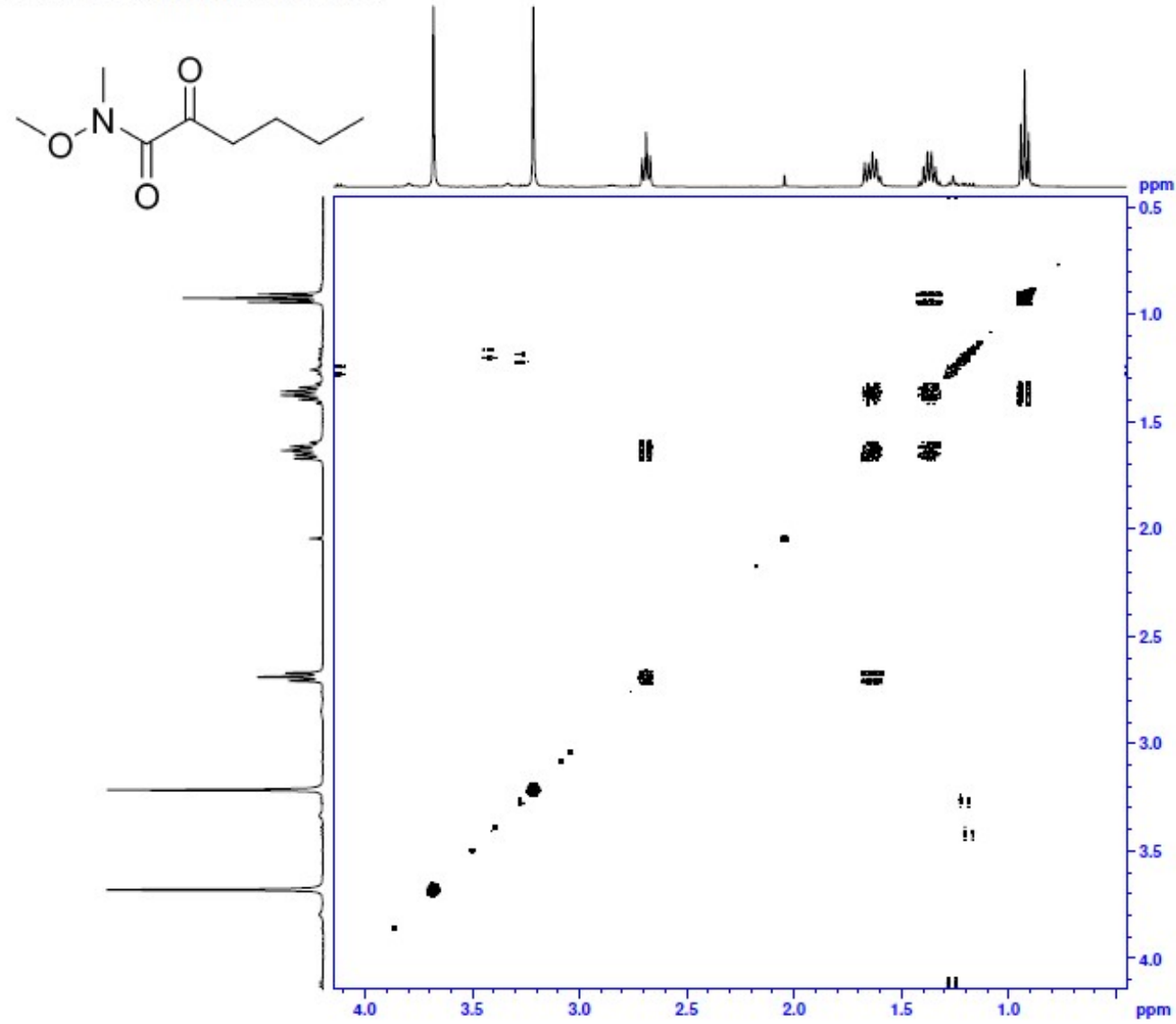




\section{Ethyl 2-oxohexanoate (precursor of 46).}<smiles>CCCCC(=O)C(=O)OCCCCOC(=O)C(=O)OCC</smiles>

This compound is known and was synthesised following similar procedure used for the synthesis of $N$-methoxy- $N$-methyl-2-oxohexanamide using magnesium turnings $(0.200 \mathrm{~g}$, $8.23 \mathrm{mmol}, 1.1 \mathrm{eq})$, n-butyl bromide $(0.90 \mathrm{~mL}, 1.14 \mathrm{~g}, 8.3 \mathrm{mmol}, 1.1 \mathrm{eq})$ and diethyl oxalate $(0.98 \mathrm{~mL}, 7.21 \mathrm{mmol}, 1.0 \mathrm{eq})$ and THF $(8 \mathrm{~mL})$ to generate crude material which was further purified by column chromatography $(10 \%$ EtOAc in petroleum ether) to afford the product as a clear oil $(0.480 \mathrm{~g}, 3.03 \mathrm{mmol}, 42 \%)$.

TLC: $\mathrm{R}_{\mathrm{f}}$ ca 0.5 (8:2, hexane: EtOAc), UV-active and strong $\mathrm{KMnO}_{4}$;

HRMS (ESI) m/z: $[\mathrm{M}+\mathrm{Na}]^{+}$Calcd for $\mathrm{C}_{8} \mathrm{H}_{14} \mathrm{O}_{3} \mathrm{Na} 181.0835$; Found 181.0835 (error 0.3 ppm);

${ }^{1} \mathrm{H}$ NMR (500 MHz, $\left.\mathrm{CDCl}_{3}\right) \delta$ 4.34-4.29 (2H, m, $\underline{\mathrm{CH}}_{2} \mathrm{CH}_{3}$ of ester group), 2.83 (2H, t, $J=7.4$ $\left.\mathrm{Hz}, \mathrm{COC}_{2} \mathrm{CH}_{2}\right), 1.66-1.59\left(2 \mathrm{H}, \mathrm{m}, \mathrm{COCH}_{2} \underline{\mathrm{CH}}_{2}\right), 1.40-1.32\left(5 \mathrm{H}, \mathrm{m}, \mathrm{C}_{2} \mathrm{CH}_{3}\right.$ of butyl group $+\mathrm{CH}_{2} \underline{\mathrm{C}}_{3}$ of ester group), $0.95-0.91$ (3H, m, $\mathrm{CH}_{2} \underline{\mathrm{C}}_{3}$ of butyl group);

${ }^{13} \mathrm{C}$ NMR (126 MHz, $\left.\mathrm{CDCl}_{3}\right) \delta 194.92$ (C=O ketone), 161.4, 62.4, 39.1, 25.2, 22.2, 14.1, 13.7;

$m / z(\mathrm{ESI}) 181.2\left[(\mathrm{M}+\mathrm{Na})^{+}, 100 \%\right]$

Blöcher, R.; Rodarte Ramírez, A.; Castro-Escarpulli, G.; Curiel-Quesada, E.; Reyes-Arellano, A. Design, Synthesis, and Evaluation of Alkyl-Quinoxalin-2(1H)-One Derivatives as AntiQuorum Sensing Molecules, Inhibiting Biofilm Formation in Aeromonas caviae Sch3. Molecules 2018, 23, 3075-3086. 
Ethyl 2-oxohexanoate.

${ }^{1} \mathrm{H}$ NMR $\left(500 \mathrm{MHz}, \mathrm{CDCl}_{3}\right)$.

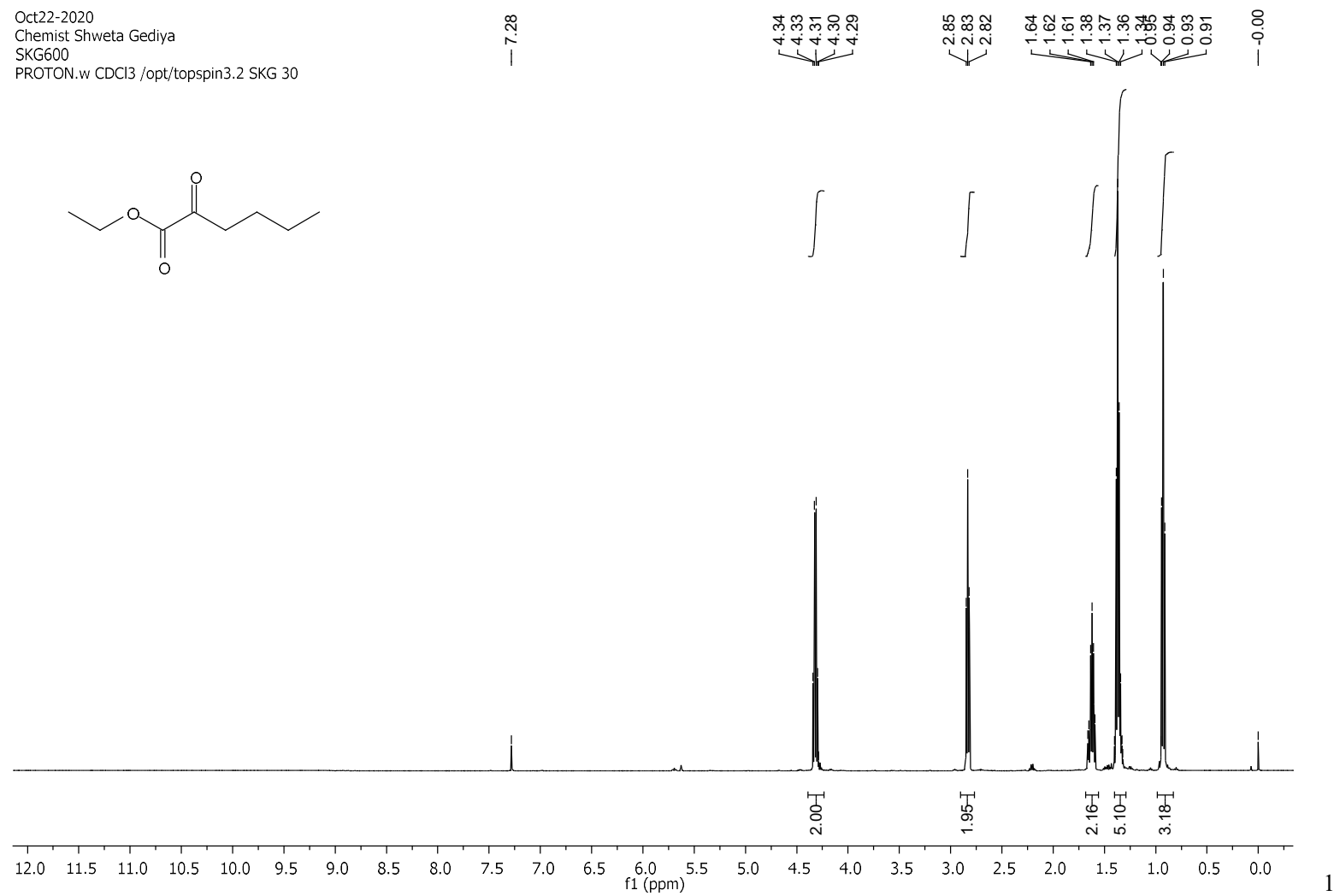

${ }^{13} \mathrm{C}$ NMR (126 MHz, $\left.\mathrm{CDCl}_{3}\right)$.

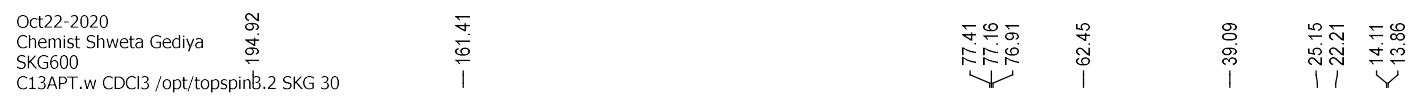
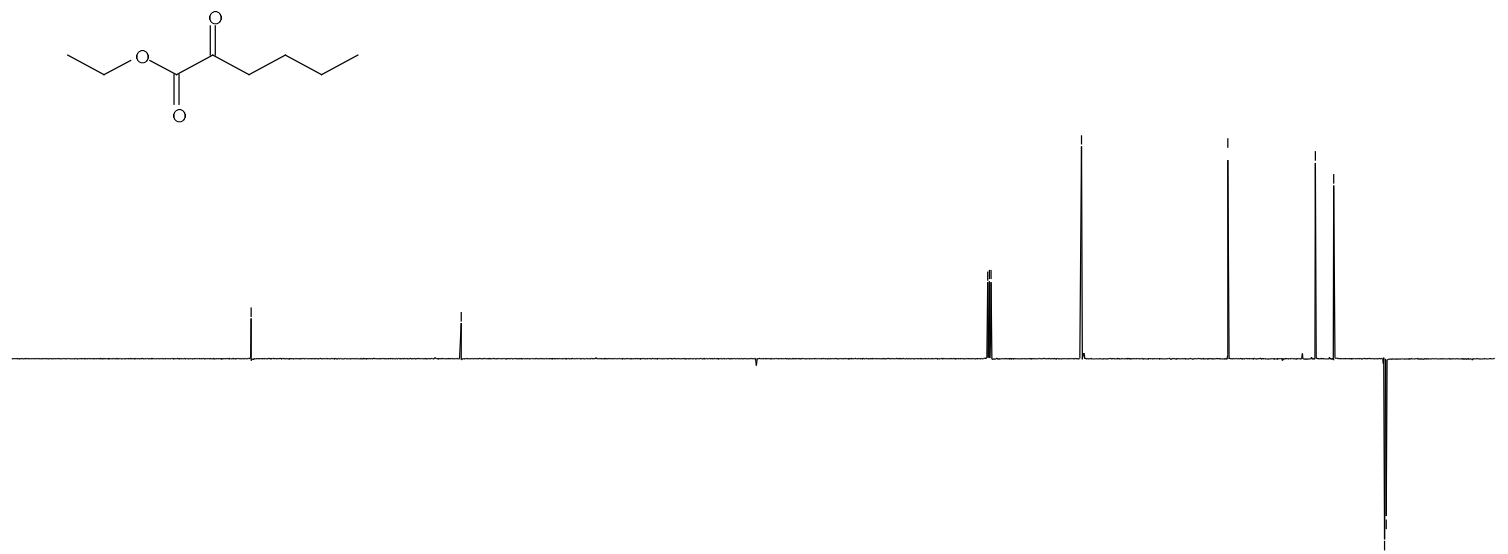

\begin{tabular}{llllllllllllllllllllllll}
\hline 230 & 220 & 210 & 200 & 190 & 180 & 170 & 160 & 150 & 140 & 130 & $\underset{f 1}{120}(\mathrm{ppm})$ & 110 & 100 & 90 & 80 & 70 & 60 & 50 & 40 & 30 & 20 & 10 & 0
\end{tabular} 
COSY.

Chemist Shweta Gediya

SKG600

COSY.w CDCl3/opt/topspin3.2 SKG 30

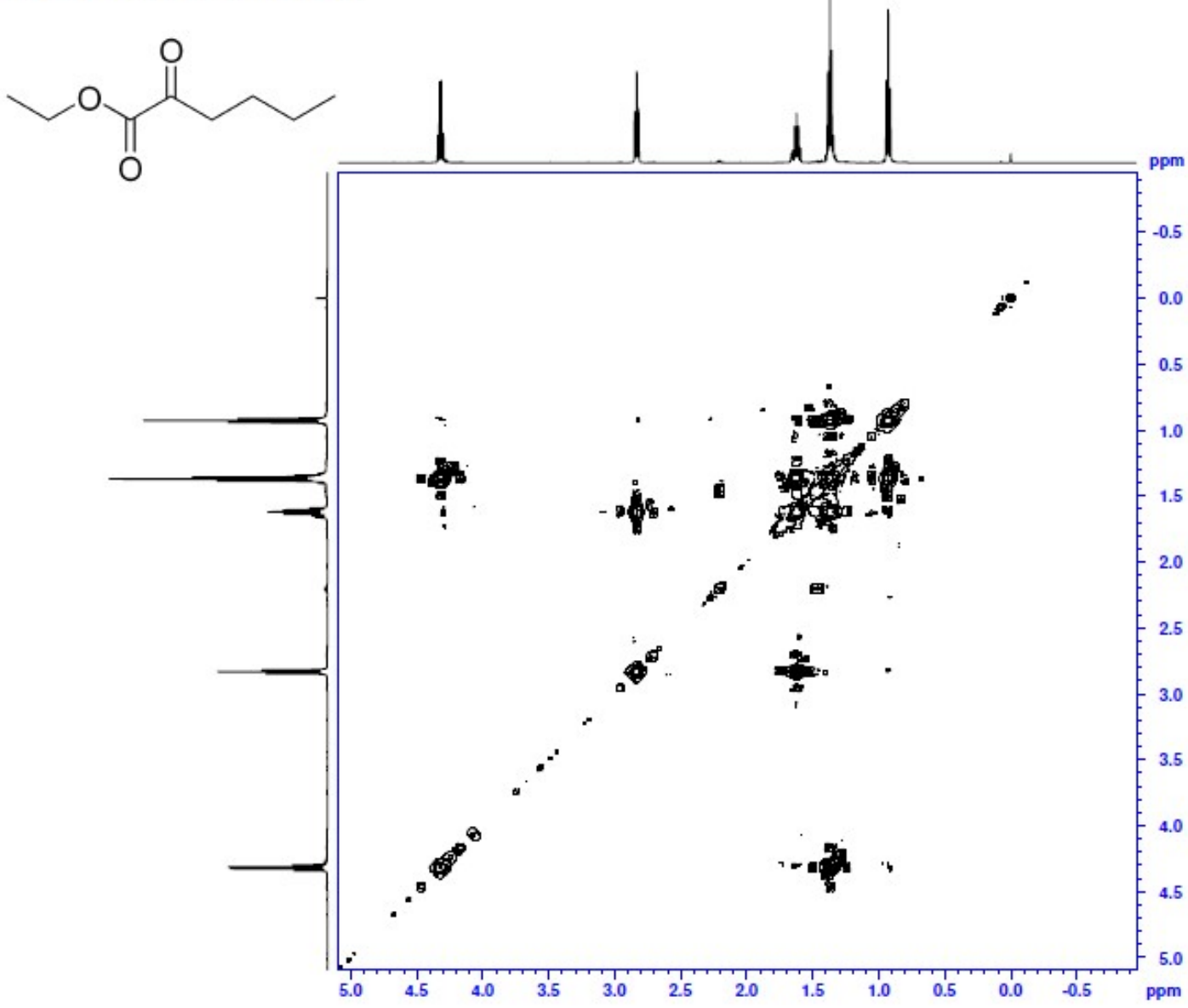


Ethyl 3,5-dioxo-5-(phenylamino)pentanoate (precursor of 47).

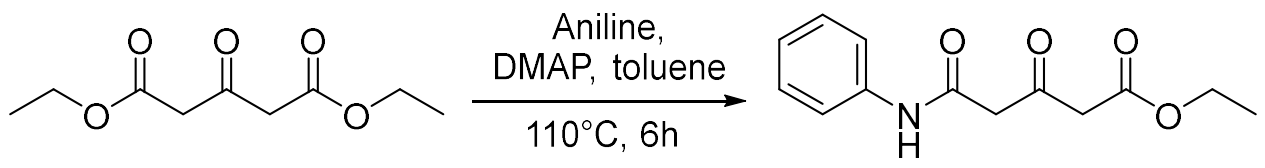

This compound is known and was prepared using reported procedure. A solution of diethyl 3oxoglutarate (0.968 g, $4.79 \mathrm{mmol}, 1.2 \mathrm{eq})$ and 4-dimethylaminopyridine (DMAP) (43 mg, $0.35 \mathrm{mmol}, 0.070 \mathrm{eq})$ in toluene $(20 \mathrm{~mL})$ was heated to $110^{\circ} \mathrm{C}$ followed by addition of aniline $(0.372 \mathrm{~g}, 3.99 \mathrm{mmol}, 1.0 \mathrm{eq})$ at the same temperature and the resulting reaction mixture was refluxed at $110{ }^{\circ} \mathrm{C}$ for $6 \mathrm{~h}$. The reaction mixture was concentrated under reduced pressure. The obtained residue was suspended in water $(50 \mathrm{~mL})$ and extracted with ethyl acetate $(3 \times 30 \mathrm{~mL})$. The combined organic layers were washed with brine solution $(50 \mathrm{~mL})$. The organic layer was dried over $\mathrm{MgSO}_{4}$, filtered and filtrate was concentrated under reduced pressure to give crude material which was further purified by column chromatography (10$20 \%$ EtOAc in petroleum ether) to afford the product as a white solid $(0.420 \mathrm{~g}, 1.68 \mathrm{mmol}$, $43 \%)$. The title compound was obtained as keto/enol tautomer as per ${ }^{1} \mathrm{H}$ NMR.

TLC: $\mathrm{R}_{\mathrm{f}}$ ca 0.4 (7:3, hexane: EtOAc), UV-active and strong $\mathrm{KMnO}_{4}$;

HRMS (ESI) m/z: [M+Na] ${ }^{+}$Calcd for $\mathrm{C}_{13} \mathrm{H}_{15} \mathrm{NO}_{4} \mathrm{Na} 272.0893$; Found 272.0890 (error -1.3 ppm);

${ }^{1} \mathrm{H}$ NMR (500 MHz, $\mathrm{CDCl}_{3}$ ) (keto/enol, 4:1(both enol forms) $\delta 13.64(0.1 \mathrm{H} \mathrm{s}$, br.s., $\mathrm{OH}$ of enol form 1), 12.50 (0.1H s, br.s., $\mathrm{OH}$ of enol form 2), $8.80(1 \mathrm{H}$, br.s., CONH), $7.52(2 \mathrm{H}, \mathrm{d}, J$ $=7.7 \mathrm{~Hz}, \mathrm{ArH}), 7.32(2 \mathrm{H}, \mathrm{t}, J=7.9 \mathrm{~Hz}, \mathrm{ArH}), 7.12(1 \mathrm{H}, \mathrm{t}, J=7.4 \mathrm{~Hz}, \mathrm{ArH}), 5.23(0.1 \mathrm{H}, \mathrm{s}$, $=\mathrm{C} \underline{\mathrm{H}}$, of enol form 1), $5.16(0.1 \mathrm{H}, \mathrm{s},=\mathrm{C} \underline{\mathrm{H}}$, of enol form 2$), 4.21\left(2 \mathrm{H}, \mathrm{q}, J=7.2 \mathrm{~Hz}, \mathrm{C}_{2} \mathrm{CH}_{3}\right)$, $3.71\left(1.6 \mathrm{H}, \mathrm{s}, \mathrm{CH}_{2}\right.$ of keto form 1), $3.63\left(1.6 \mathrm{H}, \mathrm{s}, \mathrm{CH}_{2}\right.$ of keto form 1), $3.31\left(0.2 \mathrm{H}, \mathrm{s}, \mathrm{CH}_{2}\right.$ of enol form 1), $3.22\left(0.2 \mathrm{H}, \mathrm{s}, \mathrm{CH}_{2}\right.$ of enol form 1), $1.28\left(3 \mathrm{H}, \mathrm{t}, J=7.2 \mathrm{~Hz}, \mathrm{CH}_{2} \mathrm{CH}_{3}\right)$;

${ }^{13} \mathrm{C}$ NMR (126 MHz, $\mathrm{CDCl}_{3}$ ) (keto/enol, 4:1 (both enol form)) $\delta 199.4,172.6,169.8,166.8$, $164.9,163.2$, 137.5, 129.1, 124.8, 120.3, 93.6, 92.9, 61.9, 61.6, 60.8, 50.1, 49.8, 44.4, 41.3, 14.3, 14.2, 14.1;

$m / z(\mathrm{ESI}) 272.3\left[(\mathrm{M}+\mathrm{Na})^{+}, 100 \%\right]$.

Li, W.; Ma, X.; Fan, W.; Tao, X.; Li, X.; Xie, X.; Zhang, Z. Ru-Catalyzed Asymmetric Hydrogenation of 3-Oxoglutaric Acid Derivatives via Solvent-Assisted Pinpoint Recognition of Carbonyls in Close Chemical Propinquity, Org. Lett. 2011, 13, 15, 3876-3879. 
Ethyl 3,5-dioxo-5-(phenylamino)pentanoate.

${ }^{1} \mathrm{H}$ NMR $\left(500 \mathrm{MHz}, \mathrm{CDCl}_{3}\right)$.

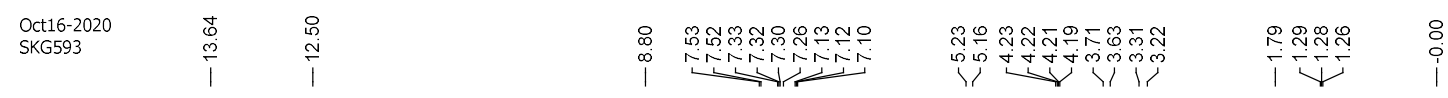<smiles>CCOC(=O)CC(=O)CC(=O)Nc1ccccc1</smiles>

keto form

enol form

$\iint \mid \int$
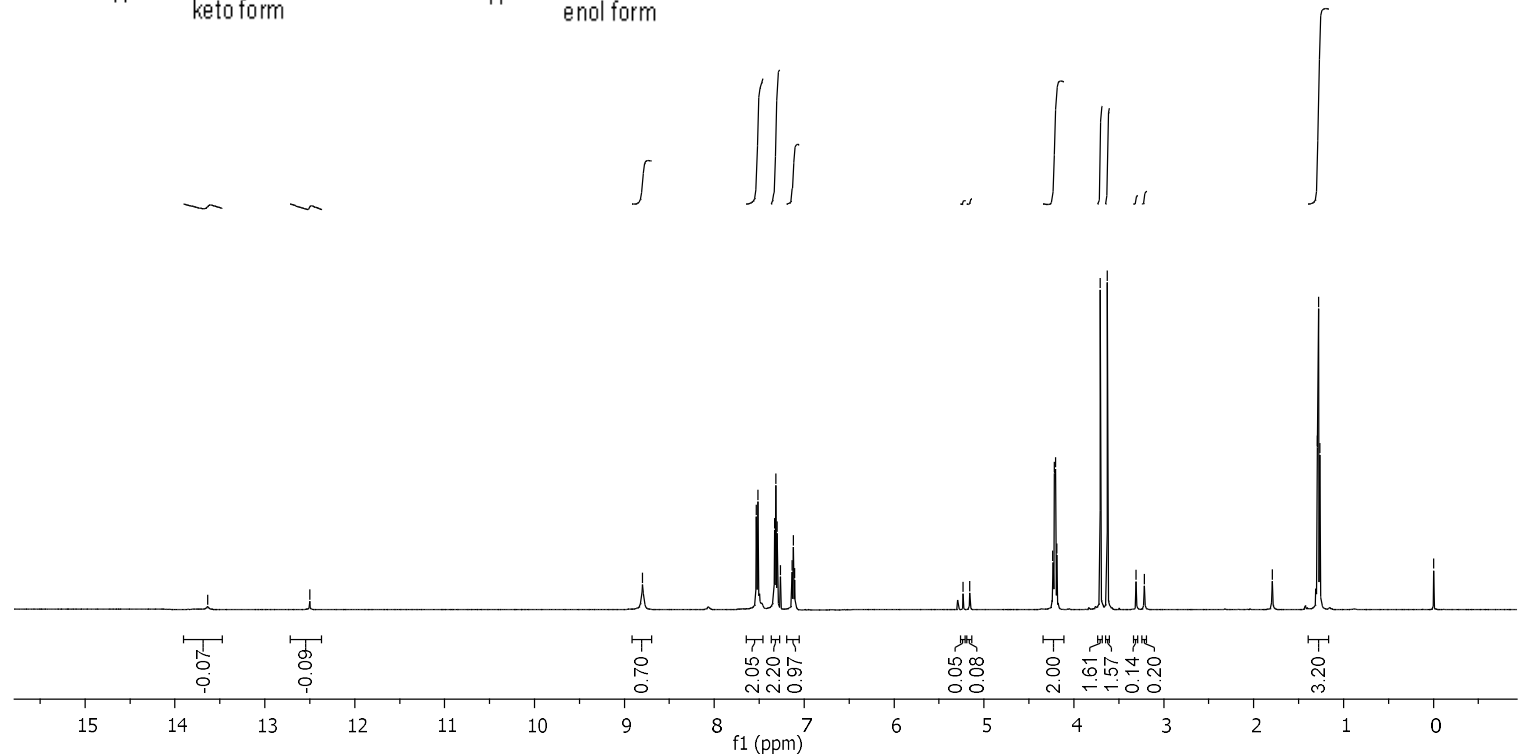

${ }^{13} \mathrm{C}$ NMR (126 MHz, $\left.\mathrm{CDCl}_{3}\right)$.

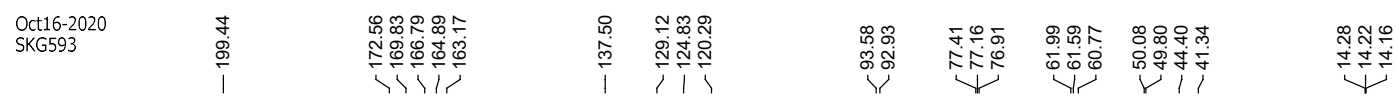<smiles>CC=CC(=CC(O)=CC(=O)OCC)C(=O)Nc1ccccc1</smiles>

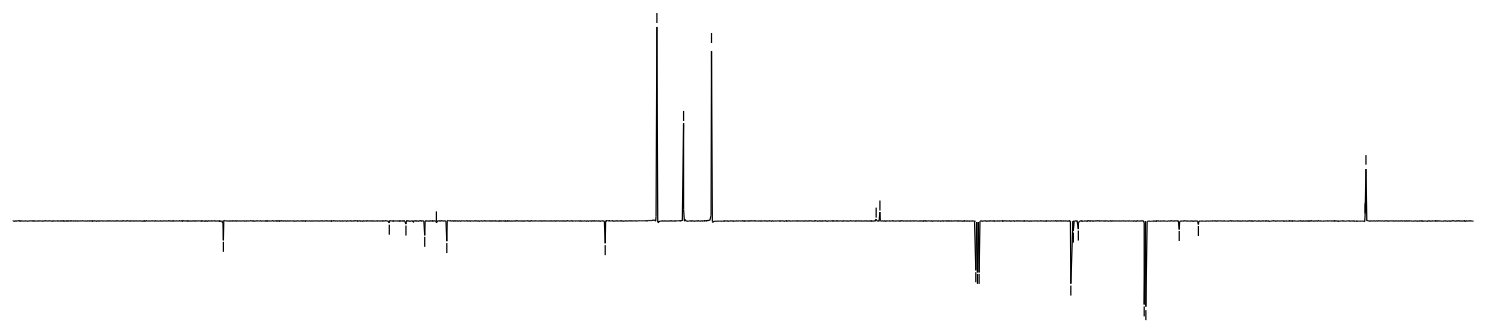

$\begin{array}{lllllllllllllllllllllllllllllllllll}230 & 220 & 210 & 200 & 190 & 180 & 170 & 160 & 150 & 140 & 130 & \underset{\mathrm{f} 1}{120}(\mathrm{ppm}) & 110 & 100 & 90 & 80 & 70 & 60 & 50 & 40 & 30 & 20 & 10 & 0\end{array}$ 
COSY.

Chemist Shweta Gediya

SKG593 US 1 .

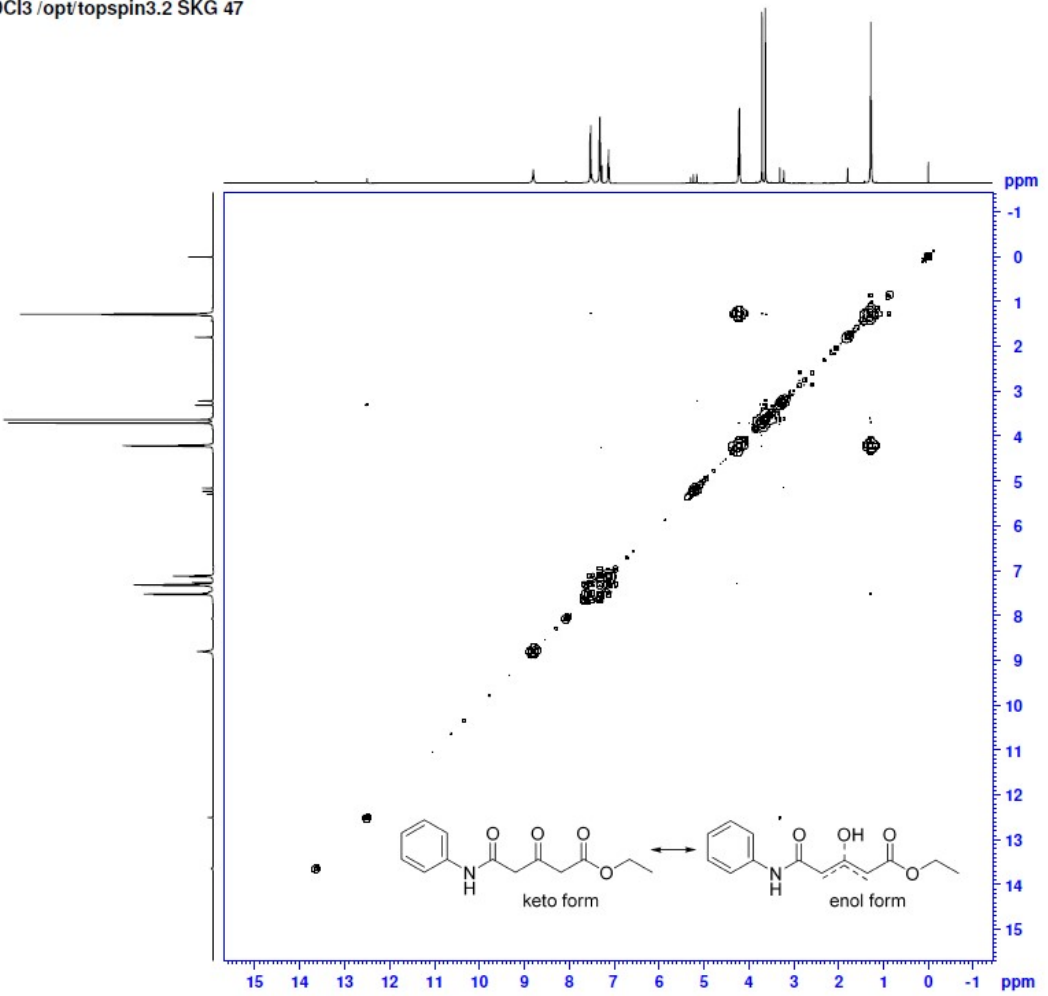




\section{Section on reductions to alcohols.}

\section{General procedure C for Asymmetric Transfer Hydrogenation ATH).}

To a stirred solution of substituted $\alpha$-ketoamide derivatives in DCM was added $(R, R)-3 \mathrm{C}$ tethered catalyst and FA: TEA and the resulting reaction mixture was stirred at room temperature for $24 \mathrm{~h}$. The reaction mixture was quenched with water and the aqueous layer was extracted with DCM. The combined organic layers were washed with brine, dried over $\mathrm{MgSO}_{4}$, and concentrated under reduced pressure to generate crude product, which was purified by flash chromatography on silica gel $(60-80 \%$ EtOAc in pet ether) to afford $\alpha$ hydroxyamide derivatives. In all cases the ATH reactions were followed until 100\% conversion was observed by TLC. In some cases, a trace of ketone was observed in the subsequent HPLC analysis and this is indicated on the spectra.

\section{General Procedure D for the Synthesis of Racemic Alcohols.}

Substituted ketone derivatives (1.0 eq) were dissolved in $\mathrm{MeOH}$ : water (5:1) and $\mathrm{NaBH}_{4}(2.0$ eq) was added portion wise. The solution was stirred at room temperature overnight. The solvent was removed under reduced pressure and the residue was suspended in water. The aqueous layer was extracted with EtOAc. The organic layers were combined, dried over $\mathrm{MgSO}_{4}$, filtered, and the solvent was removed under reduced pressure to afford racemic alcohols. This was the default method for the preparation of racemic standards of reduction products. In the cases of compounds $19,20,32,33,34,38,39,40,41,42,45,46,47$ and 54 , the use of $\mathrm{NaBH}_{4}$ led to a side reaction (typically ester reduction) therefore in these cases a 1:1 mixture of each enantiomer of catalyst 2 was used in an ATH reaction, following procedure $\mathrm{C}$. 


\section{2-Hydroxy- $N^{1}, N^{4}$-dimethyl- $N^{1}, N^{4}$-diphenylsuccinamide 5.}<smiles>CN(C(=O)CC(O)C(=O)N(C)c1ccccc1)c1ccccc1</smiles>

This compound is novel and was prepared following general procedure $\mathrm{C}$ using $N^{1}, N^{4}$ dimethyl-2-oxo- $N^{1}, N^{4}$-diphenylsuccinamide $(0.155 \mathrm{~g}, 0.500 \mathrm{mmol}, 1.0 \mathrm{eq}), 3 \mathrm{C}$ tethered catalyst $(R, R)-2(4.7 \mathrm{mg}, 7.5 \mu \mathrm{mol}, 0.015 \mathrm{eq})$ and FA:TEA $(0.3 \mathrm{~mL})$ in DCM $(3 \mathrm{~mL})$ to give 5 as a brown oil $(0.105 \mathrm{~g}, 0.338 \mathrm{mmol}, 68 \%)$.

TLC: $\mathrm{R}_{\mathrm{f}}$ ca 0.3 (4:6, hexane: EtOAc), non UV-active, Strong $\mathrm{KMnO}_{4}$ and $\mathrm{PMA}$-reactive; HRMS (ESI) m/z: [M+Na] $]^{+}$Calcd for $\mathrm{C}_{18} \mathrm{H}_{20} \mathrm{~N}_{2} \mathrm{O}_{3} \mathrm{Na}$ 335.1366; Found 335.1361 (error 1.5 ppm);

$U_{\max } 3345,2921,1632,1592,1492,1384,1273,1073,780,698 \mathrm{~cm}^{-1}$;

Enantiomeric excess determined by HPLC analysis (Chiralpak IG, $250 \mathrm{~mm} \times 4.6 \mathrm{~mm}$ column, hexane: $\mathrm{PrOH} 75: 25,1 \mathrm{~mL} / \mathrm{min}, \lambda=210 \mathrm{~nm}, \mathrm{~T}=25^{\circ} \mathrm{C}$ ), $27.3 \mathrm{~min}$ and $36.3 \mathrm{~min}, 4 \%$ ee;

${ }^{1} \mathrm{H}$ NMR (500 MHz, $\left.\mathrm{CDCl}_{3}\right) \delta$ 7.43-7.32 (6H, m, ArH), 7.19-7.12 (4H, m, ArH), 4.51 (1H, d, $J=3.4 \mathrm{~Hz}, \mathrm{C} \underline{\mathrm{HOH}}), 3.96(1 \mathrm{H}, \mathrm{d}, J=6.8 \mathrm{~Hz}, \mathrm{CHO} \underline{\mathrm{H}}), 3.27\left(3 \mathrm{H}, \mathrm{s}, \mathrm{NCH}_{3}\right), 3.21(3 \mathrm{H}, \mathrm{s}$, $\left.\mathrm{NCH}_{3}\right), 2.27\left(1 \mathrm{H}, \mathrm{dd}, \mathrm{J}=15.5,7.5 \mathrm{~Hz}, \mathrm{C}_{\mathrm{a}} \mathrm{H}_{\mathrm{b}}\right), 2.19\left(1 \mathrm{H}, \mathrm{dd}, \mathrm{J}=15.2,2.6 \mathrm{~Hz}, \mathrm{CH}_{\mathrm{a}} \underline{\mathrm{H}}_{\mathrm{b}}\right)$;

${ }^{13} \mathrm{C}$ NMR $\left(126 \mathrm{MHz}, \mathrm{CDCl}_{3}\right) \delta 172.8,170.4,143.7,142.6,130.05,129.9,128.4,128.1$, 127.6, 127.4, 66.4, 38.2, 37.8, 37.4;

$\mathrm{m} / \mathrm{z}(\mathrm{ESI}) 335.1\left[(\mathrm{M}+\mathrm{Na})^{+}, 100 \%\right]$. 
2-Hydroxy- $N^{1}, N^{4}$-dimethyl- $N^{1}, N^{4}$-diphenylsuccinamide 5.

${ }^{1} \mathrm{H}$ NMR $\left(500 \mathrm{MHz}, \mathrm{CDCl}_{3}\right)$.

Dec17-2020
Chemist Shweta Gediya

PROTON.w CDCl3 /opt/topspin3.2 SKG 52

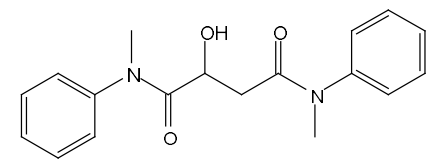

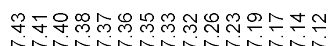

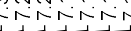

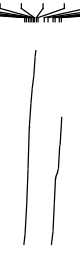

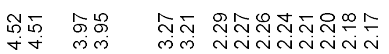

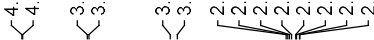

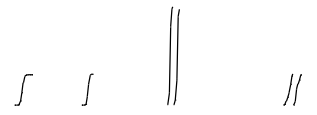

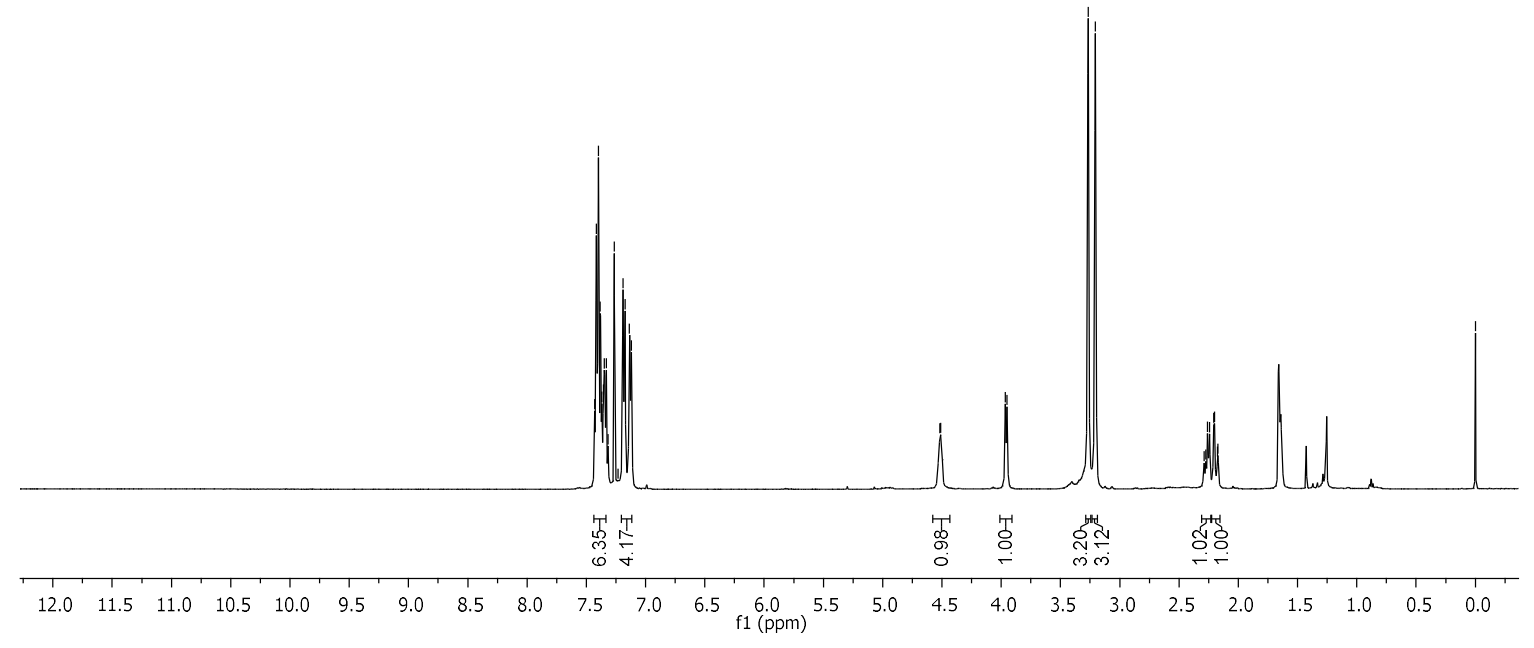

${ }^{13} \mathrm{C}$ NMR (126 MHz, $\left.\mathrm{CDCl}_{3}\right)$.

Dec17-2020

Shweta Gediya

C13APTlong.w CDCI3

ำ

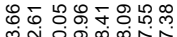

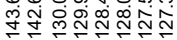

पर

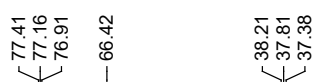<smiles>CN(C(=O)CC(O)C(=O)N(C)c1ccccc1)c1ccccc1</smiles>

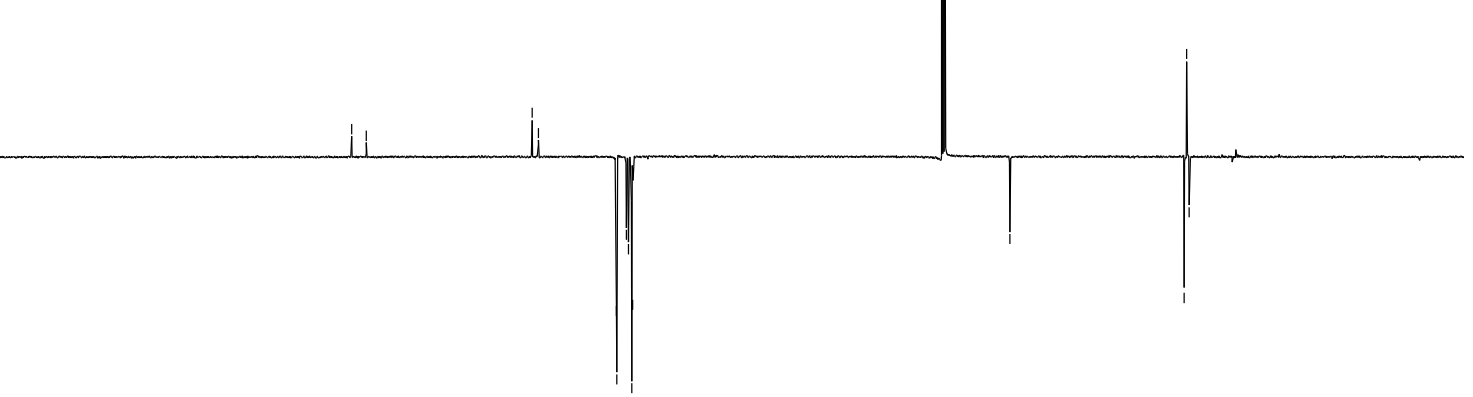

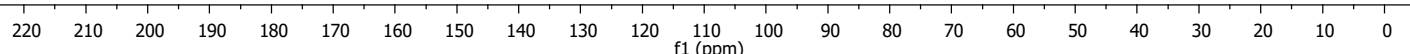


COSY.

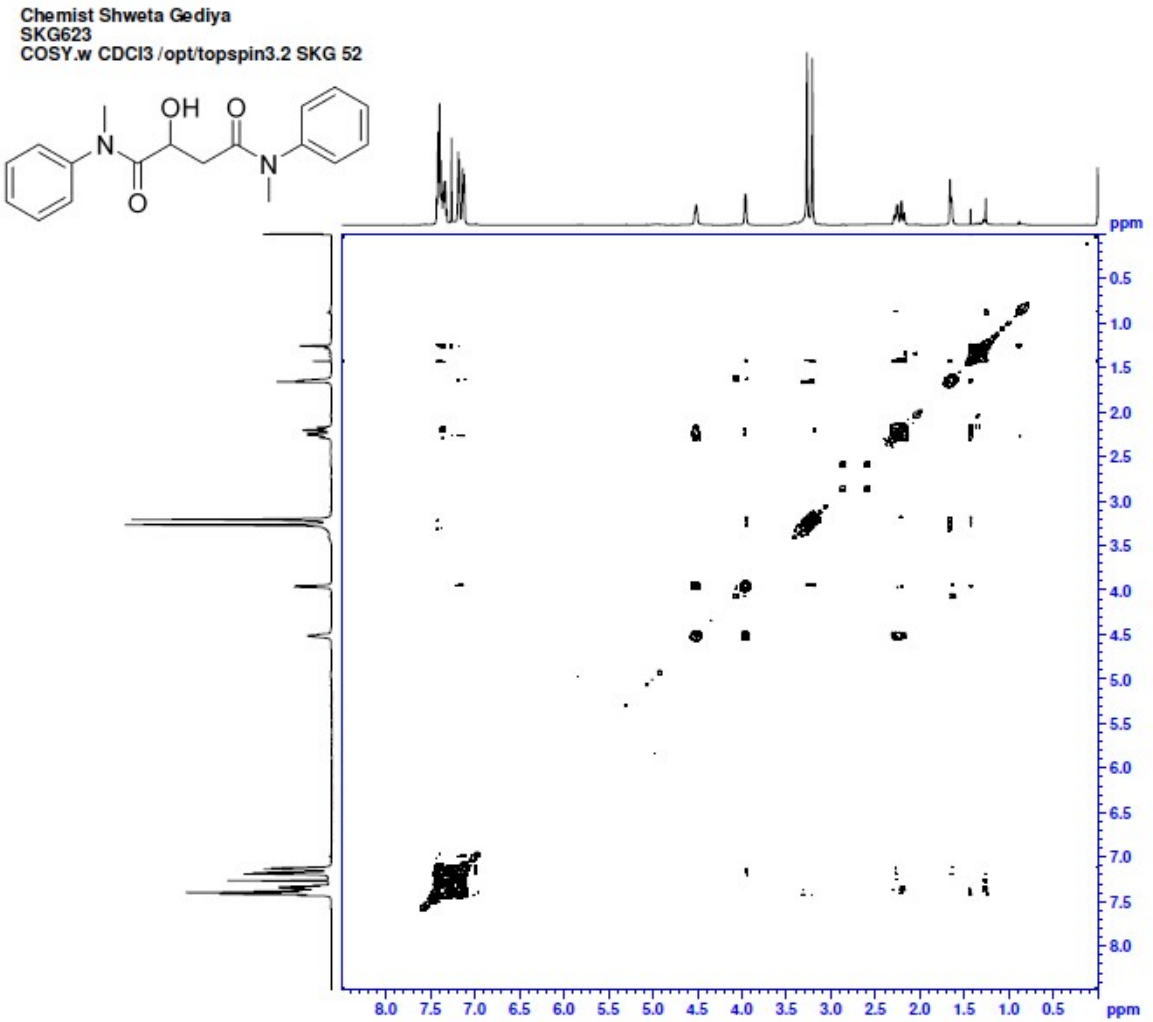

HSQC.

Chemist Shweta Gediya

SKG623

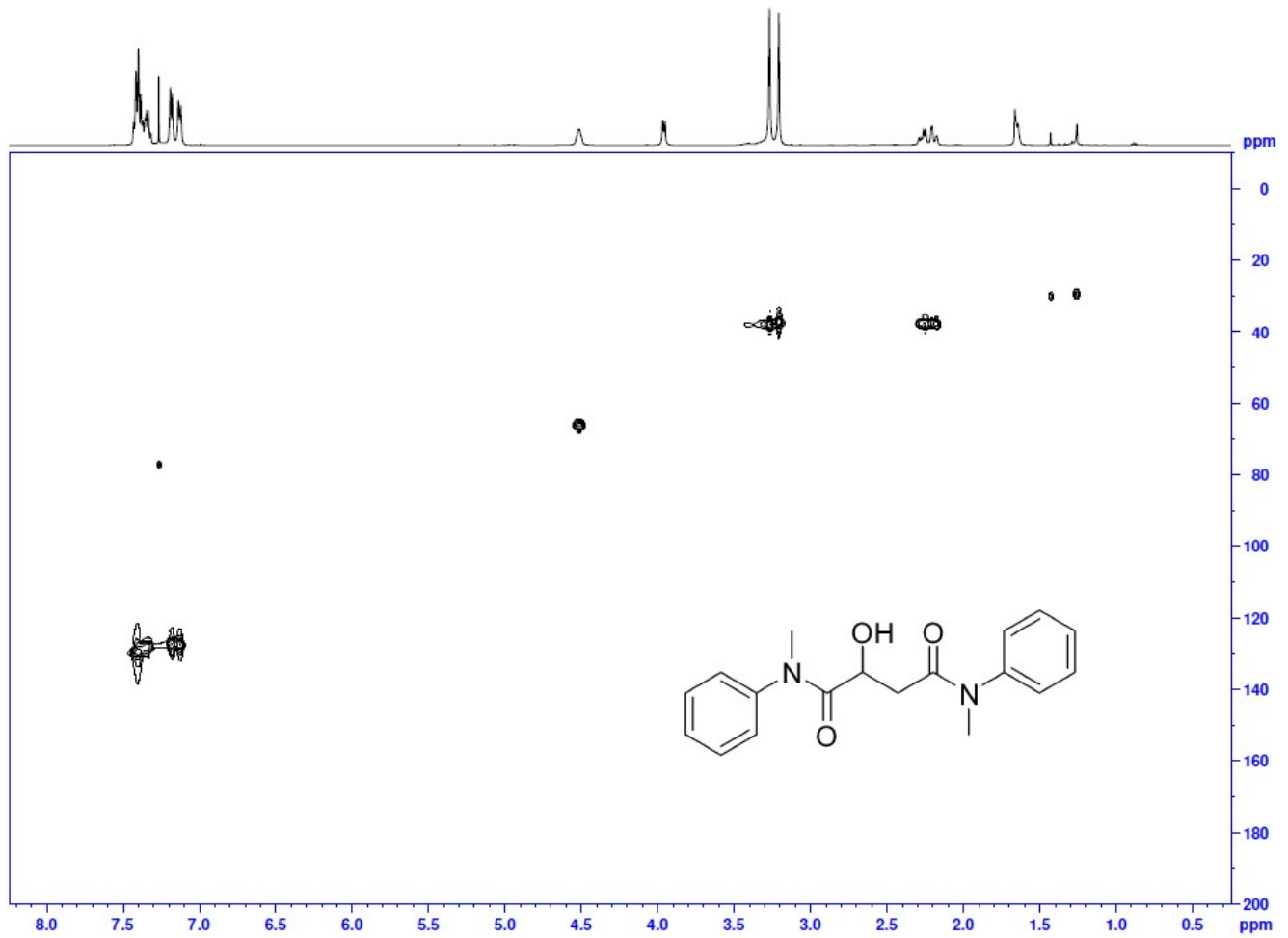




\section{Chiral HPLC of ketone precursor.}

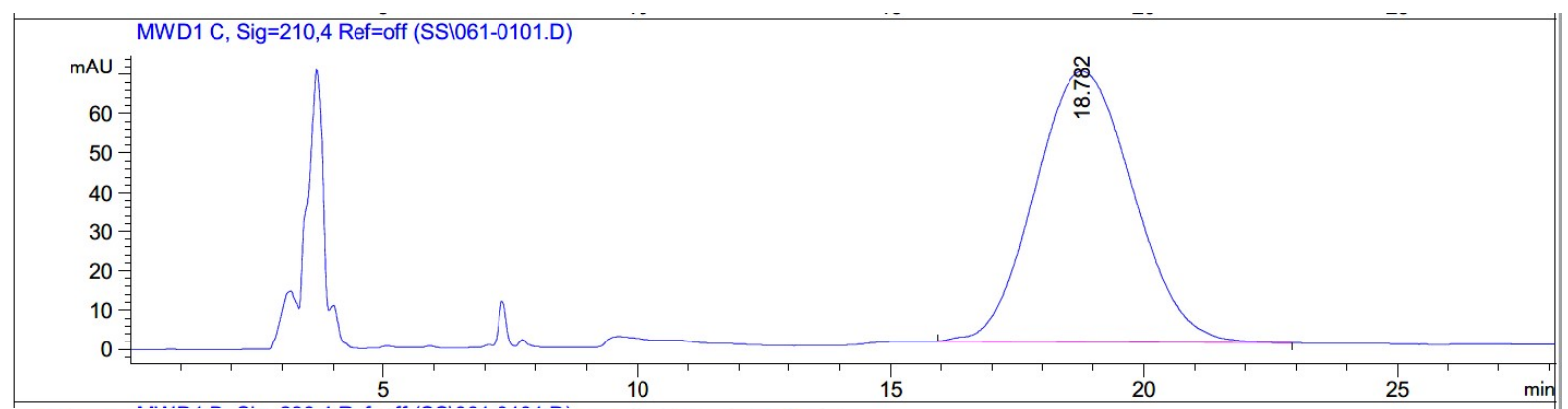

Signal 2: MWD1 C, Sig=210, 4 Ref=off

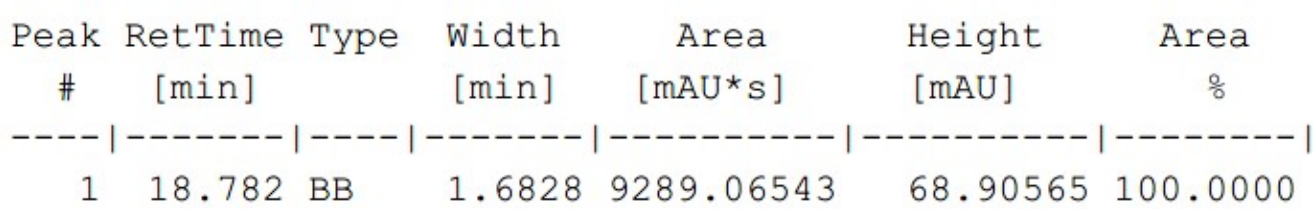

Chiral HPLC of racemic alcohol.

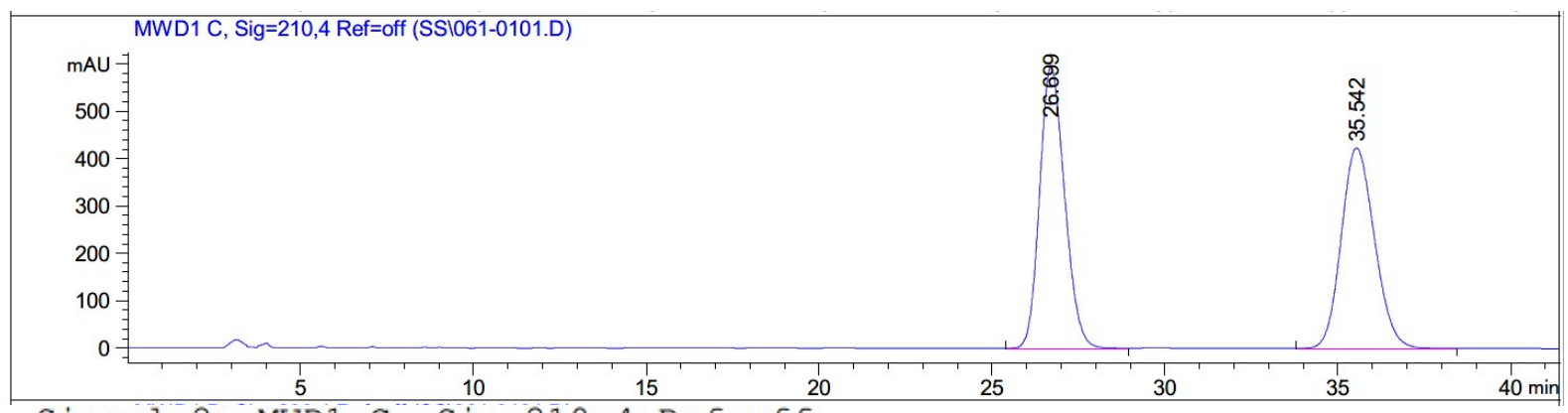

\begin{tabular}{|c|c|c|c|c|c|c|}
\hline $\begin{array}{c}\text { Peak } \\
\#\end{array}$ & $\begin{array}{c}\text { RetTime } \\
\text { [min] }\end{array}$ & Type & $\begin{array}{l}\text { Width } \\
{[\min ]}\end{array}$ & $\begin{array}{c}\text { Area } \\
{\left[\mathrm{mAU}{ }^{\star} \mathrm{s}\right]}\end{array}$ & $\begin{array}{l}\text { Height } \\
{[\mathrm{mAU}]}\end{array}$ & $\begin{array}{c}\text { Area } \\
\frac{\circ}{\delta}\end{array}$ \\
\hline---1 & |------ & & ----- & -------- & ---------- & ----- \\
\hline 1 & 26.699 & $\mathrm{BB}$ & 0.7803 & $2.98624 \mathrm{e} 4$ & 594.21887 & 51.3351 \\
\hline 2 & 35.542 & BB & 1.0383 & $2.83091 \mathrm{e} 4$ & 423.29126 & 48.6649 \\
\hline
\end{tabular}


Chiral HPLC of ATH product. 4\% ee.

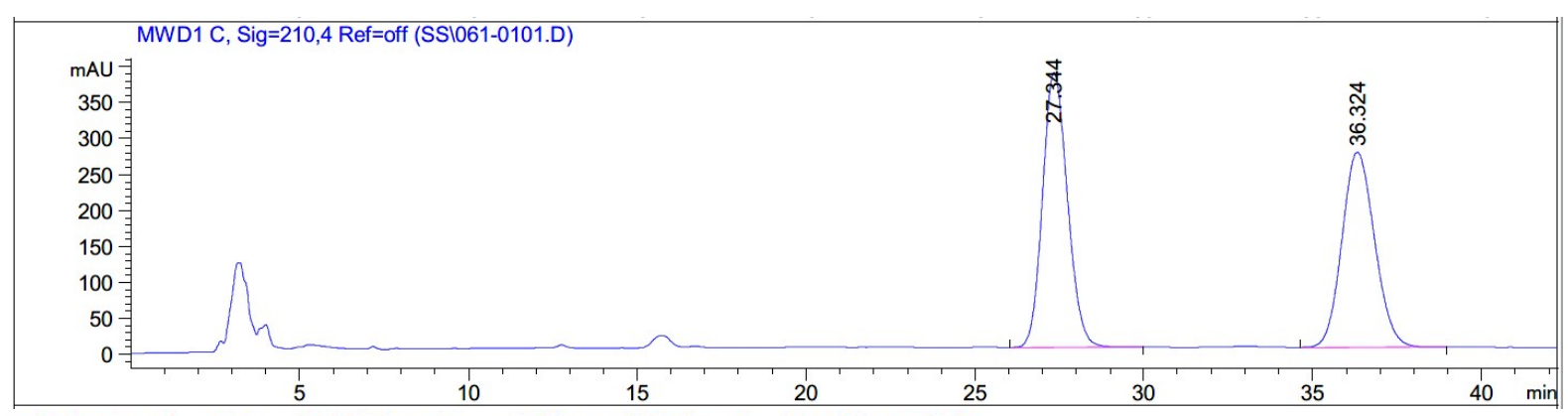

Signal 2: MWD1 C, Sig=210,4 Ref=off

\begin{tabular}{|c|c|c|c|c|c|c|}
\hline $\begin{array}{c}\text { Peak } \\
\#\end{array}$ & $\begin{array}{c}\text { RetTime } \\
\text { [min] }\end{array}$ & Type & $\begin{array}{l}\text { Width } \\
\text { [min] }\end{array}$ & $\begin{array}{c}\text { Area } \\
{\left[\mathrm{mAU}^{\star} \mathrm{s}\right]}\end{array}$ & $\begin{array}{l}\text { Height } \\
{[\mathrm{mAU}]}\end{array}$ & $\begin{array}{c}\text { Area } \\
\quad \frac{\circ}{0}\end{array}$ \\
\hline & & & & 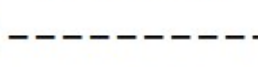 & 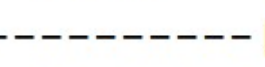 & \\
\hline 1 & 27.344 & BB & 0.7960 & $1.96064 \mathrm{e} 4$ & 382.49280 & 51.7542 \\
\hline 2 & 36.324 & BB & 1.0470 & $1.82773 \mathrm{e} 4$ & 271.64682 & 48.2458 \\
\hline
\end{tabular}


(R)- $N^{1}$-Benzyl-2-hydroxy- $N^{1}, N^{4}$-dimethyl- $N^{4}$-phenylsuccinamide 6 .<smiles>CN(Cc1ccccc1)C(=O)C(O)CC(=O)N(C)c1ccccc1</smiles>

This compound is novel and was prepared following general procedure $\mathrm{C}$ using $N^{1}$-benzyl$N^{1}, N^{4}$-dimethyl-2-oxo- $N^{4}$-phenylsuccinamide $(81.0 \mathrm{mg}, 0.250 \mathrm{mmol}, 1.0 \mathrm{eq}), 3 \mathrm{C}$ tethered catalyst $(R, R)-2(2.3 \mathrm{mg}, 3.8 \mu \mathrm{mol}, 0.015 \mathrm{eq})$ and FA:TEA $(0.15 \mathrm{~mL})$ in DCM $(1.5 \mathrm{~mL})$ to give 6 as pale yellow liquid (75.6 $\mathrm{mg}, 0.233 \mathrm{mmol}, 93 \%)$.

TLC: $\mathrm{R}_{\mathrm{f}}$ ca 0.2 (6:4, hexane: EtOAc), UV-active and PMA-reactive;

HRMS (ESI) m/z: [M+Na] ${ }^{+}$Calcd for $\mathrm{C}_{19} \mathrm{H}_{22} \mathrm{~N}_{2} \mathrm{O}_{3} \mathrm{Na}$ 349.1523; Found 349.1520 (error 0.8 ppm);

$U_{\max } 3385,2923,1632,1592,1494,1383,1118,1075,731 \mathrm{~cm}^{-1}$;

Enantiomeric excess determined by HPLC analysis (Chiralpak IG, $250 \mathrm{~mm} \times 4.6 \mathrm{~mm}$ column, hexane: $\mathrm{iPrOH} 80: 201 \mathrm{~mL} / \mathrm{min}, \lambda=210 \mathrm{~nm}, \mathrm{~T}=25^{\circ} \mathrm{C}$ ), $S$-enantiomer $39.6 \mathrm{~min}, R$ enantiomer $42.8 \min .77 \%$ ee $(R),[\alpha]_{\mathrm{D}}^{22}=+17\left(\mathrm{c}=0.1, \mathrm{CHCl}_{3}\right)$;

${ }^{1} \mathrm{H}$ NMR (400 MHz, $\mathrm{CDCl}_{3}$ ) (Rotamers, ca. 2:1) $\delta$ 7.44-7.14 (10H, m, ArH), 4.88-4.81 (1H, m, $\mathrm{C} \underline{\mathrm{HOH}}), 4.68-4.46\left(2 \mathrm{H}, \mathrm{m}, \mathrm{CH}_{2} \mathrm{Ph}\right), 4.32(1 \mathrm{H}, \mathrm{d}, J=7.5 \mathrm{~Hz}, \mathrm{CHO} \underline{\mathrm{H}}), 3.30\left(2 \mathrm{H}, \mathrm{s}, \mathrm{NCH}_{3}\right)$, $3.27\left(1 \mathrm{H}, \mathrm{s}, \mathrm{NCH}_{3}\right), 2.93\left(2 \mathrm{H}, \mathrm{s}, \mathrm{NCH}_{3}\right), 2.87\left(1 \mathrm{H}, \mathrm{s}, \mathrm{NCH}_{3}\right), 2.54-2.47\left(1 \mathrm{H}, \mathrm{m}, \mathrm{C}_{\mathrm{a}} \mathrm{H}_{\mathrm{b}}\right)$, 2.37-2.33 (1H, m, $\left.\mathrm{CH}_{\mathrm{a}} \underline{\mathrm{H}_{\mathrm{b}}}\right)$;

${ }^{13} \mathrm{C}$ NMR (101 MHz, $\mathrm{CDCl}_{3}$ ) (Rotamers, ca. 2:1) $\delta$ 173.1, 173.0, 171.3, 170.8, 143.6, 143.5, $136.5,136.2$, 129.9, 128.9, 128.7, 128.1, 128.0, 127.8, 127.6, 127.5, 127.4, 126.8, 67.1, 66.8, $52.7,51.5,38.3,37.5,37.4,34.3,33.9$; $m / z(\mathrm{ESI}) 349.1\left[(\mathrm{M}+\mathrm{Na})^{+}, 100 \%\right]$. 
$N^{1}$-Benzyl-2-hydroxy- $N^{1}, N^{4}$-dimethyl- $N^{4}$-phenylsuccinamide 6.

${ }^{1} \mathrm{H}$ NMR $\left(400 \mathrm{MHz}, \mathrm{CDCl}_{3}\right)$.

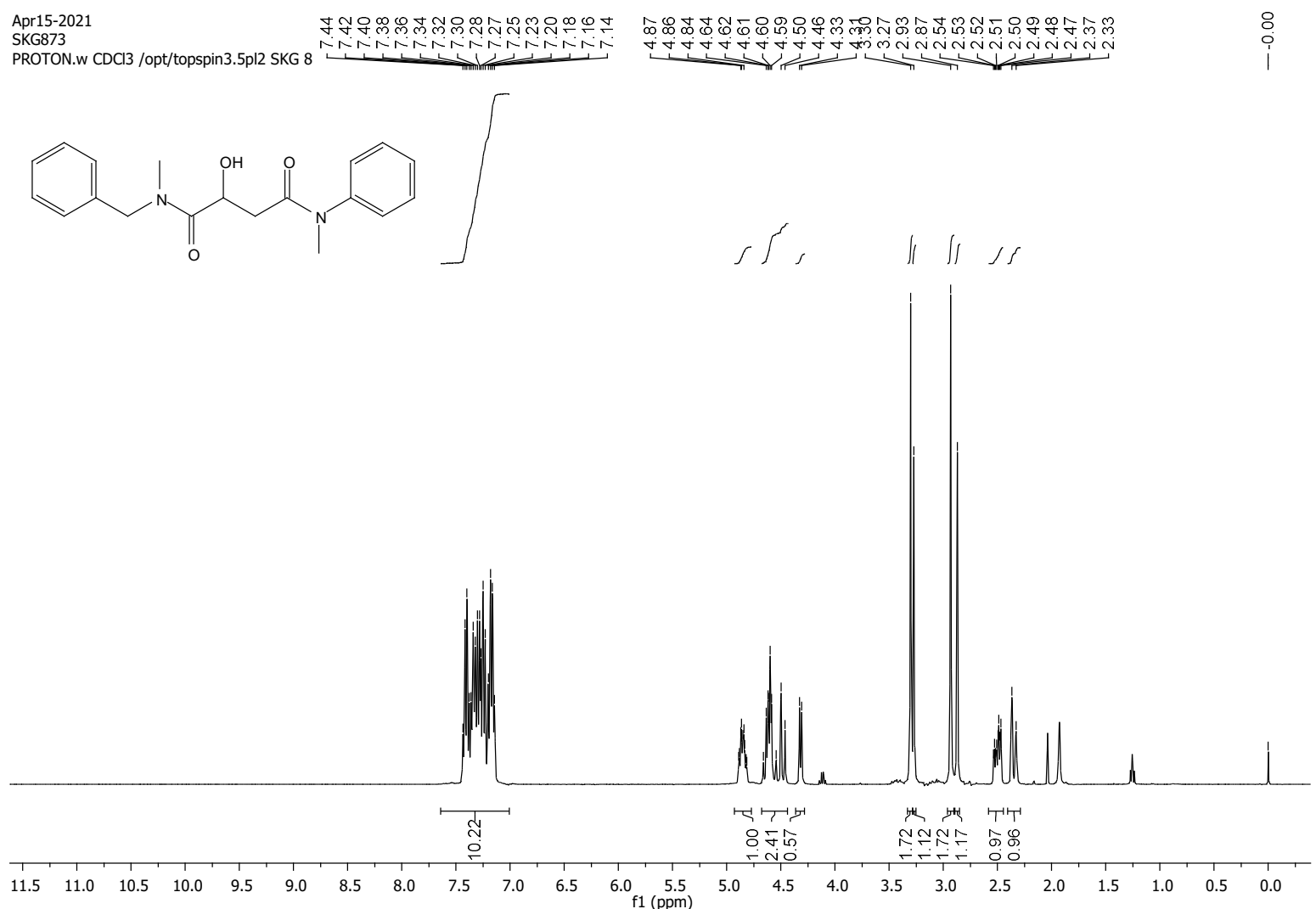

${ }^{13} \mathrm{C}$ NMR (101 MHz, $\left.\mathrm{CDCl}_{3}\right)$.

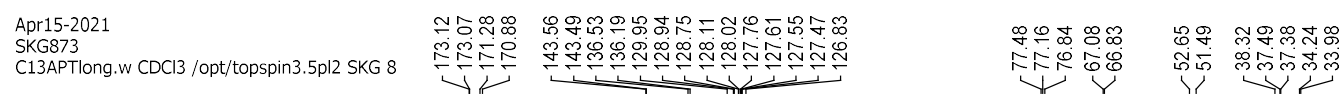

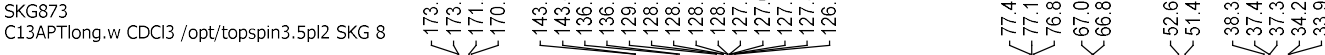<smiles>CN(Cc1ccccc1)C(=O)C(O)CC(=O)N(C)c1ccccc1</smiles>

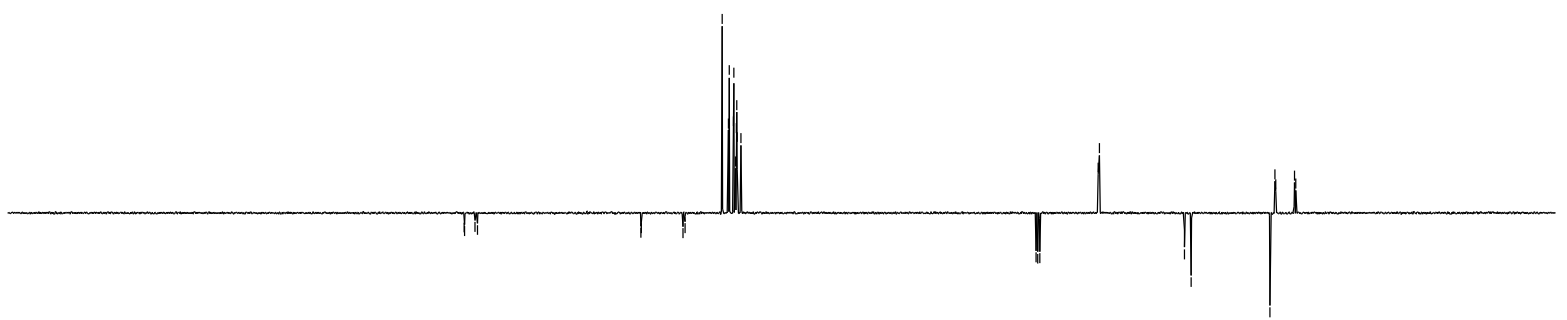

$\begin{array}{llllllllllllllllllllllllllll}240 & 230 & 220 & 210 & 200 & 190 & 180 & 170 & 160 & 150 & 140 & 130 & \begin{array}{l}120 \\ \mathrm{fpm})\end{array} & 110 & 100 & 90 & 80 & 70 & 60 & 50 & 40 & 30 & 20 & 10 & 0\end{array}$ 
COSY.

SKG873

COSY.w CDCl3 /opt/topspin3.5pl2 SKG 8

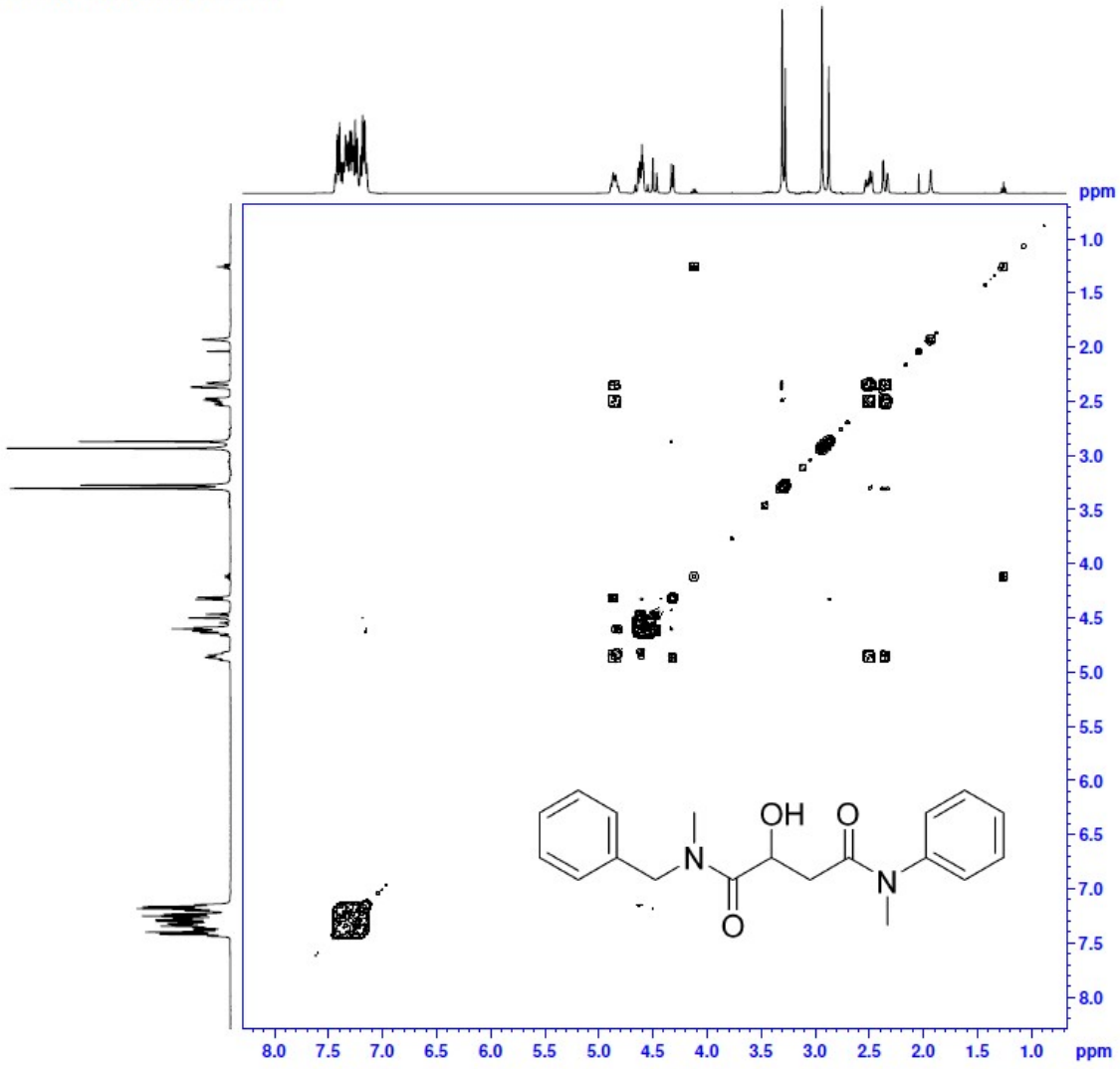

HSQC.

SKG873
HSQC.w CDCl3 /opttopspin3.5pl2 SKG 8

洲

sher
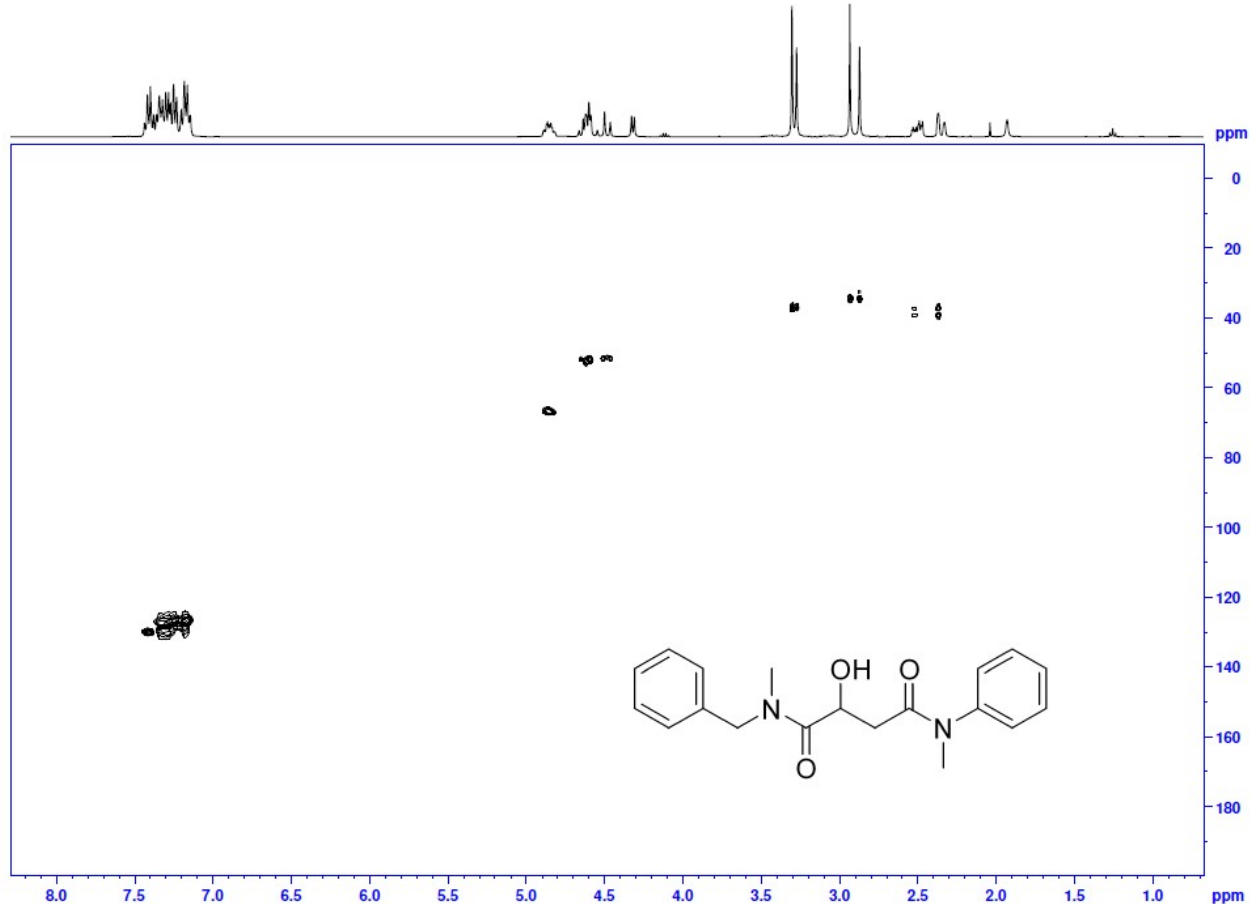


\section{Chiral HPLC of ketone precursor.}

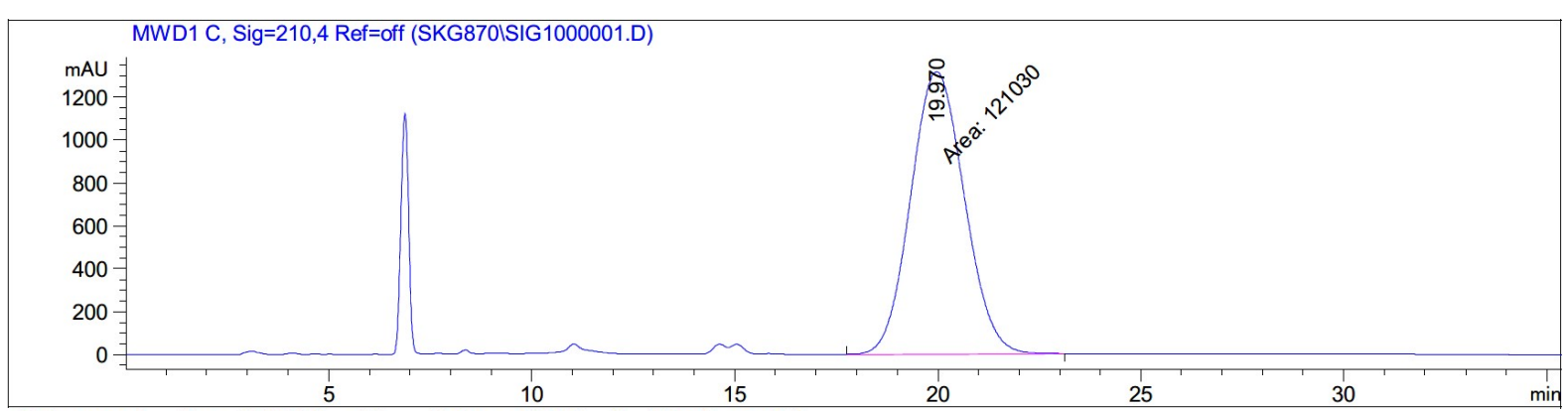

Signal 2: MWD1 C, Sig=210,4 Ref=off

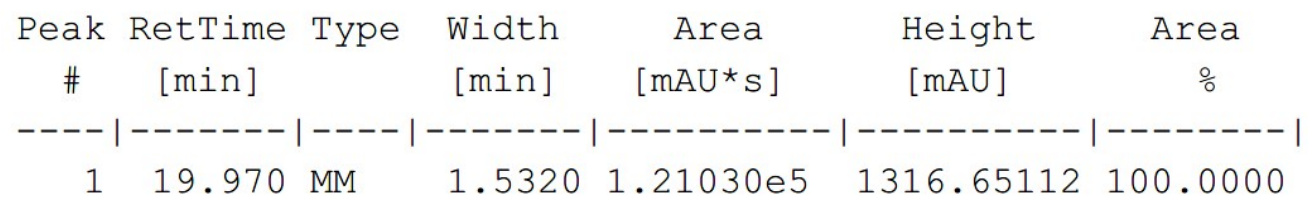

Chiral HPLC of racemic alcohol.

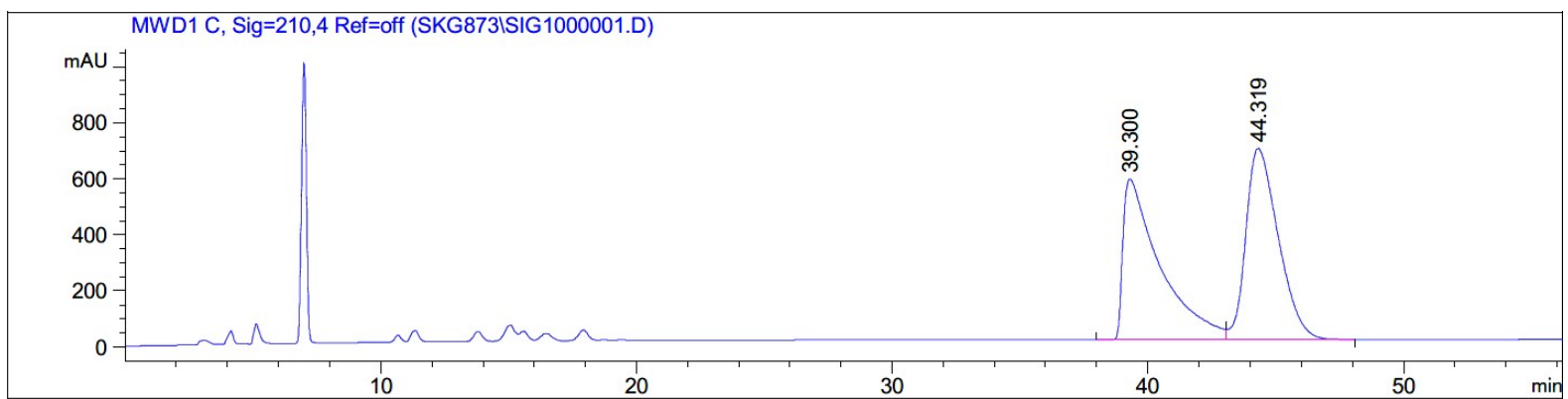

Signal 2: MWD1 C, Sig=210, 4 Ref=off

\begin{tabular}{|c|c|c|c|c|c|c|}
\hline $\begin{array}{c}\text { eak } \\
\#\end{array}$ & $\begin{array}{c}\text { RetTime } \\
\text { [min] }\end{array}$ & Type & $\begin{array}{l}\text { Width } \\
\text { [min] }\end{array}$ & $\begin{array}{c}\text { Area } \\
{\left[\mathrm{mAU}{ }^{\star} \mathrm{s}\right]}\end{array}$ & $\begin{array}{l}\text { Height } \\
\text { [mAU] }\end{array}$ & $\begin{array}{c}\text { Area } \\
\quad \%\end{array}$ \\
\hline & & & & & & - \\
\hline 1 & & & & 5.773 & $57<$ & 48 \\
\hline 2 & & & & $6.03088 \mathrm{e} 4$ & 684.16626 & 0905 \\
\hline
\end{tabular}


Chiral HPLC of ATH product. $77 \%$ ee.

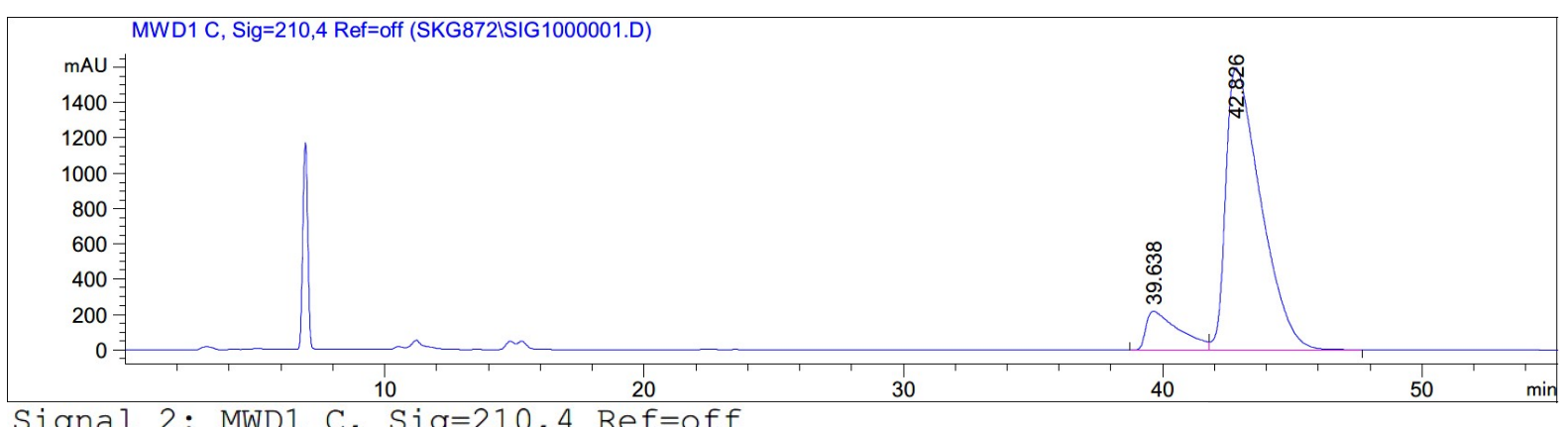

Signal 2: MWD1 C, Sig=210,4 Ref=off

\begin{tabular}{|c|c|c|c|c|c|c|}
\hline $\begin{array}{c}\text { Peak } \\
\#\end{array}$ & $\begin{array}{c}\text { RetTime } \\
{[\min ]}\end{array}$ & Type & $\begin{array}{l}\text { Width } \\
\text { [min] }\end{array}$ & $\begin{array}{c}\text { Area } \\
{[\mathrm{mAU} * \mathrm{~s}]}\end{array}$ & $\begin{array}{l}\text { Height } \\
{[\mathrm{mAU}]}\end{array}$ & $\begin{array}{c}\text { Area } \\
\frac{\circ}{0}\end{array}$ \\
\hline & - & & -1 & 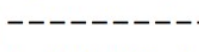 & --- & -- \\
\hline 1 & 39.638 & BV & 1.2115 & $1.90558 \mathrm{e} 4$ & 216.79692 & 253 \\
\hline 2 & 42.826 & VB & 1.3155 & $1.49202 \mathrm{e} 5$ & 1597.66663 & 88.6747 \\
\hline
\end{tabular}


(R)- $N^{1}, N^{4}$-Dibenzyl-2-hydroxy- $N^{1}, N^{4}$-dimethylsuccinamide 7.<smiles>CN(Cc1ccccc1)C(=O)C[C@H](O)C(=O)N(C)Cc1ccccc1</smiles>

This compound is novel and was prepared following general procedure $\mathrm{C}$ using $N^{1}, N^{4}$ dibenzyl- $N^{1}, N^{4}$-dimethyl-2-oxosuccinamide $(84.5 \mathrm{mg}, 0.250 \mathrm{mmol}, 1.0 \mathrm{eq}), 3 \mathrm{C}$ tethered catalyst $(R, R)-2(2.3 \mathrm{mg}, 3.8 \mu \mathrm{mol}, 0.015 \mathrm{eq})$ and FA:TEA $(0.15 \mathrm{~mL})$ in DCM $(1.5 \mathrm{~mL})$ to give 7 as pale yellow liquid (78.3 $\mathrm{mg}, 0.231 \mathrm{mmol}, 92 \%)$.

TLC: $\mathrm{R}_{\mathrm{f}}$ ca 0.2 (1:9, hexane: EtOAc), UV-active and PMA-reactive;

HRMS (ESI) m/z: [M+Na] $]^{+}$Calcd for $\mathrm{C}_{20} \mathrm{H}_{24} \mathrm{~N}_{2} \mathrm{O}_{3} \mathrm{Na} 363.1679$; Found 363.1674 (error 1.3 ppm);

$U_{\max } 3386,2927,1622,1493,1451,1400,1077,727,696 \mathrm{~cm}^{-1}$;

Enantiomeric excess determined by HPLC analysis (Chiralpak IG, $250 \mathrm{~mm} \times 4.6 \mathrm{~mm}$ column, hexane: $\operatorname{PrOH} 65: 351 \mathrm{~mL} / \mathrm{min}, \lambda=210 \mathrm{~nm}, \mathrm{~T}=25^{\circ} \mathrm{C}$ ), $S$-enantiomer $26.6 \mathrm{~min}, R$ enantiomer $32.3 \min .94 \%$ ee $(R),[\alpha]_{\mathrm{D}}^{22}=+0.29\left(\mathrm{c}=0.05, \mathrm{CHCl}_{3}\right)$;

${ }^{1} \mathrm{H}$ NMR $\left(500 \mathrm{MHz}, \mathrm{CDCl}_{3}\right)$ (mixture of rotamers) $\delta$ 7.35-7.16 (10H, m, ArH), 4.97-4.92 $(1 \mathrm{H}, \mathrm{m}, \mathrm{C} \underline{\mathrm{HOH}}), 4.78-4.48\left(4 \mathrm{H}, \mathrm{m}, \mathrm{CH}_{2} \mathrm{Ph}\right) 3.05\left(1 \mathrm{H}, \mathrm{s}, \mathrm{NCH}_{3}\right), 3.00\left(0.5 \mathrm{H}, \mathrm{m}, \mathrm{NCH}_{3}\right), 2.97-$ $2.84\left(5.5 \mathrm{H}, \mathrm{m}, \mathrm{NCH}_{3}+\mathrm{C}_{\underline{a}} \mathrm{H}_{\mathrm{b}}\right), 2.69-2.62\left(1 \mathrm{H}, \mathrm{m}, \mathrm{CH}_{\mathrm{a}} \underline{\mathrm{H}_{\mathrm{b}}}\right)$;

${ }^{13} \mathrm{C} \mathrm{NMR}\left(126 \mathrm{MHz}, \mathrm{CDCl}_{3}\right)$ (mixture of rotamers) 172.8, 172.7, 171.9, 171.8, 171.6, 171.5, $136.9,136.8,136.7,136.6,136.4,136.3,136.2,136.1,128.9,128.8,128.7,128.7,128.6$, $128.0,127.9,127.7,127.6,127.5,127.4,126.9,126.5,67.2,67.1,67.0,66.9,53.5,53.4,52.8$, 52.7, 51.5, 50.9, 50.8, 37.4, 37.3, 37.2, 37.1, 35.2, 35.1, 34.5, 34.4, 34.0, 33.8, 33.9; $m / z(\mathrm{ESI}) 363.2\left[(\mathrm{M}+\mathrm{Na})^{+}, 100 \%\right]$. 
$N^{1}, N^{4}$-Dibenzyl-2-hydroxy- $N^{1}, N^{4}$-dimethylsuccinamide 7 .

${ }^{1} \mathrm{H}$ NMR $\left(500 \mathrm{MHz}, \mathrm{CDCl}_{3}\right)$.

Apr28-2021

Chemist Shweta Gediya

SKG909 PROTON.w CDCl3 /opt/topspin3.2 SKG 18
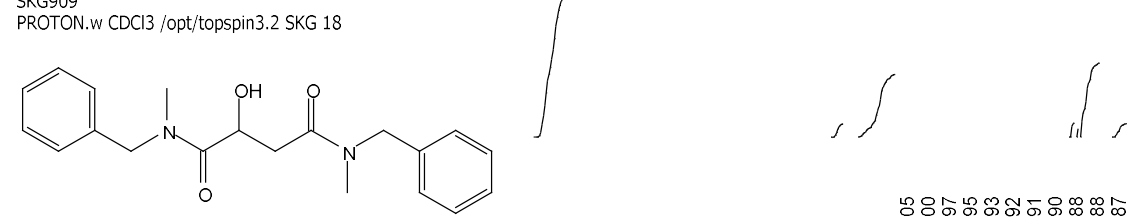

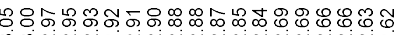

선

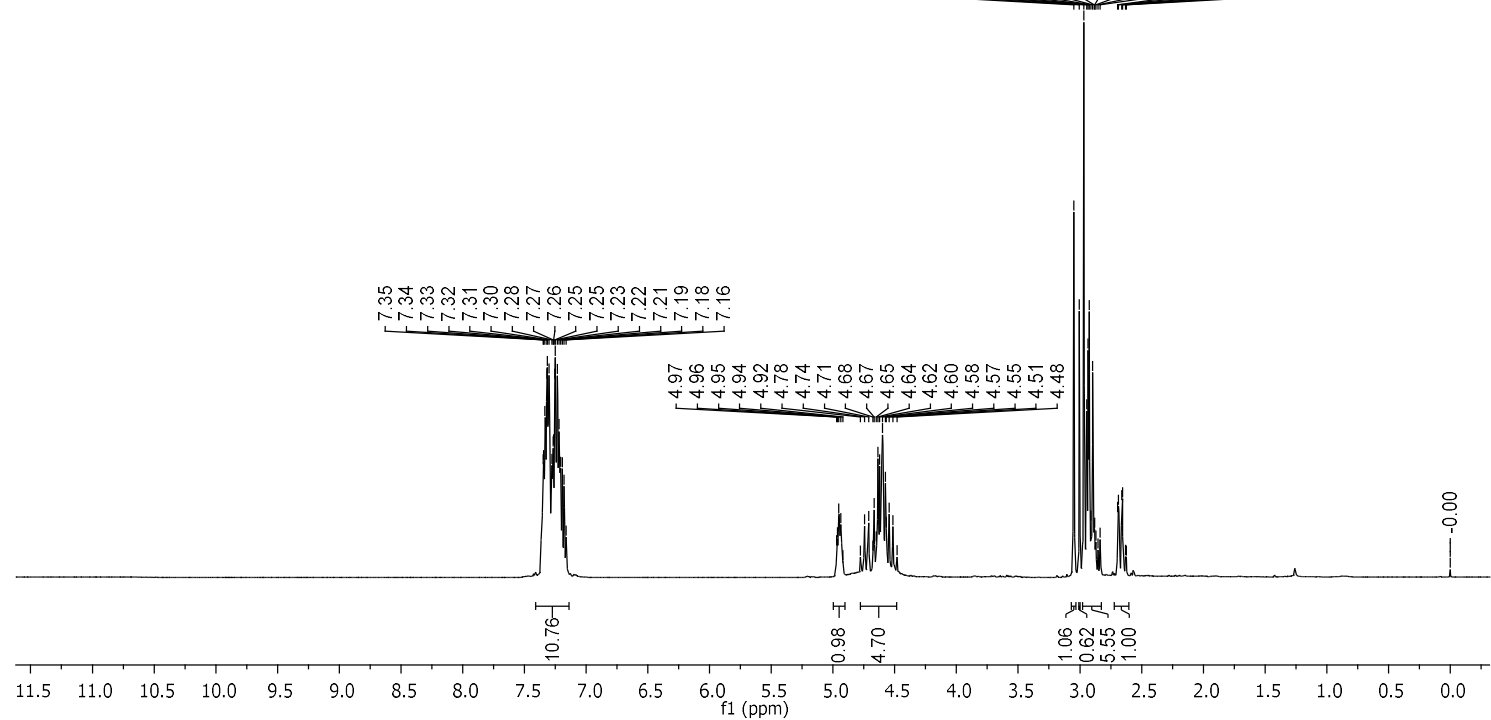

${ }^{13} \mathrm{C}$ NMR $\left(126 \mathrm{MHz}, \mathrm{CDCl}_{3}\right)$.

Apr28-2021

Shweta Gediya

C13APTIong.w $\mathrm{CDCl}$

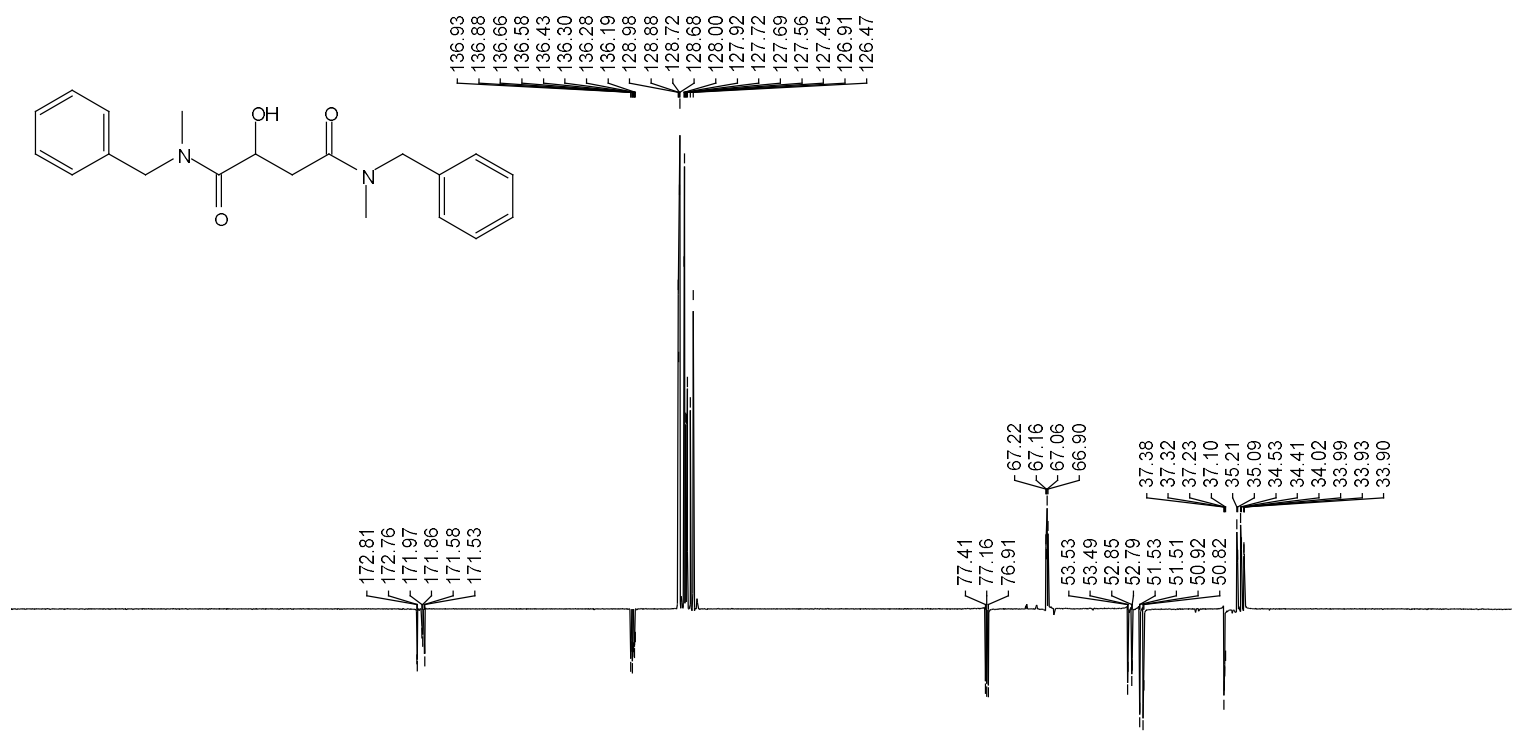

$\begin{array}{lllllllllllllllllllllllllllll}240 & 2.30 & 220 & 210 & 200 & 190 & 180 & 170 & 160 & 150 & 140 & 130 & \begin{array}{c}120 \\ \mathrm{f} 1(\mathrm{ppm})\end{array} & 100 & 90 & 80 & 70 & 60 & 50 & 40 & 30 & 20 & 10 & 0 & -10\end{array}$ 
COSY.

Chemist Shweta Gediya

COSY.w CDCl3 /opt/topspin3.2 SKG 18

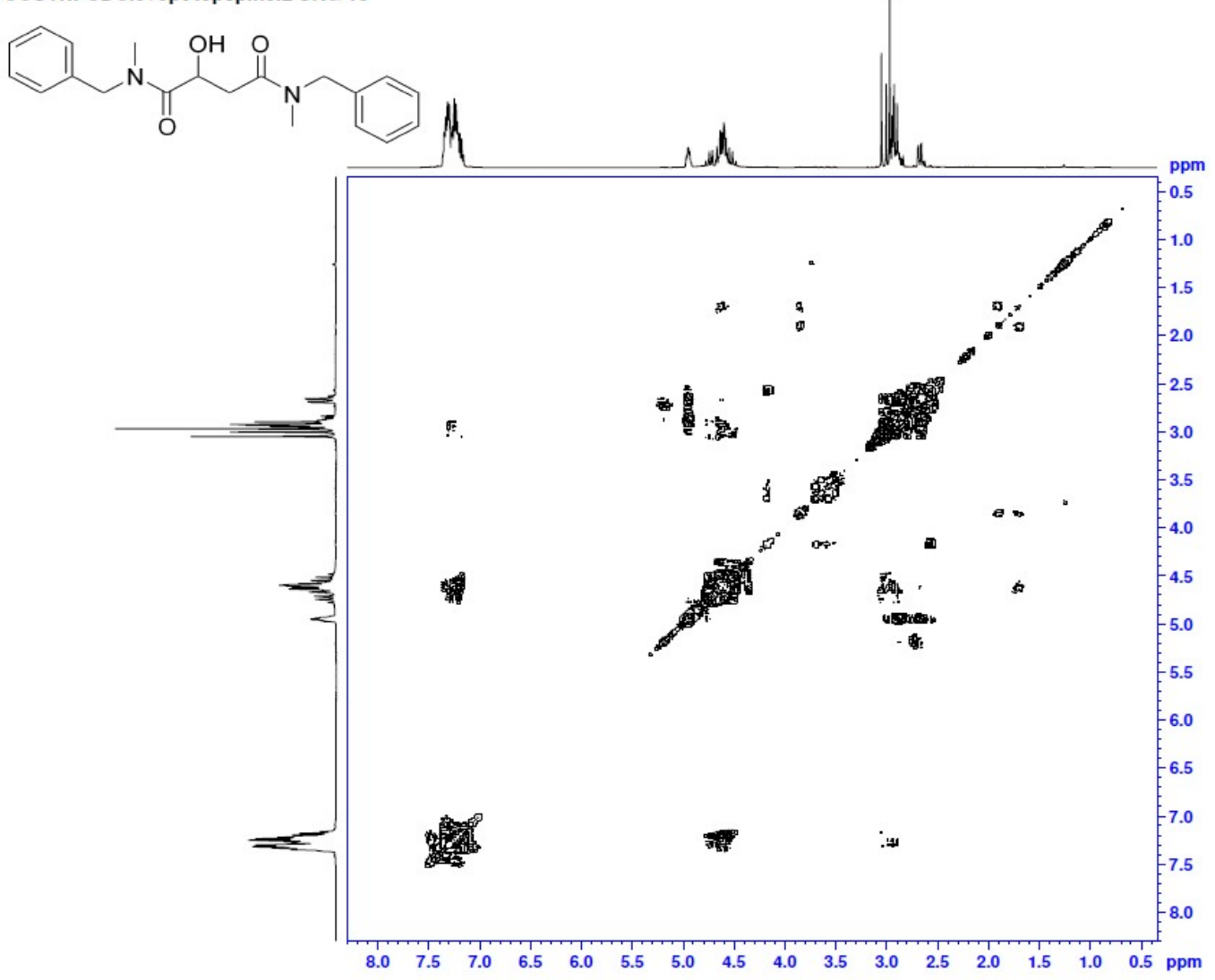

HSQC.

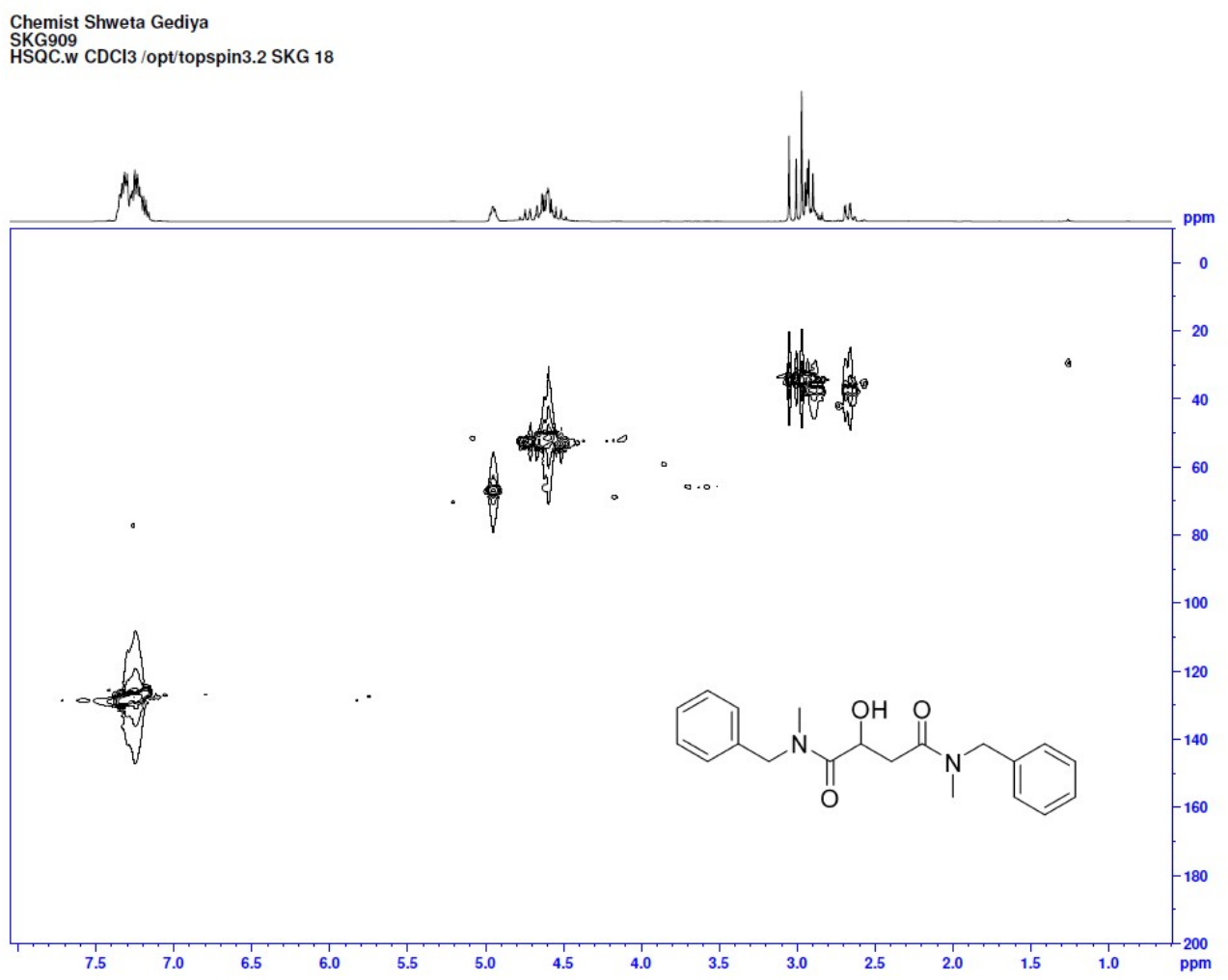

S207 


\section{Chiral HPLC of ketone precursor.}

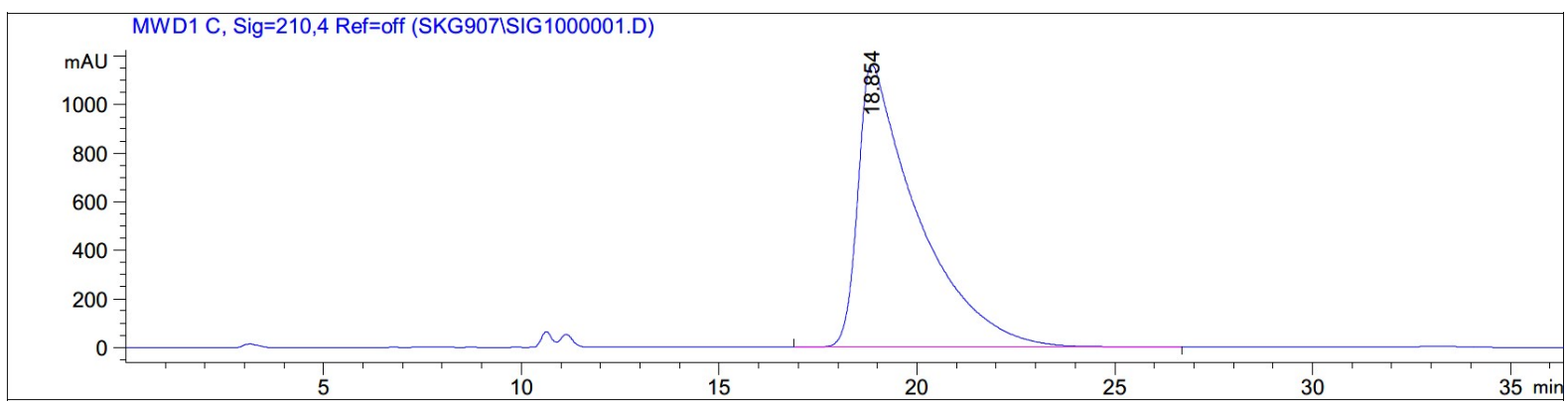

Signal 2: MWD1 C, Sig=210,4 Ref=off

\begin{tabular}{|c|c|c|c|c|c|c|}
\hline $\begin{array}{c}\text { Peak } \\
\#\end{array}$ & $\begin{array}{c}\text { RetTime } \\
\text { [min] }\end{array}$ & Type & $\begin{array}{c}\text { Width } \\
{[\mathrm{min}]}\end{array}$ & $\begin{array}{c}\text { Area } \\
{\left[\mathrm{mAU}^{\star} \mathrm{s}\right]}\end{array}$ & $\begin{array}{l}\text { Height } \\
{[\mathrm{mAU}]}\end{array}$ & $\begin{array}{c}\text { Area } \\
\quad \%\end{array}$ \\
\hline & ----- & & & --- & --- & 1 \\
\hline
\end{tabular}


Chiral HPLC of racemic alcohol.

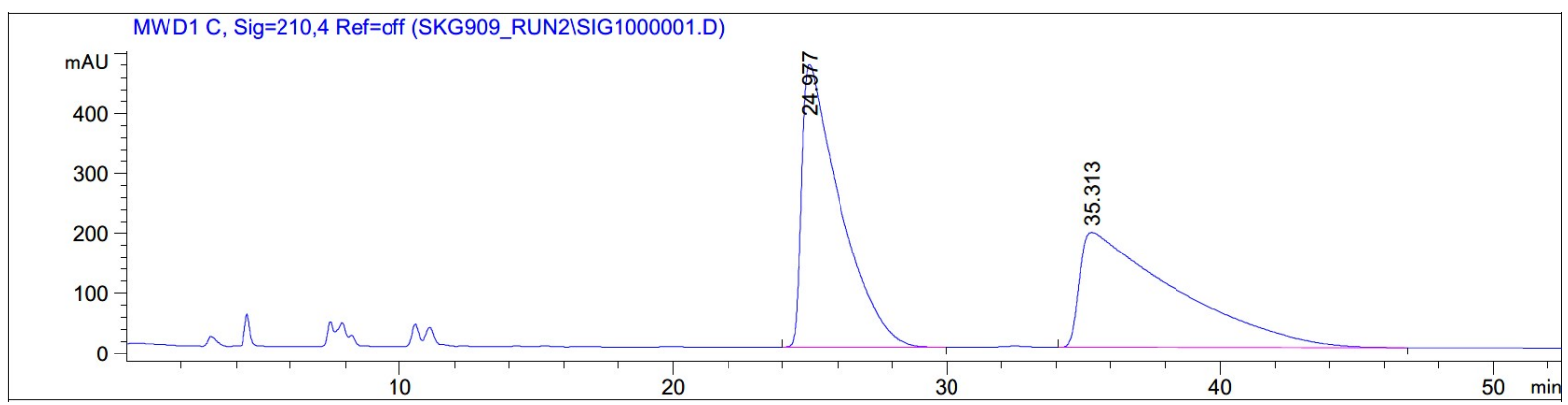

Signal 2: MWD1 C, Sig=210,4 Ref=off

\begin{tabular}{|c|c|c|c|c|c|c|}
\hline $\begin{array}{c}\text { Peak } \\
\#\end{array}$ & $\begin{array}{c}\text { RetTime } \\
\text { [min] }\end{array}$ & Type & $\begin{array}{l}\text { Width } \\
\text { [min] }\end{array}$ & $\begin{array}{c}\text { Area } \\
{\left[\mathrm{mAU}{ }^{\star} \mathrm{S}\right]}\end{array}$ & $\begin{array}{l}\text { Height } \\
{[\mathrm{mAU}]}\end{array}$ & $\begin{array}{c}\text { Area } \\
\frac{\circ}{0}\end{array}$ \\
\hline & -1 & & --1 & --ー--ー- & -ー-ー--ー- & $--------\mid$ \\
\hline 1 & 24.977 & BB & 1.3344 & $4.58942 \mathrm{e} 4$ & 470.33017 & 49.8903 \\
\hline 2 & 35.313 & $\mathrm{BB}$ & 2.9985 & $4.60960 \mathrm{e} 4$ & 191.48669 & 50.1097 \\
\hline
\end{tabular}

Chiral HPLC of ATH product. 94\% ee.

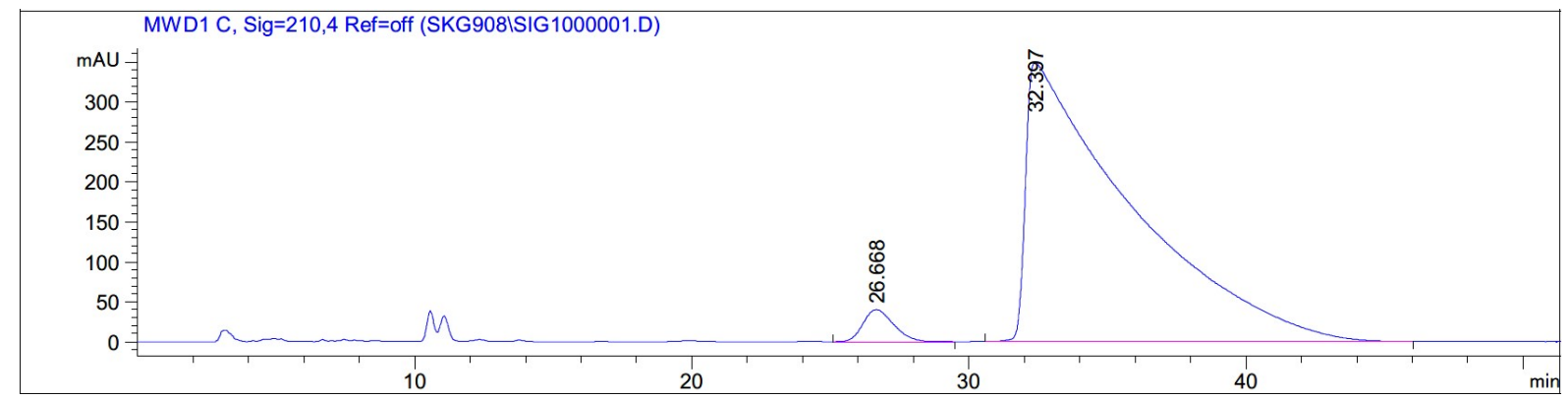

Signal 2: MWD1 C, Sig=210,4 Ref=off

\begin{tabular}{|c|c|c|c|c|c|c|}
\hline $\begin{array}{c}\text { Peak } \\
\#\end{array}$ & $\begin{array}{c}\text { RetTime } \\
\text { [min] }\end{array}$ & Type & $\begin{array}{l}\text { Width } \\
\text { [min] }\end{array}$ & $\begin{array}{c}\text { Area } \\
{\left[\mathrm{mAU}{ }^{\star} \mathrm{s}\right]}\end{array}$ & $\begin{array}{l}\text { Height } \\
{[\mathrm{mAU}]}\end{array}$ & $\begin{array}{c}\text { Area } \\
\frac{\circ}{0}\end{array}$ \\
\hline & ----- & & - & ----------- & ---------- & -------- \\
\hline 1 & 26.668 & BB & 1.1046 & 3047.00659 & 39.91579 & 3.2225 \\
\hline 2 & 32.397 & $\mathrm{BB}$ & 3.2092 & $9.15079 \mathrm{e} 4$ & 349.20761 & 96.7775 \\
\hline
\end{tabular}


(R)-2-Hydroxy- $N^{1}, N^{1}, N^{1}$-trimethyl- $N^{4}$-phenylsuccinamide 8.<smiles>CN(C)C(=O)[C@H](O)CC(=O)N(C)c1ccccc1</smiles>

This compound is novel and was prepared following general procedure C using $N^{1}, N^{1}, N^{4}$ trimethyl-2-oxo- $N^{4}$-phenylsuccinamide $(62.0 \mathrm{mg}, 0.250 \mathrm{mmol}, 1.0 \mathrm{eq}), 3 \mathrm{C}$ tethered catalyst $(R, R)-2(2.3 \mathrm{mg}, 3.8 \mu \mathrm{mol}, 0.015 \mathrm{eq})$ and FA:TEA $(0.15 \mathrm{~mL})$ in DCM $(1.5 \mathrm{~mL})$ to give 8 as a pale yellow oil (57.5 mg, $0.230 \mathrm{mmol}, 92 \%)$.

TLC: $\mathrm{R}_{\mathrm{f}}$ ca 0.3 (5:5, hexane: EtOAc), UV-active and PMA-reactive; HRMS (ESI) m/z: [M+Na] ${ }^{+}$Calcd for $\mathrm{C}_{13} \mathrm{H}_{18} \mathrm{~N}_{2} \mathrm{O}_{3} \mathrm{Na} 273.1210$; Found 273.1209 (error 0.3 ppm);

$U_{\max } 3348,2935,1648,1621,1592,1496,1385,1057,699,622 \mathrm{~cm}^{-1}$;

Enantiomeric excess determined by HPLC analysis (Chiralpak IG, $250 \mathrm{~mm} \times 4.6 \mathrm{~mm}$ column, hexane: $\operatorname{PrOH} 75: 251 \mathrm{~mL} / \mathrm{min}, \lambda=210 \mathrm{~nm}, \mathrm{~T}=25^{\circ} \mathrm{C}$ ), $S$-enantiomer $23.1 \mathrm{~min}, R$ enantiomer $29.7 \mathrm{~min} .76 \%$ ee $(R),[\alpha]_{\mathrm{D}}^{22}=+14\left(\mathrm{c}=0.25, \mathrm{CHCl}_{3}\right)$;

${ }^{1} \mathrm{H}$ NMR $\left(500 \mathrm{MHz}, \mathrm{CDCl}_{3}\right)$ (mixture of rotamers, ca 19:1) $\delta 7.41(2 \mathrm{H}, \mathrm{t}, J=7.6 \mathrm{~Hz}, \mathrm{ArH})$, $7.34(1 \mathrm{H}, \mathrm{t}, J=7.4 \mathrm{~Hz}, \mathrm{ArH}), 7.24$ (2H, d, $J=7.4 \mathrm{~Hz}, \mathrm{ArH}), 4.84-4.80$ (1H, m, CㅂOH), 4.28 $(1 \mathrm{H}, \mathrm{d}, J=7.4 \mathrm{~Hz}, \mathrm{CHO} \underline{\mathrm{H}}), 3.39\left(0.15 \mathrm{H}, \mathrm{s}, \mathrm{NCH}_{3}\right), 3.30\left(2.85 \mathrm{H}, \mathrm{s}, \mathrm{NCH}_{3}\right), 3.11(0.15 \mathrm{H}, \mathrm{s}$, $\left.\mathrm{NCH}_{3}\right), 3.03\left(2.85 \mathrm{H}, \mathrm{s}, \mathrm{NCH}_{3}\right), 2.97\left(0.15 \mathrm{H}, \mathrm{s}, \mathrm{NCH}_{3}\right), 2.93\left(2.85 \mathrm{H}, \mathrm{s}, \mathrm{NCH}_{3}\right) 2.44(1 \mathrm{H}, \mathrm{dd}, J$ $\left.=15.7,8.1 \mathrm{~Hz}, \underline{\mathrm{C}}_{\mathrm{a}} \mathrm{H}_{\mathrm{b}}\right), 2.29\left(1 \mathrm{H}, \mathrm{dd}, J=15.7,2.8 \mathrm{~Hz}, \mathrm{CH}_{\mathrm{a}} \underline{\mathrm{H}}_{\mathrm{b}}\right)$;

${ }^{13} \mathrm{C} \mathrm{NMR}\left(126 \mathrm{MHz}, \mathrm{CDCl}_{3}\right.$ ) (mixture of rotamers) $\delta 172.9,170.9,143.6,129.9,128.1$, 127.6, 66.7, 38.3, 37.5, 36.7, 35.9;

$m / z(\mathrm{ESI}) 273.1\left[(\mathrm{M}+\mathrm{Na})^{+}, 100 \%\right]$. 
2-Hydroxy- $N^{1}, N^{1}, N^{4}$-trimethyl- $N^{4}$-phenylsuccinamide 8.

${ }^{1} \mathrm{H} \mathrm{NMR}\left(500 \mathrm{MHz}, \mathrm{CDCl}_{3}\right)$.

Apr22-2021

Chemist Shweta Gediya

PROTON.w CDCl3 /opt/topspin3.2 SKG 7

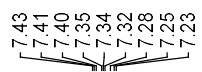

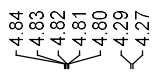

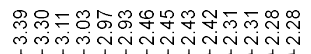

8
0
$i$
1<smiles>CC(CC(=O)N(C)c1ccccc1)C(=O)N(C)C</smiles>
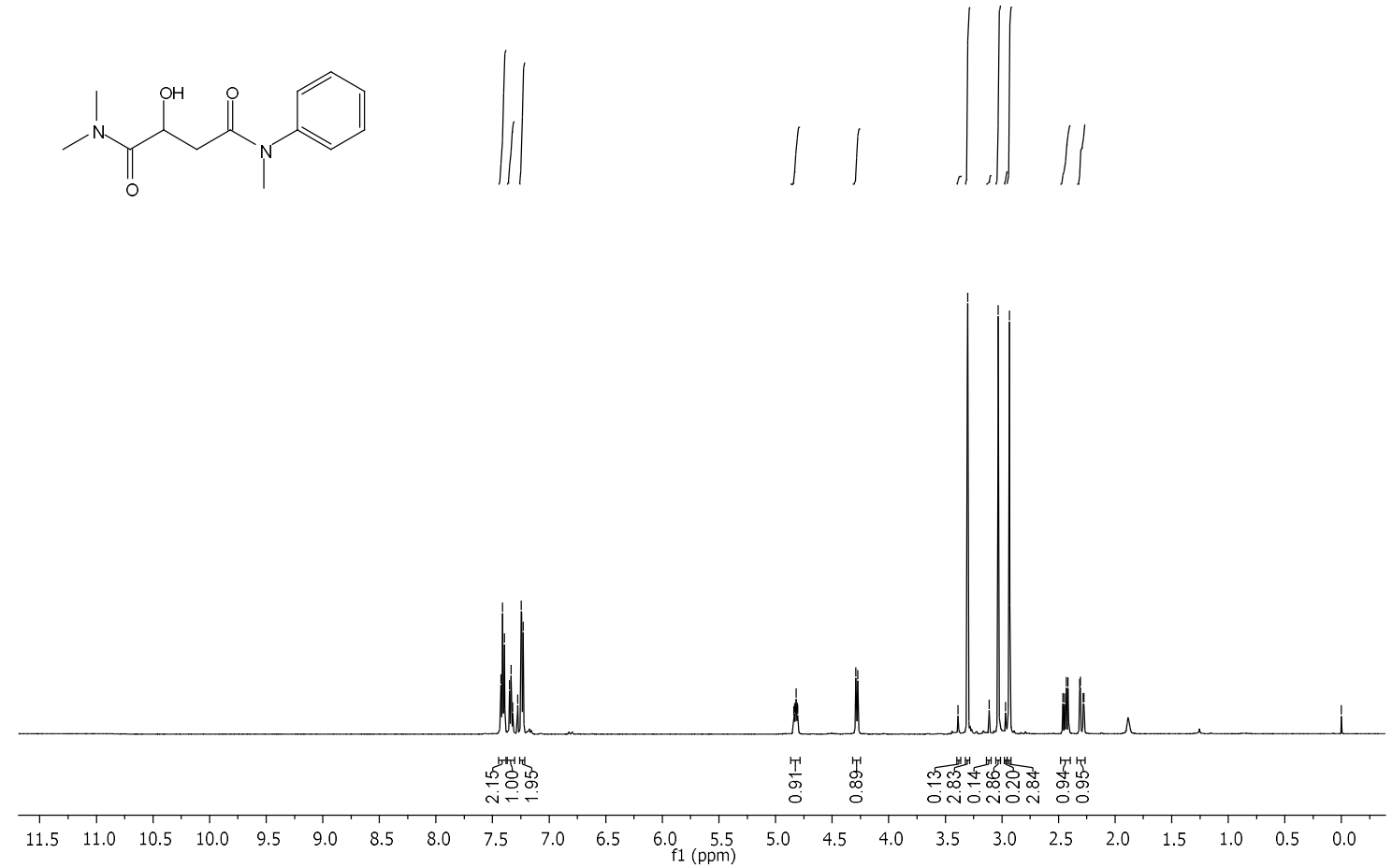

${ }^{13} \mathrm{C} \mathrm{NMR}\left(126 \mathrm{MHz}, \mathrm{CDCl}_{3}\right)$.

Apr22-2021

SKG898

C13APT.w CDCl3 /opt/topspin3.2 SKG

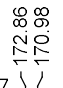

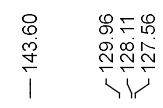

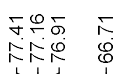

원

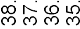

पil

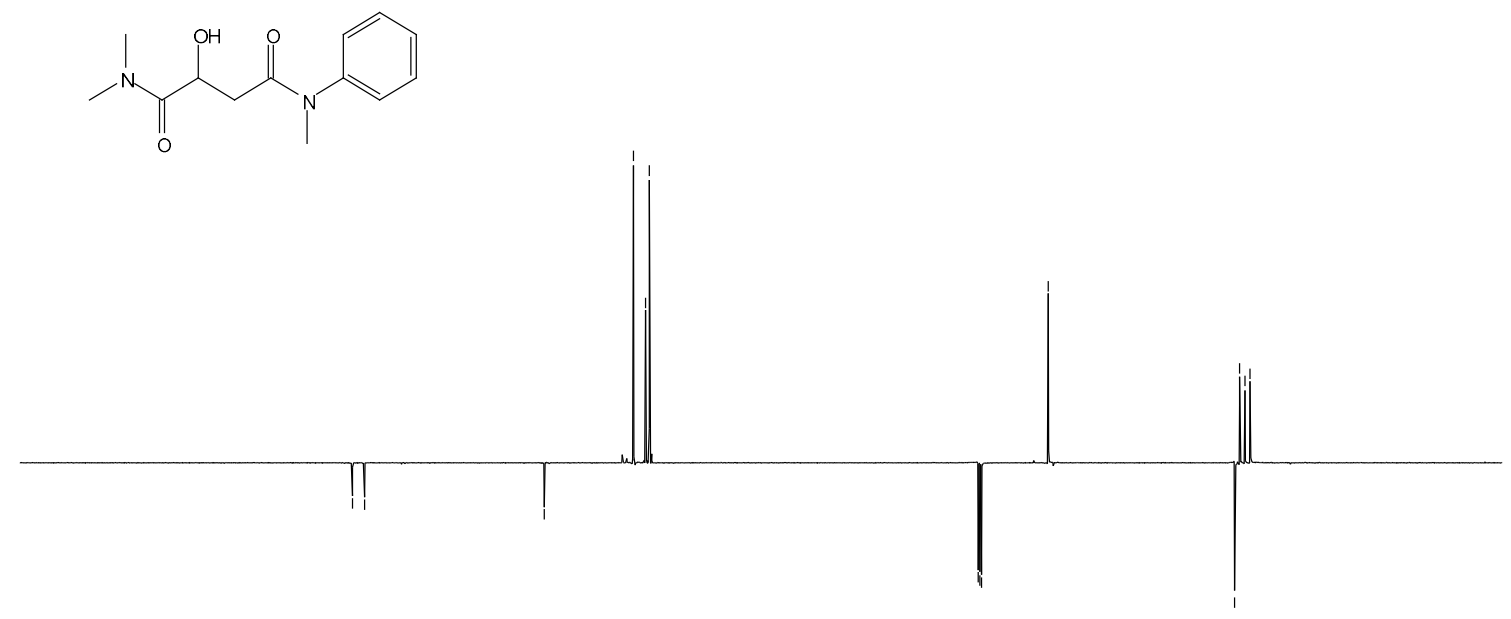

$\begin{array}{lllllllllllllllllllllllllllllllllll}220 & 210 & 200 & 190 & 180 & 170 & 160 & 150 & 140 & 130 & 120 & 110 & 100 & 90 & 80 & 70 & 60 & 50 & 40 & 30 & 20 & 10 & 0\end{array}$ 
COSY.

Chemist Shweta Gediya

COSY.w $\mathrm{CDCl}_{3}$ /opt/topspin3.2 SKG 7

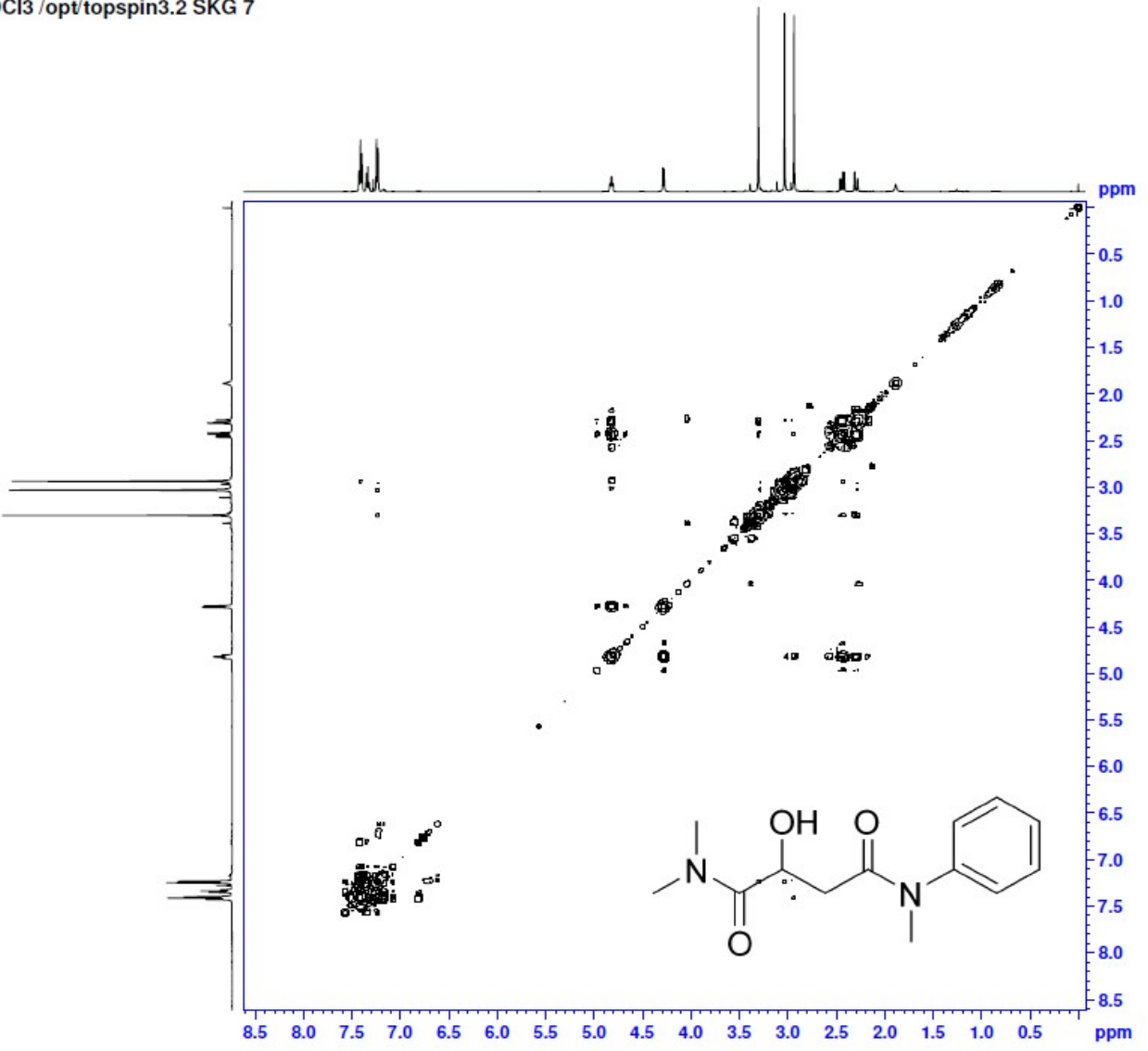

HSQC.

Chemist Shweta Gediya

SKG898 $\mathrm{HDCl}_{3} / \mathrm{opt} /$ topspin3.2 SKG 7

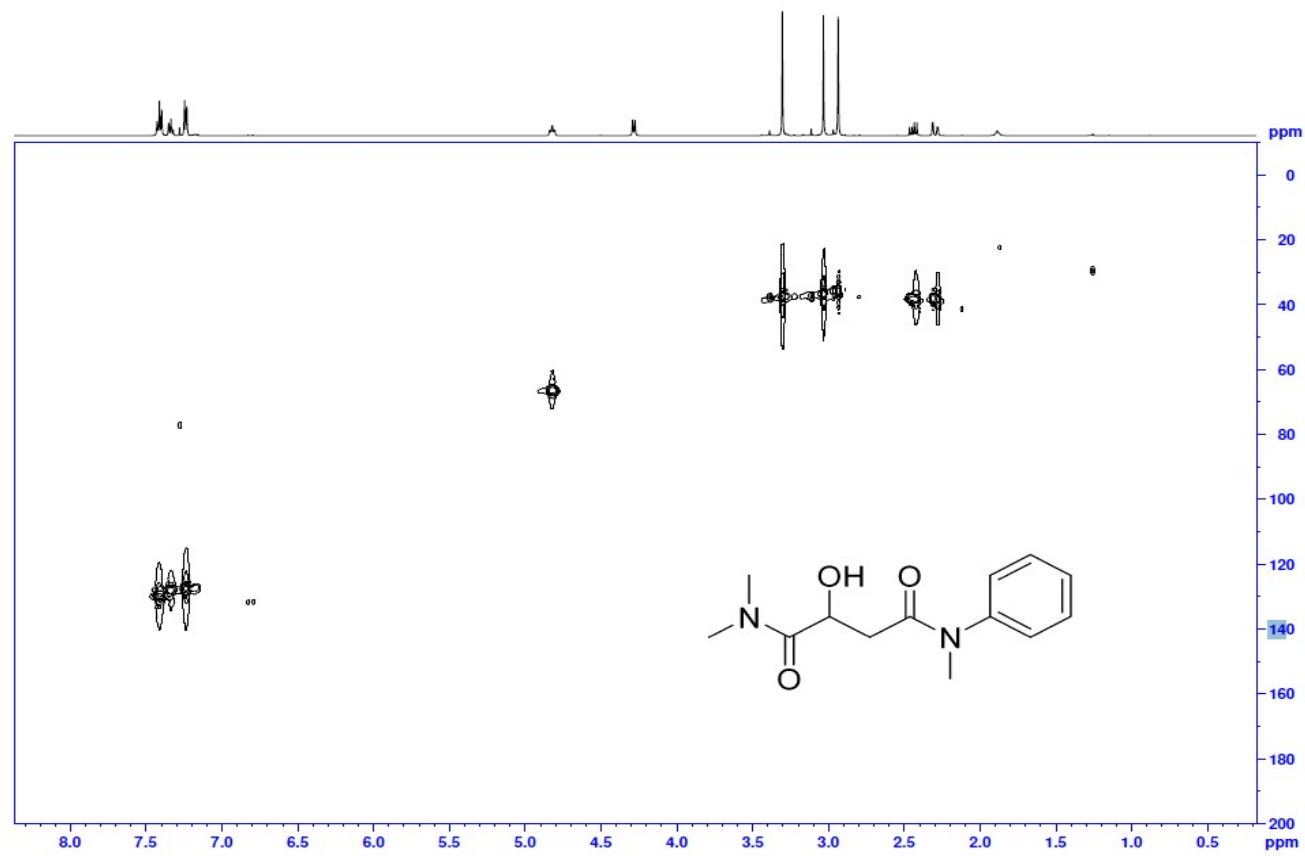




\section{Chiral HPLC of ketone precursor.}

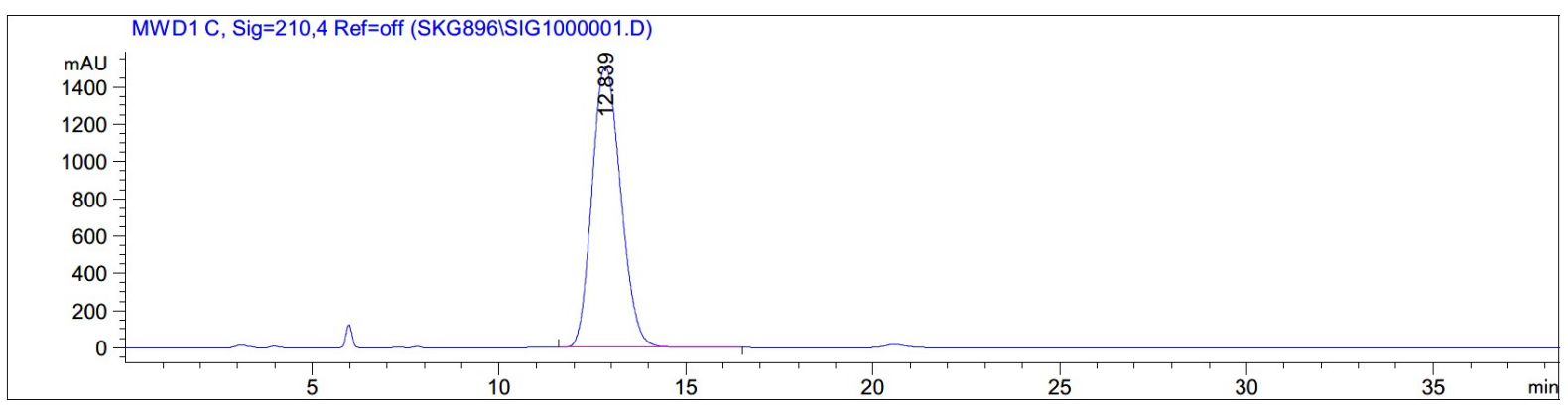

Signal 2: MWD1 C, Sig=210,4 Ref=off

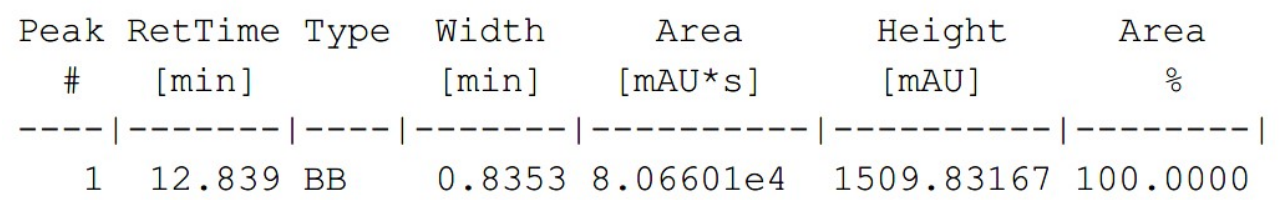




\section{Chiral HPLC of racemic alcohol.}

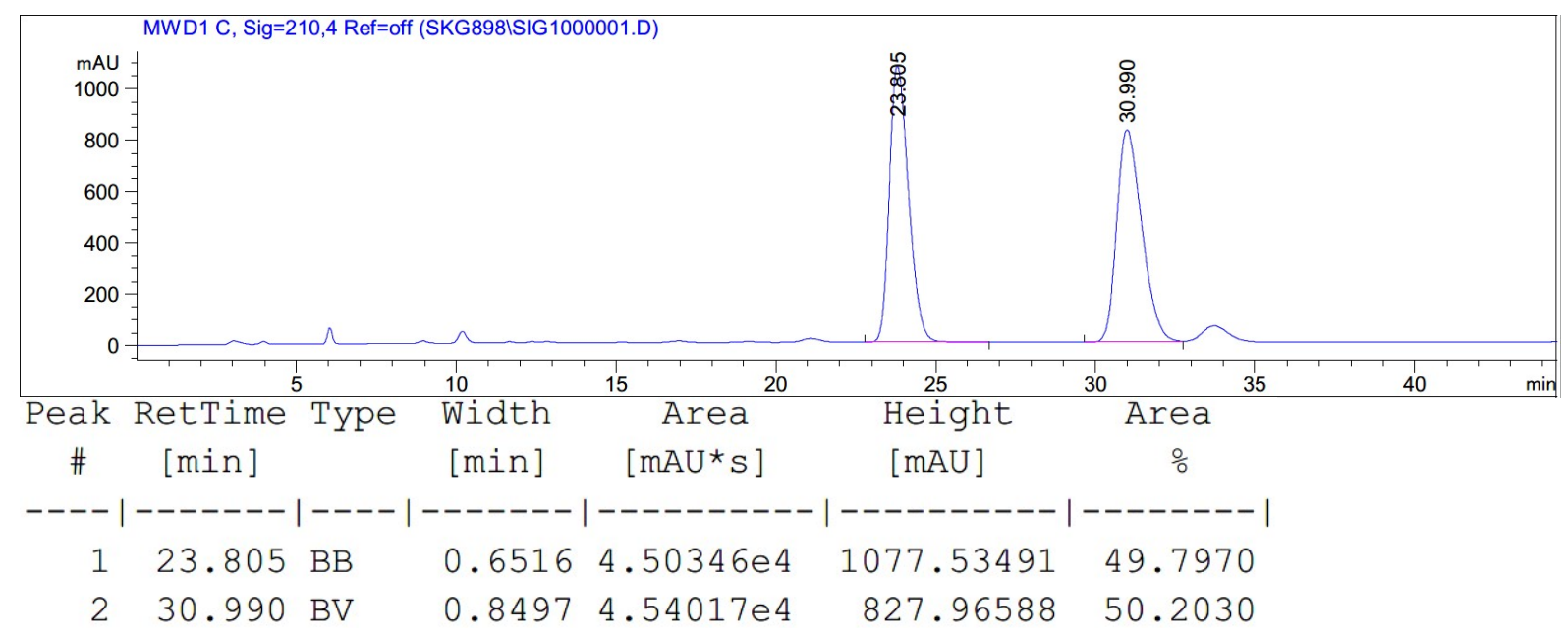

Chiral HPLC of ATH product. 76\% ee.

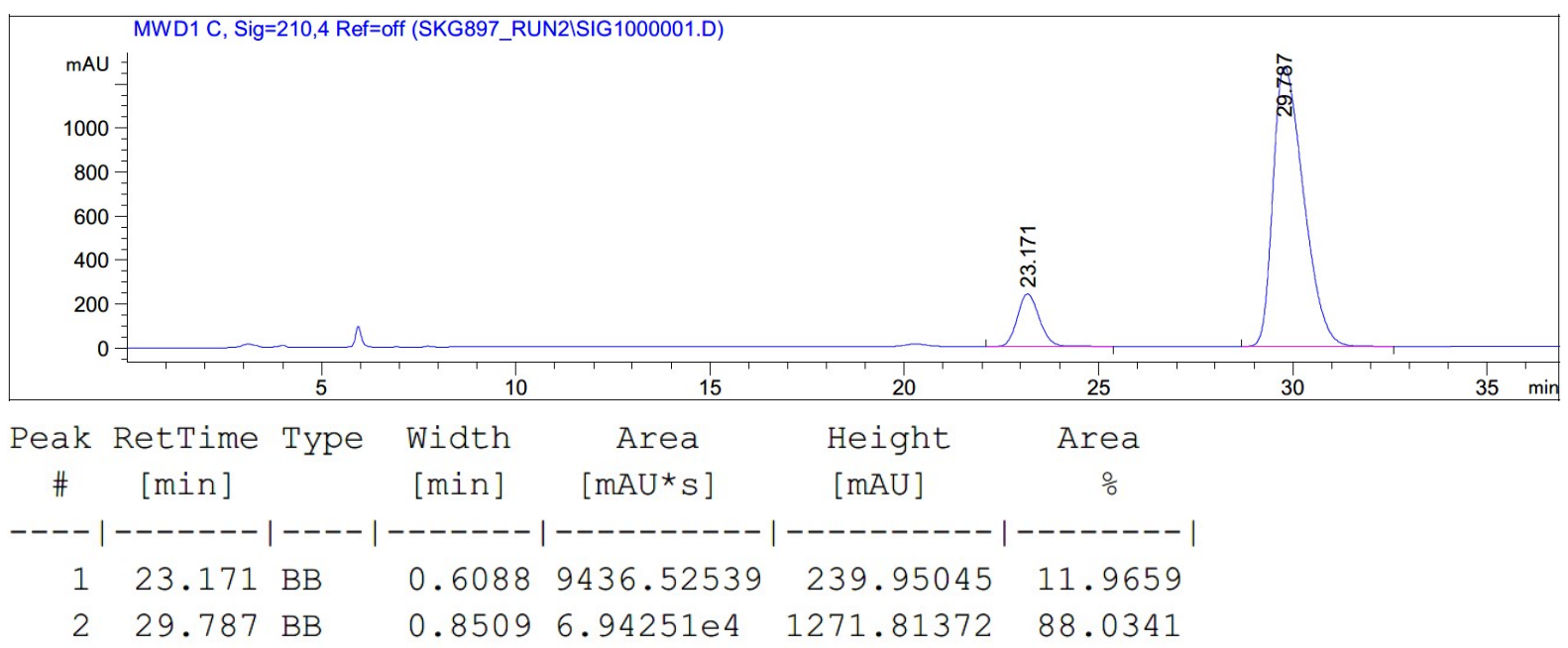


(R)- $N^{1}$-cyclohexyl-2-hydroxy- $N^{1}, N^{4}$-dimethyl- $N^{4}$-phenylsuccinamide 9.<smiles>CN(C(=O)C[C@@H](O)C(=O)N(C)C1CCCCC1)c1ccccc1</smiles>

This compound is novel and was prepared following general procedure $\mathrm{C}$ using $N^{1}$ cyclohexyl- $N^{1}, N^{4}$-dimethyl-2-oxo- $N^{4}$-phenylsuccinamide (79.0 mg, $\left.0.250 \mathrm{mmol}, 1.0 \mathrm{eq}\right), 3 \mathrm{C}$ tethered catalyst $(R, R)-2(2.3 \mathrm{mg}, 3.8 \mu \mathrm{mol}, 0.015 \mathrm{eq})$ and FA:TEA $(0.15 \mathrm{~mL})$ in DCM $(1.5$ $\mathrm{mL})$ to give 9 as a white solid $(72.1 \mathrm{mg}, 0.226 \mathrm{mmol}, 91 \%)$.

TLC: $\mathrm{R}_{\mathrm{f}}$ ca 0.2 (1:9, hexane: EtOAc), non UV-active, Strong $\mathrm{KMnO}_{4}$ and PMA-reactive; HRMS (ESI) m/z: [M+Na] $]^{+}$Calcd for $\mathrm{C}_{18} \mathrm{H}_{26} \mathrm{~N}_{2} \mathrm{O}_{3} \mathrm{Na}$ 341.1836; Found 341.1831 (error 1.4 ppm);

MP: $88-89^{\circ} \mathrm{C}$;

$U_{\max } 3300,2930,2857,1646,1616,1595,1410,1120,1079,781,704,541 \mathrm{~cm}^{-1}$;

Enantiomeric excess determined by HPLC analysis (Chiralpak IG, $250 \mathrm{~mm} \times 4.6 \mathrm{~mm}$ column, hexane: $\left.\mathrm{iPrOH} 75: 25,1 \mathrm{~mL} / \mathrm{min}, \lambda=210 \mathrm{~nm}, \mathrm{~T}=25^{\circ} \mathrm{C}\right), S$-enantiomer $22.6 \mathrm{~min}, R$ enantiomer $27.2 \min .79 \%$ ee $(R),[\alpha]_{\mathrm{D}}^{22}=+310\left(\mathrm{c}=0.01, \mathrm{CHCl}_{3}\right)$;

${ }^{1} \mathrm{H}$ NMR (500 MHz, $\mathrm{CDCl}_{3}$ ) (Rotamers, 1:1) $\delta$ 7.42-7.23 (5H, m, ArH), $4.82(1 \mathrm{H}, \mathrm{q}, J=10.3$ $\mathrm{Hz}, \mathrm{C} \underline{\mathrm{HOH}}), 4.40(0.5 \mathrm{H}, \mathrm{d}, J=7.4 \mathrm{~Hz}, \mathrm{CHO} \underline{\mathrm{H}}), 4.31-4.47(0.5 \mathrm{H}, \mathrm{m}, \mathrm{CH}$ of cyclohexyl), 4.21 $(0.5 \mathrm{H}, \mathrm{d}, J=7.4 \mathrm{~Hz}, \mathrm{CHOH}), 3.69-3.63\left(0.5 \mathrm{H}, \mathrm{m}, \mathrm{CH}\right.$ of cyclohexyl), $3.31\left(3 \mathrm{H}, \mathrm{s}, \mathrm{NCH}_{3}\right)$, $2.85\left(1.5 \mathrm{H}, \mathrm{s}, \mathrm{NCH}_{3}\right), 2.79\left(1.5 \mathrm{H}, \mathrm{s}, \mathrm{NCH}_{3}\right), 2.48-2.39\left(1 \mathrm{H}, \mathrm{m}, \mathrm{CH}_{\mathrm{a}} \mathrm{H}_{\mathrm{b}}\right), 2.27-2.19(1 \mathrm{H}, \mathrm{m}$, $\left.\mathrm{CH}_{\mathrm{a}} \underline{\mathrm{H}}_{\mathrm{b}}\right), 1.86-1.63$ (4H, m, $\mathrm{CH}_{2}$ of cyclohexyl), 1.55-1.23 (5H, m, $\mathrm{CH}_{2}$ of cyclohexyl), 1.12$1.03\left(1 \mathrm{H}, \mathrm{m}, \mathrm{CH}_{2}\right.$ of cyclohexyl);

${ }^{13} \mathrm{C}$ NMR (126 MHz, $\mathrm{CDCl}_{3}$ ) (Rotamers 1:1) $\delta$ 172.6, 172.5, 171.0, 170.7, 143.7, 129.9, $128.1,127.6,127.5,66.9,66.8,55.8,53.4,38.9,38.5,37.6,37.5,30.9,30.7,29.8,29.5,28.9$, $27.8,25.7,25.6,25.5,25.3$;

$m / z(\mathrm{ESI}) 341.2\left[(\mathrm{M}+\mathrm{Na})^{+}, 100 \%\right]$. 
$N^{1}$-Cyclohexyl-2-hydroxy- $N^{1}, N^{4}$-dimethyl- $N^{4}$-phenylsuccinamide 9.

${ }^{1} \mathrm{H}$ NMR $\left(500 \mathrm{MHz}, \mathrm{CDCl}_{3}\right)$.

Nov26-2020

Nov26-2020
Chemist Shweta Gediya
SKG670

PROTON.w CDCI3 /opt/topspin3.2 SKG 54
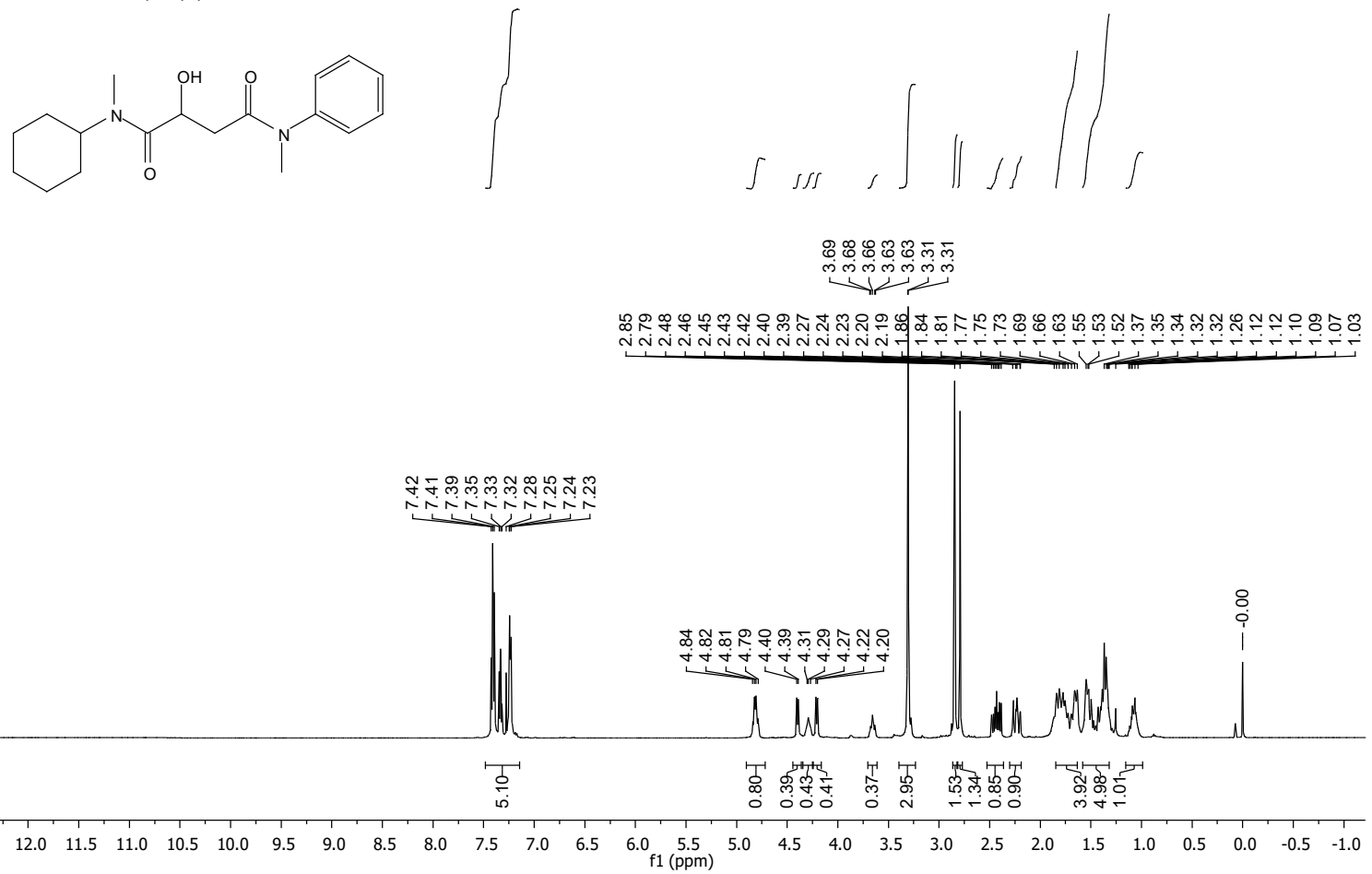

$\begin{array}{lllllllllllllllllllllllllllll}12.0 & 11.5 & 11.0 & 10.5 & 10.0 & 9.5 & 9.0 & 8.5 & 8.0 & 7.5 & 7.0 & 6.5 & 6.0 & 5.5 & 5.0 & 4.5 & 4.0 & 3.5 & 3.0 & 2.5 & 2.0 & 1.5 & 1.0 & 0.5 & 0.0 & -0.5 & -1.0\end{array}$

${ }^{13} \mathrm{C}$ NMR (126 MHz, $\left.\mathrm{CDCl}_{3}\right)$.

\begin{tabular}{|c|c|c|c|c|c|c|}
\hline $\begin{array}{l}\text { Nov26-2020 } \\
\text { Shweta Gediya } \\
\text { SKG670 } \\
\text { C13APTlong.w } \mathrm{CDCl} 3\end{array}$ & 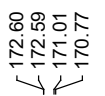 & 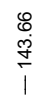 & 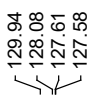 & 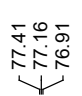 & $\begin{array}{l}\infty \\
\infty \infty \\
0.0 \\
00 \\
y\end{array}$ & 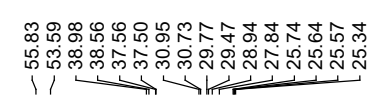 \\
\hline
\end{tabular}<smiles>CN(C(=O)CC(O)C(=O)N(C)C1CCCCC1)c1ccccc1</smiles>

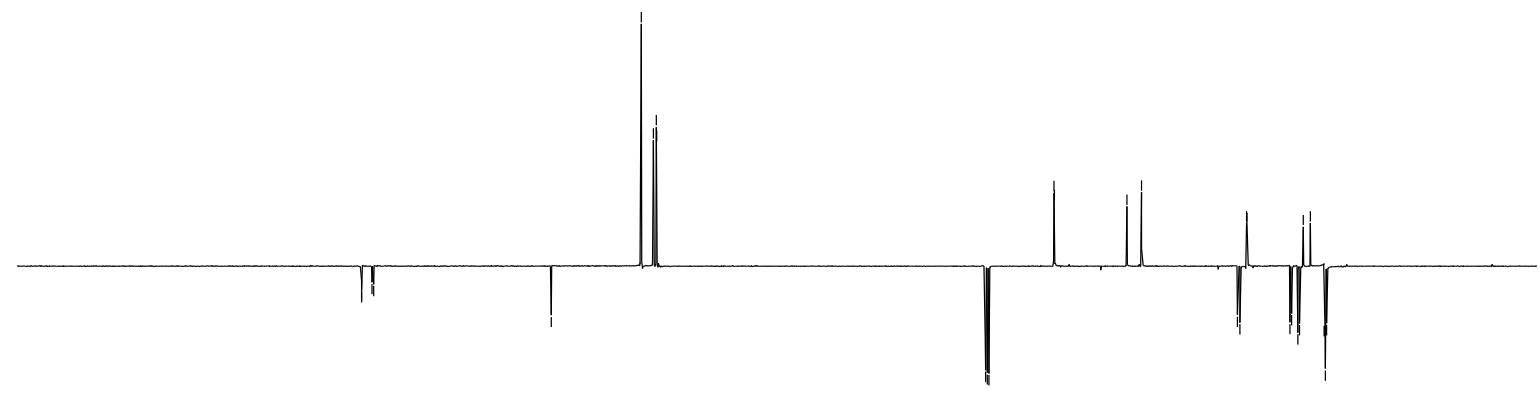

$\begin{array}{llllllllllllllllllllllllllllllllllll}220 & 210 & 200 & 190 & 180 & 170 & 160 & 150 & 140 & 130 & 120 & 110 & 100 & 90 & 80 & 70 & 60 & 50 & 40 & 30 & 20 & 10 & 0\end{array}$ 
COSY.

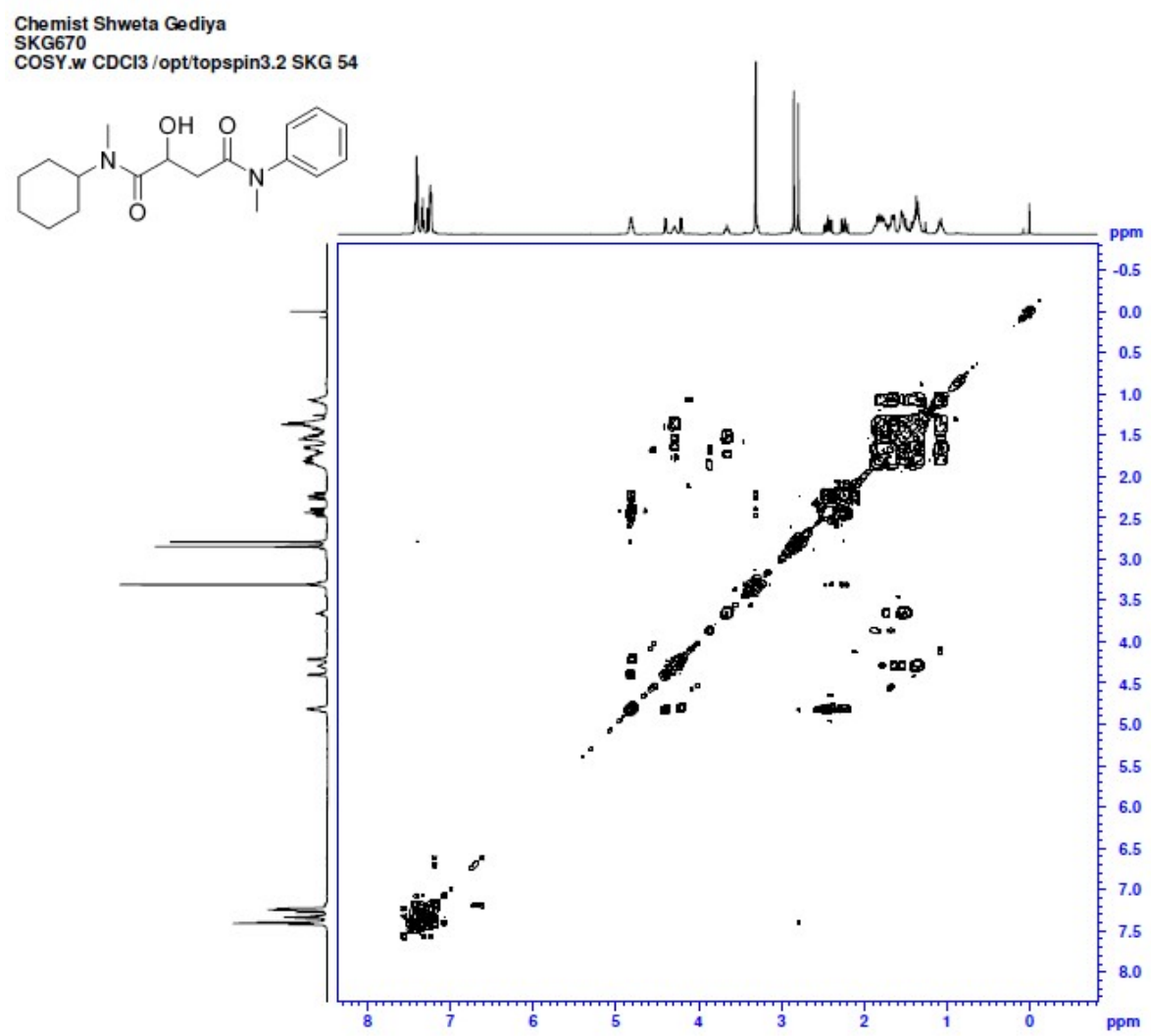

HSQC.

Chemist Shweta Gediya

HSQC.w CDCl3 /opt/topspin3.2 SKG 54

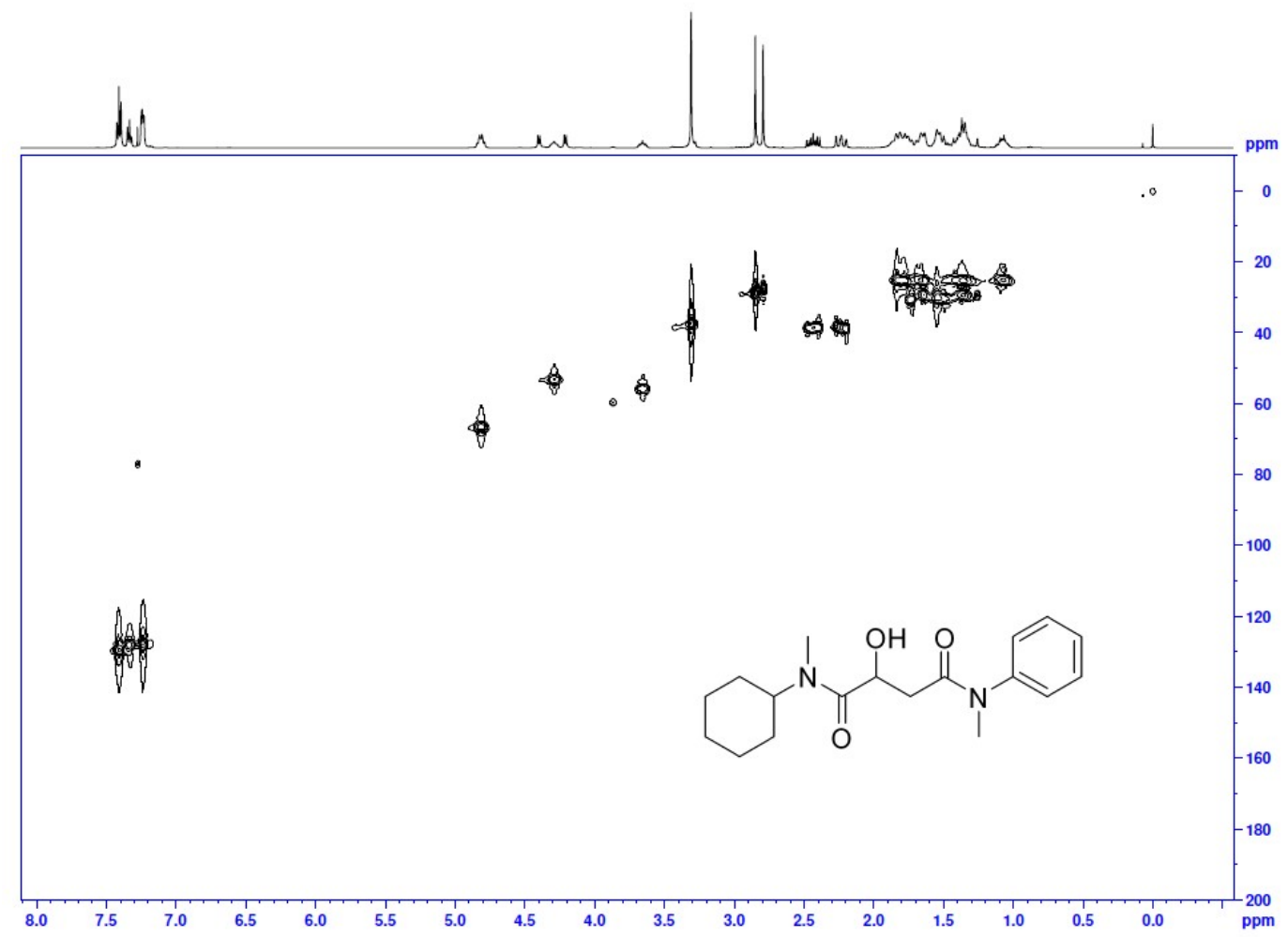




\section{Chiral HPLC of ketone precursor.}

[9080

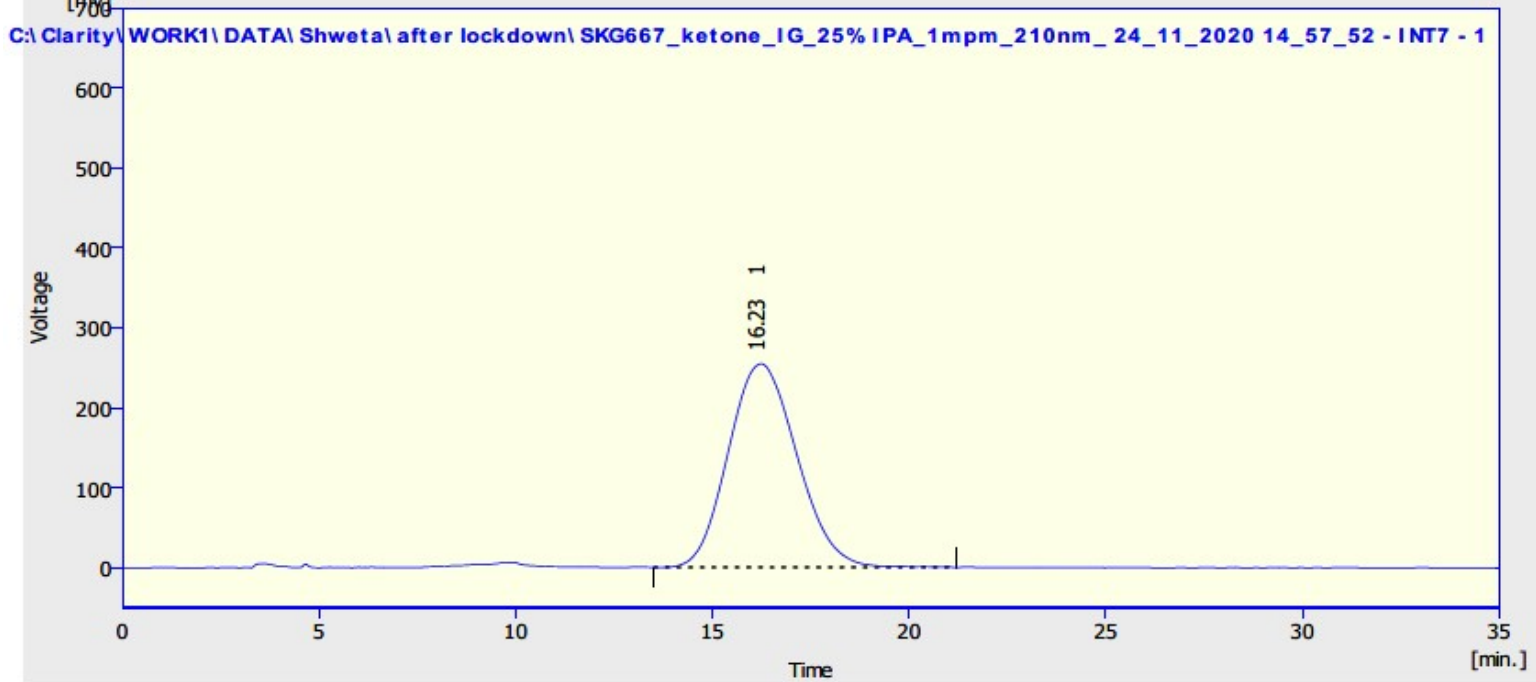

\begin{tabular}{|c|c|c|c|c|c|c|c|}
\hline & $\begin{array}{c}\text { Reten. Time } \\
{[\mathrm{min}]}\end{array}$ & $\begin{array}{c}\text { Area } \\
{[\mathrm{mV} . \mathrm{s}]}\end{array}$ & $\begin{array}{c}\text { Height } \\
{[\mathrm{mV}]}\end{array}$ & $\begin{array}{c}\text { Area } \\
{[\%]}\end{array}$ & $\begin{array}{c}\text { Height } \\
{[\%]}\end{array}$ & $\begin{array}{c}\text { W05 } \\
{[\mathrm{min}]}\end{array}$ & $\begin{array}{c}\text { Compound } \\
\text { Name }\end{array}$ \\
\hline 1 & 16.233 & 30895.760 & 254.933 & 100.0 & 100.0 & 1.91 & \\
\hline & Total & 30895.760 & 254.933 & 100.0 & 100.0 & & \\
\hline
\end{tabular}

\section{Chiral HPLC of racemic alcohol.}

[9060,

I Clarity \WORK1\ DATAI Shwet al after lockdownI SKG670_racem ic_IG_25\% I PA_1 mpm_210nm_24_11_2020 14_57_52 - I NT7 - 1

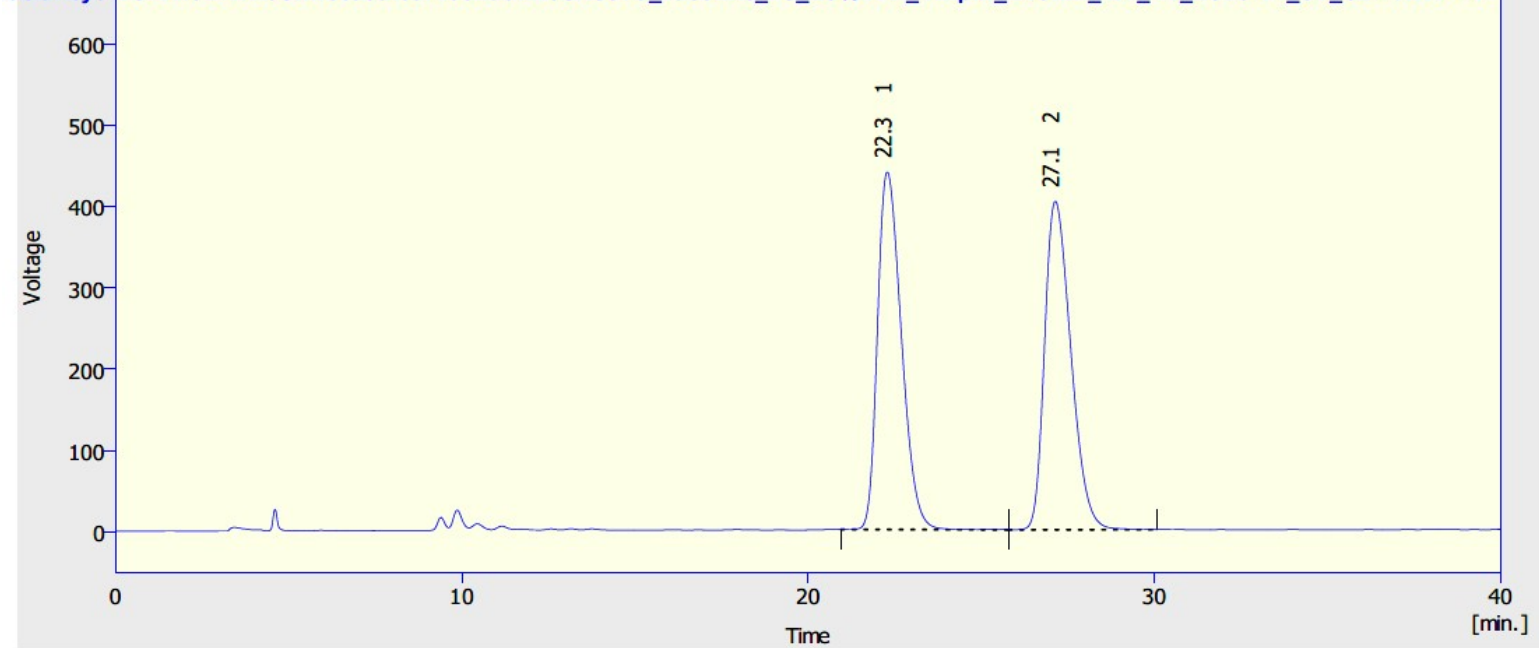

\begin{tabular}{|c|c|c|r|r|r|r|r|}
\hline & $\begin{array}{c}\text { Reten. Time } \\
{[\mathrm{min}]}\end{array}$ & $\begin{array}{c}\text { Area } \\
{[\mathrm{mV} . \mathrm{s}]}\end{array}$ & $\begin{array}{c}\text { Height } \\
{[\mathrm{mV}]}\end{array}$ & $\begin{array}{c}\text { Area } \\
{[\%]}\end{array}$ & $\begin{array}{c}\text { Height } \\
{[\%]}\end{array}$ & $\begin{array}{c}\text { W05 } \\
{[\mathrm{min}]}\end{array}$ & $\begin{array}{c}\text { Compound } \\
\text { Name }\end{array}$ \\
\hline 1 & 22.287 & 21094.228 & 440.977 & 49.7 & 52.1 & 0.75 & \\
\hline 2 & 27.143 & 21371.384 & 405.080 & 50.3 & 47.9 & 0.82 & \\
& Total & 42465.612 & 846.058 & 100.0 & 100.0 & & \\
\hline
\end{tabular}


Chiral HPLC of ATH product. 79\% ee.

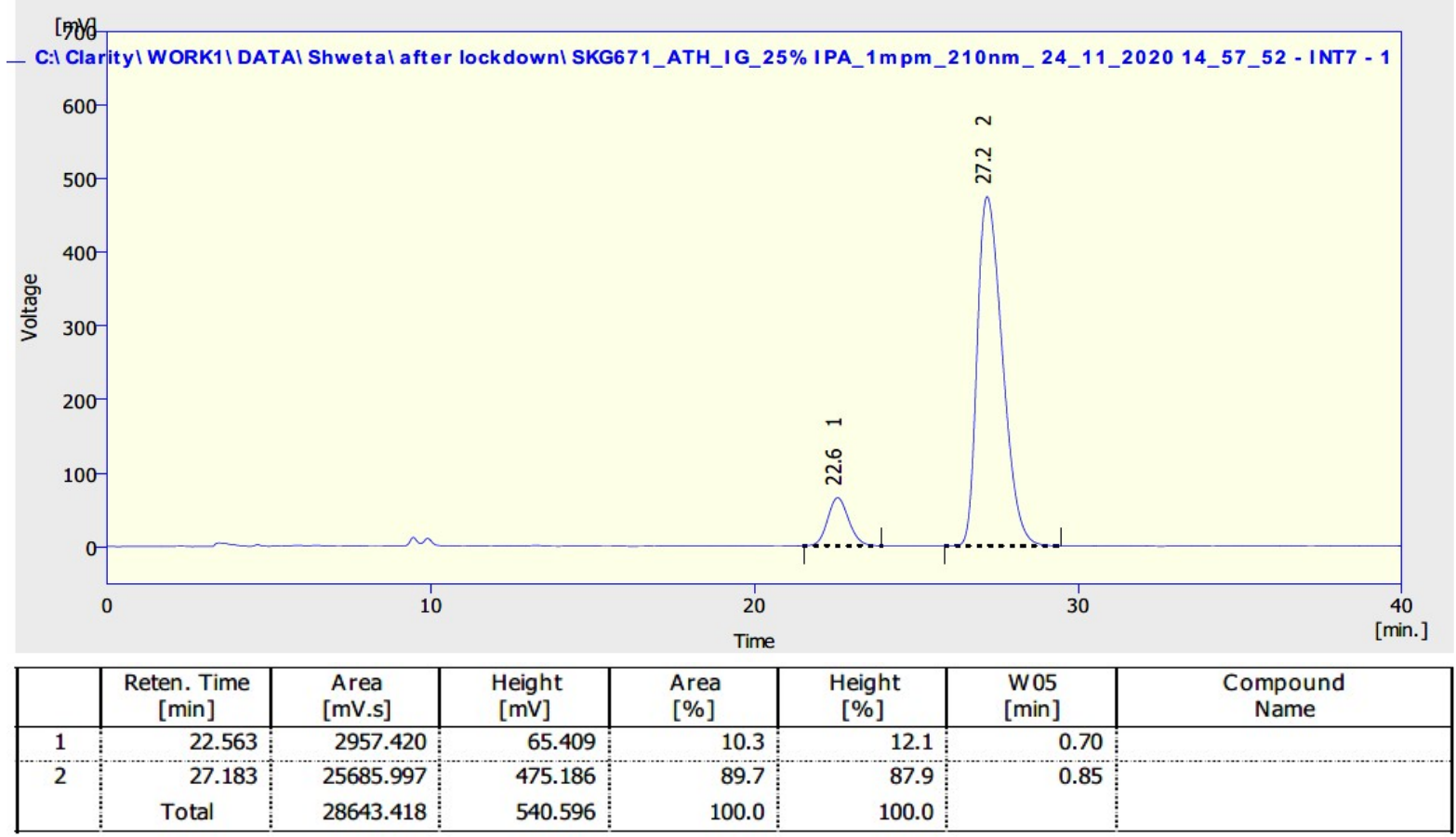


(R)- $N^{1}, N^{4}$-Dicyclohexyl-2-hydroxy- $N^{1}, N^{4}$-dimethylsuccinamide 10.<smiles>CN(C(=O)C[C@@H](O)C(=O)N(C)C1CCCCC1)C1CCCCC1</smiles>

This compound is novel and was prepared following general procedure $\mathrm{C}$ using $N^{1}, N^{4}$ dicyclohexyl- $N^{1}, N^{4}$-dimethyl-2-oxosuccinamide ( $\left.80.5 \mathrm{mg}, 0.250 \mathrm{mmol}, 1.0 \mathrm{eq}\right), 3 \mathrm{C}$ tethered catalyst $(R, R)-2(2.3 \mathrm{mg}, 3.8 \mu \mathrm{mol}, 0.015 \mathrm{eq})$ and FA:TEA $(0.15 \mathrm{~mL})$ in DCM $(1.5 \mathrm{~mL})$ to give 10 as a colourless liquid (72.3 $\mathrm{mg}, 0.223 \mathrm{mmol}, 89 \%)$.

TLC: $\mathrm{R}_{\mathrm{f}}$ ca 0.25 (1:9, hexane: EtOAc), non UV-active, Strong $\mathrm{KMnO}_{4}$ and PMA-reactive; HRMS (ESI) m/z: [M+Na] $]^{+}$Calcd for $\mathrm{C}_{18} \mathrm{H}_{32} \mathrm{~N}_{2} \mathrm{O}_{3} \mathrm{Na} 347.2305$; Found 347.2304 (error 0.3 ppm);

$U_{\max } 3400,2927,2855,2240,1617,1405,1320,1256,1087,919,726 \mathrm{~cm}^{-1}$;

Enantiomeric excess determined by HPLC analysis (Chiralpak IG, $250 \mathrm{~mm} \times 4.6 \mathrm{~mm}$ column, hexane: $\left.\mathrm{iPrOH} 90: 10,1 \mathrm{~mL} / \mathrm{min}, \lambda=210 \mathrm{~nm}, \mathrm{~T}=25^{\circ} \mathrm{C}\right), S$-enantiomer $56.9 \mathrm{~min}, R$ enantiomer $58.4 \min .95 \%$ ee $(R),[\alpha]_{\mathrm{D}}^{22}=+6.1\left(\mathrm{c}=0.1, \mathrm{CHCl}_{3}\right)$;

${ }^{1} \mathrm{H}$ NMR (500 MHz, $\mathrm{CDCl}_{3}$ ) (equal mixture of four rotamers) $\delta 4.85-4.79(1 \mathrm{H}, \mathrm{m}, \mathrm{C} \underline{\mathrm{HOH}})$, $4.64(0.25 \mathrm{H}, \mathrm{d}, J=6.5 \mathrm{~Hz}, \mathrm{CHO} \underline{\mathrm{H}}), 4.54(0.25 \mathrm{H}, \mathrm{d}, J=6.5 \mathrm{~Hz}, \mathrm{CHO} \underline{\mathrm{H}}), 4.46-4.41(0.75 \mathrm{H}$, $\mathrm{m}, \mathrm{CHO} \underline{\mathrm{H}}(0.25 \mathrm{H})+\mathrm{C} \underline{\mathrm{H}}$ of cyclohexyl $(0.5 \mathrm{H}), 4.39-4.32(0.75 \mathrm{H}, \mathrm{m}, \mathrm{CHO} \underline{\mathrm{H}}(0.25 \mathrm{H})+\mathrm{C} \underline{\mathrm{H}}$ of cyclohexyl $(0.5 \mathrm{H}), 3.82-3.77(0.5 \mathrm{H}, \mathrm{m}, \mathrm{C} \underline{\mathrm{H}}$ of cyclohexyl), 3.65-3.58 $(0.5 \mathrm{H}, \mathrm{m}, \mathrm{C} \underline{\mathrm{H}}$ of cyclohexyl), $2.93\left(0.75 \mathrm{H}, \mathrm{m}, \mathrm{NCH}_{3}\right), 2.91\left(0.75 \mathrm{H}, \mathrm{m}, \mathrm{NCH}_{3}\right), 2.85\left(0.75 \mathrm{H}, \mathrm{m}, \mathrm{NCH}_{3}\right), 2.84$ $\left(0.75 \mathrm{H}, \mathrm{m}, \mathrm{NCH}_{3}\right), 2.83-2.81\left(3 \mathrm{H}, \mathrm{m}, \mathrm{NCH}_{3}\right), 2.80-2.68\left(1 \mathrm{H}, \mathrm{m}, \mathrm{C}_{\mathrm{a}} \mathrm{H}_{\mathrm{b}}\right)$ 2.52-2.42 $(1 \mathrm{H}, \mathrm{m}$, $\left.\mathrm{CH}_{\mathrm{a}} \underline{\mathrm{H}}\right), 1.81-1.78$ (6H, m, $\mathrm{CH}_{2}$ of cyclohexyl), 1.65-1.63 (5H, m, $\mathrm{CH}_{2}$ of cyclohexyl), $1.53-$ $1.47\left(1 \mathrm{H}, \mathrm{m}, \mathrm{CH}_{2}\right.$ of cyclohexyl), 1.53-1.23 (6H, m, $\mathrm{CH}_{2}$ of cyclohexyl), 1.10-1.02 (2H, m, $\mathrm{CH}_{2}$ of cyclohexyl);

${ }^{13} \mathrm{C} \mathrm{NMR}\left(126 \mathrm{MHz}, \mathrm{CDCl}_{3}\right.$ ) (mixture of four rotamers) $\delta 172.5,172.4,172.4,172.3,171.1$, $170.9,170.8,170.6,67.2,67.1,66.9,56.7,55.9,53.6,53.5,52.6,52.5,38.5,38.3,37.9,37.6$, 30.9, 30.8, 29.9, 29.8, 29.7, 29.5, 29.2, 29.1, 27.8. 27.4, 27.3, 25.9, 25.7, 25.6. 25.4, 25.3; $\mathrm{m} / z(\mathrm{ESI}) 347.2\left[(\mathrm{M}+\mathrm{Na})^{+}, 100 \%\right]$. 
$N^{1}, N^{4}$-Dicyclohexyl-2-hydroxy- $N^{1}, N^{4}$-dimethylsuccinamide 10.

${ }^{1} \mathrm{H}$ NMR $\left(500 \mathrm{MHz}, \mathrm{CDCl}_{3}\right)$.

$$
\begin{aligned}
& \text { Dec02-2020 } \\
& \text { SKG678 }
\end{aligned}
$$
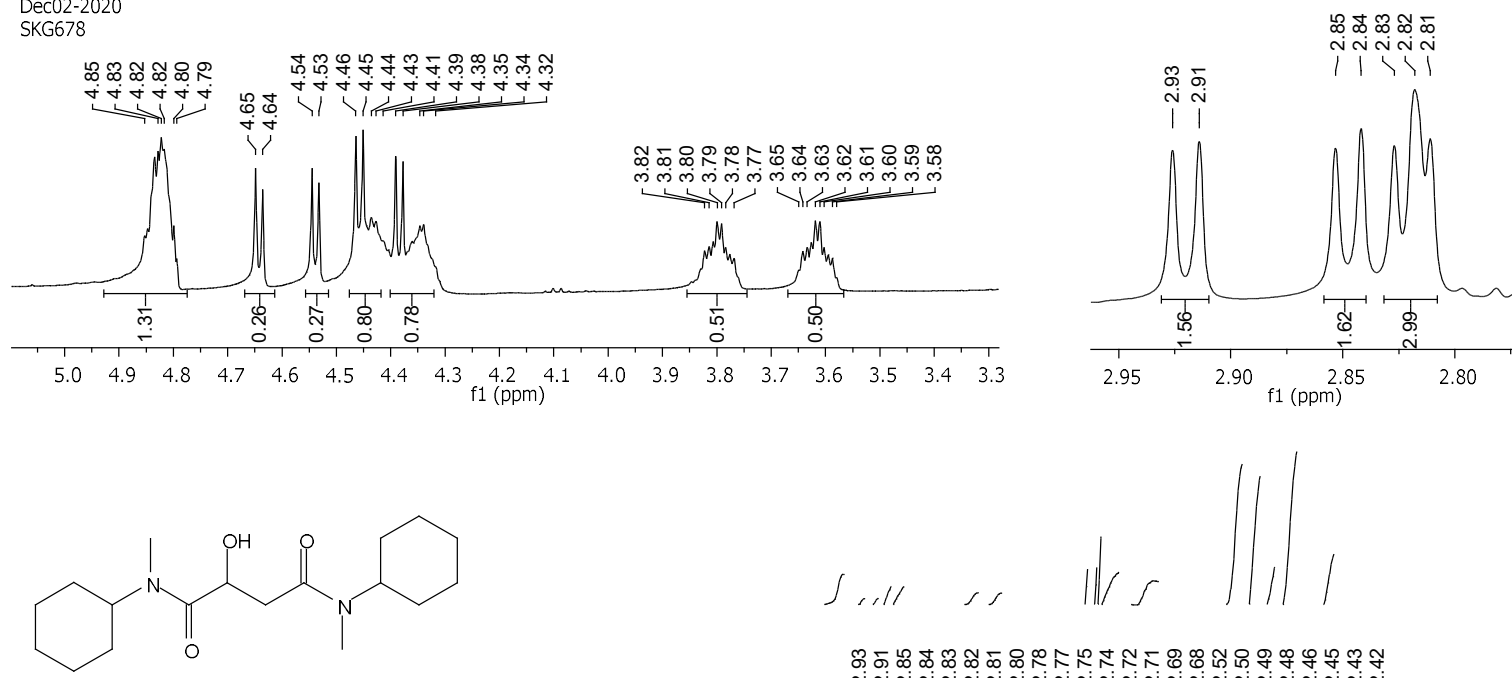

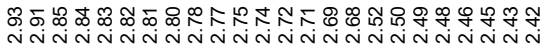

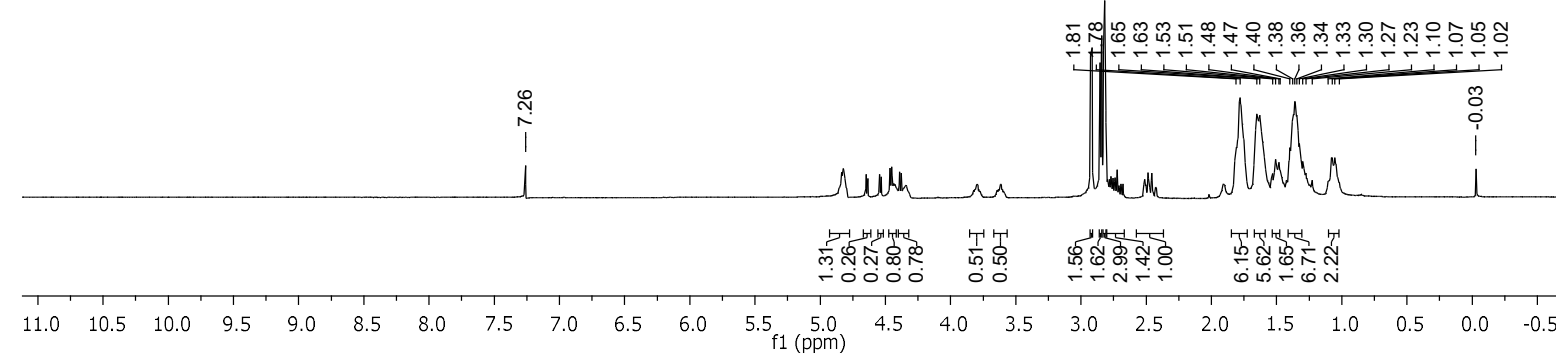

${ }^{13} \mathrm{C} \mathrm{NMR}\left(126 \mathrm{MHz}, \mathrm{CDCl}_{3}\right)$.

Dec02-2020

Shweta Gediya

C13APTlong.w $\mathrm{CDCl}$<smiles>CN(C(=O)CC(O)C(=O)N(C)C1CCCCC1)C1CCCCC1</smiles>

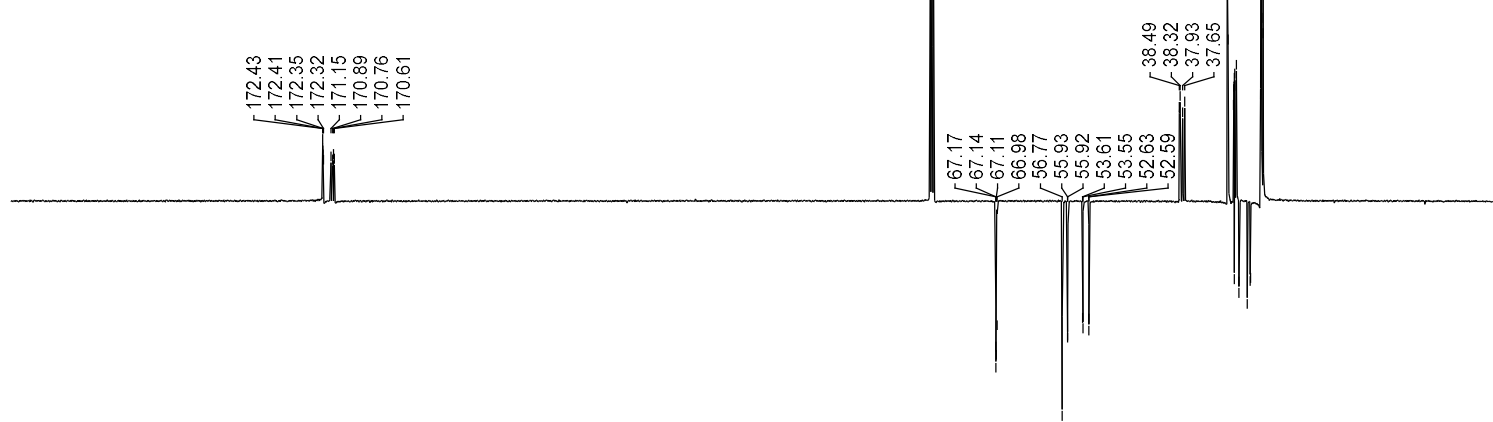

$\begin{array}{llllllllllllllllllllllllllllllllllllll} & 220 & 210 & 200 & 190 & 180 & 170 & 160 & 150 & 140 & 130 & 120 & 110 & 100 & 90 & 80 & 70 & 60 & 50 & 40 & 30 & 20 & 10 & 0 & -10\end{array}$ 
COSY.

\section{Chemist Shweta Gediya}

COSY.w CDCl3/opt/topspin3.2 SKG 12

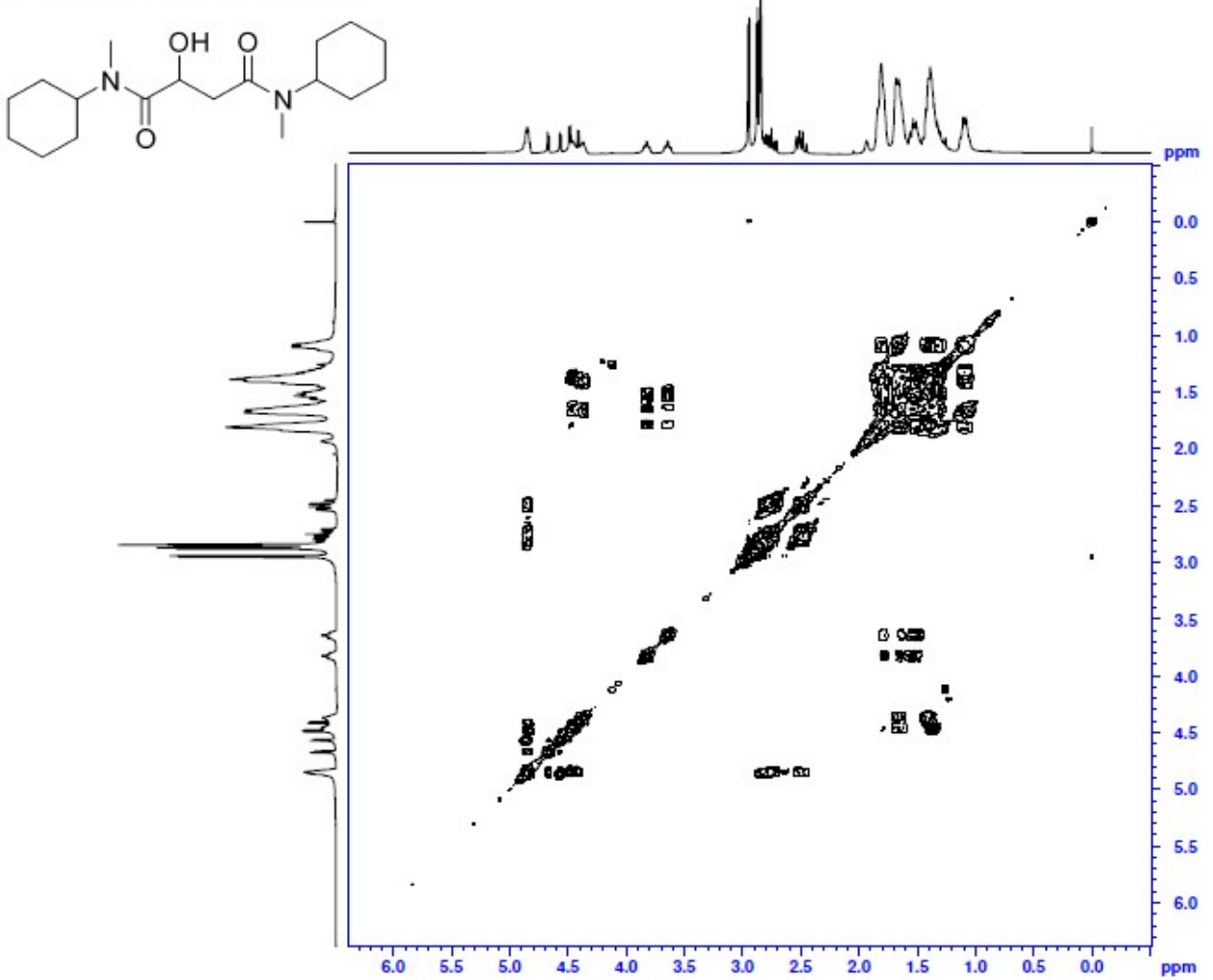

HSQC.

Chemist Shweta Gediya

HSGC.w CDCl3/opt/topspin3.2 SKG 12

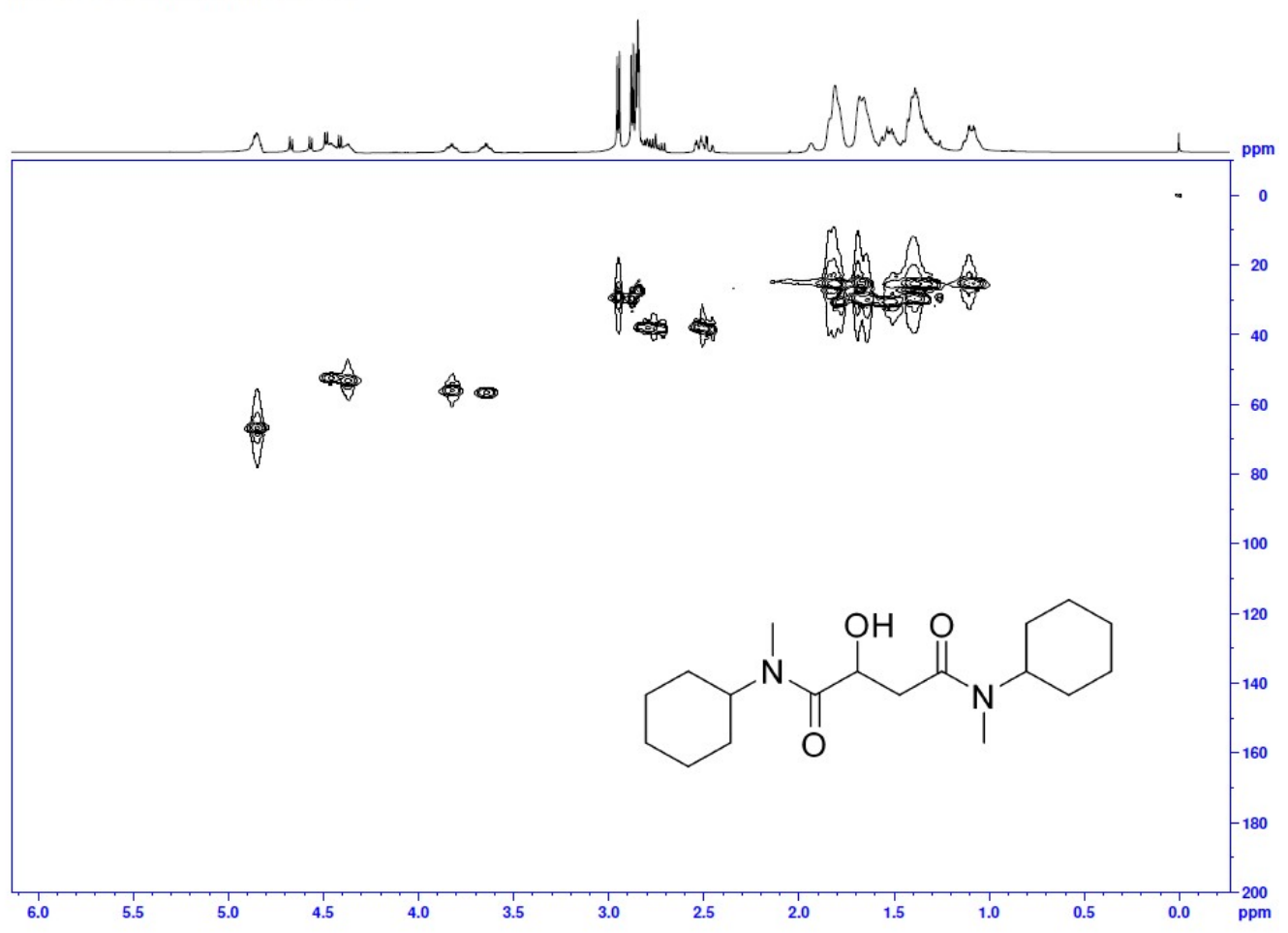




\section{Chiral HPLC of ketone precursor.}

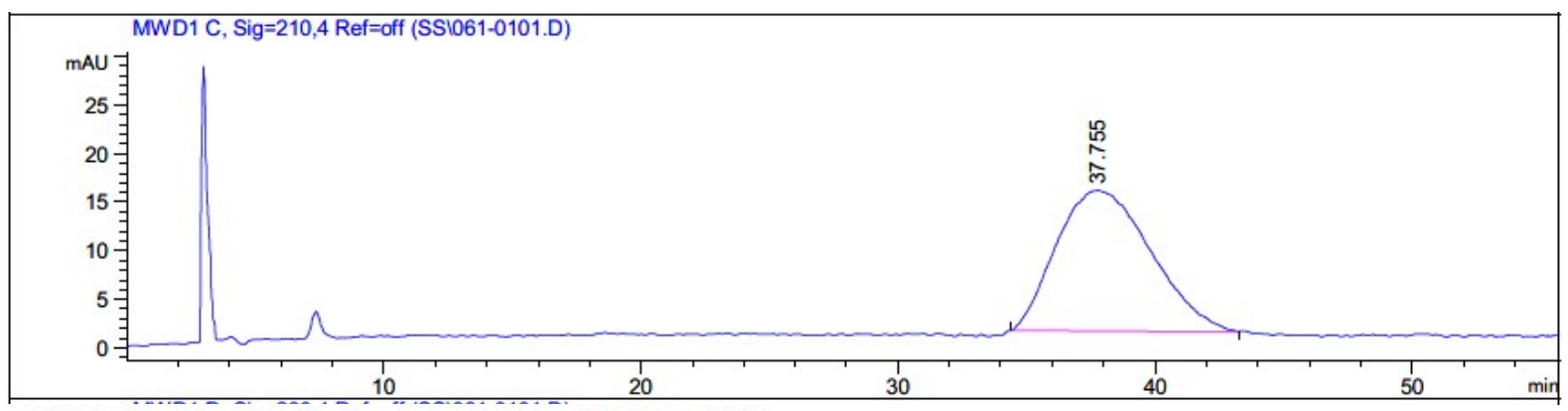

Signal 2: MWD1 C, Sig=210, 4 Ref=off

$\begin{array}{cccccc}\begin{array}{c}\text { Peak RetTime Type } \\ \#\end{array} \begin{array}{c}\text { Width } \\ \text { [min] }\end{array} & \begin{array}{c}\text { Area } \\ {[\mathrm{min}]}\end{array} & \begin{array}{c}\text { Height } \\ {\left[\mathrm{mAU}^{*} \mathrm{~S}\right]}\end{array} & \begin{array}{c}\text { Area } \\ {[\mathrm{mAU}]}\end{array} & 8 \\ ---\mid & & & \end{array}$

\section{Chiral HPLC of racemic alcohol.}

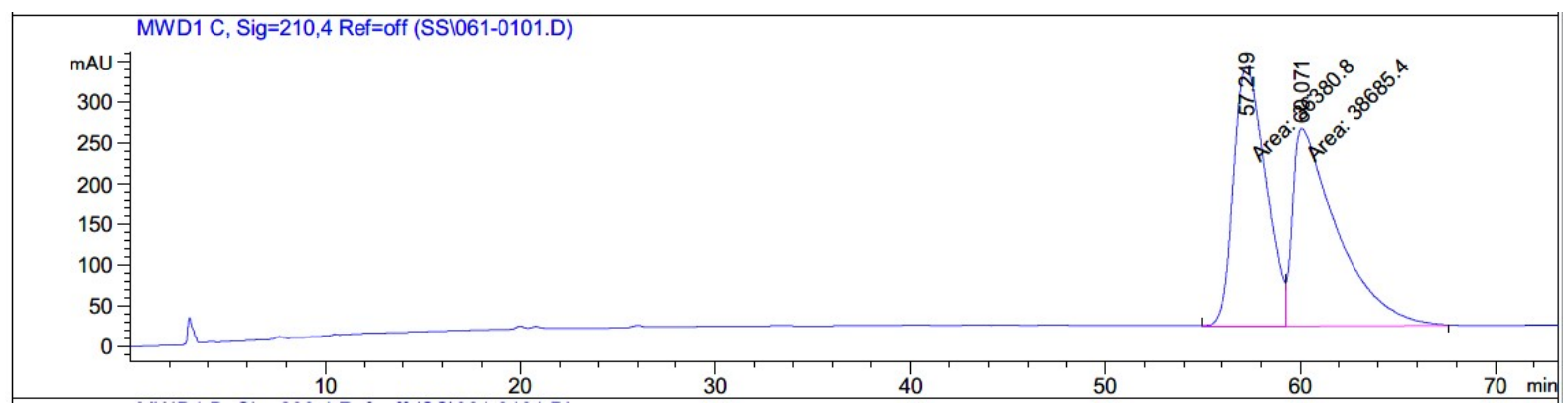

Signal 2: MWD1 C, Sig=210, 4 Ref=off

$\begin{array}{cccccc}\begin{array}{c}\text { Peak RetTime Type } \\ \# \\ \text { [min] }\end{array} & \begin{array}{c}\text { Width } \\ {[\mathrm{min}]}\end{array} & \begin{array}{c}\text { Area } \\ {\left[\mathrm{mAU}{ }^{*} \mathrm{~S}\right]}\end{array} & \begin{array}{c}\text { Height } \\ {[\mathrm{mAU}]}\end{array} & \begin{array}{c}\text { Area } \\ \%\end{array} \\ ----|------|----\mid & -------|---------| & ---------|------| \\ 1 & 57.249 \mathrm{MF} & 1.9027 & 3.63808 \mathrm{e} 4 & 318.68121 & 48.4649 \\ 2 & 60.071 \mathrm{FM} & 2.6633 & 3.86854 \mathrm{e} 4 & 242.09129 & 51.5351\end{array}$


Chiral HPLC of ATH product. 95\% ee.

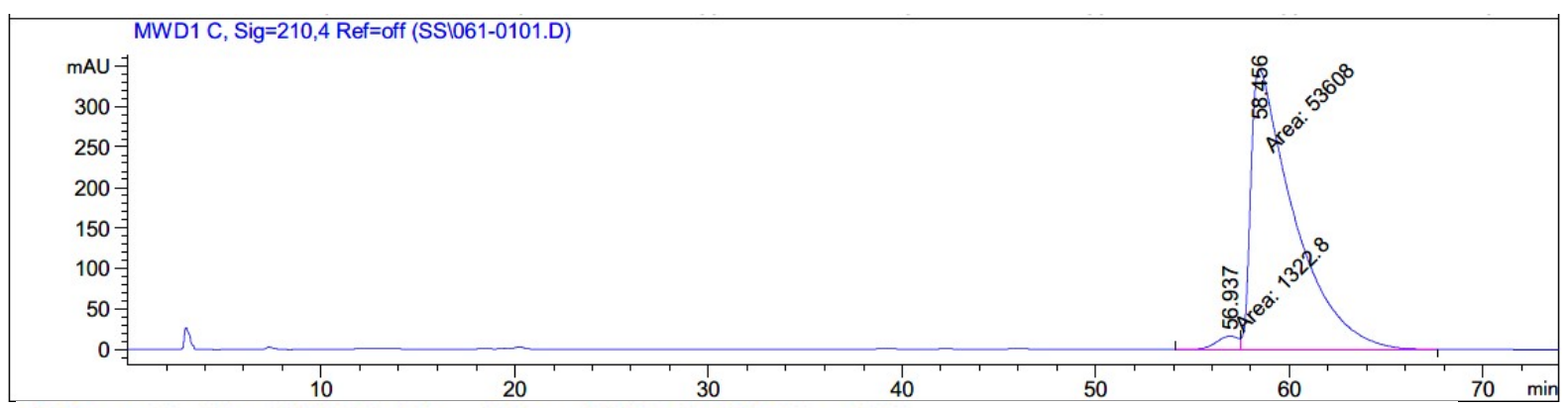

Signal 2: MWD1 C, Sig=210,4 Ref=off

\begin{tabular}{|c|c|c|c|c|c|c|}
\hline eak & $\begin{array}{c}\text { RetTime } \\
\text { [min] }\end{array}$ & Type & $\begin{array}{l}\text { Width } \\
\text { [min] }\end{array}$ & $\begin{array}{c}\text { Area } \\
{\left[\mathrm{mAU}^{\star} \mathrm{s}\right]}\end{array}$ & $\begin{array}{l}\text { Height } \\
\text { [mAU] }\end{array}$ & $\begin{array}{c}\text { Area } \\
\frac{\%}{\circ}\end{array}$ \\
\hline & & & & 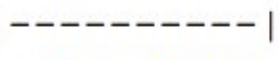 & - & \\
\hline 1 & 56 & $\mathrm{MF}$ & 8 & 1322.80029 & 1672 & 81 \\
\hline ? & 58.456 & FM & 342 & $5.36080 \mathrm{e} 4$ & 345.73801 & 5919 \\
\hline
\end{tabular}


(R)- $N^{1}$-Allyl-2-hydroxy- $N^{1}, N^{4}$-dimethyl- $N^{4}$-phenylsuccinamide 11.<smiles>C=CCN(C)C(=O)C(O)CC(=O)N(C)c1ccccc1</smiles>

This compound is novel and was prepared following general procedure $\mathrm{C}$ using $N^{1}$-allyl$N^{1}, N^{4}$-dimethyl-2-oxo- $N^{4}$-phenylsuccinamide $(0.137 \mathrm{~g}, 0.500 \mathrm{mmol}, 1.0 \mathrm{eq}), 3 \mathrm{C}$ tethered catalyst $(R, R)-2(4.7 \mathrm{mg}, 7.5 \mu \mathrm{mol}, 0.015 \mathrm{eq})$ and FA:TEA $(0.3 \mathrm{~mL})$ in DCM (3 mL) to give 11 as a clear liquid $(0.125 \mathrm{~g}, 0.452 \mathrm{mmol}, 90 \%)$.

TLC: $\mathrm{R}_{\mathrm{f}}$ ca 0.2 (1:9, hexane: EtOAc), UV-active, Strong $\mathrm{KMnO}_{4}$ and PMA-reactive; HRMS (ESI) m/z: [M+Na] ${ }^{+}$Calcd for $\mathrm{C}_{15} \mathrm{H}_{20} \mathrm{~N}_{2} \mathrm{O}_{3} \mathrm{Na} 299.1366$; Found 299.1362 (error -1.3 ppm);

$U_{\max } 3393,2928,1629,1592,1494,1384,1120,1074,773,699 \mathrm{~cm}^{-1}$

Enantiomeric excess determined by HPLC analysis (Chiralpak IG, $250 \mathrm{~mm} \times 4.6 \mathrm{~mm}$ column, hexane: $\mathrm{iPrOH} 80: 20,1 \mathrm{~mL} / \mathrm{min}, \lambda=210 \mathrm{~nm}, \mathrm{~T}=25^{\circ} \mathrm{C}$ ), $S$-enantiomer $29.0 \mathrm{~min}, R$ enantiomer $35.4 \min .77 \%$ ee $(R),[\alpha]_{\mathrm{D}}^{22}=+4.5\left(\mathrm{c}=0.1, \mathrm{CHCl}_{3}\right)$;

${ }^{1} \mathrm{H}$ NMR (500 MHz, $\mathrm{CDCl}_{3}$ ) (Rotamers, 1:1) $\delta 7.43$-7.23 (5H, m, ArH), 5.80 -5.66 (1H, m, $\left.\mathrm{CH}_{2}=\mathrm{C}_{\underline{H C H}}\right), 5.22-5.15\left(2 \mathrm{H}, \mathrm{m}, \mathrm{C}_{2}=\mathrm{CHCH}_{2}\right), 4.85-4.81(0.5 \mathrm{H}, \mathrm{m}, \mathrm{C} \underline{\mathrm{HOH}}), 4.76-4.73$ $(0.5 \mathrm{H}, \mathrm{m}, \mathrm{C} \underline{\mathrm{HOH}}), 4.43(0.5 \mathrm{H}, \mathrm{d}, J=7.5 \mathrm{~Hz}, \mathrm{CHO} \underline{\mathrm{H}}), 4.26(0.5 \mathrm{H}, \mathrm{d}, J=7.5 \mathrm{~Hz}, \mathrm{CHO} \underline{\mathrm{H}})$, 4.02-3.89 (2H, m, $\left.\mathrm{CH}_{2}=\mathrm{CHC}_{2}\right), 3.30\left(1.5 \mathrm{H}, \mathrm{s}, \mathrm{NCH}_{3}\right), 3.30\left(1.5 \mathrm{H}, \mathrm{s}, \mathrm{NCH}_{3}\right), 2.98(1.5 \mathrm{H}, \mathrm{m}$, $\mathrm{NCH}_{3}$ of allylamine), $2.89\left(1.5 \mathrm{H}, \mathrm{m}, \mathrm{NCH}_{3}\right.$ of allylamine) $2.49-2.44\left(1 \mathrm{H}, \mathrm{m}, \mathrm{C}_{\mathrm{a}} \mathrm{H}_{\mathrm{b}}\right), 2.34-$ $2.29\left(1 \mathrm{H}, \mathrm{m}, \mathrm{CH}_{\mathrm{a}} \underline{\mathrm{H}} \mathrm{b}\right)$;

${ }^{13} \mathrm{C}$ NMR (126 MHz, $\mathrm{CDCl}_{3}$ ) (Rotamers, 1:1) $\delta$ 173.0, 172.7, 171.2, 170.9, 143.6, 143.5, $132.6,132.3,129.9,128.1,127.6,127.5,117.9,117.5,66.9,66.8,51.5,50.6,38.5,38.3,37.5$, $37.4,34.2,33.8$;

$m / z(\mathrm{ESI}) 299.1\left[(\mathrm{M}+\mathrm{Na})^{+}, 100 \%\right]$. 
$N^{1}$-allyl-2-hydroxy- $N^{1}, N^{4}$-dimethyl- $N^{4}$-phenylsuccinamide 11.

${ }^{1} \mathrm{H}$ NMR $\left(500 \mathrm{MHz}, \mathrm{CDCl}_{3}\right)$.

Nov17-2020

Chemist Shweta Gediya

SKG650

PROTON.w CDCl3 /opt/topspin3.2 SKG 15

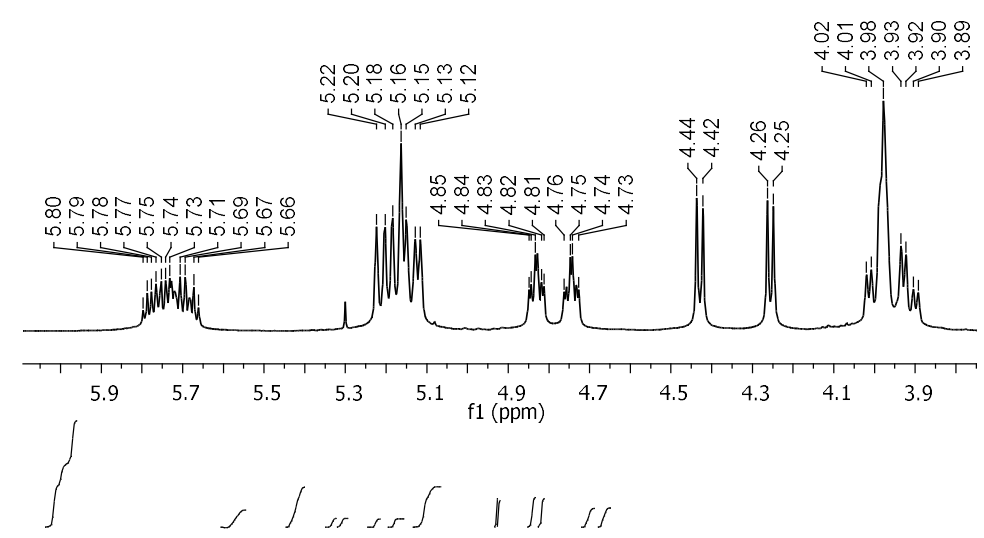

$\left.\right|_{O} ^{(1)}$

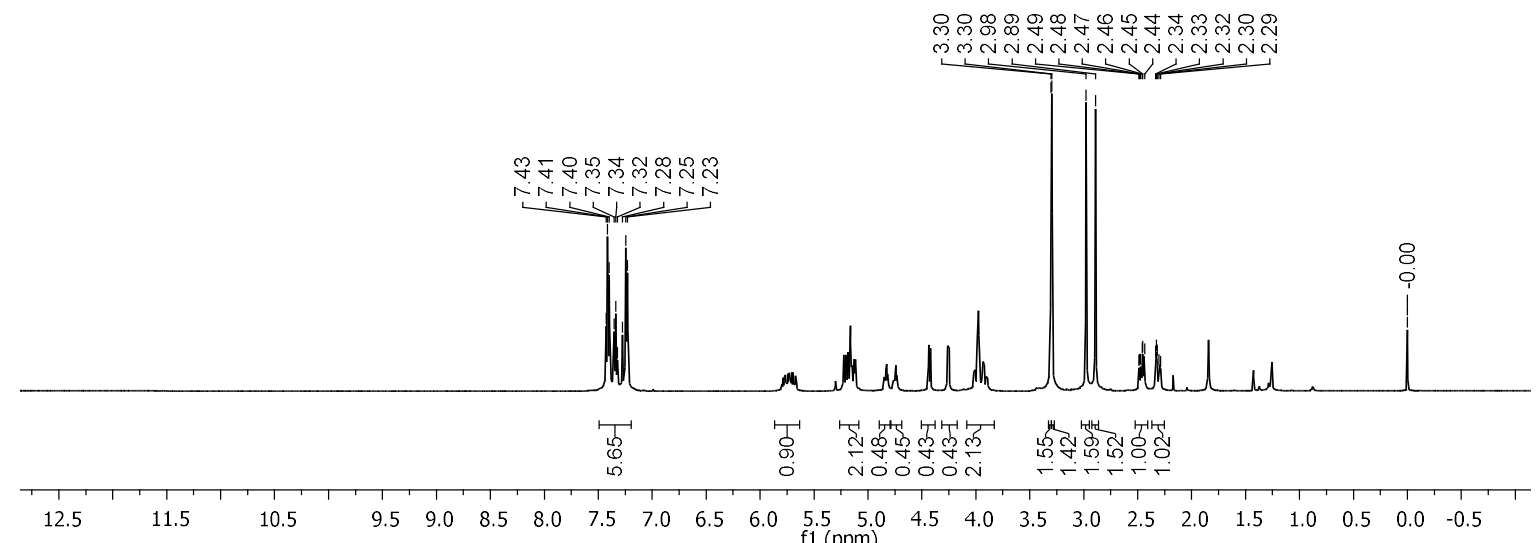

${ }^{13} \mathrm{C}$ NMR (126 MHz, $\left.\mathrm{CDCl}_{3}\right)$.

Nov17-2020
Shweta Gediya
SKG650
C13APTlong.w CDCl3
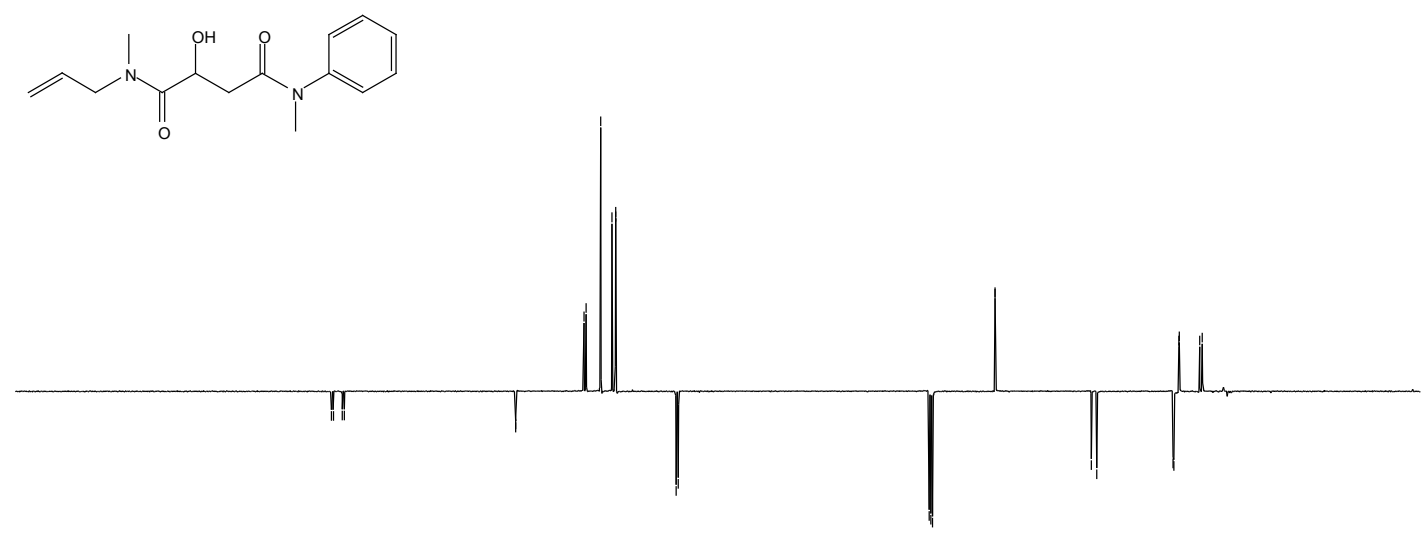

$\begin{array}{lllllllllllllllllllllllll}220 & 210 & 200 & 190 & 180 & 170 & 160 & 150 & 140 & 130 & 120 & 110 & 100 & 90 & 80 & 70 & 60 & 50 & 40 & 30 & 20 & 10 & 0\end{array}$ 
COSY.

Chemist Shweta Gediya

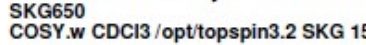

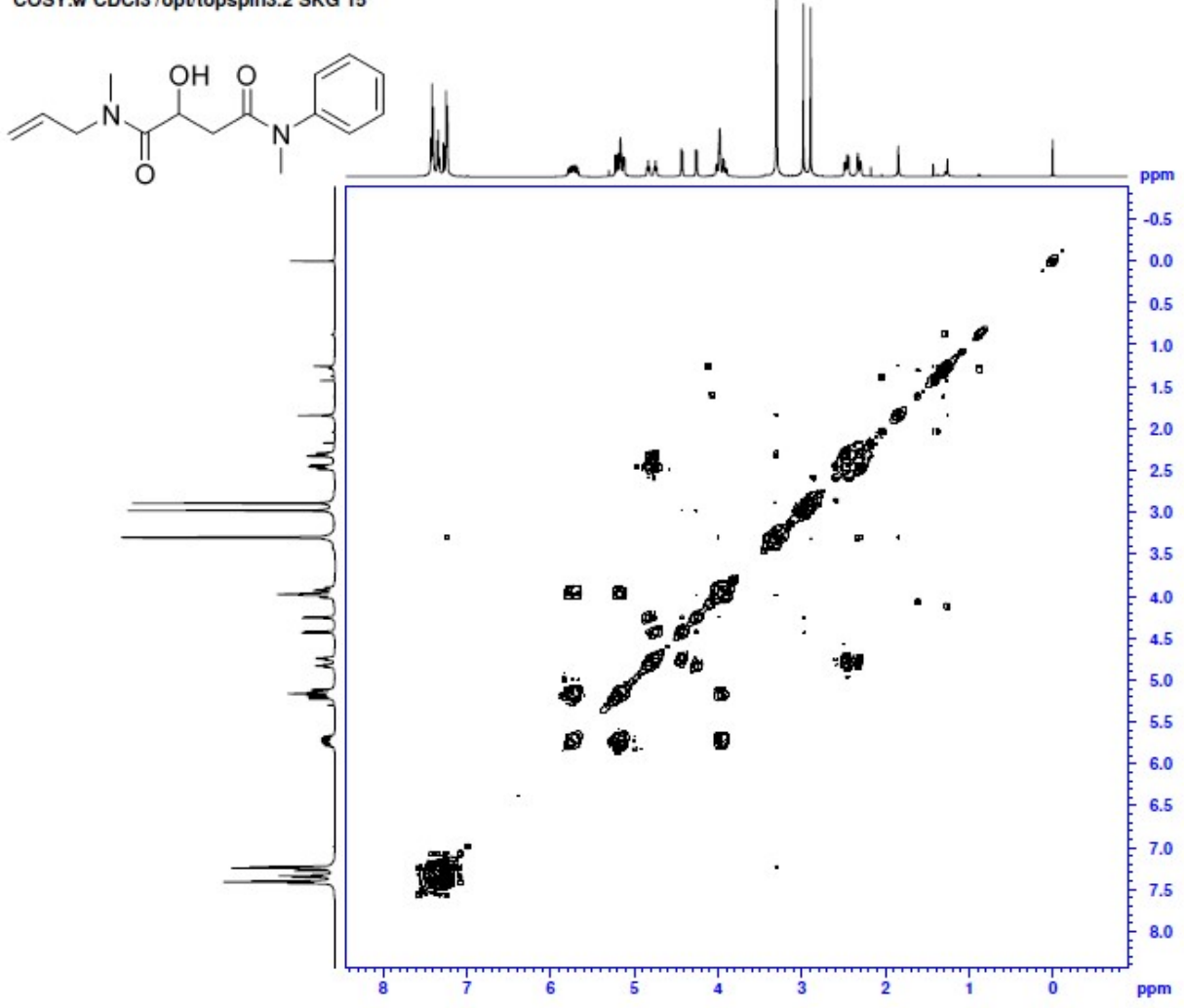

HSQC.

Chemist Shweta Gediya

HSQC.w CDCl3 /opt/topspin3.2 SKG 15

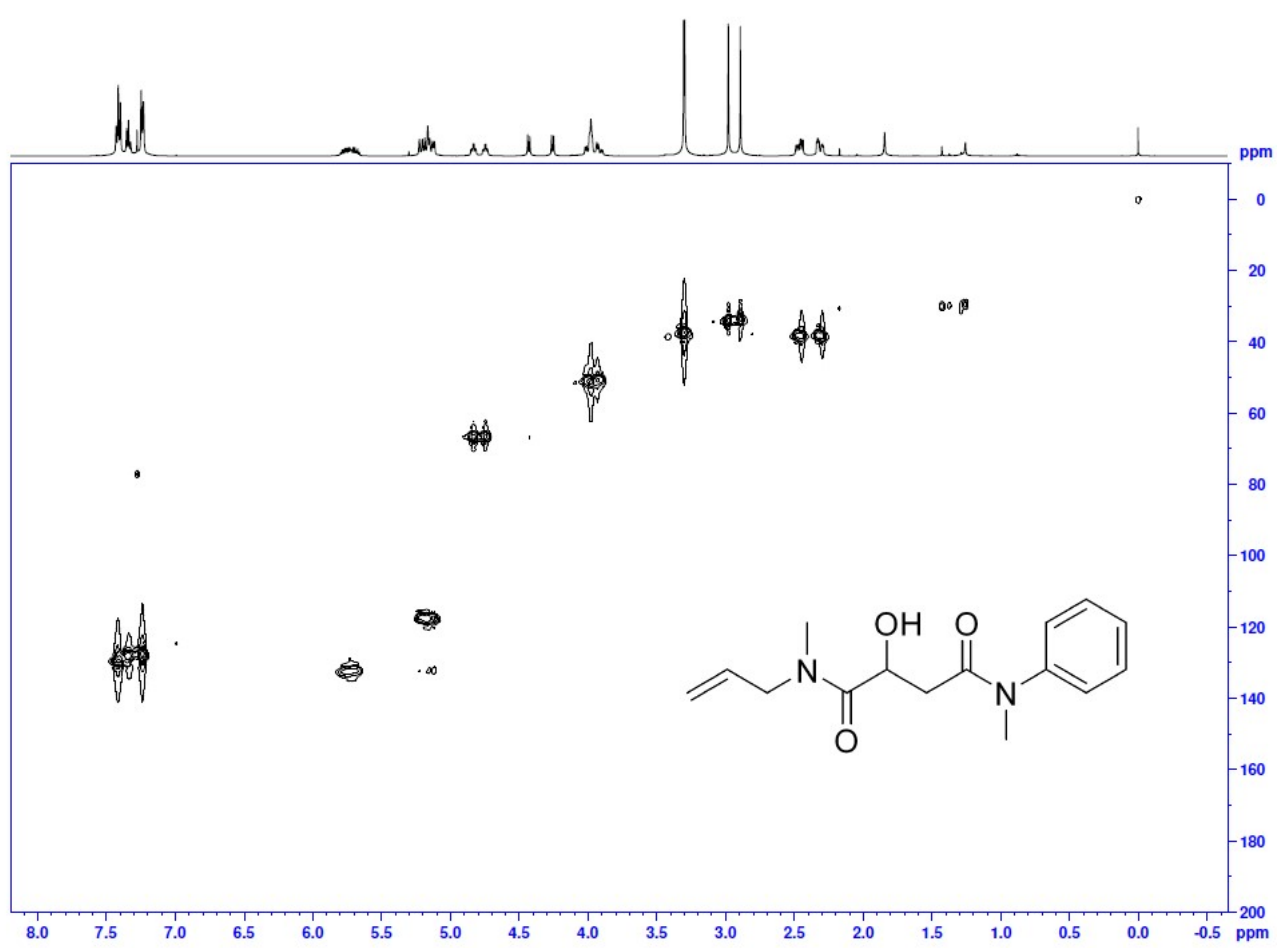




\section{Chiral HPLC of ketone precursor.}

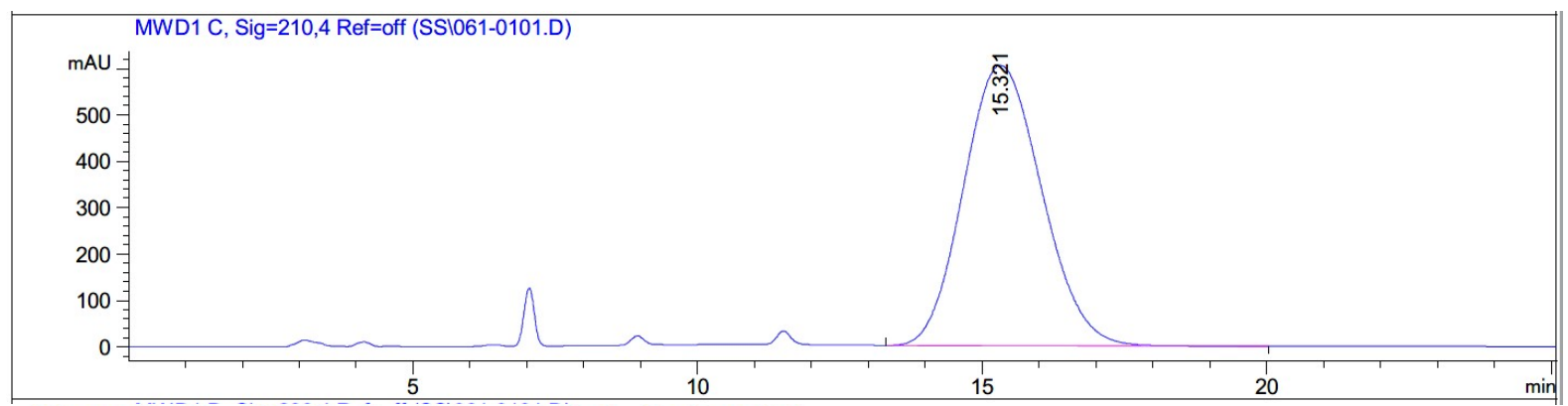

Signal 2: MWD1 C, Sig=210,4 Ref=off

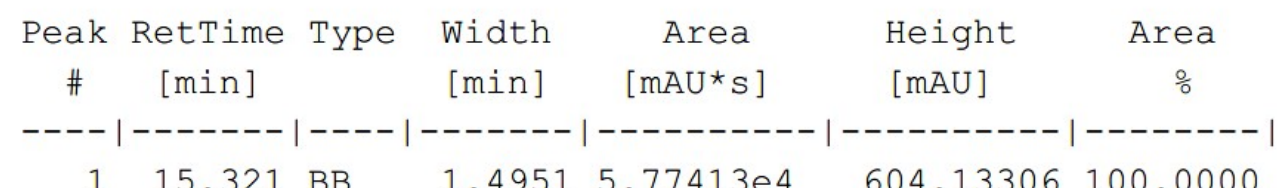

$1 \quad 15.321 \mathrm{BB} \quad 1.49515 .77413 \mathrm{e} 4 \quad 604.13306100 .0000$

\section{Chiral HPLC of racemic alcohol.}

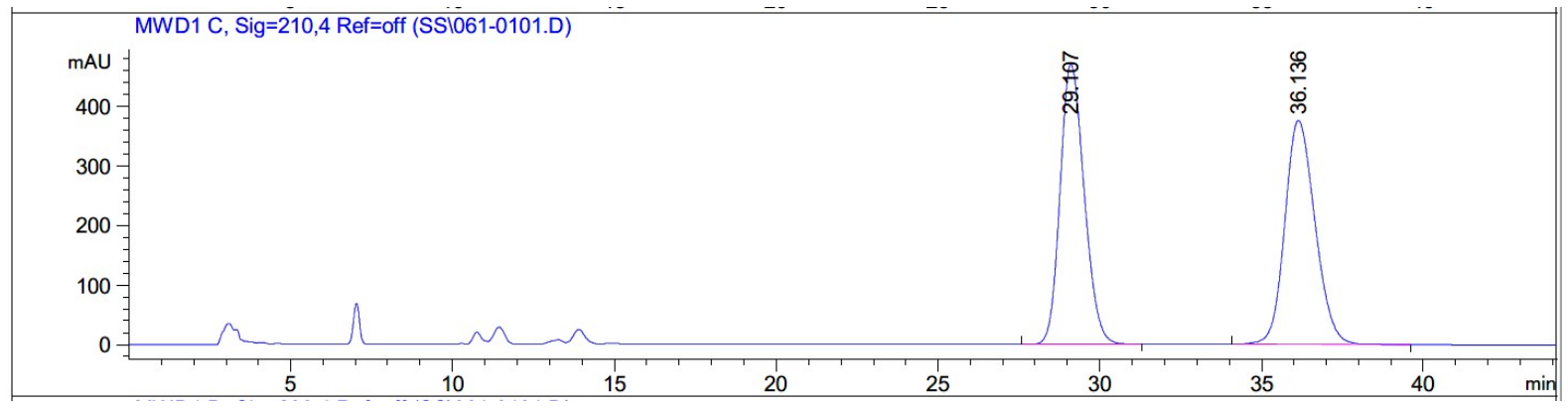

Signal 2: MWD1 C, Sig=210,4 Ref=off

\begin{tabular}{|c|c|c|c|c|c|c|}
\hline $\begin{array}{c}\text { eak } \\
\#\end{array}$ & $\begin{array}{c}\text { RetTime } \\
\text { [min] }\end{array}$ & Type & $\begin{array}{l}\text { Width } \\
\text { [min] }\end{array}$ & $\begin{array}{c}\text { Area } \\
{\left[\mathrm{mAU}^{\star} \mathrm{s}\right]}\end{array}$ & $\begin{array}{l}\text { Height } \\
{[\mathrm{mAU}]}\end{array}$ & $\begin{array}{c}\text { Area } \\
\frac{\circ}{0}\end{array}$ \\
\hline & ----- & & ------ & $\mid---------$ & 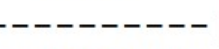 & -------- \\
\hline 1 & 29.107 & $\mathrm{BB}$ & 0.8057 & $2.43305 \mathrm{e} 4$ & 470.22705 & 49.9823 \\
\hline 2 & 36.136 & BB & 1.0017 & $2.43478 \mathrm{e} 4$ & 375.86481 & 0177 \\
\hline
\end{tabular}


Chiral HPLC of ATH product. 77\% ee.

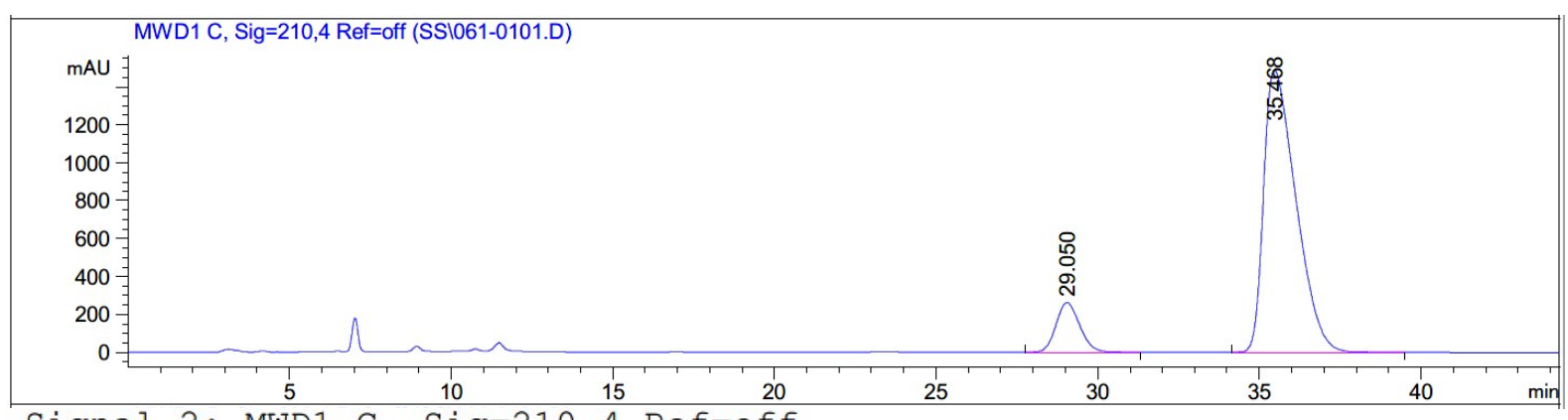

Signal 2: MWD1 C, Sig=210,4 Ref=off

\begin{tabular}{|c|c|c|c|c|c|c|}
\hline $\begin{array}{c}\text { Peak } \\
\#\end{array}$ & $\begin{array}{c}\text { RetTime } \\
\text { [min] }\end{array}$ & Type & $\begin{array}{l}\text { Width } \\
\text { [min] }\end{array}$ & $\begin{array}{c}\text { Area } \\
{\left[\mathrm{mAU}^{*} \mathrm{~s}\right]}\end{array}$ & $\begin{array}{l}\text { Height } \\
\text { [mAU] }\end{array}$ & $\begin{array}{c}\text { Area } \\
\frac{\circ}{0}\end{array}$ \\
\hline \multicolumn{7}{|c|}{$---|------|----|-------|---------|---------|-------$} \\
\hline 1 & 29.050 & $\mathrm{BB}$ & 0.7913 & $1.32666 \mathrm{e} 4$ & 260.86496 & 11.4237 \\
\hline 2 & 35.468 & $\mathrm{BB}$ & 1.0323 & $1.02866 \mathrm{e} 5$ & 1488.45654 & 88.5763 \\
\hline
\end{tabular}


(R)-2-Hydroxy- $N^{1}, N^{4}$-dimethyl- $N^{4}$-phenyl- $N^{1}$-(prop-2-yn-1-yl)succinamide 12.<smiles>C#CCN(C)C(=O)[C@H](O)CC(=O)N(C)c1ccccc1</smiles>

This compound is novel and was prepared following general procedure $\mathrm{C}$ using $N^{1}, N^{4}$ dimethyl-2-oxo- $N^{4}$-phenyl- $N^{l}$-(prop-2-yn-1-yl)succinamide (68.5 mg, $0.250 \mathrm{mmol}, 1.0 \mathrm{eq}$ ), 3C tethered catalyst $(R, R)-2(2.3 \mathrm{mg}, 3.8 \mu \mathrm{mol}, 0.015 \mathrm{eq})$ and FA:TEA $(0.15 \mathrm{~mL})$ in DCM $(1.5 \mathrm{~mL})$ to give 12 as a colourless liquid $(59.4 \mathrm{mg}, 0.217 \mathrm{mmol}, 87 \%)$.

TLC: $\mathrm{R}_{\mathrm{f}}$ ca 0.2 (1:9, hexane: EtOAc), UV-active, Strong $\mathrm{KMnO}_{4}$ and PMA-reactive;

HRMS (ESI) m/z: [M+Na] ${ }^{+}$Calcd for $\mathrm{C}_{15} \mathrm{H}_{18} \mathrm{~N}_{2} \mathrm{O}_{3} \mathrm{Na} 297.1210$; Found 297.1198 (error 4.0 ppm);

$U_{\max } 3399,3290,2928,1632,1592,1494,1385,1119,1076,1061,699 \mathrm{~cm}^{-1}$;

Enantiomeric excess determined by HPLC analysis (Chiralpak IG, $250 \mathrm{~mm} \times 4.6 \mathrm{~mm}$ column, hexane: $\mathrm{iPrOH} 80: 20,1 \mathrm{~mL} / \mathrm{min}, \lambda=210 \mathrm{~nm}, \mathrm{~T}=25^{\circ} \mathrm{C}$ ), $S$-enantiomer $31.1 \mathrm{~min}, R$ enantiomer $40.9 \min .88 \%$ ee $(R),[\alpha]_{\mathrm{D}}^{22}=+3.5\left(\mathrm{c}=0.1, \mathrm{CHCl}_{3}\right)$;

${ }^{1} \mathrm{H}$ NMR (400 MHz, $\mathrm{CDCl}_{3}$ ) (Rotamers, 2:1) $\delta$ 7.44-7.40 (2H, m, ArH), 7.36-7.32 (1H, m, ArH), 7.27-7.18 (2H, m, ArH), 4.18-4.73 (1H, m, $\mathrm{C} \underline{H O H}), 4.45-4.07$ (3H, m, $\mathrm{CH} \equiv \mathrm{CC}_{\underline{H}}+$ $\mathrm{CHO} \underline{\mathrm{H}}), 3.30\left(2 \mathrm{H}, \mathrm{s}, \mathrm{NCH}_{3}\right), 3.27\left(1 \mathrm{H}, \mathrm{s}, \mathrm{NCH}_{3}\right), 3.12\left(2 \mathrm{H}, \mathrm{s}, \mathrm{NCH}_{3}\right), 2.98\left(1 \mathrm{H}, \mathrm{s}, \mathrm{NCH}_{3}\right)$, 2.61-2.04 (3H, m, $\left.\mathrm{C} \underline{\mathrm{H}} \equiv \mathrm{CCH}_{2}+\mathrm{CH}_{\mathrm{a}} \mathrm{H}_{\mathrm{b}}\right)$;

${ }^{13} \mathrm{C}$ NMR (126 MHz, $\mathrm{CDCl}_{3}$ ) (Rotamers, 2:1) $\delta$ 172.6, 172.3, 171.8, 171.2, 143.5, 143.4, $130.0,129.9,128.2,127.8,127.5,127.4,127.2$, 78.2, 78.1, 73.1, 72.4, 67.5, 67.2, 38.8, 37.8, 37.6, 37.5, 37.4, 37.3, 37.2, 37.0, 34.1, 33.6;

$m / z(\mathrm{ESI}) 297.1\left[(\mathrm{M}+\mathrm{Na})^{+}, 100 \%\right]$. 
$N^{1}$-Allyl-2-hydroxy- $N^{1}, N^{4}$-dimethyl- $N^{4}$-phenylsuccinamide 12.

${ }^{1} \mathrm{H}$ NMR $\left(400 \mathrm{MHz}, \mathrm{CDCl}_{3}\right)$.

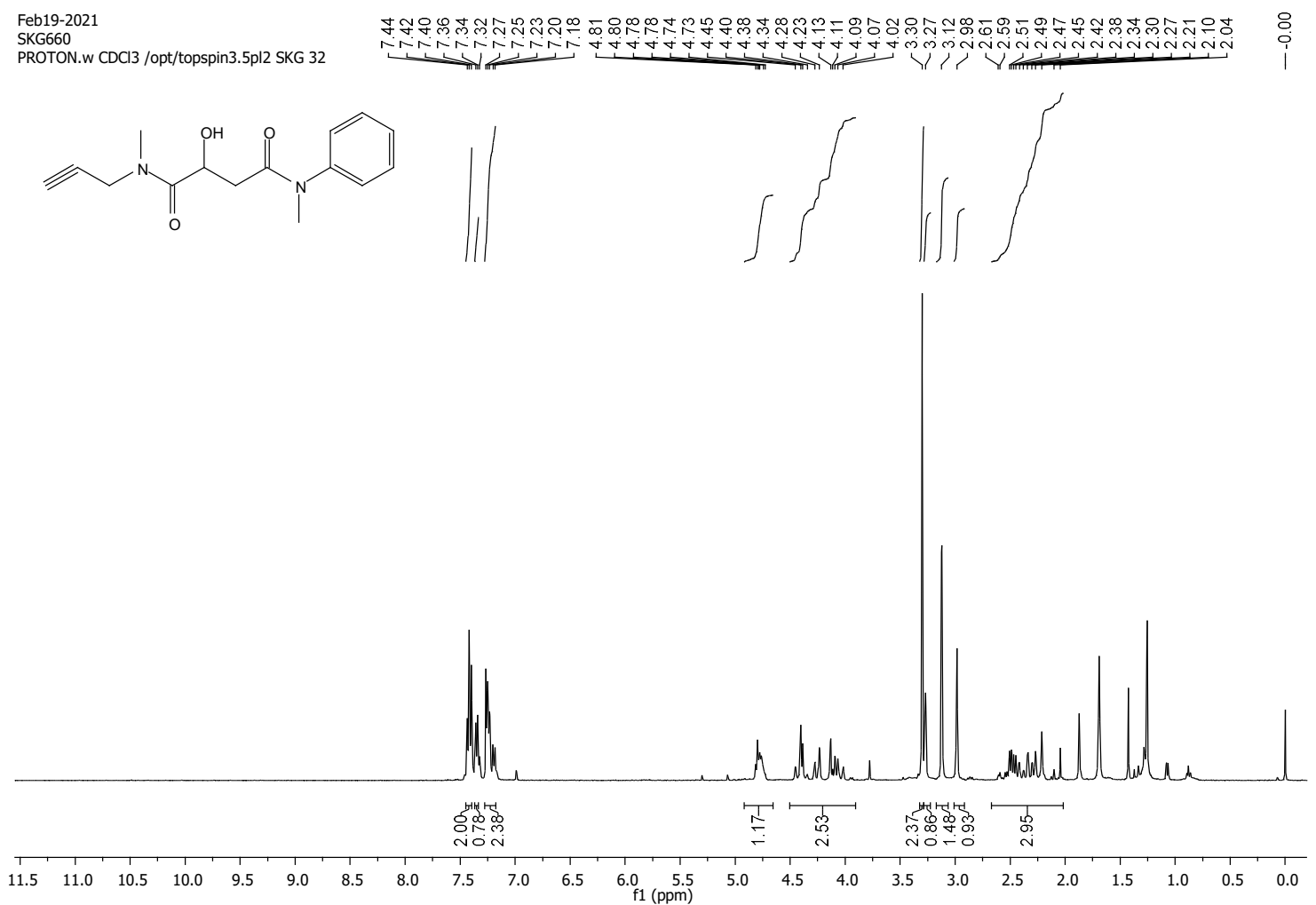

${ }^{13} \mathrm{C}$ NMR $\left(126 \mathrm{MHz}, \mathrm{CDCl}_{3}\right)$.

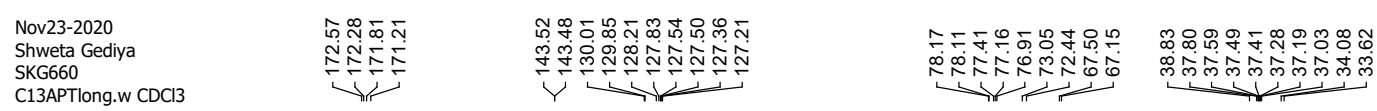

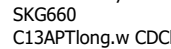<smiles>C#CCN(C)C(=O)C(O)CC(=O)N(C)c1ccccc1</smiles>

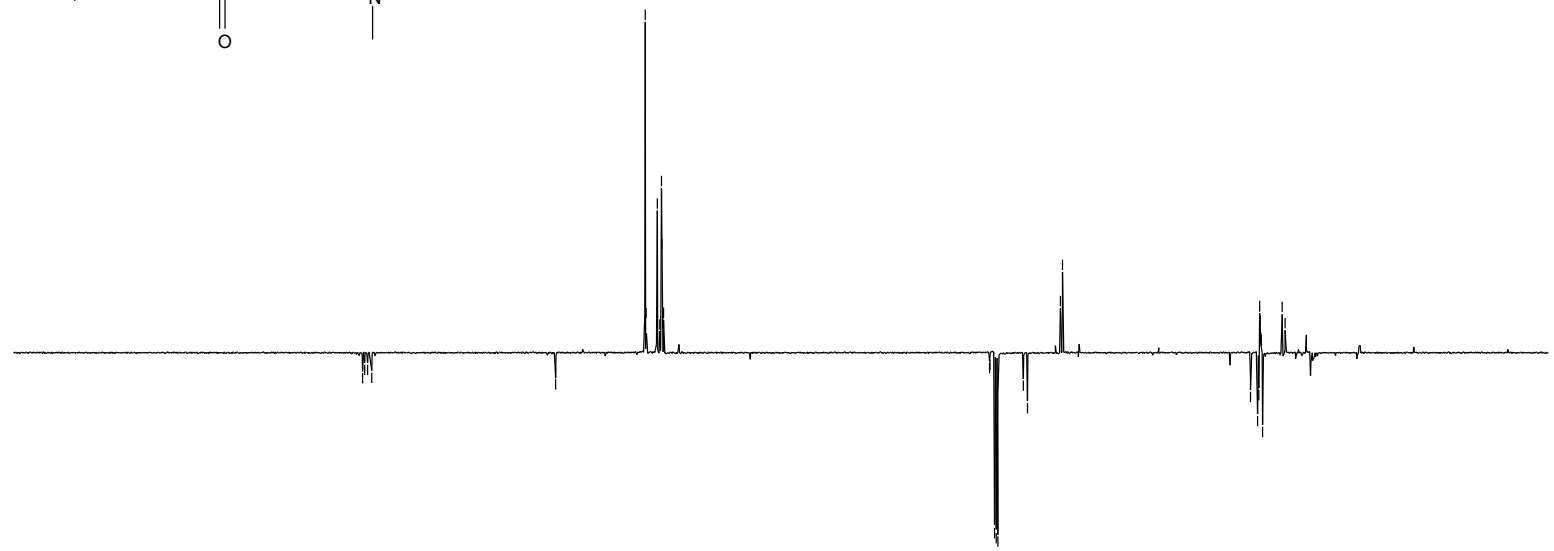

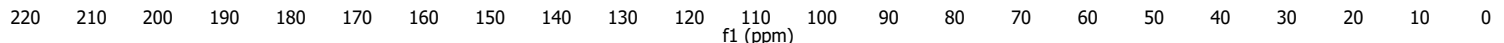


COSY.

SKG660

COSY.w CDCl3 /opt/topspin3.5pl2 SKG 32

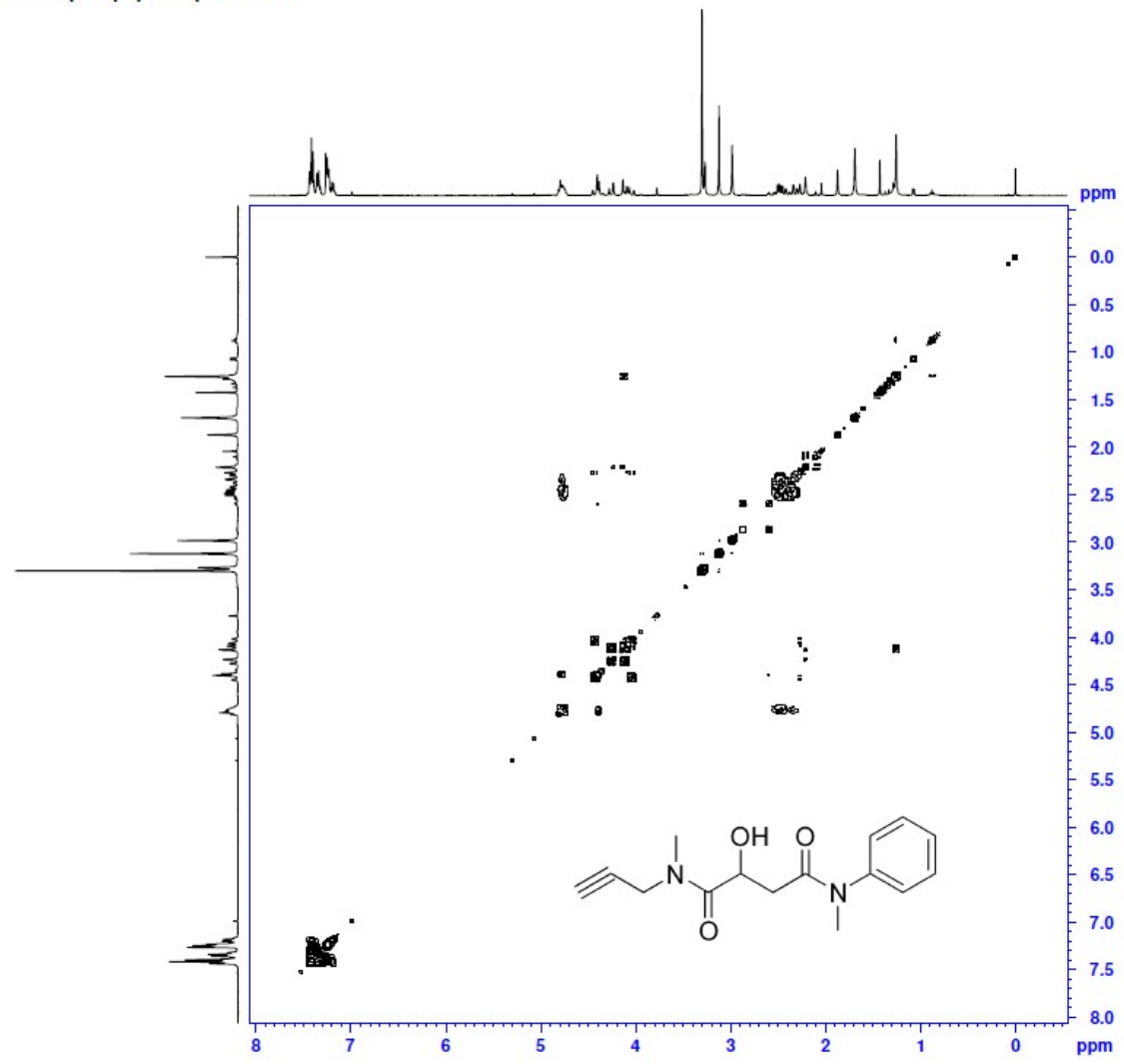

HSQC.

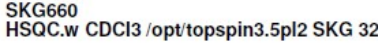

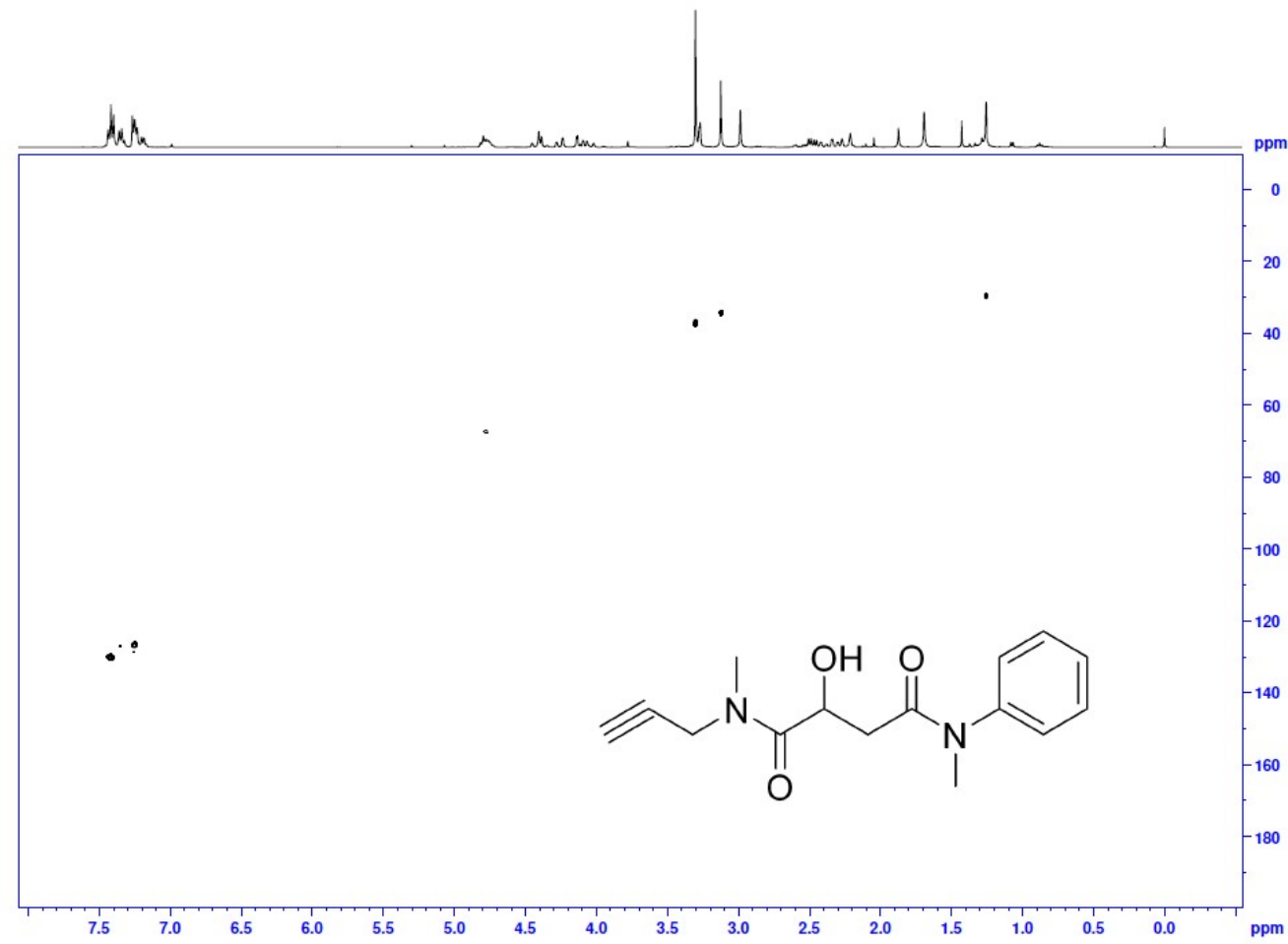




\section{Chiral HPLC of ketone precursor.}

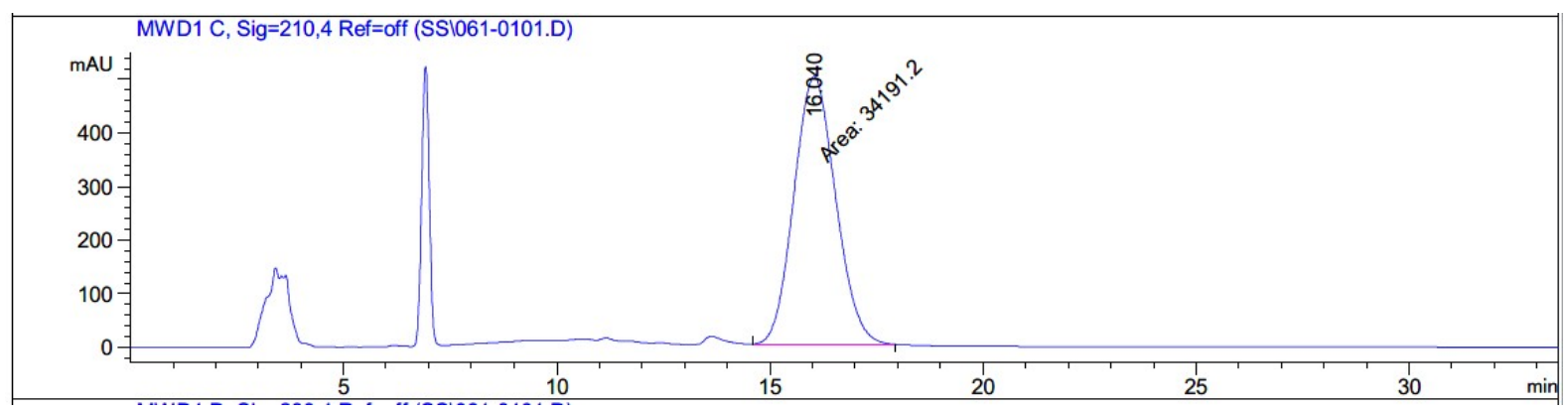

Signal 2: MWD1 C, Sig=210,4 Ref=off

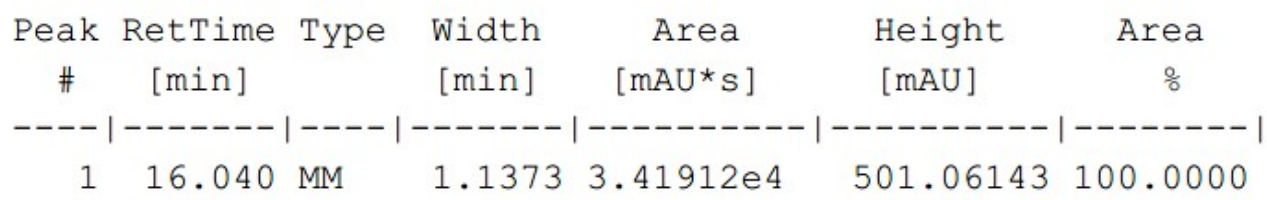

Chiral HPLC of racemic alcohol.

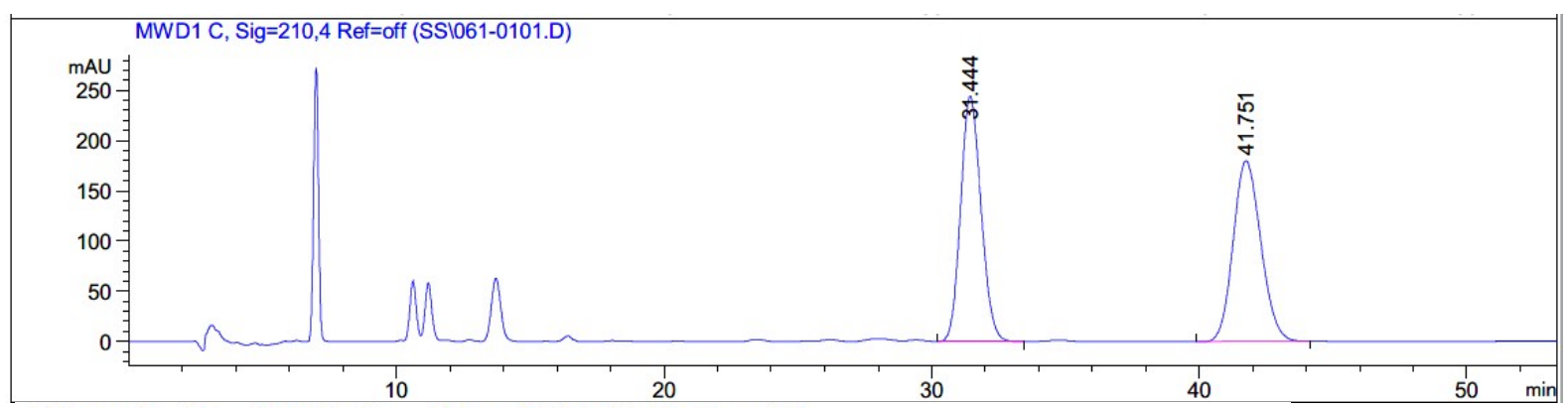

Signal 2: MWD1 C, Sig=210, 4 Ref=off

\begin{tabular}{|c|c|c|c|c|c|c|}
\hline $\begin{array}{c}\text { Peak } \\
\#\end{array}$ & $\begin{array}{c}\text { RetTime } \\
\text { [min] }\end{array}$ & Type & $\begin{array}{l}\text { Width } \\
\text { [min] }\end{array}$ & $\begin{array}{c}\text { Area } \\
{\left[\mathrm{mAU}^{\star} \mathrm{s}\right]}\end{array}$ & $\begin{array}{l}\text { Height } \\
{[\mathrm{mAU}]}\end{array}$ & $\begin{array}{c}\text { Area } \\
\frac{\circ}{\delta}\end{array}$ \\
\hline- & & & & -- & --1 & ---1 \\
\hline 1 & 31.44 & BB & 197 & $1.29246 \mathrm{e} 4$ & 244.11180 & 642 \\
\hline 2 & 41.751 & $\mathrm{BB}$ & 179 & $1.29431 \mathrm{e} 4$ & 179.78641 & 50.0358 \\
\hline
\end{tabular}


Chiral HPLC of ATH product. $88 \%$ ee.

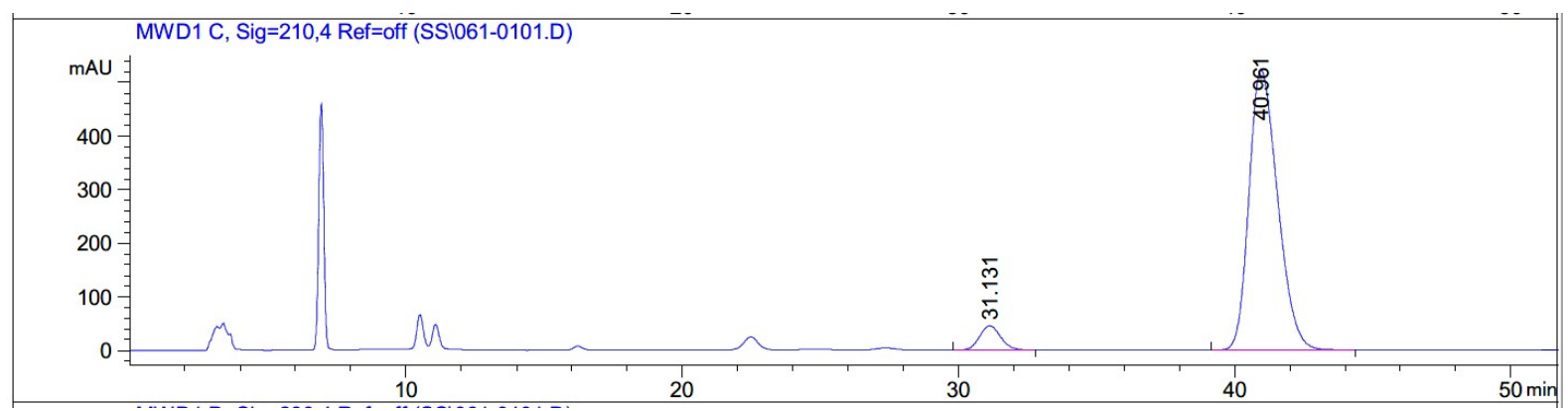

Signal 2: MWD1 C, Sig=210,4 Ref=off

\begin{tabular}{|c|c|c|c|c|c|c|}
\hline $\begin{array}{c}\text { Peak } \\
\#\end{array}$ & $\begin{array}{c}\text { RetTime } \\
\text { [min] }\end{array}$ & Type & $\begin{array}{l}\text { Width } \\
\text { [min] }\end{array}$ & $\begin{array}{c}\text { Area } \\
{\left[\mathrm{mAU}{ }^{\star} \mathrm{S}\right]}\end{array}$ & $\begin{array}{l}\text { Height } \\
\text { [mAU] }\end{array}$ & $\begin{array}{c}\text { Area } \\
\frac{\circ}{0}\end{array}$ \\
\hline & & & & & & --1 \\
\hline 1 & 1 & BB & & 2355 . & 45.05431 & 77 \\
\hline 2 & 0.961 & D & & $3.75^{\circ}$ & 522.19519 & 0923 \\
\hline
\end{tabular}


(R)- $N^{1}, N^{4}$-Diallyl-2-hydroxy- $N^{1}, N^{4}$-dimethylsuccinamide 13.<smiles>C=CCN(C)C(=O)C[C@H](O)C(=O)N(C)CC=C</smiles>

This compound is novel and was prepared following general procedure $\mathrm{C}$ using $N^{1}, N^{4}$-diallyl$N^{1}, N^{4}$-dimethyl-2-oxosuccinamide $(59.5 \mathrm{mg}, 0.250 \mathrm{mmol}, 1.0 \mathrm{eq}), 3 \mathrm{C}$ tethered catalyst $(R, R)$ $2(2.3 \mathrm{mg}, 3.8 \mu \mathrm{mol}, 0.015 \mathrm{eq})$ and FA:TEA $(0.15 \mathrm{~mL})$ in DCM $(1.5 \mathrm{~mL})$ to give $\mathbf{1 3}$ as a pale yellow liquid (55.2 mg, $0.231 \mathrm{mmol}, 93 \%)$.

TLC: $\mathrm{R}_{\mathrm{f}}$ ca 0.2 (1:9, hexane: EtOAc), non UV-active, Strong $\mathrm{KMnO}_{4}$ and PMA-reactive; HRMS (ESI) m/z: [M+Na] $]^{+}$Calcd for $\mathrm{C}_{12} \mathrm{H}_{20} \mathrm{~N}_{2} \mathrm{O}_{3} \mathrm{Na}$ 263.1366; Found 263.1368 (error -0.7 ppm);

$U_{\max } 3390,2926,1618,1484,1439,1399,1268,1077,990,919,790 \mathrm{~cm}^{-1}$;

Enantiomeric excess determined by HPLC analysis (Chiralpak IG, $250 \mathrm{~mm} \times 4.6 \mathrm{~mm}$ column, hexane: $\left.\mathrm{iPrOH} 90: 10,1 \mathrm{~mL} / \mathrm{min}, \lambda=210 \mathrm{~nm}, \mathrm{~T}=25^{\circ} \mathrm{C}\right), S$-enantiomer $56.6 \mathrm{~min}, R$ enantiomer $62.2 \min .94 \%$ ee $(R),[\alpha]_{\mathrm{D}}{ }^{22}=+13.3\left(\mathrm{c}=0.1, \mathrm{CHCl}_{3}\right)$;

${ }^{1} \mathrm{H}$ NMR (500 MHz, $\left.\mathrm{CDCl}_{3}\right)$ (equal mixture of four rotamers) $\delta 5.83-5.68(2 \mathrm{H}, \mathrm{m}$, $\left.\mathrm{CH}_{2}=\mathrm{C}_{\underline{H C H}}\right), 5.21-5.12\left(4 \mathrm{H}, \mathrm{m}, \underline{\mathrm{C}}_{2}=\mathrm{CH} \mathrm{CH}\right)^{2}, 4.86-4.82(0.5 \mathrm{H}, \mathrm{m}, \mathrm{C} \underline{\mathrm{HOH}}), 4.78-4.75$ $(0.5 \mathrm{H}, \mathrm{m}, \mathrm{C} \underline{\mathrm{HOH}}), 4.60(0.25 \mathrm{H}, \mathrm{d}, J=6.7 \mathrm{~Hz}, \mathrm{CHO} \underline{\mathrm{H}}), 4.52(0.25 \mathrm{H}, \mathrm{d}, J=6.7 \mathrm{~Hz}, \mathrm{CHO} \underline{\mathrm{H}})$, $4.45(0.25 \mathrm{H}, \mathrm{d}, J=6.7 \mathrm{~Hz}, \mathrm{CHO} \underline{\mathrm{H}}), 4.38(0.25 \mathrm{H}, \mathrm{d}, J=6.7 \mathrm{~Hz}, \mathrm{CHO} \underline{\mathrm{H}}), 4.11-3.83$ (4H, m, $\left.\mathrm{CH}_{2}=\mathrm{CHCl}_{2}\right), 3.05\left(0.75 \mathrm{H}, \mathrm{s}, \mathrm{NCH}_{3}\right), 3.03\left(0.75 \mathrm{H}, \mathrm{s}, \mathrm{NCH}_{3}\right), 2.97\left(0.75 \mathrm{H}, \mathrm{s}, \mathrm{NCH}_{3}\right), 2.96$ $\left(0.75 \mathrm{H}, \mathrm{s}, \mathrm{NCH}_{3}\right), 2.93\left(0.75 \mathrm{H}, \mathrm{s}, \mathrm{NCH}_{3}\right), 2.93\left(0.75 \mathrm{H}, \mathrm{s}, \mathrm{NCH}_{3}\right), 2.92\left(0.75 \mathrm{H}, \mathrm{s}, \mathrm{NCH}_{3}\right)$, $2.91\left(0.75 \mathrm{H}, \mathrm{s}, \mathrm{NCH}_{3}\right), 2.83-2.69\left(1 \mathrm{H}, \mathrm{m}, \underline{\mathrm{C}}_{\underline{a}} \mathrm{H}_{\mathrm{b}}\right), 2.59-2.50\left(1 \mathrm{H}, \mathrm{m}, \mathrm{CH}_{\mathrm{a}} \underline{\mathrm{H}}_{\mathrm{b}}\right)$;

${ }^{13} \mathrm{C} \mathrm{NMR}\left(126 \mathrm{MHz}, \mathrm{CDCl}_{3}\right)$ (mixture of four rotamers) $\delta 172.7,172.6,172.5,172.4,171.8$, $171.5,171.4,171.2,132.9,132.8,132.6,132.3,132.2,117.8,117.7,117.5,117.4,116.9$, 67.1, 67.0, 66.9, 52.4, 51.7, 50.7, 50.6, 50.1, 37.4, 37.1, 35.2, 35.1, 34.5, 34.4, 33.8, 33.7; $m / z(\mathrm{ESI}) 263.1\left[(\mathrm{M}+\mathrm{Na})^{+}, 100 \%\right]$. 
$\boldsymbol{N}^{1}, N^{4}$-Diallyl-2-hydroxy-dimethylsuccinamide 13.

${ }^{1} \mathrm{H}$ NMR (500 MHz, $\mathrm{CDCl}_{3}$ ).
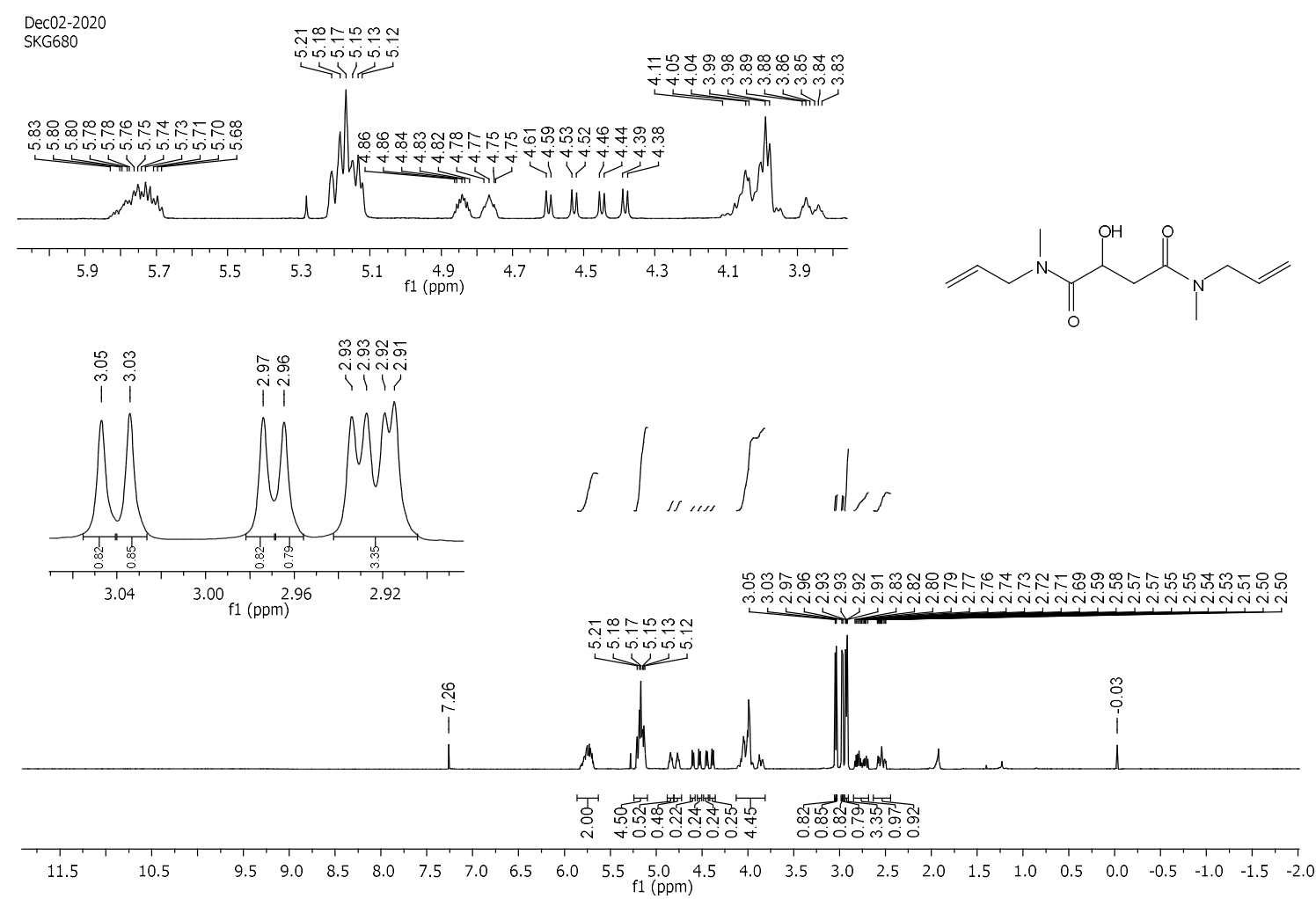

${ }^{13} \mathrm{C} \mathrm{NMR}\left(126 \mathrm{MHz}, \mathrm{CDCl}_{3}\right)$.

Dec02-2020

Chemist Shweta Gediya

SKG680

C13APT.w CDCl3 /opt/topspin3.2 SKG 13

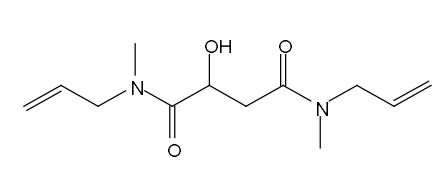

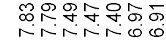
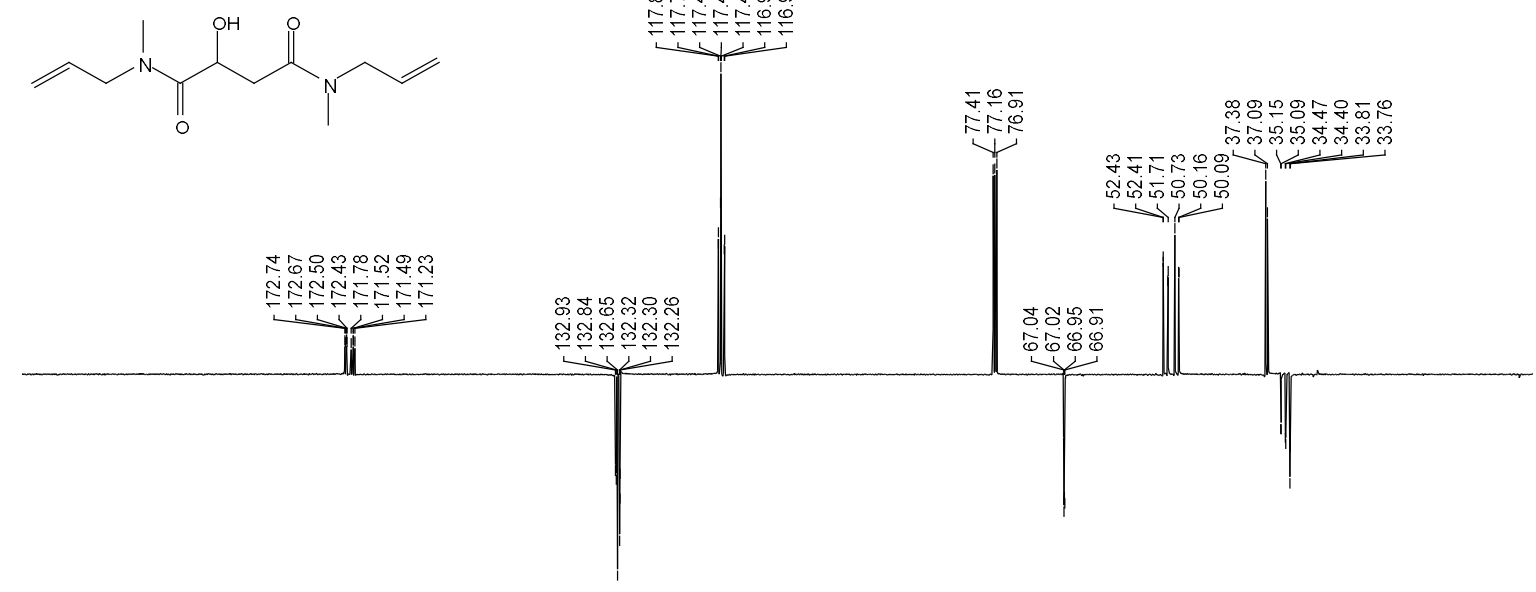

$\begin{array}{rllllllllllllllllllllllllllll}220 & 210 & 200 & 190 & 180 & 170 & 160 & 150 & 140 & 130 & 120 & 110 & 100 & 90 & 80 & 70 & 60 & 50 & 40 & 30 & 20 & 10 & 0\end{array}$


COSY.

Chemist Shweta Gediya

SKG680
COSY.w CDCI3/opt/topspin3.2 SKG 13
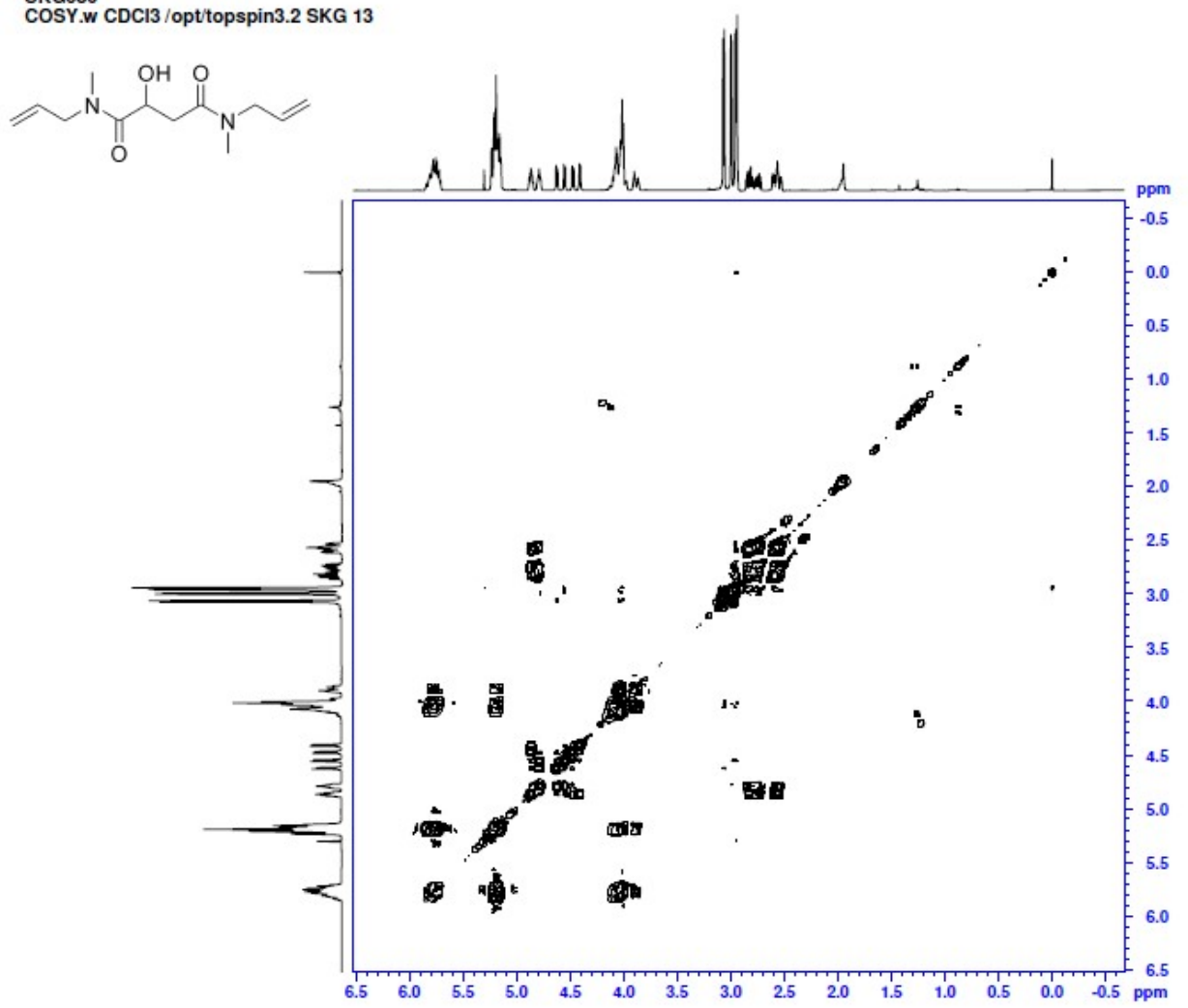

HSQC.

Chemist Shweta Gediya HSG6.w CDCl3 /opt/topspin3.2 SKG 13

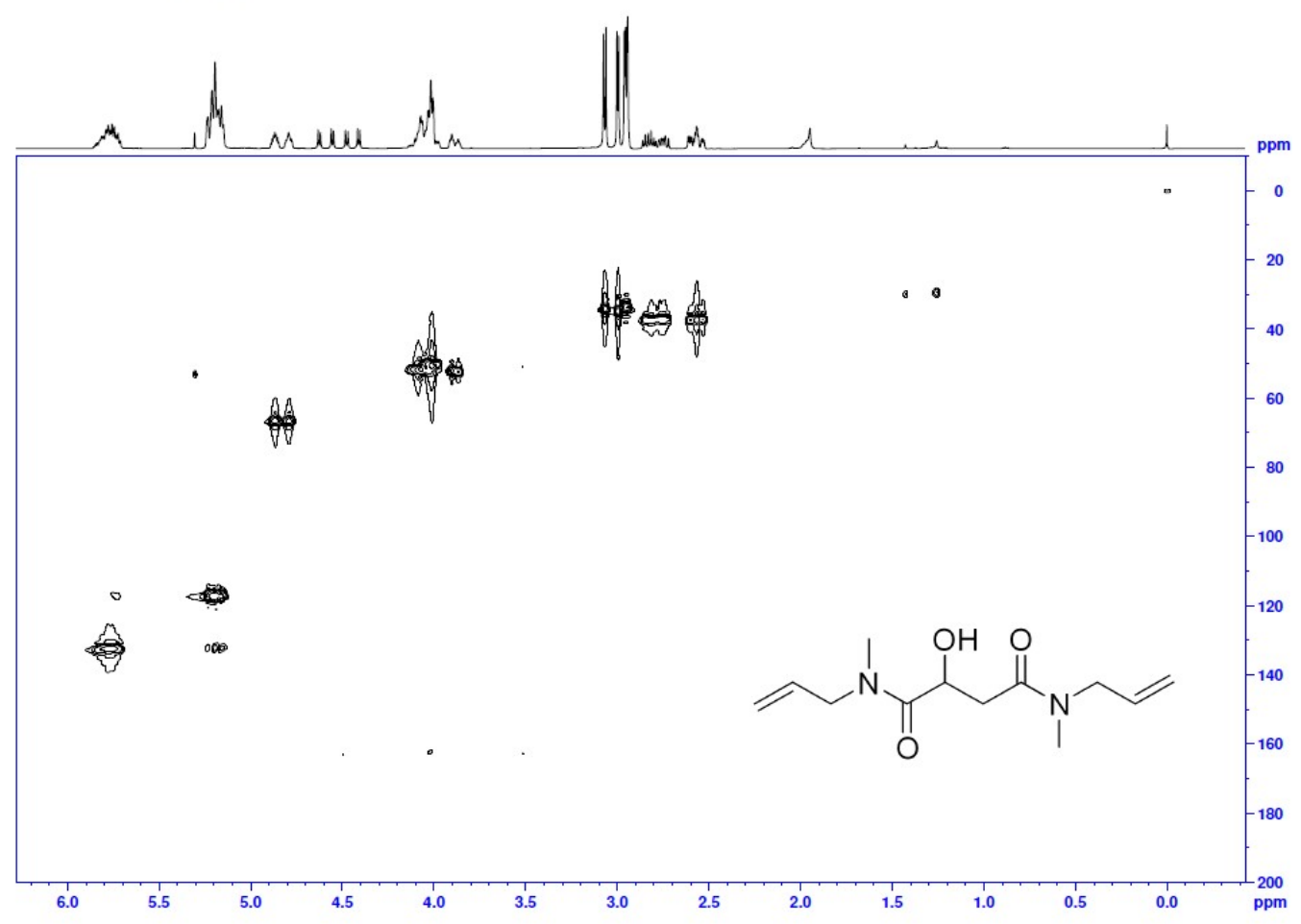

S237 
Chiral HPLC of ketone precursor.

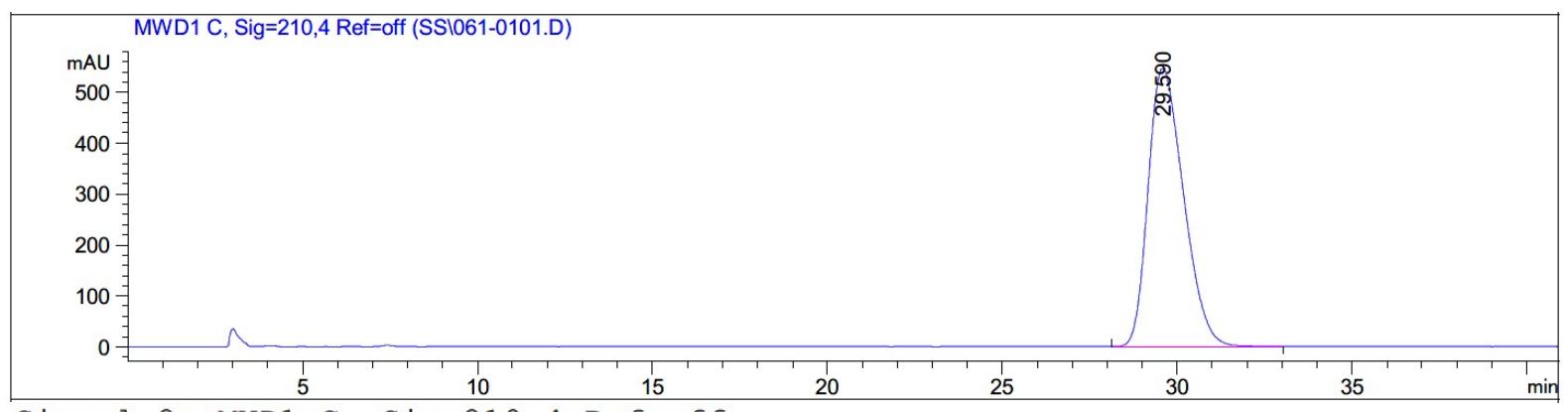

Signal 2: MWD1 C, Sig=210,4 Ref=off

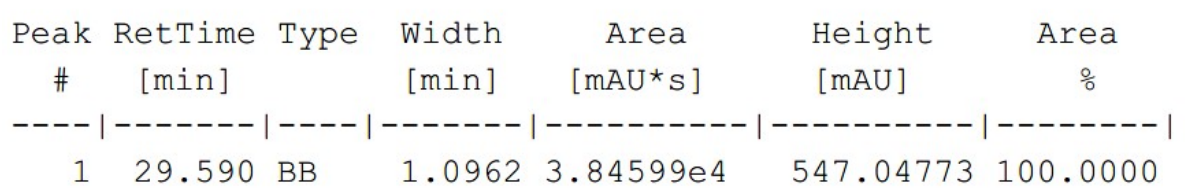

\section{Chiral HPLC of racemic alcohol.}

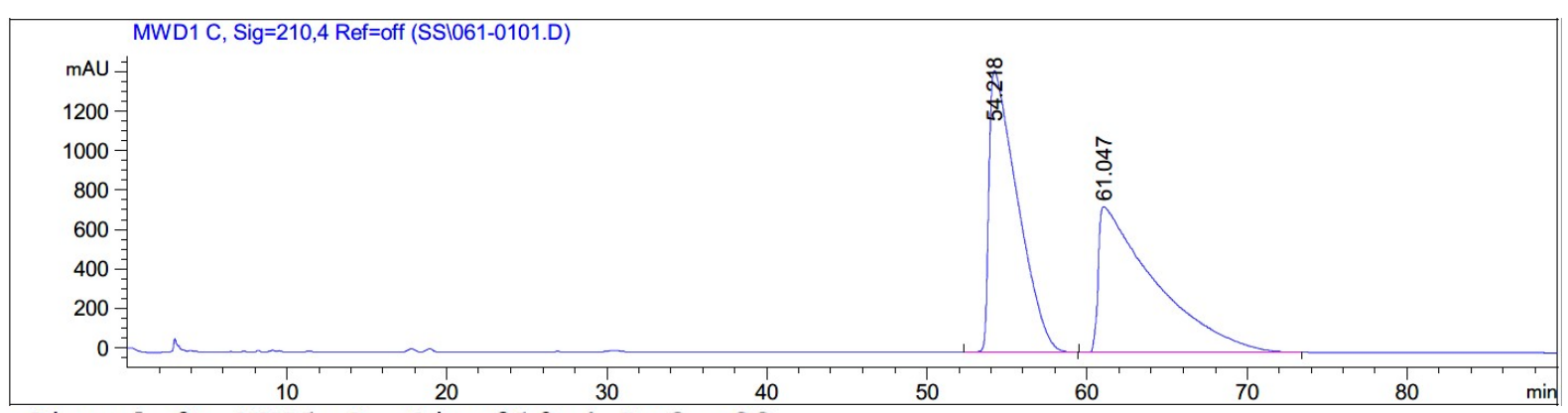

Signal 2: MWD1 C, Sig=210,4 Ref=off

\begin{tabular}{|c|c|c|c|c|c|c|}
\hline $\begin{array}{c}\text { Peak } \\
\#\end{array}$ & $\begin{array}{c}\text { RetTime } \\
\text { [min] }\end{array}$ & Type & $\begin{array}{l}\text { Width } \\
\text { [min] }\end{array}$ & $\begin{array}{c}\text { Area } \\
{\left[\mathrm{mAU}{ }^{\star} \mathrm{S}\right]}\end{array}$ & $\begin{array}{l}\text { Height } \\
{[\mathrm{mAU}]}\end{array}$ & $\begin{array}{c}\text { Area } \\
\frac{\circ}{0}\end{array}$ \\
\hline-- & ------ & & ------1 & ---------- & $|----------|$ & $--------\mid$ \\
\hline 1 & 54.218 & $\mathrm{BB}$ & 1.6383 & $1.76291 \mathrm{e} 5$ & 1426.02234 & 49.4505 \\
\hline 2 & 61.047 & $\mathrm{BB}$ & 2.9852 & $1.80209 e 5$ & 737.37592 & 50.5495 \\
\hline
\end{tabular}


Chiral HPLC of ATH product. 94\% ee.

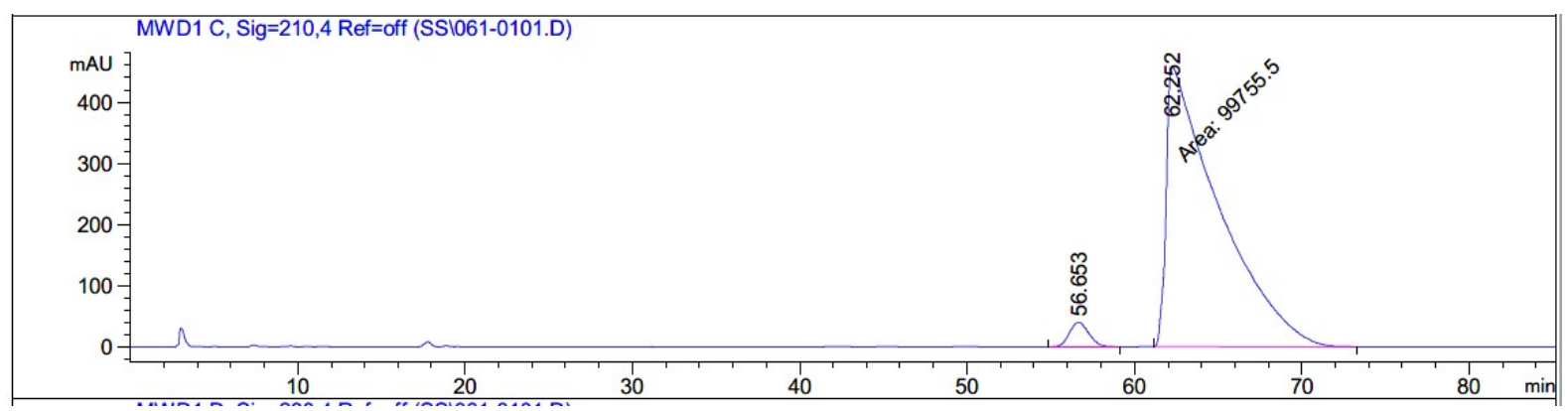

Signal 2: MWD1 C, Sig=210,4 Ref=off

\begin{tabular}{|c|c|c|c|c|c|c|}
\hline $\begin{array}{c}\text { eak } \\
\#\end{array}$ & $\begin{array}{c}\text { RetTime } \\
\text { [min] }\end{array}$ & Type & $\begin{array}{l}\text { Width } \\
\text { [min] }\end{array}$ & $\begin{array}{c}\text { Area } \\
{\left[\mathrm{mAU}^{\star} \mathrm{s}\right]}\end{array}$ & $\begin{array}{l}\text { Height } \\
{[\mathrm{mAU}]}\end{array}$ & $\begin{array}{c}\text { Area } \\
\%\end{array}$ \\
\hline & & & & --- & --- & --- \\
\hline 1 & & 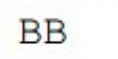 & & 3231 & 04 & 375 \\
\hline 2 & 62.252 & Me & 97 & $9.97555 \mathrm{e} 4$ & 456.78818 & 8625 \\
\hline
\end{tabular}




\section{2-Hydroxy- $N^{4}$-methyl- $N^{1}, N^{1}, N^{4}$-triphenylsuccinamide 14 .}<smiles>CN(C(=O)CC(O)C(=O)Nc1ccccc1)c1ccccc1</smiles>

This compound is novel and was prepared following general procedure $\mathrm{C}$ using $N^{4}$-methyl-2oxo- $N^{1}, N^{1}, N^{4}$-triphenylsuccinamide $(93.5 \mathrm{mg}, 0.250 \mathrm{mmol}, 1.0 \mathrm{eq}), 3 \mathrm{C}$ tethered catalyst $(R, R)-2(2.3 \mathrm{mg}, 3.8 \mu \mathrm{mol}, 0.015 \mathrm{eq})$ and FA:TEA $(0.15 \mathrm{~mL})$ in DCM $(1.5 \mathrm{~mL})$ to give 14 as a colourless semi solid material (84.2 $\mathrm{mg}, 0.226 \mathrm{mmol}, 90 \%)$.

TLC: $\mathrm{R}_{\mathrm{f}}$ ca 0.2 (7:3, hexane: EtOAc), UV-active, Strong $\mathrm{KMnO}_{4}$ and PMA-reactive; HRMS (ESI) m/z: [M+Na] ${ }^{+}$Calcd for $\mathrm{C}_{23} \mathrm{H}_{22} \mathrm{~N}_{2} \mathrm{O}_{3} \mathrm{Na}$ 397.1523; Found 397.1524 (error -0.3 ppm);

$U_{\max } 3368,2927,1641,1589,1491,1387,1282,1119,1062,699 \mathrm{~cm}^{-1}$

Enantiomeric excess determined by HPLC analysis (Chiralpak IG, $250 \mathrm{~mm} \times 4.6 \mathrm{~mm}$ column, hexane: $\left.\mathrm{iPrOH} 85: 15,1 \mathrm{~mL} / \mathrm{min}, \lambda=210 \mathrm{~nm}, \mathrm{~T}=25^{\circ} \mathrm{C}\right), S$-enantiomer $61.4 \mathrm{~min}, R$ enantiomer $65.7 \mathrm{~min} .31 \%$ ee $(R),[\alpha]_{\mathrm{D}}^{22}=-8\left(\mathrm{c}=0.1, \mathrm{CHCl}_{3}\right)$;

${ }^{1} \mathrm{H}$ NMR $\left(400 \mathrm{MHz}, \mathrm{CDCl}_{3}\right) \delta 7.41-7.21(15 \mathrm{H}, \mathrm{m}, \mathrm{ArH}), 4.66(1 \mathrm{H}, \mathrm{s}, \mathrm{C} \underline{\mathrm{HOH}}), 4.02(1 \mathrm{H}, \mathrm{s}$, $\mathrm{CHO} \underline{\mathrm{H}}), 3.24\left(3 \mathrm{H}, \mathrm{s}, \mathrm{NCH}_{3}\right), 2.43-2.29\left(2 \mathrm{H}, \mathrm{m}, \mathrm{CH}_{\mathrm{a}} \mathrm{H}_{\mathrm{b}}\right)$;

${ }^{13} \mathrm{C}$ NMR (101 MHz, $\left.\mathrm{CDCl}_{3}\right) \delta 173.3,170.3,143.7,129.9,129.4,128.1,127.6,127.4,125.9$, $121.1,117.9,67.2,37.8,37.4$;

$m / z(\mathrm{ESI}) 397.1\left[(\mathrm{M}+\mathrm{Na})^{+}, 100 \%\right]$. 
2-Hydroxy- $N^{4}$-methyl- $N^{1}, N^{1}, N^{4}$-triphenylsuccinamide 14.

${ }^{1} \mathrm{H}$ NMR (400 MHz, $\mathrm{CDCl}_{3}$ ).
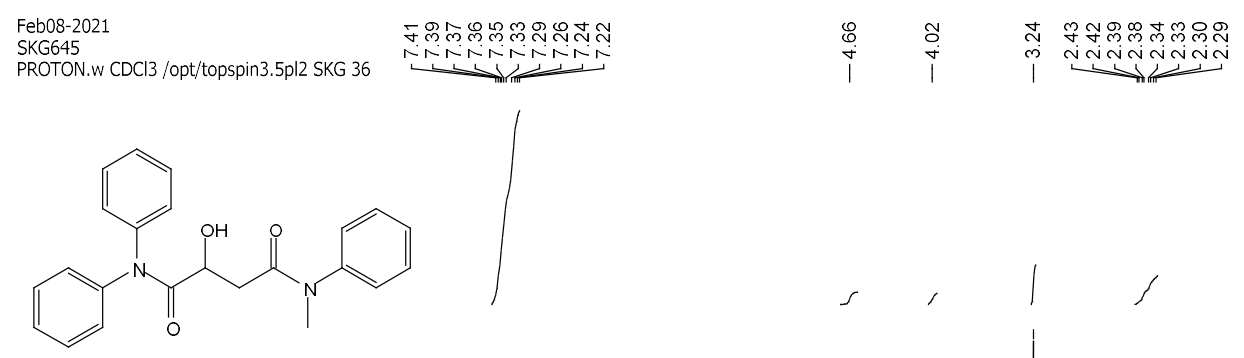

$\stackrel{\circ}{\circ}$

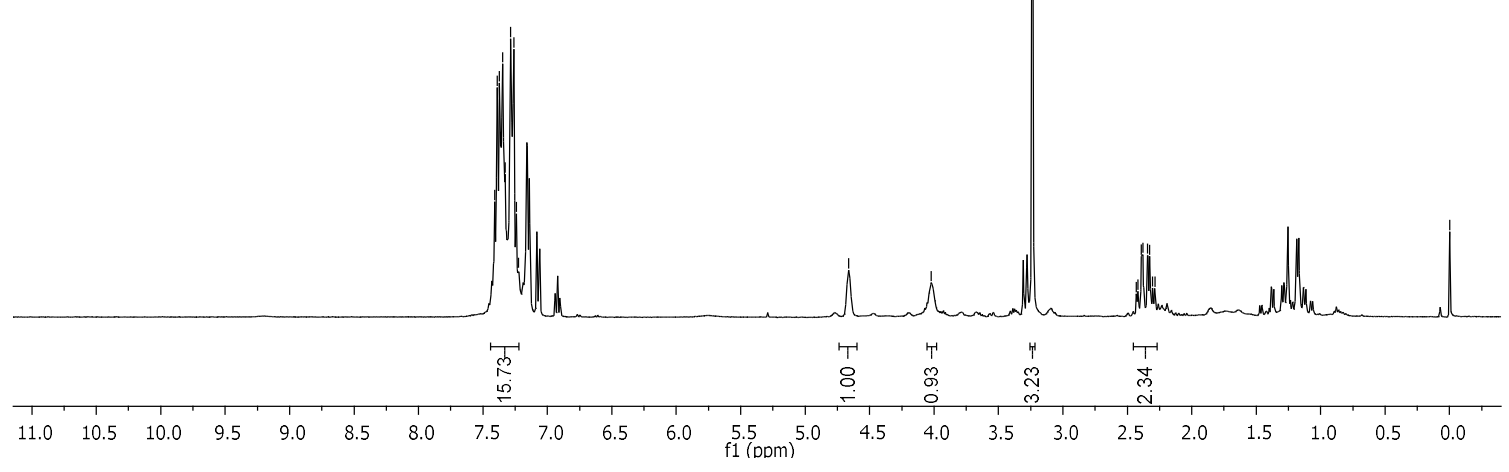

${ }^{13} \mathrm{C}$ NMR (101 MHz, $\left.\mathrm{CDCl}_{3}\right)$.

Feb08-2021
SKG645

C13APTlong.w CDCl3 /opt/topspin3.5pl2 SKG 36

ำ

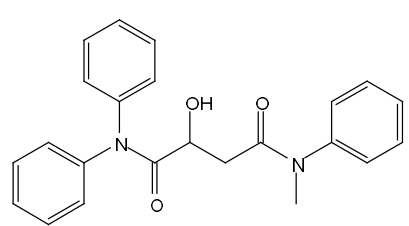

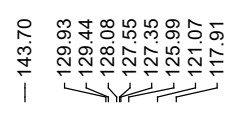

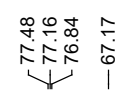

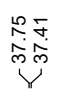
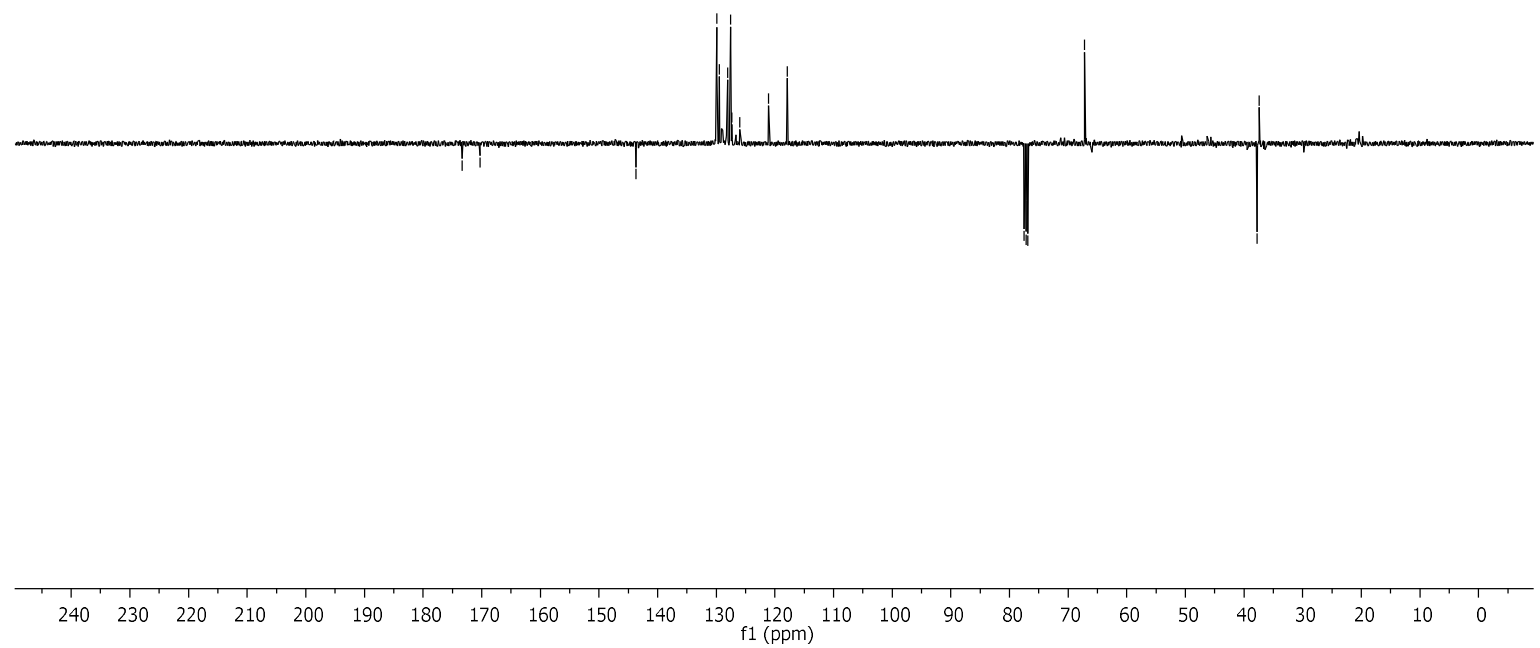

S241 
COSY.

SKG645

COSY.w CDCl3 /opt/topspin3.5pl2 SKG 36

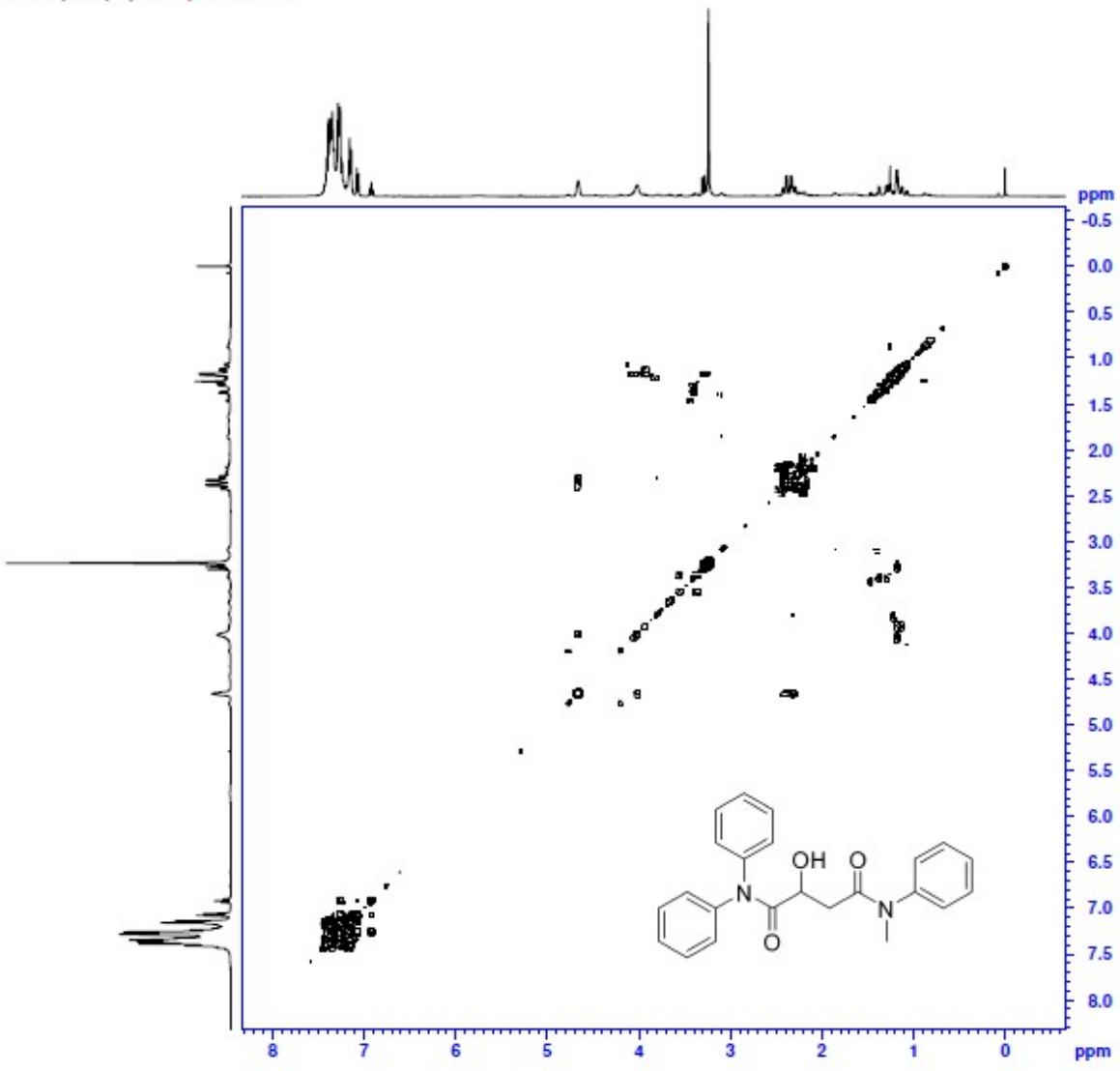

HSQC.

SKG645
HSQC.w CDCl3 /opt/topspin 3.5pl2 SKG 36

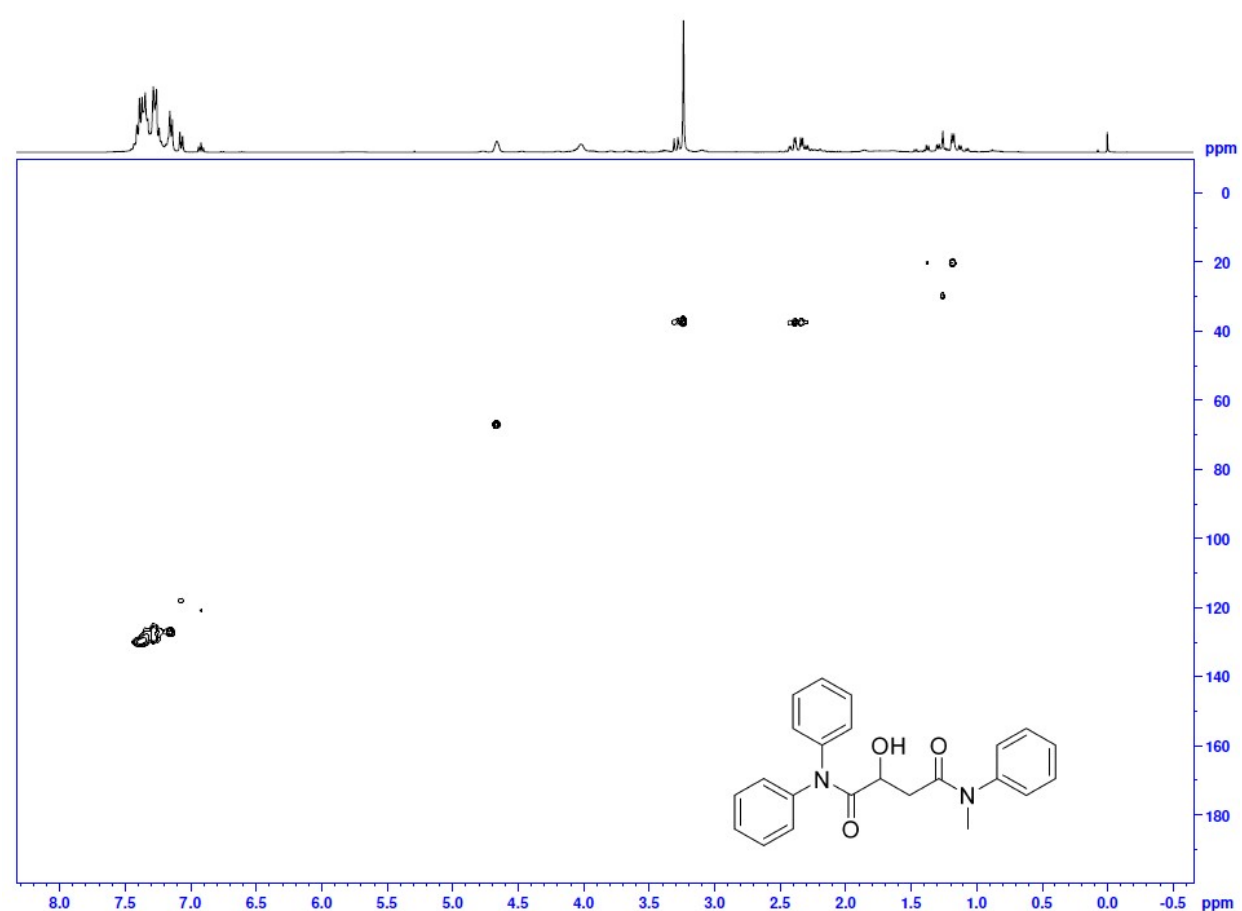




\section{Chiral HPLC of ketone precursor.}

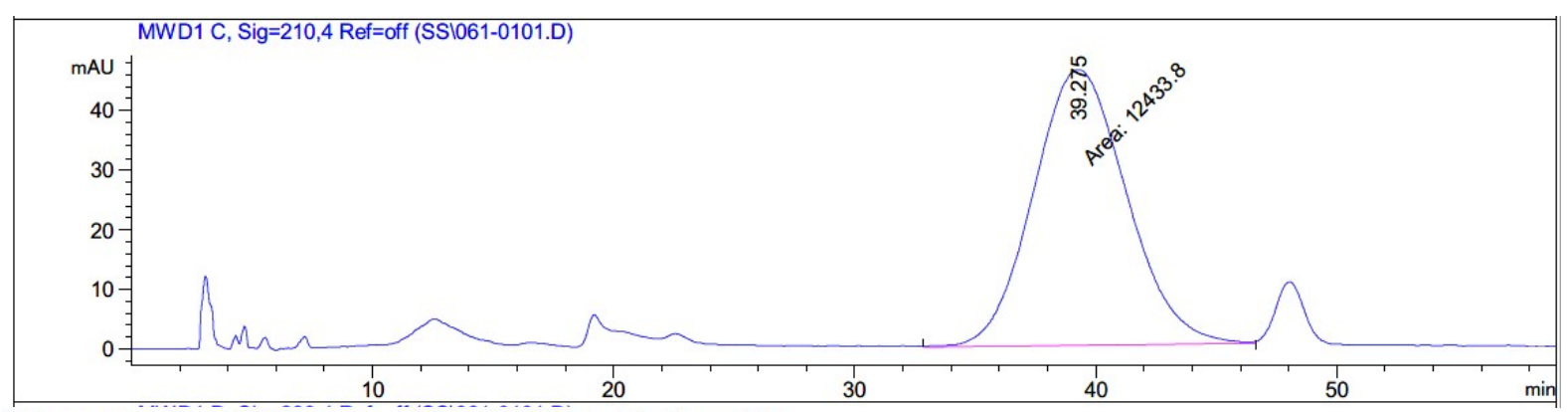

Signal 2: MWD1 C, Sig=210,4 Ref=off

\begin{tabular}{|c|c|c|c|c|c|c|}
\hline $\begin{array}{c}\text { Peak } \\
\#\end{array}$ & $\begin{array}{c}\text { RetTime } \\
\text { [min] }\end{array}$ & Type & $\begin{array}{l}\text { Width } \\
\text { [min] }\end{array}$ & $\begin{array}{c}\text { Area } \\
{\left[\mathrm{mAU}{ }^{\star} \mathrm{S}\right]}\end{array}$ & $\begin{array}{l}\text { Height } \\
{[\mathrm{mAU}]}\end{array}$ & $\begin{array}{c}\text { Area } \\
\frac{\%}{\sigma}\end{array}$ \\
\hline 1 & 9.275 & MM & 4.4873 & 1. $24338 e 4$ & 46.18122 & 100.0000 \\
\hline
\end{tabular}


Chiral HPLC of racemic alcohol.

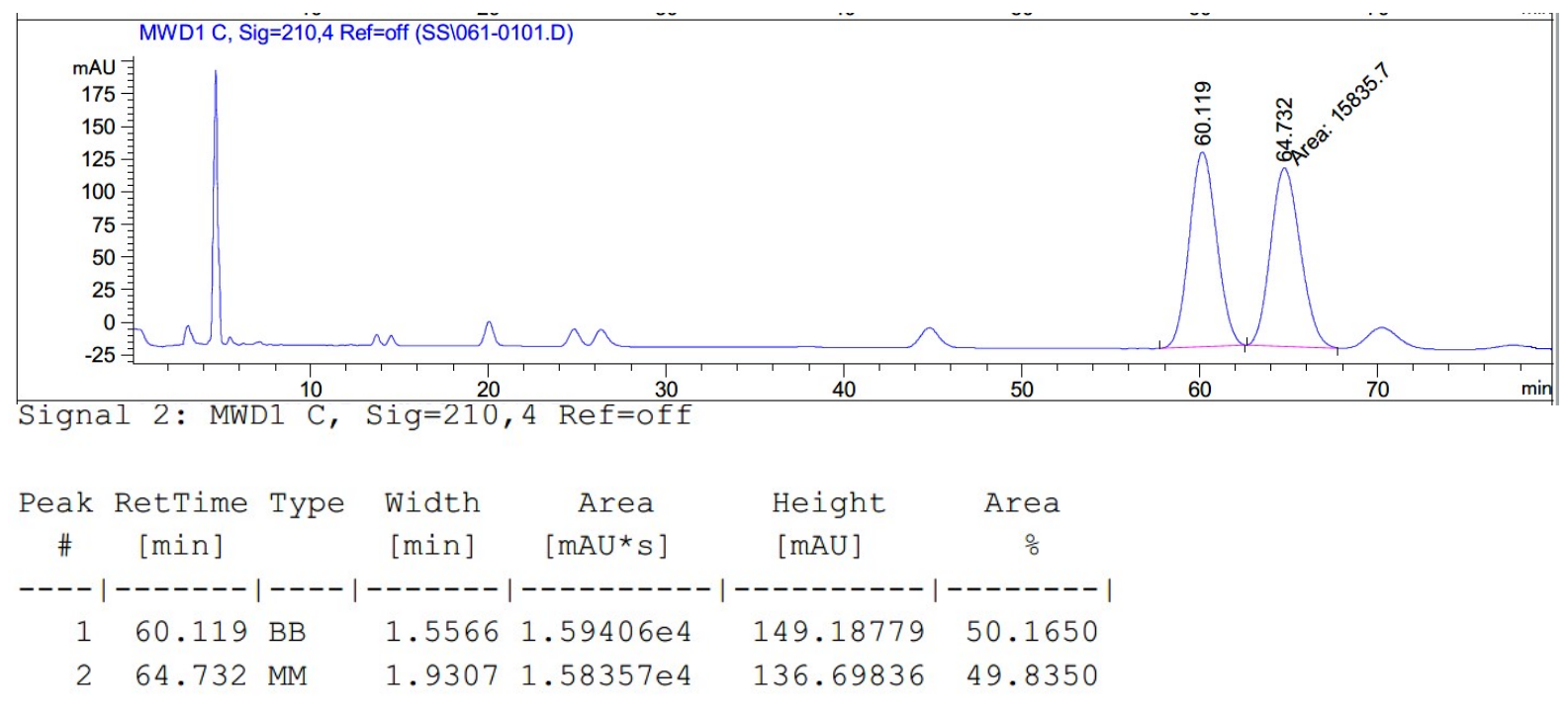

Chiral HPLC of ATH product. 31\% ee.

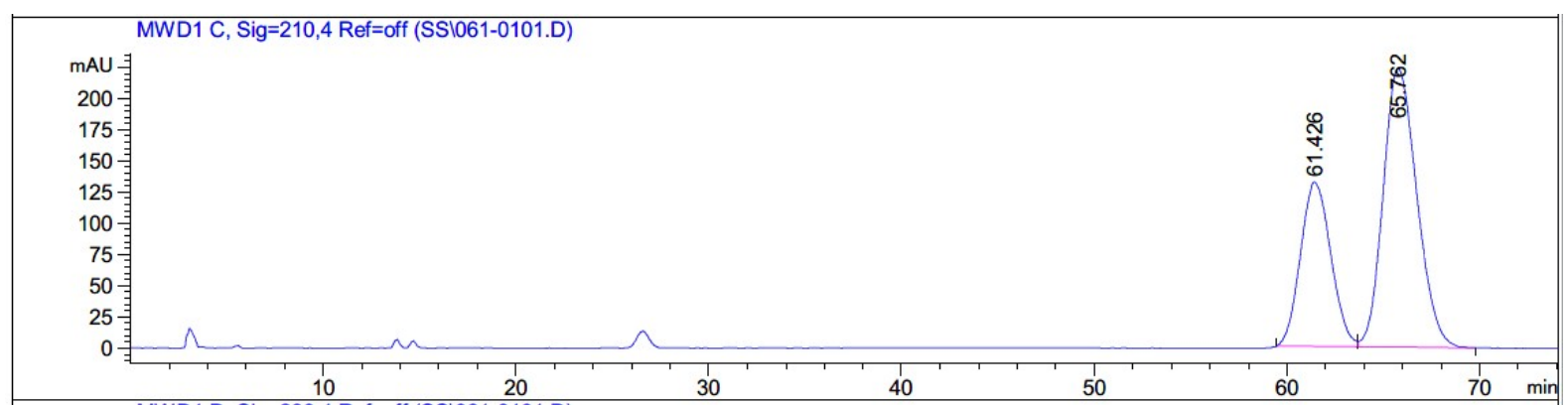

Signal 2: MWD1 C,

\begin{tabular}{|c|c|c|c|c|c|c|}
\hline $\begin{array}{c}\text { Peak } \\
\#\end{array}$ & $\begin{array}{c}\text { RetTime } \\
\text { [min] }\end{array}$ & Type & $\begin{array}{l}\text { Width } \\
\text { [min] }\end{array}$ & $\begin{array}{c}\text { Area } \\
{\left[\mathrm{mAU}^{\star} \mathrm{s}\right]}\end{array}$ & $\begin{array}{l}\text { Height } \\
{[\mathrm{mAU}]}\end{array}$ & $\begin{array}{c}\text { Area } \\
\%\end{array}$ \\
\hline & 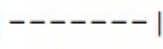 & 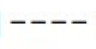 & 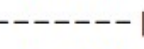 & 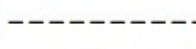 & --- & ---1 \\
\hline 1 & 61.426 & BV & 1.5772 & $1.44001 \mathrm{e} 4$ & 131.53941 & 34.6498 \\
\hline 2 & 65.762 & VB & 1.7970 & $2.71590 \mathrm{e} 4$ & 223.60840 & 65.3502 \\
\hline
\end{tabular}




\section{(R)-2-Hydroxy- $N^{4}$-methyl- $N^{1}, N^{4}$-diphenylsuccinamide 15.}<smiles>CN(C(=O)CC(O)C(=O)Nc1ccccc1)c1ccccc1</smiles>

This compound is novel and was prepared following general procedure $\mathrm{C}$ using $N^{4}$-methyl-2oxo- $N^{1}, N^{4}$-diphenylsuccinamide $(0.0740 \mathrm{~g}, 0.250 \mathrm{mmol}, 1.0 \mathrm{eq}), 3 \mathrm{C}$ tethered catalyst $(R, R)-2$ $(2.3 \mathrm{mg}, 3.8 \mu \mathrm{mol}, 0.015 \mathrm{eq})$ and FA:TEA $(0.15 \mathrm{~mL})$ in DCM $(1.5 \mathrm{~mL})$ to give $\mathbf{1 5}$ as a white solid $(0.0650 \mathrm{~g}, 0.218 \mathrm{mmol}, 87 \%)$.

TLC: $\mathrm{R}_{\mathrm{f}}$ ca 0.4 (6:4, hexane: EtOAc), UV-active, strong $\mathrm{KMnO}_{4}$, and PMA-reactive;

HRMS (ESI) m/z: [M+Na] $]^{+}$Calcd for $\mathrm{C}_{17} \mathrm{H}_{18} \mathrm{~N}_{2} \mathrm{O}_{3} \mathrm{Na} 321.1210$; Found 321.1203 (error 2.0 ppm);

MP: $131-134{ }^{\circ} \mathrm{C}$;

$U_{\max } 3294,2972,2901,1630,1592,1493,1440,1389,1095,725,692 \mathrm{~cm}^{-1}$;

Enantiomeric excess determined by HPLC analysis (Chiralpak IG, $250 \mathrm{~mm} \times 4.6 \mathrm{~mm}$ column, Hexane: $\left.\mathrm{iPrOH} 90: 10,0.7 \mathrm{~mL} / \mathrm{min}, \lambda=210 \mathrm{~nm}, \mathrm{~T}=25^{\circ} \mathrm{C}\right), R$-enantiomer $63.1 \mathrm{~min}, S$ enantiomer $66.8 \mathrm{~min} .91 \%$ ee $(R),[\alpha]_{\mathrm{D}}^{22}=-39.3\left(\mathrm{c}=0.1, \mathrm{CHCl}_{3}\right)$;

${ }^{1} \mathrm{H}$ NMR (400 MHz, $\left.\mathrm{CDCl}_{3}\right) \delta 8.87(1 \mathrm{H}, \mathrm{s}, \mathrm{CONH}), 7.56(2 \mathrm{H}, \mathrm{d}, J=7.8 \mathrm{~Hz}, \mathrm{ArH}), 7.45-7.42$ $(2 \mathrm{H}, \mathrm{m}, \mathrm{ArH}), 7.38-7.29(3 \mathrm{H}, \mathrm{m}, \mathrm{ArH}), 7.21(2 \mathrm{H}, \mathrm{d}, J=7.4 \mathrm{~Hz}, \mathrm{ArH}), 7.12-7.08(1 \mathrm{H}, \mathrm{m}$, ArH), $5.40(1 \mathrm{H}, \mathrm{d}, J=5.0 \mathrm{~Hz}, \mathrm{CHO} \underline{\mathrm{H}}), 4.47-4.43(1 \mathrm{H}, \mathrm{m}, \underline{\mathrm{CHOH}}), 3.28\left(3 \mathrm{H}, \mathrm{s}, \mathrm{NCH}_{3}\right), 2.70$ $\left(1 \mathrm{H}, \mathrm{dd}, J=17.0,4.2 \mathrm{~Hz}, \mathrm{CH}_{\mathrm{a}} \mathrm{H}_{\mathrm{b}}\right), 2.60\left(1 \mathrm{H}, \mathrm{dd}, J=17.0,7.3 \mathrm{~Hz}, \mathrm{CH}_{\mathrm{a}} \mathrm{H}_{\mathrm{b}}\right)$;

${ }^{13} \mathrm{C} \mathrm{NMR}\left(101 \mathrm{MHz}, \mathrm{CDCl}_{3}\right) \delta 172.6,170.7,142.8,137.5,130.2,129.1,128.6,127.2,124.5$, 119.7, 69.6, 37.4, 37.1;

$m / z(\mathrm{ESI}) 321.2\left[(\mathrm{M}+\mathrm{Na})^{+}, 100 \%\right]$. 
2-Hydroxy- $N^{4}$-methyl- $N^{1}, N^{4}$-diphenylsuccinamide 15.

${ }^{1} \mathrm{H}$ NMR (400 MHz, $\left.\mathrm{CDCl}_{3}\right)$.

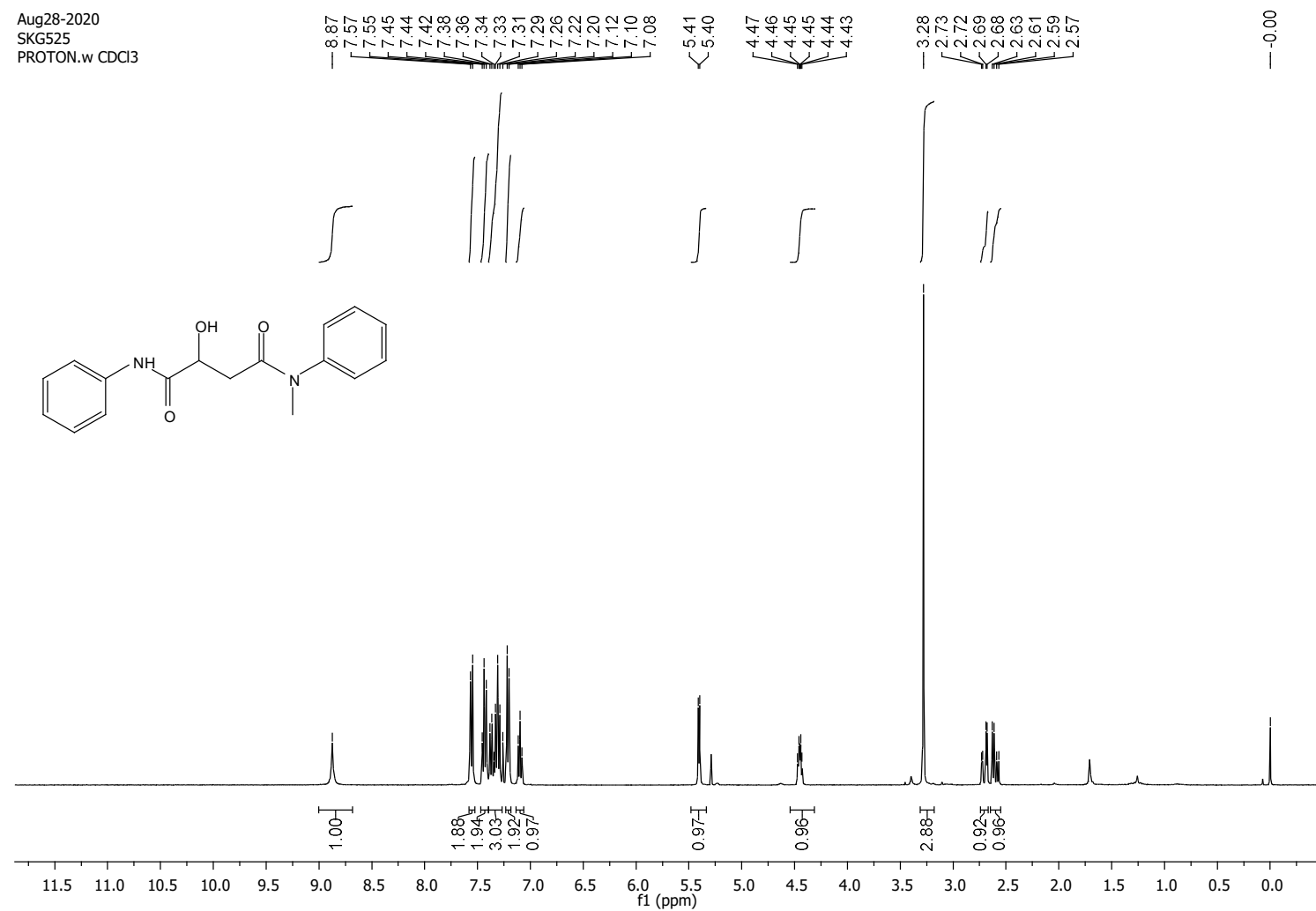

${ }^{13} \mathrm{C}$ NMR (101 MHz, $\left.\mathrm{CDCl}_{3}\right)$.

Aug28-2020
SKG525

C13APTlong.w CDCl3

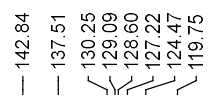

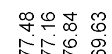

N是

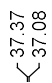<smiles>CN(C(=O)CC(O)C(=O)Nc1ccccc1)c1ccccc1</smiles>

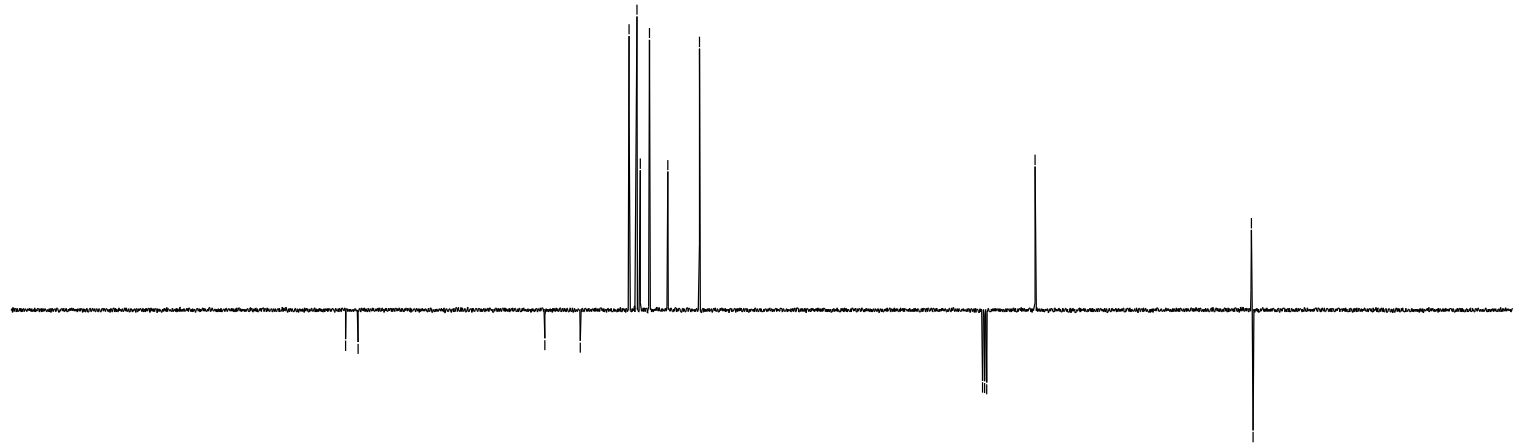

$\begin{array}{lllllllllllllllllllllllllllllllllll}220 & 210 & 200 & 190 & 180 & 170 & 160 & 150 & 140 & 130 & 120 & 110 & 100 & 90 & 80 & 70 & 60 & 50 & 40 & 30 & 20 & 10 & 0\end{array}$ 
COSY.

SKG525 pure

COSY.w CDCl3/opt/topspin3.5pl2 SKG 12
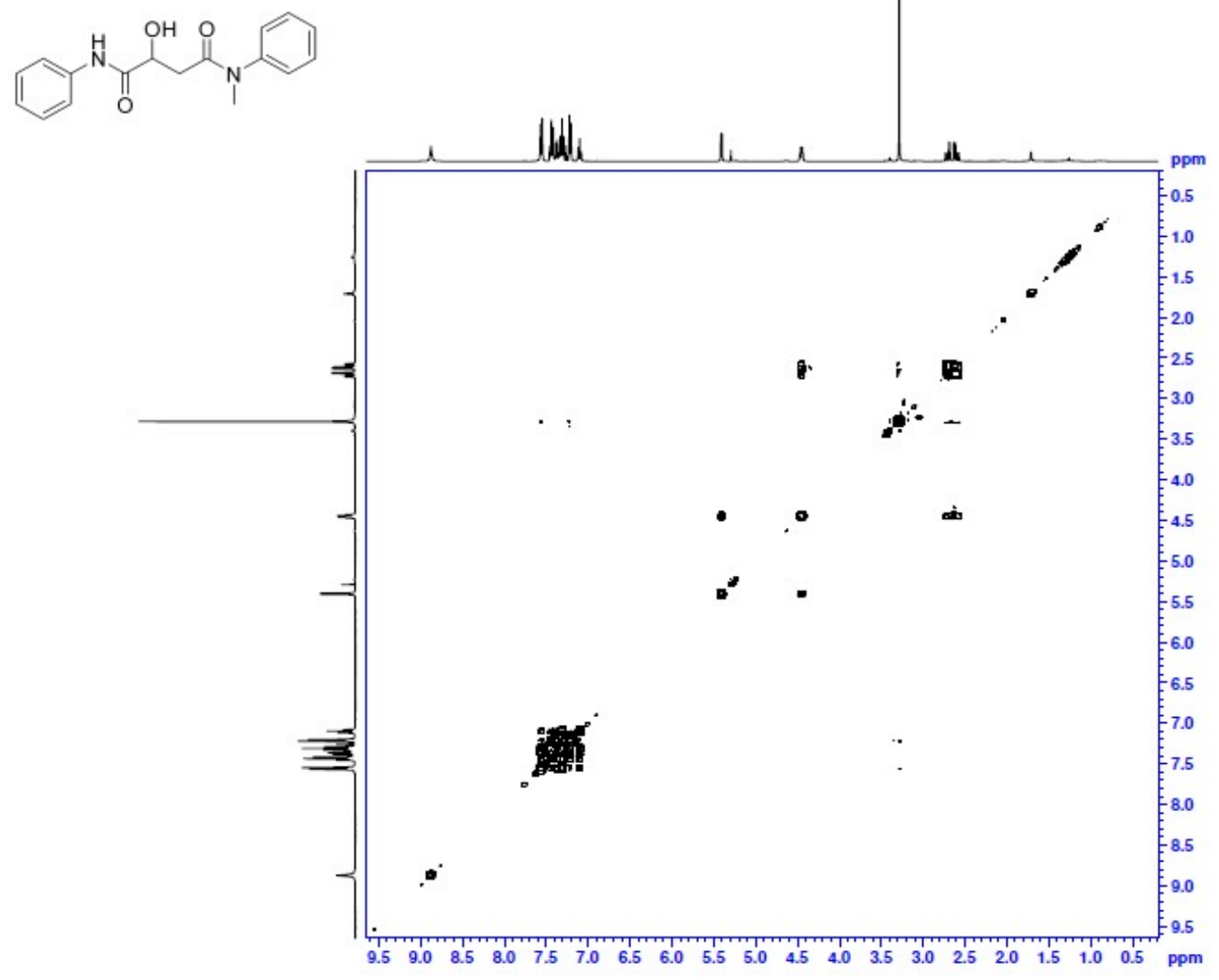

HSQC.

SKG525 pure
HSQC.w CDCl3 /opt/topspin3.5pl2 SKG 12

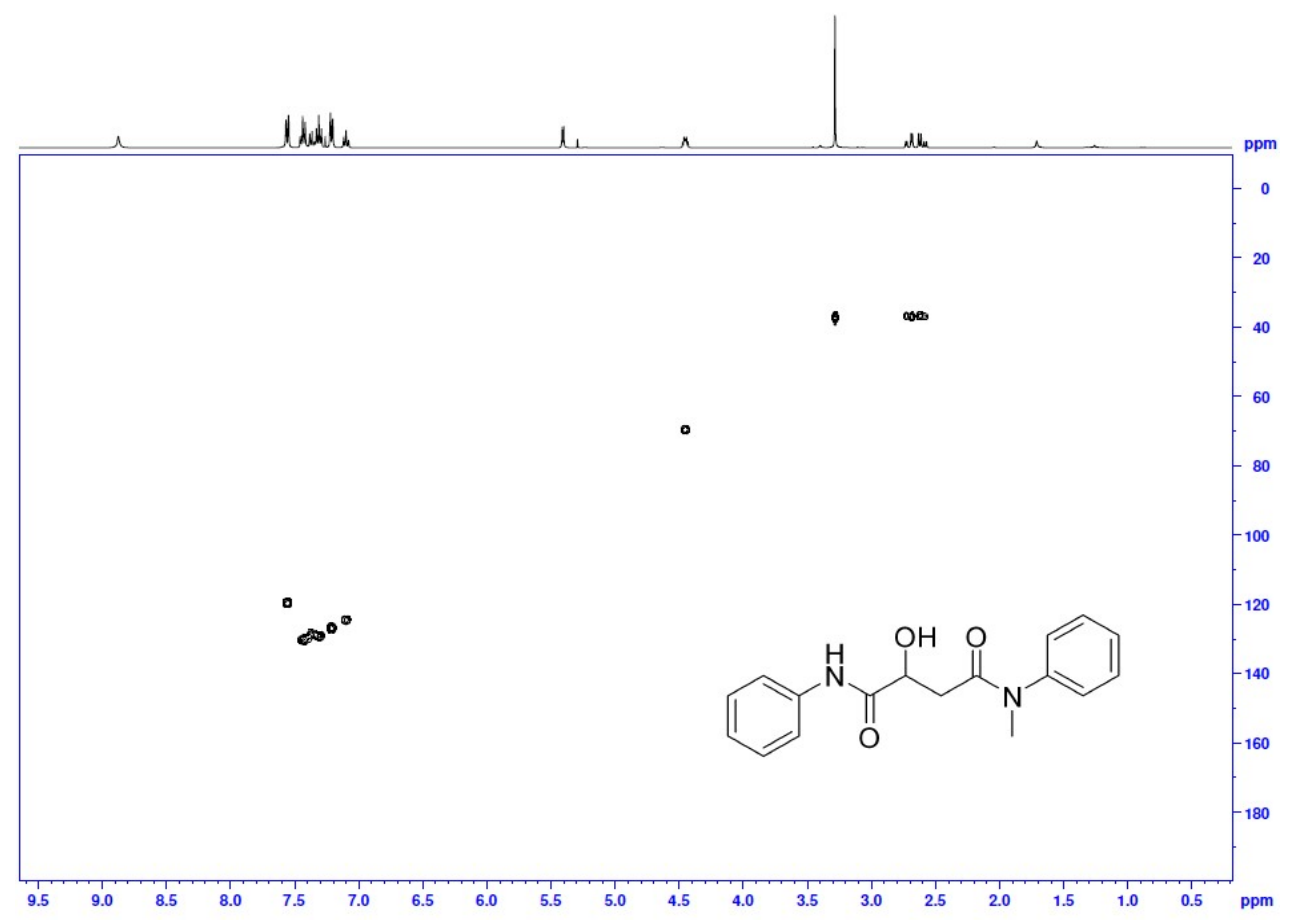

S247 


\section{Chiral HPLC of ketone precursor.}

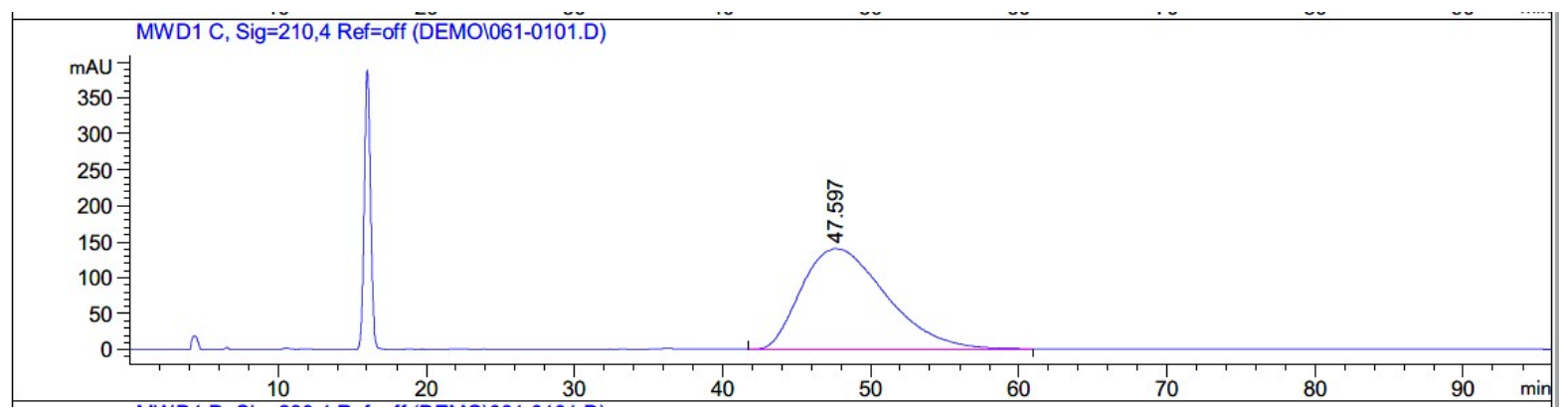

Signal 2: MWD1 C, Sig=210,4 Ref=off

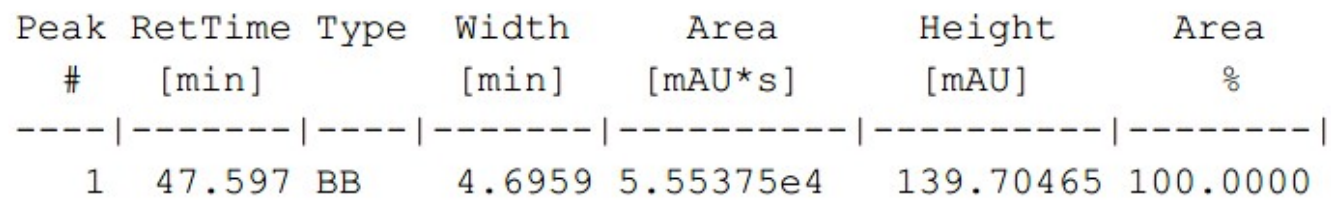

Chiral HPLC of racemic alcohol.

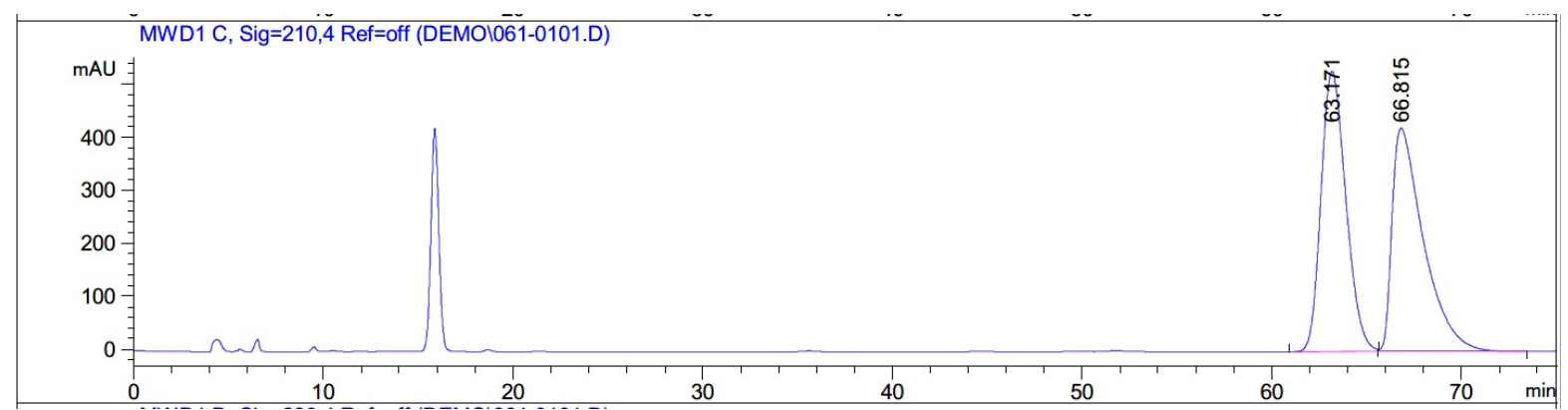

Signal 2: MWD1 C, Sig=210,4 Ref=off

\begin{tabular}{|c|c|c|c|c|c|c|}
\hline $\begin{array}{c}\text { Peak } \\
\#\end{array}$ & $\begin{array}{c}\text { RetTime } \\
\text { [min] }\end{array}$ & Type & $\begin{array}{l}\text { Width } \\
\text { [min] }\end{array}$ & $\begin{array}{c}\text { Area } \\
{\left[\mathrm{mAU}^{*} \mathrm{~s}\right]}\end{array}$ & $\begin{array}{l}\text { Height } \\
\text { [mAU] }\end{array}$ & $\begin{array}{c}\text { Area } \\
\quad \%\end{array}$ \\
\hline & & & & ---- & -- & --- \\
\hline 1 & 631 & $3 \mathrm{~V}$ & 1 & $4.80874 \mathrm{e} 4$ & 98529 & 29 \\
\hline 0 & 15 & D & 32 & $4.84680 \mathrm{e} 4$ & 421.60934 & 971 \\
\hline
\end{tabular}


Chiral HPLC of ATH product. 91\% ee

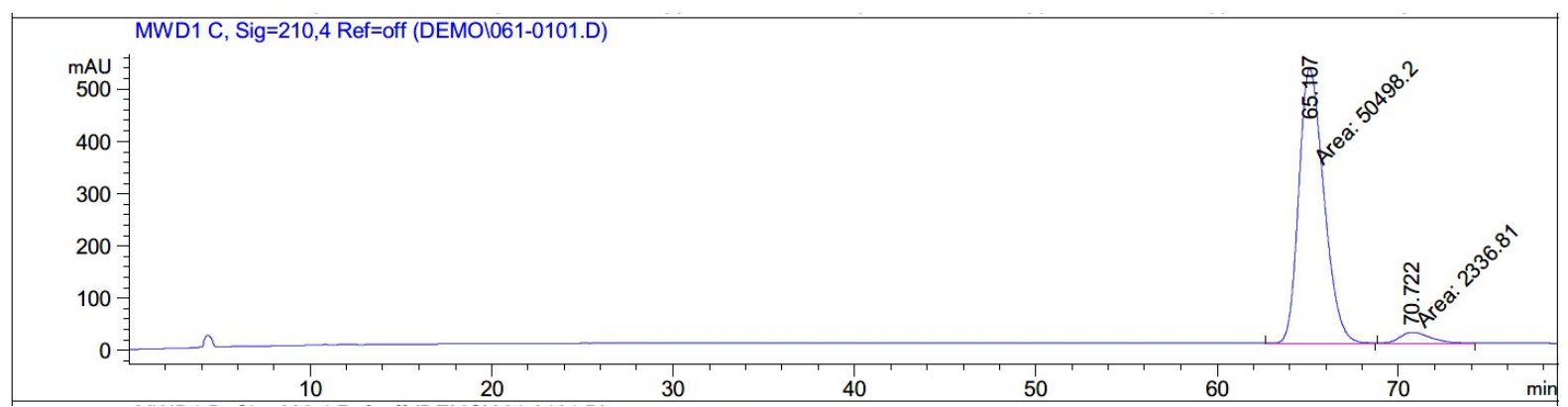

Signal 2: MWD1 C, Sig=210, 4 Ref=off

\begin{tabular}{|c|c|c|c|c|c|c|}
\hline $\begin{array}{c}\text { Peak } \\
\#\end{array}$ & $\begin{array}{c}\text { RetTime } \\
\text { [min] }\end{array}$ & Type & $\begin{array}{l}\text { Width } \\
\text { [min] }\end{array}$ & $\begin{array}{c}\text { Area } \\
{\left[\mathrm{mAU}^{*} \mathrm{~s}\right]}\end{array}$ & $\begin{array}{l}\text { Height } \\
\text { [MAU] }\end{array}$ & $\begin{array}{c}\text { Area } \\
\frac{\circ}{0}\end{array}$ \\
\hline- & --0 & & -- & --- & ---- & $-----\mid$ \\
\hline 1 & 65.107 & MM & 1.6003 & $5.04982 \mathrm{e} 4$ & 525.91980 & 95.5772 \\
\hline 2 & 70.722 & MM & 1.8898 & 2336.81055 & 20.60950 & 4.4228 \\
\hline
\end{tabular}


(R)-3-Hydroxy- $N$-methyl- $N$-phenyl-4-(phenylamino)-4-thioxobutanamide 16.<smiles>CN(C(=O)CC(O)C(=S)Nc1ccccc1)c1ccccc1</smiles>

This compound is novel and was prepared following general procedure $\mathrm{C}$ using $N$-methyl-3oxo- $N$-phenyl-4-(phenylamino)-4-thioxobutanamide (78.0 mg, $0.250 \mathrm{mmol}, 1.0 \mathrm{eq}), 3 \mathrm{C}$ tethered catalyst $(R, R)-2(2.3 \mathrm{mg}, 3.8 \mu \mathrm{mol}, 0.015 \mathrm{eq})$ and FA:TEA $(0.15 \mathrm{~mL})$ in DCM $(1.5$ $\mathrm{mL})$ to give 16 as a brown liquid (68.3 $\mathrm{mg}, 0.217 \mathrm{mmol}, 87 \%)$.

TLC: $\mathrm{R}_{\mathrm{f}}$ ca 0.4 (5:5, hexane: EtOAc), UV-active, Strong $\mathrm{KMnO}_{4}$ and PMA-reactive; HRMS (ESI) m/z: [M+Na] $]^{+}$Calcd for $\mathrm{C}_{17} \mathrm{H}_{18} \mathrm{~N}_{2} \mathrm{O}_{2} \mathrm{SNa} 337.0981$; Found 337.0975 (error 1.9 ppm);

$U_{\max } 3247,2917,1626,1591,1493,1382,1118.1072,692 \mathrm{~cm}^{-1}$;

Enantiomeric excess determined by HPLC analysis (Chiralpak IC, $250 \mathrm{~mm} \times 4.6 \mathrm{~mm}$ column, hexane: $\mathrm{iPrOH} 80: 20,1 \mathrm{~mL} / \mathrm{min}, \lambda=210 \mathrm{~nm}, \mathrm{~T}=25^{\circ} \mathrm{C}$ ), $R$-enantiomer $16.8 \mathrm{~min}, S$ enantiomer $30.4 \min .89 \%$ ee $(R),[\alpha]_{\mathrm{D}}^{22}=+58.3\left(\mathrm{c}=0.06, \mathrm{CHCl}_{3}\right)$;

${ }^{1} \mathrm{H}$ NMR (400 MHz, $\left.\mathrm{CDCl}_{3}\right) \delta 10.76(1 \mathrm{H}, \mathrm{s}, \mathrm{NH}), 7.87(2 \mathrm{H}, \mathrm{d}, J=8.1 \mathrm{~Hz}, \mathrm{ArH}), 7.47$-7.36 (5H, m, ArH), 7.27-7.21 (3H, m, ArH), 5.89 (1H, s, CHOH $), 4.69-4.66$ (1H, m, C $\left(3 \mathrm{H}, \mathrm{s}, \mathrm{NCH}_{3}\right), 2.85-2.84\left(2 \mathrm{H}, \mathrm{m}, \mathrm{CH}_{\mathrm{a}} \mathrm{H}_{\mathrm{b}}\right)$;

${ }^{13} \mathrm{C} \mathrm{NMR}\left(101 \mathrm{MHz}, \mathrm{CDCl}_{3}\right) \delta 200.8,172.8,142.7,138.4,130.2,129.0,128.7,127.2,126.9$, 122.8, 75.4, 39.9, 37.4;

$m / z(\mathrm{ESI}) 337.3\left[(\mathrm{M}+\mathrm{Na})^{+}, 100 \%\right]$. 
3-Hydroxy- $N$-methyl- $N$-phenyl-4-(phenylamino)-4-thioxobutanamide 16.

${ }^{1} \mathrm{H}$ NMR $\left(400 \mathrm{MHz}, \mathrm{CDCl}_{3}\right)$.

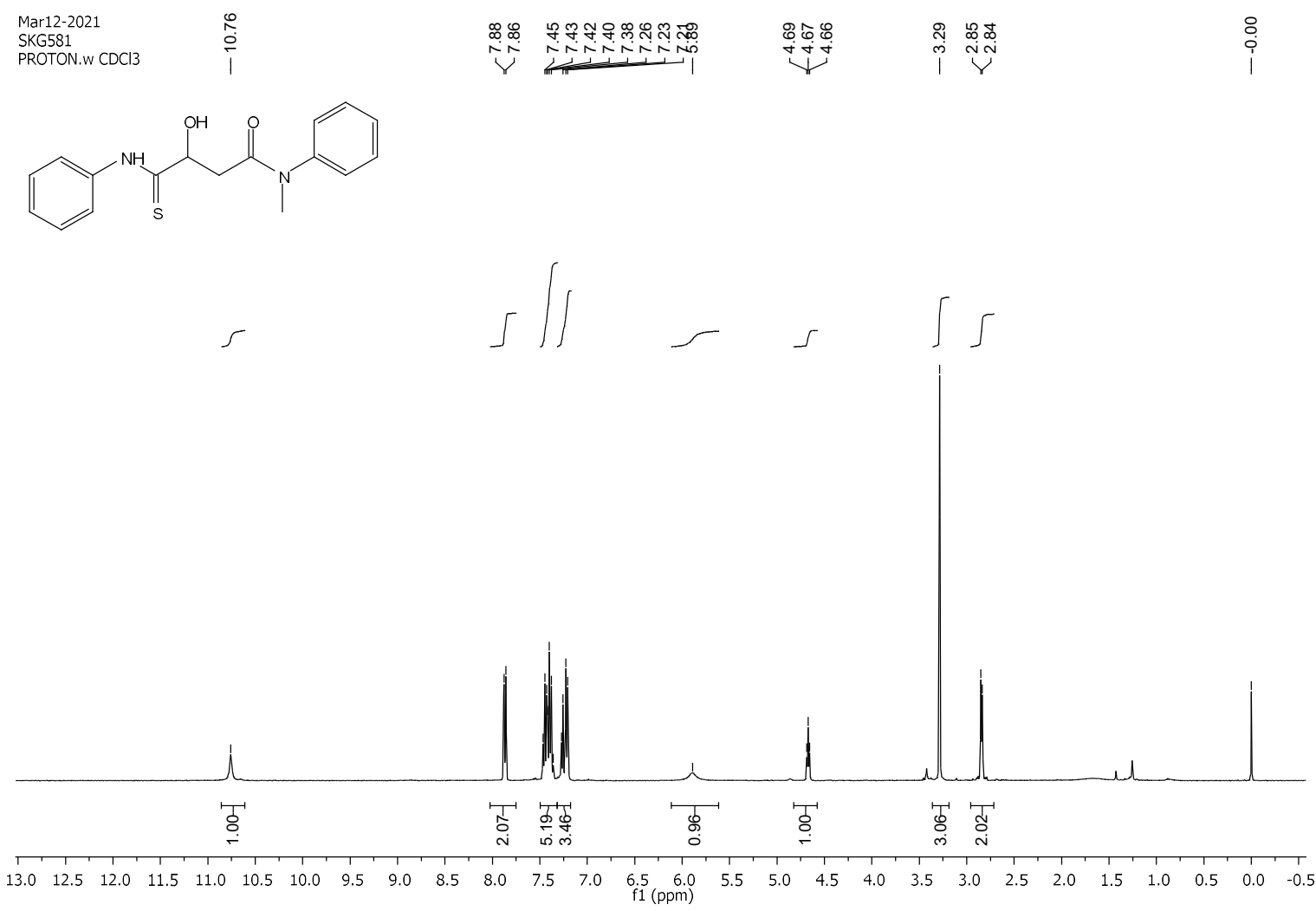

${ }^{13} \mathrm{C}$ NMR (101 MHz, $\left.\mathrm{CDCl}_{3}\right)$.

\begin{tabular}{|c|c|c|c|c|}
\hline $\begin{array}{l}\text { Mar12-2021 } \\
\text { SKG581 } \\
\text { C13APTlong.w CDCI3 }\end{array}$ & $\begin{array}{l}\text { L } \\
\stackrel{2}{0} \\
\stackrel{0}{1} \\
1\end{array}$ & $\stackrel{\infty}{\stackrel{\infty}{\Sigma}}$ & 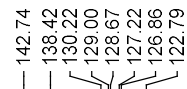 & 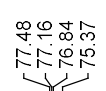 \\
\hline
\end{tabular}<smiles>CN(C(=O)CC(O)C(=S)Nc1ccccc1)c1ccccc1</smiles>

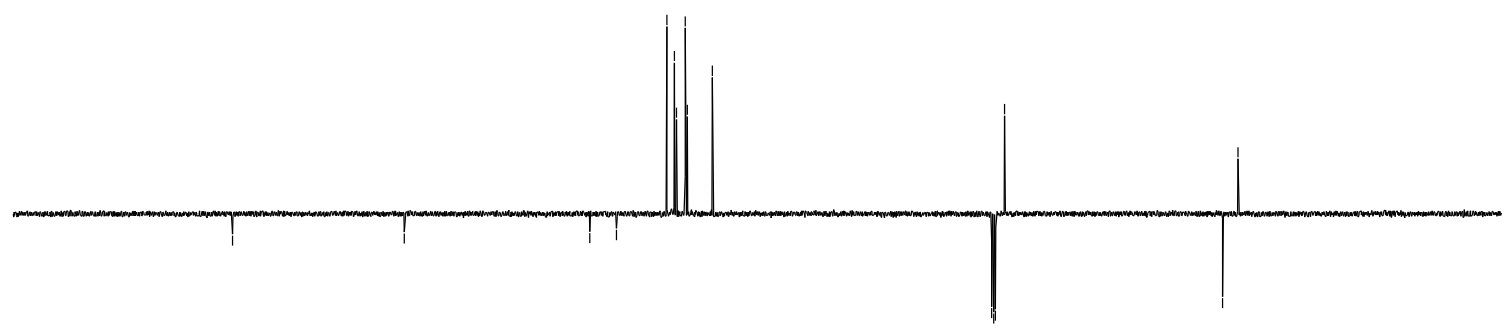

$\begin{array}{llllllllllllllllllllllll}230 & 220 & 210 & 200 & 190 & 180 & 170 & 160 & 150 & 140 & 130 & 120 & 110 & 100 & 90 & 80 & 70 & 60 & 50 & 40 & 30 & 20 & 10 & 0\end{array}$ 
COSY.

SKG581

COSY.w $\mathrm{CDCl} 3$ /opt/topspin3.5pl2 SKG 32

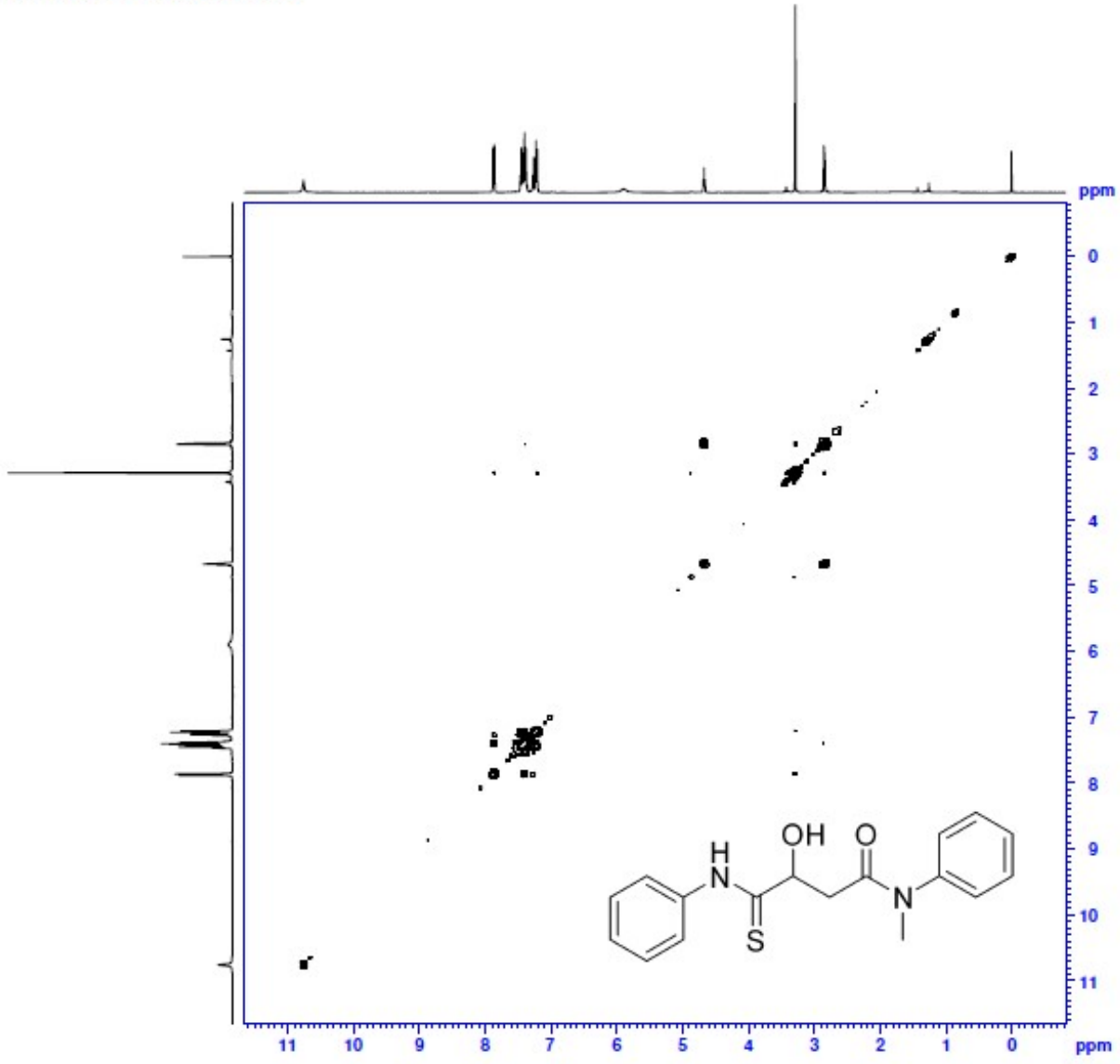

HSQC.

SKG581
HSQC.w CDCl3 /optttopspin3.5pl2 SKG 32

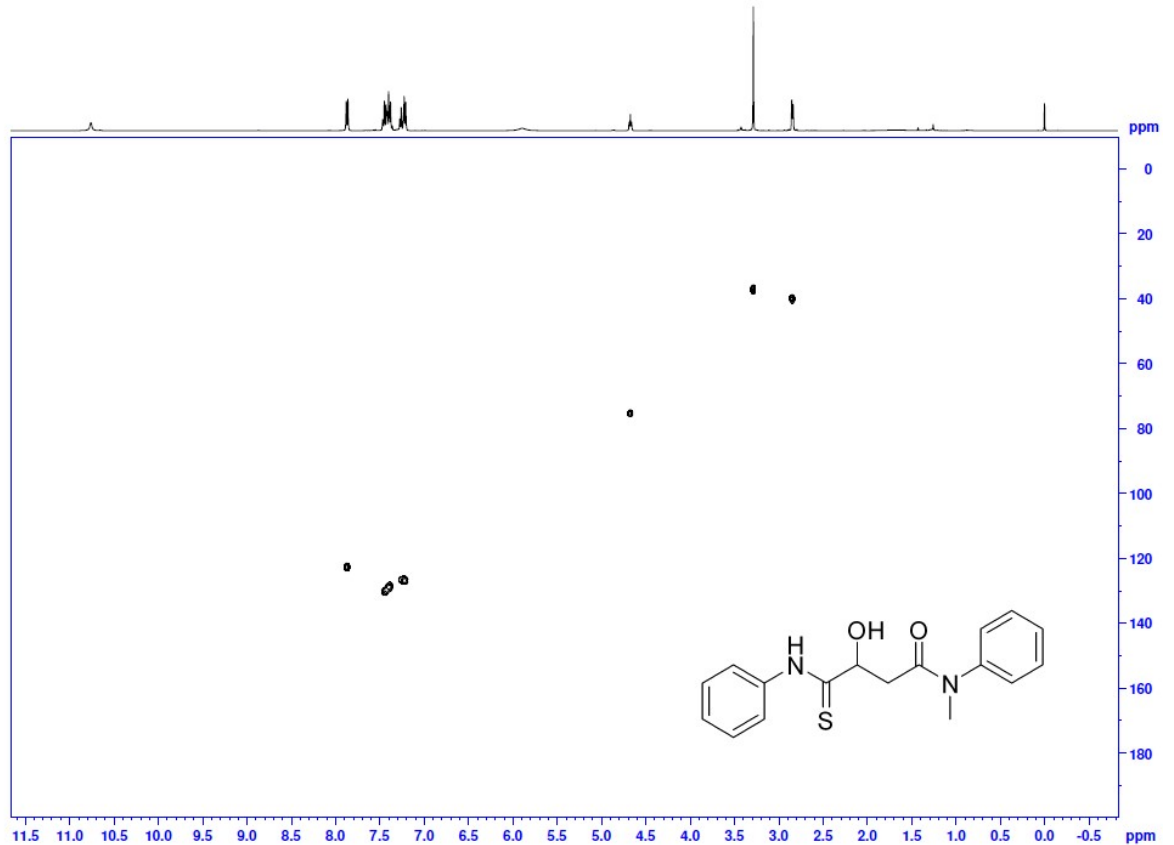




\section{Chiral HPLC of ketone precursor.}

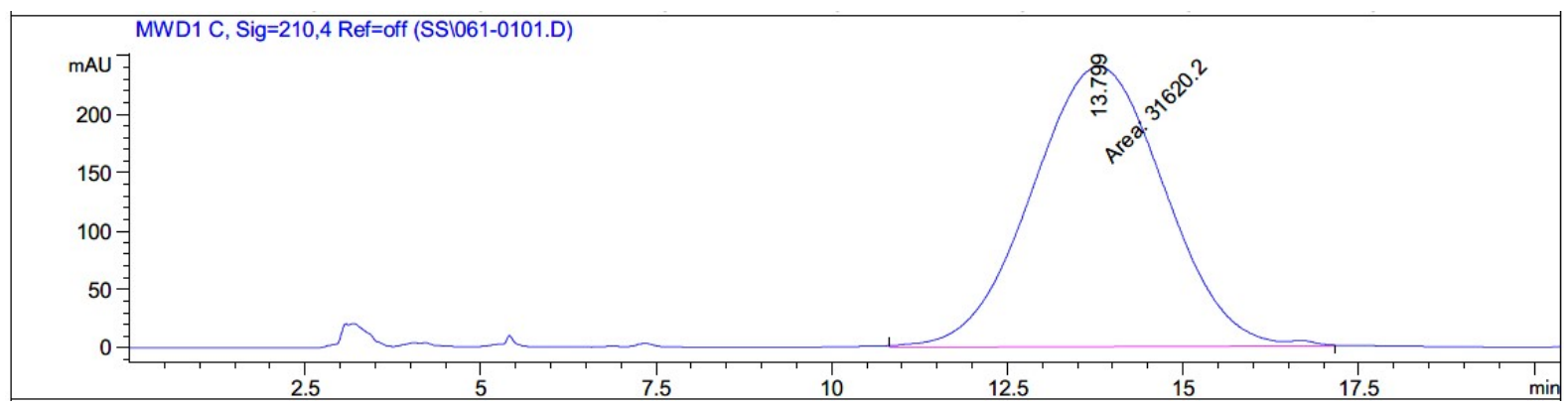

Signal 2: MWD1 C, Sig=210,4 Ref=off

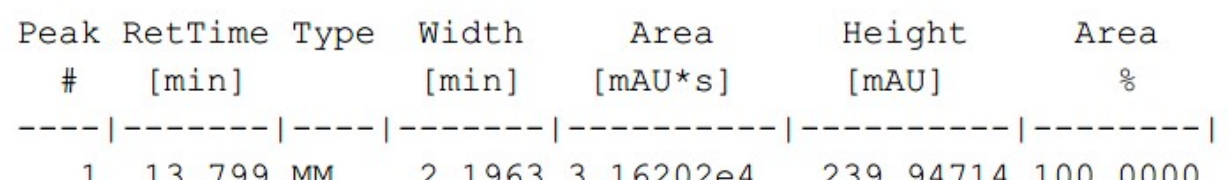

\section{Chiral HPLC of racemic alcohol.}

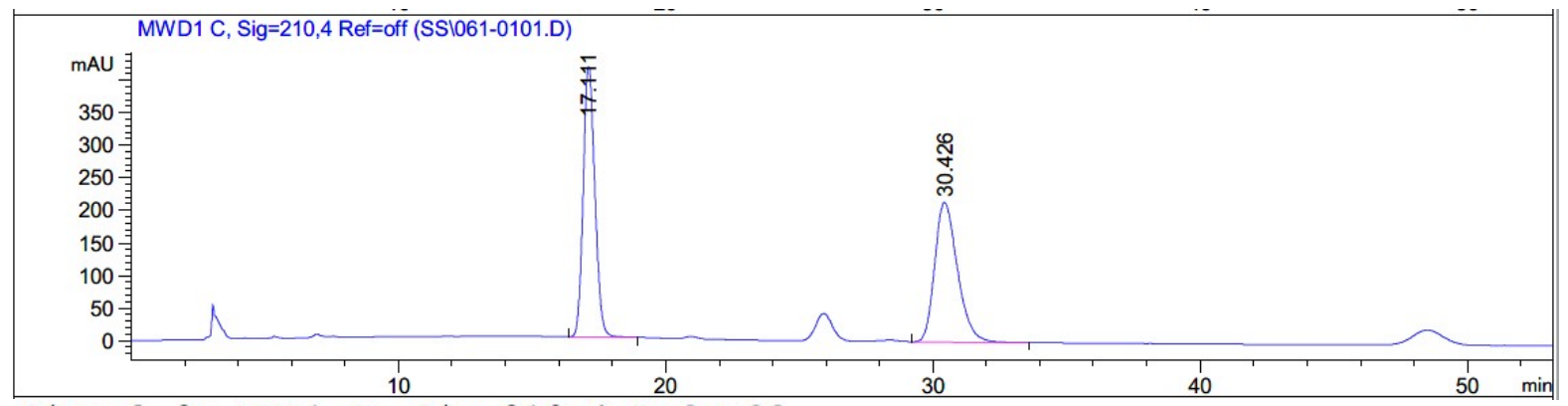

Signal 2: MWD1 C, Sig=210,4 Ref=off

\begin{tabular}{|c|c|c|c|c|c|c|}
\hline $\begin{array}{c}\text { Peak } \\
\#\end{array}$ & $\begin{array}{c}\text { RetTime } \\
{[\mathrm{min}]}\end{array}$ & Type & $\begin{array}{l}\text { Width } \\
\text { [min] }\end{array}$ & $\begin{array}{c}\text { Area } \\
{\left[\mathrm{mAU}{ }^{*} \mathrm{~s}\right]}\end{array}$ & $\begin{array}{l}\text { Height } \\
\text { [MAU] }\end{array}$ & $\begin{array}{c}\text { Area } \\
\frac{\circ}{\partial}\end{array}$ \\
\hline & & & & ---- & -------0 & $-\infty$ \\
\hline 1 & 17.1 & $\mathrm{BB}$ & 0.4719 & $1.26324 \mathrm{e} 4$ & 416.40439 & 49.8968 \\
\hline 2 & 30.426 & $\mathrm{BB}$ & 0.9071 & $1.26846 \mathrm{e} 4$ & 214.76314 & 50.1032 \\
\hline
\end{tabular}


HPLC of ATH product. 89\% ee.

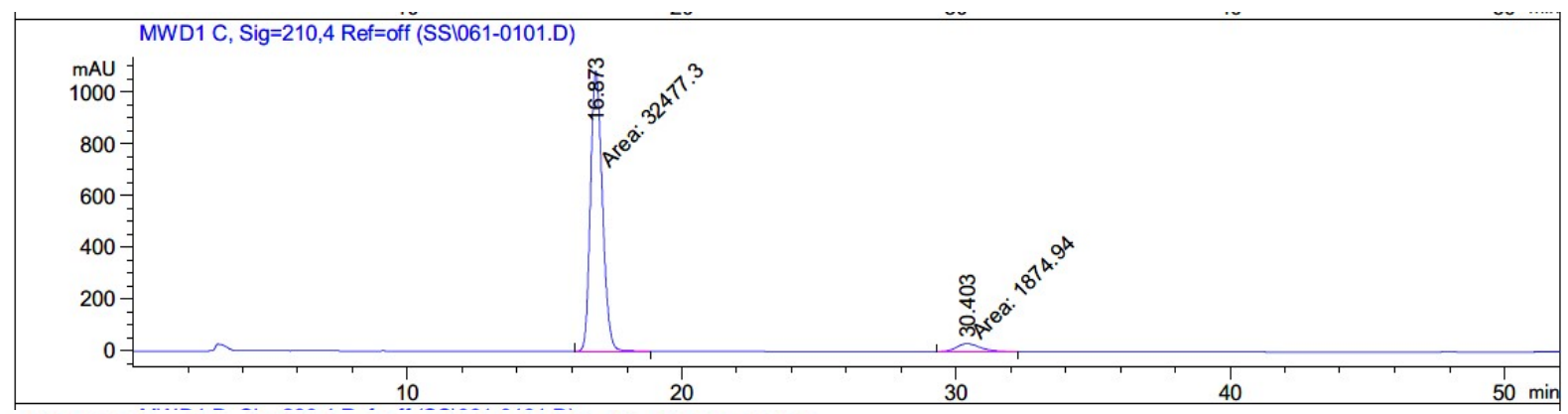

Signal 2: MWD1 C, Sig=210, 4 Ref=off

$\begin{array}{cccccc}\begin{array}{c}\text { Peak RetTime Type } \\ \# \\ \text { [min] }\end{array} & \begin{array}{c}\text { Width } \\ {[\mathrm{min}]}\end{array} & \begin{array}{c}\text { Area } \\ {[\mathrm{mAU} \text { S }]}\end{array} & \begin{array}{c}\text { Height } \\ {[\mathrm{mAU}]}\end{array} & \begin{array}{c}\text { Area } \\ \%\end{array} \\ ---|------|----\mid & -------|---------|---------\mid & -------\mid \\ 1 & 16.873 \mathrm{MM} & 0.4995 & 3.24773 e 4 & 1083.65210 & 94.5420 \\ 2 & 30.403 \mathrm{MM} & 1.0328 & 1874.94153 & 30.25796 & 5.4580\end{array}$




\section{2-Hydroxy- $N^{1}, N^{5}$-dimethyl- $N^{1}, N^{5}$-diphenylpentanediamide 17.}<smiles>CN(C(=O)CCC(O)C(=O)N(C)c1ccccc1)c1ccccc1</smiles>

This compound is novel and was prepared following general procedure $\mathrm{C}$ using $N^{1}, N^{5}$ dimethyl-2-oxo- $N^{1}, N^{5}$-diphenylpentanediamide ( $\left.81.0 \mathrm{mg}, 0.25 \mathrm{mmol}, 1.0 \mathrm{eq}\right), 3 \mathrm{C}$ tethered catalyst $(R, R)-2(2.3 \mathrm{mg}, 3.8 \mu \mathrm{mol}, 0.015 \mathrm{eq})$ and FA:TEA $(0.15 \mathrm{~mL})$ in DCM $(1.5 \mathrm{~mL})$ to give 17 as a clear oil (74.3 $\mathrm{mg}, 0.228 \mathrm{mmol}, 91 \%)$.

TLC: $\mathrm{R}_{\mathrm{f}}$ ca 0.2 (6:4, hexane: EtOAc), UV-active, Strong $\mathrm{KMnO}_{4}$ and PMA-reactive; HRMS (ESI) m/z: [M+Na] $]^{+}$Calcd for $\mathrm{C}_{19} \mathrm{H}_{22} \mathrm{~N}_{2} \mathrm{O}_{3} \mathrm{Na}$ 349.1523; Found 349.1522 (error 0.1 ppm);

$U_{\max } 3441,2917,1649,1632,1592,1494,1419,1361,1279,1087,775,699 \mathrm{~cm}^{-1}$;

Enantiomeric excess determined by HPLC analysis (Chiralpak IG, $250 \mathrm{~mm} \times 4.6 \mathrm{~mm}$ column, hexane: $\operatorname{PrOH} 75: 25,1 \mathrm{~mL} / \mathrm{min}, \lambda=210 \mathrm{~nm}, \mathrm{~T}=25^{\circ} \mathrm{C}$ ), $42.8 \mathrm{~min}$ and $48.0 \mathrm{~min}, 16 \%$ ee;

${ }^{1} \mathrm{H}$ NMR (500 MHz, $\left.\mathrm{CDCl}_{3}\right) \delta$ 7.38-7.24 (6H, m, ArH), 7.20-7.16 (2H, m, ArH), 7.06-7.05 $(2 \mathrm{H}, \mathrm{m}, \mathrm{ArH}), 3.99(1 \mathrm{H}, \mathrm{s}, \mathrm{C} \underline{\mathrm{HOH}}), 3.20\left(3 \mathrm{H}, \mathrm{s}, \mathrm{NCH}_{3}\right), 3.13\left(3 \mathrm{H}, \mathrm{s}, \mathrm{NCH}_{3}\right), 2.09-2.03(1 \mathrm{H}$, $\left.\mathrm{m}, \mathrm{C}_{\mathrm{a}} \mathrm{H}_{\mathrm{b}}\right), 1.88-1.83\left(1 \mathrm{H}, \mathrm{m}, \mathrm{CH}_{\mathrm{a}} \underline{\mathrm{H}_{\mathrm{b}}}\right), 1.60-1.59\left(2 \mathrm{H}, \mathrm{m}, \mathrm{CH}_{2}\right)(\mathrm{OH}$ not observed or obscured);

${ }^{13} \mathrm{C}$ NMR $\left(126 \mathrm{MHz}, \mathrm{CDCl}_{3}\right) \delta 174.3,172.4,144.1,142.3,130.1,129.9,128.4,127.9,127.6$, 127.4, 67.6, 38.2, 37.4, 30.0, 29.4;

$\mathrm{m} / z(\mathrm{ESI}) 349.1\left[(\mathrm{M}+\mathrm{Na})^{+}, 100 \%\right]$. 
2-Hydroxy- $N^{1}, N^{5}$-dimethyl- $N^{1}, N^{5}$-diphenylpentanediamide 17.

${ }^{1} \mathrm{H}$ NMR $\left(500 \mathrm{MHz}, \mathrm{CDCl}_{3}\right)$.

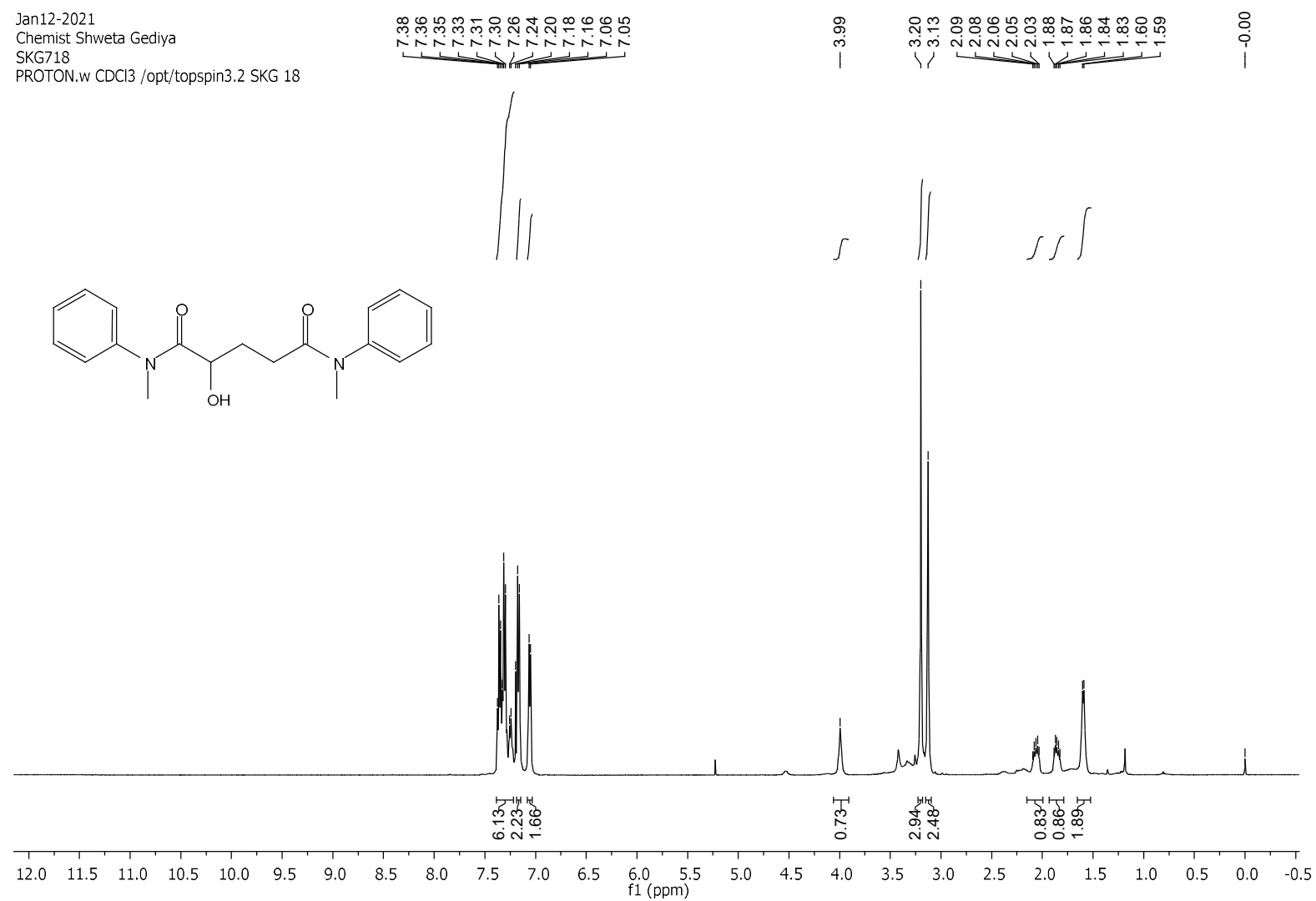

${ }^{13} \mathrm{C}$ NMR (126 MHz, $\left.\mathrm{CDCl}_{3}\right)$.

\begin{tabular}{|c|c|c|c|c|}
\hline $\begin{array}{l}\text { Jan12-2021 } \\
\text { Shweta Gediya } \\
\text { SKG718 } \\
\text { C13APTlong.w CDCI3 }\end{array}$ & 龒导 & 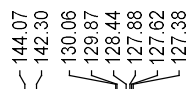 & 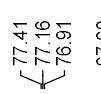 & 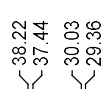 \\
\hline
\end{tabular}

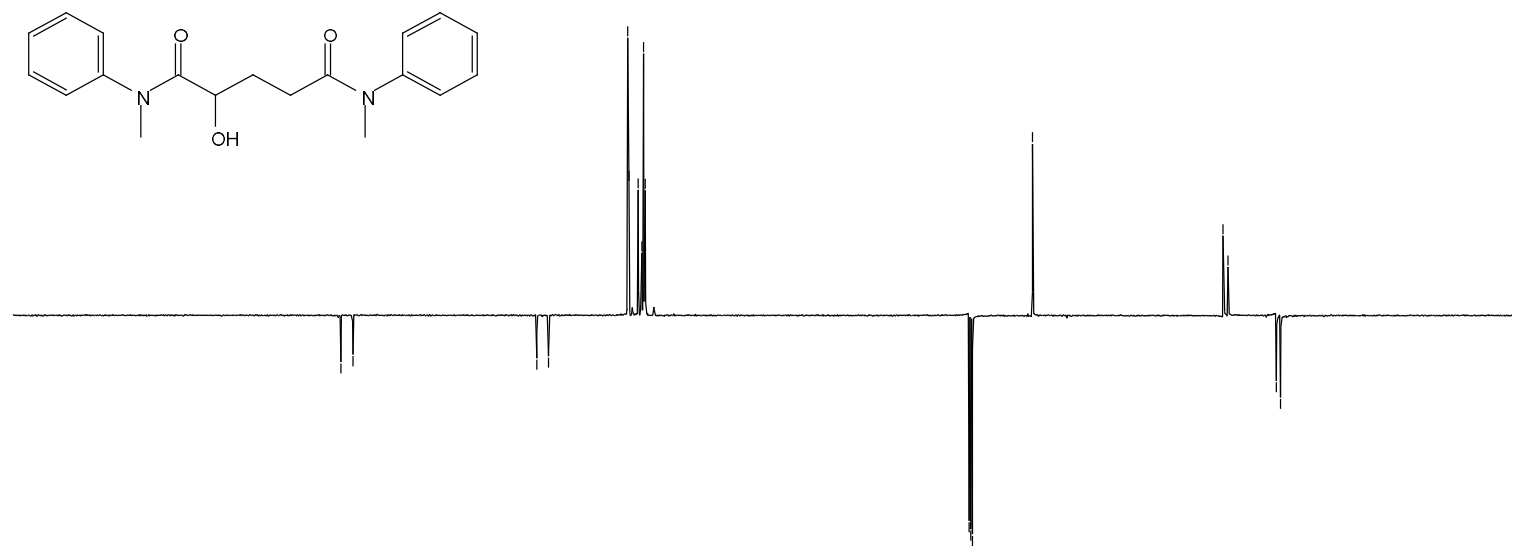

$\begin{array}{llllllllllllllllllllllllllllllllllllllllllll}220 & 210 & 200 & 190 & 180 & 170 & 160 & 150 & 140 & 130 & 120 & 110 & 100 & 90 & 80 & 70 & 60 & 50 & 40 & 30 & 20 & 10 & 0\end{array}$ 
COSY.

Chemist Shweta Gediya
SKG718

SKG718
COSY.w CDCl3/opt/topspin3.2 SKG 18

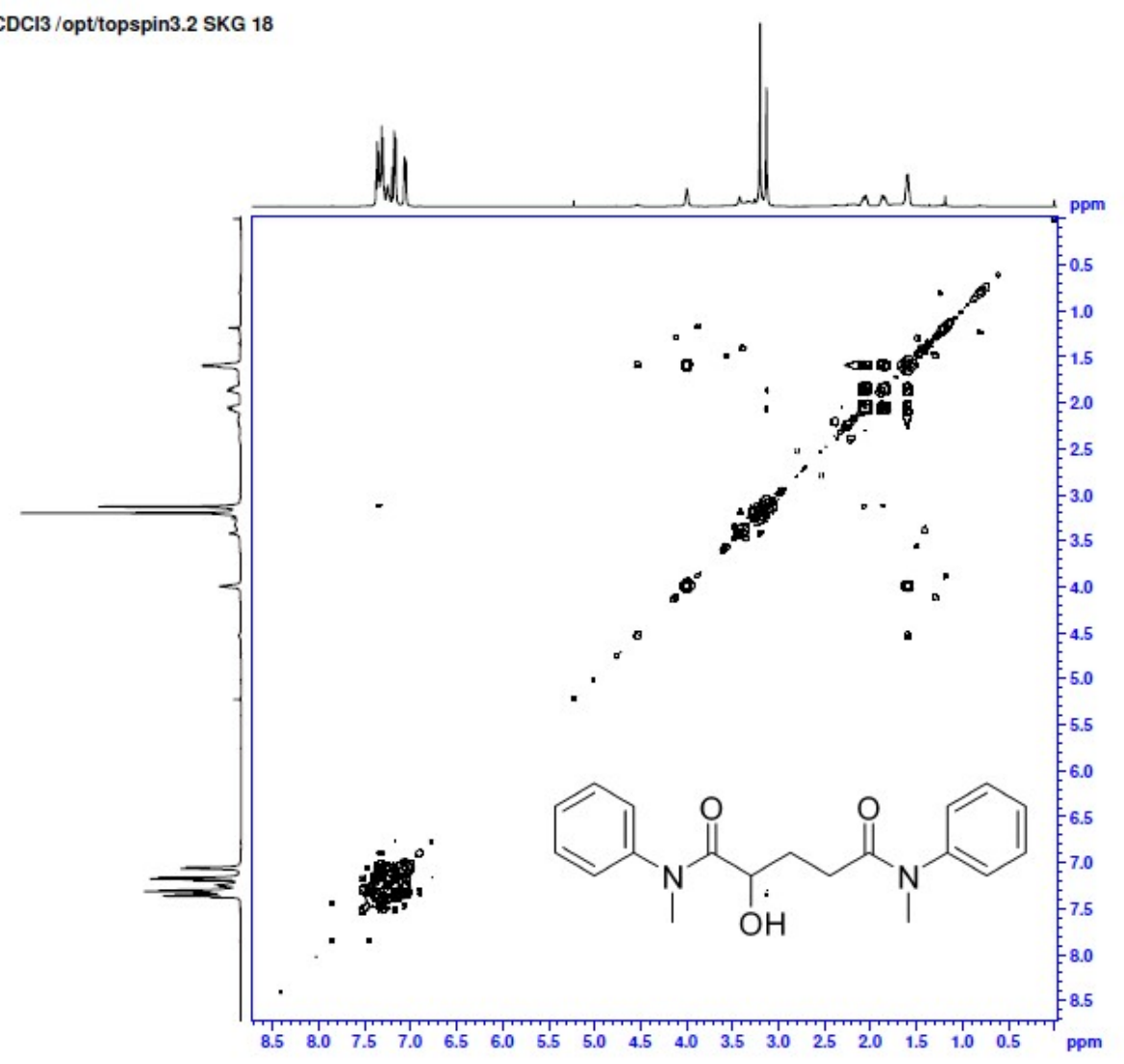

HSQC.

Chemist Shweta Gediya

HSQC.w $\mathrm{CDCl}$ /opt/topspin3.2 SKG 18

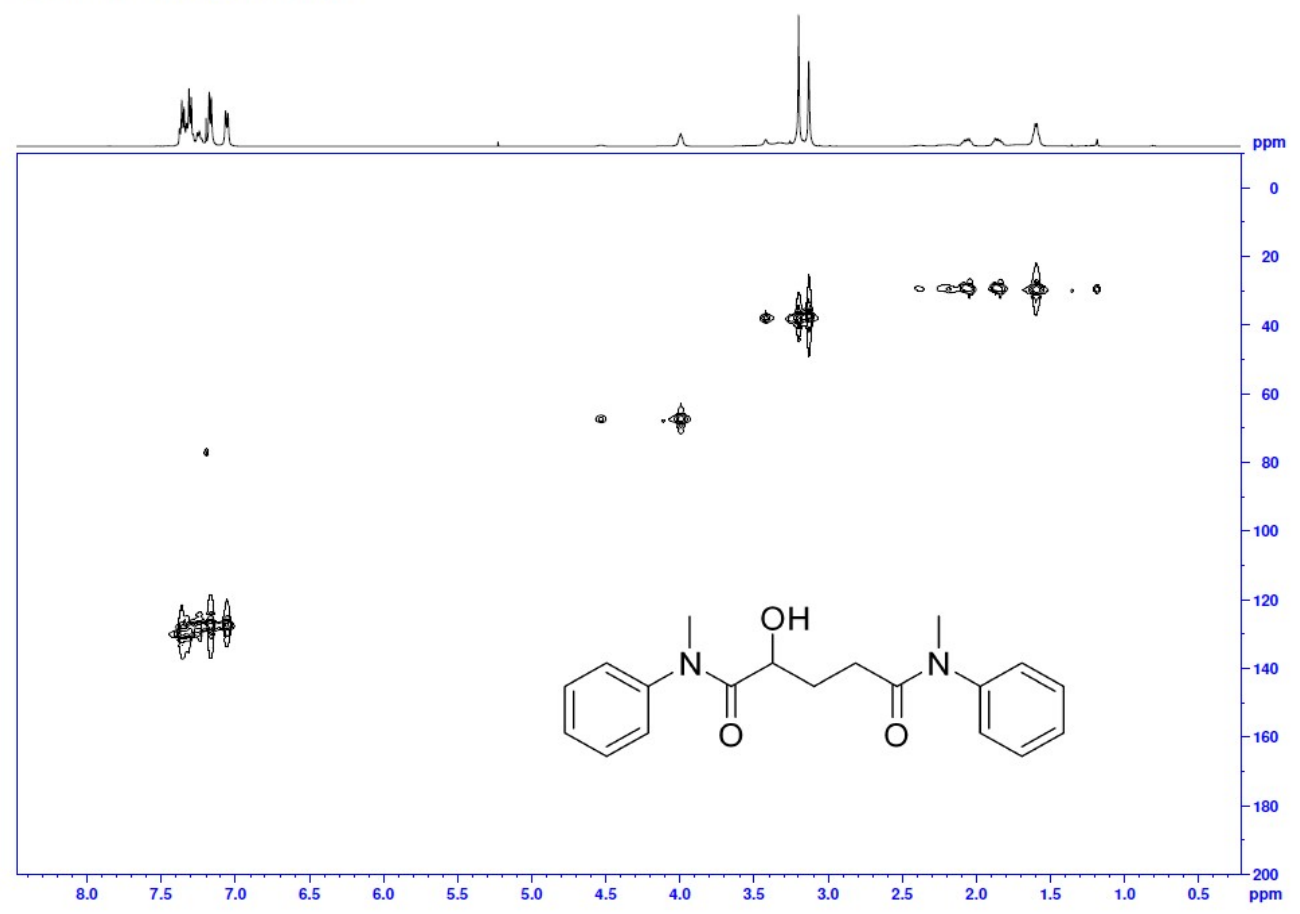

S257 


\section{Chiral HPLC of ketone precursor.}

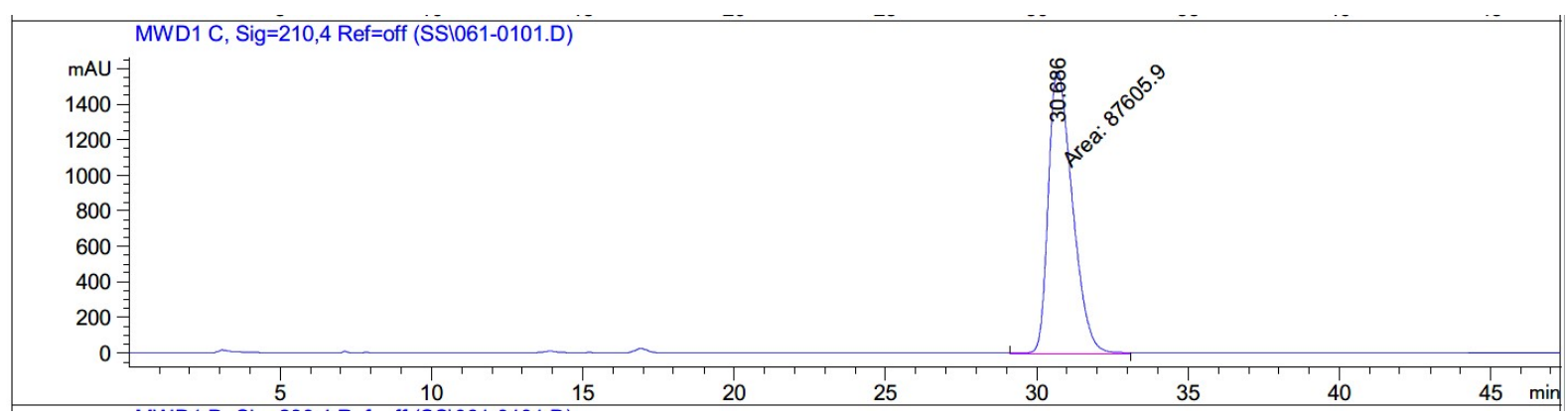

Signal 2: MWD1 C, Sig=210,4 Ref=off

\begin{tabular}{|c|c|c|c|c|c|c|}
\hline $\begin{array}{c}\text { Peak } \\
\#\end{array}$ & $\begin{array}{c}\text { RetTime } \\
\text { [min] }\end{array}$ & Type & $\begin{array}{l}\text { Width } \\
\text { [min] }\end{array}$ & $\begin{array}{c}\text { Area } \\
{\left[\mathrm{mAU}^{\star} \mathrm{s}\right]}\end{array}$ & $\begin{array}{l}\text { Height } \\
\text { [mAU] }\end{array}$ & $\begin{array}{c}\text { Area } \\
\quad \%\end{array}$ \\
\hline 1 & & $\mathrm{IM}$ & & $.76059 \mathrm{e}$ & 158 & \\
\hline
\end{tabular}


Chiral HPLC of racemic alcohol.

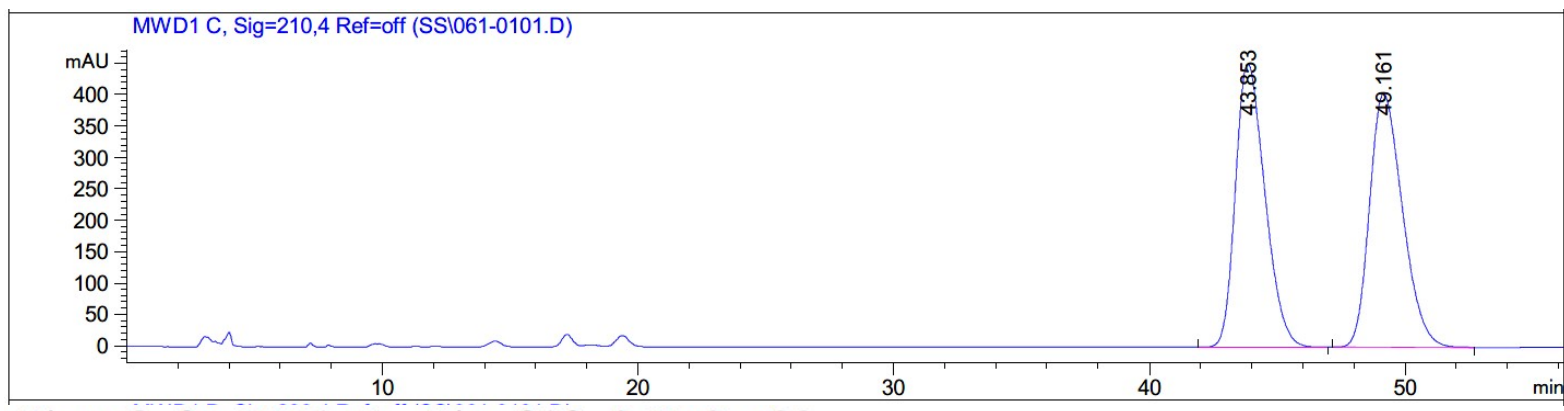

Signal 2: MWD1 C, Sig=210,4 Ref=off

\begin{tabular}{|c|c|c|c|c|c|c|}
\hline $\begin{array}{c}\text { Peak } \\
\quad \#\end{array}$ & $\begin{array}{c}\text { RetTime } \\
\text { [min] }\end{array}$ & Type & $\begin{array}{l}\text { Width } \\
{[\mathrm{min}]}\end{array}$ & $\begin{array}{c}\text { Area } \\
{\left[m A U^{\star} s\right]}\end{array}$ & $\begin{array}{l}\text { Height } \\
{[\mathrm{mAU}]}\end{array}$ & $\begin{array}{c}\text { Area } \\
\frac{\circ}{0}\end{array}$ \\
\hline & & & & -1 & - & -- \\
\hline 1 & 43.853 & BB & 25 & $3.62050 \mathrm{e} 4$ & 451.03693 & 9961 \\
\hline 2 & 49.161 & BB & 1.3640 & $3.62107 e 4$ & 403.86896 & 50.0039 \\
\hline
\end{tabular}

\section{Chiral HPLC of ATH product $16 \%$ ee.}

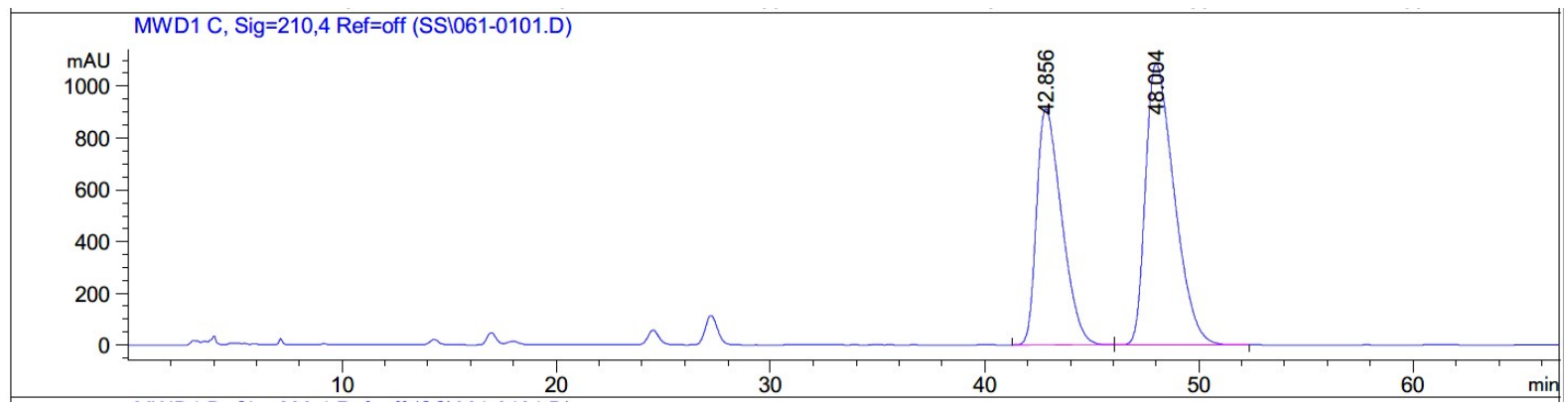

Signal 2: MWD1 C, Sig=210,4 Ref=off

\begin{tabular}{|c|c|c|c|c|c|c|}
\hline $\begin{array}{c}\text { Peak } \\
\text { \# }\end{array}$ & $\begin{array}{c}\text { RetTime } \\
\text { [min] }\end{array}$ & Type & $\begin{array}{l}\text { Width } \\
{[\mathrm{min}]}\end{array}$ & $\begin{array}{c}\text { Area } \\
{\left[\mathrm{mAU}^{\star} \mathrm{s}\right]}\end{array}$ & $\begin{array}{l}\text { Height } \\
\text { [mAU] }\end{array}$ & $\begin{array}{c}\text { Area } \\
\frac{\circ}{0}\end{array}$ \\
\hline \multicolumn{7}{|c|}{$----|-------|----|-------|----------|----------|--------\mid$} \\
\hline 1 & 42.856 & $\mathrm{BB}$ & 1.2484 & $7.41211 e 4$ & 911.79681 & 42.2548 \\
\hline 2 & 48.004 & BB & 1.4271 & $1.01294 \mathrm{e} 5$ & 1085.06750 & 57.7452 \\
\hline
\end{tabular}




\section{(R)-2 Hydroxy- $N^{1}, N^{4}$-diphenylsuccinamide 18.}<smiles>O=C(CC(O)C(=O)Nc1ccccc1)Nc1ccccc1</smiles>

This compound is novel and was prepared following general procedure $\mathrm{C}$ using 2-oxo- $N^{1}, N^{4}$ diphenylsuccinamide $(70.5 \mathrm{mg}, 0.250 \mathrm{mmol}, 1.0 \mathrm{eq}), 3 \mathrm{C}$ tethered catalyst $(R, R)-2(2.3 \mathrm{mg}$, $3.8 \mu \mathrm{mol}, 0.015 \mathrm{eq})$ and FA:TEA $(0.15 \mathrm{~mL})$ in DCM $(1.5 \mathrm{~mL})$ to give $\mathbf{1 8}$ as a white solid $(63.1 \mathrm{~g}, 0.222 \mathrm{mmol}, 89 \%)$.

TLC: $\mathrm{R}_{\mathrm{f}}$ ca 0.3 (4:6, hexane: EtOAc), UV-active, Strong $\mathrm{KMnO}_{4}$ and PMA-reactive; MP: $199-201^{\circ} \mathrm{C}$;

HRMS (ESI) m/z: [M+Na] $]^{+}$Calcd for $\mathrm{C}_{16} \mathrm{H}_{16} \mathrm{~N}_{2} \mathrm{O}_{3} \mathrm{Na}$ 307.1053; Found 307.1051 (error 0.6 ppm);

$U_{\max } 3346,3284,2973,1640,1594,1542,1443,1305,1250,1079,751,691 \mathrm{~cm}^{-1}$;

Enantiomeric excess determined by HPLC analysis (Chiralpak IC, $250 \mathrm{~mm} \times 4.6 \mathrm{~mm}$ column, hexane: $\mathrm{iPrOH} 75: 25,1 \mathrm{~mL} / \mathrm{min}, \lambda=210 \mathrm{~nm}, \mathrm{~T}=25^{\circ} \mathrm{C}$ ), $S$-enantiomer $6.6 \mathrm{~min}, R$-enantiomer $7.3 \min .95 \%$ ee $(R),[\alpha]_{\mathrm{D}}^{22}=+77.5(\mathrm{c}=0.04, \mathrm{MeOH})$;

${ }^{1} \mathrm{H}$ NMR (500 MHz, DMSO-d $\left.d_{6}\right) \delta 9.98(1 \mathrm{H}, \mathrm{s}, \mathrm{CONH}), 9.79(1 \mathrm{H}, \mathrm{s}, \mathrm{CONH}), 7.72(2 \mathrm{H}, \mathrm{d}, J=$ $7.8 \mathrm{~Hz}, \mathrm{ArH}), 7.62(2 \mathrm{H}, \mathrm{d}, J=7.8 \mathrm{~Hz}, \mathrm{ArH}), 7.32-7.29$ (4H, m, ArH), 7.08-7.02 (2H, m, ArH), $6.02(1 \mathrm{H}, \mathrm{d}, J=6.0 \mathrm{~Hz}, \mathrm{CHO} \underline{\mathrm{H}}), 4.55-4.52(1 \mathrm{H}, \mathrm{m}, \mathrm{C} \underline{\mathrm{HOH}}), 2.80$ (1H, dd, $J=14.7$, $\left.4.0 \mathrm{~Hz}, \mathrm{CH}_{\mathrm{a}} \mathrm{H}_{\mathrm{b}}\right), 2.64\left(1 \mathrm{H}, \mathrm{dd}, J=14.7,8.8 \mathrm{~Hz}, \mathrm{CH}_{\mathrm{a}} \underline{\mathrm{H}}_{\mathrm{b}}\right)$;

${ }^{13} \mathrm{C}$ NMR (126 MHz, DMSO-d6) $\delta 172.2,168.7,139.2$, 138.6, 128.7, 128.6, 123.5, 123.1, 119.7, 119.6, 68.9, 41.8; $m / z(\mathrm{ESI}) 307.1\left[(\mathrm{M}+\mathrm{Na})^{+}, 100 \%\right]$. 
2-Hydroxy- $N^{1}, N^{4}$-diphenylsuccinamide 18.

${ }^{1} \mathrm{H}$ NMR $\left(500 \mathrm{MHz}, \mathrm{DMSO}-d_{6}\right)$.

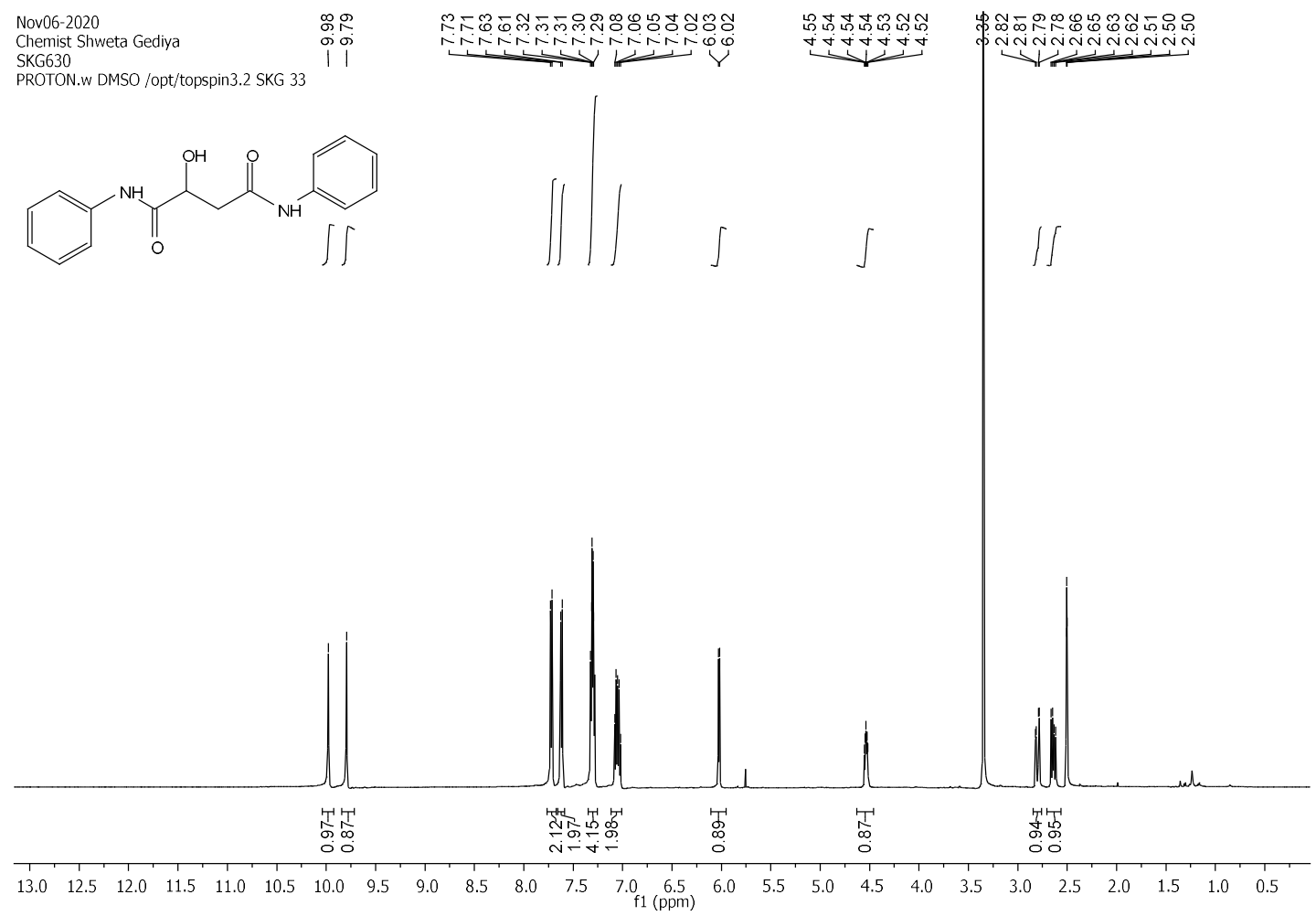

${ }^{13} \mathrm{C}$ NMR $\left(126 \mathrm{MHz}, \mathrm{DMSO}-d_{6}\right)$.

\begin{tabular}{|c|}
\hline $\begin{array}{l}\text { Nov06-2020 } \\
\text { Shweta Gediya } \\
\text { SKG630 } \\
\text { C13APTlong.w DMSO }\end{array}$ \\
\hline
\end{tabular}<smiles>O=C(CC(O)C(=O)Nc1ccccc1)Nc1ccccc1</smiles>

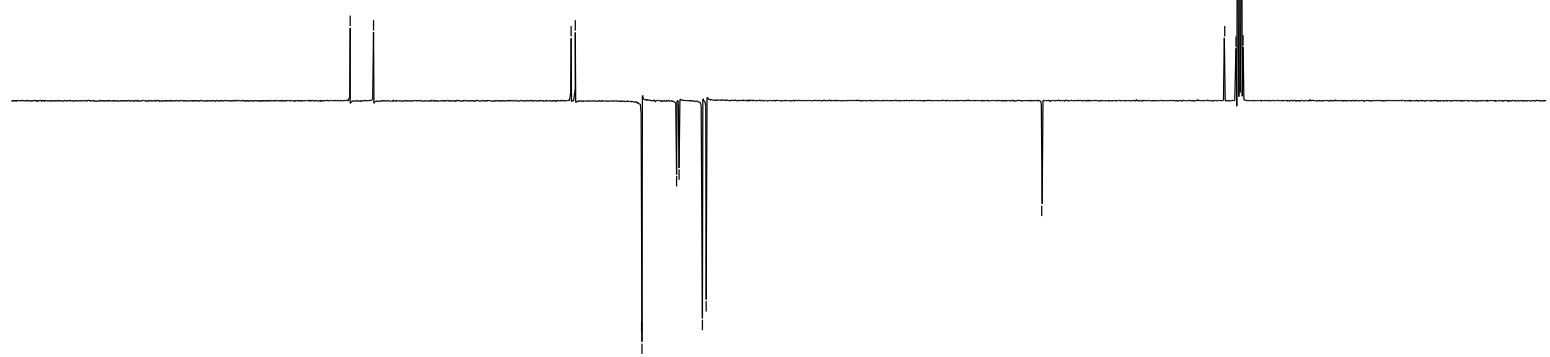

$\begin{array}{lllllllllllllllllllllllllllllllll}220 & 210 & 200 & 190 & 180 & 170 & 160 & 150 & 140 & 130 & 120 & 110 & 100 & 90 & 80 & 70 & 60 & 50 & 40 & 30 & 20 & 10 & 0\end{array}$ 
COSY.

SKG630
COSY.w DMSO /opt/topspin3.5pl2 SKG 6

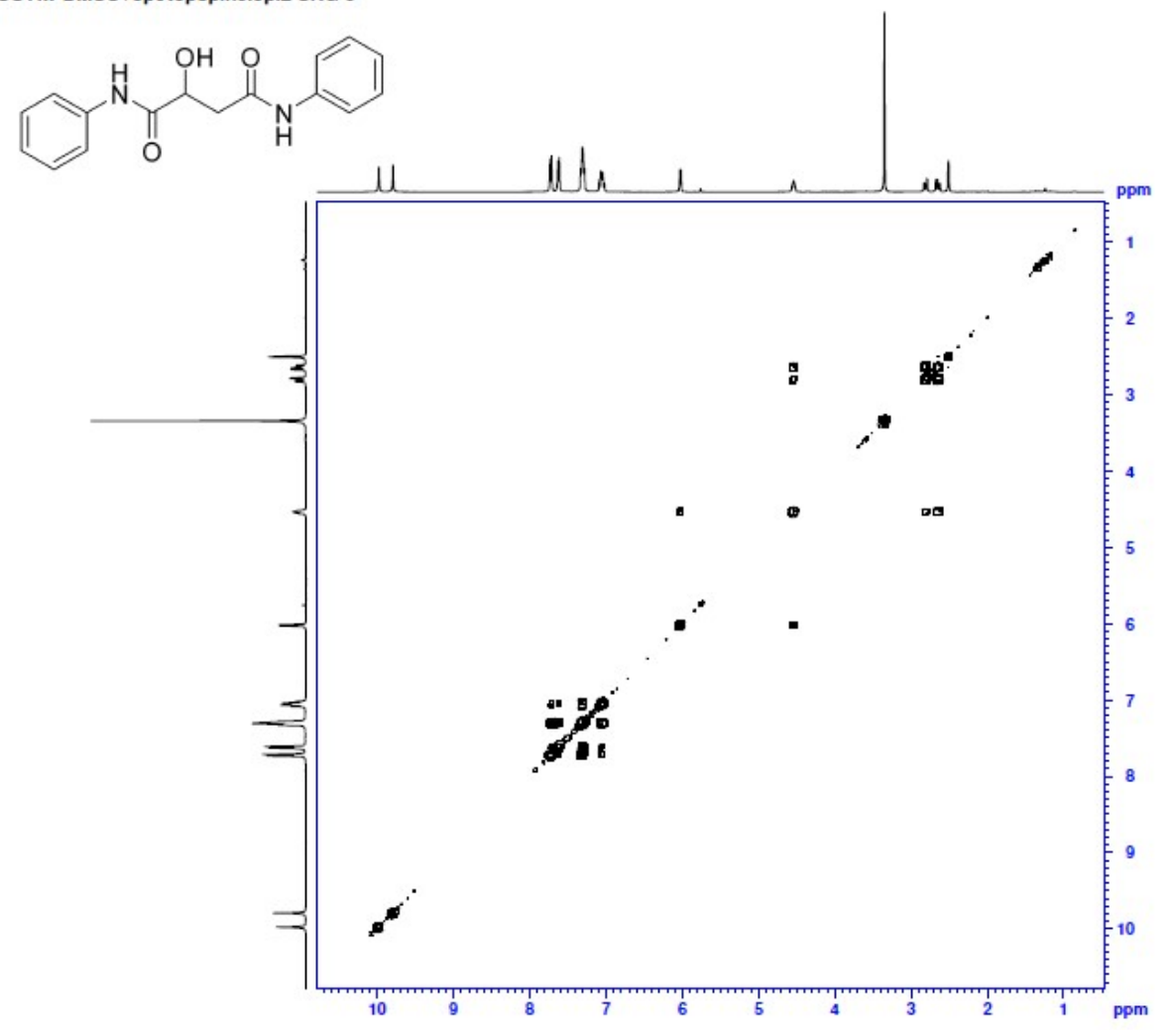

HSQC.

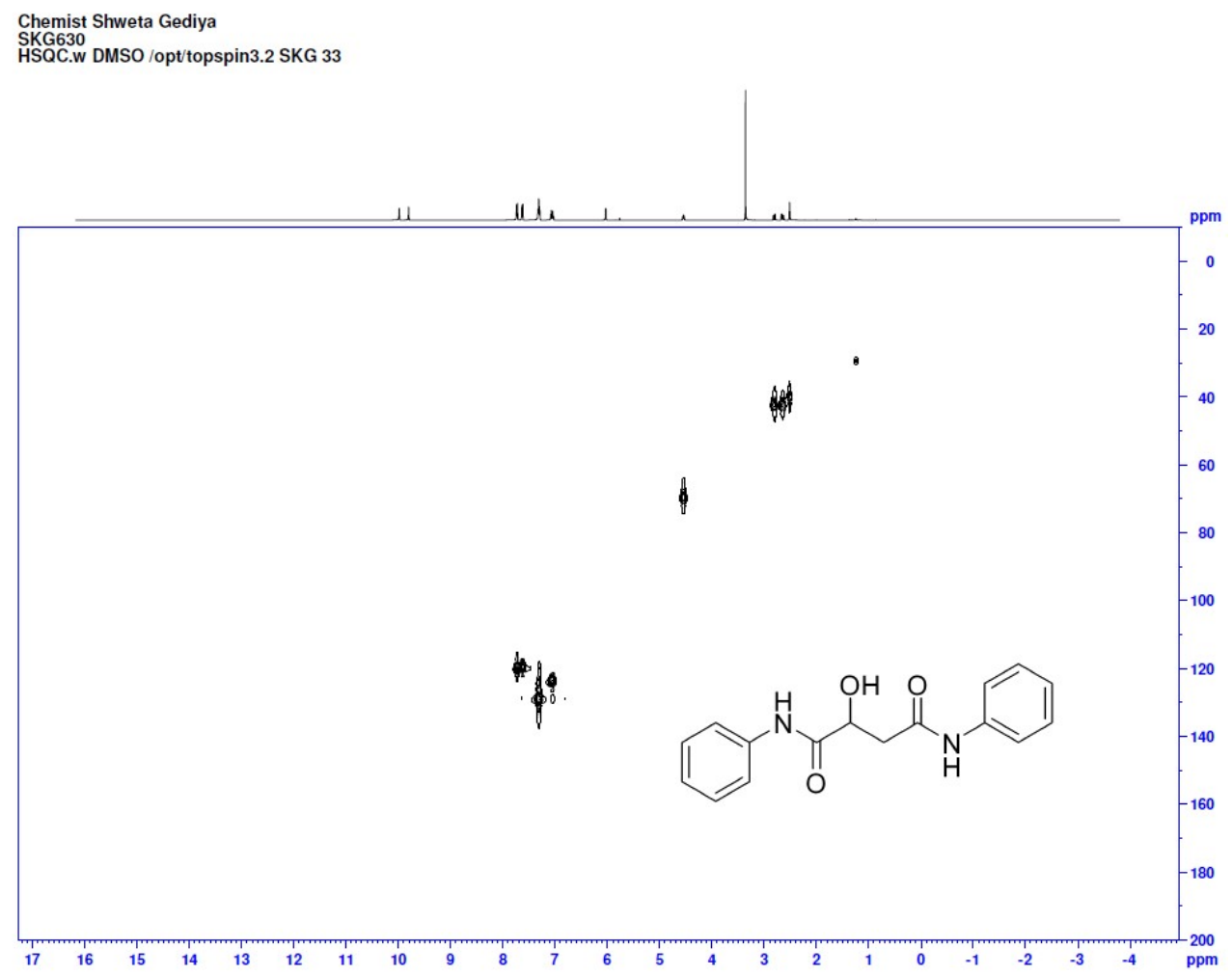

S262 


\section{Chiral HPLC of ketone precursor.}

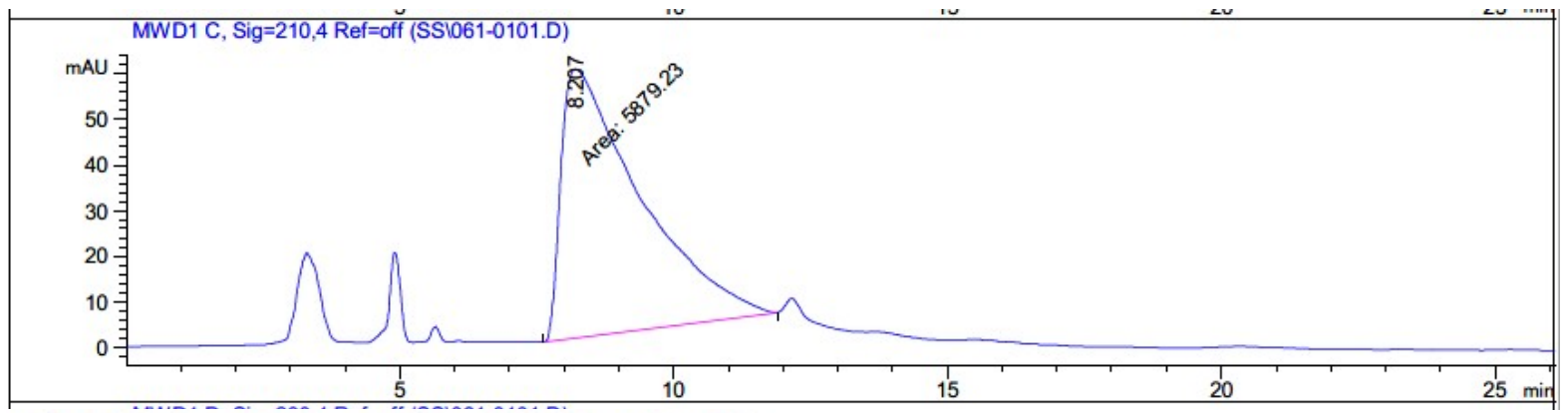

Signal 2: MWD1 C, Sig=210,4 Ref=off

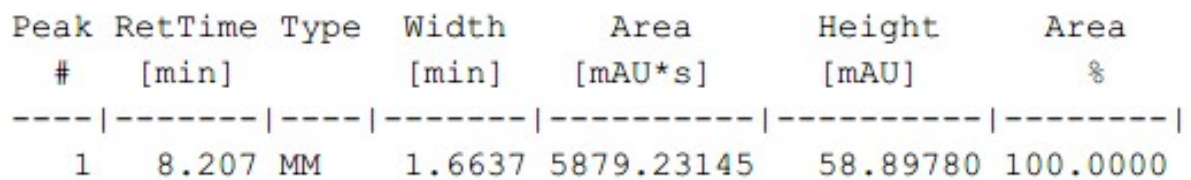

Chiral HPLC of racemic alcohol.

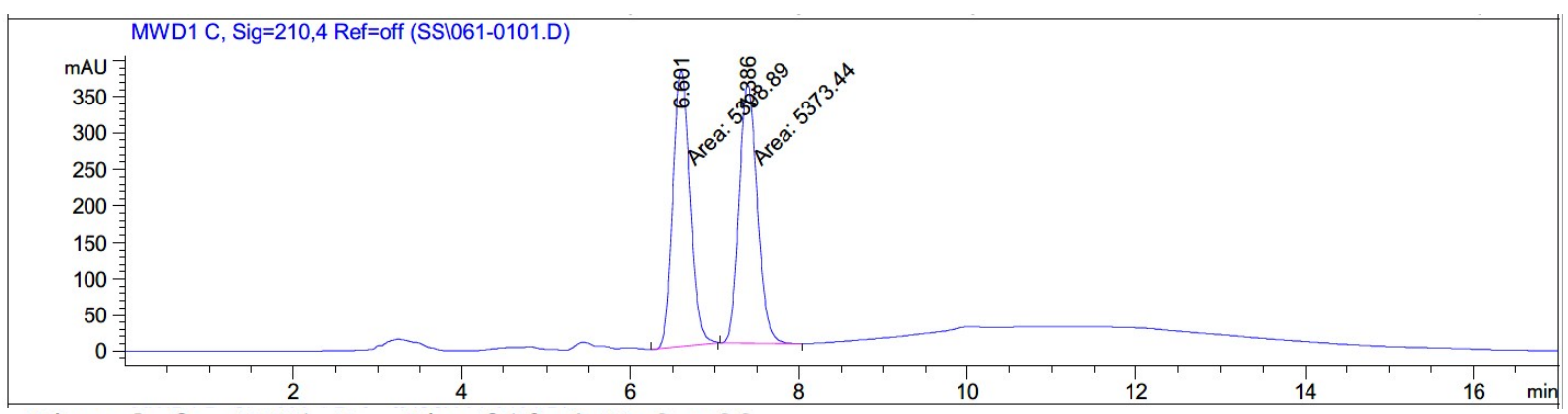

Signal 2: MWD1 C, Sig=210,4 Ref=off

\begin{tabular}{|c|c|c|c|c|c|c|}
\hline $\begin{array}{c}\text { eak } \\
\#\end{array}$ & $\begin{array}{c}\text { RetTime } \\
\text { [min] }\end{array}$ & Type & $\begin{array}{l}\text { Width } \\
\text { [min] }\end{array}$ & $\begin{array}{c}\text { Area } \\
{\left[\mathrm{mAU}{ }^{\star} \mathrm{S}\right]}\end{array}$ & $\begin{array}{l}\text { Height } \\
{[\mathrm{mAU}]}\end{array}$ & $\begin{array}{c}\text { Area } \\
\quad \%\end{array}$ \\
\hline & $----n$ & & & ---- & --------- & ---1 \\
\hline 1 & 6 & & , & 5398.89160 & 984 & 81 \\
\hline 2 & 7.386 & M & 517 & 5373.44141 & 355.82620 & 3819 \\
\hline
\end{tabular}


Chiral HPLC of ATH product. 95\% ee.

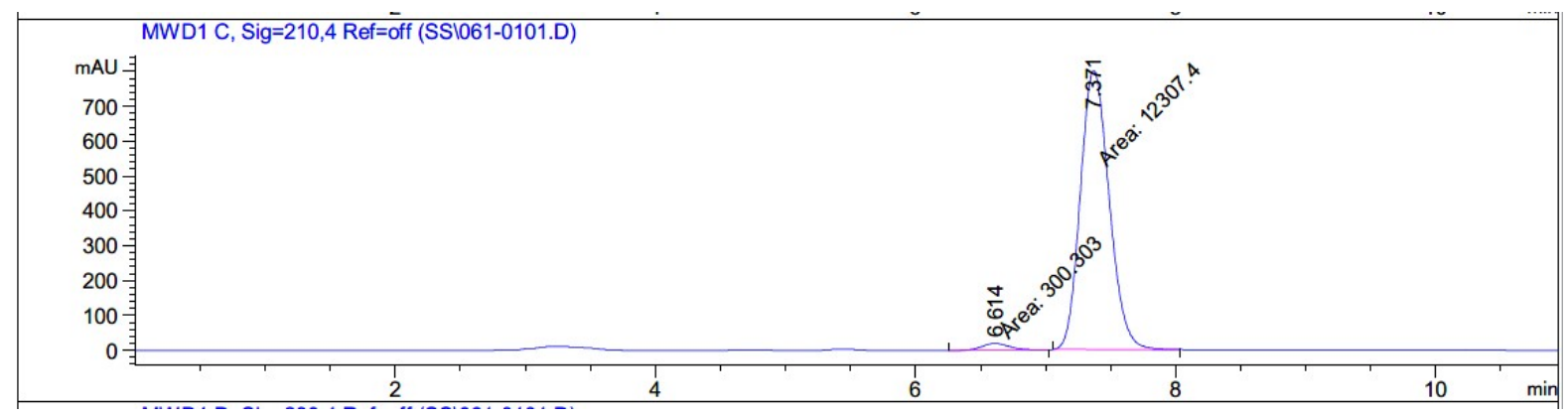

Signal 2: MWD1 C, Sig=210,4 Ref=off

\begin{tabular}{|c|c|c|c|c|c|c|}
\hline $\begin{array}{c}\text { Peak } \\
\quad \#\end{array}$ & $\begin{array}{c}\text { RetTime } \\
\text { [min] }\end{array}$ & Type & $\begin{array}{l}\text { Width } \\
\text { [min] }\end{array}$ & $\begin{array}{c}\text { Area } \\
{\left[\mathrm{mAU}{ }^{*} \mathrm{~s}\right]}\end{array}$ & $\begin{array}{l}\text { Height } \\
\text { [mAU] }\end{array}$ & $\begin{array}{c}\text { Area } \\
\quad \%\end{array}$ \\
\hline & & & -1 & -- & _ & \\
\hline 1 & .614 & $\mathrm{MM}$ & 0.2518 & 300.30289 & 19.87688 & 319 \\
\hline 2 & 7.371 & $\mathrm{MM}$ & 0.2559 & $1.23074 \mathrm{e} 4$ & 801.71936 & 6181 \\
\hline
\end{tabular}


(S)-2 Hydroxy- $N^{1}, N^{4}$-diphenylsuccinamide 18 from (S)-2-hydroxysuccinic acid.<smiles>O=C(C[C@H](O)C(=O)Nc1ccccc1NC(=O)C[C@@H](O)C(=O)O)Nc1ccccc1</smiles>

To a stirred solution of (S)-2-hydroxysuccinic acid (0.500 g, $3.73 \mathrm{mmol}, 1.0 \mathrm{eq})$, aniline (1.04 g, $11.2 \mathrm{mmol}, \quad 3.0 \mathrm{eq}$ and 1-[bis(dimethylamino)methylene]-1H-1,2,3-triazolo[4,5b]pyridinium 3-oxide hexafluorophosphate $(2.83 \mathrm{~g}, 7.46 \mathrm{mmol}, 2.0 \mathrm{eq})$ in $N, N$ dimethylformamide $(10 \mathrm{~mL})$ was added $N, N$-Diisopropylethylamine $(1.93 \mathrm{~g}, 14.9 \mathrm{mmol}, 4.0$ eq) and resulting reaction mixture was stirred at room temperature for $24 \mathrm{~h}$. The reaction mixture was poured into ice cold water $(100 \mathrm{~mL})$ and the obtained solid material was filtered and dried and under vacuum to generate crude product which was further purified by column chromatography to afford 18 as a white solid (0.605 g, $0.213 \mathrm{mmol}, 57 \%)$.

(S)-Enantiomer standard prepared from (S)-2-hydroxysuccinic acid ((S)-malic acid).

(S)-2 Hydroxy- $N^{1}, N^{4}$-diphenylsuccinamide.

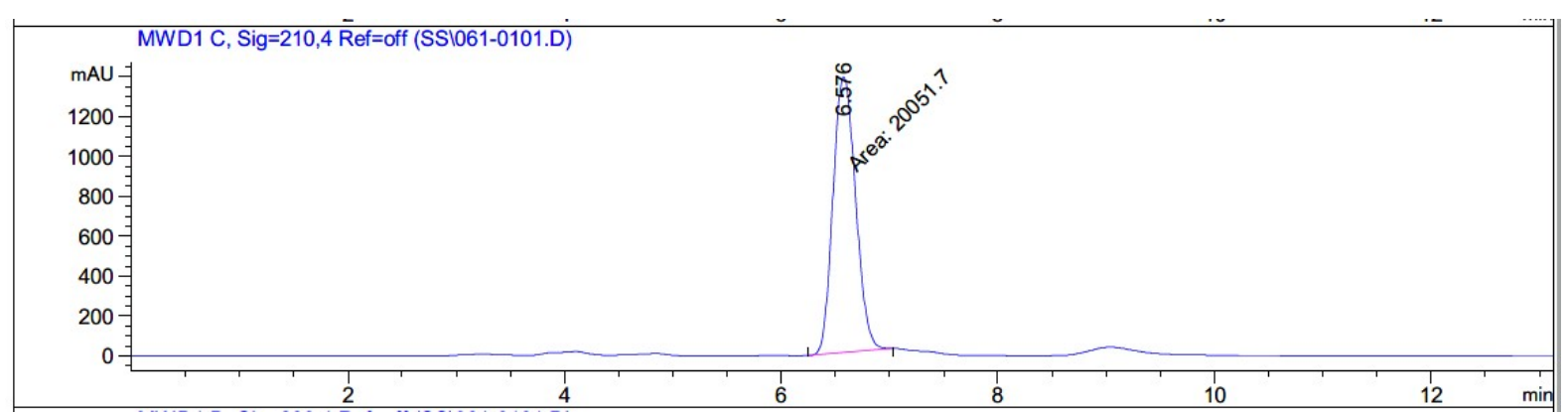

Signal 2: MWD1 C, Sig=210,4 Ref=off

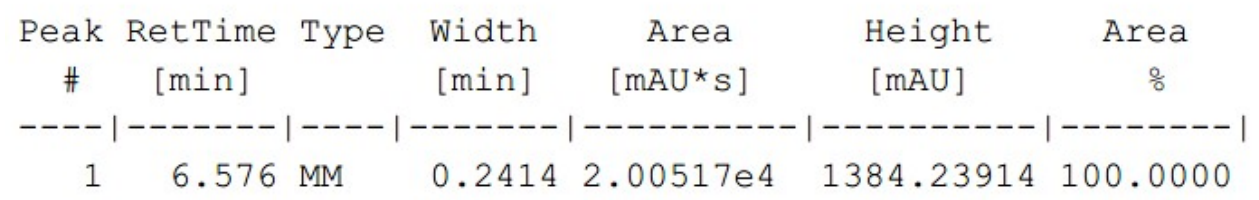


Methyl (R)-2-hydroxy-4-(methyl (phenyl)amino)-4-oxobutanoate 19.<smiles>COC(=O)C(O)CC(=O)N(C)c1ccccc1</smiles>

This compound is novel and was prepared following general procedure C using Methyl 4(methyl(phenyl)amino)-2,4-dioxobutanoate $(58.8 \mathrm{mg}, 0.250 \mathrm{mmol}, 1.0 \mathrm{eq}), 3 \mathrm{C}$ tethered catalyst $(R, R)-2(2.3 \mathrm{mg}, 3.8 \mu \mathrm{mol}, 0.015 \mathrm{eq})$ and FA:TEA $(0.15 \mathrm{~mL})$ in DCM $(1.5 \mathrm{~mL})$ to give 19 as a clear liquid (45.2 $\mathrm{mg}, 0.191 \mathrm{mmol}, 76 \%)$.

TLC: $\mathrm{R}_{\mathrm{f}}$ ca 0.4 (5:5, hexane: EtOAc), non UV-active, Strong $\mathrm{KMnO}_{4}$ and PMA-reactive;

HRMS (ESI) m/z: [M+Na] ${ }^{+}$Calcd for $\mathrm{C}_{12} \mathrm{H}_{15} \mathrm{NO}_{4} \mathrm{Na} 260.0893$; Found 260.0896 (error -1.1 ppm);

$U_{\max } 3445,2915,1634,1590,1493,1389,1115,1042,700 \mathrm{~cm}^{-1}$;

Enantiomeric excess determined by HPLC analysis (Chiralpak IC, $250 \mathrm{~mm} \times 4.6 \mathrm{~mm}$ column, hexane: $\left.\mathrm{iPrOH} 70: 30,1 \mathrm{~mL} / \mathrm{min}, \lambda=210 \mathrm{~nm}, \mathrm{~T}=25^{\circ} \mathrm{C}\right), S$-enantiomer $53.9 \mathrm{~min}, R$ enantiomer $80.9 \min .77 \%$ ee $(R),[\alpha]_{\mathrm{D}}^{22}=-18.6\left(\mathrm{c}=0.04, \mathrm{CHCl}_{3}\right)$;

${ }^{1} \mathrm{H}$ NMR (400 MHz, $\mathrm{CDCl}_{3}$ ) (Rotamers) $\delta$ 7.46-7.33 (3H, m, ArH), 7.22-7.16 (2H, m, ArH), $4.41(1 \mathrm{H}, \mathrm{dd}, J=9.7,4.8 \mathrm{~Hz}, \mathrm{C} \underline{\mathrm{HOH}}), 3.97(1 \mathrm{H}, \mathrm{d}, J=6.0 \mathrm{~Hz}, \mathrm{CHO} \underline{\mathrm{H}}), 3.77\left(3 \mathrm{H}, \mathrm{s}, \mathrm{OCH}_{3}\right)$, $3.27\left(2.5 \mathrm{H}, \mathrm{s}, \mathrm{NCH}_{3}\right), 3.27\left(0.5 \mathrm{H}, \mathrm{s}, \mathrm{NCH}_{3}\right), 2.60\left(2 \mathrm{H}, \mathrm{m}, \mathrm{CH}_{\mathrm{a}} \mathrm{H}_{\mathrm{b}}\right)$;

${ }^{13} \mathrm{C} \mathrm{NMR}\left(101 \mathrm{MHz}, \mathrm{CDCl}_{3}\right.$ ) (Rotamers) $\delta 174.1,170.6,143.3,130.1,128.3,127.4,68.2$, 52.6, 37.8, 37.3;

$m / z(\mathrm{ESI}) 260.1\left[(\mathrm{M}+\mathrm{Na})^{+}, 100 \%\right]$. 
Methyl-2-hydroxy-4-(methyl (phenyl)amino)-4-oxobutanoate 19.

${ }^{1} \mathrm{H}$ NMR (400 MHz, $\mathrm{CDCl}_{3}$ ).

Sep23-2020

SKG552
PROTON.w CDCl3 /opt/topspin3.5pl2 SKG 21

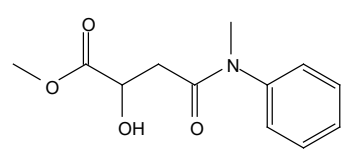

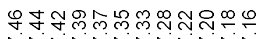

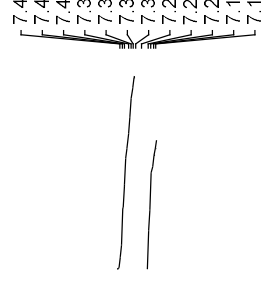

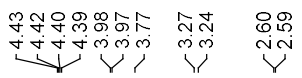

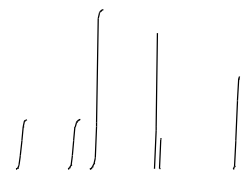

$\stackrel{8}{i}$

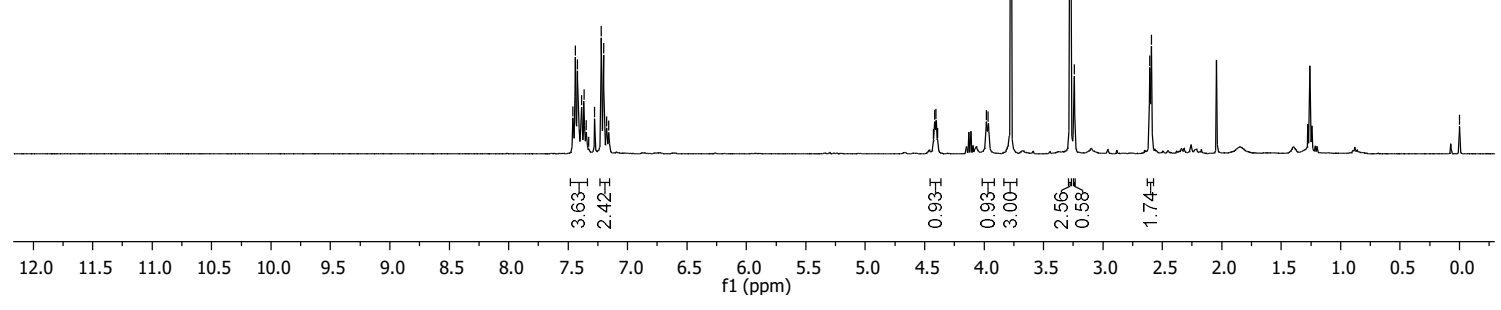

${ }^{13} \mathrm{C}$ NMR (101 MHz, $\left.\mathrm{CDCl}_{3}\right)$.

Sep23-2020

SKG552

C13APTlong.w $\mathrm{CDCl} 3$

少兵

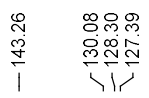

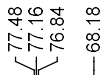
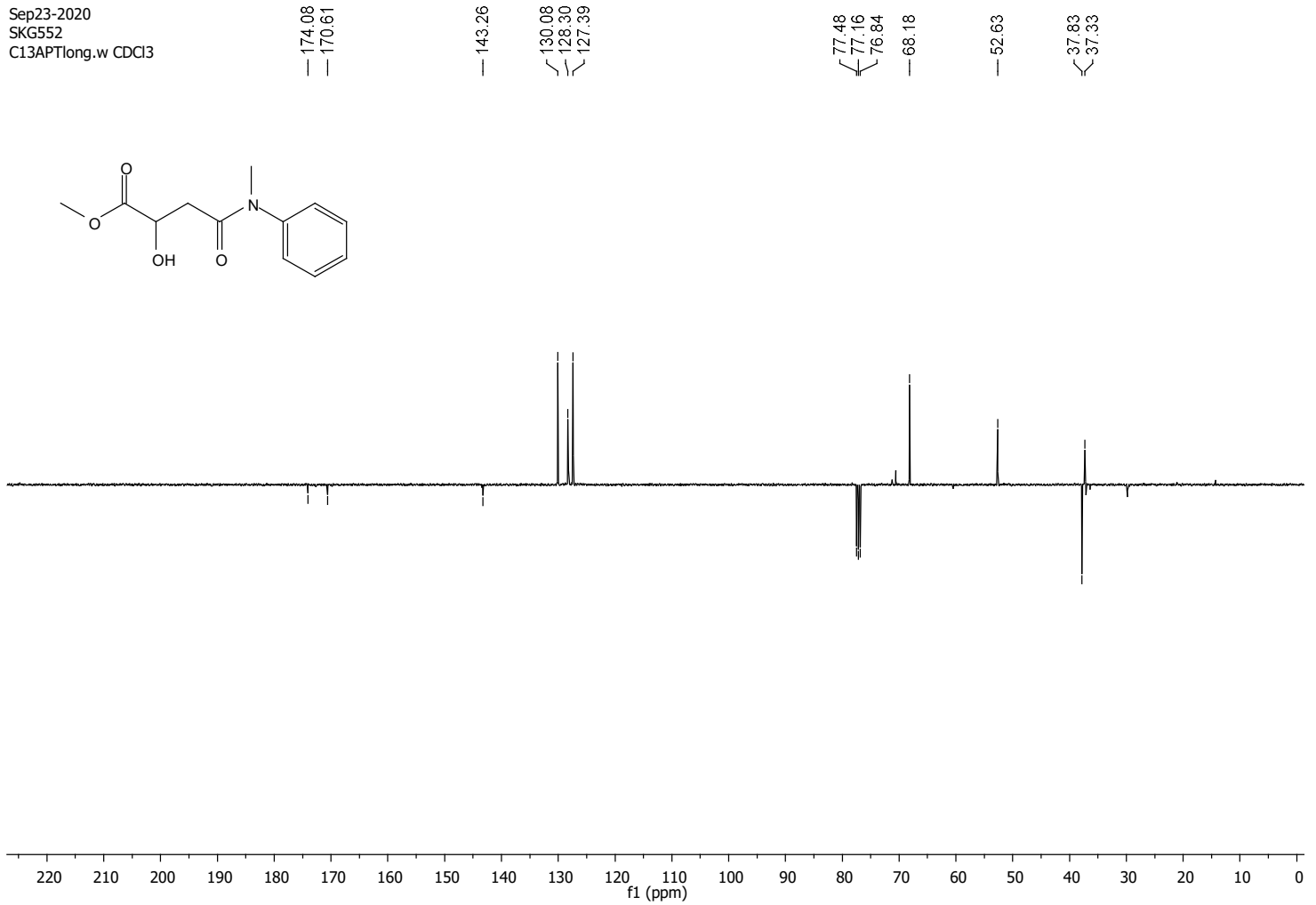

S267 
COSY.

SKG552

CDCl3/opt/topspin3.5pl2 SKG 21

$\overbrace{O H}^{O H}$

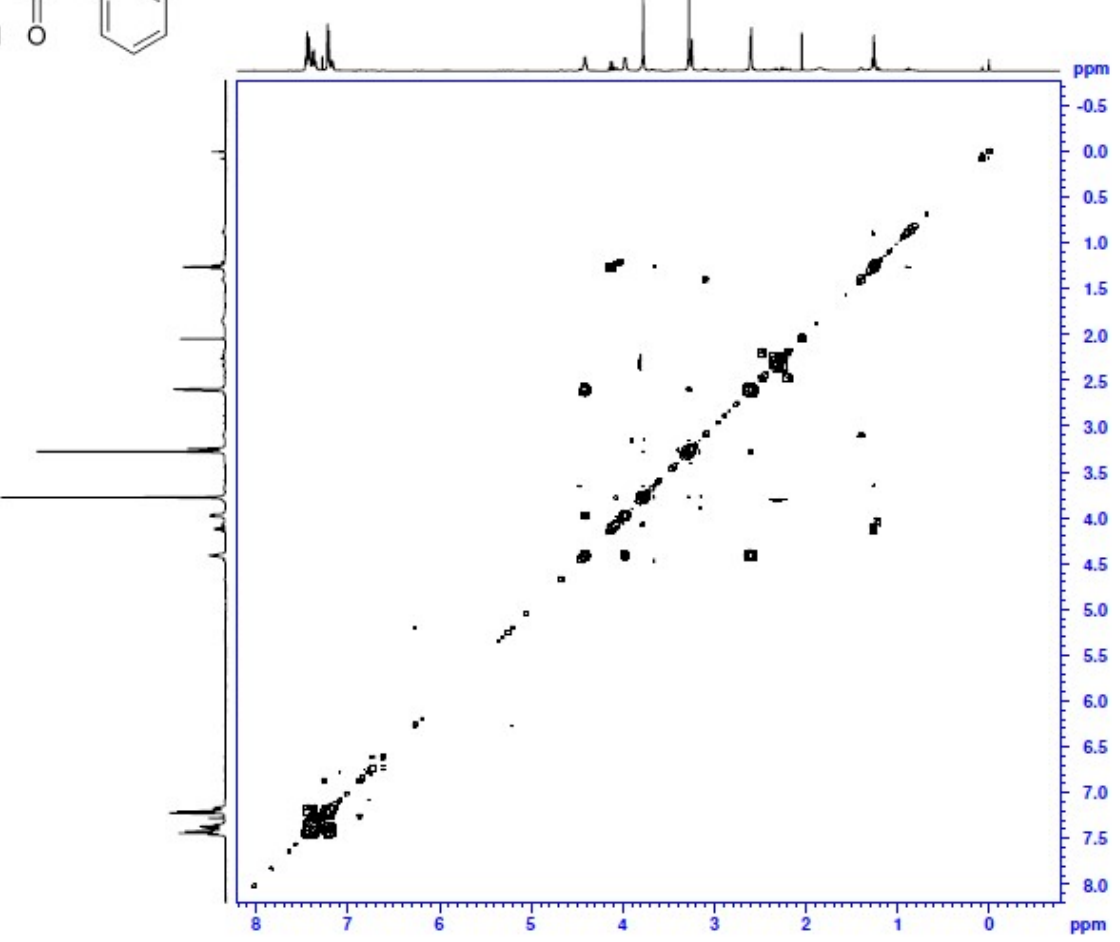

HSQC.

HSQC.w CDCl3 /opttopspin3.5pl2 SKG 21

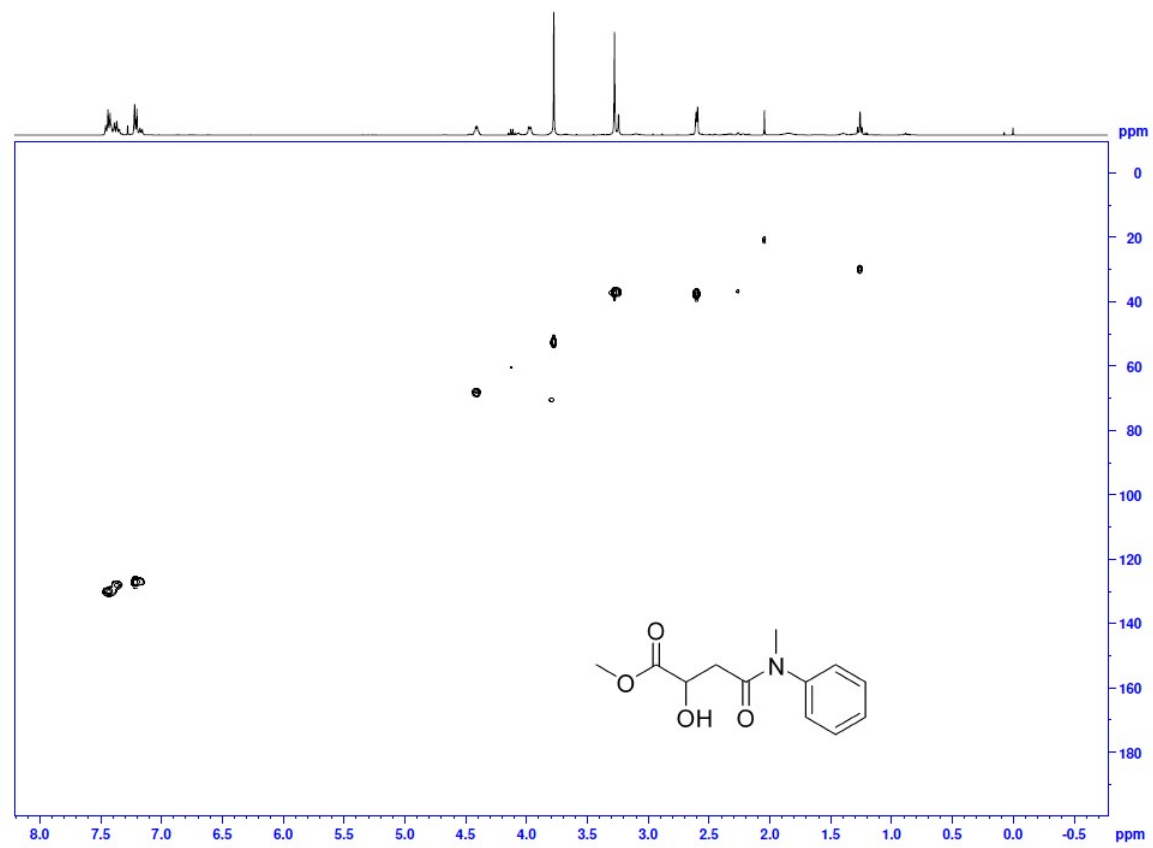


Chiral HPLC of ketone precursor.

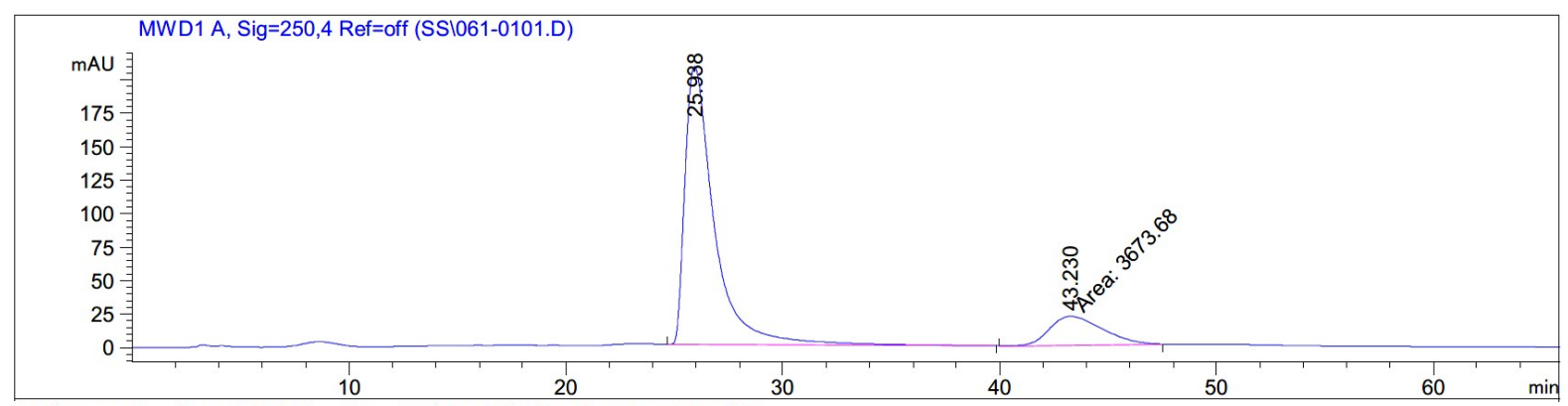

Signal 1: MWD1 A, Sig=250,4 Ref=off

\begin{tabular}{|c|c|c|c|c|c|c|}
\hline $\begin{array}{l}\text { Peak } \\
\quad \#\end{array}$ & $\begin{array}{c}\text { RetTime } \\
\text { [min] }\end{array}$ & Type & $\begin{array}{l}\text { Width } \\
\text { [min] }\end{array}$ & $\begin{array}{c}\text { Area } \\
{\left[\mathrm{mAU}^{\star} \mathrm{s}\right]}\end{array}$ & $\begin{array}{l}\text { Height } \\
\text { [mAU] }\end{array}$ & $\begin{array}{c}\text { Area } \\
\frac{\circ}{0}\end{array}$ \\
\hline 1 & 25.938 & BB & 1.4040 & $1.95164 \mathrm{e} 4$ & 207.75436 & 84.1584 \\
\hline 2 & 43.230 & MM & 2.8208 & 3673.67529 & 21.70594 & 15.8416 \\
\hline
\end{tabular}

\section{Chiral HPLC of racemic alcohol.}

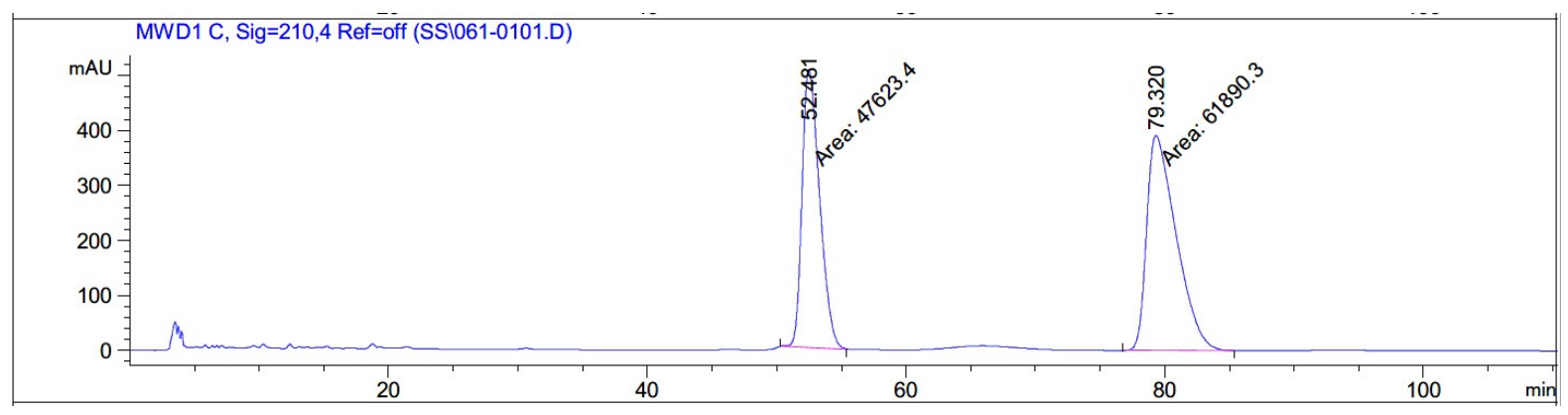

Signal 2: MWD1 C, Sig=210,4 Ref=off

\begin{tabular}{|c|c|c|c|c|c|c|}
\hline $\begin{array}{c}\text { Peak } \\
\#\end{array}$ & $\begin{array}{c}\text { RetTime } \\
\text { [min] }\end{array}$ & Type & $\begin{array}{l}\text { Width } \\
\text { [min] }\end{array}$ & $\begin{array}{c}\text { Area } \\
{\left[\mathrm{mAU}{ }^{\star} \mathrm{s}\right]}\end{array}$ & $\begin{array}{l}\text { Height } \\
\text { [mAU] }\end{array}$ & $\begin{array}{c}\text { Area } \\
\frac{\circ}{0}\end{array}$ \\
\hline & & & 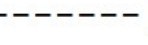 & -1 & -1 & -- \\
\hline 1 & 52.481 & MM & 1.5725 & $4.76234 \mathrm{e} 4$ & 504.76489 & 43.4863 \\
\hline 2 & 79.320 & MM & 2.6386 & $6.18903 e 4$ & 390.93237 & 56.5137 \\
\hline
\end{tabular}


Chiral HPLC of ATH product. $77 \%$ ee.

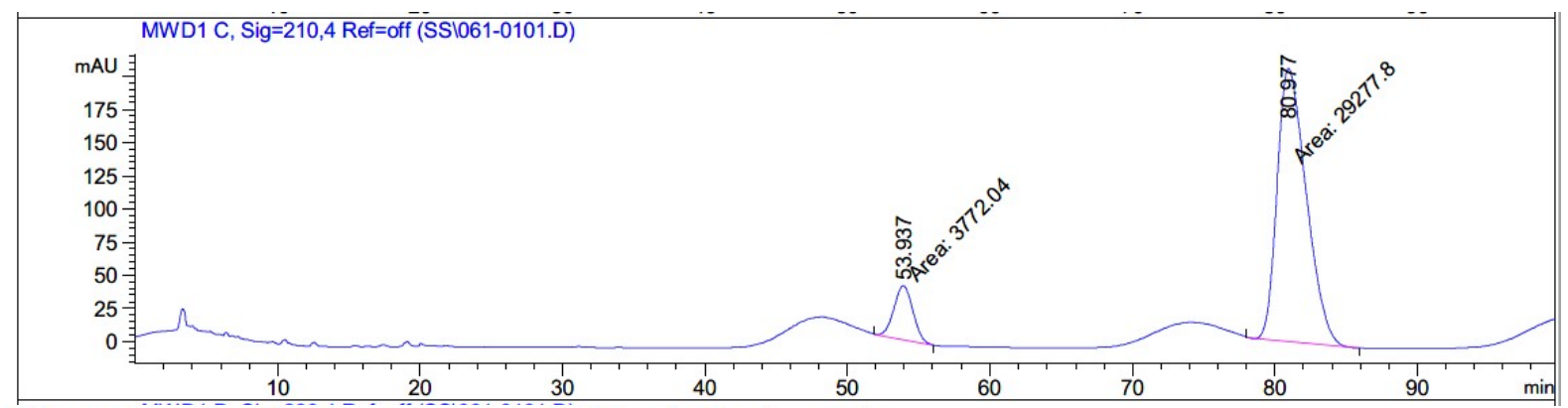

Signal 2: MWD1 C, Sig=210,4 Ref=off

\begin{tabular}{|c|c|c|c|c|c|c|}
\hline $\begin{array}{c}\text { Peak } \\
\#\end{array}$ & $\begin{array}{c}\text { RetTime } \\
\text { [min] }\end{array}$ & Type & $\begin{array}{c}\text { Width } \\
\text { [min] }\end{array}$ & $\begin{array}{c}\text { Area } \\
{\left[\mathrm{mAU}^{\star} \mathrm{s}\right]}\end{array}$ & $\begin{array}{l}\text { Height } \\
{[\mathrm{mAU}]}\end{array}$ & $\begin{array}{c}\text { Area } \\
\frac{\circ}{0}\end{array}$ \\
\hline & & & & -2 & ---- & \\
\hline 1 & 53 & MM & 39 & 3772.03638 & 71972 & 132 \\
\hline 2 & 80.977 & MM & 722 & $2.92778 e 4$ & 205.70323 & 5868 \\
\hline
\end{tabular}


Ethyl (R)-2-hydroxy-4-(methyl(phenyl)amino)-4-oxobutanoate 20.<smiles>CCOC(=O)C(O)CC(=O)N(C)c1ccccc1</smiles>

This compound is novel and was prepared following general procedure $\mathrm{C}$ using ethyl 4(methyl(phenyl)amino)-2,4-dioxobutanoate $(0.062 \mathrm{~g}, 0.25 \mathrm{mmol}, 1.0 \mathrm{eq}), 3 \mathrm{C}$ tethered catalyst $(R, R)-2(2.3 \mathrm{mg}, 3.8 \mu \mathrm{mol}, 0.015 \mathrm{eq})$ and FA:TEA $(0.15 \mathrm{~mL})$ in DCM $(1.5 \mathrm{~mL})$ to give 20 as a brown liquid (0.050 g, $0.20 \mathrm{mmol}, 80 \%)$.

TLC: $\mathrm{R}_{\mathrm{f}}$ ca 0.4 (2:8, hexane: EtOAc), non UV-active, Strong $\mathrm{KMnO}_{4}$ and PMA-reactive;

HRMS (ESI) m/z: [M+Na] $]^{+}$Calcd for $\mathrm{C}_{13} \mathrm{H}_{17} \mathrm{NO}_{4} \mathrm{Na} 274.1050$; Found 274.1055 (error -1.9 ppm);

$U_{\max } 3427,2980,1731,1638,1593,1495,1389,1197,1099,699 \mathrm{~cm}^{-1}$;

Enantiomeric excess determined by HPLC analysis (Chiralpak IC, $250 \mathrm{~mm} \times 4.6 \mathrm{~mm}$ column, Hexane: $\mathrm{iPrOH} 80: 20,1 \mathrm{~mL} / \mathrm{min}, \lambda=210 \mathrm{~nm}, \mathrm{~T}=25{ }^{\circ} \mathrm{C}$ ), $S$-enantiomer $81.5 \mathrm{~min}, R$ enantiomer $99.2 \mathrm{~min} .84 \%$ ee $(R),[\alpha]_{\mathrm{D}}^{22}=-11.5\left(\mathrm{c}=0.1, \mathrm{CHCl}_{3}\right)$;

${ }^{1} \mathrm{H}$ NMR (400 MHz, $\mathrm{CDCl}_{3}$ ) $\delta$ 7.45-7.34 (3H, m, ArH), 7.20 (2H, d, J=7.6 Hz, ArH), 4.38 $(1 \mathrm{H}, \mathrm{s}, \mathrm{C} \underline{\mathrm{HOH}}), 4.26-4.18$ (2H, m, $\left.\underline{\mathrm{CH}}_{2} \mathrm{CH}_{3}\right), 3.89$ (1H, s, $\left.\mathrm{CHO} \underline{\mathrm{H}}\right), 3.27$ (3H, s, $\left.\mathrm{NCH}_{3}\right), 2.59-$ $2.58\left(2 \mathrm{H}, \mathrm{m}, \mathrm{CH}_{\mathrm{a}} \mathrm{H}_{\mathrm{b}}\right), 1.28\left(3 \mathrm{H}, \mathrm{t}, J=7.1 \mathrm{~Hz}, \mathrm{CH}_{2} \underline{\mathrm{CH}_{3}}\right)$;

${ }^{13} \mathrm{C}$ NMR $\left(101 \mathrm{MHz}, \mathrm{CDCl}_{3}\right) \delta 173.7,170.6,143.4,130.1,128.3,127.4,68.3,61.7,37.9$, 37.4, 14.3;

$m / z(\mathrm{ESI}) 274.1\left[(\mathrm{M}+\mathrm{Na})^{+}, 100 \%\right]$. 
Ethyl 2-hydroxy-4-(methyl(phenyl)amino)-4-oxobutanoate 20.

${ }^{1} \mathrm{H}$ NMR (400 MHz, $\mathrm{CDCl}_{3}$ ).
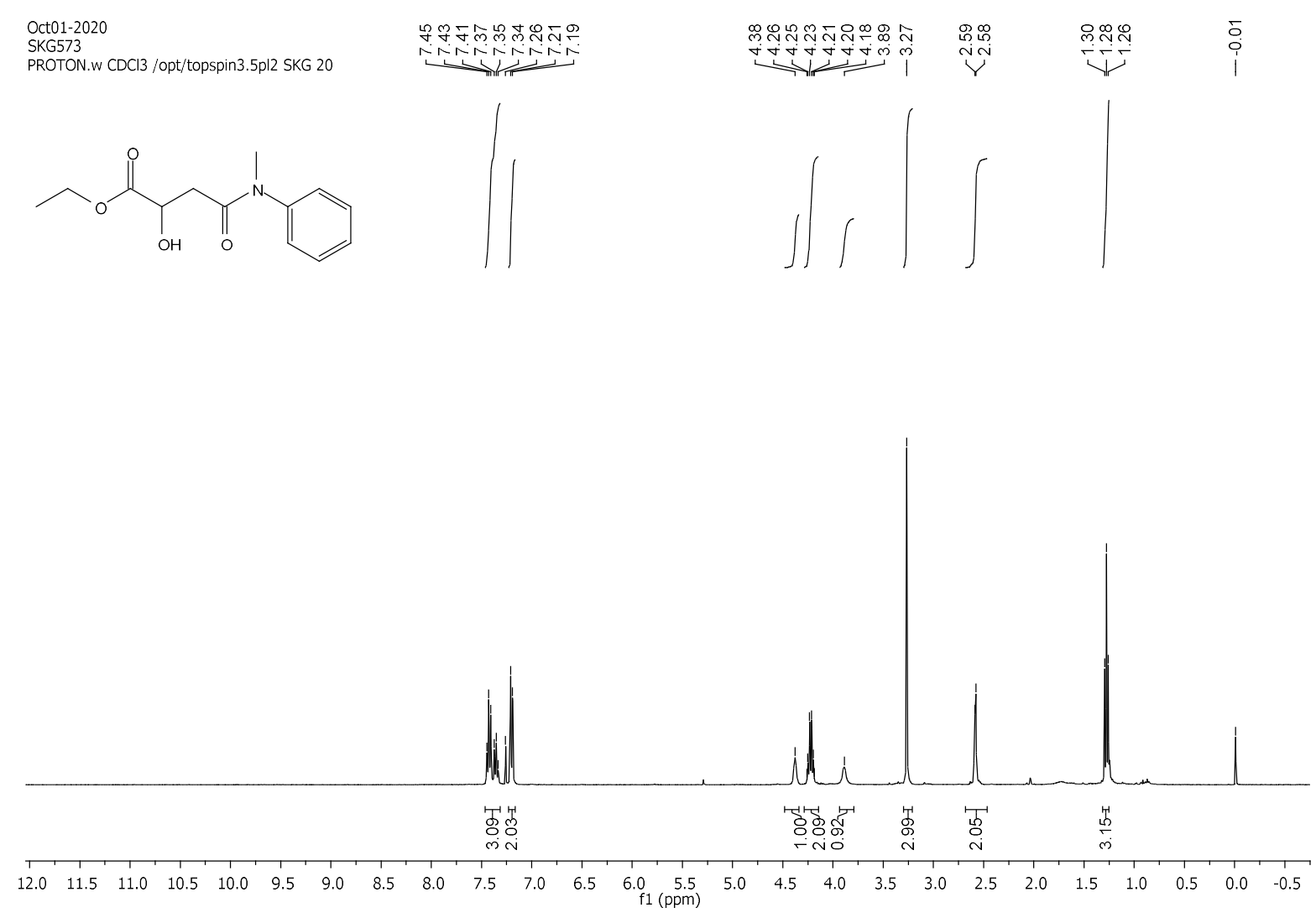

${ }^{13} \mathrm{C}$ NMR (101 MHz, $\left.\mathrm{CDCl}_{3}\right)$.

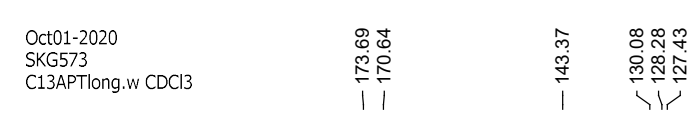

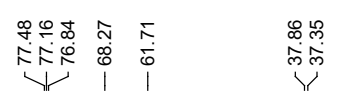

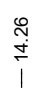
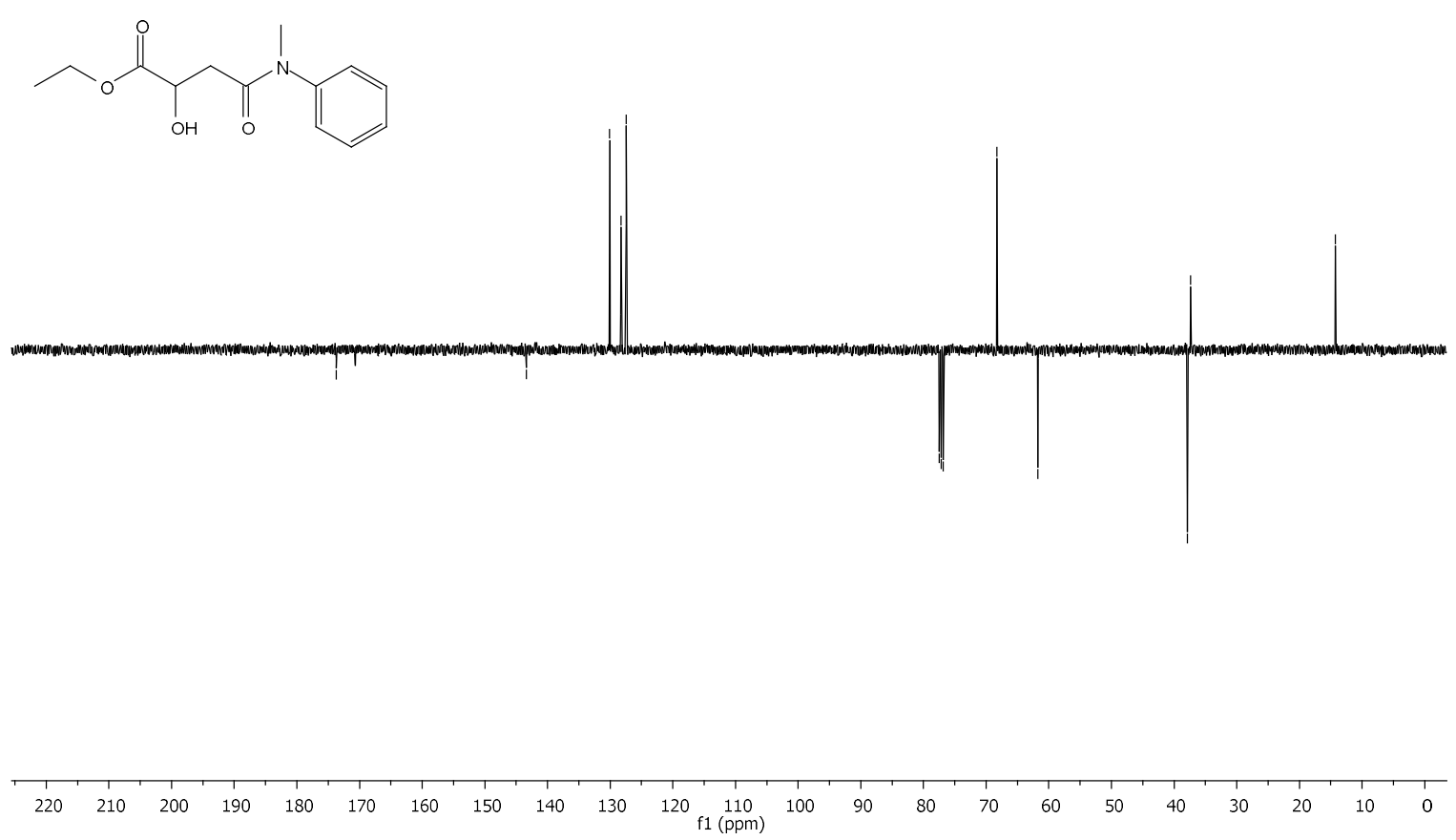

S272 
COSY.

SKG573

COSY, $\mathrm{CDCl} 3$ /opt/topspin3.5pl2 SKG 20

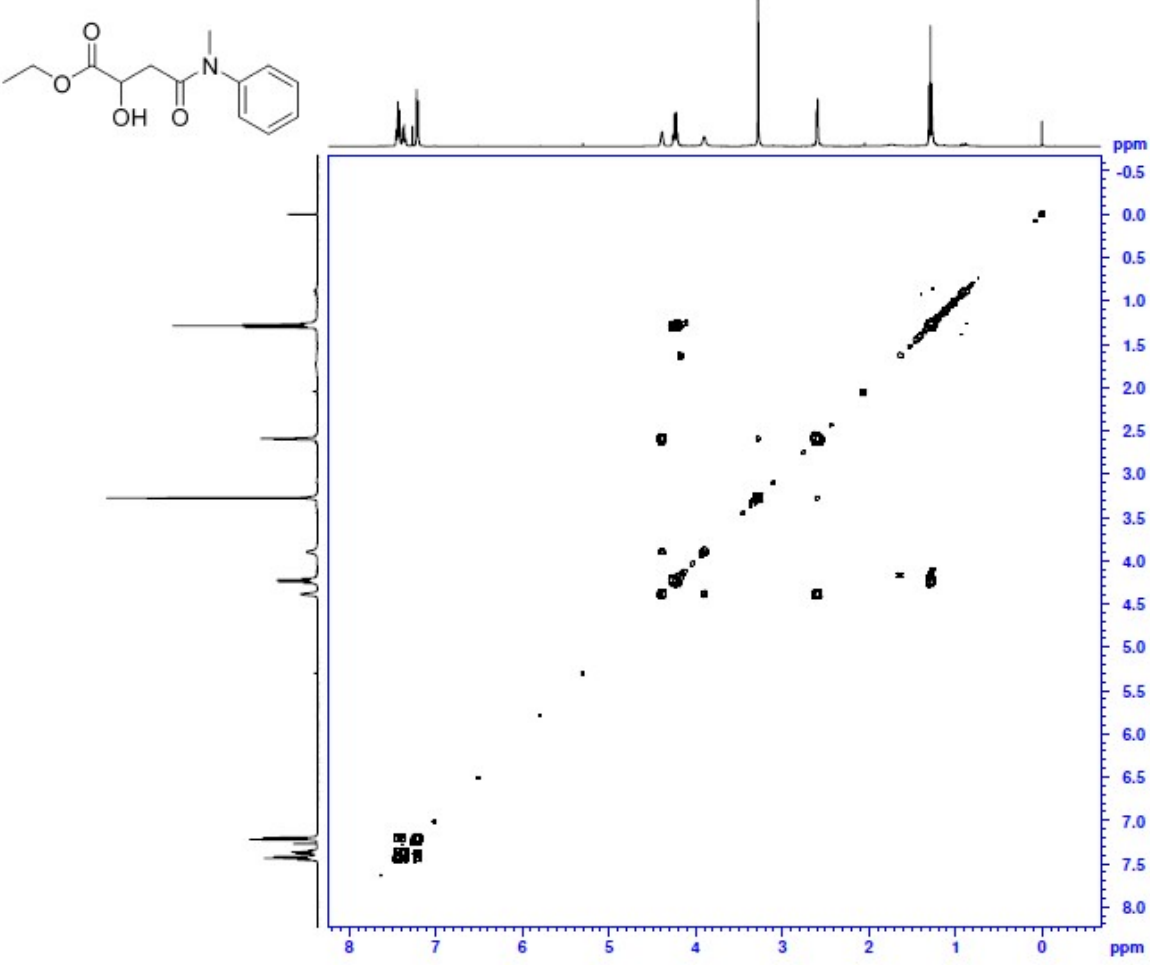

HSQC.

SKG573
HSQC.w CDCl3/opt/topspin3.5pl2 SKG 20

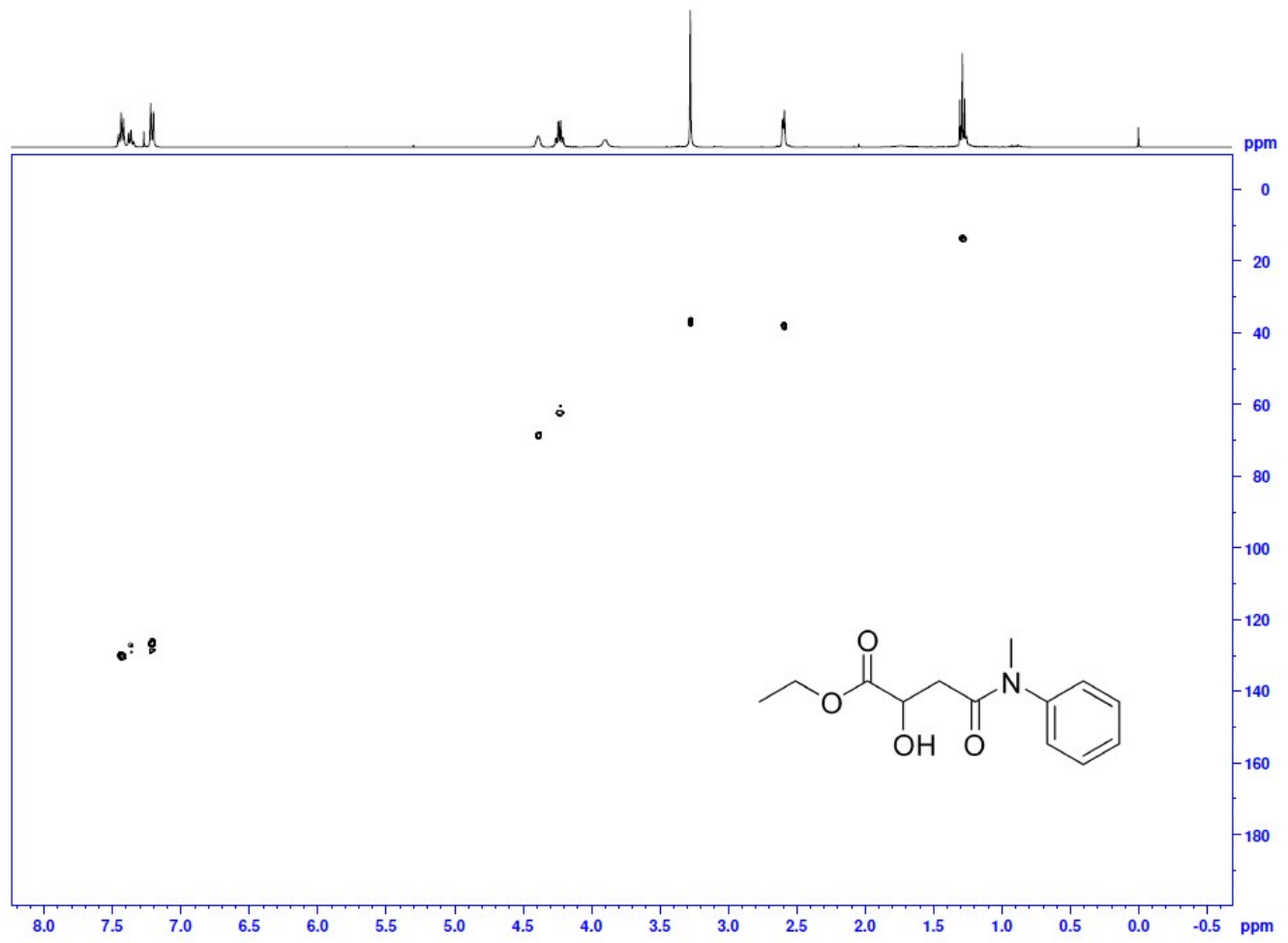




\section{Chiral HPLC of ketone precursor.}

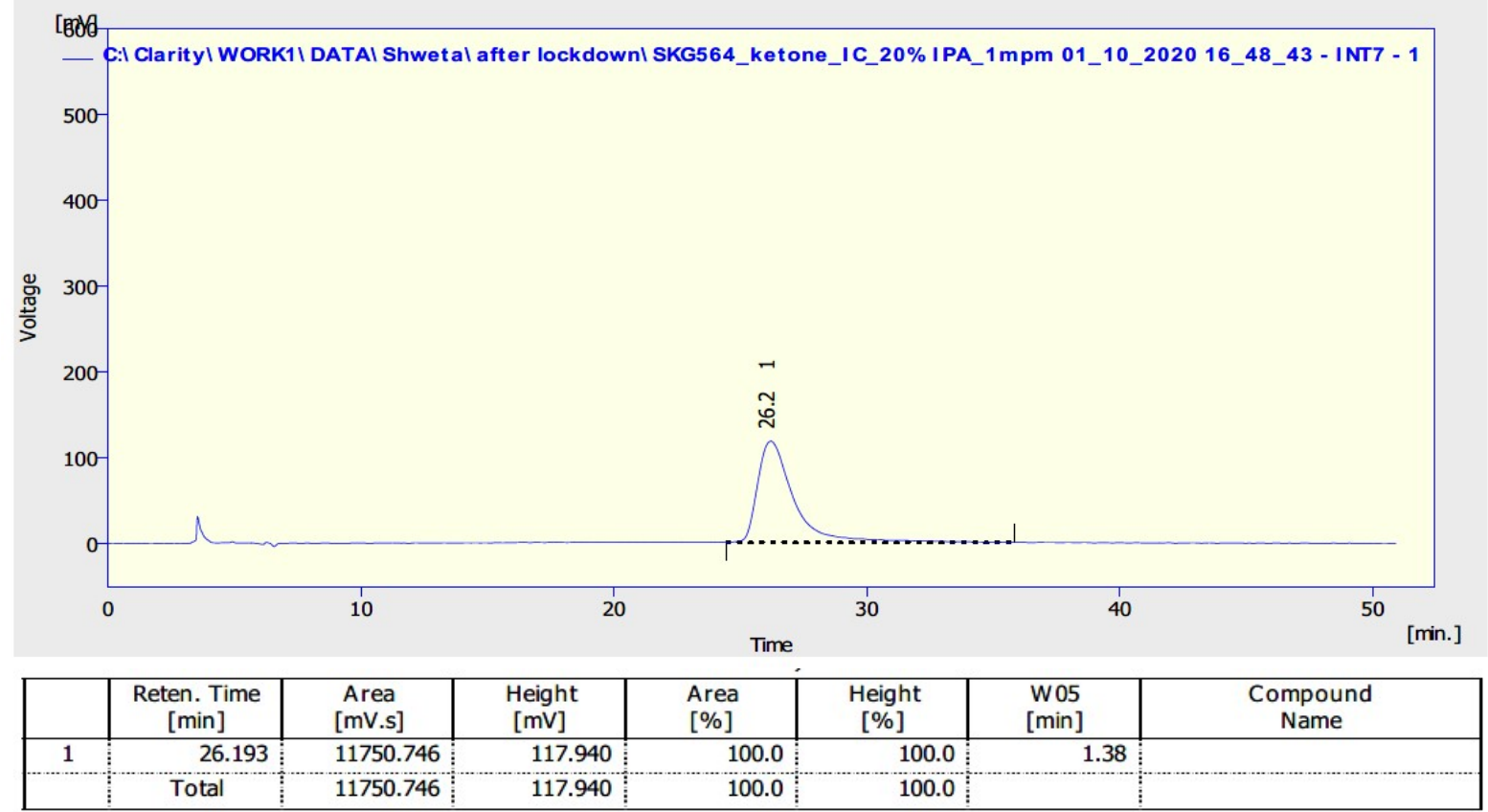

\section{Chiral HPLC of racemic alcohol.}

[3006

$\therefore$ Clarity\ WORK1 I DATAI Shwetal after lockdownI SKG574_racemic_IC_20\% IPA_1mpm_210nm_29_09_2020 11_29_33 - INT7 - 1

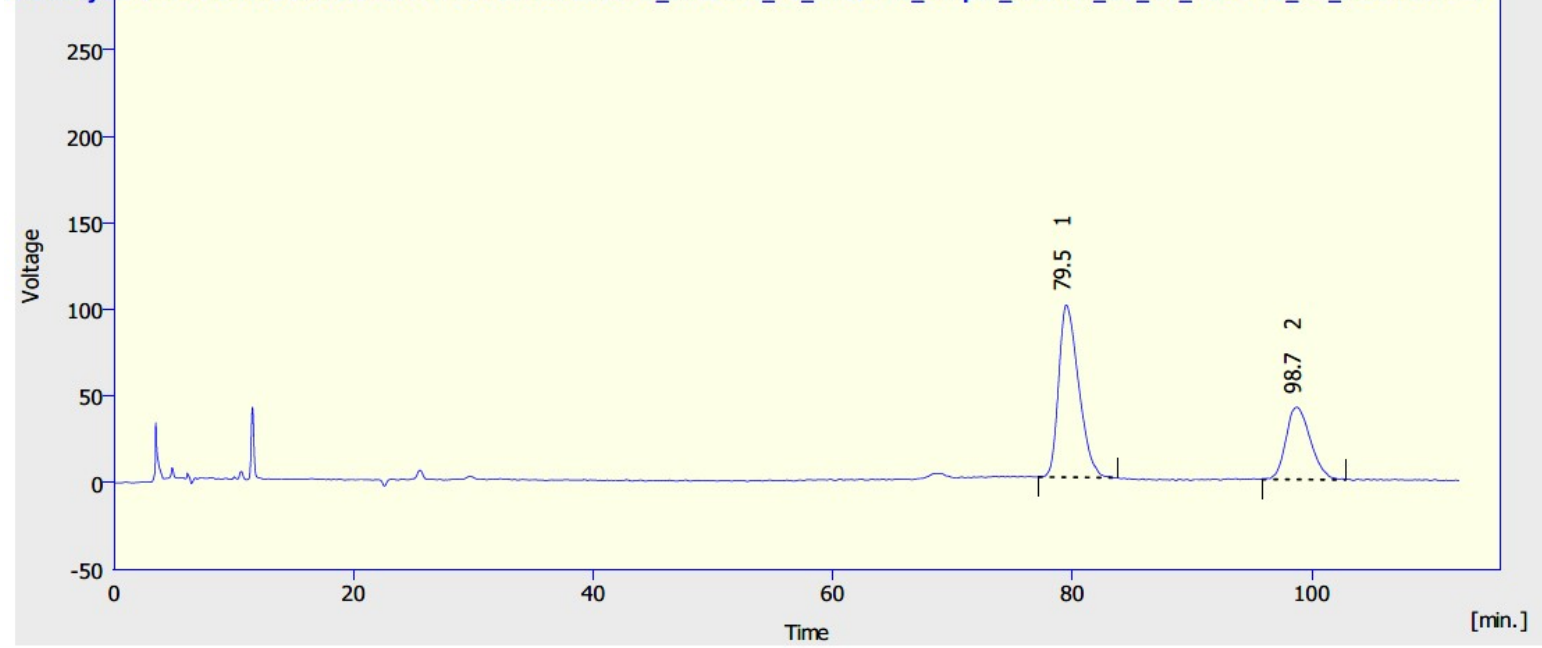

\begin{tabular}{|c|c|c|c|c|c|c|c|}
\hline & $\begin{array}{c}\text { Reten. Time } \\
{[\mathrm{min}]}\end{array}$ & $\begin{array}{c}\text { Area } \\
{[\mathrm{mV} . \mathrm{s}]}\end{array}$ & $\begin{array}{c}\text { Height } \\
{[\mathrm{mV}]}\end{array}$ & $\begin{array}{c}\text { Area } \\
{[\%]}\end{array}$ & $\begin{array}{c}\text { Height } \\
{[\%]}\end{array}$ & $\begin{array}{c}\text { W05 } \\
{[\mathrm{min}]}\end{array}$ & $\begin{array}{c}\text { Compound } \\
\text { Name }\end{array}$ \\
\hline 1 & 79.497 & 11558.902 & 99.691 & 66.0 & 70.4 & 1.80 \\
\hline 2 & 98.740 & 5943.092 & 41.892 & 34.0 & 29.6 & 2.21 & \\
& Total & 17501.995 & 141.583 & 100.0 & 100.0 & & \\
\hline
\end{tabular}


Chiral HPLC of ATH product. $84 \%$ ee.

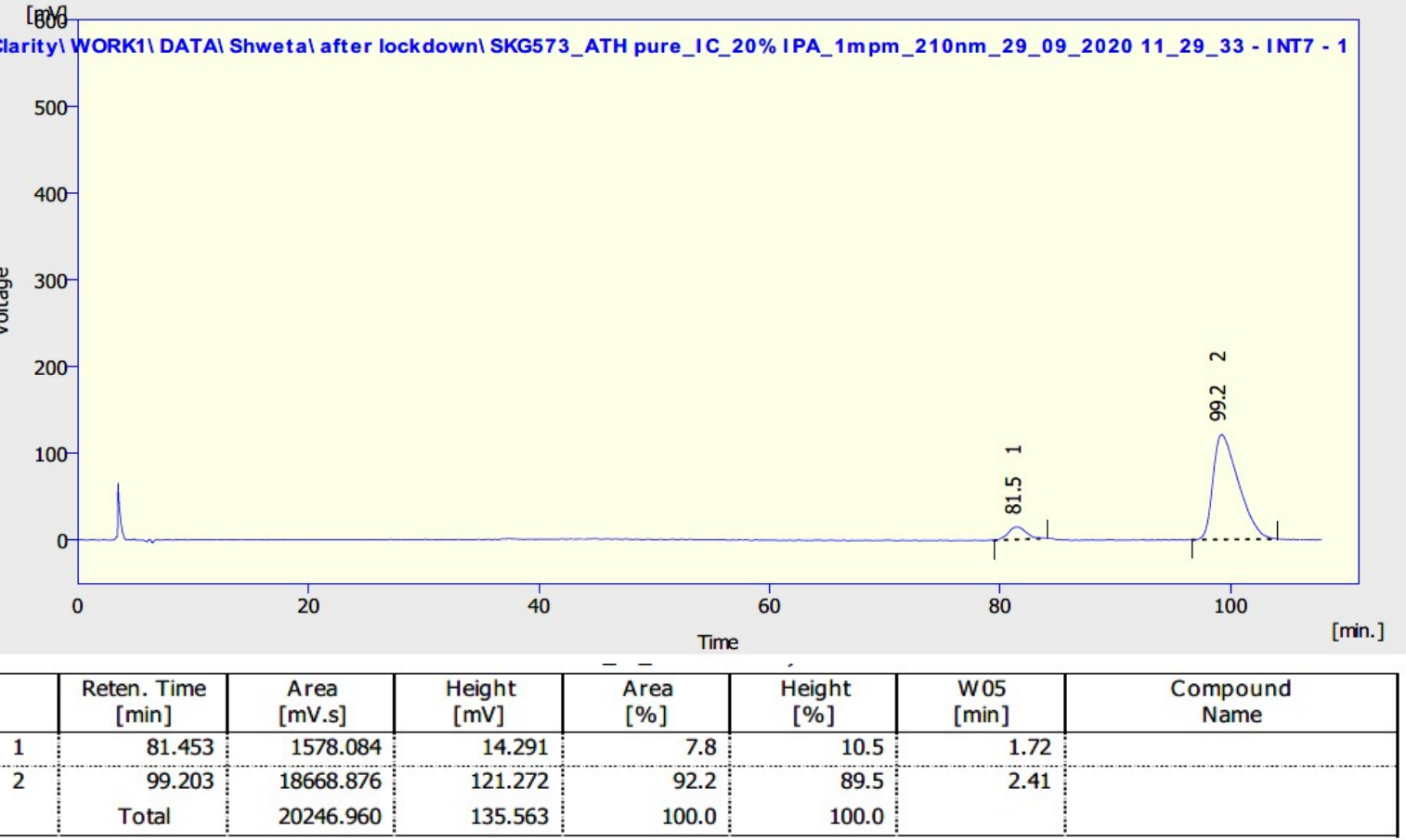




\section{(R)-2-Hydroxy- $N$-methyl-4-oxo- $N$-phenyl-4-(piperidin-1-yl)butanamide 21.}<smiles>CN(C(=O)[C@H](O)CC(=O)N1CCCCC1)c1ccccc1</smiles>

This compound is novel and was prepared following general procedure $\mathrm{C}$ using $N$-methyl2,4-dioxo- $N$-phenyl-4-(piperidin-1-yl)butanamide (0.144 g, $0.500 \mathrm{mmol}, 1.0 \mathrm{eq})$, 3C tethered catalyst $(R, R)-2(4.7 \mathrm{mg}, 7.5 \mu \mathrm{mol}, 0.015 \mathrm{eq})$ and FA:TEA $(0.3 \mathrm{~mL})$ in DCM $(3 \mathrm{~mL})$ to give 21 as a white solid (0.122 g, $0.420 \mathrm{mmol}, 84 \%)$.

TLC: $\mathrm{R}_{\mathrm{f}}$ ca 0.4 (2:8, hexane: EtOAc), UV-active, strong $\mathrm{KMnO}_{4}$, and PMA-reactive; HRMS (ESI) m/z: [M+Na] $]^{+}$Calcd for $\mathrm{C}_{16} \mathrm{H}_{22} \mathrm{~N}_{2} \mathrm{O}_{3} \mathrm{Na} 313.1523$; Found 313.1517 (error 1.7 ppm);

MP: $117-120{ }^{\circ} \mathrm{C}$;

$U_{\max } 3365,2918,2858,1655,1614,1596,1451,1256,1045,704 \mathrm{~cm}^{-1}$;

Enantiomeric excess determined by HPLC analysis (Chiralpak IG, 250mm $\times 4.6 \mathrm{~mm}$ column, hexane: $\left.\mathrm{iPrOH} 80: 20,1 \mathrm{~mL} / \mathrm{min}, \lambda=210 \mathrm{~nm}, \mathrm{~T}=25{ }^{\circ} \mathrm{C}\right), R$-enantiomer $43.4 \mathrm{~min}, S$ enantiomer $47.3 \min .89 \%$ ee $(R),[\alpha]_{\mathrm{D}}^{22}=-4.5\left(\mathrm{c}=0.1, \mathrm{CHCl}_{3}\right)$;

${ }^{1} \mathrm{H}$ NMR (400 MHz, $\left.\mathrm{CDCl}_{3}\right)$ 7.45-7.29 (5H, m, ArH), 4.56 (1H, d, J=4.4 Hz, CㅂO), 3.95 $(1 \mathrm{H}, \mathrm{d}, J=5.9 \mathrm{~Hz}, \mathrm{CHO} \underline{\mathrm{H}}), 3.54-3.52\left(2 \mathrm{H}, \mathrm{m}, \mathrm{NCH}_{2}\right.$ of piperidine), $3.32\left(3 \mathrm{H}, \mathrm{s}, \mathrm{NCH}_{3}\right), 3.25$ $\left(2 \mathrm{H}, \mathrm{s}, \mathrm{NCH}_{2}\right.$ of piperidine), 2.59-2.48 $\left(2 \mathrm{H}, \mathrm{m}, \mathrm{CH}_{\mathrm{a}} \mathrm{H}_{\mathrm{b}}\right), 1.68-1.49\left(6 \mathrm{H}, \mathrm{m}, \mathrm{CH}_{2}\right.$ of piperidine). ${ }^{13} \mathrm{C}$ NMR $\left(101 \mathrm{MHz}, \mathrm{CDCl}_{3}\right) \delta 172.5,168.6,142.7,129.9,128.2,127.4,66.2,46.7,42.7$, $38.1,37.1,26.4,25.5,24.5$;

$m / z(\mathrm{ESI}) 313.1\left[(\mathrm{M}+\mathrm{Na})^{+}, 100 \%\right]$. 
2-Hydroxy- $N$-methyl-4-oxo- $N$-phenyl-4-(piperidin-1-yl)butanamide 21.

${ }^{1} \mathrm{H}$ NMR (400 MHz, $\mathrm{CDCl}_{3}$ ).

Aug28-2020

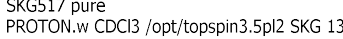

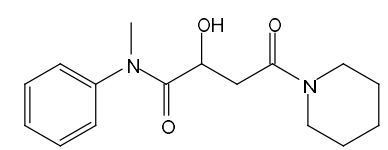

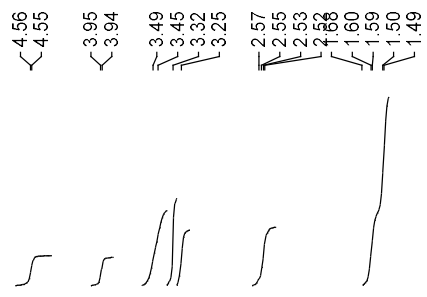

8
0
$i$
$i$

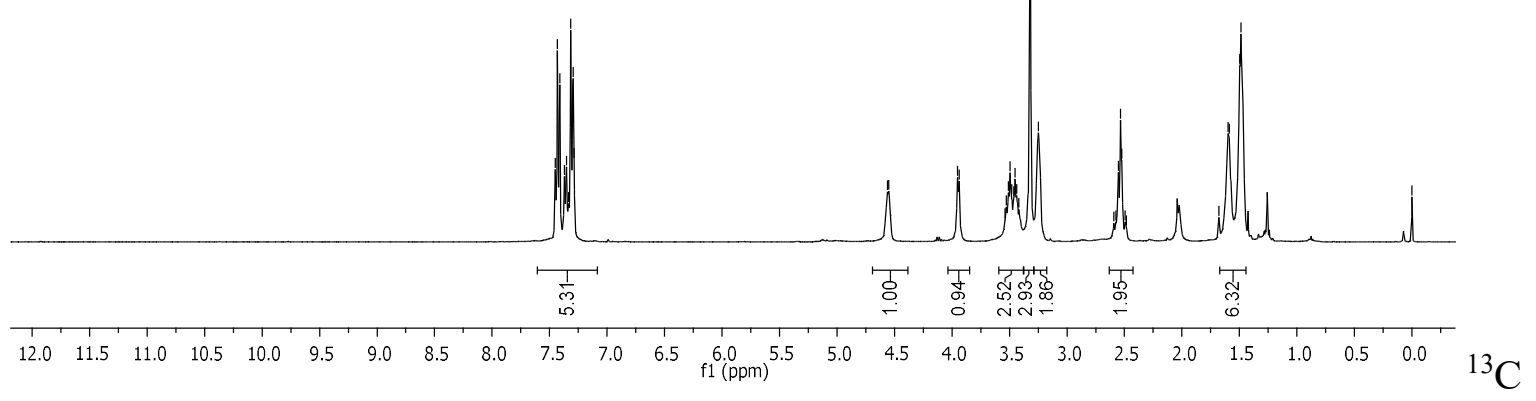

NMR (101 MHz, $\mathrm{CDCl}_{3}$ ).

Aug28-2020

C13APTlong.w CDCl3

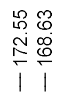

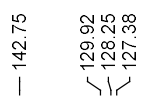

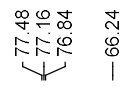

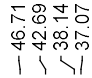

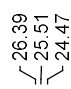
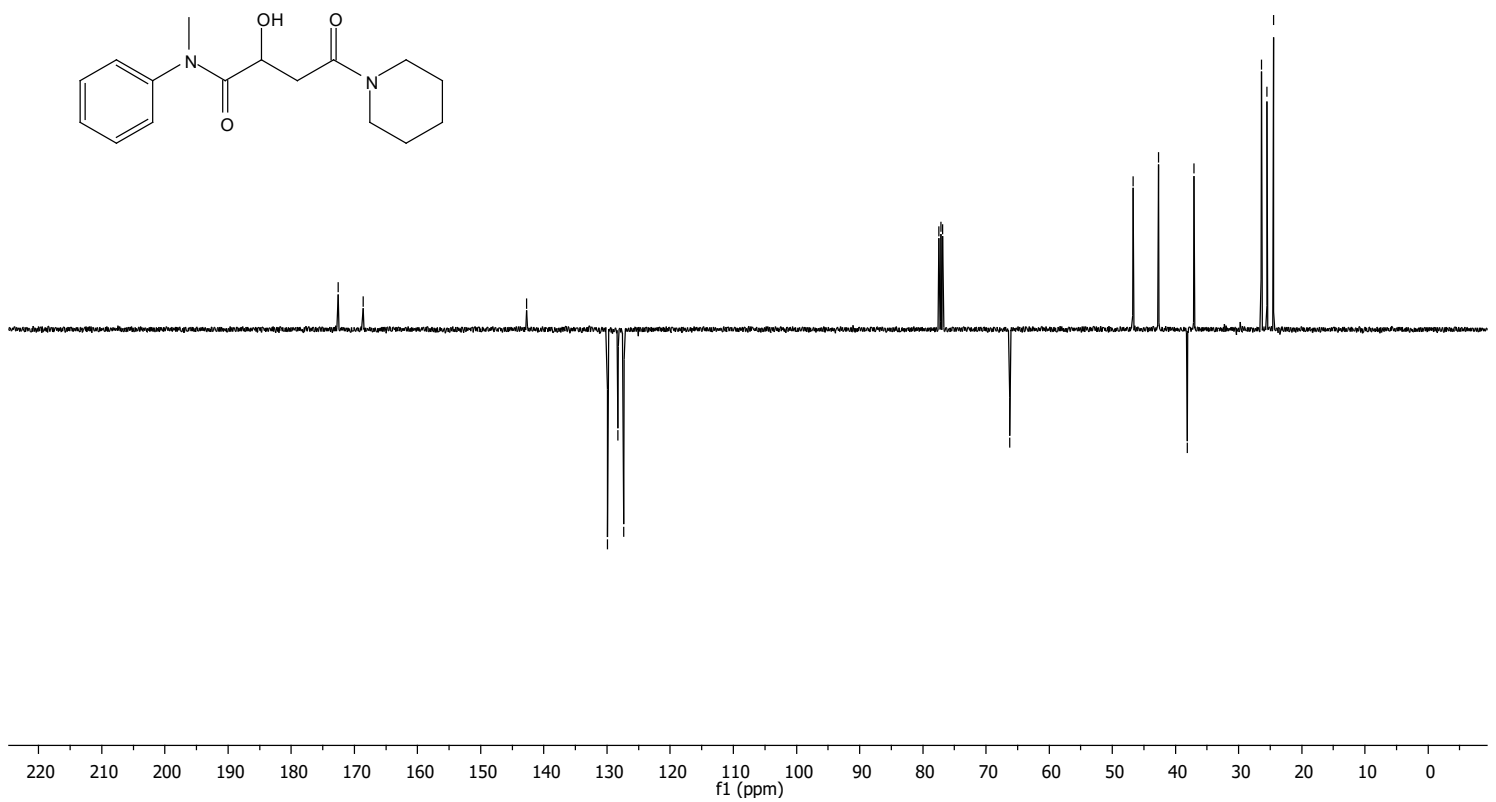

S277 
COSY.

SKG517 pure
COSY.w CDCl3/opt/topspin3.5pl2 SKG 13

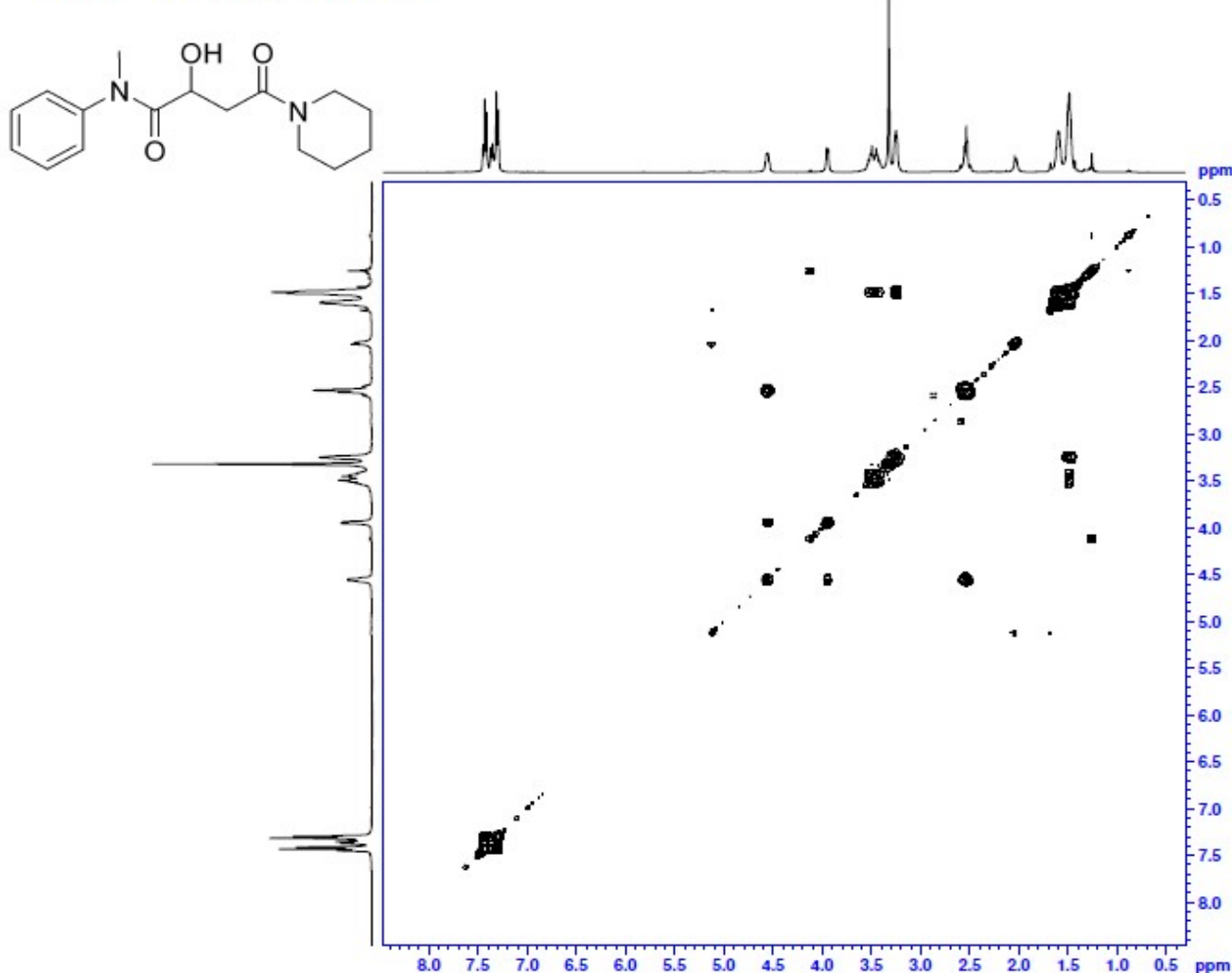

HSQC.

SKG517 pure
HSQC.w CDCl3 /opt/topspin3.5pl2 SKG 13

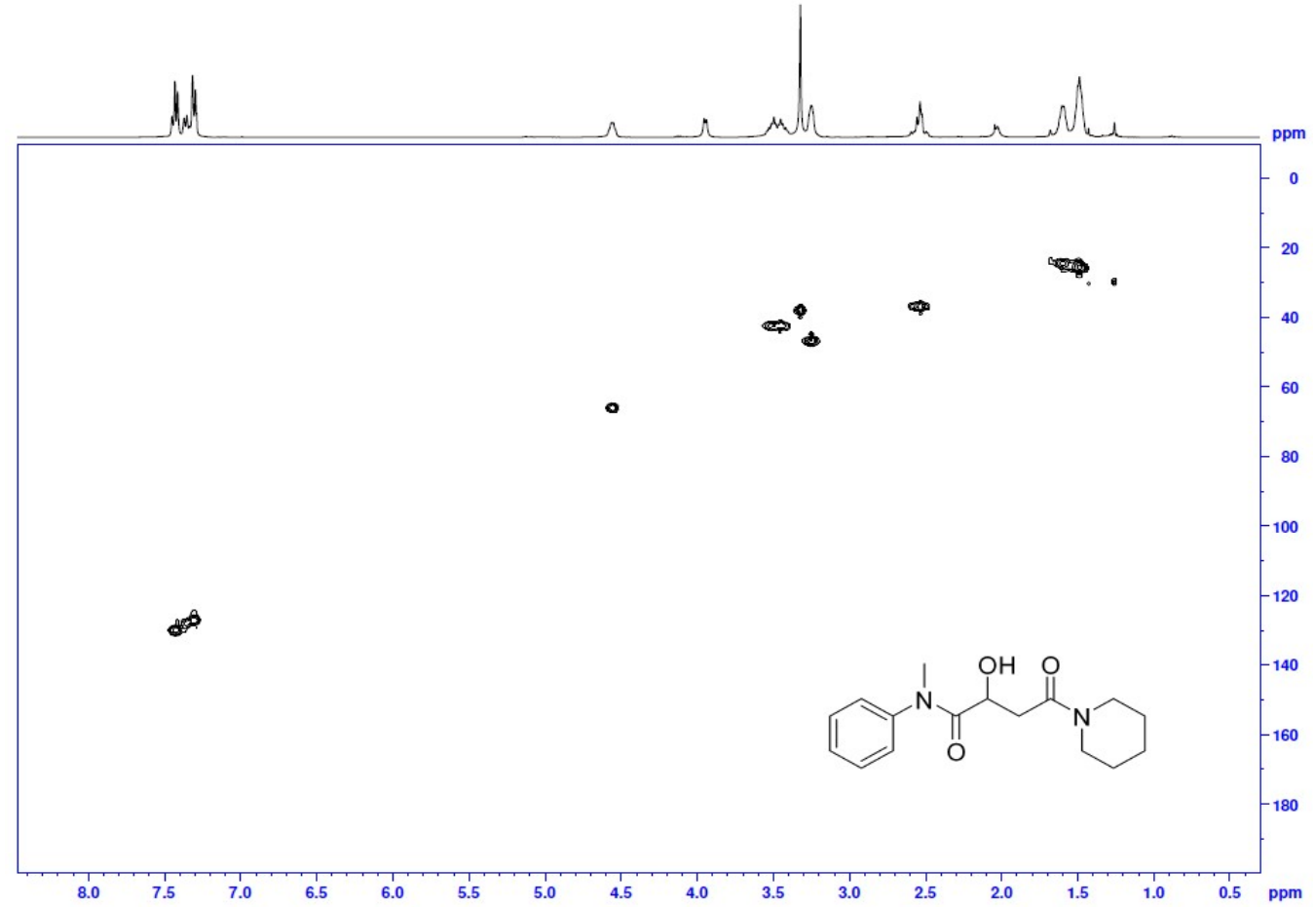




\section{Chiral HPLC of ketone precursor.}

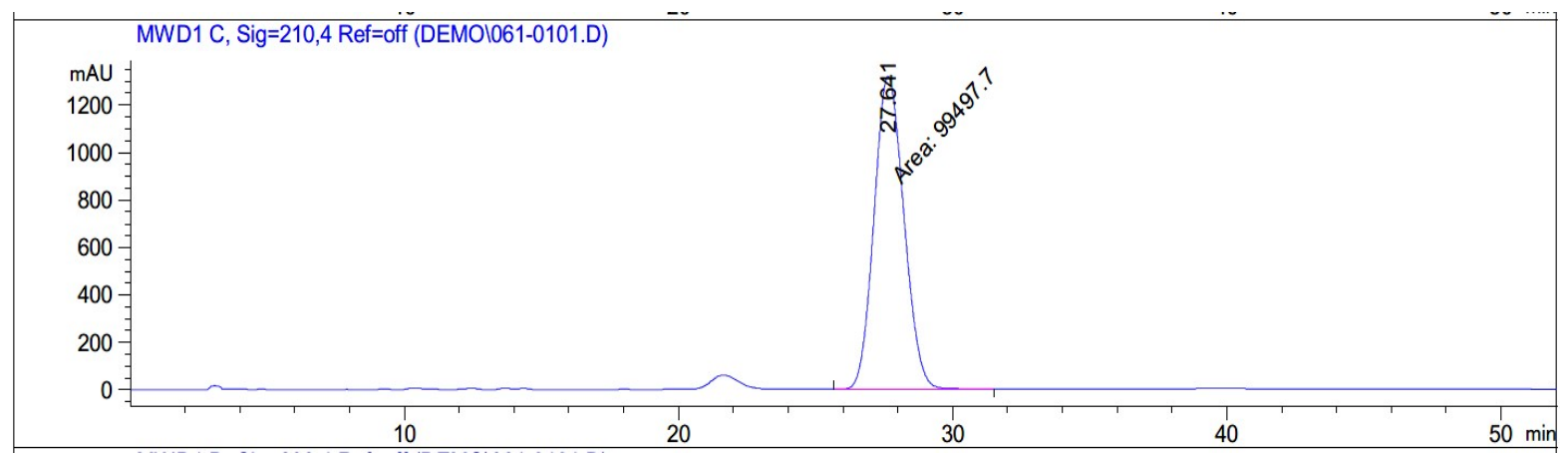

Signal 2: MWD1 C, Sig=210,4 Ref=off

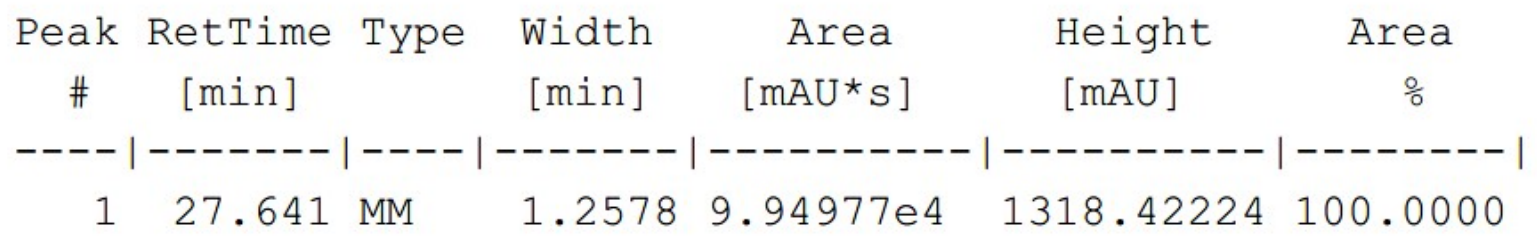

\section{Chiral HPLC of racemic alcohol.}

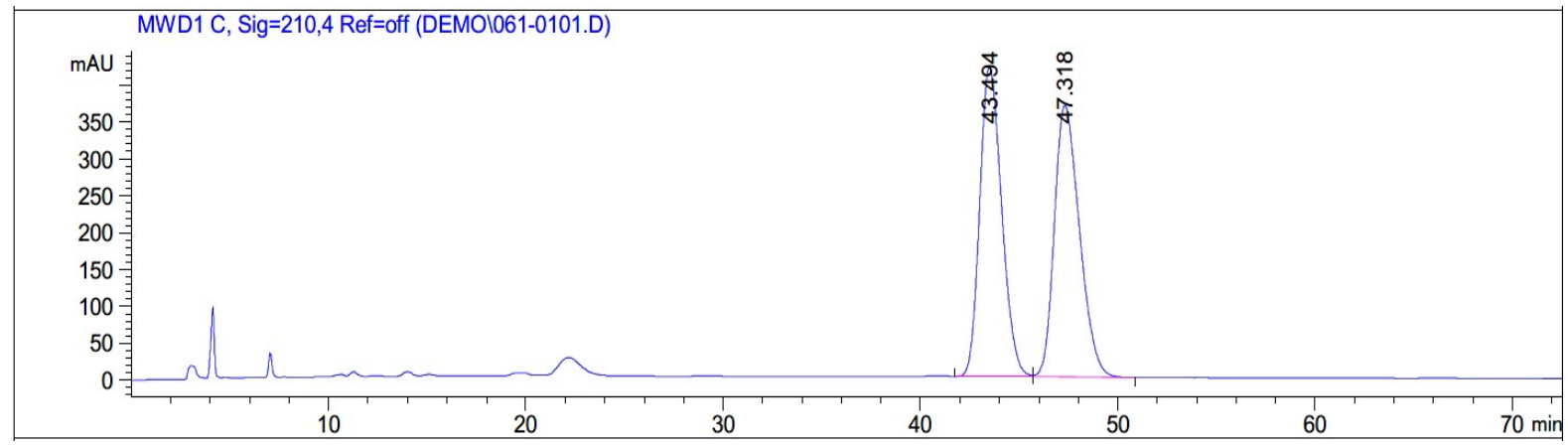

Signal 2: MWD1 C, Sig=210,4 Ref=off

\begin{tabular}{|c|c|c|c|c|c|c|}
\hline $\begin{array}{c}\text { Peak } \\
\quad \#\end{array}$ & $\begin{array}{l}\text { RetTime } \\
\text { [min] }\end{array}$ & Type & $\begin{array}{l}\text { Width } \\
\text { [min] }\end{array}$ & $\begin{array}{c}\text { Area } \\
{\left[\mathrm{mAU}^{\star} \mathrm{s}\right]}\end{array}$ & $\begin{array}{l}\text { Height } \\
\text { [mAU] }\end{array}$ & $\begin{array}{c}\text { Area } \\
\frac{\circ}{0}\end{array}$ \\
\hline 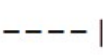 & 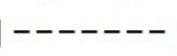 & & 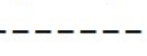 & -------1 & - - - - - - & $-------\mid$ \\
\hline 1 & 43.494 & BV & 1.2044 & $3.24076 \mathrm{e} 4$ & 419.89951 & 49.9009 \\
\hline 2 & 47.318 & VB & 1.3680 & $3.25363 e 4$ & 367.83243 & 50.0991 \\
\hline
\end{tabular}


Chiral HPLC of ATH product. 89\% ee.

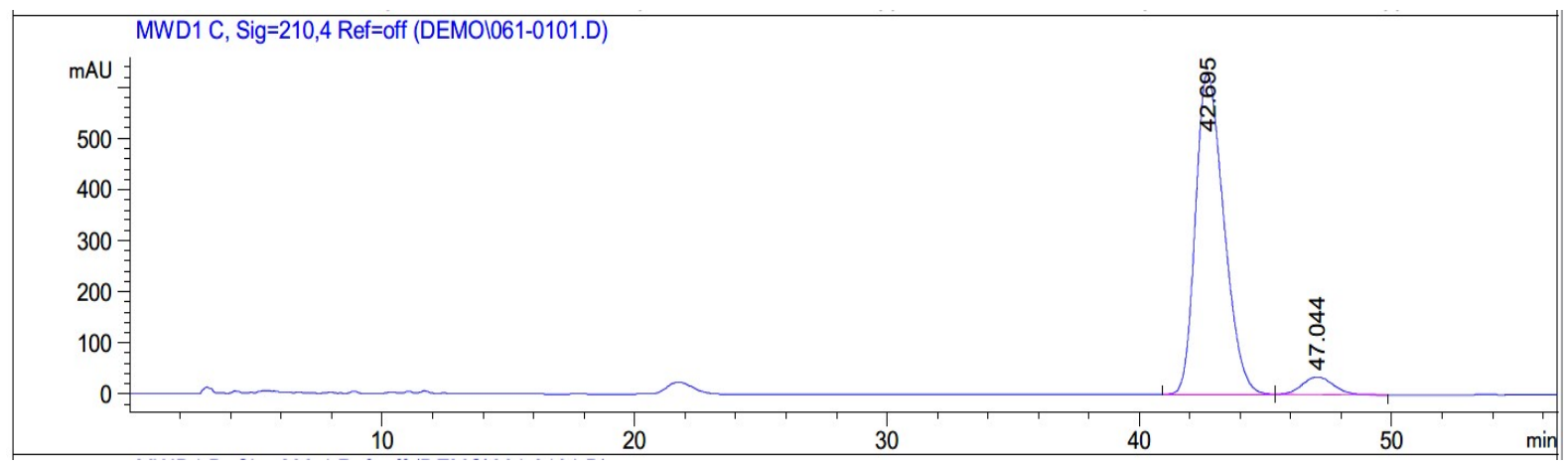

Signal 2: MWD1 C, Sig=210,4 Ref=off

\begin{tabular}{|c|c|c|c|c|c|c|}
\hline $\begin{array}{c}\text { Peak } \\
\#\end{array}$ & $\begin{array}{c}\text { RetTime } \\
\text { [min] }\end{array}$ & Type & $\begin{array}{l}\text { Width } \\
\text { [min] }\end{array}$ & $\begin{array}{c}\text { Area } \\
{\left[\mathrm{mAU}^{\star} \mathrm{s}\right]}\end{array}$ & $\begin{array}{l}\text { Height } \\
{[\mathrm{mAU}]}\end{array}$ & $\begin{array}{c}\text { Area } \\
\quad \%\end{array}$ \\
\hline- & ------ & - & ----- & ---- & $-----\mid$ & --- \\
\hline 1 & 42.695 & BB & 1.1962 & $4.89725 e 4$ & 627.74847 & 94.4034 \\
\hline 2 & 47.044 & $\mathrm{BB}$ & 1.2135 & 2903.30420 & 34.43969 & 5.5966 \\
\hline
\end{tabular}




\section{(R)-2-Hydroxy-1,4-di(piperidin-1-yl)butane-1,4-dione 22.}<smiles>O=C(CC(O)C(=O)N1CCCCC1)N1CCCCC1</smiles>

This compound is novel and was prepared following general procedure $\mathrm{C}$ using 1,4di(piperidin-1-yl)butane-1,2,4-trione (133 mg, $0.500 \mathrm{mmol}, 1.0 \mathrm{eq}), 3 \mathrm{C}$ tethered catalyst $(R, R)-2(4.7 \mathrm{mg}, 7.5 \mu \mathrm{mol}, 0.015 \mathrm{eq})$ and FA:TEA $(0.3 \mathrm{~mL})$ in DCM $(3 \mathrm{~mL})$ to give 22 as a clear semisolid material (110 mg, $0.410 \mathrm{mmol}, 82 \%)$.

TLC: $\mathrm{R}_{\mathrm{f}}$ ca 0.2 (4:6, hexane: EtOAc), not UV-active, light $\mathrm{KMnO}_{4}$, and strong PMA-reactive; HRMS (ESI) m/z: [M+Na] ${ }^{+}$Calcd for $\mathrm{C}_{14} \mathrm{H}_{24} \mathrm{~N}_{2} \mathrm{O}_{3} \mathrm{Na} 291.1679$; Found 291.1674 (error 1.6 ppm);

$U_{\max } 3419,2931,2849,1624,1439,1371,1254,1189,852,542 \mathrm{~cm}^{-1}$

Enantiomeric excess determined by HPLC analysis (Chiralpak IG, $250 \mathrm{~mm} \times 4.6 \mathrm{~mm}$ column, hexane: iPrOH 80:20, $1 \mathrm{~mL} / \mathrm{min}, \lambda=210 \mathrm{~nm}, \mathrm{~T}=25{ }^{\circ} \mathrm{C}$ ), $R$-enantiomer $33.8 \mathrm{~min}, S$ enantiomer $36.9 \min .92 \%$ ee $(R),[\alpha]_{\mathrm{D}}^{22}=-5.5\left(\mathrm{c}=0.1, \mathrm{CHCl}_{3}\right)$;

${ }^{1} \mathrm{H}$ NMR (500 MHz, $\left.\mathrm{CDCl}_{3}\right) \delta$ 4.84- $4.81(1 \mathrm{H}, \mathrm{m}, \mathrm{C} \underline{\mathrm{HOH}}), 4.42(1 \mathrm{H}, \mathrm{d}, J=6.5 \mathrm{~Hz}, \mathrm{OH})$, 3.68-3.394 (8H, m, $\mathrm{NCH}_{2}$ of piperidine), $2.73\left(1 \mathrm{H}, \mathrm{dd}, J=15.5,8.5 \mathrm{~Hz}, \mathrm{C}_{\mathrm{a}} \mathrm{H}_{\mathrm{b}}\right), 2.46(1 \mathrm{H}$, $\left.\mathrm{dd}, \mathrm{J}=15.5,2.8 \mathrm{~Hz}, \mathrm{CH}_{\mathrm{a}} \underline{\mathrm{H}}_{\mathrm{b}}\right), 1.68-1.50\left(12 \mathrm{H}, \mathrm{m}, \mathrm{CH}_{2}\right.$ of piperidine);

${ }^{13} \mathrm{C}$ NMR $\left(126 \mathrm{MHz}, \mathrm{CDCl}_{3}\right) \delta 171.2,169.3,66.6,47.1,46.3,43.7,42.9,37.6,26.5,26.4$, 25.6, 24.6, 24.5;

$m / z(\mathrm{ESI}) 291.1\left[(\mathrm{M}+\mathrm{Na})^{+}, 100 \%\right]$. 
2-Hydroxy-1,4-di(piperidin-1-yl)butane-1,4-dione 22.

${ }^{1} \mathrm{H}$ NMR $\left(500 \mathrm{MHz}, \mathrm{CDCl}_{3}\right)$.

Jul23-2020
Chemist Shweta Gediya
SKG479
PROTON.w CDCI3 /opt/topspin3.2 SKG 49

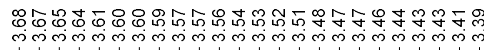
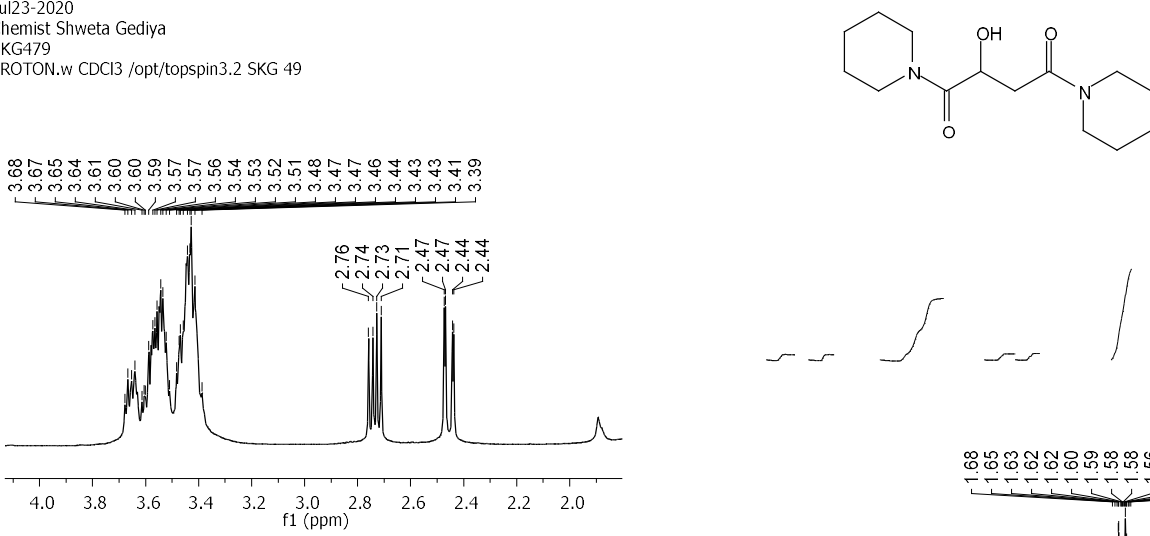

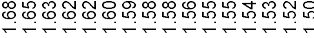

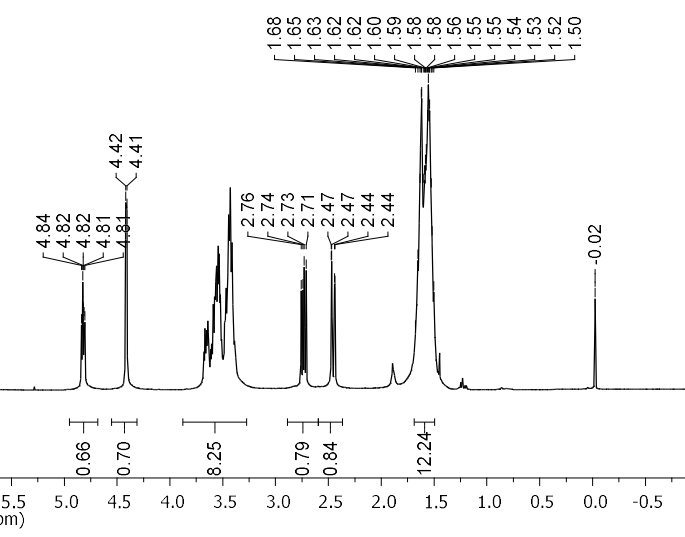

${ }^{13} \mathrm{C}$ NMR (126 MHz, $\left.\mathrm{CDCl}_{3}\right)$.

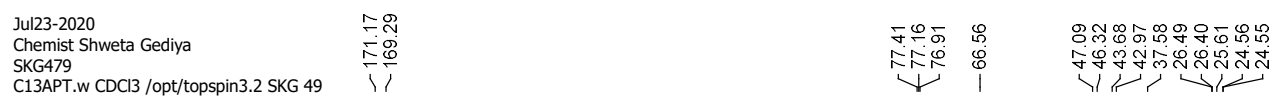<smiles>O=C(CC(O)C(=O)N1CCCCC1)N1CCCCC1</smiles>

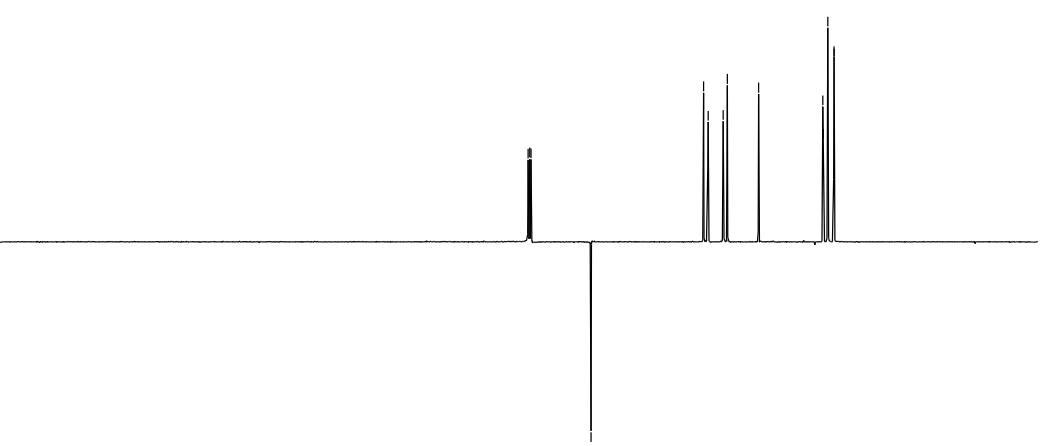

$\begin{array}{lllllllllllllllllllllllllll}230 & 220 & 210 & 200 & 190 & 180 & 170 & 160 & 150 & 140 & 130 & 120 & 110 & 100 & 90 & 80 & 70 & 60 & 50 & 40 & 30 & 20 & 10 & 0 & -10\end{array}$ 
COSY.

Chemist Shweta Gediya

SKG 479
COSY.w CDCl3/opt/topspin3.2 SKG 49

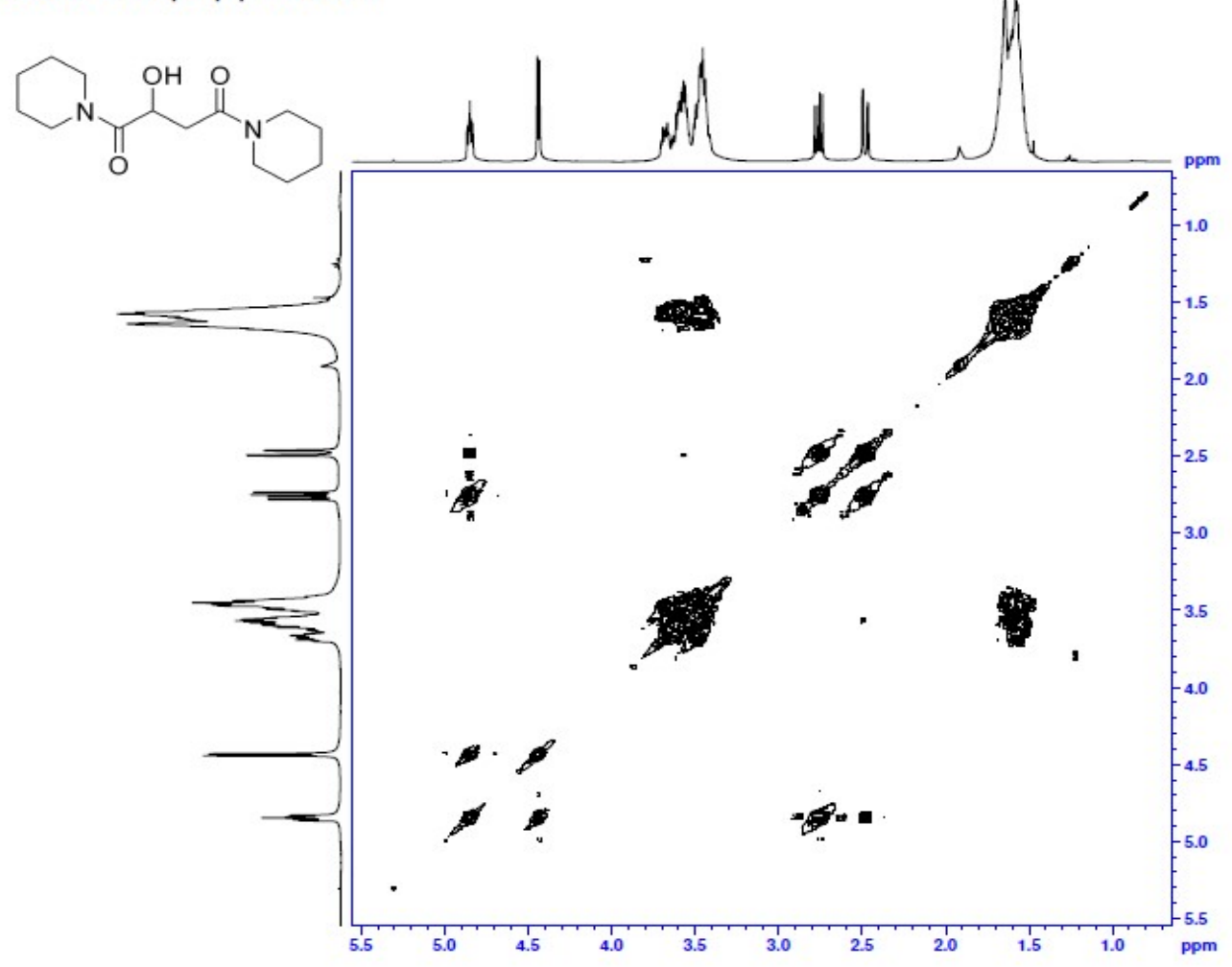

HSQC.

Chemist Shweta Gediya

HSG 479 C.w CDCl3 /opttopspin 3.2 SKG 49

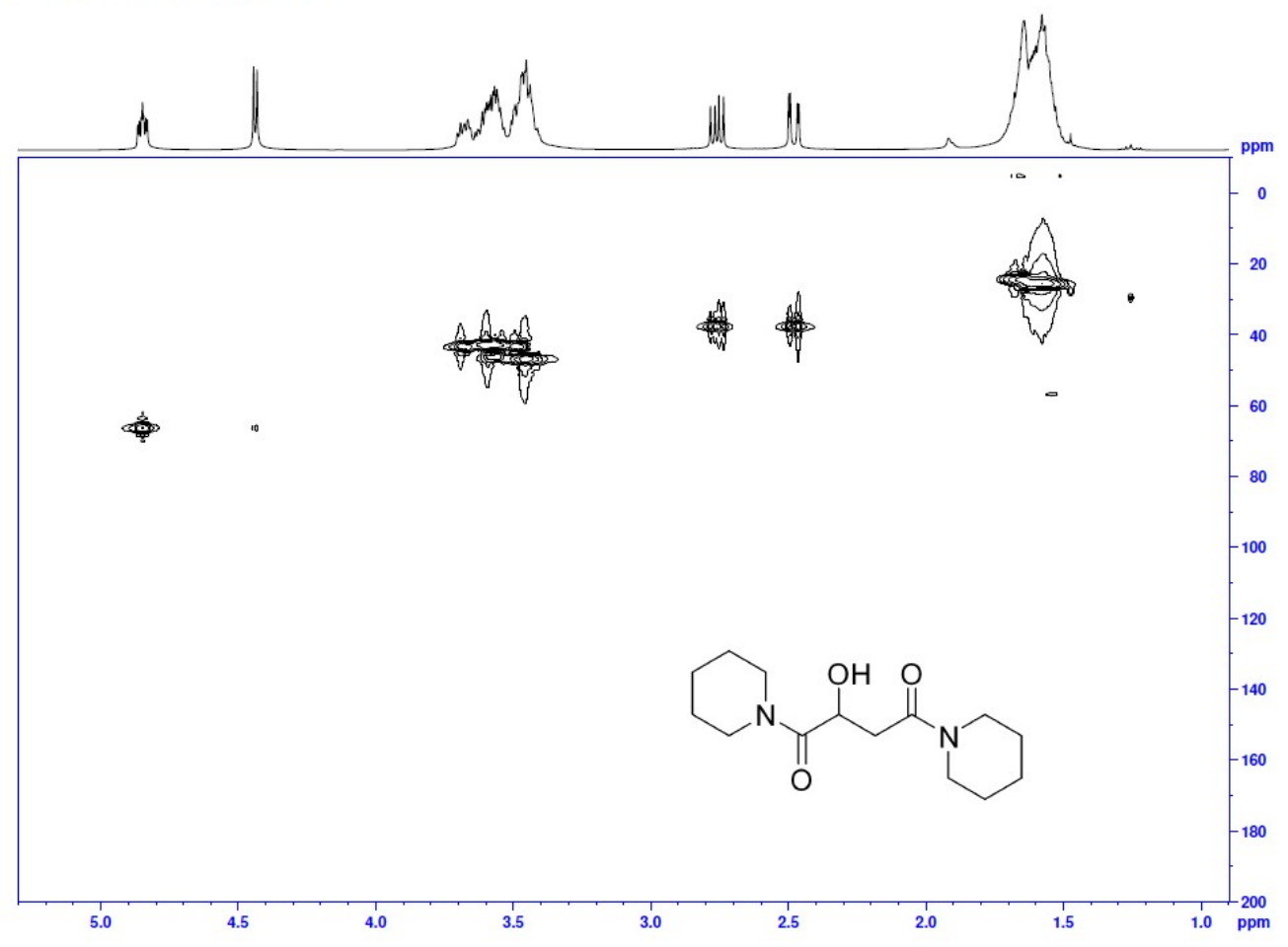

S283 


\section{HPLC of Ketone precursor.}

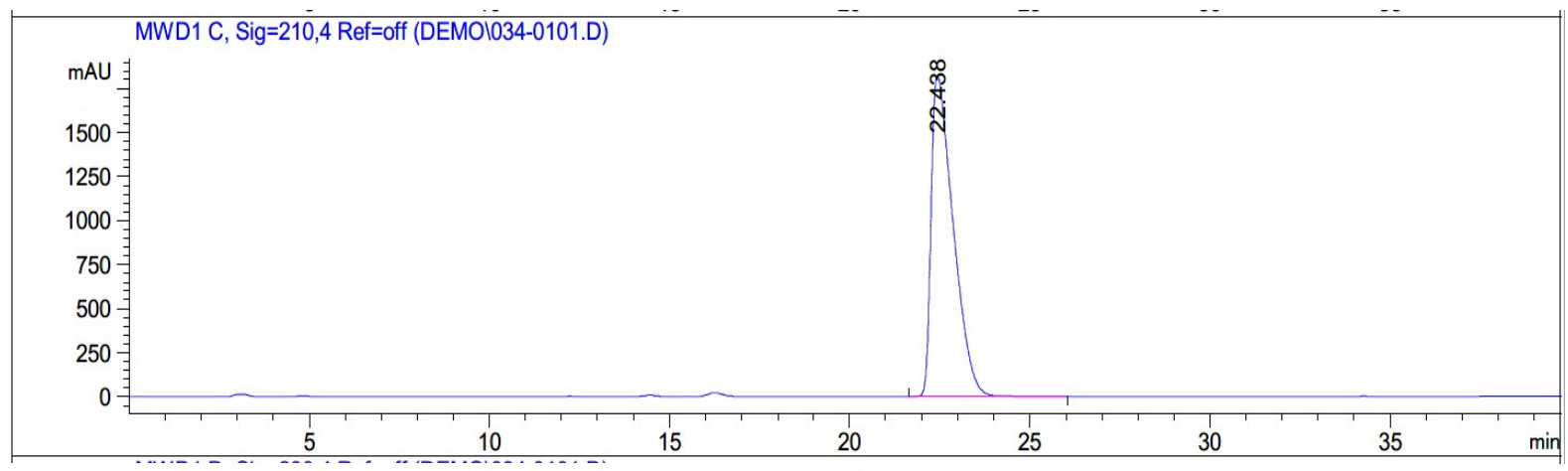

Signal 2: MWD1 C, Sig=210,4 Ref=off

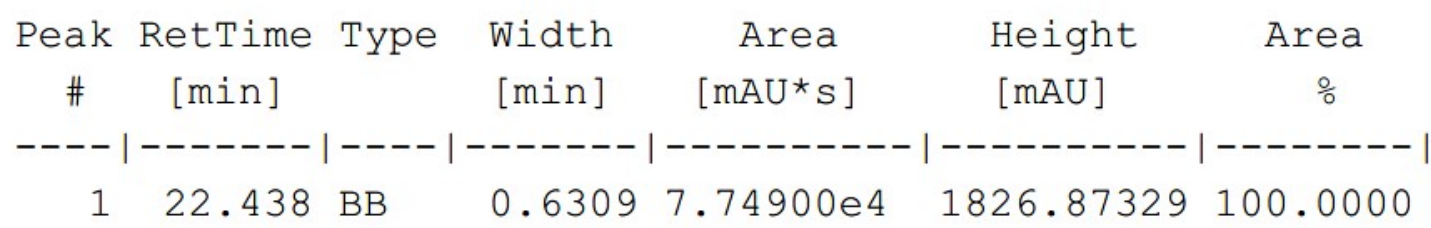

HPLC of racemic alcohol.

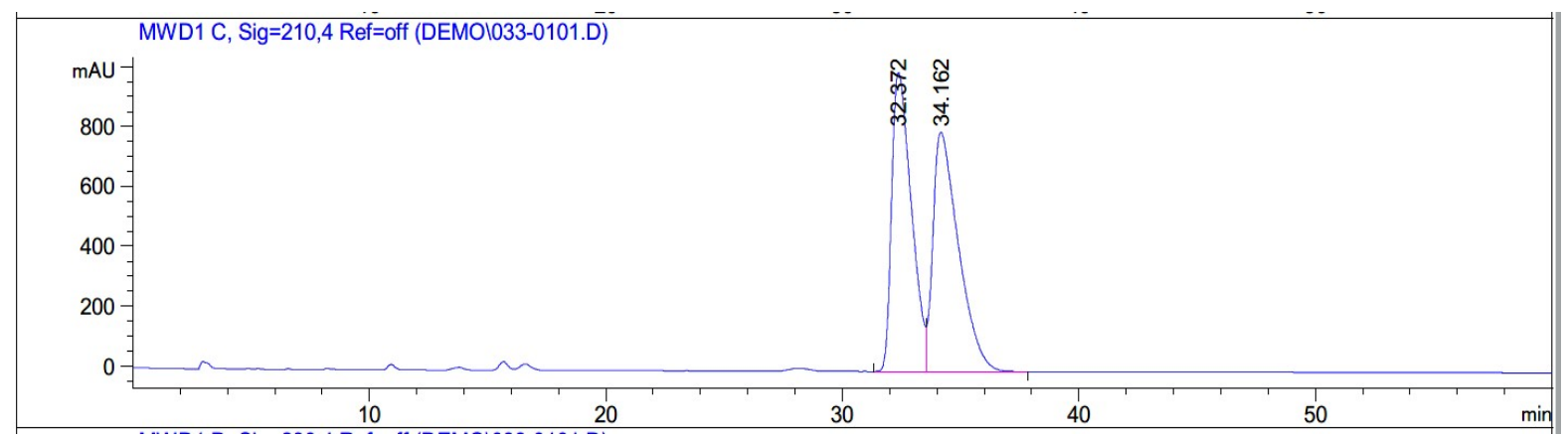

Signal 2: MWD1 C, Sig=210,4 Ref=off

\begin{tabular}{|c|c|c|c|c|c|c|}
\hline eak & $\begin{array}{c}\text { RetTime } \\
\text { [min] }\end{array}$ & ype & $\begin{array}{l}\text { Width } \\
\text { [min] }\end{array}$ & $\begin{array}{c}\text { Area } \\
{\left[\mathrm{mAU}{ }^{\star} \mathrm{s}\right]}\end{array}$ & $\begin{array}{l}\text { Height } \\
\text { [mAU] }\end{array}$ & $\begin{array}{c}\text { Area } \\
\quad \frac{\circ}{0}\end{array}$ \\
\hline \multicolumn{7}{|c|}{$---|-------|----|-------|----------|----------|--------\mid$} \\
\hline 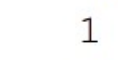 & & & & & & 06 \\
\hline 2 & & & 87 & $6.02331 e 4$ & 799.68976 & 1494 \\
\hline
\end{tabular}


Chiral HPLC of ATH product. $92 \%$ ee.

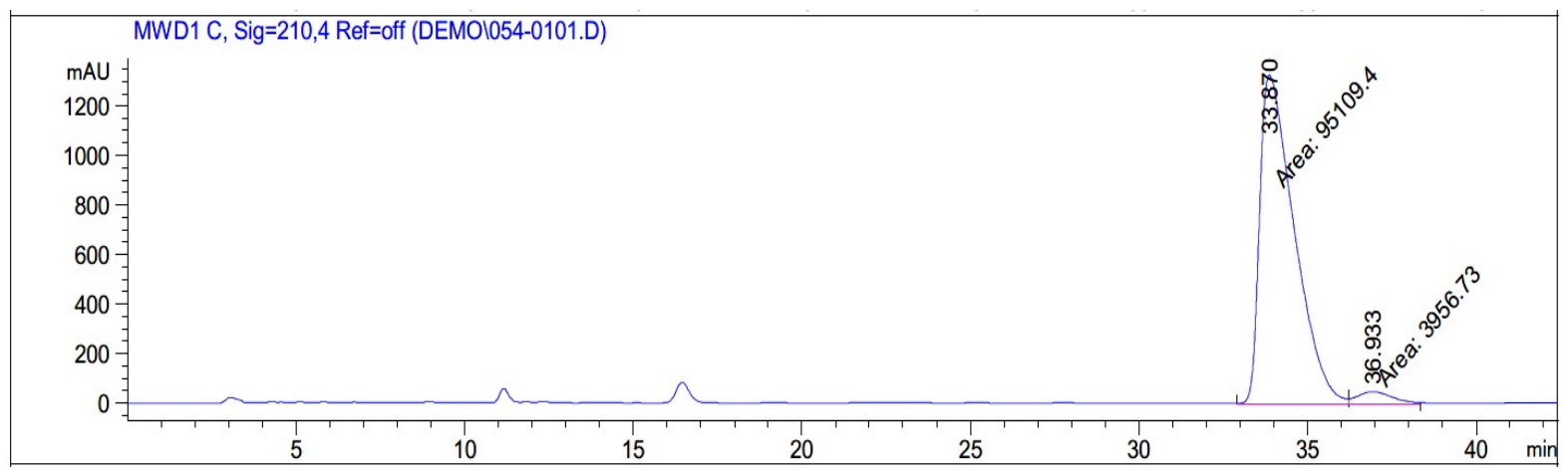

Signal 2: MWD1 C, Sig=210, 4 Ref=off

\begin{tabular}{|c|c|c|c|c|c|c|}
\hline $\begin{array}{c}\text { Peak } \\
\#\end{array}$ & $\begin{array}{c}\text { RetTime } \\
\text { [min] }\end{array}$ & Type & $\begin{array}{l}\text { Width } \\
\text { [min] }\end{array}$ & $\begin{array}{c}\text { Area } \\
{\left[\mathrm{mAU}^{*} \mathrm{~s}\right]}\end{array}$ & $\begin{array}{l}\text { Height } \\
{[\mathrm{mAU}]}\end{array}$ & $\begin{array}{c}\text { Area } \\
\frac{\circ}{0}\end{array}$ \\
\hline & & & & . & $1--0$ & -- \\
\hline 1 & & & & 9.51 & 1332 & 060 \\
\hline 2 & 36.933 & M & 99 & 3956.72754 & 75933 & 940 \\
\hline
\end{tabular}


(R)-2-Hydroxy-1-morpholino-4-(piperidin-1-yl)butane-1,4-dione 23.<smiles>O=C(C[C@H](O)C(=O)N1CCOCC1)N1CCCCC1</smiles>

This compound is novel and was prepared following general procedure $\mathrm{C}$ using 1morpholino-4-(piperidin-1-yl)butane-1,2,4-trione (0.135 g, $0.500 \mathrm{mmol}, 1.0 \mathrm{eq}), 3 \mathrm{C}$ tethered catalyst $(R, R)-2(4.7 \mathrm{mg}, 7.5 \mu \mathrm{mol}, 0.015 \mathrm{eq})$ and FA:TEA $(0.3 \mathrm{~mL})$ in DCM $(3 \mathrm{~mL})$ to give 23 as a clear semisolid material (0.115 g, $0.429 \mathrm{mmol}, 86 \%)$.

TLC: $\mathrm{R}_{\mathrm{f}}$ ca 0.2 (1:9, hexane: EtOAc), not UV-active, light $\mathrm{KMnO}_{4}$, and strong PMA-reactive; HRMS (ESI) m/z: [M+Na] $]^{+}$Calcd for $\mathrm{C}_{13} \mathrm{H}_{22} \mathrm{~N}_{2} \mathrm{O}_{4} \mathrm{Na} 293.1472$; Found 293.1465 (error 2.4 ppm);

$U_{\max } 3411,2923,2856,1609,1441,1271,1226,11111024,572 \mathrm{~cm}^{-1}$;

Enantiomeric excess determined by HPLC analysis (Chiralpak IG, $250 \mathrm{~mm} \times 4.6 \mathrm{~mm}$ column, hexane: $\left.\mathrm{iPrOH} 90: 10,1 \mathrm{~mL} / \mathrm{min}, \lambda=210 \mathrm{~nm}, \mathrm{~T}=25^{\circ} \mathrm{C}\right), R$-enantiomer $105.9 \mathrm{~min}, S$ enantiomer $112.0 \min .92 \%$ ee $(R),[\alpha]_{\mathrm{D}}^{22}=-4.5\left(\mathrm{c}=0.1, \mathrm{CHCl}_{3}\right)$;

${ }^{1} \mathrm{H}$ NMR (400 MHz, $\left.\mathrm{CDCl}_{3}\right) \delta$ 4.76-4.74 (2H, m, $\left.\underline{\mathrm{HOH}}+\mathrm{OH}\right), 3.72-3.67\left(6 \mathrm{H}, \mathrm{m}, \mathrm{CH}_{2}\right.$ of morpholine), 3.57-3.51 (4H, m, $\mathrm{CH}_{2}$ of morpholine/piperidine), 3.45-3.35 (2H, m, $\mathrm{NCH}_{2}$ of piperidine), $2.80\left(1 \mathrm{H}, \mathrm{dd}, J=15.9,7.6 \mathrm{~Hz}, \mathrm{C}_{\underline{\mathrm{a}}} \mathrm{H}_{\mathrm{b}}\right), 2.55\left(1 \mathrm{H}, \mathrm{dd}, J=15.9,2.8 \mathrm{~Hz}, \mathrm{CH}_{\mathrm{a}} \underline{\mathrm{H}_{\mathrm{b}}}\right)$, 1.71-1.49 (6H, m, $\mathrm{CH}_{2}$ of piperidine);

${ }^{13} \mathrm{C}$ NMR $\left(101 \mathrm{MHz}, \mathrm{CDCl}_{3}\right) \delta 171.1,169.8,66.9,66.8,46.7,46.1,42.9,42.8,36.5,26.4$, 25.6, 24.5;

$m / z(\mathrm{ESI}) 293.1\left[(\mathrm{M}+\mathrm{Na})^{+}, 100 \%\right]$. 
(R)- 2-Hydroxy-1-morpholino-4-(piperidin-1-yl)butane-1,4-dione 23.

${ }^{1} \mathrm{H}$ NMR (400 MHz, $\mathrm{CDCl}_{3}$ ).

Sep11-2020

SKG507 B
PROTON.w CDCl3 /opt/topspin3.5pl2 SKG 59

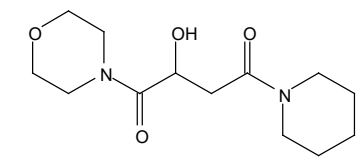

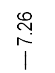

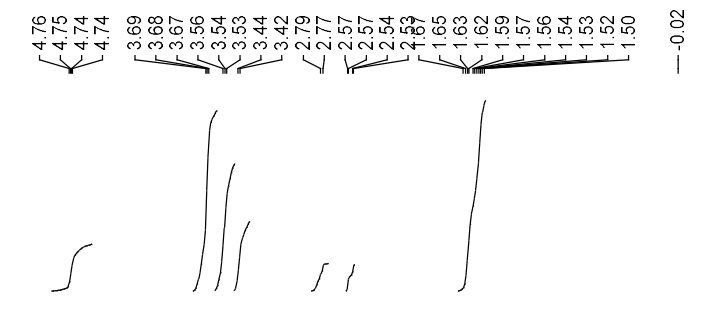

${ }^{13} \mathrm{C}$ NMR $\left(101 \mathrm{MHz}, \mathrm{CDCl}_{3}\right)$.

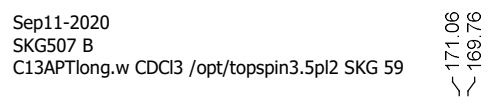

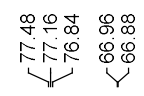

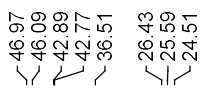
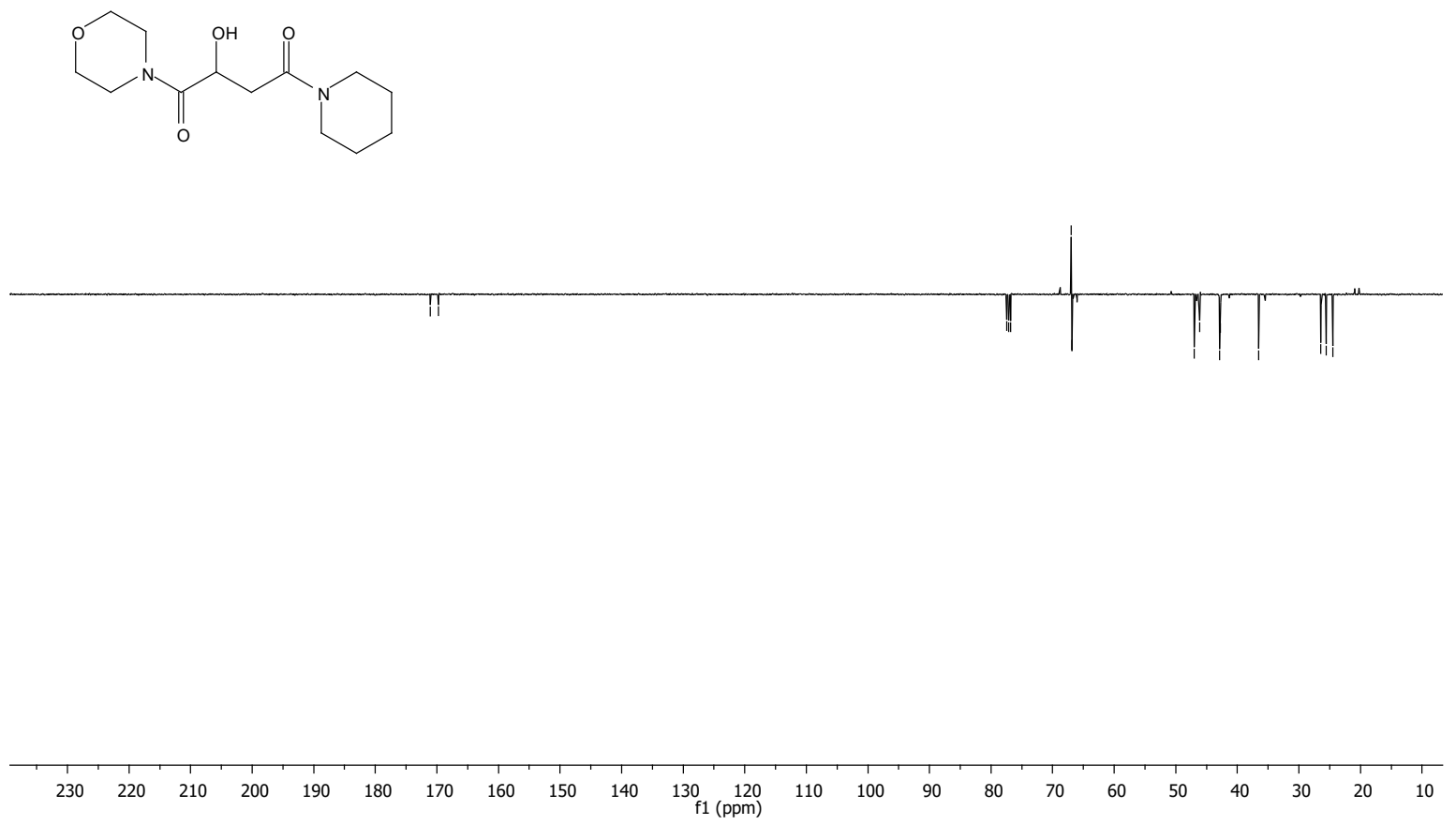

S287 
COSY.

SKG507 B

COSY.w CDCl3/opt/topspin3.5pl2 SKG 59

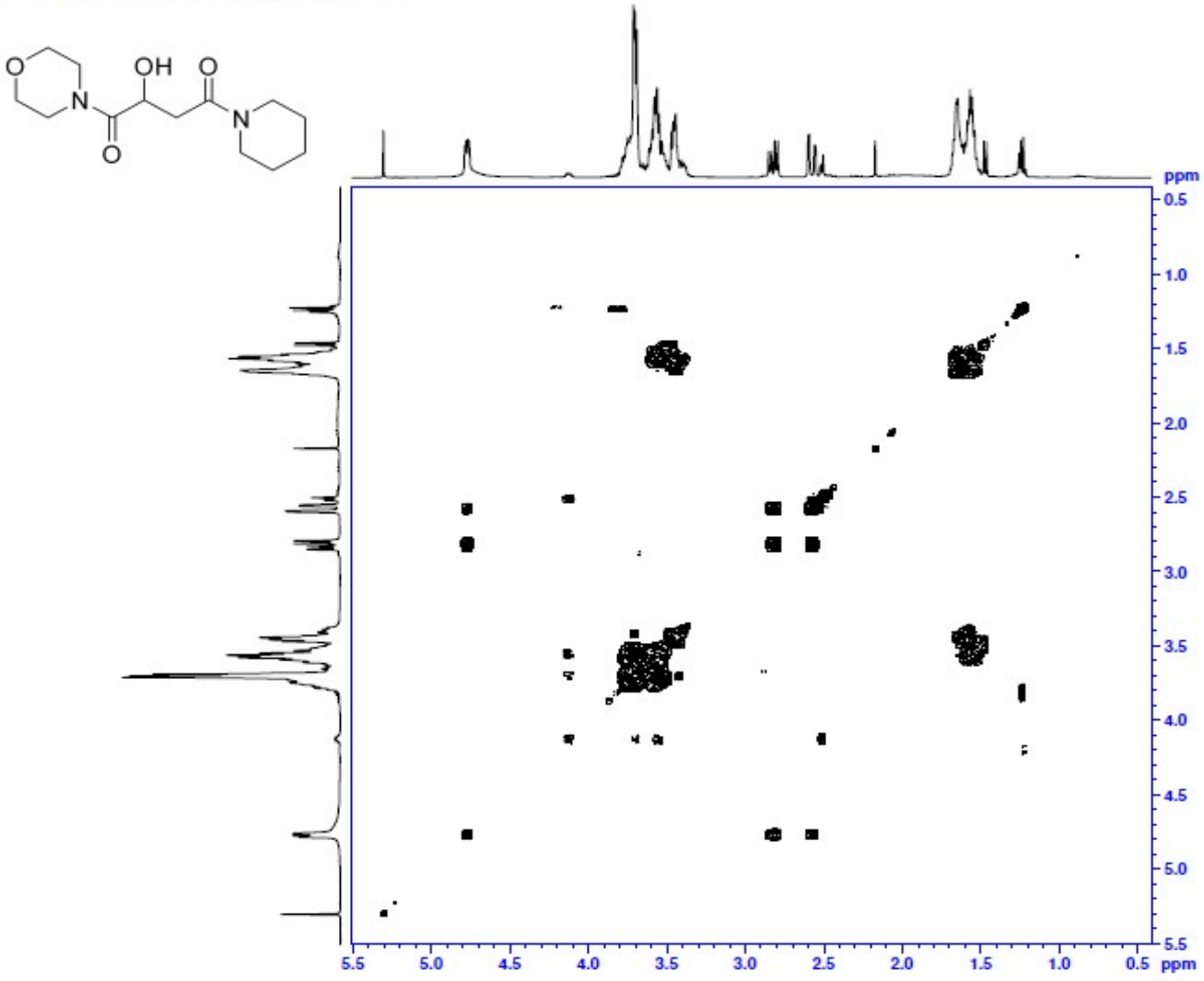

HSQC.

HSQC.W CDCl3/opt/topspin3.5pl2 SKG 59

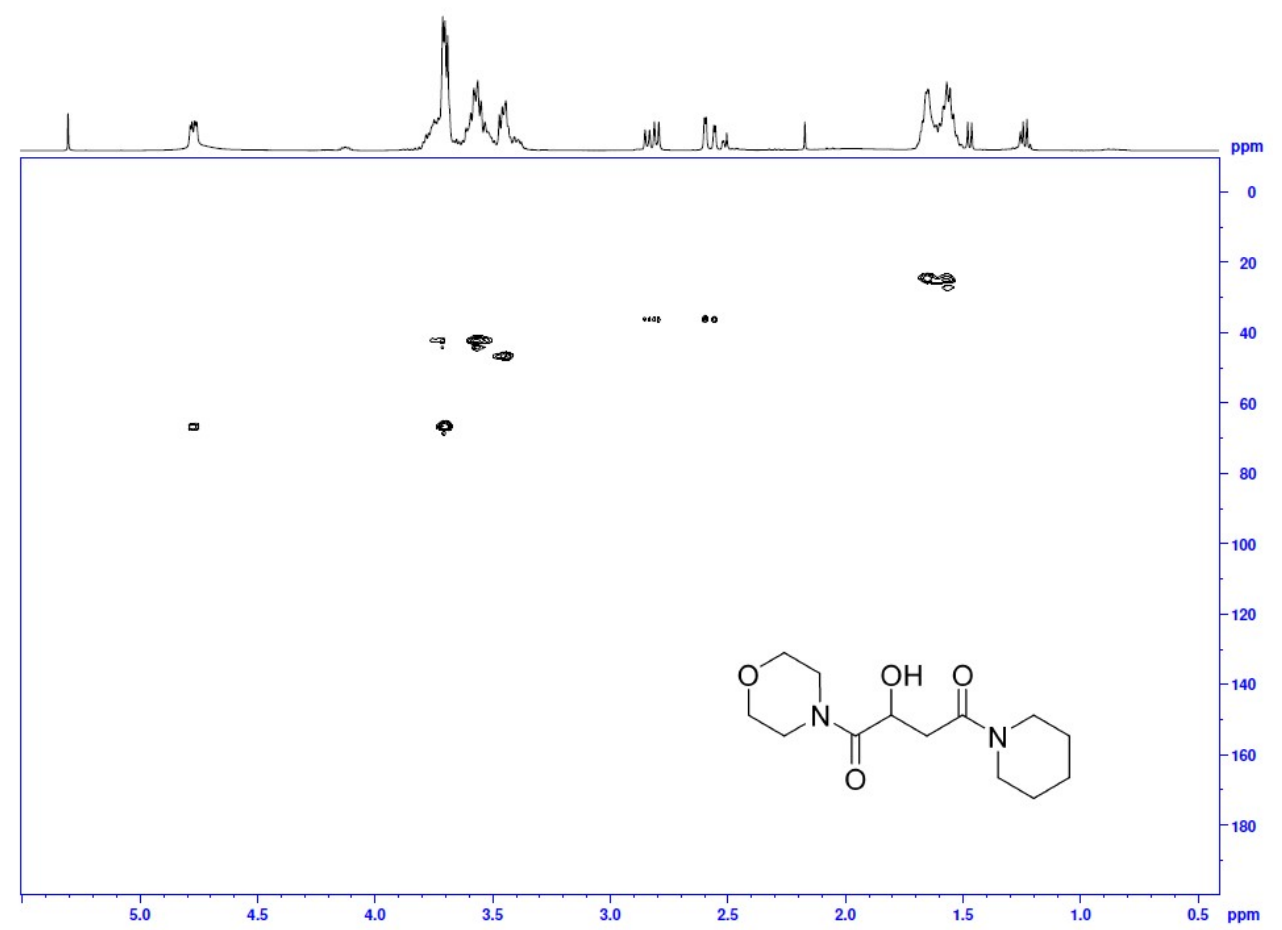




\section{Chiral HPLC of ketone precursor.}

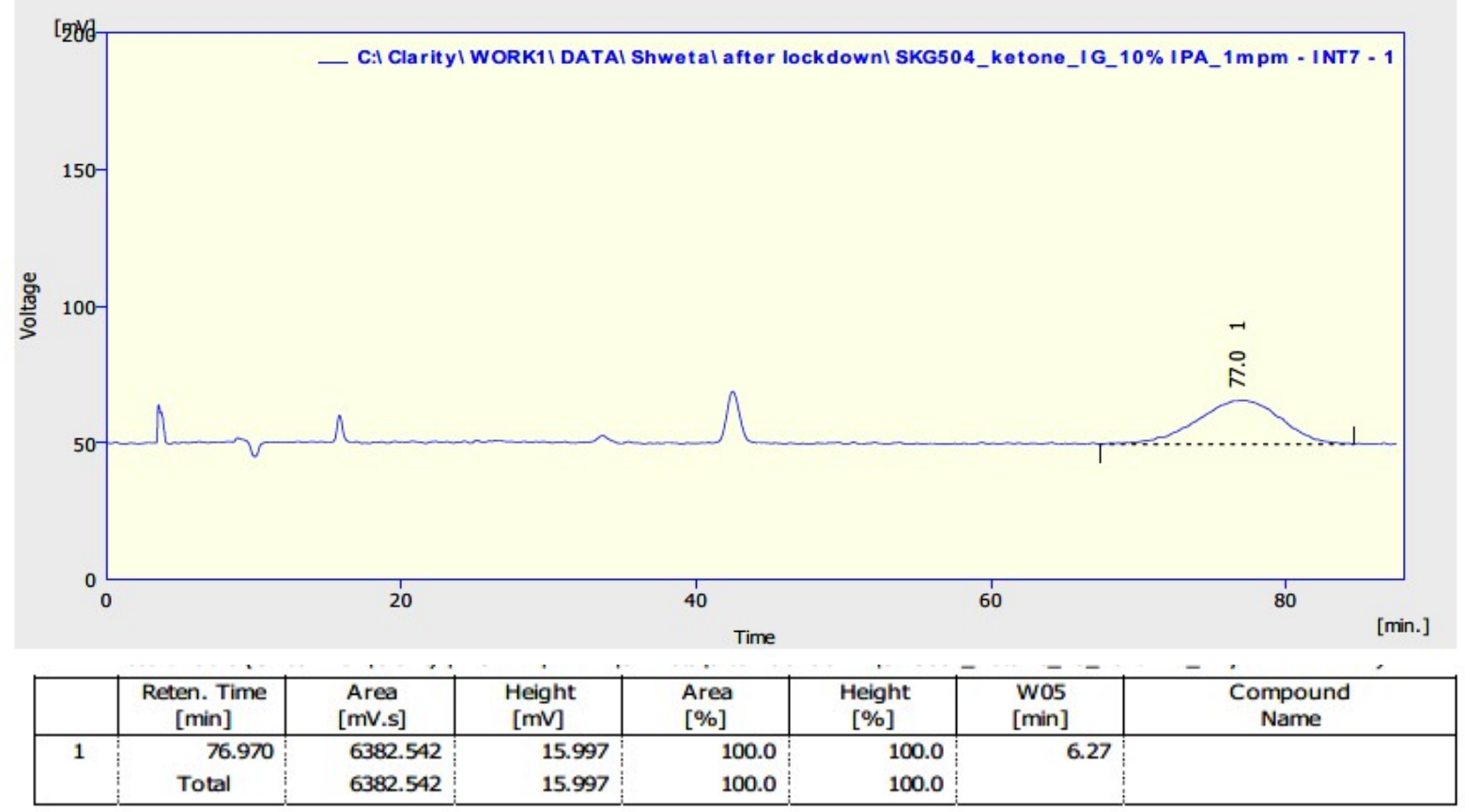

Chiral HPLC of racemic alcohol.

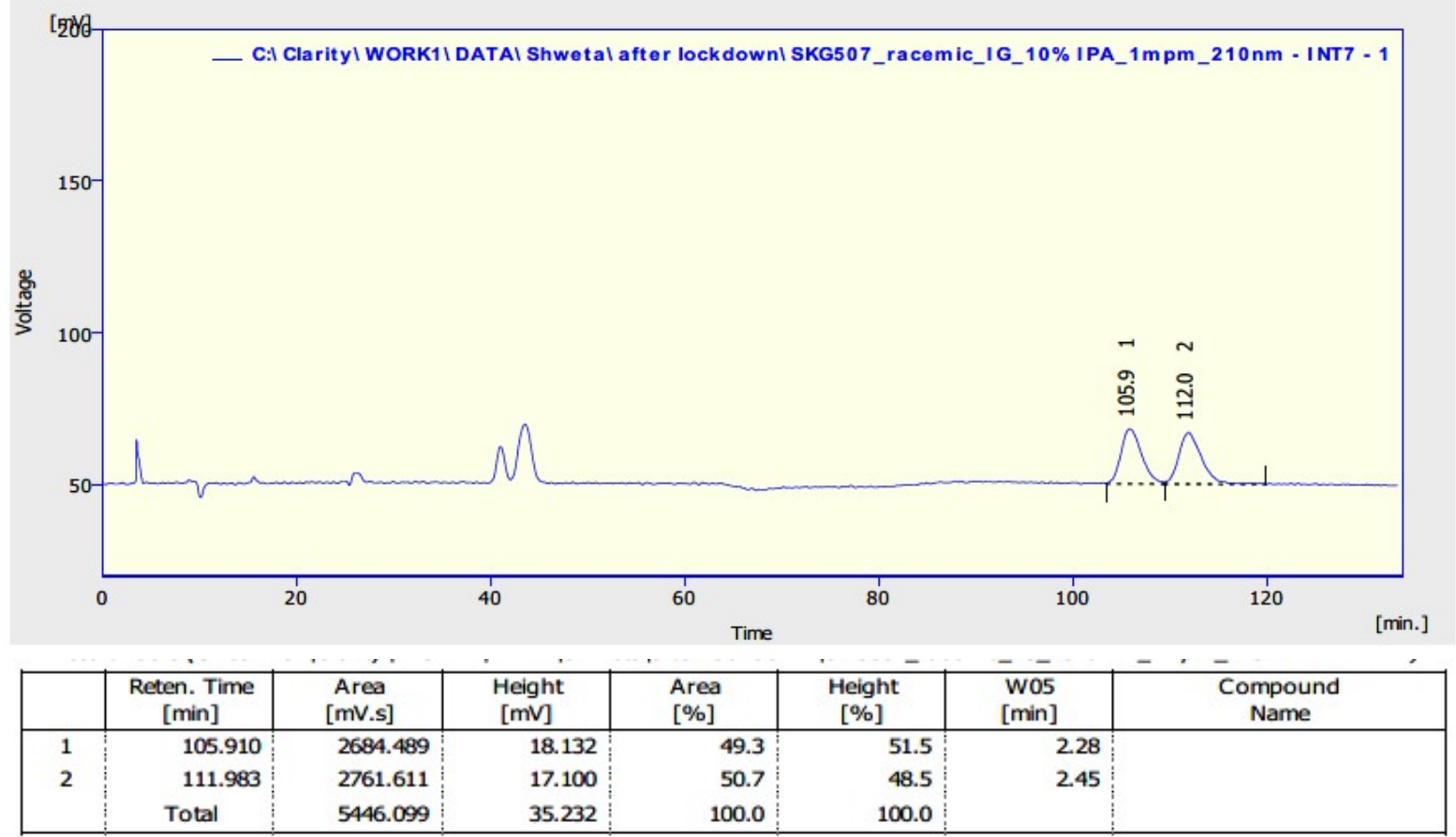


Chiral HPLC of ATH product. 92\% ee.

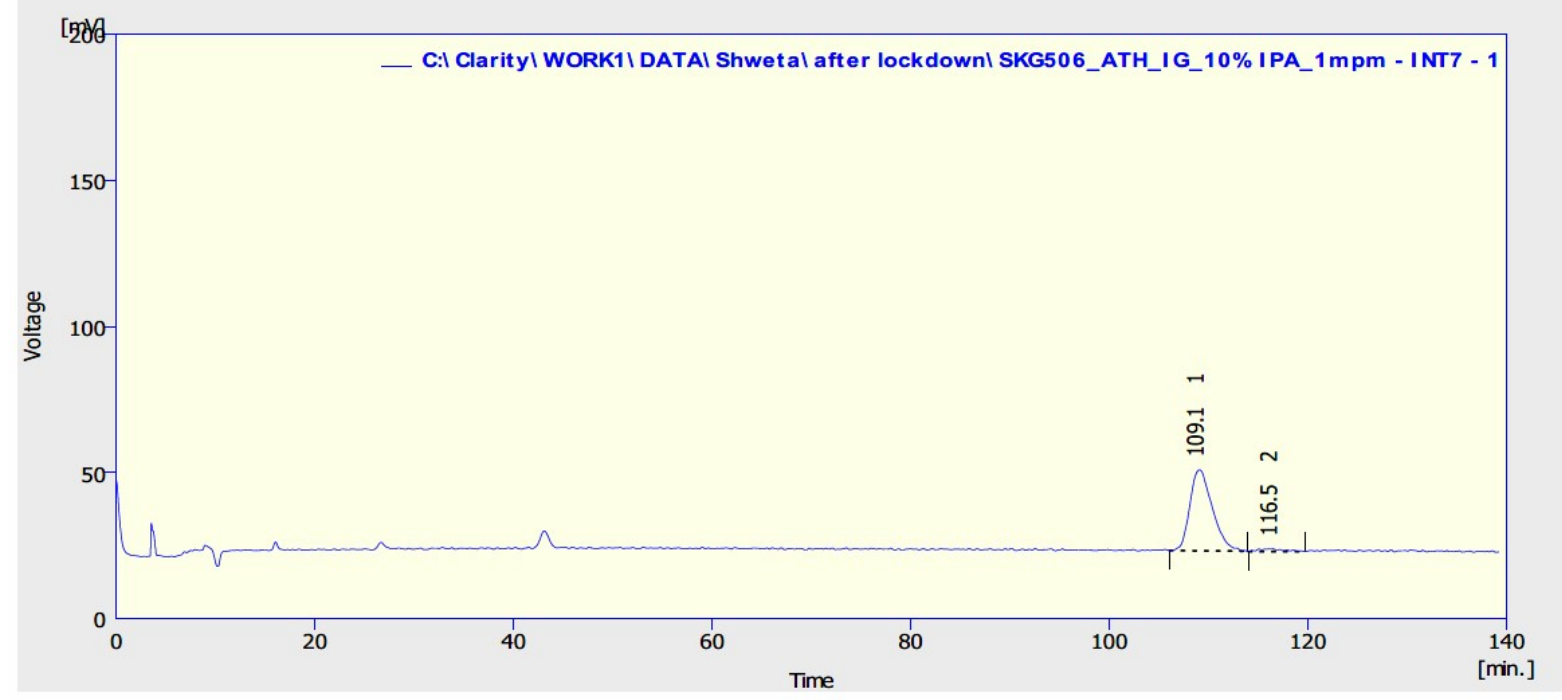

\begin{tabular}{|c|c|c|c|c|c|c|c|}
\hline & $\begin{array}{l}\text { Reten. Time } \\
\text { [min] }\end{array}$ & $\begin{array}{c}\text { Area } \\
\text { [mV.s] }\end{array}$ & $\begin{array}{c}\text { Height } \\
{[\mathrm{mV}]}\end{array}$ & $\begin{array}{l}\text { Area } \\
{[\%]}\end{array}$ & $\begin{array}{l}\text { Height } \\
{[\%]}\end{array}$ & $\begin{array}{l}\text { W05 } \\
\text { [min] }\end{array}$ & $\begin{array}{c}\text { Compound } \\
\text { Name }\end{array}$ \\
\hline 1 & 109.077 & 4371.151 & 27.693 & 96.0 & 96.5 & 2.43 & \\
\hline \multirow[t]{2}{*}{2} & 116.467 & 181.909 & 1.018 & 4.0 & 3.5 & 2.49 & \\
\hline & Total & 4553.060 & 28.711 & 100.0 & 100.0 & & \\
\hline
\end{tabular}




\section{(R)-2-Hydroxy-4-oxo- $N$-phenyl-4-(piperidin-1-yl)butanamide 24.}<smiles>O=C(Nc1ccccc1)C(O)CC(=O)N1CCCCC1</smiles>

This compound is novel and was prepared following general procedure $\mathrm{C}$ using 2,4-dioxo- $\mathrm{N}$ phenyl-4-(piperidin-1-yl)butanamide $(68.5 \mathrm{mg}, 0.250 \mathrm{mmol}, 1.0 \mathrm{eq}), 3 \mathrm{C}$ tethered catalyst $(R, R)-2(2.3 \mathrm{mg}, 3.8 \mu \mathrm{mol}, 0.015 \mathrm{eq})$ and FA:TEA $(0.15 \mathrm{~mL})$ in DCM $(1.5 \mathrm{~mL})$ to give 24 as a yellow solid (61.1 mg, $0.221 \mathrm{mmol}, 88 \%)$.

TLC: $\mathrm{R}_{\mathrm{f}}$ ca 0.2 (8:2, hexane: EtOAc), UV-active, strong $\mathrm{KMnO}_{4}$, and PMA-reactive; MP: $120-123^{\circ} \mathrm{C}$;

HRMS (ESI) m/z: [M+Na] ${ }^{+}$Calcd for $\mathrm{C}_{15} \mathrm{H}_{20} \mathrm{~N}_{2} \mathrm{O}_{3} \mathrm{Na}$ 299.1366; Found 299.1362 (error 1.4 ppm);

$U_{\max } 3295,2923,1660,1619,1515,1255,1104,755 \mathrm{~cm}^{-1}$;

Enantiomeric excess determined by HPLC analysis (Chiralpak IC, $250 \mathrm{~mm} \times 4.6 \mathrm{~mm}$ column, hexane: $\left.\mathrm{iPrOH} 80: 20,1 \mathrm{~mL} / \mathrm{min}, \lambda=254 \mathrm{~nm}, \mathrm{~T}=25{ }^{\circ} \mathrm{C}\right), S$-enantiomer $23.5 \mathrm{~min}, R$ enantiomer $29.1 \mathrm{~min} .97 \%$ ee $(R),[\alpha]_{\mathrm{D}}^{22}=+8\left(\mathrm{c}=0.025, \mathrm{CHCl}_{3}\right)$;

${ }^{1} \mathrm{H}$ NMR (500 MHz, $\left.\mathrm{CDCl}_{3}\right) \delta 8.91(1 \mathrm{H}, \mathrm{s}, \mathrm{CONH}), 7.59$ (2H, d, $\left.J=7.8 \mathrm{~Hz}, \mathrm{ArH}\right), 7.33(2 \mathrm{H}$, $\mathrm{t}, J=7.8 \mathrm{~Hz}, \operatorname{ArH}), 7.12(1 \mathrm{H}, \mathrm{t}, J=7.4 \mathrm{~Hz}, \mathrm{ArH}), 5.47(1 \mathrm{H}, \mathrm{d}, J=4.4 \mathrm{~Hz}, \mathrm{CHO} \underline{\mathrm{H}}), 4.56-4.53$ $(1 \mathrm{H}, \mathrm{m}, \mathrm{C} \underline{\mathrm{HOH}}), 3.60-3.52\left(2 \mathrm{H}, \mathrm{m}, \mathrm{NCH}_{2}\right.$ of piperidine), 3.42-3.40 (2H, m, $\mathrm{NCH}_{2}$ of piperidine), $2.99\left(1 \mathrm{H}, \mathrm{dd}, J=16.6,4.1 \mathrm{~Hz}, \mathrm{C}_{\underline{a}} \mathrm{H}_{\mathrm{b}}\right), 2.82\left(1 \mathrm{H}, \mathrm{dd}, J=16.6,7.5 \mathrm{~Hz}, \mathrm{CH}_{\mathrm{a}} \underline{\mathrm{H}}\right)$, 1.67-1.53 (6H, m, $\mathrm{CH}_{2}$ of piperidine);

${ }^{13} \mathrm{C} \mathrm{NMR}\left(126 \mathrm{MHz}, \mathrm{CDCl}_{3}\right) \delta 171.0,170.3,137.5,129.1,124.5,119.8,69.5,46.8,42.9$, 35.7, 26.4, 25.6, 24.4;

$m / z(\mathrm{ESI}) 299.2\left[(\mathrm{M}+\mathrm{Na})^{+}, 100 \%\right]$. 
2-Hydroxy-4-oxo-N-phenyl-4-(piperidin-1-yl)butanamide 24.

${ }^{1} \mathrm{H}$ NMR $\left(500 \mathrm{MHz}, \mathrm{CDCl}_{3}\right)$.

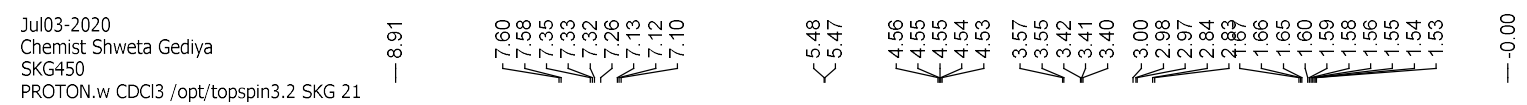<smiles>O=C(Nc1ccccc1)C(O)CC(=O)N1CCCCC1</smiles>

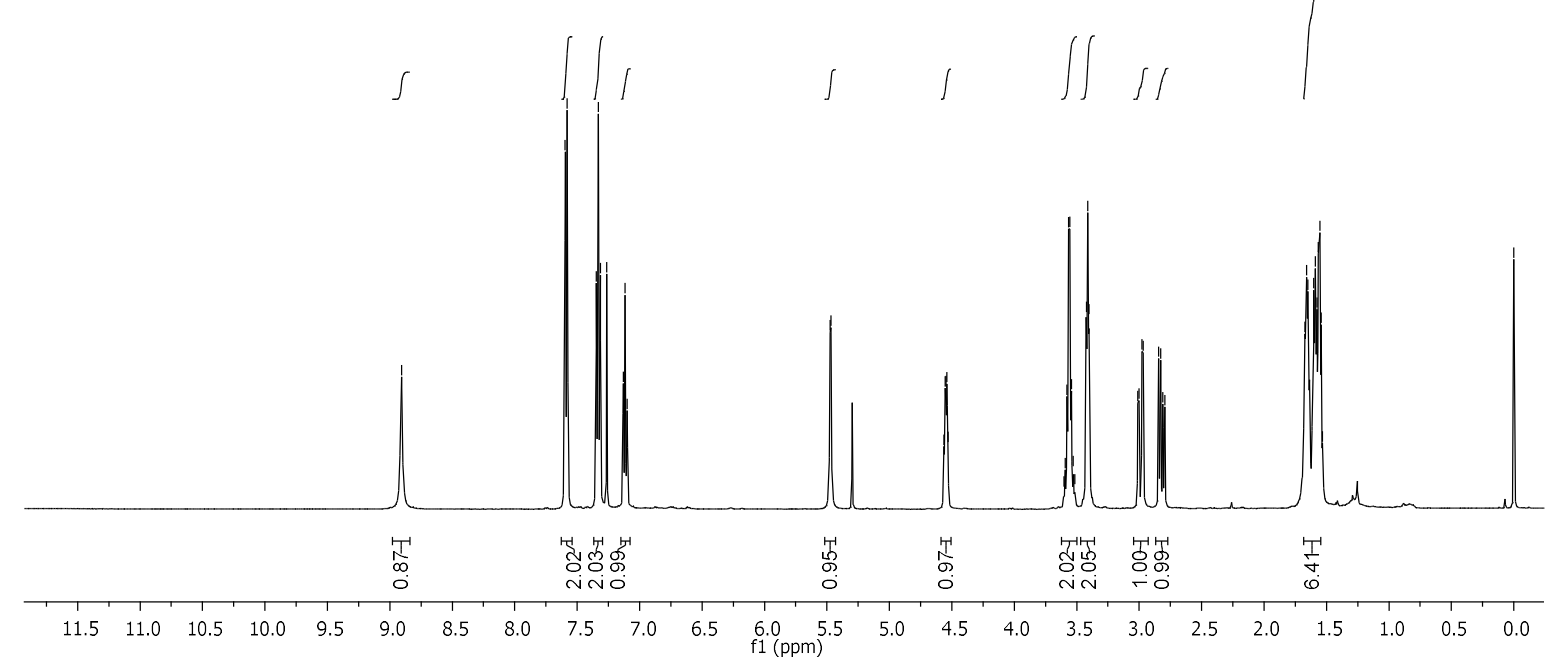

${ }^{13} \mathrm{C}$ NMR $\left(126 \mathrm{MHz}, \mathrm{CDCl}_{3}\right)$.

\begin{tabular}{|c|c|c|c|c|}
\hline $\begin{array}{l}\text { Jul03-2020 } \\
\text { Chemist Shweta Gediya } \\
\text { SKG } 450 \\
\text { C13APT.w CDCI3 /opt/topspin3.2 SKG } 21\end{array}$ & 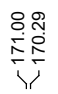 & 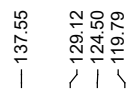 & 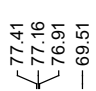 & 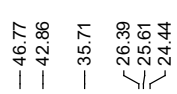 \\
\hline
\end{tabular}

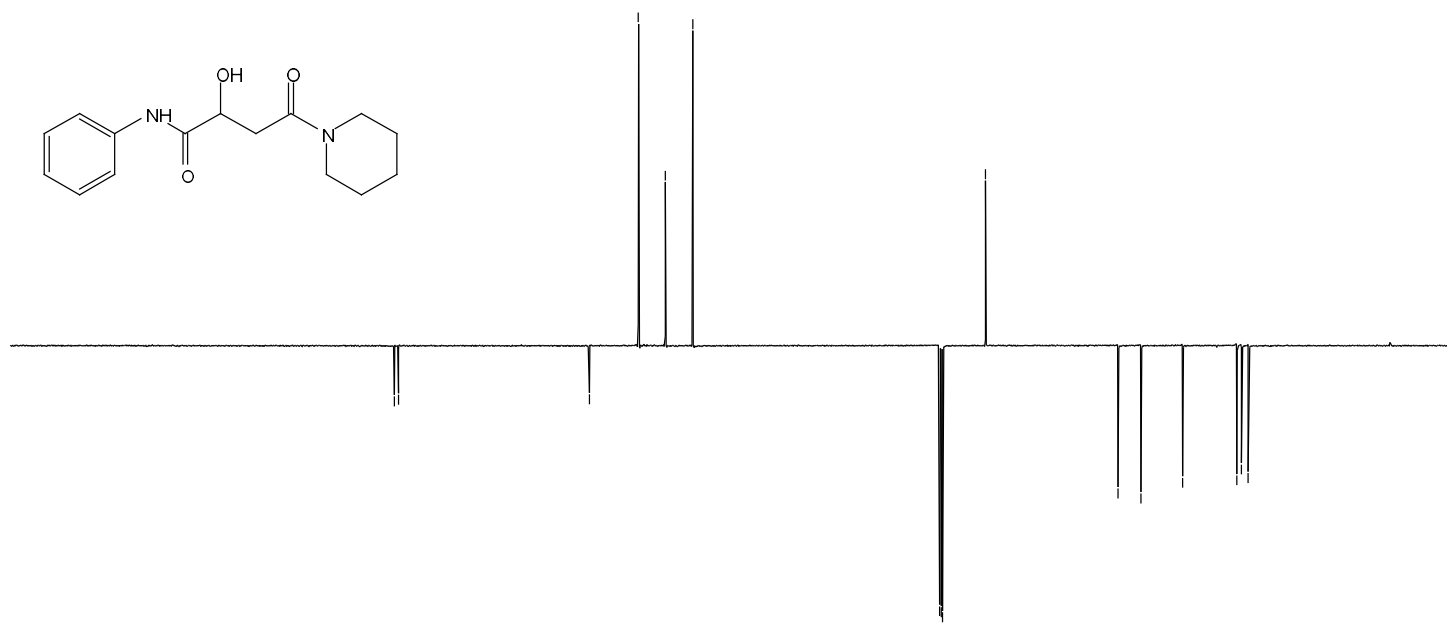

$\begin{array}{llllllllllllllllllllllllllllll}230 & 220 & 210 & 200 & 190 & 180 & 170 & 160 & 150 & 140 & 130 & 120 & 110 & 100 & 90 & 80 & 70 & 60 & 50 & 40 & 30 & 20 & 10 & 0 & -10\end{array}$ 
COSY.

Chemist Shweta Gediya

COSY.w CDCl3/opt/topspin3.2 SKG 21

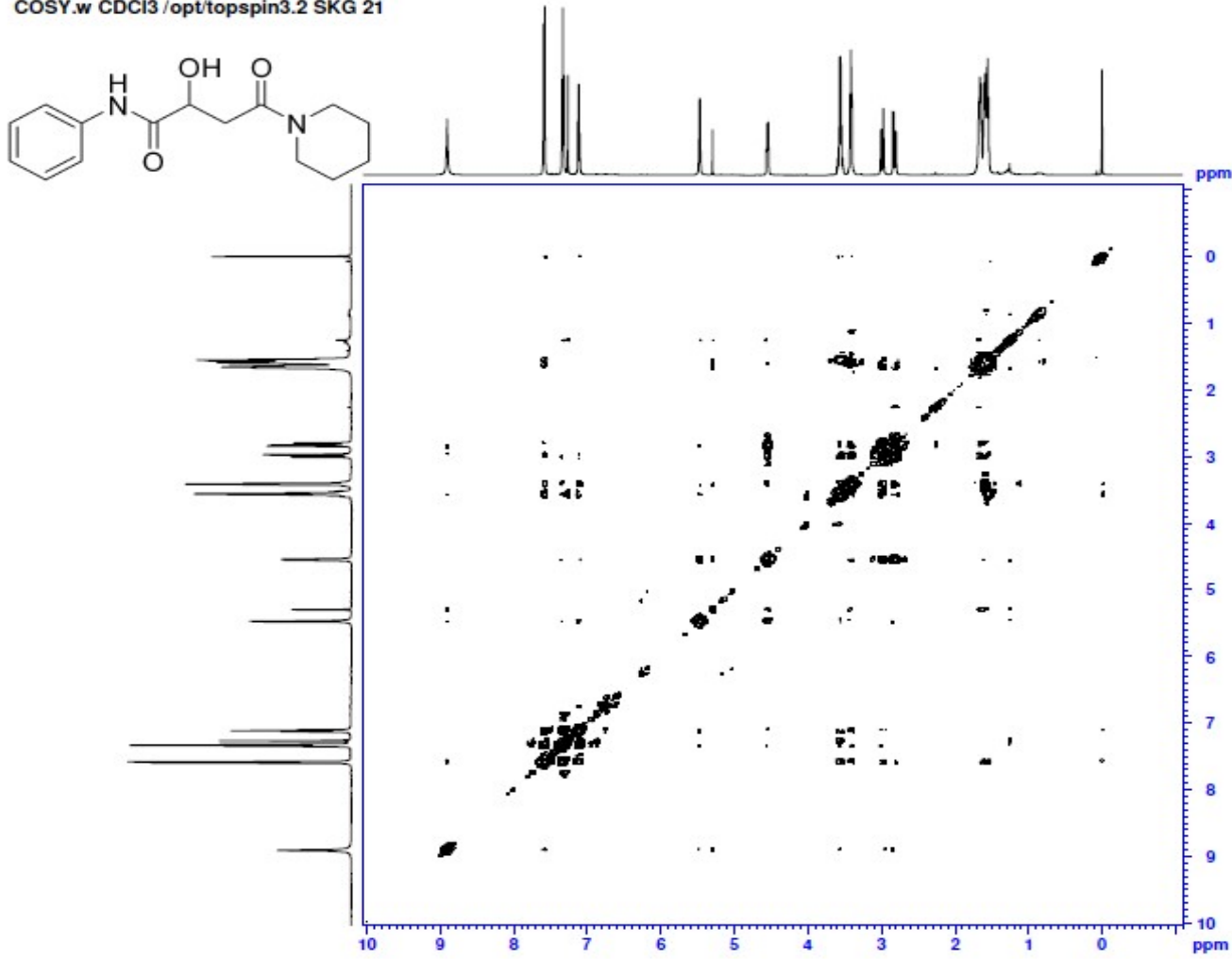

HSQC.

HSQC.w CDCl3 /opt/topspin3.2 SKG 21

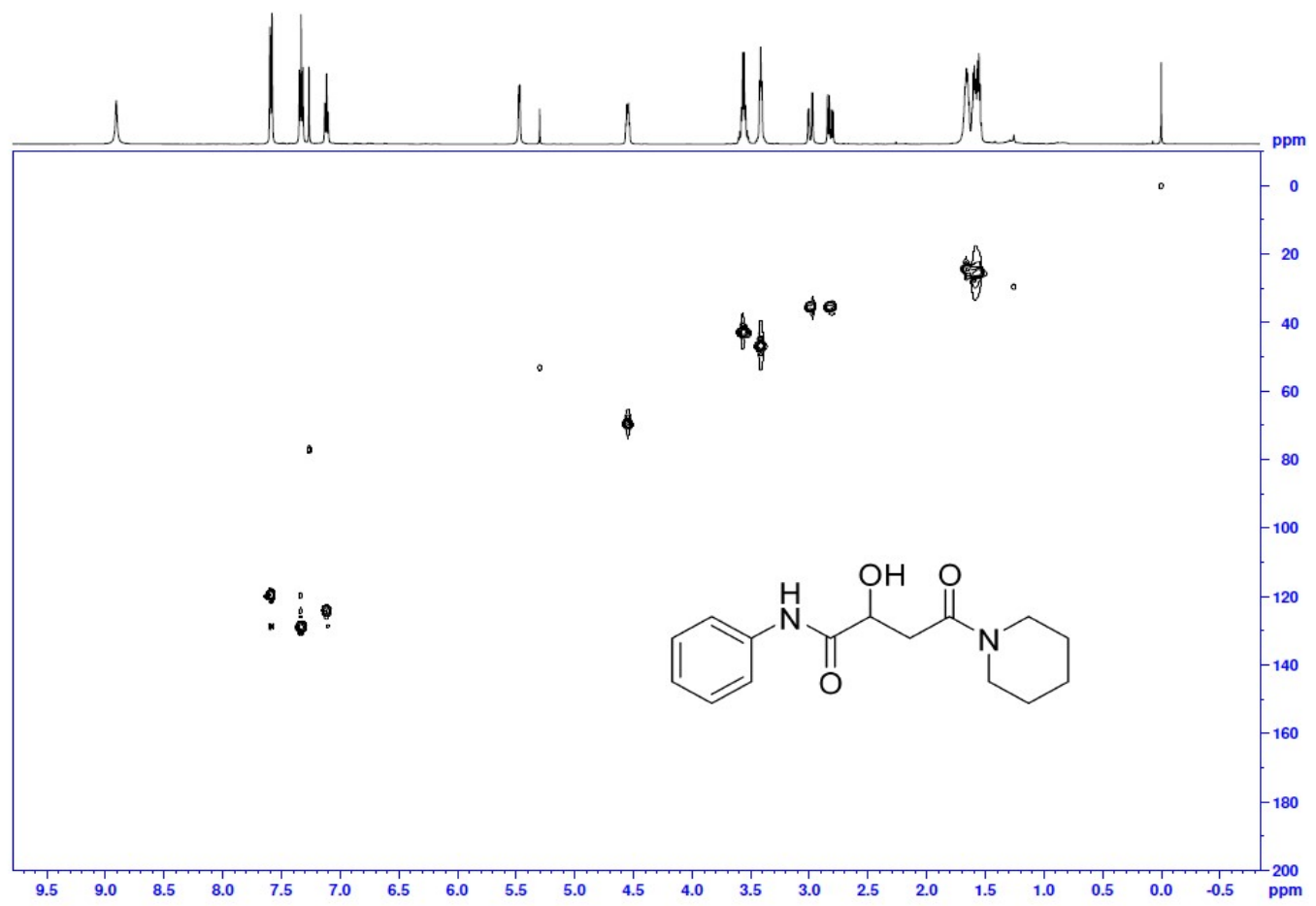




\section{Chiral HPLC of Ketone precursor.}

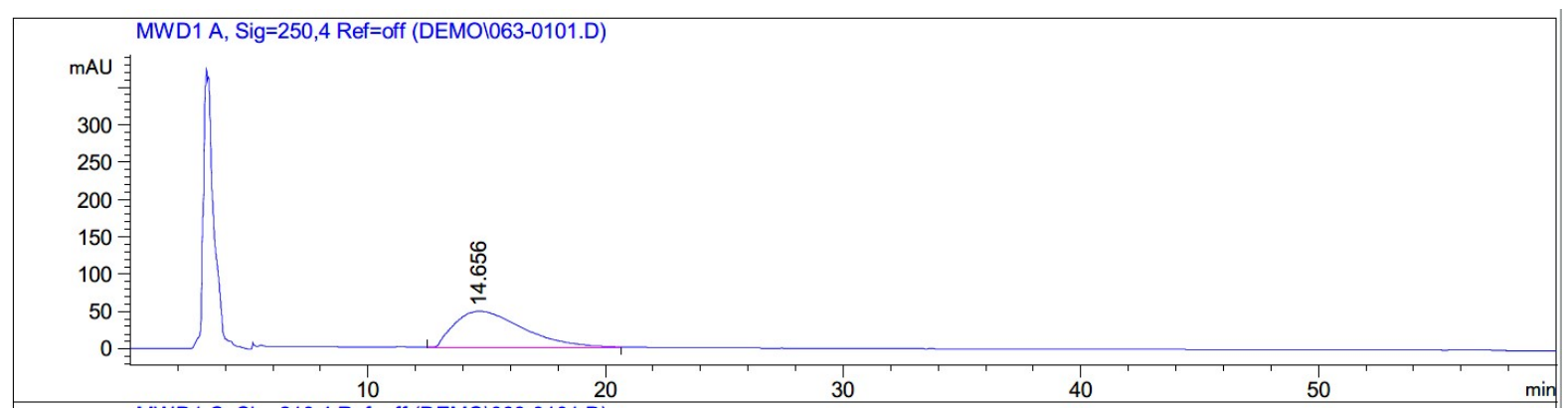

Signal 1: MWD1 A, Sig=250,4 Ref=off

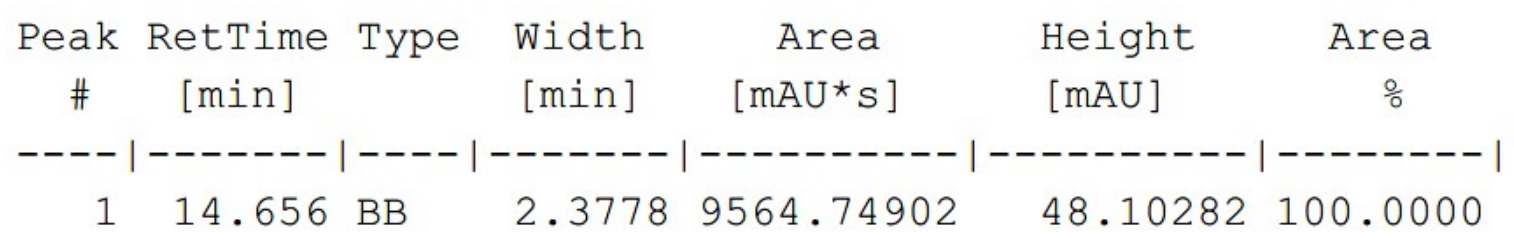

\section{Chiral HPLC of racemic alcohol.}

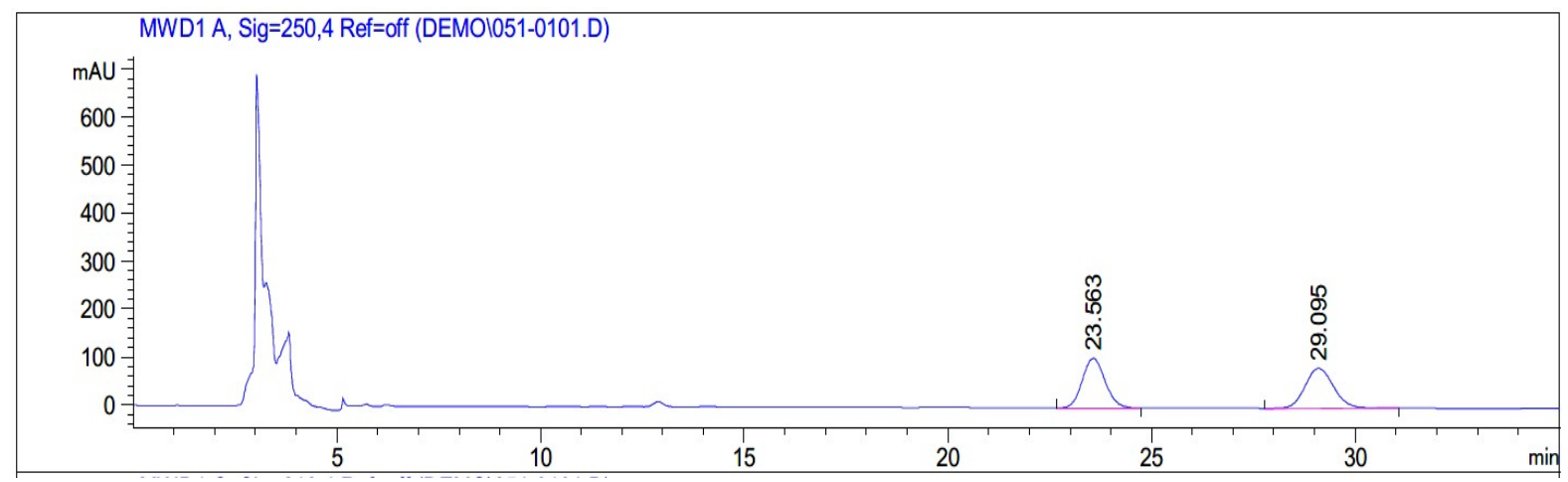

Signal 1: MWD1 A, Sig=250,4 Ref=off

\begin{tabular}{|c|c|c|c|c|c|c|}
\hline $\begin{array}{c}\text { Peak } \\
\#\end{array}$ & $\begin{array}{c}\text { RetTime } \\
\text { [min] }\end{array}$ & Type & $\begin{array}{l}\text { Width } \\
\text { [min] }\end{array}$ & $\begin{array}{c}\text { Area } \\
{\left[\mathrm{mAU}{ }^{\star} \mathrm{s}\right]}\end{array}$ & $\begin{array}{l}\text { Height } \\
{[\mathrm{mAU}]}\end{array}$ & $\begin{array}{c}\text { Area } \\
\frac{\circ}{0}\end{array}$ \\
\hline & & & & ---------- & --- & $----\mid$ \\
\hline 1 & 23.563 & VB & 0.6043 & 4026.45190 & 103.39489 & 6390 \\
\hline 2 & 29.095 & BV & 0.7614 & 4085.01733 & 83.69572 & 50.3610 \\
\hline
\end{tabular}


Chiral HPLC of ATH product. 97\% ee.

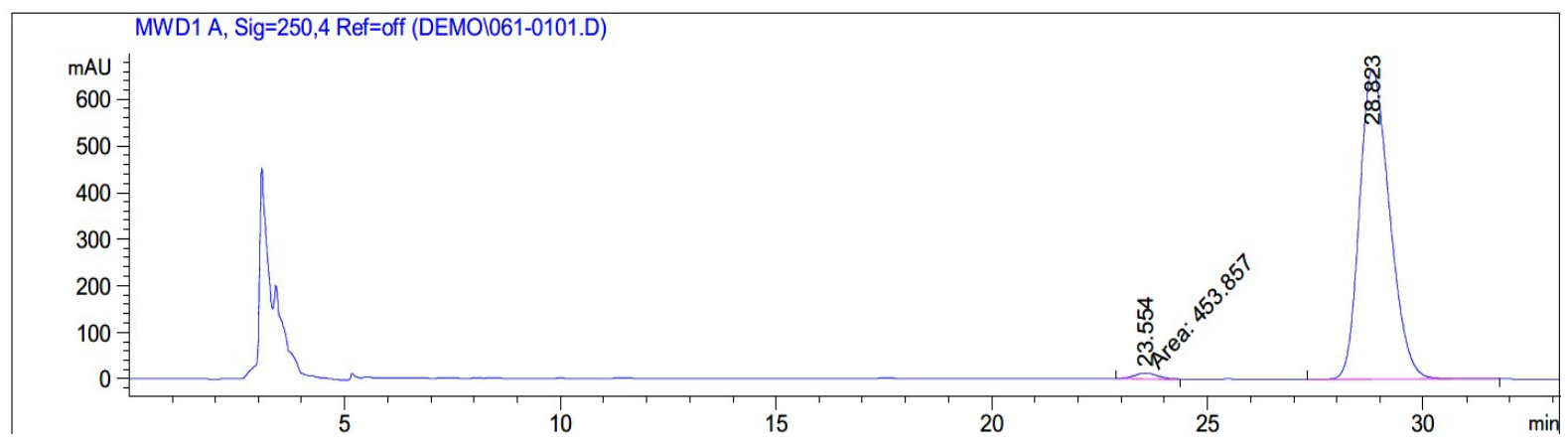

Signal 1: MWD1 A, Sig=250,4 Ref=off

\begin{tabular}{|c|c|c|c|c|c|c|}
\hline $\begin{array}{c}\text { Peak } \\
\#\end{array}$ & $\begin{array}{c}\text { RetTime } \\
\text { [min] }\end{array}$ & Type & $\begin{array}{l}\text { Width } \\
\text { [min] }\end{array}$ & $\begin{array}{c}\text { Area } \\
{\left[\mathrm{mAU}{ }^{\star} \mathrm{s}\right]}\end{array}$ & $\begin{array}{l}\text { Height } \\
{[\mathrm{mAU}]}\end{array}$ & $\begin{array}{c}\text { Area } \\
\quad \%\end{array}$ \\
\hline : & --ー-ー & -1 & - & --------- & $----------\mid$ & ----1 \\
\hline 1 & 23.554 & MM & 0.6302 & 453.85706 & 12.00227 & 1.3935 \\
\hline 2 & 28.823 & $\mathrm{BB}$ & 0.7478 & $3.21162 \mathrm{e} 4$ & 664.58191 & 98.6065 \\
\hline
\end{tabular}


(R)-N-(4-Chlorophenyl)-2-hydroxy-4-oxo-4-(piperidin-1-yl)butanamide 25.<smiles>O=C(Nc1ccc(Cl)cc1)[C@H](O)CC(=O)N1CCCCC1</smiles>

This compound is novel and was prepared following general procedure $\mathrm{C}$ using $\mathrm{N}$-(4chlorophenyl)-2,4-dioxo-4-(piperidin-1-yl)butanamide (77.0 mg, $0.250 \mathrm{mmol}, 1.0 \mathrm{eq}$ ), 3C tethered catalyst $(R, R)-2(2.3 \mathrm{mg}, 3.8 \mu \mathrm{mol}, 0.015 \mathrm{eq})$ and FA:TEA $(0.15 \mathrm{~mL})$ in DCM $(1.5$ $\mathrm{mL})$ to give 25 as a white solid $(68.1 \mathrm{~g}, 0.219 \mathrm{mmol}, 88 \%)$.

TLC: $\mathrm{R}_{\mathrm{f}}$ ca 0.4 (1:9, hexane: EtOAc), UV-active, Strong $\mathrm{KMnO}_{4}$ and PMA-reactive; MP: $159-161^{\circ} \mathrm{C}$;

HRMS (ESI) m/z: $[\mathrm{M}+\mathrm{H}]^{+}$Calcd for $\mathrm{C}_{15} \mathrm{H}_{20}{ }^{35} \mathrm{ClN}_{2} \mathrm{O}_{3} 311.1157$; Found 311.1152 (error 1.7 ppm);

$U_{\max } 3335,3262,2924,1665,1619,1588,1442,1396,1219,1105,1087,816,507 \mathrm{~cm}^{-1}$;

Enantiomeric excess determined by HPLC analysis (Chiralpak IG, $250 \mathrm{~mm} \times 4.6 \mathrm{~mm}$ column, hexane: $\mathrm{iPrOH} 80: 20,1 \mathrm{~mL} / \mathrm{min}, \lambda=210 \mathrm{~nm}, \mathrm{~T}=25{ }^{\circ} \mathrm{C}$ ), $R$-enantiomer $23.6 \mathrm{~min}, S$ enantiomer $27.8 \min .97 \%$ ee $(R),[\alpha]_{\mathrm{D}}^{22}=-0.75\left(\mathrm{c}=0.1, \mathrm{CHCl}_{3}\right)$;

${ }^{1} \mathrm{H}$ NMR (400 MHz, $\left.\mathrm{CDCl}_{3}\right) \delta 8.95(1 \mathrm{H}, \mathrm{s}, \mathrm{CONH}), 7.56-7.53(2 \mathrm{H}, \mathrm{m}, \mathrm{ArH}), 7.31-7.28(2 \mathrm{H}$, $\mathrm{m}, \mathrm{ArH}), 5.48(1 \mathrm{H}, \mathrm{d}, J=4.8 \mathrm{~Hz}, \mathrm{CHO} \underline{\mathrm{H}}), 4.55-4.51(1 \mathrm{H}, \mathrm{m}, \mathrm{C} \underline{\mathrm{HOH}}), 3.61-3.51(2 \mathrm{H}, \mathrm{m}$, $\mathrm{NCH}_{2}$ of piperidine), 3.42-3.40 $\left(2 \mathrm{H}, \mathrm{m}, \mathrm{NCH}_{2}\right.$ of piperidine), $2.97(1 \mathrm{H}, \mathrm{dd}, J=16.7,4.2 \mathrm{~Hz}$, $\left.\mathrm{C}_{\underline{\mathrm{H}}} \mathrm{H}_{\mathrm{b}}\right), 2.82\left(1 \mathrm{H}, \mathrm{dd}, J=16.7,7.4 \mathrm{~Hz}, \mathrm{CH}_{\mathrm{a}} \underline{\mathrm{H}}_{\mathrm{b}}\right), 1.70-1.52\left(6 \mathrm{H}, \mathrm{m}, \mathrm{CH}_{2}\right.$ of piperidine);

${ }^{13} \mathrm{C}$ NMR $\left(101 \mathrm{MHz}, \mathrm{CDCl}_{3}\right) \delta 171.1,170.2,136.2,129.4,129.1,121.0,68.5,46.8,42.9$, 35.6, 26.4, 25.6, 24.4;

$m / z(\mathrm{ESI}) 311.2\left[\left(\mathrm{M}\left({ }^{35} \mathrm{Cl}\right)+\mathrm{H}\right)^{+}, 100 \%\right]$. 
N-(4-Chlorophenyl)-2-hydroxy-4-oxo-4-(piperidin-1-yl)butanamide 25.

${ }^{1} \mathrm{H}$ NMR (400 MHz, $\mathrm{CDCl}_{3}$ ).

Sep16-2020
SKG546
PROTON.w CDCI3 /opt/topspin3.5pI2 SKG $19 \stackrel{\text { \% }}{\infty}$

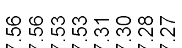

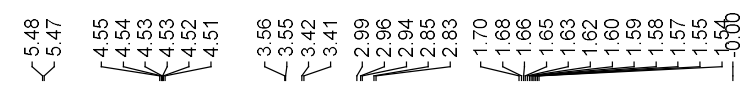
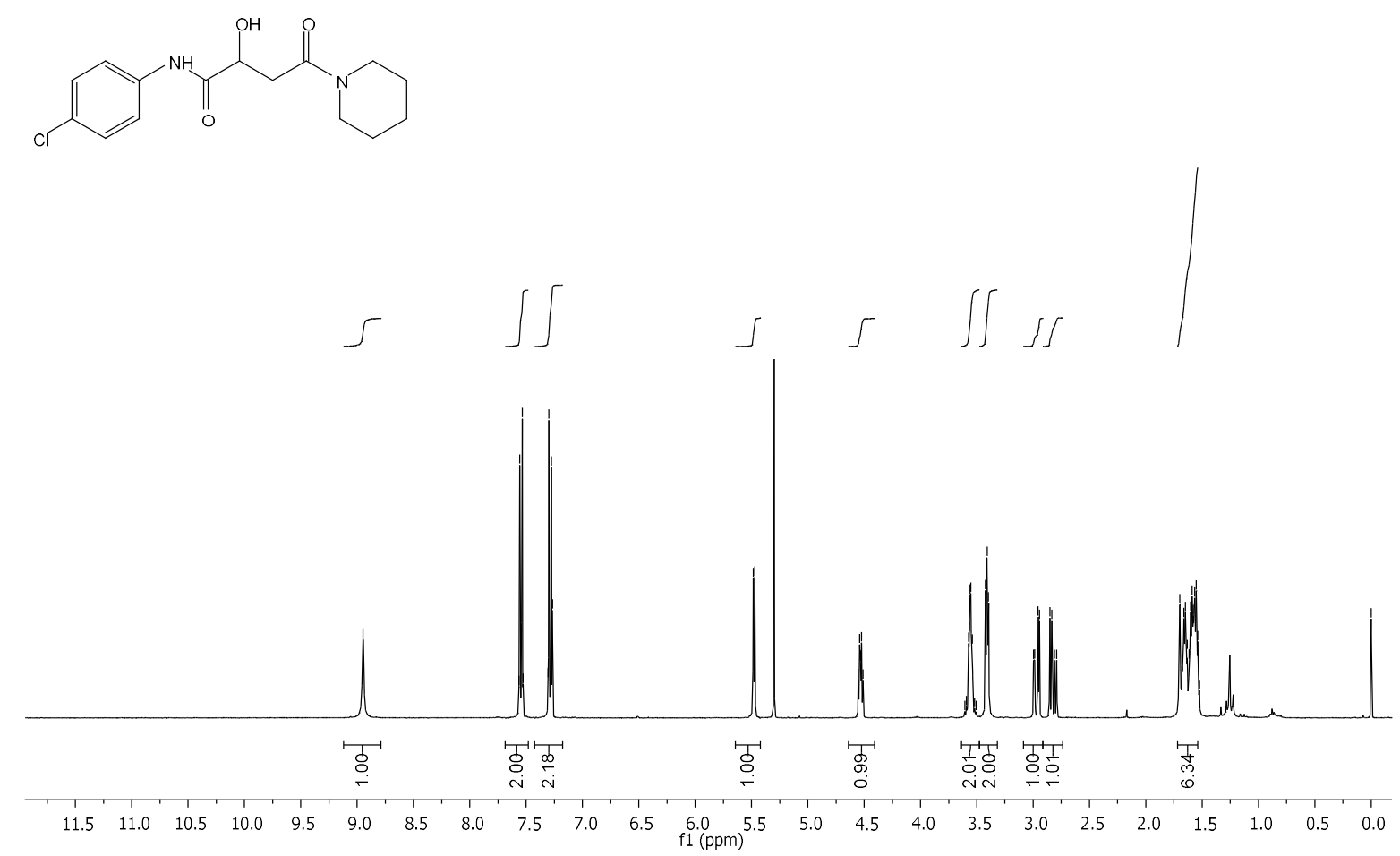

${ }^{13} \mathrm{C}$ NMR (101 MHz, $\left.\mathrm{CDCl}_{3}\right)$.

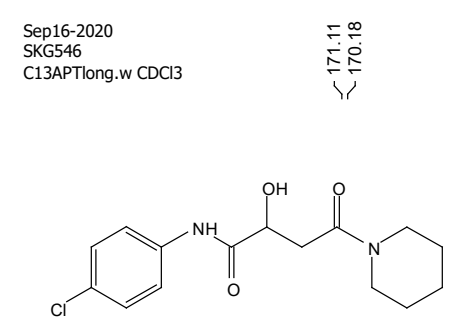

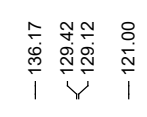

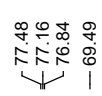

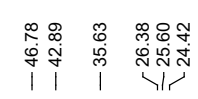
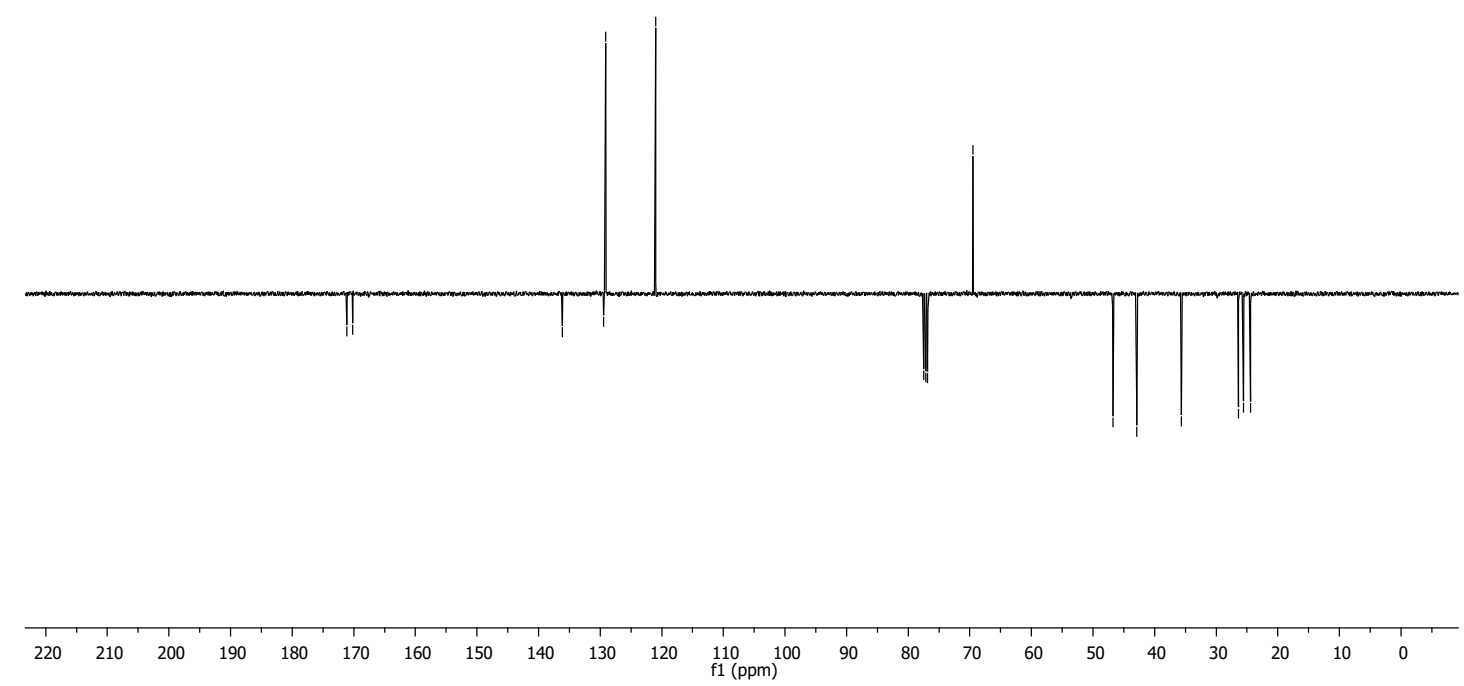

S297 
COSY.

SKG546

COSY.w CDCl3/opt/topspin3.5pl2 SKG 19

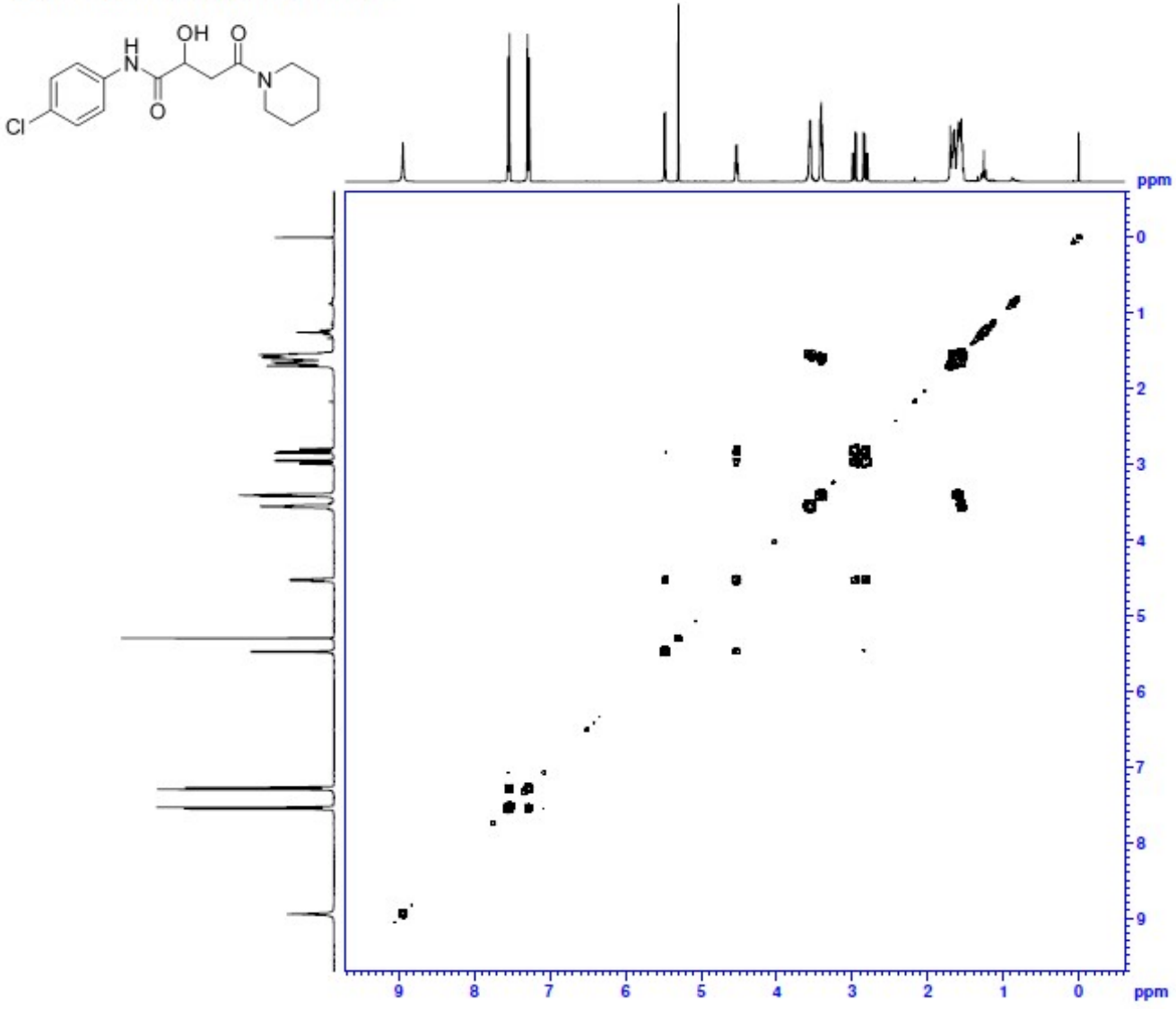

HSQC.

SKG556
HSQC.w CDCl3 /opt/topspin3.5pl2 SKG 19

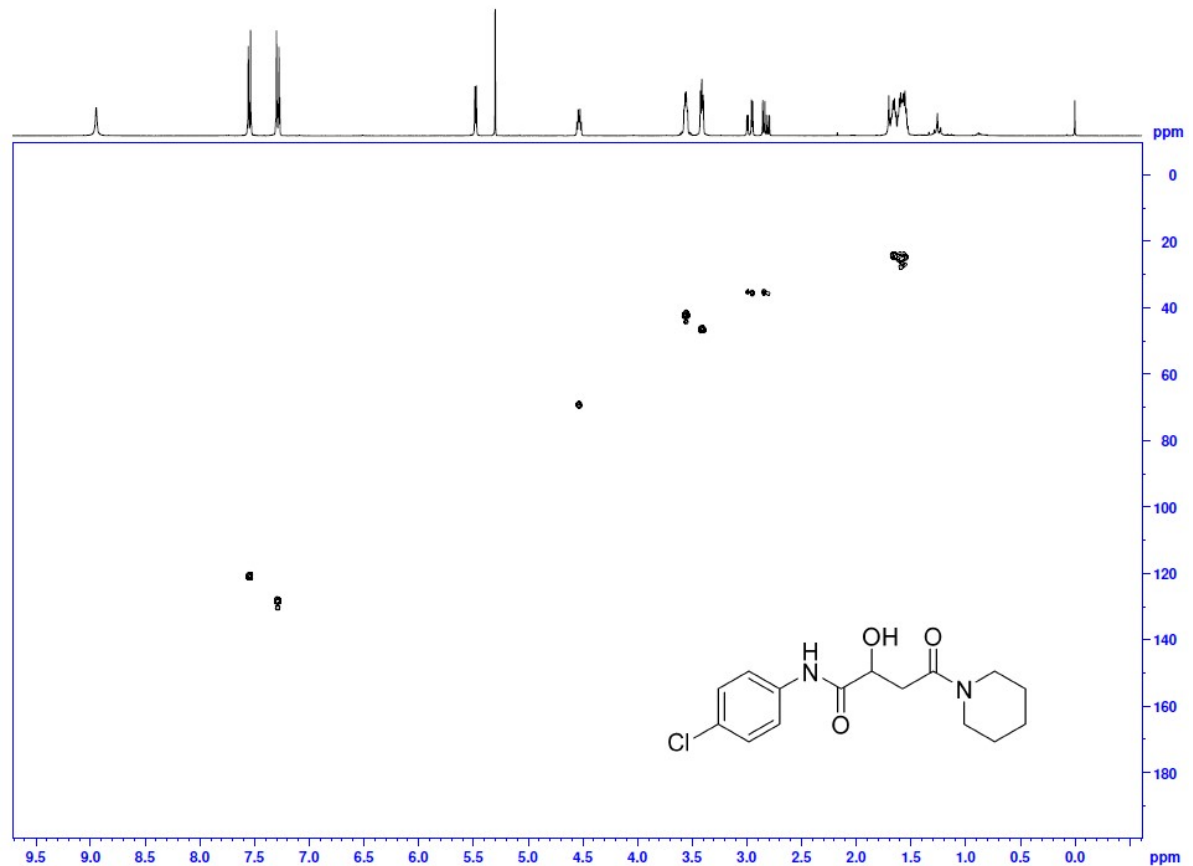




\section{Chiral HPLC of ketone precursor.}

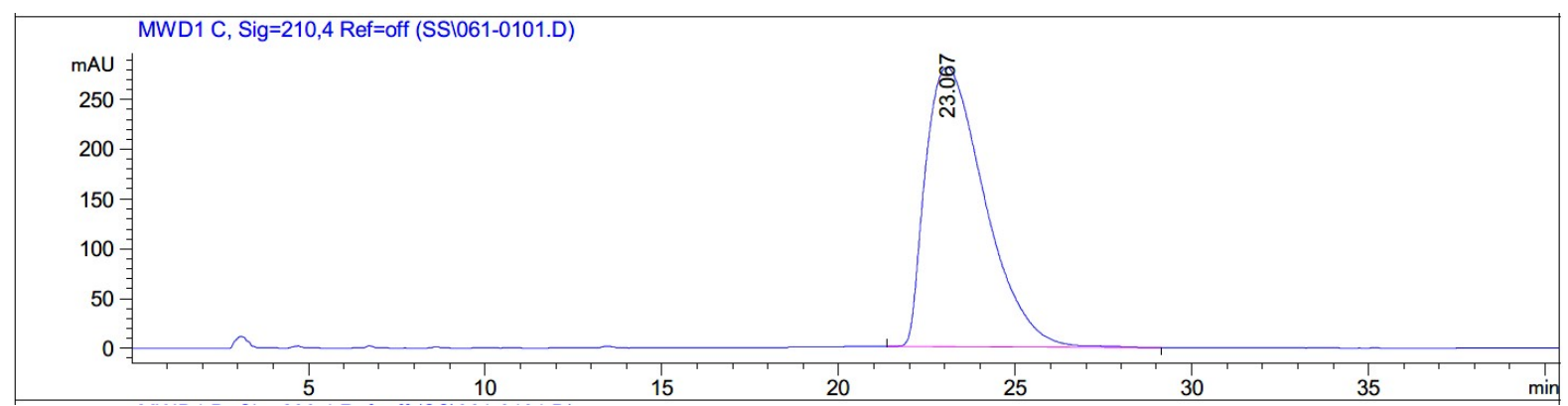

Signal 2: MWD1 C, Sig=210,4 Ref=off

\begin{tabular}{|c|c|c|c|c|c|c|}
\hline $\begin{array}{c}\text { Peak } \\
\#\end{array}$ & $\begin{array}{c}\text { RetTime } \\
\text { [min] }\end{array}$ & Type & $\begin{array}{c}\text { Width } \\
\text { [min] }\end{array}$ & $\begin{array}{c}\text { Area } \\
{\left[\mathrm{mAU}^{*} \mathrm{~S}\right]}\end{array}$ & $\begin{array}{l}\text { Height } \\
\text { [mAU] }\end{array}$ & $\begin{array}{c}\text { Area } \\
\frac{\circ}{0}\end{array}$ \\
\hline 1 & 23.067 & $\mathrm{BB}$ & 1832 & $3.26475 \mathrm{e}$ & 280 & 000 \\
\hline
\end{tabular}

Chiral HPLC of racemic alcohol.

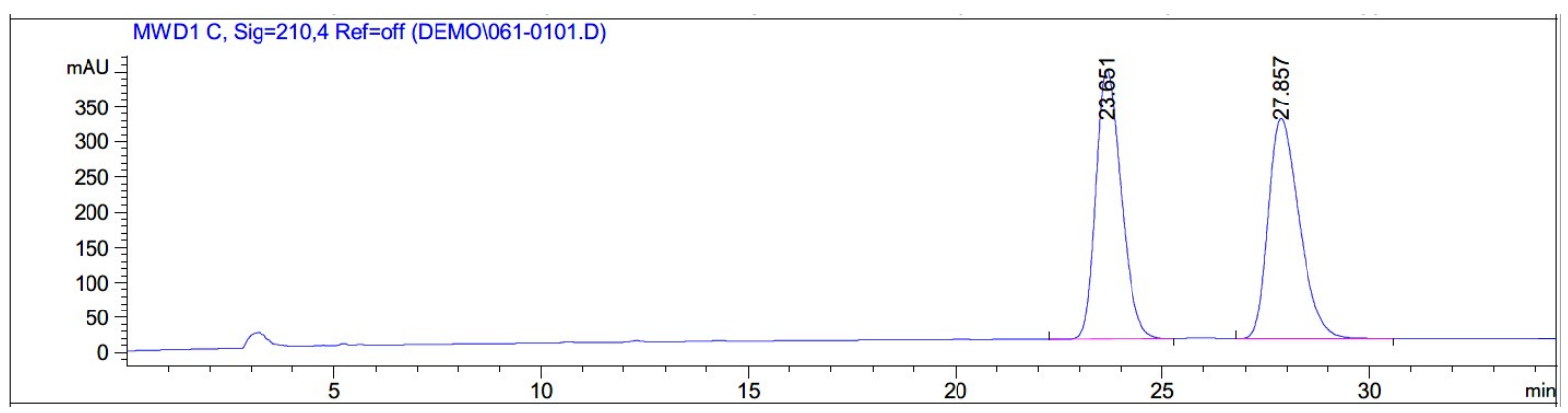

Signal 2: MWD1 C, Sig=210,4 Ref=off

\begin{tabular}{|c|c|c|c|c|c|c|}
\hline $\begin{array}{c}\text { Peak } \\
\#\end{array}$ & $\begin{array}{c}\text { RetTime } \\
\text { [min] }\end{array}$ & Type & $\begin{array}{l}\text { Width } \\
\text { [min] }\end{array}$ & $\begin{array}{c}\text { Area } \\
{\left[\mathrm{mAU}^{\star} \mathrm{s}\right]}\end{array}$ & $\begin{array}{l}\text { Height } \\
\text { [mAU] }\end{array}$ & $\begin{array}{c}\text { Area } \\
\quad \frac{\circ}{0}\end{array}$ \\
\hline--- & ------- & 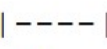 & ----- & - & --- & ---1 \\
\hline 1 & 23.651 & BB & 0.6659 & $1.63686 \mathrm{e} 4$ & 383.53671 & 49.9460 \\
\hline 2 & 27.857 & $\mathrm{BB}$ & 0.8096 & $1.64041 \mathrm{e} 4$ & 312.90869 & 50.0540 \\
\hline
\end{tabular}


Chiral HPLC of ATH product. 97\% ee.

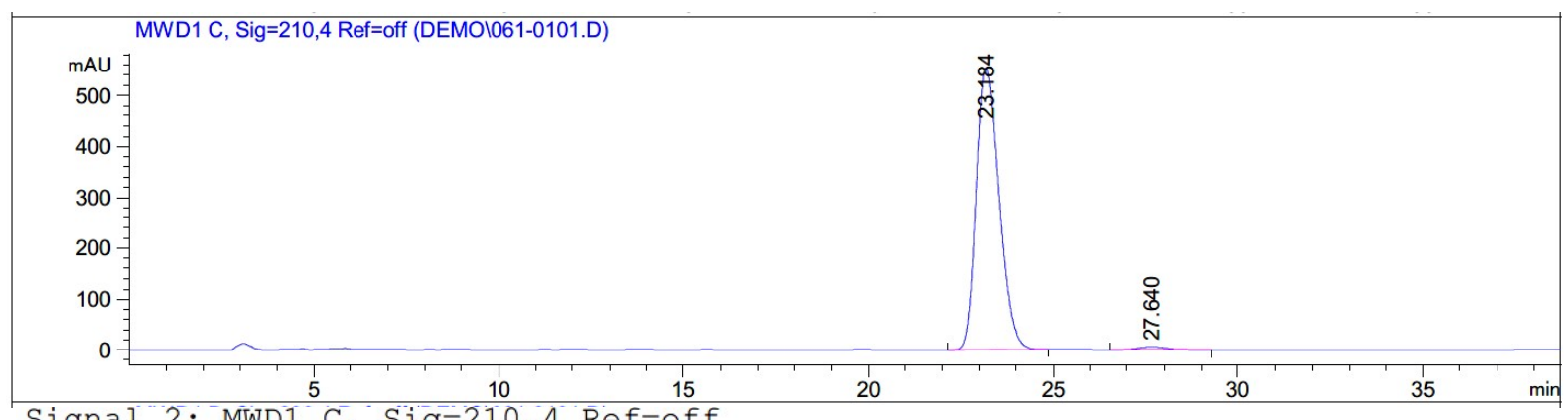

\begin{tabular}{|c|c|c|c|c|c|c|}
\hline $\begin{array}{c}\text { Peak } \\
\#\end{array}$ & $\begin{array}{l}\text { RetTime } \\
\text { [min] }\end{array}$ & Type & $\begin{array}{l}\text { Width } \\
\text { [min] }\end{array}$ & $\begin{array}{c}\text { Area } \\
{[\mathrm{mAU} * \mathrm{~s}]}\end{array}$ & $\begin{array}{l}\text { Height } \\
{[\mathrm{mAU}]}\end{array}$ & $\begin{array}{c}\text { Area } \\
\frac{\circ}{0}\end{array}$ \\
\hline-0 & & & & -- & --- & - -- \\
\hline 1 & 23.184 & BB & 0.6522 & $2.32953 e 4$ & 554.36951 & 98.6896 \\
\hline 2 & 27.640 & $\mathrm{BB}$ & 0.6528 & 309.32169 & 5.98658 & 1.3104 \\
\hline
\end{tabular}


(R)-N-(2-Chlorophenyl)-2-hydroxy-4-oxo-4-(piperidin-1-yl)butanamide 26.<smiles>O=C(Nc1ccccc1Cl)[C@H](O)CC(=O)N1CCCCC1</smiles>

This compound is novel and was prepared following general procedure $\mathrm{C}$ using $\mathrm{N}$-(2chlorophenyl)-2,4-dioxo-4-(piperidin-1-yl)butanamide (77.2 mg, $0.250 \mathrm{mmol}, 1.0 \mathrm{eq}), 3 \mathrm{C}$ tethered catalyst $(R, R)-2(2.3 \mathrm{mg}, 3.8 \mu \mathrm{mol}, 0.015 \mathrm{eq})$ and FA:TEA $(0.15 \mathrm{~mL})$ in DCM $(1.5$ $\mathrm{mL})$ to give 26 as a clear oil $(72.5 \mathrm{mg}, 0.233 \mathrm{mmol}, 93 \%)$.

TLC: $\mathrm{R}_{\mathrm{f}}$ ca 0.4 (4:6, hexane: EtOAc), UV-active, Strong $\mathrm{KMnO}_{4}$ and PMA-reactive; HRMS (ESI) m/z: [M+Na] ${ }^{+}$Calcd for $\mathrm{C}_{15} \mathrm{H}_{19}{ }^{35} \mathrm{ClN}_{2} \mathrm{O}_{3} \mathrm{Na} 333.0976$; Found 333.0971 (error $1.5 \mathrm{ppm})$;

$U_{\max } 3352,2935,2856,1688,1614,1516,1438,1298,1032,751 \mathrm{~cm}^{-1}$;

Enantiomeric excess determined by HPLC analysis (Chiralpak IG, $250 \mathrm{~mm} \times 4.6 \mathrm{~mm}$ column, hexane: $\mathrm{iPrOH} 80: 20,0.6 \mathrm{~mL} / \mathrm{min}, \lambda=210 \mathrm{~nm}, \mathrm{~T}=25^{\circ} \mathrm{C}$ ), $S$-enantiomer $30.9 \mathrm{~min}, R$ enantiomer $32.2 \min .98 \%$ ee $(R),[\alpha]_{\mathrm{D}}^{22}=-15\left(\mathrm{c}=0.05, \mathrm{CHCl}_{3}\right)$;

${ }^{1} \mathrm{H}$ NMR (500 MHz, $\left.\mathrm{CDCl}_{3}\right) \delta 9.42(1 \mathrm{H}, \mathrm{s}, \mathrm{CONH}), 8.42(1 \mathrm{H}, \mathrm{dd}, J=8.3,1.4 \mathrm{~Hz}, \mathrm{ArH}), 7.38$ $(1 \mathrm{H}, \mathrm{dd}, J=8.0,1.3 \mathrm{~Hz}, \mathrm{ArH}), 7.29-7.25$ (1H, m, ArH), 7.07-7.03 (1H, $J=7.9,1.5 \mathrm{~Hz}$, $\mathrm{ArH}), 5.51(1 \mathrm{H}, \mathrm{d}, J=4.9 \mathrm{~Hz}, \mathrm{CHO} \underline{\mathrm{H}}), 4.61-4.57(1 \mathrm{H}, \mathrm{m}, \mathrm{CHOH}), 3.61-3.52\left(2 \mathrm{H}, \mathrm{m}, \mathrm{NCH}_{2}\right.$ of piperidine), 3.45-3.37 (2H, m, $\mathrm{NCH}_{2}$ of piperidine), $3.01(1 \mathrm{H}, \mathrm{dd}, J=16.6,3.7 \mathrm{~Hz}$, $\left.\mathrm{C}_{\underline{\mathrm{a}}} \mathrm{H}_{\mathrm{b}}\right), 2.84\left(1 \mathrm{H}, \mathrm{dd}, J=16.6,7.8 \mathrm{~Hz}, \mathrm{CH}_{\mathrm{a}} \underline{\mathrm{H}}\right), 1.69-1.53\left(6 \mathrm{H}, \mathrm{m}, \mathrm{CH}_{2}\right.$ of piperidine);

${ }^{13} \mathrm{C} \mathrm{NMR}\left(126 \mathrm{MHz}, \mathrm{CDCl}_{3}\right) \delta 171.3,170.1,135.4,129.3,127.7,124.8,123.5,121.3,69.9$, 46.7, 42.9, 35.5, 26.4, 25.6, 24.5;

$m / z(\mathrm{ESI}) 333.1\left[\left(\mathrm{M}\left({ }^{35} \mathrm{Cl}\right)+\mathrm{Na}\right)^{+}, 100 \%\right], 335.1\left[\left(\mathrm{M}\left({ }^{37} \mathrm{Cl}\right)+\mathrm{Na}\right)^{+}, 35 \%\right]$. 
$\mathrm{N}$-(2-Chlorophenyl)-2-hydroxy-4-oxo-4-(piperidin-1-yl)butanamide 26.

${ }^{1} \mathrm{H}$ NMR $\left(500 \mathrm{MHz}, \mathrm{CDCl}_{3}\right)$.

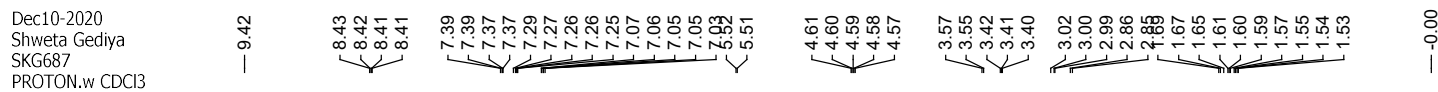<smiles>O=C(Nc1ccccc1Cl)C(O)CC(=O)N1CCCCC1</smiles>

$5 \quad 5 \quad \int S$
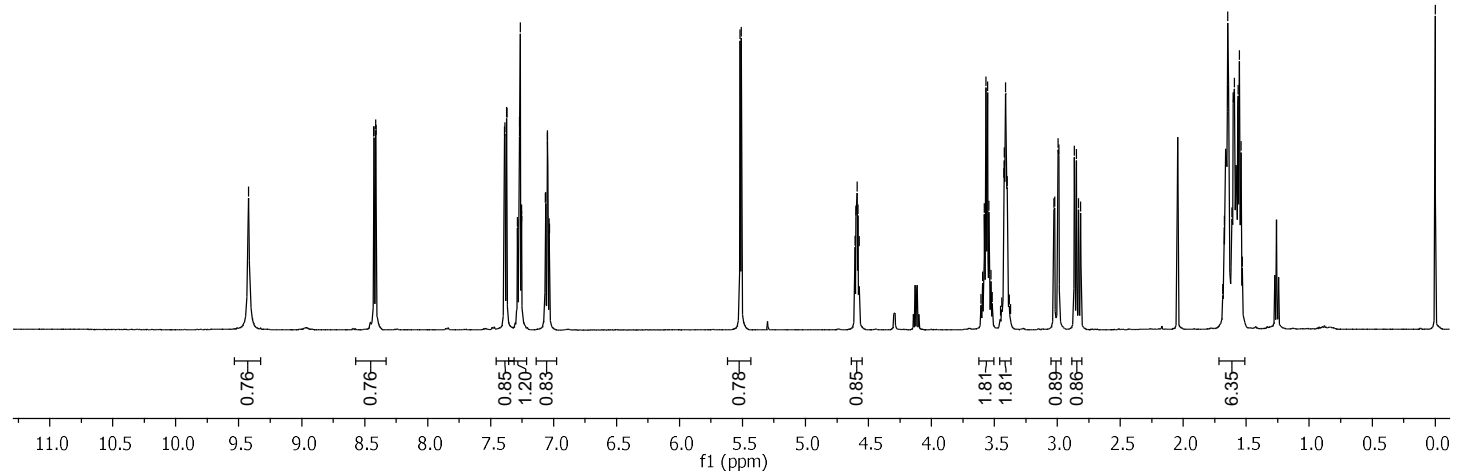

${ }^{13} \mathrm{C} \mathrm{NMR}\left(126 \mathrm{MHz}, \mathrm{CDCl}_{3}\right)$.

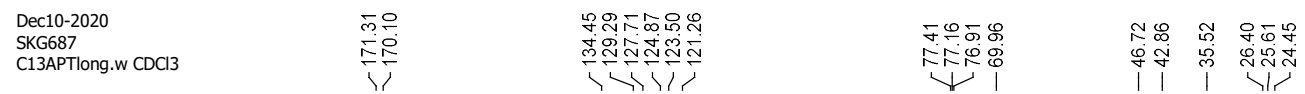<smiles>O=C(Nc1ccccc1Cl)C(O)CC(=O)N1CCCCC1</smiles>

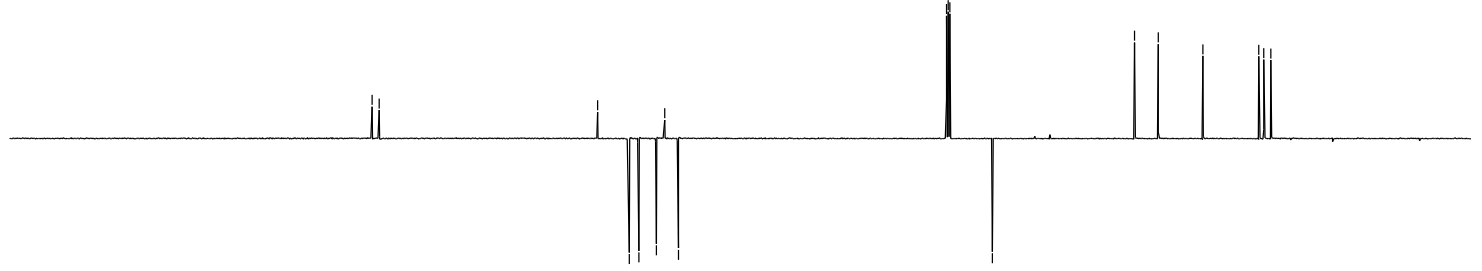

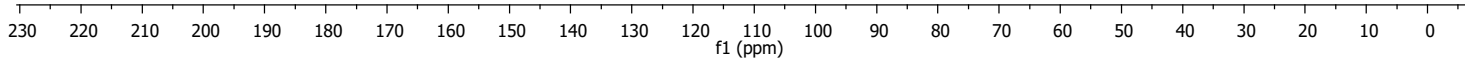


COSY.

Chemist Shweta Gediya

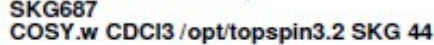

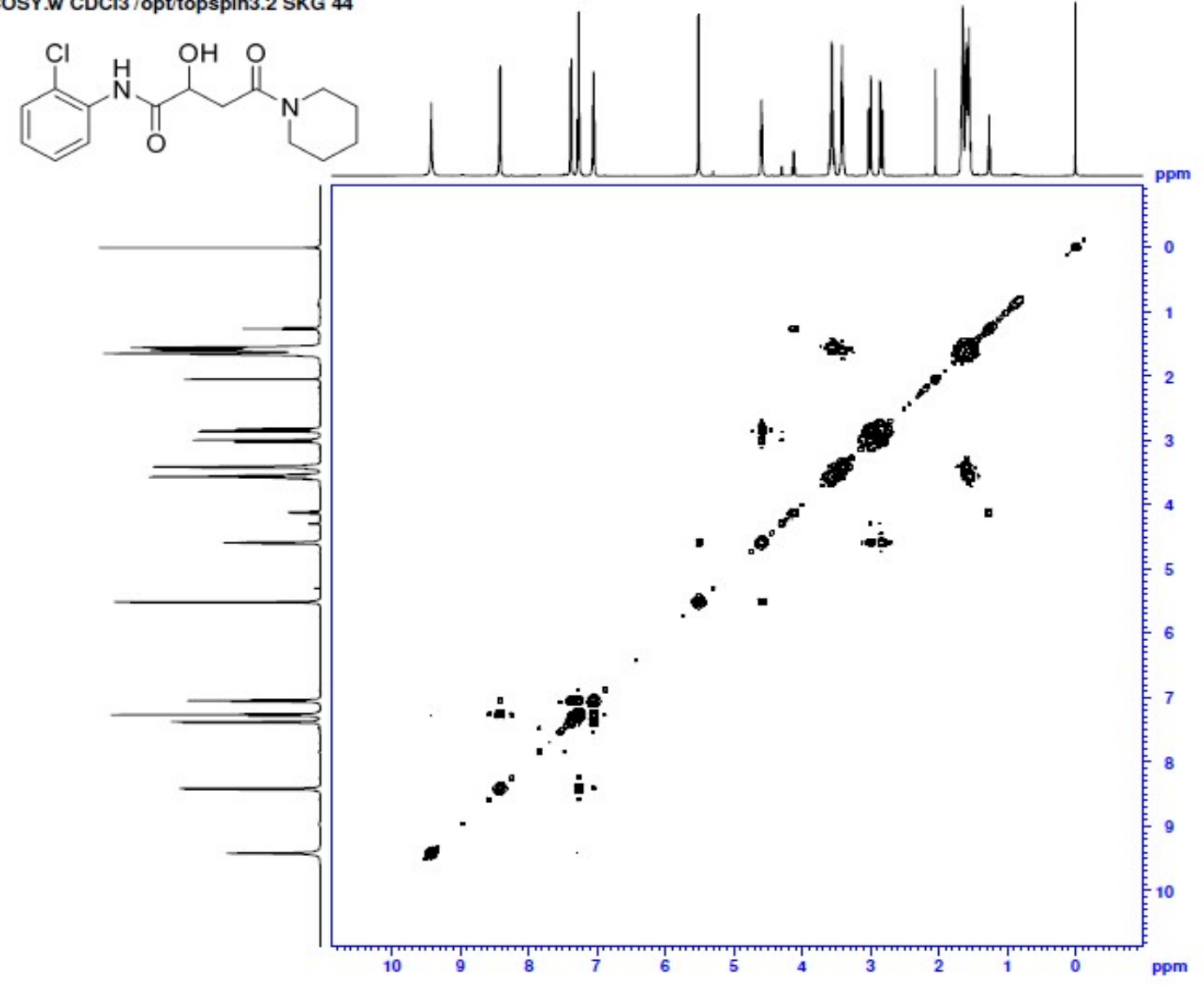

HSQC.

HSQC.w CDCl3 /opt/topspin3.2 SKG 44

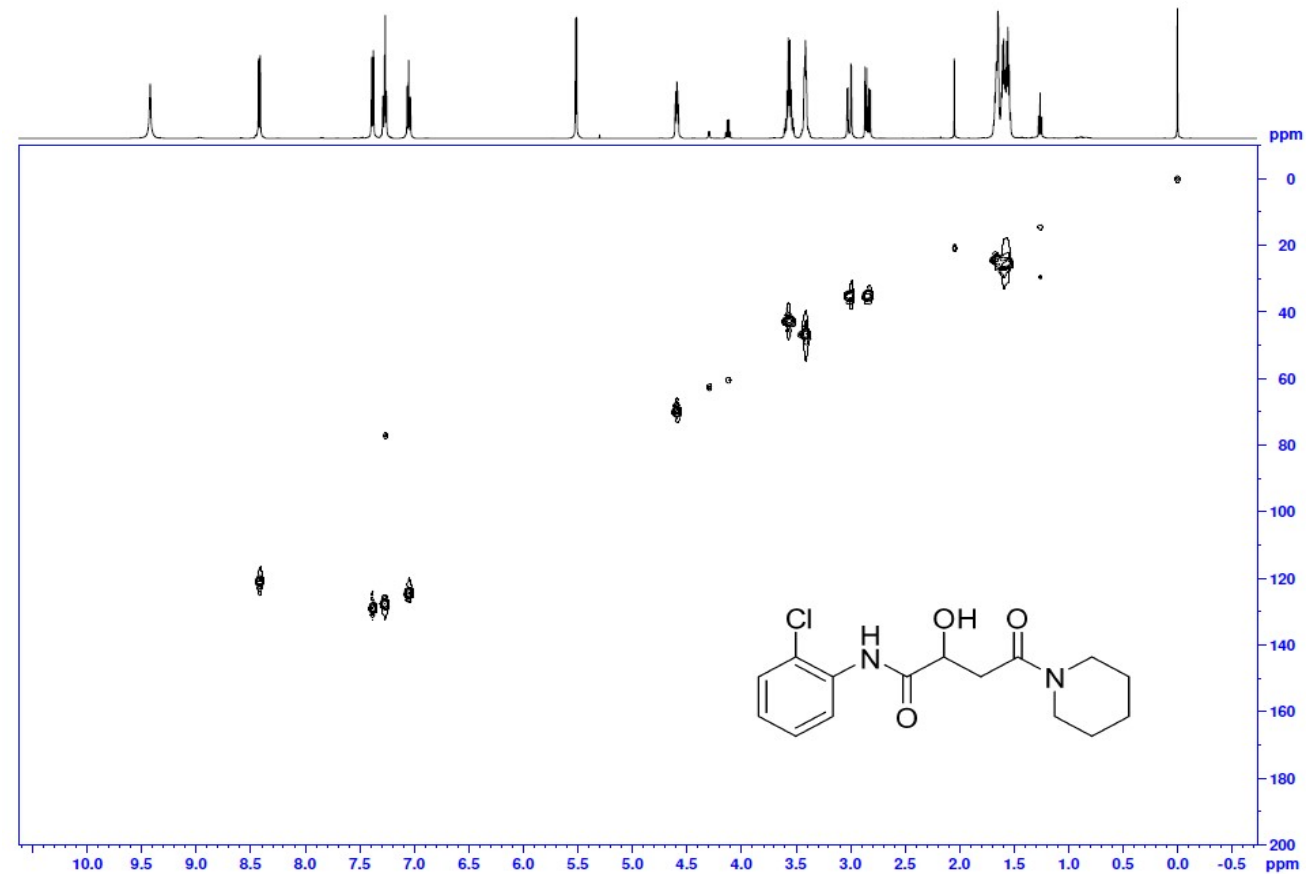




\section{Chiral HPLC of ketone precursor.}

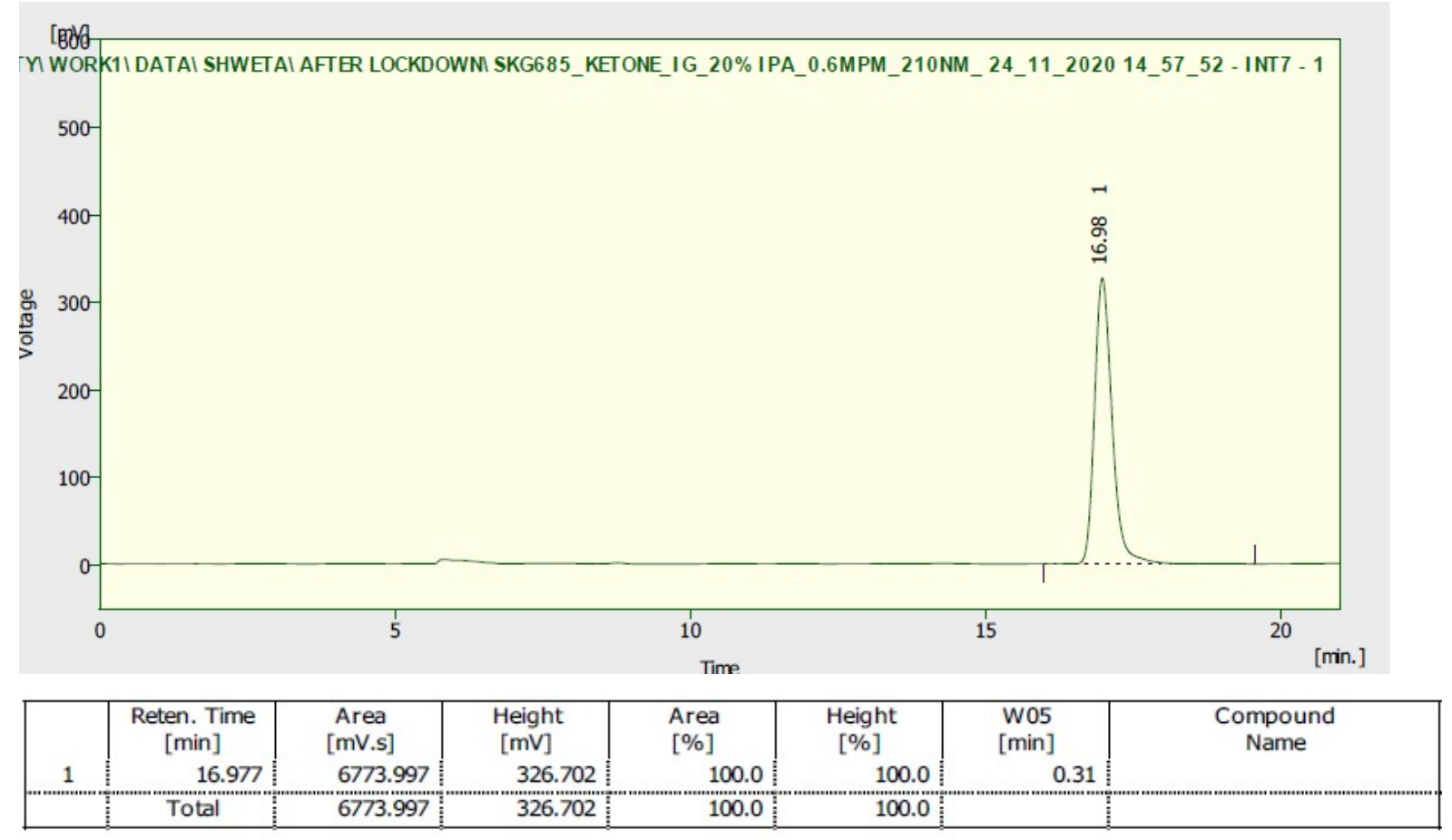

\section{Chiral HPLC of racemic alcohol.}

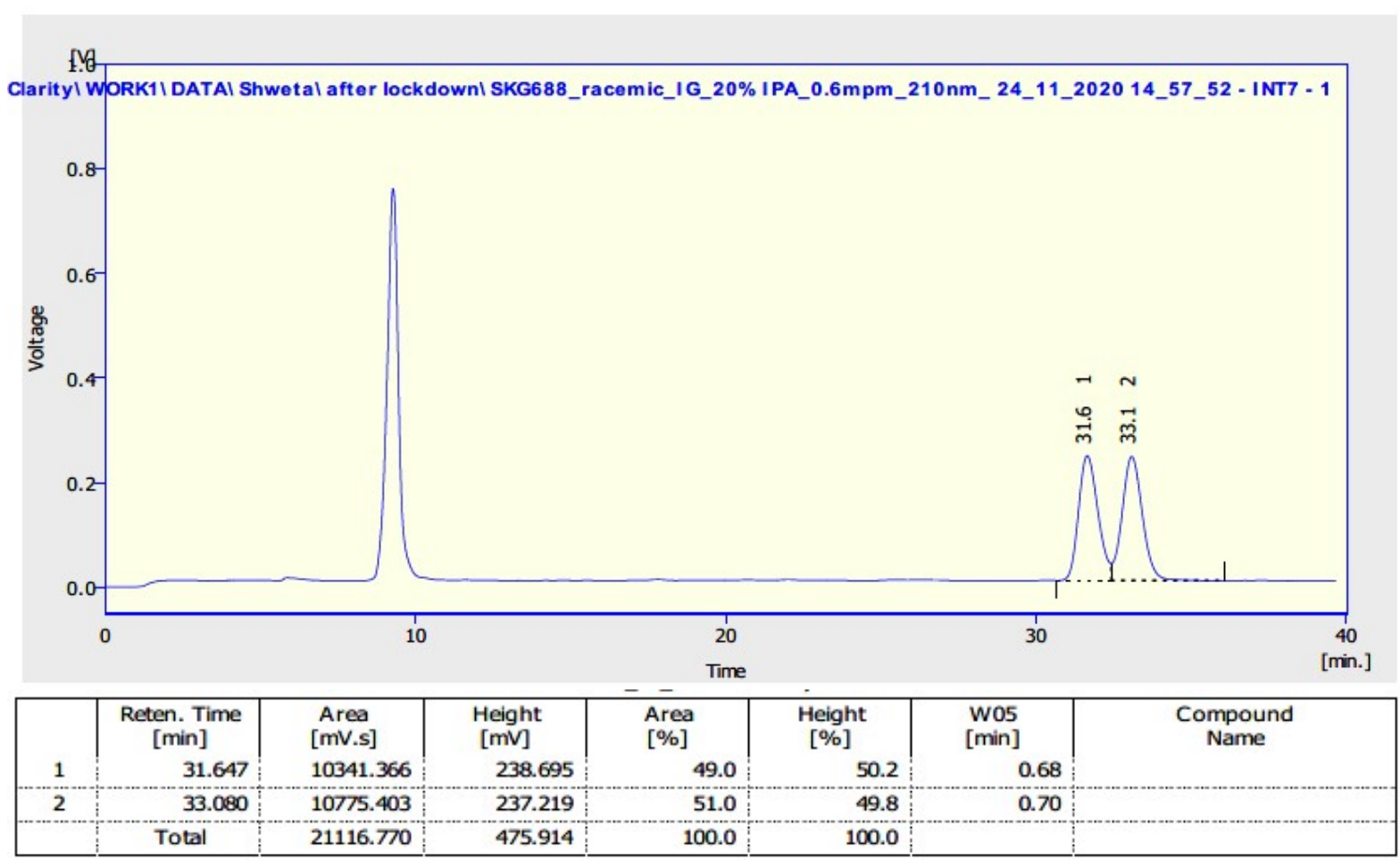


Chiral HPLC of ATH product. $98 \%$ ee.

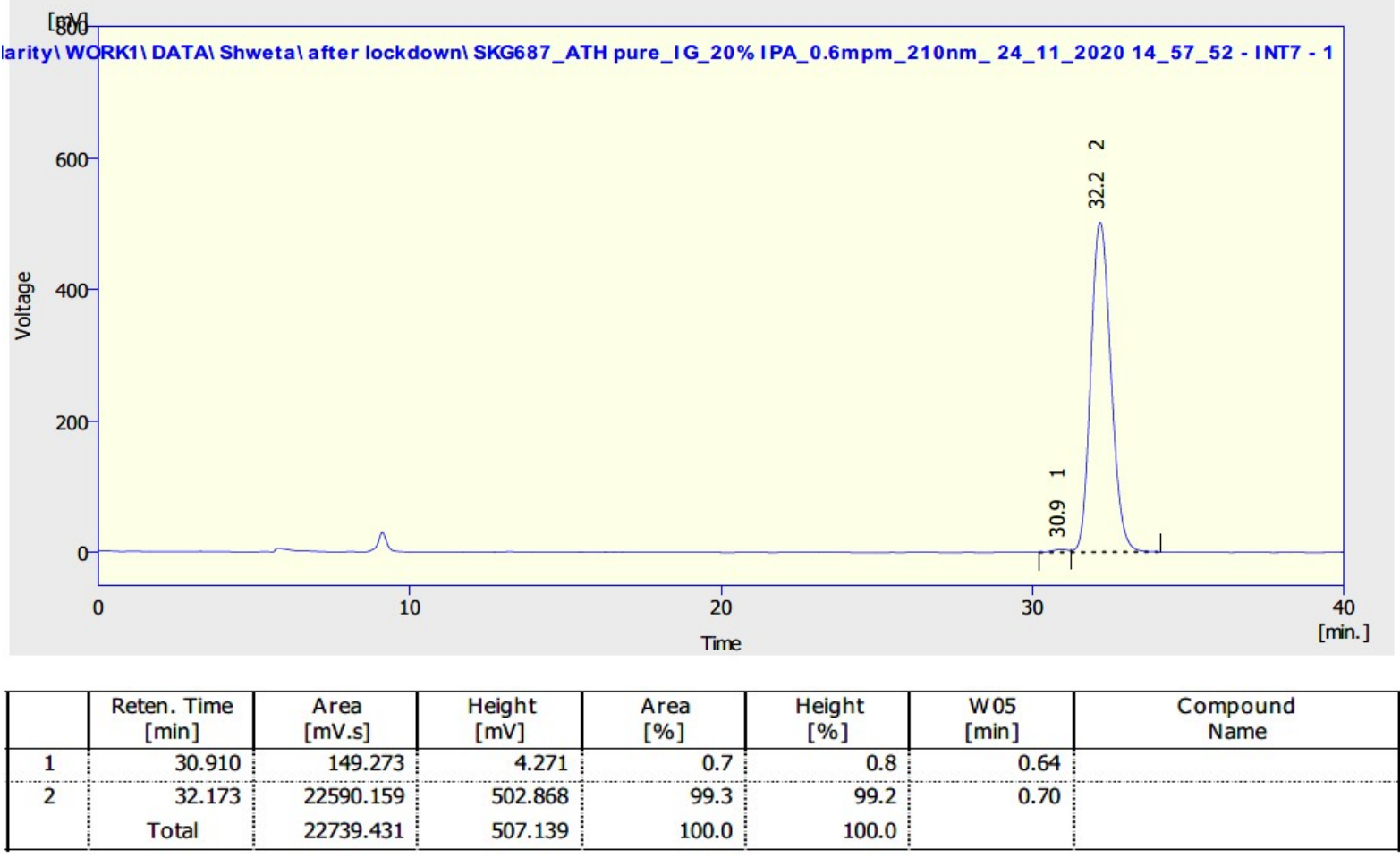


(R)-N-(2,6-Diisopropylphenyl)-2-hydroxy-4-oxo-4-(piperidin-1-yl)butanamide 27.<smiles>CC(C)c1cccc(C(C)C)c1NC(=O)[C@H](O)CC(=O)N1CCCCC1</smiles>

This compound is novel and was prepared following general procedure $\mathrm{C}$ using $\mathrm{N}$ - $(2,6-$ diisopropylphenyl)-2,4-dioxo-4-(piperidin-1-yl)butanamide ( $89.5 \mathrm{mg}, 0.250 \mathrm{mmol}, 1.0 \mathrm{eq}$ ), 3C tethered catalyst $(R, R)-2(2.3 \mathrm{mg}, 3.8 \mu \mathrm{mol}, 0.015 \mathrm{eq})$ and FA:TEA $(0.15 \mathrm{~mL})$ in DCM $(1.5 \mathrm{~mL})$ to give 27 as a yellow semi solid material $(81.7 \mathrm{mg}, 0.227 \mathrm{mmol}, 91 \%)$.

TLC: $\mathrm{R}_{\mathrm{f}}$ ca 0.2 (7:3, hexane: EtOAc), UV-active, Strong $\mathrm{KMnO}_{4}$ and $\mathrm{PMA}$-reactive; MP: $103-105^{\circ} \mathrm{C}$;

HRMS (ESI) m/z: [M+Na] ${ }^{+}$Calcd for $\mathrm{C}_{21} \mathrm{H}_{32} \mathrm{~N}_{2} \mathrm{O}_{3} \mathrm{Na}$ 383.2292; Found 383.2295 (error -0.9 ppm);

$U_{\max } 3376,3293,2961,1731,1673,1613,1497,1407,1254,1081,1027,730 \mathrm{~cm}^{-1}$;

Enantiomeric excess determined by HPLC analysis (Chiralpak IG, $250 \mathrm{~mm} \times 4.6 \mathrm{~mm}$ column, hexane: $\left.\mathrm{iPrOH} 90: 10,1 \mathrm{~mL} / \mathrm{min}, \lambda=210 \mathrm{~nm}, \mathrm{~T}=25^{\circ} \mathrm{C}\right), R$-enantiomer $14.2 \mathrm{~min}, S$ enantiomer $15.3 \min .99 \%$ ee $(R),[\alpha]_{\mathrm{D}}^{22}=-3.5\left(\mathrm{c}=0.1, \mathrm{CHCl}_{3}\right)$;

${ }^{1} \mathrm{H}$ NMR $\left(500 \mathrm{MHz}, \mathrm{CDCl}_{3}\right) \delta 8.39(1 \mathrm{H}, \mathrm{s}, \mathrm{CONH}), 7.30-7.26(1 \mathrm{H}, \mathrm{m}, \mathrm{ArH}), 7.19-7.17(2 \mathrm{H}$, m, ArH), $5.71(1 \mathrm{H}, \mathrm{s}, \mathrm{CHO} \underline{\mathrm{H}}), 4.64(1 \mathrm{H}, \mathrm{s}, \mathrm{C} \underline{\mathrm{HOH}}), 3.65-3.49\left(2 \mathrm{H}, \mathrm{m}, \mathrm{NCH}_{2}\right.$ of piperidine), 3.45-3.48 (2H, m, $\mathrm{NCH}_{2}$ of piperidine), 3.09-3.02 (2H, m, $\mathrm{CH}$ of isopropyl group), 2.98-2.89 $\left(2 \mathrm{H}, \mathrm{m}, \mathrm{CH}_{\mathrm{a}} \mathrm{H}_{\mathrm{b}}\right), 1.67-1.56\left(6 \mathrm{H}, \mathrm{m}, \mathrm{CH}_{2}\right.$ of piperidine $), 1.21-1.18\left(12 \mathrm{H}, \mathrm{m}, \mathrm{CH}_{3}\right.$ of isopropyl group);

${ }^{13} \mathrm{C}$ NMR $\left(126 \mathrm{MHz}, \mathrm{CDCl}_{3}\right) \delta 172.5,170.1,146.2,130.4,128.4,123.6,69.7,48.9,42.8$, $35.2,28.8,26.4,25.6,24.5,23.8,23.7$;

$\mathrm{m} / z(\mathrm{ESI}) 383.2\left[(\mathrm{M}+\mathrm{Na})^{+}, 100 \%\right]$. 
Use of other catalysts:

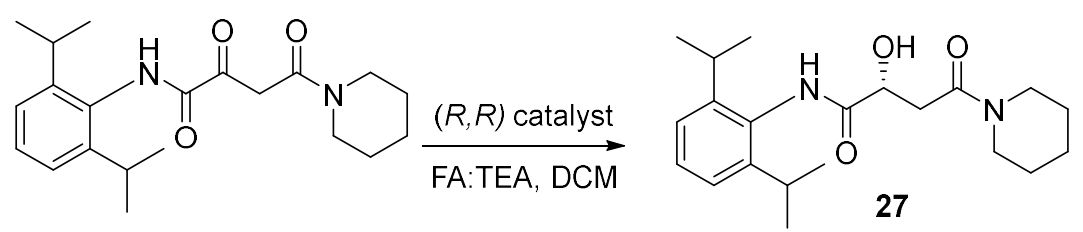

\begin{tabular}{|l|l|l|l|l|l|}
\hline Sr no & Catalyst & Time & Con (\%) & Ee(\%) & Yield (\%) \\
\hline 1 & & & & \\
\hline & & & & \\
\hline
\end{tabular}


N-(2,6-diisopropylphenyl)-2-hydroxy-4-oxo-4-(piperidin-1-yl)butanamide 27.

${ }^{1} \mathrm{H} \mathrm{NMR}\left(500 \mathrm{MHz}, \mathrm{CDCl}_{3}\right)$.

Nov17-2020

Chemist Shweta Gediya

PROTON.w CDCl3 /opt/topspin3.2 SKG 13

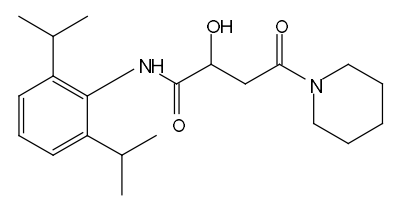

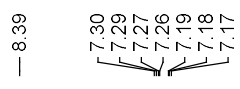

$\begin{array}{lllllll}11 & 4 & 4\end{array}$

$c_{1}^{2}$

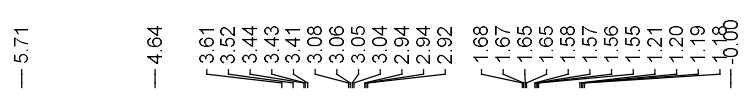

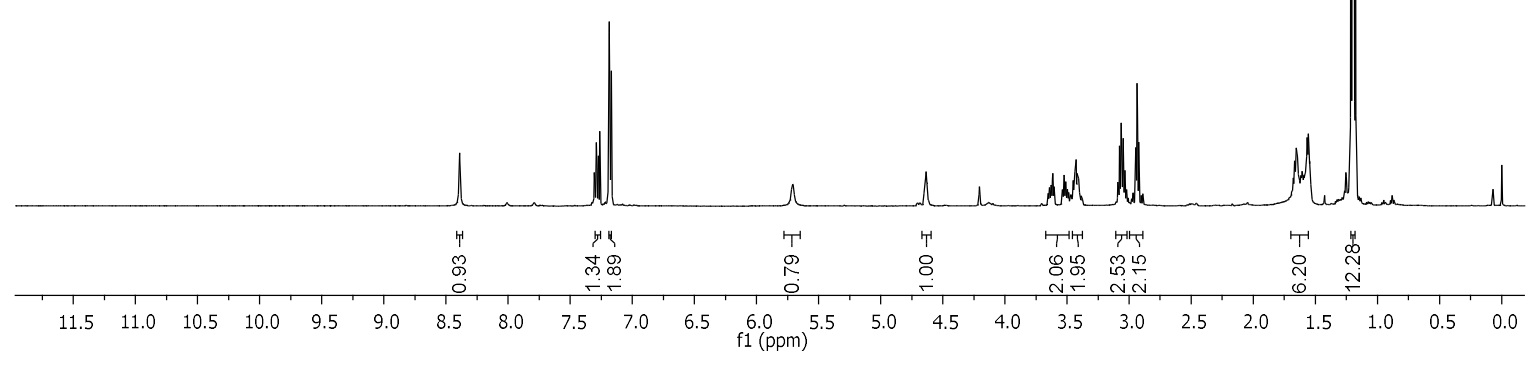

${ }^{13} \mathrm{C}$ NMR (126 MHz, $\left.\mathrm{CDCl}_{3}\right)$.

Nov17-2020

Nov17-2020
Chemist Shweta Gediya
SKG649
C13APT.w CDCl3 /opt/topspin3.2 SKG 13

Nov17-2020
Chemist Shweta Gediya
SKG649
C13APT.w CDCl3 /opt/topspin3.2 SKG 13

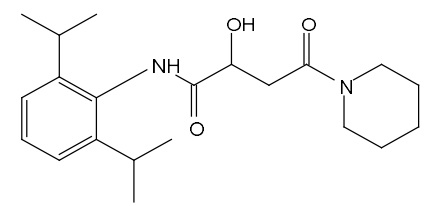

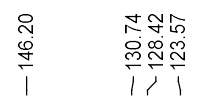

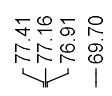

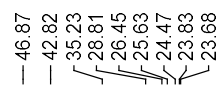
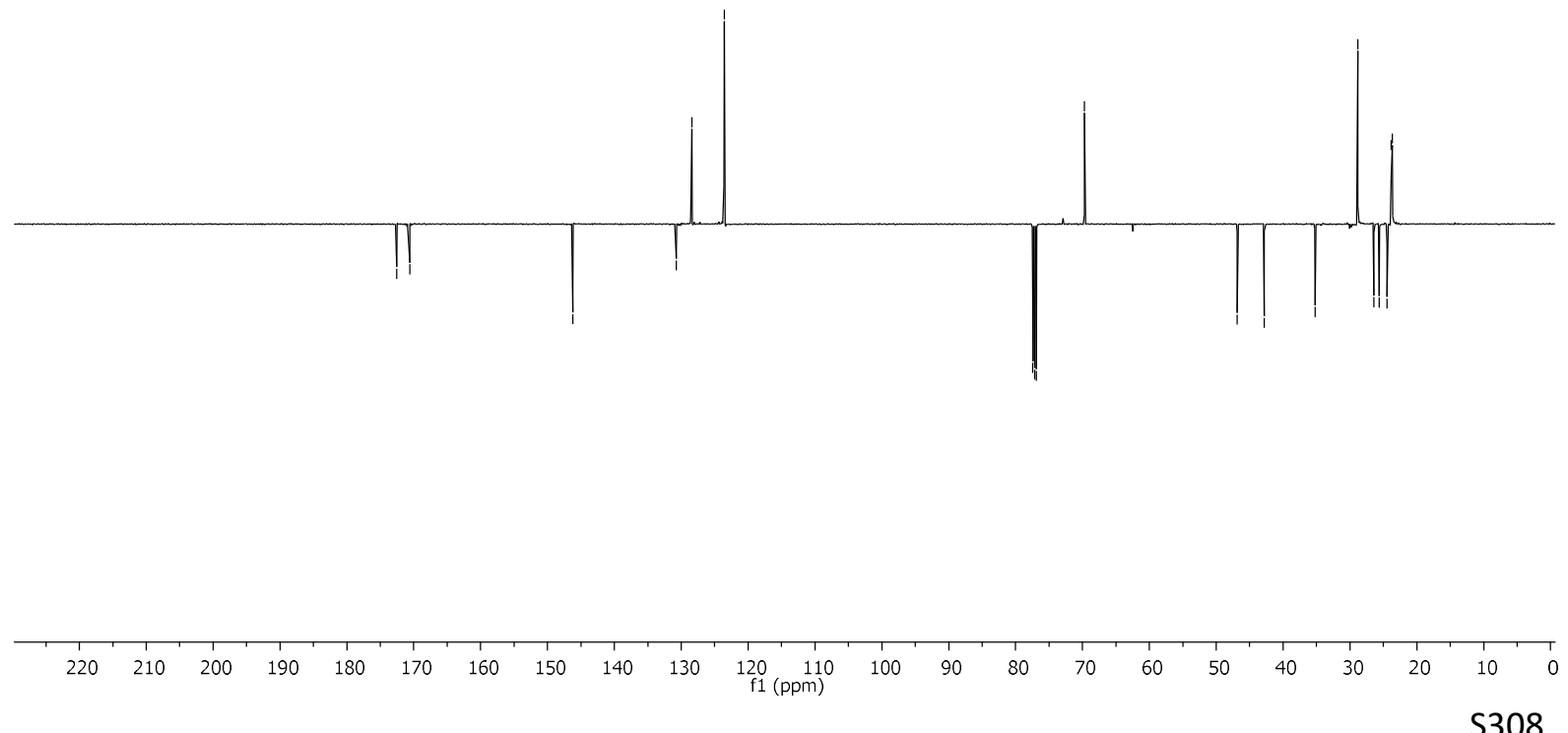

S308 
COSY.

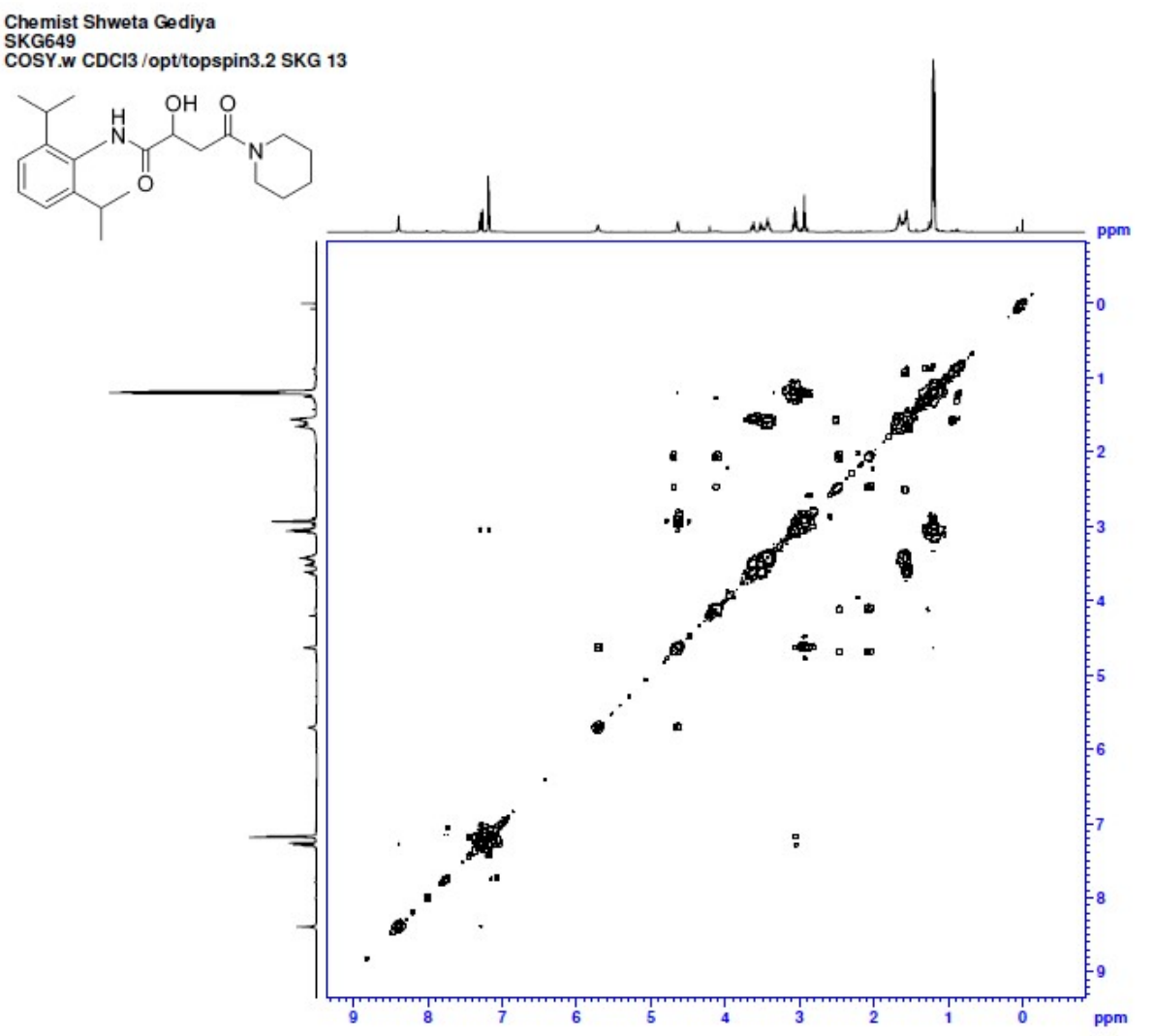

HSQC.

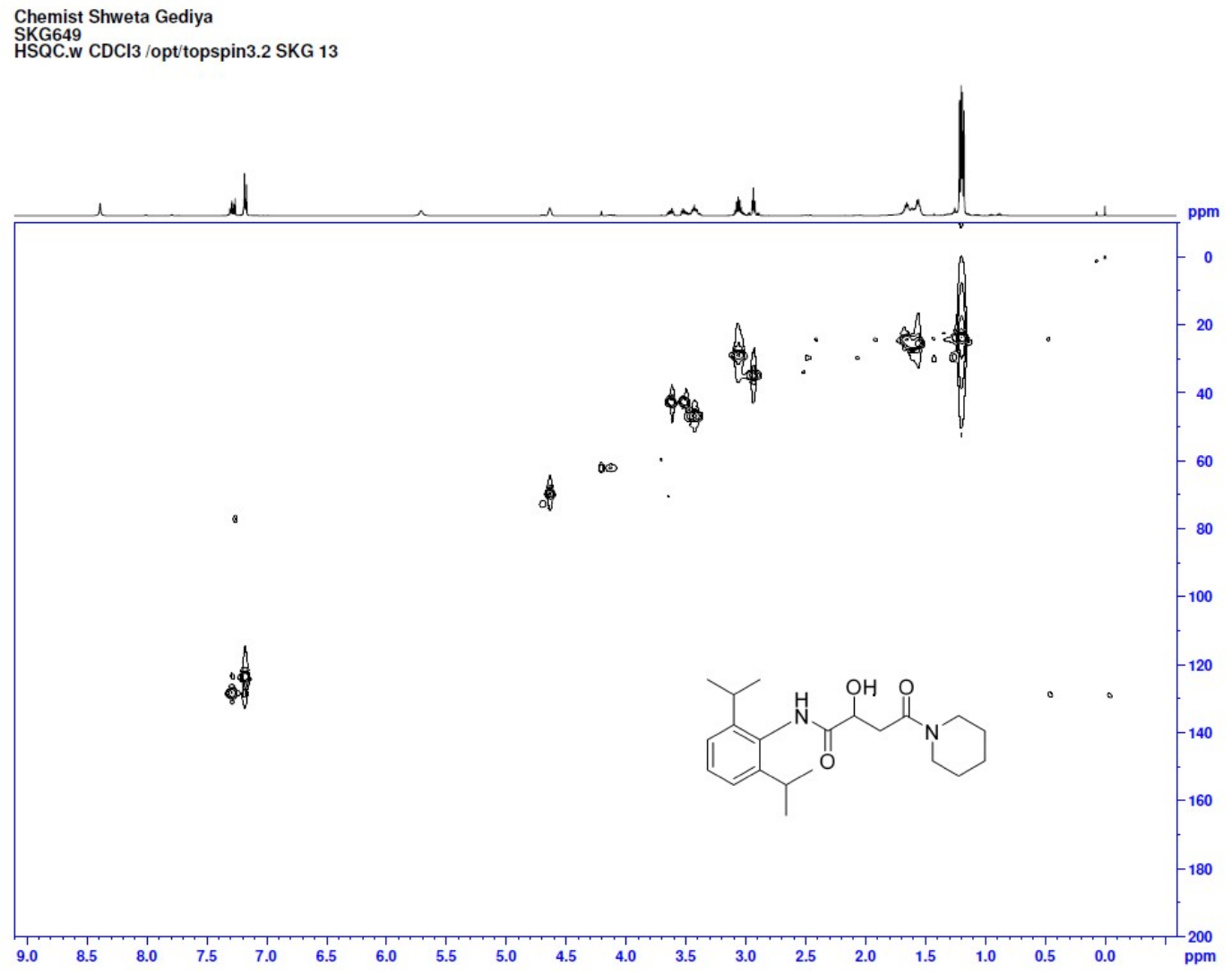

S309 


\section{Chiral HPLC of ketone precursor.}

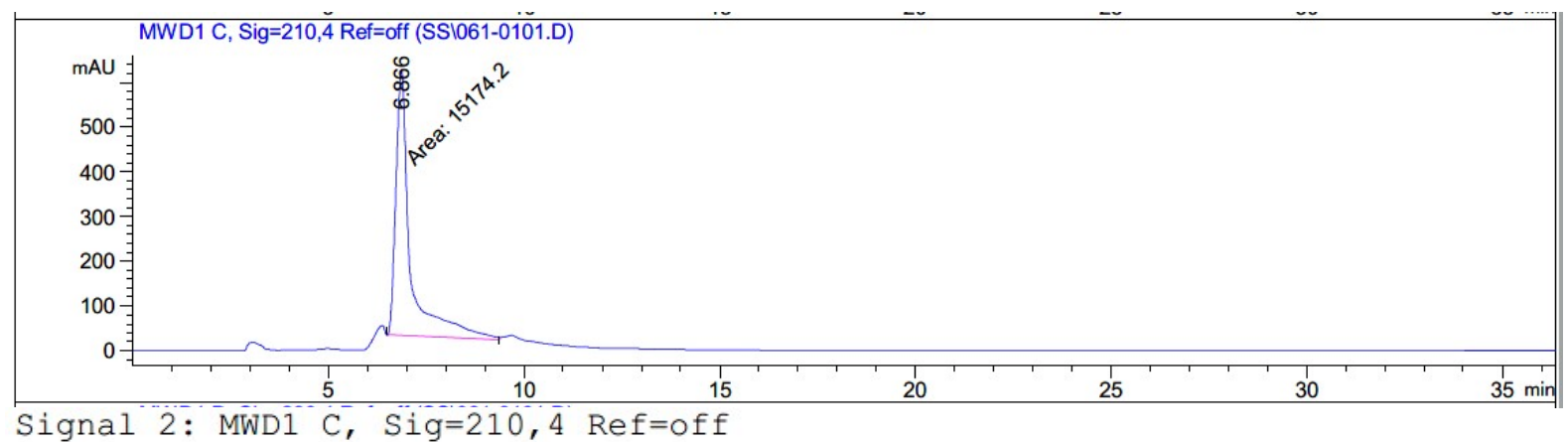

Signal 2: MẄD1 C, Sig=210, 4 Ref=off

\begin{tabular}{|c|c|c|c|c|c|c|}
\hline $\begin{array}{c}\text { Peak } \\
\#\end{array}$ & $\begin{array}{c}\text { RetTime } \\
\text { [min] }\end{array}$ & Type & $\begin{array}{l}\text { Width } \\
\text { [min] }\end{array}$ & $\begin{array}{c}\text { Area } \\
{\left[\mathrm{mAU}{ }^{\star} \mathrm{s}\right]}\end{array}$ & $\begin{array}{l}\text { Height } \\
\text { [mAU] }\end{array}$ & $\begin{array}{c}\text { Area } \\
\frac{\%}{\partial}\end{array}$ \\
\hline 1 & 6.866 & $\mathrm{MM}$ & 0.4268 & $.51742 e 4$ & 592.59576 & 100.0000 \\
\hline
\end{tabular}

\section{Chiral HPLC of racemic alcohol.}

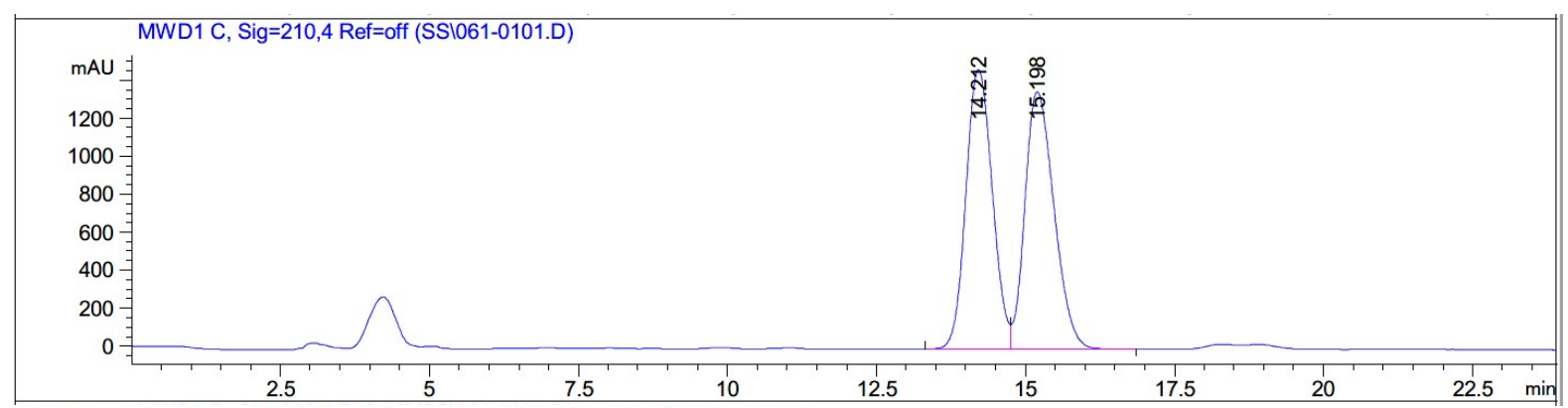

Signal 2: MWD1 C, Sig=210,4 Ref=off

\begin{tabular}{|c|c|c|c|c|c|c|}
\hline $\begin{array}{c}\text { Peak } \\
\#\end{array}$ & $\begin{array}{c}\text { RetTime } \\
\text { [min] }\end{array}$ & Type & $\begin{array}{l}\text { Width } \\
\text { [min] }\end{array}$ & $\begin{array}{c}\text { Area } \\
{\left[\mathrm{mAU}{ }^{\star} \mathrm{s}\right]}\end{array}$ & $\begin{array}{l}\text { Height } \\
{[\mathrm{mAU}]}\end{array}$ & $\begin{array}{c}\text { Area } \\
\quad \%\end{array}$ \\
\hline & & & 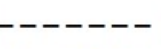 & 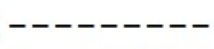 & -- & --- \\
\hline 1 & 14.212 & BV & 0.4860 & $4.53937 e 4$ & 1470.95264 & 49.6231 \\
\hline 2 & 5.198 & $\mathrm{VB}$ & 0.5329 & $4.60833 \mathrm{e} 4$ & 1354.52783 & 50.3769 \\
\hline
\end{tabular}


Chiral HPLC of ATH product. 99\% ee.

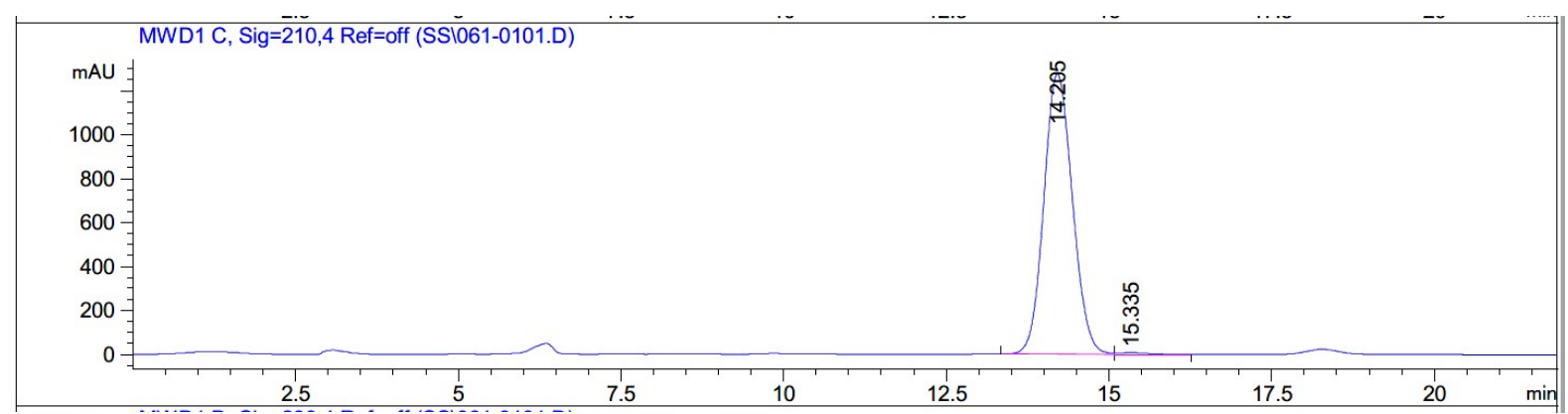

Signal 2: MWD1 C, Sig=210,4 Ref=off

\begin{tabular}{|c|c|c|c|c|c|c|}
\hline $\begin{array}{c}\text { Peak } \\
\#\end{array}$ & $\begin{array}{c}\text { RetTime } \\
\text { [min] }\end{array}$ & Type & $\begin{array}{l}\text { Width } \\
\text { [min] }\end{array}$ & $\begin{array}{c}\text { Area } \\
{\left[\mathrm{mAU}{ }^{\star} \mathrm{s}\right]}\end{array}$ & $\begin{array}{l}\text { Height } \\
\text { [mAU] }\end{array}$ & $\begin{array}{c}\text { Area } \\
\frac{\circ}{0}\end{array}$ \\
\hline-- & ----- & & --- & $\mid----------$ & $|----------|$ & $-------\mid$ \\
\hline 1 & 14.205 & BV & 0.4807 & $3.90070 \mathrm{e} 4$ & 1275.97302 & 99.4491 \\
\hline 2 & 15.335 & VB & 0.4486 & 216.06374 & 7.06950 & 0.5509 \\
\hline
\end{tabular}

A small amount of ketone is visible in this analysis. 
(R)-N-Cyclohexyl-2-hydroxy-4-oxo-4-(piperidin-1-yl)butanamide 28.<smiles>O=C(NC1CCCCC1)[C@H](O)CC(=O)N1CCCCC1</smiles>

This compound is novel and was prepared following general procedure $\mathrm{C}$ using $\mathrm{N}$ cyclohexyl-2,4-dioxo-4-(piperidin-1-yl)butanamide (0.070 g, $0.250 \mathrm{mmol}, 1.0 \mathrm{eq}), 3 \mathrm{C}$ tethered catalyst $(R, R)-2(2.3 \mathrm{mg}, 3.8 \mu \mathrm{mol}, 0.015 \mathrm{eq})$ and FA:TEA $(0.15 \mathrm{~mL})$ in DCM $(1.5$ $\mathrm{mL})$ to give 28 as a clear liquid $(0.059 \mathrm{~g}, 0.209 \mathrm{mmol}, 84 \%)$.

TLC: $\mathrm{R}_{\mathrm{f}}$ ca 0.4 (3:7, hexane: EtOAc), not UV-active, light $\mathrm{KMnO}_{4}$, and strong PMA-reactive; HRMS (ESI) m/z: [M+Na] $]^{+}$Calcd for $\mathrm{C}_{15} \mathrm{H}_{26} \mathrm{~N}_{2} \mathrm{O}_{3} \mathrm{Na} 305.1836$; Found 305.1832 (error 1.1 ppm);

$U_{\max } 3484,3326,2925,2850,1613,1540,1472,1244,1094,851 \mathrm{~cm}^{-1}$;

Enantiomeric excess determined by HPLC analysis (Chiralpak IG, $250 \mathrm{~mm} \times 4.6 \mathrm{~mm}$ column, hexane: $\left.\mathrm{iPrOH} 80: 20,1 \mathrm{~mL} / \mathrm{min}, \lambda=210 \mathrm{~nm}, \mathrm{~T}=25^{\circ} \mathrm{C}\right), S$-enantiomer $16.0 \mathrm{~min}, R$ enantiomer $17.1 \mathrm{~min} .97 \%$ ee $(R),[\alpha]_{\mathrm{D}}^{22}=-6.25\left(\mathrm{c}=0.1, \mathrm{CHCl}_{3}\right)$;

${ }^{1} \mathrm{H}$ NMR (500 MHz, $\left.\mathrm{CDCl}_{3}\right) \delta 6.89(1 \mathrm{H}, \mathrm{d}, J=7.4 \mathrm{~Hz}, \mathrm{CONH}), 5.23(1 \mathrm{H}, \mathrm{s}, \mathrm{CHO} \underline{\mathrm{H}}), 4.40-$ $4.38(1 \mathrm{H}, \mathrm{m}, \mathrm{C} \underline{\mathrm{HOH}}), 3.79-3.72\left(1 \mathrm{H}, \mathrm{m}, \mathrm{CH}\right.$ of cyclohexyl), 3.59-3.51 $\left(2 \mathrm{H}, \mathrm{m}, \mathrm{NCH}_{2}\right.$ of piperidine), 3.43-3.46 (2H, m, $\mathrm{NCH}_{2}$ of piperidine), $2.93\left(1 \mathrm{H}, \mathrm{dd}, J=16.6,3.7 \mathrm{~Hz}, \mathrm{C}_{\mathrm{a}} \mathrm{H}_{\mathrm{b}}\right)$, $2.67\left(1 \mathrm{H}, \mathrm{dd}, J=16.5,8.0 \mathrm{~Hz}, \mathrm{CH}_{\mathrm{a}} \underline{\mathrm{H}_{\mathrm{b}}}\right), 1.91-1.87\left(2 \mathrm{H}, \mathrm{m}, \mathrm{CH}_{2}\right.$ of cyclohexyl), 1.73-1.52 (8H, m, $\mathrm{CH}_{2}$ of cyclohexyl/piperidine), 1.42-1.14 (6H, m, $\mathrm{CH}_{2}$ of cyclohexyl/piperidine);

${ }^{13} \mathrm{C} \mathrm{NMR}\left(126 \mathrm{MHz}, \mathrm{CDCl}_{3}\right) \delta 171.1,170.5,69.3,47.9,46.7,42.8,35.8,33.1,33.0,26.4$, 25.6, 24.9, 24.5;

$m / z(\mathrm{ESI}) 305.3\left[(\mathrm{M}+\mathrm{Na})^{+}, 100 \%\right]$. 
N-Cyclohexyl-2-hydroxy-4-oxo-4-(piperidin-1-yl)butanamide 28.

${ }^{1} \mathrm{H}$ NMR (500 MHz, $\left.\mathrm{CDCl}_{3}\right)$.

Aug07-2020

Chemist Shweta Gediya

PROTON.w CDCl3 /opt/topspin3.2 SKG 53

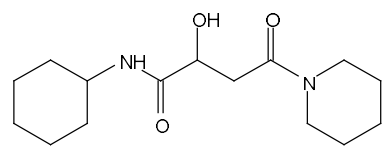

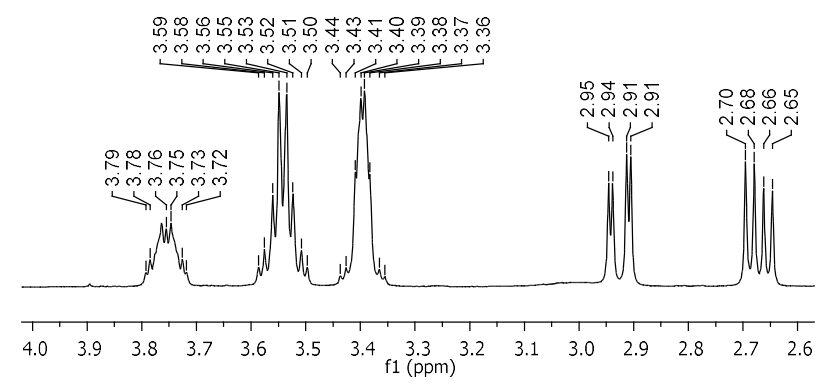

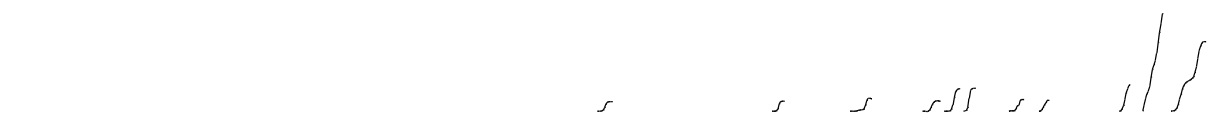

${ }^{13} \mathrm{C}$ NMR (126 MHz, $\left.\mathrm{CDCl}_{3}\right)$.

Aug07-2020
Chemist Shweta Gediya
SKG491
C13APT.w CDCI3 /opt/topspin3.2 SKG 53

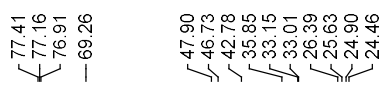
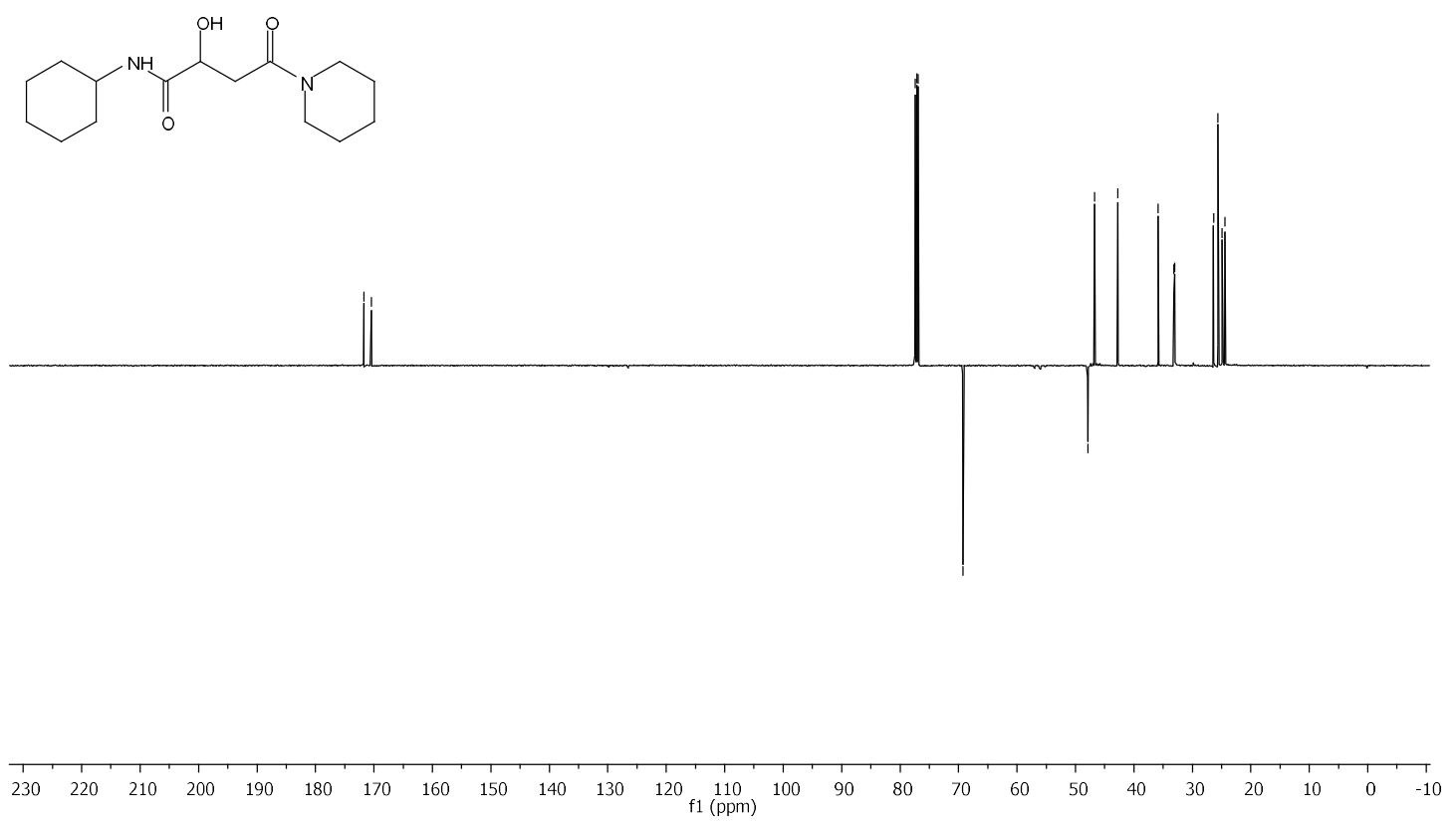

S313 
COSY.

\section{Chemist Shweta Gediya}

COSY.w $\mathrm{CDCl} 3 /$ opt/topspin3.2 SKG 53

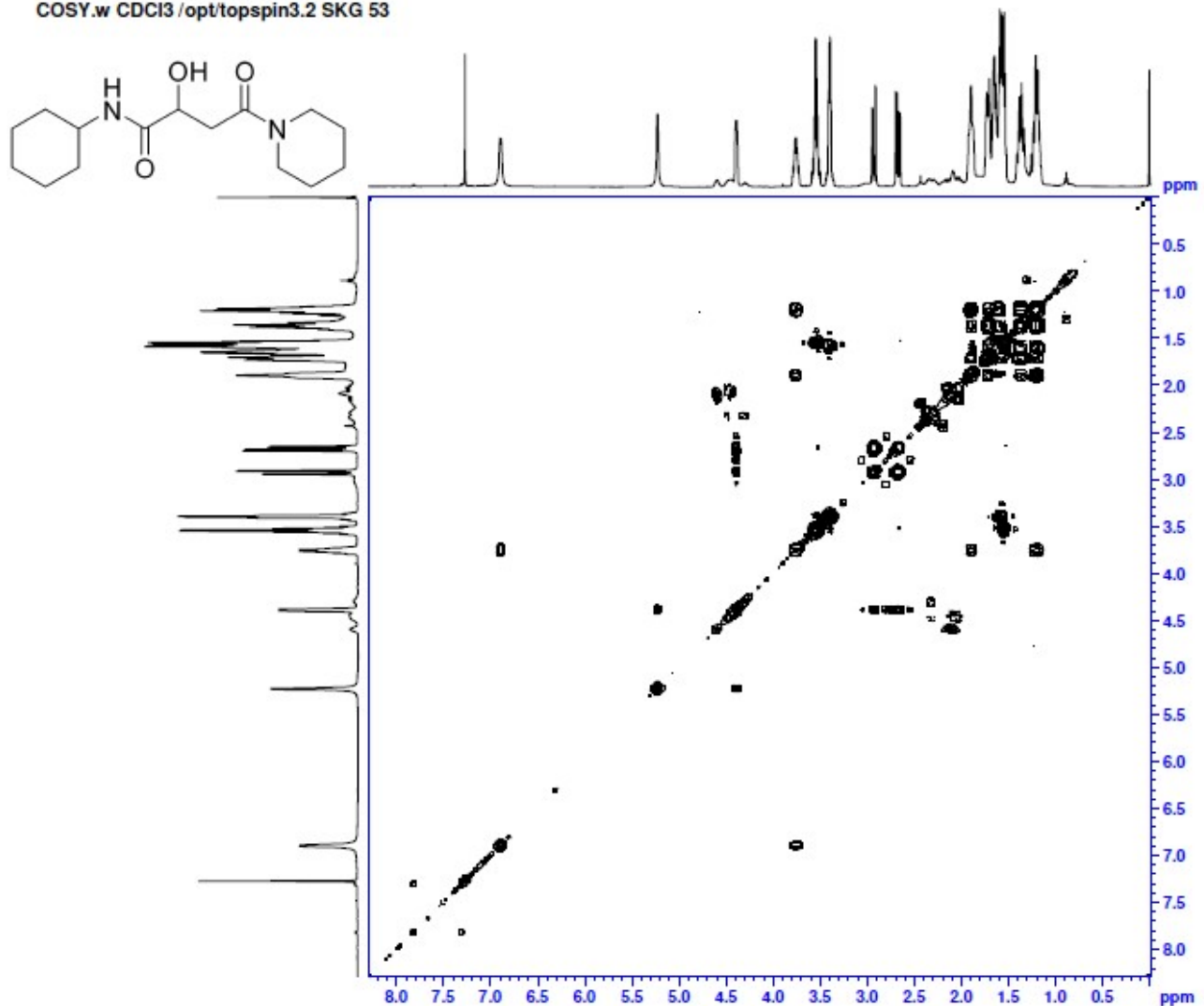

HSQC.

Chemist Shweta Gediya

HSQC.w CDCl3 /opttopspin3.2 SKG 53

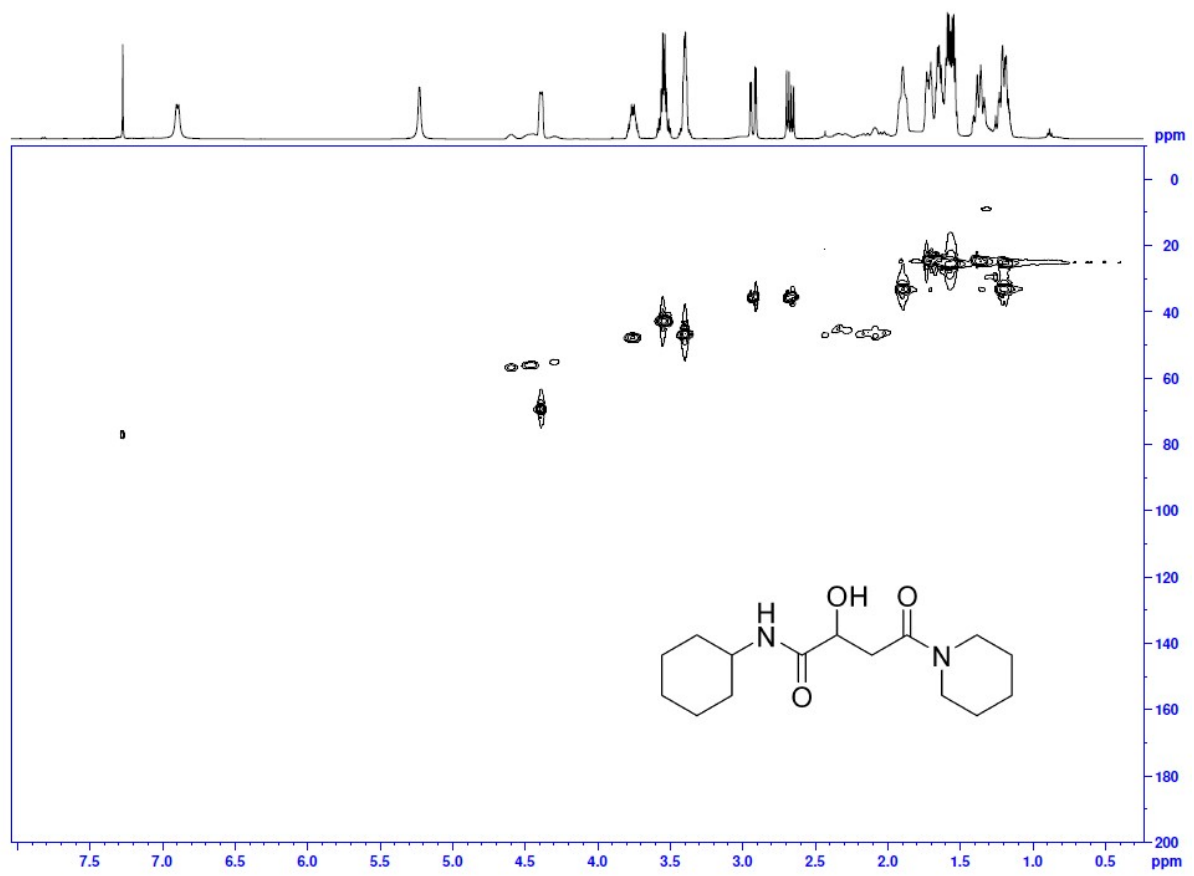




\section{Chiral HPLC of Ketone precursor.}

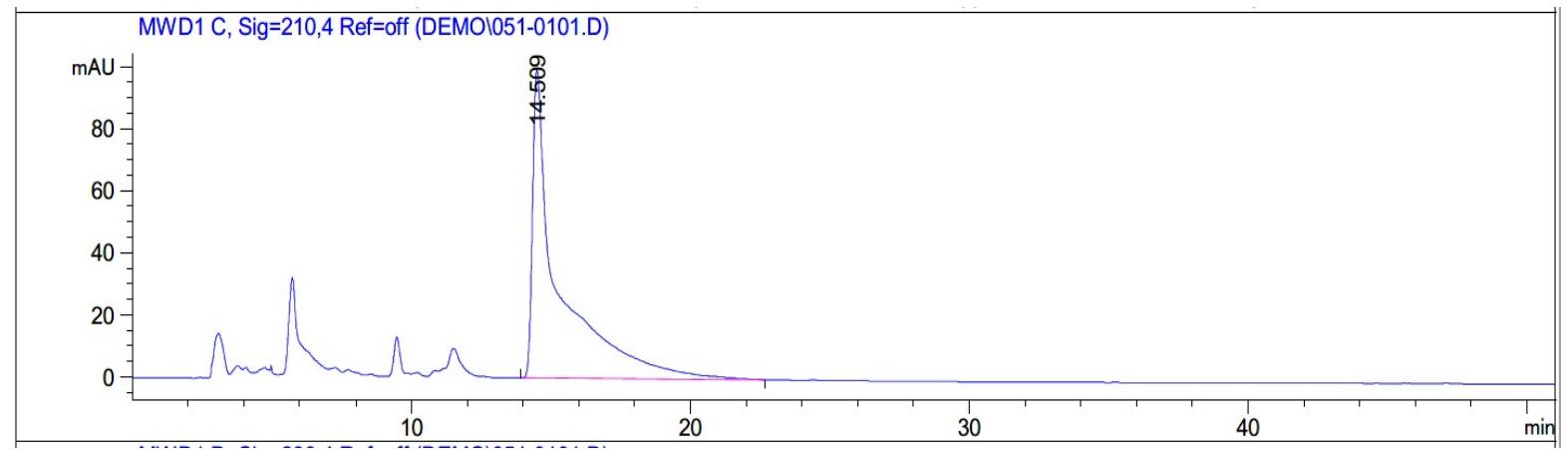

Signal 2: MWD1 C, Sig=210,4 Ref=off

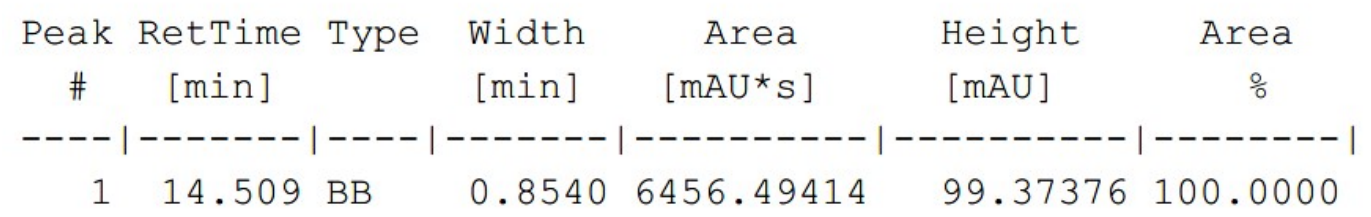

\section{Chiral HPLC of racemic alcohol.}

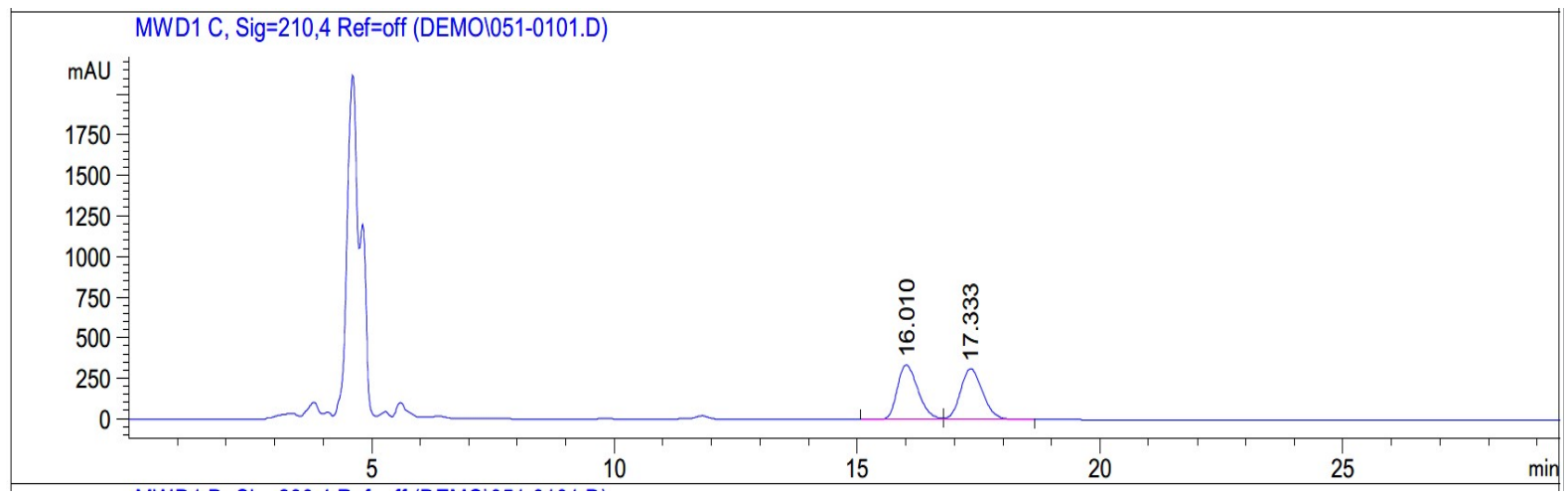

Signal 2: MWD1 C, Sig=210,4 Ref=off

\begin{tabular}{|c|c|c|c|c|c|c|}
\hline $\begin{array}{c}\text { Peak } \\
\#\end{array}$ & $\begin{array}{c}\text { RetTime } \\
\text { [min] }\end{array}$ & Type & $\begin{array}{l}\text { Width } \\
\text { [min] }\end{array}$ & $\begin{array}{c}\text { Area } \\
{\left[\mathrm{mAU}^{\star} \mathrm{s}\right]}\end{array}$ & $\begin{array}{l}\text { Height } \\
{[\mathrm{mAU}]}\end{array}$ & $\begin{array}{c}\text { Area } \\
\quad \%\end{array}$ \\
\hline & & & & & & \\
\hline $\begin{array}{l}1 \\
2\end{array}$ & 17.333 & $\begin{array}{l}\text { BV } \\
\text { VB }\end{array}$ & $\begin{array}{l}0.4605 \\
0.4935\end{array}$ & $\begin{array}{l}9982.99121 \\
9986.84375\end{array}$ & $\begin{array}{l}336.12946 \\
313.66306\end{array}$ & $\begin{array}{l}49.9904 \\
50.0096\end{array}$ \\
\hline
\end{tabular}




\section{Chiral HPLC of ATH product, 97\%.}

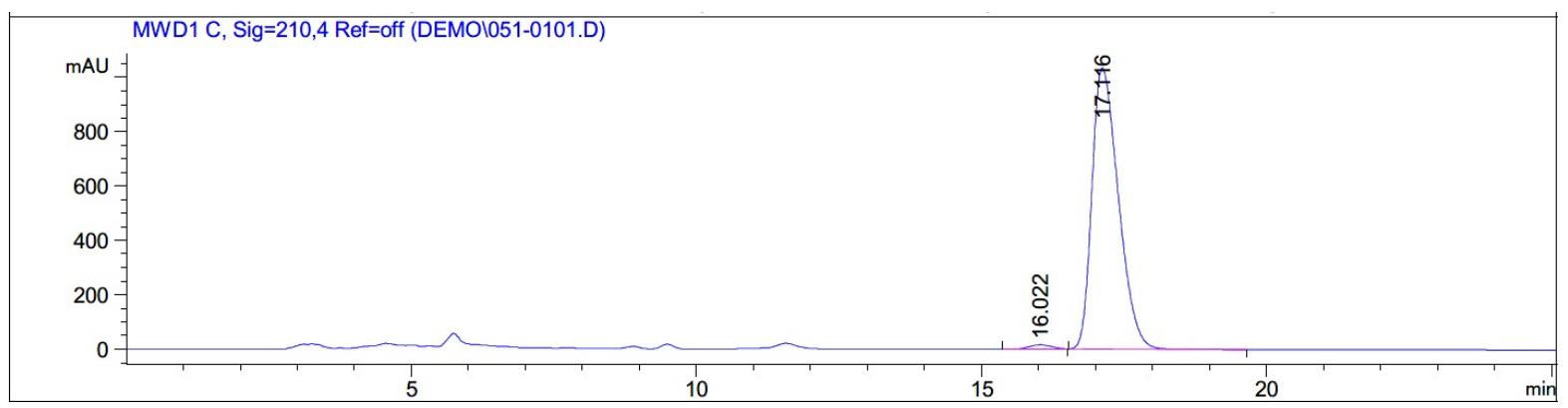

Signal 2: MWD1 C, Sig=210,4 Ref=off

\begin{tabular}{|c|c|c|c|c|c|c|}
\hline eak & $\begin{array}{c}\text { RetTime } \\
\text { [min] }\end{array}$ & Type & $\begin{array}{l}\text { Width } \\
\text { [min] }\end{array}$ & $\begin{array}{c}\text { Area } \\
{\left[\mathrm{mAU}{ }^{\star} \mathrm{s}\right]}\end{array}$ & $\begin{array}{l}\text { Height } \\
\text { [mAU] }\end{array}$ & $\begin{array}{c}\text { Area } \\
\quad \%\end{array}$ \\
\hline & & & & & 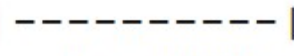 & \\
\hline 1 & & BV & 443 & 476.5 & 01 & 4 \\
\hline & 6 & & & $3.34670 \mathrm{e} 4$ & 663 & 9 \\
\hline
\end{tabular}

A small amount of ketone is visible in this analysis. 
(R)-2-Hydroxy- $N$-methyl-4-oxo-4-(piperidin-1-yl)butanamide 29.<smiles>CNC(=O)[C@H](O)CC(=O)N1CCCCC1</smiles>

This compound is novel and was prepared following general procedure $\mathrm{C}$ using $N$-methyl2,4-dioxo-4-(piperidin-1-yl)butanamide $(53.0 \mathrm{mg}, 0.250 \mathrm{mmol}, 1.0 \mathrm{eq}), 3 \mathrm{C}$ tethered catalyst $(R, R)-2(2.3 \mathrm{mg}, 3.8 \mu \mathrm{mol}, 0.015 \mathrm{eq})$ and FA:TEA $(0.15 \mathrm{~mL})$ in DCM $(1.5 \mathrm{~mL})$ to give 29 as a semisolid material (38.2 $\mathrm{mg}, 0.178 \mathrm{mmol}, 71 \%)$.

TLC: $\mathrm{R}_{\mathrm{f}}$ ca 0.3 (1:9, hexane: EtOAc), not UV-active, light $\mathrm{KMnO}_{4}$, and strong PMA-reactive; HRMS (ESI) m/z: [M+Na] ${ }^{+}$Calcd for $\mathrm{C}_{10} \mathrm{H}_{18} \mathrm{~N}_{2} \mathrm{O}_{3} \mathrm{Na} 237.1210$; Found 237.1217 (error -3.0 ppm);

$U_{\max } 3368,3297,2933,1718,1660,1625,1523,1260,1078,619 \mathrm{~cm}^{-1}$;

Enantiomeric excess determined by HPLC analysis (Chiralpak IG, $250 \mathrm{~mm} \times 4.6 \mathrm{~mm}$ column, Hexane: $\left.\mathrm{iPrOH} 75: 25,1 \mathrm{~mL} / \mathrm{min}, \lambda=210 \mathrm{~nm}, \mathrm{~T}=25{ }^{\circ} \mathrm{C}\right), R$-enantiomer $18.5 \mathrm{~min}, S$ enantiomer $23.6 \min .95 \%$ ee $(R),[\alpha]_{\mathrm{D}}^{22}=+7.75\left(\mathrm{c}=0.1, \mathrm{CHCl}_{3}\right)$;

${ }^{1} \mathrm{H}$ NMR (400 MHz, $\left.\mathrm{CDCl}_{3}\right) \delta 7.00(1 \mathrm{H}, \mathrm{s}, \mathrm{CONH}), 5.23(1 \mathrm{H}, \mathrm{s}, \mathrm{CHO} \underline{\mathrm{H}}), 4.41(1 \mathrm{H}, \mathrm{s}$, $\mathrm{C} \underline{\mathrm{HOH}}), 3.59-3.49\left(2 \mathrm{H}, \mathrm{m}, \mathrm{NCH}_{2}\right.$ of piperidine), 3.40-3.37 (2H, m, $\mathrm{NCH}_{2}$ of piperidine), $2.93\left(1 \mathrm{H}, \mathrm{dd}, J=16.6,3.7 \mathrm{~Hz}, \underline{\mathrm{C}}_{\mathrm{a}} \mathrm{H}_{\mathrm{b}}\right), 2.85\left(3 \mathrm{H}, \mathrm{d}, J=4.9 \mathrm{~Hz}, \mathrm{NHC}_{3}\right), 2.70(1 \mathrm{H}, \mathrm{dd}, J=$ 16.6, $\left.7.8 \mathrm{~Hz}, \mathrm{CH}_{\mathrm{a}} \underline{\mathrm{H}} \mathrm{b}\right), 1.66-1.53\left(6 \mathrm{H}, \mathrm{m}, \mathrm{CH}_{2}\right.$ of piperidine);

${ }^{13} \mathrm{C} \mathrm{NMR}\left(101 \mathrm{MHz}, \mathrm{CDCl}_{3}\right) \delta 173.4,170.4,69.4,46.7,42.8,35.8,26.4,25.9,25.6,24.5$;

$m / z(\mathrm{ESI}) 237.3\left[(\mathrm{M}+\mathrm{Na})^{+}, 10 \%\right], 451.4\left[(2 \mathrm{M}+\mathrm{Na})^{+}, 100 \%\right]$. 
2-Hydroxy- $N$-methyl-4-oxo-4-(piperidin-1-yl)butanamide 29.

${ }^{1} \mathrm{H}$ NMR (400 MHz, $\left.\mathrm{CDCl}_{3}\right)$.

Aug18-2020
SKG514

PROTON.w CDCl3 /opt/topspin3.5pl2 SKG 20

$\stackrel{i}{i}$

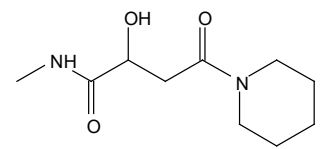

$\stackrel{\infty}{\infty}$

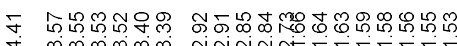

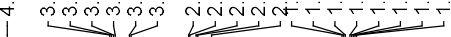

1

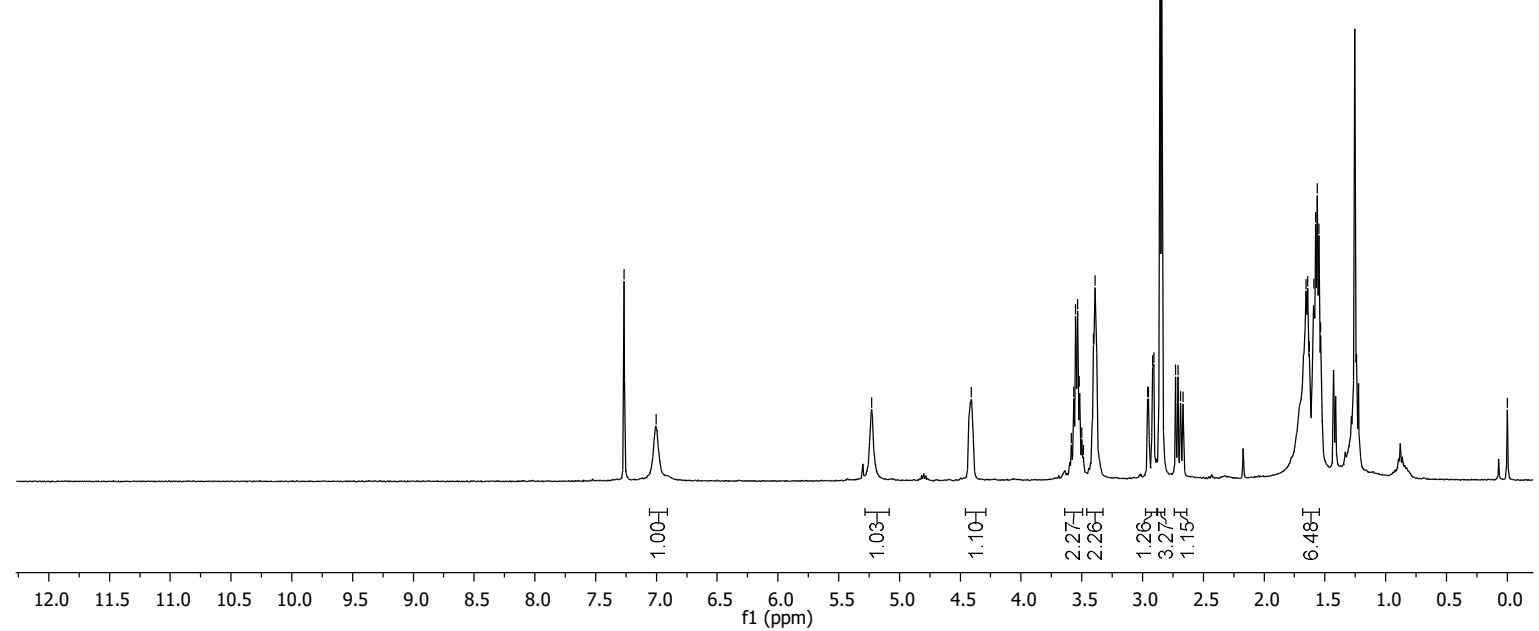

${ }^{13} \mathrm{C}$ NMR $\left(101 \mathrm{MHz}, \mathrm{CDCl}_{3}\right)$.<smiles>CNC(=O)C(O)CC(=O)N1CCCCC1</smiles>

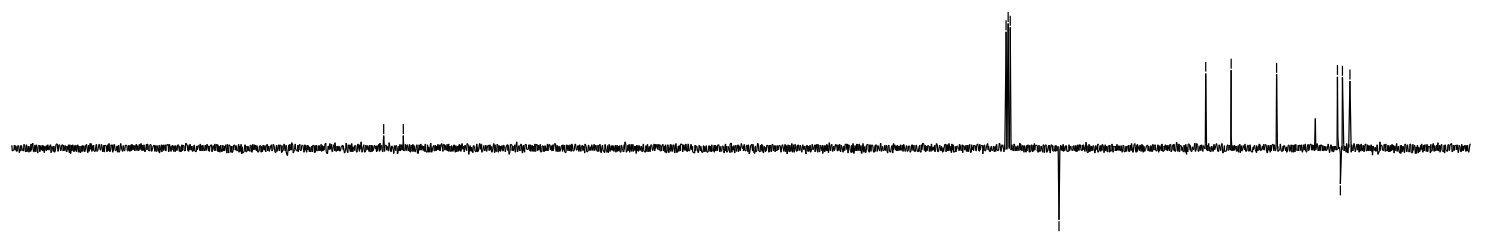

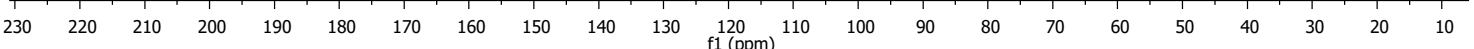


COSY.

SKG514
COSY.w CDCl3/opt/topspin3.5pl2 SKG 20

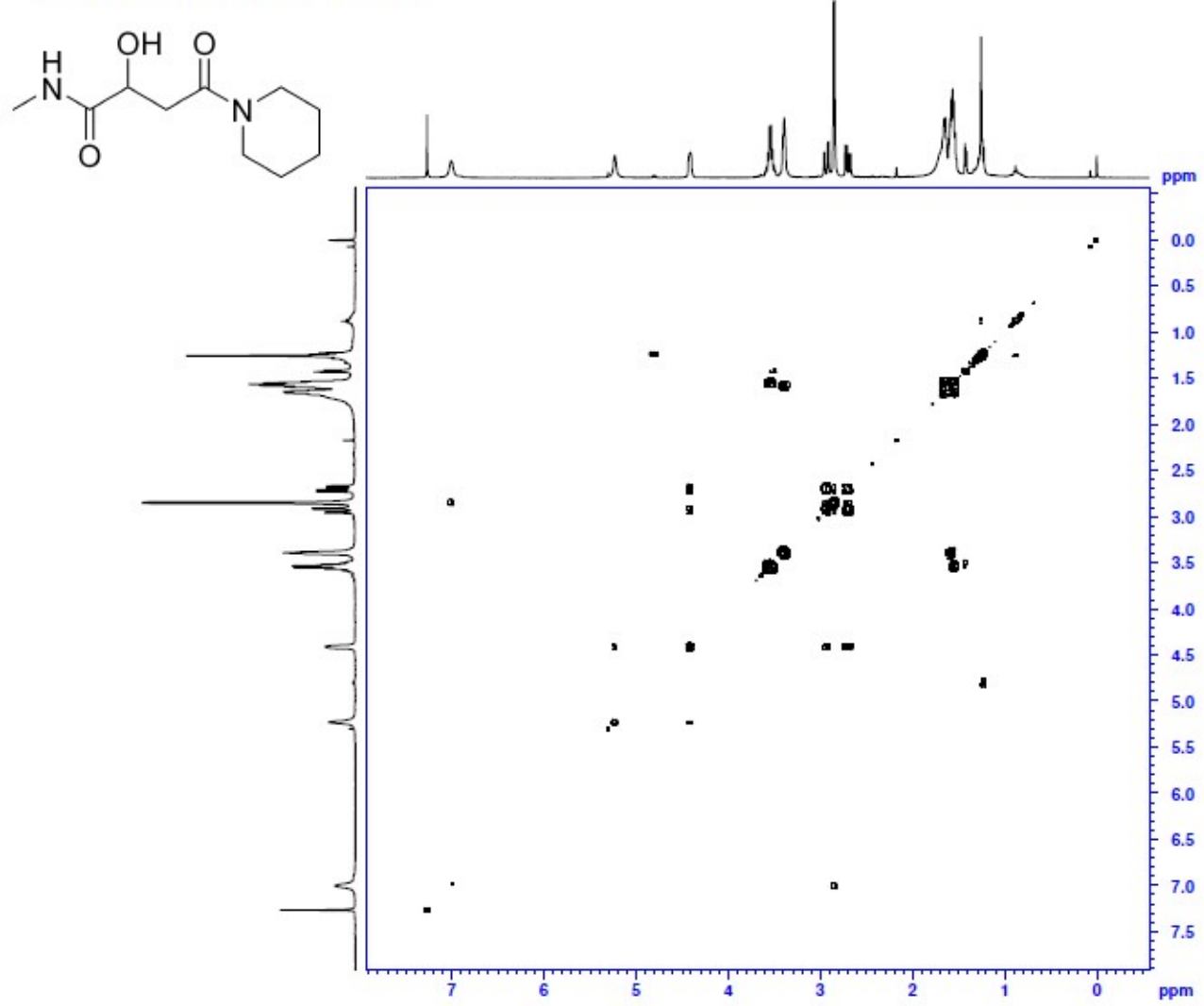

\section{Chiral HPLC of ketone precursor.}

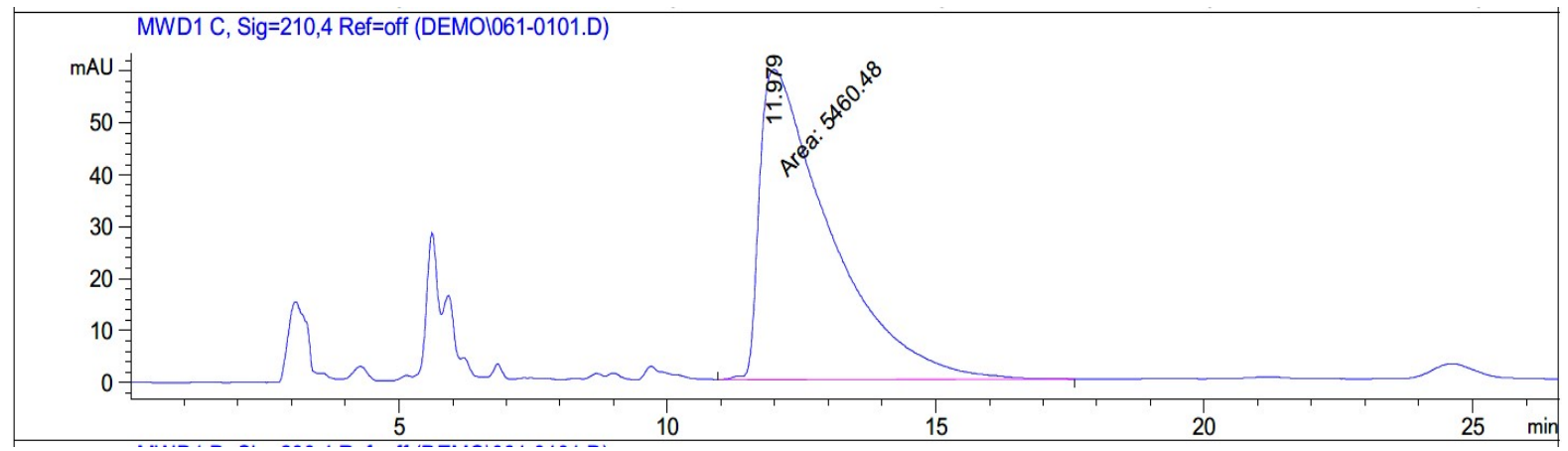

Signal 2: MWD1 C, Sig=210,4 Ref=off

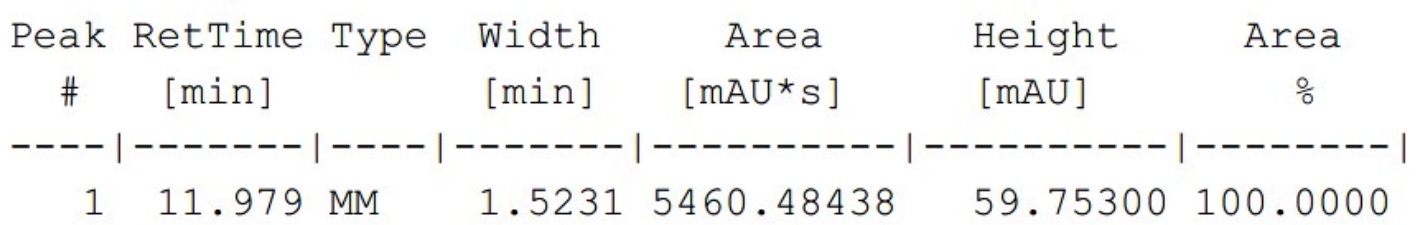




\section{Chiral HPLC of racemic alcohol.}

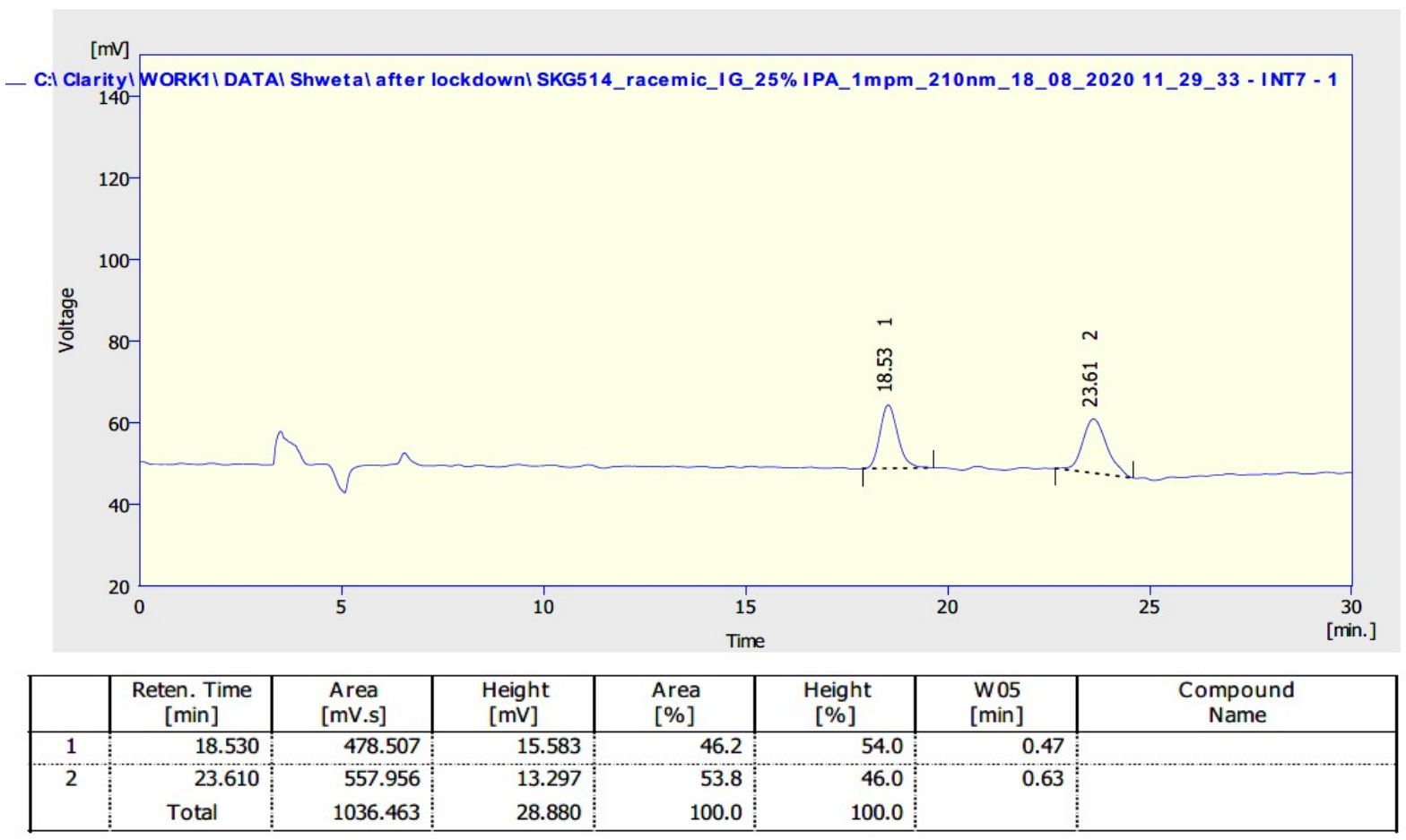

\section{Chiral HPLC of ATH product. 95\% ee.}

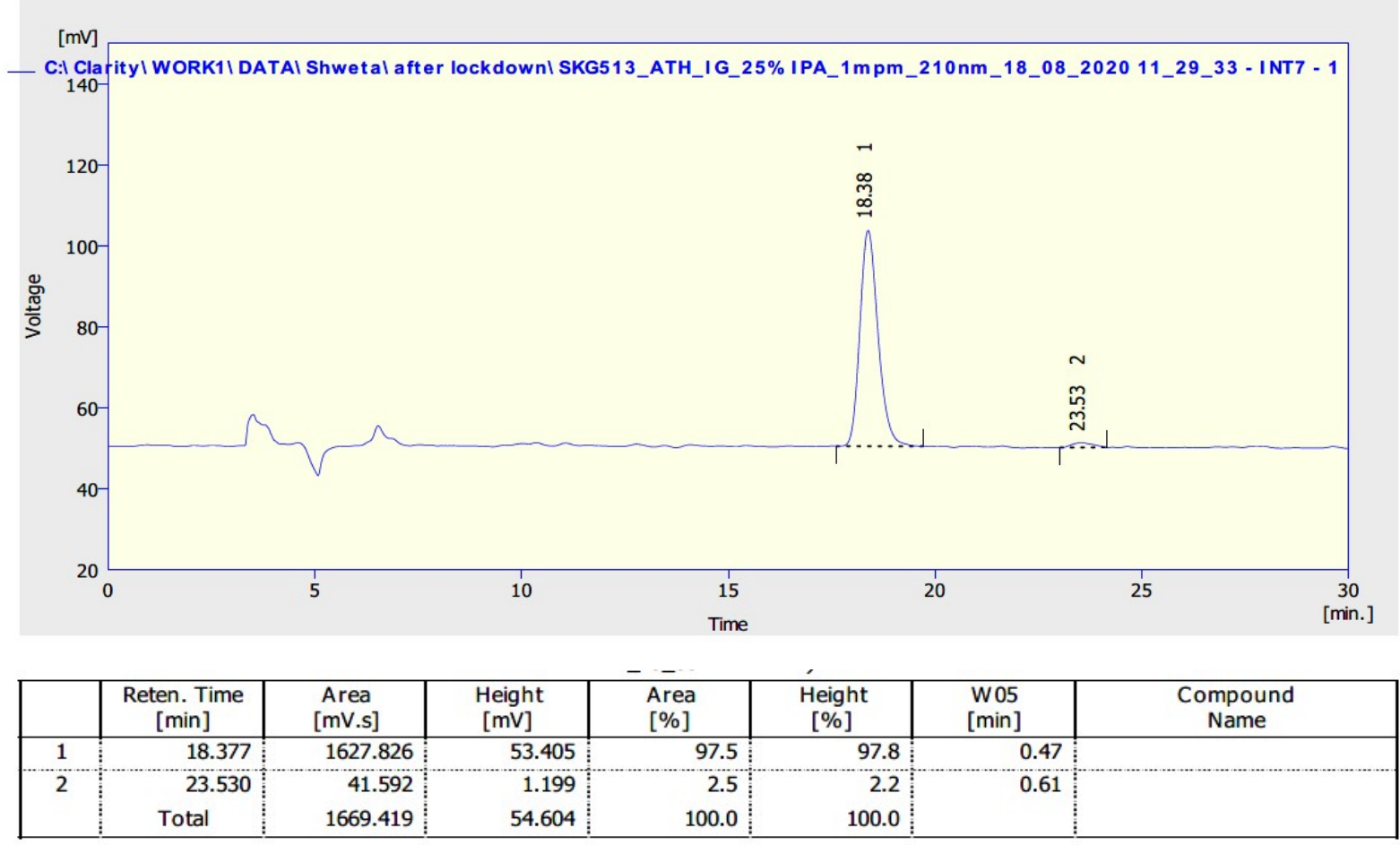


(R)-N-Cyclopropyl-2-hydroxy-4-oxo-4-(piperidin-1-yl)butanamide 30.<smiles>O=C(NC1CC1)[C@H](O)CC(=O)N1CCCCC1</smiles>

This compound is novel and was prepared following general procedure $\mathrm{C}$ using $\mathrm{N}$ cyclopropyl-2,4-dioxo-4-(piperidin-1-yl)butanamide (0.119 g, $0.500 \mathrm{mmol}, 1.0 \mathrm{eq})$, 3C tethered catalyst $(R, R)-2(4.7 \mathrm{mg}, 7.5 \mu \mathrm{mol}, 0.015 \mathrm{eq})$ and FA:TEA $(0.3 \mathrm{~mL})$ in DCM $(3 \mathrm{~mL})$ to give 30 as an off white $(0.105 \mathrm{~g}, 0.437 \mathrm{mmol}, 88 \%)$.

TLC: $\mathrm{R}_{\mathrm{f}}$ ca 0.4 (1:9, hexane: EtOAc), non UV-active, light $\mathrm{KMnO}_{4}$, and strong PMAreactive;

MP: $124-127{ }^{\circ} \mathrm{C}$;

HRMS (ESI) m/z: [M+Na] $]^{+}$Calcd for $\mathrm{C}_{12} \mathrm{H}_{20} \mathrm{~N}_{2} \mathrm{O}_{3} \mathrm{Na}$ 263.1366; Found 263.1373 (error -2.7 ppm);

$U_{\max } 3339,3289,2933,1643,1623,1510,1441,1270,1219,1103,1054,609 \mathrm{~cm}^{-1}$;

Enantiomeric excess determined by HPLC analysis (Chiralpak IG, $250 \mathrm{~mm} \times 4.6 \mathrm{~mm}$ column, hexane: $\mathrm{iPrOH} 90: 10,0.6 \mathrm{~mL} / \mathrm{min}, \lambda=210 \mathrm{~nm}, \mathrm{~T}=25{ }^{\circ} \mathrm{C}$ ), $R$-enantiomer $88.8 \mathrm{~min}, S$ enantiomer $93.7 \min .98 \%$ ee $(R),[\alpha]_{\mathrm{D}}^{22}=+10.2\left(\mathrm{c}=0.1, \mathrm{CHCl}_{3}\right)$;

${ }^{1} \mathrm{H}$ NMR $\left(400 \mathrm{MHz}, \mathrm{CDCl}_{3}\right)^{1} \mathrm{H}$ NMR $\left(400 \mathrm{MHz}, \mathrm{CDCl}_{3}\right) \delta 7.02(1 \mathrm{H}, \mathrm{s}, \mathrm{CONH}), 5.21(1 \mathrm{H}, \mathrm{d}$, $J=2.9 \mathrm{~Hz}, \mathrm{CHO} \underline{\mathrm{H}}), 4.37-4.35$ (1H, m, $\underline{\mathrm{HOH}}), 3.57-3.51$ (2H, m, $\mathrm{NCH}_{2}$ of piperidine), 3.39$3.36\left(2 \mathrm{H}, \mathrm{m}, \mathrm{NCH}_{2}\right.$ of piperidine), $2.90\left(1 \mathrm{H}, \mathrm{dd}, J=16.6,3.8 \mathrm{~Hz}, \mathrm{CH}_{\mathrm{a}} \mathrm{H}_{\mathrm{b}}\right), 2.76-2.64(2 \mathrm{H}, \mathrm{m}$, CㅌNH-cyclopropyl $\left.+\mathrm{CH}_{\mathrm{a}} \underline{\mathrm{H}_{\mathrm{b}}}\right), 1.64-1.50\left(6 \mathrm{H}, \mathrm{m}, \mathrm{CH}_{2}\right.$ of piperidine $), 0.79-0.74\left(2 \mathrm{H}, \mathrm{m}, \mathrm{CH}_{2}-\right.$ cyclopropyl), 0.55-0.48 (2H, m, $\mathrm{CH}_{2}$-cyclopropyl);

${ }^{13} \mathrm{C}$ NMR $\left(101 \mathrm{MHz}, \mathrm{CDCl}_{3}\right) \delta 174.3,170.4,69.3,46.7,42.8,35.7,26.4,25.6,24.5,22.3$, $6.5,6.4$

$m / z(\mathrm{ESI}) 263.1\left[(\mathrm{M}+\mathrm{Na})^{+}, 100 \%\right]$. 
N-Cyclopropyl-2-hydroxy-4-oxo-4-(piperidin-1-yl)butanamide 30.

${ }^{1} \mathrm{H}$ NMR $\left(400 \mathrm{MHz}, \mathrm{CDCl}_{3}\right)$.

Sep10-2020

PROTON.W CDCI3

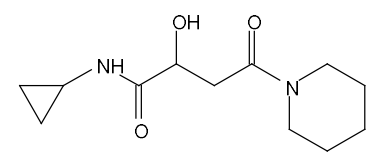

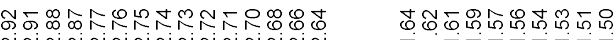

L

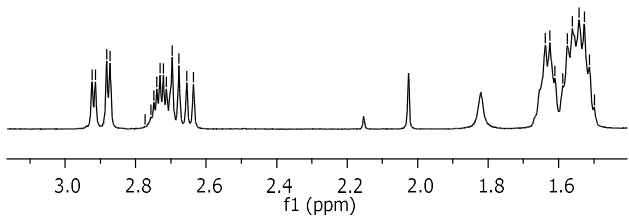

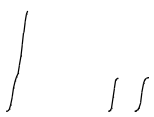

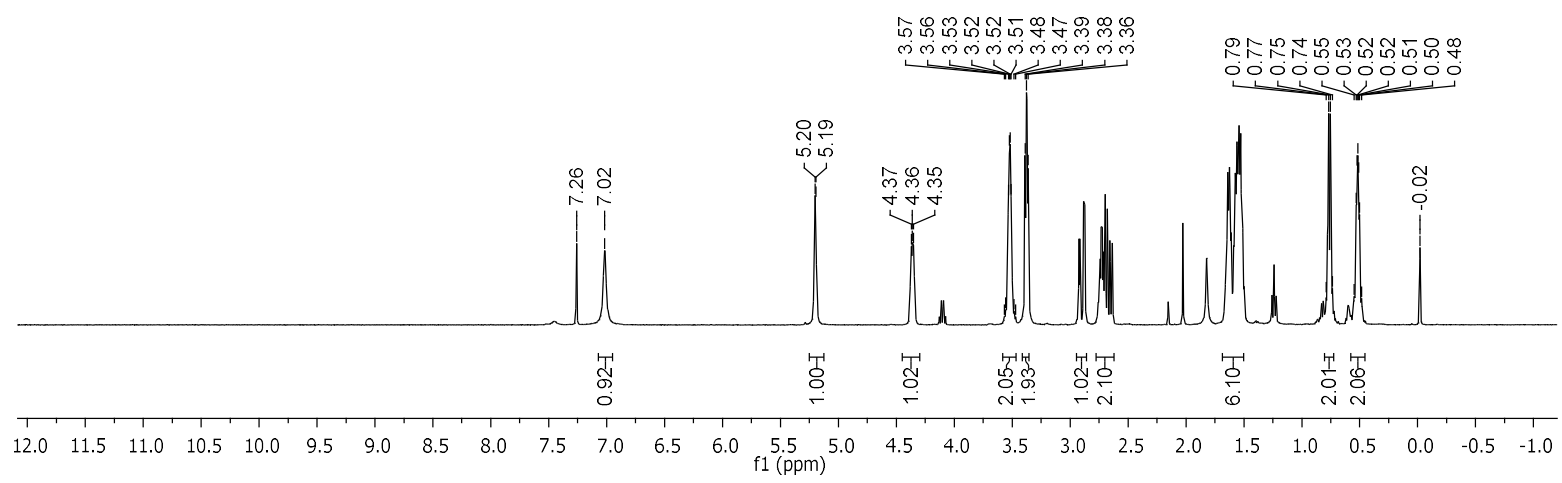

${ }^{13} \mathrm{C}$ NMR (101 MHz, $\left.\mathrm{CDCl}_{3}\right)$.

Sep10-2020

SKG535
C13APTIong.w CDCI3

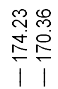

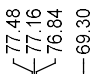

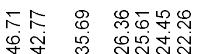

i i
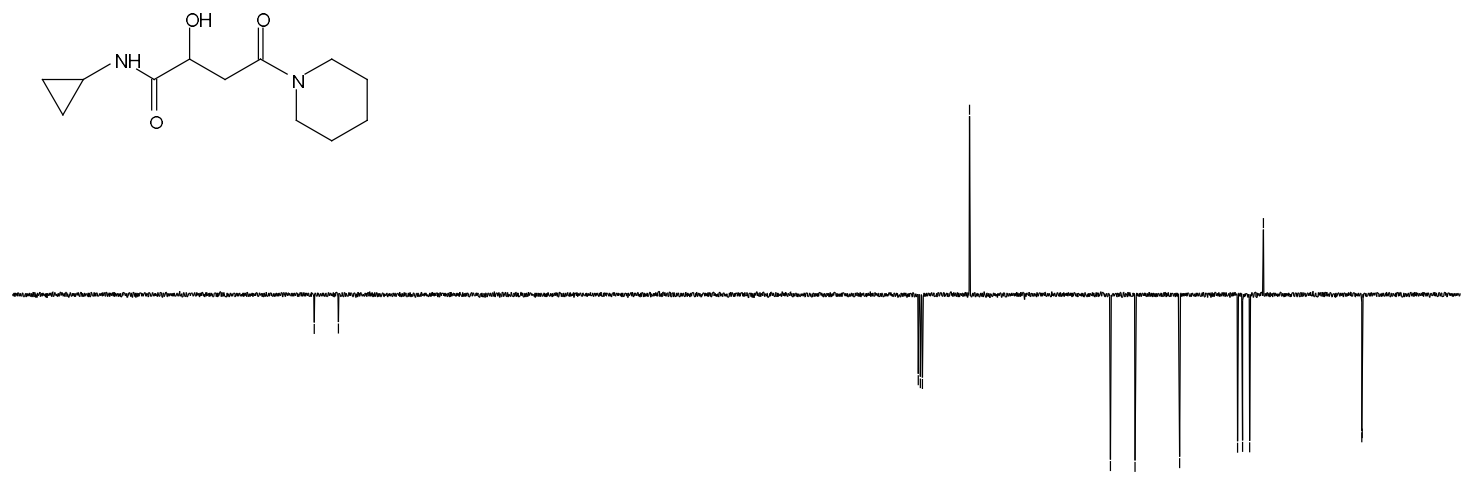

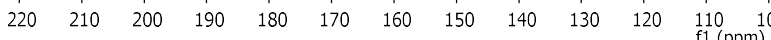


COSY.

SKG535 pure on the form

SKG536 pure on the tube

COSY.w CDCl3/opttopspin3.5pl2 SKG 23

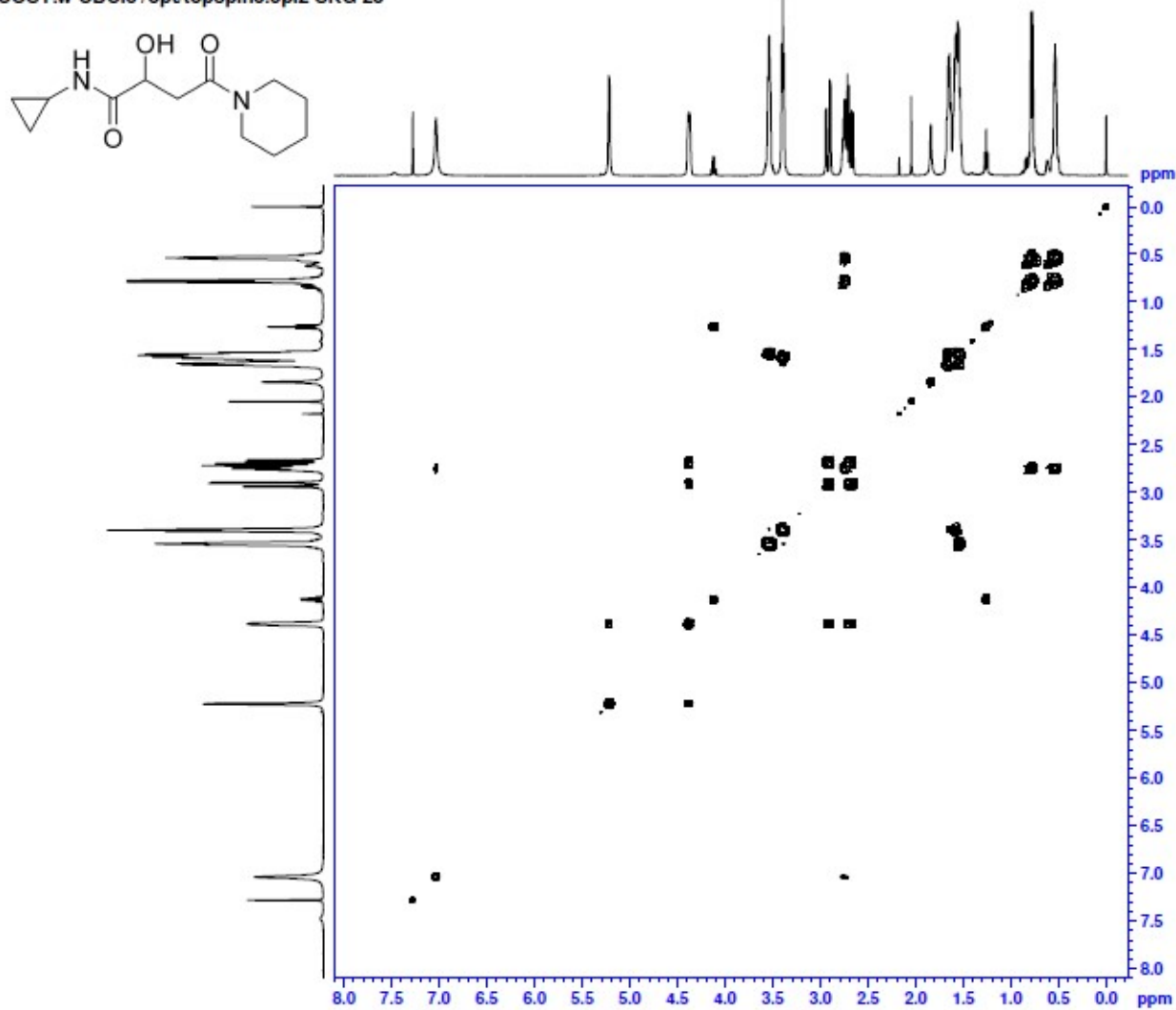

HSQC.

SKG535 pure on the form

SKG536 pure on the tube
HSQC.W CDCl3 / opt topspin 3.5 pl2 SKG 23

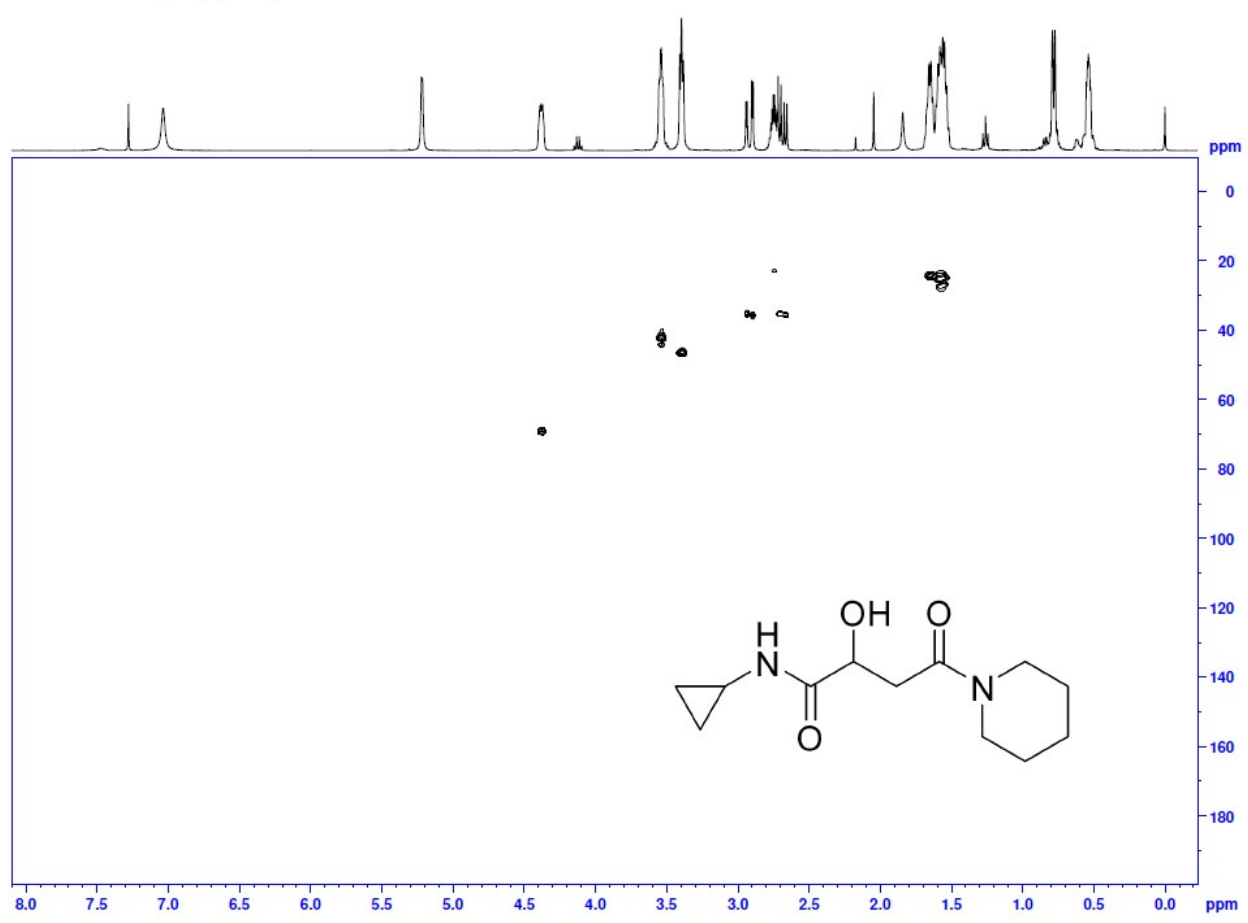




\section{Chiral HPLC of ketone precursor.}

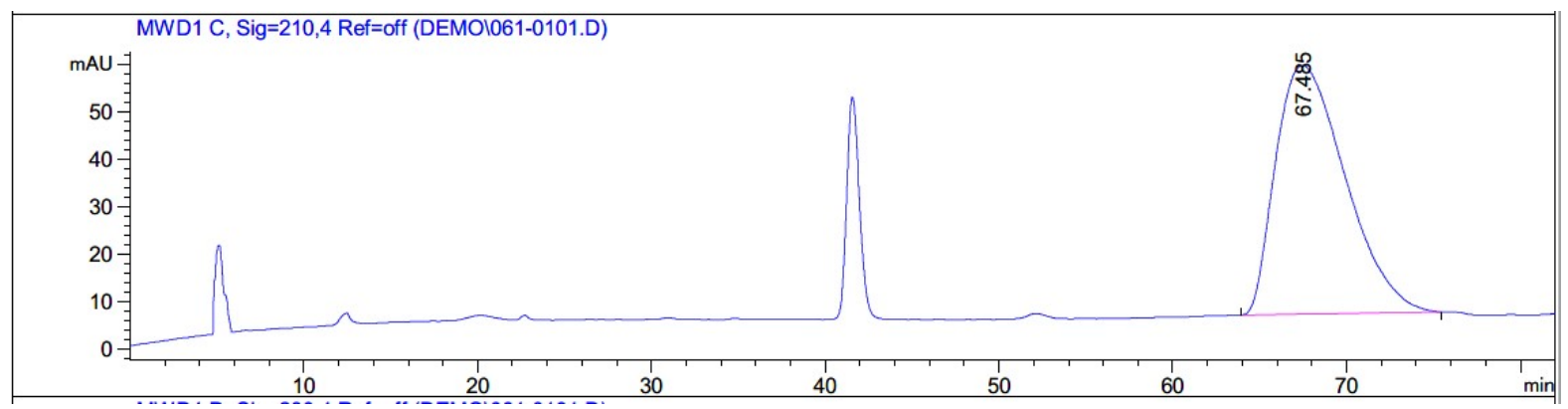

Signal 2: MWD1 C, Sig=210, 4 Ref=off

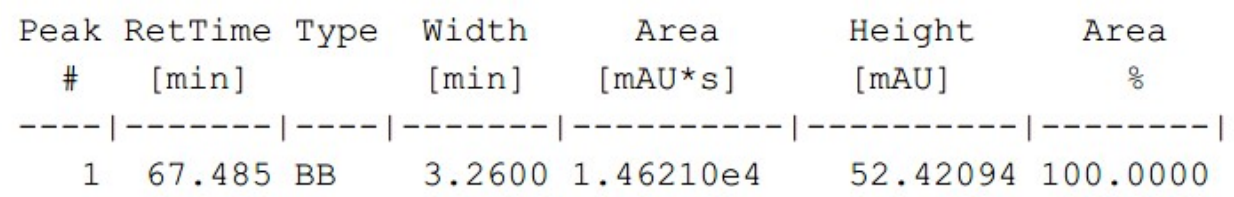

\section{Chiral HPLC of racemic alcohol.}

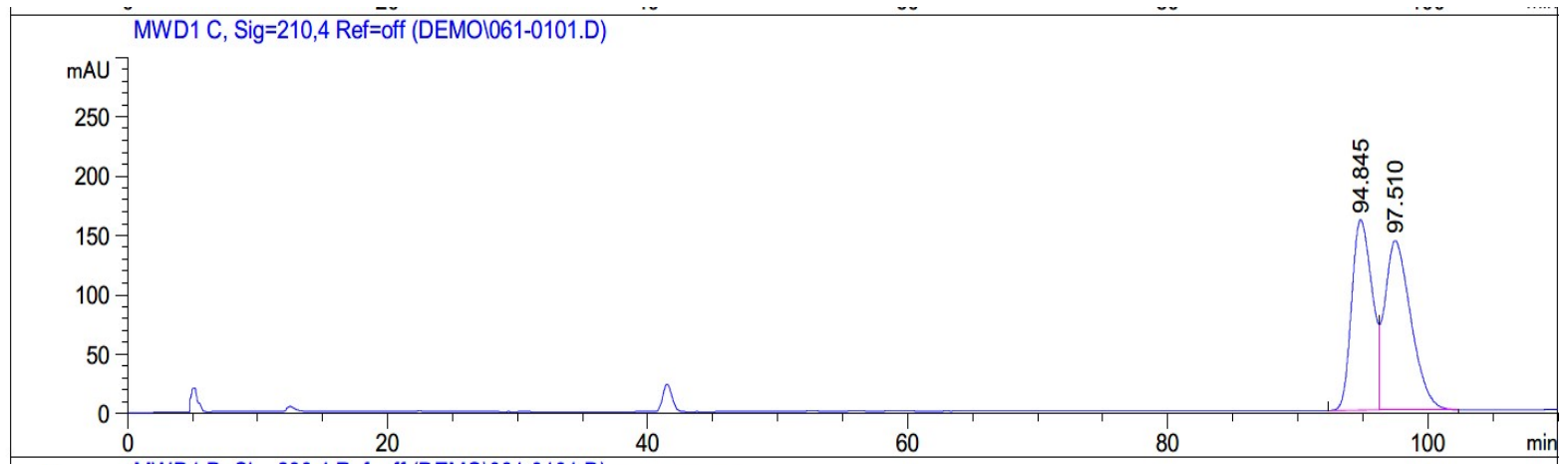

Signal 2: MWD1 C, Sig=210,4 Ref=off

\begin{tabular}{|c|c|c|c|c|c|c|}
\hline $\begin{array}{c}\text { eak } \\
\#\end{array}$ & $\begin{array}{c}\text { RetTime } \\
\text { [min] }\end{array}$ & Type & $\begin{array}{l}\text { Width } \\
\text { [min] }\end{array}$ & $\begin{array}{c}\text { Area } \\
{\left[m A U^{*} \mathrm{~s}\right]}\end{array}$ & $\begin{array}{l}\text { Height } \\
\text { [mAU] }\end{array}$ & $\begin{array}{c}\text { Area } \\
\frac{\circ}{0}\end{array}$ \\
\hline & & & & 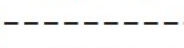 & --- & ---1 \\
\hline 1 & 94 & BV & 21 & $1.77258 \mathrm{e} 4$ & 160.54665 & 247 \\
\hline 2 & 97.510 & B & 1.9486 & $2.00492 \mathrm{e} 4$ & 142.74930 & 0753 \\
\hline
\end{tabular}


Chiral HPLC of ATH product. $98 \%$ ee.

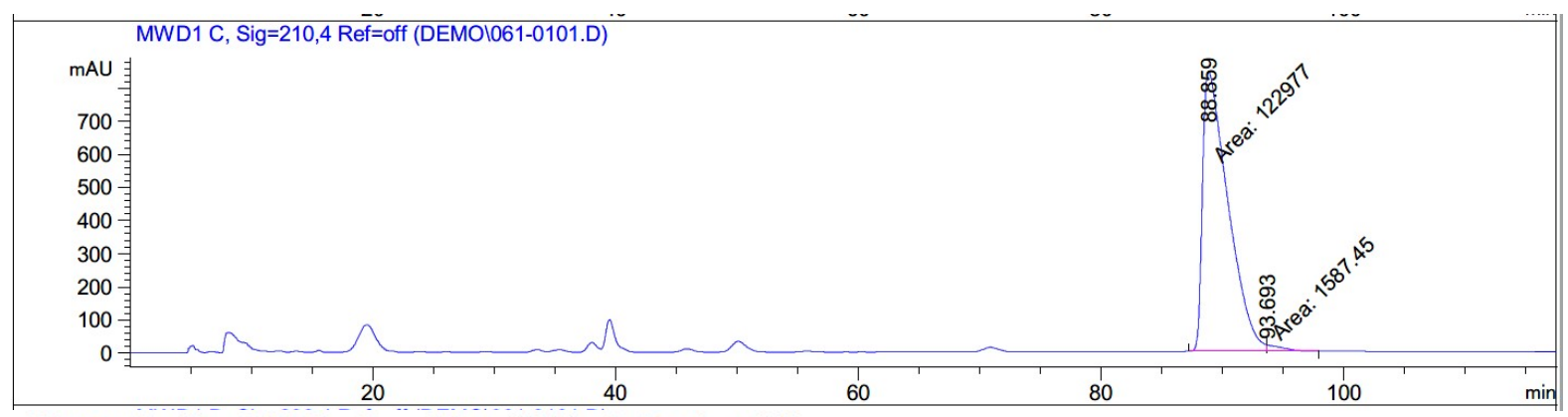

Signal 2: MWD1 C, Sig=210,4 Ref=off

\begin{tabular}{|c|c|c|c|c|c|c|}
\hline $\begin{array}{c}\text { eak } \\
\#\end{array}$ & $\begin{array}{c}\text { RetTime } \\
\text { [min] }\end{array}$ & Type & $\begin{array}{l}\text { Width } \\
\text { [min] }\end{array}$ & $\begin{array}{c}\text { Area } \\
{\left[\mathrm{mAU}{ }^{*} \mathrm{~s}\right]}\end{array}$ & $\begin{array}{l}\text { Height } \\
\text { [mAU] }\end{array}$ & $\begin{array}{c}\text { Area } \\
\frac{\circ}{0}\end{array}$ \\
\hline & - & & & |---- & ------- & \\
\hline 1 & 88.859 & MF & 2.4274 & $1.22977 \mathrm{e} 5$ & 844.35150 & 7256 \\
\hline 2 & 93 & FM & 1.4411 & 1587.45251 & 18.35942 & 1.2744 \\
\hline
\end{tabular}




\section{(R)-2-Hydroxy-5-oxo- $N$-phenyl-5-(piperidin-1-yl)pentanamide 31.}<smiles>CC(C)(CC(=O)N1CCCCC1)C(=O)Nc1ccccc1</smiles>

This compound is novel and was prepared following general procedure $\mathrm{C}$ using 2,5-dioxo- $\mathrm{N}$ phenyl-5-(piperidin-1-yl)pentanamide $(72.0 \mathrm{mg}, 0.250 \mathrm{mmol}, 1.0 \mathrm{eq}), 3 \mathrm{C}$ tethered catalyst $(R, R)-2(2.3 \mathrm{mg}, 3.8 \mu \mathrm{mol}, 0.015 \mathrm{eq})$ and FA:TEA $(0.15 \mathrm{~mL})$ in DCM $(1.5 \mathrm{~mL})$ to give 31 as a white solid (57.2 mg, $0.197 \mathrm{mmol}, 79 \%)$.

TLC: $\mathrm{R}_{\mathrm{f}}$ ca 0.3 (1:9, hexane: EtOAc), UV-active, strong $\mathrm{KMnO}_{4}$, and PMA-reactive; HRMS (ESI) m/z: [M+Na] $]^{+}$Calcd for $\mathrm{C}_{16} \mathrm{H}_{22} \mathrm{~N}_{2} \mathrm{O}_{3} \mathrm{Na} 313.1523$; Found 313.1514 (error 2.8 ppm);

MP: $105-108^{\circ} \mathrm{C}$;

$U_{\max } 3339,2902,2858,1667,16011518,1438,1252,1066,1036,758,687 \mathrm{~cm}^{-1}$;

Enantiomeric excess determined by HPLC analysis (Chiralpak IG, 250mm $\times 4.6 \mathrm{~mm}$ column, hexane: iPrOH 80:20, $\left.1 \mathrm{~mL} / \mathrm{min}, \lambda=210 \mathrm{~nm}, \mathrm{~T}=25^{\circ} \mathrm{C}\right), R$-enantiomer $20.2 \mathrm{~min}, S$ enantiomer $21.9 \min .94 \%$ ee $(R),[\alpha]_{\mathrm{D}}{ }^{22}=-8.8\left(\mathrm{c}=0.1, \mathrm{CHCl}_{3}\right)$;

${ }^{1} \mathrm{H}$ NMR (400 MHz, $\left.\mathrm{CDCl}_{3}\right) \delta 9.06(1 \mathrm{H}, \mathrm{s}, \mathrm{CONH}), 7.62(2 \mathrm{H}, \mathrm{d}, J=8.0 \mathrm{~Hz}, \mathrm{ArH}), 7.33(2 \mathrm{H}$, t, $J=7.8 \mathrm{~Hz}, \mathrm{ArH}), 7.11(1 \mathrm{H}, \mathrm{t}, J=7.4 \mathrm{~Hz}, \mathrm{ArH}), 6.95(1 \mathrm{H}, \mathrm{d}, J=2.1 \mathrm{~Hz}, \mathrm{CHO} \underline{\mathrm{H}}), 4.24-4.22$ $(1 \mathrm{H}, \mathrm{m}, \mathrm{C} \underline{\mathrm{HOH}}), 3.63-3.52\left(2 \mathrm{H}, \mathrm{m}, \mathrm{NCH}_{2}\right.$ of piperidine $), 3.42-3.40\left(2 \mathrm{H}, \mathrm{m}, \mathrm{NCH}_{2}\right.$ of piperidine), 2.66-2.61 (2H, m, $\left.\mathrm{CH}_{\mathrm{a}} \mathrm{H}_{\mathrm{b}} \underline{\mathrm{H}}_{2}\right), 2.37-2.33\left(1 \mathrm{H}, \mathrm{m}, \mathrm{C}_{\underline{\mathrm{a}}} \mathrm{H}_{\mathrm{b}}\right), 2.24-2.16(1 \mathrm{H}, \mathrm{m}$, $\left.\mathrm{CH}_{\mathrm{a}} \underline{\mathrm{H}_{\mathrm{b}}}\right), 1.67-1.56\left(6 \mathrm{H}, \mathrm{m}, \mathrm{CH}_{2}\right.$ of piperidine).

${ }^{13} \mathrm{C}$ NMR $\left(101 \mathrm{MHz}, \mathrm{CDCl}_{3}\right) \delta 172.7,172.4,137.7,129.1,124.3,119.6,73.1,47.2,43.5$, 30.8, 28.7, 26.4, 25.6, 24.4;

$m / z(\mathrm{ESI}) 313.1\left[(\mathrm{M}+\mathrm{Na})^{+}, 100 \%\right]$. 
2-Hydroxy-5-oxo- $N$-phenyl-5-(piperidin-1-yl)pentanamide 31.

${ }^{1} \mathrm{H}$ NMR (400 MHz, $\left.\mathrm{CDCl}_{3}\right)$.

Sep11-2020

PROTON.w CDCI3

잉

i ninkningo

$\overbrace{\mathrm{S}}$ $\underbrace{7}$

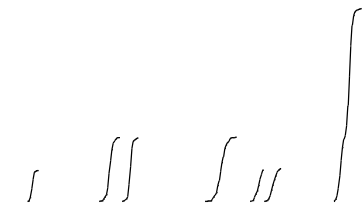

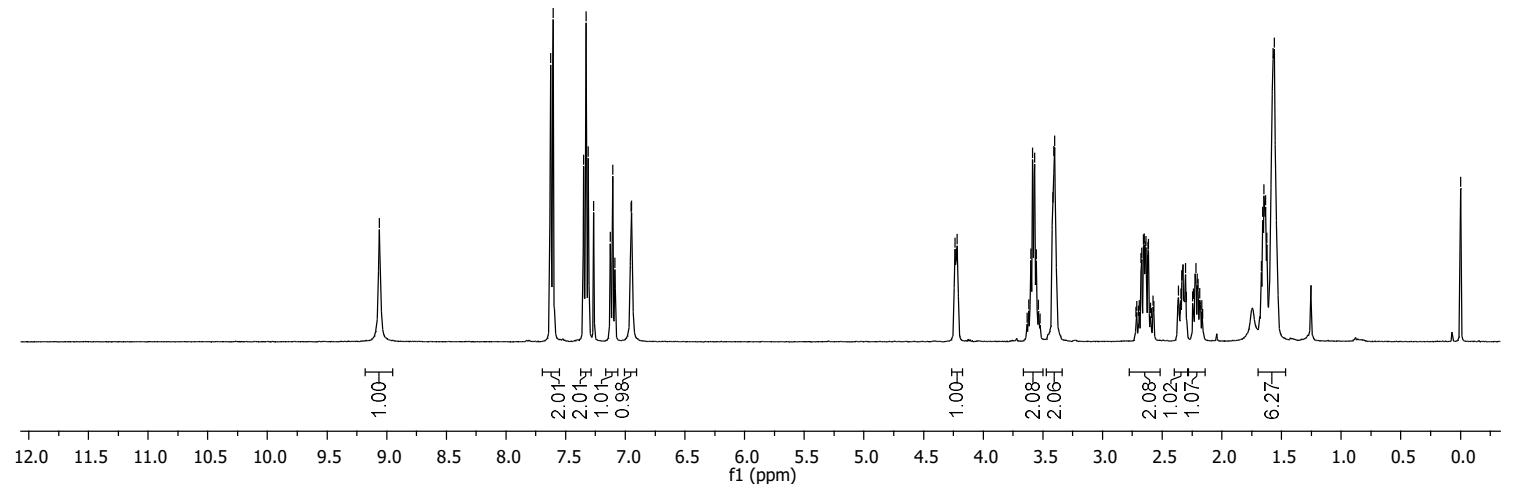

${ }^{13} \mathrm{C}$ NMR (101 MHz, $\left.\mathrm{CDCl}_{3}\right)$.

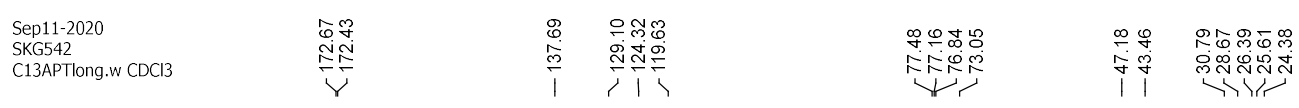<smiles>O=C(Nc1ccccc1)C(O)CCC(=O)N1CCCCC1</smiles>

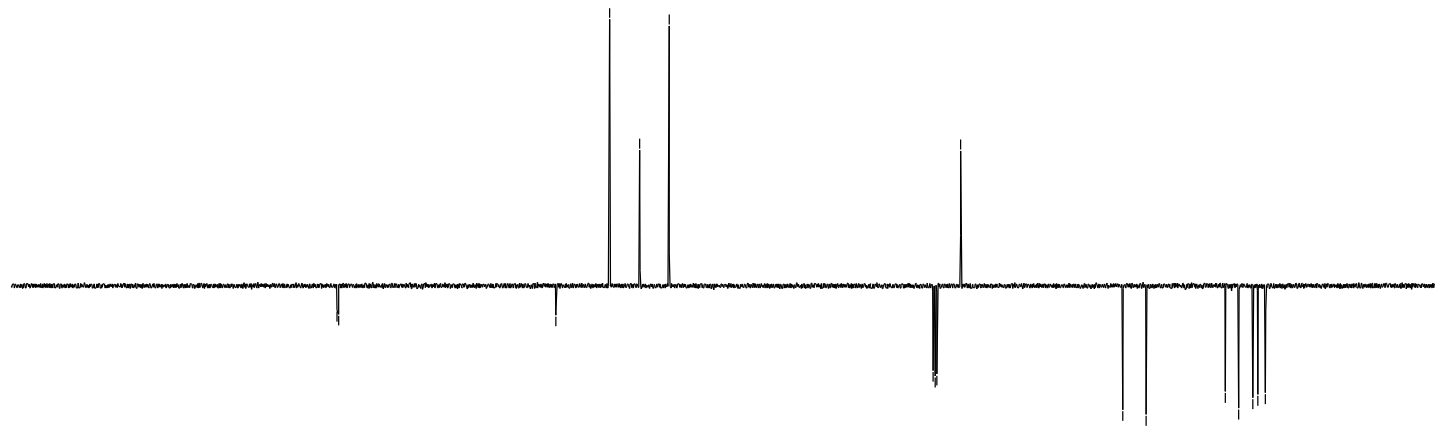

$\begin{array}{llllllllllllllllllllllllll}220 & 210 & 200 & 190 & 180 & 170 & 160 & 150 & 140 & 130 & 120 & 110 & 100 & 90 & 80 & 70 & 60 & 50 & 40 & 30 & 20 & 10 & 0\end{array}$ 
COSY.

SKG542

COSY.w CDCl3/opttopspin3.5pl2 SKG 24

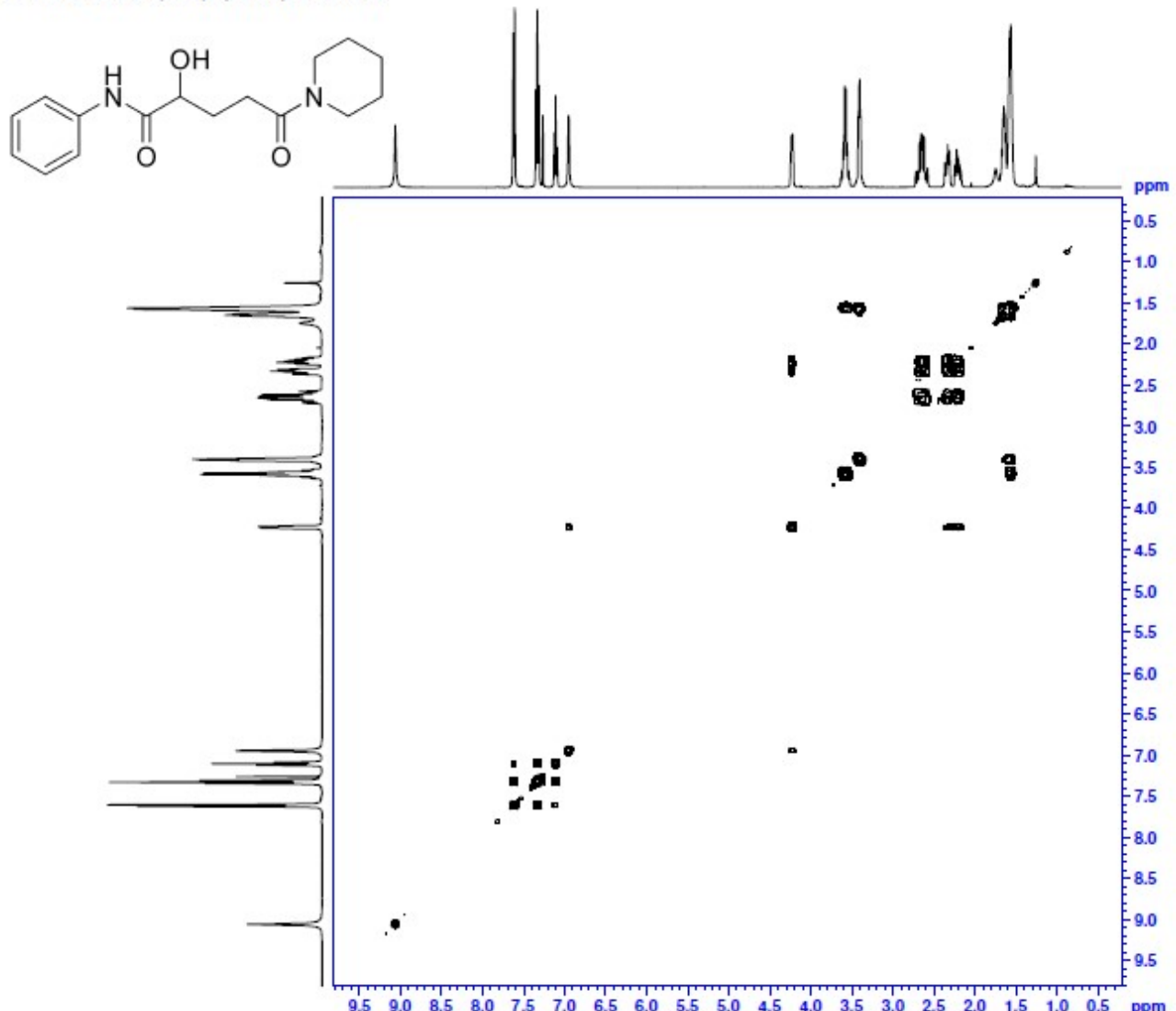

HSQC.

SKG542
HSQC.w CDCl3 /opttopspin3.5pl2 SKG 24

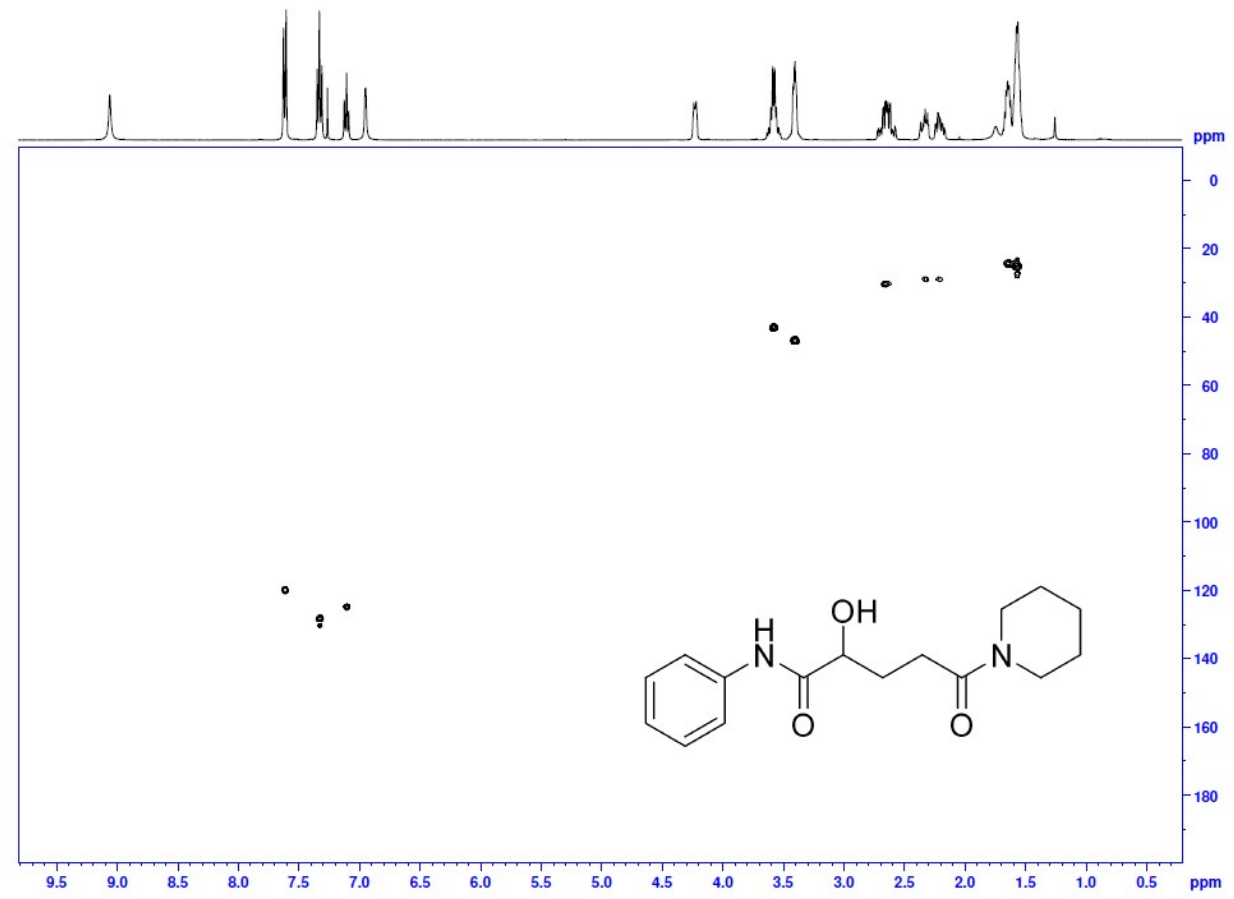




\section{Chiral HPLC of ketone precursor.}

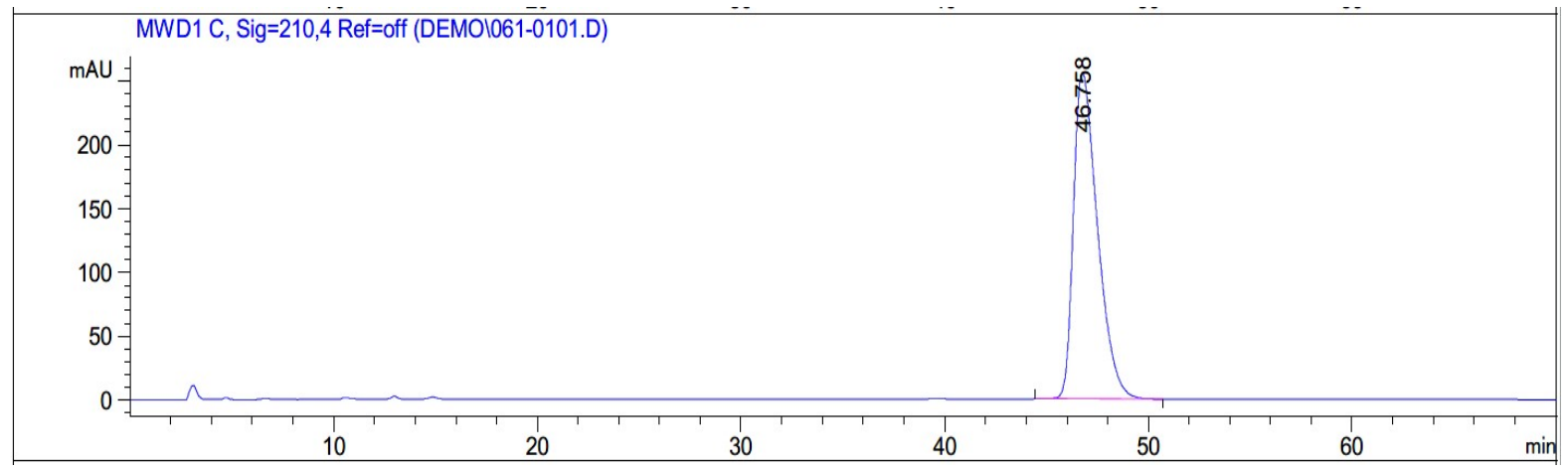

Signal 2: MWD1 C, Sig=210,4 Ref=off

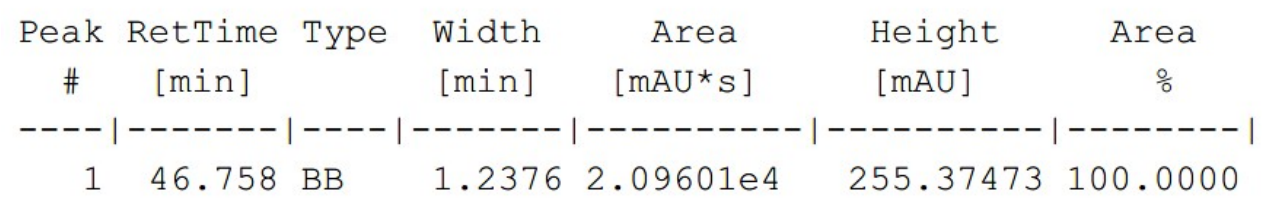

Chiral HPLC of racemic alcohol.

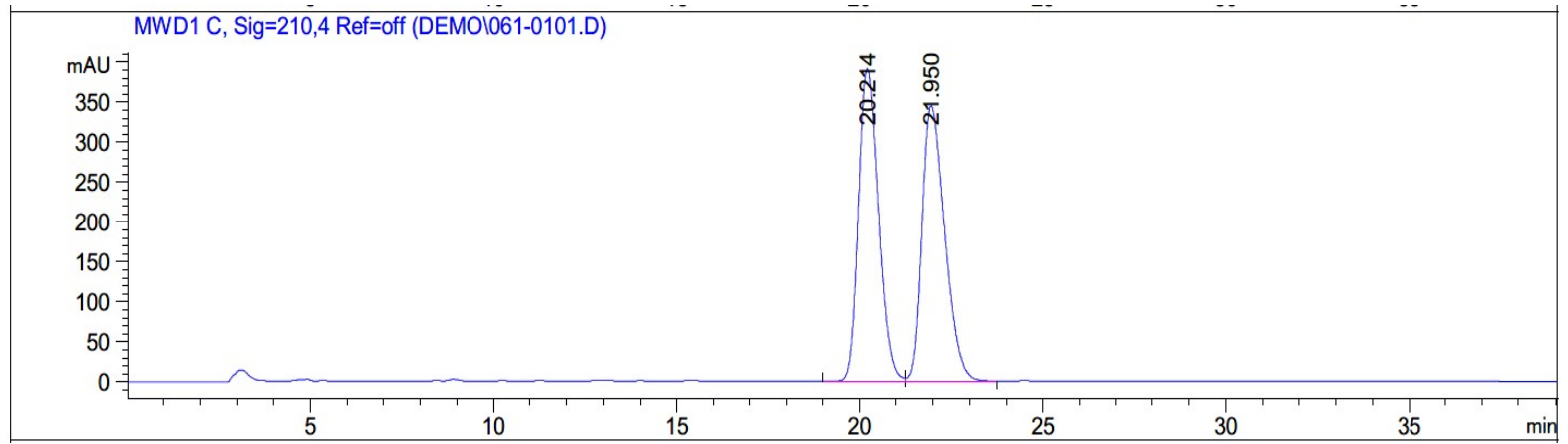

Signal 2: MWD1 C, Sig=210,4 Ref=off

\begin{tabular}{|c|c|c|c|c|c|c|}
\hline $\begin{array}{c}\text { Peak } \\
\#\end{array}$ & $\begin{array}{c}\text { RetTime } \\
\text { [min] }\end{array}$ & Type & $\begin{array}{l}\text { Width } \\
\text { [min] }\end{array}$ & $\begin{array}{c}\text { Area } \\
{\left[\mathrm{mAU}^{\star} \mathrm{s}\right]}\end{array}$ & $\begin{array}{l}\text { Height } \\
{[\mathrm{mAU}]}\end{array}$ & $\begin{array}{c}\text { Area } \\
\quad \%\end{array}$ \\
\hline -- & -ー-ー-ー & & --- & --- & -- & ----- \\
\hline 1 & 20.214 & BV & 0.5888 & $1.47736 \mathrm{e} 4$ & 390.99658 & 49.9049 \\
\hline 2 & 21.950 & VB & 0.6620 & $1.48299 \mathrm{e} 4$ & 344.66833 & 50.0951 \\
\hline
\end{tabular}


Chiral HPLC of ATH product. 94\% ee.

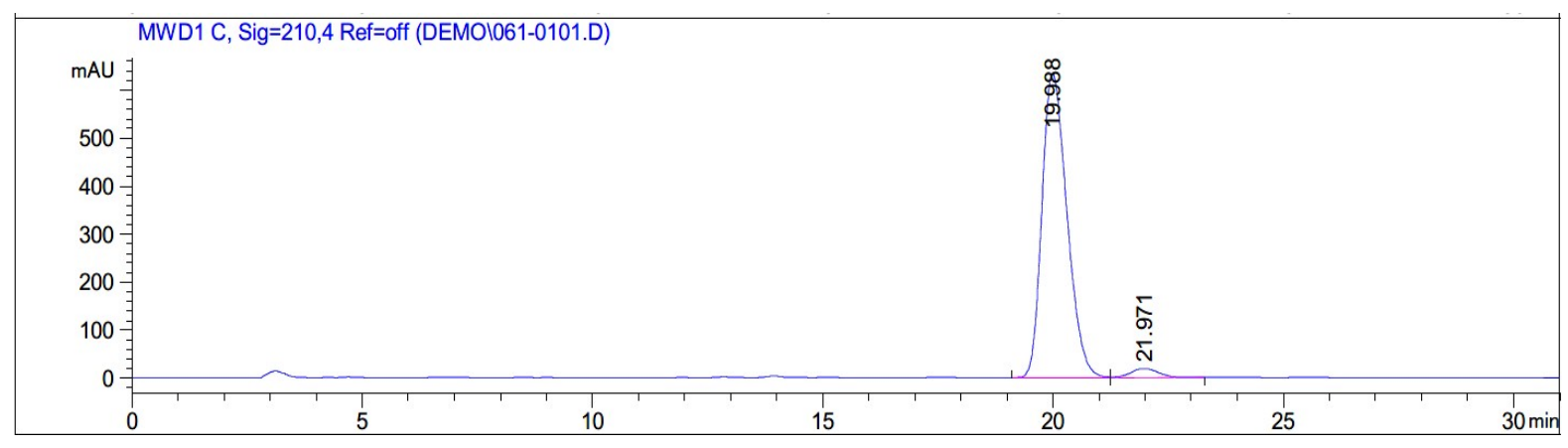

Signal 2: MWD1 C, Sig=210,4 Ref=off

\begin{tabular}{|c|c|c|c|c|c|c|}
\hline $\begin{array}{c}\text { Peak } \\
\quad \#\end{array}$ & $\begin{array}{c}\text { RetTime } \\
\text { [min] }\end{array}$ & Type & $\begin{array}{l}\text { Width } \\
\text { [min] }\end{array}$ & $\begin{array}{c}\text { Area } \\
{\left[m A U^{\star} \mathrm{s}\right]}\end{array}$ & $\begin{array}{l}\text { Height } \\
{[\mathrm{mAU}]}\end{array}$ & $\begin{array}{c}\text { Area } \\
\frac{\circ}{0}\end{array}$ \\
\hline & $\mid--$ & & --- & | ---------- & ------- & $--------\mid$ \\
\hline 1 & 19.988 & BV & 0.5856 & $2.39332 \mathrm{e} 4$ & 635.11566 & 96.7499 \\
\hline 2 & 21.971 & VB & 0.6457 & 803.98248 & 19.15391 & 3.2501 \\
\hline
\end{tabular}


Ethyl (R)-2-hydroxy-4-oxo-4-(piperidin-1-yl)butanoate 32.<smiles>CCOC(=O)C(O)CC(=O)N1CCCCC1</smiles>

This compound is novel and was prepared following general procedure $\mathrm{C}$ using ethyl 2,4dioxo-4-(piperidin-1-yl)butanoate (114 g, $0.500 \mathrm{mmol}, 1.0 \mathrm{eq}), 3 \mathrm{C}$ tethered catalyst $(R, R)-2$ (4.7 $\mathrm{mg}, 7.5 \mu \mathrm{mol}, 0.015 \mathrm{eq})$ and FA:TEA $(0.3 \mathrm{~mL})$ in DCM $(3 \mathrm{~mL})$ to give 32 as a clear semisolid material (104 mg, $0.454 \mathrm{mmol}, 91 \%)$.

TLC: $\mathrm{R}_{\mathrm{f}}$ ca 0.4 (4:6, hexane: EtOAc), UV-active, Strong $\mathrm{KMnO}_{4}$ and PMA-reactive; HRMS (ESI) m/z: $[\mathrm{M}+\mathrm{Na}]^{+}$Calcd for $\mathrm{C}_{11} \mathrm{H}_{19} \mathrm{NO}_{4} \mathrm{Na} 252.1206$; Found 252.1209 (error -1.1 ppm);

$U_{\max } 3429,2933,1734,1616,1443,1251,1206,1183,1124,1026,853 \mathrm{~cm}^{-1}$;

Enantiomeric excess determined by HPLC analysis (Chiralpak IG, 250mm $\times 4.6 \mathrm{~mm}$ column, hexane: $\left.\mathrm{iPrOH} 80: 20,1 \mathrm{~mL} / \mathrm{min}, \lambda=210 \mathrm{~nm}, \mathrm{~T}=25^{\circ} \mathrm{C}\right), S$-enantiomer $22.5 \mathrm{~min}, R$ enantiomer $24.4 \min .98 \%$ ee $(R),[\alpha]_{\mathrm{D}}^{22}=-2\left(\mathrm{c}=0.1, \mathrm{CHCl}_{3}\right)$;

${ }^{1} \mathrm{H}$ NMR $\left(500 \mathrm{MHz}, \mathrm{CDCl}_{3}\right) \delta 4.49(1 \mathrm{H}, \mathrm{dd}, J=10.3,6.0 \mathrm{~Hz}, \mathrm{C} \underline{\mathrm{HOH}}), 4.26(2 \mathrm{H}, \mathrm{q}, J=7.1$ $\mathrm{Hz}, \mathrm{C}_{2} \mathrm{CH}_{3}$ of ester), $4.16(1 \mathrm{H}, \mathrm{d}, J=6.8 \mathrm{~Hz}, \mathrm{CHO} \underline{\mathrm{H}}), 3.56-3.54\left(2 \mathrm{H}, \mathrm{m}, \mathrm{NCH}_{2}\right.$ of piperidine), 3.49-3.37 (2H, m, $\mathrm{NCH}_{2}$ of piperidine), 2.89-2.81 $\left(2 \mathrm{H}, \mathrm{m}, \mathrm{CH}_{\mathrm{a}} \mathrm{H}_{\mathrm{b}}\right), 1.67-1.52$ $\left(6 \mathrm{H}, \mathrm{m}, \mathrm{CH}_{2}\right.$ of piperidine), $1.31\left(3 \mathrm{H}, \mathrm{t}, J=7.1 \mathrm{~Hz}, \mathrm{CH}_{2} \mathrm{CH}_{3}\right.$ of ester);

${ }^{13} \mathrm{C}$ NMR $\left(126 \mathrm{MHz}, \mathrm{CDCl}_{3}\right) \delta 173.4,168.8,68.2,61.5,46.5,42.7,36.6,26.3,25.5,24.2$, 14.2;

$m / z(\mathrm{ESI}) 252.1\left[(\mathrm{M}+\mathrm{Na})^{+}, 60 \%\right], 481.1\left[(2 \mathrm{M}+\mathrm{Na})^{+}, 100 \%\right]$. 
Ethyl 2-hydroxy-4-oxo-4-(piperidin-1-yl)butanoate 32.

${ }^{1} \mathrm{H}$ NMR $\left(500 \mathrm{MHz}, \mathrm{CDCl}_{3}\right)$.

Dec17-2020

Chemist Shweta Gediya

PROTON.w CDCl3 /opt/topspin3.2 SKG 55

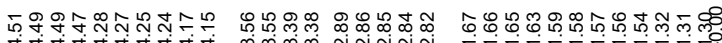<smiles>CCOC(=O)C(O)CC(=O)N1CCCCC1</smiles>
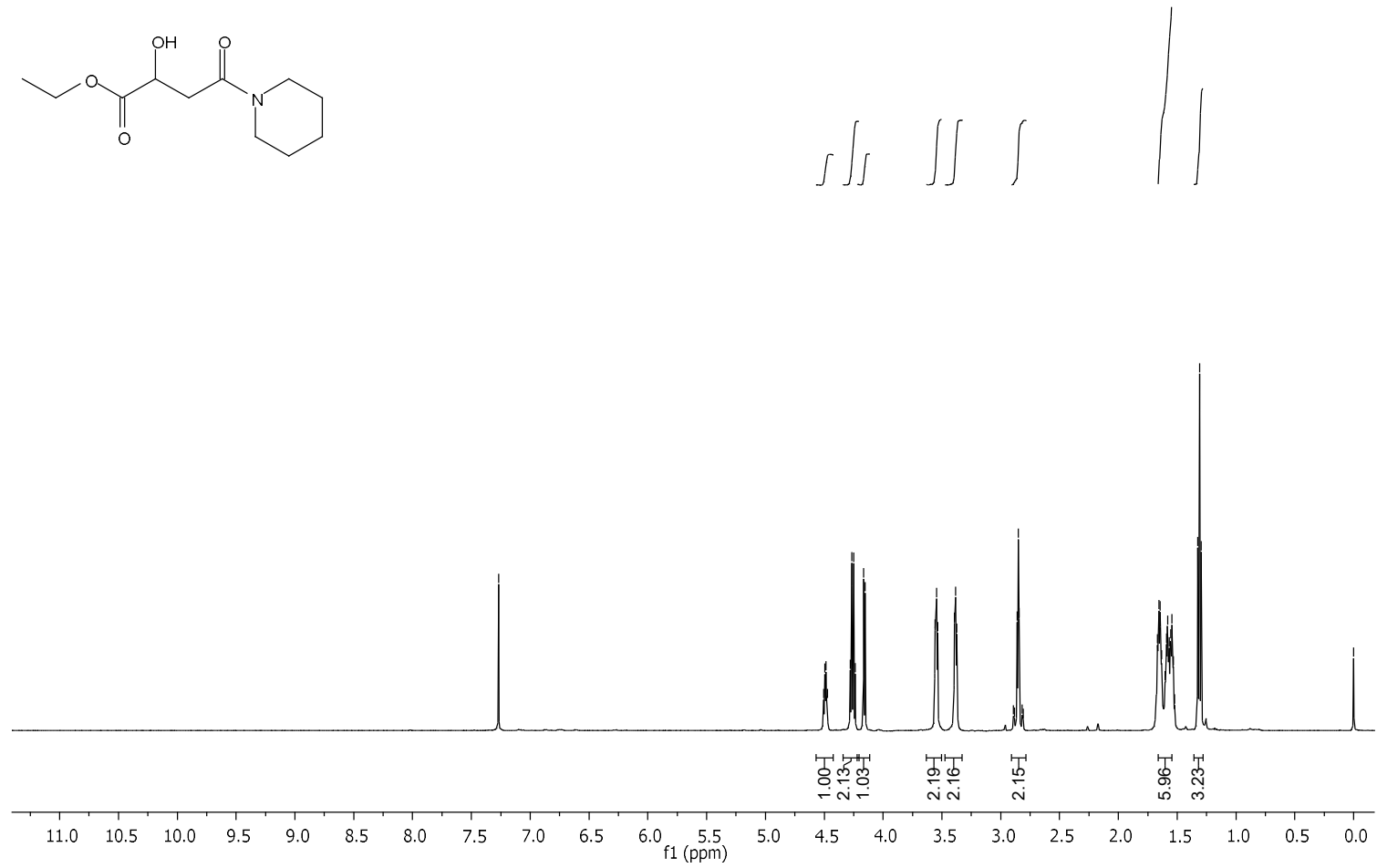

${ }^{13} \mathrm{C} \mathrm{NMR}\left(126 \mathrm{MHz}, \mathrm{CDCl}_{3}\right)$.

\begin{tabular}{|c|c|}
\hline $\begin{array}{l}\text { Dec17-2020 } \\
\text { Shweta Gediya } \\
\text { SKG701 } \\
\text { C13APTlong.w CDCl3 }\end{array}$ & 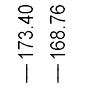 \\
\hline
\end{tabular}

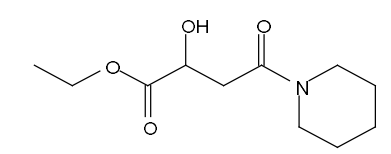

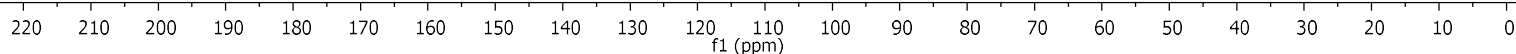


COSY.

Chemist Shweta Gediya

COSY.w CDCI3/opt/topspin3.2 SKG 55

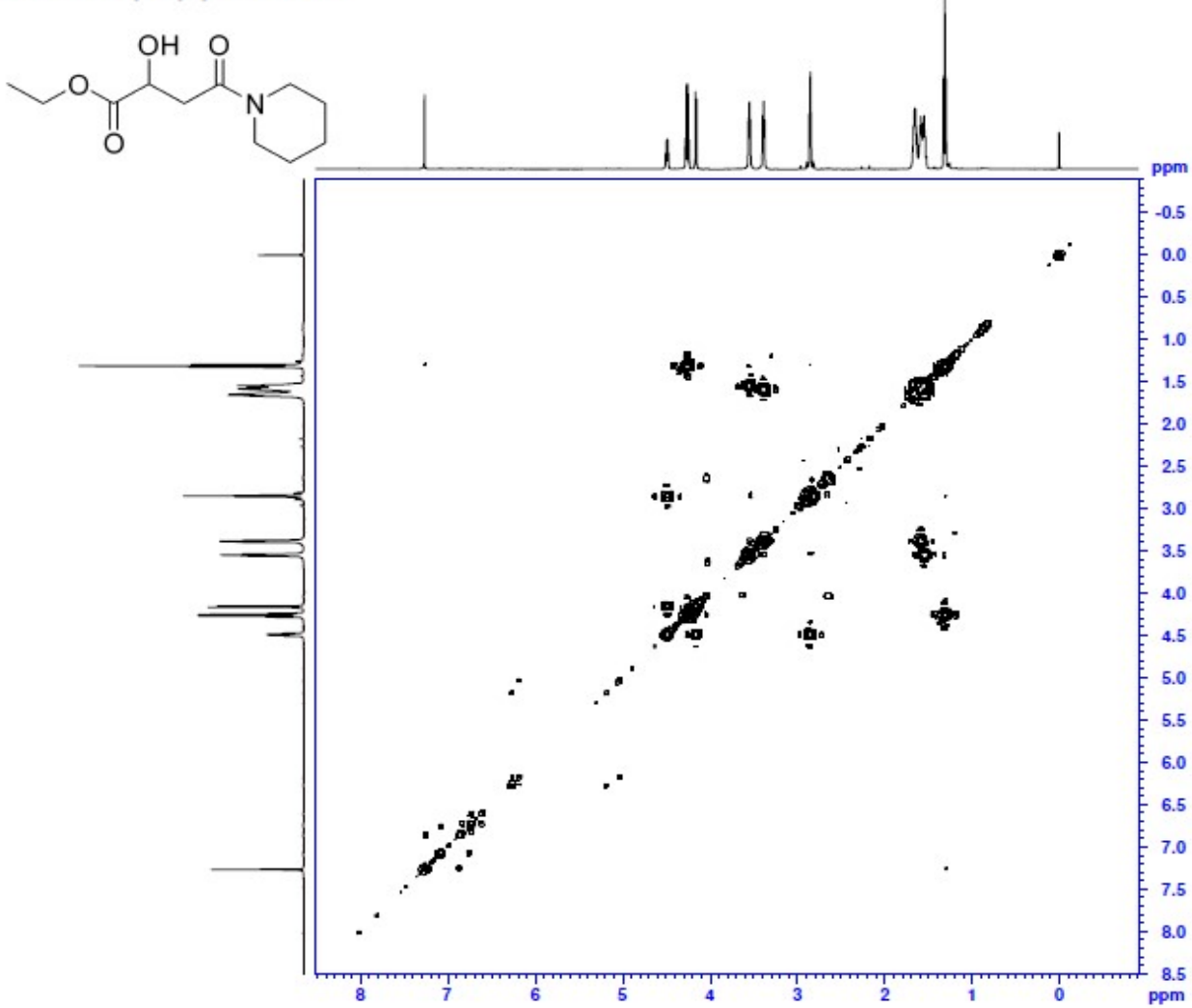

HSQC.

Chemist Shweta Gediya

HSQC.w CDCl3 /opt/topspin3.2 SKG 55

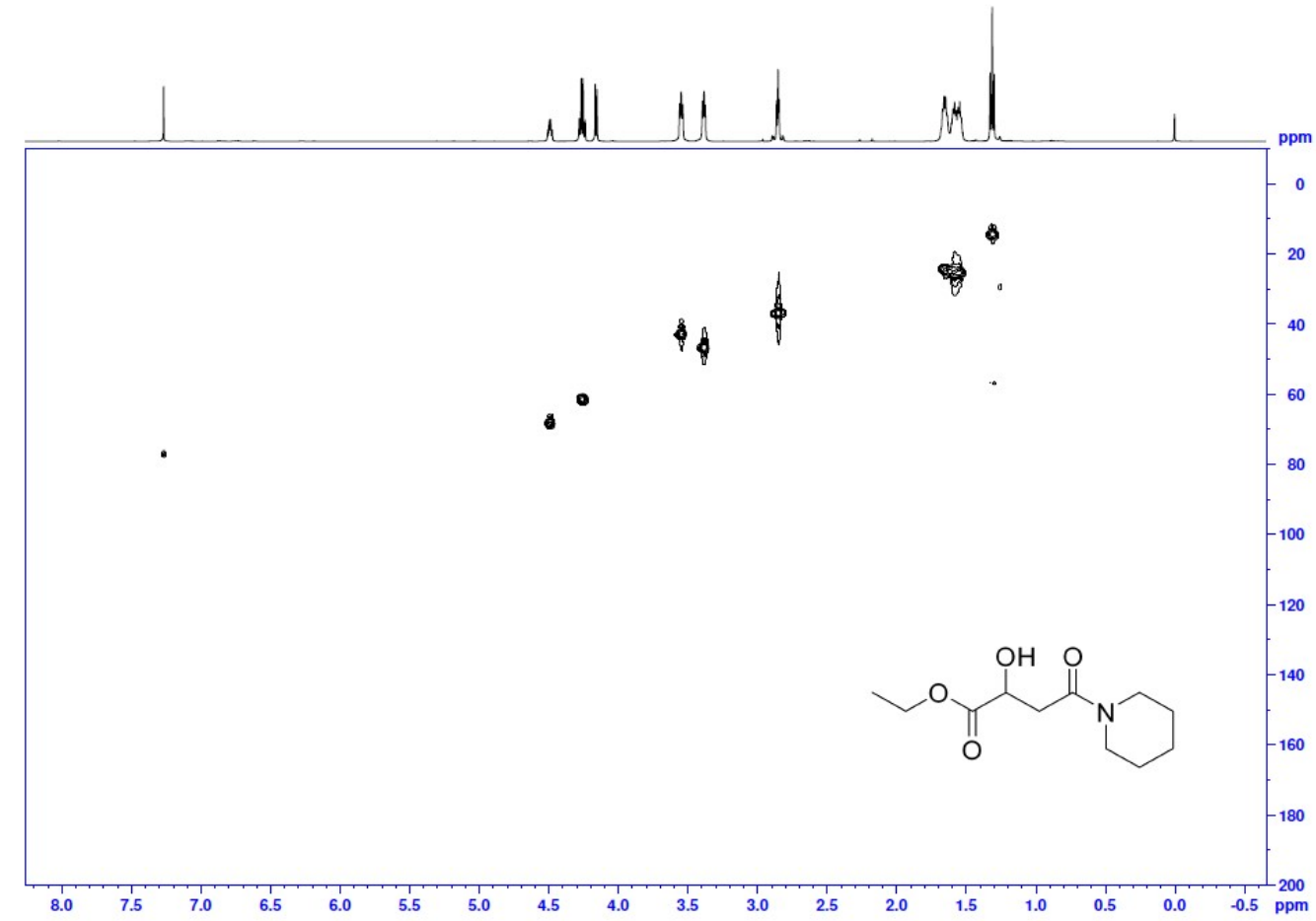




\section{Chiral HPLC of ketone precursor.}

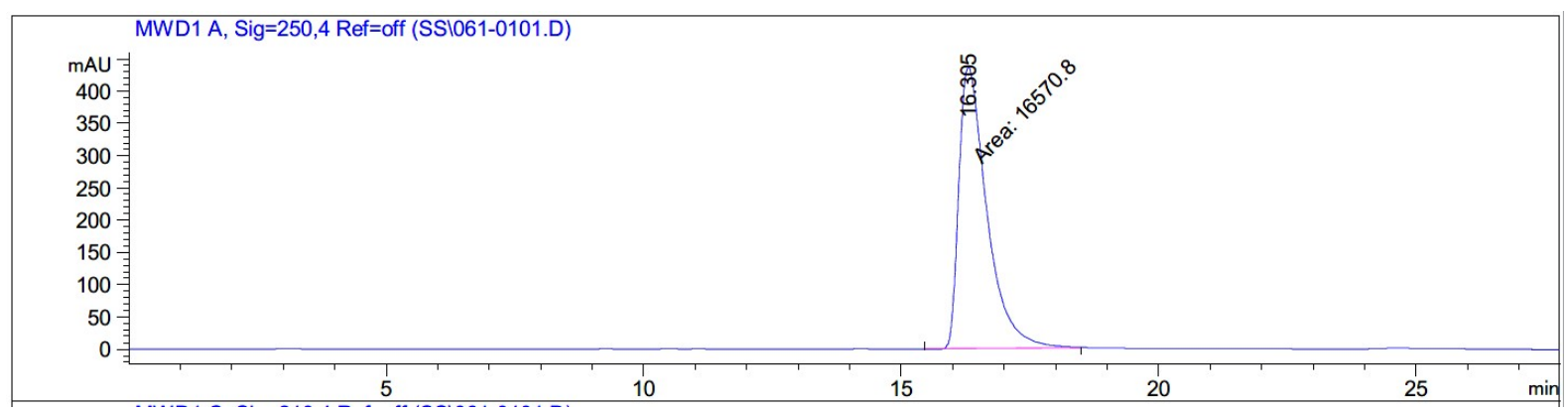

Signal 1: MWD1 A, Sig=250,4 Ref=off

\begin{tabular}{|c|c|c|c|c|c|c|}
\hline $\begin{array}{l}\text { eak } \\
\#\end{array}$ & $\begin{array}{c}\text { RetTime } \\
\text { [min] }\end{array}$ & Type & $\begin{array}{l}\text { Width } \\
\text { [min] }\end{array}$ & $\begin{array}{c}\text { Area } \\
{\left[m A U^{\star} s\right]}\end{array}$ & $\begin{array}{l}\text { Height } \\
{[\mathrm{mAU}]}\end{array}$ & $\begin{array}{c}\text { Area } \\
\frac{\circ}{\circ}\end{array}$ \\
\hline & 05 & & $0>0$ & $.65708 e$ & 43 & 10 \\
\hline
\end{tabular}


Chiral HPLC of racemic alcohol.

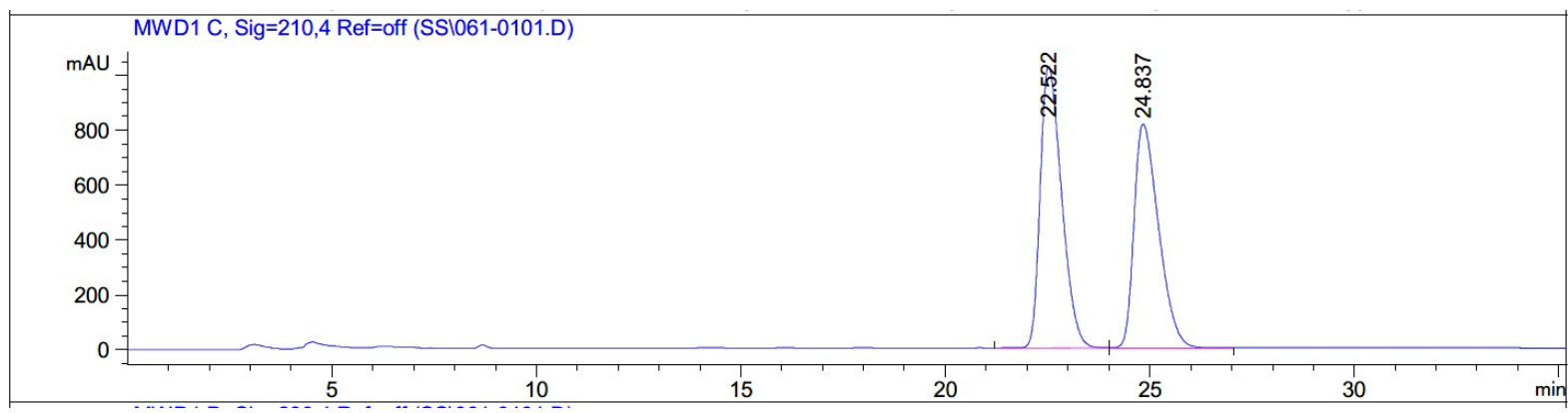

Signal 2: MWD1 C, Sig=210,4 Ref=off

\begin{tabular}{|c|c|c|c|c|c|c|}
\hline $\begin{array}{c}\text { Peak } \\
\#\end{array}$ & $\begin{array}{c}\text { RetTime } \\
\text { [min] }\end{array}$ & Type & $\begin{array}{l}\text { Width } \\
\text { [min] }\end{array}$ & $\begin{array}{c}\text { Area } \\
{\left[\mathrm{mAU}^{\star} \mathrm{s}\right]}\end{array}$ & $\begin{array}{l}\text { Height } \\
{[\mathrm{mAU}]}\end{array}$ & $\begin{array}{c}\text { Area } \\
\frac{\circ}{\circ}\end{array}$ \\
\hline- & - & & --1 & -------- & --------- & $-----\mid$ \\
\hline 1 & 22.522 & $\mathrm{BB}$ & 0.5639 & $3.72965 \mathrm{e} 4$ & 1026.40430 & 52.3326 \\
\hline 2 & 24.837 & $\mathrm{BB}$ & 0.6399 & 3. $39718 e 4$ & 815.62311 & 47.6674 \\
\hline
\end{tabular}

Chiral HPLC of ATH product. 98\% ee.

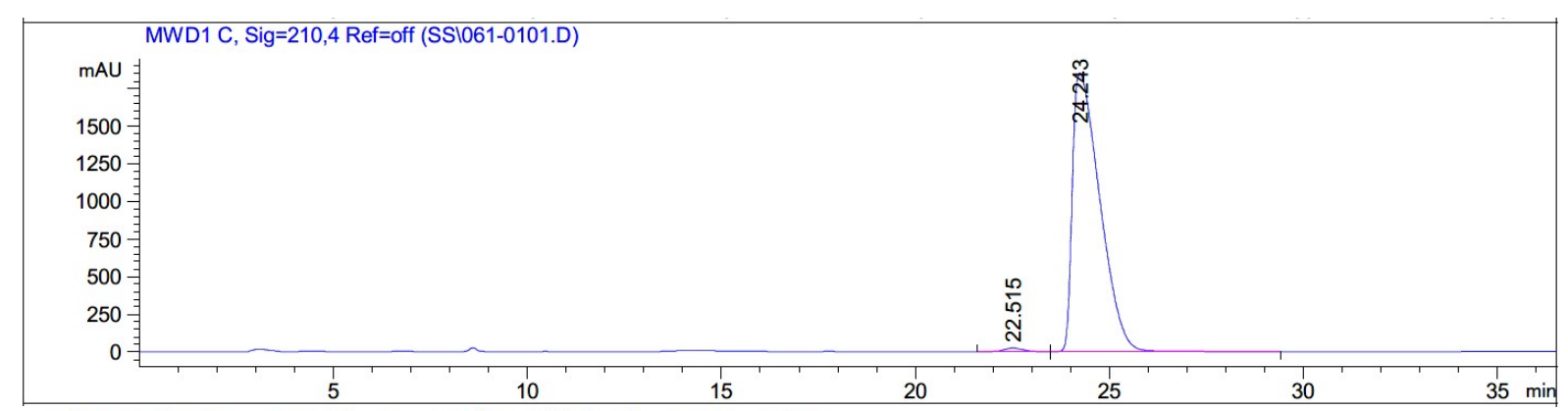

Signal 2: MWD1 C, Sig=210,4 Ref=off

\begin{tabular}{|c|c|c|c|c|c|c|}
\hline $\begin{array}{c}\text { Peak } \\
\#\end{array}$ & $\begin{array}{c}\text { RetTime } \\
\text { [min] }\end{array}$ & Type & $\begin{array}{l}\text { Width } \\
\text { [min] }\end{array}$ & $\begin{array}{c}\text { Area } \\
{\left[\mathrm{mAU}{ }^{*} \mathrm{~s}\right]}\end{array}$ & $\begin{array}{l}\text { Height } \\
{[\mathrm{mAU}]}\end{array}$ & $\begin{array}{c}\text { Area } \\
\text { 응 }\end{array}$ \\
\hline- & 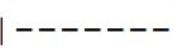 & 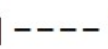 & 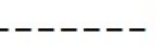 & ---------- & ---------- & $----\mid$ \\
\hline 1 & 22.515 & $\mathrm{BB}$ & 0.5292 & 827.86633 & 24.31878 & 0.9030 \\
\hline 2 & 24.243 & $\mathrm{BB}$ & 0.7640 & $9.08502 \mathrm{e} 4$ & 1853.12561 & 99.0970 \\
\hline
\end{tabular}


Ethyl (R)-4-((2,6-diisopropylphenyl)amino)-3-hydroxy-4-oxobutanoate 33.<smiles>CCOC(=O)C[C@H](O)C(=O)Nc1c(C(C)C)cccc1C(C)C</smiles>

This compound is novel and was prepared following general procedure $\mathrm{C}$ using ethyl 4-((2,6diisopropylphenyl)amino)-3,4-dioxobutanoate (79.7 $\mathrm{mg}, 0.250 \mathrm{mmol}, 1.0 \mathrm{eq}), 3 \mathrm{C}$ tethered catalyst $(R, R)-2(2.3 \mathrm{mg}, 3.8 \mu \mathrm{mol}, 0.015 \mathrm{eq})$ and FA:TEA $(0.15 \mathrm{~mL})$ in DCM $(1.5 \mathrm{~mL})$ to give 33 as a white solid (70.9 $\mathrm{mg}, 0.221 \mathrm{mmol}, 88 \%$ ).

MP: $148-150{ }^{\circ} \mathrm{C}$;

TLC: $\mathrm{R}_{\mathrm{f}}$ ca 0.2 (8:2, hexane: EtOAc), UV-active and PMA-reactive;

HRMS (ESI) m/z: [M+Na] $]^{+}$Calcd for $\mathrm{C}_{18} \mathrm{H}_{27} \mathrm{NO}_{4} \mathrm{Na}$ 344.1832; Found 344.1829 (error 1.0 ppm);

$U_{\max } 3325,3204,2965,1738,1658,1510,1256,1109,1047,805 \mathrm{~cm}^{-1}$;

Enantiomeric excess determined by HPLC analysis (Chiralpak IC, $250 \mathrm{~mm} \times 4.6 \mathrm{~mm}$ column, hexane: $\left.\mathrm{iPrOH} 85: 15,1 \mathrm{~mL} / \mathrm{min}, \lambda=210 \mathrm{~nm}, \mathrm{~T}=25^{\circ} \mathrm{C}\right), S$-enantiomer $6.9 \mathrm{~min}, R$-enantiomer $8.4 \min .98 \%$ ee $(R),[\alpha]_{\mathrm{D}}^{22}=-73.8\left(\mathrm{c}=0.04, \mathrm{CHCl}_{3}\right)$;

${ }^{1} \mathrm{H}$ NMR $\left(500 \mathrm{MHz}, \mathrm{CDCl}_{3}\right) \delta 8.17(1 \mathrm{H}, \mathrm{s}, \mathrm{CONH}), 7.31-7.26(1 \mathrm{H}, \mathrm{m}, \mathrm{ArH}), 7.18(2 \mathrm{H}, \mathrm{d}, J=$ $7.7 \mathrm{~Hz}, \mathrm{ArH}), 4.67-4.65(1 \mathrm{H}, \mathrm{m}, \mathrm{C} \underline{\mathrm{HOH}}), 4.21\left(2 \mathrm{H}, \mathrm{q}, J=7.1 \mathrm{~Hz}, \mathrm{OC}_{2} \mathrm{CH}_{3}\right), 3.14$ (1H, br.s, $\mathrm{CHO} \underline{\mathrm{H}}), 3.08-2.99\left(3 \mathrm{H}, \mathrm{m}, \mathrm{CH}\right.$ of isopropyl $\left.+\mathrm{C}_{\underline{a}} \mathrm{H}_{\mathrm{b}}\right), 2.85(1 \mathrm{H}, \mathrm{dd}, J=17.3,8.0 \mathrm{~Hz}$ $\left.\mathrm{CH}_{\mathrm{a}} \underline{\mathrm{H}} \mathrm{b}\right), 1.29\left(3 \mathrm{H}, \mathrm{t}, J=7.1 \mathrm{~Hz}, \mathrm{OCH}_{2} \mathrm{C}_{3}\right), 1.21-1.19\left(12 \mathrm{H}, \mathrm{m}, \mathrm{CH}_{3}\right.$ of isopropyl);

${ }^{13} \mathrm{C}$ NMR $\left(126 \mathrm{MHz}, \mathrm{CDCl}_{3}\right) \delta 173.2,171.5,146.1,130.4,128.3,123.6,68.9,61.4,38.2$, $28.8,23.7,23.6,14.2$;

$m / z(\mathrm{ESI}) 344.2\left[(\mathrm{M}+\mathrm{Na})^{+}, 100 \%\right]$. 
Ethyl 4-((2,6-diisopropylphenyl)amino)-3-hydroxy-4-oxobutanoate 33.

${ }^{1} \mathrm{H}$ NMR (500 MHz, $\mathrm{CDCl}_{3}$ ).

Jan12-2021

Chemist Shweta Gediya

SKG727
PROTON.w CDCl3 /opt/topspin3.2 SKG 20
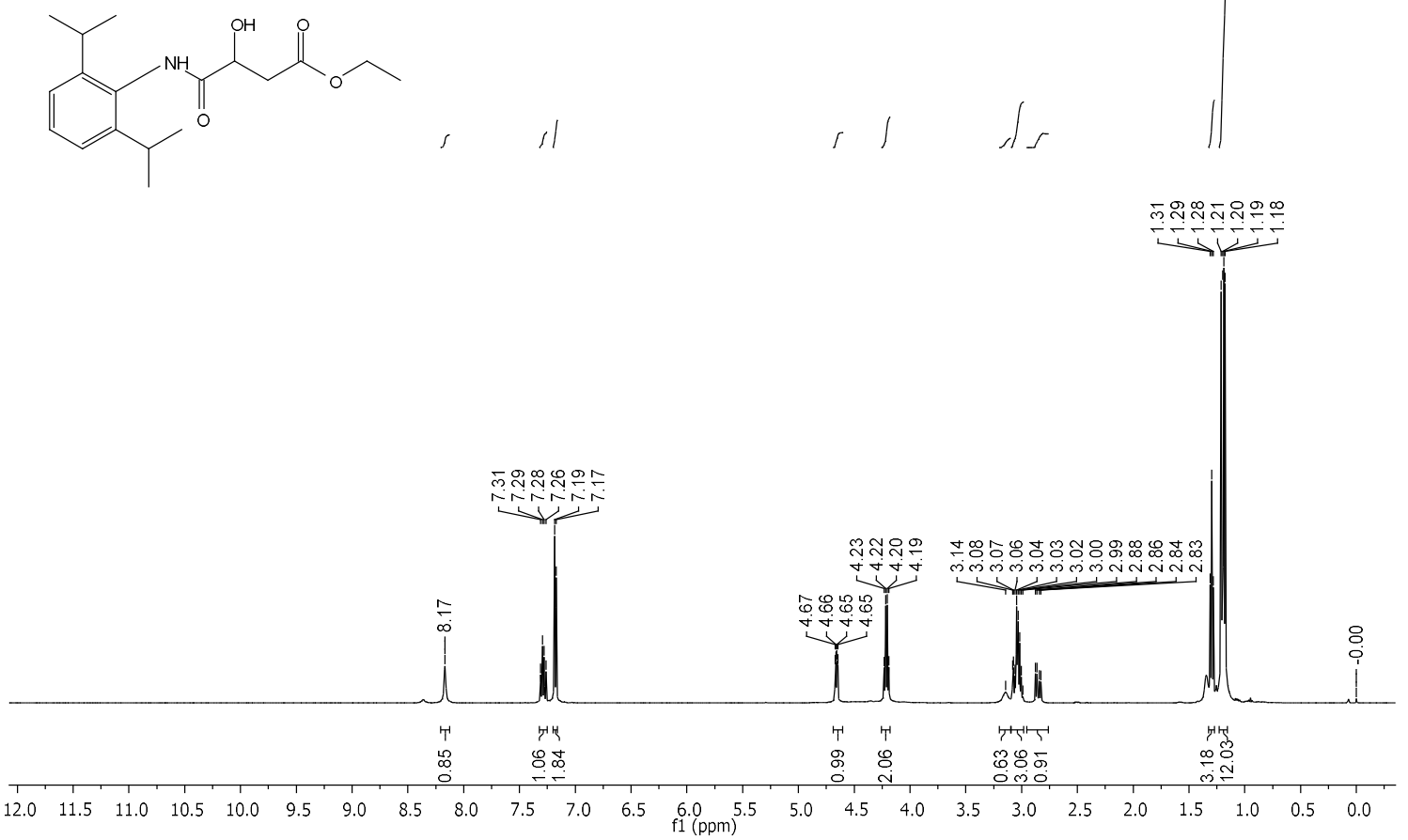

${ }^{13} \mathrm{C}$ NMR (126 MHz, $\left.\mathrm{CDCl}_{3}\right)$.

Jan12-2021

Chemist Shweta Gediya

C13APT.w CDCl3 /opt/topspin3.2 SKG 20

$\frac{5}{N}+$
$\frac{1}{2} \frac{1}{5}$
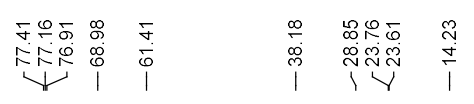
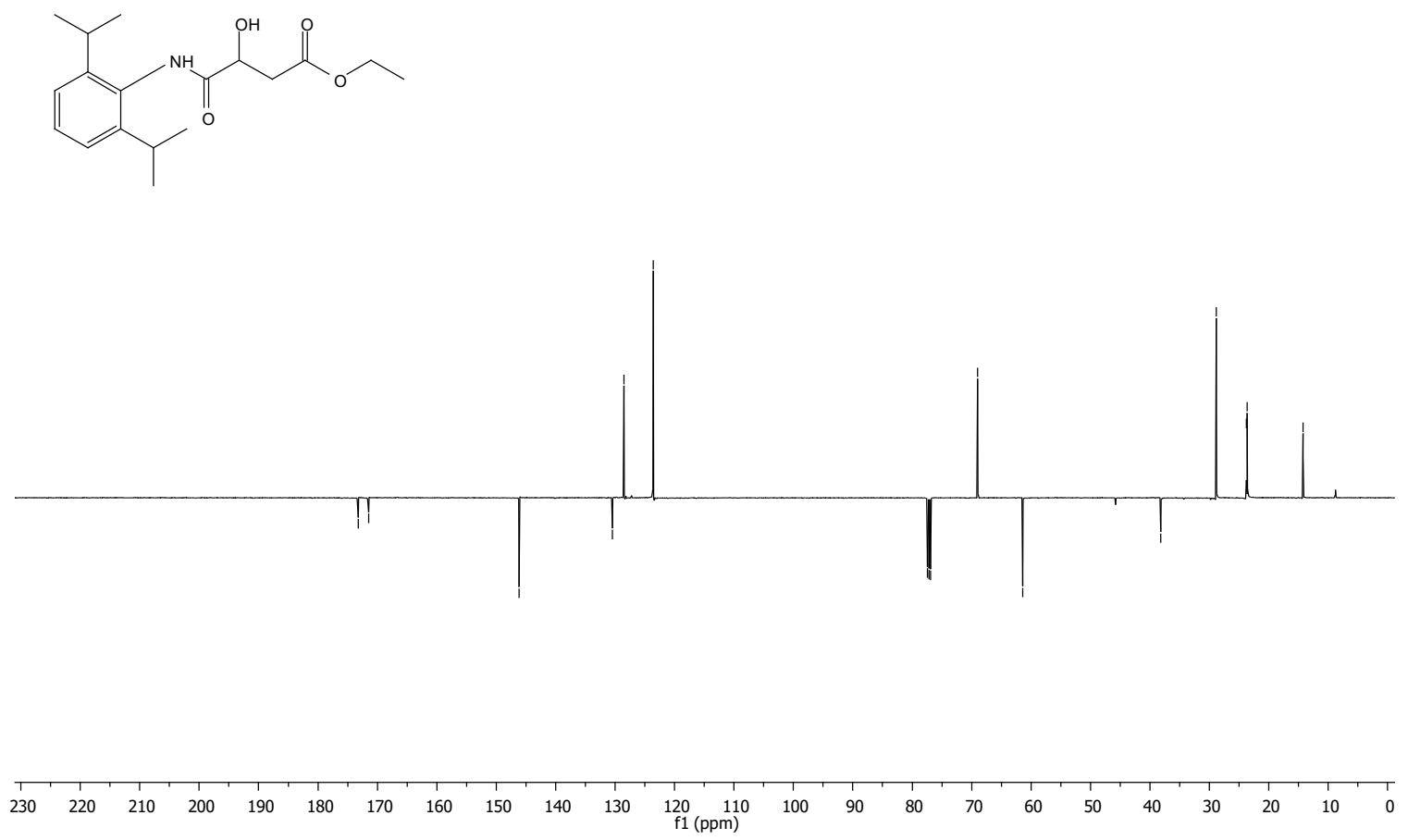

S337 
COSY.

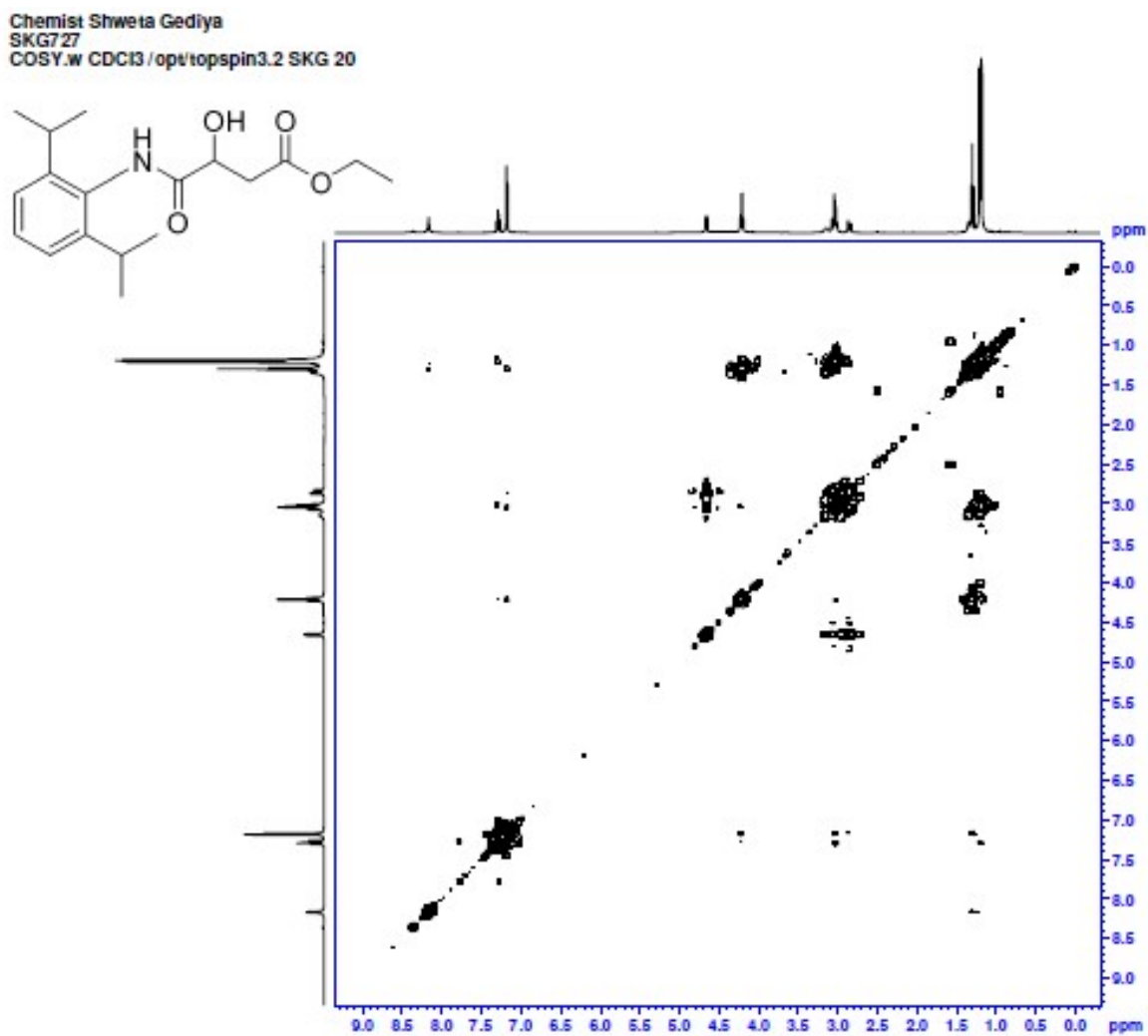

HSQC.

Chemist Shweta Gediya

HSQC.w CDCl3 /opt/topspin3.2 SKG 20

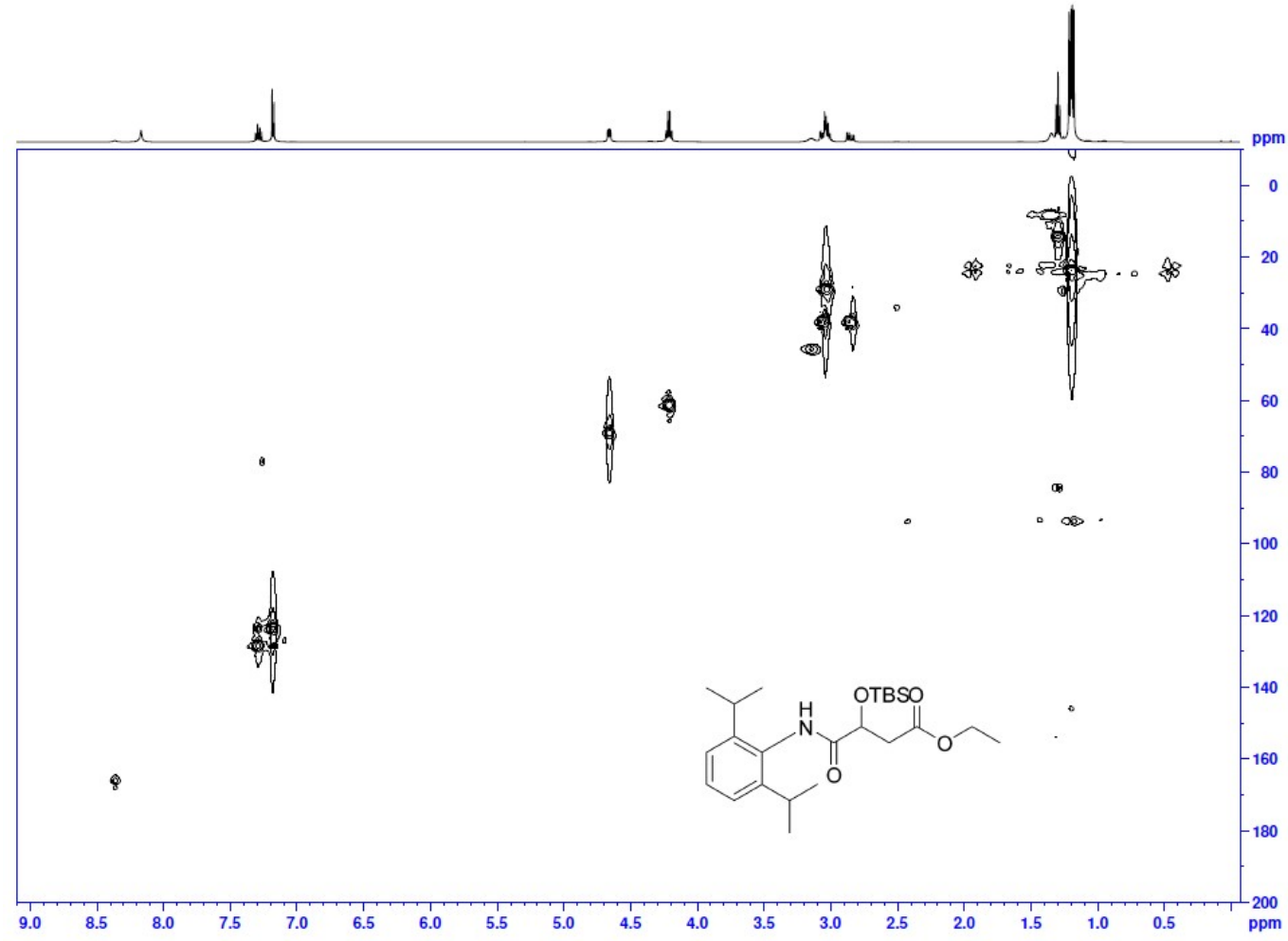




\section{Chiral HPLC of ketone}

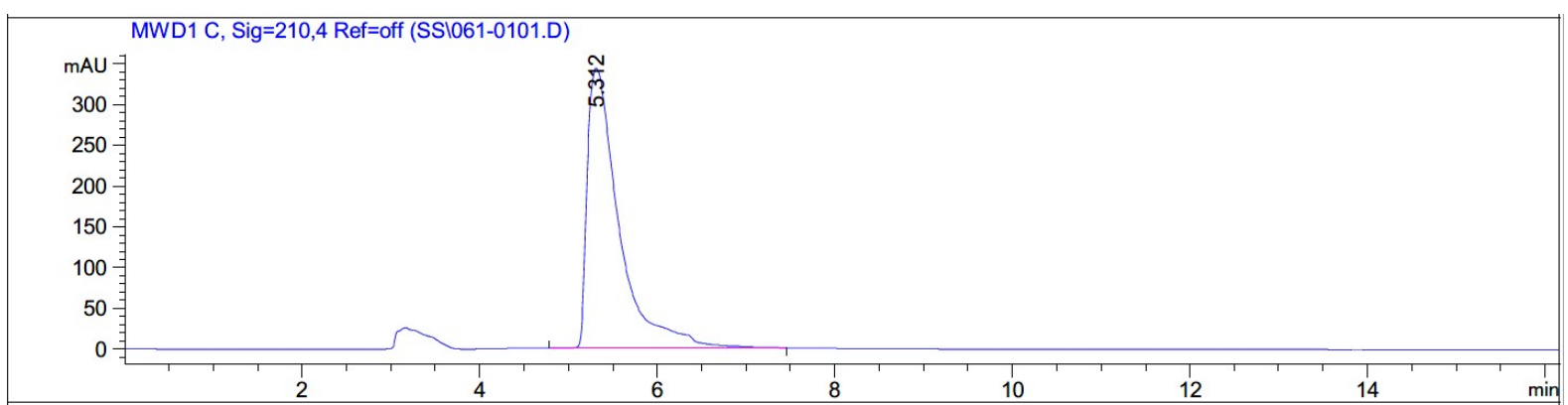

Signal 2: MWD1 C, Sig=210,4 Ref=off

\begin{tabular}{|c|c|c|c|c|c|c|}
\hline $\begin{array}{c}\text { Peak } \\
\#\end{array}$ & $\begin{array}{c}\text { RetTime } \\
\text { [min] }\end{array}$ & Type & $\begin{array}{l}\text { Width } \\
\text { [min] }\end{array}$ & $\begin{array}{c}\text { Area } \\
{\left[\mathrm{mAU}{ }^{*} \mathrm{~s}\right]}\end{array}$ & $\begin{array}{l}\text { Height } \\
\text { [mAU] }\end{array}$ & $\begin{array}{c}\text { Area } \\
8\end{array}$ \\
\hline & ---- & & ------ & ---------- & -------- & $-------\mid$ \\
\hline 1 & 5.312 & BB & 0.3700 & 8526.26758 & 343.05850 & 100.0000 \\
\hline
\end{tabular}

Chiral HPLC of racemic standard.

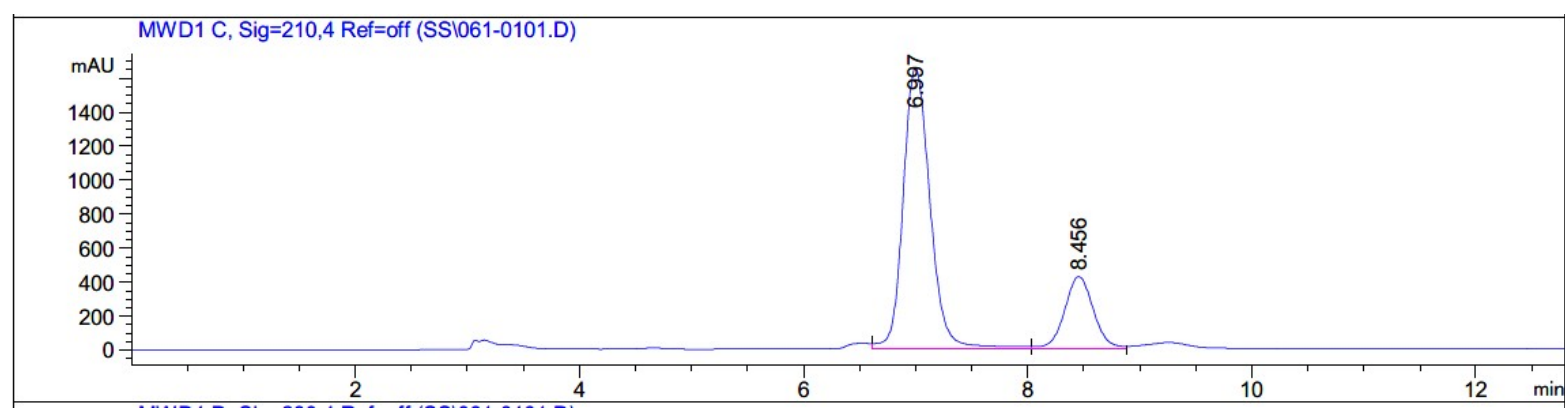

Signal 2: MWD1 C, Sig=210,4 Ref=off

\begin{tabular}{|c|c|c|c|c|c|c|}
\hline $\begin{array}{c}\text { Peak } \\
\#\end{array}$ & $\begin{array}{c}\text { RetTime } \\
\text { [min] }\end{array}$ & Type & $\begin{array}{l}\text { Width } \\
\text { [min] }\end{array}$ & $\begin{array}{c}\text { Area } \\
{\left[\mathrm{mAU}{ }^{*} \mathrm{~S}\right]}\end{array}$ & $\begin{array}{l}\text { Height } \\
\text { [mAU] }\end{array}$ & $\begin{array}{c}\text { Area } \\
\frac{\%}{\%}\end{array}$ \\
\hline & & & & ---- & $|----------|$ & ------- \\
\hline 1 & 6.997 & VV & 0.2608 & $2.78581 \mathrm{e} 4$ & 1651.09094 & 78.3837 \\
\hline 2 & 8.456 & VV & 0.2785 & 7682.56250 & 425.83914 & 21.6163 \\
\hline
\end{tabular}


Chiral HPLC of AH product, $98 \%$ ee.

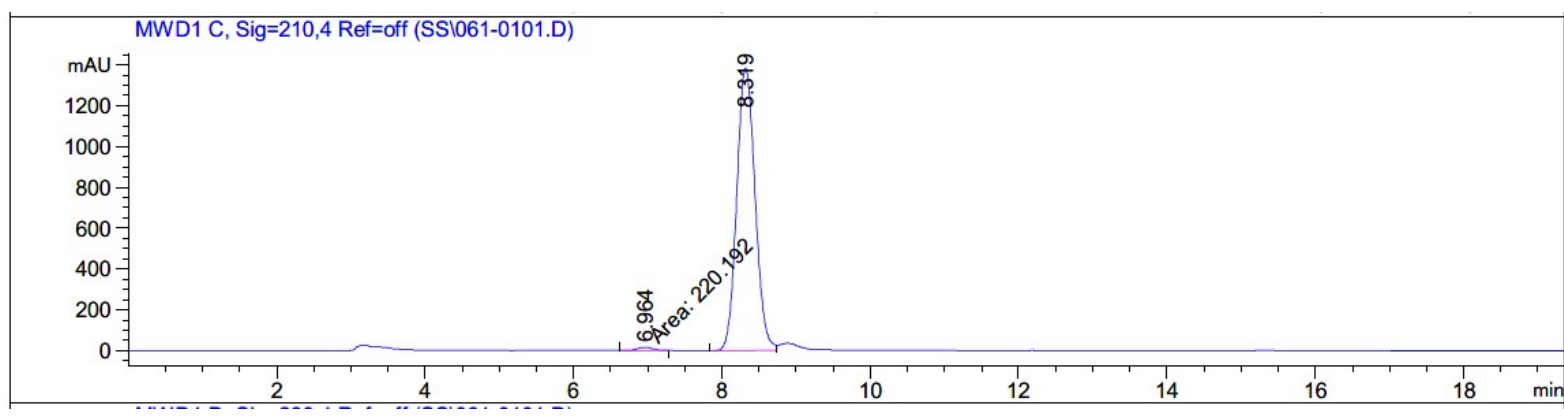

Signal 2: MWD1 C, Sig=210,4 Ref=off

\begin{tabular}{|c|c|c|c|c|c|c|}
\hline $\begin{array}{c}\text { Peak } \\
\#\end{array}$ & $\begin{array}{c}\text { RetTime } \\
\text { [min] }\end{array}$ & Type & $\begin{array}{l}\text { Width } \\
\text { [min] }\end{array}$ & $\begin{array}{c}\text { Area } \\
{\left[\mathrm{mAU}^{\star} \mathrm{s}\right]}\end{array}$ & $\begin{array}{l}\text { Height } \\
{[\mathrm{mAU}]}\end{array}$ & $\begin{array}{c}\text { Area } \\
\%\end{array}$ \\
\hline-- & ----- & & -2 & ---------- & ---- & ----- \\
\hline 1 & 6.964 & MM & 0.2416 & 220.19193 & 15.19075 & 0.9193 \\
\hline 2 & 8.319 & BV & 0.2679 & $2.37318 \mathrm{e} 4$ & 1385.11829 & 99.0807 \\
\hline
\end{tabular}


Ethyl (R)-5-((2,6-diisopropylphenyl)amino)-4-hydroxy-5-oxopentanoate 34 .<smiles>CCOC(=O)CC[C@H](O)C(=O)Nc1c(C(C)C)cccc1C(C)C</smiles>

This compound is novel and was prepared following general procedure $\mathrm{C}$ using ethyl 4-((2,6diisopropylphenyl)amino)-3,4-dioxobutanoate ( $83.3 \mathrm{mg}, 0.250 \mathrm{mmol}, 1.0 \mathrm{eq}), 3 \mathrm{C}$ tethered catalyst $(R, R)-2(2.3 \mathrm{mg}, 3.8 \mu \mathrm{mol}, 0.015 \mathrm{eq})$ and FA:TEA $(0.15 \mathrm{~mL})$ in DCM $(1.5 \mathrm{~mL})$ to give 34 as a yellow oil (70.9 mg, $0.221 \mathrm{mmol}, 88 \%)$.

TLC: $\mathrm{R}_{\mathrm{f}}$ ca 0.3 (6:4, hexane: EtOAc), UV-active and PMA-reactive;

HRMS (ESI) m/z: [M+Na] ${ }^{+}$Calcd for $\mathrm{C}_{19} \mathrm{H}_{29} \mathrm{NO}_{4} \mathrm{Na} 358.1989$; Found 358.1984 (error 1.3 ppm);

$U_{\max } 3325,3245,2961,1735,1652,1506,1159,1103,805 \mathrm{~cm}^{-1}$;

Enantiomeric excess determined by HPLC analysis (Chiralpak IC, $250 \mathrm{~mm} \times 4.6 \mathrm{~mm}$ column, hexane: $\left.\operatorname{PrOH} 90: 10,1 \mathrm{~mL} / \mathrm{min}, \lambda=210 \mathrm{~nm}, \mathrm{~T}=25{ }^{\circ} \mathrm{C}\right), R$-enantiomer $12.6 \mathrm{~min}, R$ enantiomer $18.4 \min .68 \%$ ee $(R),[\alpha]_{\mathrm{D}}^{22}=+15\left(\mathrm{c}=0.05, \mathrm{CHCl}_{3}\right)$;

${ }^{1} \mathrm{H}$ NMR $\left(500 \mathrm{MHz}, \mathrm{CDCl}_{3}\right) \delta 8.20(1 \mathrm{H}, \mathrm{s}, \mathrm{CONH}), 7.31-7.26(1 \mathrm{H}, \mathrm{m}, \mathrm{ArH}), 7.18(2 \mathrm{H}, \mathrm{d}, J=$ $7.7 \mathrm{~Hz}, \mathrm{ArH}), 4.87(1 \mathrm{H}, \mathrm{s}, \mathrm{CHO} \underline{\mathrm{H}}), 4.36-4.35(1 \mathrm{H}, \mathrm{m}, \mathrm{C} \underline{\mathrm{HOH}}), 4.23-4.17\left(2 \mathrm{H}, \mathrm{m}, \mathrm{C}_{2} \mathrm{CH}_{3}\right.$ of ester group), 3.07-2.99 (2H, m, CH of isopropyl), 2.73-2.67 (1H, m, $\left.\mathrm{C}_{\mathrm{a}} \mathrm{H}_{\mathrm{b}} \mathrm{COOEt}\right), 2.63-$ $2.57\left(1 \mathrm{H}, \mathrm{m}, \mathrm{CH}_{\mathrm{a}} \underline{\mathrm{H}}_{\mathrm{b}} \mathrm{COOEt}\right), 2.36-2.30\left(1 \mathrm{H}, \mathrm{m}, \mathrm{C}_{\underline{\mathrm{H}}} \mathrm{H}_{\mathrm{b}} \mathrm{CH}_{2} \mathrm{COOEt}\right), 2.16-2.09(1 \mathrm{H}, \mathrm{m}$, $\left.\mathrm{CH}_{\mathrm{a}} \underline{\mathrm{H}}_{\mathrm{b}} \mathrm{CH}_{2} \mathrm{COOEt}\right), 1.30\left(3 \mathrm{H}, \mathrm{t}, J=7.1 \mathrm{~Hz}, \mathrm{CH}_{2} \underline{\mathrm{CH}}_{3}\right.$ of ester group), 1.22-1.28 (12H, m, $\mathrm{CH}_{3}$ of isopropyl);

${ }^{13} \mathrm{C}$ NMR $\left(126 \mathrm{MHz}, \mathrm{CDCl}_{3}\right) \delta 175.9,172.8,146.1,130.7,128.5,123.6,72.7,61.5,31.4$, 29.2, 28.9, 23.8, 23.6, 14.3; $m / z(\mathrm{ESI}) 358.2\left[(\mathrm{M}+\mathrm{Na})^{+}, 100 \%\right]$. 
Ethyl 5-((2,6-diisopropylphenyl)amino)-4-hydroxy-5-oxopentanoate 34.

${ }^{1} \mathrm{H}$ NMR $\left(500 \mathrm{MHz}, \mathrm{CDCl}_{3}\right)$.

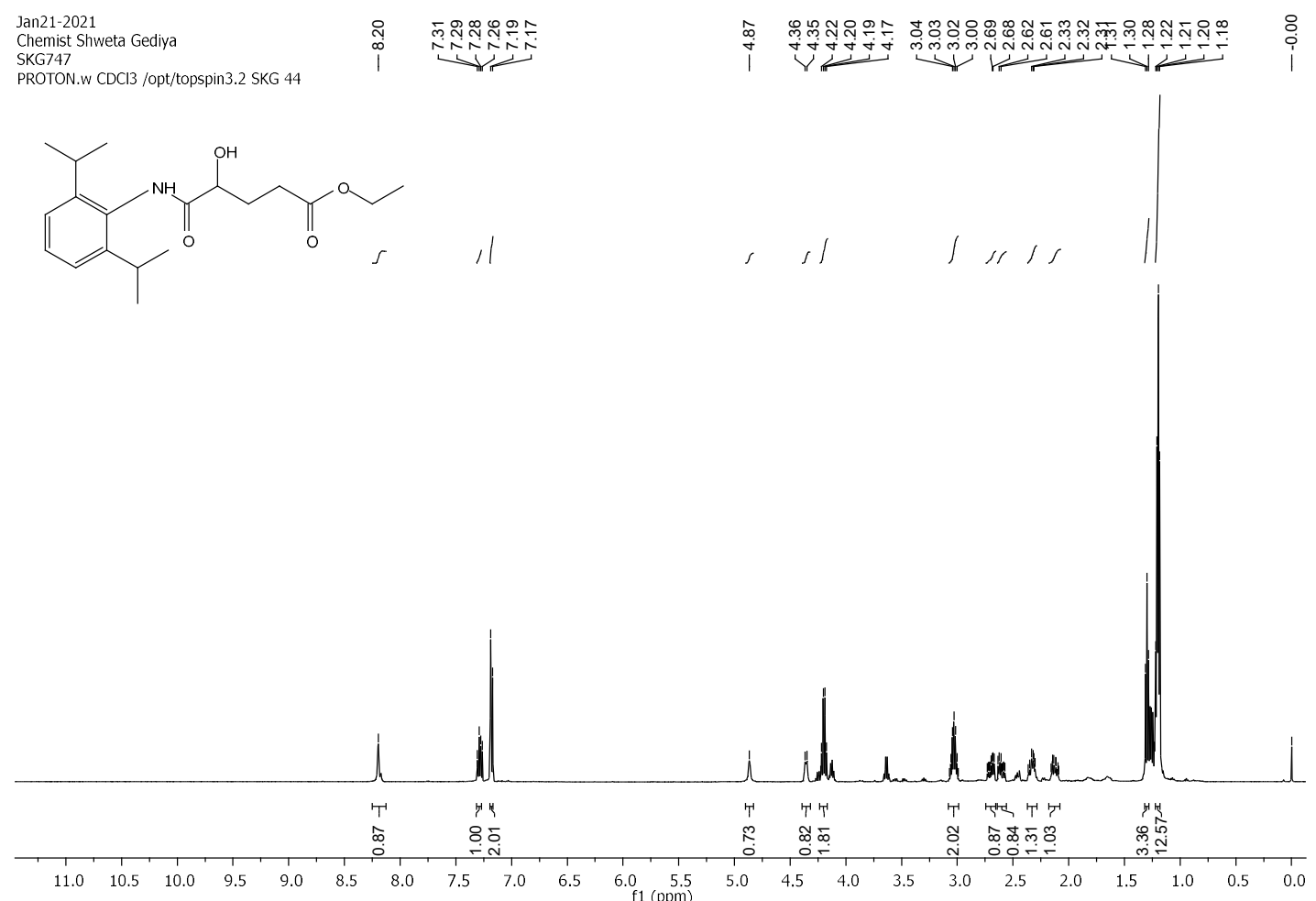

${ }^{13} \mathrm{C} \mathrm{NMR}\left(126 \mathrm{MHz}, \mathrm{CDCl}_{3}\right)$.

Jan21-2021<smiles>CCOC(=O)CCC(O)C(=O)Nc1c(C(C)C)cccc1C(C)C</smiles>

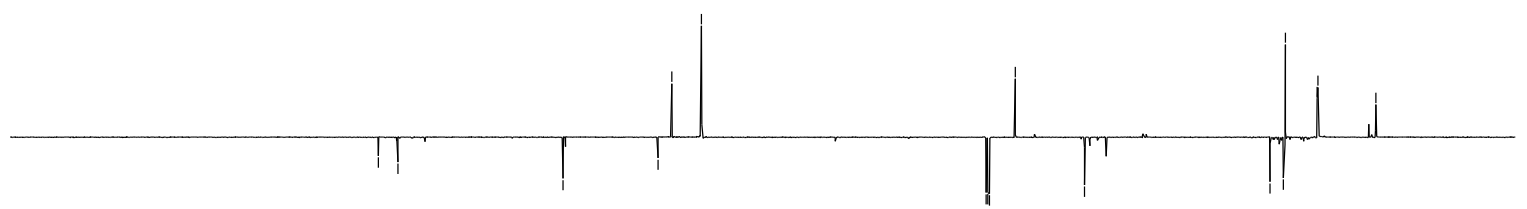

$\begin{array}{llllllllllllllllllllllll}230 & 220 & 210 & 200 & 190 & 180 & 170 & 160 & 150 & 140 & 130 & 120 & 110 & 100 & 90 & 80 & 70 & 60 & 50 & 40 & 30 & 20 & 10 & 0\end{array}$ 
COSY.

Chemist Shweta Gediya

COSY.w $\mathrm{CDCl} 3$ /opt/topspin3.2 SKG 44

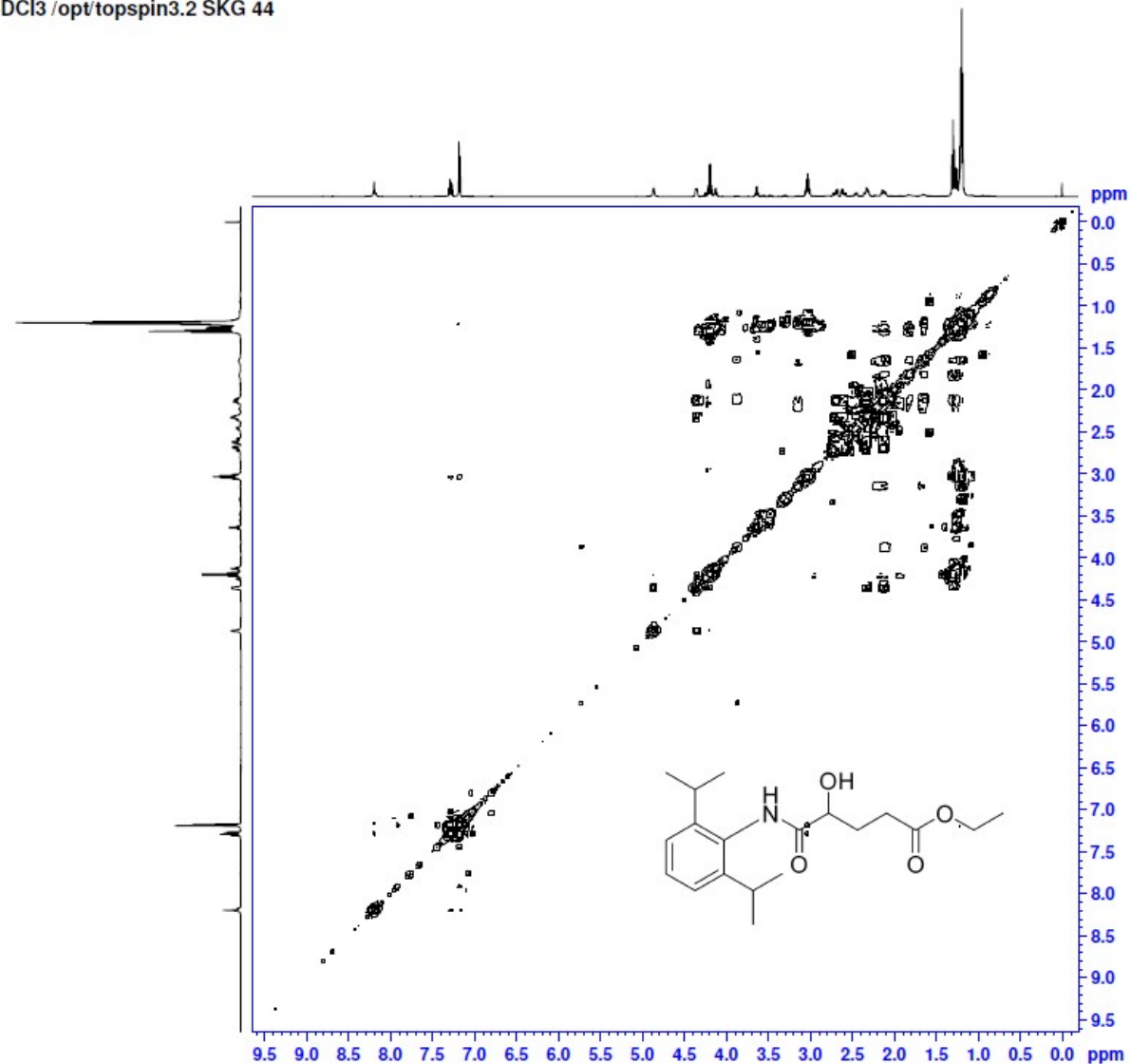

HSQC.

Chemist Shweta Gediya

HSQC.w CDCl3/opt/topspin3.2 SKG 44

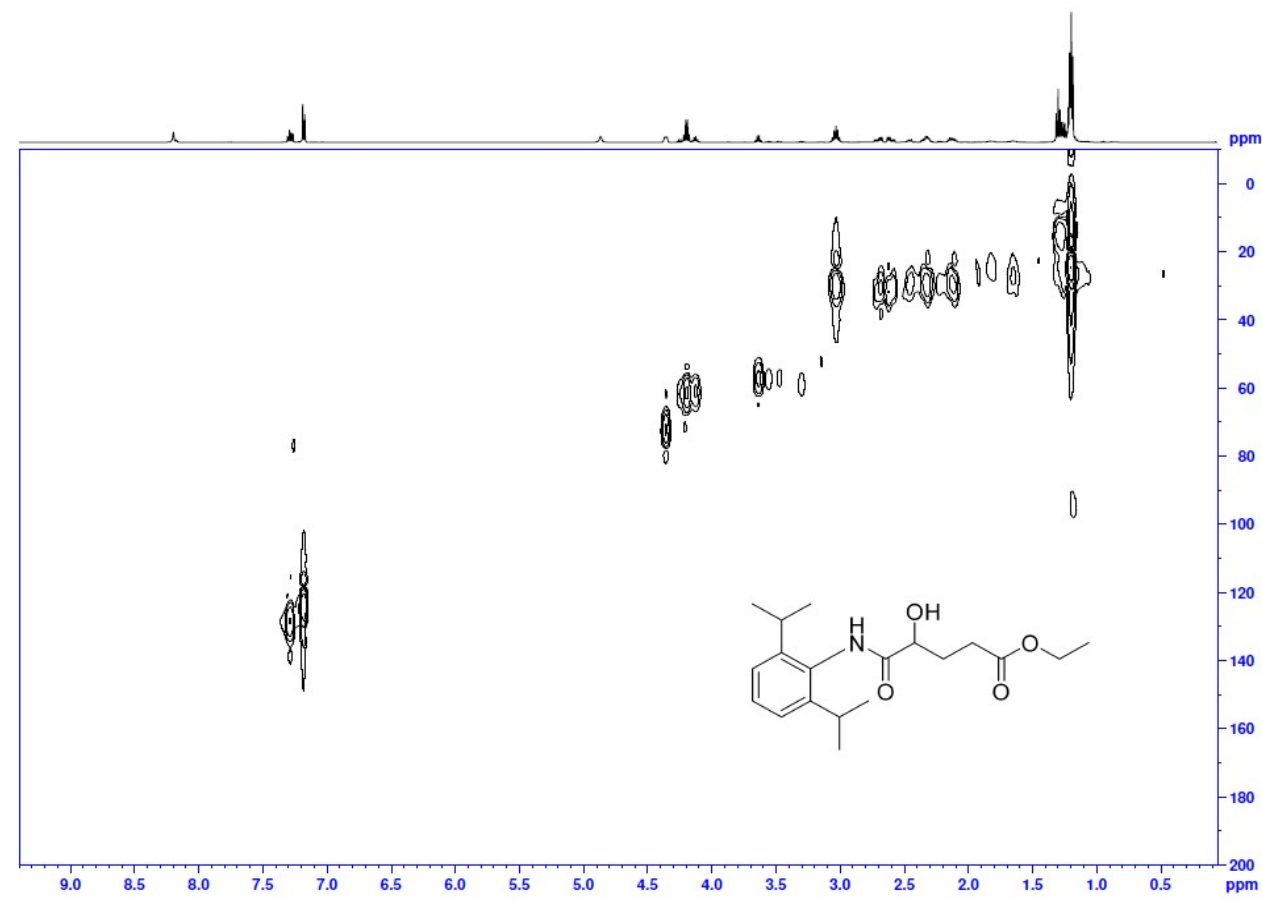




\section{Chiral HPLC of ketone precursor.}

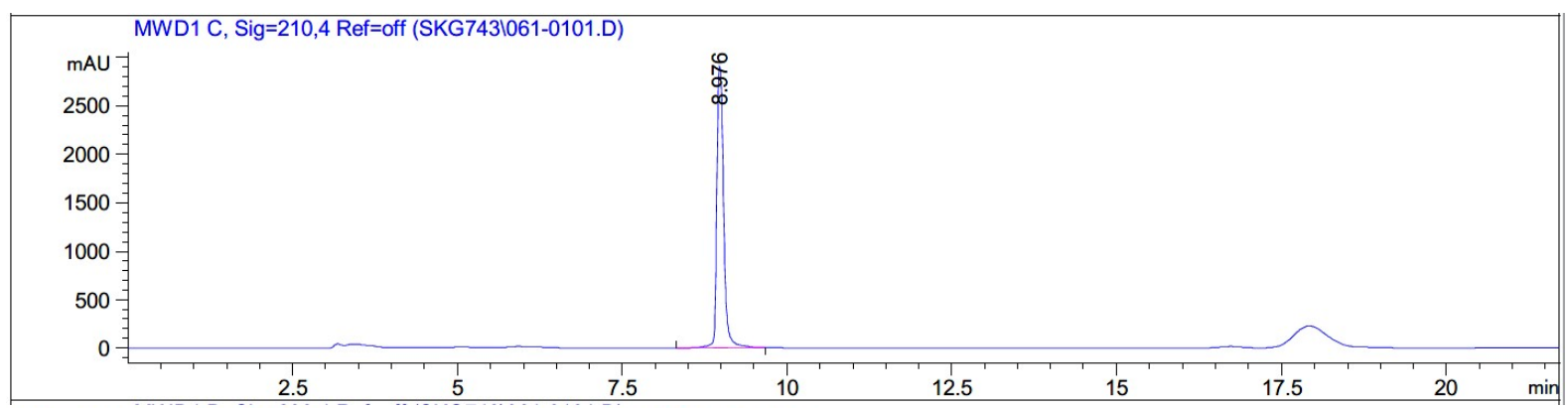

Signal 2: MWD1 C, Sig=210,4 Ref=off

\begin{tabular}{|c|c|c|c|c|c|c|}
\hline $\begin{array}{c}\text { Peak } \\
\quad \#\end{array}$ & $\begin{array}{c}\text { RetTime } \\
\text { [min] }\end{array}$ & Type & $\begin{array}{l}\text { Width } \\
\text { [min] }\end{array}$ & $\begin{array}{c}\text { Area } \\
{\left[\mathrm{mAU}^{*} \mathrm{~S}\right]}\end{array}$ & $\begin{array}{l}\text { Height } \\
{[\mathrm{mAU}]}\end{array}$ & $\begin{array}{c}\text { Area } \\
\frac{\circ}{0}\end{array}$ \\
\hline 1 & 8.976 & $\mathrm{BB}$ & 33 & $.06971 e 4$ & 561 & \\
\hline
\end{tabular}




\section{Chiral HPLC of racemic alcohol.}

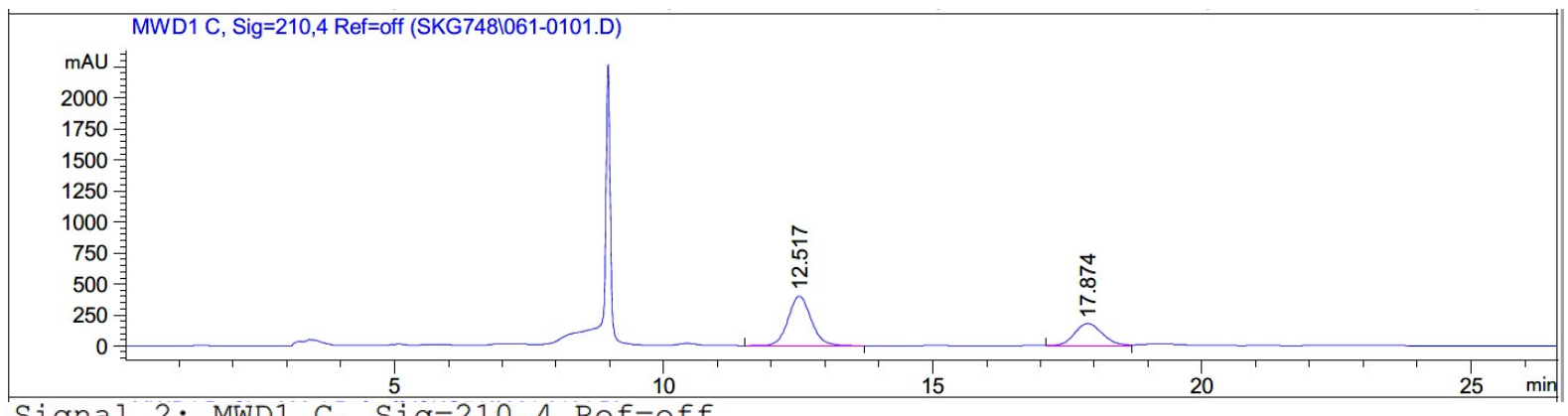

Signal 2: MWD1 C, Sig=210,4 Ref=off

\begin{tabular}{|c|c|c|c|c|c|c|}
\hline $\begin{array}{c}\text { Peak } \\
\#\end{array}$ & $\begin{array}{c}\text { RetTime } \\
\text { [min] }\end{array}$ & Type & $\begin{array}{l}\text { Width } \\
\text { [min] }\end{array}$ & $\begin{array}{c}\text { Area } \\
{\left[\mathrm{mAU}{ }^{\star} \mathrm{s}\right]}\end{array}$ & $\begin{array}{l}\text { Height } \\
{[\mathrm{mAU}]}\end{array}$ & $\begin{array}{c}\text { Area } \\
\frac{\circ}{0}\end{array}$ \\
\hline 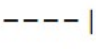 & & & & ---- & -- & -- \\
\hline 1 & 12.517 & $\mathrm{BB}$ & 0.4536 & $1.18083 \mathrm{e} 4$ & 400.93875 & 64.7927 \\
\hline 2 & 17.874 & VV & 0.5605 & 6416.45605 & 178.85754 & 35.2073 \\
\hline
\end{tabular}

\section{Chiral HPLC of ATH product. $68 \%$ ee.}

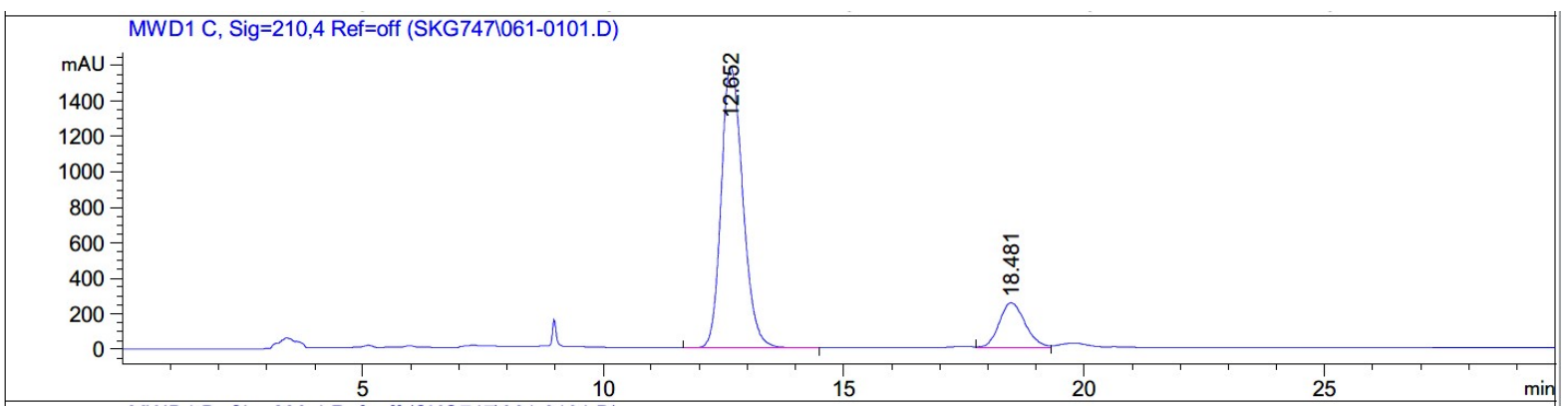

Signal 2: MWD1 C, Sig=210,4 Ref=off

\begin{tabular}{|c|c|c|c|c|c|c|}
\hline $\begin{array}{c}\text { eak } \\
\#\end{array}$ & $\begin{array}{c}\text { RetTime } \\
\text { [min] }\end{array}$ & Type & $\begin{array}{l}\text { Width } \\
\text { [min] }\end{array}$ & $\begin{array}{c}\text { Area } \\
{\left[\mathrm{mAU}^{\star} \mathrm{s}\right]}\end{array}$ & $\begin{array}{l}\text { Height } \\
{[\mathrm{mAU}]}\end{array}$ & $\begin{array}{c}\text { Area } \\
\frac{\circ}{0}\end{array}$ \\
\hline & & & & -- & -7 & \\
\hline 1 & & & & 4.9 & 902 & 83 \\
\hline & 481 & VV & & 9604.46191 & 253.60895 & 13 \\
\hline
\end{tabular}

A small amount of ketone is visible in this analysis. 
(R)-N-(2,6-Diisopropylphenyl)-2-hydroxyhexanamide 35 .<smiles>CCCC[C@H](O)C(=O)Nc1c(C(C)C)cccc1C(C)C</smiles>

This compound is novel and was prepared following general procedure $\mathrm{C}$ using $N-(2,6-$ diisopropylphenyl)-2-oxohexanamide $(72.8 \mathrm{mg}, 0.250 \mathrm{mmol}, 1.0 \mathrm{eq}), 3 \mathrm{C}$ tethered catalyst $(R, R)-2(2.3 \mathrm{mg}, 3.8 \mu \mathrm{mol}, 0.015 \mathrm{eq})$ and FA:TEA $(0.15 \mathrm{~mL})$ in DCM $(1.5 \mathrm{~mL})$ to give 35 as a brown solid (62.3 mg, $0.214 \mathrm{mmol}, 86 \%)$.

TLC: $\mathrm{R}_{\mathrm{f}}$ ca 0.2 (6:4, hexane: EtOAc), UV-active, Strong $\mathrm{KMnO}_{4}$ and PMA-reactive; MP: $168-171^{\circ} \mathrm{C}$;

HRMS (ESI) m/z: $[\mathrm{M}+\mathrm{Na}]^{+}$Calcd for $\mathrm{C}_{18} \mathrm{H}_{29} \mathrm{NO}_{2} \mathrm{Na} 314.2090$; Found 314.2090 (error 0.2 ppm);

$U_{\max } 3328,2959,1654,1502,1462,1256,1088,804,743,664,623 \mathrm{~cm}^{-1}$;

Enantiomeric excess determined by HPLC analysis (Chiralpak AD-H, 250mm $\times 4.6 \mathrm{~mm}$ column, hexane: iPrOH 95:5, $\left.0.5 \mathrm{~mL} / \mathrm{min}, \lambda=210 \mathrm{~nm}, \mathrm{~T}=25{ }^{\circ} \mathrm{C}\right), R$-enantiomer $11.5 \mathrm{~min}, S$ enantiomer $15.6 \mathrm{~min}, 97 \%$ ee $(R),[\alpha]_{\mathrm{D}}^{22}=+35\left(\mathrm{c}=0.1, \mathrm{CHCl}_{3}\right)$;

${ }^{1} \mathrm{H}$ NMR $\left(400 \mathrm{MHz}, \mathrm{CDCl}_{3}\right) \delta 7.77(1 \mathrm{H}, \mathrm{s}, \mathrm{CONH}), 7.32-7.28(1 \mathrm{H}, \mathrm{m}, \mathrm{ArH}), 7.19-7.17$ (2H, $\mathrm{m}, \mathrm{ArH}), 4.36-4.32(1 \mathrm{H}, \mathrm{m}, \mathrm{CHOH}), 3.08-2.99(2 \mathrm{H}, \mathrm{m}, \mathrm{CH}$ of isopropyl $), 2.63(1 \mathrm{H}, \mathrm{s}$, $\mathrm{CHO} \underline{\mathrm{H}}), 2.03$-1.95 $\left(1 \mathrm{H}, \mathrm{m}, \mathrm{C}_{\underline{a}} \mathrm{H}_{\mathrm{b}}\right), 1.84-1.71\left(1 \mathrm{H}, \mathrm{m}, \mathrm{CH}_{\mathrm{a}} \underline{\mathrm{H}_{\mathrm{b}}}\right), 1.53-1.47\left(2 \mathrm{H}, \mathrm{m}, \mathrm{CH}_{2}\right)$, 1.44-1.36 (2H, m, $\left.\mathrm{CH}_{2}\right), 1.22-1.19\left(12 \mathrm{H}, \mathrm{m}, \mathrm{CH}_{3}\right.$ of isopropyl), $0.95\left(3 \mathrm{H}, \mathrm{t}, J=7.2 \mathrm{~Hz}, \mathrm{CH}_{3}\right)$; ${ }^{13} \mathrm{C}$ NMR $\left(101 \mathrm{MHz}, \mathrm{CDCl}_{3}\right) \delta 173.0,146.2,128.5,123.6,72.9,34.9,28.9,27.5,23.8,23.7$, 22.6, 14.2; $m / z(\mathrm{ESI}) 314.2\left[(\mathrm{M}+\mathrm{Na})^{+}, 100 \%\right]$. 
$N$-(2,6-Diisopropylphenyl)-2-hydroxyhexanamide 35 .

${ }^{1} \mathrm{H}$ NMR (400 MHz, $\left.\mathrm{CDCl}_{3}\right)$.

Feb19-2021

SKG664

PROTON.w CDCl3 /opt/topspin3.5pl2 SKG 35

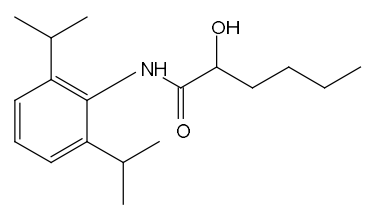

$$
\text { sh s s s ill }
$$

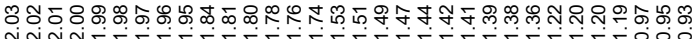

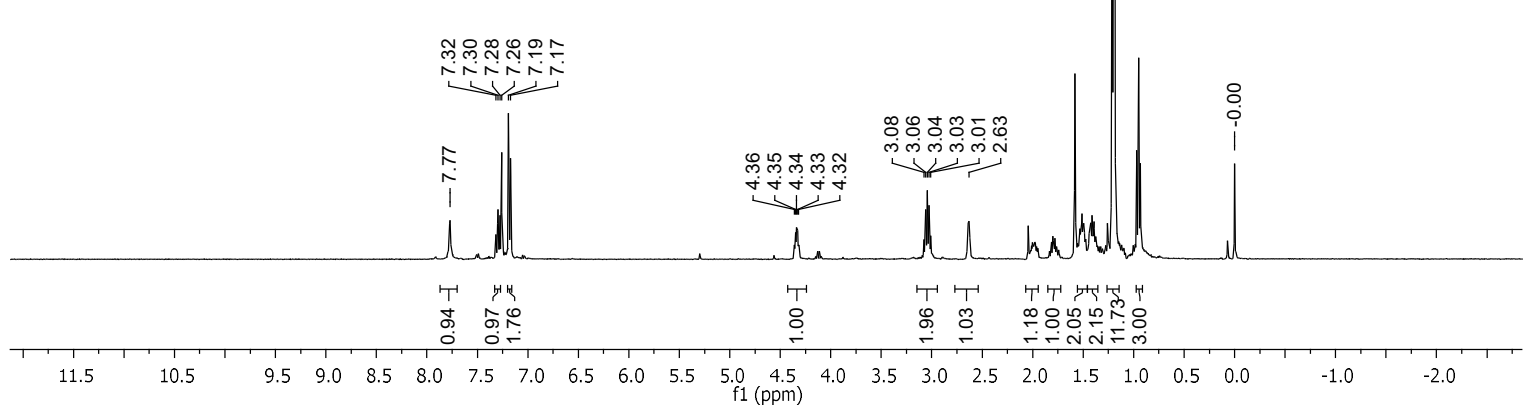

${ }^{13} \mathrm{C}$ NMR (101 MHz, $\left.\mathrm{CDCl}_{3}\right)$.

Feb19-2021

SKG664

C13APTlong.w CDCl3 /opt/topspin3.5pl2 SKG 35

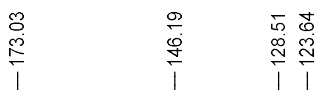

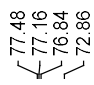

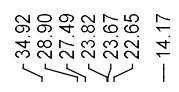
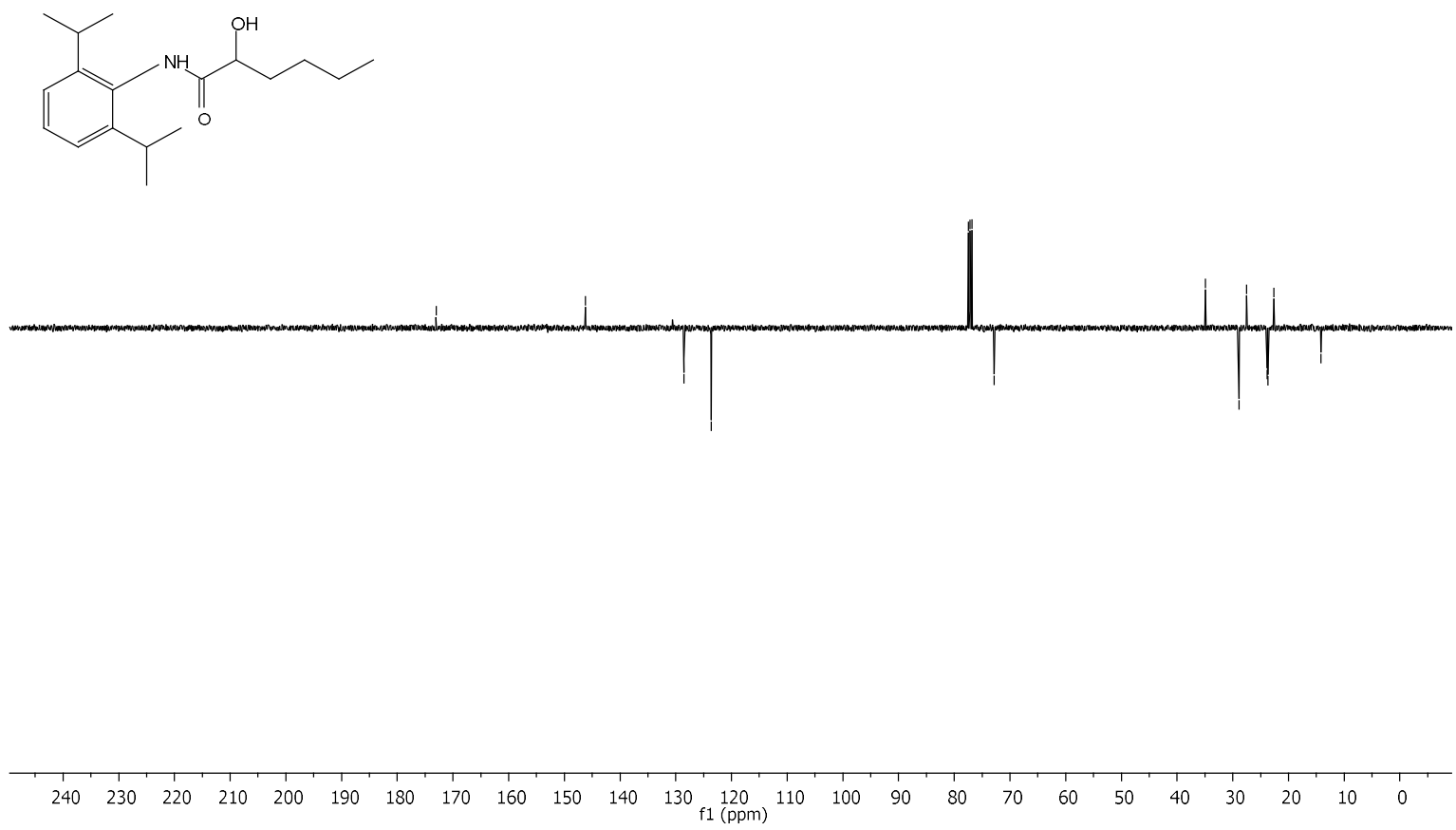

S347 
COSY.

SKG664

COSY.w $\mathrm{CDCl} 3$ /opt/topspin3.5pl2 SKG 35

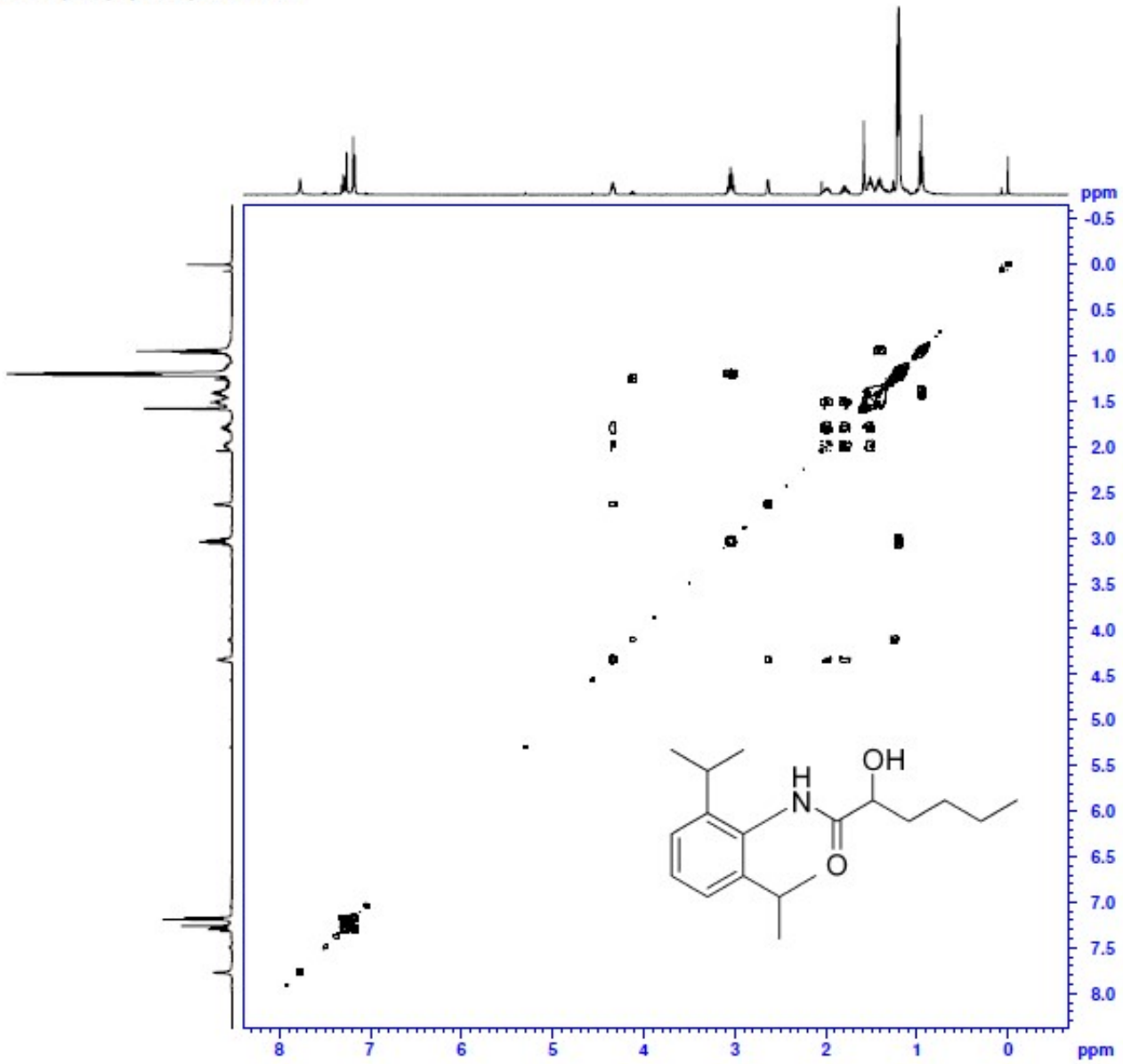

Chiral HPLC of ketone precursor.

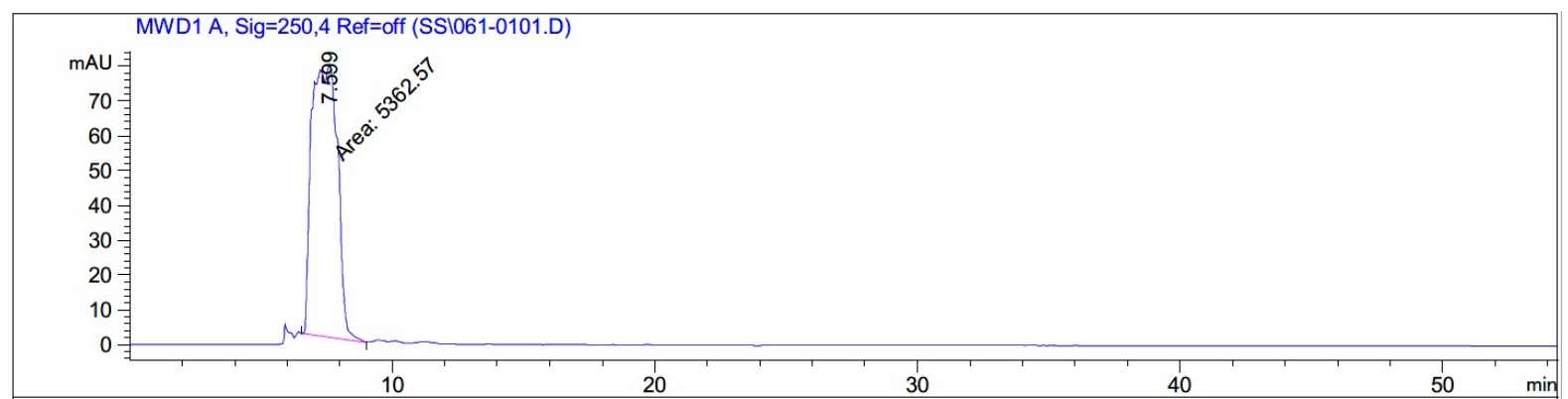

Signal 1: MWD1 A, Sig=250,4 Ref=off

\begin{tabular}{|c|c|c|c|c|c|c|}
\hline $\begin{array}{l}\text { eak } \\
\#\end{array}$ & $\begin{array}{c}\text { RetTime } \\
\text { [min] }\end{array}$ & Type & $\begin{array}{l}\text { Width } \\
\text { [min] }\end{array}$ & $\begin{array}{c}\text { Area } \\
{\left[\mathrm{mAU}^{\star} \mathrm{s}\right]}\end{array}$ & $\begin{array}{l}\text { Height } \\
\text { [mAU] }\end{array}$ & $\begin{array}{c}\text { Area } \\
\%\end{array}$ \\
\hline 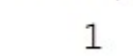 & 599 & & 3 & 5362.56592 & 197 & 100 \\
\hline
\end{tabular}


Chiral HPLC of racemic alcohol.

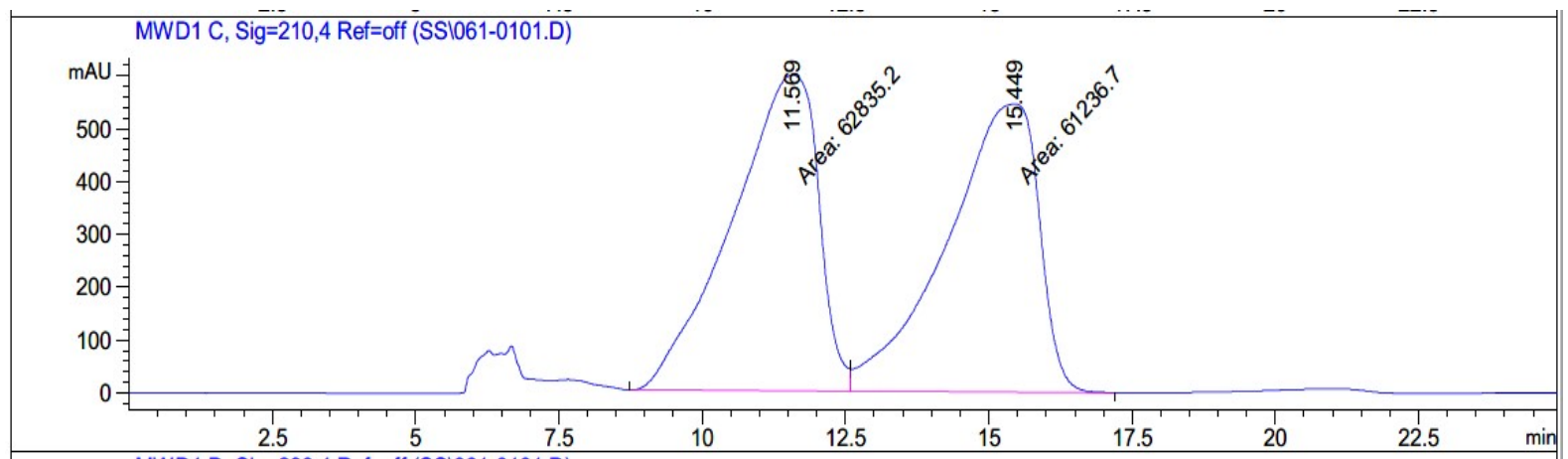

Signal 2: MWD1 C, Sig=210, 4 Ref=off

\begin{tabular}{|c|c|c|c|c|c|c|}
\hline $\begin{array}{c}\text { Peak } \\
\quad \#\end{array}$ & $\begin{array}{c}\text { RetTime } \\
\text { [min] }\end{array}$ & Type & $\begin{array}{l}\text { Width } \\
{[\mathrm{min}]}\end{array}$ & $\begin{array}{c}\text { Area } \\
{\left[\mathrm{mAU}^{*} \mathrm{~s}\right]}\end{array}$ & $\begin{array}{l}\text { Height } \\
{[\mathrm{mAU}]}\end{array}$ & $\begin{array}{c}\text { Area } \\
\frac{8}{0}\end{array}$ \\
\hline 1 & 11.569 & MF & & $6.28352 \mathrm{e} 4$ & 597.88513 & 442 \\
\hline 2 & 15.449 & FM & 1.8718 & $6.12367 \mathrm{e} 4$ & 545.26727 & 49.3558 \\
\hline
\end{tabular}

Chiral HPLC of ATH product. 97\% ee.

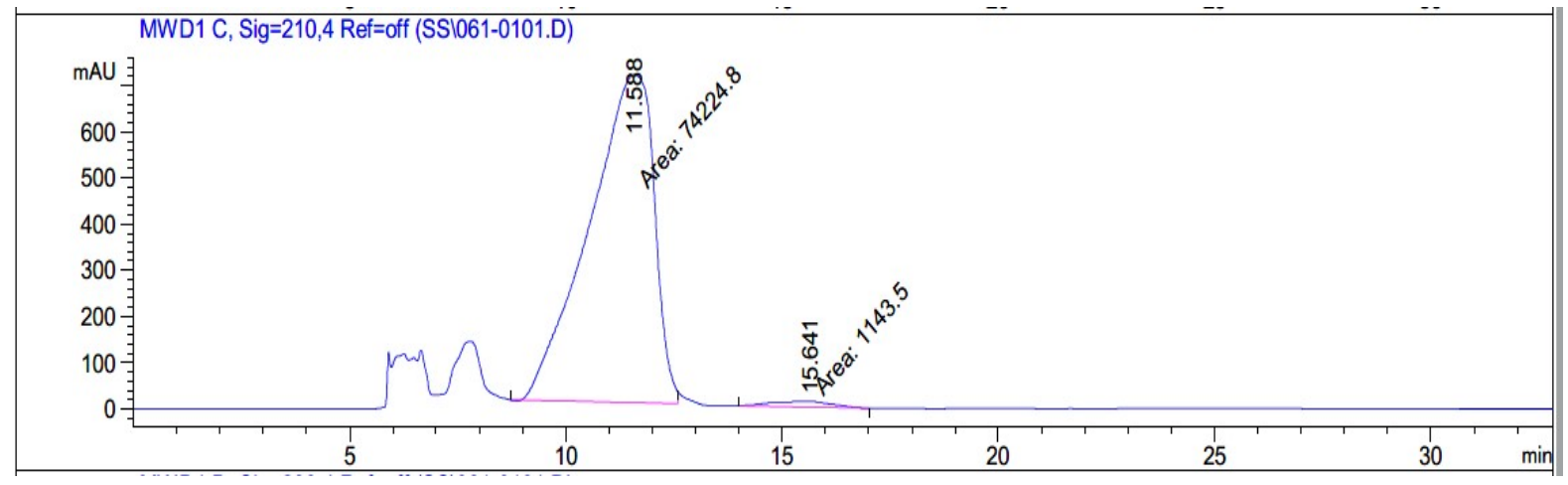

Signal 2: MWD1 C, Sig=210,4 Ref=off

\begin{tabular}{|c|c|c|c|c|c|c|}
\hline $\begin{array}{c}\text { Peak } \\
\#\end{array}$ & $\begin{array}{c}\text { RetTime } \\
\text { [min] }\end{array}$ & Type & $\begin{array}{c}\text { Width } \\
{[\mathrm{min}]}\end{array}$ & $\begin{array}{c}\text { Area } \\
{\left[\mathrm{mAU}^{*} \mathrm{~s}\right]}\end{array}$ & $\begin{array}{l}\text { Height } \\
{[\mathrm{mAU}]}\end{array}$ & $\begin{array}{c}\text { Area } \\
\quad \%\end{array}$ \\
\hline & & & & 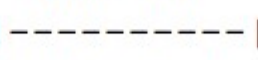 & 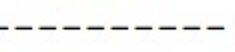 & --- \\
\hline 1 & & MF & & 7.42 & 710.95251 & 1828 \\
\hline 2 & 641 & M & 059 & 1143.49524 & 12.65536 & 1.5172 \\
\hline
\end{tabular}

A small amount of ketone is visible in this analysis. 
(R)-N-(2,6-Diisopropylphenyl)-2-hydroxy-3-phenylpropanamide 36.<smiles>CC(C)c1cccc(C(C)C)c1NC(=O)[C@H](O)Cc1ccccc1</smiles>

This compound is novel and was prepared following general procedure $\mathrm{C}$ using $\mathrm{N}-(2,6-$ diisopropylphenyl)-2-oxo-3-phenylpropanamide ( $80.7 \mathrm{mg}, 0.250 \mathrm{mmol}, 1.0 \mathrm{eq}$ ), 3C tethered catalyst $(R, R)-2(2.3 \mathrm{mg}, 3.8 \mu \mathrm{mol}, 0.015 \mathrm{eq})$ and FA:TEA $(0.15 \mathrm{~mL})$ in DCM $(1.5 \mathrm{~mL})$ to give 36 as a white solid (72.3 $\mathrm{mg}, 0.222 \mathrm{mmol}, 89 \%)$.

TLC: $R_{\mathrm{f}}$ ca 0.2 (7:3, hexane: EtOAc), UV-active and PMA-reactive;

MP: $202-204{ }^{\circ} \mathrm{C}$;

HRMS (ESI) m/z: $[\mathrm{M}+\mathrm{Na}]^{+}$Calcd for $\mathrm{C}_{21} \mathrm{H}_{27} \mathrm{NO}_{2} \mathrm{Na}$ 348.1934; Found 348.1928 (error 1.6 ppm);

$U_{\max } 3336,3264,2962,2866,1652,1497,1094,805,696 \mathrm{~cm}^{-1}$;

Enantiomeric excess determined by HPLC analysis (Chiralcel OD-H, $250 \mathrm{~mm} \times 4.6 \mathrm{~mm}$, hexane: $\mathrm{iPrOH} 90: 10,0.5 \mathrm{~mL} / \mathrm{min}, \lambda=210 \mathrm{~nm}, \mathrm{~T}=25^{\circ} \mathrm{C}$ ), $S$-enantiomer $13.0 \mathrm{~min}, R$ enantiomer $14.9 \min .98 \%$ ee $(R),[\alpha]_{\mathrm{D}}^{22}=+227.5\left(\mathrm{c}=0.02, \mathrm{CHCl}_{3}\right)$;

${ }^{1} \mathrm{H}$ NMR (500 MHz, CDCl $) \delta 7.86(1 \mathrm{H}, \mathrm{s}, \mathrm{CONH}), 7.38-7.26(6 \mathrm{H}, \mathrm{m}, \mathrm{ArH}), 7.17(2 \mathrm{H}, \mathrm{d}, J=$ $7.7 \mathrm{~Hz}, \mathrm{ArH}), 4.55-4.53(1 \mathrm{H}, \mathrm{m}, \mathrm{C} \underline{\mathrm{HOH}}), 3.37\left(1 \mathrm{H}, \mathrm{dd}, \mathrm{J}=14.0,3.8 \mathrm{~Hz}, \mathrm{C}_{\mathrm{a}} \mathrm{H}_{\mathrm{b}}\right), 3.05(1 \mathrm{H}$, $\left.\mathrm{dd}, \mathrm{J}=14.0,8.4 \mathrm{~Hz}, \mathrm{CH}_{\mathrm{a}} \underline{\mathrm{H}} \mathrm{b}\right), 2.97-2.89\left(2 \mathrm{H}, \mathrm{m}, \mathrm{CH}\right.$ of isopropyl), 1.18-1.16 (12H, m, $\mathrm{CH}_{3}$ of isopropyl);

${ }^{13} \mathrm{C} \mathrm{NMR}\left(126 \mathrm{MHz}, \mathrm{CDCl}_{3}\right) \delta 171.8,146.1,136.8,130.5,129.8,129.1,128.5,127.4,123.6$, 73.5, 40.9, 28.8, 23.8, 23.7;

$m / z(\mathrm{ESI}) 348.2\left[(\mathrm{M}+\mathrm{Na})^{+}, 100 \%\right]$. 
N-(2,6-Diisopropylphenyl)-2-hydroxy-3-phenylpropanamide 36.

${ }^{1} \mathrm{H}$ NMR $\left(500 \mathrm{MHz}, \mathrm{CDCl}_{3}\right)$.

Jan08-2021

Shweta Gediya

PROTON.w CDCl3 /opt/topspin3.2 SKG 14

œ

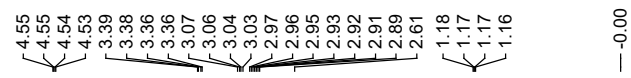<smiles>CC(C)c1cccc(C(C)C)c1NC(=O)C(O)Cc1ccccc1</smiles>

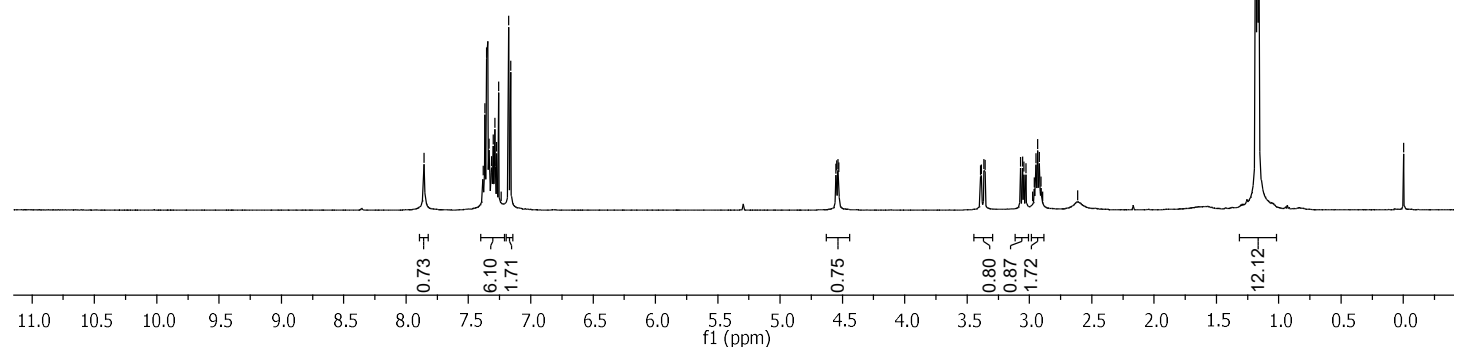

${ }^{13} \mathrm{C}$ NMR (126 MHz, $\left.\mathrm{CDCl}_{3}\right)$.

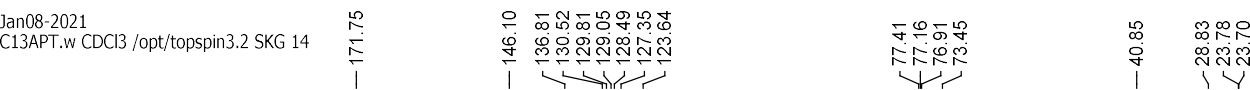
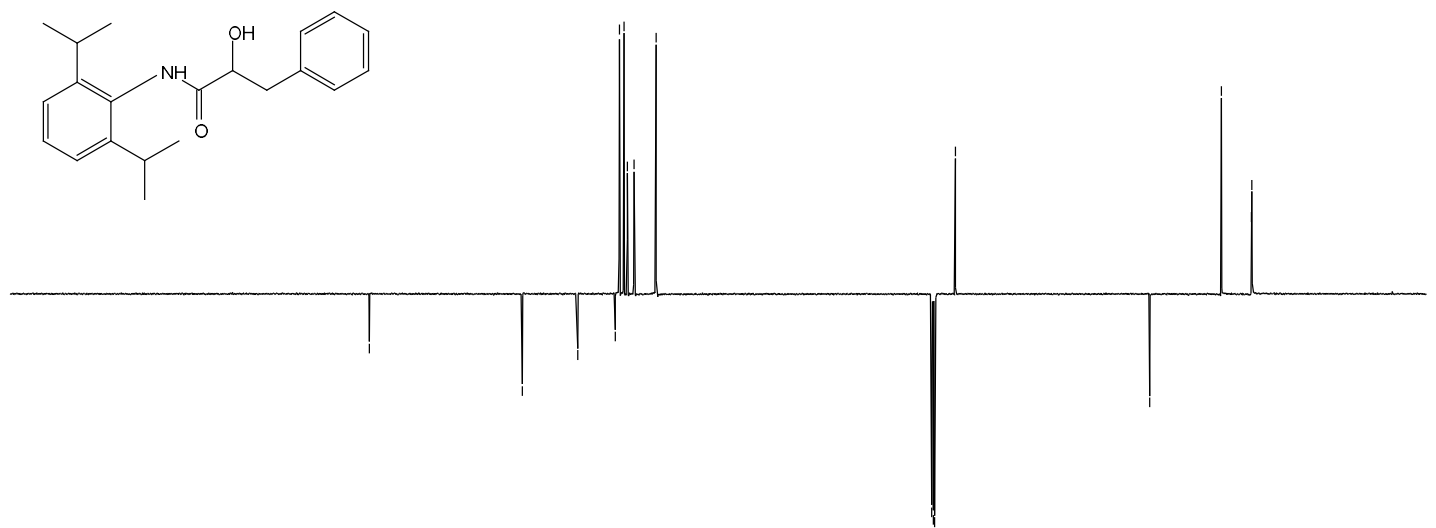

\begin{tabular}{lllllllllllllllllllllllllllllllll}
\hline 230 & 220 & 210 & 200 & 190 & 180 & 170 & 160 & 150 & 140 & 130 & 120 & 110 & 100 & 90 & 80 & 70 & 60 & 50 & 40 & 30 & 20 & 10 & 0
\end{tabular} 
COSY.

Chemist Shweta Gediya

COSY.w CDCl3 /opt/topspin3.2 SKG 14

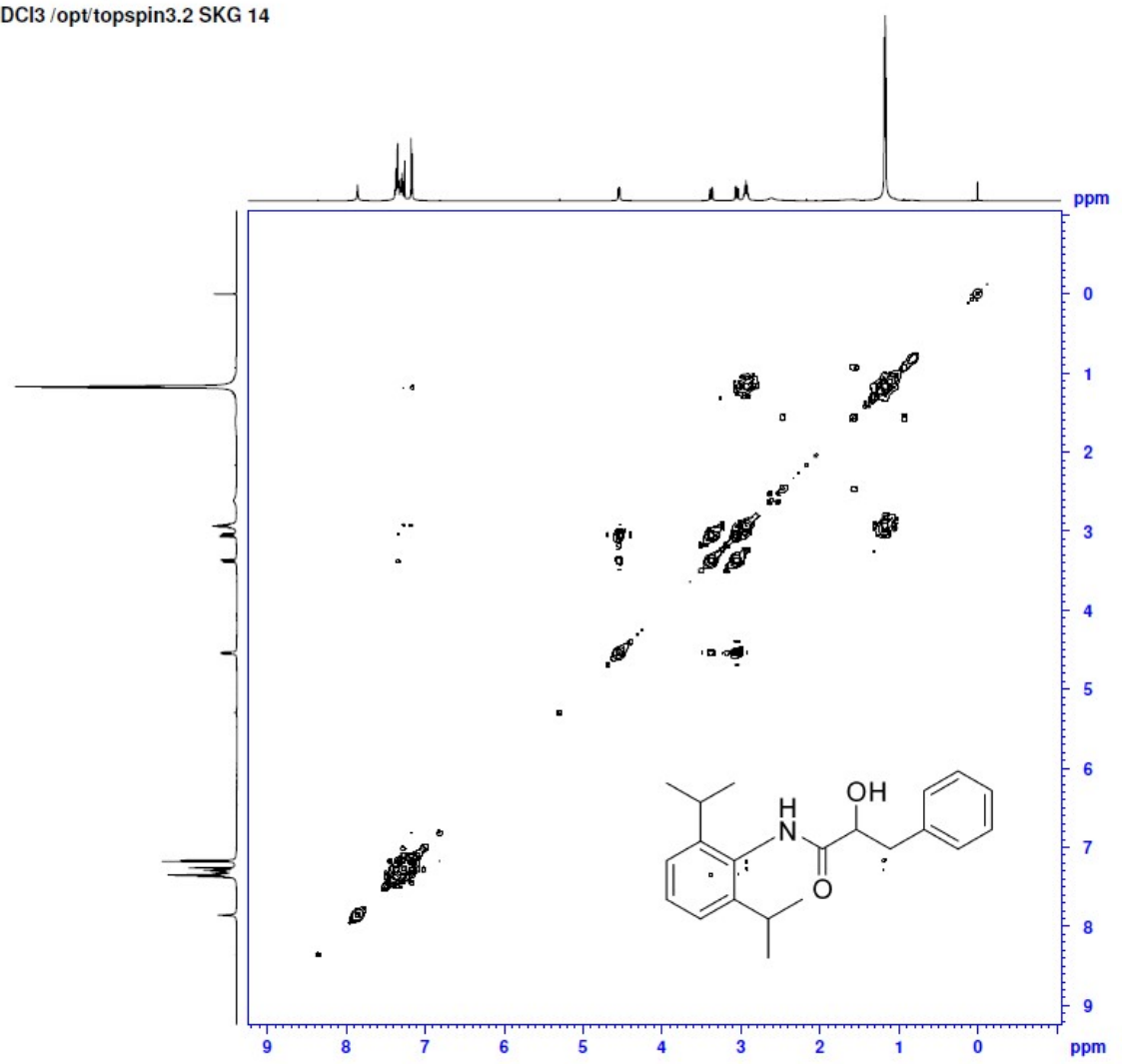

HSQC.

Chemist Shweta Gediya

HSQC.w CDCl3/opt/topspin3.2 SKG 14

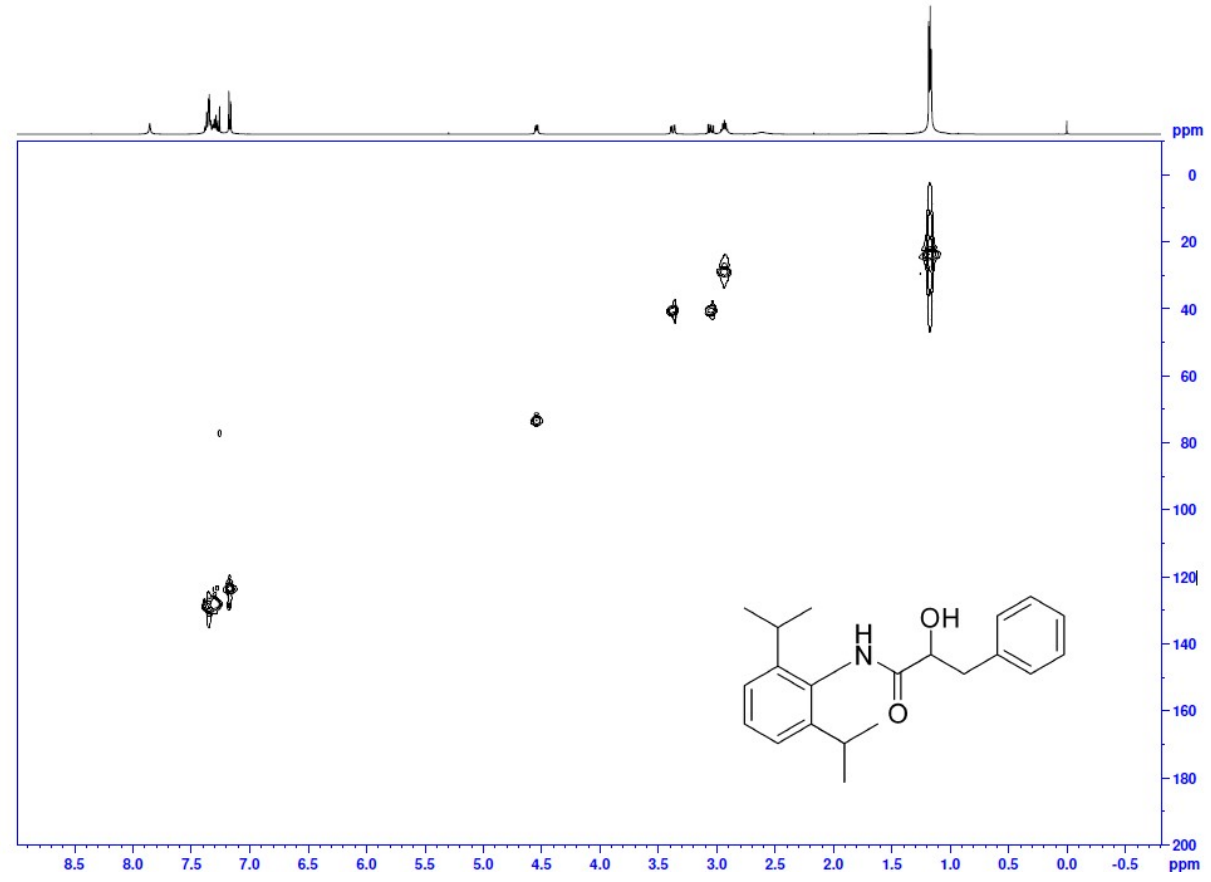




\section{Chiral HPLC of ketone precursor.}

I.⿴囗十

arity। WORK1। DATAI Shwetal after lockdownI SKG721_ketone_OD-H_10\% IPA_0.5m pm_old HPLC 08 _01_2021 11_14_05 - INT7 - 1

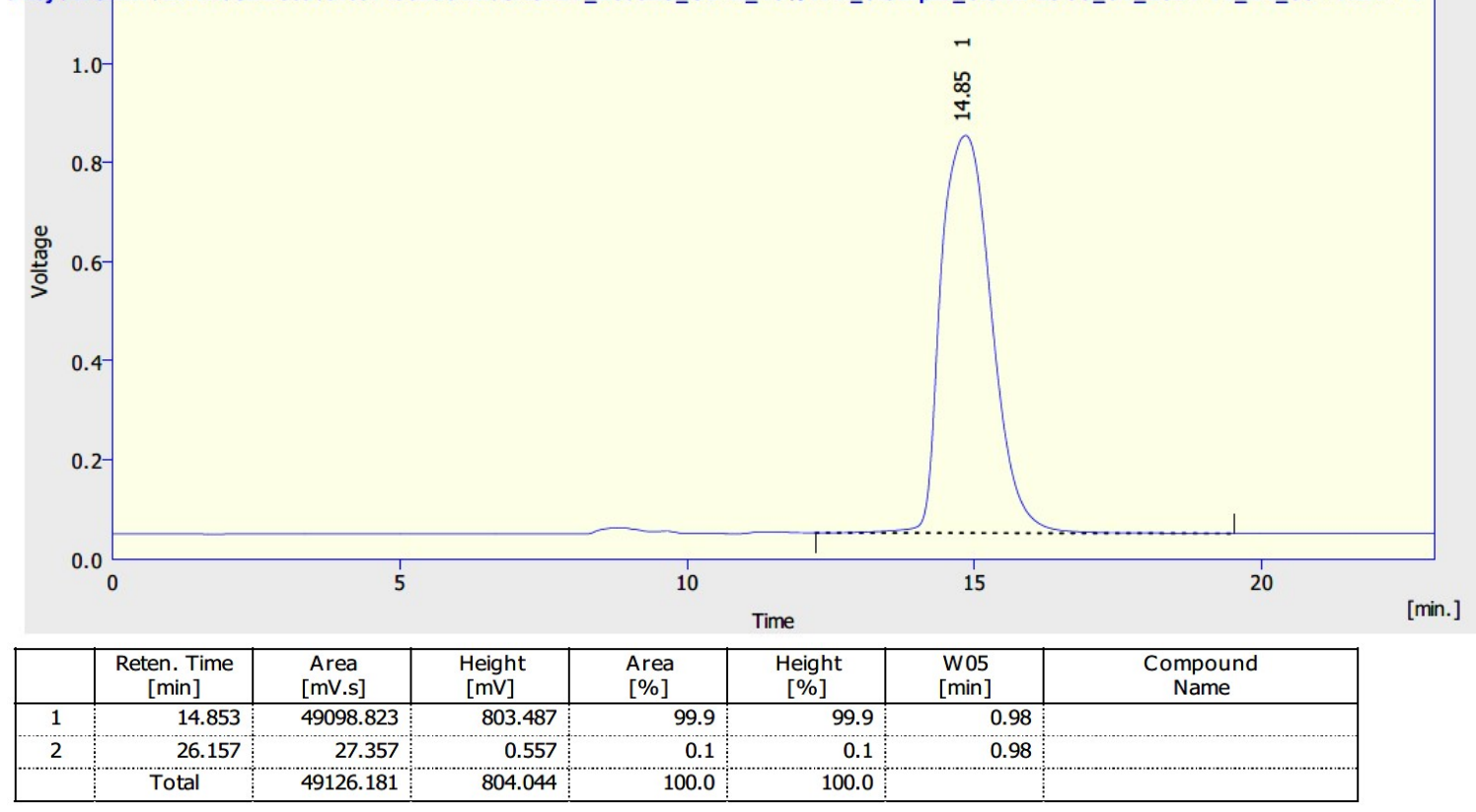

\section{Chiral HPLC of racemic alcohol.}

[980

iityl WORK1। DATAI Shwet al after lockdown! SKG724_racem ic_OD-H_10\% I PA_0.5m pm_old HPLC 08 _01_2021 11_14_05 - INT7 - 1

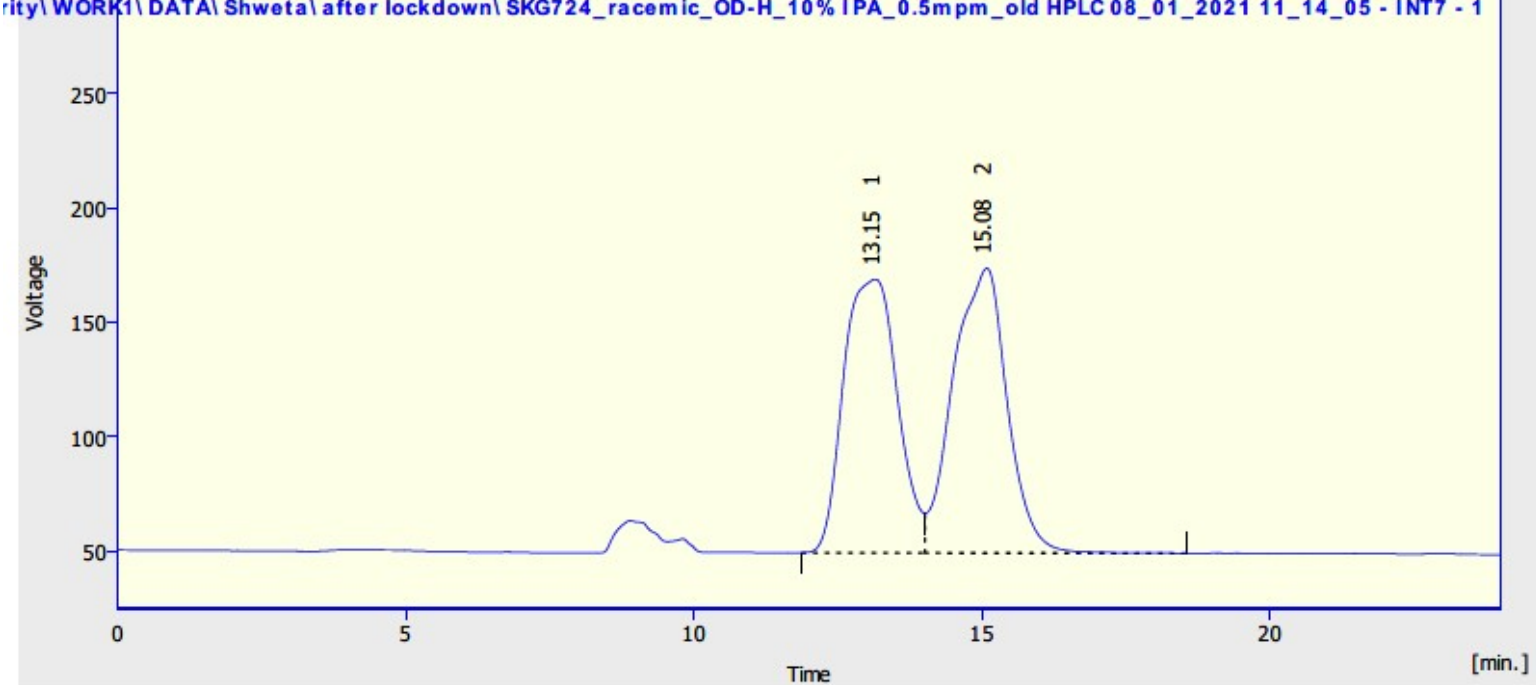

\begin{tabular}{|c|c|c|c|r|r|r|r|}
\hline & $\begin{array}{c}\text { Reten. Time } \\
{[\mathrm{min}]}\end{array}$ & $\begin{array}{c}\text { Area } \\
{[\mathrm{mV} . \mathrm{s}]}\end{array}$ & $\begin{array}{c}\text { Height } \\
{[\mathrm{mV}]}\end{array}$ & $\begin{array}{c}\text { Area } \\
{[\%]}\end{array}$ & $\begin{array}{c}\text { Height } \\
{[\%]}\end{array}$ & $\begin{array}{c}\text { W05 } \\
{[\mathrm{min}]}\end{array}$ & $\begin{array}{c}\text { Compound } \\
\text { Name }\end{array}$ \\
\hline 1 & 13.153 & 7764.125 & 119.469 & 48.8 & 49.0 & 1.05 & \\
\hline 2 & 15.080 & 8141.252 & 124.474 & 51.2 & 51.0 & 1.04 & \\
& Total & 15905.377 & 243.943 & 100.0 & 100.0 & & \\
\hline
\end{tabular}


Chiral HPLC of ATH product. $98 \%$ ee.

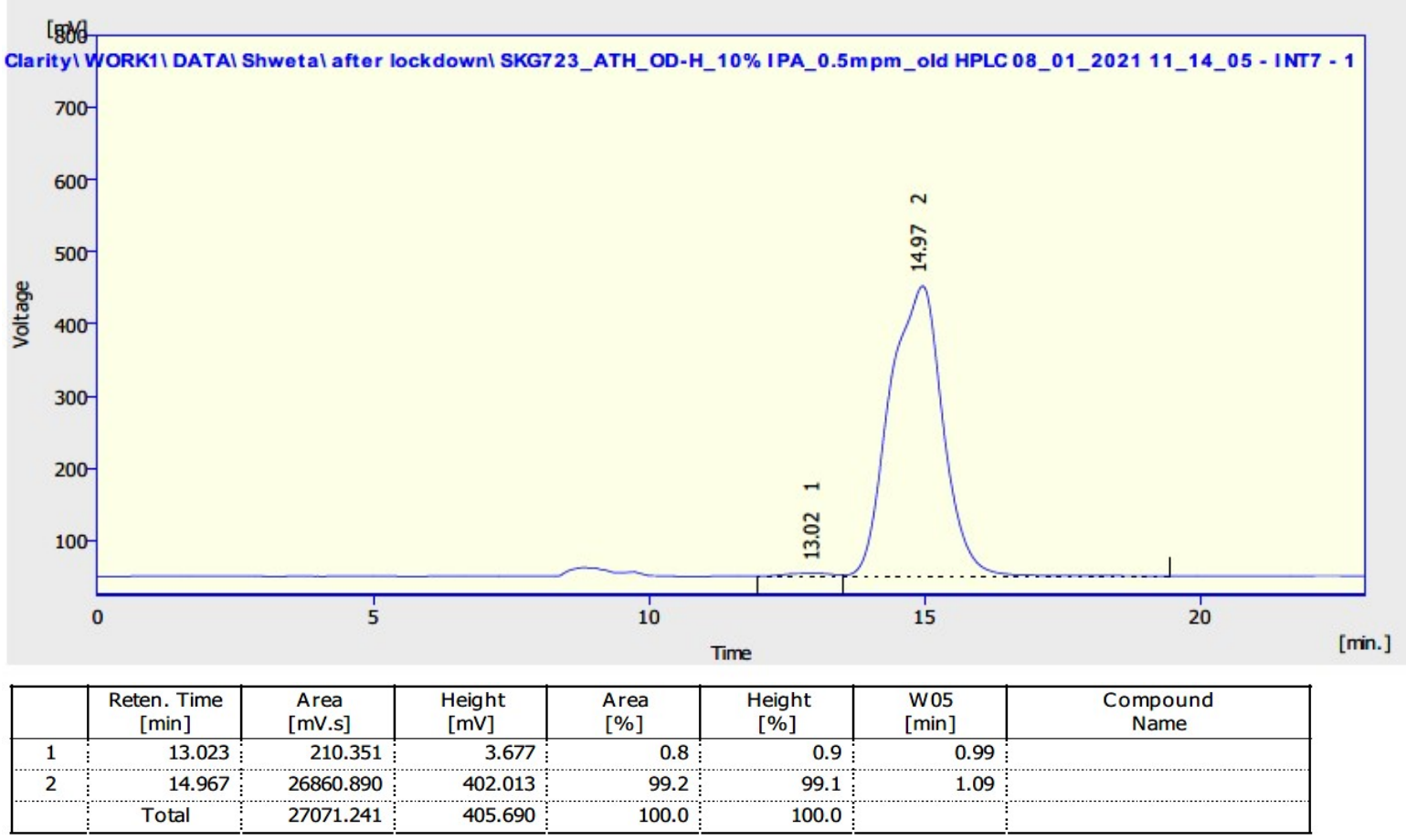




\section{$N$-(2,6-Diisopropylphenyl)-2-hydroxy-2-phenylacetamide 37.}<smiles>CC(C)c1cccc(C(C)C)c1NC(=O)C(O)c1ccccc1</smiles>

This compound is novel and was prepared following general procedure $\mathrm{C}$ using $\mathrm{N}-(2,6-$ diisopropylphenyl)-2-oxo-2-phenylacetamide (77.3 $\mathrm{mg}, 0.250 \mathrm{mmol}, 1.0 \mathrm{eq}), 3 \mathrm{C}$ tethered catalyst $(R, R)-2(2.3 \mathrm{mg}, 3.8 \mu \mathrm{mol}, 0.015 \mathrm{eq})$ and FA:TEA $(0.15 \mathrm{~mL})$ in DCM $(1.5 \mathrm{~mL})$ to give 37 as a yellow solid (64.3 $\mathrm{mg}, 0.206 \mathrm{mmol}, 83 \%$ ).

TLC: $\mathrm{R}_{\mathrm{f}}$ ca 0.3 (7:3, hexane: EtOAc), UV-active, Strong $\mathrm{KMnO}_{4}$ and PMA-reactive; HRMS (ESI) m/z: $[\mathrm{M}+\mathrm{Na}]^{+}$Calcd for $\mathrm{C}_{20} \mathrm{H}_{25} \mathrm{NO}_{2} \mathrm{Na}$ 334.1777; Found 334.1776 (error 0.5 ppm);

MP: $150-152{ }^{\circ} \mathrm{C}$;

$U_{\max } 3327,2960,1656,1502,1470,1199,1172,1063,802,691,512 \mathrm{~cm}^{-1}$;

Enantiomeric excess determined by HPLC analysis (Chiralpak AD-H, 250mm $\times 4.6 \mathrm{~mm}$ column, hexane: $\mathrm{iPrOH} 90: 10,0.6 \mathrm{~mL} / \mathrm{min}, \lambda=210 \mathrm{~nm}, \mathrm{~T}=25^{\circ} \mathrm{C}$ ), $S$-enantiomer $9.7 \mathrm{~min}, R$ enantiomer $13.5 \mathrm{~min}, 23 \%$ ee $(R),[\alpha]_{\mathrm{D}}^{22}=+75\left(\mathrm{c}=0.02, \mathrm{CHCl}_{3}\right)$; ${ }^{1} \mathrm{H}$ NMR (400 MHz, $\left.\mathrm{CDCl}_{3}\right) \delta 8.42-8.35(1 \mathrm{H}, \mathrm{m}, \mathrm{CONH}), 7.53(2 \mathrm{H}, \mathrm{d}, J=7.5 \mathrm{~Hz}, \mathrm{ArH})$, 7.43-7.48 (3H, m, ArH), 7.28-7.22 (1H, m, ArH), $7.12(2 \mathrm{H}, \mathrm{d}, J=7.6 \mathrm{~Hz}, \operatorname{ArH}), 5.24(1 \mathrm{H}, \mathrm{s}$, $\mathrm{C} \underline{\mathrm{HOH}}), 3.94(1 \mathrm{H}, \mathrm{s}, \mathrm{CHO} \underline{\mathrm{H}}), 3.12-3.05(0.33 \mathrm{H}, \mathrm{m}, \mathrm{CH}$ of isopropyl $), 2.77-2.74(1.67 \mathrm{H}, \mathrm{m}$, $\mathrm{CH}$ of isopropyl), $1.23\left(2 \mathrm{H}, \mathrm{d}, J=6.9 \mathrm{~Hz}, \mathrm{CH}_{3}\right.$ of isopropyl), $1.11\left(5 \mathrm{H}, \mathrm{d}, J=6.8 \mathrm{~Hz}, \mathrm{CH}_{3}\right.$ of isopropyl), $1.02\left(5 \mathrm{H}, \mathrm{d}, J=6.8 \mathrm{~Hz}, \mathrm{CH}_{3}\right.$ of isopropyl). ${ }^{13} \mathrm{C} \mathrm{NMR}\left(126 \mathrm{MHz}, \mathrm{CDCl}_{3}\right) \delta 171.7$, 146.2 , 139.7, 134.9, 131.6, 130.2, 129.1, 129.0, 128.8, 128.7, 126.8, 123.8, 123.6, 74.4, 29.1, 28.7, 23.8, 23.7, 23.5; $m / z(\mathrm{ESI}) 334.2\left[(\mathrm{M}+\mathrm{Na})^{+}, 100 \%\right]$. 
N-(2,6-Diisopropylphenyl)-2-hydroxy-2-phenylacetamide 37.

${ }^{1} \mathrm{H}$ NMR (400 MHz, $\left.\mathrm{CDCl}_{3}\right)$.

Mar12-2021

PROTON.w CDCl3 /opt/topspin3.5pl2 SKG 33<smiles>C#CC#CC</smiles>

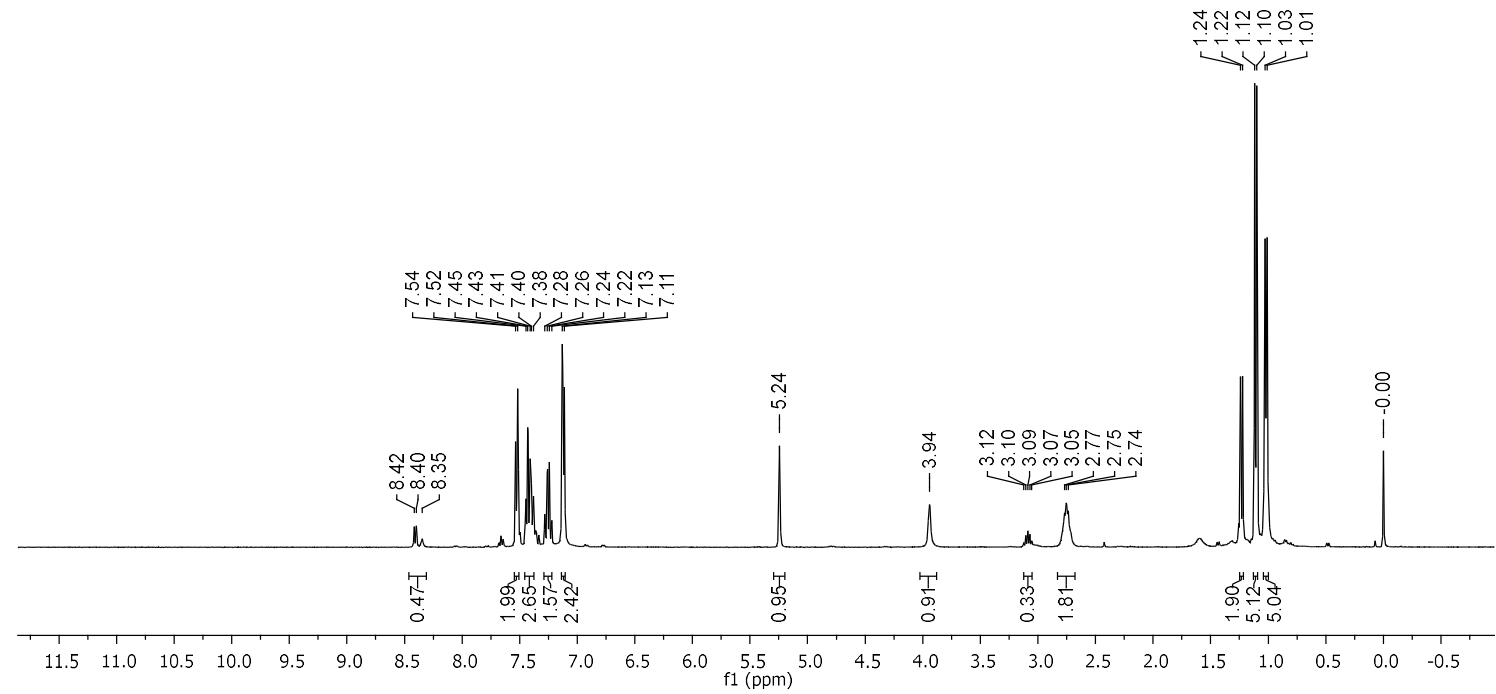

${ }^{13} \mathrm{C}$ NMR (126 MHz, $\left.\mathrm{CDCl}_{3}\right)$.

May17-2021

Shweta Gediya

C13APTlong.w CDCl3

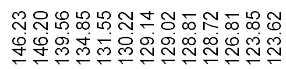

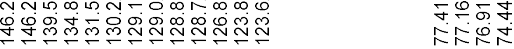

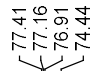

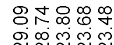

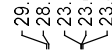
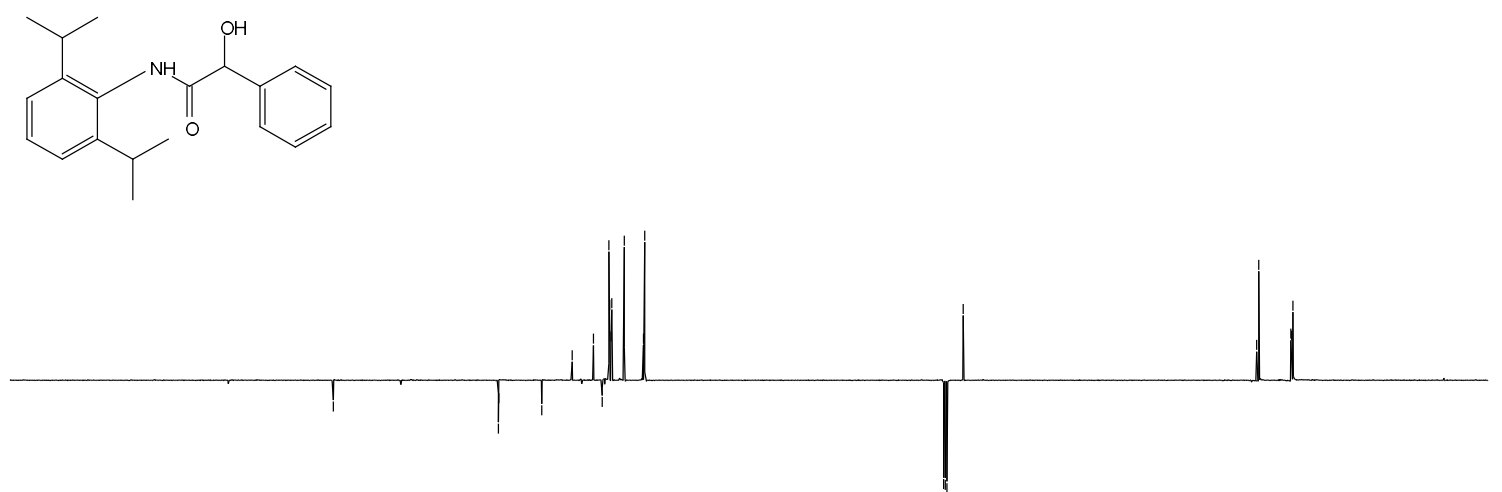

$\begin{array}{lllllllllllllllllllllllllllllll}220 & 210 & 200 & 190 & 180 & 170 & 160 & 150 & 140 & 130 & 120 & \underset{f 1}{110}(\mathrm{ppm}) & 100 & 90 & 80 & 70 & 60 & 50 & 40 & 30 & 20 & 10 & 0\end{array}$ 
COSY.

SKG692

COSY.w CDCl3/opt/topspin3.5pl2 SKG 33

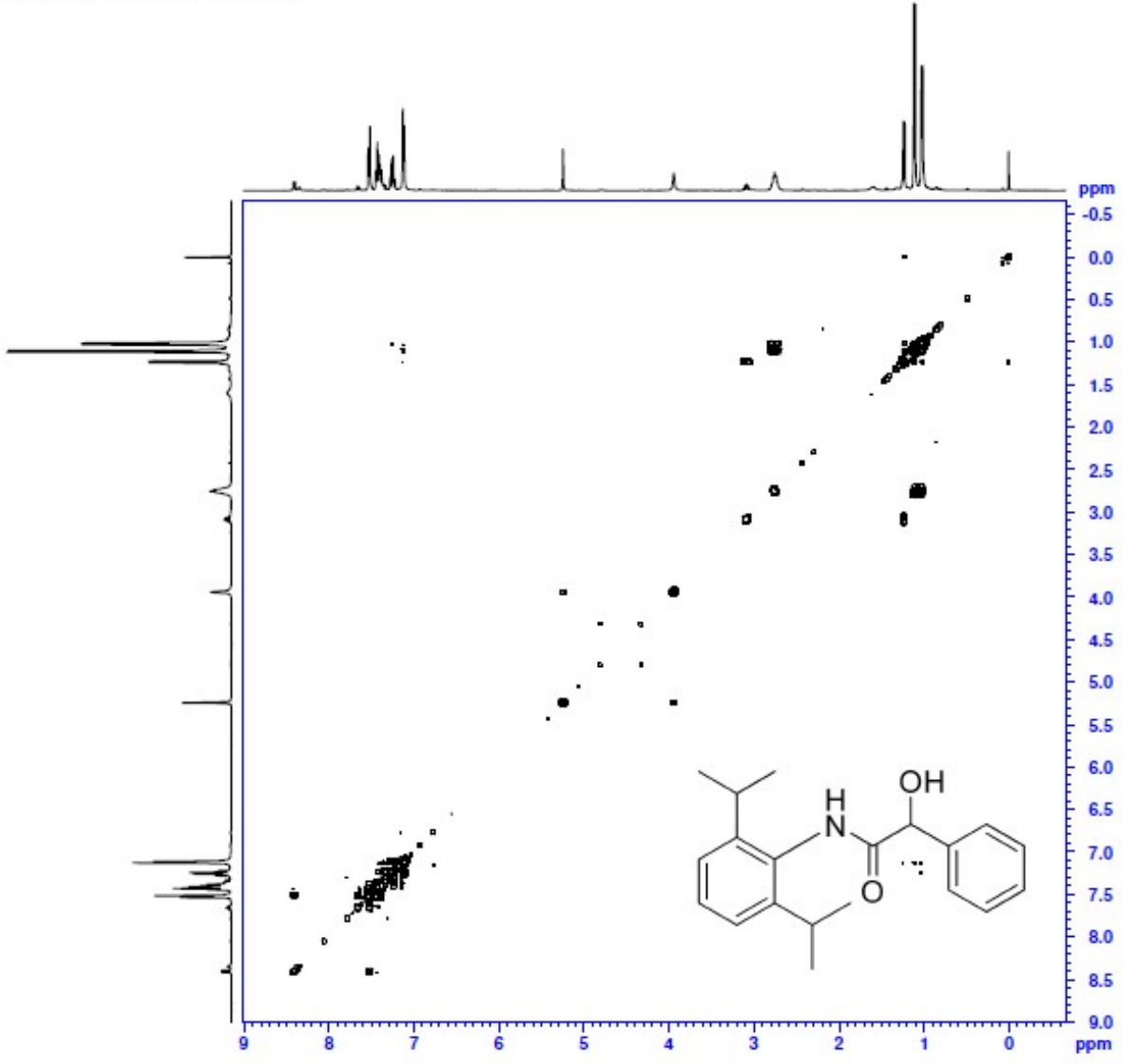

HSQC.

Chemist Shweta Gediya

HSQC.W CDCl3 /opt/topspin3.2 SKG 57

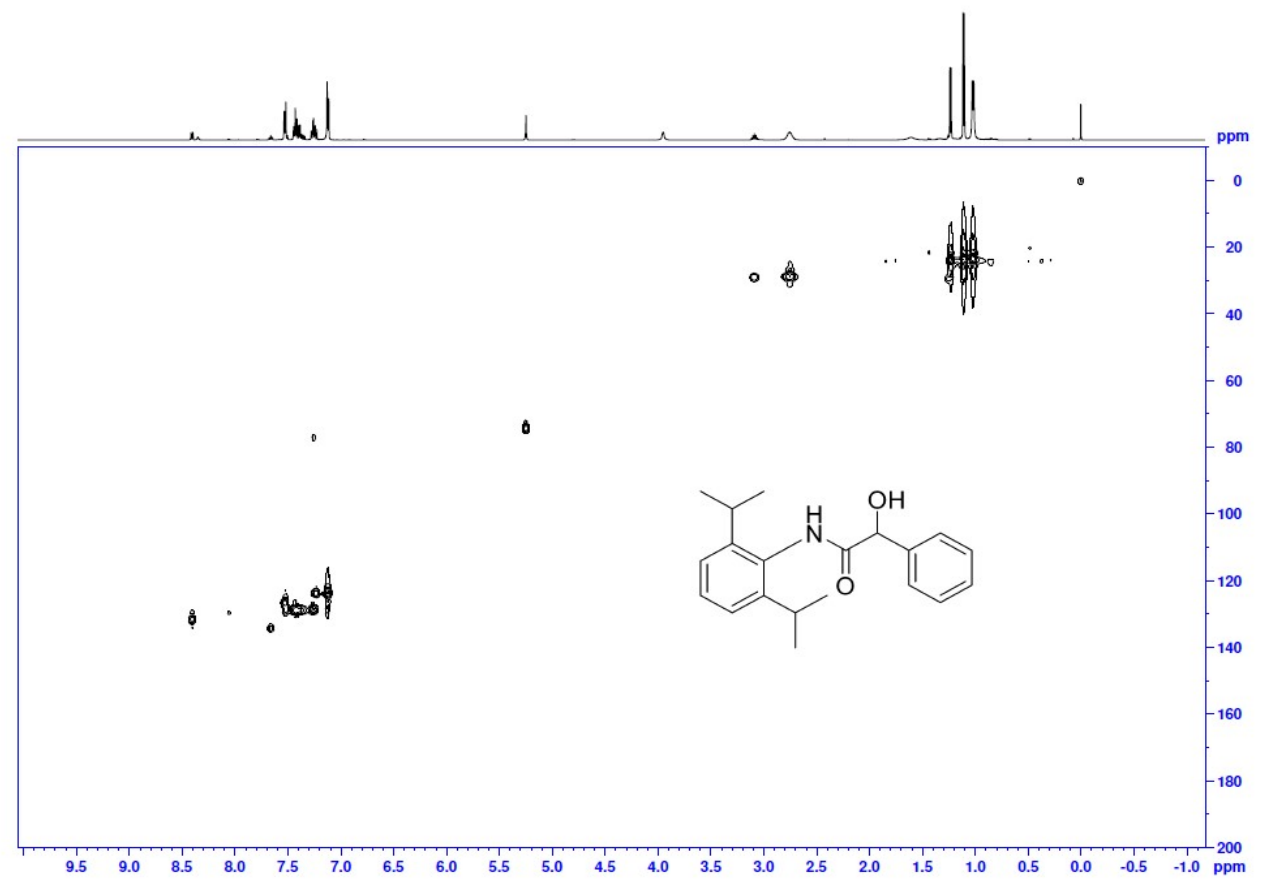




\section{Chiral HPLC of ketone precursor.}

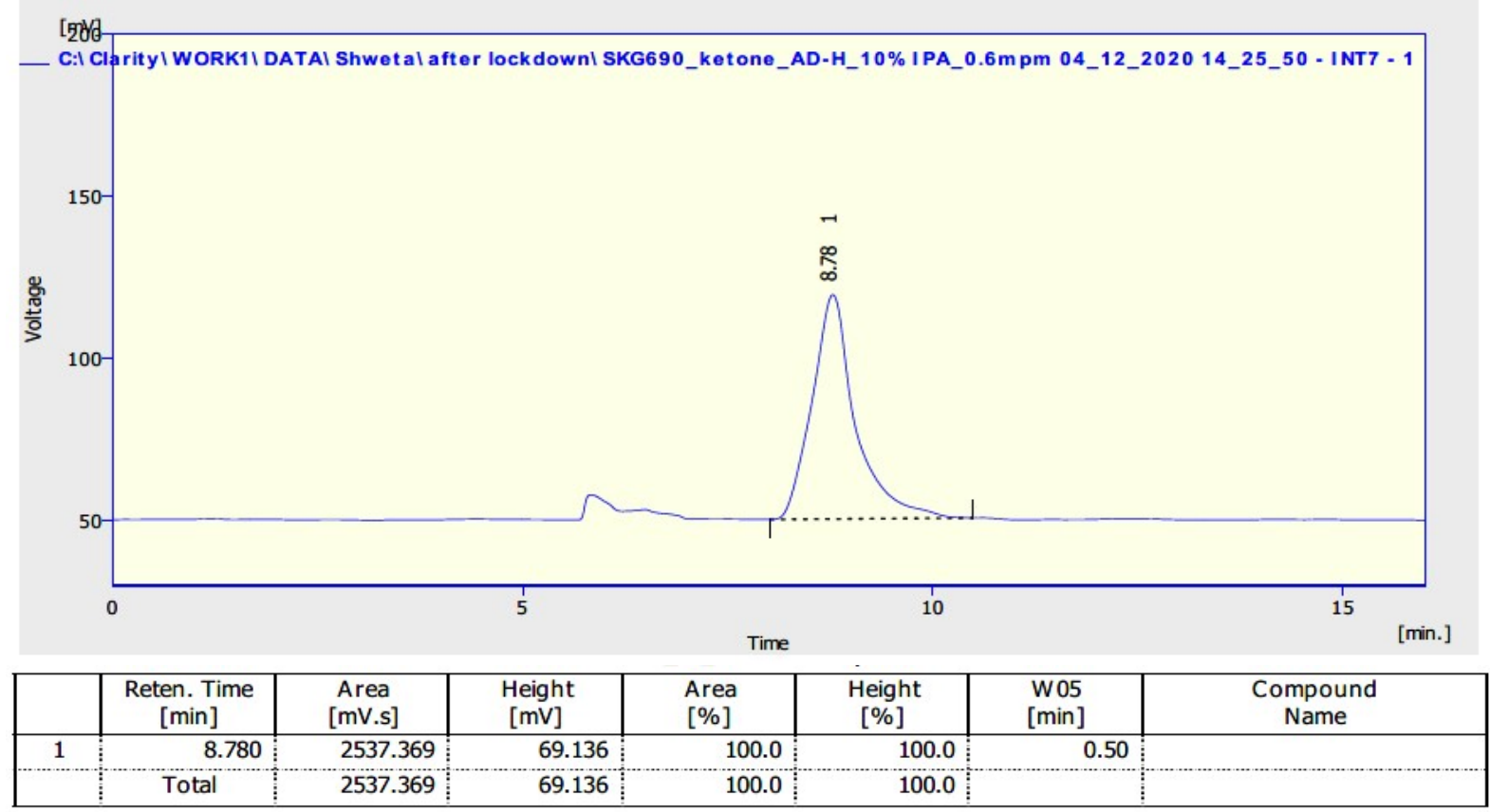




\section{Chiral HPLC of racemic alcohol.}

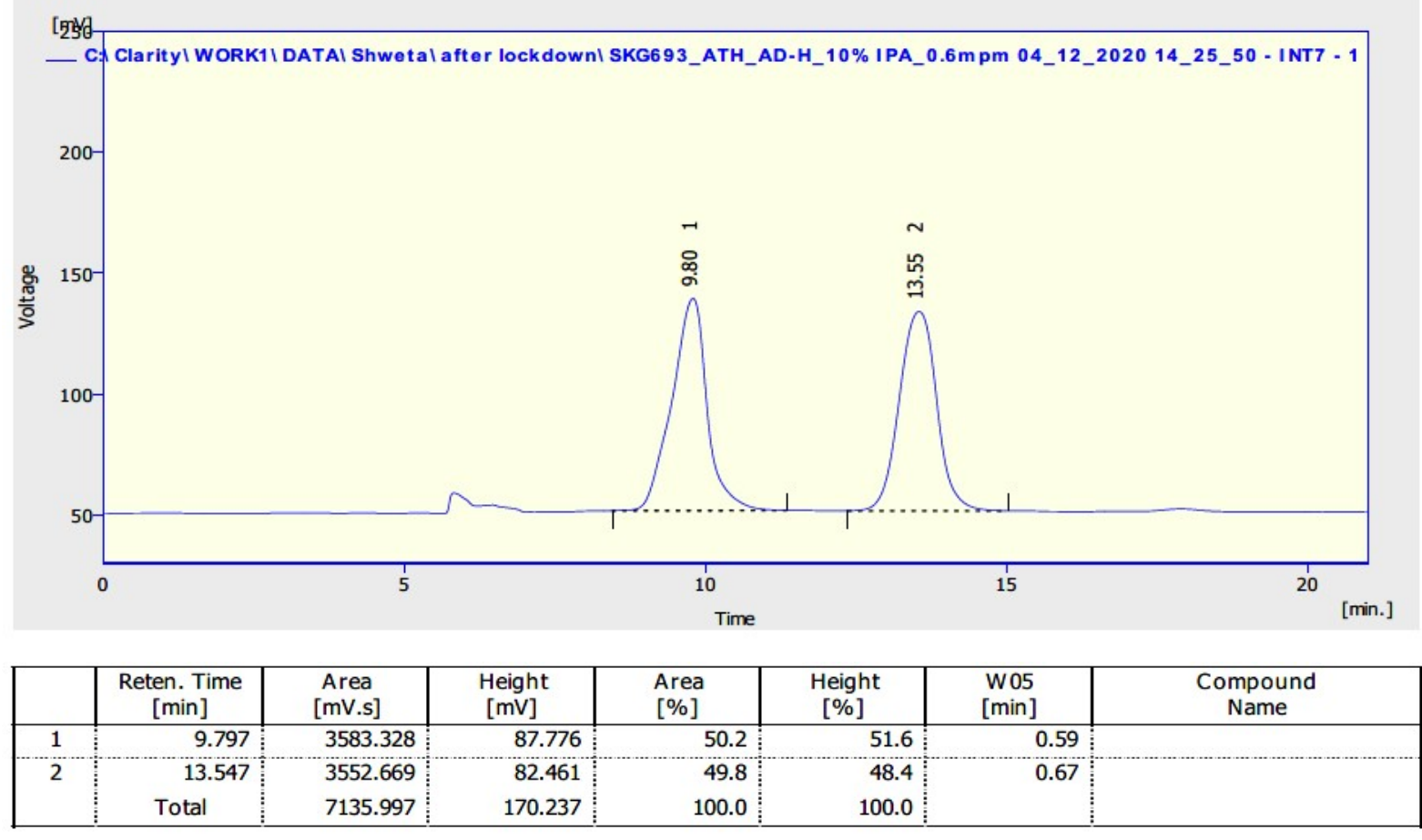

Chiral HPLC of ATH product. 23\% ee.

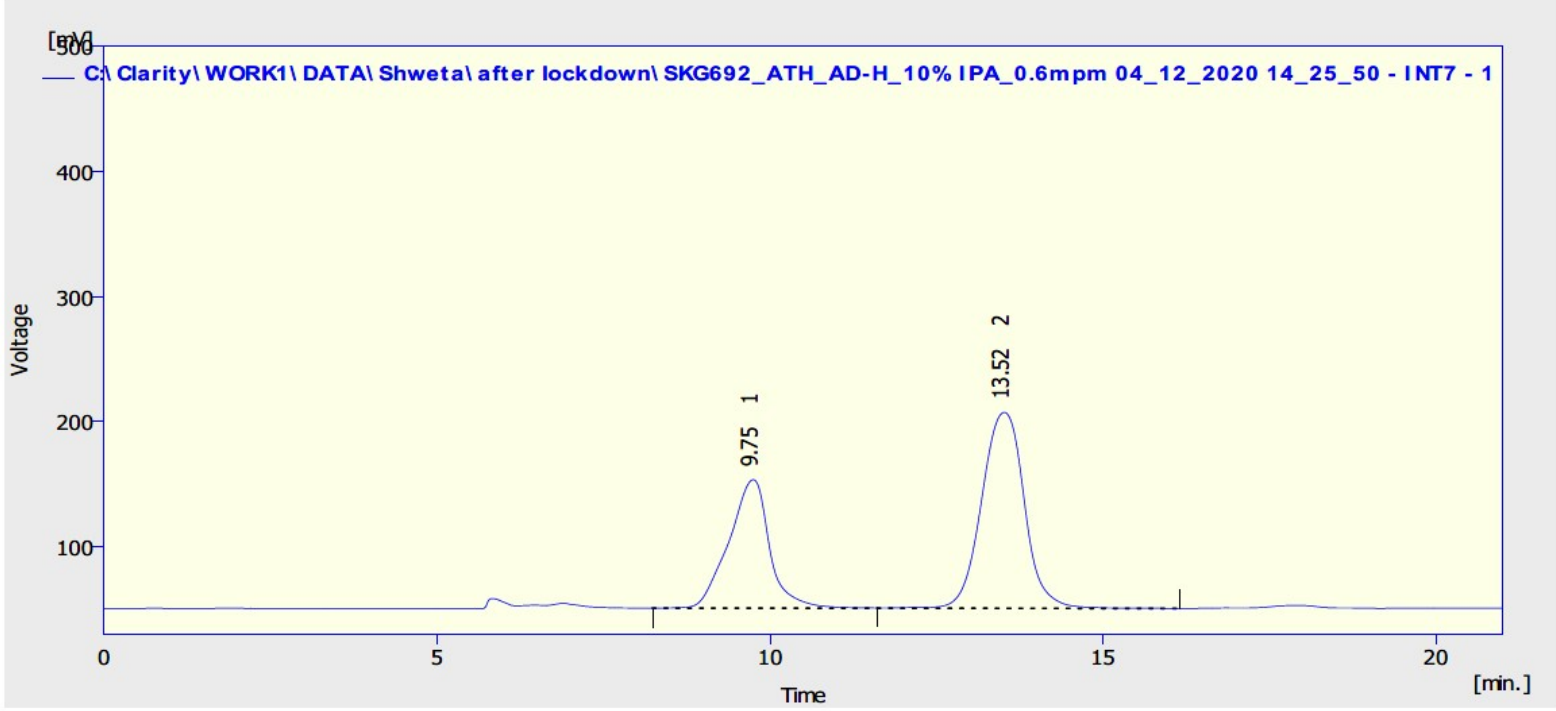

\begin{tabular}{|c|c|c|c|c|c|c|c|}
\hline & $\begin{array}{c}\text { Reten. Time } \\
{[\mathrm{min}]}\end{array}$ & $\begin{array}{c}\text { Area } \\
{[\mathrm{mV} . \mathrm{s}]}\end{array}$ & $\begin{array}{c}\text { Height } \\
{[\mathrm{mV}]}\end{array}$ & $\begin{array}{c}\text { Area } \\
{[\%]}\end{array}$ & $\begin{array}{c}\text { Height } \\
{[\%]}\end{array}$ & $\begin{array}{c}\text { W05 } \\
{[\mathrm{min}]}\end{array}$ & $\begin{array}{c}\text { Compound } \\
\text { Name }\end{array}$ \\
\hline 1 & 9.750 & 4375.996 & 102.912 & 38.4 & 39.6 & 0.62 & \\
\hline 2 & 13.520 & 7012.536 & 156.858 & 61.6 & 60.4 & 0.69 & \\
& Total & 11388.532 & 259.771 & 100.0 & 100.0 & & \\
\hline
\end{tabular}


Ethyl (R)-3-hydroxy-4-(methyl(phenyl)amino)-4-oxobutanoate 38.<smiles>CCOC(=O)CC(O)C(=O)N(C)c1ccccc1</smiles>

This compound is novel and was prepared following general procedure $\mathrm{C}$ using ethyl 4(methyl(phenyl)amino)-3,4-dioxobutanoate $(62.6 \mathrm{mg}, 0.250 \mathrm{mmol}, 1.0 \mathrm{eq}), 3 \mathrm{C}$ tethered catalyst $(R, R)-2(2.3 \mathrm{mg}, 3.75 \mu \mathrm{mol}, 0.015 \mathrm{eq})$ and FA:TEA $(0.15 \mathrm{~mL})$ in DCM $(1.5 \mathrm{~mL})$ to give 38 as a white solid (56.2 $\mathrm{mg}, 0.223 \mathrm{mmol}, 92 \%)$.

TLC: $\mathrm{R}_{\mathrm{f}}$ ca 0.2 (8:2, hexane: EtOAc), UV-active and PMA-reactive;

HRMS (ESI) m/z: [M+Na] $]^{+}$Calcd for $\mathrm{C}_{13} \mathrm{H}_{17} \mathrm{NO}_{4} \mathrm{Na} 274.1050$; Found 274.1054 (error -1.5 ppm);

$U_{\max } 3416,2981,1729,1647,1594,1495,1370,1164,1029,699 \mathrm{~cm}^{-1}$;

Enantiomeric excess determined by HPLC analysis (Chiralpak IG, $250 \mathrm{~mm} \times 4.6 \mathrm{~mm}$ column, hexane: $\mathrm{iPrOH} 80: 20,1 \mathrm{~mL} / \mathrm{min}, \lambda=210 \mathrm{~nm}, \mathrm{~T}=25^{\circ} \mathrm{C}$ ), $R$-enantiomer $21.0 \mathrm{~min}, S$ enantiomer $24.8 \min .54 \%$ ee $(R),[\alpha]_{\mathrm{D}}^{22}=+8.8\left(\mathrm{c}=0.04, \mathrm{CHCl}_{3}\right)$;

${ }^{1} \mathrm{H}$ NMR (500 MHz, $\left.\mathrm{CDCl}_{3}\right) \delta 7.46(2 \mathrm{H}, \mathrm{t}, J=7.5 \mathrm{~Hz}, \mathrm{ArH}), 7.39(1 \mathrm{H}, \mathrm{t}, J=7.2 \mathrm{~Hz}, \mathrm{ArH})$, $7.26(2 \mathrm{H}, \mathrm{t}, J=7.0 \mathrm{~Hz}, \mathrm{ArH}), 4.56-4.52(1 \mathrm{H}, \mathrm{m}, \mathrm{C} \underline{\mathrm{HOH}}), 4.09-4.05\left(2 \mathrm{H}, \mathrm{m}, \mathrm{C}_{2} \mathrm{CH}_{3}\right), 3.71$ $(1 \mathrm{H}, \mathrm{d}, J=6.5 \mathrm{~Hz}, \mathrm{CHO} \underline{\mathrm{H}}), 3.34\left(3 \mathrm{H}, \mathrm{s},-\mathrm{NCH}_{3}\right), 2.44\left(1 \mathrm{H}, \mathrm{dd}, J=15.4,3.6 \mathrm{~Hz}, \mathrm{C}_{\underline{\mathrm{a}}} \mathrm{H}_{\mathrm{b}}\right)$, $2.31\left(1 \mathrm{H}, \mathrm{dd}, J=15.4,7.8 \mathrm{~Hz}, \mathrm{CH}_{2} \underline{\mathrm{H}_{\mathrm{b}}}\right), 1.19\left(3 \mathrm{H}, \mathrm{t}, J=7.1 \mathrm{~Hz}, \mathrm{CH}_{2} \underline{\mathrm{C}}_{3}\right)$;

${ }^{13} \mathrm{C}$ NMR $\left(126 \mathrm{MHz}, \mathrm{CDCl}_{3}\right) \delta 172.7,170.4,142.1,130.2,128.7,127.4,65.7,60.8,39.6$, $38.3,14.2$;

$m / z(\mathrm{ESI}) 274.1\left[(\mathrm{M}+\mathrm{Na})^{+}, 100 \%\right]$ 
Ethyl 3-hydroxy-4-(methyl(phenyl)amino)-4-oxobutanoate 38.

${ }^{1} \mathrm{H}$ NMR $\left(500 \mathrm{MHz}, \mathrm{CDCl}_{3}\right)$.
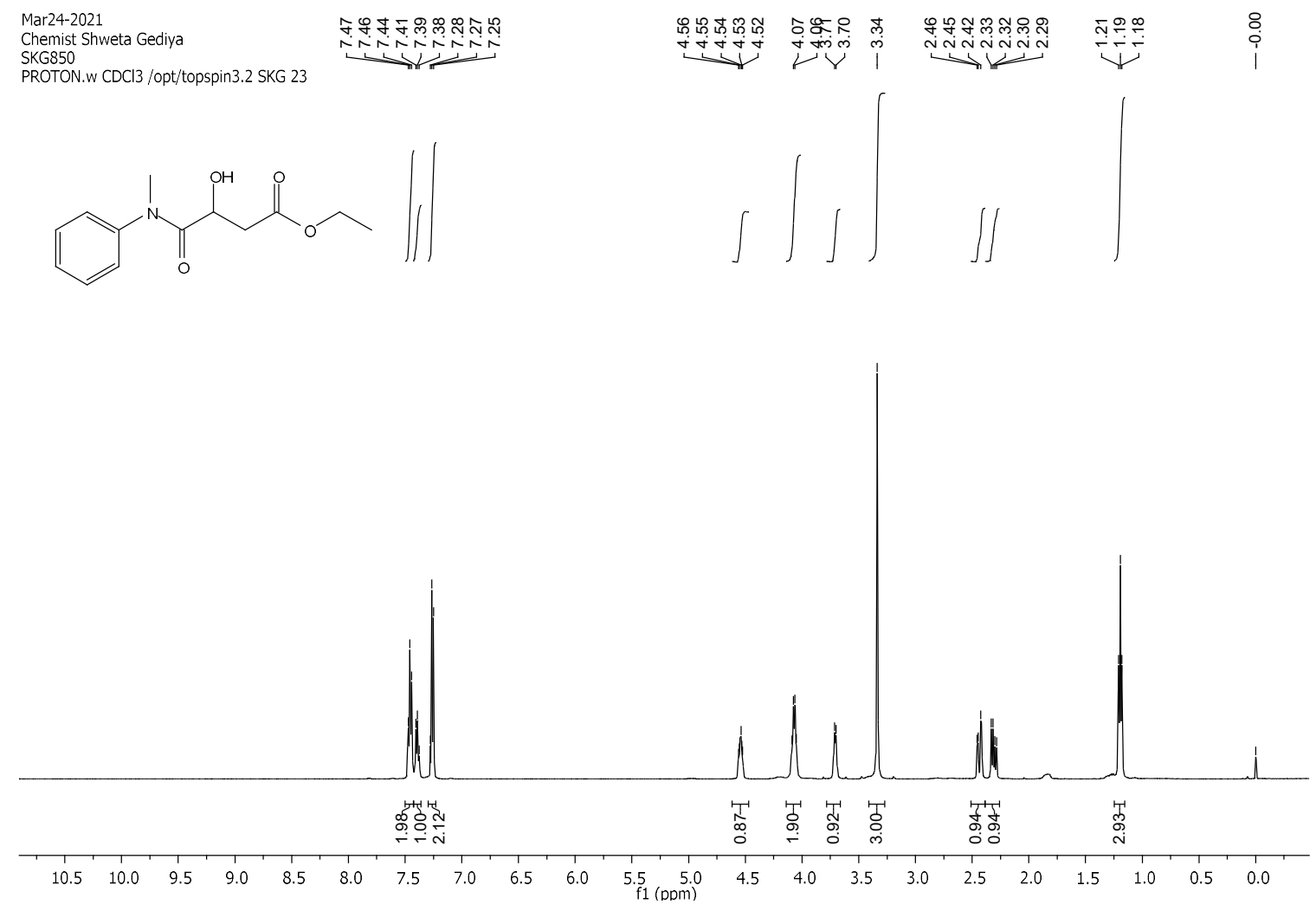

${ }^{13} \mathrm{C} \mathrm{NMR}\left(126 \mathrm{MHz}, \mathrm{CDCl}_{3}\right)$.

$\begin{array}{llllll}\text { Mar24-2021 } & \text { Chemist Shweta Gediya }\end{array} \begin{array}{llll}\text { SKG850 } \\ \text { C13APT.w CDC13 /opt/topspin3.2 SKG } 23\end{array}$

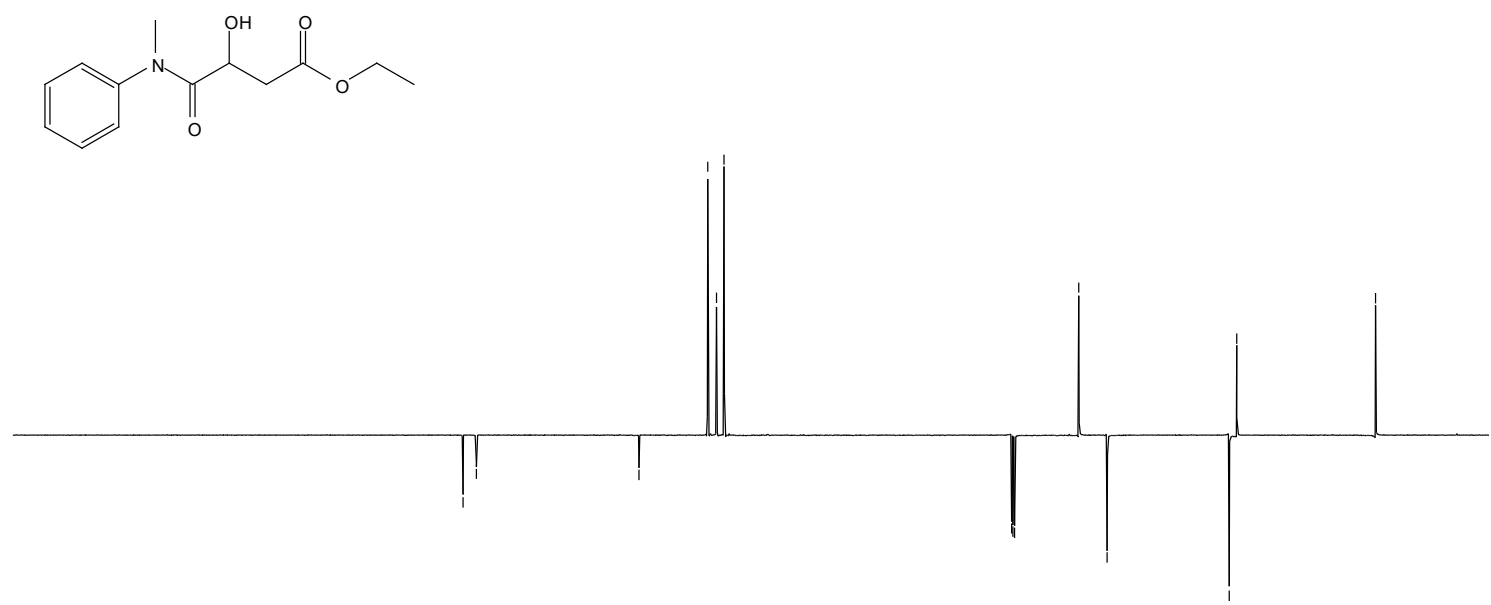

$\begin{array}{rlllllllllllllllllllllllllllllllllll}250 & 240 & 230 & 220 & 210 & 200 & 190 & 180 & 170 & 160 & 150 & 140 & 130 & 120 & 110 & 100 & 90 & 80 & 70 & 60 & 50 & 40 & 30 & 20 & 10 & 0\end{array}$ 
COSY.

SKG850

COSY.w CDCl3 /opt/topspin3.5pl2 SKG 24

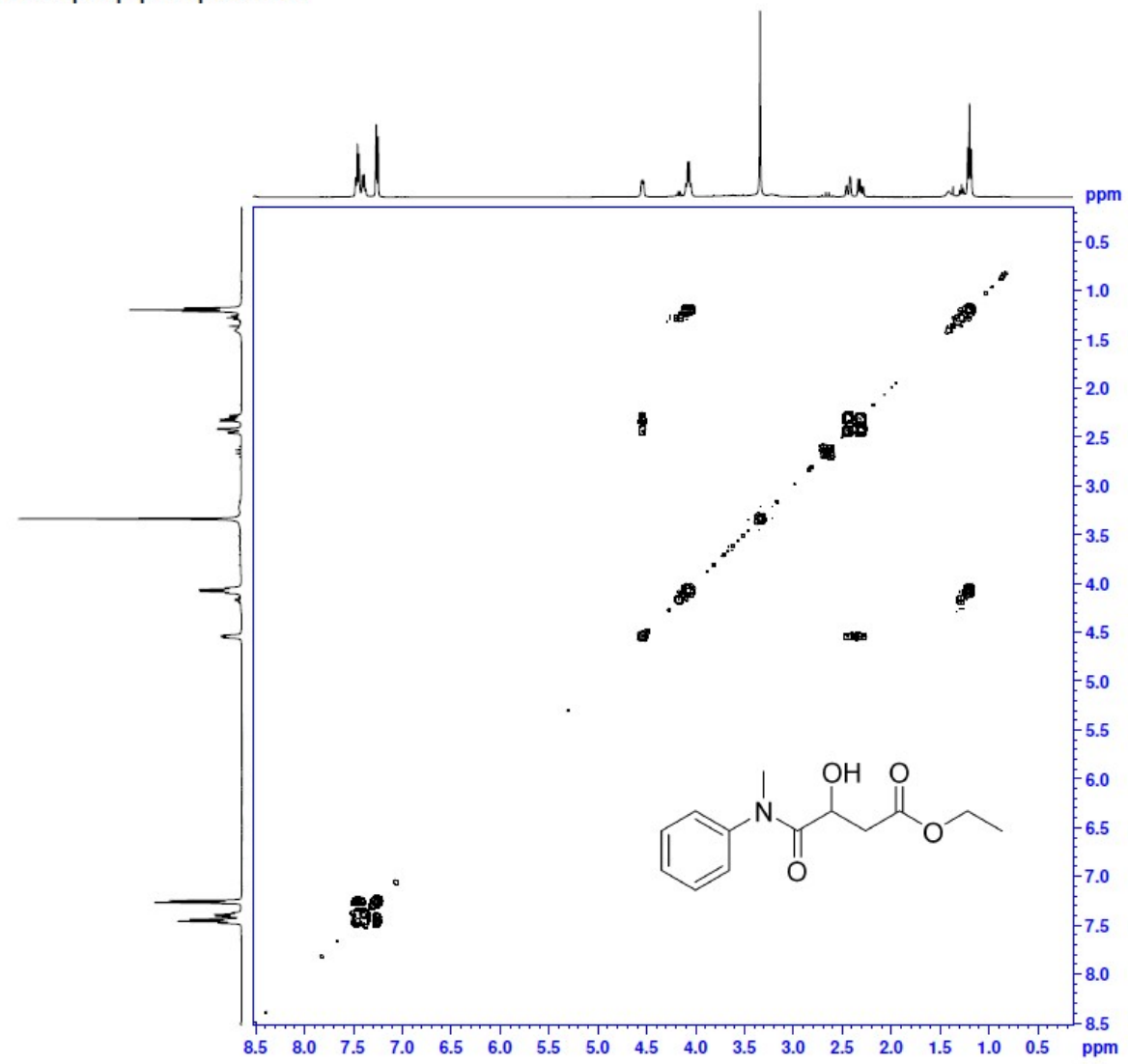

HSQC.

Chemist Shweta Gediya

HSQC.w CDCl3/opttopspin3.2 SKG 23

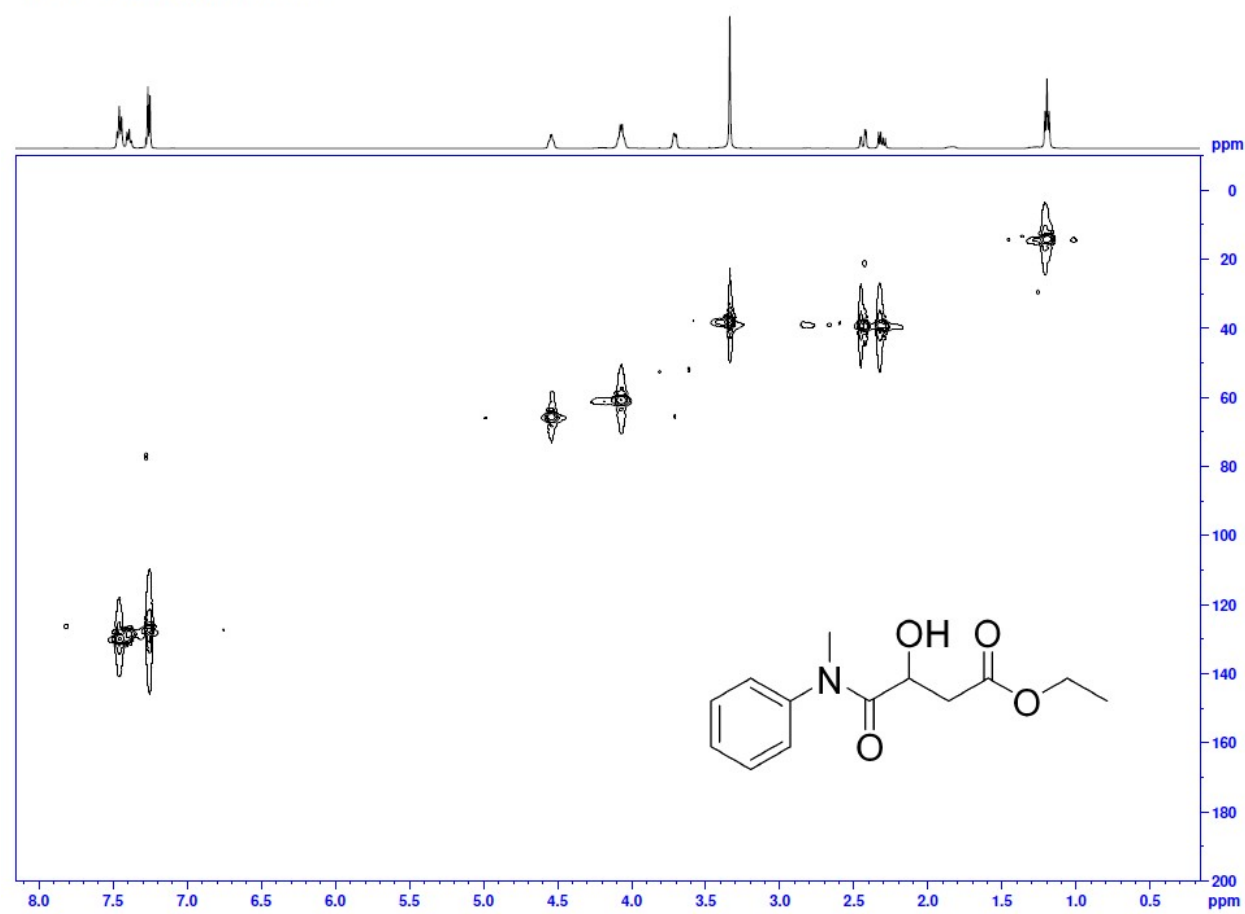




\section{Chiral HPLC of ketone.}

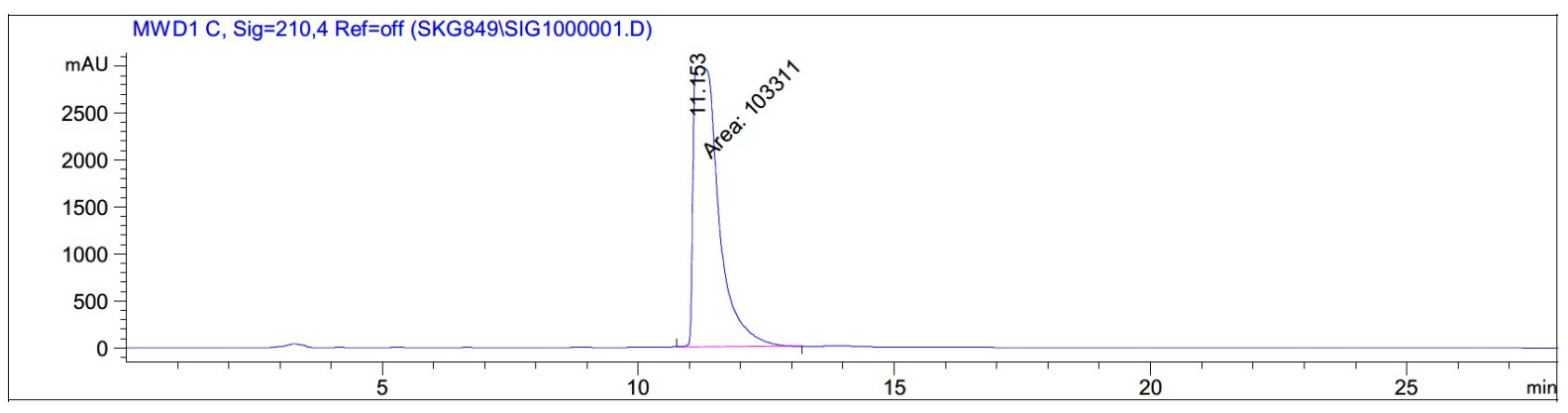

Signal 2: MWD1 C, Sig=210,4 Ref=off

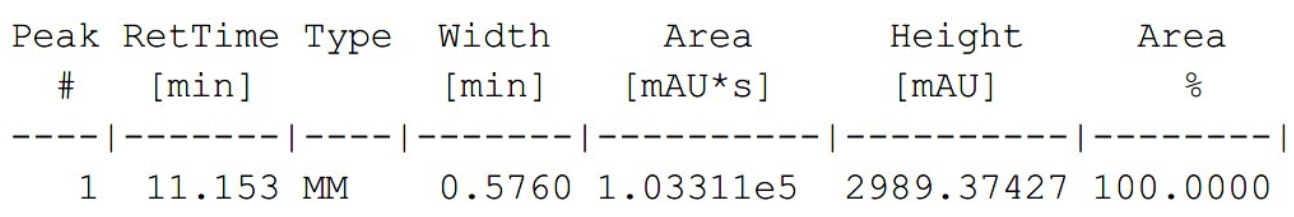

\section{Chiral HPLC of racemic standard.}

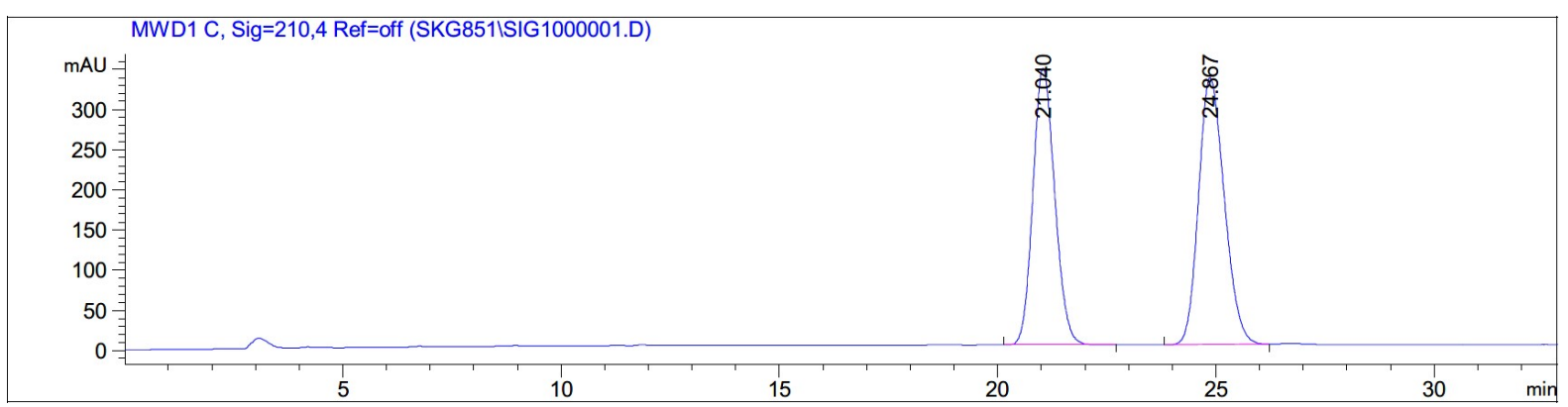

Signal 2: MWD1 C, Sig=210,4 Ref=off

\begin{tabular}{|c|c|c|c|c|c|c|}
\hline $\begin{array}{c}\text { Peak } \\
\#\end{array}$ & $\begin{array}{l}\text { RetTime } \\
\text { [min] }\end{array}$ & Type & $\begin{array}{l}\text { Width } \\
\text { [min] }\end{array}$ & $\begin{array}{c}\text { Area } \\
{\left[\mathrm{mAU}^{\star} \mathrm{S}\right]}\end{array}$ & $\begin{array}{l}\text { Height } \\
{[\mathrm{mAU}]}\end{array}$ & $\begin{array}{c}\text { Area } \\
\frac{\circ}{0}\end{array}$ \\
\hline & & & & & - & -1 \\
\hline 1 & 21.04 & BB & 0.5396 & $1.19937 \mathrm{e} 4$ & 344.99115 & 46.7501 \\
\hline 2 & 24.867 & $\mathrm{BB}$ & 0.6421 & $1.36612 \mathrm{e} 4$ & 331.95737 & 53.2499 \\
\hline
\end{tabular}


Chiral HPLC of ATH product. 54\% ee.

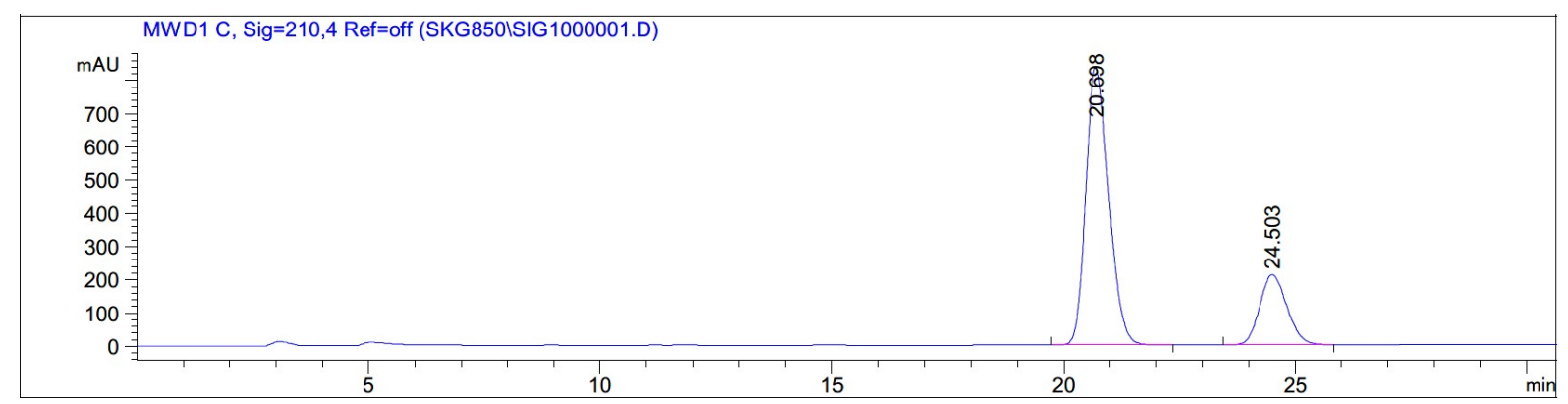

Signal 2: MWD1 C, Sig=210,4 Ref=off

\begin{tabular}{|c|c|c|c|c|c|c|}
\hline $\begin{array}{c}\text { eak } \\
\text { \# }\end{array}$ & $\begin{array}{c}\text { RetTime } \\
\text { [min] }\end{array}$ & Type & $\begin{array}{l}\text { Width } \\
\text { [min] }\end{array}$ & $\begin{array}{c}\text { Area } \\
{\left[\mathrm{mAU}{ }^{*} \mathrm{~S}\right]}\end{array}$ & $\begin{array}{l}\text { Height } \\
\text { [mAU] }\end{array}$ & $\begin{array}{c}\text { Area } \\
\quad \%\end{array}$ \\
\hline \multicolumn{7}{|c|}{$----|-------|----|-------|----------|----------|--------\mid$} \\
\hline 1 & 20.698 & $\mathrm{BB}$ & 0.5378 & $2.88302 e 4$ & 837.08197 & 77.1616 \\
\hline 2 & 24.503 & $\mathrm{BB}$ & 0.6316 & 8533.23145 & 211.07811 & 22.8384 \\
\hline
\end{tabular}


Ethyl (R)-4-(benzyl(methyl)amino)-3-hydroxy-4-oxobutanoate 39 (2 mmol scale).<smiles>CCOC(=O)CC(O)C(=O)N(C)Cc1ccccc1</smiles>

This compound is novel and was prepared following general procedure $\mathrm{C}$ using ethyl 4(benzyl(methyl)amino)-3,4-dioxobutanoate $(0.530 \mathrm{~g}, 2.00 \mathrm{mmol}, 1.0 \mathrm{eq}), 3 \mathrm{C}$ tethered catalyst $(R, R)-2(21.6 \mathrm{mg}, 0.030 \mathrm{mmol}, 0.015 \mathrm{eq})$ and FA:TEA $(1.2 \mathrm{~mL})$ in DCM $(12 \mathrm{~mL})$ to give 39 as clear oil (0.495 $\mathrm{mg}, 1.87 \mathrm{mmol}, 93 \%)$.

TLC: $\mathrm{R}_{\mathrm{f}}$ ca 0.4 (3:7, hexane: EtOAc), UV active and PMA-reactive;

HRMS (ESI) m/z: $[\mathrm{M}+\mathrm{Na}]^{+}$Calcd for $\mathrm{C}_{14} \mathrm{H}_{19} \mathrm{NO}_{4} \mathrm{Na} 288.1206$; Found 288.1204 (error 0.7 ppm);

$U_{\max } 3410,3399,2928,1728,1610,1452,1375,1256,1166,1079,698 \mathrm{~cm}^{-1}$;

Enantiomeric excess determined by HPLC analysis (Chiralpak IG, $250 \mathrm{~mm} \times 4.6 \mathrm{~mm}$ column, hexane: iPrOH 85:15, $1 \mathrm{~mL} / \mathrm{min}, \lambda=210 \mathrm{~nm}, \mathrm{~T}=25^{\circ} \mathrm{C}$ ), $R$-enantiomer $20.9 \mathrm{~min}, S$ enantiomer $23.3 \min .90 \%$ ee $(R),[\alpha]_{\mathrm{D}}^{22}=+4.33\left(\mathrm{c}=0.3, \mathrm{CHCl}_{3}\right)$;

${ }^{1} \mathrm{H}$ NMR $\left(500 \mathrm{MHz}, \mathrm{CDCl}_{3}\right)$ (mixture of rotamers) $\delta$ 7.38-7.18 (5H, m, ArH), 4.89-4.84 (1H, m, $\mathrm{C} \underline{\mathrm{HOH}}), 4.69-4.63\left(1 \mathrm{H}, \mathrm{m} \mathrm{CH} \mathrm{CH}_{2} \mathrm{Ph}\right), 4.58-4.51\left(1 \mathrm{H}, \mathrm{m}, \mathrm{CH}_{2} \mathrm{Ph}\right), 4.20-4.16\left(2 \mathrm{H}, \mathrm{m}, \mathrm{CH}_{2}\right.$ of ester group), 4.06-4.01 (1H, m, $\mathrm{CHO} \underline{\mathrm{H}}), 2.95\left(3 \mathrm{H}, \mathrm{s},-\mathrm{NCH}_{3}\right), 2.69-2.56\left(2 \mathrm{H}, \mathrm{m}, \mathrm{CH}_{\mathrm{a}} \mathrm{H}_{\mathrm{b}}\right)$, $1.29-1.25\left(3 \mathrm{H}, \mathrm{m}, \mathrm{CH}_{3}\right.$ of ester group);

${ }^{13} \mathrm{C} \mathrm{NMR}\left(126 \mathrm{MHz}, \mathrm{CDCl}_{3}\right.$ ) (mixture of rotamers) $\delta 172.9,172.8,171.1,170.9,136.3$, $135.6,129.2$, 128.9, 128.2, 128.1, 127.9, 126.8, 65.9, 65.7, 61.2, 61.1, 52.6, 51.7, 49.2, 39.9, 34.1, 14.3;

$m / z(\mathrm{ESI}) 288.1\left[(\mathrm{M}+\mathrm{Na})^{+}, 100 \%\right]$.

Comparison of results at different scales.

\begin{tabular}{|l|l|l|l|l|}
\hline Reaction scale & Time & Conversion & ee & Yield \\
\hline $0.25 \mathrm{mmol}$ & $24 \mathrm{~h}$ & $100 \%$ & $90 \%$ & $91 \%$ \\
\hline $2.00 \mathrm{mmol}$ (above) & $24 \mathrm{~h}$ & $100 \%$ & $90 \%$ & $93 \%$ \\
\hline
\end{tabular}


Ethyl 4-(benzyl(methyl)amino)-3-hydroxy-4-oxobutanoate 39.

${ }^{1} \mathrm{H}$ NMR $\left(500 \mathrm{MHz}, \mathrm{CDCl}_{3}\right)$.

Apr26-2021

Chemist Shweta Gediya

PROTON.w CDCl3 /opt/topspin3.2 SKG 13
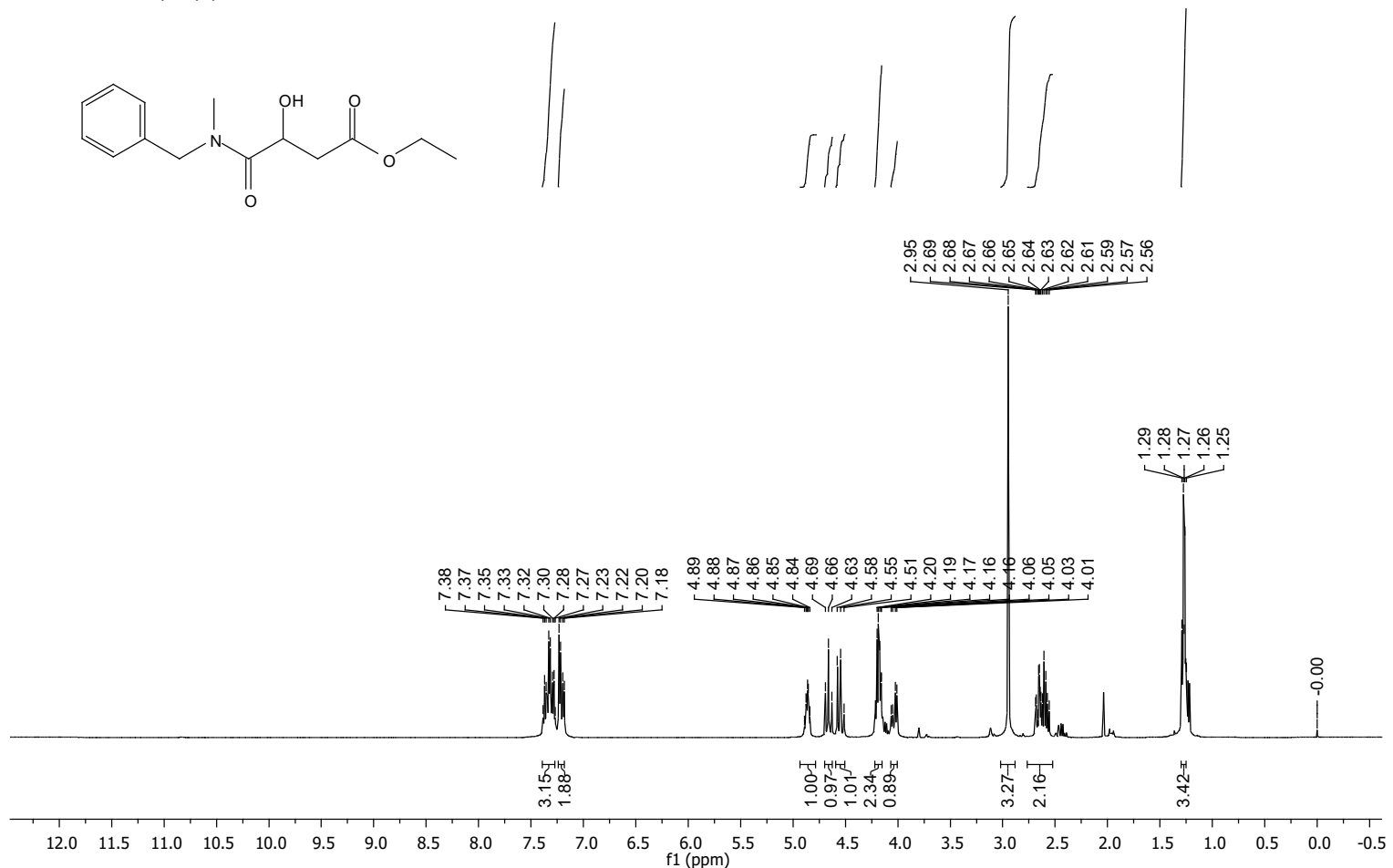

${ }^{13} \mathrm{C} \mathrm{NMR}\left(126 \mathrm{MHz}, \mathrm{CDCl}_{3}\right)$.

\begin{tabular}{|c|c|c|c|c|}
\hline $\begin{array}{l}\text { Apr15-2021 } \\
\text { SKG889 } \\
\text { C13APTlong.w.CDCl3 }\end{array}$ & 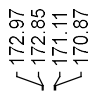 & 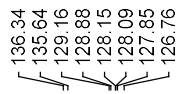 & 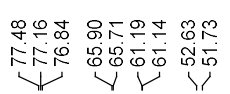 & 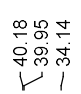 \\
\hline
\end{tabular}<smiles>CCOC(=O)CC(O)C(=O)N(C)Cc1ccccc1</smiles>

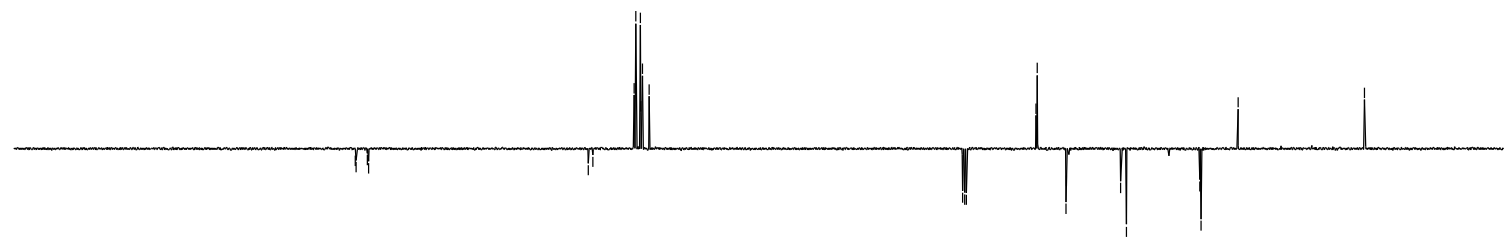

$\begin{array}{lllllllllllllllllllllllllllllllllllllllll}220 & 210 & 200 & 190 & 180 & 170 & 160 & 150 & 140 & 130 & 120 & 110 & 100 & 90 & 80 & 70 & 60 & 50 & 40 & 30 & 20 & 10 & 0\end{array}$ 
COSY.

SKG889

. $\mathrm{CDCl} 3$ /opt/topspin3.5pl2 SKG 9

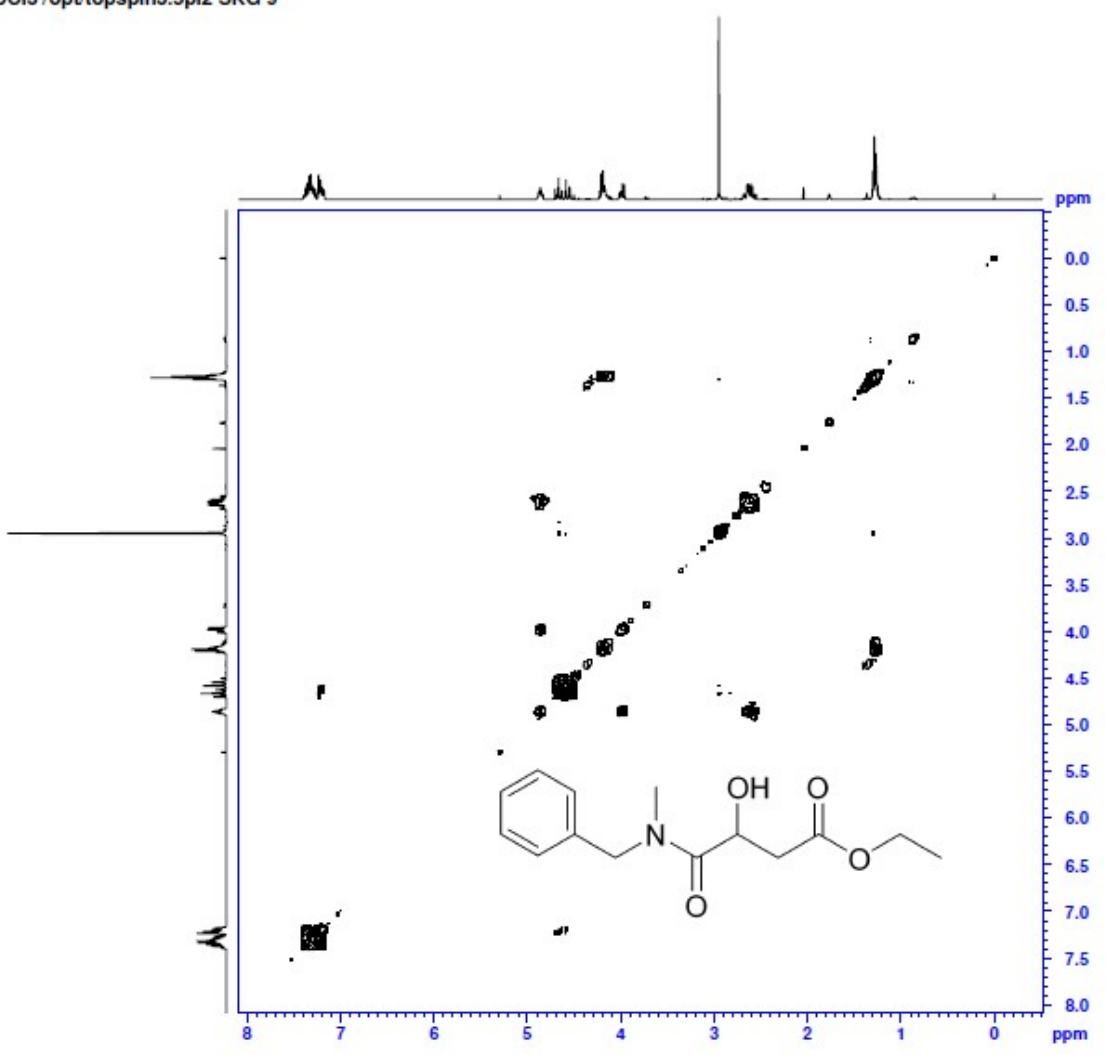

HSQC.

SKG889
HSQC.W CDCl3/opttopspin3.5pl2 SKG 9

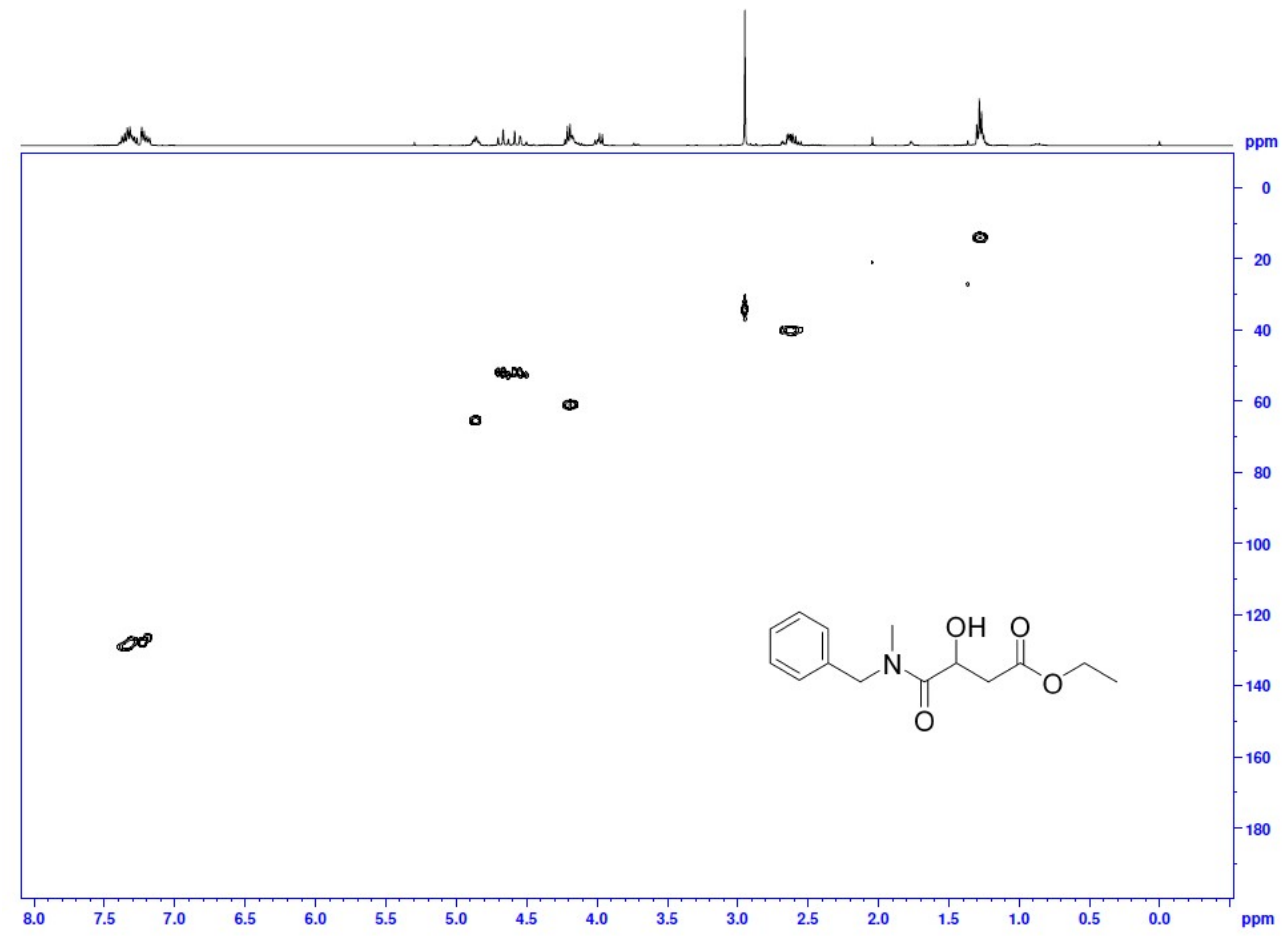




\section{Chiral HPLC of ketone.}

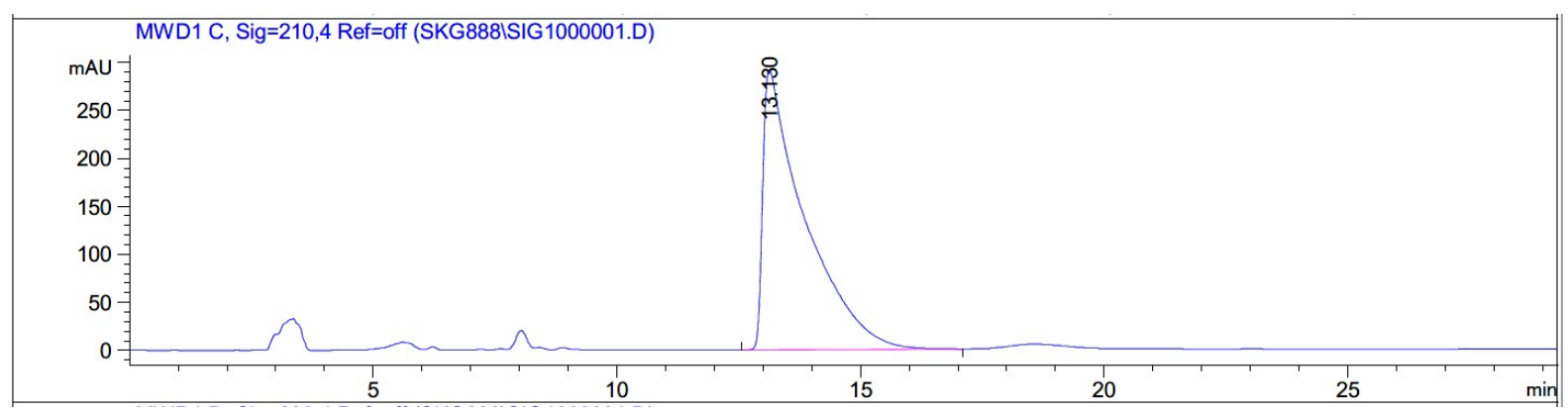

Signal 2: MWD1 C, Sig=210,4 Ref=off

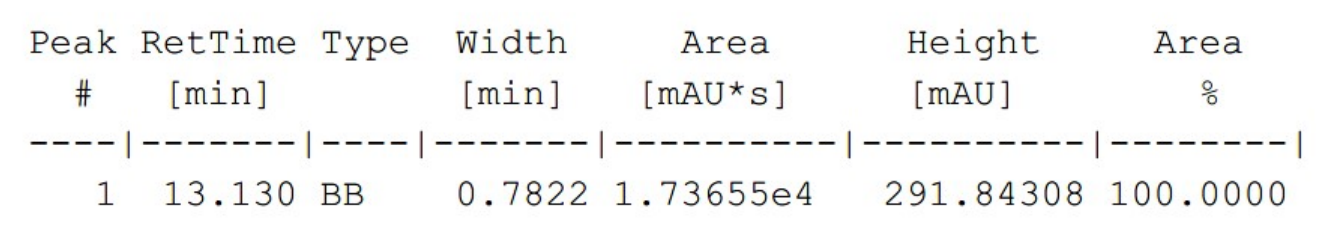

\section{Chiral HPLC of racemic standard.}

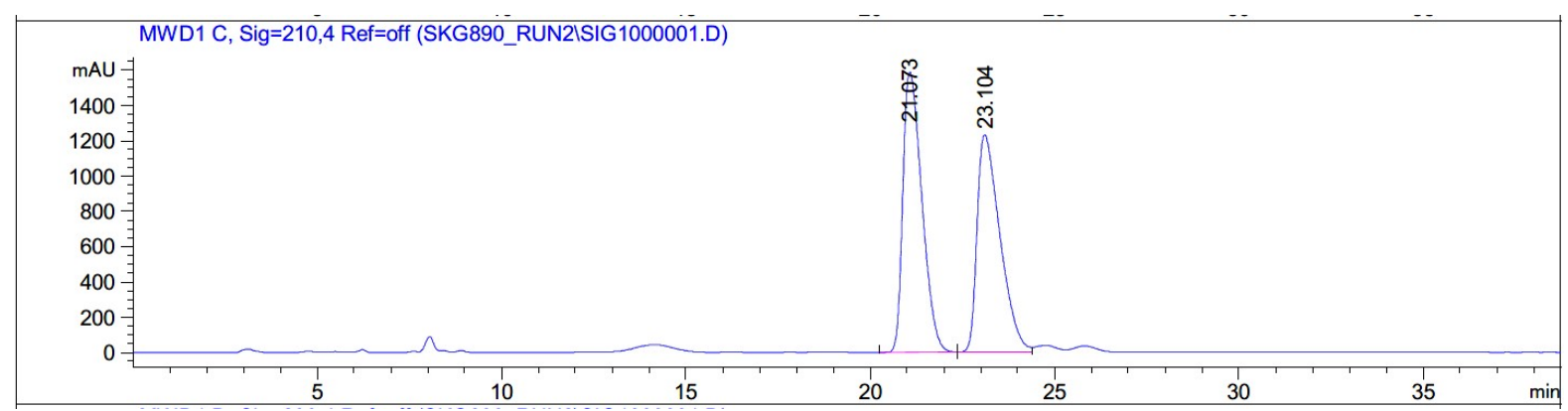

Signal 2: MWD1 C, Sig=210,4 Ref=off

\begin{tabular}{|c|c|c|c|c|c|c|}
\hline $\begin{array}{c}\text { eak } \\
\#\end{array}$ & $\begin{array}{c}\text { RetTime } \\
\text { [min] }\end{array}$ & Type & $\begin{array}{l}\text { Width } \\
\text { [min] }\end{array}$ & $\begin{array}{c}\text { Area } \\
{\left[m A U^{\star} s\right]}\end{array}$ & $\begin{array}{l}\text { Height } \\
{[\mathrm{mAU}]}\end{array}$ & $\begin{array}{c}\text { Area } \\
\frac{\circ}{0}\end{array}$ \\
\hline \multicolumn{7}{|c|}{$--|-------|----|-------|----------|----------|--------$} \\
\hline 1 & & & & $5.67390 e 4$ & 1587.83069 & \\
\hline 2 & 23.104 & BV & 0 . & $5.30612 e 4$ & 1230.15430 & 48.3252 \\
\hline
\end{tabular}


Chiral HPLC of ATH product. $90 \%$ ee.

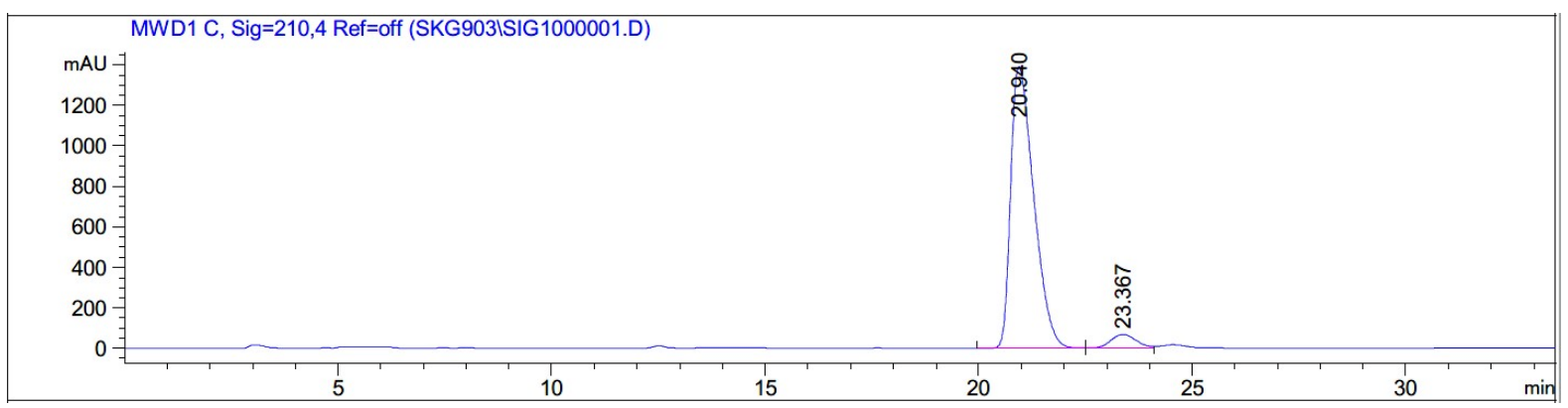

Signal 2: MWD1 C, Sig=210,4 Ref=off

\begin{tabular}{|c|c|c|c|c|c|c|}
\hline $\begin{array}{c}\text { Peak } \\
\#\end{array}$ & $\begin{array}{c}\text { RetTime } \\
\text { [min] }\end{array}$ & Type & $\begin{array}{l}\text { Width } \\
\text { [min] }\end{array}$ & $\begin{array}{c}\text { Area } \\
{\left[\mathrm{mAU}{ }^{\star} \mathrm{s}\right]}\end{array}$ & $\begin{array}{l}\text { Height } \\
{[\mathrm{mAU}]}\end{array}$ & $\begin{array}{c}\text { Area } \\
\frac{\circ}{0}\end{array}$ \\
\hline & $\mid----1$ & & & $----------\mid$ & $|----------|$ & $-------\mid$ \\
\hline 1 & 20.940 & $\mathrm{BV}$ & 0.5771 & $5.21377 e 4$ & 1391.66345 & 94.7884 \\
\hline 2 & 23.367 & VV & 0.6482 & 2866.60205 & 68.22710 & 5.2116 \\
\hline
\end{tabular}

A small amount of ketone is visible in this analysis. 
Ethyl (R)-3-hydroxy-4-oxo-4-(piperidin-1-yl)butanoate 40.<smiles>CCOC(=O)CC(O)C(=O)N1CCCCC1</smiles>

This compound is novel and was prepared following general procedure $\mathrm{C}$ using ethyl 4morpholino-3,4-dioxobutanoate (113.5 mg, $0.500 \mathrm{mmol}, 1.0 \mathrm{eq}), 3 \mathrm{C}$ tethered catalyst $(R, R)-2$ (4.7 $\mathrm{mg}, 7.5 \mu \mathrm{mol}, 0.015 \mathrm{eq})$ and FA:TEA $(0.3 \mathrm{~mL})$ in DCM $(3 \mathrm{~mL})$ to give $\mathbf{4 0}$ as clear oil (108 mg, $0.471 \mathrm{mmol}, 94 \%)$.

TLC: $R_{f}$ ca 0.2 (1:9, hexane: EtOAc), UV active and PMA-reactive;

HRMS (ESI) m/z: $[\mathrm{M}+\mathrm{Na}]^{+}$Calcd for $\mathrm{C}_{11} \mathrm{H}_{19} \mathrm{NO}_{4} \mathrm{Na}$ 252.1206; Found 252.1200 (error 2.5 ppm);

$U_{\max } 3415,2936,2859,1730,1631,1445,1254,1162,1008 \mathrm{~cm}^{-1}$;

Enantiomeric excess determined by HPLC analysis (Chiralpak IC, $250 \mathrm{~mm} \times 4.6 \mathrm{~mm}$ column, hexane: iPrOH 80:20, $1 \mathrm{~mL} / \mathrm{min}, \lambda=210 \mathrm{~nm}, \mathrm{~T}=25^{\circ} \mathrm{C}$ ), $S$-enantiomer $44.9 \mathrm{~min}, R$ enantiomer $64.4 \min .94 \%$ ee $(R),[\alpha]_{\mathrm{D}}^{22}=+10.1\left(\mathrm{c}=0.1, \mathrm{CHCl}_{3}\right)$;

${ }^{1} \mathrm{H}$ NMR $\left(500 \mathrm{MHz}, \mathrm{CDCl}_{3}\right) \delta$ 4.78-4.74 $(1 \mathrm{H}, \mathrm{m}, \mathrm{C} \underline{\mathrm{HOH}}), 4.17(2 \mathrm{H}, \mathrm{q}, J=7.1 \mathrm{~Hz}$, $\left.\mathrm{OC}_{2}{ }_{2} \mathrm{CH}_{3}\right), 4.03(1 \mathrm{H}, \mathrm{d}, J=7.6 \mathrm{~Hz}, \mathrm{CHO} \underline{\mathrm{H}}), 3.66-3.61\left(1 \mathrm{H}, \mathrm{m}, \mathrm{NCH}_{2}\right.$ of piperidine), $3.51-$ $3.46\left(1 \mathrm{H}, \mathrm{m}, \mathrm{NCH}_{2}\right.$ of piperidine $), 3.43-3.38\left(1 \mathrm{H}, \mathrm{m}, \mathrm{NCH}_{2}\right.$ of piperidine $), 3.35-3.30(1 \mathrm{H}, \mathrm{m}$, $\mathrm{NCH}_{2}$ of piperidine), $2.54\left(1 \mathrm{H}, \mathrm{dd}, J=15.6,3.1 \mathrm{~Hz}, \underline{\mathrm{C}}_{\mathrm{a}} \mathrm{H}_{\mathrm{b}}\right), 2.46(1 \mathrm{H}, \mathrm{dd}, J=15.6,8.7 \mathrm{~Hz}$, $\left.\mathrm{CH}_{\mathrm{a}} \underline{\mathrm{H}} \mathrm{b}\right), 1.68-1.55\left(6 \mathrm{H}, \mathrm{m}, \mathrm{CH}_{2}\right.$ of piperidine), 1.27-1.24 (3H, m, $\left.\mathrm{OCH}_{2} \underline{\mathrm{C}}_{3}\right)$;

${ }^{13} \mathrm{C}$ NMR $\left(126 \mathrm{MHz}, \mathrm{CDCl}_{3}\right) \delta 170.9,170.8,65.3,61.1,46.0,43.8,40.4,26.3,25.5,24.4$, 14.2;

$m / z(\mathrm{ESI}) 252.1\left[(\mathrm{M}+\mathrm{Na})^{+}, 100 \%\right]$. 
Ethyl 3-hydroxy-4-oxo-4-(piperidin-1-yl)butanoate 40.

${ }^{1} \mathrm{H}$ NMR $\left(500 \mathrm{MHz}, \mathrm{CDCl}_{3}\right)$.

Mar25-2021

Chemist Shweta Gediy

PROTON.w CDCl3 /opt/topspin3.2 SKG 28
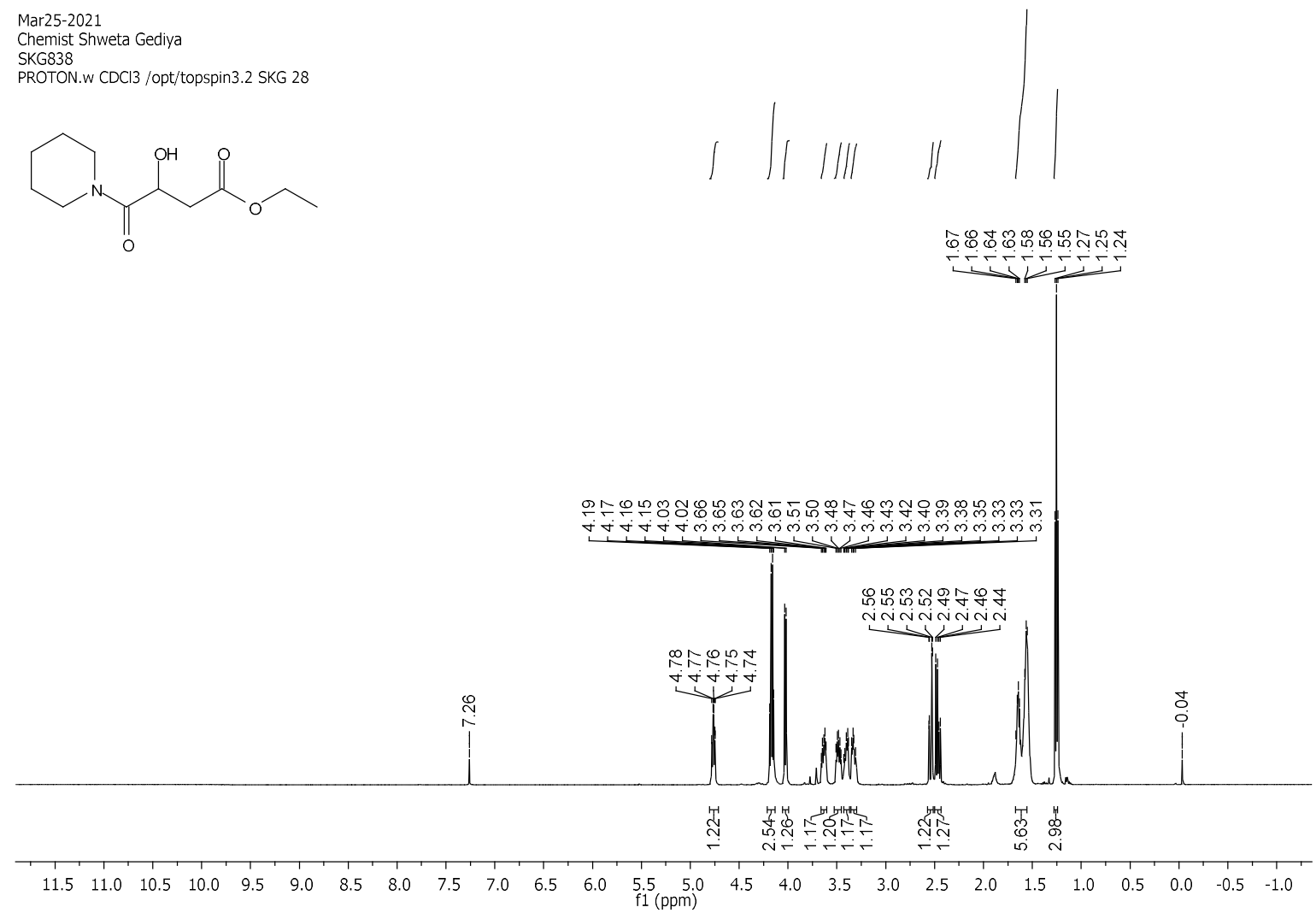

${ }^{13} \mathrm{C}$ NMR (126 MHz, $\left.\mathrm{CDCl}_{3}\right)$.

Mar25-2021
Chemist Shweta Gediya
SKG838

C13APT.w CDCl3 /opt/topspin3.2 SKG 28
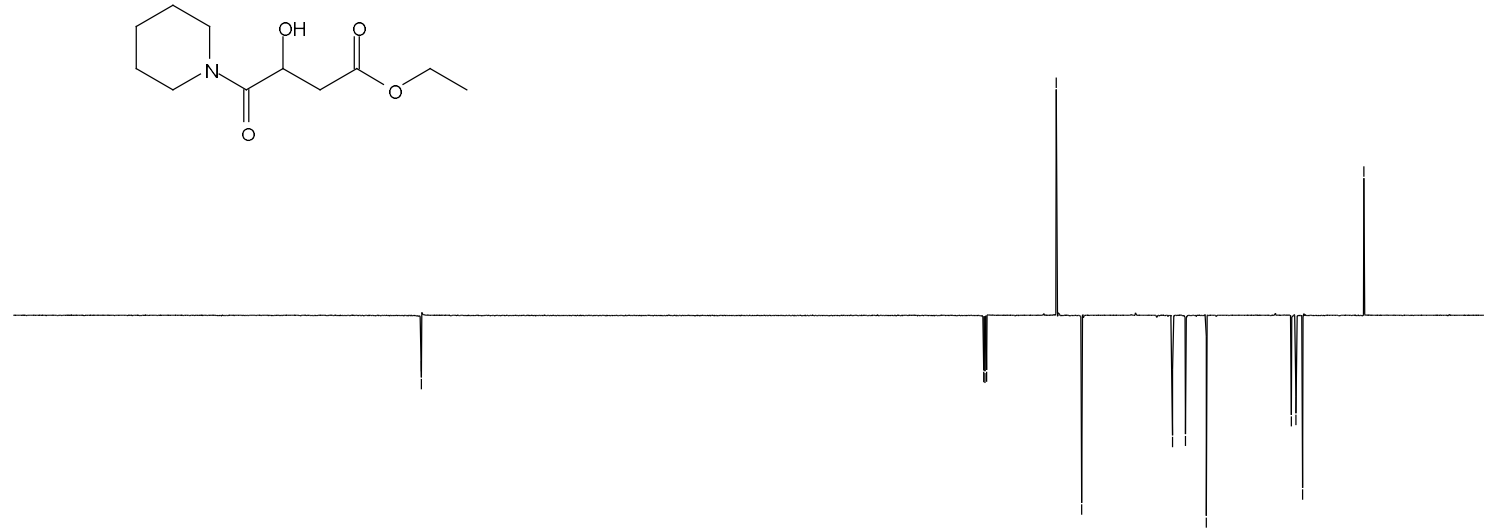
COSY.

Chemist Shweta Gediya

COSY.w CDCl3 /opt/topspin3.2 SKG 28

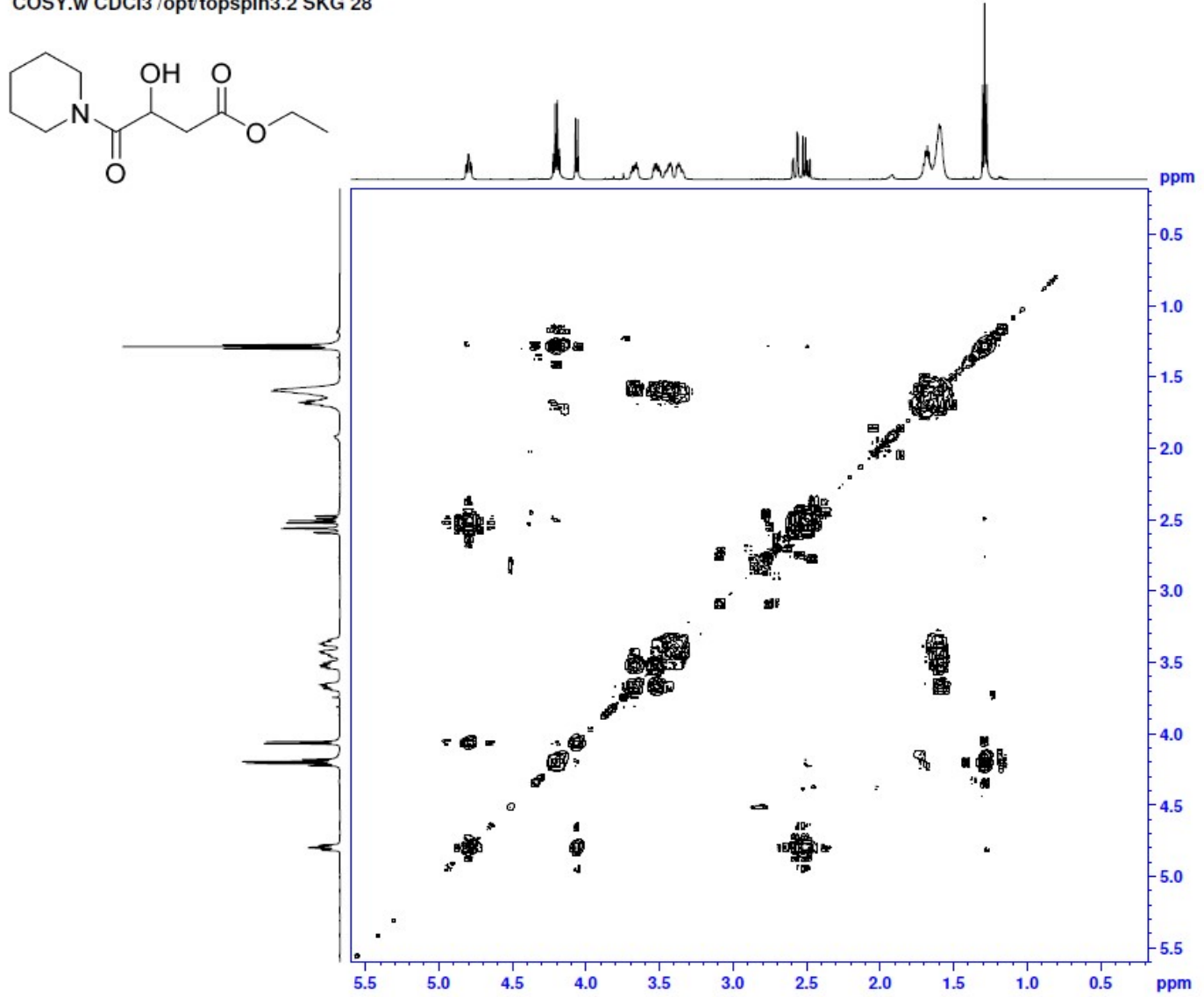

HSQC.

Chemist Shweta Gediya

HSGC.w CDCl3/opt/topspin 3.2 SKG 28

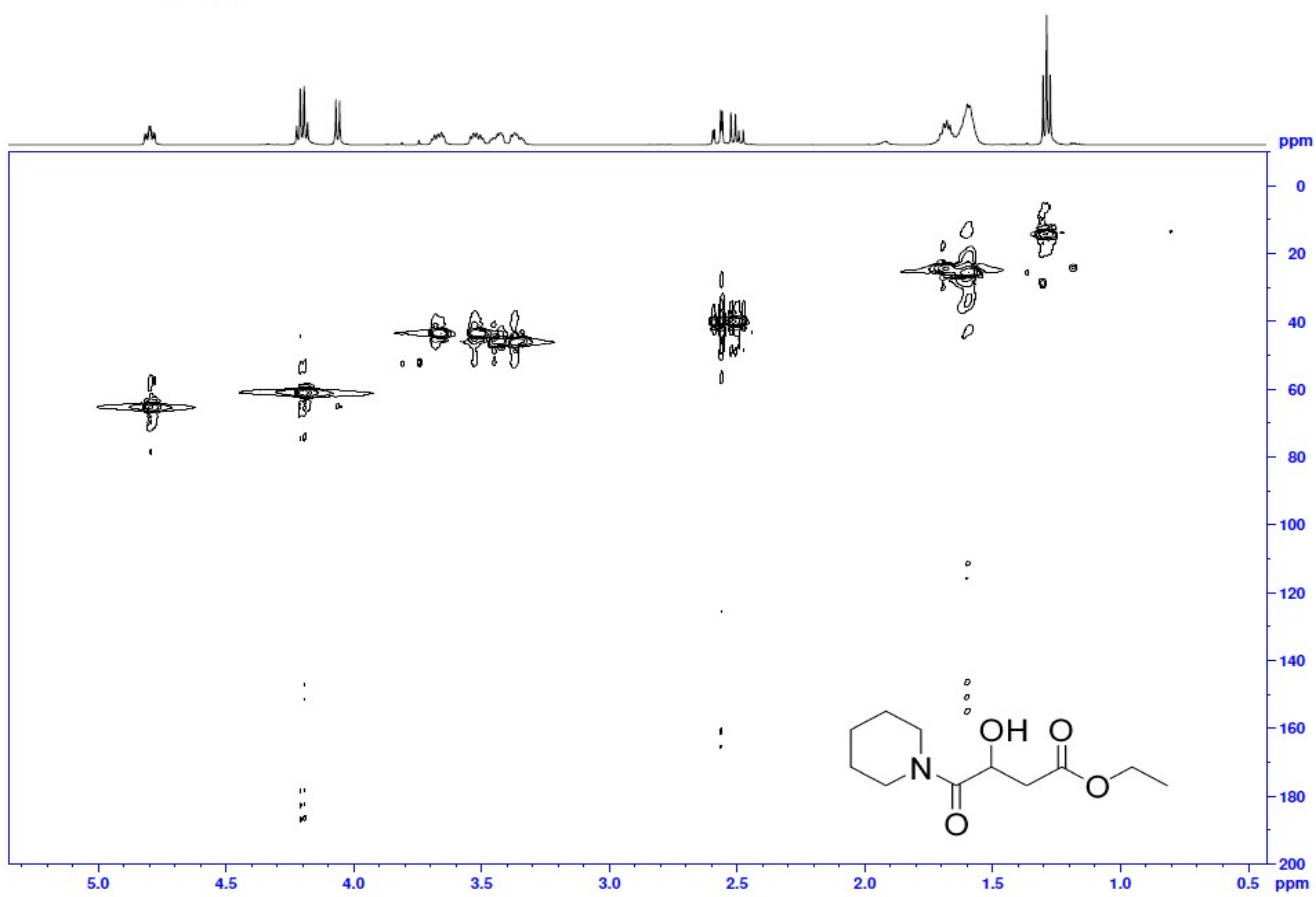




\section{Chiral HPLC of ketone.}

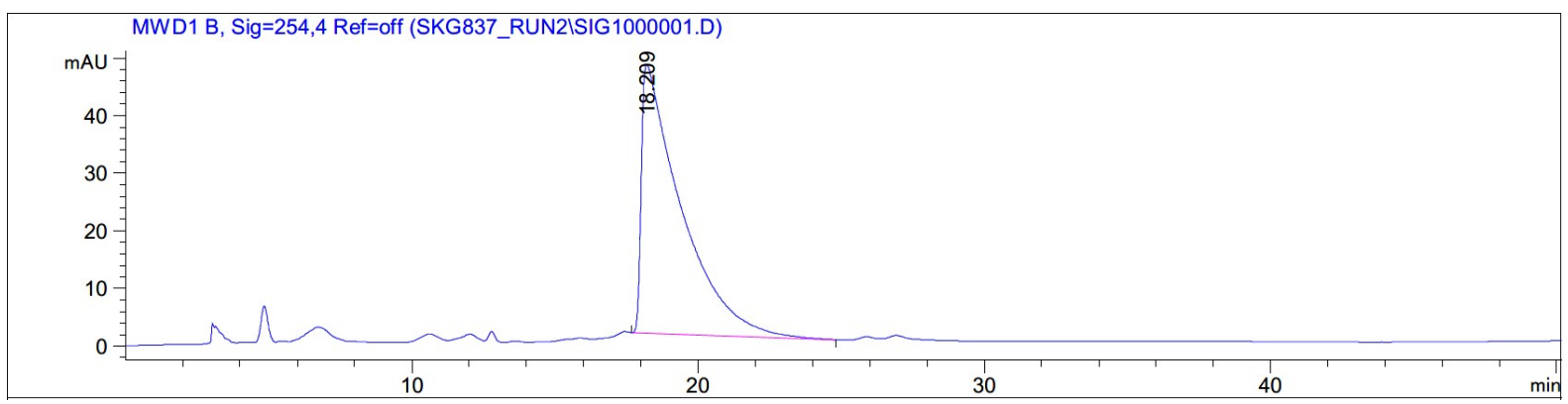

Signal 1: MWD1 B, Sig=254,4 Ref=off

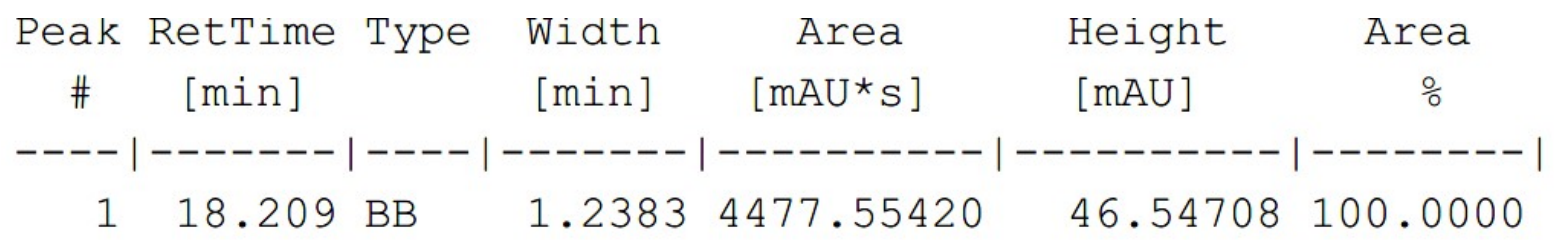

\section{Chiral HPLC of racemic standard.}

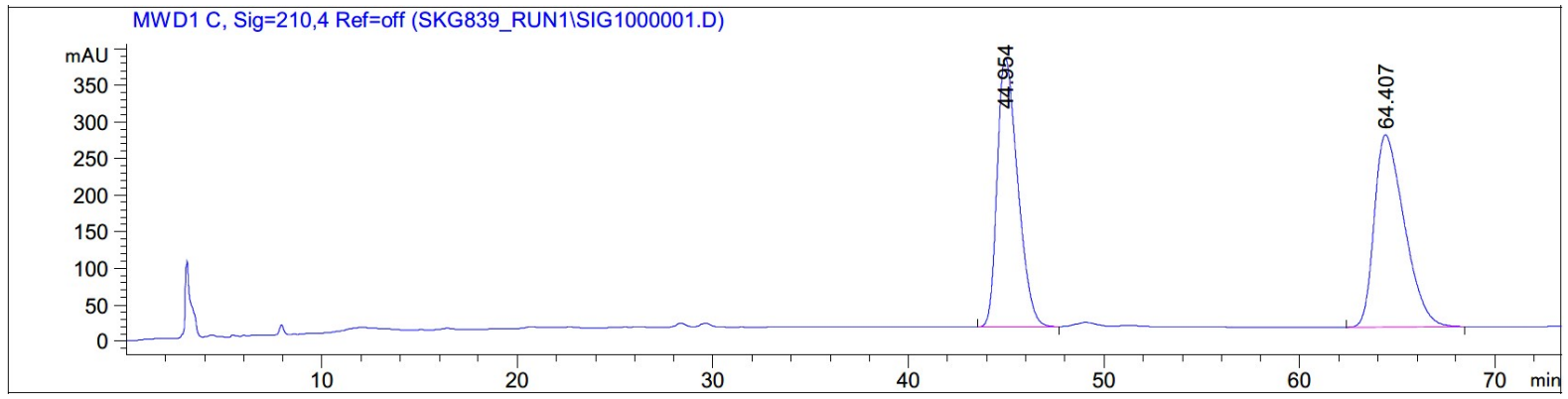

Signal 2: MWD1 C, Sig=210, 4 Ref=off

\begin{tabular}{|c|c|c|c|c|c|c|}
\hline $\begin{array}{c}\text { eak } \\
\text { \# }\end{array}$ & $\begin{array}{l}\text { RetTime } \\
\text { [min] }\end{array}$ & Type & $\begin{array}{c}\text { Width } \\
\text { [min] }\end{array}$ & $\begin{array}{c}\text { Area } \\
{\left[\mathrm{mAU}^{*} \mathrm{~s}\right]}\end{array}$ & $\begin{array}{l}\text { Height } \\
\text { [mAU] }\end{array}$ & $\begin{array}{c}\text { Area } \\
\frac{\circ}{0}\end{array}$ \\
\hline & & & & & & \\
\hline 1 & & & & 2.67 & 77 & 48 \\
\hline 2 & 64. & & 38 & $2.80767 e 4$ & 71829 & 200 \\
\hline
\end{tabular}


Chiral HPLC of ATH product. 94\% ee.

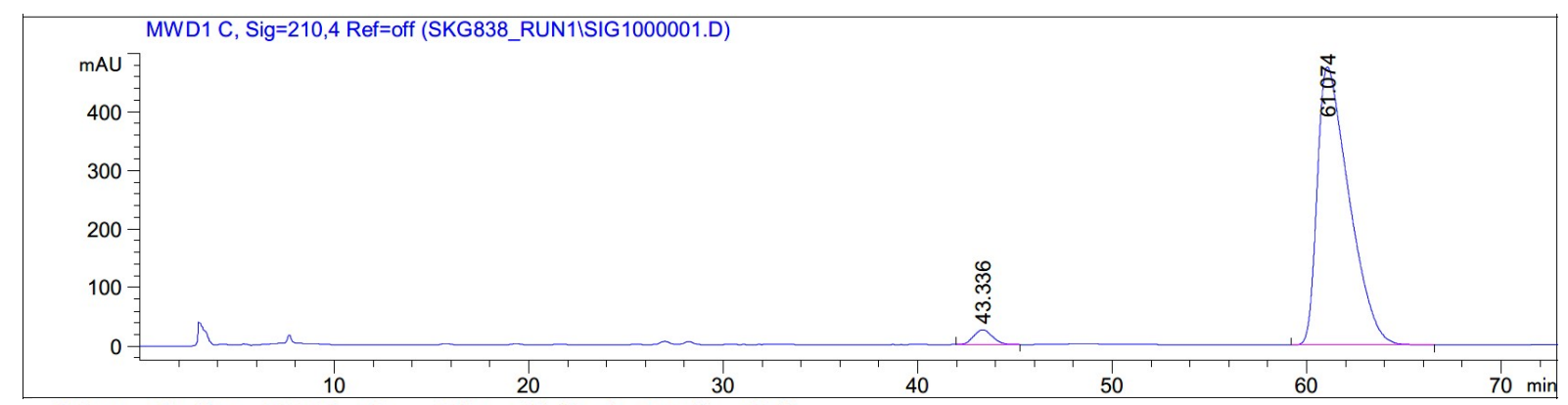

Signal 2: MWD1 C, Sig=210,4 Ref=off

\begin{tabular}{|c|c|c|c|c|c|c|}
\hline $\begin{array}{c}\text { Peak } \\
\text { \# }\end{array}$ & $\begin{array}{c}\text { RetTime } \\
\text { [min] }\end{array}$ & Type & $\begin{array}{l}\text { Width } \\
\text { [min] }\end{array}$ & $\begin{array}{c}\text { Area } \\
{\left[\mathrm{mAU}^{*} \mathrm{~s}\right]}\end{array}$ & $\begin{array}{l}\text { Height } \\
\text { [mAU] }\end{array}$ & $\begin{array}{c}\text { Area } \\
\frac{\circ}{0}\end{array}$ \\
\hline & & & & & & \\
\hline 1 & 43.336 & $\mathrm{BB}$ & 0.9883 & 1679.80957 & 24.98712 & 3.0258 \\
\hline 2 & 61.074 & $\mathrm{BB}$ & 1.6259 & $5.38357 e 4$ & 474.40533 & 96.9742 \\
\hline
\end{tabular}


Ethyl (R)-3-hydroxy-4-morpholino-4-oxobutanoate 41.<smiles>CCOC(=O)CC(O)C(=O)N1CCOCC1</smiles>

This compound is novel and was prepared following general procedure $\mathrm{C}$ using ethyl 4morpholino-3,4-dioxobutanoate $(113 \mathrm{mg}, 0.500 \mathrm{mmol}, 1.0 \mathrm{eq}), 3 \mathrm{C}$ tethered catalyst $(R, R)-2$ (4.7 $\mathrm{mg}, 7.5 \mu \mathrm{mol}, 0.015 \mathrm{eq})$ and FA:TEA $(0.3 \mathrm{~mL})$ in DCM $(3 \mathrm{~mL})$ to give 41 as a pale yellow semisolid material (104.3 $\mathrm{mg}, 0.454 \mathrm{mmol}, 90 \%)$.

TLC: $\mathrm{R}_{\mathrm{f}}$ ca 0.2 (6:4, hexane: EtOAc), UV active and PMA-reactive;

HRMS (ESI) m/z: [M+Na] ${ }^{+}$Calcd for $\mathrm{C}_{10} \mathrm{H}_{17} \mathrm{NO}_{5} \mathrm{Na} 254.0999$; Found 254.1009 (error -3.8 ppm);

$U_{\max } 3392,2977,2858,1727,1637,1143,1271,1161,1111,1017,847 \mathrm{~cm}^{-1}$;

Enantiomeric excess determined by HPLC analysis (Chiralpak IG, $250 \mathrm{~mm} \times 4.6 \mathrm{~mm}$ column, hexane: $\operatorname{PrOH} 80: 20,1 \mathrm{~mL} / \mathrm{min}, \lambda=210 \mathrm{~nm}, \mathrm{~T}=25{ }^{\circ} \mathrm{C}$ ), $R$-enantiomer $15.1 \mathrm{~min}, S$ enantiomer $21.4 \min .92 \%$ ee $(R),[\alpha]_{\mathrm{D}}^{22}=-8\left(\mathrm{c}=0.1, \mathrm{CHCl}_{3}\right)$;

${ }^{1} \mathrm{H}$ NMR (500 MHz, $\left.\mathrm{CDCl}_{3}\right) \delta$ 4.76-4.72 (1H, m, $\left.\mathrm{C} \underline{\mathrm{HOH}}\right), 4.21-4.15$ (2H, m $\left.\underline{\mathrm{C}}_{2} \mathrm{CH}_{3}\right), 3.96$ $(1 \mathrm{H}, \mathrm{d}, J=7.9 \mathrm{~Hz}, \mathrm{CHO} \underline{\mathrm{H}}), 3.75-3.68\left(5 \mathrm{H}, \mathrm{m}, \mathrm{CH}_{2}\right.$ of morpholine), 3.56-3.54 (2H, m, $\mathrm{CH}_{2}$ of morpholine), 3.49-3.44 (1H, m, $\mathrm{CH}_{2}$ of morpholine), 2.62-2.54 (2H, m, $\left.\mathrm{CH}_{\mathrm{a}} \mathrm{H}_{\mathrm{b}}\right), 1.27(3 \mathrm{H}, \mathrm{t}, J$ $\left.=7.1 \mathrm{~Hz}, \mathrm{CH}_{2} \mathrm{CH}_{3}\right)$;

${ }^{13} \mathrm{C} \mathrm{NMR}\left(126 \mathrm{MHz}, \mathrm{CDCl}_{3}\right) \delta 171.2,171.1,66.8,66.6,65.6,61.2,45.8,42.9,39.8,14.2$; $m / z(\mathrm{ESI}) 254.1\left[(\mathrm{M}+\mathrm{Na})^{+}, 100 \%\right]$. 
Ethyl 3-hydroxy-4-morpholino-4-oxobutanoate 41.

${ }^{1} \mathrm{H}$ NMR $\left(500 \mathrm{MHz}, \mathrm{CDCl}_{3}\right)$.

Mar25-2021

Chemist Shweta Gediya

PROTON.w CDCl3 /opt/topspin3.2 SKG 26<smiles>CCOC(=O)CC(O)C(=O)N1CCOCC1</smiles><smiles>CC#CCCCC</smiles>

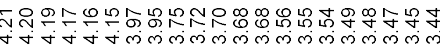

年

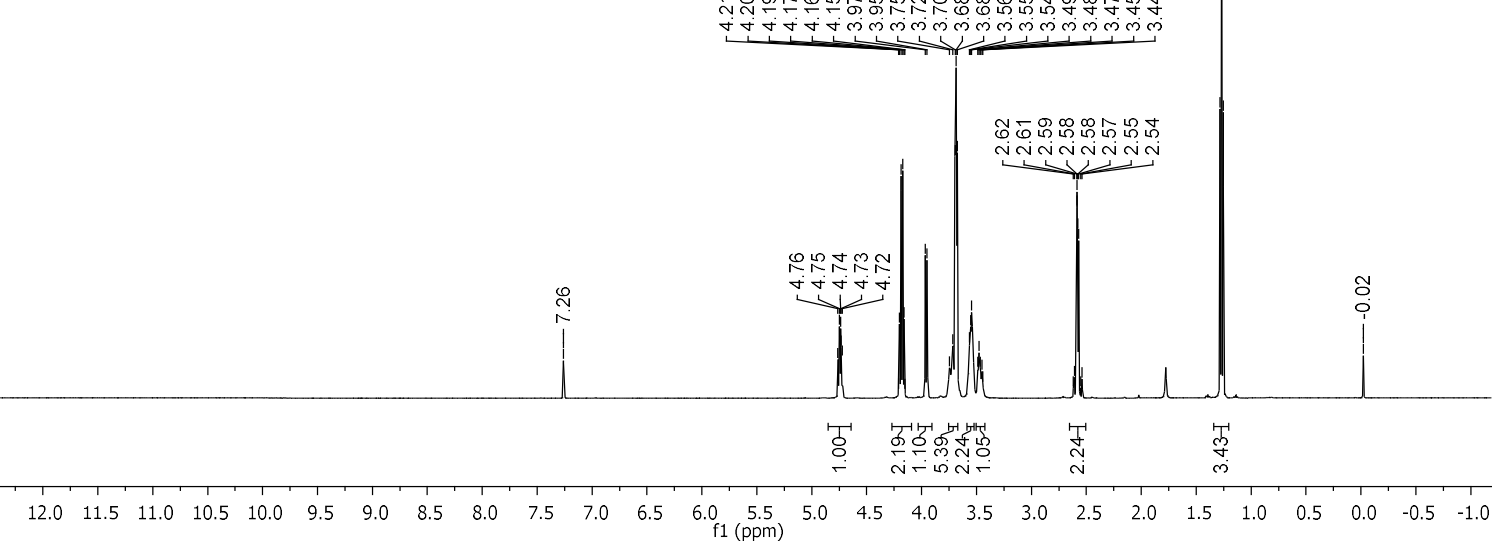

${ }^{13} \mathrm{C} \mathrm{NMR}\left(126 \mathrm{MHz}, \mathrm{CDCl}_{3}\right)$.

$\begin{array}{ll}\text { Mar25-2021 } & \text { SKG863 Gediya } \\ \text { Shweta Gediya } & \text { C13APT.w CDCl3 }\end{array}$<smiles>CCOC(=O)CC(O)C(=O)N1CCOCC1</smiles>

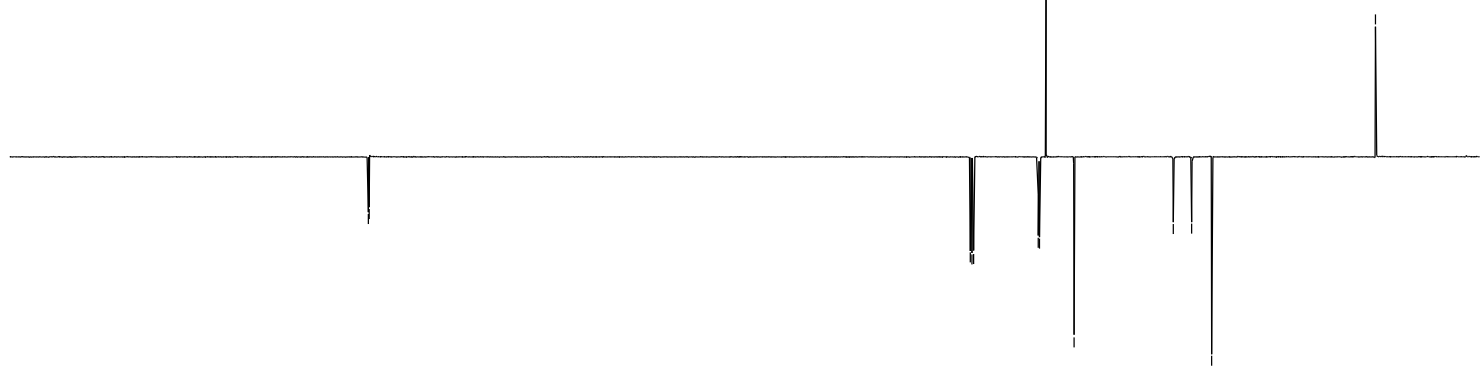

$\begin{array}{lllllllllllllllllllllll}220 & 210 & 200 & 190 & 180 & 170 & 160 & 150 & 140 & 130 & 120 & 110 & 100 & 90 & 80 & 70 & 60 & 50 & 40 & 30 & 20 & 10 & 0\end{array}$ 
COSY.

Chemist Shweta Gediya

COSY.w CDCl3 /opt/topspin3.2 SKG 26

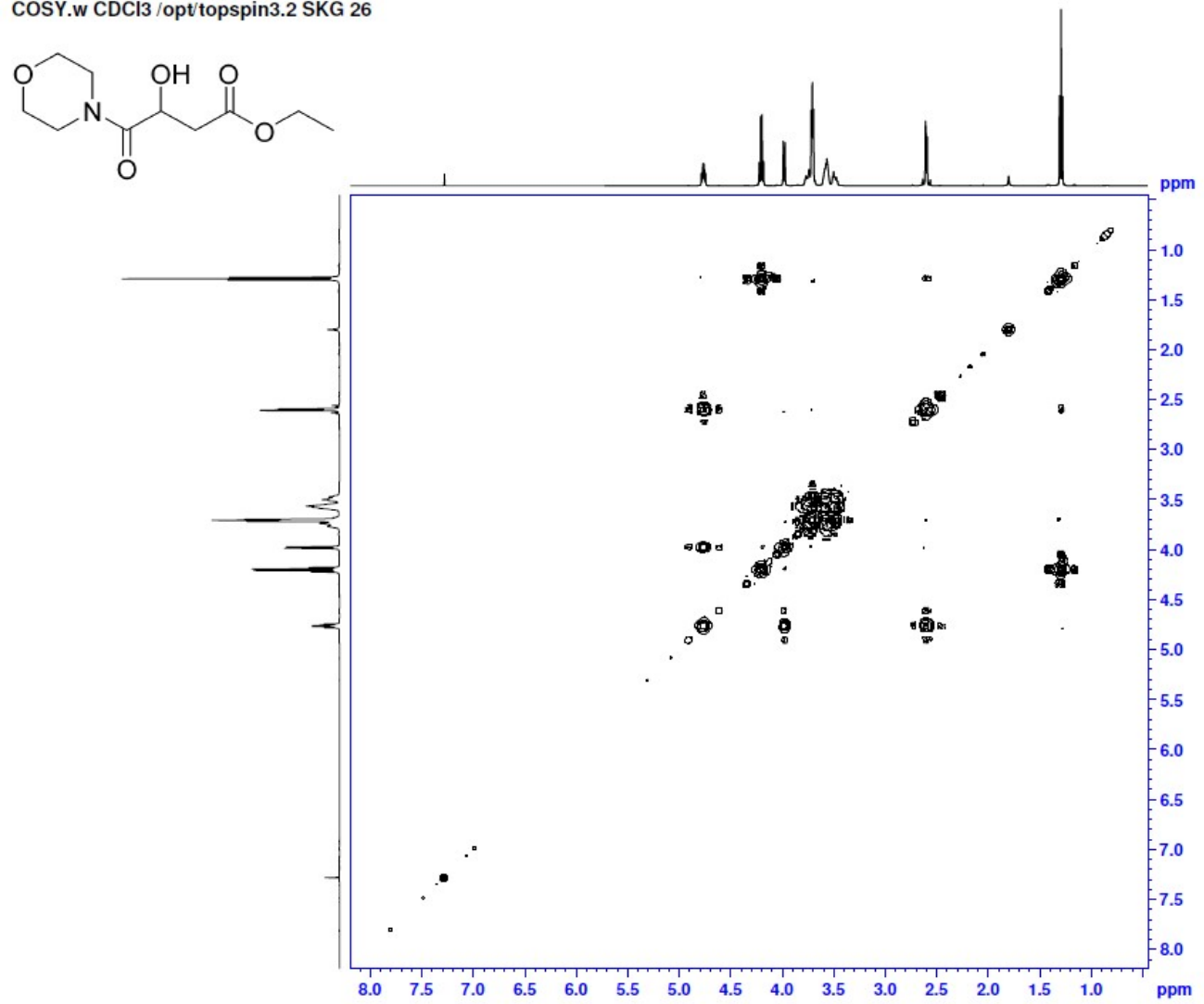

HSQC.

Chemist Shweta Gediya

HSGC.w CDCl3 /opt/topspin 3.2 SKG 26

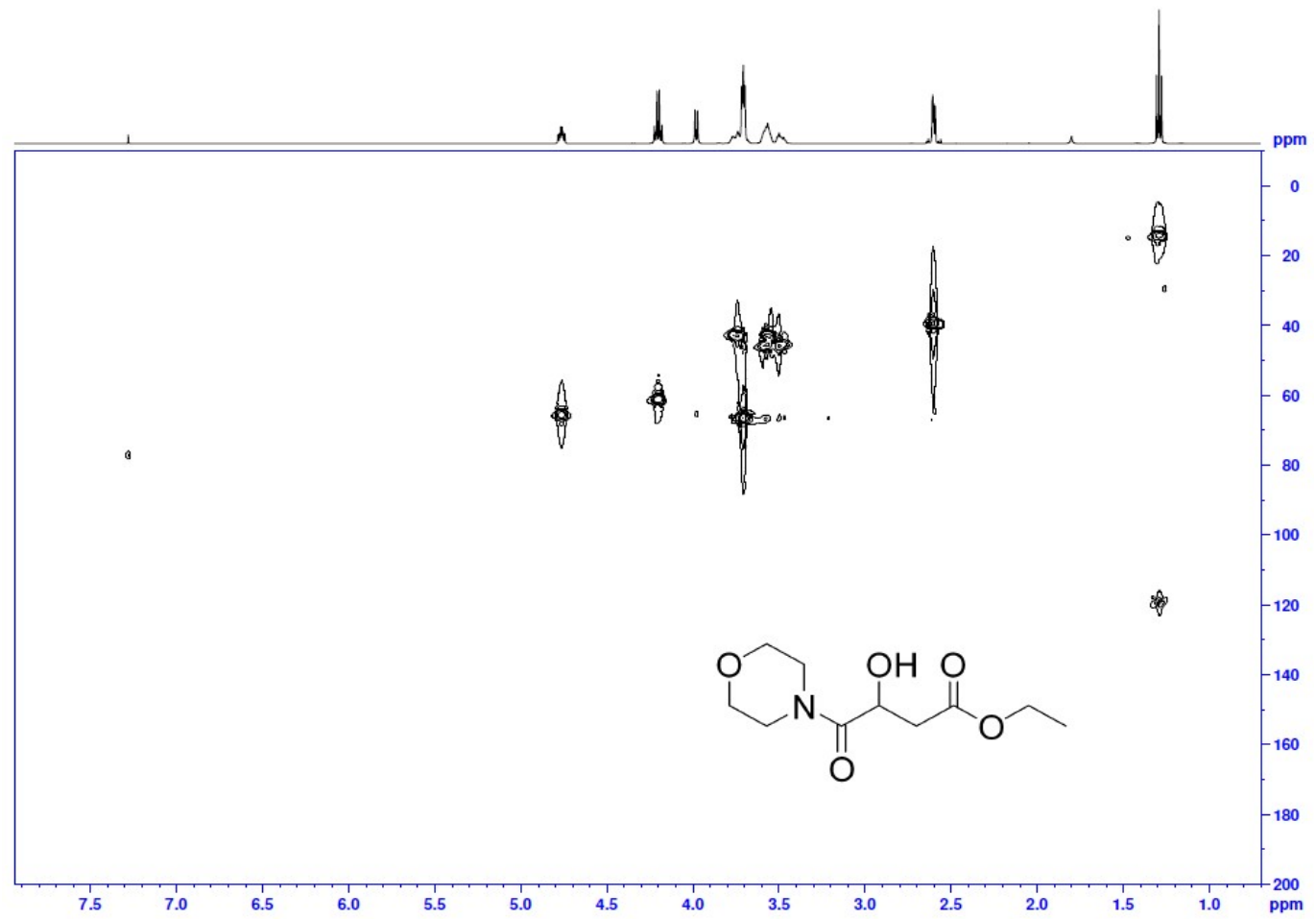




\section{Chiral HPLC of ketone.}

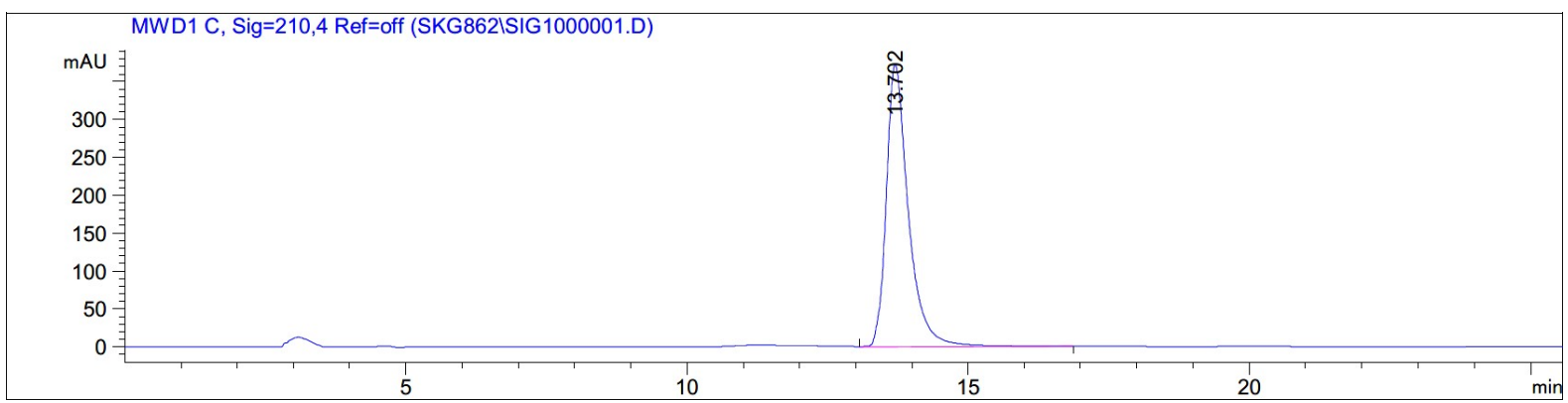

Signal 2: MWD1 C, Sig=210,4 Ref=off

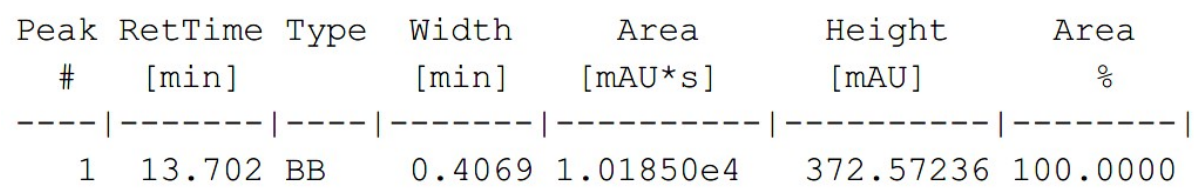




\section{Chiral HPLC of Racemic standard.}

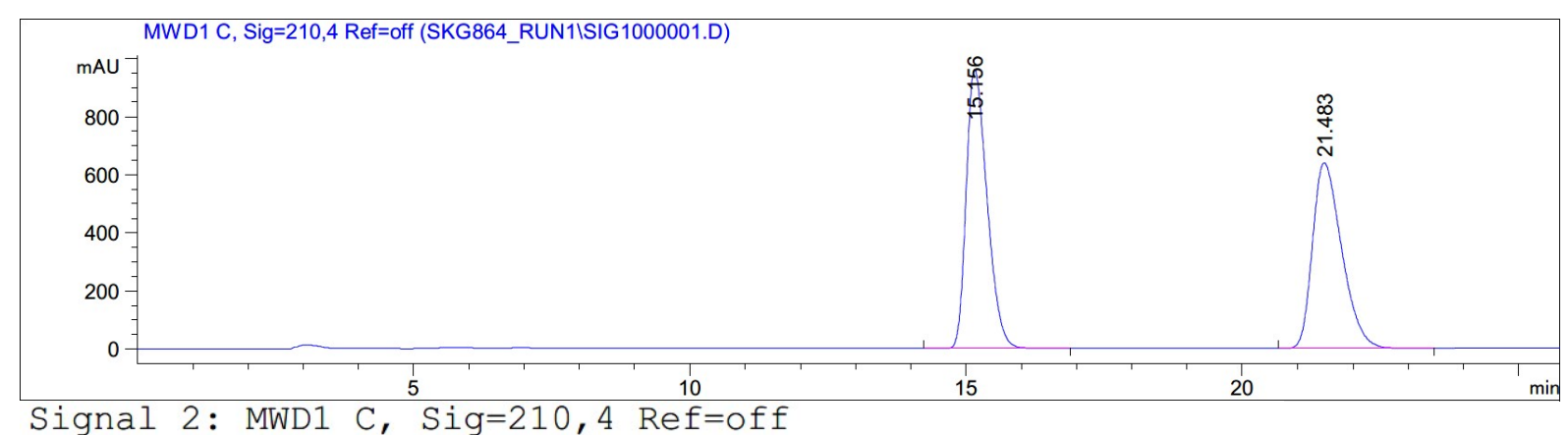

Signal 2: MWD1 C, Sig=210, 4 Ref=off

\begin{tabular}{|c|c|c|c|c|c|c|}
\hline $\begin{array}{c}\text { Peak } \\
\quad \#\end{array}$ & $\begin{array}{c}\text { RetTime } \\
\text { [min] }\end{array}$ & Type & $\begin{array}{l}\text { Width } \\
\text { [min] }\end{array}$ & $\begin{array}{c}\text { Area } \\
{\left[\mathrm{mAU}^{*} \mathrm{~s}\right]}\end{array}$ & $\begin{array}{l}\text { Height } \\
{[\mathrm{mAU}]}\end{array}$ & $\begin{array}{c}\text { Area } \\
\frac{\circ}{0}\end{array}$ \\
\hline 1 & & & & 520 & & 674 \\
\hline 2 & 21.483 & $\mathrm{BB}$ & 0.5695 & $2.35274 \mathrm{e} 4$ & 639.06293 & 48.2326 \\
\hline
\end{tabular}

Chiral HPLC of ATH product. 92\% ee.

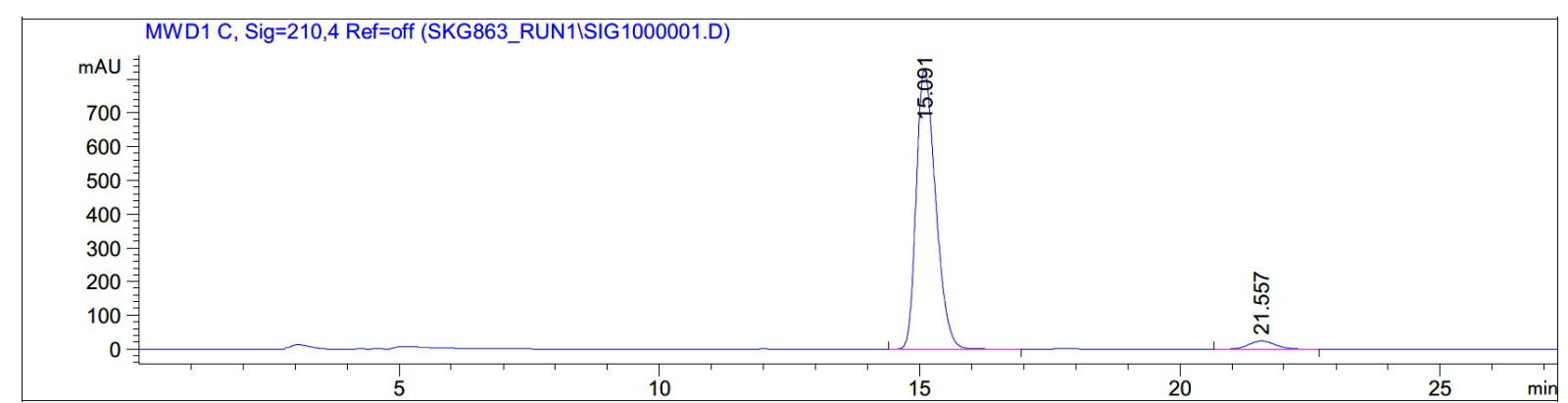

Signal 2: MWD1 C, Sig=210, 4 Ref=off

\begin{tabular}{|c|c|c|c|c|c|c|}
\hline $\begin{array}{c}\text { Peak } \\
\quad \#\end{array}$ & $\begin{array}{c}\text { RetTime } \\
\text { [min] }\end{array}$ & Type & $\begin{array}{l}\text { Width } \\
\text { [min] }\end{array}$ & $\begin{array}{c}\text { Area } \\
{\left[\mathrm{mAU}{ }^{\star} \mathrm{s}\right]}\end{array}$ & $\begin{array}{l}\text { Height } \\
{[\mathrm{mAU}]}\end{array}$ & $\begin{array}{c}\text { Area } \\
\quad \%\end{array}$ \\
\hline 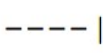 & & & 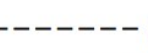 & ----- & --------- & ------- \\
\hline 1 & 15.09 & BB & 0.3994 & $2.14012 \mathrm{e} 4$ & 828.53766 & 96.1184 \\
\hline 2 & 21.557 & $\mathrm{BB}$ & 0.5517 & 864.25549 & 24.13674 & 3.8816 \\
\hline
\end{tabular}


Ethyl (S)-3-hydroxy-4-oxo-4-(propylamino)butanoate 42.<smiles>CCCNC(=O)[C@H](O)CC(=O)OCC</smiles>

This compound is novel and was prepared following general procedure $\mathrm{C}$ using ethyl 3, 4dioxo-4-(propylamino) butanoate $(50.0 \mathrm{mg}, 0.250 \mathrm{mmol}, 1.0 \mathrm{eq}), 3 \mathrm{C}$ tethered catalyst $(S, S)-2$ (2.3 $\mathrm{mg}, 3.8 \mu \mathrm{mol}, 0.015 \mathrm{eq})$ and FA:TEA $(0.15 \mathrm{~mL})$ in DCM $(1.5 \mathrm{~mL})$ to give $\mathbf{4 2}$ as clear liquid (45.2 mg, $0.223 \mathrm{mmol}, 89 \%)$.

TLC: $\mathrm{R}_{\mathrm{f}}$ ca 0.4 (1:9, hexane: EtOAc) and PMA-reactive;

HRMS (ESI) m/z: [M+Na] $]^{+}$Calcd for $\mathrm{C}_{9} \mathrm{H}_{17} \mathrm{NO}_{4} \mathrm{Na} 226.1050$; Found 226.1045 (error 2.3 ppm);

$U_{\max } 3364,2967,1732,1647,1536,1372,1258,1162,1080 \mathrm{~cm}^{-1}$;

Enantiomeric excess determined by HPLC analysis (Chiralpak IC, $250 \mathrm{~mm} \times 4.6 \mathrm{~mm}$ column, hexane: $\operatorname{PrOH} 80: 20,1 \mathrm{~mL} / \mathrm{min}, \lambda=210 \mathrm{~nm}, \mathrm{~T}=25{ }^{\circ} \mathrm{C}$ ), $S$-enantiomer $20.4 \mathrm{~min}, R$ enantiomer $25.7 \min .90 \%$ ee $(S),[\alpha]_{\mathrm{D}}^{22}=+2.5\left(\mathrm{c}=0.1, \mathrm{CHCl}_{3}\right)$;

${ }^{1} \mathrm{H}$ NMR (400 MHz, $\left.\mathrm{CDCl}_{3}\right) \delta 6.88(1 \mathrm{H}, \mathrm{s}, \mathrm{CONH}), 4.43-4.40(1 \mathrm{H}, \mathrm{m}, \mathrm{C} \underline{\mathrm{HOH}}), 4.16(2 \mathrm{H}, \mathrm{q}$, $J=7.1 \mathrm{~Hz}, \mathrm{C}_{2} \mathrm{CH}_{3}$ of ester), $4.05(1 \mathrm{H}, \mathrm{s}, \mathrm{OH}), 3.25-3.20\left(2 \mathrm{H}, \mathrm{m}, \mathrm{CH}_{2}\right), 2.94(1 \mathrm{H}, \mathrm{dd}, J=$ $\left.17.2,3.6 \mathrm{~Hz}, \mathrm{CH}_{\mathrm{a}} \mathrm{H}_{\mathrm{b}}\right), 2.68\left(1 \mathrm{H}, \mathrm{dd}, J=17.2,8.2 \mathrm{~Hz}, \mathrm{CH}_{\mathrm{a}} \underline{\mathrm{H}} \mathrm{b}\right), 1.58-1.49\left(2 \mathrm{H}, \mathrm{m}, \mathrm{C}_{2} \mathrm{CH}_{3}\right.$ of propyl), $1.26\left(3 \mathrm{H}, \mathrm{t}, J=7.1 \mathrm{~Hz}, \mathrm{CH}_{2} \underline{\mathrm{CH}}_{3}\right.$ of ester), $0.91\left(3 \mathrm{H}, \mathrm{t}, J=7.4 \mathrm{~Hz}, \mathrm{CH}_{2} \underline{\mathrm{C}}_{3}\right.$ of propyl);

${ }^{13} \mathrm{C}$ NMR (101 MHz, $\left.\mathrm{CDCl}_{3}\right) \delta 173.0,171.9,68.6,61.3,40.9,38.4,22.9,14.2,11.4$;

$m / z(\mathrm{ESI}) 226.1\left[(\mathrm{M}+\mathrm{Na})^{+}, 100 \%\right]$. 
Ethyl 3-hydroxy-4-oxo-4-(propylamino)butanoate 42.

${ }^{1} \mathrm{H}$ NMR $\left(400 \mathrm{MHz}, \mathrm{CDCl}_{3}\right)$.

Feb10-2021

SKG788 w CDCl3/opt/topspin3.5p12 SKG 18
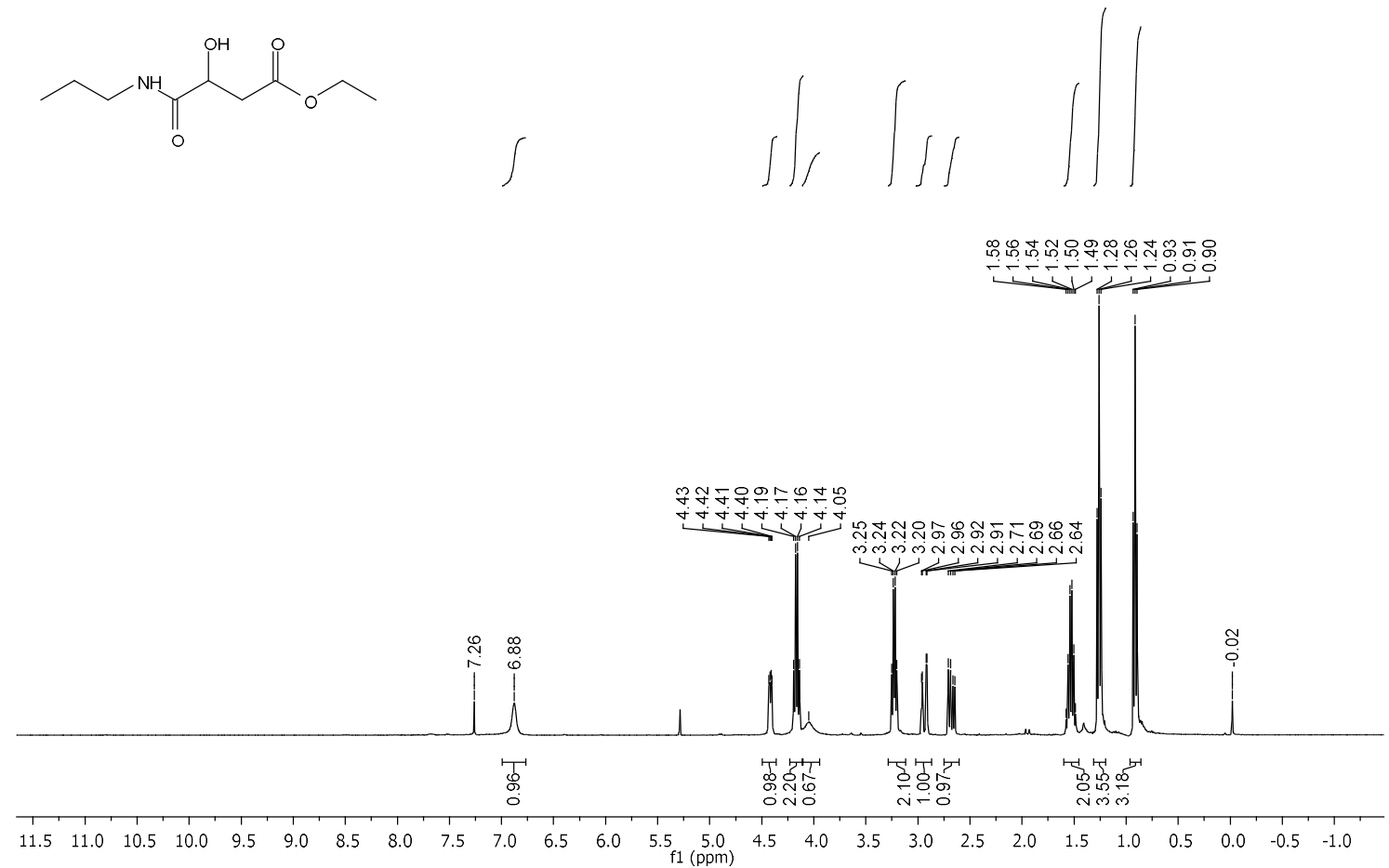

${ }^{13} \mathrm{C}$ NMR $\left(101 \mathrm{MHz}, \mathrm{CDCl}_{3}\right)$.

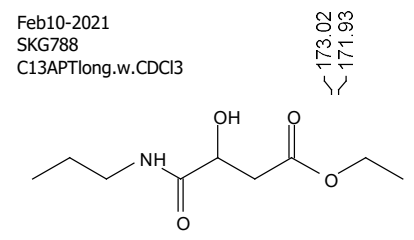

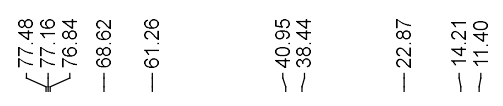

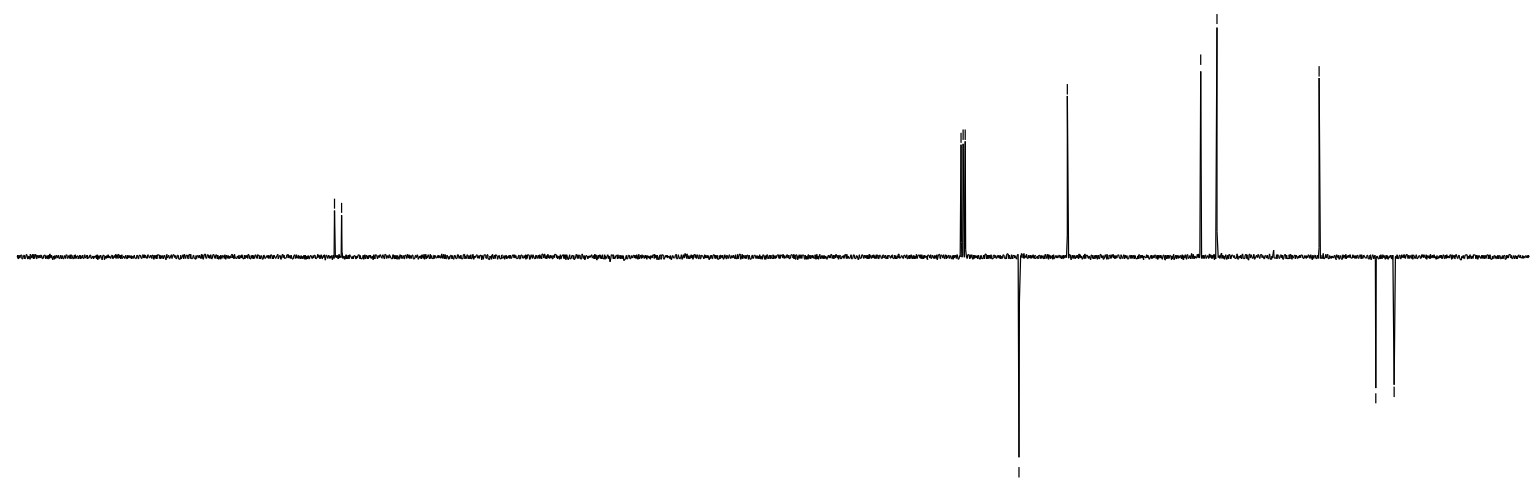

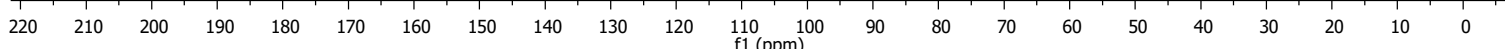


COSY.

SKG788

COSY.w CDCl3 /opt/topspin3.5pl2 SKG 18

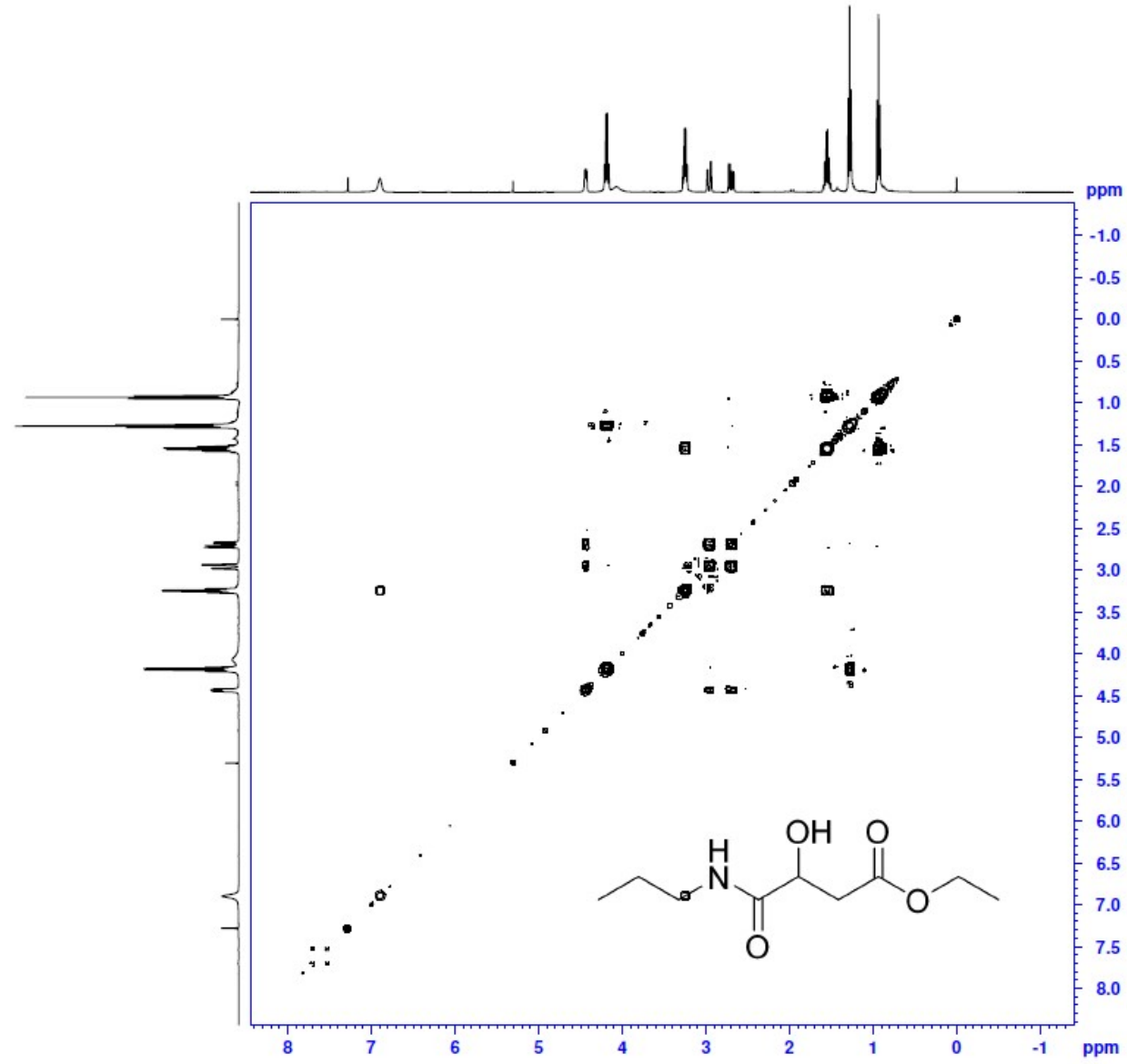

HSQC.

SKG788
HSOC.w CDCl3 /opt'topspin3.5pl2 SKG 18

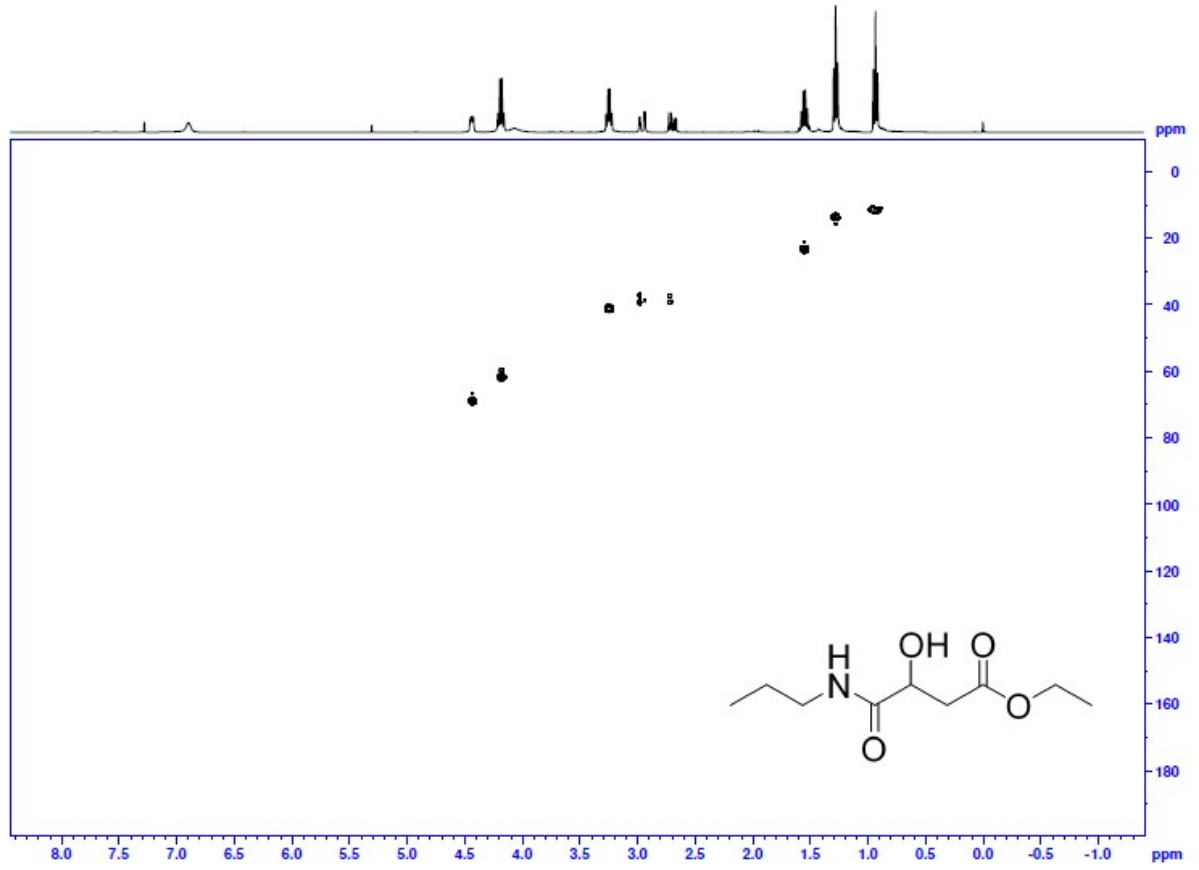




\section{Chiral HPLC of ketone.}

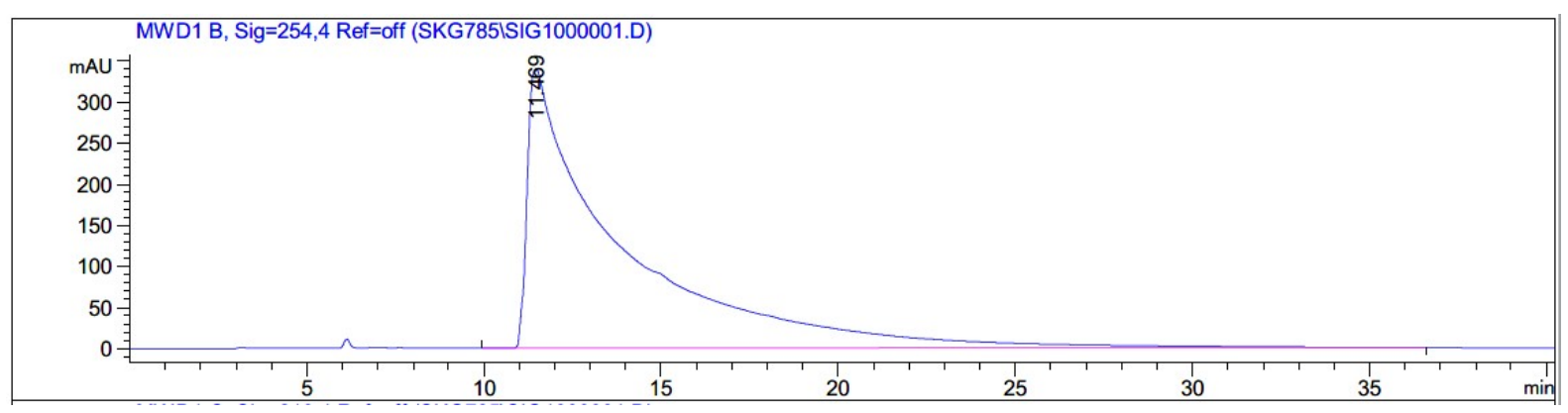

Signal 1: MWD1 B, Sig=254,4 Ref=off

\begin{tabular}{|c|c|c|c|c|c|c|}
\hline $\begin{array}{c}\text { Peak } \\
\#\end{array}$ & $\begin{array}{c}\text { RetTime } \\
\text { [min] }\end{array}$ & Type & $\begin{array}{l}\text { Width } \\
\text { [min] }\end{array}$ & $\begin{array}{c}\text { Area } \\
{\left[\mathrm{mAU}^{\star} \mathrm{s}\right]}\end{array}$ & $\begin{array}{l}\text { Height } \\
{[\mathrm{mAU}]}\end{array}$ & $\begin{array}{c}\text { Area } \\
\%\end{array}$ \\
\hline & 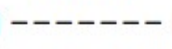 & & --- & --- & --- & -- \\
\hline 1 & 11.469 & BB & 2.2530 & $6.15681 \mathrm{e} 4$ & 339.61438 & 0000 \\
\hline
\end{tabular}

\section{Chiral HPLC of racemic standard.}

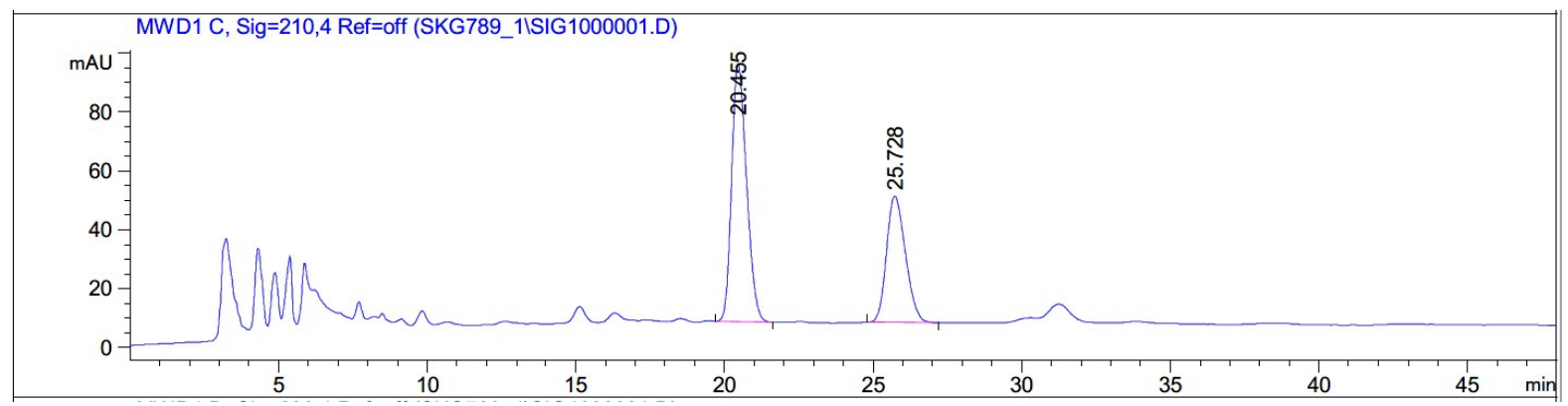

Signal 2: MWD1 C, Sig=210,4 Ref=off

\begin{tabular}{|c|c|c|c|c|c|c|}
\hline $\begin{array}{c}\text { Peak } \\
\#\end{array}$ & $\begin{array}{c}\text { RetTime } \\
\text { [min] }\end{array}$ & Type & $\begin{array}{l}\text { Width } \\
\text { [min] }\end{array}$ & $\begin{array}{c}\text { Area } \\
{\left[\mathrm{mAU}{ }^{\star} \mathrm{s}\right]}\end{array}$ & $\begin{array}{l}\text { Height } \\
\text { [mAU] }\end{array}$ & $\begin{array}{c}\text { Area } \\
\quad \%\end{array}$ \\
\hline & 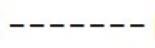 & & --- & ---------- & ------- & $-------\mid$ \\
\hline 1 & 20.455 & $\mathrm{BB}$ & 0.5434 & 3049.93042 & 87.34168 & 61.6539 \\
\hline 2 & 25.728 & $\mathrm{BB}$ & 0.6792 & 1896.92664 & 42.95952 & 38.3461 \\
\hline
\end{tabular}


Chiral HPLC of ATH product. $90 \%$ ee

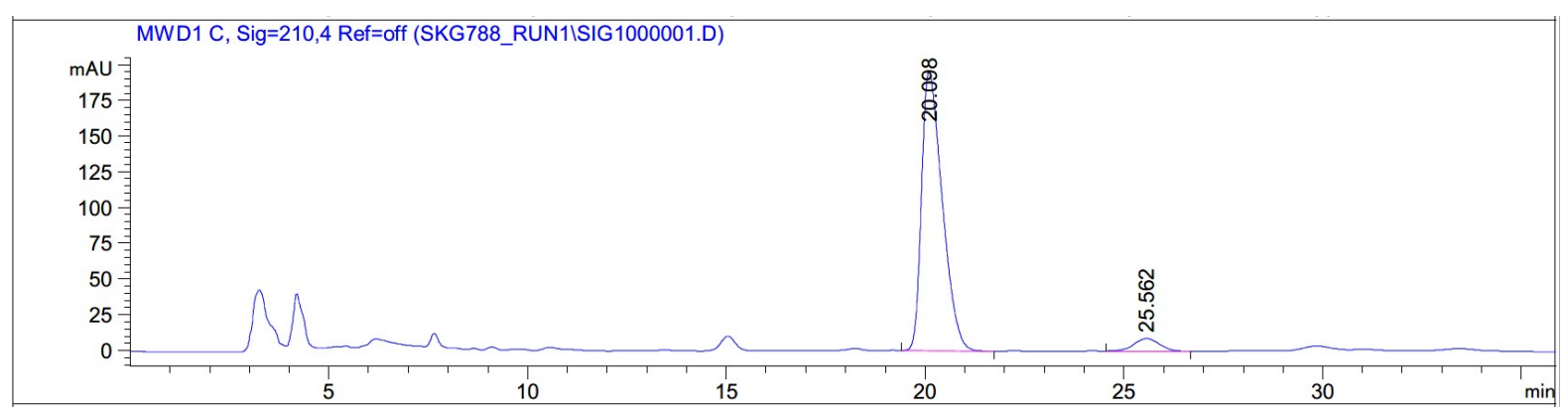

Signal 2: MWD1 C, Sig=210,4 Ref=off

\begin{tabular}{|c|c|c|c|c|c|c|}
\hline $\begin{array}{c}\text { Peak } \\
\#\end{array}$ & $\begin{array}{c}\text { RetTime } \\
\text { [min] }\end{array}$ & Type & $\begin{array}{l}\text { Width } \\
\text { [min] }\end{array}$ & $\begin{array}{c}\text { Area } \\
{\left[\mathrm{mAU}{ }^{*} \mathrm{~s}\right]}\end{array}$ & $\begin{array}{l}\text { Height } \\
{[\mathrm{mAU}]}\end{array}$ & $\begin{array}{c}\text { Area } \\
\quad \%\end{array}$ \\
\hline 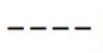 & & & & ---------- & ---------- & $--------\mid$ \\
\hline 1 & 20.098 & VB & 0.5507 & 7076.48389 & 195.31850 & 94.7924 \\
\hline 2 & 25.562 & $\mathrm{BB}$ & 0.6455 & 388.76294 & 8.83292 & 5.2076 \\
\hline
\end{tabular}




\section{(R)-2-Hydroxy- $N$-phenylhexanamide 43.}<smiles>CCCC[C@H](O)C(=O)Nc1ccccc1</smiles>

This compound is reported; however, it has not been fully characterized previously. This compound was prepared following general procedure $\mathrm{C}$ using 2-oxo- $N$-phenylhexanamide (51.3 mg, $0.250 \mathrm{mmol}, 1.0 \mathrm{eq}), 3 \mathrm{C}$ tethered catalyst $(R, R)-2(2.3 \mathrm{mg}, 3.8 \mu \mathrm{mol}, 0.015 \mathrm{eq})$ and FA:TEA $(0.15 \mathrm{~mL})$ in DCM $(1.5 \mathrm{~mL})$ to give 43 as a yellow semi solid material $(42.5 \mathrm{mg}$, $0.205 \mathrm{mmol}, 82 \%)$.

TLC: $\mathrm{R}_{\mathrm{f}}$ ca 0.4 (7:3, hexane: EtOAc), UV-active, Strong $\mathrm{KMnO}_{4}$ and PMA-reactive; HRMS (ESI) m/z: [M+Na] $]^{+}$Calcd for $\mathrm{C}_{12} \mathrm{H}_{17} \mathrm{NO}_{2} \mathrm{Na} 230.1151$; Found 230.1158 (error -2.7 ppm);

$U_{\max } 3281,2953,2867,1649,1598,1543,1442,1327,1083,751,691 \mathrm{~cm}^{-1}$;

Enantiomeric excess determined by HPLC analysis (Chiralcel OD-H, $250 \mathrm{~mm} \times 4.6 \mathrm{~mm}$ column hexane: $\left.\mathrm{iPrOH} 90: 10,1 \mathrm{~mL} / \mathrm{min}, \lambda=210 \mathrm{~nm}, \mathrm{~T}=25^{\circ} \mathrm{C}\right), S$-enantiomer $9.7 \mathrm{~min}, R$ enantiomer $23.1 \min .93 \%$ ee $(R),[\alpha]_{\mathrm{D}}^{22}=+217.5\left(\mathrm{c}=0.02, \mathrm{CHCl}_{3}\right)$;

${ }^{1} \mathrm{H}$ NMR (500 MHz, CDCl 3$) \delta 8.40(1 \mathrm{H}, \mathrm{s}, \mathrm{CONH}), 7.57(2 \mathrm{H}, \mathrm{d}, J=8.1 \mathrm{~Hz}, \mathrm{ArH}), 7.34(2 \mathrm{H}$, t, $J=7.9 \mathrm{~Hz}, \mathrm{ArH}), 7.12(1 \mathrm{H}, \mathrm{t}, J=7.4 \mathrm{~Hz}, \mathrm{ArH}), 4.26$ (1H, dd, $J=7.6,3.3 \mathrm{~Hz}, \mathrm{CHOH}), 2.63$ $(1 \mathrm{H}, \mathrm{s}, \mathrm{CHO} \underline{\mathrm{H}}), 1.98-1.92\left(1 \mathrm{H}, \mathrm{m}, \mathrm{C}_{\underline{\mathrm{a}}} \mathrm{H}_{\mathrm{b}}\right), 1.79-1.71\left(1 \mathrm{H}, \mathrm{m}, \mathrm{CH}_{\mathrm{a}} \underline{\mathrm{H}_{\mathrm{b}}}\right), 1.48-1.35(4 \mathrm{H}, \mathrm{m}$, $\left.\mathrm{CH}_{2}\right), 0.92\left(3 \mathrm{H}, \mathrm{t}, J=7.2 \mathrm{~Hz}, \mathrm{CH}_{3}\right)$;

${ }^{13} \mathrm{C} \mathrm{NMR}\left(126 \mathrm{MHz}, \mathrm{CDCl}_{3}\right) \delta 171.9,137.4,129.2,124.6,119.9,72.9,34.7,27.3,22.6,14.1$; $m / z(\mathrm{ESI}) 230.0\left[(\mathrm{M}+\mathrm{Na})^{+}, 30 \%\right], 437.3\left[(2 \mathrm{M}+\mathrm{Na})^{+}, 100 \%\right]$.

Onomura, O.; Mitsuda, M.; Nguyen, M. T. T.; Demizu, Y.“Asymmetric tosylation of racemic 2-hydroxyalkanamides with chiral copper catalyst", Tetrahedron Lett. 2007, 48, 9080-9084. 


\section{2-Hydroxy- $N$-phenylhexanamide 43.}

${ }^{1} \mathrm{H}$ NMR $\left(500 \mathrm{MHz}, \mathrm{CDCl}_{3}\right)$.

Sep24-2020

Chemist Shweta Gediya

PROTON.w CDCl3 /opt/topspin3.2 SKG 33<smiles>CCCCC(O)C(=O)Nc1ccccc1</smiles><smiles>CCCCC</smiles>

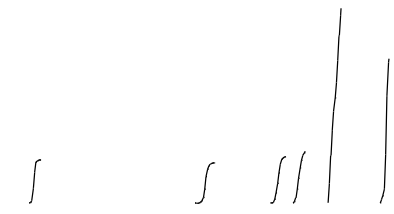

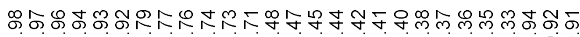

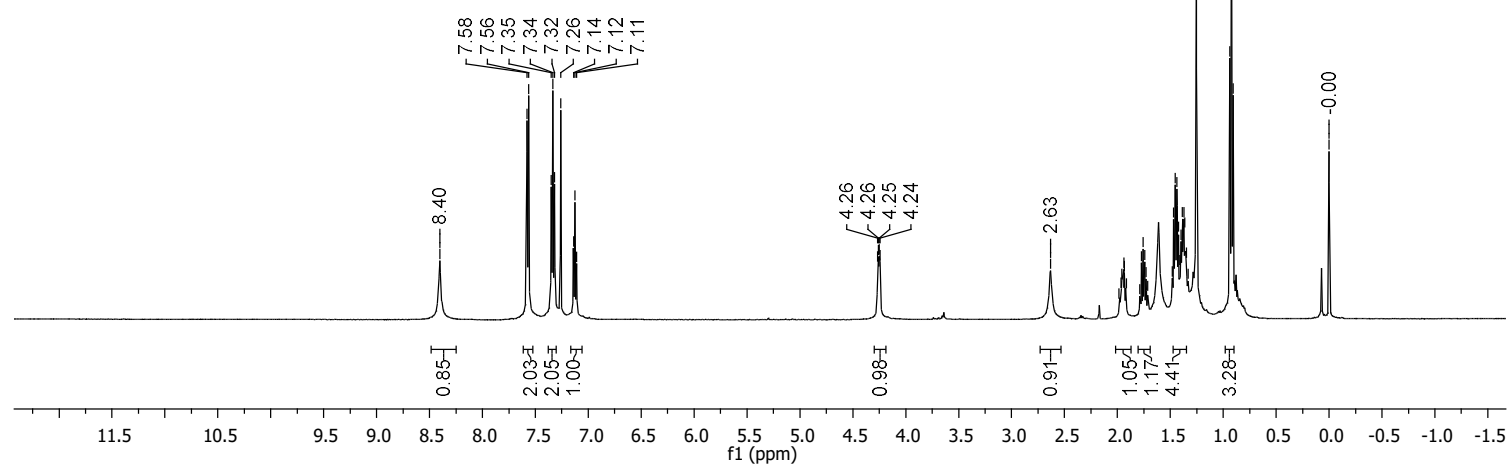

${ }^{13} \mathrm{C}$ NMR $\left(126 \mathrm{MHz}, \mathrm{CDCl}_{3}\right)$.

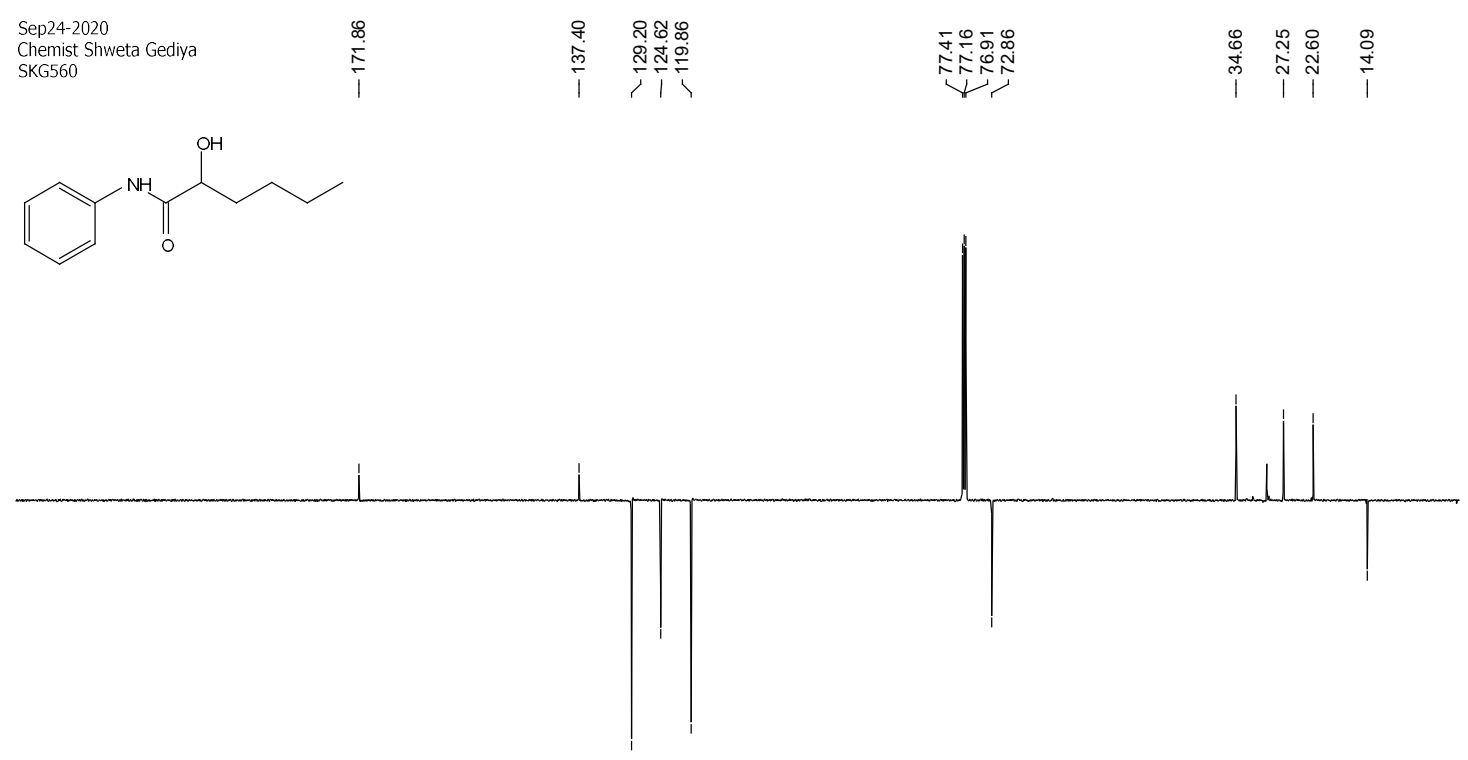

$\begin{array}{lllllllllllllllllllllll}220 & 210 & 200 & 190 & 180 & 170 & 160 & 150 & 140 & 130 & 120 & 110 & 100 & 90 & 80 & 70 & 60 & 50 & 40 & 30 & 20 & 10 & 0\end{array}$


COSY.

Chemist Shweta Gediya

COSY.w CDCl3/opt/topspin3.2 SKG 33

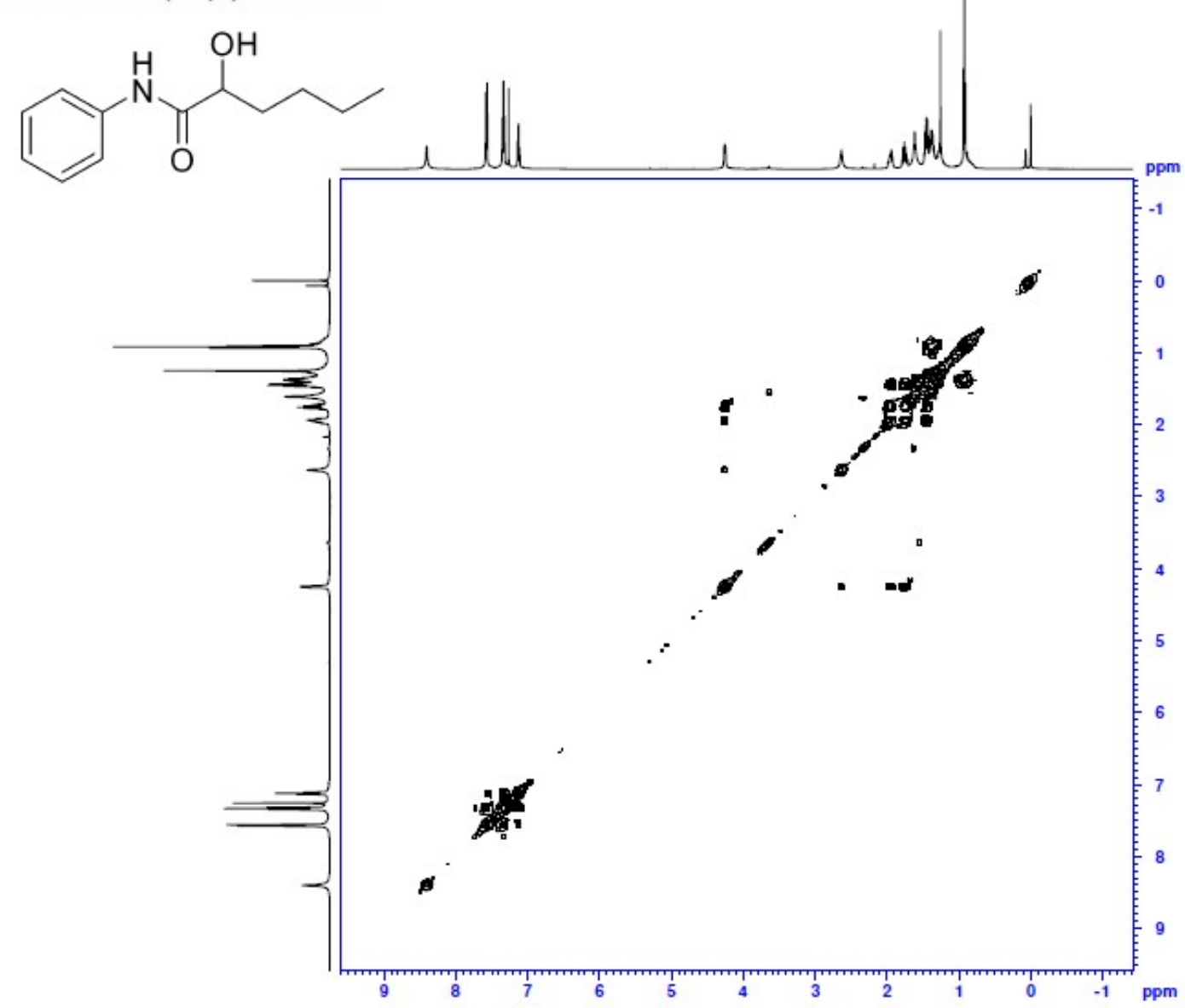

Chiral HPLC of ketone precursor.

[3060

4 Clarity $\backslash$ WIORK1। DATAI Shwetal after lockdownl SKG555_ketone_OD-H_10\% IPA_1mpm_210nm_18_08_2020 11_29_33 - INT7 - 1

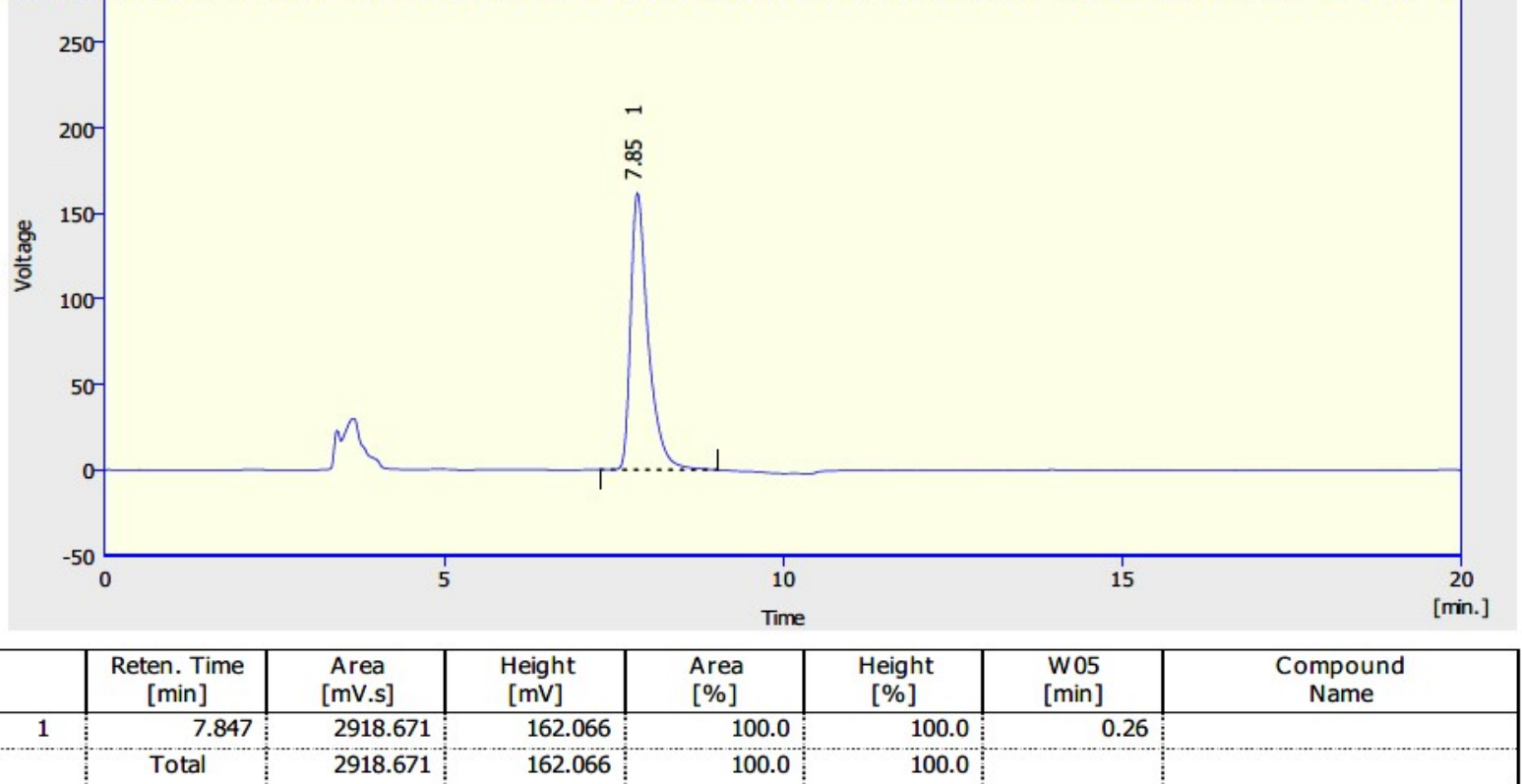




\section{Chiral HPLC of racemic alcohol.}

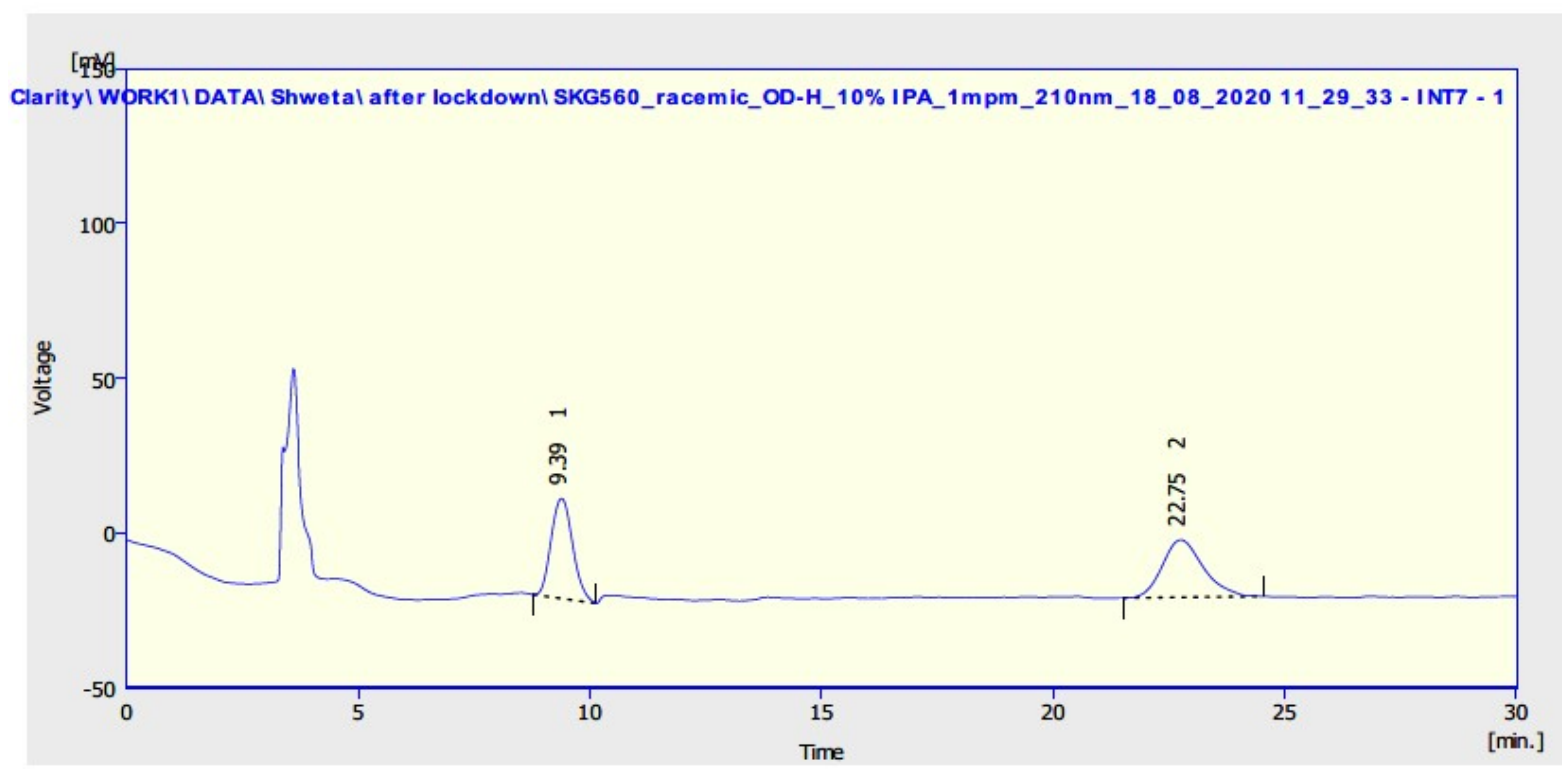

\begin{tabular}{|c|c|c|c|r|r|r|r|}
\hline & $\begin{array}{c}\text { Reten. Time } \\
{[\mathrm{min}]}\end{array}$ & $\begin{array}{c}\text { Area } \\
{[\mathrm{mV} . \mathrm{s}]}\end{array}$ & $\begin{array}{c}\text { Height } \\
{[\mathrm{mV}]}\end{array}$ & $\begin{array}{c}\text { Area } \\
{[\%]}\end{array}$ & $\begin{array}{c}\text { Height } \\
{[\%]}\end{array}$ & $\begin{array}{c}\text { W05 } \\
{[\mathrm{min}]}\end{array}$ & $\begin{array}{c}\text { Compound } \\
\text { Name }\end{array}$ \\
\hline 1 & 9.390 & 1044.472 & 32.424 & 47.7 & 63.6 & 0.51 & \\
\hline 2 & 22.750 & 1144.951 & 18.582 & 52.3 & 36.4 & 0.94 & \\
& Total & 2189.422 & 51.006 & 100.0 & 100.0 & & \\
\hline
\end{tabular}

\section{Chiral HPLC of ATH product.. 93\%}

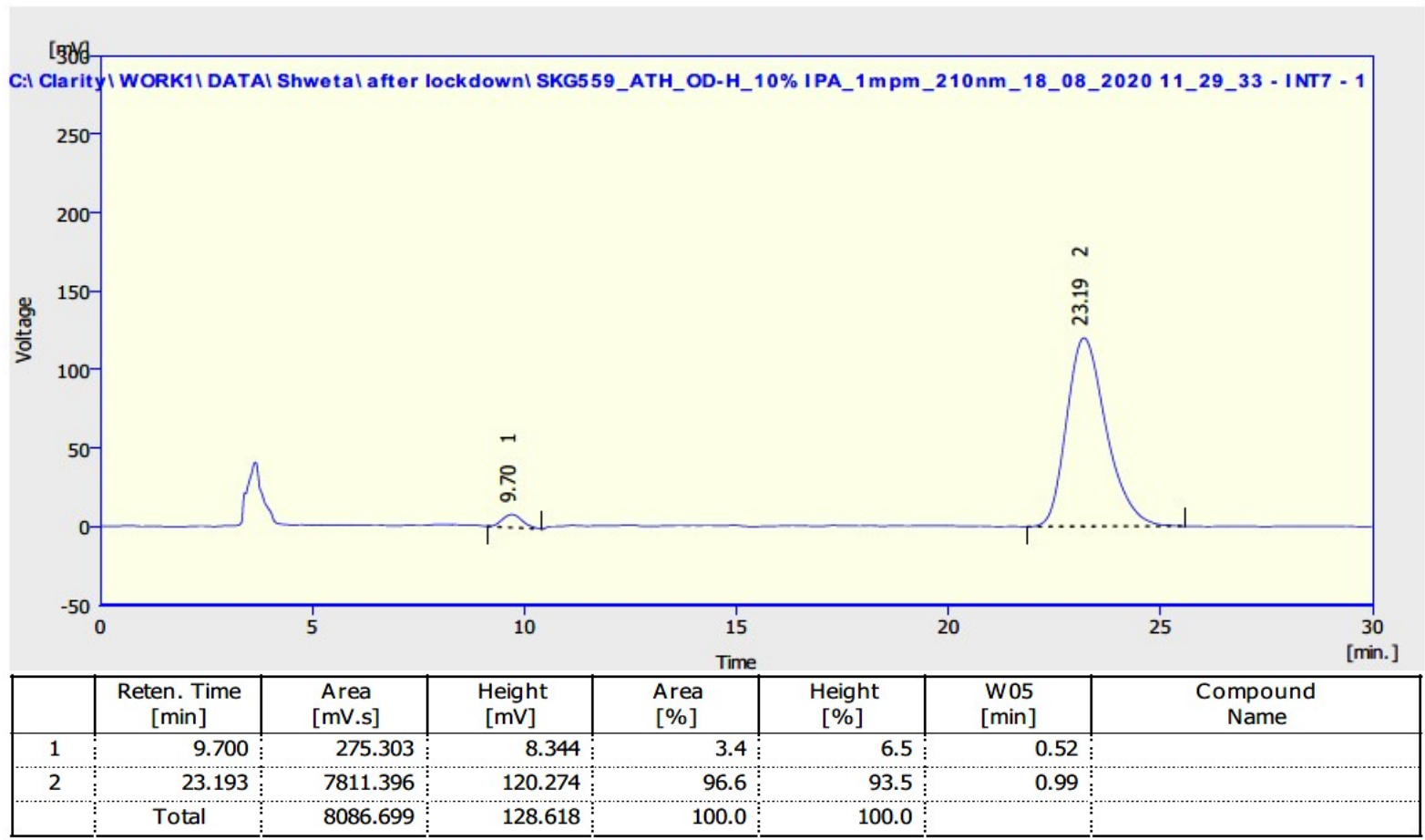


(R)-N-Ethyl-2-hydroxyhexanamide 44.<smiles>CCCCC(O)C(=O)NCC</smiles>

This compound has been reported however it has not been characterised previously.

This compound was prepared following general procedure C $N$-ethyl-2-oxohexanamide (39.3 $\mathrm{mg}, 0.250 \mathrm{mmol}, 1.0 \mathrm{eq}), 3 \mathrm{C}$ tethered catalyst $(R, R)-2(2.3 \mathrm{mg}, 3.8 \mu \mathrm{mol}, 0.015 \mathrm{eq})$ and FA:TEA $(0.15 \mathrm{~mL})$ in DCM $(1.5 \mathrm{~mL})$ to give 44 as a yellow oil $(34.2 \mathrm{mg}, 0.215 \mathrm{mmol}, 86 \%)$. TLC: $\mathrm{R}_{\mathrm{f}}$ ca 0.3 (7:3, hexane: EtOAc), non UV-active, Strong $\mathrm{KMnO}_{4}$ and PMA-reactive; HRMS (ESI) m/z: $[\mathrm{M}+\mathrm{Na}]^{+}$Calcd for $\mathrm{C}_{8} \mathrm{H}_{17} \mathrm{NO}_{2} \mathrm{Na} 182.1151$; Found 182.1151 (error 0.1 ppm);

$U_{\max } 3304,1642,1594,1534,1493,1442,1397,1252,1232,1002,698,553 \mathrm{~cm}^{-1}$;

Enantiomeric excess determined by HPLC analysis (Chiralpak AD-H, 250mm $\times 4.6 \mathrm{~mm}$ column, hexane: iPrOH 93:7, $1 \mathrm{~mL} / \mathrm{min}, \lambda=210 \mathrm{~nm}, \mathrm{~T}=25^{\circ} \mathrm{C}$ ), $R$-enantiomer $7.0 \mathrm{~min}, S$ enantiomer $8.1 \mathrm{~min} .90 \%$ ee $(R),[\alpha]_{\mathrm{D}}^{22}=+7\left(\mathrm{c}=0.1, \mathrm{CHCl}_{3}\right)$;

${ }^{1} \mathrm{H}$ NMR (500 MHz, $\left.\mathrm{CDCl}_{3}\right) \delta 6.61(1 \mathrm{H}, \mathrm{s}, \mathrm{CONH}), 4.08(1 \mathrm{H}, \mathrm{dd}, J=7.6,3.3 \mathrm{~Hz}, \mathrm{C} \underline{\mathrm{HOH}})$, 3.36-3.28 (2H, m, $\mathrm{CH}_{2}$ of ethylamine), $3.18(1 \mathrm{H}, \mathrm{s}, \mathrm{CHOH}), 1.86-1.80\left(1 \mathrm{H}, \mathrm{m}, \mathrm{CH}_{\mathrm{a}} \mathrm{H}_{\mathrm{b}}\right), 1.66-$ $1.60\left(1 \mathrm{H}, \mathrm{m}, \mathrm{CH}_{\mathrm{a}} \mathrm{H}_{\mathrm{b}}\right), 1.43-1.27\left(4 \mathrm{H}, \mathrm{m}, \mathrm{CH}_{2}\right), 1.16\left(3 \mathrm{H}, \mathrm{t}, J=7.3 \mathrm{~Hz}, \mathrm{CH}_{3}\right.$ of ethylamine $)$, $0.91\left(3 \mathrm{H}, \mathrm{t}, J=7.1 \mathrm{~Hz}, \mathrm{CH}_{3}\right.$ of n-butyl group);

${ }^{13} \mathrm{C} \mathrm{NMR}\left(126 \mathrm{MHz}, \mathrm{CDCl}_{3}\right) \delta 174.4,72.2,34.7,34.1,27.2,22.6,14.9,14.1$;

$m / z(\mathrm{ESI}) 182.1\left[(\mathrm{M}+\mathrm{Na})^{+}, 100 \%\right]$. 
N-Ethyl-2-hydroxyhexanamide 44.

${ }^{1} \mathrm{H}$ NMR (500 MHz, $\mathrm{CDCl}_{3}$ ).

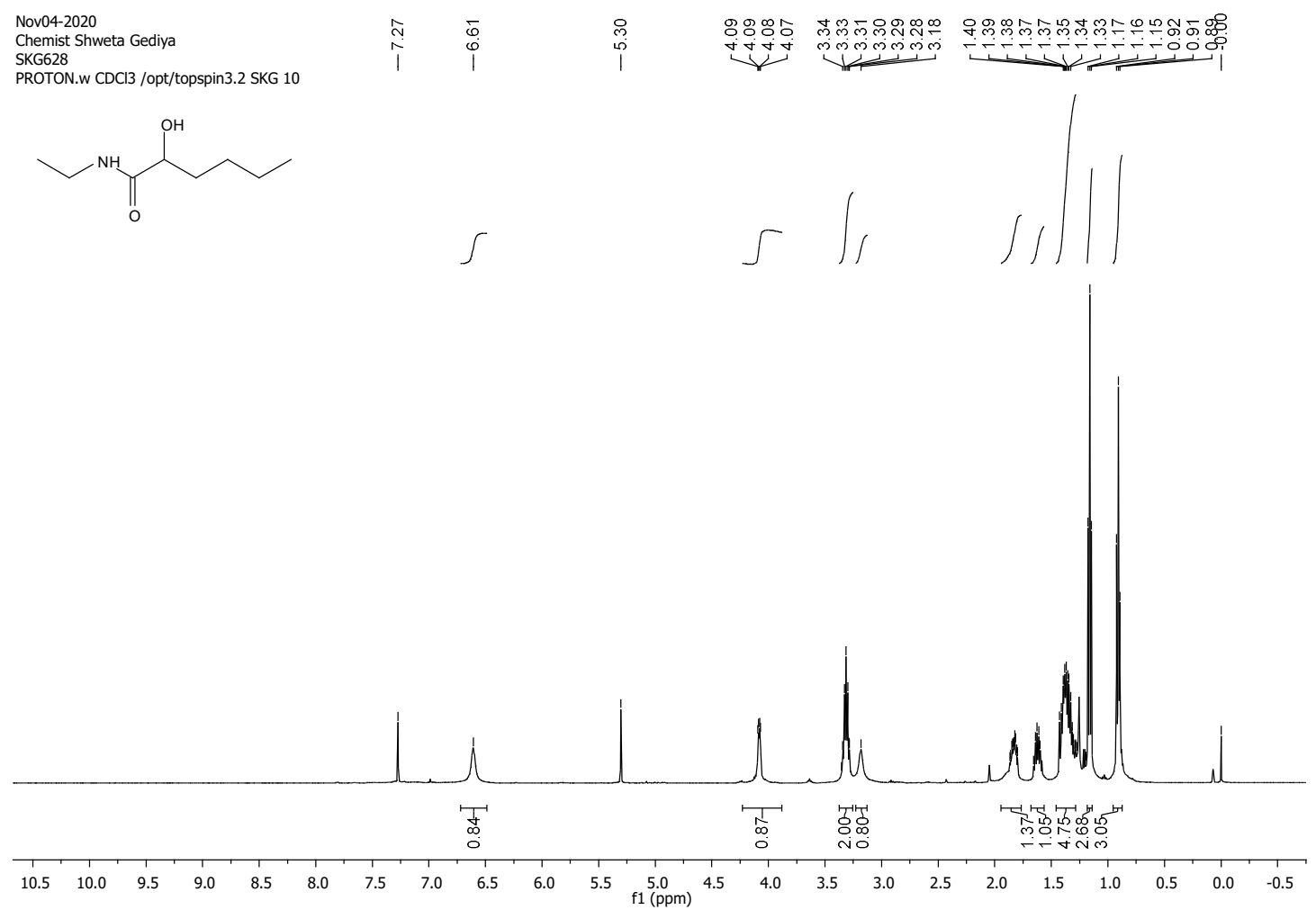

$\left.{ }^{13} \mathrm{C} \mathrm{NMR} \mathrm{(126} \mathrm{MHz,} \mathrm{CDCl}_{3}\right)$.

Nov04-2020

Shweta Gediya

SKG628 13 APTlong.w CDCl3

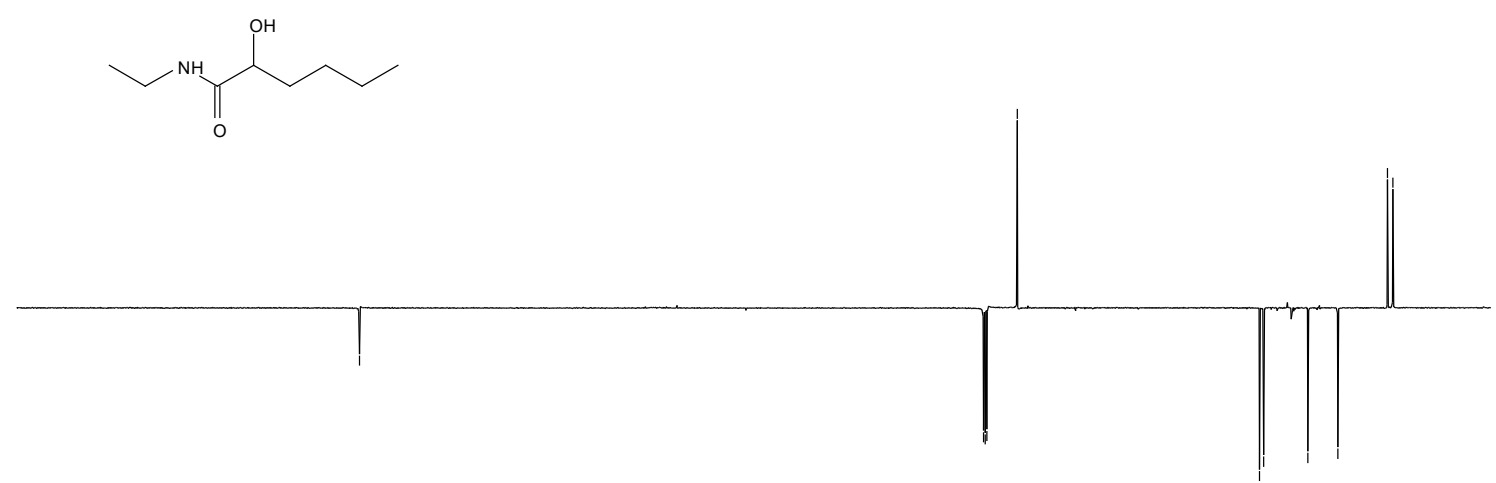

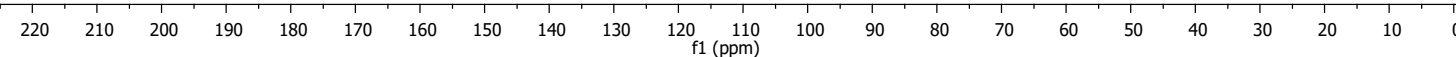


COSY.

Chemist Shweta Gediya

SKG628

COSY.w CDCl3/opt/topspin3.2 SKG 10

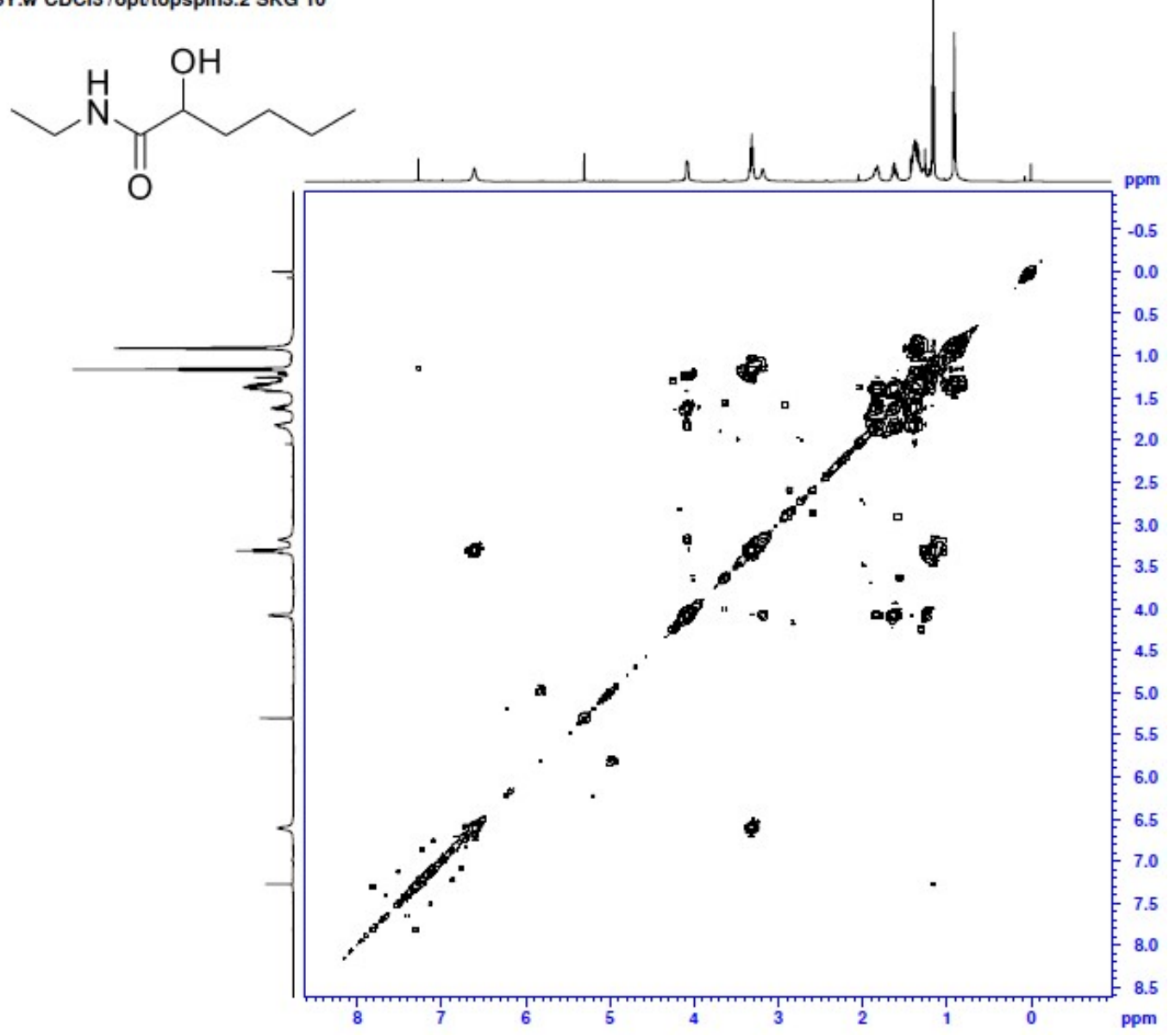

\section{Chiral HPLC of ketone precursor.}

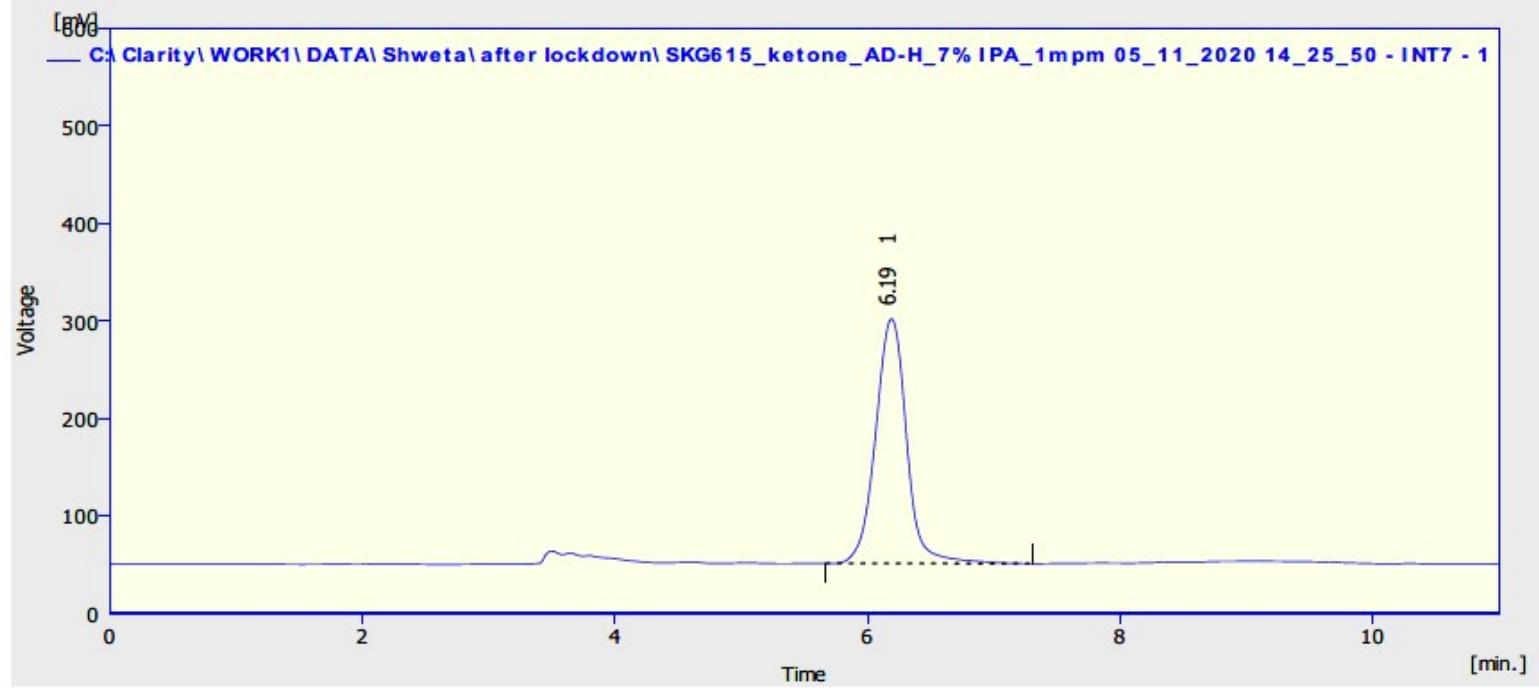

\begin{tabular}{|c|c|c|c|c|c|c|c|}
\hline & $\begin{array}{c}\text { Reten. Time } \\
{[\mathrm{min}]}\end{array}$ & $\begin{array}{c}\text { Area } \\
{[\mathrm{mV} . \mathrm{s}]}\end{array}$ & $\begin{array}{c}\text { Height } \\
{[\mathrm{mV}]}\end{array}$ & $\begin{array}{c}\text { Area } \\
{[\%]}\end{array}$ & $\begin{array}{c}\text { Height } \\
{[\%]}\end{array}$ & $\begin{array}{c}\text { W05 } \\
{[\mathrm{min}]}\end{array}$ & $\begin{array}{c}\text { Compound } \\
\text { Name }\end{array}$ \\
\hline 1 & 6.190 & 4281.337 & 250.974 & 100.0 & 100.0 & 0.26 & \\
\hline & Total & 4281.337 & 250.974 & 100.0 & 100.0 & & \\
\hline
\end{tabular}




\section{Chiral HPLC of racemic alcohol.}

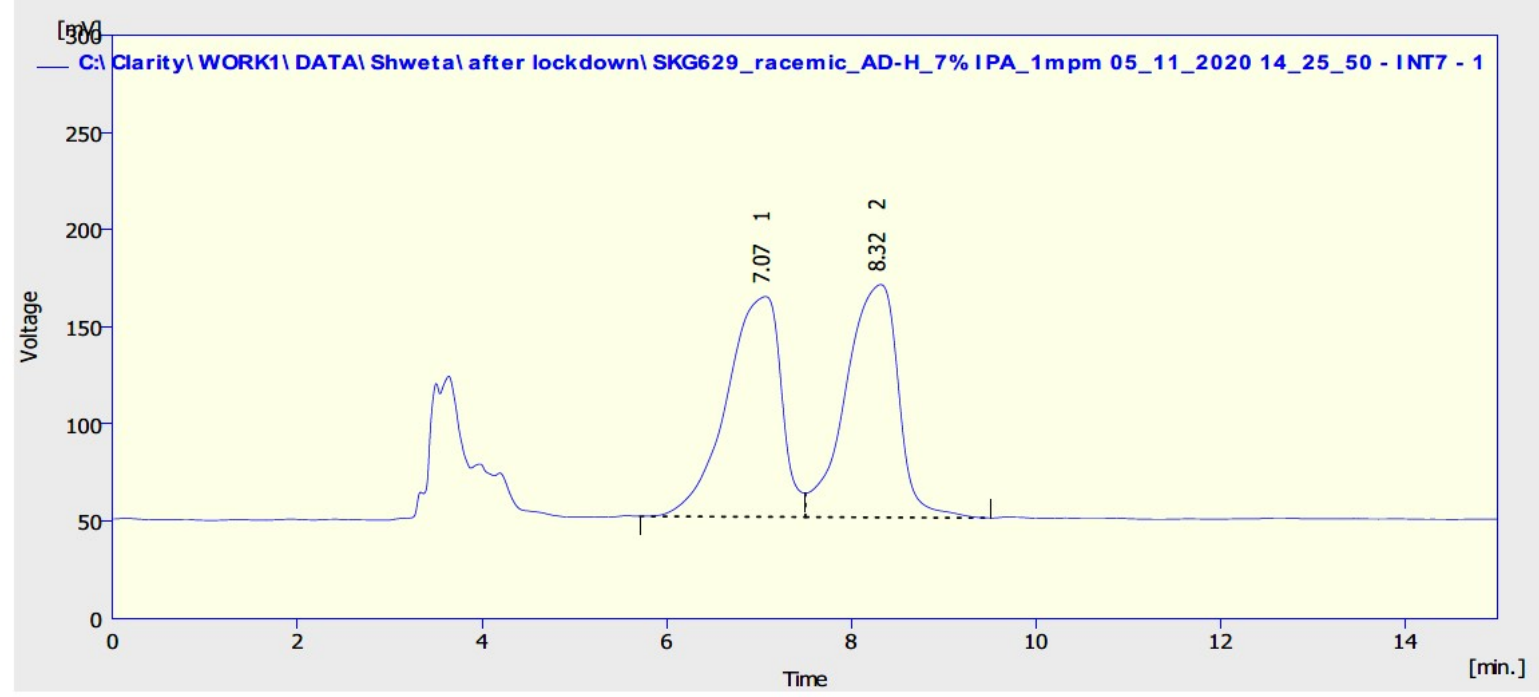

\begin{tabular}{|c|c|c|c|c|c|c|c|}
\hline & $\begin{array}{l}\text { Reten. Time } \\
\text { [min] }\end{array}$ & $\begin{array}{c}\text { Area } \\
\text { [mV.s] }\end{array}$ & $\begin{array}{c}\text { Height } \\
{[\mathrm{mV}]}\end{array}$ & $\begin{array}{l}\text { Area } \\
{[\%]}\end{array}$ & $\begin{array}{c}\text { Height } \\
{[\%]}\end{array}$ & $\begin{array}{l}\text { W05 } \\
\text { [min] }\end{array}$ & $\begin{array}{c}\text { Compound } \\
\text { Name }\end{array}$ \\
\hline 2 & 8.320 & 4755.555 & 119.949 & 50.3 & 51.4 & 0.61 & \\
\hline
\end{tabular}

Chiral HPLC of ATH product. 90\% ee.

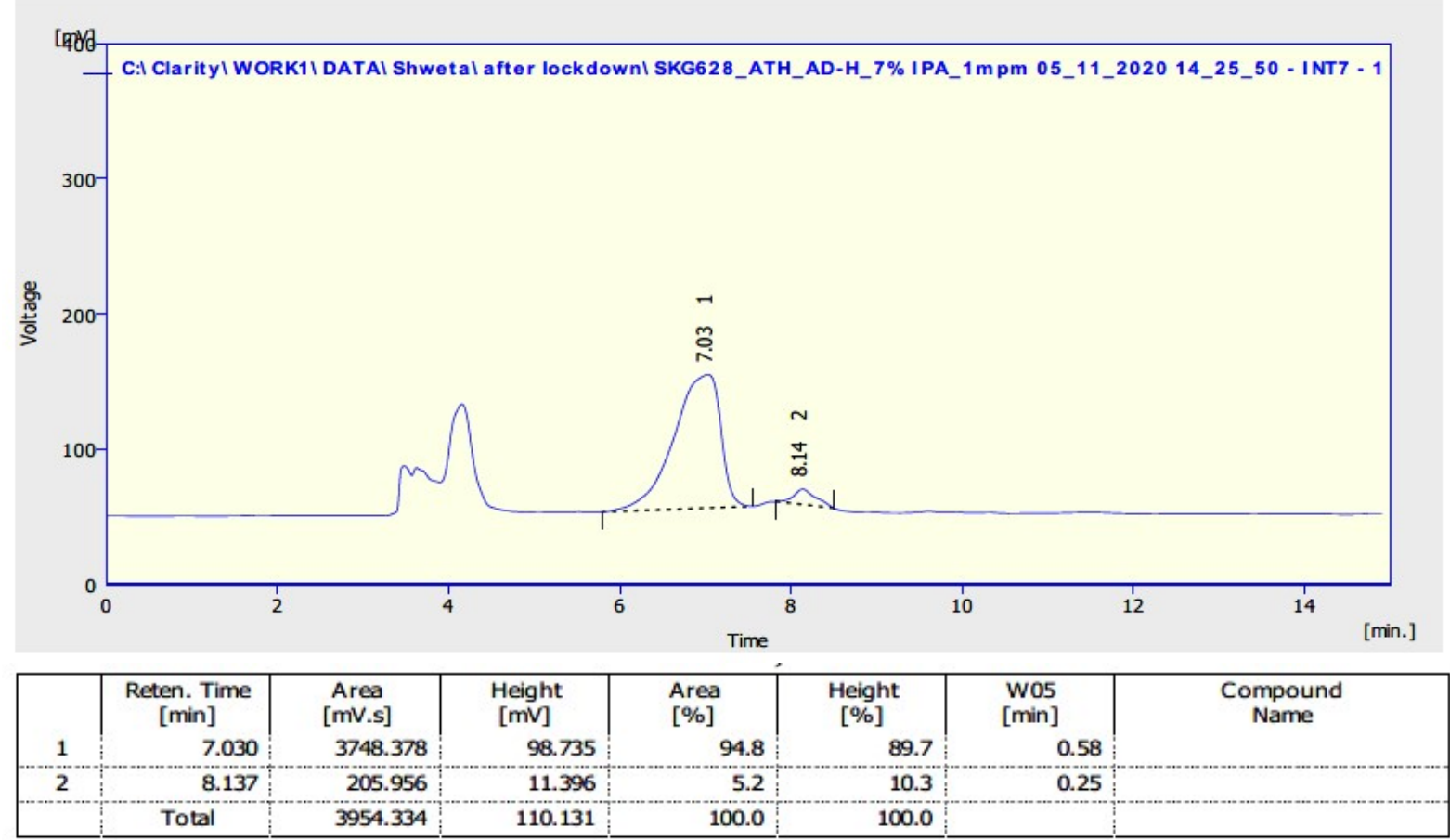




\section{(R)-2-Hydroxy- $N$-methoxy- $N$-methylhexanamide 45.}<smiles>CCCCC(O)C(=O)N(C)OC</smiles>

This compound has been reported and fully characterized.

This compound was prepared following general procedure $\mathrm{C}$ using $N$-methoxy- $N$-methyl-2oxohexanamide (43.3 mg, $0.250 \mathrm{mmol}, 1.0 \mathrm{eq}), 3 \mathrm{C}$ tethered catalyst $(R, R)-2(2.3 \mathrm{mg}, 3.8$ $\mu \mathrm{mol}, 0.015 \mathrm{eq})$ and FA:TEA $(0.15 \mathrm{~mL})$ in DCM $(1.5 \mathrm{~mL})$ to give 45 as a brown liquid (36.1 $\mathrm{mg}, 0.206 \mathrm{mmol}, 82 \%)$.

TLC: $\mathrm{R}_{\mathrm{f}}$ ca 0.3 (7:3, hexane: EtOAc), non UV-active, Strong $\mathrm{KMnO}_{4}$ and PMA-reactive; HRMS (ESI) m/z: $[\mathrm{M}+\mathrm{Na}]^{+}$Calcd for $\mathrm{C}_{8} \mathrm{H}_{17} \mathrm{NO}_{3} \mathrm{Na}$ 198.1101; Found 198.1108 (error -3.6 ppm);

Enantiomeric excess determined by HPLC analysis (Chiralpak IC, $250 \mathrm{~mm} \times 4.6 \mathrm{~mm}$ column, hexane: $\left.\mathrm{iPrOH} 90: 10,1 \mathrm{~mL} / \mathrm{min}, \lambda=210 \mathrm{~nm}, \mathrm{~T}=25^{\circ} \mathrm{C}\right), R$-enantiomer $17.1 \mathrm{~min}, S$ enantiomer $19.1 \mathrm{~min} .82 \%$ ee $(R),[\alpha]_{\mathrm{D}}^{22}=+41.3\left(\mathrm{c}=0.04, \mathrm{CHCl}_{3}\right)$;

${ }^{1} \mathrm{H}$ NMR (400 MHz, $\left.\mathrm{CDCl}_{3}\right) \delta 4.39(1 \mathrm{H}, \mathrm{s}, \mathrm{C} \underline{\mathrm{HOH}}), 3.72\left(3 \mathrm{H}, \mathrm{s}, \mathrm{OCH}_{3}\right), 3.25\left(3 \mathrm{H}, \mathrm{s}, \mathrm{NCH}_{3}\right)$, 1.75-1.68 (1H, m, $\left.\underline{\mathrm{CH}}_{\mathrm{a}} \mathrm{H}_{\mathrm{b}}\right), 1.55-1.24\left(5 \mathrm{H}, \mathrm{m}, \mathrm{CH}_{\mathrm{a}} \underline{\mathrm{H}_{\mathrm{b}} \mathrm{CH}_{2} \mathrm{CH}_{2}}\right), 0.91-0.87\left(3 \mathrm{H}, \mathrm{m}, \mathrm{CH}_{3}\right)$;

${ }^{13} \mathrm{C}$ NMR (101 MHz, $\left.\mathrm{CDCl}_{3}\right) \delta 175.4,68.7,61.4,34.5,32.5,27.2,22.5,14.0$; $m / z(\mathrm{ESI}) 198.1\left[(\mathrm{M}+\mathrm{Na})^{+}, 60 \%\right], 373.2\left[(2 \mathrm{M}+\mathrm{Na})^{+}, 100 \%\right]$.

The data matches the reported data.

Murata, T.; Kawanishi, T.; Sekiguchi, A.; Ishikawa, R.; Ono, K.; Nakata, K.; Shiina, I. Kinetic Resolution of Racemic 2-Hydroxyamides Using a Diphenylacetyl Component as an Acyl Source and a Chiral Acyl-Transfer Catalyst, Molecules 2018, 23, 2003, 1-19. 
2-Hydroxy- $N$-methoxy- $N$-methylhexanamide 45.

${ }^{1} \mathrm{H}$ NMR (400 MHz, $\left.\mathrm{CDCl}_{3}\right)$.

Oct30-2020

PROTON.w CDCl3/opt/topspin3.5pl2 SKG 28

$\stackrel{\substack{\text { i } \\ \text { i }}}{1}$<smiles>CCCCC(O)C(=O)N(C)OC</smiles>

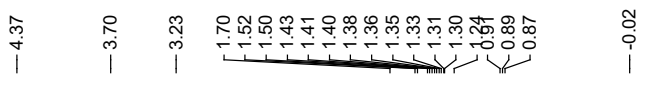

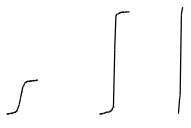

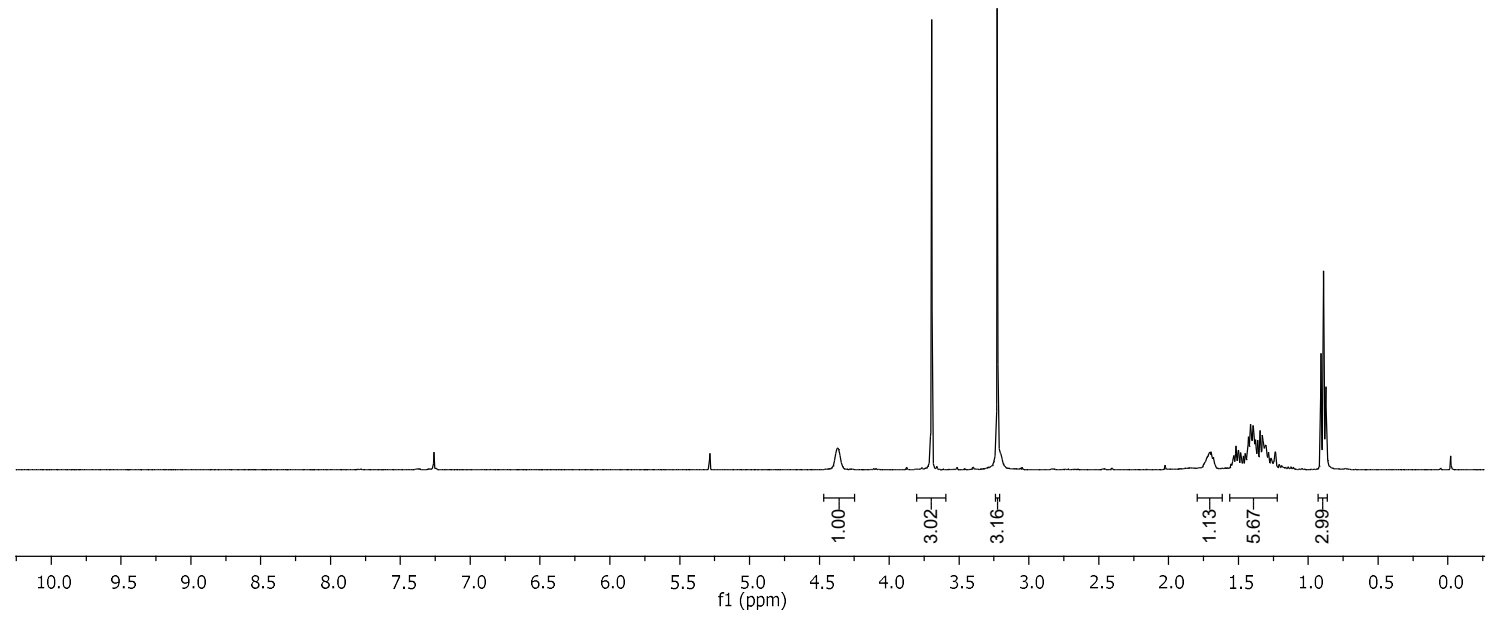

${ }^{13} \mathrm{C}$ NMR (101 MHz, $\left.\mathrm{CDCl}_{3}\right)$.

Oct30-2020
SKG619

C13APTlong.w CDCl3

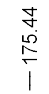

$\overbrace{0}^{-N_{0}}$

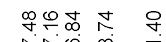

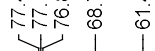

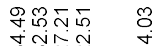

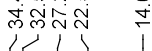

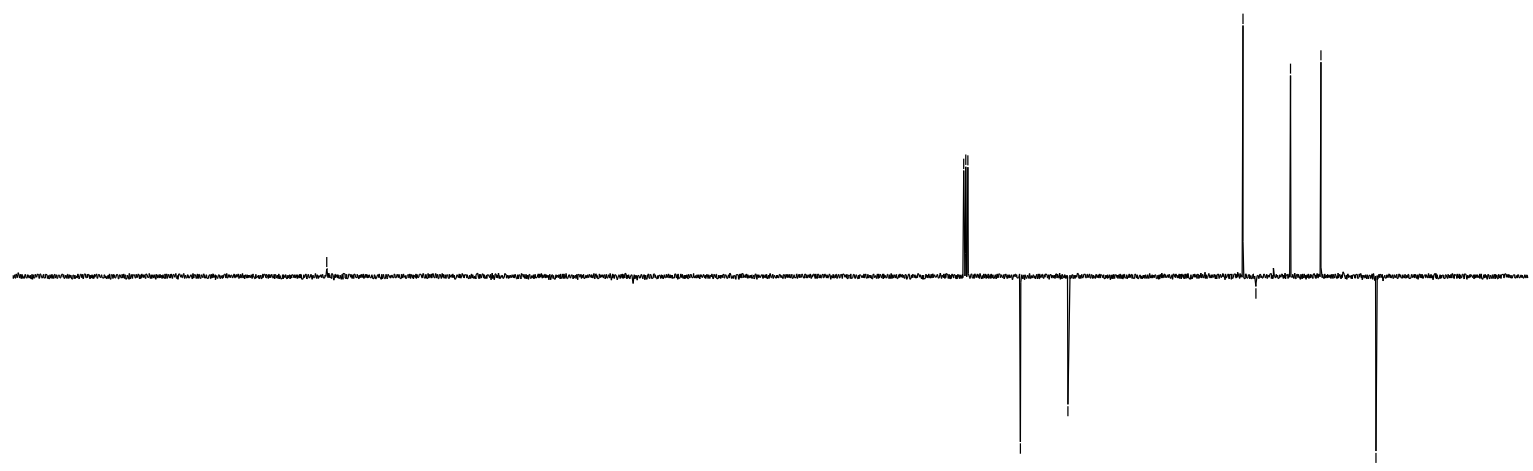

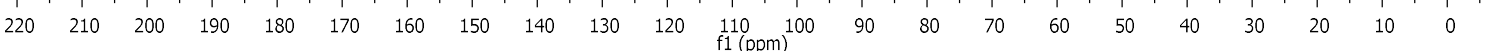


COSY.

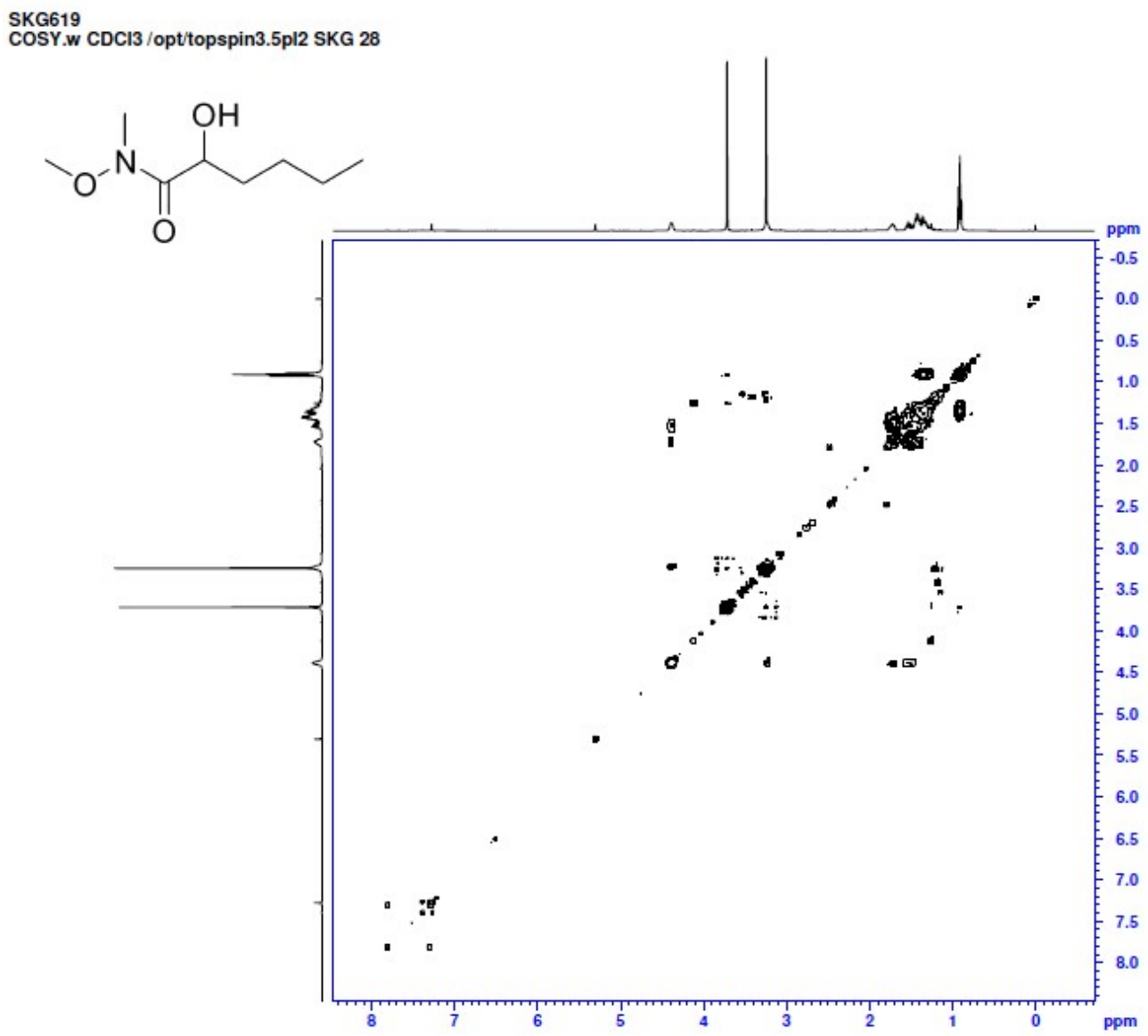

\section{Chiral HPLC of ketone precursor.}

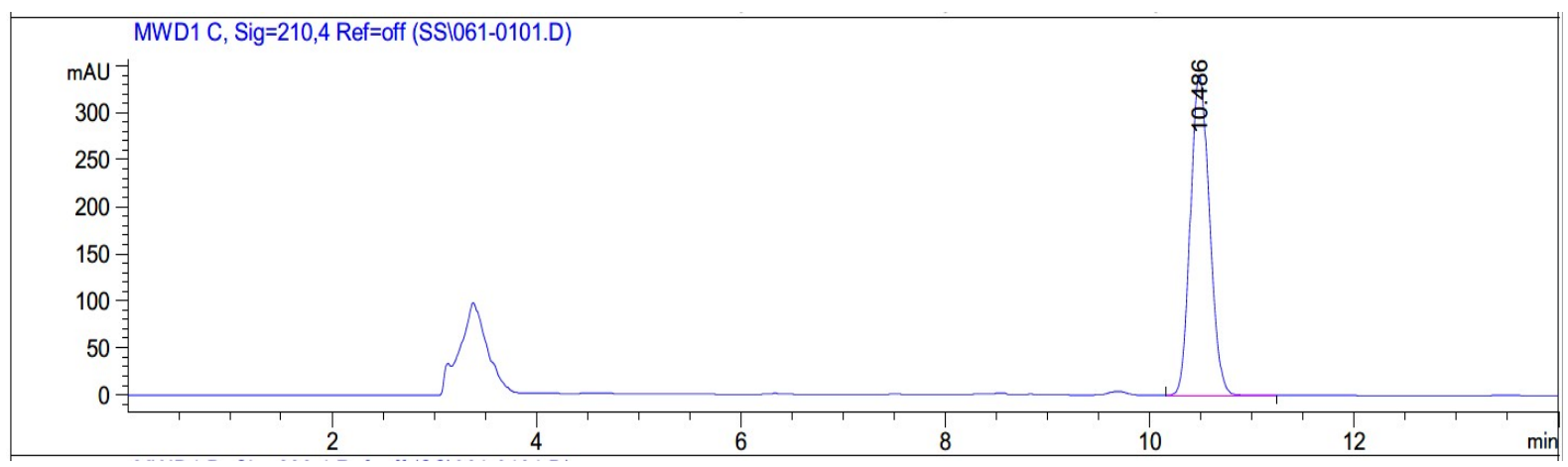

Signal 2: MWD1 C, Sig=210,4 Ref=off

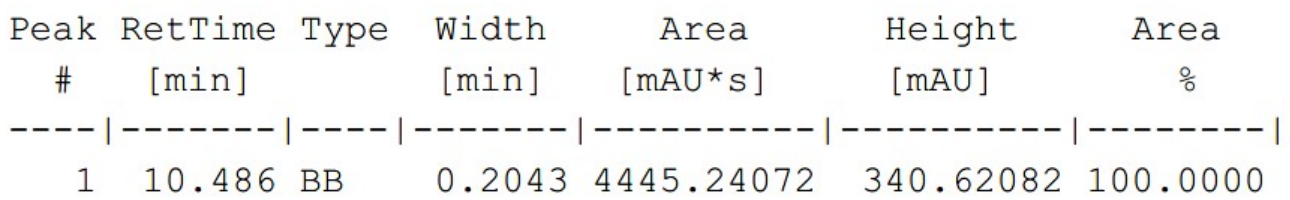


Chiral HPLC of racemic alcohol.

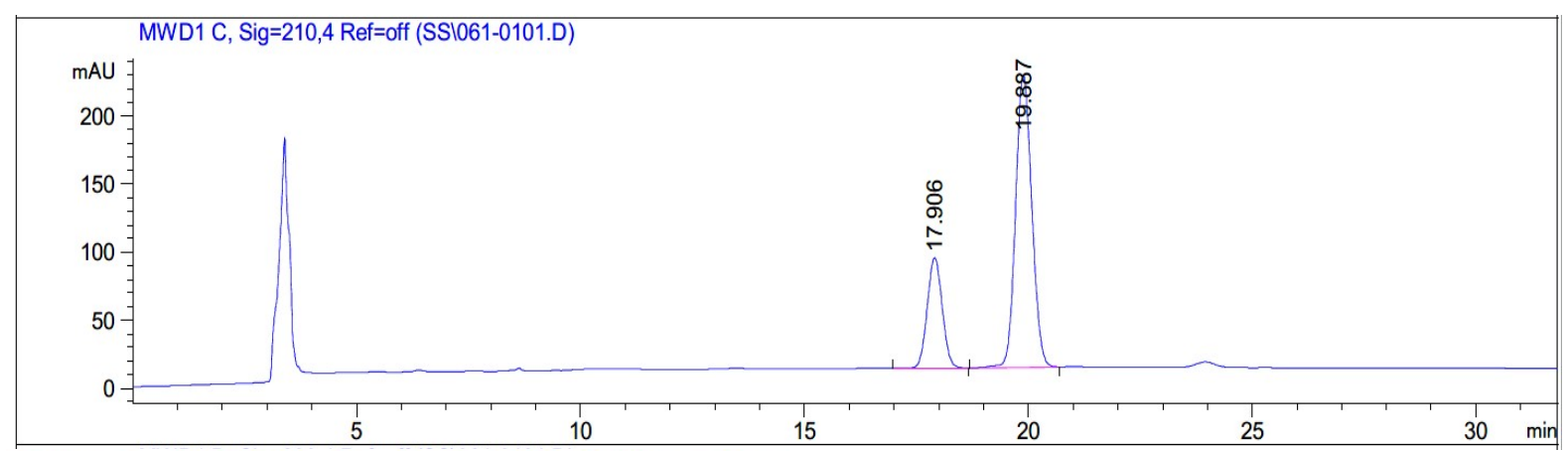

Signal 2: MWD1 C, Sig=210,4 Ref=off

\begin{tabular}{|c|c|c|c|c|c|c|}
\hline $\begin{array}{c}\text { Peak } \\
\#\end{array}$ & $\begin{array}{c}\text { RetTime } \\
\text { [min] }\end{array}$ & Type & $\begin{array}{l}\text { Width } \\
\text { [min] }\end{array}$ & $\begin{array}{c}\text { Area } \\
{\left[\mathrm{mAU}{ }^{*} \mathrm{~s}\right]}\end{array}$ & $\begin{array}{l}\text { Height } \\
{[\mathrm{mAU}]}\end{array}$ & $\begin{array}{c}\text { Area } \\
\frac{\circ}{0}\end{array}$ \\
\hline & - & & -- & ------ & -ー-ー-ー-ー & ---- \\
\hline 1 & 906 & $\mathrm{BB}$ & 0.3633 & 1886.37146 & 81.13036 & 25.8166 \\
\hline 2 & 19.887 & $\mathrm{BB}$ & 0.3937 & 5420.43115 & 215.34433 & 74.1834 \\
\hline
\end{tabular}

Chiral HPLC of ATH product. $82 \%$ ee.

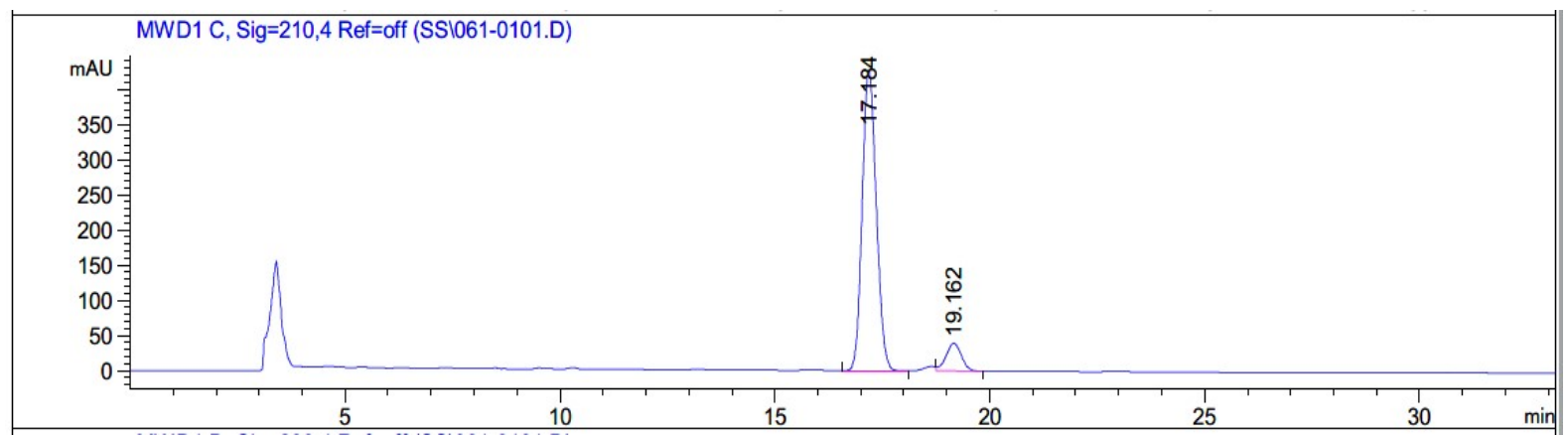

Signal 2: MWD1 C, Sig=210,4 Ref=off

\begin{tabular}{|c|c|c|c|c|c|c|}
\hline $\begin{array}{c}\text { Peak } \\
\#\end{array}$ & $\begin{array}{c}\text { RetTime } \\
\text { [min] }\end{array}$ & Type & $\begin{array}{l}\text { Width } \\
\text { [min] }\end{array}$ & $\begin{array}{c}\text { Area } \\
{\left[\mathrm{mAU}^{*} \mathrm{~s}\right]}\end{array}$ & $\begin{array}{l}\text { Height } \\
{[\mathrm{mAU}]}\end{array}$ & $\begin{array}{c}\text { Area } \\
\%\end{array}$ \\
\hline & & & & & & \\
\hline 1 & $t$ & $\mathrm{BB}$ & & 9710. & 427 & 17 \\
\hline 2 & 19 & B & 37 & 981.29980 & 9.52174 & .783 \\
\hline
\end{tabular}




\section{Ethyl (R)-2-hydroxyhexanoate 46.}<smiles>CCCCC(O)C(=O)OCC</smiles>

This compound is known; however, it has not been fully characterized previously.

This compound was prepared following general procedure $\mathrm{C}$ using ethyl 2-oxohexanoate (39.5 mg, $0.500 \mathrm{mmol}, 1.0 \mathrm{eq}), 3 \mathrm{C}$ tethered catalyst $(R, R)-2(4.7 \mathrm{mg}, 7.5 \mu \mathrm{mol}, 0.015 \mathrm{eq})$ and FA:TEA $(0.3 \mathrm{~mL})$ in DCM (3 mL) to give 46 as a clear oil (62.6 mg, $0.393 \mathrm{mmol}, 79 \%)$.

TLC: $\mathrm{R}_{\mathrm{f}}$ ca 0.5 (6:4, hexane: EtOAc), non UV-active, Strong $\mathrm{KMnO}_{4}$ and PMA-reactive;

Enantiomeric excess determined by chiral GC analysis (CROMPAC CYCLODEXTRIN- $\beta$ $236 \mathrm{M}-19,50 \mathrm{~m} \times 0.25 \mathrm{~mm} \times 0.25 \mu \mathrm{m}$, gas hydrogen, $\mathrm{T}=90{ }^{\circ} \mathrm{C}, \mathrm{P}=16$ psi, FID temp 250 ${ }^{\circ} \mathrm{C}$, injector temp $220{ }^{\circ} \mathrm{C}$ ), $R$-enantiomer $9.6 \mathrm{~min}, S$-enantiomer $12.4 \mathrm{~min} .81 \%$ ee $(R),[\alpha]_{\mathrm{D}}{ }^{22}$ $=+1.5(\mathrm{c}=0.1, \mathrm{EtOH})$, Literature $[\alpha]_{\mathrm{D}}^{25}+5.5^{\circ}(\mathrm{c}=5.0, \mathrm{EtOH}), R$-enantiomer.

${ }^{1} \mathrm{H}$ NMR $\left(500 \mathrm{MHz}, \mathrm{CDCl}_{3}\right) \delta 4.22-4.13\left(3 \mathrm{H}, \mathrm{m}, \underline{\mathrm{C}}_{2} \mathrm{CH}_{3}\right.$ of ester group $\left.+\mathrm{C} \underline{\mathrm{HOH}}\right), 2.86(1 \mathrm{H}$, s, $\mathrm{CHO} \underline{\mathrm{H}}), 1.79-1.73\left(1 \mathrm{H}, \mathrm{m}, \underline{\mathrm{C}}_{\mathrm{a}} \mathrm{H}_{\mathrm{b}}\right), 1.64-1.57\left(1 \mathrm{H}, \mathrm{m}, \mathrm{CHa} \underline{H_{\mathrm{b}}}\right), 1.45-1.19\left(7 \mathrm{H}, \mathrm{m}, \mathrm{CH}_{2}+\right.$ $\mathrm{CH}_{2} \underline{\mathrm{CH}}_{3}$ of ester group), 0.88 (3H, t, $J=7.1 \mathrm{~Hz}, \mathrm{CH}_{3}$ of n-butyl group);

${ }^{13} \mathrm{C}$ NMR $\left(126 \mathrm{MHz}, \mathrm{CDCl}_{3}\right) \delta 175.5,70.5,61.6,34.2,26.9,22.5,14.3,14.0$; $m / z(\mathrm{ESI}) 183.1\left[(\mathrm{M}+\mathrm{Na})^{+}, 100 \%\right]$.

Tang, L.; Li Deng, L. Dynamic Kinetic Resolution via Dual-Function Catalysis of Modified Cinchona Alkaloids: Asymmetric Synthesis of $\alpha$-Hydroxy Carboxylic Acids, J. Am. Chem. Soc. 2002, 124, 12, 2870-2871. 
Ethyl 2-hydroxyhexanoate 46.

${ }^{1} \mathrm{H}$ NMR $\left(500 \mathrm{MHz}, \mathrm{CDCl}_{3}\right)$.

Oct23-2020

Chemist Shweta Gediya

PROTON.w CDCl3 /opt/topspin3.2 SKG 37

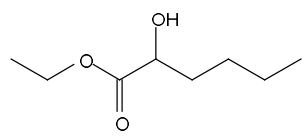

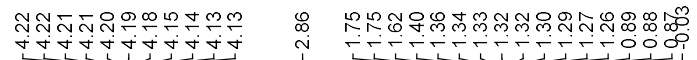

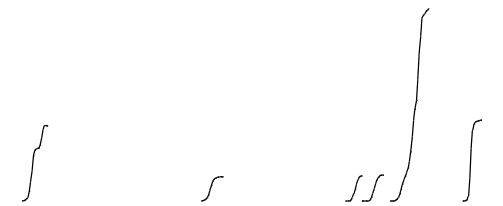

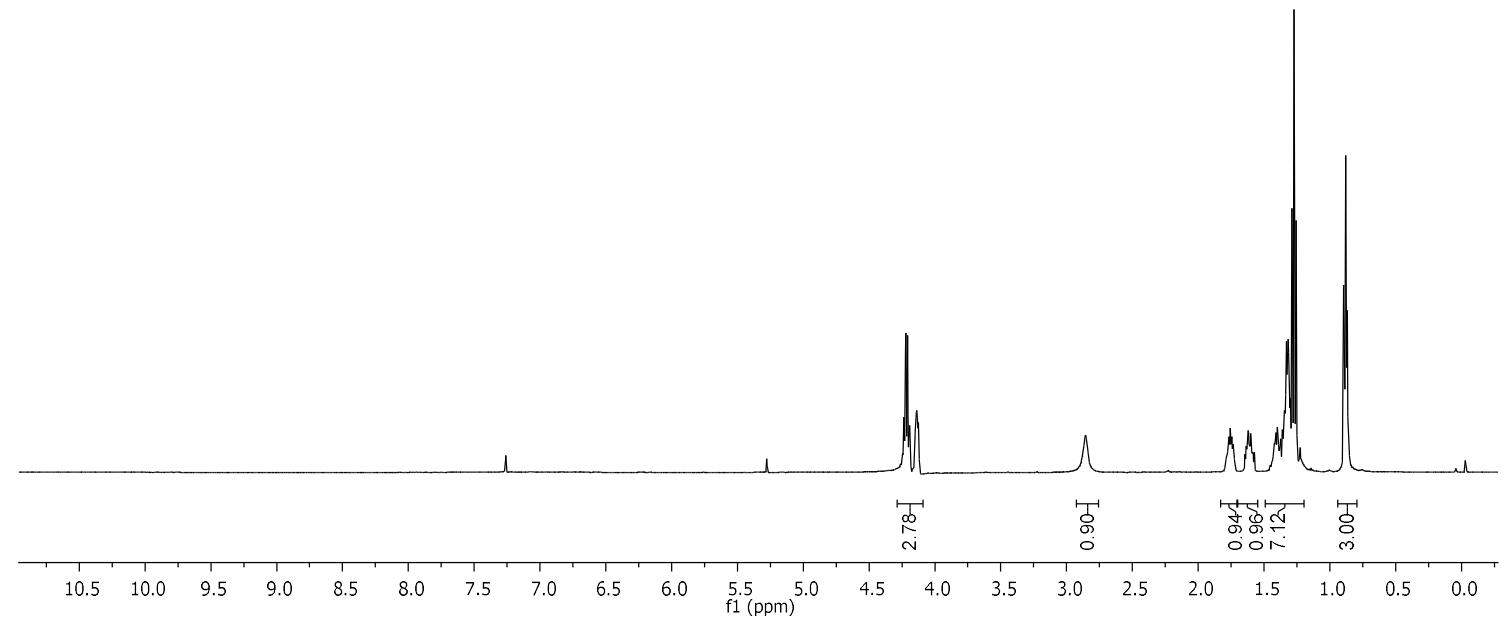

${ }^{13} \mathrm{C}$ NMR $\left(126 \mathrm{MHz}, \mathrm{CDCl}_{3}\right)$.

\begin{tabular}{|c|c|c|}
\hline $\begin{array}{l}\text { Oct23-2020 } \\
\text { Shweta Gediya } \\
\text { SKG608 } \\
\text { C13APTlong.w CDCl3 }\end{array}$ & 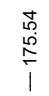 & 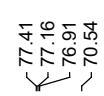 \\
\hline
\end{tabular}
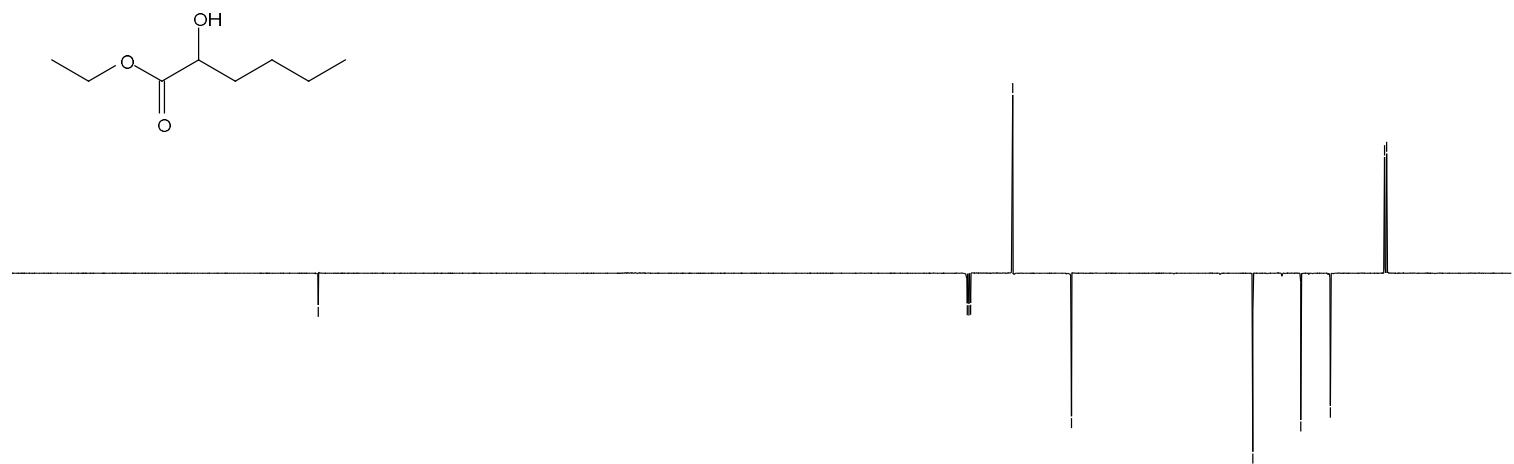

$\begin{array}{llllllllllllllllllllllllllll}220 & 210 & 200 & 190 & 180 & 170 & 160 & 150 & 140 & 130 & 120 & 110 & 100 & 90 & 80 & 70 & 60 & 50 & 40 & 30 & 20 & 10 & 0\end{array}$ 
COSY.

Chemist Shweta Gediya

COSY.w CDCl3/opt/topspin3.2 SKG 37

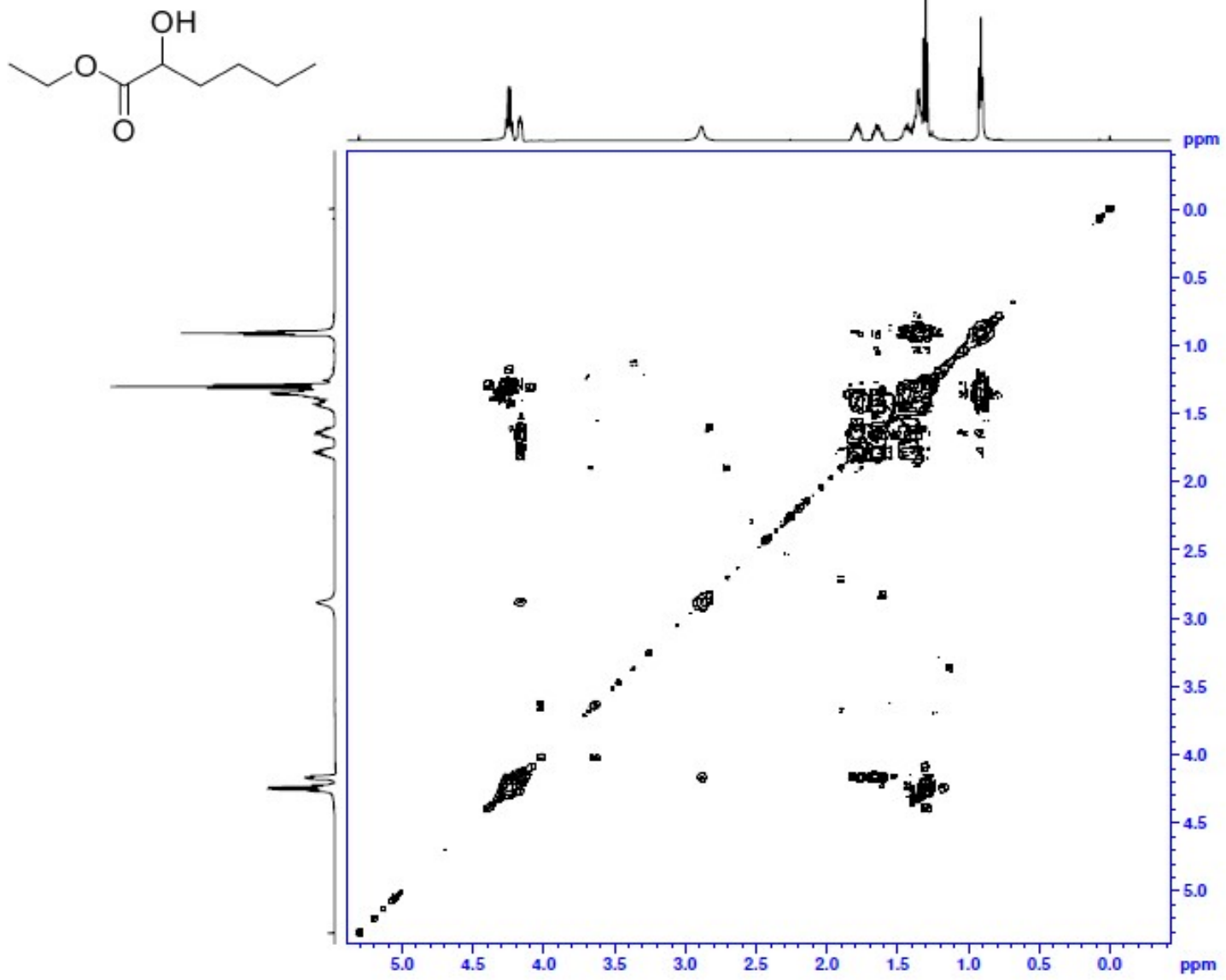

Chiral GC of ketone precursor.

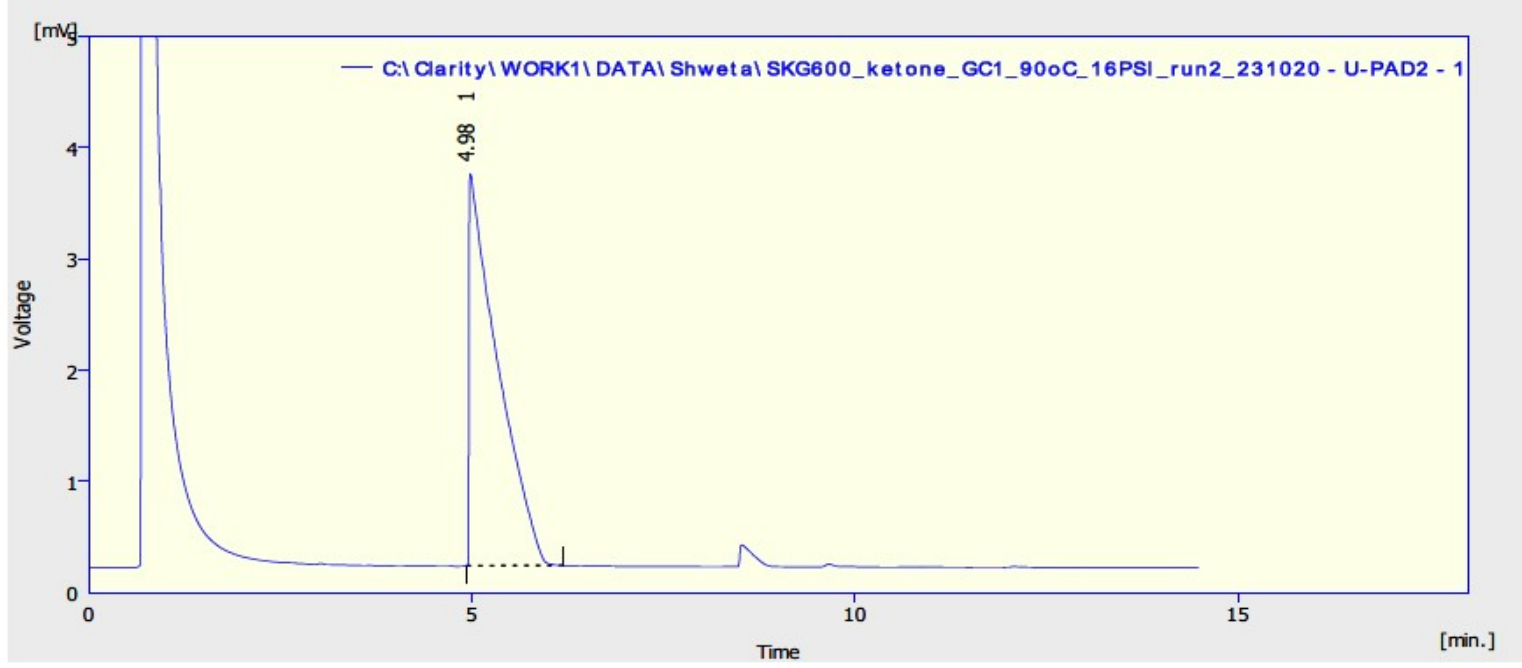

\begin{tabular}{|c|c|c|c|c|c|c|c|}
\hline & $\begin{array}{c}\text { Reten. Time } \\
{[\mathrm{min}]}\end{array}$ & $\begin{array}{c}\text { Area } \\
{[\mathrm{mV} . \mathrm{s}]}\end{array}$ & $\begin{array}{l}\text { Height } \\
{[\mathrm{mV}]}\end{array}$ & $\begin{array}{l}\text { Area } \\
{[\%]}\end{array}$ & $\begin{array}{c}\text { Height } \\
{[\%]}\end{array}$ & $\begin{array}{c}\text { W05 } \\
\text { [min] }\end{array}$ & $\begin{array}{c}\text { Compound } \\
\text { Name }\end{array}$ \\
\hline 1 & 4.980 & 903.052 & 35.229 & 100.0 & 100.0 & 0.39 & \\
\hline & Total & 903.052 & 35.229 & 100.0 & 100.0 & & \\
\hline
\end{tabular}




\section{Chiral GC of racemic alcohol.}

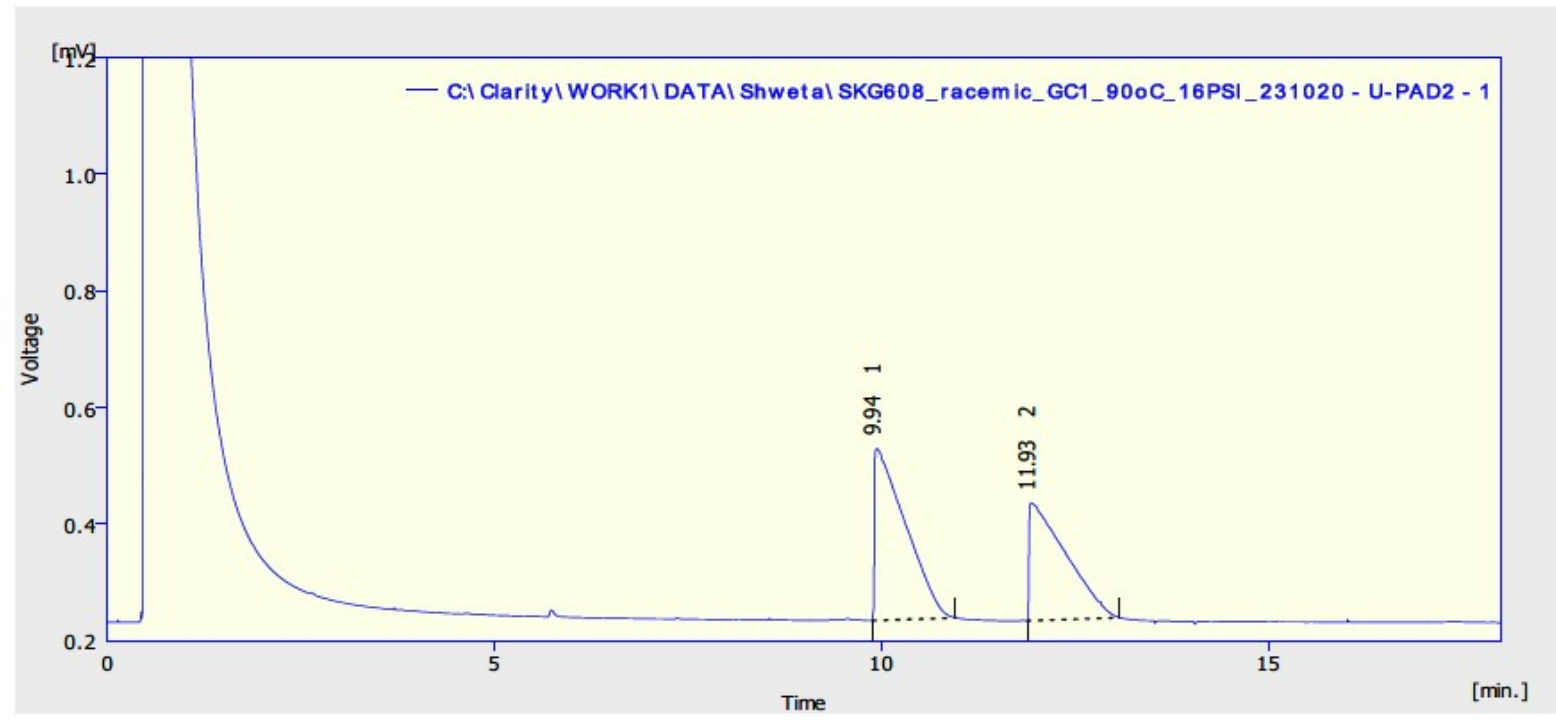

\section{Chiral GC of ATH product. $81 \%$ ee.}

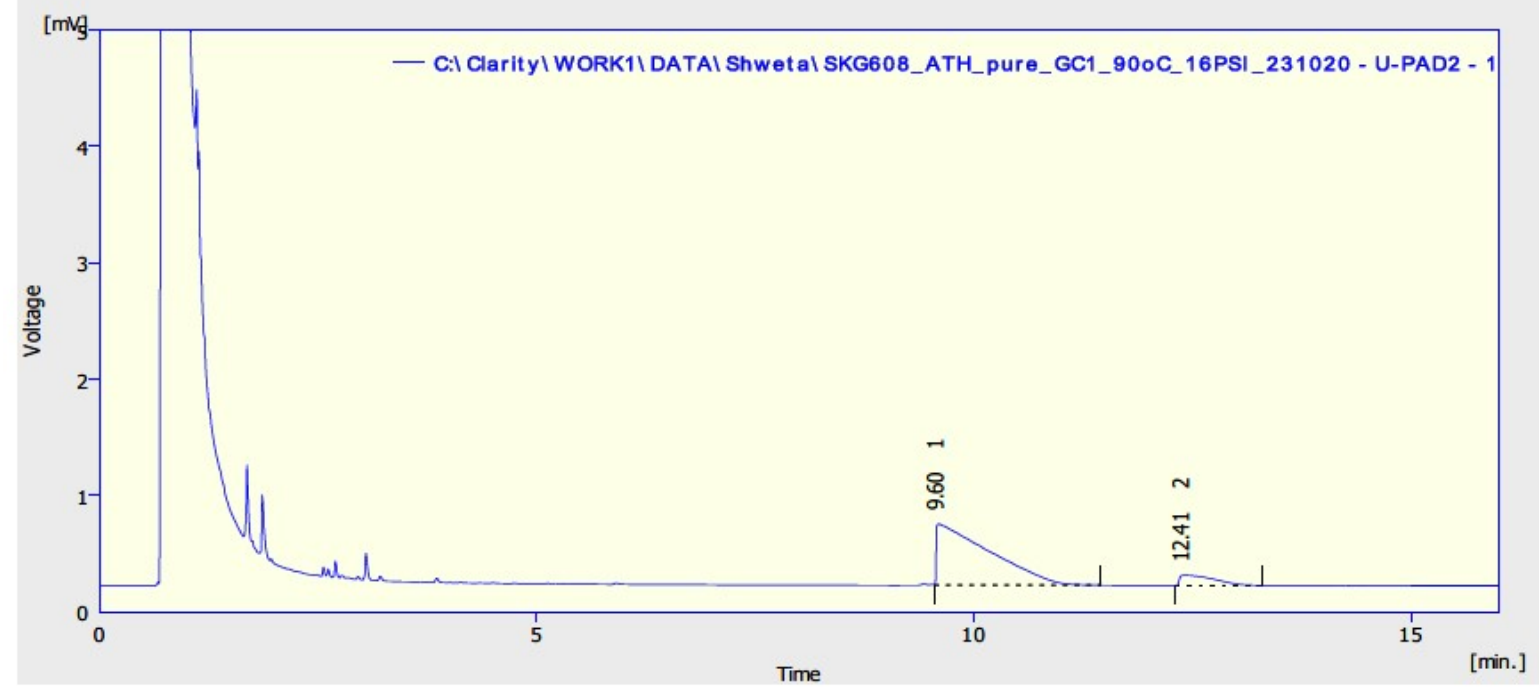

\begin{tabular}{|c|c|c|c|c|c|c|c|}
\hline & $\begin{array}{l}\text { Reten. Time } \\
\text { [min] }\end{array}$ & $\begin{array}{c}\text { Area } \\
{[\mathrm{mV} . \mathrm{s}]}\end{array}$ & $\begin{array}{l}\text { Height } \\
{[\mathrm{mV}]}\end{array}$ & $\begin{array}{l}\text { Area } \\
{[\%]}\end{array}$ & $\begin{array}{c}\text { Height } \\
{[\%]}\end{array}$ & $\begin{array}{l}\text { W05 } \\
{[\mathrm{min}]}\end{array}$ & $\begin{array}{c}\text { Compound } \\
\text { Name }\end{array}$ \\
\hline 1 & 9.604 & 222.104 & 5.261 & 90.3 & 85.6 & 0.69 & \\
\hline \multirow[t]{2}{*}{2} & 12.412 & 23.946 & 0.887 & 9.7 & 14.4 & 0.46 & \\
\hline & Total & 246.050 & 6.149 & 100.0 & 100.0 & & \\
\hline
\end{tabular}


Ethyl (R)-3-hydroxy-5-oxo-5-(phenylamino)pentanoate 47.<smiles>CCOC(=O)CC(O)CC(=O)Nc1ccccc1</smiles>

This compound is known and has been fully characterized previously.

This compound was prepared following general procedure $\mathrm{C}$ using ethyl 3,5-dioxo-5(phenylamino)pentanoate $(66.2 \mathrm{mg}, 0.250 \mathrm{mmol}, 1.0 \mathrm{eq}), 3 \mathrm{C}$ tethered catalyst $(R, R)-2(2.3$ $\mathrm{mg}, 3.75 \mu \mathrm{mol}, 0.015 \mathrm{eq})$ and FA:TEA $(0.15 \mathrm{~mL})$ in DCM $(1.5 \mathrm{~mL})$ to give 47 as a semisolid material (52.0 mg, $0.207 \mathrm{mmol}, 83 \%)$.

TLC: $\mathrm{R}_{\mathrm{f}}$ ca 0.3 (6:4, hexane: EtOAc), UV-active, Strong $\mathrm{KMnO}_{4}$ and PMA-reactive; HRMS (ESI) m/z: $[\mathrm{M}+\mathrm{Na}]^{+}$Calcd for $\mathrm{C}_{13} \mathrm{H}_{17} \mathrm{NO}_{4} \mathrm{Na} 274.1050$; Found 274.1049 (error 0.2 ppm);

$U_{\max } 3485,3260,2928,1732,1683,1596,1544,1412,1298,1155,1095,752 \mathrm{~cm}^{-1}$;

Enantiomeric excess determined by HPLC analysis (Chiralpak AD-H, 250mm $\times 4.6 \mathrm{~mm}$ column, Hexane: iPrOH 91:9, $0.8 \mathrm{~mL} / \mathrm{min}, \lambda=210 \mathrm{~nm}, \mathrm{~T}=25^{\circ} \mathrm{C}$ ), $21.2 \mathrm{~min}$ and $25.6 \mathrm{~min}$, $4 \%$ ee;

${ }^{1} \mathrm{H}$ NMR (500 MHz, $\left.\mathrm{CDCl}_{3}\right) \delta 8.32(1 \mathrm{H}, \mathrm{s}, \mathrm{NH}), 7.51(2 \mathrm{H}, \mathrm{d}, J=7.7 \mathrm{~Hz}, \mathrm{ArH}), 7.30(2 \mathrm{H}, \mathrm{m}$, ArH), $7.10(1 \mathrm{H}, \mathrm{t}, J=7.4 \mathrm{~Hz}, \mathrm{ArH}), 4.51-4.46(1 \mathrm{H}, \mathrm{m}, \mathrm{C} \underline{\mathrm{HOH}}), 4.18(2 \mathrm{H}, \mathrm{q}, J=7.1 \mathrm{~Hz}$, $\left.\mathrm{CH}_{2}\right), 4.14(1 \mathrm{H}, \mathrm{d}, J=2.9 \mathrm{~Hz}, \mathrm{OH}), 2.62-2.54\left(4 \mathrm{H}, \mathrm{m}, \mathrm{CH}_{2}\right), 1.28\left(3 \mathrm{H}, \mathrm{t}, J=7.1 \mathrm{~Hz}, \mathrm{CH}_{3}\right)$; ${ }^{13} \mathrm{C} \mathrm{NMR}\left(126 \mathrm{MHz}, \mathrm{CDCl}_{3}\right) \delta 172.5,169.4,137.8,129.1,124.5,120.1,65.4,61.2,43.3$, 40.6, 14.3;

$m / z(\mathrm{ESI}) 274.1\left[(\mathrm{M}+\mathrm{Na})^{+}, 100 \%\right]$.

Li, W.; Ma, X.; Fan, W.; Tao, X.; Li, X.; Xie, X.; Zhang, Z. Ru-Catalyzed Asymmetric Hydrogenation of 3-Oxoglutaric Acid Derivatives via Solvent-Assisted Pinpoint Recognition of Carbonyls in Close Chemical Propinquity, Org. Lett. 2011, 13, 15, 3876-3879. 
Ethyl-3-hydroxy-5-oxo-5-(phenylamino)pentanoate 47.

${ }^{1} \mathrm{H}$ NMR $\left(500 \mathrm{MHz}, \mathrm{CDCl}_{3}\right)$.

Oct16-2020
Chemist Shweta Gediya
SKG601
PROTON.w CDC13 /opt/topspin3.2 SKG 44

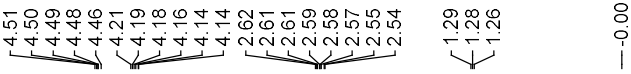

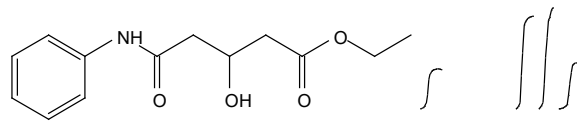

$\int 1$
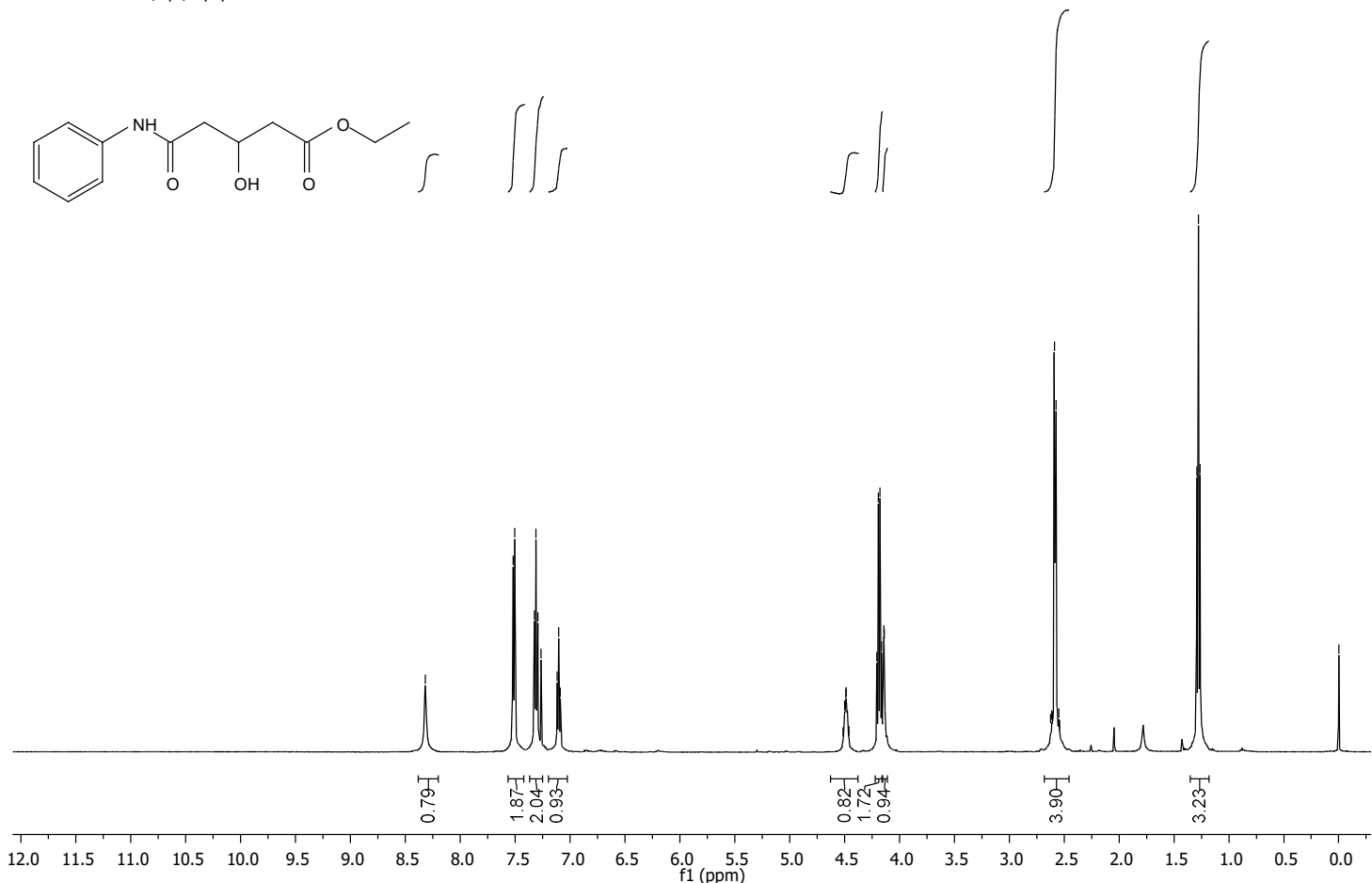

${ }^{13} \mathrm{C} \mathrm{NMR}\left(126 \mathrm{MHz}, \mathrm{CDCl}_{3}\right)$.

Oct16-2020

Chemist Shweta Gediya

热

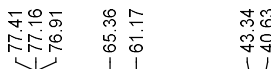

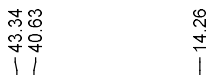<smiles>CCOC(=O)CC(O)CC(=O)Nc1ccccc1</smiles>

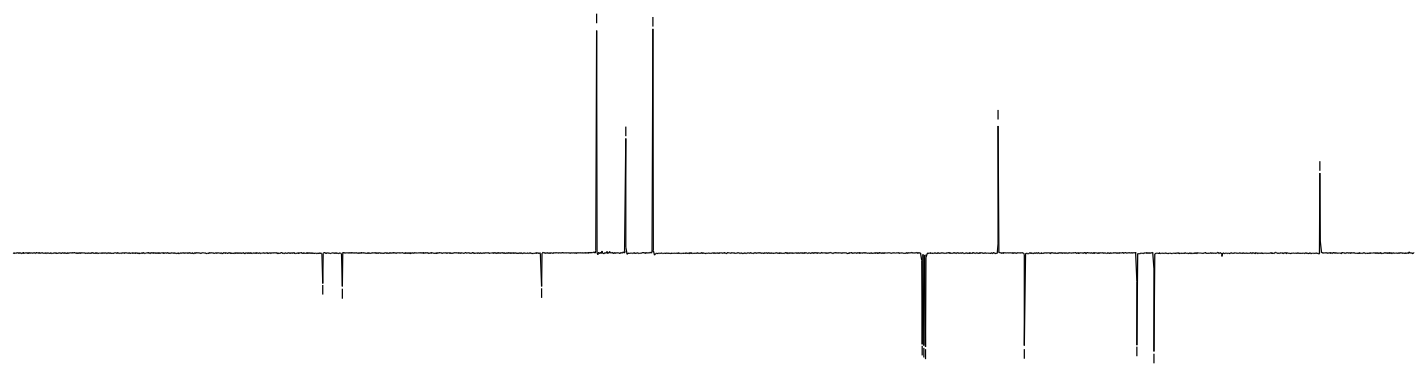

$\begin{array}{llllllllllllllllllllllllll}220 & 210 & 200 & 190 & 180 & 170 & 160 & 150 & 140 & 130 & 120 & 110 & 100 & 90 & 80 & 70 & 60 & 50 & 40 & 30 & 20 & 10 & 0\end{array}$ 
COSY.

Chemist Shweta Gediya

COSY.w CDCI3/opt/topspin3.2 SKG 44

$\stackrel{\mathrm{N}}{\mathrm{N}} \prod_{\mathrm{OH}}^{\mathrm{H}} \prod_{\mathrm{O}}^{\mathrm{O}}$

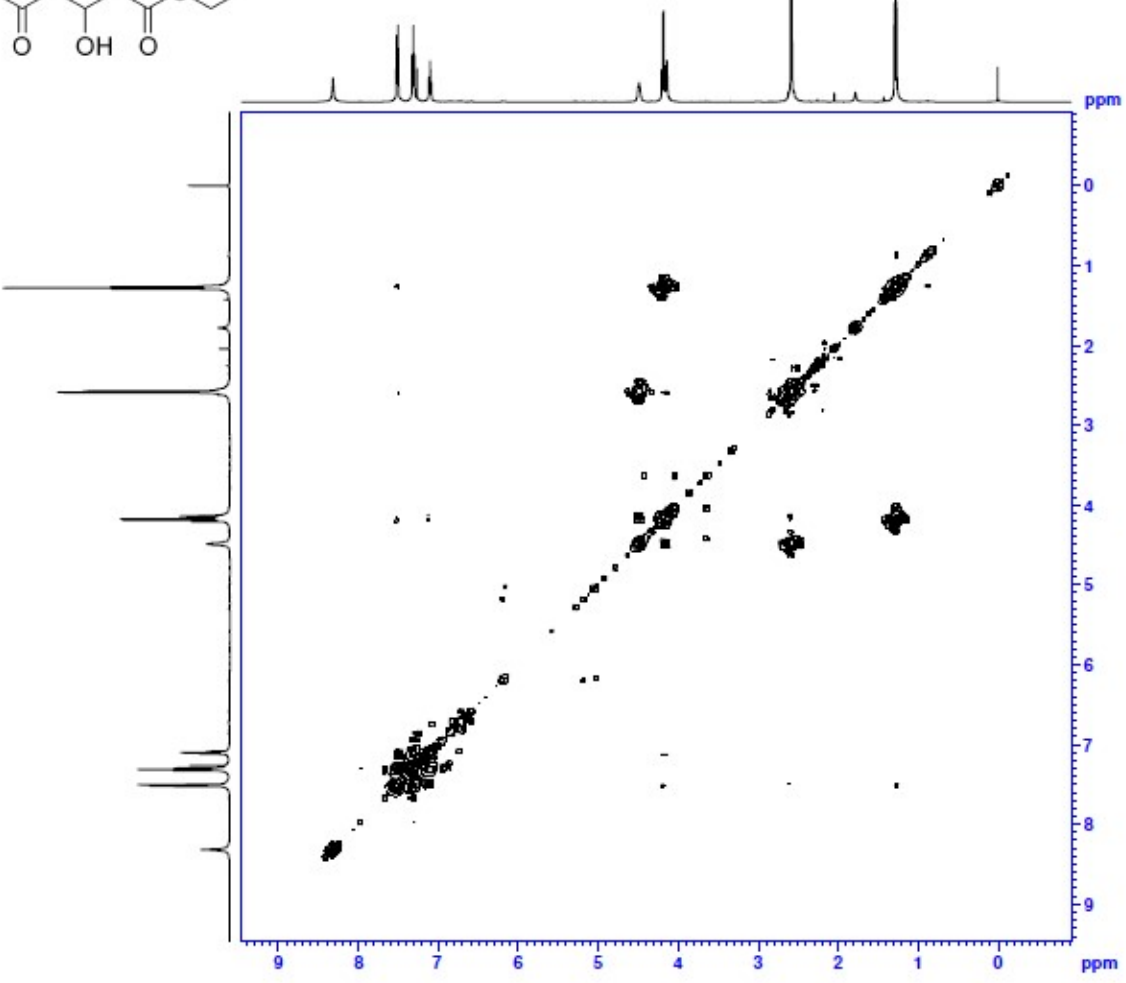

Chiral HPLC of ketone.

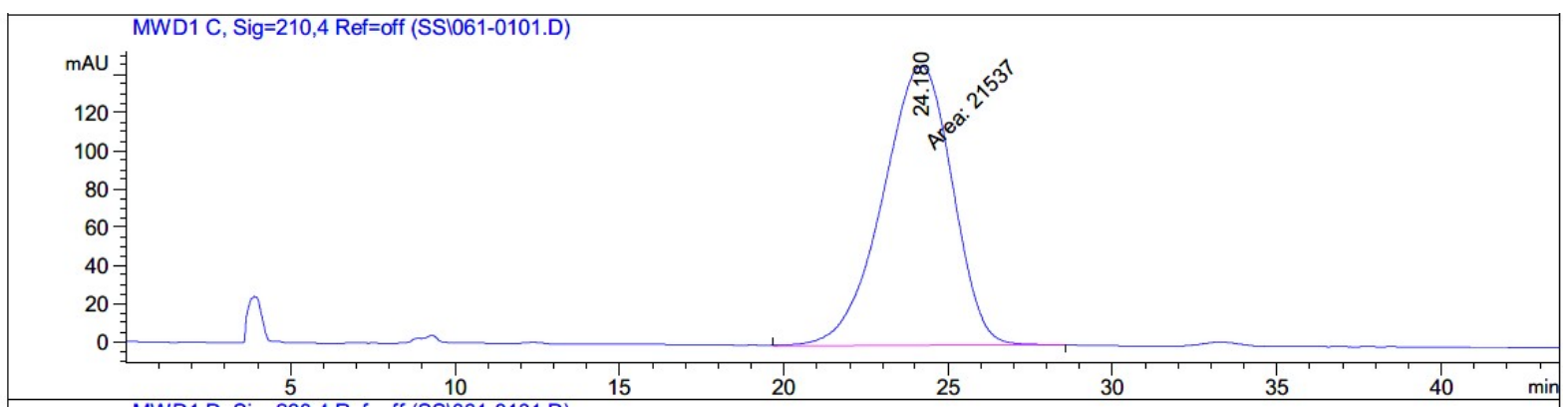

Signal 2: MWD1 C, Sig=210,4 Ref=off

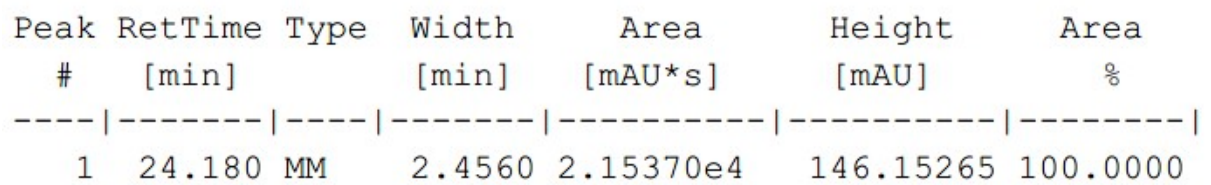




\section{Chiral HPLC of racemic standard.}

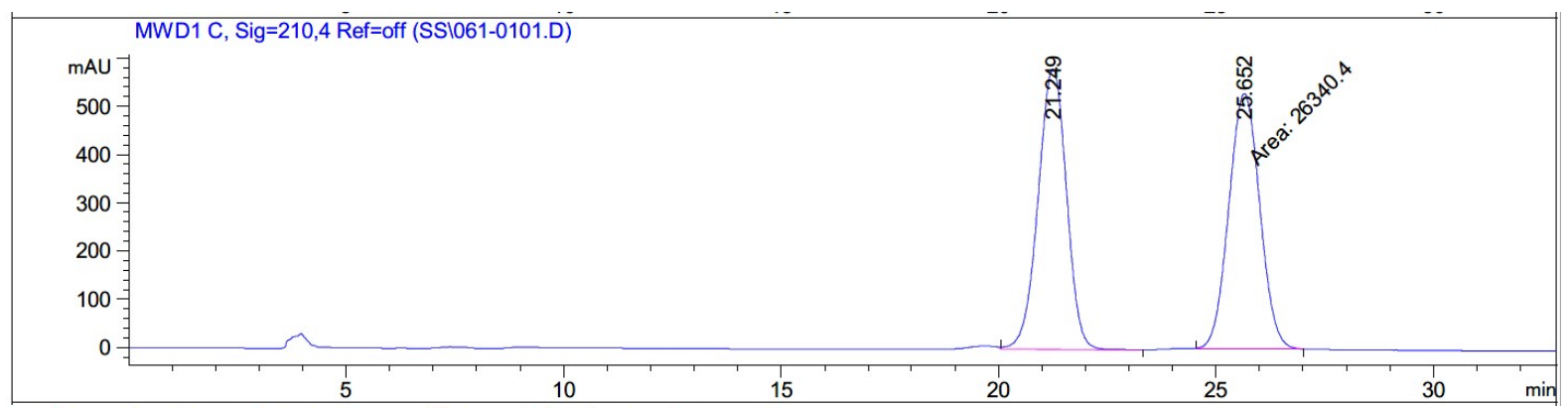

Signal 2: MWD1 C, Sig=210,4 Ref=off

\begin{tabular}{|c|c|c|c|c|c|c|}
\hline $\begin{array}{c}\text { Peak } \\
\#\end{array}$ & $\begin{array}{c}\text { RetTime } \\
\text { [min] }\end{array}$ & Type & $\begin{array}{l}\text { Width } \\
\text { [min] }\end{array}$ & $\begin{array}{c}\text { Area } \\
{\left[\mathrm{mAU}^{\star} \mathrm{s}\right]}\end{array}$ & $\begin{array}{l}\text { Height } \\
{[\mathrm{mAU}]}\end{array}$ & $\begin{array}{c}\text { Area } \\
\frac{\circ}{0}\end{array}$ \\
\hline & & & & --------- & ---------- & $--------\mid$ \\
\hline 1 & 21.249 & VB & 0.7148 & $2.64435 e 4$ & 581.22144 & 50.0976 \\
\hline 2 & 25.652 & MM & 0.8280 & $2.63404 \mathrm{e} 4$ & 530.22406 & 49.9024 \\
\hline
\end{tabular}

Chiral HPLC of ATH product, $4 \%$ ee.

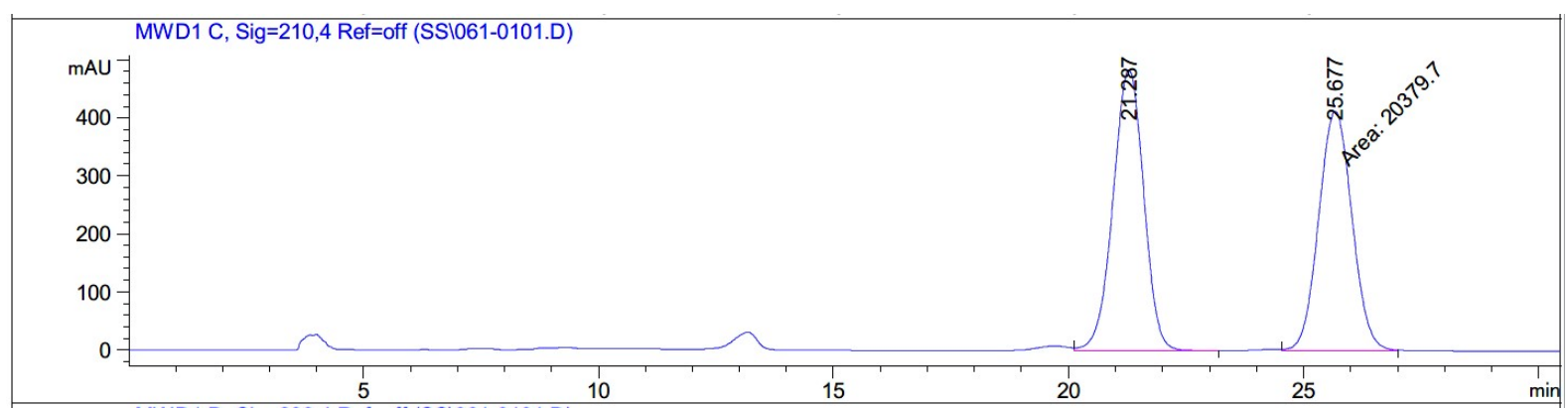

Signal 2: MWD1 C, Sig=210,4 Ref=off

\begin{tabular}{|c|c|c|c|c|c|c|}
\hline $\begin{array}{c}\text { Peak } \\
\#\end{array}$ & $\begin{array}{c}\text { RetTime } \\
\text { [min] }\end{array}$ & Type & $\begin{array}{l}\text { Width } \\
\text { [min] }\end{array}$ & $\begin{array}{c}\text { Area } \\
{\left[\mathrm{mAU}{ }^{\star} \mathrm{s}\right]}\end{array}$ & $\begin{array}{l}\text { Height } \\
{[\mathrm{mAU}]}\end{array}$ & $\begin{array}{c}\text { Area } \\
\%\end{array}$ \\
\hline & -- & & & ---- & -------- & ------- \\
\hline 1 & 21.287 & VB & 46 & $2.20248 e 4$ & 484.27899 & 9398 \\
\hline 2 & 25.677 & MM & 0.8232 & $2.03797 e 4$ & 412.61255 & 48.0602 \\
\hline
\end{tabular}




\section{Section on Dynamic Kinetic Resolution (DKR).}

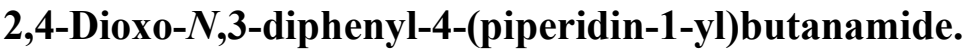<smiles>O=C(Nc1ccccc1)C(=O)C(C(=O)N1CCCCC1)c1ccccc1</smiles>

This compound is novel and was prepared following general procedure B using methyl 2oxo-2-(phenylamino)acetate (500 mg, $2.79 \mathrm{mmol}, 1.0 \mathrm{eq})$ in anhydrous THF (10 mL), 2phenyl-1-(piperidin-1-yl)ethan-1-one (648 mg, $3.19 \mathrm{mmol}, 1.2 \mathrm{eq})$ in anhydrous THF (10 $\mathrm{mL}$ ) and LDA (1M solution in THF, $8.37 \mathrm{~mL}, 8.37 \mathrm{mmol}, 2.3 \mathrm{eq})$ to give crude material which was further purified by manual column chromatography (10-30\% EtOAc in petroleum ether) to afford the product as a clear liquid $(180 \mathrm{mg}, 0.51 \mathrm{mmol}, 18 \%)$. The title compound was obtained as a keto/enol tautomer (1:1) as per ${ }^{1} \mathrm{H}$ NMR.

HRMS (ESI) m/z: [M+Na] $]^{+}$Calcd for $\mathrm{C}_{21} \mathrm{H}_{22} \mathrm{~N}_{2} \mathrm{O}_{3} \mathrm{Na} 373.1523$; Found 373.1515

${ }^{1} \mathrm{H}$ NMR $\left(400 \mathrm{MHz}, \mathrm{CDCl}_{3}\right)$ (keto/enol, 1:1) $\delta 9.13(0.5 \mathrm{H}, \mathrm{s}, \mathrm{NH}$ keto), $8.69(0.5 \mathrm{H}, \mathrm{s}, \mathrm{H}$ enol), 7.67 - 7.54 (2H, m, ArH), $7.43-7.25$ (7H, m, ArH), $7.20-7.12$ (1H, m, ArH), 5.88 $(0.5 \mathrm{H}, \mathrm{s},=\mathrm{CH}$ enol $), 4.14-4.10(0.5 \mathrm{H}, \mathrm{m}, \mathrm{CH}$ keto $), 3.71-3.57\left(2 \mathrm{H}, \mathrm{m}, \mathrm{CH}_{2}\right.$ piperidine $)$, $3.45-3.36\left(2 \mathrm{H}, \mathrm{m}, \mathrm{CH}_{2}\right.$ piperidine $), 1.72-1.48\left(6 \mathrm{H}, \mathrm{m}, \mathrm{CH}_{2}\right.$ piperidine);

${ }^{13} \mathrm{C}$ NMR (101 MHz, $\mathrm{CDCl}_{3}$ ) (keto/enol, 1:1) $\delta 192.4$ (C=O keto), 166.7 (C=O amide), 158.1 $(\mathrm{C}=\mathrm{O}$ amide $), 136.4,132.4,129.4,129.1,128.9,128.2,125.2,124.9,120.0,119.8(\mathrm{C}=\mathrm{CH}$, enol), 55.9, 47.7, 44.9, 43.1, 26.9, 25.8, 25.6, 25.3, 24.5, 24.3;

$m / z(\mathrm{ESI}) 373.2\left[(\mathrm{M}+\mathrm{Na})^{+}, 100 \%\right]$. 
2,4-Dioxo-N,3-diphenyl-4-(piperidin-1-yl)butanamide.

${ }^{1} \mathrm{H}$ NMR (400 MHz, $\left.\mathrm{CDCl}_{3}\right)$.

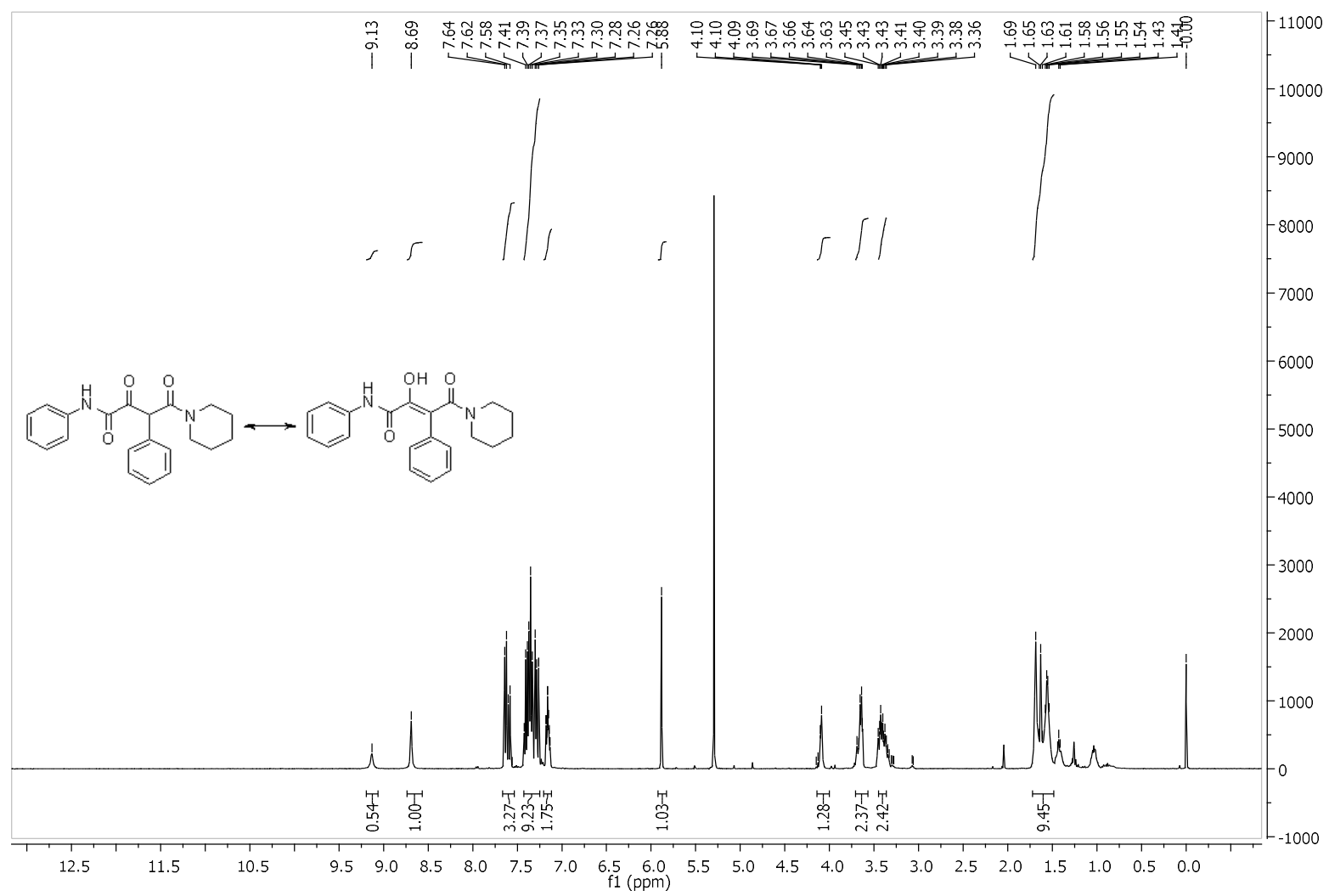

${ }^{13} \mathrm{C}$ NMR (101 MHz, $\left.\mathrm{CDCl}_{3}\right)$.

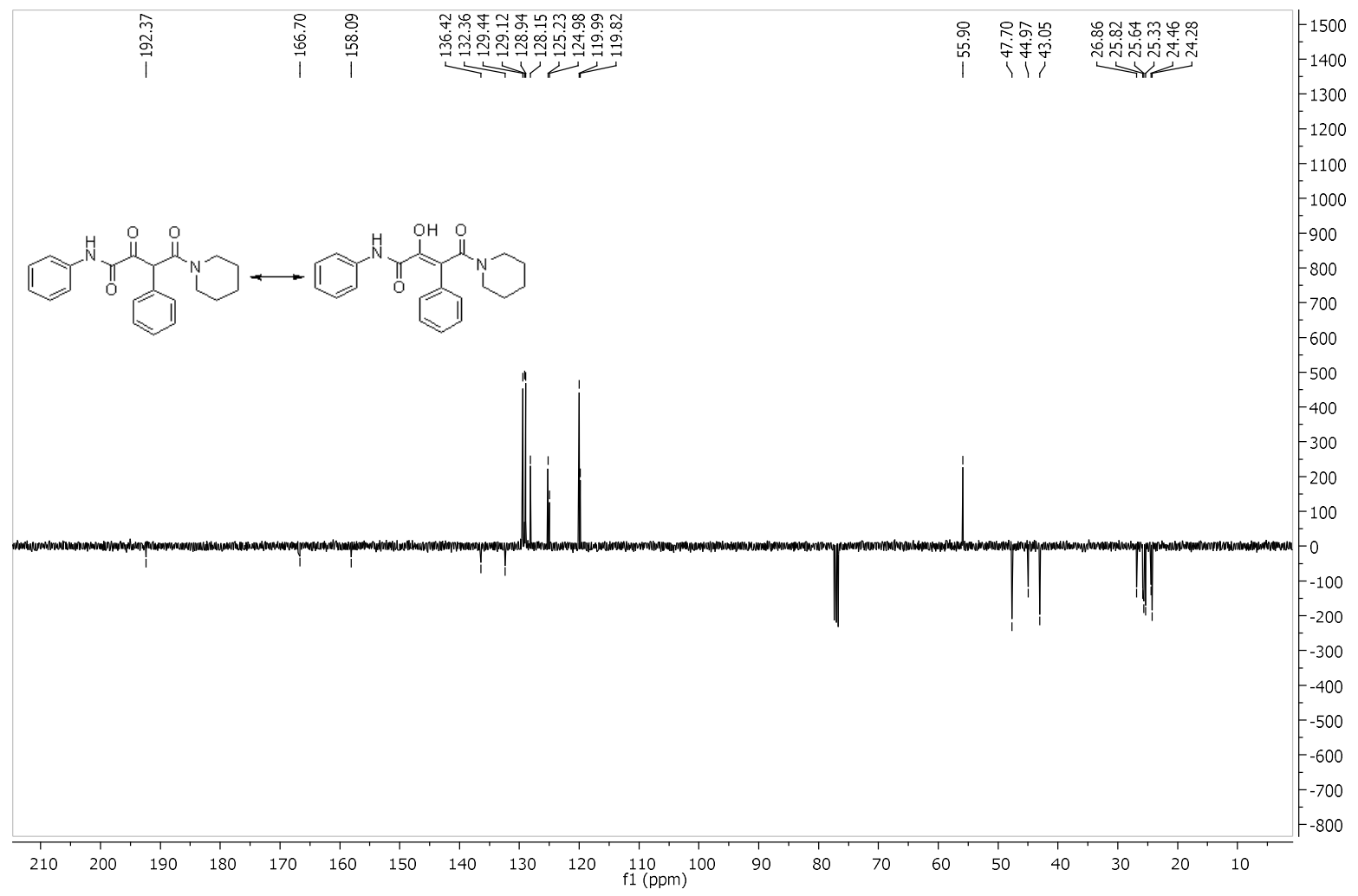

S406 
Racemic and chiral 2-hydroxy-4-oxo- $N, 3-$ diphenyl-4-(piperidin-1-yl)butanamide 48.<smiles>O=C(Nc1ccccc1)C(O)C(C(=O)N1CCCCC1)c1ccccc1</smiles>

This compound is novel.

This compound was prepared in racemic form following this procedure: 2,4-dioxo- $N, 3-$ diphenyl-4-(piperidin-1-yl)butanamide (70 mg, $0.20 \mathrm{mmol}, 1.0$ equiv) was dissolved in $\mathrm{MeOH}(5 \mathrm{~mL})$ at $\mathrm{rt}$ and $\mathrm{NaBH}_{4}(16 \mathrm{mg}, 0.4 \mathrm{mmol}, 2.0$ equiv) was added. The reaction was stirred at $\mathrm{rt}$ for $2 \mathrm{~h}$. After the completion of reaction, it was quenched by water $(5 \mathrm{~mL})$ and extracted with ethyl acetate $(8 \mathrm{~mL})$. The organic layer was dried with $\mathrm{Na}_{2} \mathrm{SO}_{4}$ and concentrated to obtain a residue which was purified by flash chromatography on silica gel. A mixture of diastereomers was isolated by flash chromatography (pet ether/ EtOAc: 80:20) as a white solid (42 mg, $0.12 \mathrm{mmol}, 60 \%)$.

This compound was prepared following general procedure $\mathrm{C}$ using 2,4-dioxo- $N, 3$-diphenyl-4(piperidin-1-yl)butanamide (105 mg, $0.30 \mathrm{mmol}, 1.0 \mathrm{eq}), 3 \mathrm{C}$ tethered catalyst $(R, R)-2(1.86$ $\left.\mathrm{mg}, 2.9 \times 10^{-3} \mathrm{mmol}, 1 \mathrm{~mol} \%\right)$ and FA:TEA $(0.2 \mathrm{~mL})$ in DCM $(2 \mathrm{~mL})$ to give 48 as a colourless oil (93 mg, $0.26 \mathrm{mmol}, 88 \%$ ).

HRMS (ESI) m/z: [M+Na] ${ }^{+}$Calcd for $\mathrm{C}_{21} \mathrm{H}_{24} \mathrm{~N}_{2} \mathrm{O}_{3} \mathrm{Na}$ 375.1679; Found 375.1673;

$U_{\max } 3419,2931,2849,1624,1439,1371,1254,1189,852,542 \mathrm{~cm}^{-1}$;

Enantiomeric excess and diastereomeric ratio determined by HPLC analysis (CHIRALPAK IC column, hexane 90:10 $\mathrm{iPrOH}, 1.0 \mathrm{~mL} / \mathrm{min}, \mathrm{T}=25^{\circ} \mathrm{C}, \lambda=250 \mathrm{~nm}$, Ketone $10.8, t_{\mathrm{R}}=20.9$ min (major, d1), 36.8 min (minor, d2), 40.8 min (major, d1), 45.8 min (minor, d2). 71:29 dr, $86 \%$ ee $(\mathrm{d} 1), 91 \%$ ee $(\mathrm{d} 2)$.

${ }^{1} \mathrm{H}$ NMR (400 MHz, $\mathrm{CDCl}_{3}$ ) (Mixture of diastereomers) $\delta 9.21$ (0.67H, s, NH Major), 9.13 (0.33H, s, NH Minor), 7.62 - 7.52 (2H, m, ArH), $7.39-7.23$ (7H, m, ArH), 7.18 - 7.05 (1H, m, ArH), $6.35(0.67 \mathrm{H}, \mathrm{d}, J=9.3 \mathrm{~Hz}, \mathrm{C} \underline{\mathrm{HOH}}$ Major), 5.89 (0.33H, s, C $(0.33 \mathrm{H}, \mathrm{d}, J=9.9 \mathrm{~Hz}, \mathrm{OH}$ Minor), $4.73(0.67 \mathrm{H}, \mathrm{d}, J=2.2 \mathrm{~Hz}, \mathrm{OH}$ Major $), 4.52(0.33 \mathrm{H}, \mathrm{d}, J=$ $2.2 \mathrm{~Hz}$, CHPh Minor), 4.37 (0.67H, dd, $J=9.3,2.2 \mathrm{~Hz}$, C파 $\mathrm{Hz}$, piperidine), $3.70-3.58(1 \mathrm{H}, \mathrm{m}$, piperidine), $3.43-3.16$ (2H, m, piperidine), $1.73-1.43$ (6H, m, piperidine), $1.31-1.18(1 \mathrm{H}, \mathrm{m}$, piperidine);

${ }^{13} \mathrm{C} \mathrm{NMR}\left(101 \mathrm{MHz}, \mathrm{CDCl}_{3}\right.$ ) (Mixture of diastereomers) $\delta 171.8,171.7,171.1,170.9,137.5$, $136.3,129.2$, 129.1, 129.0, 128.9, 128.8, 128.5, 128.3, 127.7, 125.0, 124.5, 124.3, 120.2, 
$119.8,119.8,76.2,73.7,49.7,48.4,47.7,47.2,46.9,45.0,42.9,26.8,25.8,25.7,25.6,25.5$, 25.4, 24.5, 24.2;

$m / z(\mathrm{ESI}) 375.3\left[(\mathrm{M}+\mathrm{Na})^{+}, 100 \%\right]$. 
Diastereomers).

${ }^{\mathbf{1}} \mathbf{H}$ NMR $\left(400 \mathrm{MHz}, \mathrm{CDCl}_{3}\right)$.

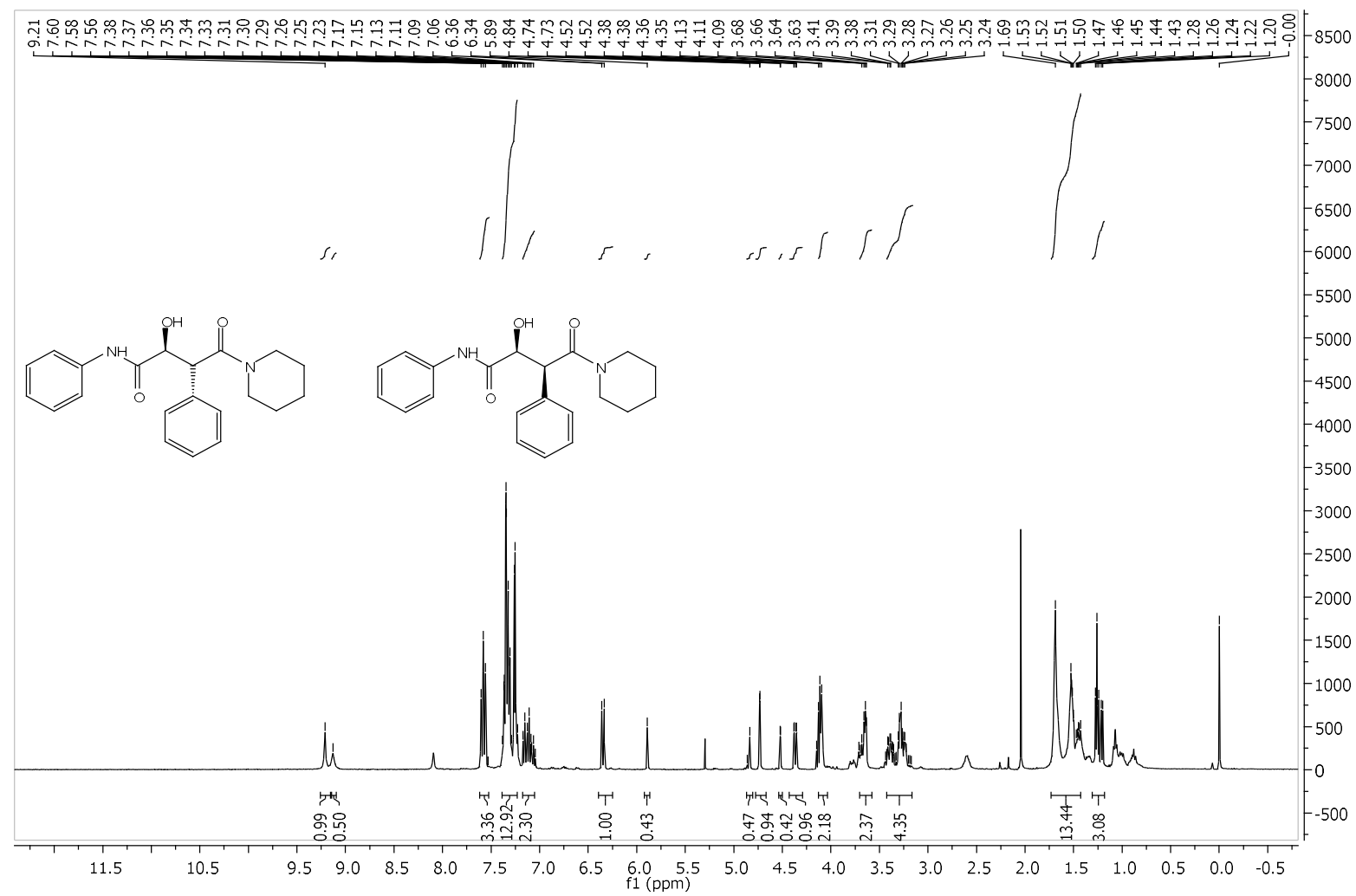

${ }^{13}$ C NMR (101 MHz, $\left.\mathrm{CDCl}_{3}\right)$.

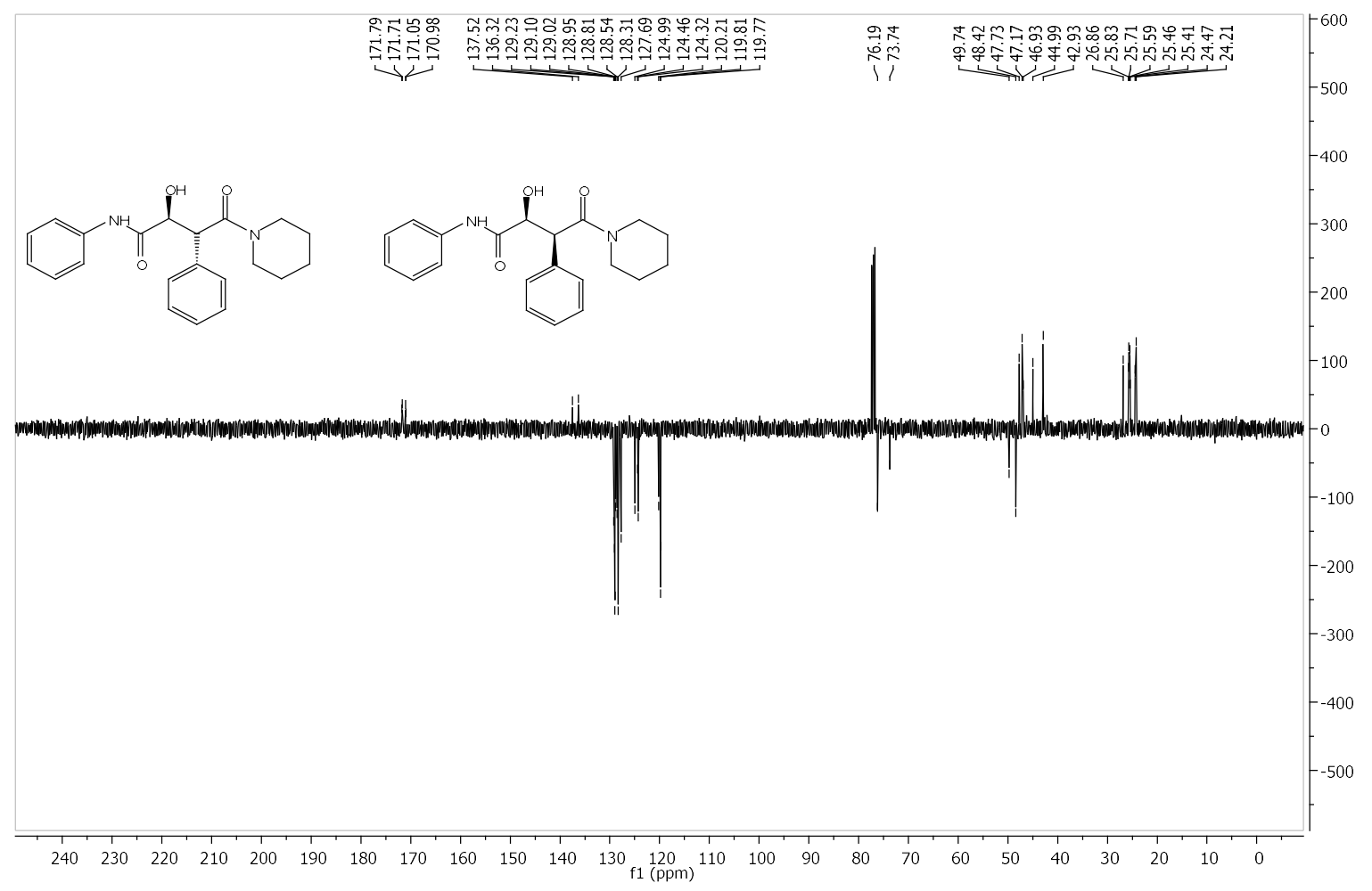


Chiral HPLC of racemic 2-hydroxy-4-oxo- $N, 3-$ diphenyl-4-(piperidin-1-yl)butanamide 48.

Data File C:\CHEM32\1\DATA \DEMO\086-0101.D

Sample Name: VKV 473802010 Rac IC

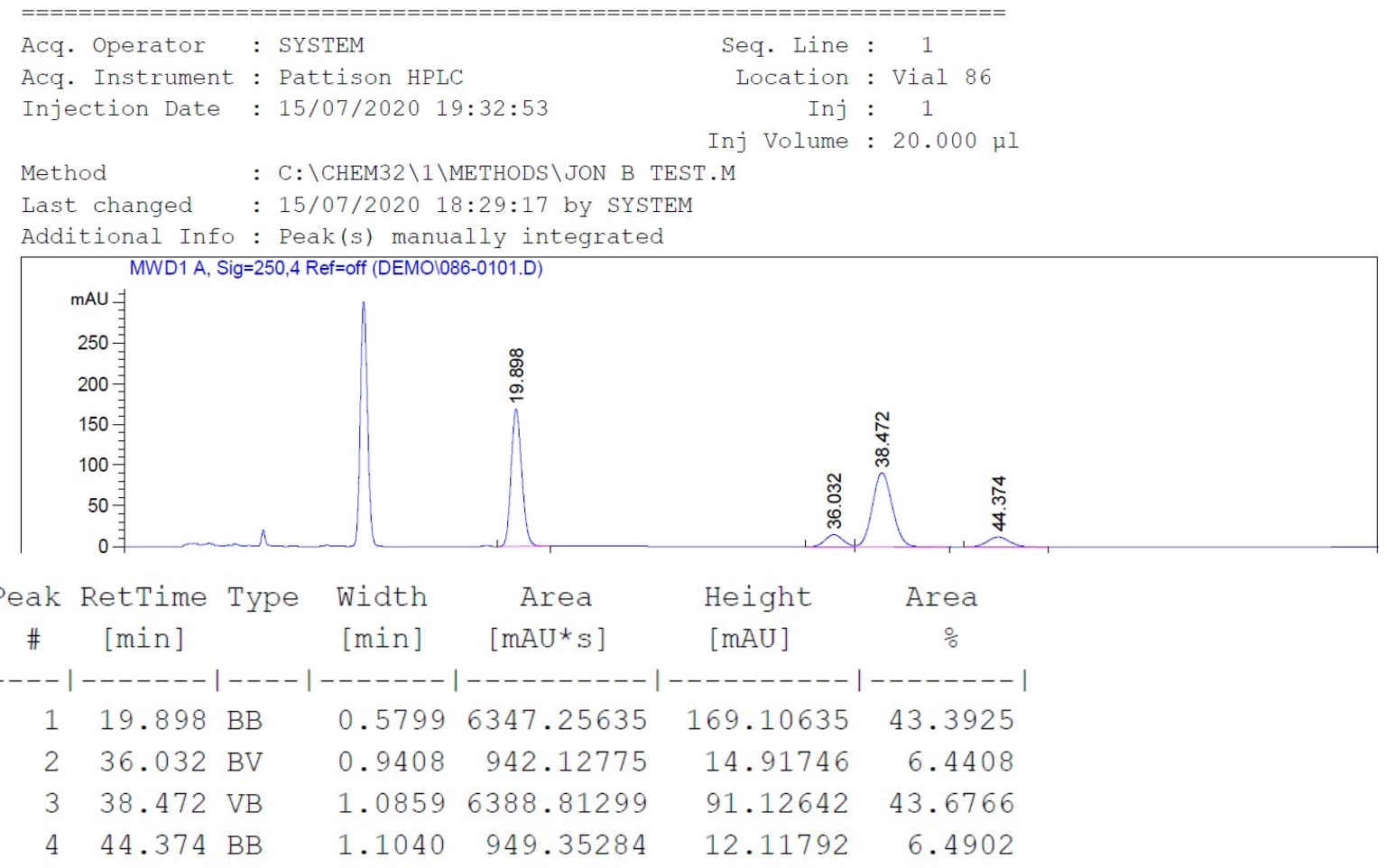


Chiral HPLC of ATH 2-hydroxy-4-oxo- $N, 3-$ diphenyl-4-(piperidin-1-yl)butanamide showing dr.

Data File C: \CHEM32\1\DATA \DEMO\061-0101.D Sample Name: VKV 672 ATH 901010 IC

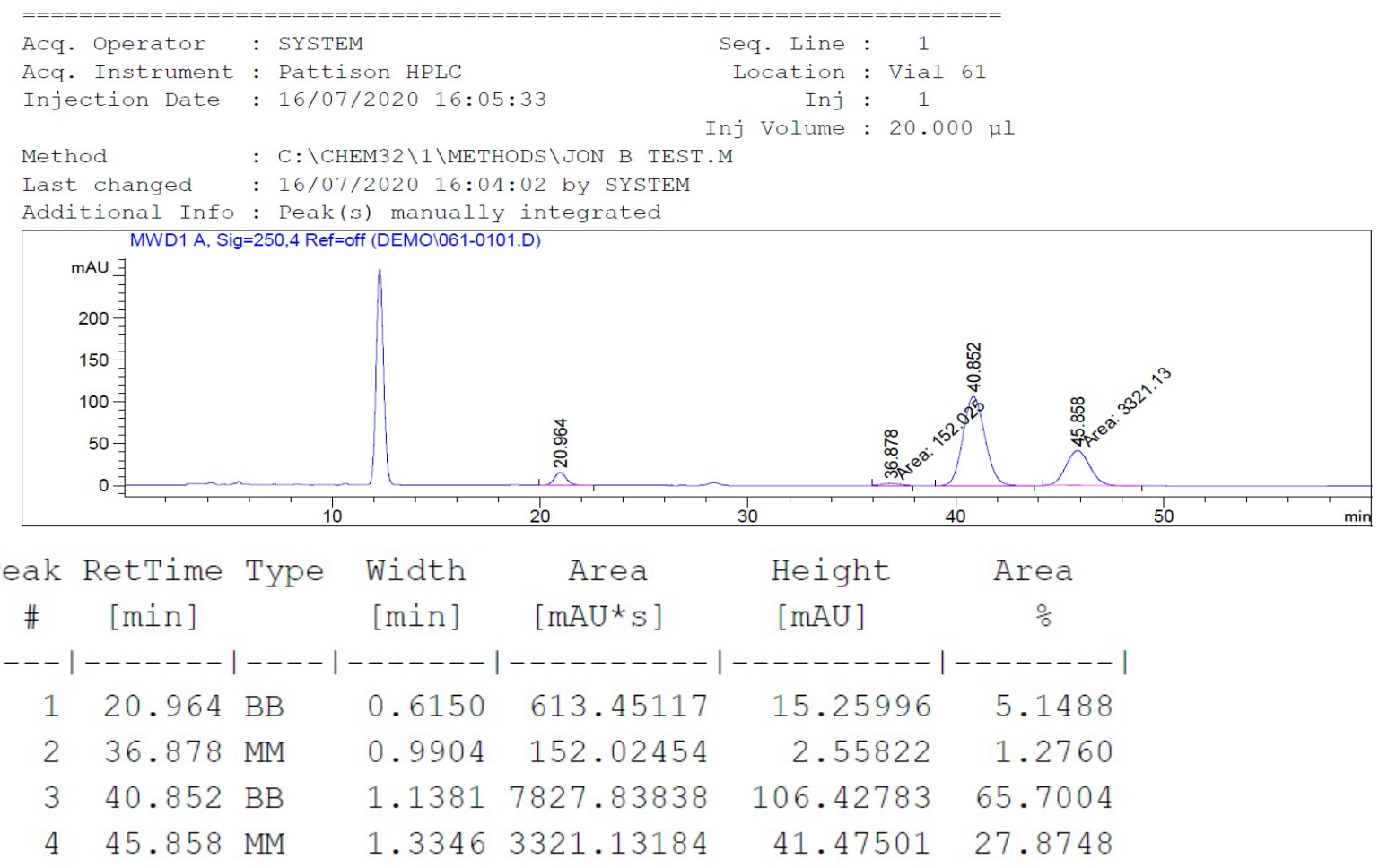


Chiral HPLC of ATH 2-hydroxy-4-oxo- $N, 3-$ diphenyl-4-(piperidin-1-yl)butanamide showing ee for d1.

Data File C: \CHEM32\1\DATA \DEMO\061-0101.D

Sample Name: VKV 672 ATH 901010 IC
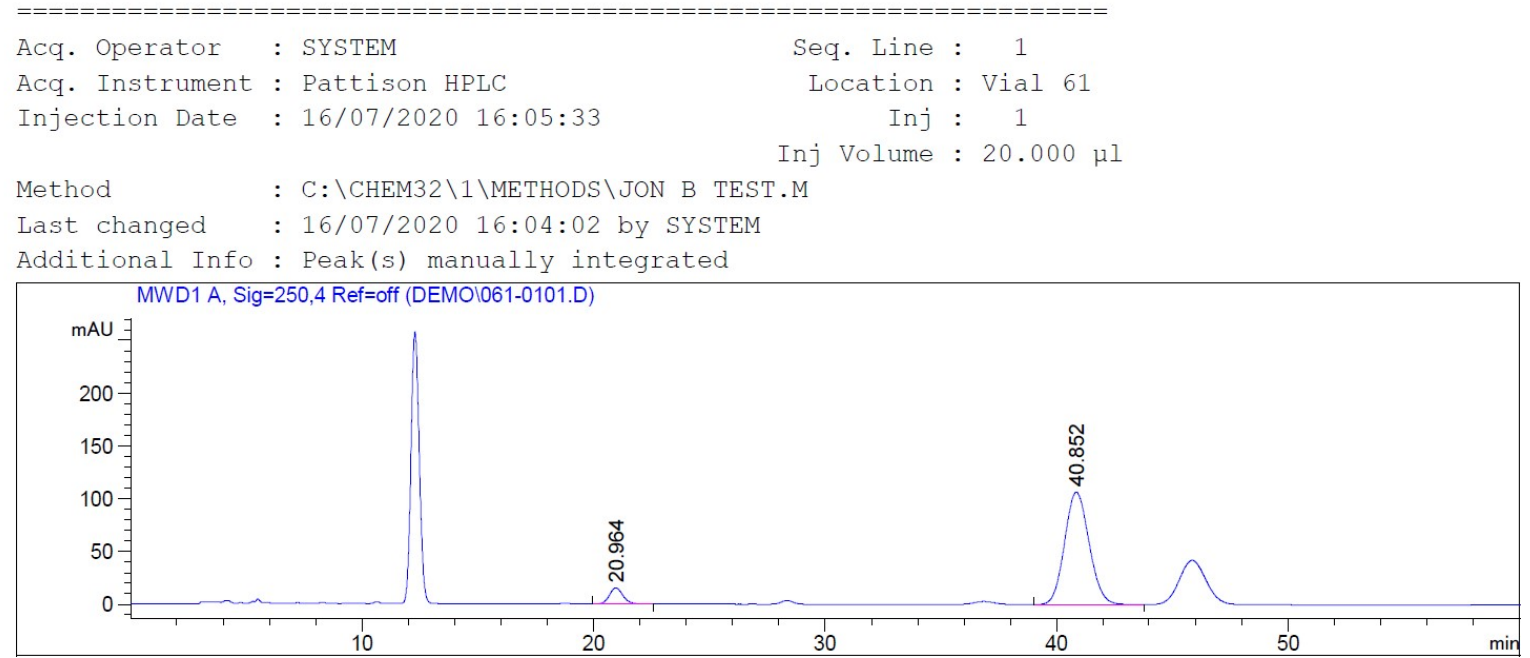

\begin{tabular}{|c|c|c|c|c|c|c|}
\hline $\begin{array}{c}\text { Peak } \\
\#\end{array}$ & $\begin{array}{c}\text { RetTime } \\
\text { [min] }\end{array}$ & Type & $\begin{array}{l}\text { Width } \\
\text { [min] }\end{array}$ & $\begin{array}{c}\text { Area } \\
{\left[\mathrm{mAU}^{\star} \mathrm{s}\right]}\end{array}$ & $\begin{array}{l}\text { Height } \\
\text { [mAU] }\end{array}$ & $\begin{array}{c}\text { Area } \\
\%\end{array}$ \\
\hline & ------ & & ----- & $\mid----------$ & --------- & -------- \\
\hline 1 & 20.964 & $\mathrm{BB}$ & .6150 & 613.45117 & 15.25996 & 7.2673 \\
\hline 2 & 40.852 & $\mathrm{RP}$ & .1381 & 7827.83838 & 106.42783 & 92.7327 \\
\hline
\end{tabular}


Chiral HPLC of ATH 2-hydroxy-4-oxo- $N, 3-$ diphenyl-4-(piperidin-1-yl)butanamide showing ee for $\mathrm{d} 2$.

Data File C:\CHEM32\1\DATA \DEMO\061-0101.D

Sample Name: VKV 672 ATH 901010 IC
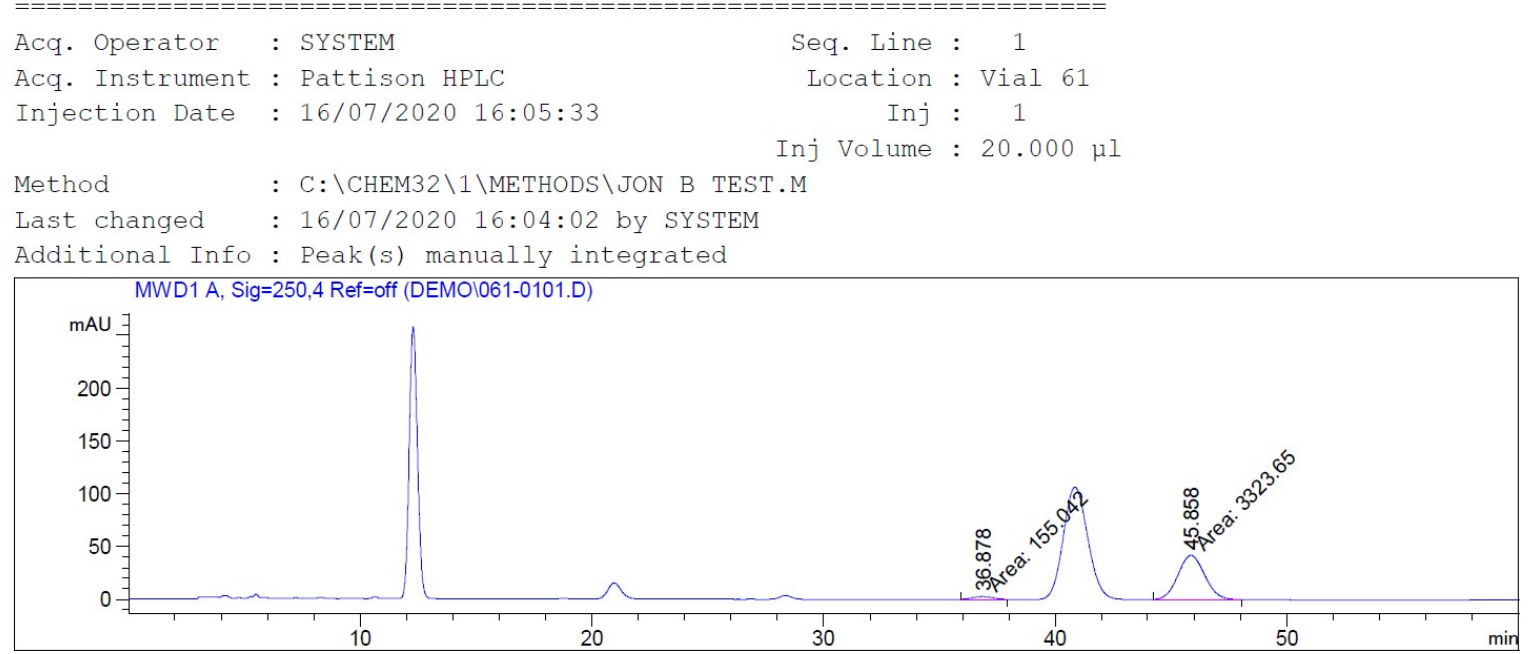

\begin{tabular}{|c|c|c|c|c|c|c|}
\hline $\begin{array}{c}\text { eak } \\
\#\end{array}$ & $\begin{array}{c}\text { RetTime } \\
\text { [min] }\end{array}$ & Type & $\begin{array}{l}\text { Width } \\
{[\mathrm{min}]}\end{array}$ & $\begin{array}{c}\text { Area } \\
{\left[\mathrm{mAU}^{*} \mathrm{~s}\right]}\end{array}$ & $\begin{array}{l}\text { Height } \\
{[\mathrm{mAU}]}\end{array}$ & $\begin{array}{c}\text { Area } \\
\frac{\circ}{\circ}\end{array}$ \\
\hline & & & & . & & 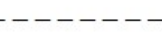 \\
\hline 1 & 36.878 & MM & 1.0005 & 155.04218 & 2.58263 & 4.4569 \\
\hline 2 & 45.858 & MM & 264 & 3323.65210 & 41.76245 & 95.5431 \\
\hline
\end{tabular}




\section{Chiral HPLC of ketone; 2,4-dioxo- $N$,3-diphenyl-4-(piperidin-1-yl)butanamide.}

Data File C:\CHEM32\1\DATA \DEMO \085-0101.D

Sample Name: VKV 471802010 Ketone IC
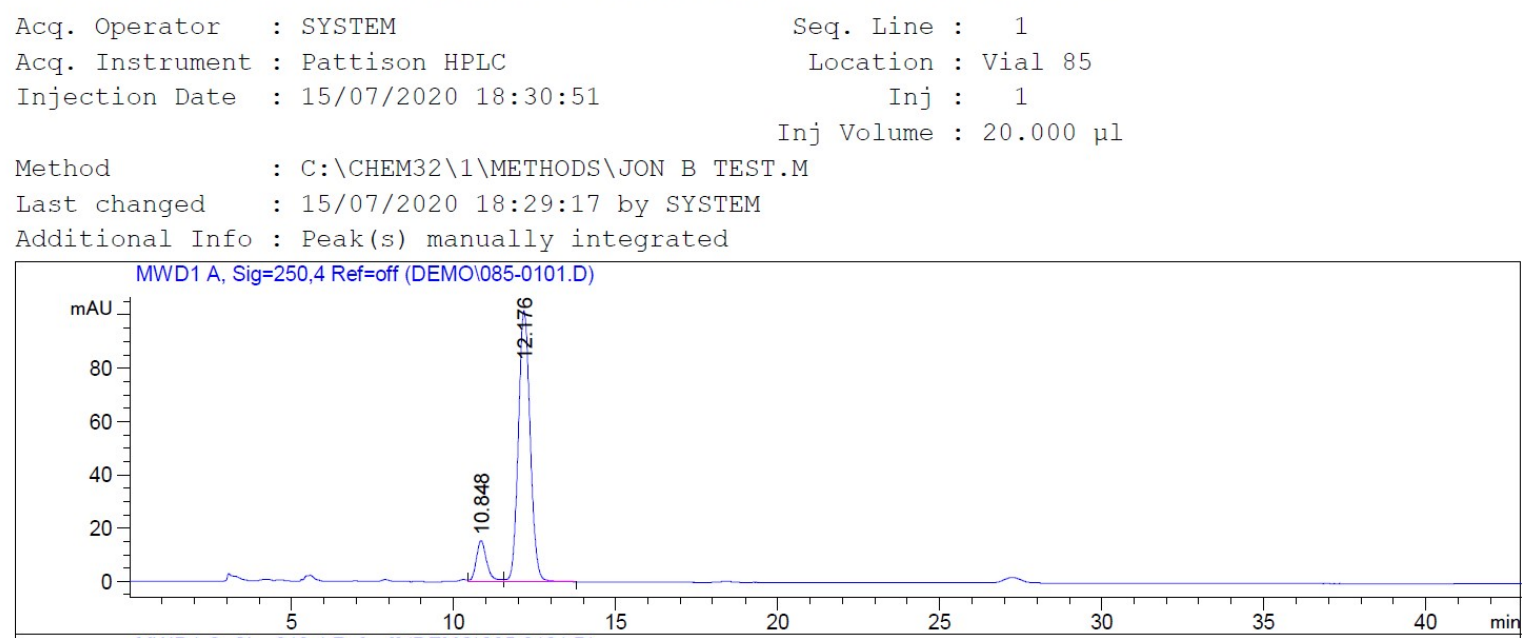

\begin{tabular}{|c|c|c|c|c|c|c|}
\hline eak & $\begin{array}{c}\text { RetTime } \\
\text { [min] }\end{array}$ & Type & $\begin{array}{l}\text { Width } \\
\text { [min] }\end{array}$ & $\begin{array}{c}\text { Area } \\
{\left[\mathrm{mAU}{ }^{\star} \mathrm{s}\right]}\end{array}$ & $\begin{array}{l}\text { Height } \\
\text { [mAU] }\end{array}$ & $\begin{array}{c}\text { Area } \\
\quad \%\end{array}$ \\
\hline & & & & --- & ---- & ---1 \\
\hline 1 & & & & 348.12286 & 0778 & 03 \\
\hline 2 & 76 & & 41 & 2579.67627 & 65299 & 097 \\
\hline
\end{tabular}




\section{3-Methyl-2,4-dioxo- $N$-phenyl-4-(piperidin-1-yl)butanamide.}<smiles>CC(C(=O)Nc1ccccc1)C(=O)N1CCCCC1</smiles>

This compound is novel and was prepared following the general procedure B using methyl 2oxo-2-(phenylamino)acetate $(500 \mathrm{mg}, 2.79 \mathrm{mmol}, 1.0 \mathrm{eq})$ in anhydrous THF (10 mL), 1(piperidin-1-yl)propan-1-one (420 mg, $3.07 \mathrm{mmol}, 1.1 \mathrm{eq})$ in anhydrous THF (10 mL) and LDA (1M solution in THF, $5.58 \mathrm{~mL}, 5.58 \mathrm{mmol}, 2.0 \mathrm{eq})$ to give crude material which was further purified by manual column chromatography (25-30\% EtOAc in petroleum ether) to afford the product as a white solid (140 $\mathrm{mg}, 0.48 \mathrm{mmol}, 17 \%)$.

HRMS (ESI) m/z: [M+Na] ${ }^{+}$Calcd for $\mathrm{C}_{16} \mathrm{H}_{20} \mathrm{~N}_{2} \mathrm{O}_{3} \mathrm{Na} 311.1366$; Found 311.1364;

${ }^{1} \mathrm{H}$ NMR $\left(400 \mathrm{MHz}, \mathrm{CDCl}_{3}\right) \delta 8.68(1 \mathrm{H}, \mathrm{s}, \mathrm{NH}), 7.61(2 \mathrm{H}, \mathrm{d}, J=8.0 \mathrm{~Hz}, \mathrm{ArH}), 7.34(2 \mathrm{H}, \mathrm{t}, J$ $=7.9 \mathrm{~Hz}, \mathrm{ArH}), 7.19-7.09(1 \mathrm{H}, \mathrm{m}, \mathrm{ArH}), 4.59\left(1 \mathrm{H}, \mathrm{q}, J=7.1 \mathrm{~Hz}, \mathrm{C}^{\mathrm{HCH}} 3\right), 3.63-3.48(4 \mathrm{H}$, m, piperidine), $1.73-1.57$ (6H, m, piperidine), $1.38\left(3 \mathrm{H}, \mathrm{d}, J=7.1 \mathrm{~Hz}, \mathrm{CHCH}_{3}\right)$;

${ }^{13} \mathrm{C}$ NMR $\left(101 \mathrm{MHz}, \mathrm{CDCl}_{3}\right) \delta 194.5,169.3,157.7,136.4,129.1,125.2,119.9,47.8,43.5$, 42.9, 26.4, 25.4, 24.5, 12.0;

$m / z(\mathrm{ESI}) 311.3\left[(\mathrm{M}+\mathrm{Na})^{+}, 100 \%\right]$. 
3-Methyl-2,4-dioxo-N-phenyl-4-(piperidin-1-yl)butanamide.

${ }^{1} \mathbf{H}$ NMR (400 MHz, $\left.\mathrm{CDCl}_{3}\right)$.

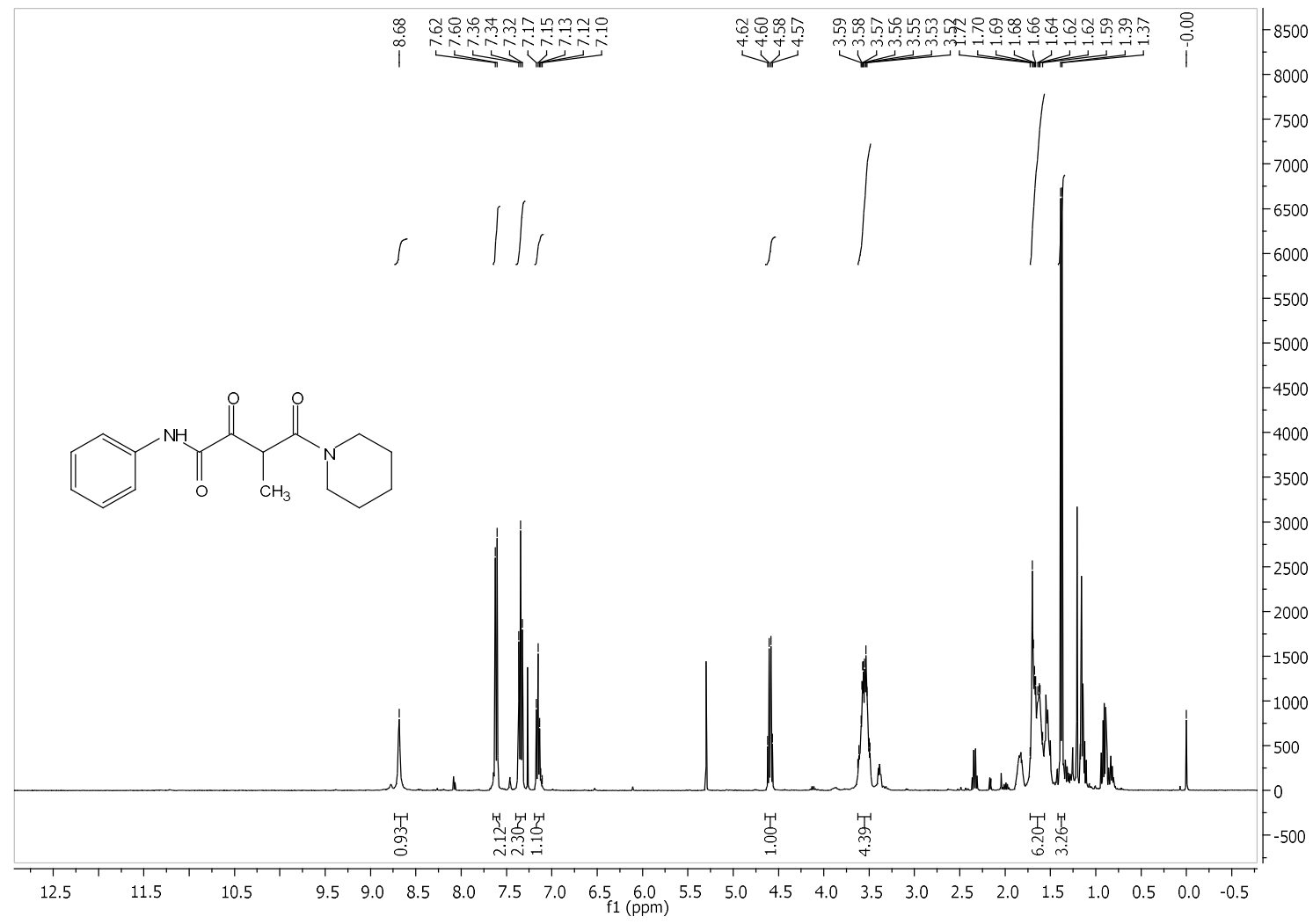

${ }^{13}$ C NMR (101 MHz, $\left.\mathrm{CDCl}_{3}\right)$.

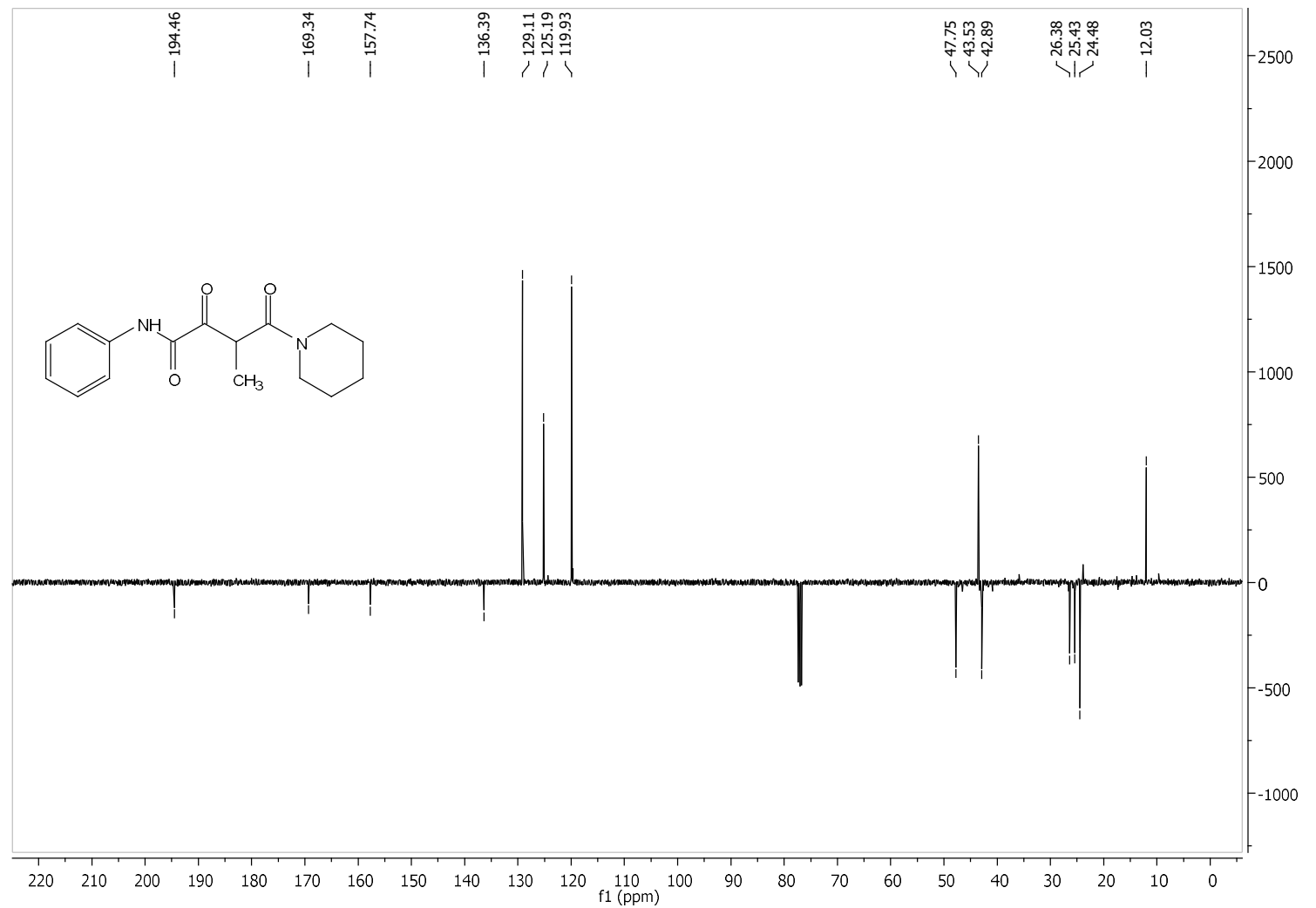




\section{Racemic and chiral 2-hydroxy-3-methyl-4-oxo- $N$-phenyl-4-(piperidin-1-yl)butanamide}

49.<smiles>CC(C(=O)N1CCCCC1)C(O)C(=O)Nc1ccccc1</smiles>

This compound is Novel.

This compound was prepared in racemic form following this procedure: 3-Methyl-2,4-dioxo$\mathrm{N}$-phenyl-4-(piperidin-1-yl)butanamide (57 $\mathrm{mg}, 0.20 \mathrm{mmol}, 1.0$ equiv) was dissolved in $\mathrm{MeOH}(5 \mathrm{~mL})$ at $\mathrm{rt}$ and $\mathrm{NaBH}_{4}(16 \mathrm{mg}, 0.40 \mathrm{mmol}, 2.0$ equiv) was added. The reaction was stirred at $\mathrm{rt}$ for $2 \mathrm{~h}$. After the completion of the reaction, it was quenched by water $(5 \mathrm{~mL})$ and extracted with ethyl acetate $(8 \mathrm{~mL})$. The organic layer was dried with $\mathrm{Na}_{2} \mathrm{SO}_{4}$ and concentrated to obtain a residue which was purified by flash chromatography on silica gel. A mixture of diastereomers was isolated by flash chromatography (pet ether/ EtOAc: 70:30) as a colourless oil (35 mg, $0.12 \mathrm{mmol}, 61 \%)$.

This compound is novel and was prepared in asymmetric form following the general procedure $\mathrm{C}$ using : 3-methyl-2,4-dioxo-N-phenyl-4-(piperidin-1-yl)butanamide (57 mg, 0.20 mmol, 1.0 equiv), 3C tethered catalyst $(R, R)-2\left(1.2 \mathrm{mg}, 1.9 \times 10^{-3} \mathrm{mmol}, 1 \mathrm{~mol} \%\right)$ and FA:TEA $(0.3 \mathrm{~mL})$ in DCM $(2 \mathrm{~mL})$ to give 49 as a clear colourless oil $(49 \mathrm{mg}, 0.17 \mathrm{mmol}$, $85 \%)$.

HRMS (ESI) m/z: [M+Na] ${ }^{+}$Calcd for $\mathrm{C}_{16} \mathrm{H}_{22} \mathrm{~N}_{2} \mathrm{O}_{3} \mathrm{Na}$ 313.1523; Found 313.1510; $U_{\max } 3419,2931,2849,1624,1439,1371,1254,1189,852,542 \mathrm{~cm}^{-1}$;

Enantiomeric excess and diastereomeric ratio determined by HPLC analysis (CHIRALPAK IC column, hexane 90:10 $\mathrm{iPrOH}, 1.0 \mathrm{~mL} / \mathrm{min}, \mathrm{T}=25^{\circ} \mathrm{C}, \lambda=250 \mathrm{~nm}$, Ketone 24.6 and 30.4, $t_{\mathrm{R}}=24.3 \min$ (major, d1), $29.2 \min$ (minor, d2), $32.9 \min$ (major, d2), $58.4 \min$ (minor, d1). $33: 67 \mathrm{dr}, 98 \%$ ee $(\mathrm{d} 1), 68 \%$ ee $(\mathrm{d} 2)$;

${ }^{1} \mathrm{H}$ NMR $\left(400 \mathrm{MHz}, \mathrm{CDCl}_{3}\right) \delta 9.17(1 \mathrm{H}, \mathrm{s}, \mathrm{NH}), 7.57(2 \mathrm{H} . \mathrm{d}, J=7.9 \mathrm{~Hz}, \mathrm{ArH}), 7.32(2 \mathrm{H}, \mathrm{t}, J$ $=7.9 \mathrm{~Hz}, \mathrm{ArH}), 7.10(1 \mathrm{H}, \mathrm{t}, J=7.4 \mathrm{~Hz}, \mathrm{ArH}), 6.22(1 \mathrm{H}, \mathrm{d}, J=8.5 \mathrm{~Hz}, \mathrm{C} \underline{\mathrm{HOH}}), 4.15(1 \mathrm{H}, \mathrm{d}, J$ $\left.=6.2 \mathrm{~Hz}, \mathrm{C}_{\mathrm{HCH}}\right), 3.57-3.43(4 \mathrm{H}, \mathrm{m}$, piperidine $), 1.65-1.46(6 \mathrm{H}, \mathrm{m}$, piperidine $), 1.37$ $\left(3 \mathrm{H}, \mathrm{d}, J=7.4 \mathrm{~Hz}, \mathrm{CHC}_{3}\right)$;

${ }^{13} \mathrm{C}$ NMR $\left(101 \mathrm{MHz}, \mathrm{CDCl}_{3}\right) \delta$ 174.7, 171.4, 137.5, 128.9, 124.3, 119.7, 75.30, 47.2, 42.8, 35.6, 26.6, 25.6, 24.4, 15.2;

$m / z(\mathrm{ESI}) 313.1\left[(\mathrm{M}+\mathrm{Na})^{+}, 100 \%\right]$. 
2-Hydroxy-3-methyl-4-oxo-N-phenyl-4-(piperidin-1-yl)butanamide

49 (Mixture of Diastereomers).

${ }^{1}$ H NMR (400 MHz, $\left.\mathrm{CDCl}_{3}\right)$.

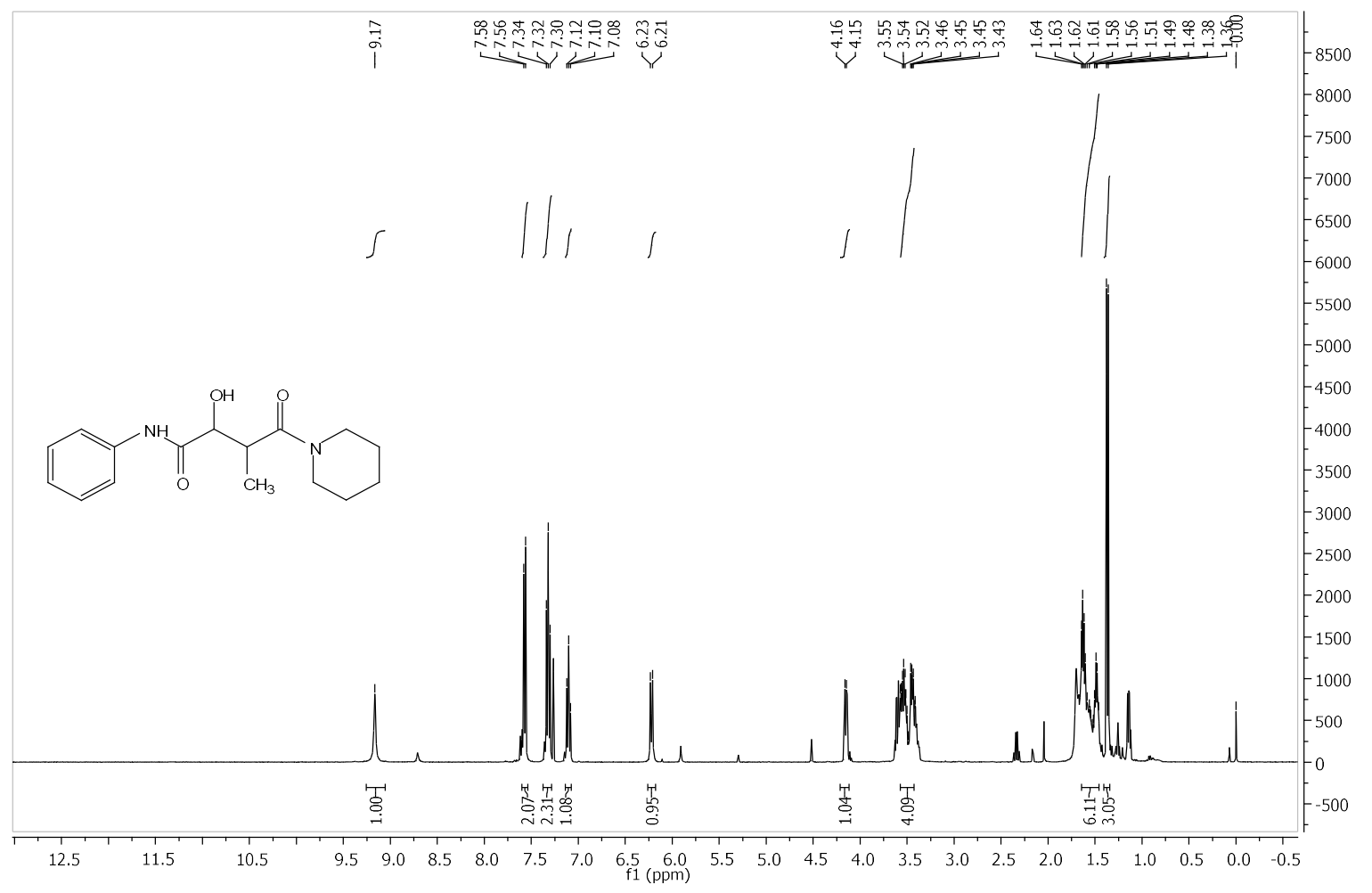

${ }^{13}$ C NMR (101 MHz, $\left.\mathrm{CDCl}_{3}\right)$.

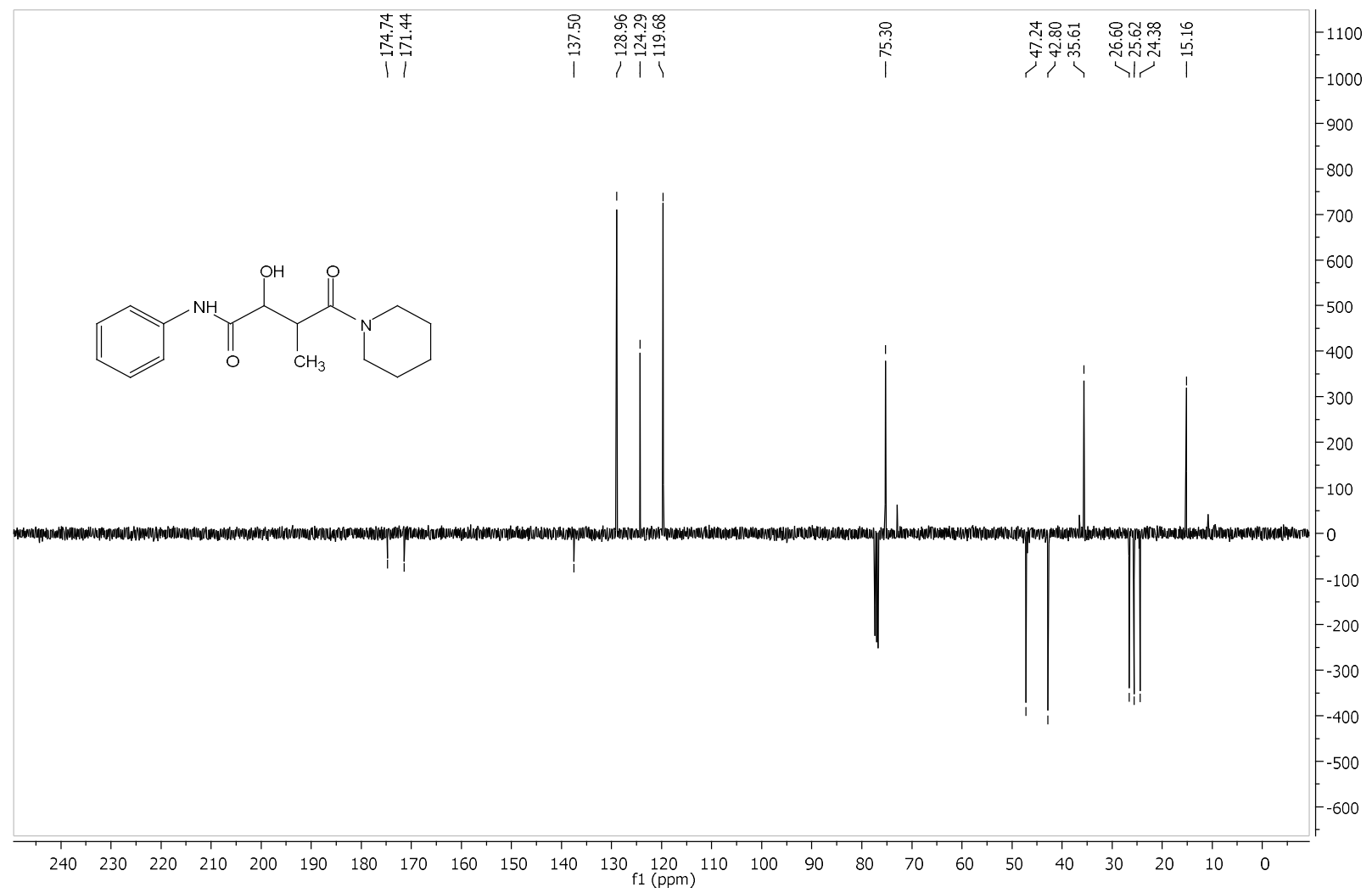

S418 


\section{Chiral HPLC of racemic 2-hydroxy-3-methyl-4-oxo-N-phenyl-4-(piperidin-1-}

\section{yl)butanamide.}

Data File C: \CHEM32\1\DATA \DEMO\022-0101.D

Sample Name: VKV 745 Rac 901010 IC
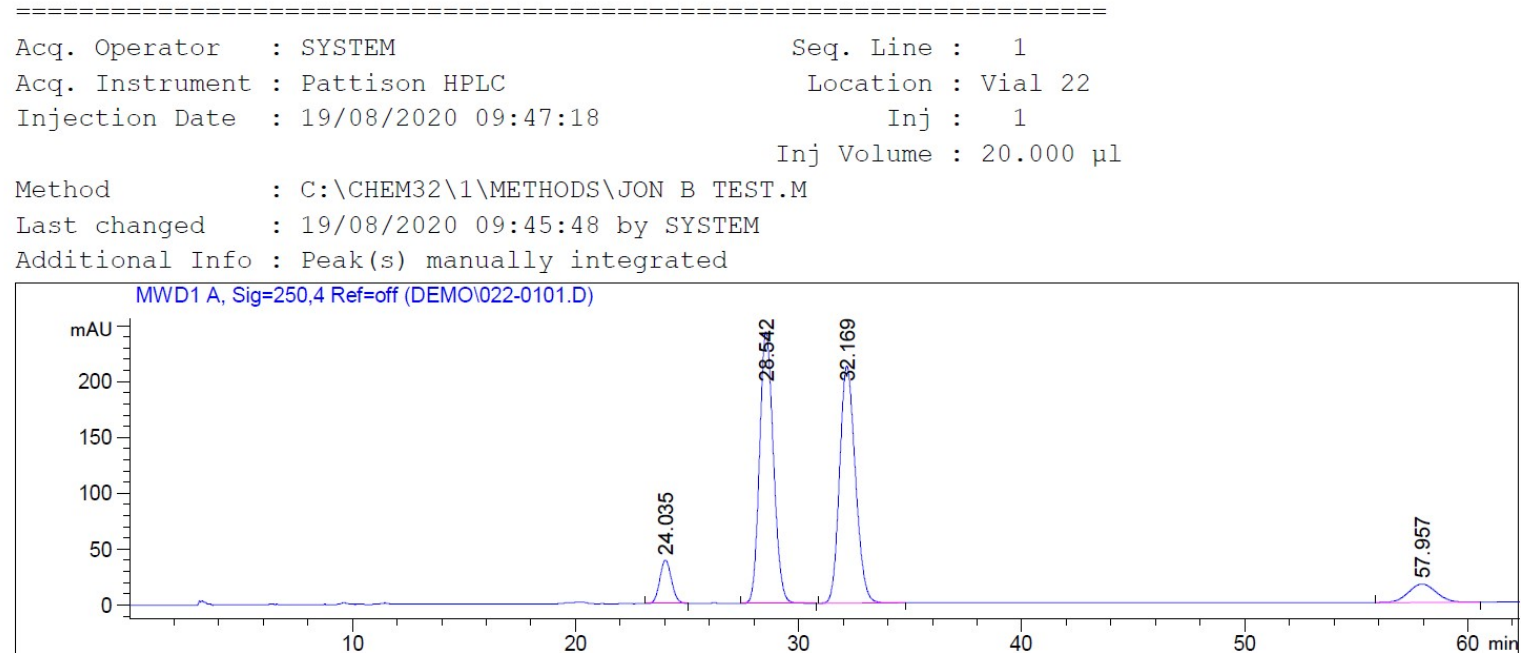

\begin{tabular}{|c|c|c|c|c|c|c|}
\hline ak & $\begin{array}{c}\text { RetTime } \\
\text { [min] }\end{array}$ & Type & $\begin{array}{l}\text { Width } \\
\text { [min] }\end{array}$ & $\begin{array}{c}\text { Area } \\
{\left[\mathrm{mAU}{ }^{\star} \mathrm{s}\right]}\end{array}$ & $\begin{array}{l}\text { Height } \\
{[\mathrm{mAU}]}\end{array}$ & $\begin{array}{c}\text { Area } \\
\%\end{array}$ \\
\hline & & & & $1--$ & - & 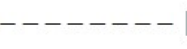 \\
\hline 1 & 24.035 & $\mathrm{BB}$ & 0.5620 & 1405.68262 & 38.86271 & 5.9243 \\
\hline 2 & 28.542 & $\mathrm{BB}$ & 0.6731 & $1.04524 e 4$ & 242.36308 & 44.0519 \\
\hline 3 & 32.169 & $\mathrm{BB}$ & 0.7679 & $1.04446 \mathrm{e} 4$ & 211.58893 & 44.0192 \\
\hline 4 & 57.957 & $\mathrm{BB}$ & 1.1696 & 1424.71814 & 16.36643 & 6.0045 \\
\hline
\end{tabular}




\section{Chiral HPLC of ATH of 2-hydroxy-3-methyl-4-oxo-N-phenyl-4-(piperidin-1-}

yl)butanamide showing dr.

Jata File C: \CHEM32\1\DATA \DEMO\093-0101.D

sample Name: VKV 747 ATH 901010 IC

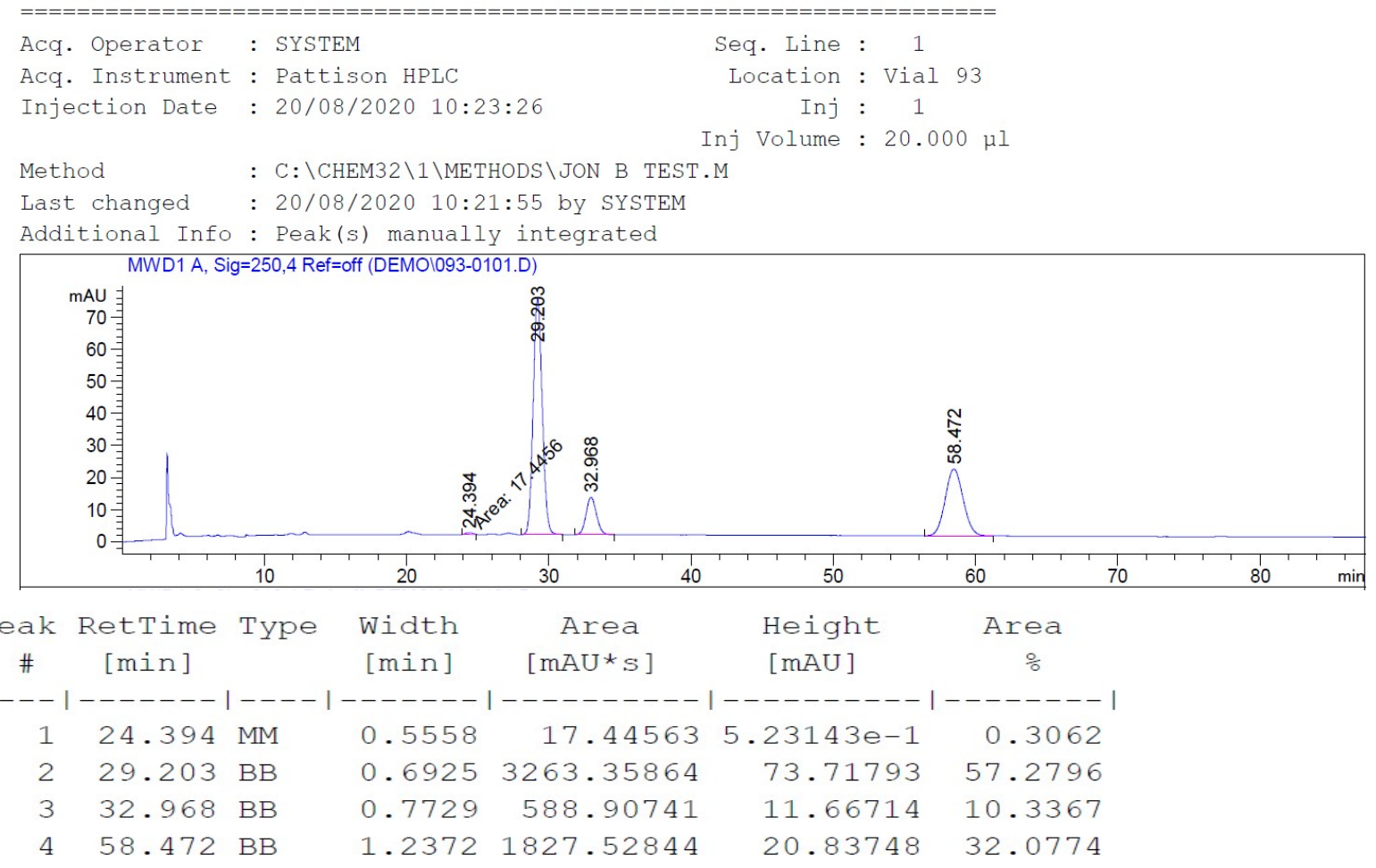




\section{Chiral HPLC of ATH of 2-hydroxy-3-methyl-4-oxo-N-phenyl-4-(piperidin-1-} yl)butanamide showing ee for $\mathrm{d} 2$.

Data File C: \CHEM32\1\DATA \DEMO\093-0101.D

Sample Name: VKV 747 ATH 901010 IC
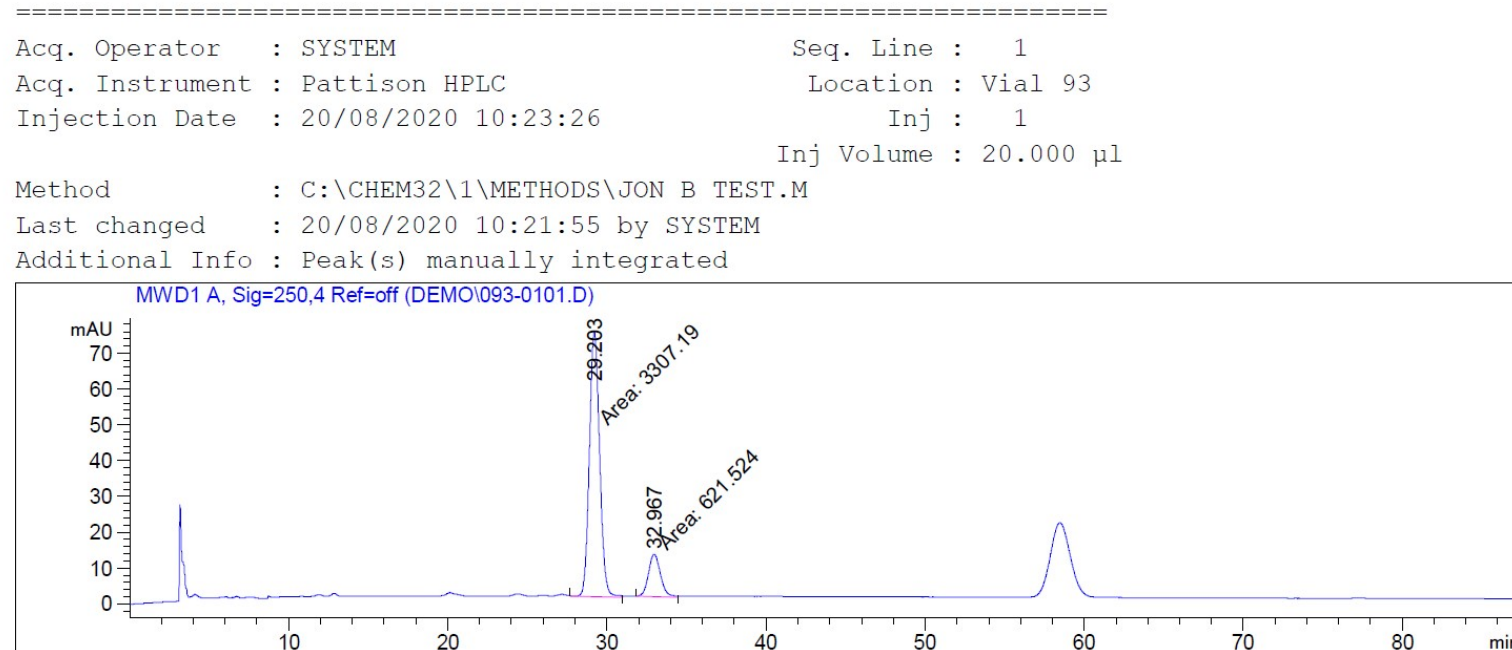

\begin{tabular}{|c|c|c|c|c|c|c|}
\hline eak & $\begin{array}{c}\text { RetTime } \\
\text { [min] }\end{array}$ & Type & $\begin{array}{l}\text { Width } \\
\text { [min] }\end{array}$ & $\begin{array}{c}\text { Area } \\
{\left[\mathrm{mAU}^{*} \mathrm{~s}\right]}\end{array}$ & $\begin{array}{l}\text { Height } \\
{[\mathrm{mAU}]}\end{array}$ & $\begin{array}{c}\text { Area } \\
\quad \%\end{array}$ \\
\hline & & & & ---- & 1 & \\
\hline 1 & & & & 3307.19165 & 685 & 00 \\
\hline 2 & 67 & & & 621.52380 & 4568 & 3200 \\
\hline
\end{tabular}


HPLC of ATH of 2-hydroxy-3-methyl-4-oxo-N-phenyl-4-(piperidin-1-yl)butanamide showing ee for d1.

Jata File C:\CHEM32\1\DATA \DEMO\093-0101.D

sample Name: VKV 747 ATH 901010 IC

\begin{tabular}{|c|c|c|c|}
\hline Acq. Operator & : SYSTEM & Seq. Line & 1 \\
\hline Acq. Instrument & : Pattison HPLC & Location & : Vial 93 \\
\hline Injection Date & $: 20 / 08 / 202010: 23: 26$ & $\begin{array}{r}\text { Inj } \\
\text { Inj Volume }\end{array}$ & $\begin{array}{l}: \quad 1 \\
: 20.000 \mu l\end{array}$ \\
\hline
\end{tabular}

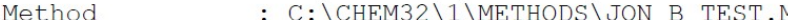

Last changed : 20/08/2020 10:21:55 by SYSTEM

Additional Info: Peak(s) manually integrated

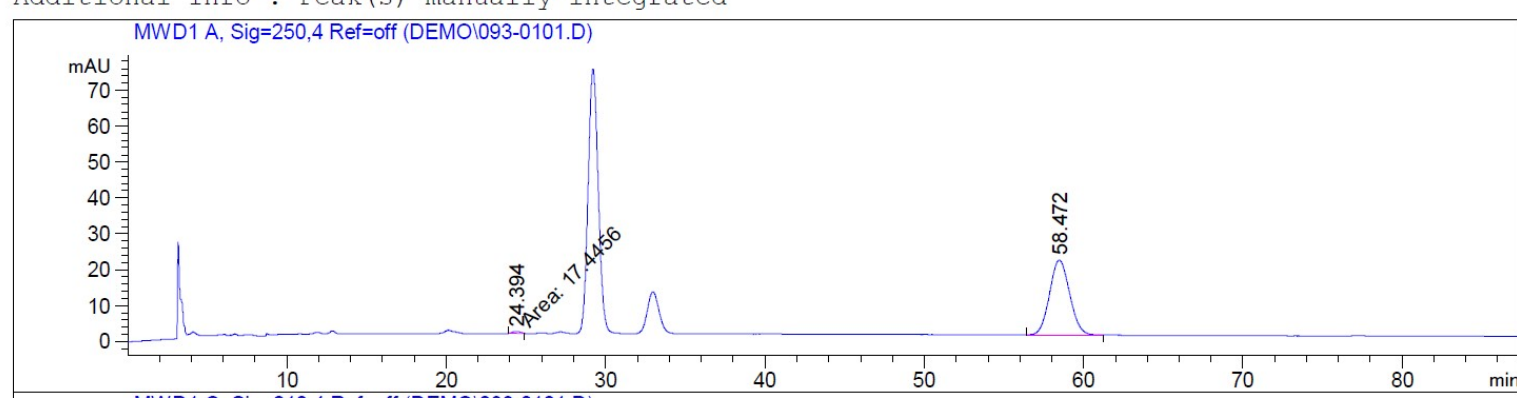

\begin{tabular}{|c|c|c|c|c|c|c|}
\hline eak & $\begin{array}{c}\text { RetTime } \\
\text { [min] }\end{array}$ & Type & $\begin{array}{l}\text { Width } \\
\text { [min] }\end{array}$ & $\begin{array}{c}\text { Area } \\
{\left[m A U^{*} \mathrm{~s}\right]}\end{array}$ & $\begin{array}{l}\text { Height } \\
{[\mathrm{mAU}]}\end{array}$ & $\begin{array}{c}\text { Area } \\
\div\end{array}$ \\
\hline & & & & -- & 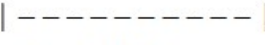 & \\
\hline 1 & 24 & MM & & 17.44563 & $3 e-1$ & 456 \\
\hline 2 & 72 & & 72 & 1827.52844 & 3748 & 99.0544 \\
\hline
\end{tabular}


HPLC of 3-methyl-2,4-dioxo-N-phenyl-4-(piperidin-1-yl)butanamide precursor ketone.

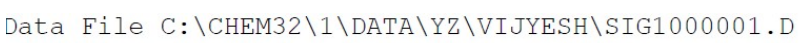

Sample Name: VKV 741 Ketone 901010 IC
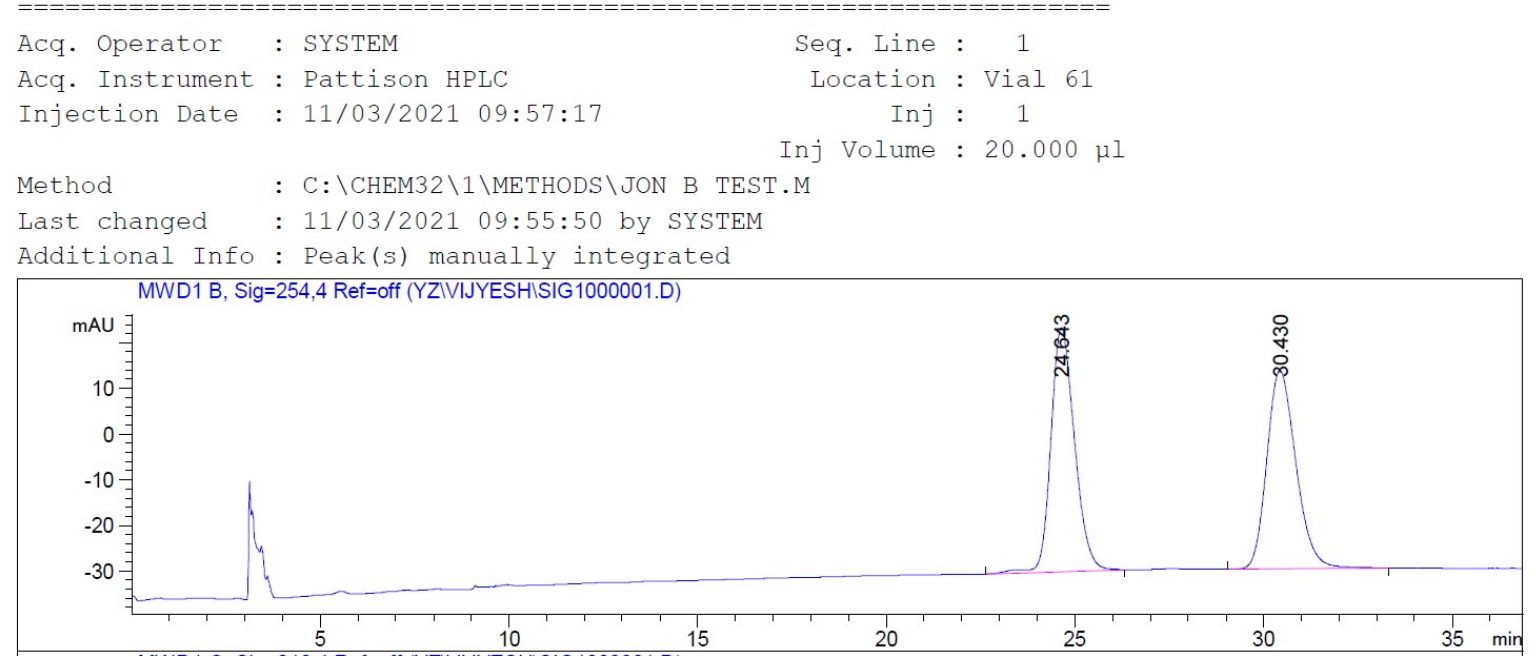

\begin{tabular}{|c|c|c|c|c|c|c|}
\hline $\begin{array}{c}\text { Peak } \\
\#\end{array}$ & $\begin{array}{c}\text { RetTime } \\
\text { [min] }\end{array}$ & Type & $\begin{array}{l}\text { Width } \\
\text { [min] }\end{array}$ & $\begin{array}{c}\text { Area } \\
{\left[\mathrm{mAU}^{*} \mathrm{~s}\right]}\end{array}$ & $\begin{array}{l}\text { Height } \\
\text { [mAU] }\end{array}$ & $\begin{array}{c}\text { Area } \\
\quad \%\end{array}$ \\
\hline & & & & & & \\
\hline 1 & 543 & $\mathrm{BB}$ & 709 & 2330.86401 & 3.43750 & 012 \\
\hline 2 & 30.430 & $\mathrm{BB}$ & 0.8294 & 2330.74878 & 43.48126 & 9988 \\
\hline
\end{tabular}




\section{3-Benzyl-2,4-dioxo- $N$-phenyl-4-(piperidin-1-yl)butanamide.}<smiles>O=C(Nc1ccccc1)C(=O)C(Cc1ccccc1)C(=O)N1CCCCC1</smiles>

This compound is novel and was prepared following the general procedure B using methyl 2oxo-2-(phenylamino)acetate (608 mg, $3.40 \mathrm{mmol}, 1.0 \mathrm{eq})$ in anhydrous THF (10 mL) , 3phenyl-1-(piperidin-1-yl)propan-1-one (800 mg, $3.69 \mathrm{mmol}, 1.1 \mathrm{eq})$ in anhydrous THF (10 $\mathrm{mL}$ ) and LDA (1M solution in THF, $6.80 \mathrm{~mL}, 6.80 \mathrm{mmol}, 2.0 \mathrm{eq})$ to give crude material which was further purified by manual column chromatography (13-15\% EtOAc in petroleum ether) to afford the product as a white solid (400 $\mathrm{mg}, 1.09 \mathrm{mmol}, 32 \%)$.

HRMS (ESI) m/z: [M+Na] ${ }^{+}$Calcd for $\mathrm{C}_{22} \mathrm{H}_{24} \mathrm{~N}_{2} \mathrm{O}_{3} \mathrm{Na} 387.1679$; Found 387.1664;

${ }^{1} \mathrm{H}$ NMR (400 MHz, $\left.\mathrm{CDCl}_{3}\right) \delta 8.68(1 \mathrm{H}, \mathrm{s}, \mathrm{NH}), 7.58(2 \mathrm{H}, \mathrm{d}, J=7.7 \mathrm{~Hz}, \mathrm{ArH}), 7.37$ - 7.13 (8H, m, ArH), 4.85 (1H, dd, $\left.J=8.7,6.1 \mathrm{~Hz}, \underline{\mathrm{HCH}}_{2}\right), 3.59$ - 3.51 (2H, m, piperidine), 3.37 3.28 (2H, m, piperidine), $2.95\left(1 \mathrm{H}, \mathrm{dd}, J=18.0,9.6 \mathrm{~Hz}, \mathrm{CHC}_{2}\right), 2.62(1 \mathrm{H}, \mathrm{dd}, J=9.0,7.0$ $\left.\mathrm{Hz}, \mathrm{CHC}_{2}\right), 1.61-1.48$ (6H, m, piperidine);

${ }^{13} \mathrm{C} \mathrm{NMR}\left(101 \mathrm{MHz}, \mathrm{CDCl}_{3}\right) \delta 193.2,167.0,157.4,138.3,136.3,129.2,129.2,128.6,128.5$, $126.8,126.1,125.3,119.9,50.8,47.7,43.0,34.1,31.6,25.3,24.3$;

$m / z(\mathrm{ESI}) 387.3\left[(\mathrm{M}+\mathrm{Na})^{+}, 100 \%\right]$. 
3-benzyl-2,4-dioxo- $N$-phenyl-4-(piperidin-1-yl)butanamide.

${ }^{1} \mathbf{H}$ NMR (400 MHz, $\left.\mathrm{CDCl}_{3}\right)$.

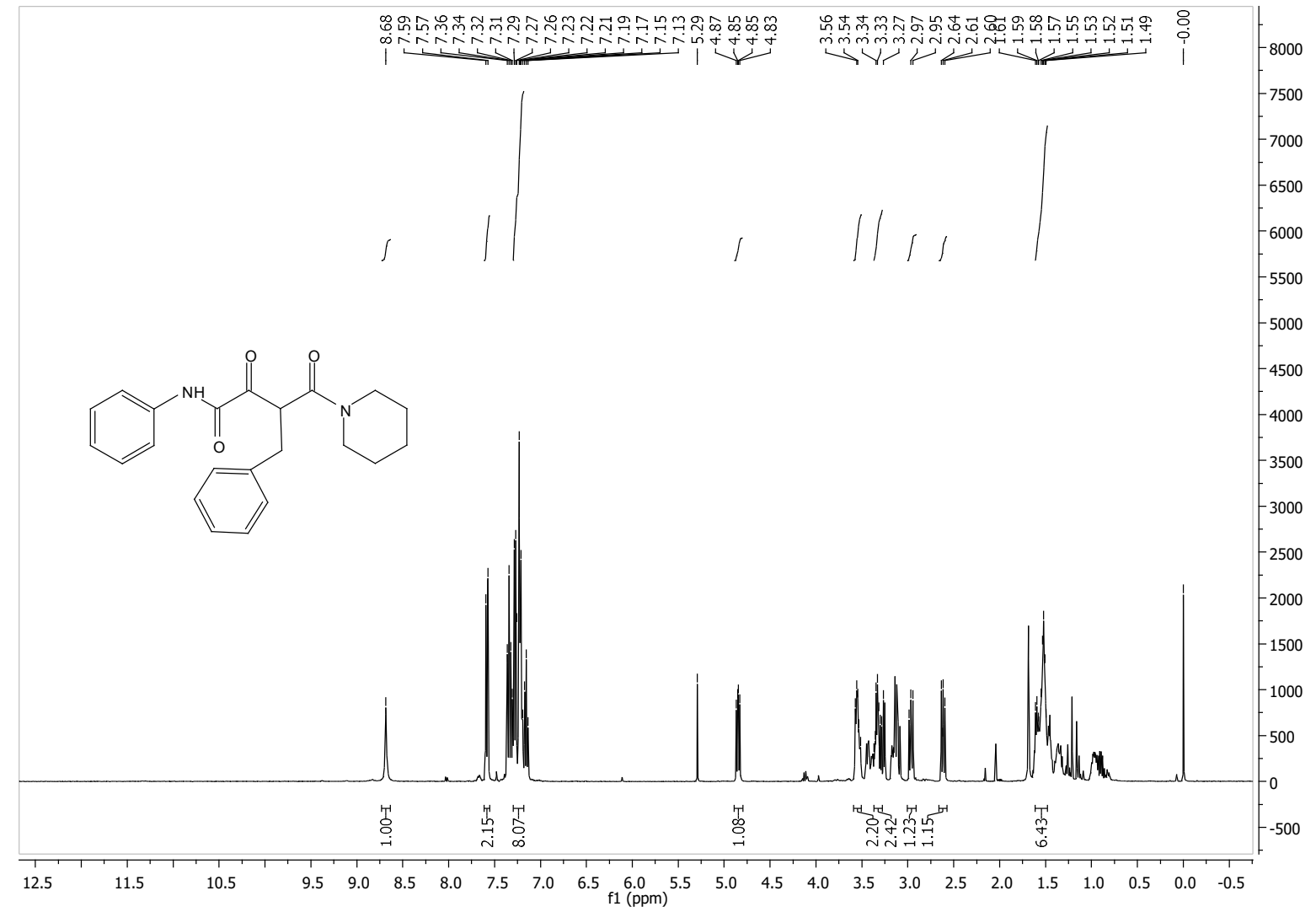

${ }^{13}$ C NMR (101 MHz, $\left.\mathrm{CDCl}_{3}\right)$.

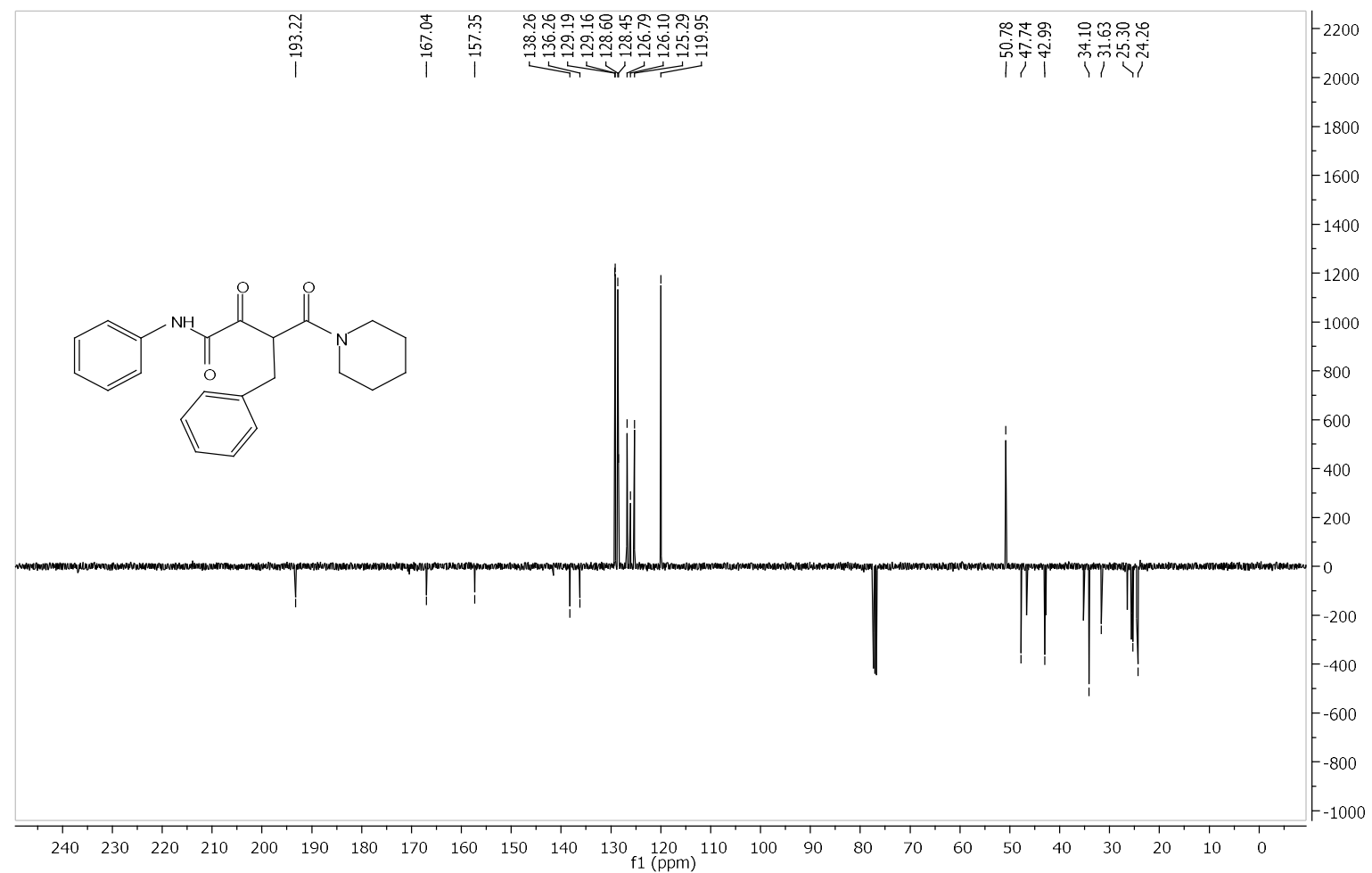




\section{Racemic and chiral 3-benzyl-2-hydroxy-4-oxo- $N$-phenyl-4-(piperidin-1-yl)butanamide}

50.<smiles>O=C(Nc1ccccc1)[C@H](O)C(Cc1ccccc1)C(=O)N1CCCCC1</smiles>

This compound is Novel.

This compound was prepared in racemic form following this procedure: 3-benzyl-2,4-dioxo$\mathrm{N}$-phenyl-4-(piperidin-1-yl)butanamide (72 $\mathrm{mg}, 0.2 \mathrm{mmol}, 1.0$ equiv) was dissolved in MeOH (5 mL) at $\mathrm{rt}$ and $\mathrm{NaBH}_{4}(16 \mathrm{mg}, 0.4 \mathrm{mmol}, 2.0$ equiv) was added. The reaction was stirred at $\mathrm{rt}$ for $2 \mathrm{~h}$. After the completion of the reaction, it was quenched by water $(5 \mathrm{~mL})$ and extracted with ethyl acetate $(8 \mathrm{~mL})$. The organic layer was dried with $\mathrm{Na}_{2} \mathrm{SO}_{4}$ and concentrated to obtain a residue which is purified by flash chromatography on silica gel. A mixture of diastereomers was isolated by flash chromatography (pet ether/ EtOAc: 80:20) as a white solid (60 mg, $0.16 \mathrm{mmol}, 83 \%$ ).

This compound is novel and was prepared in asymmetric form following the general procedure C using : 3-benzyl-2,4-dioxo-N-phenyl-4-(piperidin-1-yl)butanamide (182 mg, $0.50 \mathrm{mmol}, 1.0$ equiv), $3 \mathrm{C}$ tethered catalyst $(R, R)-2\left(3.1 \mathrm{mg}, 5.0 \times 10^{-3} \mathrm{mmol}, 1 \mathrm{~mol} \%\right)$ and FA:TEA $(0.5 \mathrm{~mL})$ in DCM (3 mL) to give 50 as a white solid (163 $\mathrm{mg}, 0.44 \mathrm{mmol}, 89 \%)$. HRMS (ESI) m/z: [M+Na] ${ }^{+}$Calcd for $\mathrm{C}_{22} \mathrm{H}_{26} \mathrm{~N}_{2} \mathrm{O}_{3} \mathrm{Na} 389.1836$; Found 389.1827; $U_{\max } 3419,2931,2849,1624,1439,1371,1254,1189,852,542 \mathrm{~cm}^{-1}$;

Enantiomeric excess and diastereomeric ratio determined by HPLC analysis (CHIRALPAK IC column, hexane 90:10 $\mathrm{PrOH}, 0.7 \mathrm{~mL} / \mathrm{min}, \mathrm{T}=25^{\circ} \mathrm{C}, \lambda=250 \mathrm{~nm}$, Ketone 41.6 and 46.8, $t_{\mathrm{R}}=23.3 \min$ (major, d1), $30.2 \min$ (minor, d2), $32.1 \min$ (major, d2), $48.4 \min$ (minor, $\mathrm{d} 1$ ). $43: 57 \mathrm{dr}, 99 \%$ ee $(\mathrm{d} 1), 86 \%$ ee $(\mathrm{d} 2)$;

${ }^{1} \mathrm{H}$ NMR $\left(400 \mathrm{MHz}, \mathrm{CDCl}_{3}\right)$ (Mixture of diastereomers, ca. 55:45) $\delta 8.82(0.55 \mathrm{H}, \mathrm{s}, \mathrm{NH}$, Major), $8.71(0.45 \mathrm{H}, \mathrm{s}, \mathrm{NH}$, Minor), $7.55(0.55 \mathrm{H}, \mathrm{d}, J=7.7 \mathrm{~Hz}, \mathrm{ArH}$, Major), $7.46(0.45 \mathrm{H}, \mathrm{d}$, $J=7.7 \mathrm{~Hz}, \mathrm{ArH}$, Minor), $7.29-7.03(9 \mathrm{H}, \mathrm{m}, \mathrm{ArH}), 6.50(0.55 \mathrm{H}, \mathrm{d}, J=8.8 \mathrm{~Hz}, \mathrm{C} \underline{\mathrm{HOH}}$, Major), $6.18(0.45 \mathrm{H}, \mathrm{s}, \mathrm{C} \underline{\mathrm{HOH}}, \mathrm{Minor}), 4.36(0.45 \mathrm{H}, \mathrm{d}, J=2.0 \mathrm{~Hz}, \mathrm{CHO} \underline{\mathrm{H}}$, Minor) 4.14 $(0.55 \mathrm{H}, \mathrm{dd}, J=8.8,2.3 \mathrm{~Hz}, \mathrm{CHO} \underline{\mathrm{H}}, \mathrm{Major}), 3.78-3.70$ (0.55H, m, $\left.\mathrm{CHCH}_{2} \mathrm{Major}\right), 3.65-$ $3.58\left(0.45 \mathrm{H}, \mathrm{m}, \mathrm{C}^{\mathrm{HCH}}{ }_{2}\right.$ Minor $), 3.51-3.45(1 \mathrm{H}, \mathrm{m}$, piperidine $), 3.35-3.24(2 \mathrm{H}, \mathrm{m}$, piperidine), $3.00-2.96\left(1 \mathrm{H}, \mathrm{m}\right.$, piperidine), $2.88\left(0.55 \mathrm{H}, \mathrm{d}, J=8.4 \mathrm{~Hz}, \mathrm{CHC}_{2} \underline{\mathrm{H}}_{2}\right.$ Major$), 2.73$ $-2.66\left(0.9 \mathrm{H}, \mathrm{m}, \mathrm{CHC} \underline{\mathrm{H}}_{2}\right.$ Minor), $2.53\left(0.55 \mathrm{H}, \mathrm{d}, J=7.7 \mathrm{~Hz}, \mathrm{CHC}_{2} \underline{\mathrm{H}}_{2}\right.$ Major$), 1.56-1.21(6 \mathrm{H}$, $\mathrm{m}$, piperidine); 
${ }^{13} \mathrm{C}$ NMR (101 MHz, $\mathrm{CDCl}_{3}$ ) (Mixture of Diastereomers) $\delta 173.5,173.1,171.4,170.4,138.3$, $137.4,129.4,129.2,129.1,129.0,128.7,128.5,128.5,128.5,126.8,126.7,126.1,124.5$, 124.4, 119.8, 119.7, 74.0, 72.9, 47.2, 46.9, 46.6, 43.1, 42.7, 42.2, 36.9, 35.2, 33.3, 31.6, 26.4, 25.9, 25.6, 25.5, 25.3, 24.6, 24.2, 24.0;

$\mathrm{m} / z(\mathrm{ESI}) 389.3\left[(\mathrm{M}+\mathrm{Na})^{+}, 100 \%\right]$. 
3-Benzyl-2-hydroxy-4-oxo-N-phenyl-4-(piperidin-1-yl)butanamide 50.

${ }^{1} \mathbf{H}$ NMR $\left(400 \mathrm{MHz}, \mathrm{CDCl}_{3}\right)$.

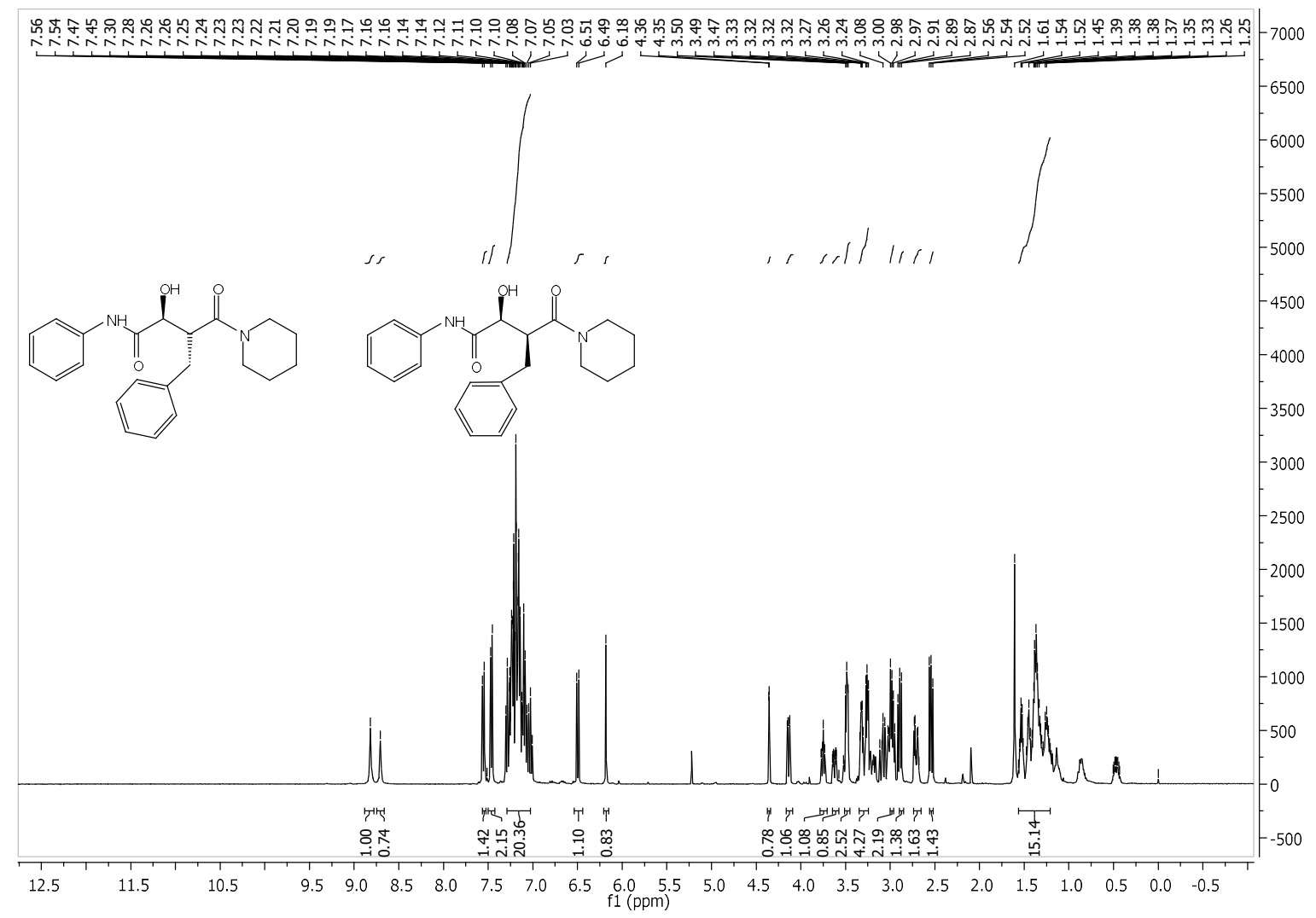

${ }^{13} \mathbf{C}$ NMR (101 MHz, $\left.\mathrm{CDCl}_{3}\right)$.

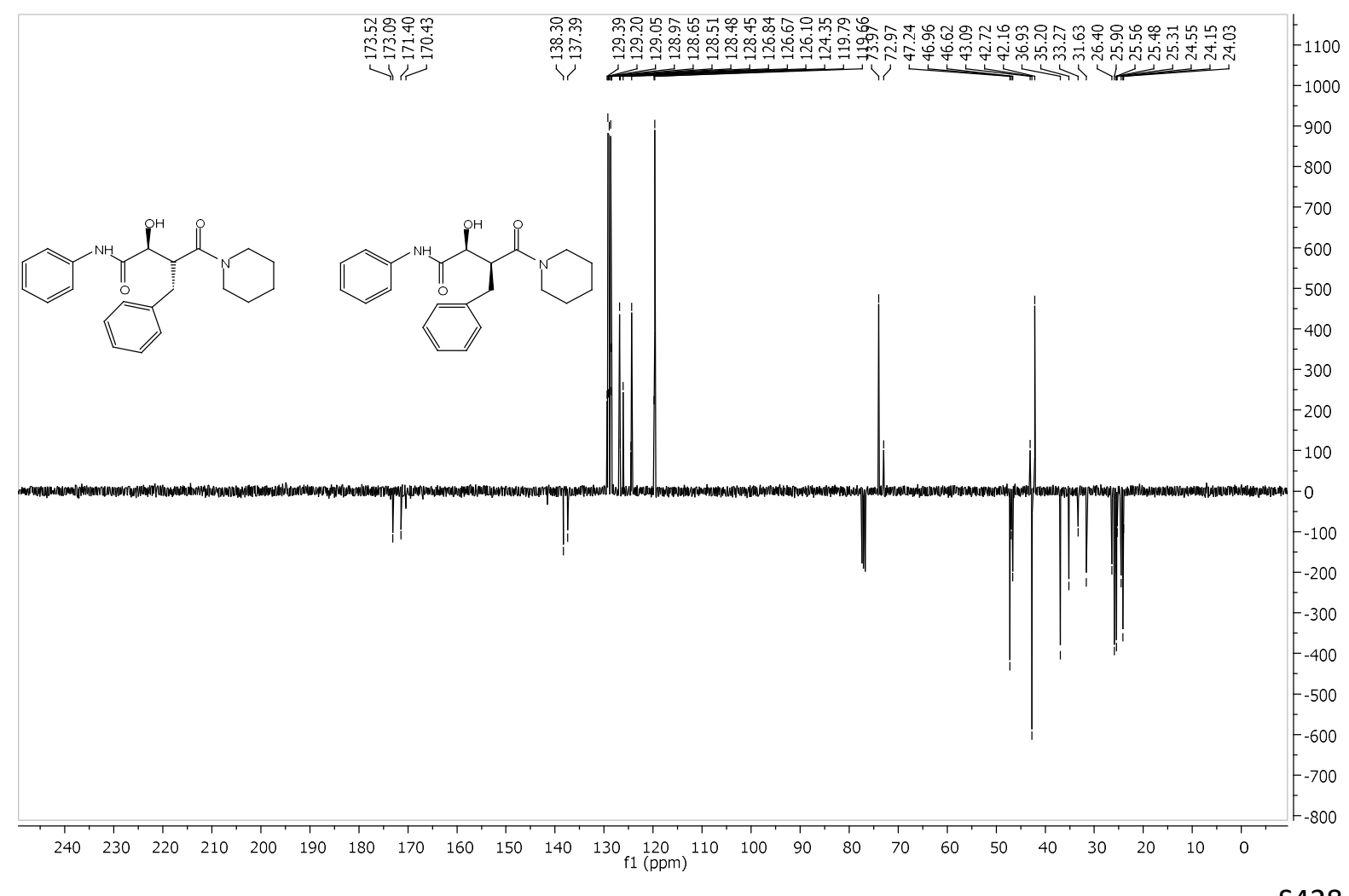




\section{Chiral HPLC of racemic 3-benzyl-2-hydroxy-4-oxo-N-phenyl-4-(piperidin-1-}

\section{yl)butanamide 50.}

Data File C:\CHEM32\1\DATA \DEMO\061-0101.D

Sample Name: VKV 765901007 Rac
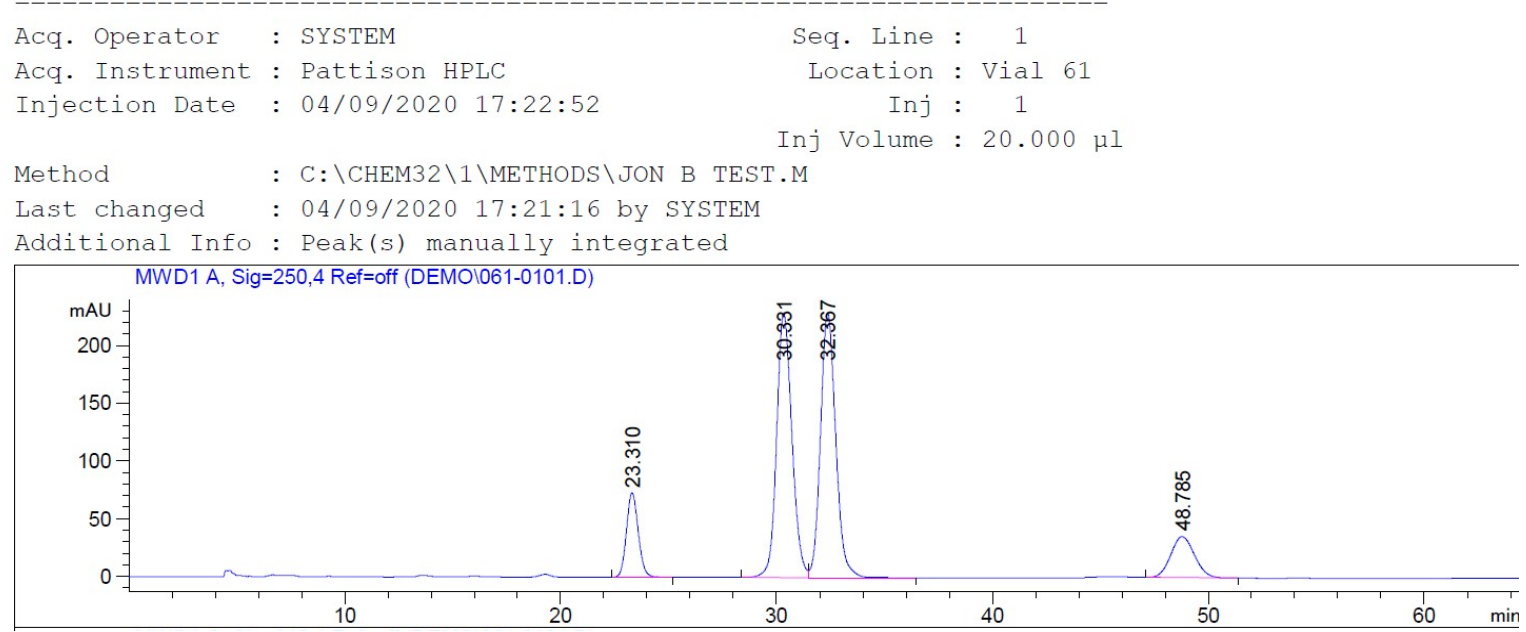

\begin{tabular}{|c|c|c|c|c|c|c|}
\hline $\begin{array}{c}\text { eak } \\
\#\end{array}$ & $\begin{array}{c}\text { RetTime } \\
\text { [min] }\end{array}$ & Type & $\begin{array}{l}\text { Width } \\
\text { [min] }\end{array}$ & $\begin{array}{c}\text { Area } \\
{\left[\mathrm{mAU}^{*} \mathrm{~s}\right]}\end{array}$ & $\begin{array}{l}\text { Height } \\
\text { [mAU] }\end{array}$ & $\begin{array}{c}\text { Area } \\
\text { \% }\end{array}$ \\
\hline & & & & & . & 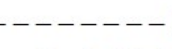 \\
\hline 1 & 23.310 & $\mathrm{BB}$ & 0.5906 & 2772.83423 & 73.08577 & 9.9004 \\
\hline 2 & 30.331 & BV & 0.7582 & $1.12741 e 4$ & 229.06892 & 40.2539 \\
\hline 3 & 32.367 & VB & 0.7577 & 1.12340 e4 & 229.26846 & 40.1107 \\
\hline 4 & 48.785 & $\mathrm{BB}$ & 1.1402 & 2726.52686 & 35.57121 & 9.7350 \\
\hline
\end{tabular}




\section{Chiral HPLC of ATH of 3-benzyl-2-hydroxy-4-oxo-N-phenyl-4-(piperidin-1-}

\section{yl)butanamide.}

\section{Showing dr}

Jata File C:\CHEM32\1\DATA \DEMO\062-0101.D

sample Name: VKV 766901007 ATH
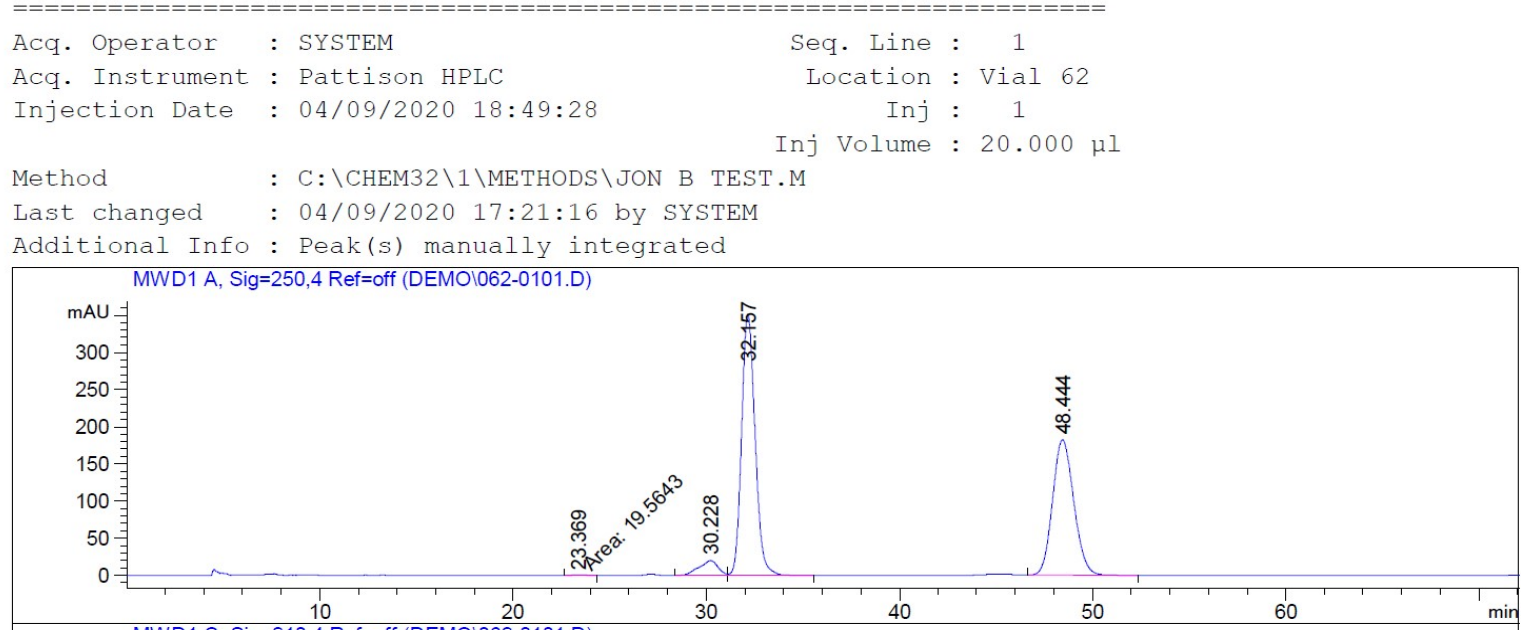

\begin{tabular}{|c|c|c|c|c|c|c|}
\hline $\begin{array}{c}\text { eak } \\
\#\end{array}$ & $\begin{array}{c}\text { RetTime } \\
\text { [min] }\end{array}$ & Type & $\begin{array}{l}\text { Width } \\
\text { [min] }\end{array}$ & $\begin{array}{c}\text { Area } \\
{\left[\mathrm{mAU}^{\star} \mathrm{s}\right]}\end{array}$ & $\begin{array}{l}\text { Height } \\
{[\mathrm{mAU}]}\end{array}$ & $\begin{array}{c}\text { Area } \\
\quad \%\end{array}$ \\
\hline \multicolumn{7}{|c|}{$----|-------|----|-------|----------|----------|--------\mid$} \\
\hline 1 & 23.369 & MM & 0.8344 & 19.56431 & $3.90779 e-1$ & 0.0607 \\
\hline 2 & 30.228 & BV & 326 & 1333.61072 & 19.82920 & 4.1402 \\
\hline 3 & 32.157 & $\mathrm{VB}$ & 7525 & $1.69875 e 4$ & 351.07056 & 380 \\
\hline 4 & 48.444 & $\mathrm{BB}$ & 1. 1904 & $1.38704 e 4$ & 182.15829 & 43.0610 \\
\hline
\end{tabular}




\section{Chiral HPLC of ATH of 3-benzyl-2-hydroxy-4-oxo-N-phenyl-4-(piperidin-1-} yl)butanamide. Showing ee for $\mathrm{d} 2$.

Data File C:\CHEM32\1\DATA \DEMO\062-0101.D

Sample Name: VKV 766901007 ATH
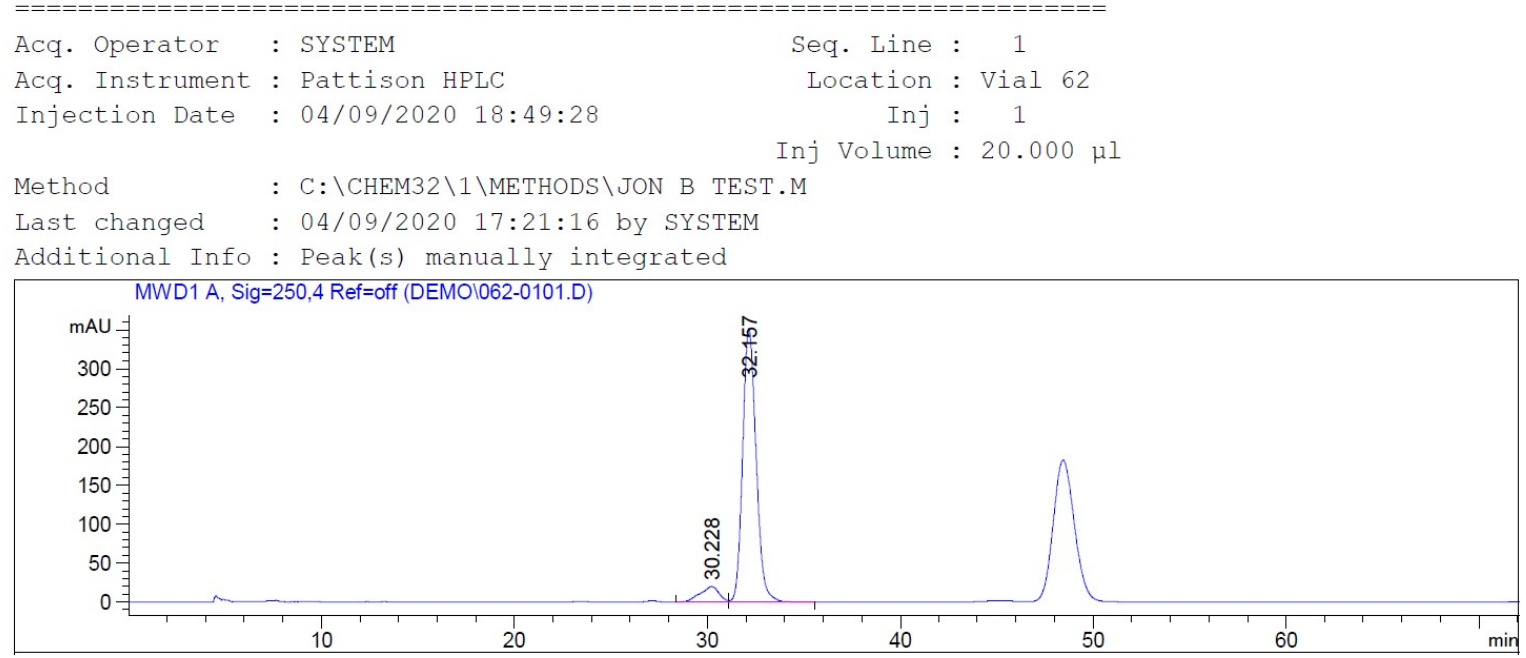

\begin{tabular}{|c|c|c|c|c|c|c|}
\hline $\begin{array}{c}\text { Peak } \\
\#\end{array}$ & $\begin{array}{c}\text { RetTime } \\
\text { [min] }\end{array}$ & Type & $\begin{array}{l}\text { Width } \\
\text { [min] }\end{array}$ & $\begin{array}{c}\text { Area } \\
{\left[\mathrm{mAU}^{\star} \mathrm{s}\right]}\end{array}$ & $\begin{array}{l}\text { Height } \\
{[\mathrm{mAU}]}\end{array}$ & $\begin{array}{c}\text { Area } \\
\quad \%\end{array}$ \\
\hline & & & - & $\mid----------$ & --------- & ---- \\
\hline 1 & 30.228 & BV & 0.9326 & 1333.61072 & 19.82920 & 7.2791 \\
\hline 2 & 32.157 & VB & 0.7525 & $1.69875 e 4$ & 351.07056 & 92.7209 \\
\hline
\end{tabular}




\section{Chiral HPLC of ATH of 3-benzyl-2-hydroxy-4-oxo-N-phenyl-4-(piperidin-1-}

\section{yl)butanamide. Showing ee for d1}

Data File C: \CHEM32\1\DATA \DEMO\062-0101.D

Sample Name: VKV 766901007 ATH
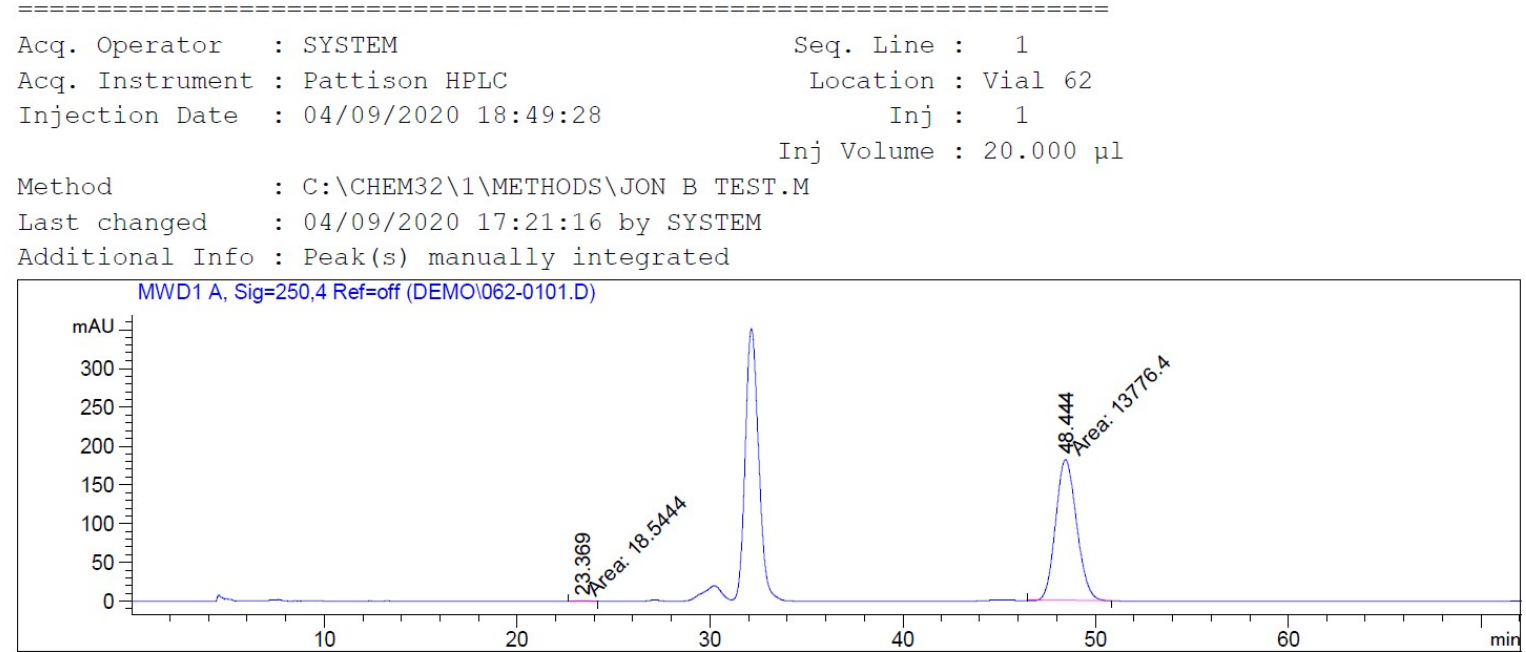

\begin{tabular}{|c|c|c|c|c|c|c|}
\hline $\begin{array}{c}\text { eak } \\
\#\end{array}$ & $\begin{array}{c}\text { RetTime } \\
\text { [min] }\end{array}$ & Type & $\begin{array}{l}\text { Width } \\
\text { [min] }\end{array}$ & $\begin{array}{c}\text { Area } \\
{\left[\mathrm{mAU}{ }^{*} \mathrm{~s}\right]}\end{array}$ & $\begin{array}{l}\text { Height } \\
\text { [mAU] }\end{array}$ & $\begin{array}{c}\text { Area } \\
\%\end{array}$ \\
\hline & & & & --------- & --- & $-------\mid$ \\
\hline 1 & 23 & & 69 & 18.54438 & $22 e-1$ & 0.1344 \\
\hline 2 & 48.444 & & $\$ 34$ & $1.37764 \mathrm{e} 4$ & 4390 & 3656 \\
\hline
\end{tabular}




\section{Chiral HPLC of 3-benzyl-2,4-dioxo-N-phenyl-4-(piperidin-1-yl)butanamide ketone.}

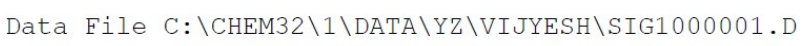

Sample Name: VKV 762 Ketone 901007 IC

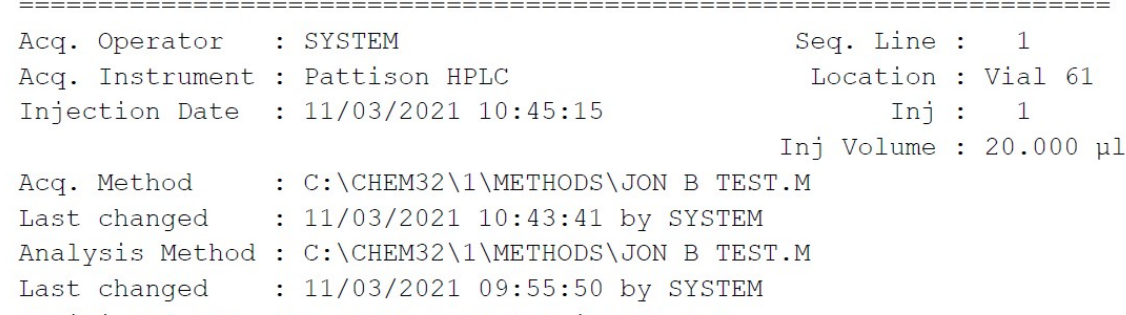

$2 \quad 46.816 \mathrm{MM} \quad 1.3506 \quad 5368.62695 \quad 66.24827 \quad 50.9274$ 


\section{Section on applications.}

tert-Butyl (S)-(1-(dimethylamino)-1-oxo-3-phenylpropan-2-yl)carbamate.<smiles>CN(C)C(=O)C(Cc1ccccc1)NC(C)(C)C</smiles>

This compound is reported and was prepared using the following procedure.

To a solution of (tert-butoxycarbonyl)-L-phenylalanine (2.65 g, $10.0 \mathrm{mmol}, 1.0 \mathrm{eq}), \mathrm{N}, \mathrm{N}$ dimethylamine hydrochloride (1.48 g, $22.0 \mathrm{mmol}, 2.2 \mathrm{eq})$ and hydroxybenzotriazole (HOBt) (1.62 g, $12.0 \mathrm{mmol}, 1.2 \mathrm{eq})$ in dichloromethane $(40 \mathrm{~mL})$ was added $N$-ethyl- $N$ '-(3dimethylaminopropyl)carbodiimide hydrochloride $(\mathrm{EDC} \mathrm{HCl})(2.30 \mathrm{~g}, 12.0 \mathrm{mmol}, 1.2 \mathrm{eq})$ and the reaction mixture was stirred at room temperature. $N, N$-Diisopropylethylamine $(4.25$ $\mathrm{mL}, 3.10 \mathrm{~g}, 24.0 \mathrm{mmol}, 2.4 \mathrm{eq}$ ) was added and resulting reaction mixture was stirred at room temperature for $24 \mathrm{~h}$. The reaction mixture was quenched with water $(100 \mathrm{~mL})$ and extracted with ethyl acetate $(3 \times 30 \mathrm{~mL})$. The combined organic layers were washed with dilute cold $\mathrm{HCl}(2 \times 20 \mathrm{~mL})$, saturated aqueous $\mathrm{NaHCO}_{3}$ solution $(2 \times 20 \mathrm{~mL})$, water $(2 \times 30 \mathrm{~mL})$ and finally with brine solution $(50 \mathrm{~mL})$. The organic layer was dried over $\mathrm{MgSO}_{4}$, filtered and filtrate was concentrated under reduced pressure to give the product as a clear semisolid material (2.89 g, $9.89 \mathrm{mmol}$, 99\%) which was used in the next step without further purification.

TLC: $\mathrm{R}_{\mathrm{f}}$ ca 0.5 (8:2, Hexane: EtOAc), strong UV active;

HRMS (ESI) m/z: [M+Na] $]^{+}$Calcd for $\mathrm{C}_{16} \mathrm{H}_{24} \mathrm{~N}_{2} \mathrm{O}_{3} \mathrm{Na} 315.1679$; Found 315.1670 (error 3.0 ppm);

${ }^{1} \mathrm{H}$ NMR (400 MHz, $\left.\mathrm{CDCl}_{3}\right) \delta$ 7.29-7.18 (5H, m, ArH), $5.43(1 \mathrm{H}, \mathrm{d}, J=8.4 \mathrm{~Hz}, \mathrm{CONH})$, $4.82(1 \mathrm{H}, \mathrm{dd}, J=14.9,8.2 \mathrm{~Hz}, \mathrm{C} \underline{\mathrm{HNH}}), 3.00-2.85\left(2 \mathrm{H}, \mathrm{m}, \mathrm{CH}_{2} \mathrm{Ph}\right), 2.85\left(3 \mathrm{H}, \mathrm{s}, \mathrm{NCH}_{3}\right), 2.61$ $\left(3 \mathrm{H}, \mathrm{s}, \mathrm{NCH}_{3}\right), 1.41\left(9 \mathrm{H}, \mathrm{s}, \mathrm{C}\left(\mathrm{CH}_{3}\right)_{3}\right.$;

${ }^{13} \mathrm{C} \mathrm{NMR}\left(101 \mathrm{MHz}, \mathrm{CDCl}_{3}\right) \delta 171.7,155.2,136.7,129.5,128.5,126.7,79.7,51.5,40.5$, $36.7,35.6,28.5$;

$m / z(\mathrm{ESI}) 315.1[(\mathrm{M}+\mathrm{Na})+, 100 \%]$.

1) Klein, J. J.; Hecht, S. Synthesis of a New Class of Bis(thiourea)hydrazide Pseudopeptides as Potential Inhibitors of $\beta$-Sheet Aggregation, Org. Lett. 2012, 14, 330-333. 2) Andrés, J. M.; Manzano, R.; Pedrosa, R. Novel Bifunctional Chiral Urea and Thiourea Derivatives as 
Organocatalysts: Enantioselective Nitro-Michael Reaction of Malonates and Diketones, Chem. Eur. J. 2008, 14, 5116 - 5119, 
tert-Butyl (S)-(1-(dimethylamino)-1-oxo-3-phenylpropan-2-yl)carbamate.

${ }^{1} \mathrm{H}$ NMR $\left(400 \mathrm{MHz}, \mathrm{CDCl}_{3}\right)$.
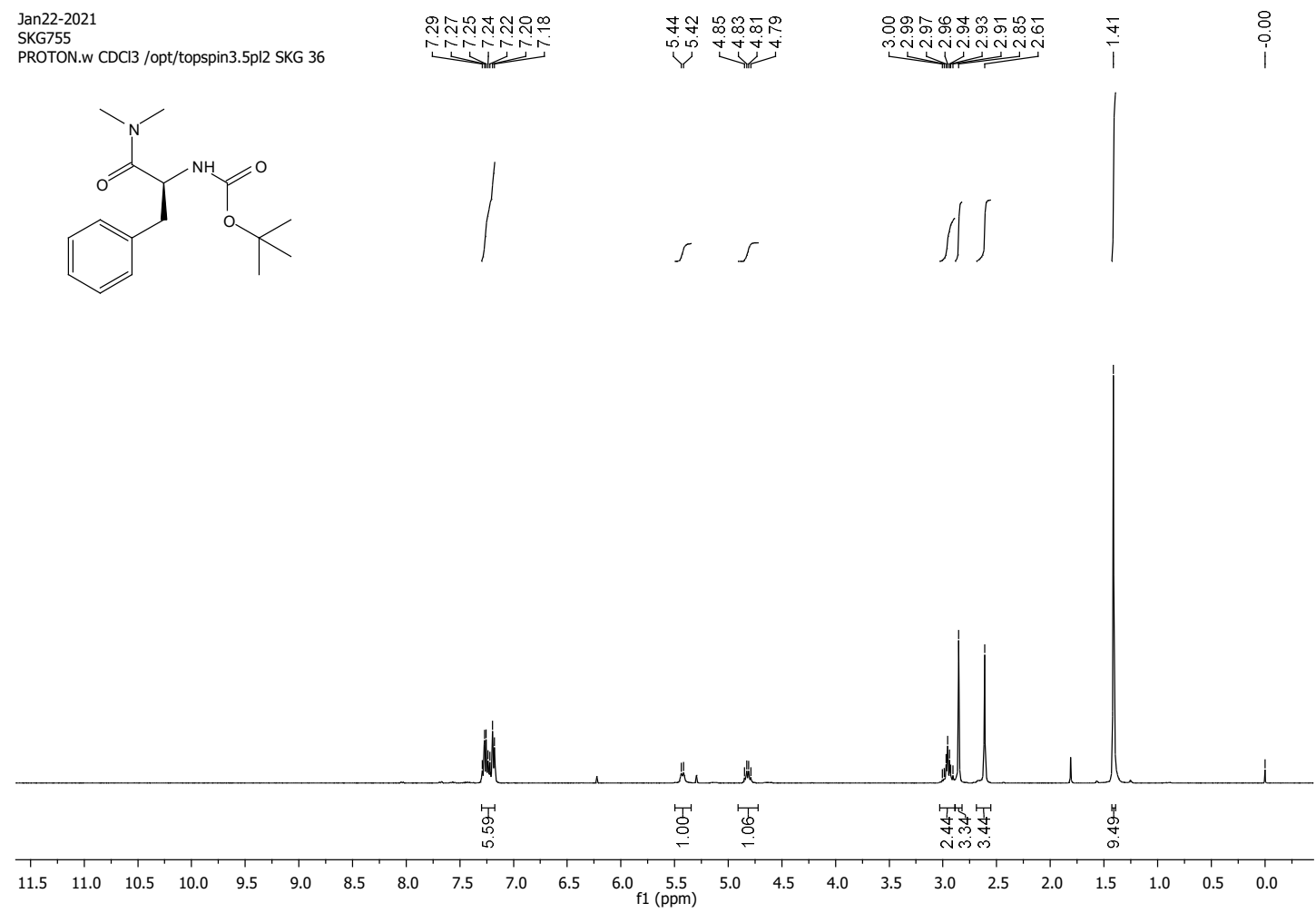

${ }^{13} \mathrm{C} \mathrm{NMR}\left(101 \mathrm{MHz}, \mathrm{CDCl}_{3}\right)$.

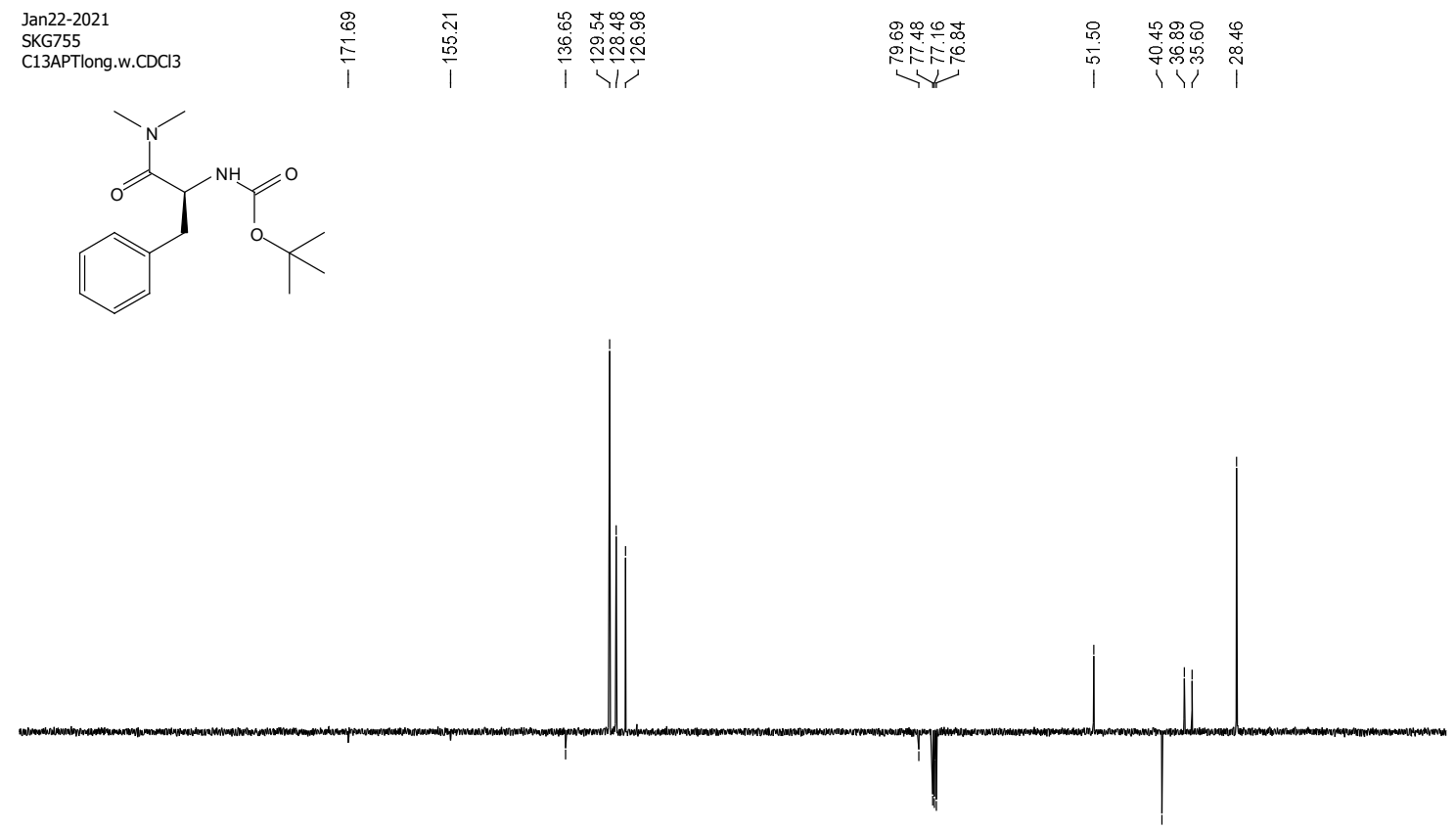

$\begin{array}{llllllllllllllllllllllllll}220 & 210 & 200 & 190 & 180 & 170 & 160 & 150 & 140 & 130 & 120 & 110 & 100 & 90 & 80 & 70 & 60 & 50 & 40 & 30 & 20 & 10 & 0\end{array}$ 


\section{(S)-2-Amino- $N, N$-dimethyl-3-phenylpropanamide hydrochloride 51.}<smiles>CN(C)C(=O)C(N)Cc1ccccc1</smiles>

This compound is reported and was prepared using the following procedure.

To a solution of tert-butyl (S)-(1-(dimethylamino)-1-oxo-3-phenylpropan-2-yl)carbamate $(2.89 \mathrm{~g}, 9.89 \mathrm{mmol}, 1.0 \mathrm{eq})$ in methanol $(30 \mathrm{~mL})$ was added $1.25 \mathrm{M} \mathrm{HCl}$ in $\mathrm{MeOH}(40 \mathrm{~mL}$, $49.4 \mathrm{mmol}, 5.0 \mathrm{eq}$ ) and resulting reaction mixture was stirred at room temperature for $24 \mathrm{~h}$. The reaction mixture was concentrated under reduced pressure. The resulting residues were triturated in n-pentane $(3 \times 20 \mathrm{~mL})$ to give $\mathbf{5 1}$ as a white solid $(1.97 \mathrm{~g}, 8.64 \mathrm{mmol}, 87 \%)$ which was used in the next step without further purification.

${ }^{1} \mathrm{H}$ NMR (400 MHz, DMSO-d $\left.d_{6}\right) \delta .50$ (2H, br.s., $\left.\mathrm{NH}_{2}\right), 7.33-7.19(5 \mathrm{H}, \mathrm{m}, \mathrm{ArH}), 4.50(1 \mathrm{H}$, $\left.\mathrm{dd}, J=8.5,5.5 \mathrm{~Hz}, \mathrm{CHCH}_{2} \mathrm{Ph}\right), 3.17\left(1 \mathrm{H}, \mathrm{dd}, J=13.2,5.3 \mathrm{~Hz}, \underline{\mathrm{HaH}}_{\mathrm{b}} \mathrm{Ph}\right), 2.93(1 \mathrm{H}, \mathrm{dd}, J=$ 13.2, 8.7 Hz, $\left.\mathrm{CHa} \underline{\mathrm{H}}_{\mathrm{b}} \mathrm{Ph}\right), 2.74\left(3 \mathrm{H}, \mathrm{s}, \mathrm{NCH}_{3}\right), 2.55\left(3 \mathrm{H}, \mathrm{s}, \mathrm{NCH}_{3}\right)$;

${ }^{13} \mathrm{C}$ NMR (101 MHz, DMSO-d $\left.d_{6}\right) \delta 167.9,134.8,129.5,128.5,127.3,50.1,36.7,36.3,35.2$; $m / z(\mathrm{ESI}) 193.1\left[(\mathrm{M}-\mathrm{Cl})^{+}, 100 \%\right]$.

The data matches the reported data.

Takeda Pharmaceutical Company Limited; Matsumoto, S.; Ono, K.; Tominari, Yusuke; K. T.; Miwa, K.; Hasuoka, A.; Imamura, S. WO2013/18929, 2013, A1, Paragraph 0654. 
(S)-2-Amino- $N, N$-dimethyl-3-phenylpropanamide hydrochloride 51. ${ }^{1} \mathrm{H}$ NMR (400 MHz, DMSO-do).

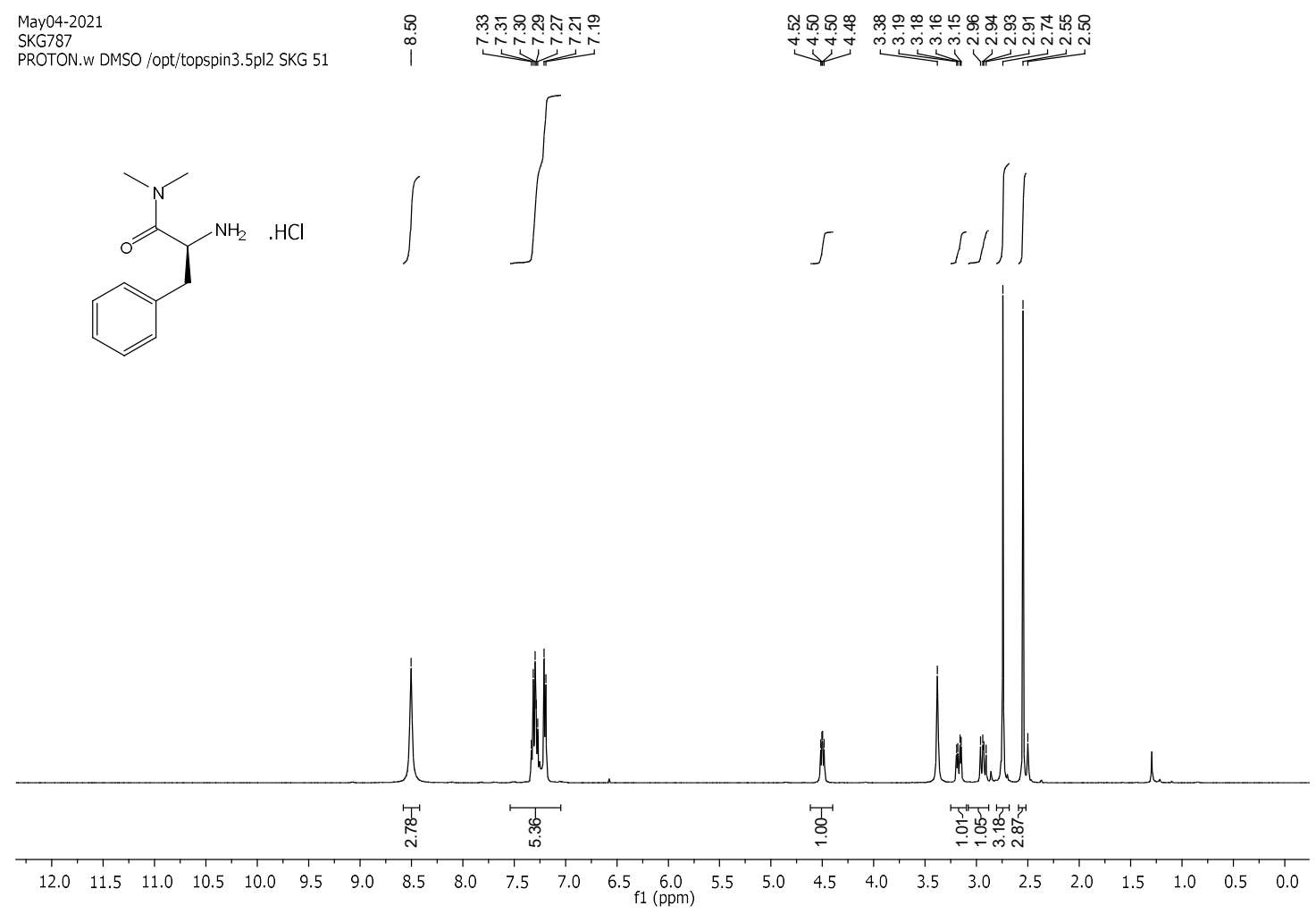

${ }^{13} \mathrm{C}$ NMR (101 MHz, DMSO-d6).

$\begin{array}{llll}\text { May04-2021 } & \infty & 04 \\ \text { KKG787 } & 0 & 0\end{array}$

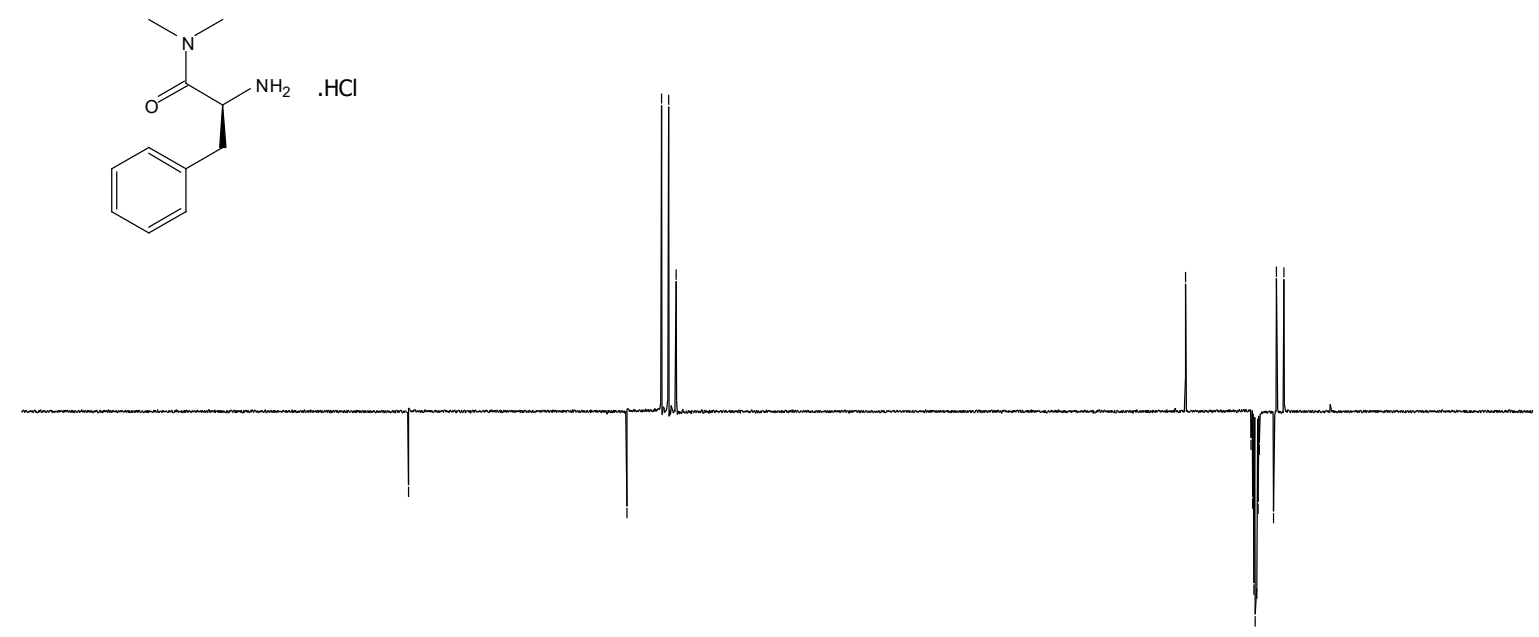

$\begin{array}{lllllllllllllllllllllllllllll} & 220 & 210 & 200 & 190 & 180 & 170 & 160 & 150 & 140 & 130 & 120 & 110 & 100 & 90 & 80 & 70 & 60 & 50 & 40 & 30 & 20 & 10 & 0\end{array}$ 


\section{COSY.}

SKG787

COSY.w DMSO /opt/topspin3.5pl2 SKG 5

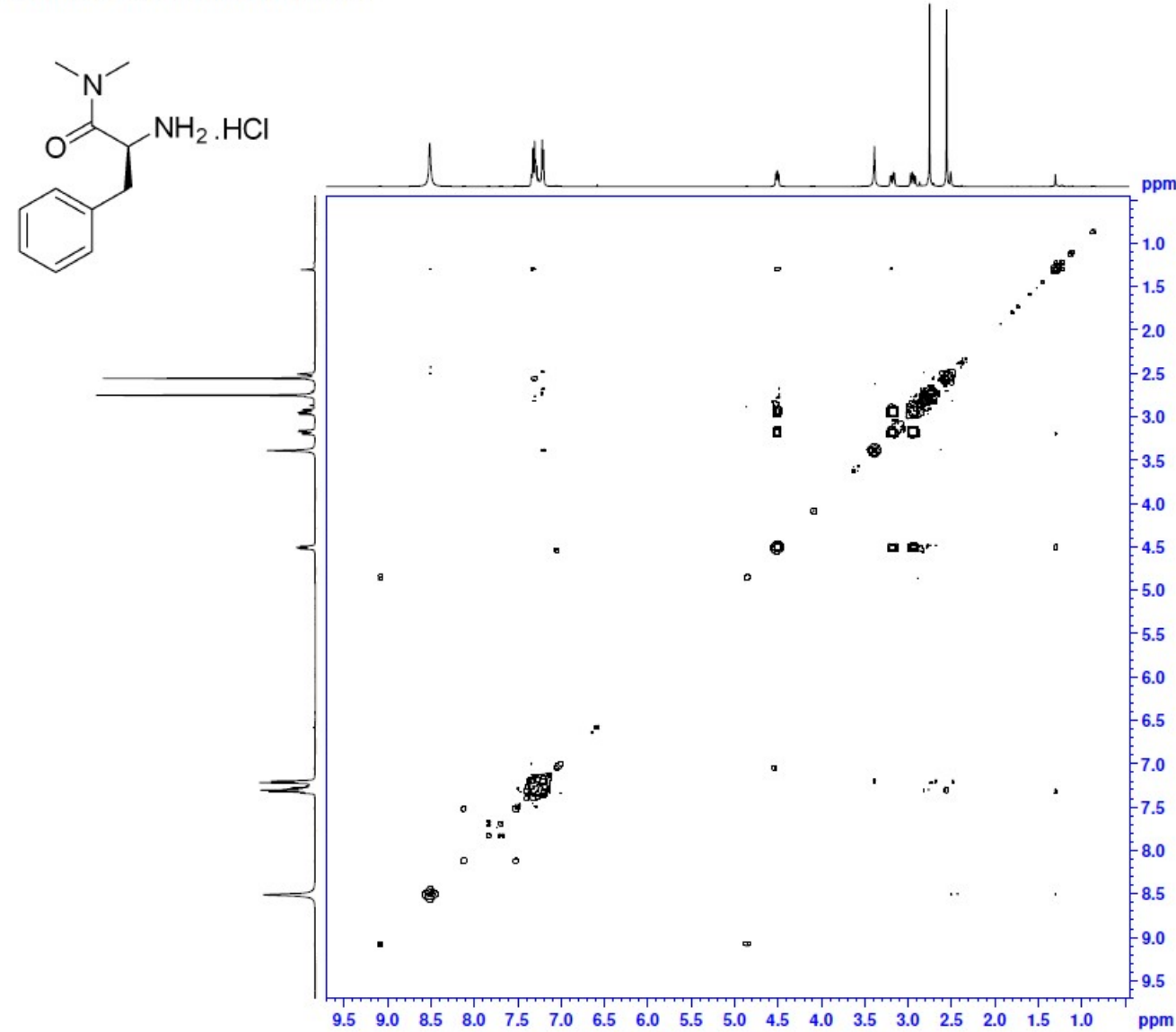


Methyl (S)-2-((1-(dimethylamino)-1-oxo-3-phenylpropan-2-yl)amino)-2-oxoacetate 52.<smiles>COC(=O)C(=O)NC(Cc1ccccc1)C(=O)N(C)C</smiles>

This compound is novel and was prepared following the general procedure A using methyl 2chloro-2-oxoacetate (0.463 g, $0.35 \mathrm{~mL}, 3.77 \mathrm{mmol}, 1.2 \mathrm{eq})$, (S)-2-amino- $N, N$-dimethyl-3phenylpropanamide hydrochloride $51(0.710 \mathrm{~g}, 3.14 \mathrm{mmol}, 1.0 \mathrm{eq})$ and triethylamine $(0.792$ $\mathrm{g}, 1.10 \mathrm{~mL}, 7.85 \mathrm{mmol}, 2.50 \mathrm{eq})$ in dichloromethane $(10 \mathrm{~mL})$ to give $\mathbf{5 2}$ as a yellow liquid (0.553 g, $1.98 \mathrm{mmol}, 64 \%)$.

TLC: $\mathrm{R}_{\mathrm{f}}$ ca 0.3 (7:3, Hexane: EtOAc), strong UV active;

HRMS (ESI) m/z: [M+Na] $]^{+}$Calcd for $\mathrm{C}_{14} \mathrm{H}_{18} \mathrm{~N}_{2} \mathrm{O}_{4} \mathrm{Na} 301.1159$; Found 301.1147 (error 3.9 ppm);

$U_{\max } 3245,2954,1738,1695,1634,1495,1270,1216,979,700 \mathrm{~cm}^{-1}$;

${ }^{1} \mathrm{H}$ NMR (400 MHz, $\left.\mathrm{CDCl}_{3}\right) \delta 7.90(1 \mathrm{H}, \mathrm{d}, J=7.9 \mathrm{~Hz}, \mathrm{CONH}), 7.31-7.23(3 \mathrm{H}, \mathrm{m}, \mathrm{ArH})$, 7.20-7.18 (2H, m, ArH), 5.15-5.09 (1H, m, $\left.\mathrm{CHCH}_{2} \mathrm{Ph}\right), 3.89\left(3 \mathrm{H}, \mathrm{s},-\mathrm{OCH}_{3}\right), 3.06-3.03(2 \mathrm{H}$, m, $\left.\mathrm{CH}_{2} \mathrm{Ph}\right), 2.88\left(3 \mathrm{H}, \mathrm{s}, \mathrm{NCH}_{3}\right), 2.62\left(3 \mathrm{H}, \mathrm{s}, \mathrm{NCH}_{3}\right)$;

${ }^{13} \mathrm{C}$ NMR $\left(101 \mathrm{MHz}, \mathrm{CDCl}_{3}\right) \delta 170.0,160.4,155.6,135.7,129.5,128.7,127.4,53.7,50.8$, 39.6, 36.9, 35.7;

$m / z(\mathrm{ESI}) 301.1[(\mathrm{M}+\mathrm{Na})+, 100 \%]$. 
Methyl (S)-2-((1-(dimethylamino)-1-oxo-3-phenylpropan-2-yl)amino)-2-oxoacetate 52. ${ }^{1} \mathrm{H}$ NMR $\left(400 \mathrm{MHz}, \mathrm{CDCl}_{3}\right)$.

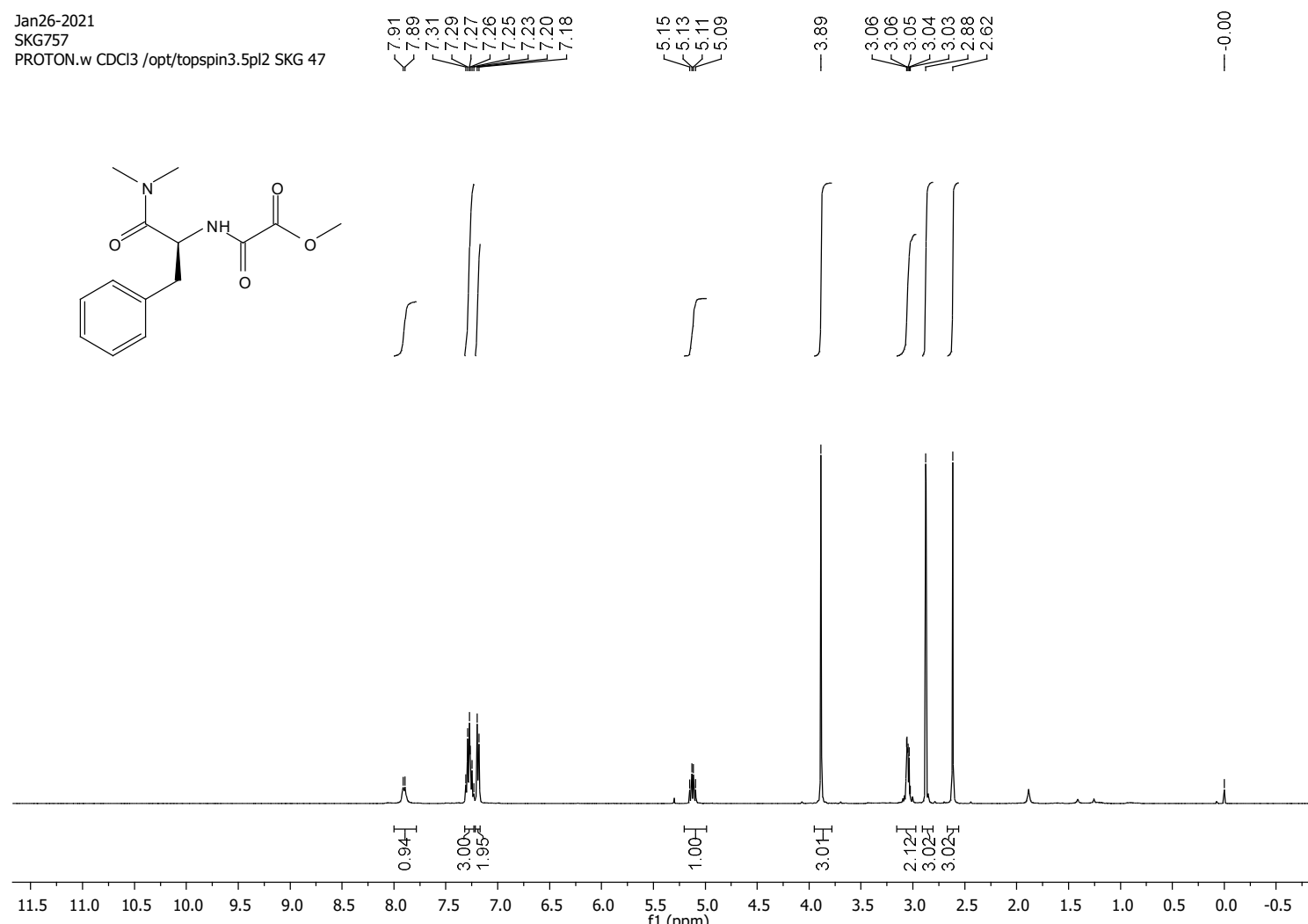

${ }^{13} \mathrm{C}$ NMR (101 MHz, $\left.\mathrm{CDCl}_{3}\right)$.

\begin{tabular}{|c|c|c|c|c|c|}
\hline $\begin{array}{l}\text { Jan26-2021 } \\
\text { SKG757 } \\
\text { C13APTlong.w.CDCl3 }\end{array}$ & $\stackrel{?}{\frac{P}{2}}$ & $\begin{array}{ll}9 & 8 \\
0 & 0 \\
0 & 0 \\
1 & 0 \\
1 & 1 \\
1\end{array}$ & 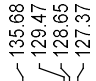 & 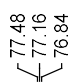 & $\begin{array}{l}\hat{0} 0 \\
070 \\
00 \\
11 \\
11\end{array}$ \\
\hline
\end{tabular}

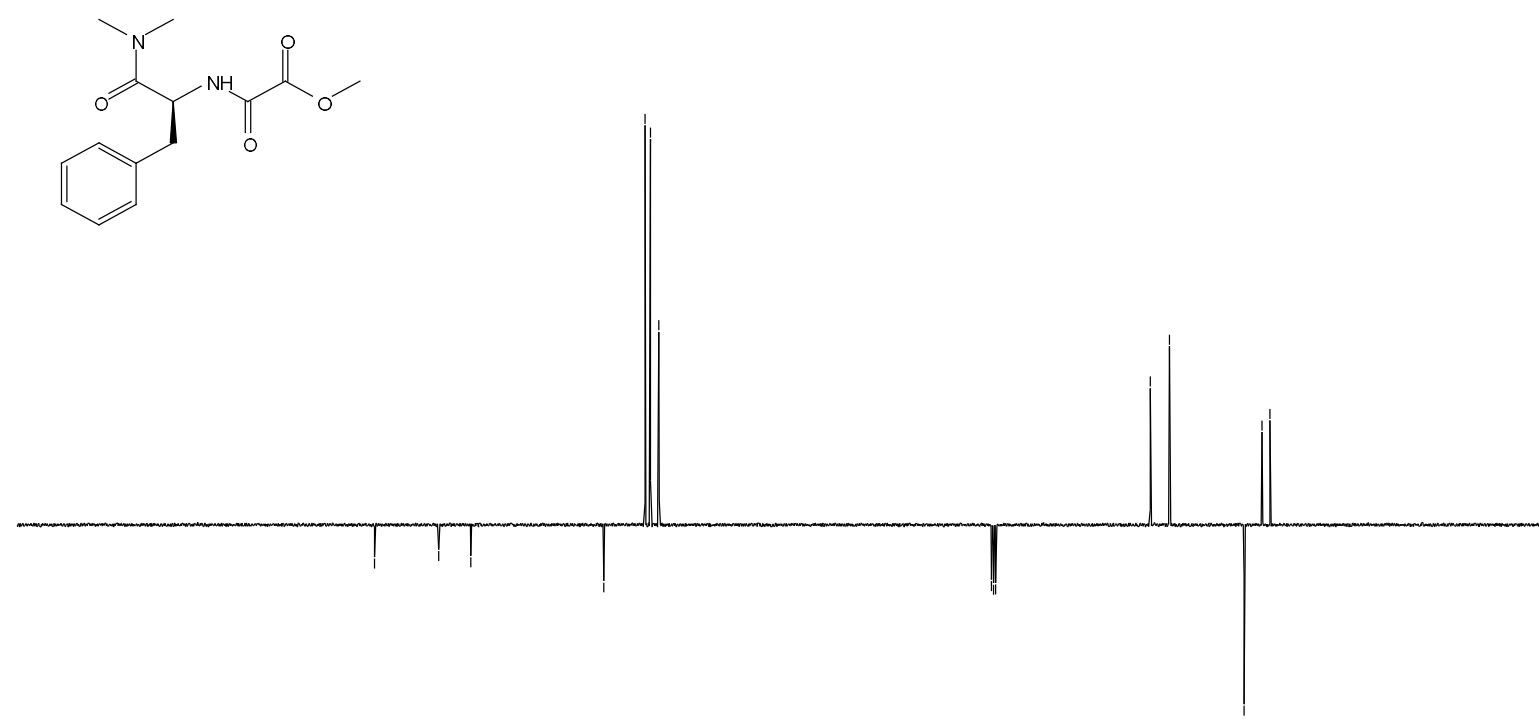

$\begin{array}{lllllllllllllllllllllllllllllllllllll}220 & 210 & 200 & 190 & 180 & 170 & 160 & 150 & 140 & 130 & 120 & 110 & 100 & 90 & 80 & 70 & 60 & 50 & 40 & 30 & 20 & 10 & 0\end{array}$ 
COSY.

SKG757

COSY.w CDCl3 /opt/topspin3.5pl2 SKG 47

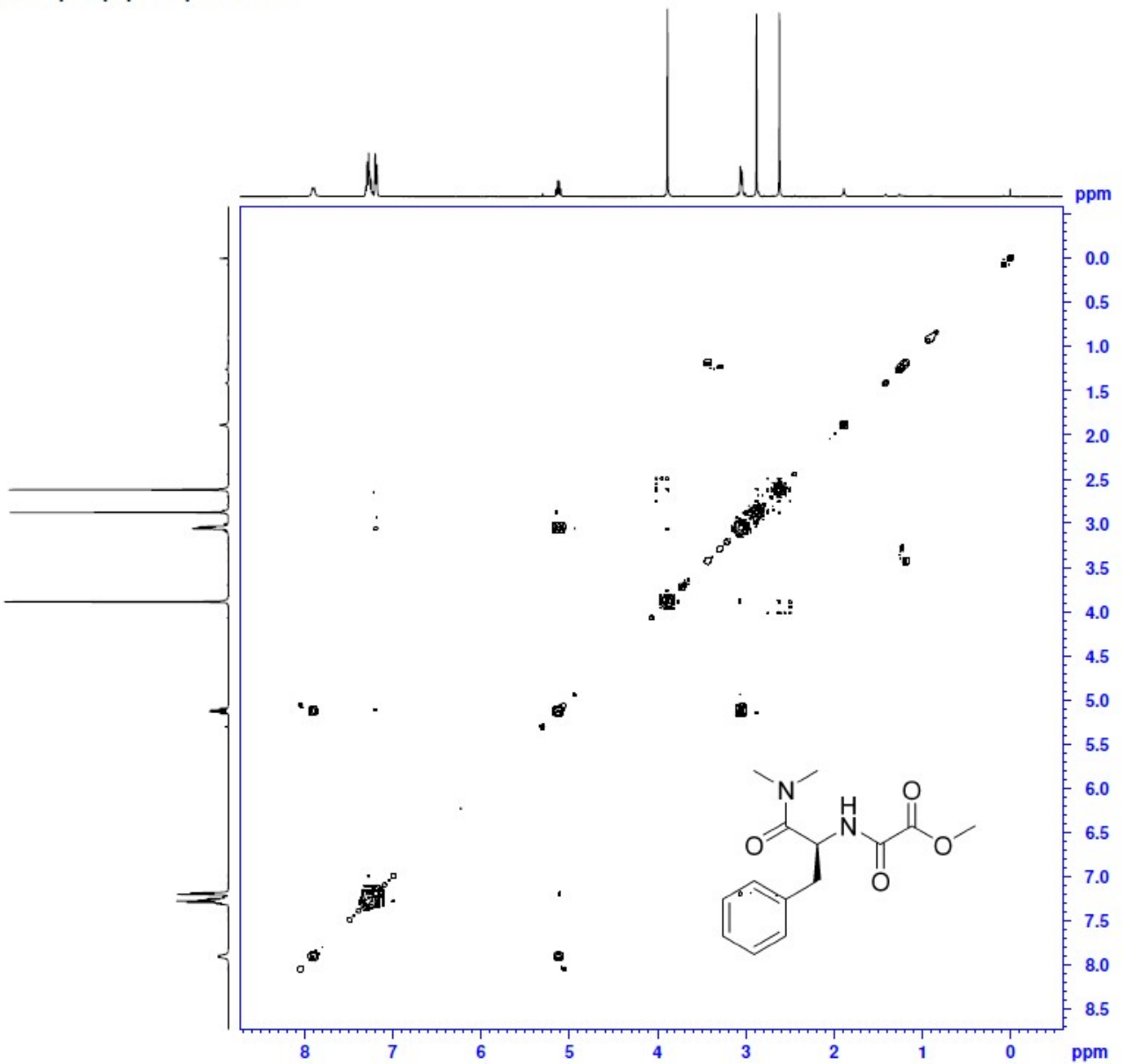


Ethyl (S)-4-((1-(dimethylamino)-1-oxo-3-phenylpropan-2-yl)amino)-3,4-dioxobutanoate 53.<smiles>CCOC(=O)CC(=O)C(=O)NC(Cc1ccccc1)C(=O)N(C)C</smiles>

This compound is novel and was prepared following general procedure B using methyl (S)-2((1-(dimethylamino)-1-oxo-3-phenylpropan-2-yl)amino)-2-oxoacetate $\quad \mathbf{5 2} \quad\left(\begin{array}{llll}0.500 & \mathrm{~g}, & 1.79\end{array}\right.$ mmol, $1.0 \mathrm{eq})$ in anhydrous THF $(6 \mathrm{~mL})$, ethyl acetate $(0.380 \mathrm{~g}, 4.31 \mathrm{mmol}, 2.4 \mathrm{eq})$ in anhydrous THF (4 mL) and LDA (1M solution in THF) $(5.37 \mathrm{~mL}, 5.37 \mathrm{mmol}, 3 \mathrm{eq})$ to give crude material which was further purified by column chromatography (30-50\% EtOAc in petroleum ether) to afford 53 as a yellow liquid (0.365 g, $1.09 \mathrm{mmol}, 61 \%)$. The title compound was obtained as keto/enol tautomer (1:1) as per ${ }^{1} \mathrm{H}$ NMR.

TLC: $\mathrm{R}_{\mathrm{f}}$ ca 0.4 (5:5, hexane: EtOAc), UV-active and strong $\mathrm{KMnO}_{4}$; HRMS (ESI) m/z: [M+Na] ${ }^{+}$Calcd for $\mathrm{C}_{17} \mathrm{H}_{22} \mathrm{~N}_{2} \mathrm{O}_{5} \mathrm{Na} 357.1421$; Found 357.1430 (error -2.6 ppm);

$U_{\max } 3441,2972,1648,1633,1594,1494,1418,1364,1279,1038,700 \mathrm{~cm}^{-1}$;

${ }^{1} \mathrm{H}$ NMR (500 MHz, $\mathrm{CDCl}_{3}$ ) (keto/enol, 1:1) $\delta 11.90(0.5 \mathrm{H}, \mathrm{s}, \mathrm{OH}$ of enol form), 7.74-7.69 $(1 \mathrm{H}, \mathrm{m}, \mathrm{CONH}), 7.30-7.24(3 \mathrm{H}, \mathrm{m}, \mathrm{ArH}), 7.20-7.17(2 \mathrm{H}, \mathrm{m}, \mathrm{ArH}), 6.03(0.5 \mathrm{H}, \mathrm{s},=\mathrm{C} \underline{\mathrm{H}}$ of enol form), $5.18\left(0.5 \mathrm{H}, \mathrm{q}, J=8.2 \mathrm{~Hz}, \mathrm{CHCH}_{2} \mathrm{Ph}\right.$ of keto form), $5.05(0.5 \mathrm{H}, \mathrm{q}, J=8.2 \mathrm{~Hz}$, $\left.\mathrm{C}_{\mathrm{HCH}} \mathrm{Ph}\right), 4.28-4.24\left(1 \mathrm{H}, \mathrm{m}, \underline{\mathrm{CH}}_{2} \mathrm{CH}_{3}\right.$ of ester group), 4.28-4.17 (1H, m, $\underline{\mathrm{C}}_{2} \mathrm{CH}_{3}$ of ester group), 3.86-3.79 (1H, m, $\mathrm{CH}_{2}$ of keto form), 3.06-3.02 $\left(2 \mathrm{H}, \mathrm{m}, \mathrm{C}_{2} \mathrm{Ph}\right), 2.87(1.5 \mathrm{H}, \mathrm{s}$, $\left.\mathrm{NCH}_{3}\right), 2.86\left(1.5 \mathrm{H}, \mathrm{s}, \mathrm{NCH}_{3}\right), 2.60\left(1.5 \mathrm{H}, \mathrm{s}, \mathrm{NCH}_{3}\right), 2.59\left(1.5 \mathrm{H}, \mathrm{s}, \mathrm{NCH}_{3}\right)$ 1.33-1.30 (1.5H, $\mathrm{m}, \mathrm{CH}_{2} \underline{\mathrm{CH}}_{3}$ of ester group), 1.27-1.24 (1.5H, m, $\mathrm{CH}_{2} \underline{\mathrm{C}}_{3}$ of ester group);

${ }^{13} \mathrm{C}$ NMR (126 MHz, $\mathrm{CDCl}_{3}$ ) (keto/enol, 1:1) $\delta 190.7$ (C=O keto), 172.4, 171.4, 169.9, 166.7, $161.3,159.9,158.6,135.9,135.7,129.5,129.4,128.6,128.5,124.3,124.2,93.9(\mathrm{C}=\underline{\mathrm{C}} \mathrm{H}$, enol), 61.6, 61.2, 50.5, 50.4, 43.5 ( $\mathrm{CH}_{2}$, keto form), 39.8, 39.7, 36.9, 36.8, 35.7, 35.6, 14.2, 14.1;

$m / z(\mathrm{ESI}) 357.1\left[(\mathrm{M}+\mathrm{Na})^{+}, 100 \%\right]$. 
Ethyl (S)-4-((1-(dimethylamino)-1-oxo-3-phenylpropan-2-yl)amino)-3,4-dioxobutanoate 53.

${ }^{1} \mathrm{H}$ NMR (500 MHz, $\left.\mathrm{CDCl}_{3}\right)$.
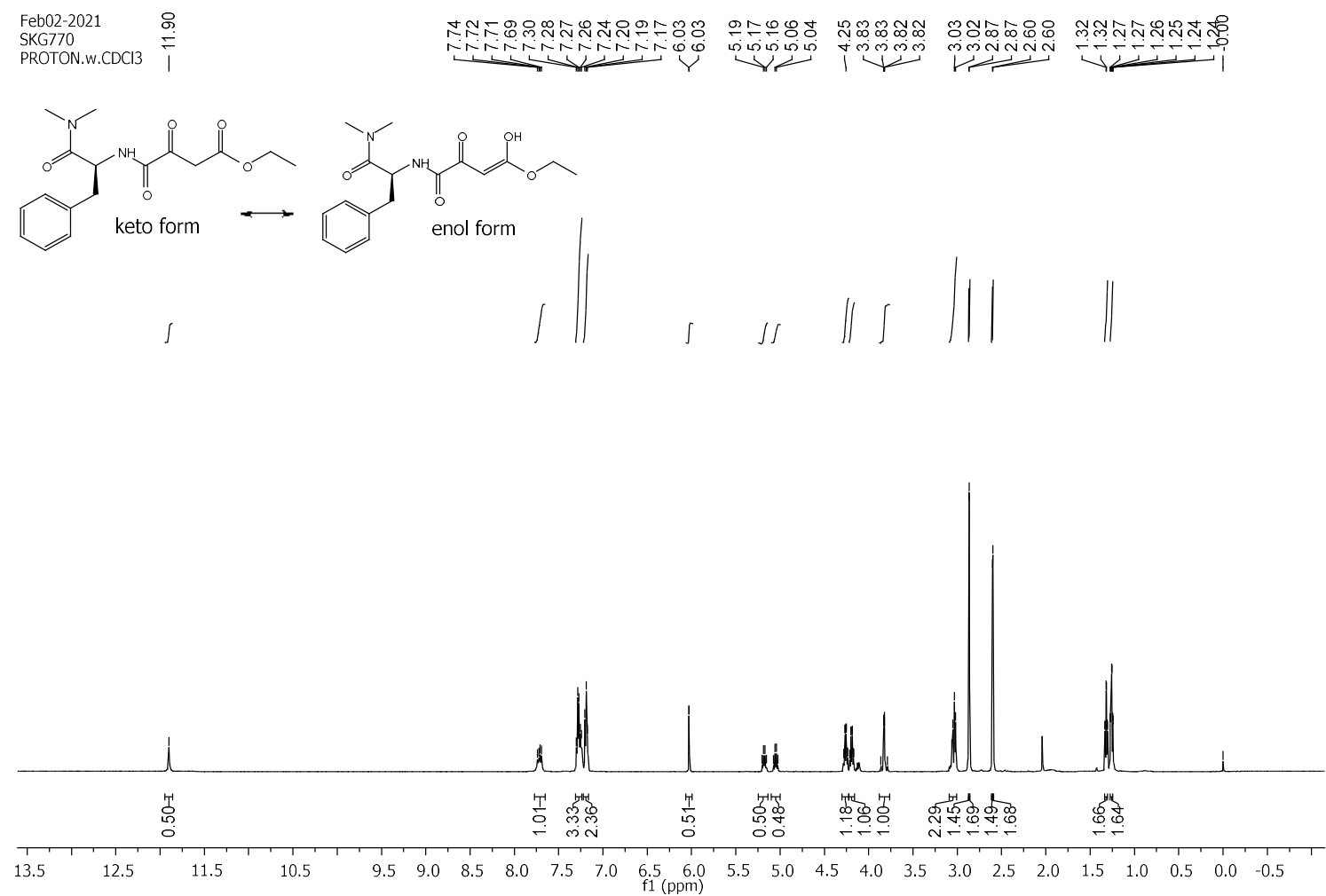

${ }^{13} \mathrm{C} \mathrm{NMR}\left(126 \mathrm{MHz}, \mathrm{CDCl}_{3}\right)$.

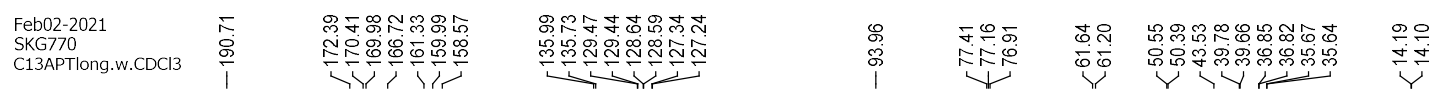<smiles>C=C(CCCC)CC(=O)C(=O)N[C@@H](Cc1ccccc1)C(=O)N(C)C</smiles>

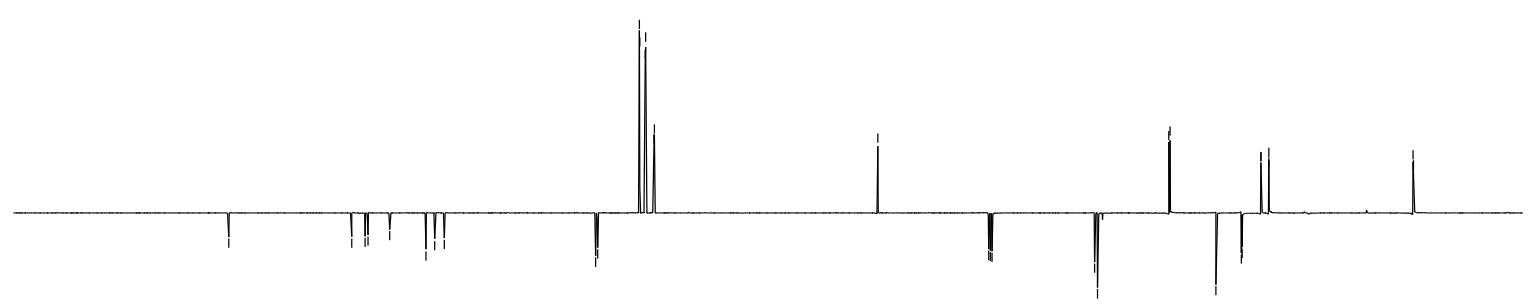

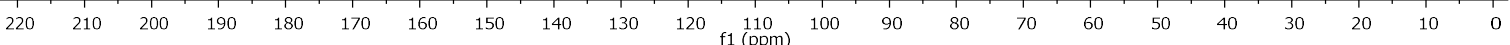


COSY.

Chemist Shweta Gediya

SKG770 $\mathrm{COSY}$.w $\mathrm{CDCl}$ /opt/topspin3.2 SKG 23

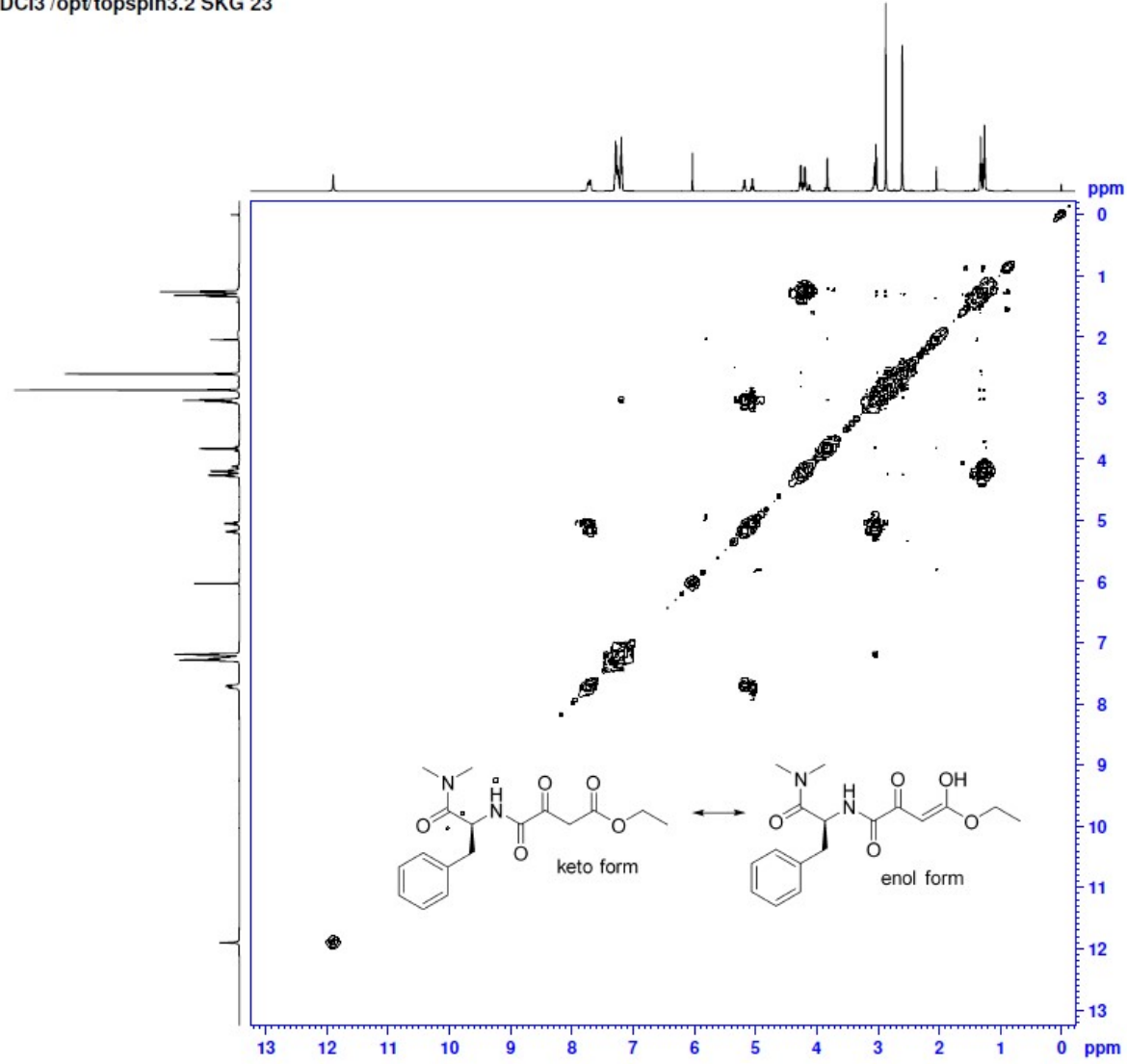

HSQC.

Chemist Shweta Gediya

SKG 230

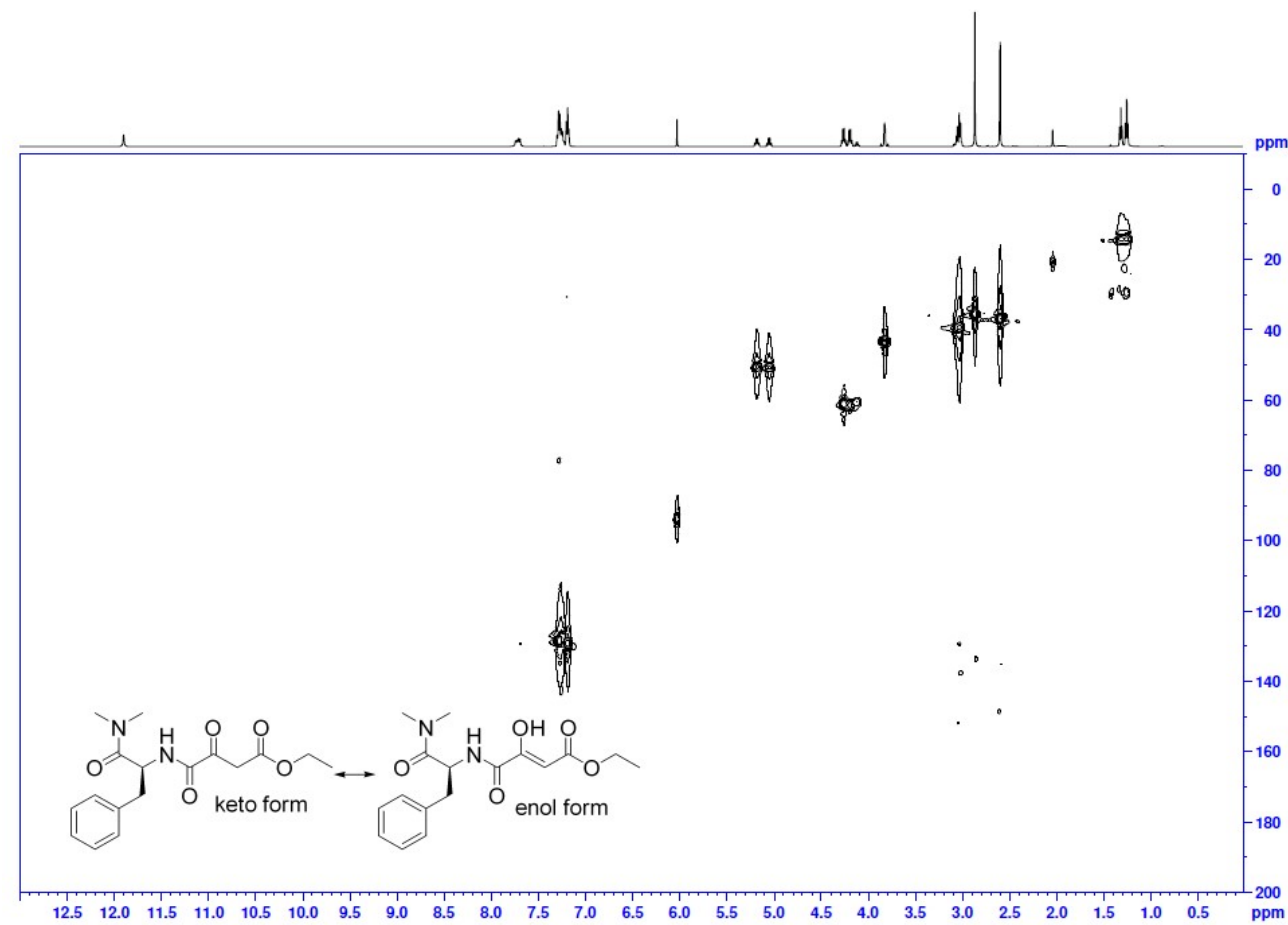


Ethyl (S)-4-((S)-1-(dimethylamino)-1-oxo-3-phenylpropan-2-yl)amino)-3-hydroxy-4oxobutanoate 54.<smiles>CCOC(=O)CC(O)C(=O)NC(Cc1ccccc1)C(=O)N(C)C</smiles>

This compound is novel and was prepared following general procedure $\mathrm{C}$ using ethyl $(S)$-4((1-(dimethylamino)-1-oxo-3-phenylpropan-2-yl)amino)-3,4-dioxobutanoate 53 (83.5 mg, $0.25 \mathrm{mmol}, 1.0 \mathrm{eq}), 3 \mathrm{C}$ tethered catalyst $(S, S)-2(2.3 \mathrm{mg}, 3.8 \mu \mathrm{mol}, 0.015 \mathrm{eq})$ and FA:TEA $(0.15 \mathrm{~mL})$ in DCM $(1.5 \mathrm{~mL})$ to give 54 as a clear liquid (71.5 mg, $0.213 \mathrm{mmol}, 85 \%)$.

TLC: $\mathrm{R}_{\mathrm{f}}$ ca 0.4 (3:7, hexane: EtOAc), UV active and PMA-reactive; HRMS (ESI) m/z: [M+Na] $]^{+}$Calcd for $\mathrm{C}_{17} \mathrm{H}_{24} \mathrm{~N}_{2} \mathrm{O}_{5} \mathrm{Na}$ 359.1577; Found 359.1567 (error 2.8 ppm);

$U_{\max } 3396,2980,2929,1732,1629,1506,1495,1257,1161,1086,700 \mathrm{~cm}^{-1}$;

Diastereomeric excess (dr) determined by HPLC analysis (Chiralpak IC, $250 \mathrm{~mm} \times 4.6 \mathrm{~mm}$ column, hexane: $\operatorname{PrOH} 80: 20,1 \mathrm{~mL} / \mathrm{min}, \lambda=210 \mathrm{~nm}, \mathrm{~T}=25{ }^{\circ} \mathrm{C}$ ), $S$-enantiomer $26.9 \mathrm{~min}, R$ enantiomer 46.7 min; Dr 99:1 $(S, S)-54 /(S, R)-54,[\alpha]_{\mathrm{D}}{ }^{22}=+11.5\left(\mathrm{c}=0.1, \mathrm{CHCl}_{3}\right)$;

Using $(R, R)$-2 catalyst: Dr 18:82 (S,S)-54/(S,R)-54.

${ }^{1} \mathrm{H}$ NMR $\left(500 \mathrm{MHz}, \mathrm{CDCl}_{3}\right.$ ) (major diastereoisomer using $\left.(S, S)-2\right) \delta 7.66(1 \mathrm{H}, \mathrm{d}, J=8.5 \mathrm{~Hz}$, CONH), 7.30 -7.27 (2H, m, ArH), 7.25-7.23 (1H, m, ArH), 7.21-7.19 (1H, m, ArH), 5.16$5.11\left(1 \mathrm{H}, \mathrm{m}, \mathrm{C}_{\underline{H}} \mathrm{H}_{2} \mathrm{Ph}\right), 4.51-4.45(2 \mathrm{H}, \mathrm{m}, \underline{\mathrm{CHOH}}+\mathrm{CHO} \underline{\mathrm{H}}), 4.21-4.15\left(2 \mathrm{H}, \mathrm{m}, \mathrm{C}_{2} \mathrm{CH}_{3}\right.$ of ester group), $3.00\left(2 \mathrm{H}, \mathrm{dd}, J=7.4,2.6 \mathrm{~Hz}, \mathrm{CH}_{2} \mathrm{Ph}\right), 2.89-2.85\left(4 \mathrm{H}, \mathrm{m}, \mathrm{C}_{\mathrm{a}} \mathrm{H}_{\mathrm{b}}+\mathrm{NCH}_{3}\right)$, $2.66-2.58\left(4 \mathrm{H}, \mathrm{m}, \mathrm{CH}_{\mathrm{a}} \underline{\mathrm{H}_{\mathrm{b}}}+\mathrm{NCH}_{3}\right), 1.27\left(3 \mathrm{H}, \mathrm{t}, J=7.1 \mathrm{~Hz}, \mathrm{CH}_{2} \underline{\mathrm{C}}_{3}\right.$ of ester group);

${ }^{13} \mathrm{C}$ NMR (126 MHz, $\mathrm{CDCl}_{3}$ ) (major diastereoisomer using $\left.(S, S)-2\right) \delta 172.2,171.7,171.3$, $136.3,129.5,128.5,127.2,68.7,61.1,49.9,39.7,38.8,37.0,35.7,14.3$;

$m / z(\mathrm{ESI}) 359.1\left[(\mathrm{M}+\mathrm{Na})^{+}, 100 \%\right]$. 
Ethyl (S)-4-((S)-1-(dimethylamino)-1-oxo-3-phenylpropan-2-yl)amino)-3-hydroxy-4oxobutanoate 54 (major diastereoisomer using $(S, S)-2$ ).

${ }^{1} \mathrm{H}$ NMR $\left(500 \mathrm{MHz}, \mathrm{CDCl}_{3}\right)$.

Feb02-2021

Chemist Shweta Gediya

PROTON.w CDCl3 /opt/topspin3.2 SKG 24

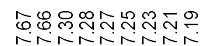

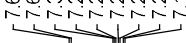

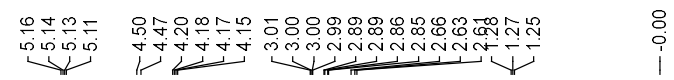<smiles>C#CCCCCC(=O)C[C@@H](O)C(=O)N[C@@H](Cc1ccccc1)C(=O)N(C)C</smiles><smiles>CC#CCCCCCCCCCCCCC</smiles>

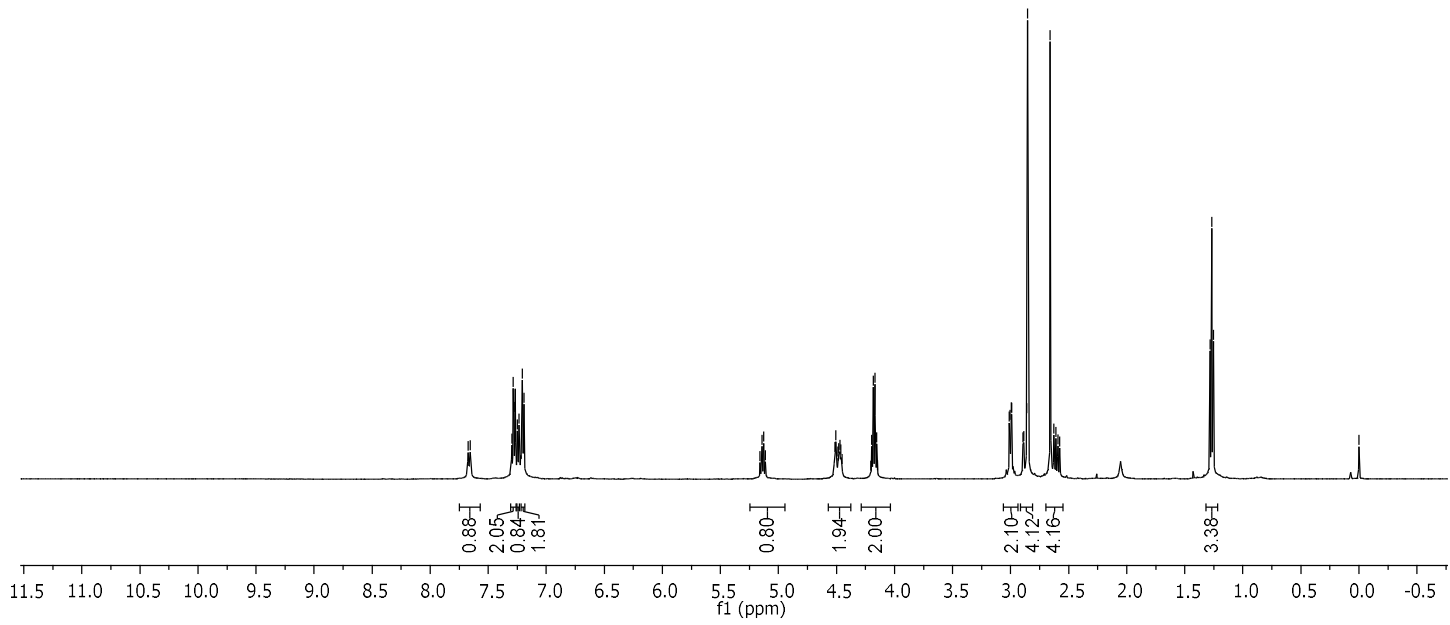

${ }^{13} \mathrm{C}$ NMR (126 MHz, $\mathrm{CDCl}_{3}$ );

Feb02-2021
SKG773

SKG773

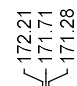

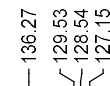<smiles>C=C([C@H](Cc1ccccc1)NC(=O)[C@H](O)CC(=O)OCC)N(C)C</smiles> 
COSY.

Chemist Shweta Gediya

COSY.w CDCl3 /opt/topspin3.2 SKG 24

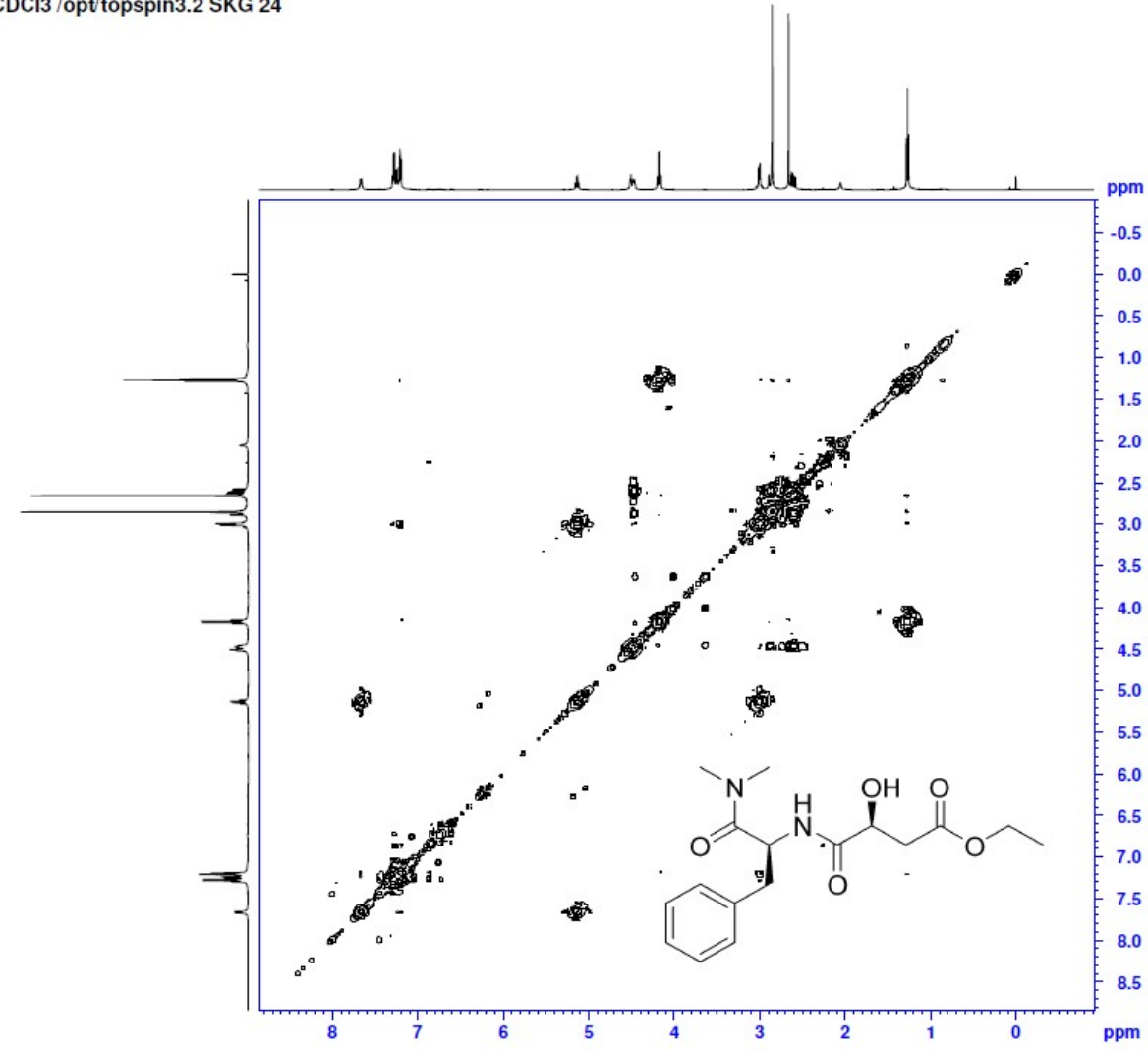

HSQC.

HSQC.w CDCl3 /opt/topspin3.2 SKG 24

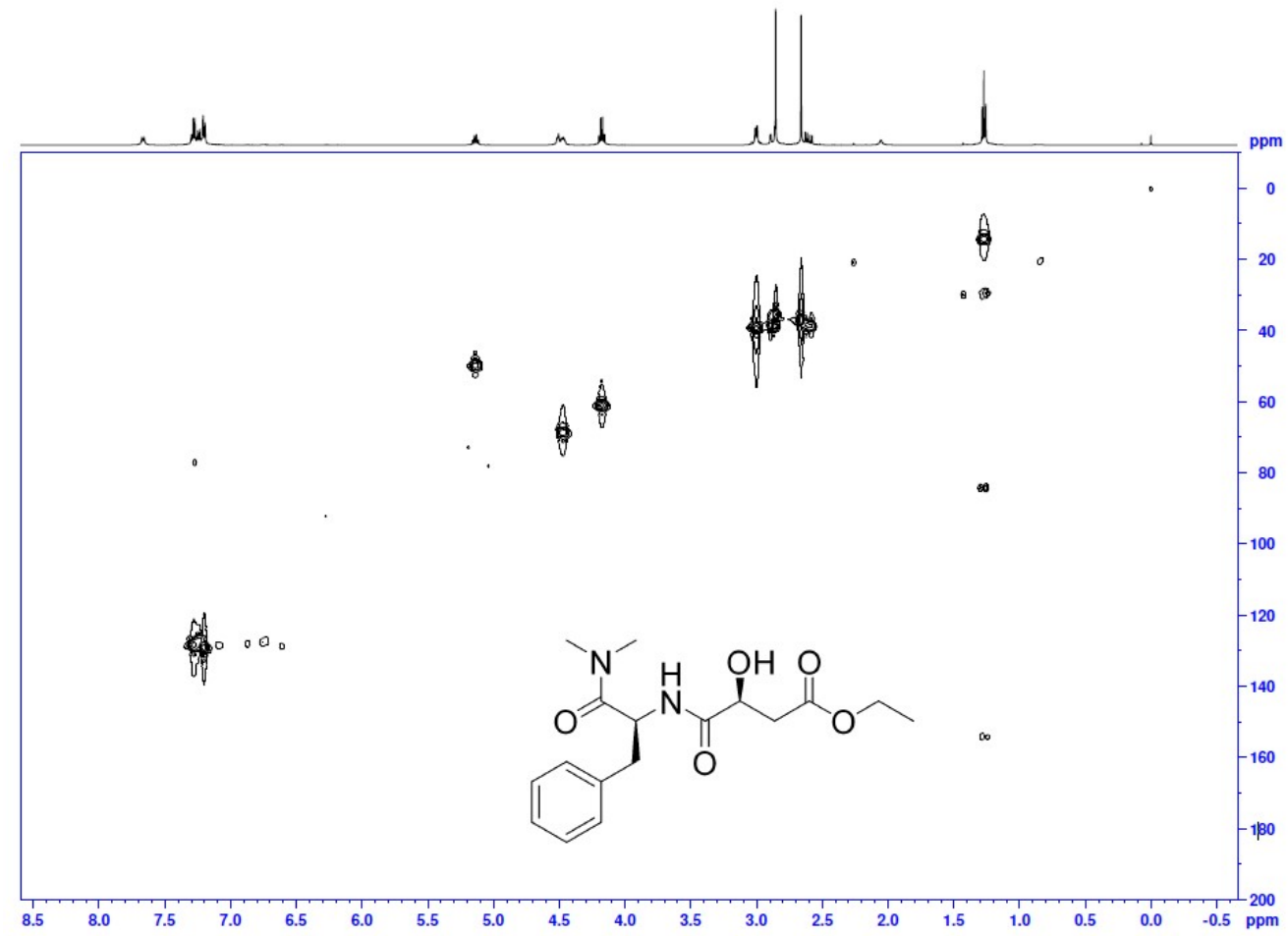


Chiral HPLC of ketone 53 (single enantiomer).

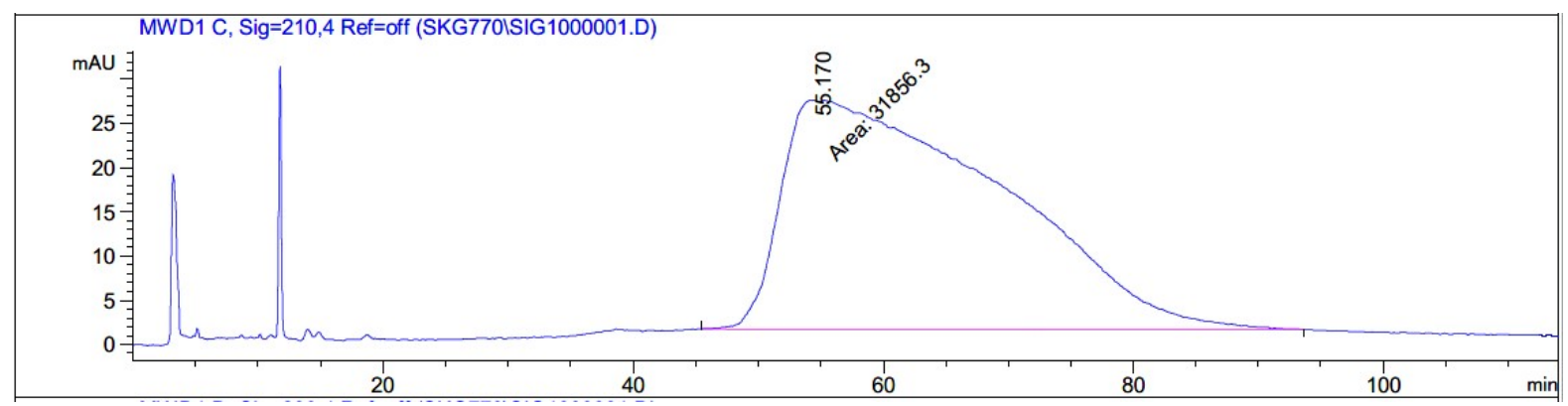

Signal 2: MWD1 C, Sig=210,4 Ref=off

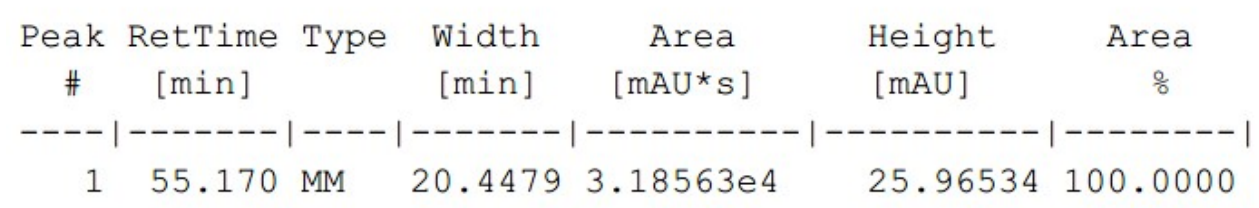

Chiral HPLC of standard of diastereoisomers formed by ATH with a 1:1 mixture of $(R, R)-$ and $(S, S)-2$, dr 64:36.

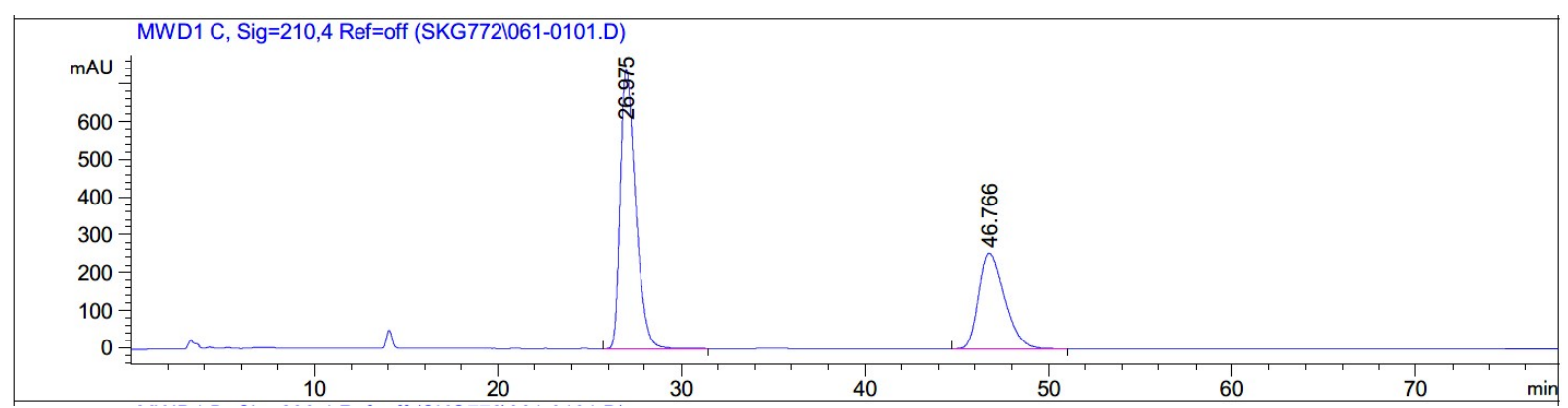

Signal 2: MWD1 C, Sig=210,4 Ref=off

\begin{tabular}{|c|c|c|c|c|c|c|}
\hline $\begin{array}{c}\text { Peak } \\
\#\end{array}$ & $\begin{array}{c}\text { RetTime } \\
\text { [min] }\end{array}$ & Type & $\begin{array}{l}\text { Width } \\
\text { [min] }\end{array}$ & $\begin{array}{c}\text { Area } \\
{\left[\mathrm{mAU}{ }^{\star} \mathrm{s}\right]}\end{array}$ & $\begin{array}{l}\text { Height } \\
{[\mathrm{mAU}]}\end{array}$ & $\begin{array}{c}\text { Area } \\
\frac{\circ}{0}\end{array}$ \\
\hline--1 & ------ & & & ---------- & ---------- & $-------\mid$ \\
\hline 1 & 26.975 & BB & 0.9017 & $4.34297 e 4$ & 738.91339 & 63.7585 \\
\hline 2 & 46.766 & $\mathrm{BB}$ & 1.4987 & $2.46862 \mathrm{e} 4$ & 252.92122 & 36.2415 \\
\hline
\end{tabular}


Chiral HPLC of ATH product using $(S, S)-2.99: 1 \mathrm{dr}(S, S)-54 /(S, R)-54$.

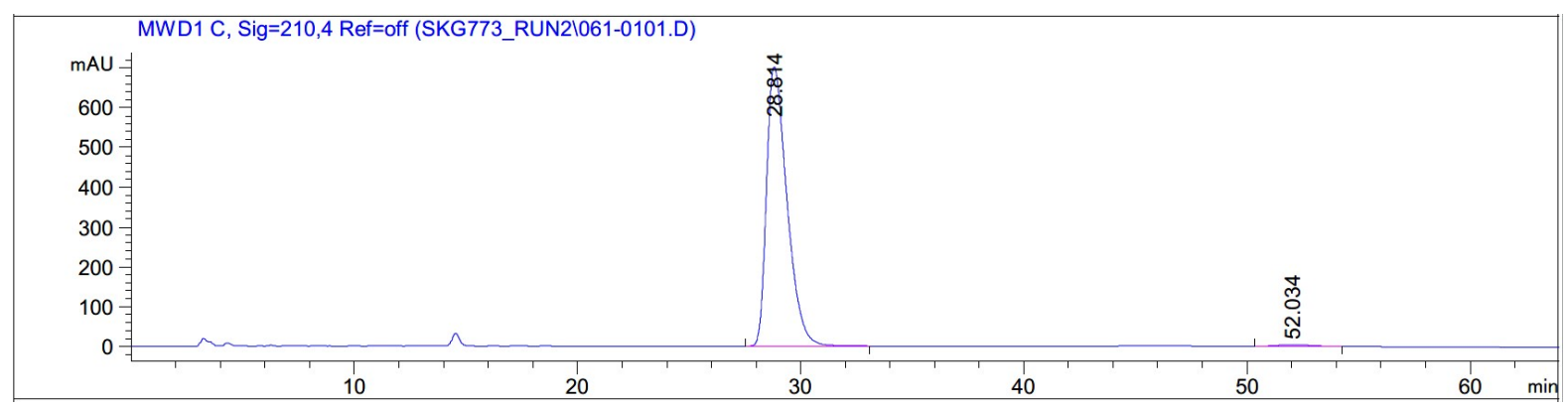

Signal 2: MWD1 C, Sig=210,4 Ref=off

\begin{tabular}{|c|c|c|c|c|c|c|}
\hline $\begin{array}{c}\text { Peak } \\
\#\end{array}$ & $\begin{array}{c}\text { RetTime } \\
{[\min ]}\end{array}$ & Type & $\begin{array}{l}\text { Width } \\
\text { [min] }\end{array}$ & $\begin{array}{c}\text { Area } \\
{\left[\mathrm{mAU}{ }^{*} \mathrm{~s}\right]}\end{array}$ & $\begin{array}{l}\text { Height } \\
\text { [mAU] }\end{array}$ & $\begin{array}{c}\text { Area } \\
\frac{\circ}{0}\end{array}$ \\
\hline & & & & -- & --- & -- \\
\hline 1 & 28.814 & 3B & 0.9732 & $4.47970 \mathrm{e} 4$ & 703.18689 & 99.0376 \\
\hline 2 & 52.034 & $\mathrm{BB}$ & 1.2357 & 435.29672 & 4.15021 & 0.9624 \\
\hline
\end{tabular}

Chiral HPLC of ATH product using (R,R)-2. 18:82 dr $(S, S)-54 /(S, R)-54$.

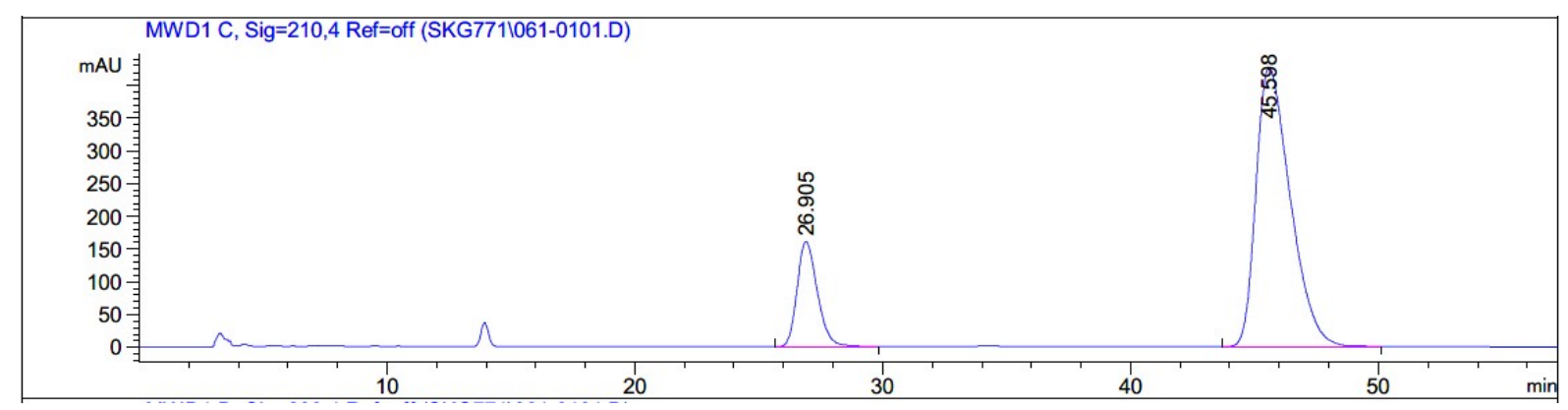

Signal 2: MWD1 C, Sig=210,4 Ref=off

\begin{tabular}{|c|c|c|c|c|c|c|}
\hline $\begin{array}{c}\text { Peak } \\
\#\end{array}$ & $\begin{array}{c}\text { RetTime } \\
\text { [min] }\end{array}$ & Type & $\begin{array}{c}\text { Width } \\
\text { [min] }\end{array}$ & $\begin{array}{c}\text { Area } \\
{\left[\mathrm{mAU}^{*} \mathrm{~s}\right]}\end{array}$ & $\begin{array}{l}\text { Height } \\
{[\mathrm{mAU}]}\end{array}$ & $\begin{array}{c}\text { Area } \\
\%\end{array}$ \\
\hline-- & ------ & & ------- & ---------- & --------- & $--------\mid$ \\
\hline 1 & 26.905 & $\mathrm{BB}$ & 0.8759 & 9175.54980 & 160.76468 & 18.2702 \\
\hline 2 & 45.598 & $\mathrm{BB}$ & 1.4828 & $4.10459 \mathrm{e} 4$ & 425.71835 & 81.7298 \\
\hline
\end{tabular}


Ethyl 3-((tert-butyldimethylsilyl)oxy)-4-((2,6-diisopropylphenyl)amino)-4-oxobutanoate.<smiles>CCOC(=O)CC([O-])C1Nc2c(C(C)C)cccc2C(C)O1</smiles>

This compound is novel. To a stirred solution of ethyl 4-((2,6-diisopropylphenyl)amino)-3hydroxy-4-oxobutanoate $33(0.270 \mathrm{~g}, 0.841 \mathrm{mmol}, 1.0 \mathrm{eq})$ in DMF (5 mL) was added tertbutyldimethylsilyl chloride (TBDMSCl) $(0.252 \mathrm{~g}, 1.68 \mathrm{mmol}, 2.0 \mathrm{eq})$ and imidazole (0.143 g, $2.10 \mathrm{mmol}, 2.5 \mathrm{eq}$ ) and the resulting reaction mixture was stirred at room temperature for $3 \mathrm{~h}$. The reaction mixture was quenched with ice cold water $(20 \mathrm{~mL})$ and extracted with ethyl acetate $(3 \times 10 \mathrm{~mL})$. The combined organic layer was washed with brine $(50 \mathrm{~mL})$. The organic layer was dried over $\mathrm{MgSO}_{4}$, filtered and filtrate was concentrated under reduced pressure to generate crude material which was further purified by column chromatography (5$10 \%$ EtOAc in petroleum ether) to afford the product as a clear oil $(0.350 \mathrm{~g}, 0.804 \mathrm{mmol}$, $96 \%)$.

TLC: $\mathrm{R}_{\mathrm{f}}$ ca 0.5 (8:2, hexane: EtOAc), UV active, strong $\mathrm{KMnO}_{4}$ active and PMA-reactive;

HRMS (ESI) m/z: [M+Na] ${ }^{+}$Calcd for $\mathrm{C}_{24} \mathrm{H}_{41} \mathrm{NO}_{4} \mathrm{SiNa} 458.2697$; Found 458.2691 (error 1.4 ppm);

$U_{\max } 3404,2957,2929,1738,1696,1492,1471,1523,1174,1098,829,779 \mathrm{~cm}^{-1}$;

${ }^{1} \mathrm{H}$ NMR (400 MHz, $\left.\mathrm{CDCl}_{3}\right) \delta 7.94$ (1H, br.s., CONH) 7.32-7.26 (1H, m, ArH), 7.20-7.18 $(2 \mathrm{H}, \mathrm{m}, \mathrm{ArH}), 4.79-4.76(1 \mathrm{H}, \mathrm{m}, \mathrm{C} \underline{\mathrm{HOTBS}}), 4.25-4.11\left(2 \mathrm{H}, \mathrm{m}, \mathrm{C}_{2} \mathrm{CH}_{3}\right), 3.11-3.01(2 \mathrm{H}, \mathrm{m}$, $\mathrm{CH}$ of isopropyl), $2.97\left(1 \mathrm{H}, \mathrm{dd}, J=15.4,4.0 \mathrm{~Hz}, \mathrm{C}_{\mathrm{a}} \mathrm{H}_{\mathrm{b}}\right), 2.78(1 \mathrm{H}, \mathrm{dd}, J=15.4,7.5 \mathrm{~Hz}$, $\left.\mathrm{CH}_{\mathrm{a}} \underline{\mathrm{H}} \mathrm{b}\right), 1.29\left(3 \mathrm{H}, \mathrm{t}, J=7.2 \mathrm{~Hz}, \mathrm{OCH}_{2} \mathrm{CH}_{3}\right), 1.23\left(6 \mathrm{H}, \mathrm{d}, J=6.9 \mathrm{~Hz}, \mathrm{CH}_{3}\right.$ of isopropyl), 1.18 $\left(6 \mathrm{H}, \mathrm{d}, J=6.9 \mathrm{~Hz}, \mathrm{CH}_{3}\right.$ of isopropyl), $0.96\left(9 \mathrm{H}, \mathrm{s}, \mathrm{CH}_{3}\right.$ of tert-butyl group), 0.21 (3H, s, $\left.\left(\mathrm{CH}_{3}\right)_{2} \mathrm{Si}\right), 0.17\left(3 \mathrm{H}, \mathrm{s},\left(\mathrm{CH}_{3}\right)_{2} \mathrm{Si}\right)$;

${ }^{13} \mathrm{C}$ NMR $\left(101 \mathrm{MHz}, \mathrm{CDCl}_{3}\right) \delta 171.9,170.4,146.2,130.4,128.5,123.7,71.2,60.9,41.3$, $28.8,25.8,23.9,23.7,14.4,-4.7,-5.1$;

$m / z(\mathrm{ESI}) 458.2\left[(\mathrm{M}+\mathrm{Na})^{+}, 100 \%\right]$. 
Ethyl 3-((tert-butyldimethylsilyl)oxy)-4-oxo-4-(piperidin-1-yl)butanoate.

${ }^{1} \mathrm{H}$ NMR (400 MHz, $\left.\mathrm{CDCl}_{3}\right)$.

Feb18-202

SKG802
PROTON.w CDCI3 /opt/topspin3.5pl2 SKG 43

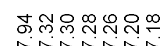

ivininin

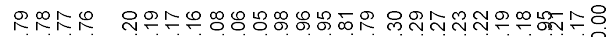

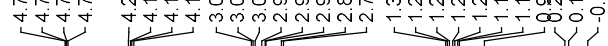

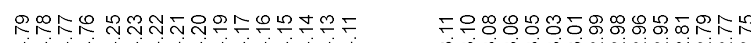

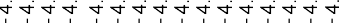
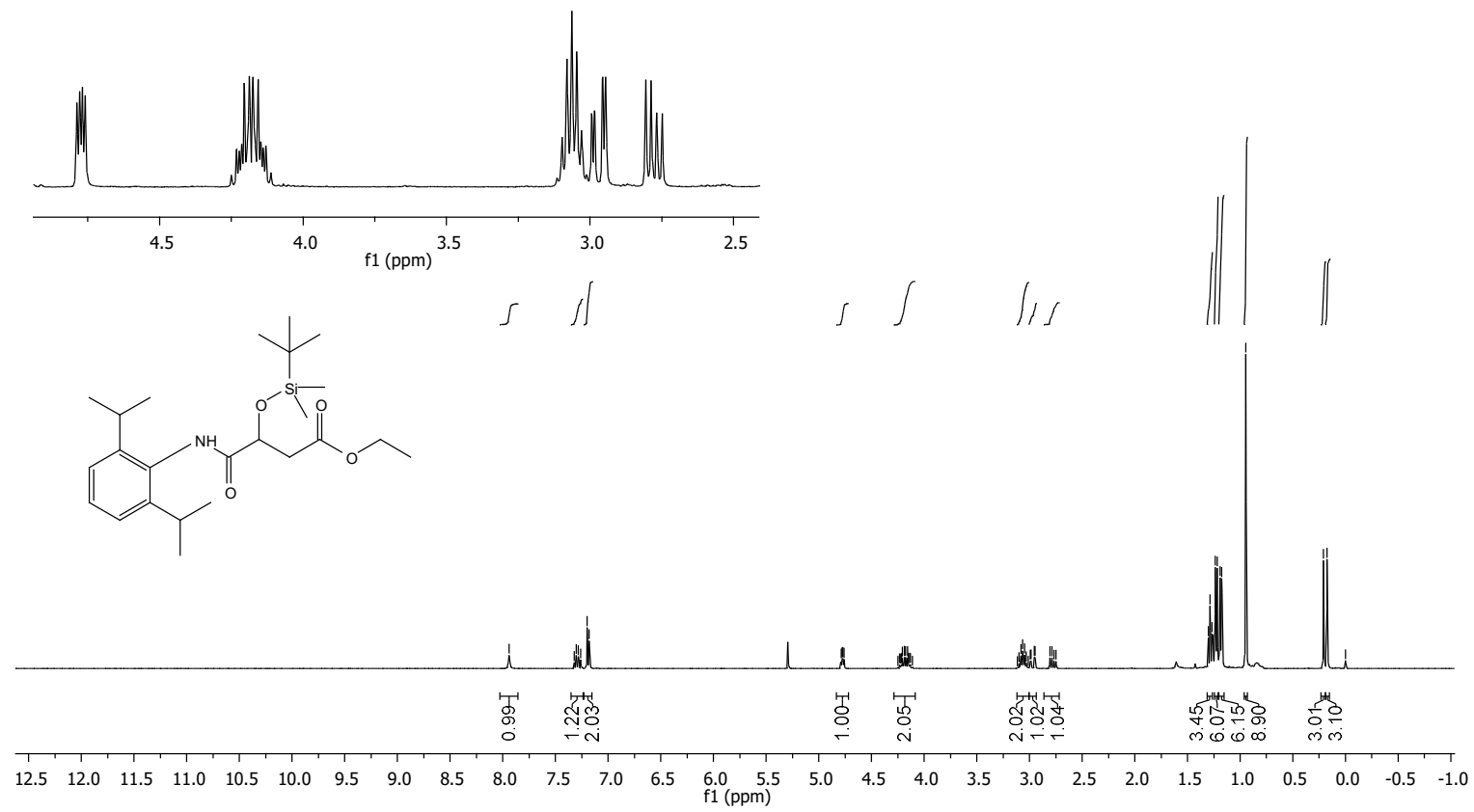

${ }^{13} \mathrm{C}$ NMR $\left(101 \mathrm{MHz}, \mathrm{CDCl}_{3}\right)$.

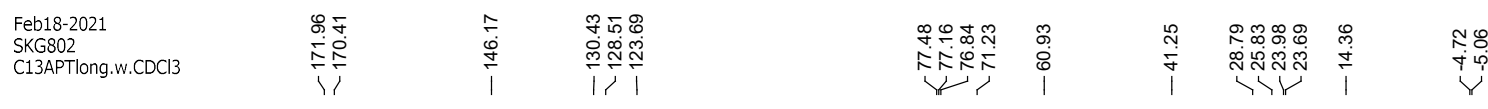

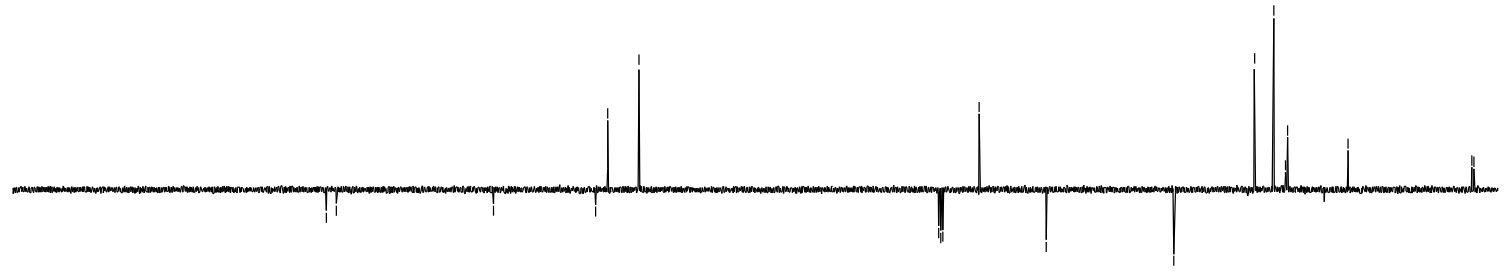

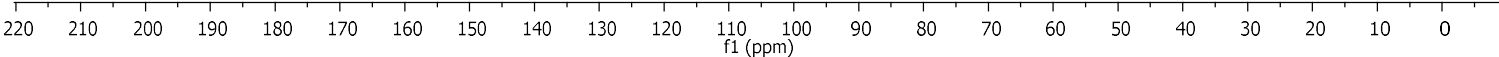


COSY.

SKG802

COSY.w CDCl3 /opt/topspin3.5pl2 SKG 43

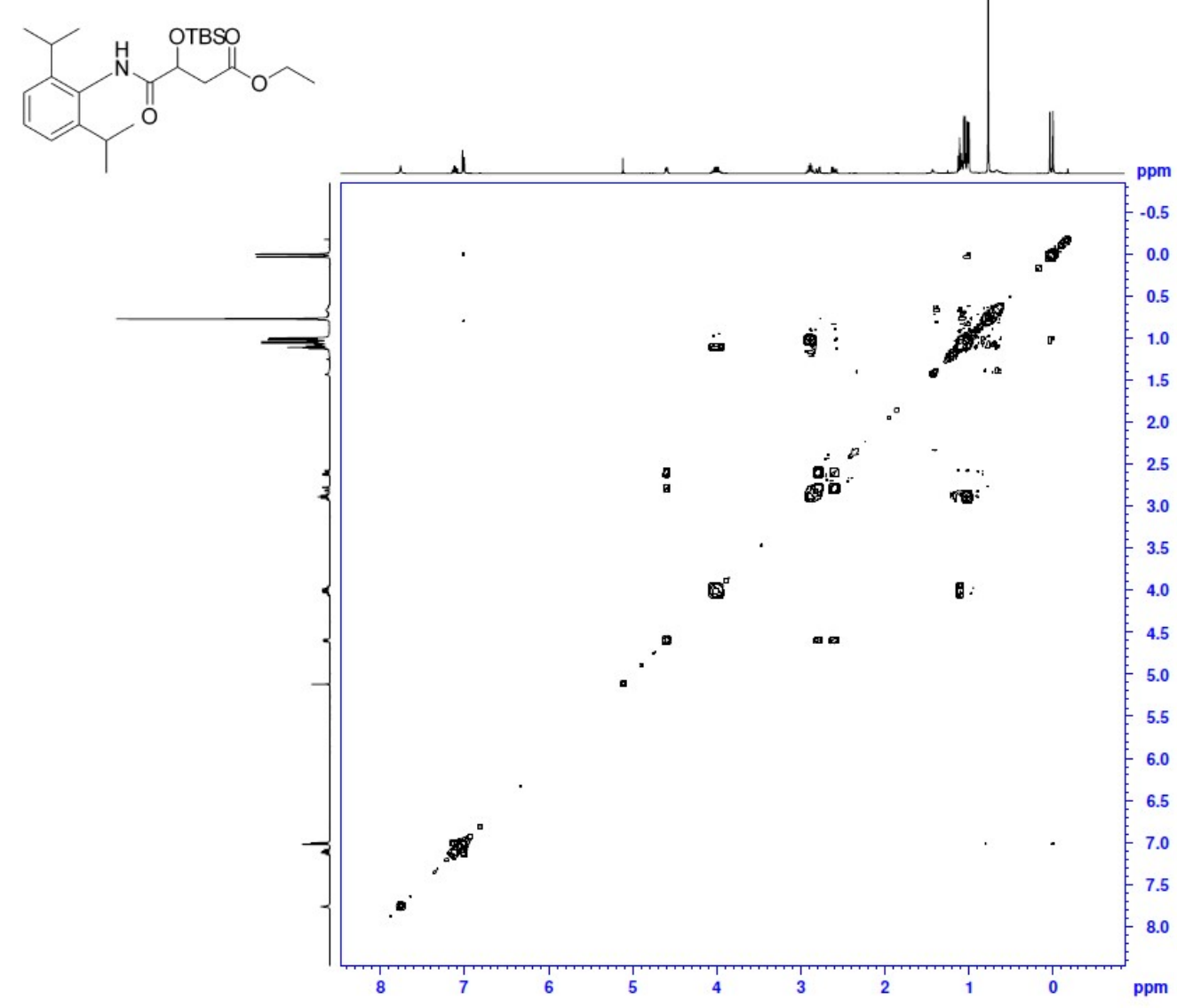


3-((tert-Butyldimethylsilyl)oxy)-4-((2,6-diisopropylphenyl)amino)-4-oxobutanoic acid.<smiles>CC(C)c1cccc2c1NC(CC(=O)O)OC2C</smiles>

This compound is novel. To a stirred solution of ethyl 3-((tert-butyldimethylsilyl)oxy)-4((2,6-diisopropylphenyl)amino)-4-oxobutanoate $(0.350 \mathrm{~g}, 0.805 \mathrm{mmol}, 1.0 \mathrm{eq})$ in methanol (5 $\mathrm{mL}$ ) was added $40 \%$ aqueous potassium hydroxide solution $(1 \mathrm{~mL})$ and the resulting reaction mixture was stirred at room temperature for $3 \mathrm{~h}$. The reaction mixture was concentrated under reduced pressure and the obtained residue was dissolved in water $(10 \mathrm{~mL})$. The aqueous layer was extracted with ethyl acetate $(20 \mathrm{~mL})$ to remove non-polar impurities. The aqueous layer was acidified with $2 \mathrm{~N} \mathrm{HCl}$ to $\mathrm{pH} 2$ and then extracted with ethyl acetate $(3 \times 15 \mathrm{~mL})$. The combined organic layer was washed with water $(50 \mathrm{~mL})$ and brine $(50 \mathrm{~mL})$. The organic layer was dried over $\mathrm{MgSO}_{4}$, filtered and filtrate was concentrated under reduced pressure to afford the product as a clear oil $(0.280 \mathrm{~g}, 0.687 \mathrm{mmol}, 86 \%)$ which was used in next step without further purification.

TLC: $\mathrm{R}_{\mathrm{f}}$ ca $0.2\left(9: 1, \mathrm{CHCl}_{3}: \mathrm{MeOH}\right), \mathrm{UV}$ active, strong $\mathrm{KMnO}_{4}$ active and PMA-reactive; HRMS (ESI) m/z: [M+Na $]^{+}$Calcd for $\mathrm{C}_{22} \mathrm{H}_{37} \mathrm{NO}_{4} \mathrm{SiNa}$ 430.2384; Found 430.2391 (error -1.5 ppm);

$U_{\max } 3349,2964,1742,1618,1530,1198,1085,793 \mathrm{~cm}^{-1}$;

${ }^{1} \mathrm{H}$ NMR (400 MHz, $\left.\mathrm{CDCl}_{3}\right) \delta$ 7.56-7.52 (1H, m, CONH), 7.32-7.16 (1H, m, ArH), 7.19-7.16 (2H, m, ArH), 4.56-4.49 (1H, m, CㅌTBS), 3.11-2.95 (4H, m, CH of isopropyl $\left.+\mathrm{CH}_{\mathrm{a}} \mathrm{H}_{\mathrm{b}}\right)$, 1.20-1.16 (12H, m, $\mathrm{CH}_{3}$ of isopropyl), 0.96-0.89 (9H, m, $\mathrm{CH}_{3}$ of tert-butyl group), 0.32-0.10 $\left(6 \mathrm{H}, \mathrm{m},\left(\mathrm{CH}_{3}\right)_{2} \mathrm{Si}\right)$;

${ }^{13} \mathrm{C}$ NMR $\left(101 \mathrm{MHz}, \mathrm{CDCl}_{3}\right) \delta 173.8,169.3,148.9,146.1,146.0,129.0,128.4,123.7,123.5$, $69.7,68.3,68.9,40.3,39.0,28.9,28.8,28.6,25.7,25.6,25.5,25.4,23.6,22.5$; $m / z(\mathrm{ESI}) 430.2\left[(\mathrm{M}+\mathrm{Na})^{+}, 100 \%\right]$. 
3-((tert-Butyldimethylsilyl)oxy)-4-((2,6-diisopropylphenyl)amino)-4-oxobutanoicacid. ${ }^{1} \mathrm{H}$ NMR $\left(400 \mathrm{MHz}, \mathrm{CDCl}_{3}\right)$.

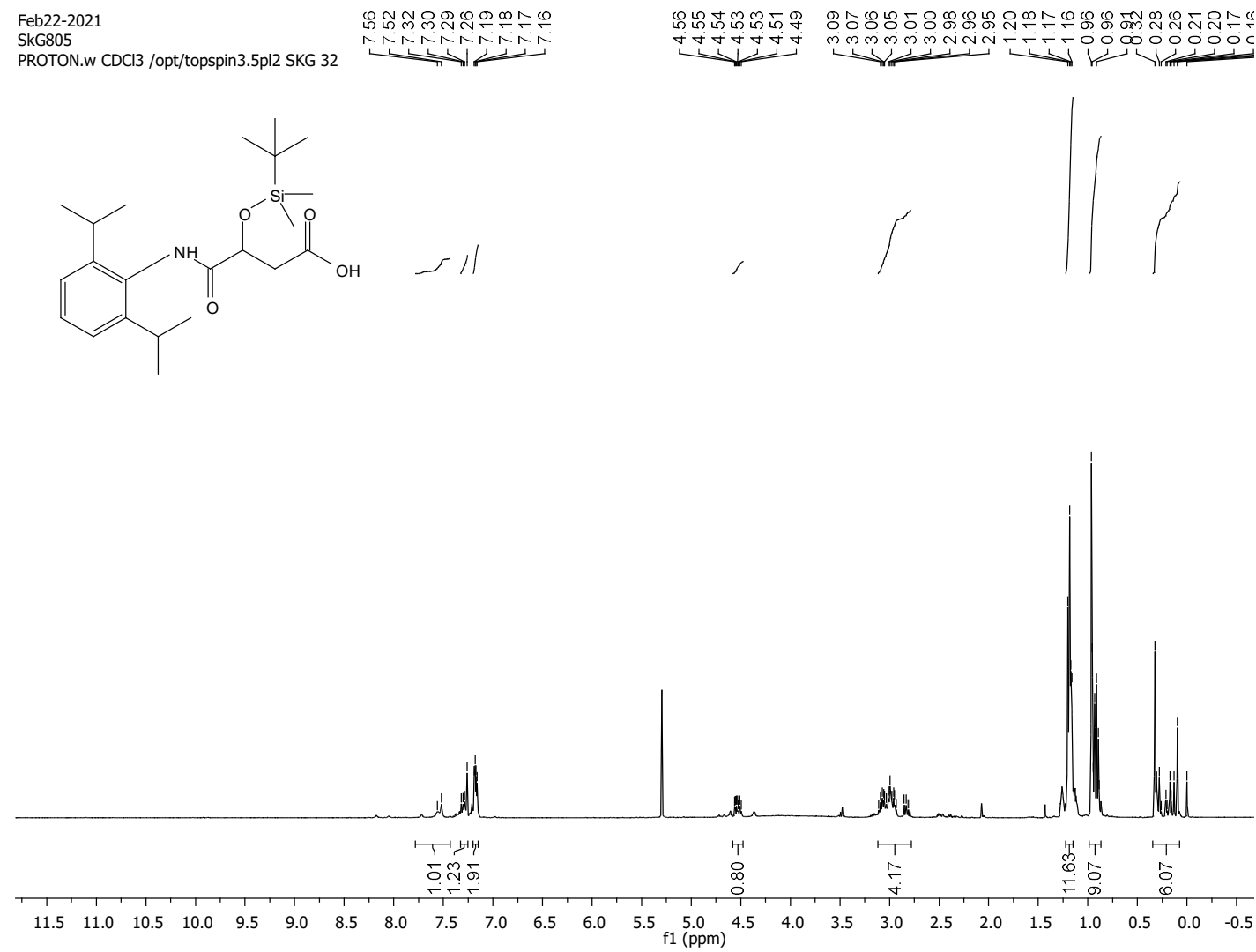

${ }^{13} \mathrm{C} \mathrm{NMR}\left(101 \mathrm{MHz}, \mathrm{CDCl}_{3}\right)$.

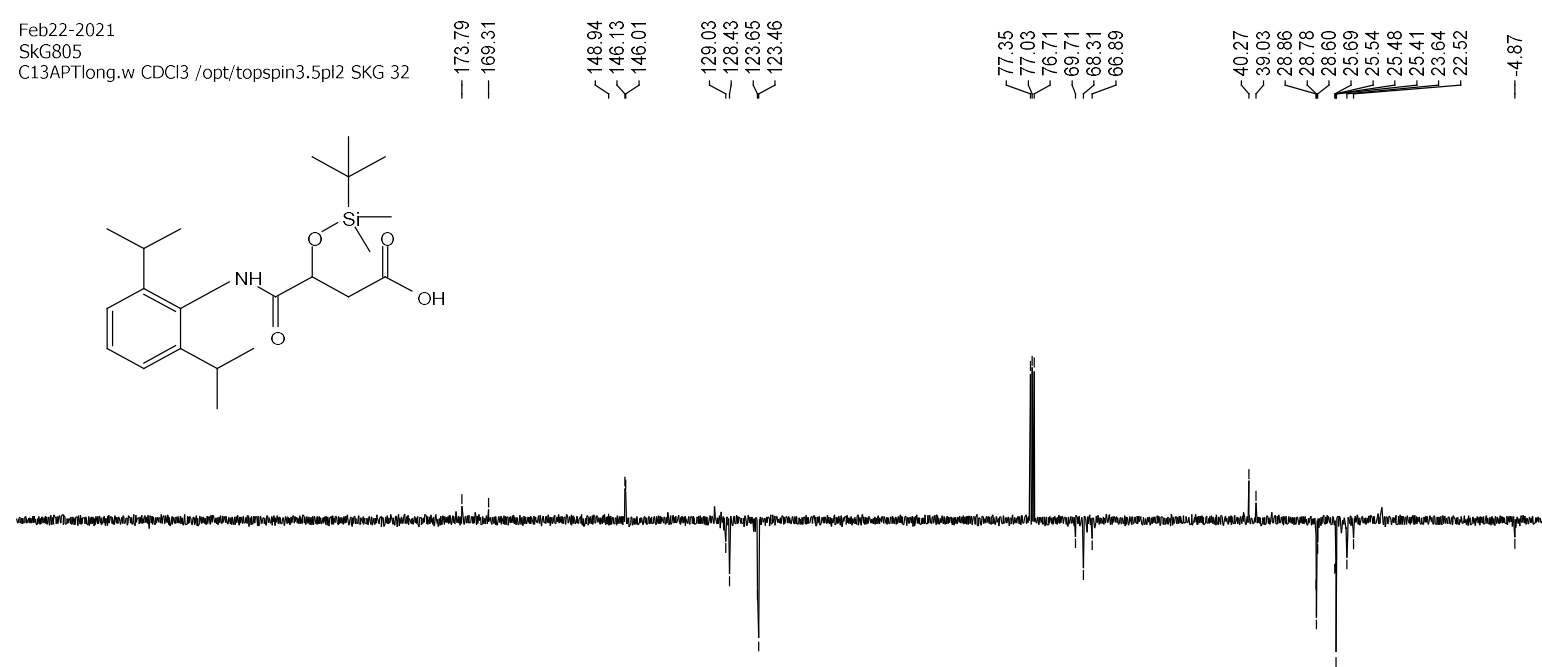

$\begin{array}{lllllllllllllllllllllllllllllll}240 & 230 & 220 & 210 & 200 & 190 & 180 & 170 & 160 & 150 & 140 & 130 & \begin{array}{l}120 \\ \mathrm{f} 1(\mathrm{ppm})\end{array} & 110 & 100 & 90 & 80 & 70 & 60 & 50 & 40 & 30 & 20 & 10 & 0\end{array}$ 
COSY.

SkG805

COSY.w CDCl3/opt/topspin3.5pl2 SKG 32

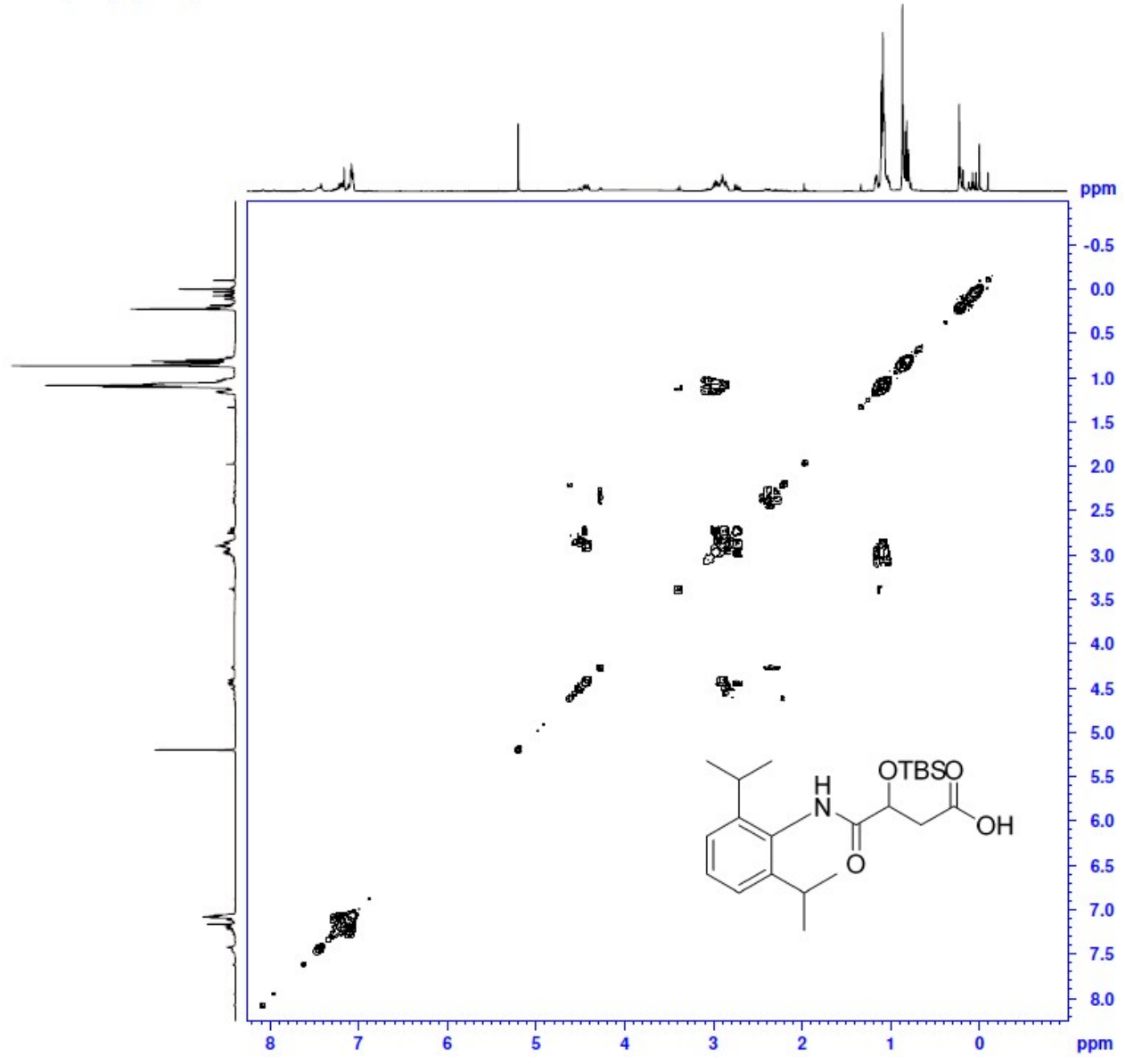


(racemic).<smiles>CCOC(=O)CC(O)C(=O)N(C)c1ccccc1</smiles>

This compound is novel. To a stirred solution of ethyl 3-hydroxy-4-(methyl(phenyl)amino)4-oxobutanoate 38 (0.500 g, $1.99 \mathrm{mmol}, 1.0 \mathrm{eq})$ in DMF (10 mL) was added tertbutyldimethylsilyl chloride (TBDMSCl) $(0.600 \mathrm{~g}, 3.98 \mathrm{mmol}, 2.0 \mathrm{eq})$ and imidazole (0.338 g, $4.97 \mathrm{mmol}, 2.5 \mathrm{eq}$ ) and the resulting reaction mixture was stirred at room temperature for $3 \mathrm{~h}$. The reaction mixture was quenched with ice cold water $(50 \mathrm{~mL})$ and extracted with ethyl acetate $(3 \times 20 \mathrm{~mL})$. The combined organic layers were washed with brine $(50 \mathrm{~mL})$. The organic layer was dried over $\mathrm{MgSO}_{4}$, filtered and filtrate was concentrated under reduced pressure to generate crude material which was further purified by column chromatography $(10-20 \%$ EtOAc in petroleum ether) to afford the product as a clear oil $(0.540 \mathrm{~g}, 1.48 \mathrm{mmol}$, $74 \%)$.

TLC: $\mathrm{R}_{\mathrm{f}}$ ca 0.5 (8:2, hexane: EtOAc), UV active, strong $\mathrm{KMnO}_{4}$ active and PMA-reactive;

HRMS (ESI) m/z: [M+Na] ${ }^{+}$Calcd for $\mathrm{C}_{19} \mathrm{H}_{31} \mathrm{NO}_{4} \mathrm{SiNa} 388.1915$; Found 388.1904 (error 2.6 ppm);

$U_{\max } 2955,2929,1733,1666,1495,1250,1177,1106,832,774,699 \mathrm{~cm}^{-1}$;

${ }^{1} \mathrm{H}$ NMR (400 MHz, $\left.\mathrm{CDCl}_{3}\right) \delta 7.54(2 \mathrm{H}, \mathrm{t}, J=7.5 \mathrm{~Hz}, \mathrm{ArH}), 7.46(1 \mathrm{H}, \mathrm{t}, J=7.1 \mathrm{~Hz}, \mathrm{ArH})$, $7.38(2 \mathrm{H}, \mathrm{d}, J=7.7 \mathrm{~Hz}, \mathrm{ArH}), 4.76(1 \mathrm{H}, \mathrm{s}, \mathrm{C} \underline{\mathrm{HOTBS}}), 4.21-4.10\left(2 \mathrm{H}, \mathrm{m}, \mathrm{C}_{2} \mathrm{CH}_{3}\right), 3.40(3 \mathrm{H}$, s, $\left.\mathrm{NCH}_{3}\right), 2.89\left(1 \mathrm{H}, \mathrm{dd}, J=15.8,5.7 \mathrm{~Hz}, \underline{C}_{\underline{\mathrm{a}}} \mathrm{H}_{\mathrm{b}}\right), 2.69\left(1 \mathrm{H}, \mathrm{dd}, J=15.7,7.0 \mathrm{~Hz}, \mathrm{CH}_{\mathrm{a}} \underline{\mathrm{H}_{\mathrm{b}}}\right)$, $1.31\left(3 \mathrm{H}, \mathrm{t}, J=7.1 \mathrm{~Hz}, \mathrm{CH}_{2} \underline{\mathrm{C}}_{3}\right), 0.93\left(9 \mathrm{H}, \mathrm{s}, \mathrm{CH}_{3}\right.$ of tert-butyl group), 0.01-0.00 (6H, m, $\left(\mathrm{CH}_{3}\right)_{2} \mathrm{Si}$, consist two singlets at 0.01 and $\left.0.00 \mathrm{ppm}\right)$;

${ }^{13} \mathrm{C}$ NMR $\left(101 \mathrm{MHz}, \mathrm{CDCl}_{3}\right) \delta 171.3,170.7,143.1,129.8,128.1,127.5,66.6,60.5,39.9$, $37.9,25.7,18.2,14.2,-4.7,-4.8$;

$m / z(\mathrm{ESI}) 388.2\left[(\mathrm{M}+\mathrm{Na})^{+}, 100 \%\right]$. 
Ethyl 3-((tert-butyldimethylsilyl)oxy)-4-(methyl(phenyl)amino)-4-oxobutanoate. ${ }^{1} \mathrm{H}$ NMR $\left(400 \mathrm{MHz}, \mathrm{CDCl}_{3}\right)$.
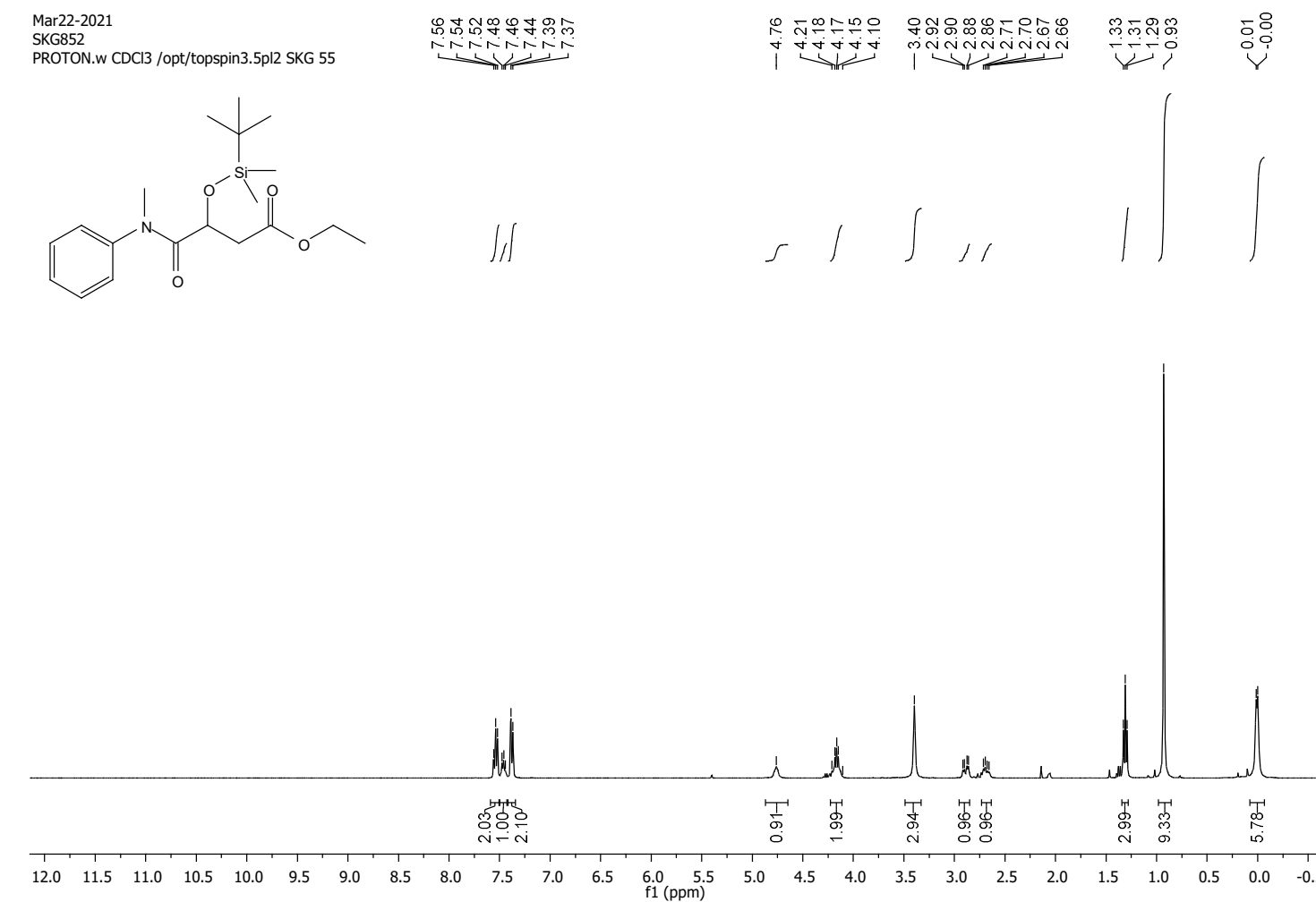

${ }^{13} \mathrm{C}$ NMR (101 MHz, $\left.\mathrm{CDCl}_{3}\right)$.

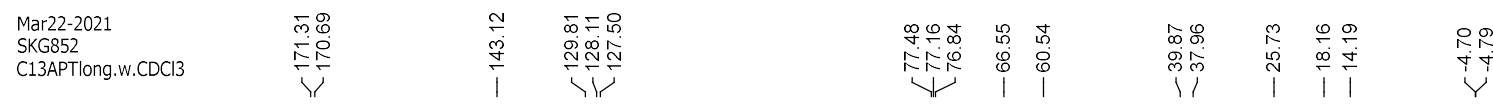<smiles>CCOC(=O)CC(O[Si](C)(C)C(C)(C)C)C(=O)N(C)c1ccccc1</smiles>

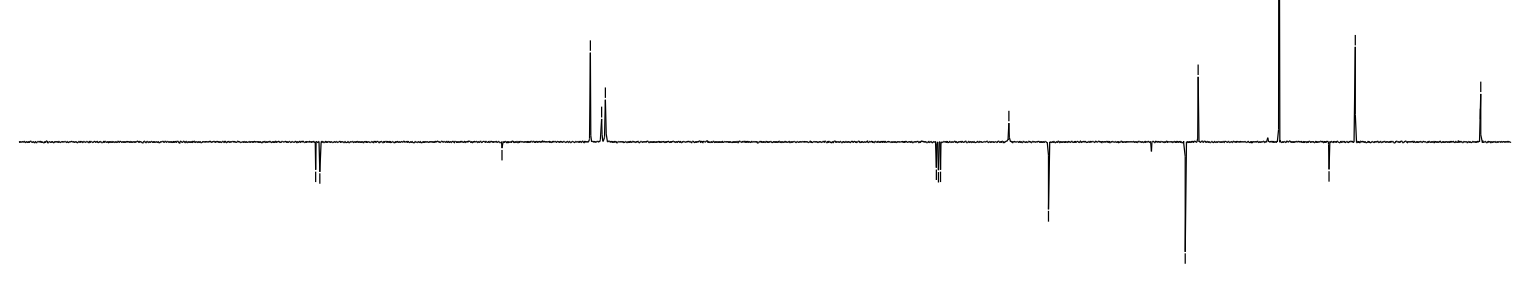

$\begin{array}{lllllllllllllllllllllllll}210 & 200 & 190 & 180 & 170 & 160 & 150 & 140 & 130 & 120 & 110 & 100 & 90 & 80 & 70 & 60 & 50 & 40 & 30 & 20 & 10 & 0\end{array}$ 
COSY.

SKG852
COSY.w CDCl3 /opt/topspin3.5pl2 SKG 55

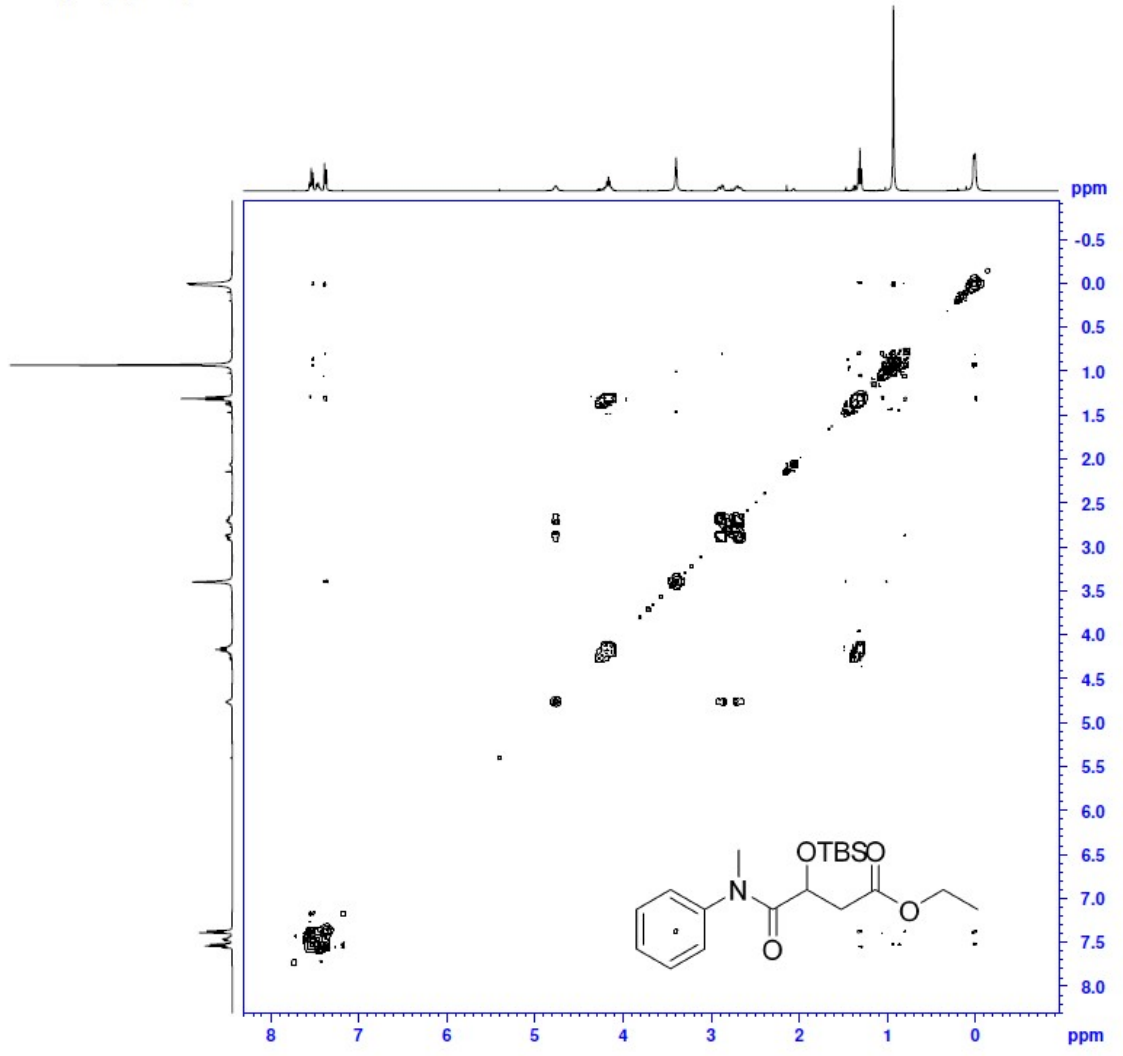

HSQC.

HSQC.w CDCl3/opt/topspin3.5pl2 SKG 55

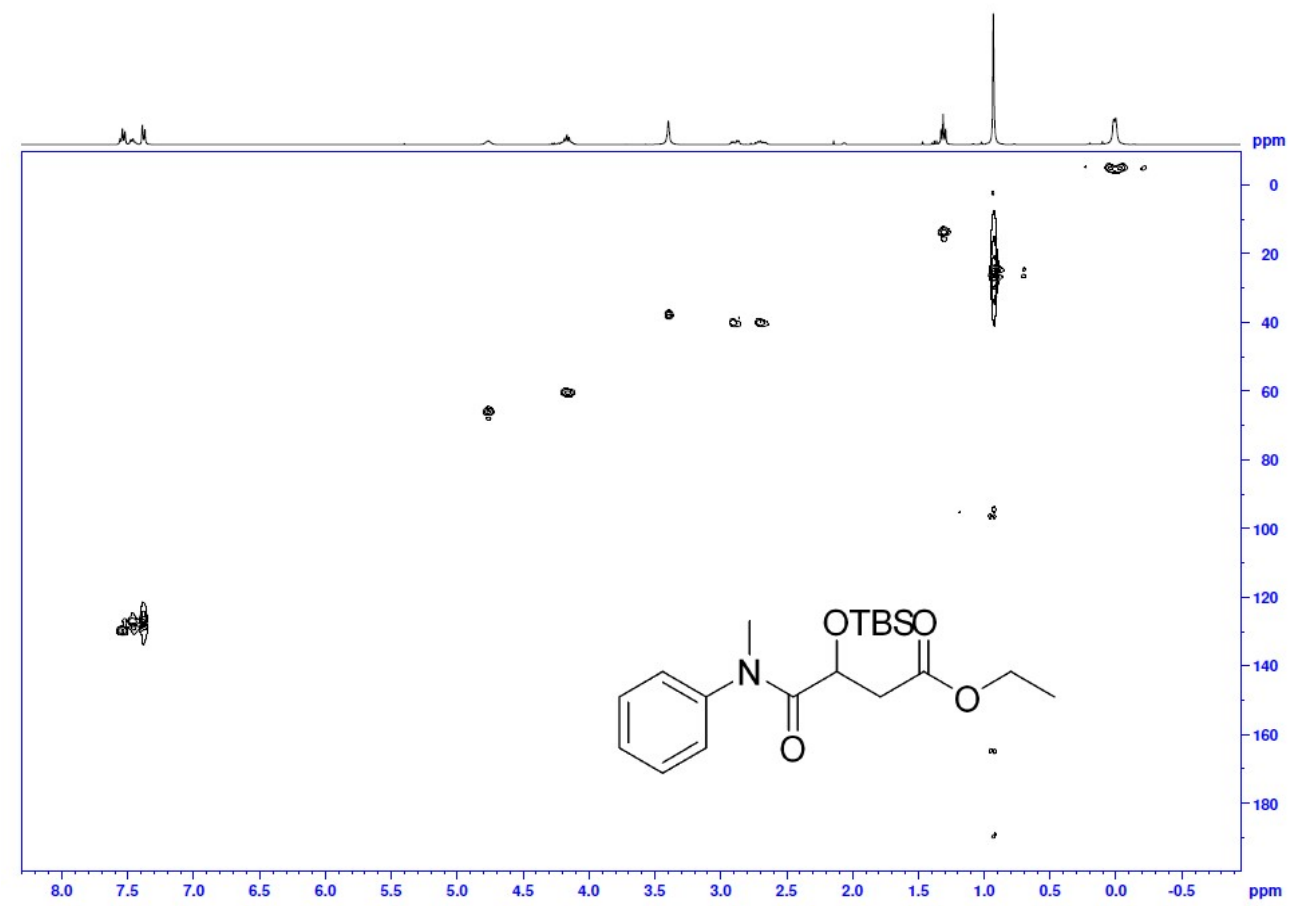


3-((tert-Butyldimethylsilyl)oxy)-4-(methyl(phenyl)amino)-4-oxobutanoic acid (racemic).<smiles>CCCCC(=O)OCC(=O)N(C)c1ccccc1</smiles>

This compound is novel. To a stirred solution of ethyl 3-((tert-butyldimethylsilyl)oxy)-4(methyl(phenyl)amino)-4-oxobutanoate $(0.500 \mathrm{~g}, 1.37 \mathrm{mmol}, 1.0 \mathrm{eq})$ in methanol $(5 \mathrm{~mL})$ was added $40 \%$ aqueous potassium hydroxide solution $(2 \mathrm{~mL})$ and the resulting reaction mixture was stirred at room temperature for $3 \mathrm{~h}$. The reaction mixture was concentrated under reduced pressure and the obtained residue was dissolved in water $(20 \mathrm{~mL})$. The aqueous layer was extracted with ethyl acetate $(20 \mathrm{~mL})$ to remove non-polar impurities. The obtained aqueous layer was acidified with $2 \mathrm{~N} \mathrm{HCl}$ to $\mathrm{pH} 2$ and then extracted with ethyl acetate $(3 \times 15 \mathrm{~mL})$. The combined organic layer was washed with water $(50 \mathrm{~mL})$ and brine $(50 \mathrm{~mL})$. The organic layer was dried over $\mathrm{MgSO}_{4}$, filtered and the filtrate was concentrated under reduced pressure to afford the product as a clear liquid $(0.394 \mathrm{~g}, 1.17 \mathrm{mmol}, 86 \%)$ which was used in the next step without further purification.

TLC: $\mathrm{R}_{\mathrm{f}}$ ca 0.2 (9:1, $\left.\mathrm{CHCl}_{3}: \mathrm{MeOH}\right), \mathrm{UV}$ active, strong $\mathrm{KMnO}_{4}$ active and PMA-reactive; HRMS (ESI) m/z: [M+Na] ${ }^{+}$Calcd for $\mathrm{C}_{17} \mathrm{H}_{27} \mathrm{NO}_{4} \mathrm{SiNa} 360.1602$; Found 360.1596 (error 2.9 ppm);

$U_{\max } 3372,2953,2928,2856,1725,1636,1593,1496,1392,1252,832,770,698 \mathrm{~cm}^{-1}$;

${ }^{1} \mathrm{H}$ NMR (400 MHz, $\mathrm{CDCl}_{3}$ ) (Rotamers) $\delta$ 7.46-7.17 (5H, m, ArH), 4.73-4.59 (0.5H, m,

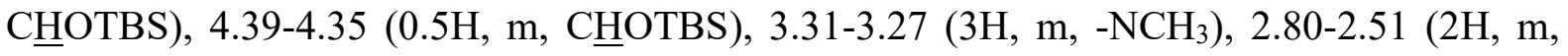
$\left.\mathrm{CH}_{\mathrm{a}} \mathrm{H}_{\mathrm{b}}\right), 0.91-0.83$ (9H, m, $\mathrm{CH}_{3}$ of tert-butyl group) 0.28-0.10 (6H, m, $\left.\left(\mathrm{CH}_{3}\right)_{2} \mathrm{Si}\right)$;

${ }^{13} \mathrm{C}$ NMR (101 MHz, $\mathrm{CDCl}_{3}$ ) (Rotamers) $\delta$ 174.0, 173.4, 172.0, 170.3, 143.4, 142.5, 130.3, $130.1,130.0,128.2,127.4,127.0,68.6,38.2$, , 37.6, 37.5, 25.8, 25.7, 25.6, 25.5, 18.1 17.3 $4.7,-4.8,-4.9,-5.0$;

$m / z(\mathrm{ESI}) 360.1\left[(\mathrm{M}+\mathrm{Na})^{+}, 100 \%\right]$. 
3-((tert-Butyldimethylsilyl)oxy)-4-(methyl(phenyl)amino)-4-oxobutanoic acid.

${ }^{1} \mathrm{H}$ NMR $\left(400 \mathrm{MHz}, \mathrm{CDCl}_{3}\right)$.

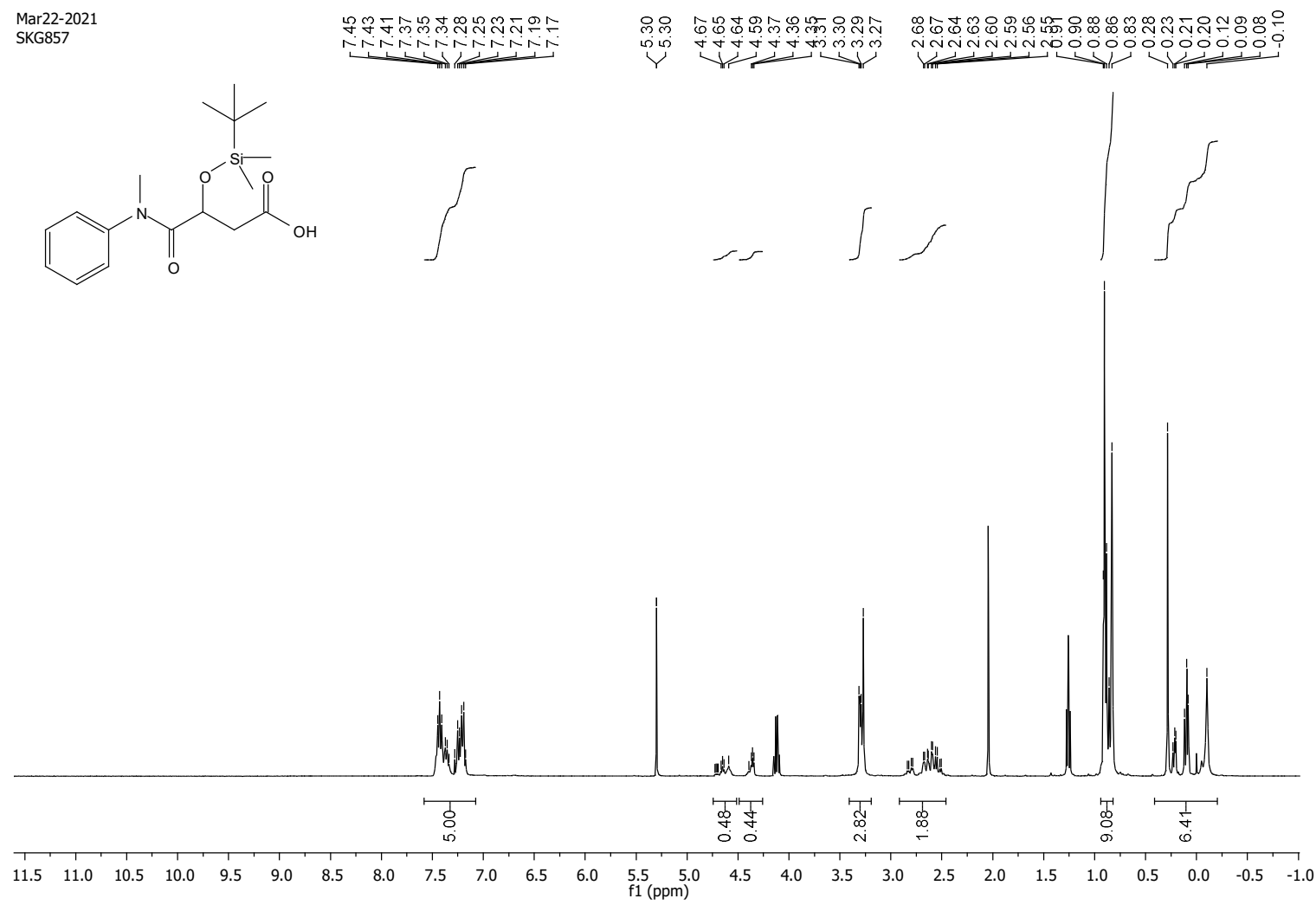

${ }^{13} \mathrm{C}$ NMR (101 MHz, $\left.\mathrm{CDCl}_{3}\right)$.

\begin{tabular}{|c|c|c|c|c|c|}
\hline $\begin{array}{l}\text { Mar22-2021 } \\
\text { SKG857 } \\
\text { C13APTlong.w.CDCl3 }\end{array}$ & 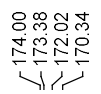 & 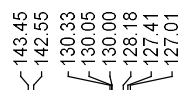 & 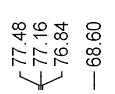 & 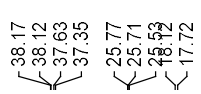 & 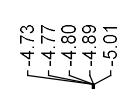 \\
\hline
\end{tabular}
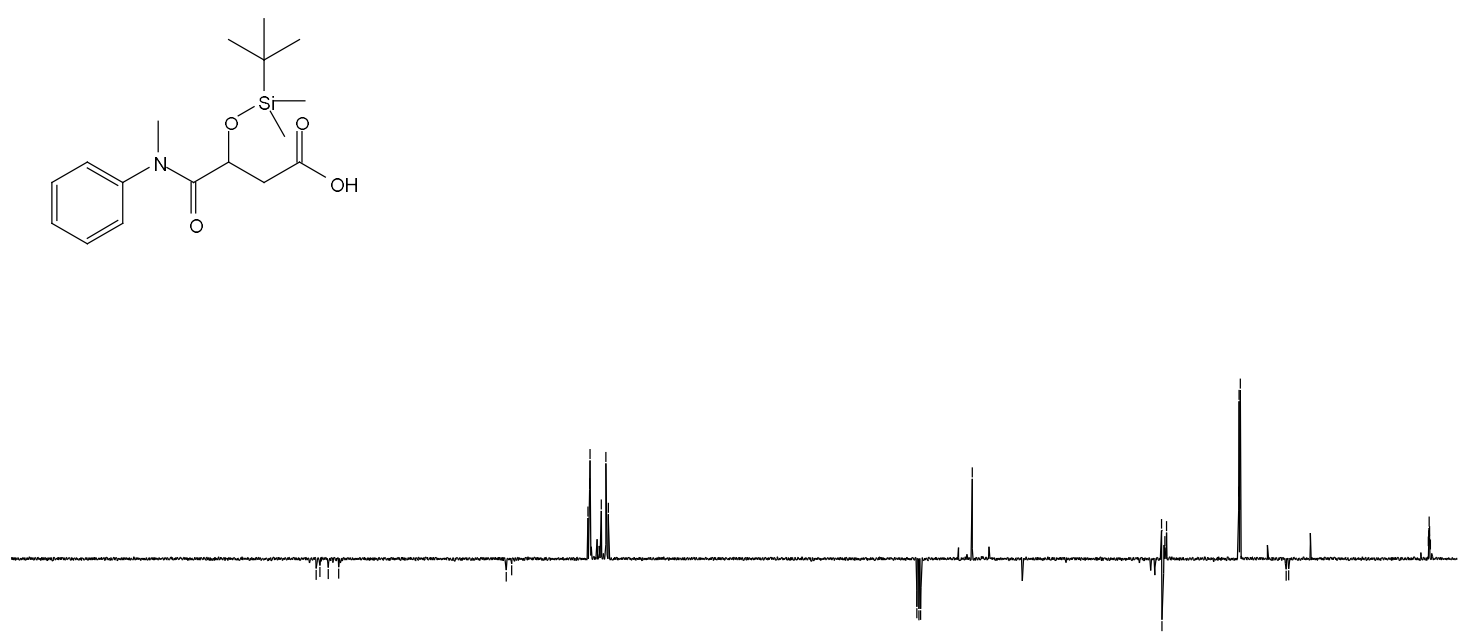

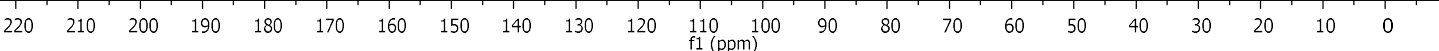


COSY.

SKG857

COSY.w CDCl3 /opt/topspin3.5pl2 SKG 4

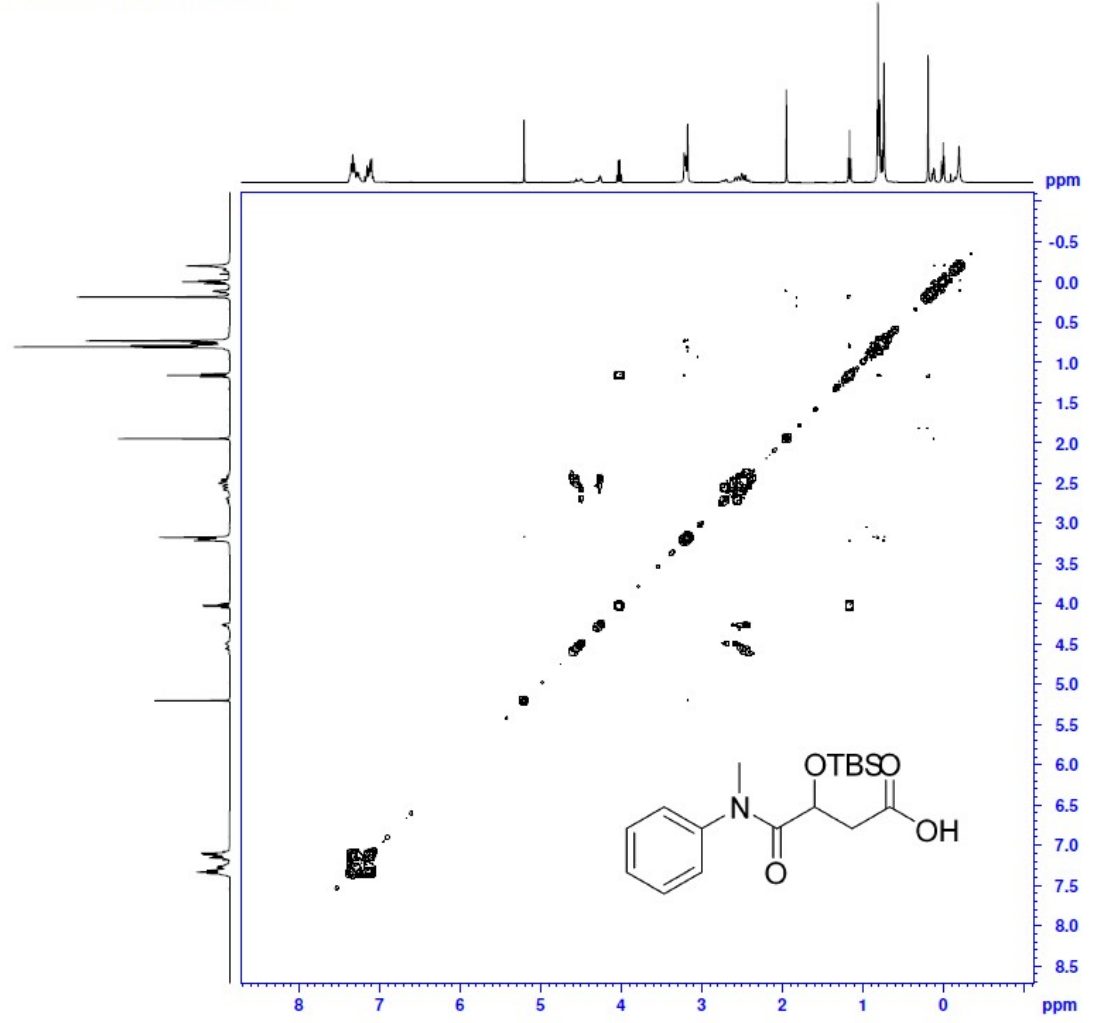

HSQC.

SKG857
HSQC.w CDCl3 /opt/topspin3.5pl2 SKG 4

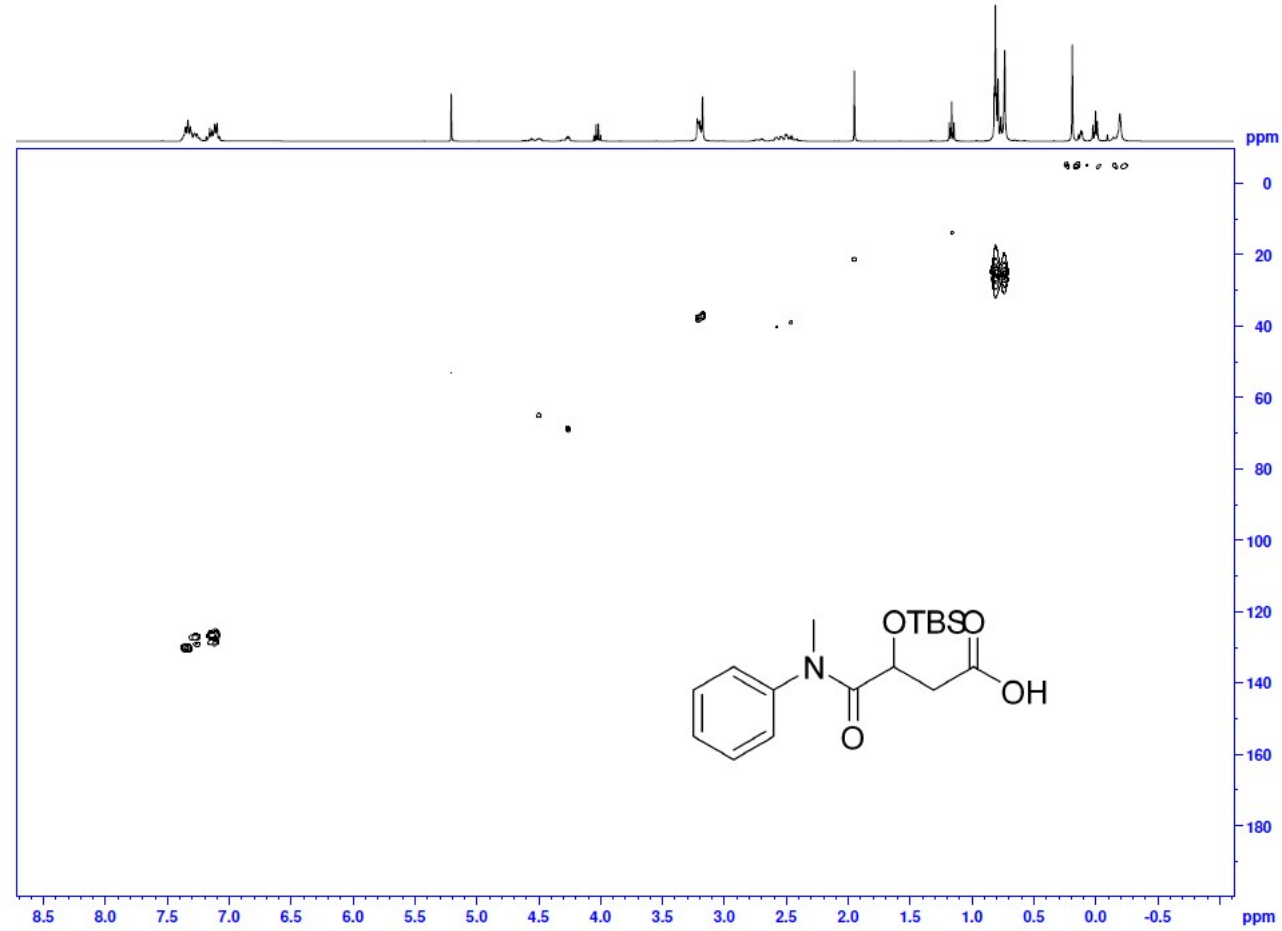


tert-Butyl

carbamate (racemic).<smiles>CN(CC(C)(C)C)C(=O)CC(O)C(=O)N(C)c1ccccc1</smiles>

This compound is novel. 3-((tert-Butyldimethylsilyl)oxy)-4-(methyl(phenyl)amino)-4oxobutanoic acid (0.100 g, $0.296 \mathrm{mmol}, 1.0 \mathrm{eq})$ was dissolved in anhydrous toluene $(3 \mathrm{~mL})$ followed by addition of triethylamine $(0.036 \mathrm{~g}, 0.356 \mathrm{mmol}, 1.2 \mathrm{eq})$ and diphenyl phosphoryl azide DPPA (0.098 g, $0.356 \mathrm{mmol}, 1.2 \mathrm{eq})$ and the resulting reaction solution was stirred at room temperature for $2 \mathrm{~h}$ during which it became yellow coloured. $t$-Butanol $(0.5 \mathrm{~mL})$ was added, and reaction mixture was heated to $90{ }^{\circ} \mathrm{C}$ at which evolution of nitrogen gas was observed. After stirring the reaction at $90{ }^{0} \mathrm{C}$ for $24 \mathrm{~h}$, the reaction solution was cooled to room temperature and quenched with saturated aqueous sodium nitrite $(10 \mathrm{~mL})$ and extracted with EtOAc $(3 \times 10 \mathrm{~mL})$. The combined organic layer was washed with Sat. $\mathrm{NaHCO}_{3}(20$ $\mathrm{mL})$, water $(50 \mathrm{~mL})$ and brine $(50 \mathrm{~mL})$. The organic layer was dried over $\mathrm{MgSO}_{4}$, filtered and the filtrate was concentrated under reduced pressure to generate crude material which was further purified by column chromatography $(20-30 \%$ EtOAc in petroleum ether) to afford the product as a clear oil $(29.6 \mathrm{mg}, 0.073 \mathrm{mmol}, 25 \%)$.

TLC: $\mathrm{R}_{\mathrm{f}}$ ca 0.3 (6:4, hexane: EtOAc), light UV active, $\mathrm{KMnO}_{4}$ active and PMA-reactive;

HRMS (ESI) m/z: [M+Na] ${ }^{+}$Calcd for $\mathrm{C}_{21} \mathrm{H}_{36} \mathrm{~N}_{2} \mathrm{O}_{4} \mathrm{SiNa} 431.2337$; Found 431.2339 (error $0.6 \mathrm{ppm})$;

$U_{\max } 3331,2953,2929,1712,1661,1495,1250,1168,1108,835,775,699 \mathrm{~cm}^{-1}$;

${ }^{1} \mathrm{H}$ NMR $\left(500 \mathrm{MHz}, \mathrm{CDCl}_{3}\right) \delta$ 7.44-7.41 (2H, m, ArH), 7.36-7.35 (1H, m, ArH), 7.26-7.22 $(2 \mathrm{H}, \mathrm{m}, \mathrm{ArH}), 4.93(1 \mathrm{H}, \mathrm{s}, \mathrm{N} \underline{\mathrm{HBoc}}), 4.28(1 \mathrm{H}, \mathrm{s}, \mathrm{C} \underline{\mathrm{HOTBS}}), 3.27\left(5 \mathrm{H}, \mathrm{s},-\mathrm{NCH}_{3}+\mathrm{CH}_{\mathrm{a}} \mathrm{H}_{\mathrm{b}}\right)$, $1.42\left(9 \mathrm{H}, \mathrm{s}, \mathrm{CH}_{3}\right.$ of tert-butyl group of $\left.\mathrm{NHBoc}\right), 0.83\left(9 \mathrm{H}, \mathrm{s}, \mathrm{CH}_{3}\right.$ of tert-butyl group), -0.08 $\left(6 \mathrm{H}, \mathrm{s},\left(\mathrm{CH}_{3}\right)_{2} \mathrm{Si}\right)$;

${ }^{13} \mathrm{C}$ NMR $\left(126 \mathrm{MHz}, \mathrm{CDCl}_{3}\right) \delta 171.1,156.0,142.9,129.9,128.3,127.6,79.3,68.3,44.4$, 37.9, 28.5, 25.9, 18.3. -4.6, -4.7;

$m / z(\mathrm{ESI}) 431.2\left[(\mathrm{M}+\mathrm{Na})^{+}, 100 \%\right]$. 
tert-Butyl

carbamate.

${ }^{1} \mathrm{H} \mathrm{NMR}\left(500 \mathrm{MHz}, \mathrm{CDCl}_{3}\right)$.

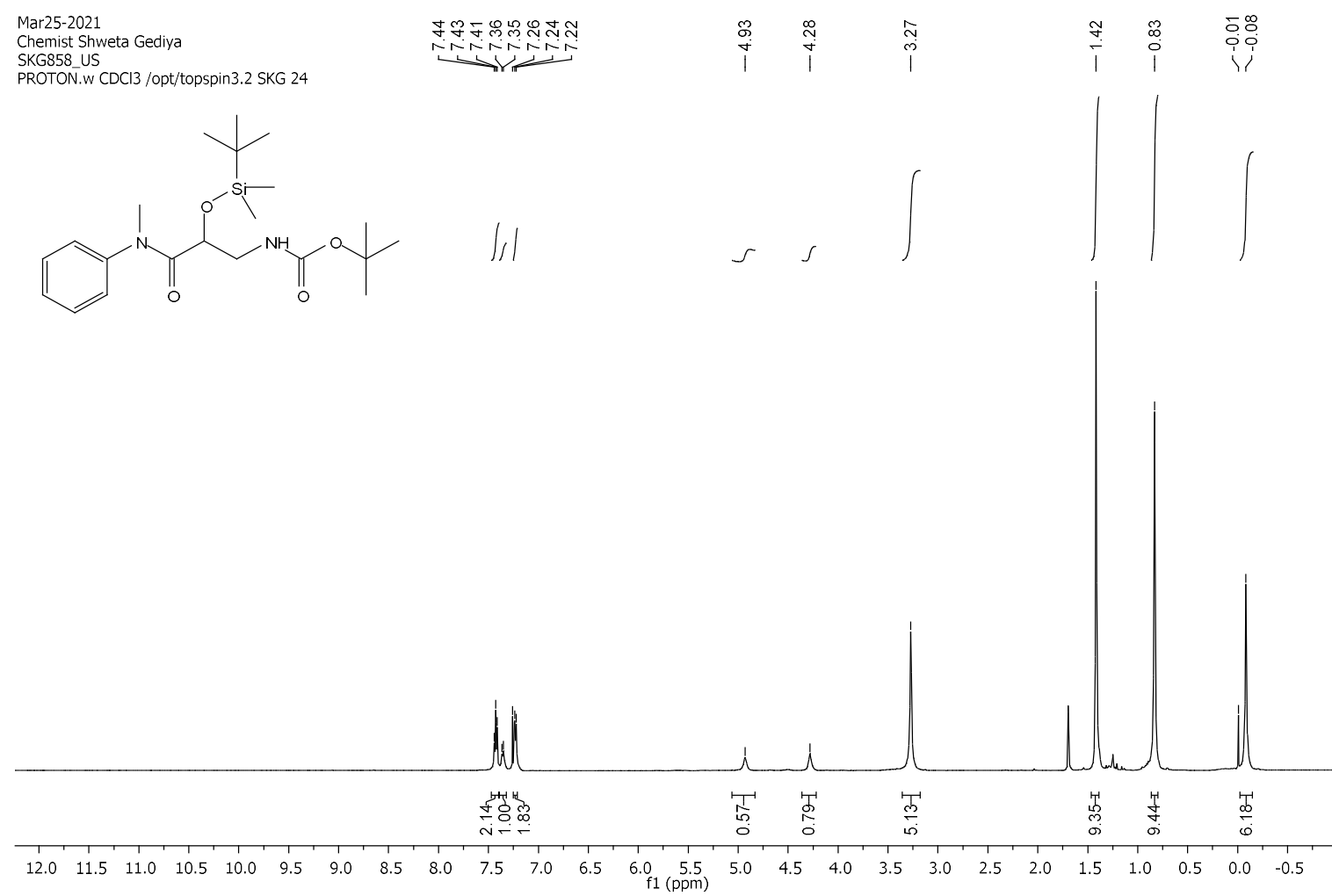

${ }^{13} \mathrm{C}$ NMR $\left(126 \mathrm{MHz}, \mathrm{CDCl}_{3}\right)$.

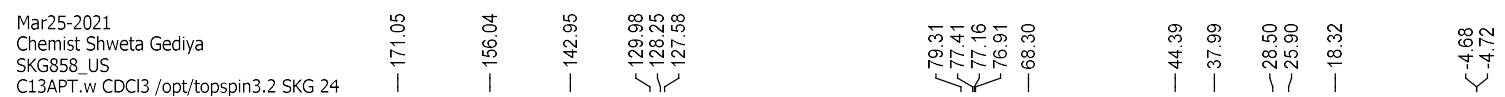<smiles>CN(C(=O)C1CN(C(=O)OC(C)(C)C)CC(C)(C(C)(C)C)C1)c1ccccc1</smiles>

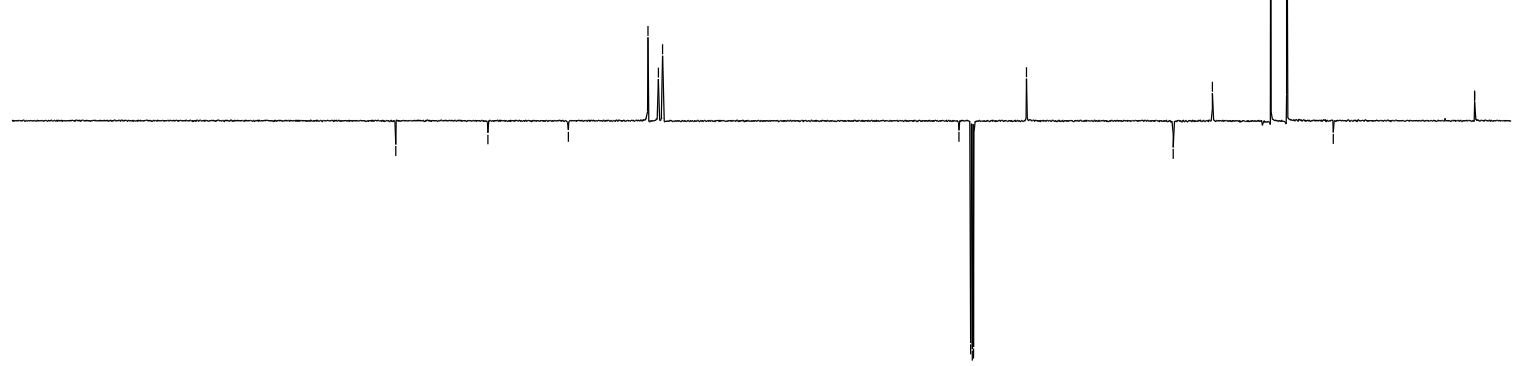

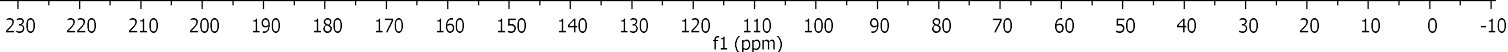


COSY.

Chemist Shweta Gediya

COSY ${ }^{2} \mathrm{CDCl}_{3} /$ opt/topspin3.2 SKG 24

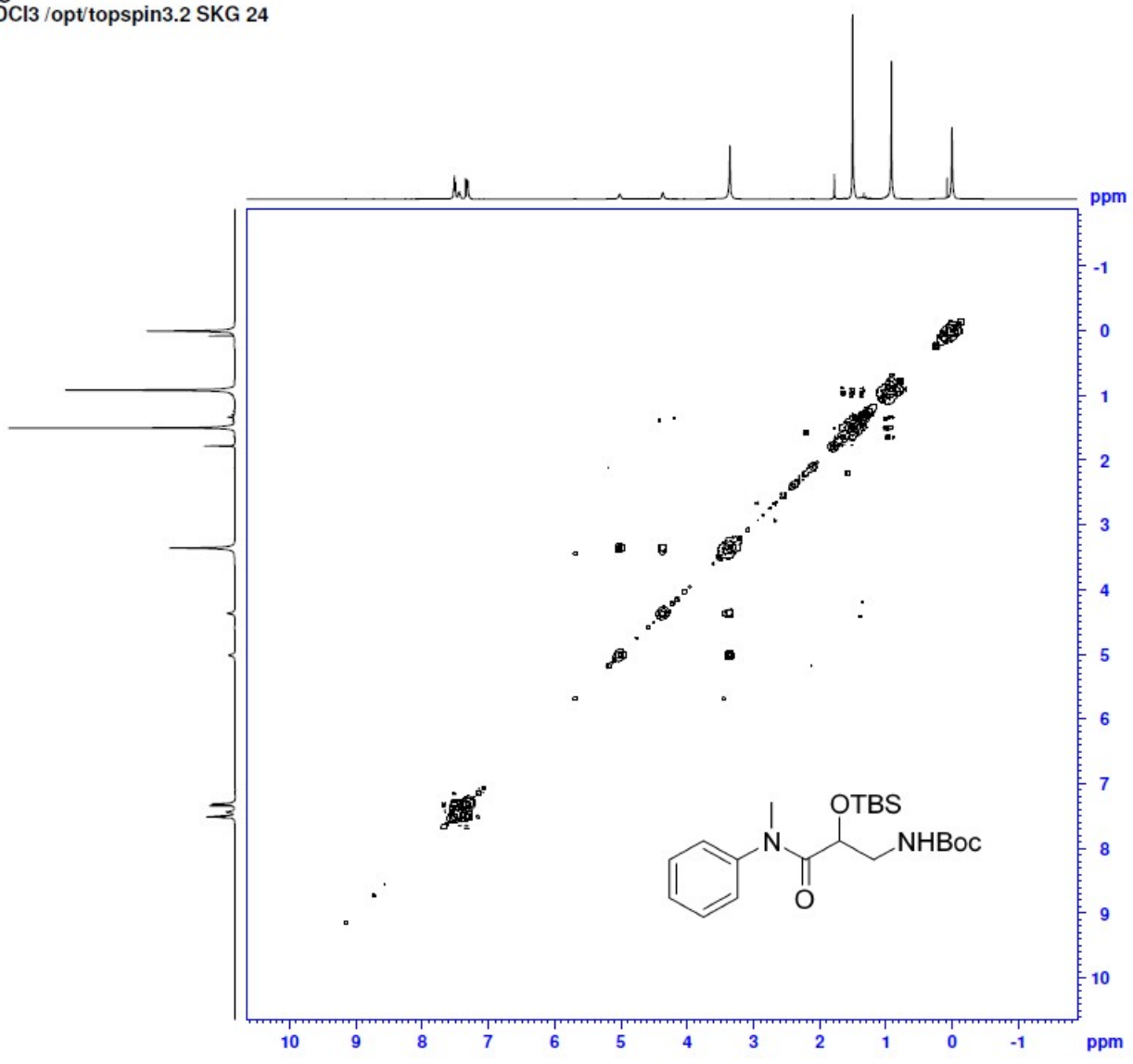

HSQC.

Chemist Shweta Gediya

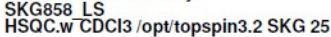

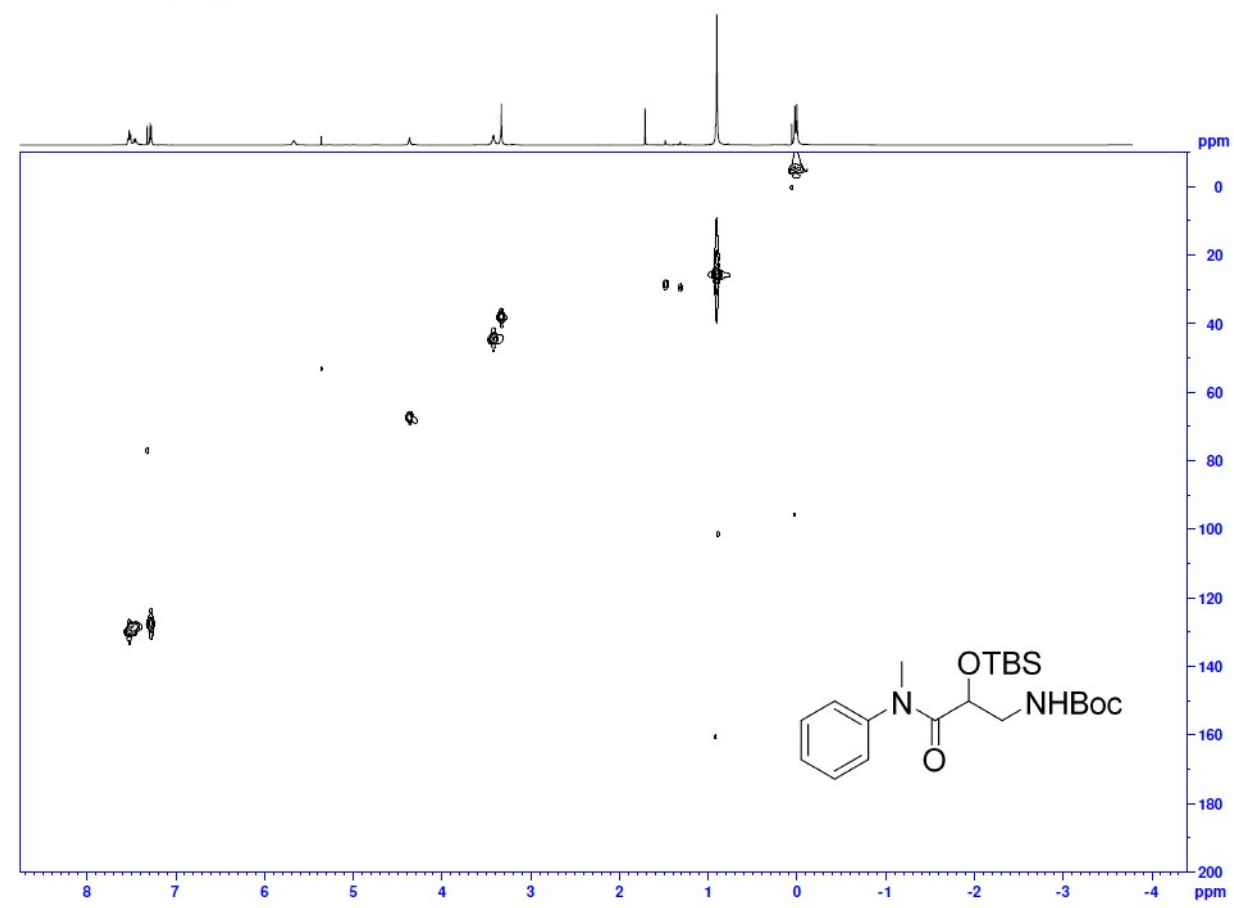


Ethyl (R)-4-(benzyl(methyl)amino)-3-((tert-butyldimethylsilyl)oxy)-4-oxobutanoate.<smiles>CCOC(=O)CC(OCC)C(=O)N(C)Cc1ccccc1</smiles>

This compound is novel. To a stirred solution of ethyl (R)-4-(benzyl(methyl)amino)-3hydroxy-4-oxobutanoate 39 (0.500 g, $1.87 \mathrm{mmol}, 1.0 \mathrm{eq})$ in DMF (10 mL) was added tertbutyldimethylsilyl chloride (TBDMSCl) $(0.561 \mathrm{~g}, 3.74 \mathrm{mmol}, 2.0 \mathrm{eq})$ and imidazole $(0.318 \mathrm{~g}$, $4.67 \mathrm{mmol}, 2.5 \mathrm{eq}$ ) and the resulting reaction mixture was stirred at room temperature for $3 \mathrm{~h}$. The reaction mixture was quenched with ice cold water $(30 \mathrm{~mL})$ and extracted with ethyl acetate $(3 \times 20 \mathrm{~mL})$. The combined organic layer was washed with brine $(50 \mathrm{~mL})$. The organic layer was dried over $\mathrm{MgSO}_{4}$, filtered and the filtrate was concentrated under reduced pressure to generate crude material which was further purified by column chromatography (5$10 \%$ EtOAc in petroleum ether) to afford the product as a clear oil $(0.525 \mathrm{~g}, 1.39 \mathrm{mmol}$, $74 \%)$.

TLC: $\mathrm{R}_{\mathrm{f}}$ ca 0.6 (7:3, hexane: EtOAc), UV active, strong $\mathrm{KMnO}_{4}$ active and PMA-reactive;

HRMS (ESI) m/z: $[\mathrm{M}+\mathrm{Na}]^{+}$Calcd for $\mathrm{C}_{20} \mathrm{H}_{33} \mathrm{NO}_{4} \mathrm{SiNa} 402.2071$; Found 402.2066 (error 1.2 ppm);

$U_{\max } 2955,2930,2856,1732,1652,1252,1173,1095,1026,831,777 \mathrm{~cm}^{-1}$;

Enantiomeric excess determined by HPLC analysis (Chiralpak IC, $250 \mathrm{~mm} \times 4.6 \mathrm{~mm}$ column, hexane: $\operatorname{PrOH} 90: 10,1 \mathrm{~mL} / \mathrm{min}, \lambda=210 \mathrm{~nm}, \mathrm{~T}=25{ }^{\circ} \mathrm{C}$ ), $R$-enantiomer $13.2 \mathrm{~min}, S$ enantiomer $24.1 \mathrm{~min} .88 \%$ ee $(R),[\alpha]_{\mathrm{D}}^{22}=+111.5\left(\mathrm{c}=0.1, \mathrm{CHCl}_{3}\right)$;

${ }^{1} \mathrm{H}$ NMR (500 MHz, $\mathrm{CDCl}_{3}$ ) (Rotamers 3:2) $\delta$ 7.34-7.18 (5H, m, ArH), 4.97-4.94 (1H, m, CㅂTBS), $4.69\left(0.8 \mathrm{H}, \mathrm{s}, \mathrm{CH}_{2} \mathrm{Ph}\right), 4.53\left(1.2 \mathrm{H}, \mathrm{s}, \mathrm{CH}_{2} \mathrm{Ph}\right), 4.15-4.04\left(2 \mathrm{H}, \mathrm{m}, \mathrm{CH}_{2}\right.$ of ester group), $3.00\left(1.8 \mathrm{H}, \mathrm{s}, \mathrm{NCH}_{3}\right), 2.84-2.78\left(2.2 \mathrm{H}, \mathrm{s}, \mathrm{NCH}_{3}(1.2 \mathrm{H})+\mathrm{C}_{\mathrm{H}} \mathrm{H}_{\mathrm{b}}(1 \mathrm{H})\right), 2.68-2.55$ $\left(1 \mathrm{H}, \mathrm{m}, \mathrm{CH}_{\mathrm{a}} \underline{\mathrm{H}}_{\mathrm{b}}\right), 1.24-1.19\left(3 \mathrm{H}, \mathrm{m}, \mathrm{CH}_{3}\right.$ of ester group), $0.82\left(9 \mathrm{H}, \mathrm{s}, \mathrm{CH}_{3}\right.$ of tert-butyl group), $0.05\left(2 \mathrm{H}, \mathrm{s},\left(\mathrm{CH}_{3}\right)_{2} \mathrm{Si}\right), 0.03\left(3 \mathrm{H}, \mathrm{s},\left(\mathrm{CH}_{3}\right)_{2} \mathrm{Si}\right), 0.00\left(1 \mathrm{H}, \mathrm{s},\left(\mathrm{CH}_{3}\right)_{2} \mathrm{Si}\right)$;

${ }^{13} \mathrm{C}$ NMR $\left(126 \mathrm{MHz}, \mathrm{CDCl}_{3}\right)$ (Rotamers 3:2) $\delta 171.9,171.6,171.4,170.8,136.9,136.7$, 128.9, 128.6, 128.2, 127.7, 127.5, 126.8, 69.6, 69.5, 60.8, 60.7, 52.8, 51.5, 45.0, 40.1, 39.8, 34.4, 34.2, 25.7, 18.2, 14.2, -4.3, -4.4, -4.8;

$m / z(\mathrm{ESI}) 402.1\left[(\mathrm{M}+\mathrm{Na})^{+}, 100 \%\right]$. 
Ethyl-4-(benzyl(methyl)amino)-3-((tert-butyldimethylsilyl)oxy)-4-oxobutanoate. ${ }^{1} \mathrm{H}$ NMR $\left(500 \mathrm{MHz}, \mathrm{CDCl}_{3}\right)$.

Apr26-2021

Chemist Shweta Gediy

SKG900

PROTON.w CDCl3 /opt/topspin3.2 SKG 14
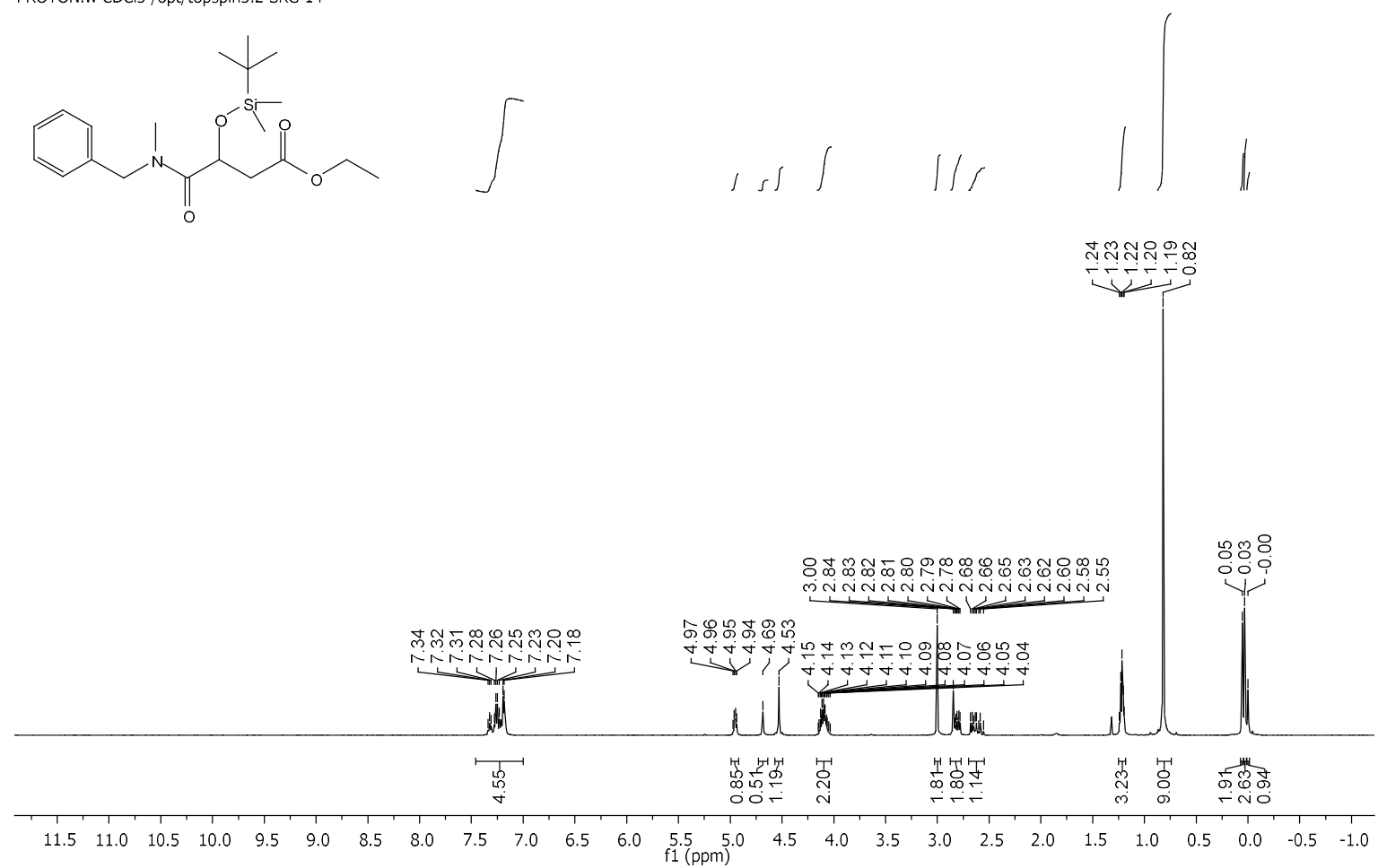

${ }^{13} \mathrm{C}$ NMR (126 MHz, $\left.\mathrm{CDCl}_{3}\right)$.

Apr26-2021
SKG900
Shweta Gediya
C13APTlong.w.CDCl3
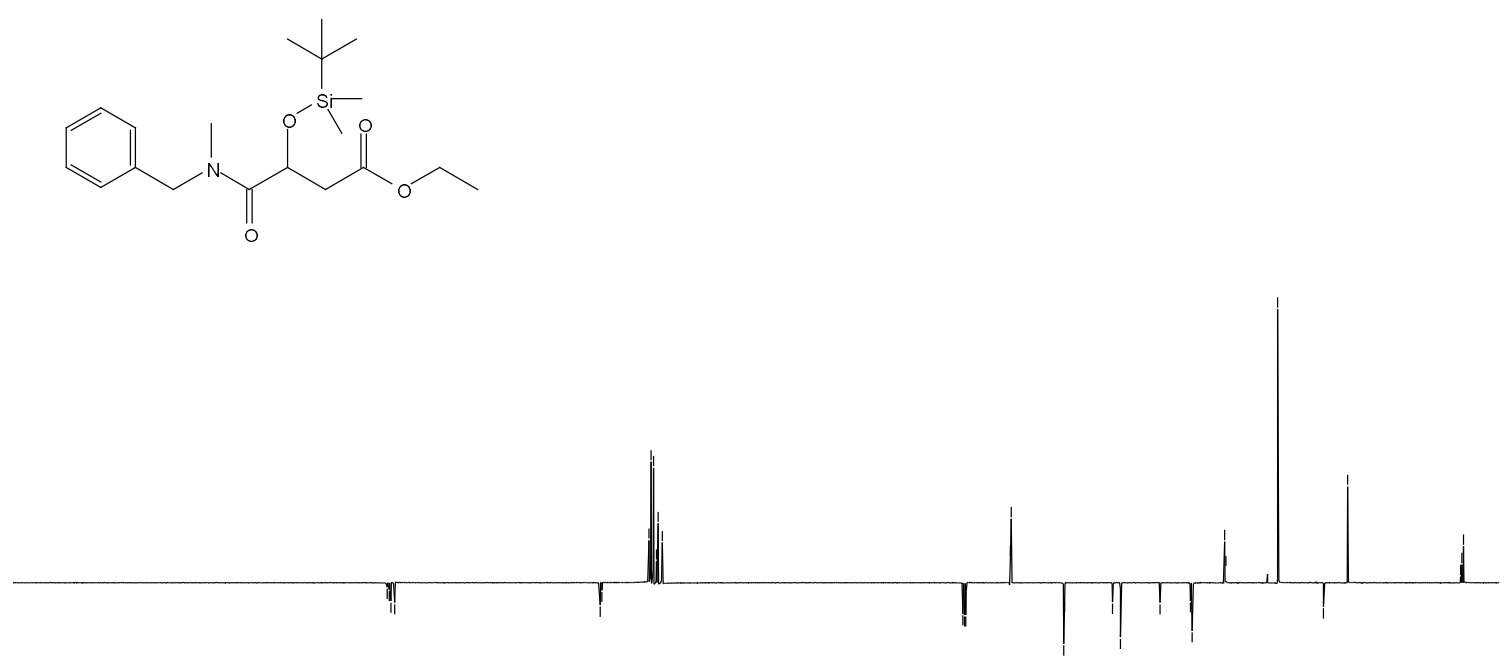

$\begin{array}{llllllllllllllllllllllllllllllllllll}230 & 220 & 210 & 200 & 190 & 180 & 170 & 160 & 150 & 140 & 130 & 120 & 110 & 100 & 90 & 80 & 70 & 60 & 50 & 40 & 30 & 20 & 10 & 0 & -10\end{array}$ 
COSY.

Chemist Shweta Gediya

COSY.w CDCl3/opt/topspin3.2 SKG 14
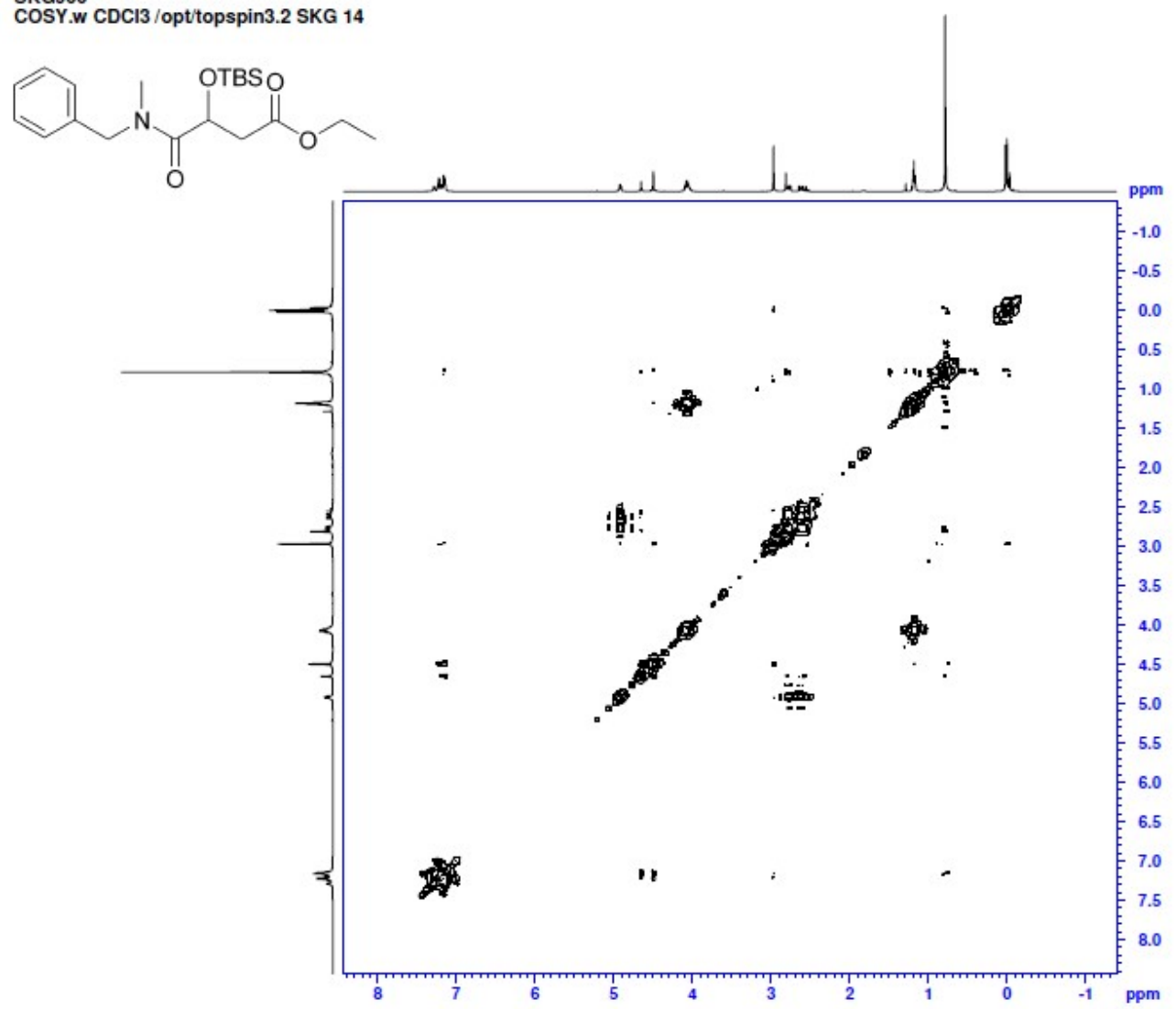

HSQC.

HSQC.w CDCl3 /opt/topspin3.2 SKG 14

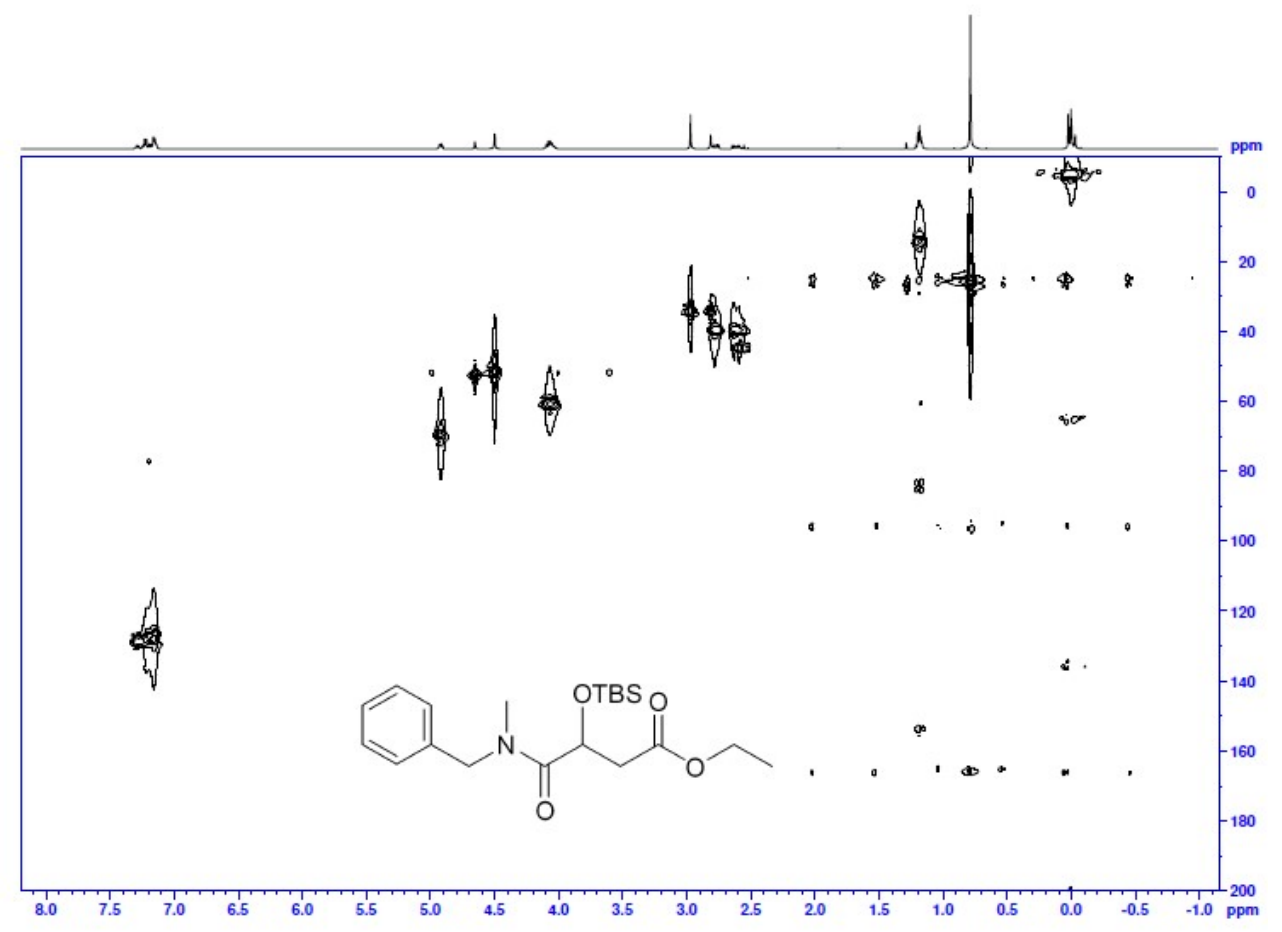

S468 


\section{Chiral HPLC of racemic standard.}

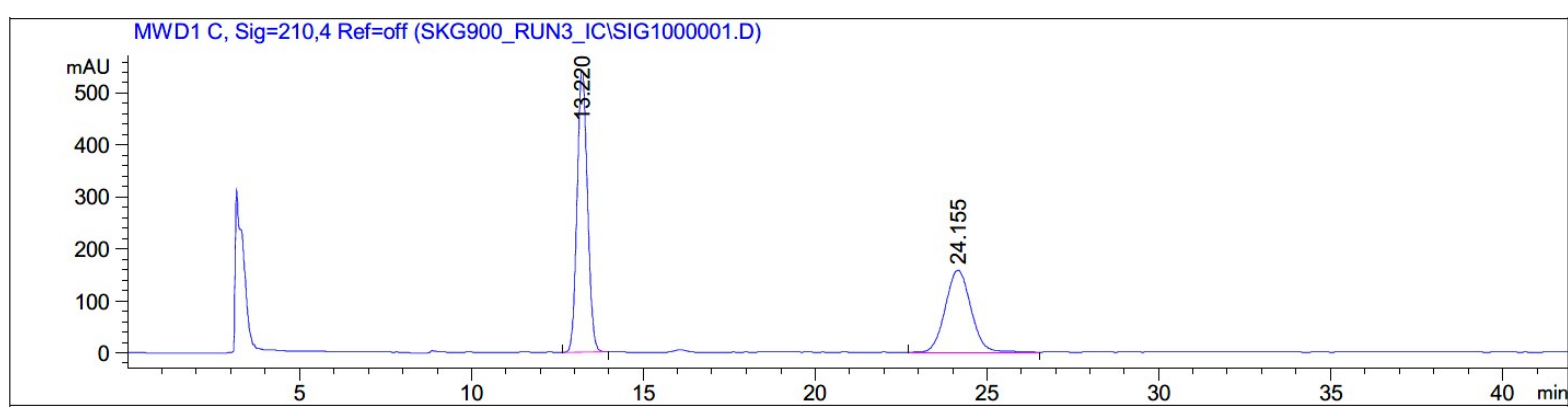

Signal 2: MWD1 C, Sig=210,4 Ref=off

\begin{tabular}{|c|c|c|c|c|c|c|}
\hline $\begin{array}{c}\text { Peak } \\
\#\end{array}$ & $\begin{array}{c}\text { RetTime } \\
\text { [min] }\end{array}$ & Type & $\begin{array}{l}\text { Width } \\
\text { [min] }\end{array}$ & $\begin{array}{c}\text { Area } \\
{\left[m A{ }^{\star} s\right]}\end{array}$ & $\begin{array}{l}\text { Height } \\
\text { [mAU] }\end{array}$ & $\begin{array}{c}\text { Area } \\
\quad \%\end{array}$ \\
\hline$\ldots$ & -- & & 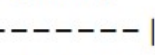 & $\mid---------$ & --- & ---1 \\
\hline 1 & 13.220 & $\mathrm{BB}$ & 0.3262 & $1.13641 \mathrm{e} 4$ & 542.88165 & 58.2566 \\
\hline 2 & 24.155 & $\mathrm{BB}$ & 0.7994 & 8142.88525 & 157.96262 & 41.7434 \\
\hline
\end{tabular}

Chiral HPLC of AH product. $88 \%$ ee.

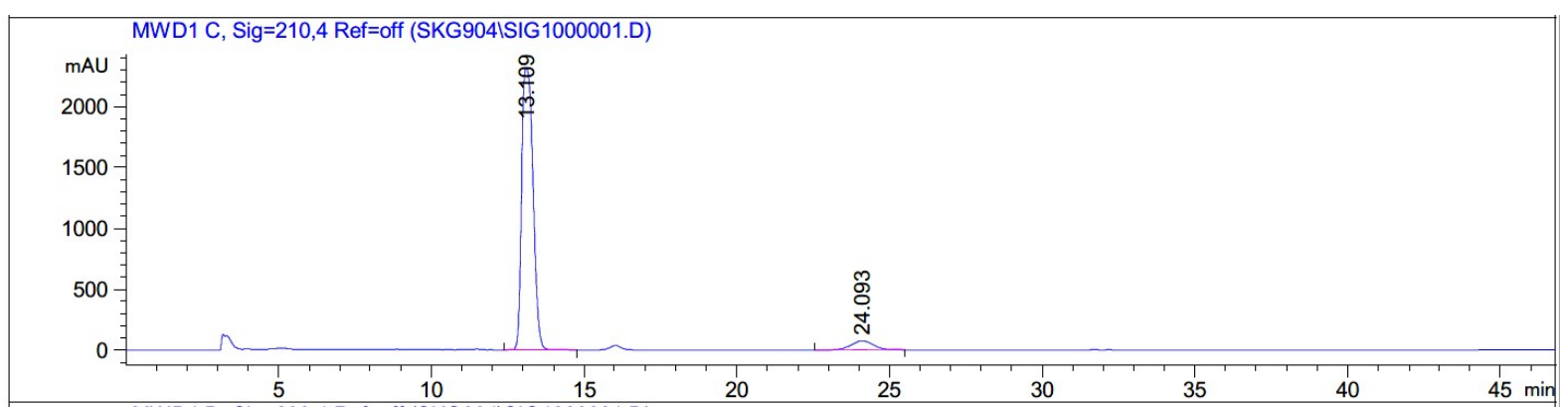

Signal 2: MWD1 C, Sig=210,4 Ref=off

\begin{tabular}{|c|c|c|c|c|c|c|}
\hline $\begin{array}{c}\text { eak } \\
\#\end{array}$ & $\begin{array}{c}\text { RetTime } \\
\text { [min] }\end{array}$ & Type & $\begin{array}{l}\text { Width } \\
\text { [min] }\end{array}$ & $\begin{array}{c}\text { Area } \\
{\left[\mathrm{mAU}^{\star} \mathrm{s}\right]}\end{array}$ & $\begin{array}{l}\text { Height } \\
\text { [mAU] }\end{array}$ & $\begin{array}{c}\text { Area } \\
\quad \%\end{array}$ \\
\hline & & & & 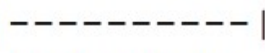 & $\mid---$ & -- \\
\hline 1 & & & & 5.9 & 52 & 12 \\
\hline 2 & 24.093 & B & 18 & 3741.39282 & 74.13622 & 9088 \\
\hline
\end{tabular}


(R)-4-(Benzyl(methyl)amino)-3-((tert-butyldimethylsilyl)oxy)-4-oxobutanoic acid 56.<smiles>CN(Cc1ccccc1)C(=O)C([OH2+])CC(=O)O</smiles>

This compound is novel. To a stirred solution of ethyl (R)-4-(benzyl(methyl)amino)-3-((tertbutyldimethylsilyl)oxy)-4-oxobutanoate $(0.470 \mathrm{~g}, 1.34 \mathrm{mmol}, 1.0 \mathrm{eq})$ in methanol $(10 \mathrm{~mL})$ was added $40 \%$ aqueous potassium hydroxide solution $(2 \mathrm{~mL})$ and the resulting reaction mixture was stirred at room temperature for $3 \mathrm{~h}$. The reaction mixture was concentrated under reduced pressure and the residue was dissolved in water $(10 \mathrm{~mL})$. The aqueous layer was extracted with ethyl acetate $(20 \mathrm{~mL})$ to remove non-polar impurities. The aqueous layer was acidified with $2 \mathrm{~N} \mathrm{HCl}$ to $\mathrm{pH} 2$ and then extracted with ethyl acetate $(3 \times 15 \mathrm{~mL})$. The combined organic layer was washed with water $(50 \mathrm{~mL})$ and brine $(50 \mathrm{~mL})$. The organic layer was dried over $\mathrm{MgSO}_{4}$, filtered and the filtrate was concentrated under reduced pressure to afford 56 as a clear oil $(0.337 \mathrm{~g}, 0.960 \mathrm{mmol}, 72 \%)$ which was used in next step without further purification.

TLC: $\mathrm{R}_{\mathrm{f}}$ ca 0.2 (9:1, $\left.\mathrm{CHCl}_{3}: \mathrm{MeOH}\right), \mathrm{UV}$ active, strong $\mathrm{KMnO}_{4}$ active and PMA-reactive; HRMS (ESI) m/z: [M+Na $]^{+}$Calcd for $\mathrm{C}_{18} \mathrm{H}_{29} \mathrm{NO}_{4} \mathrm{SiNa} 374.1758$; Found 374.1760 (error -0.5 ppm);

$U_{\max } 3393,2953,2929,2857,1716,1627,1402,1252,1174,1082,833,732,697 \mathrm{~cm}^{-1}$;

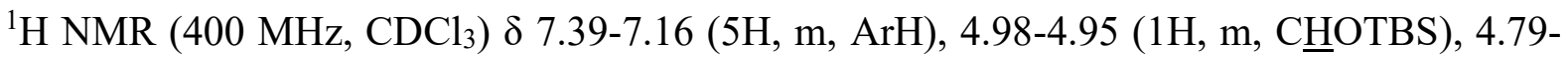
$4.52\left(2 \mathrm{H}, \mathrm{m}, \mathrm{CH}_{2} \mathrm{Ph}\right), 3.04\left(2 \mathrm{H}, \mathrm{s}, \mathrm{NCH}_{3}\right), 3.02-2.66\left(3 \mathrm{H}, \mathrm{m},-\mathrm{NCH}_{3}(1 \mathrm{H})+\mathrm{CH}_{\mathrm{a}} \mathrm{H}_{\mathrm{b}}(2 \mathrm{H})\right)$, 0.91-0.87 (9H, m, $\mathrm{CH}_{3}$ of tert-butyl group), 0.10-0.07 (6H, m, $\left.\left(\mathrm{CH}_{3}\right)_{2} \mathrm{Si}\right)$;

${ }^{13} \mathrm{C}$ NMR $\left(101 \mathrm{MHz}, \mathrm{CDCl}_{3}\right) \delta 174.4,171.9,136.6,129.1,128.8,128.2,127.7,126.7,68.6$, $53.0,51.7,40.0,39.8,34.7,34.6,25.7,18.2,-4.5$;

$\mathrm{m} / z(\mathrm{ESI}) 374.2\left[(\mathrm{M}+\mathrm{Na})^{+}, 100 \%\right]$. 
4-(Benzyl(methyl)amino)-3-((tert-butyldimethylsilyl)oxy)-4-oxobutanoic acid 56. ${ }^{1} \mathrm{H}$ NMR $\left(400 \mathrm{MHz}, \mathrm{CDCl}_{3}\right)$.

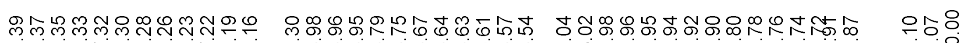
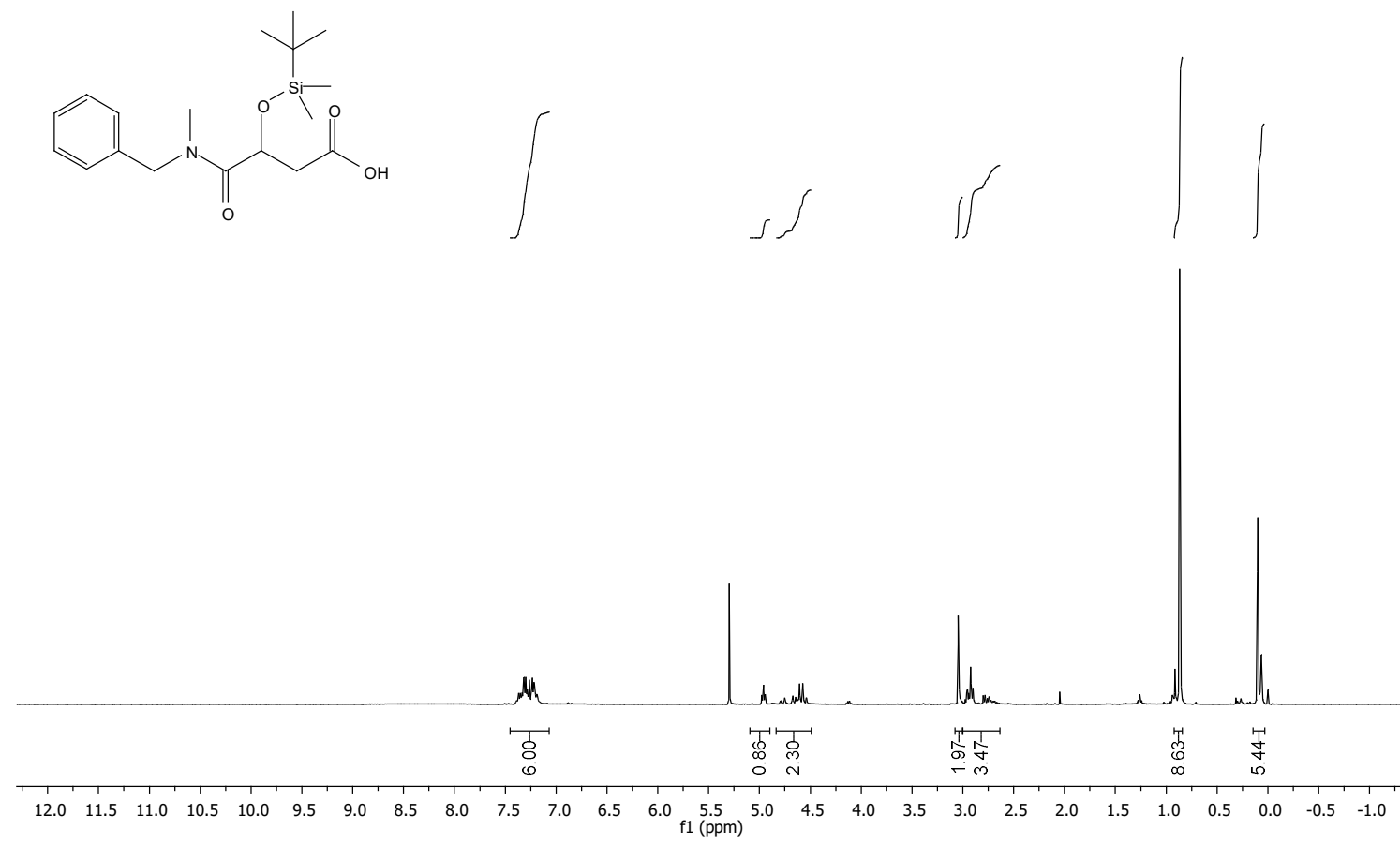

${ }^{13} \mathrm{C}$ NMR (101 MHz, $\left.\mathrm{CDCl}_{3}\right)$.

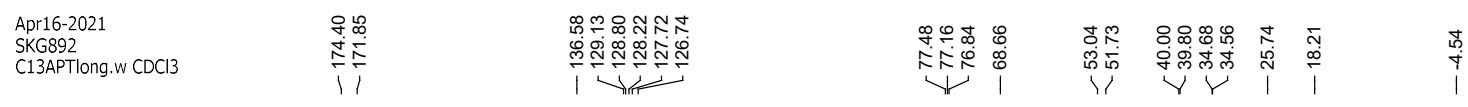
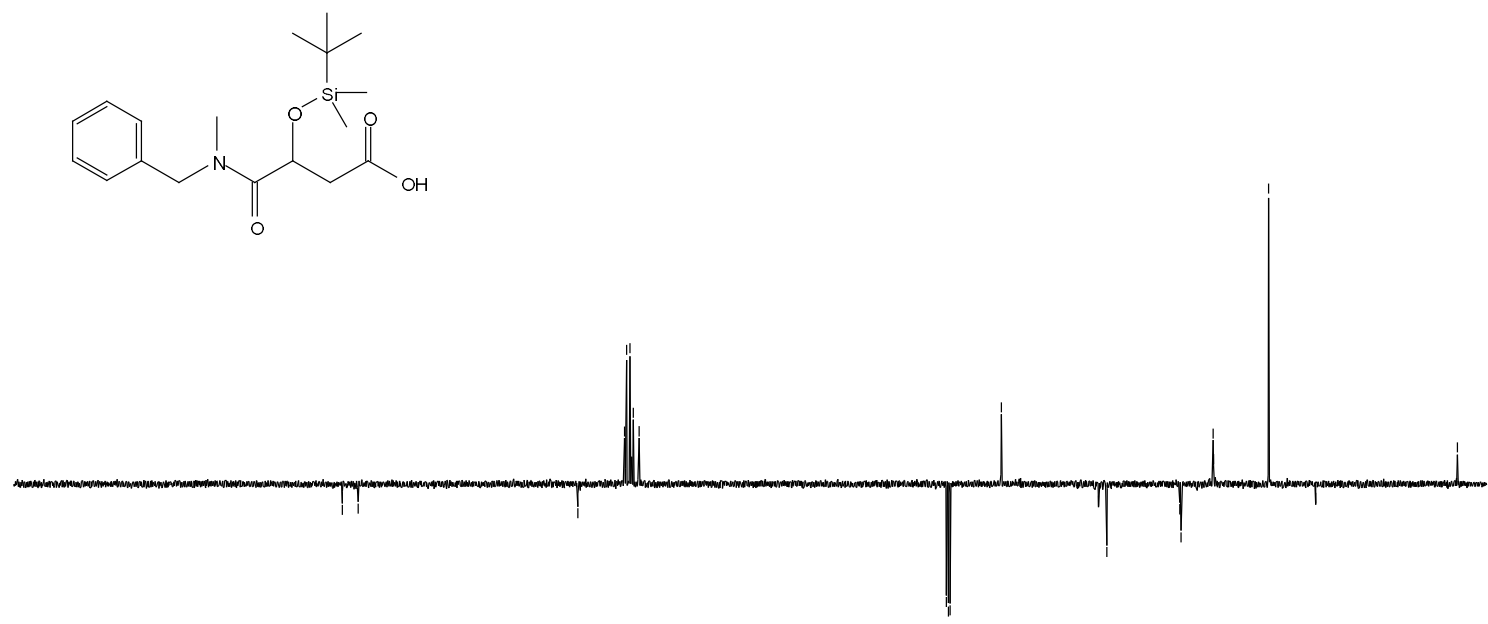

$\begin{array}{llllllllllllllllllllllllllllll}220 & 210 & 200 & 190 & 180 & 170 & 160 & 150 & 140 & 130 & 120 & 110 & 100 & 90 & 80 & 70 & 60 & 50 & 40 & 30 & 20 & 10 & 0\end{array}$ 


\section{COSY}

SKG892

COSY.w CDCl3 /opt/topspin3.5pl2 SKG 22

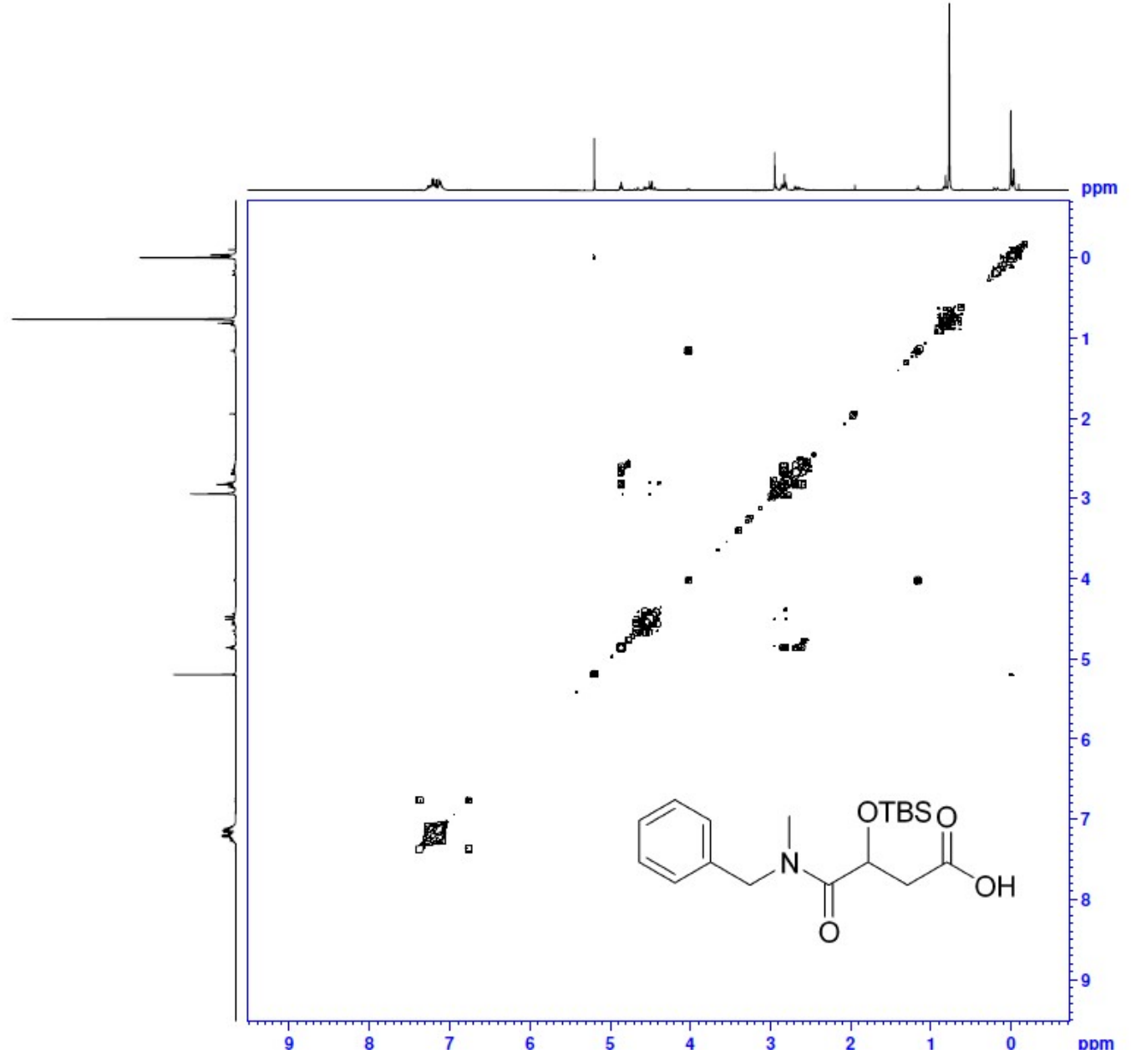


tert-Butyl-(R)-(3-(benzyl(methyl)amino)-2-((tert-butyldimethylsilyl)oxy)-3-oxopropyl) carbamate 57.<smiles>CN(Cc1ccccc1)C(=O)C(CNC(=O)O)O[SnH3]</smiles>

This compound is novel. (R)-4-(benzyl(methyl)amino)-3-((tert-butyldimethylsilyl)oxy)-4oxobutanoic acid 56 (0.150 g, $0.427 \mathrm{mmol}, 1.0 \mathrm{eq})$ was dissolved in anhydrous toluene (5 $\mathrm{mL})$ followed by addition of triethylamine $(0.052 \mathrm{~g}, 0.512 \mathrm{mmol}, 1.2 \mathrm{eq})$ and diphenyl phosphoryl azide DPPA (0.141 g, $0.512 \mathrm{mmol}, 1.2 \mathrm{eq})$ and the resulting reaction solution was stirred at room temperature for $2 \mathrm{~h}$ during which the reaction mixture became yellow coloured. $t$-Butanol $(1 \mathrm{~mL})$ was added, and reaction mixture was heated to $90{ }^{0} \mathrm{C}$ at which evolution of nitrogen gas was observed. After stirring the reaction at $90{ }^{0} \mathrm{C}$ for $24 \mathrm{~h}$, the reaction solution was cooled to room temperature and quenched with saturated aqueous sodium nitrite $(10 \mathrm{~mL})$ and extracted with EtOAc $(3 \times 10 \mathrm{~mL})$. The combined organic layer was washed with Sat. $\mathrm{NaHCO}_{3}(20 \mathrm{~mL})$, water $(50 \mathrm{~mL})$ and brine $(50 \mathrm{~mL})$. The organic layer was dried over $\mathrm{MgSO}_{4}$, filtered and the filtrate was concentrated under reduced pressure to generate crude material which was further purified by column chromatography $(20-30 \%$ EtOAc in petroleum ether) to afford 57 as a clear oil $(68.9 \mathrm{mg}, 0.163 \mathrm{mmol}, 38 \%)$.

TLC: $\mathrm{R}_{\mathrm{f}}$ ca 0.5 (7:3, hexane: EtOAc), $\mathrm{UV}$ active, $\mathrm{KMnO}_{4}$ active and PMA-reactive; HRMS (ESI) m/z: [M+Na] ${ }^{+}$Calcd for $\mathrm{C}_{22} \mathrm{H}_{38} \mathrm{~N}_{2} \mathrm{O}_{4} \mathrm{SiNa} 445.2493$; Found 445.2487 (error 1.5 ppm);

$U_{\max } 2952,2929,2856,1704,1647,1495,1364,1250,1168,1097,833,777 \mathrm{~cm}^{-1}$;

Enantiomeric excess determined by HPLC analysis (Chiralpak IG, $250 \mathrm{~mm} \times 4.6 \mathrm{~mm}$ column, hexane: iPrOH 90:10, $1 \mathrm{~mL} / \mathrm{min}, \lambda=210 \mathrm{~nm}, \mathrm{~T}=25^{\circ} \mathrm{C}$ ), $S$-enantiomer $7.3 \mathrm{~min}, R$ enantiomer $9.0 \min .89 \%$ ee $(R),[\alpha]_{\mathrm{D}}^{22}=+3.6\left(\mathrm{c}=0.25, \mathrm{CHCl}_{3}\right)$;

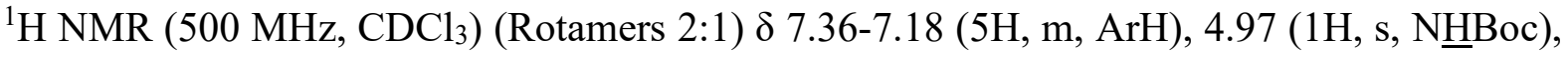
4.77-4.54 $\left(3 \mathrm{H}, \mathrm{m}, \mathrm{C} \underline{\mathrm{HOTBS}}+\mathrm{CH}_{2} \mathrm{Ph}\right), 3.52-3.44\left(1 \mathrm{H}, \mathrm{m}, \mathrm{C}_{\underline{a}} \mathrm{H}_{\mathrm{b}}\right), 3.29-3.24(1 \mathrm{H}, \mathrm{m}$, $\left.\mathrm{CH}_{\mathrm{a}} \underline{\mathrm{H}}_{\mathrm{b}}\right), 3.03\left(2 \mathrm{H}, \mathrm{s}, \mathrm{NCH}_{3}\right), 2.93\left(1 \mathrm{H}, \mathrm{m}, \mathrm{NCH}_{3}\right), 1.43\left(9 \mathrm{H}, \mathrm{s}, \mathrm{CH}_{3}\right.$ of $\left.\mathrm{NHBoc}\right), 0.90(6 \mathrm{H}, \mathrm{s}$, $\mathrm{CH}_{3}$ of tert-butyl group), $0.85\left(3 \mathrm{H}, \mathrm{s}, \mathrm{CH}_{3}\right.$ of tert-butyl group), $0.12\left(2 \mathrm{H}, \mathrm{s},\left(\mathrm{CH}_{3}\right)_{2} \mathrm{Si}\right), 0.09$ $\left(2 \mathrm{H}, \mathrm{s},\left(\mathrm{CH}_{3}\right)_{2} \mathrm{Si}\right), 0.00\left(2 \mathrm{H}, \mathrm{s},\left(\mathrm{CH}_{3}\right)_{2} \mathrm{Si}\right)$;

${ }^{13} \mathrm{C}$ NMR (126 MHz, $\mathrm{CDCl}_{3}$ ) (Rotamers 2:1) $\delta 171.5,171.2,156.1,137.0,136.9,129.0$, $128.7,128.2,127.8,127.6,126.9,70.7,70.3,52.8,51.4,44.5,44.1,34.6,34.5,28.5,25.9$, $25.8,18.5,18.4,-4.5,-4.6 ; \mathrm{m} / z(\mathrm{ESI}) 445.3\left[(\mathrm{M}+\mathrm{Na})^{+}, 100 \%\right]$. 
tert-Butyl-(3-(benzyl(methyl)amino)-2-((tert-butyldimethylsilyl)oxy)-3-oxopropyl)carbamate 57.

${ }^{1} \mathrm{H}$ NMR $\left(500 \mathrm{MHz}, \mathrm{CDCl}_{3}\right)$.

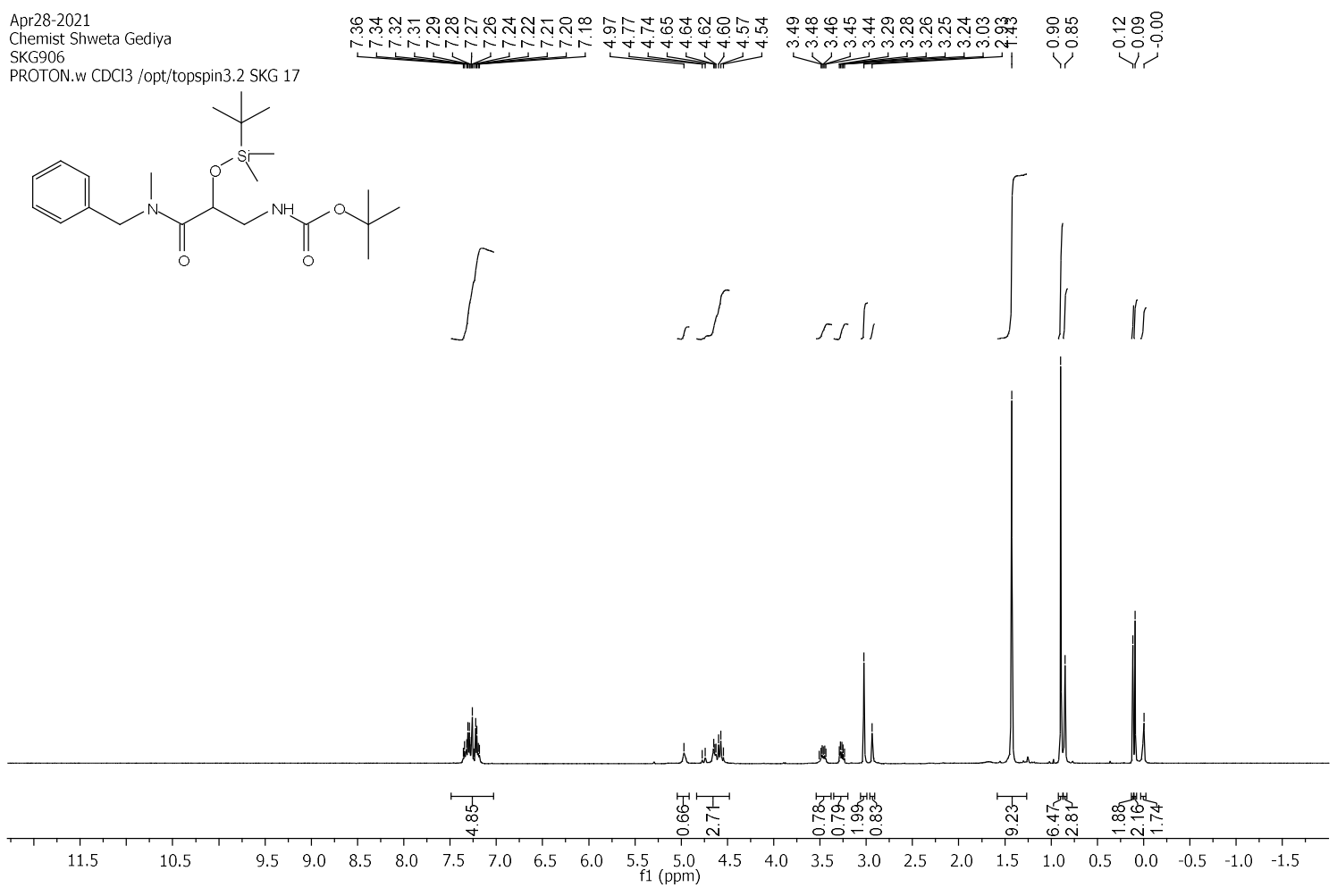

${ }^{13} \mathrm{C}$ NMR (126 MHz, $\left.\mathrm{CDCl}_{3}\right)$.

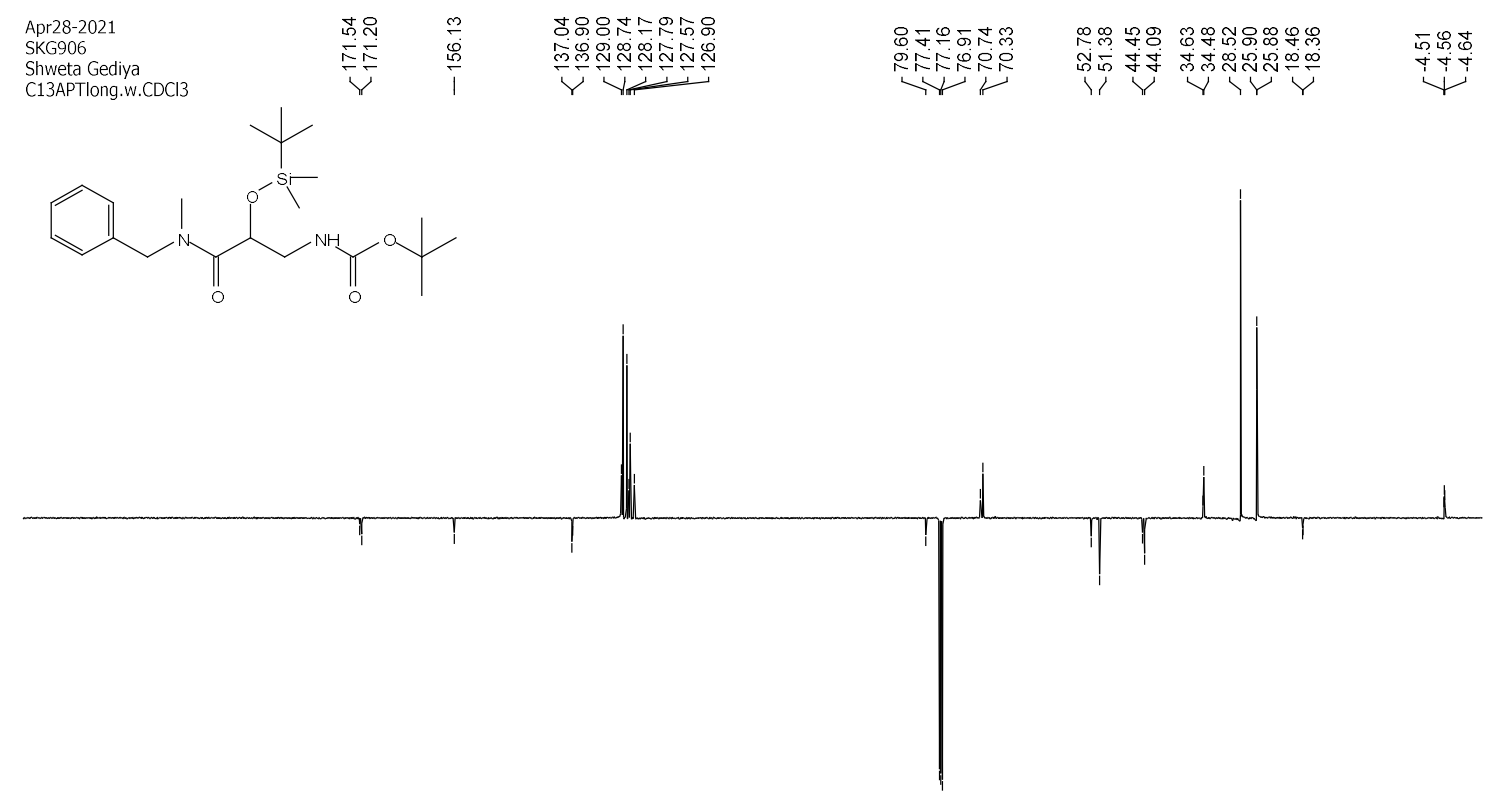

$\begin{array}{llllllllllllllllllllllllll} & 220 & 210 & 200 & 190 & 180 & 170 & 160 & 150 & 140 & 130 & 120 & \underset{f 1}{110}(\mathrm{ppm}) & 100 & 90 & 80 & 70 & 60 & 50 & 40 & 30 & 20 & 10 & 0 & -10\end{array}$ 
COSY.

Chemist Shweta Gediya

SKG906
COSY.w CDCl3 /opt/topspin3.2 SKG 17

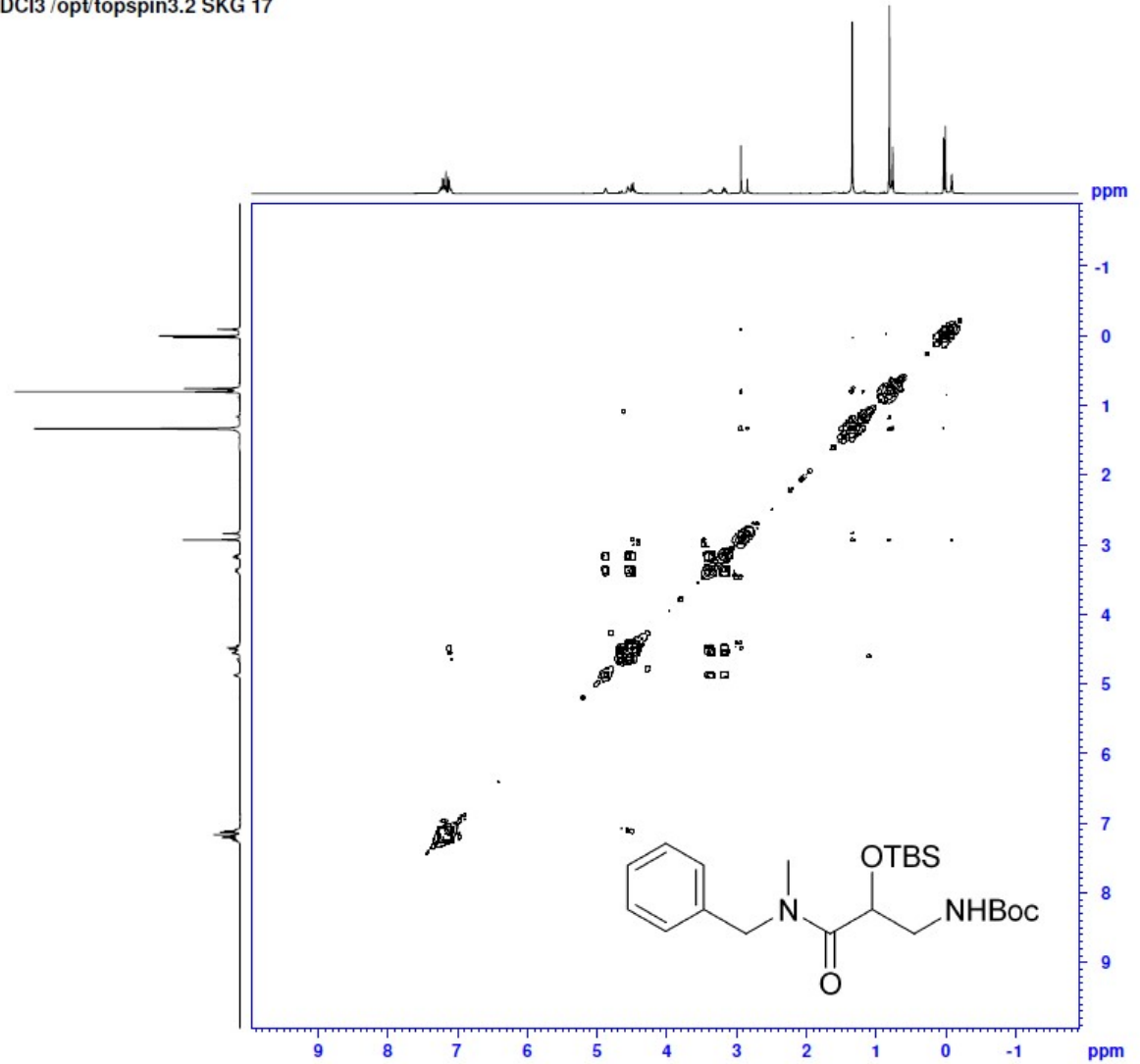

HSQC.

HSQC.w CDCl3/opt/topspin3.2 SKG 17

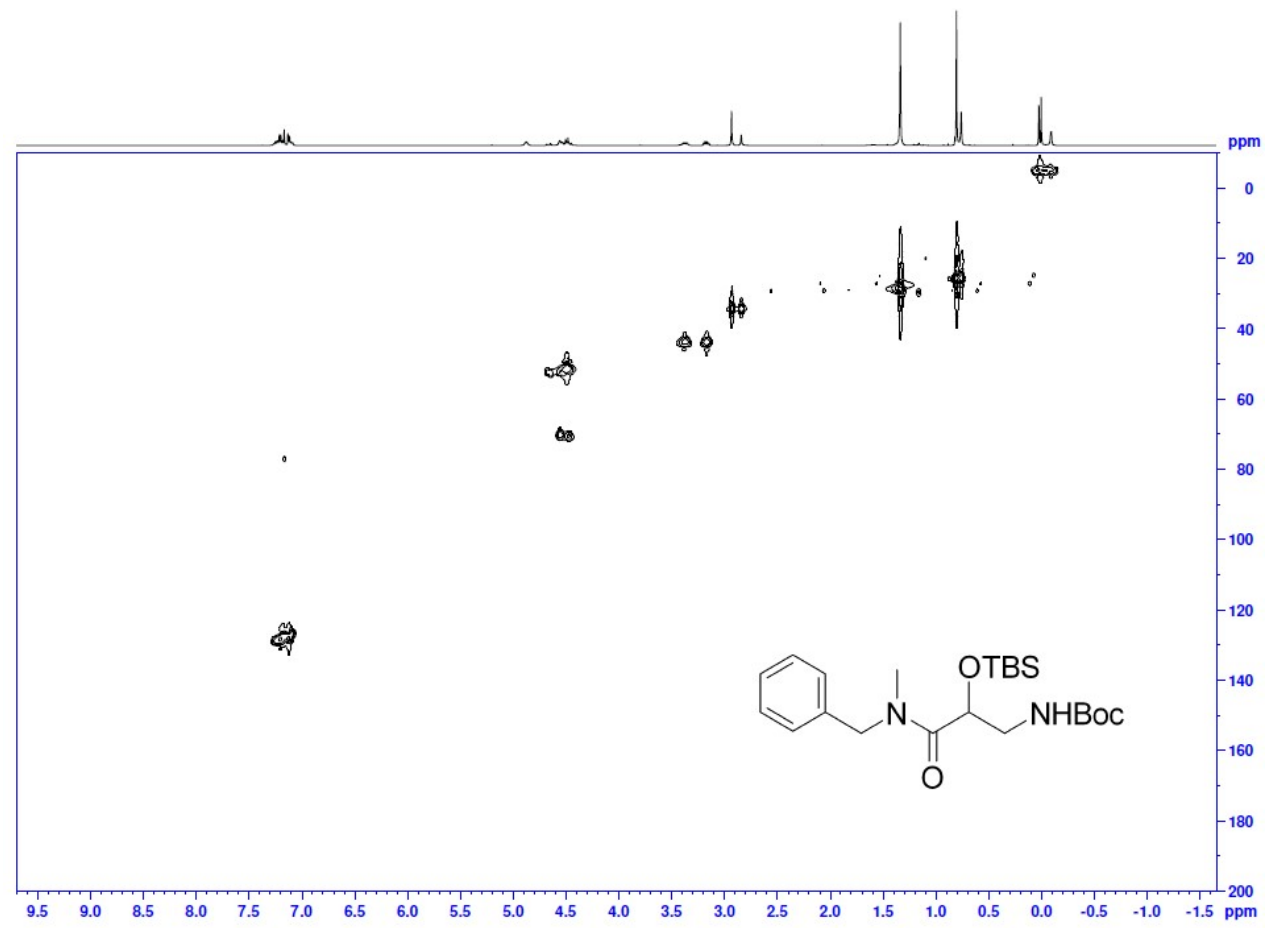




\section{Chiral HPLC of racemic standard.}

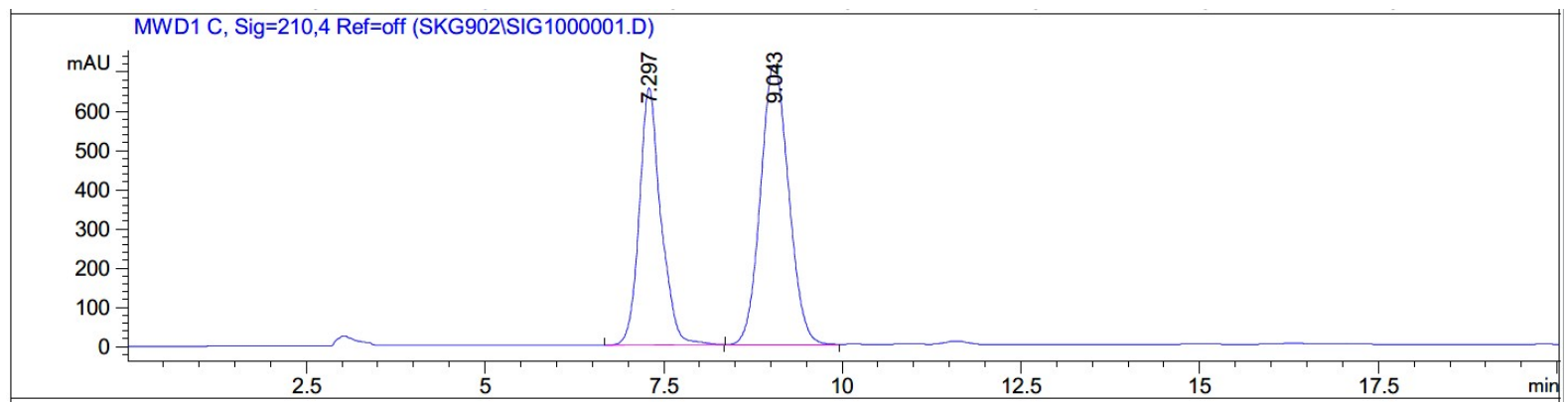

Signal 2: MWD1 C, Sig=210,4 Ref=off

\begin{tabular}{|c|c|c|c|c|c|c|}
\hline $\begin{array}{c}\text { Peak } \\
\#\end{array}$ & $\begin{array}{c}\text { RetTime } \\
\text { [min] }\end{array}$ & Type & $\begin{array}{l}\text { Width } \\
\text { [min] }\end{array}$ & $\begin{array}{c}\text { Area } \\
{\left[\mathrm{mAU}^{\star} \mathrm{s}\right]}\end{array}$ & $\begin{array}{l}\text { Height } \\
{[\mathrm{mAU}]}\end{array}$ & $\begin{array}{c}\text { Area } \\
\frac{\circ}{\circ}\end{array}$ \\
\hline & & & & --------- & ---1 & $--------\mid$ \\
\hline 1 & 7.297 & $\mathrm{BB}$ & 0.3091 & $1.37490 \mathrm{e} 4$ & 654.37738 & 41.4307 \\
\hline 2 & 9.043 & $\mathrm{BB}$ & 0.4266 & $1.94366 \mathrm{e} 4$ & 711.67865 & 58.5693 \\
\hline
\end{tabular}

\section{Chiral HPLC of ATH product.}

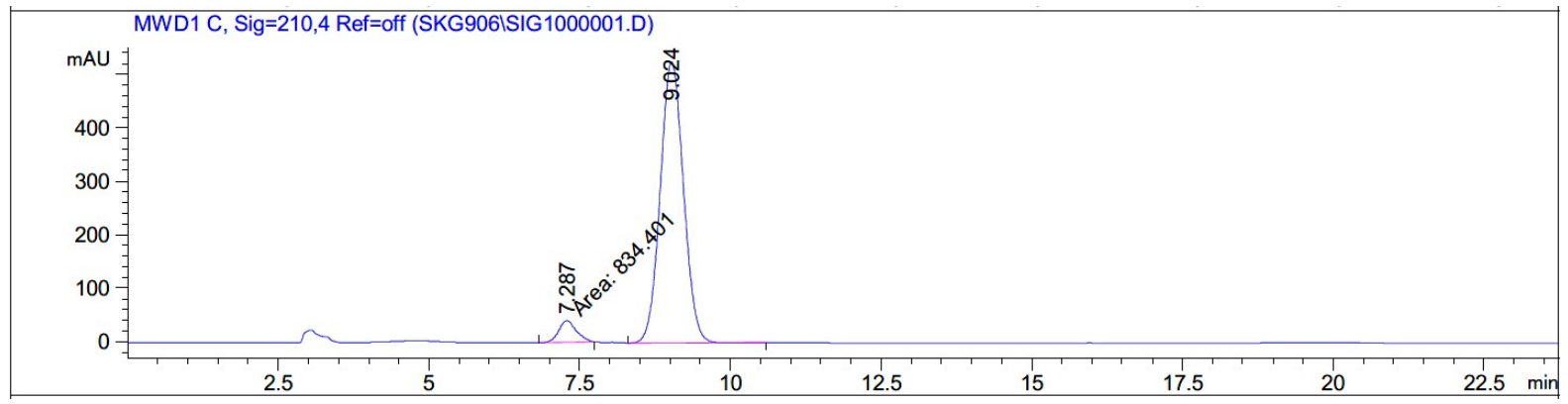

Signal 2: MWD1 C, Sig=210,4 Ref=off

\begin{tabular}{|c|c|c|c|c|c|c|}
\hline $\begin{array}{c}\text { Peak } \\
\quad \#\end{array}$ & $\begin{array}{c}\text { RetTime } \\
\text { [min] }\end{array}$ & Type & $\begin{array}{l}\text { Width } \\
\text { [min] }\end{array}$ & $\begin{array}{c}\text { Area } \\
{\left[\mathrm{mAU}^{\star} \mathrm{s}\right]}\end{array}$ & $\begin{array}{l}\text { Height } \\
{[\mathrm{mAU}]}\end{array}$ & $\begin{array}{c}\text { Area } \\
\quad \%\end{array}$ \\
\hline & & & & ------ & $---------\mid$ & $-------\mid$ \\
\hline 1 & & MM & 0.3369 & 834.40070 & 41.27611 & 807 \\
\hline 2 & 9.024 & $\mathrm{BB}$ & 0.4169 & $1.41172 \mathrm{e} 4$ & 526.30536 & 94.4193 \\
\hline
\end{tabular}


Ethyl 3-((tert-butyldimethylsilyl)oxy)-4-oxo-4-(piperidin-1-yl)butanoate (racemic).<smiles>CCOC(=O)CC(OCC)C(=O)N1CCCCC1</smiles>

This compound is novel. To a stirred solution of ethyl 3-hydroxy-4-oxo-4-(piperidin-1yl)butanoate $40(0.190 \mathrm{~g}, 0.837 \mathrm{mmol}, 1.0 \mathrm{eq})$ in DMF (5 mL) was added tertbutyldimethylsilyl chloride (TBDMSCl) $(0.251 \mathrm{~g}, 1.67 \mathrm{mmol}, 2.0 \mathrm{eq})$ and imidazole $(0.142 \mathrm{~g}$, $2.09 \mathrm{mmol}, 2.5 \mathrm{eq})$ and the resulting reaction mixture was stirred at room temperature for $3 \mathrm{~h}$. The reaction mixture was quenched with ice cold water $(20 \mathrm{~mL})$ and extracted with ethyl acetate $(3 \times 10 \mathrm{~mL})$. The combined organic layer was washed with brine $(50 \mathrm{~mL})$. The organic layer was dried over $\mathrm{MgSO}_{4}$, filtered and the filtrate was concentrated under reduced pressure to generate crude material which was further purified by column chromatography (5$10 \%$ EtOAc in petroleum ether $)$ to afford the product as a clear oil $(0.212 \mathrm{~g}, 0.618 \mathrm{mmol}$, $74 \%)$.

TLC: $\mathrm{R}_{\mathrm{f}}$ ca 0.5 (6:4, hexane: EtOAc), UV active, strong $\mathrm{KMnO}_{4}$ active and PMA-reactive;

HRMS (ESI) m/z: [M+Na] ${ }^{+}$Calcd for $\mathrm{C}_{17} \mathrm{H}_{33} \mathrm{NO}_{4} \mathrm{SiNa} 366.2071$; Found 366.2066 (error 1.5 ppm);

$U_{\max } 2931,2856,1735,1641,1443,1253,1174,1098,829,776 \mathrm{~cm}^{-1}$;

${ }^{1} \mathrm{H}$ NMR (400 MHz, $\left.\mathrm{CDCl}_{3}\right) \delta$ 4.93-4.90 (1H, m, C프BS), 4.16-4.10 (2H, m, $\mathrm{C}_{2} \mathrm{CH}_{3}$ ), 3.60-3.44 (4H, m, $\mathrm{CH}_{2}$ of piperidine), $2.74\left(1 \mathrm{H}, \mathrm{dd}, J=15.3,4.9 \mathrm{~Hz}, \mathrm{C}_{\mathrm{a}} \mathrm{H}_{\mathrm{b}}\right), 2.64(1 \mathrm{H}, \mathrm{dd}, J$ $\left.=15.3,8.7 \mathrm{~Hz}, \mathrm{CH}_{\mathrm{a}} \underline{\mathrm{H}}\right), 1.69-1.55\left(6 \mathrm{H}, \mathrm{m}, \mathrm{CH}_{2}\right.$ of piperidine $), 1.26(3 \mathrm{H}, \mathrm{t}, J=7.1 \mathrm{~Hz}$, $\left.\mathrm{CH}_{2} \mathrm{C}_{3}\right), 0.86\left(9 \mathrm{H}, \mathrm{s}, \mathrm{CH}_{3}\right.$ of tert-butyl group), $0.09\left(3 \mathrm{H}, \mathrm{s},\left(\mathrm{CH}_{3}\right)_{2} \mathrm{Si}\right), 0.07(3 \mathrm{H}, \mathrm{s}$, $\left.\left(\mathrm{CH}_{3}\right)_{2} \mathrm{Si}\right)$;

${ }^{13} \mathrm{C}$ NMR $\left(101 \mathrm{MHz}, \mathrm{CDCl}_{3}\right) \delta 170.1,169.9,70.5,60.7,46.3,43.6,40.3,26.7,25.8,25.7$, 24.7, 18.2, 14.3, -4.4, -5.1;

$m / z(\mathrm{ESI}) 366.2\left[(\mathrm{M}+\mathrm{Na})^{+}, 100 \%\right]$. 
Ethyl 3-((tert-butyldimethylsilyl)oxy)-4-oxo-4-(piperidin-1-yl)butanoate.

${ }^{1} \mathrm{H}$ NMR $\left(400 \mathrm{MHz}, \mathrm{CDCl}_{3}\right)$.

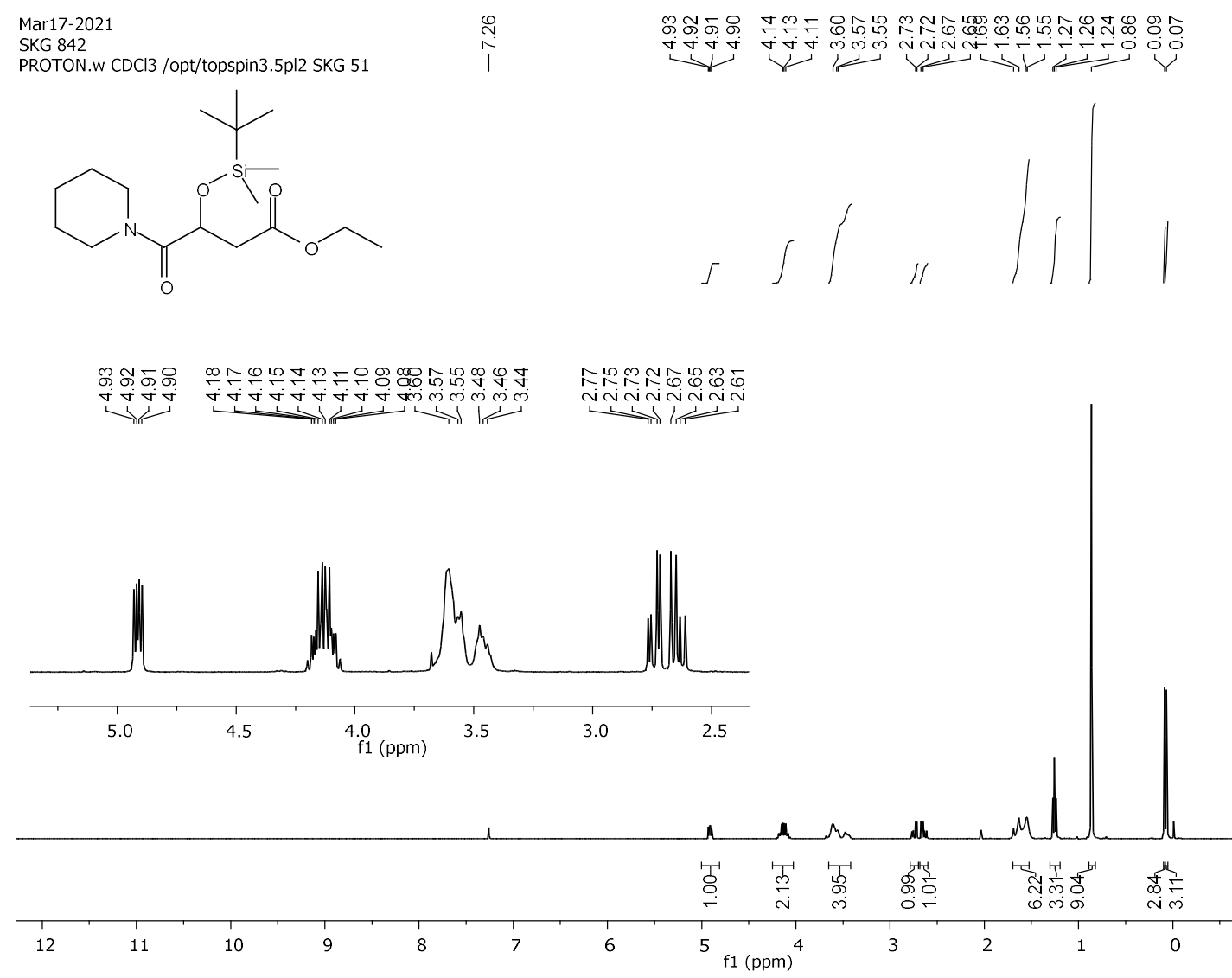

${ }^{13} \mathrm{C}$ NMR (101 MHz, $\left.\mathrm{CDCl}_{3}\right)$.

\begin{tabular}{|c|c|c|c|c|}
\hline $\begin{array}{l}\text { Mar17-2021 } \\
\text { SKG842 } \\
\text { C13APTlong.w.CDCI3 }\end{array}$ & $\begin{array}{l}\text { Ro } \\
0.0 \\
11\end{array}$ & 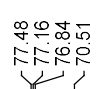 & $\begin{array}{l}0 \\
\infty \\
0 \\
0 \\
1\end{array}$ & 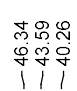 \\
\hline
\end{tabular}
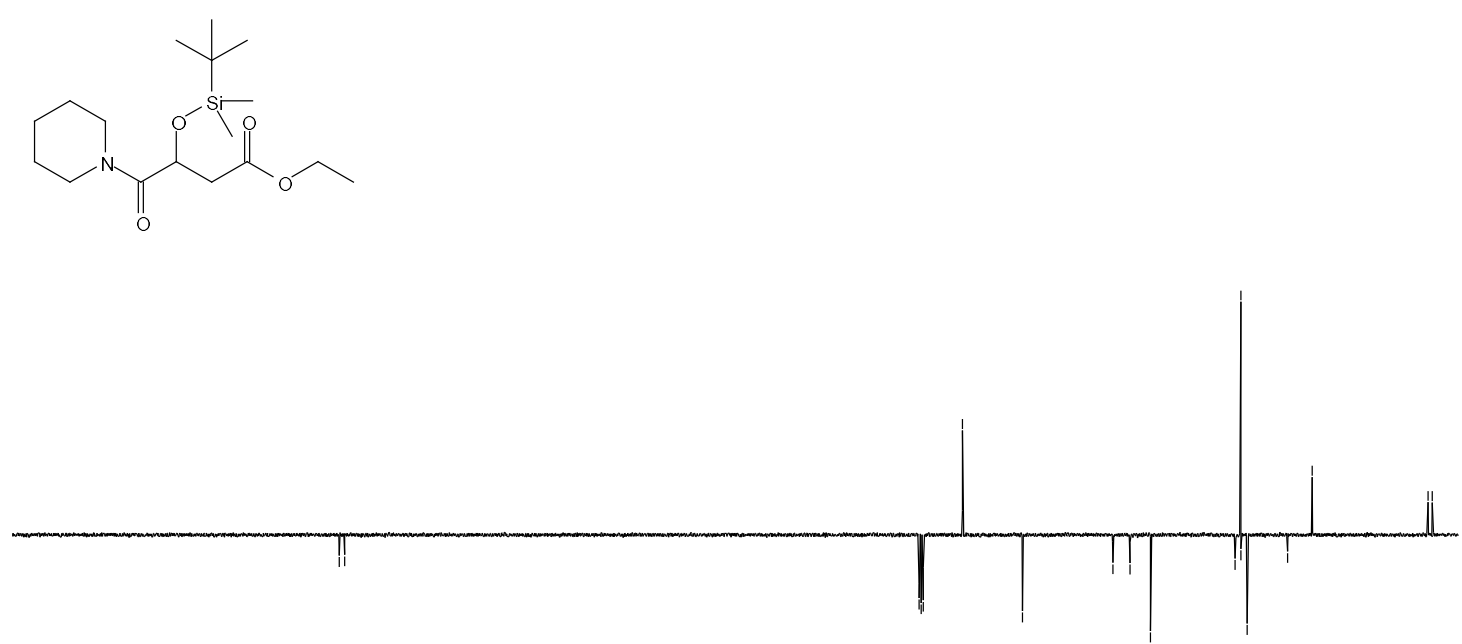

$\begin{array}{lllllllllllllllllllllll}220 & 210 & 200 & 190 & 180 & 170 & 160 & 150 & 140 & 130 & 120 & 110 & 100 & 90 & 80 & 70 & 60 & 50 & 40 & 30 & 20 & 10 & 0\end{array}$ 
COSY.

SKG 842

COSY, $\mathrm{CDCl} 3$ /opt/topspin3.5pl2 SKG 5

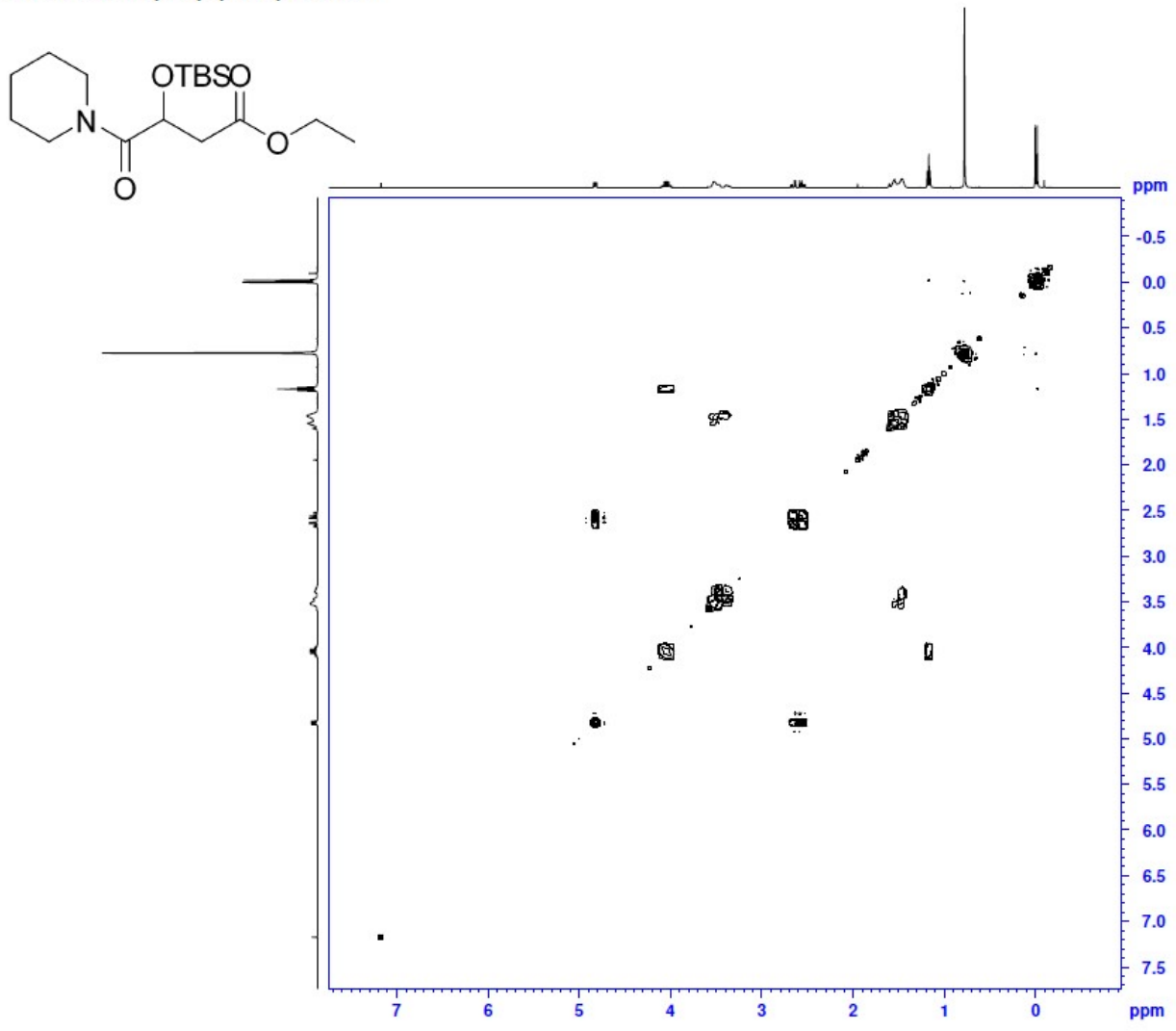

HSQC.

SKG 842
HSQC.w CDCl3 /opt/topspin3.5pl2 SKG 51

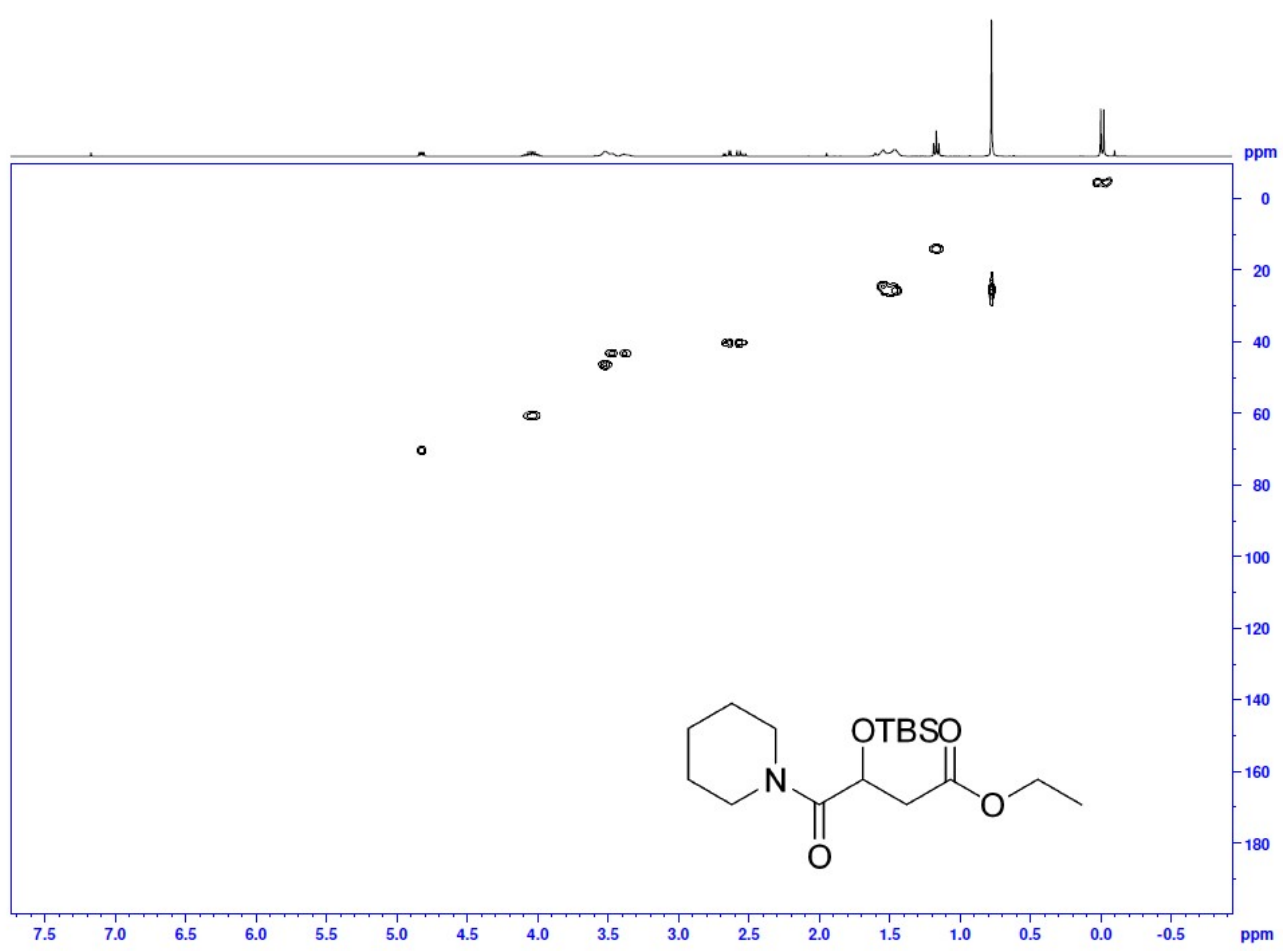


Ethyl 3-((tert-butyldimethylsilyl)oxy)-4-morpholino-4-oxobutanoate (racemic).<smiles>CCOC(=O)CC(O[GaH])C(=O)N1CCOCC1</smiles>

This compound is novel. To a stirred solution of ethyl-3-hydroxy-4-morpholino-4oxobutanoate $41(0.231 \mathrm{~g}, 1.00 \mathrm{mmol}, 1.0 \mathrm{eq})$ in DMF (5 mL) was added tertbutyldimethylsilyl chloride (TBDMSCl) $(0.301 \mathrm{~g}, 2.00 \mathrm{mmol}, 2.0 \mathrm{eq})$ and imidazole $(0.170 \mathrm{~g}$, $2.50 \mathrm{mmol}, 2.5 \mathrm{eq}$ ) and the resulting reaction mixture was stirred at room temperature for $3 \mathrm{~h}$. The reaction mixture was quenched with ice cold water $(20 \mathrm{~mL})$ and extracted with ethyl acetate $(3 \times 10 \mathrm{~mL})$. The combined organic layer was washed with brine $(50 \mathrm{~mL})$. The organic layer was dried over $\mathrm{MgSO}_{4}$, filtered and filtrate was concentrated under reduced pressure to generate crude material which was further purified by column chromatography (5$10 \%$ EtOAc in petroleum ether) to afford the product as a clear oil $(0.322 \mathrm{~g}, 0.877 \mathrm{mmol}$, $88 \%$ ).

TLC: $\mathrm{R}_{\mathrm{f}}$ ca 0.6 (6:4, hexane: EtOAc), UV active, strong $\mathrm{KMnO}_{4}$ active and PMA-reactive;

HRMS (ESI) m/z: $[\mathrm{M}+\mathrm{Na}]^{+}$Calcd for $\mathrm{C}_{16} \mathrm{H}_{31} \mathrm{NO}_{5} \mathrm{SiNa}$ 368.1864; Found 368.1856 (error 2.1 ppm);

$U_{\max } 2957,2857,1734,1645,1437,1251,1112,830,777 \mathrm{~cm}^{-1}$;

${ }^{1} \mathrm{H}$ NMR (500 MHz, $\left.\mathrm{CDCl}_{3}\right) \delta$ 4.84-4.81(1H, m, C $\left.\underline{H O T B S}\right), 4.12-4.04\left(2 \mathrm{H}, \mathrm{m}, \mathrm{C}_{2} \mathrm{CH}_{3}\right)$, $3.72-3.52\left(8 \mathrm{H}, \mathrm{m}, \mathrm{CH}_{2}\right.$ of morpholine $), 2.73-2.68\left(1 \mathrm{H}, \mathrm{m}, \mathrm{CH}_{\mathrm{a}} \mathrm{H}_{\mathrm{b}}\right), 2.60-2.55(1 \mathrm{H}, \mathrm{m}$, $\left.\mathrm{CH}_{\mathrm{a}} \underline{\mathrm{H}}_{\mathrm{b}}\right), 1.19$ (3H, t, $\left.J=7.2 \mathrm{~Hz}, \mathrm{CH}_{2} \mathrm{C}_{3}\right), 0.80-0.79$ (9H, m, $\mathrm{CH}_{3}$ of tert-butyl group), 0.03 $\left(3 \mathrm{H}, \mathrm{s},\left(\mathrm{CH}_{3}\right)_{2} \mathrm{Si}\right), 0.01\left(3 \mathrm{H}, \mathrm{m},\left(\mathrm{CH}_{3}\right)_{2} \mathrm{Si}\right)$;

${ }^{13} \mathrm{C}$ NMR $\left(126 \mathrm{MHz}, \mathrm{CDCl}_{3}\right) \delta 172.2,170.1,70.5,66.8,66.7,60.8,45.8,42.6,39.9,25.6$, $18.0,14.1,-4.6,-5.2$; $m / z(\mathrm{ESI}) 368.2\left[(\mathrm{M}+\mathrm{Na})^{+}, 100 \%\right]$. 
Ethyl 3-((tert-butyldimethylsilyl)oxy)-4-morpholino-4-oxobutanoate.

${ }^{1} \mathrm{H}$ NMR (500 MHz, $\left.\mathrm{CDCl}_{3}\right)$.

Mar29-2021

Chemist Shweta Gediy

SKG865

PROTON.w CDCl3 /opt/topspin3.2 SKG 34
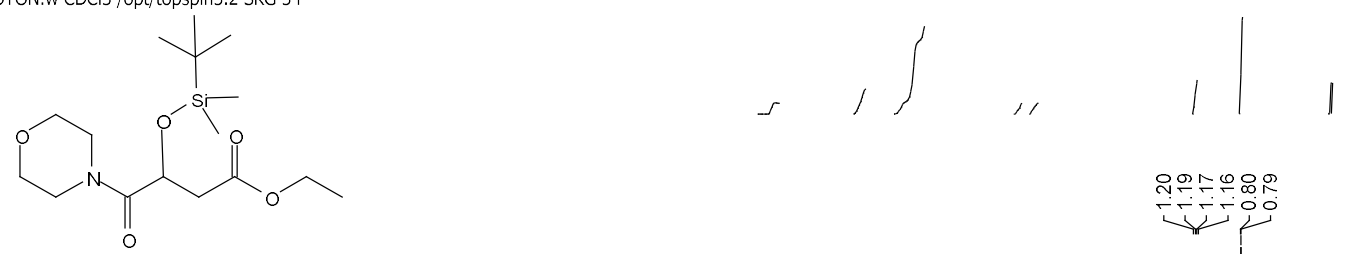

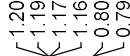
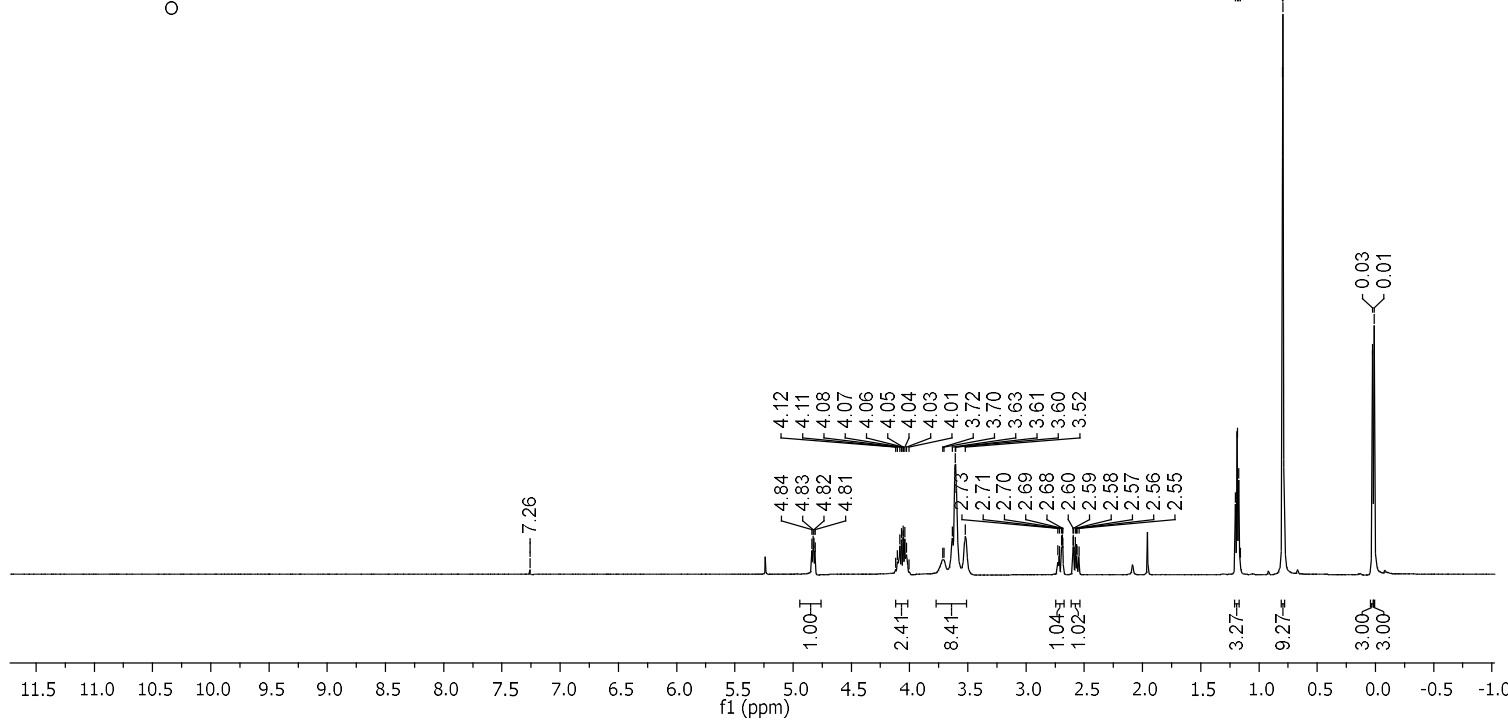

${ }^{13} \mathrm{C} \mathrm{NMR}\left(126 \mathrm{MHz}, \mathrm{CDCl}_{3}\right)$.

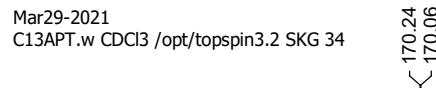

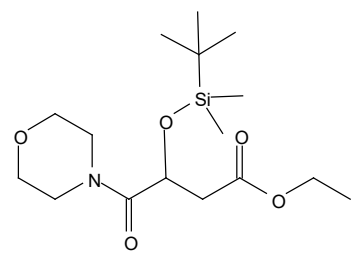

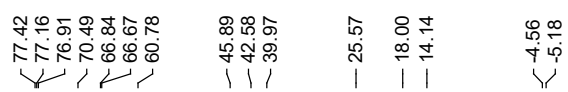

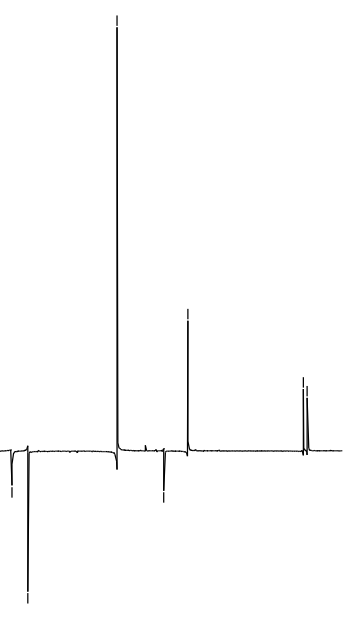

$\begin{array}{rlllllllllllllllllllllllllllll}230 & 220 & 210 & 200 & 190 & 180 & 170 & 160 & 150 & 140 & 130 & 120 & 110 & 100 & 90 & 80 & 70 & 60 & 50 & 40 & 30 & 20 & 10 & 0 & -10\end{array}$ 
COSY.

Chemist Shweta Gediya

COSY.w CDCl3 /opt/topspin3.2 SKG 34

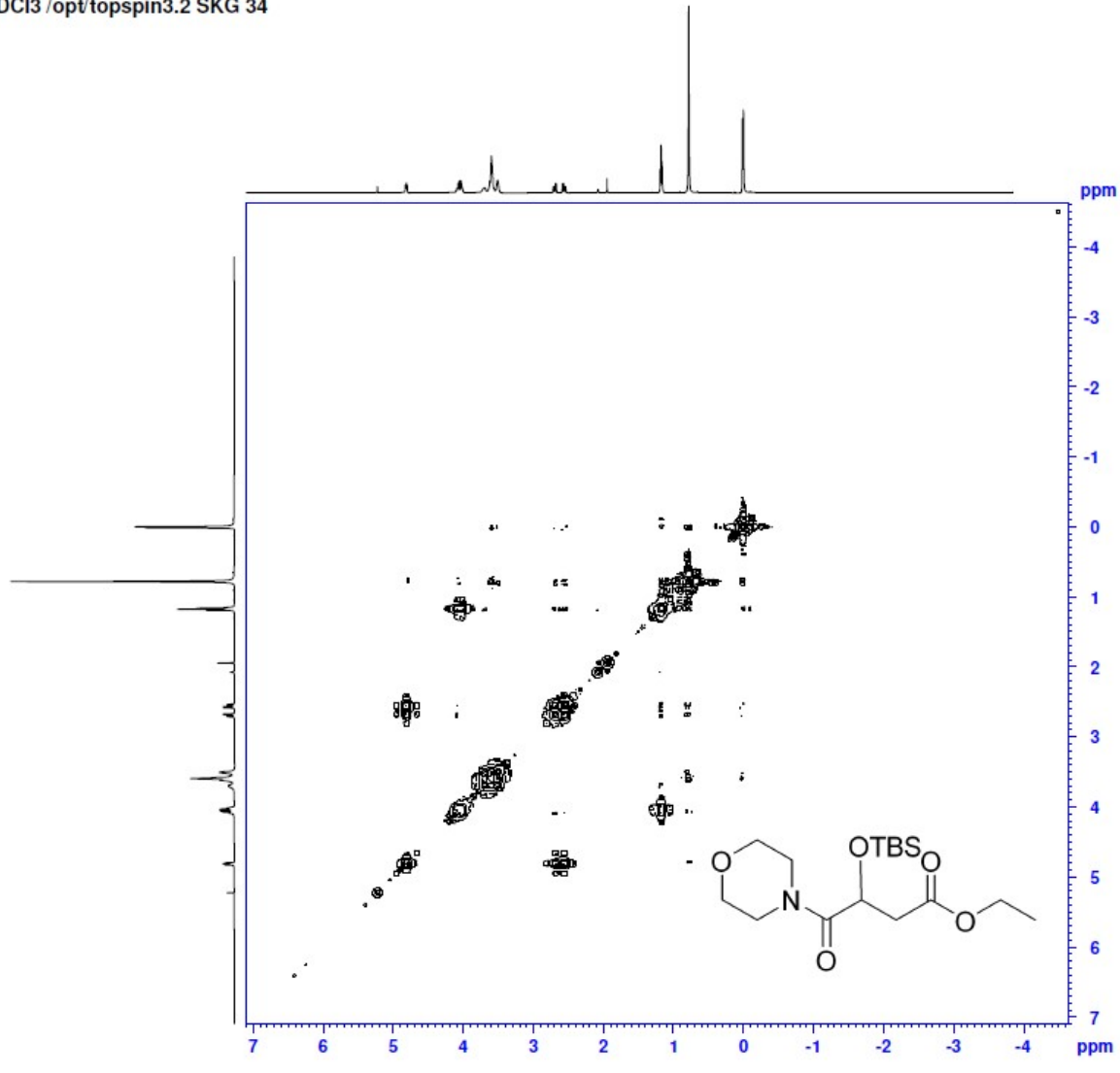

HSQC.

Chemist Shweta Gediy

SKG865

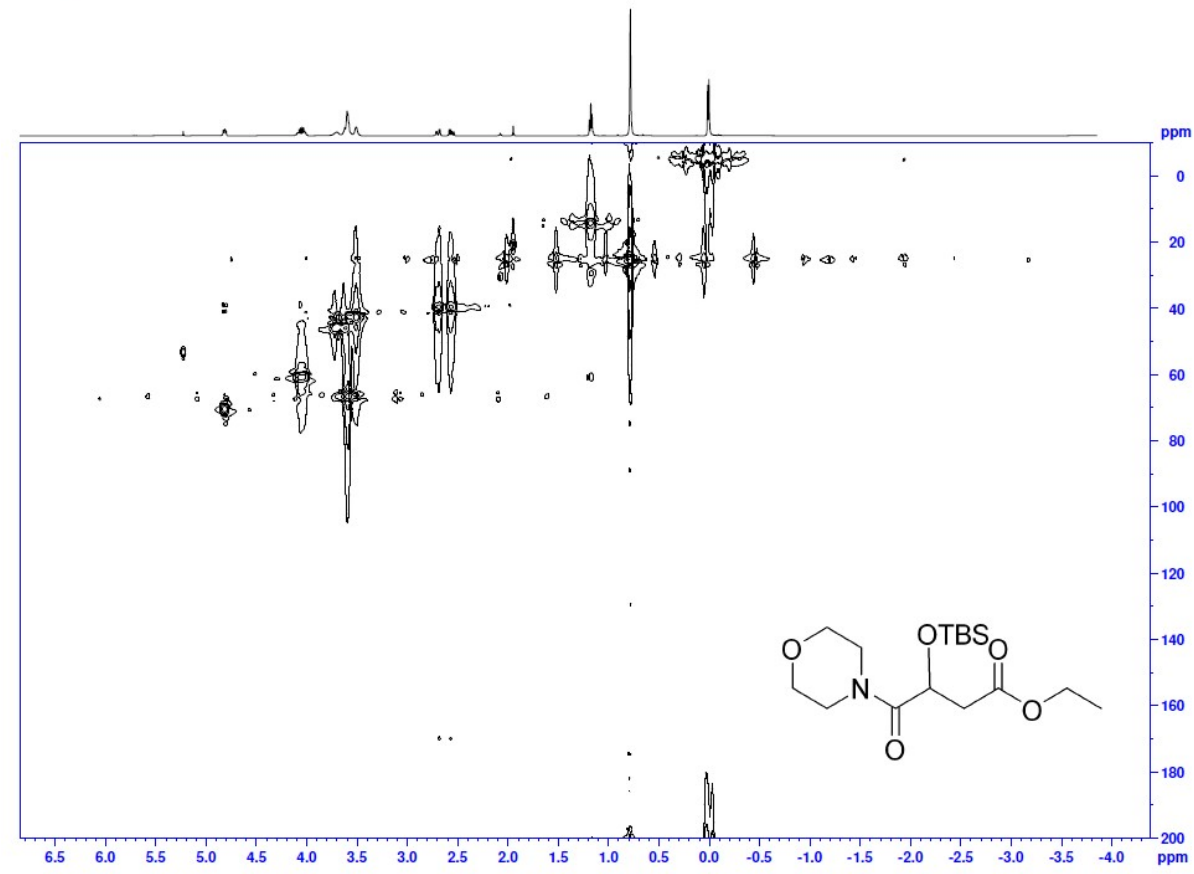


General procedure $E$ for reduction of amides.<smiles>[R]N([R])CCC(O)CCN([R])[R]</smiles>

To a stirred solution of amide (1.0 eq) in anhydrous THF was added lithium aluminum hydride $\left(\mathrm{LiAlH}_{4}\right)\left(2 \mathrm{M}\right.$ in THF) $(2.5 \mathrm{eq})$ in at $0{ }^{0} \mathrm{C}$. The resulting reaction mixture was stirred at same temperature for 15 mins then at room temperature for 15 minutes and then was stirred under reflux at $80{ }^{\circ} \mathrm{C}$ for $2 \mathrm{~h}$. Once the reaction was complete (as assessed by TLC), the reaction solution was cooled to room temperature and quenched with Rochelle salt $(20 \mathrm{~mL})$ and extracted with EtOAc $(3 \times 10 \mathrm{~mL})$. The combined organic layer was washed with water $(50 \mathrm{~mL})$ and brine $(50 \mathrm{~mL})$. The organic layer was dried over $\mathrm{MgSO}_{4}$, filtered and the filtrate was concentrated under reduced pressure to generate crude material which was further purified by column chromatography on silica gel to afford the respective amino alcohols. 


\section{(R)- 1,4-Bis(phenylamino)butan-2-ol 58.}<smiles>O[C@H](CCNc1ccccc1)CNc1ccccc1</smiles>

This compound is novel and was prepared following general procedure $\mathbf{E}$ using $(R)-(2-$ hydroxy- $N^{1}, N^{4}$-diphenylsuccinamide $18(0.150 \mathrm{~g}, 0.528 \mathrm{mmol}, 1.0 \mathrm{eq}), \mathrm{LiAlH}_{4}(2 \mathrm{M}$ in THF) $(0.66 \mathrm{~mL}, 1.32 \mathrm{mmol}, 1.2 \mathrm{eq})$ in THF $(6 \mathrm{~mL})$ to generate crude material which was further purified by column chromatography ( $30 \%$ EtOAc in petroleum ether) to afford $\mathbf{5 8}$ as a white solid (0.103 mg, $0.415 \mathrm{mmol}, 79 \%)$.

MP: $83-86{ }^{\circ} \mathrm{C}$;

TLC: $\mathrm{R}_{\mathrm{f}}$ ca 0.4 (7:3, hexane: EtOAc), UV active $\mathrm{KM}_{\mathrm{n}} \mathrm{O}_{4}$ reactive and PMA-reactive; HRMS (ESI) m/z: [M+H] $]^{+}$Calcd for $\mathrm{C}_{16} \mathrm{H}_{21} \mathrm{~N}_{2} \mathrm{O} 257.1648$; Found 257.1657 (error -3.5 ppm); $U_{\max } 3333,3233,2929,1598,1490,1271,1055,898,746,692 \mathrm{~cm}^{-1}$;

Enantiomeric excess determined by HPLC analysis (Chiralpak IG, $250 \mathrm{~mm} \times 4.6 \mathrm{~mm}$ column, hexane: iPrOH 85:15, $1 \mathrm{~mL} / \mathrm{min}, \lambda=254 \mathrm{~nm}, \mathrm{~T}=25{ }^{\circ} \mathrm{C}$ ), $S$-enantiomer $6.3 \mathrm{~min}, R$ enantiomer $6.8 \min .93 \%$ ee $(R),[\alpha]_{\mathrm{D}}^{22}=-1.37\left(\mathrm{c}=0.4, \mathrm{CHCl}_{3}\right)$;

${ }^{1} \mathrm{H}$ NMR (500 MHz, $\left.\mathrm{CDCl}_{3}\right) \delta$ 7.20-7.15 (4H, m, ArH), 6.74-6.71 (2H, m, ArH), 6.62- 6.60 $(4 \mathrm{H}, \mathrm{m}, \mathrm{ArH}), 3.96-3.92(1 \mathrm{H}, \mathrm{m}, \mathrm{C} \underline{\mathrm{HOH}}), 3.71(1 \mathrm{H}$, br.s, $\mathrm{OH}), 3.27-3.26(2 \mathrm{H}, \mathrm{m}$, $\left.\mathrm{CHCH}_{2} \mathrm{CH}_{2}\right), 3.19\left(1 \mathrm{H}, \mathrm{dd}, J=12.9,3.5 \mathrm{~Hz}, \mathrm{CHCH}_{\mathrm{a}} \mathrm{H}_{\mathrm{b}} \mathrm{NH}\right), 3.02(1 \mathrm{H}, \mathrm{dd}, J=12.9,8.2 \mathrm{~Hz}$, $\left.\mathrm{CHCH}_{\mathrm{a}} \underline{\mathrm{H}}_{\mathrm{b}} \mathrm{NH}\right), 1.83-1.69$ (2H, m, $\left.\mathrm{CHCH}_{2} \mathrm{CH}_{2}\right)$;

${ }^{13} \mathrm{C} \mathrm{NMR}\left(126 \mathrm{MHz}, \mathrm{CDCl}_{3}\right) \delta 148.3,148.2,129.4,129.3,118.1,117.9,113.40,113.4,69.6$, 50.4, 41.7, 34.0; $m / z(\mathrm{ESI}) 257.1\left[(\mathrm{M}+\mathrm{H})^{+}, 100 \%\right]$. 


\section{1,4-Bis(phenylamino)butan-2-ol 58.}

${ }^{1} \mathrm{H}$ NMR $\left(500 \mathrm{MHz}, \mathrm{CDCl}_{3}\right)$.

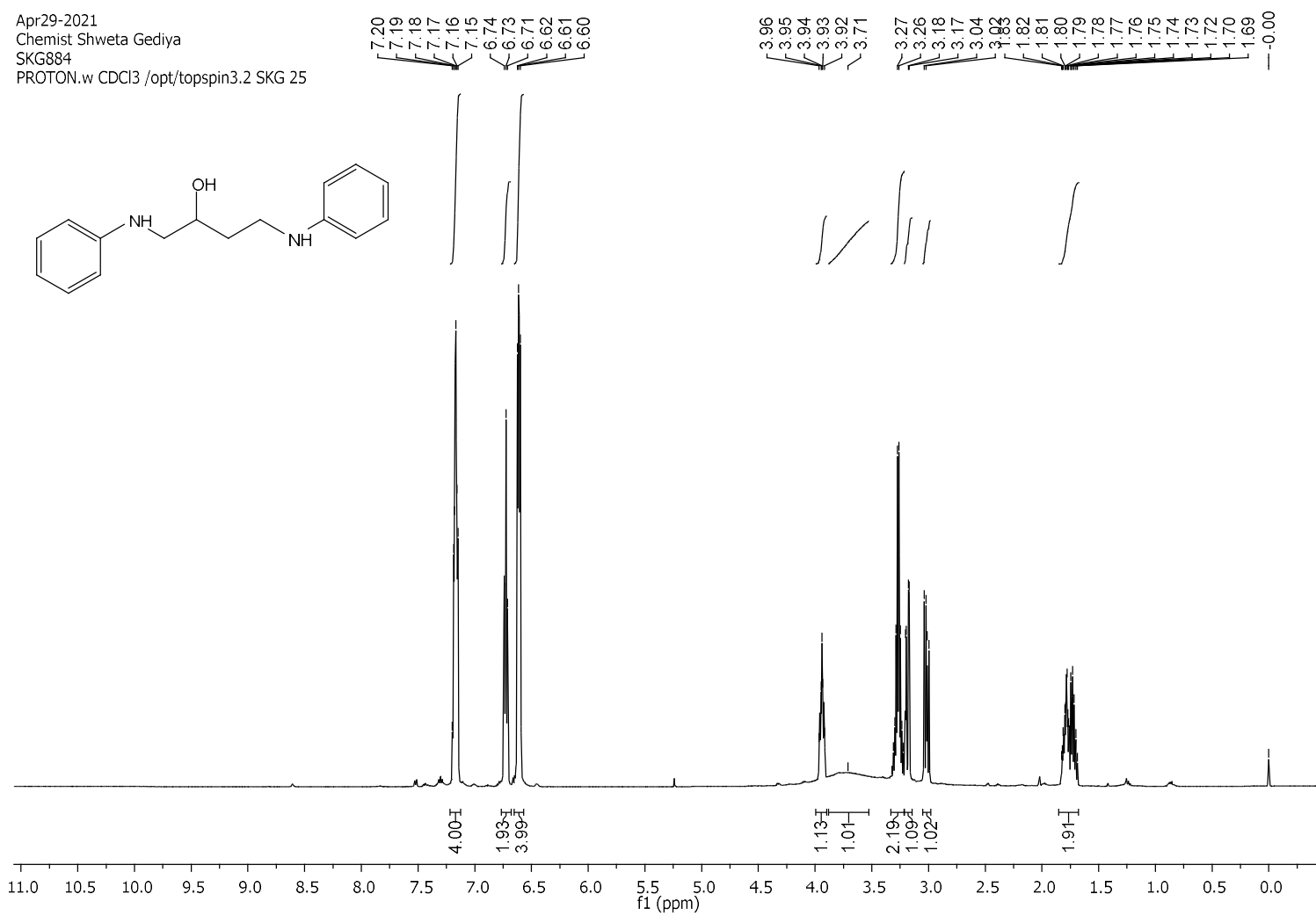

${ }^{13} \mathrm{C}$ NMR $\left(126 \mathrm{MHz}, \mathrm{CDCl}_{3}\right)$.

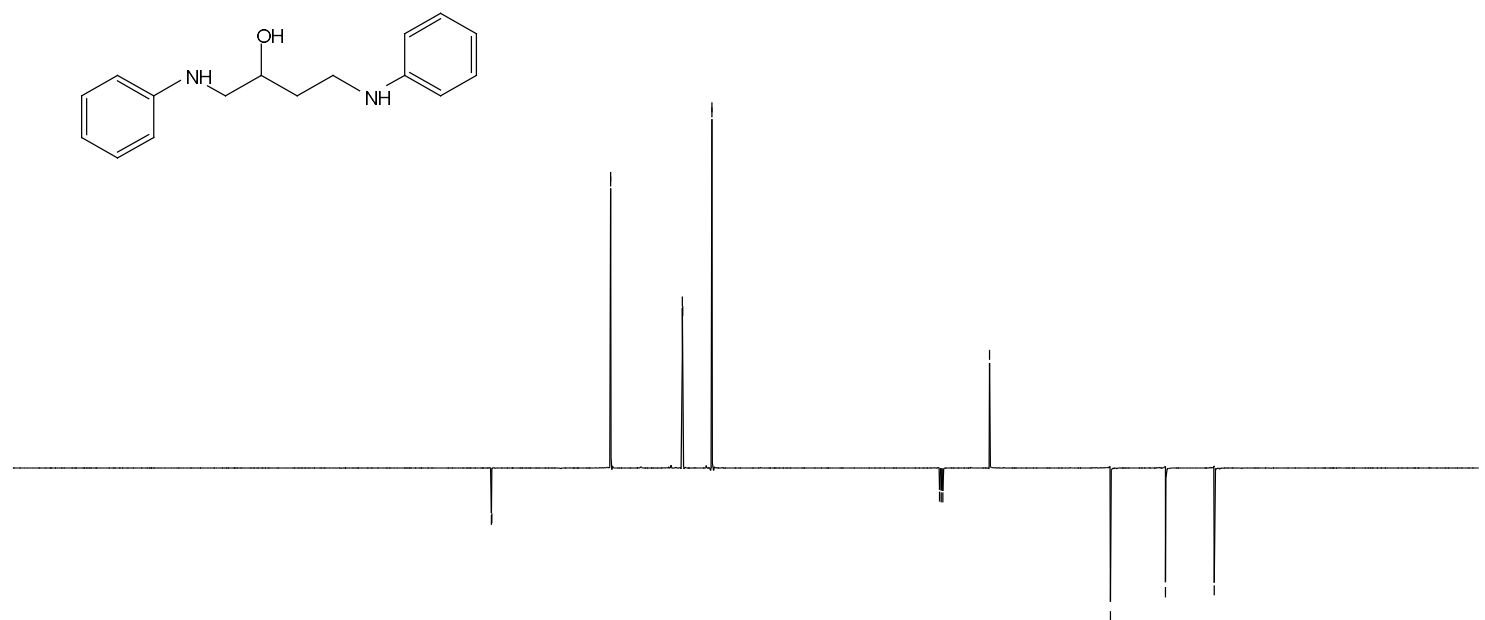


COSY.

Chemist Shweta Gediya

SKG884

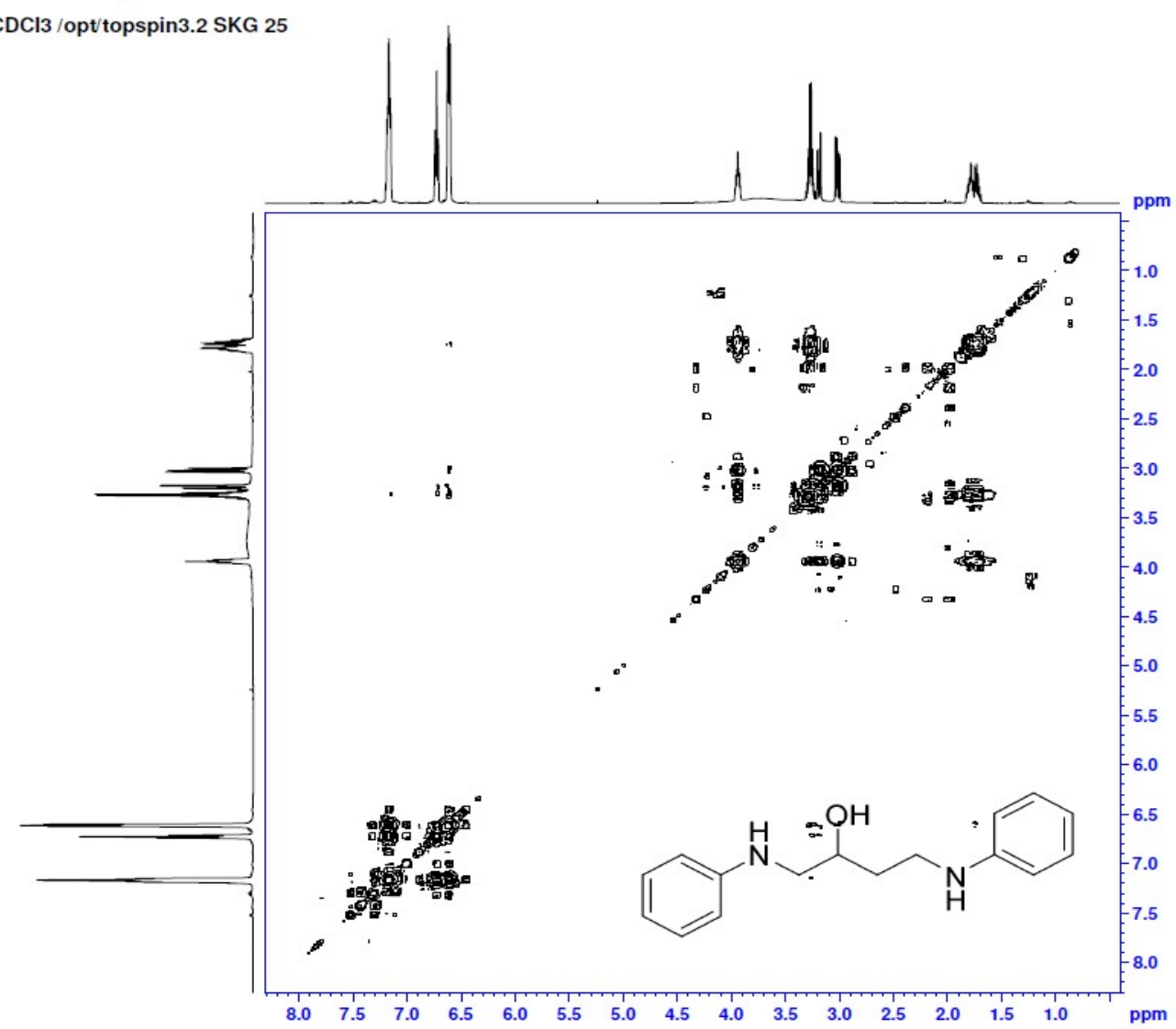

HSQC.

Chemist Shweta Gediya

HSG8.w CDCl3/opt/topspin3.2 SKG 25

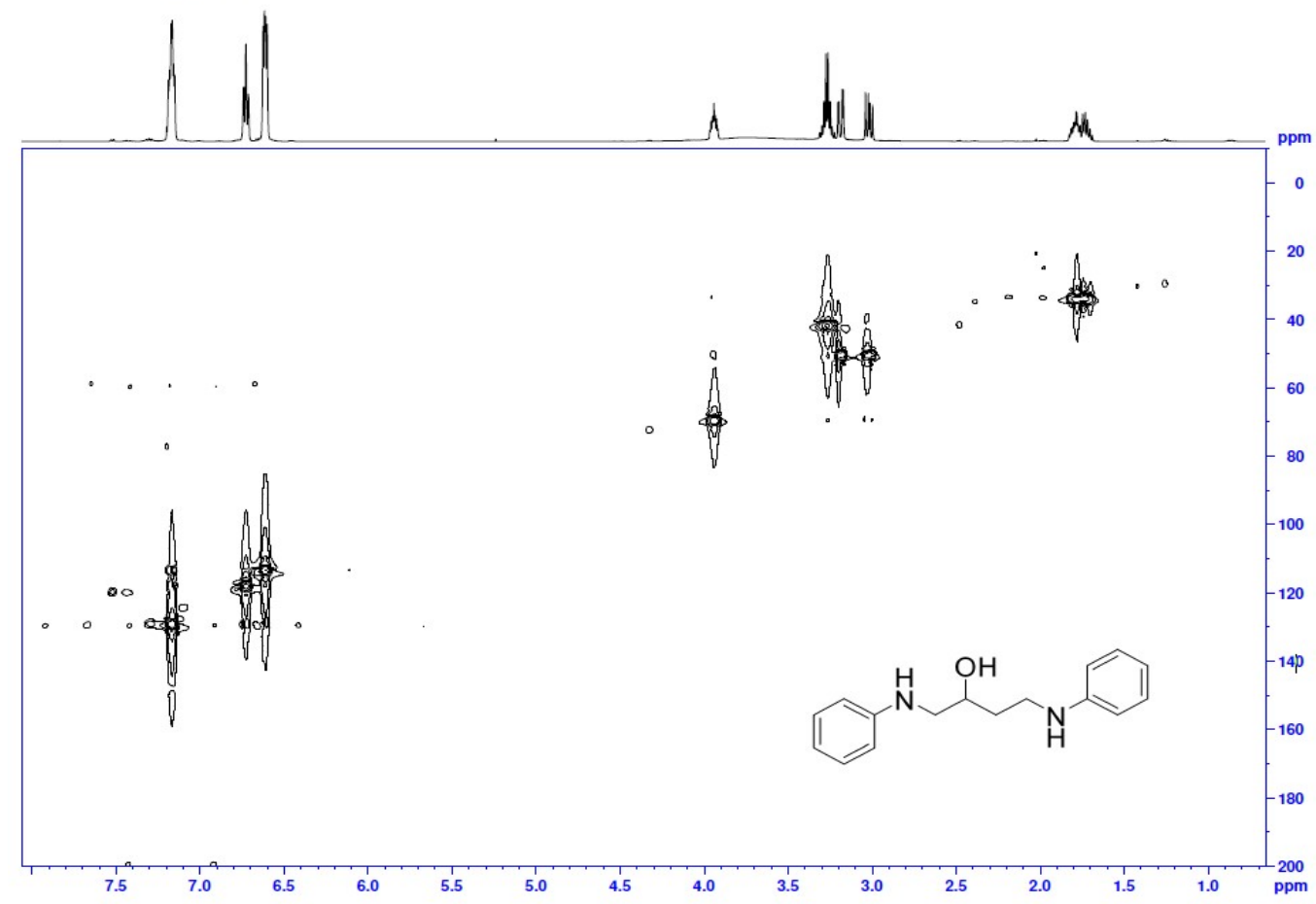


Chiral HPLC of racemic standard.

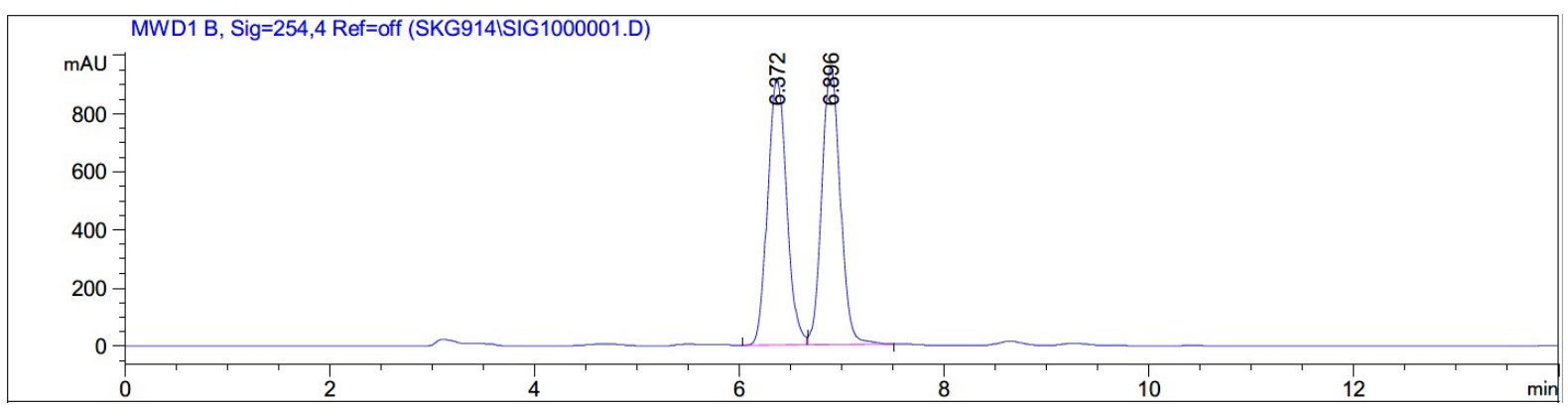

Signal 1: MWD1 B, Sig=254,4 Ref=off

\begin{tabular}{|c|c|c|c|c|c|c|}
\hline $\begin{array}{c}\text { Peak } \\
\quad \#\end{array}$ & $\begin{array}{c}\text { RetTime } \\
\text { [min] }\end{array}$ & Type & $\begin{array}{l}\text { Width } \\
\text { [min] }\end{array}$ & $\begin{array}{c}\text { Area } \\
{\left[\mathrm{mAU}^{*} \mathrm{~s}\right]}\end{array}$ & $\begin{array}{l}\text { Height } \\
{[\mathrm{mAU}]}\end{array}$ & $\begin{array}{c}\text { Area } \\
\quad \%\end{array}$ \\
\hline & & & & --------- & - & 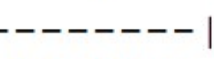 \\
\hline 1 & 6.372 & BV & 0.2002 & $1.18070 \mathrm{e} 4$ & 916.85663 & 49.6826 \\
\hline 2 & 6.896 & VB & 0.1959 & $1.19579 \mathrm{e} 4$ & 956.20630 & 50.3174 \\
\hline
\end{tabular}

\section{$R$-enantiomer, $93 \%$ ee.}

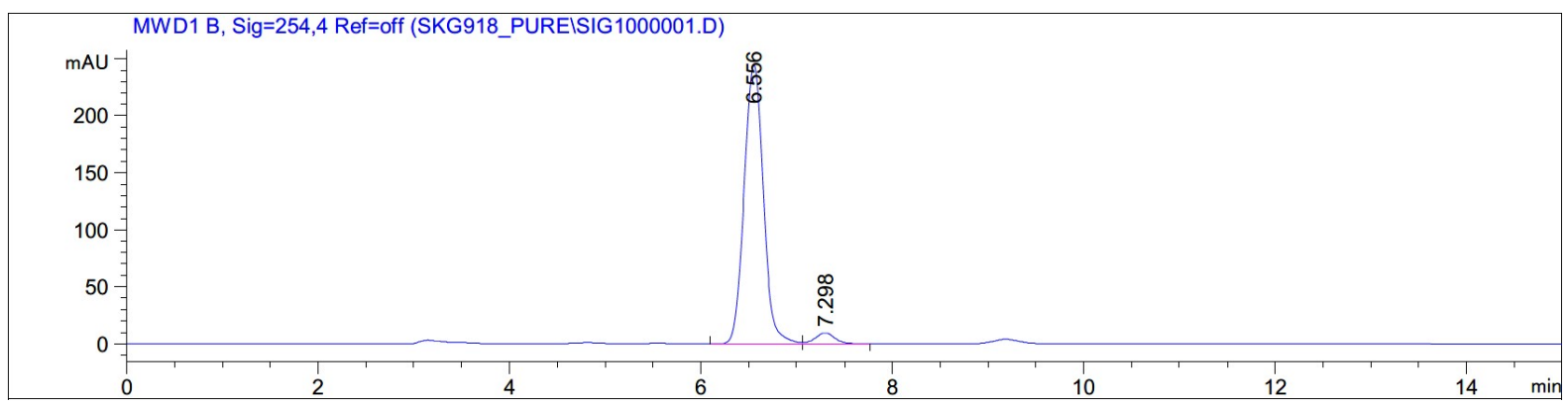

Signal 1: MWD1 B, Sig=254,4 Ref=off

\begin{tabular}{|c|c|c|c|c|c|c|}
\hline $\begin{array}{c}\text { eak } \\
\text { \# }\end{array}$ & $\begin{array}{c}\text { RetTime } \\
\text { [min] }\end{array}$ & Type & $\begin{array}{l}\text { Width } \\
\text { [min] }\end{array}$ & $\begin{array}{c}\text { Area } \\
{\left[\mathrm{mAU}{ }^{\star} \mathrm{S}\right]}\end{array}$ & $\begin{array}{l}\text { Height } \\
{[\mathrm{mAU}]}\end{array}$ & $\begin{array}{c}\text { Area } \\
\quad \%\end{array}$ \\
\hline & & & & & 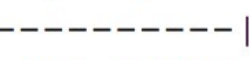 & 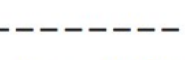 \\
\hline 1 & & & & 340 & 57 & 96 \\
\hline 2 & 7.298 & B & 38 & 130.88725 & 9.43554 & 3.7018 \\
\hline
\end{tabular}


$S$-enantiomer $97 \%$ ee (from reduction of product from $(S)$-malic acid.

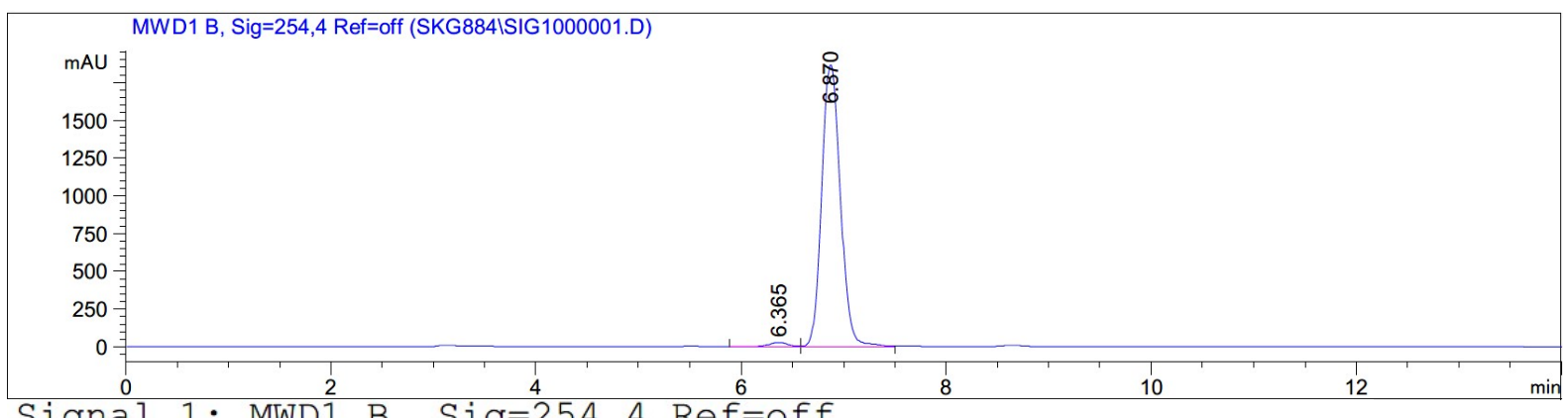

Signal 1: MWD1 B, Sig=254,4 Ref=off

\begin{tabular}{|c|c|c|c|c|c|c|}
\hline $\begin{array}{c}\text { Peak } \\
\text { \# }\end{array}$ & $\begin{array}{c}\text { RetTime } \\
\text { [min] }\end{array}$ & Type & $\begin{array}{l}\text { Width } \\
{[\min ]}\end{array}$ & $\begin{array}{c}\text { Area } \\
{\left[\mathrm{mAU}{ }^{\star} \mathrm{s}\right]}\end{array}$ & $\begin{array}{l}\text { Height } \\
{[\mathrm{mAU}]}\end{array}$ & $\begin{array}{c}\text { Area } \\
\quad \%\end{array}$ \\
\hline & --1 & & --- & --------- & $---------\mid$ & ------- \\
\hline 1 & 6 & BV & 71 & 371.26456 & 27.57075 & 1.5507 \\
\hline 2 & 6.870 & VV & 974 & $2.35698 e 4$ & 1865.61768 & 98.4493 \\
\hline
\end{tabular}




\section{(R)-1-(Phenylamino)-4-(piperidin-1-yl)butan-2-ol 59.}<smiles>OC(CCN1CCCCC1)CNc1ccccc1</smiles>

This compound is novel and was prepared following general procedure $\mathbf{E}$ using ethyl $(R)-4$ (benzyl(methyl)amino)-3-hydroxy-4-oxobutanoate 24 (83.5 g, $0.302 \mathrm{mmol}, 1.0 \mathrm{eq}), \mathrm{LiAlH}_{4}$ (2M in THF) $(0.378 \mathrm{~mL}, 0.756 \mathrm{mmol}, 1.2 \mathrm{eq})$ in THF $(6 \mathrm{~mL})$ to generate crude material which was further purified by column chromatography $\left(10 \% \mathrm{MeOH}\right.$ in $\left.\mathrm{CHCl}_{3}\right)$ to afford $\mathbf{5 9}$ as a clear oil (59.3 $\mathrm{mg}, 0.239 \mathrm{mmol}, 78 \%)$.

TLC: $\mathrm{R}_{\mathrm{f}}$ ca $0.2\left(9: 1, \mathrm{CHCl}_{3}: \mathrm{MeOH}\right), \mathrm{UV}$ active, $\mathrm{KMnO}_{4}$ reactive and PMA-reactive; HRMS (ESI) m/z: [M+H] $]^{+}$Calcd for $\mathrm{C}_{15} \mathrm{H}_{25} \mathrm{~N}_{2} \mathrm{O} 249.1961$; Found 249.1970 (error -3.5 ppm); $U_{\max } 3374,3350,2933,1601,1502,1117,1033,746,691 \mathrm{~cm}^{-1}$;

Enantiomeric excess determined by HPLC analysis (Chiralpak IG, $250 \mathrm{~mm} \times 4.6 \mathrm{~mm}$ column, hexane: iPrOH 92:8, $0.6 \mathrm{~mL} / \mathrm{min}, \lambda=254 \mathrm{~nm}, \mathrm{~T}=25{ }^{\circ} \mathrm{C}$ ), $S$-enantiomer $20.2 \mathrm{~min}, R$ enantiomer $22.4 \min .96 \%$ ee $(R),[\alpha]_{\mathrm{D}}^{22}=-12.1\left(\mathrm{c}=0.35, \mathrm{CHCl}_{3}\right)$;

${ }^{1} \mathrm{H}$ NMR (500 MHz, $\left.\mathrm{CDCl}_{3}\right) \delta$ 7.18-7.15 (2H, m, ArH), 6.69 (1H, t, J=7.3 Hz, ArH), 6.64 $(2 \mathrm{H}, \mathrm{d}, J=7.8 \mathrm{~Hz}, \mathrm{ArH}), 4.07-4.02(1 \mathrm{H}, \mathrm{m}, \mathrm{C} \underline{\mathrm{HOH}}), 3.17(1 \mathrm{H}, \mathrm{dd}, J=12.0,4.2 \mathrm{~Hz}-$ $\left.\mathrm{NHC}_{\underline{H a H}} \mathrm{CH}\right), 3.06\left(1 \mathrm{H}, \mathrm{dd}, J=12.0,7.3 \mathrm{~Hz},-\mathrm{NHCHa} \underline{\mathrm{H}}_{\mathrm{b}} \mathrm{CH}\right), 2.68-2.58(4 \mathrm{H}, \mathrm{m}$, $\mathrm{CHCH}_{\mathrm{a}} \mathrm{H}_{\mathrm{b}} \underline{\mathrm{H}}_{2}+\mathrm{NCH}_{2}$ of piperidine), 2.34 (2H, br.s, $\mathrm{NCH}_{2}$ of piperidine), 1.82-1.74 (1H, m, $\left.\mathrm{CHC}_{\underline{\mathrm{H}}} \mathrm{H}_{\mathrm{b}} \mathrm{CH}_{2}\right), 1.62-1.45\left(7 \mathrm{H}, \mathrm{m}, \mathrm{CHCHa} \underline{\mathrm{H}} \mathrm{CH} \mathrm{CH}_{2}+\mathrm{CH}_{2}\right.$ of piperidine);

${ }^{13} \mathrm{C} \mathrm{NMR}\left(126 \mathrm{MHz}, \mathrm{CDCl}_{3}\right) \delta 148.8,129.3,117.4,113.2,72.2,58.3,50.2,29.4,26.1,24.3$; $m / z(\mathrm{ESI}) 249.2\left[(\mathrm{M}+\mathrm{H})^{+}, 100 \%\right]$. 


\section{1,4-bis(benzyl(methyl)amino)butan-2-ol 59.}

${ }^{1} \mathrm{H}$ NMR (500 MHz, $\left.\mathrm{CDCl}_{3}\right)$.

May $18-2021$

Chemist Shweta Gediya

SKG951

PROTON.w CDCl3 /opt/topspin3.2 SKG 3<smiles>OC(CCN1CCCCC1)CNc1ccccc1</smiles>

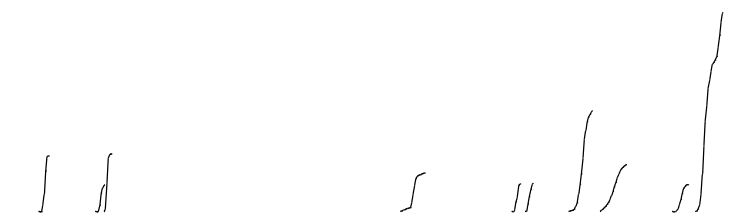

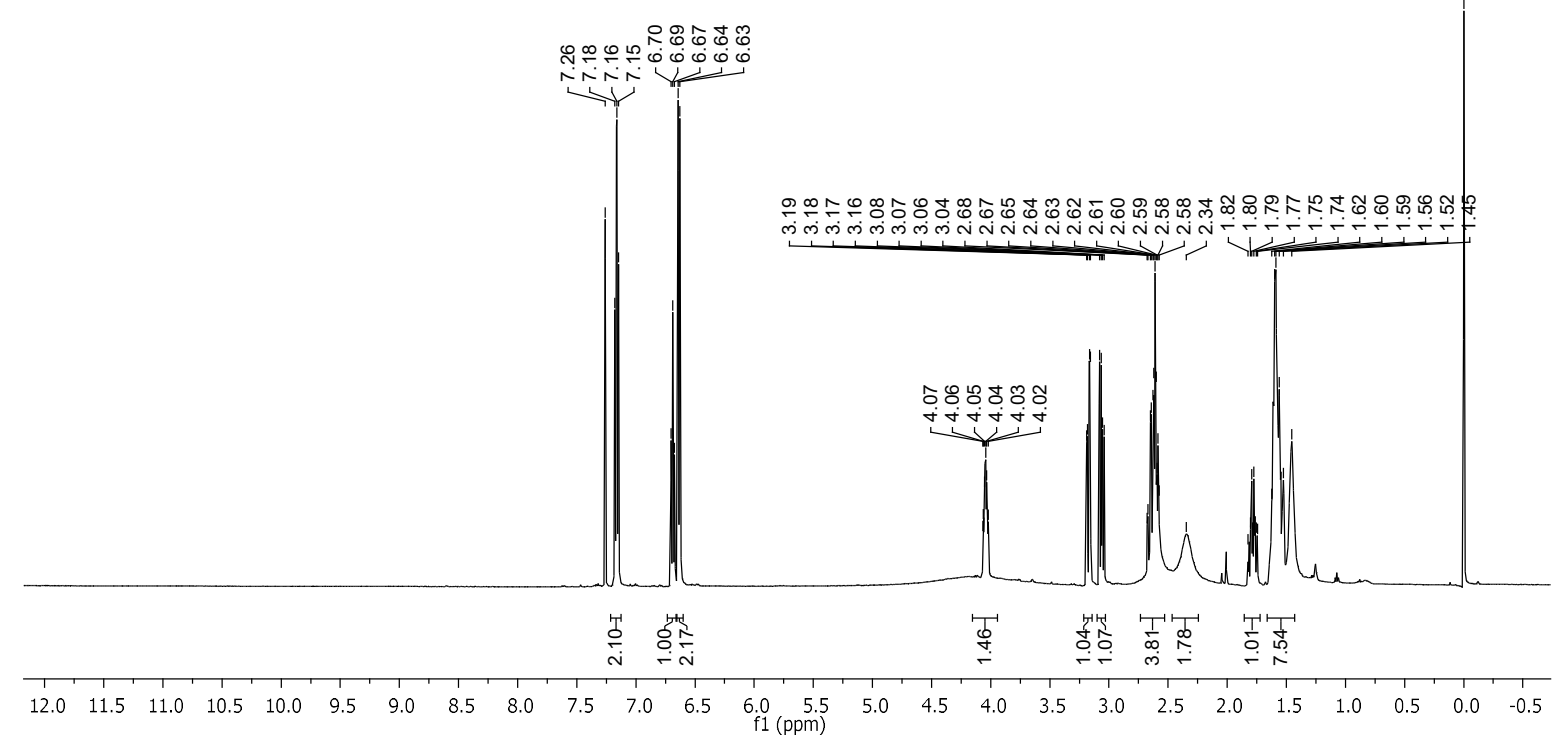

${ }^{13} \mathrm{C}$ NMR (126 MHz, $\left.\mathrm{CDCl}_{3}\right)$.

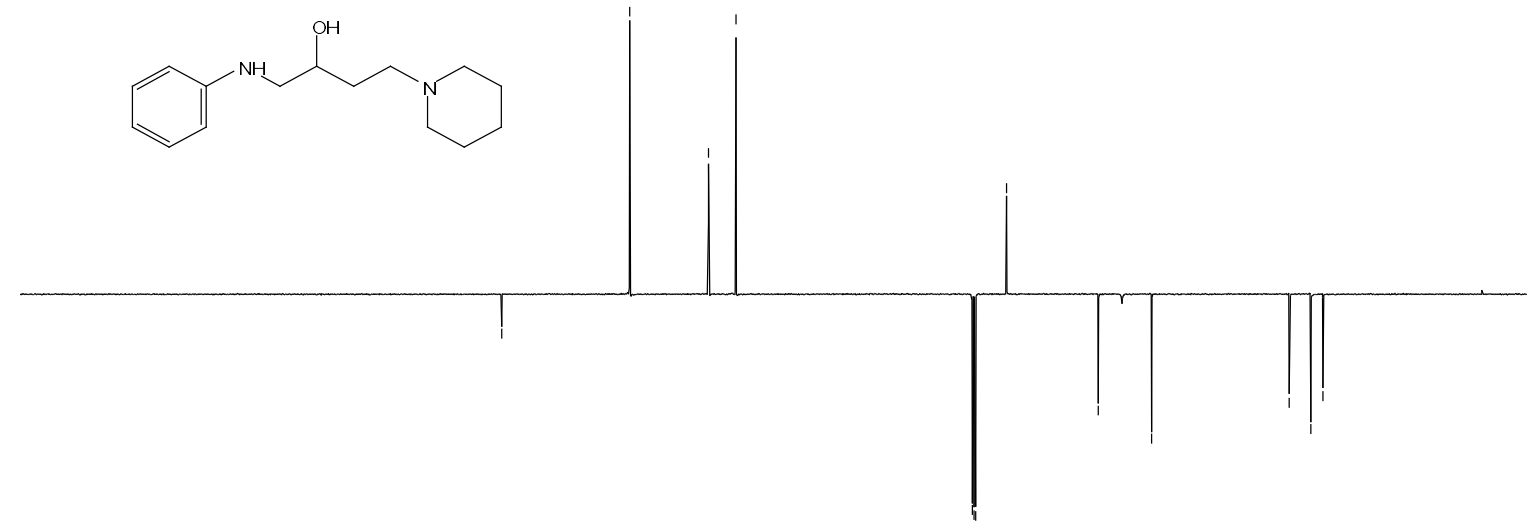

$\begin{array}{llllllllllllllllllllllllll} & 220 & 210 & 200 & 190 & 180 & 170 & 160 & 150 & 140 & 130 & 120 & 110 & 100 & 90 & 80 & 70 & 60 & 50 & 40 & 30 & 20 & 10 & 0\end{array}$ 
COSY.

Chemist Shweta Gediya

COSY.w $\mathrm{CDCl} 3$ /opt/topspin3.2 SKG 3

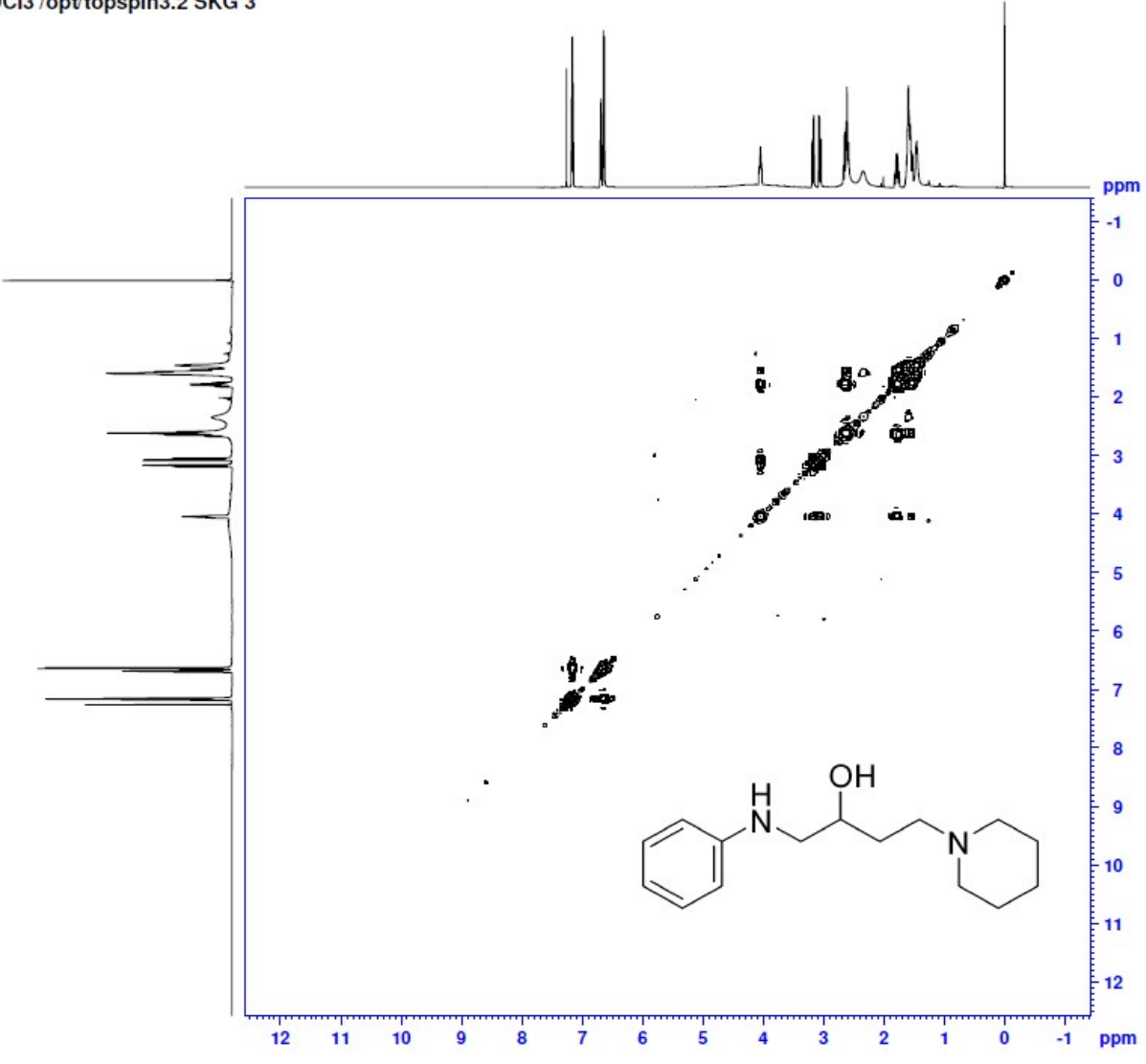

HSQC.

Chemist Shweta Gediya

SKG951 ${ }_{\text {HSQC.w }}$ CDCl3 /opt/topspin3.2 SKG 3

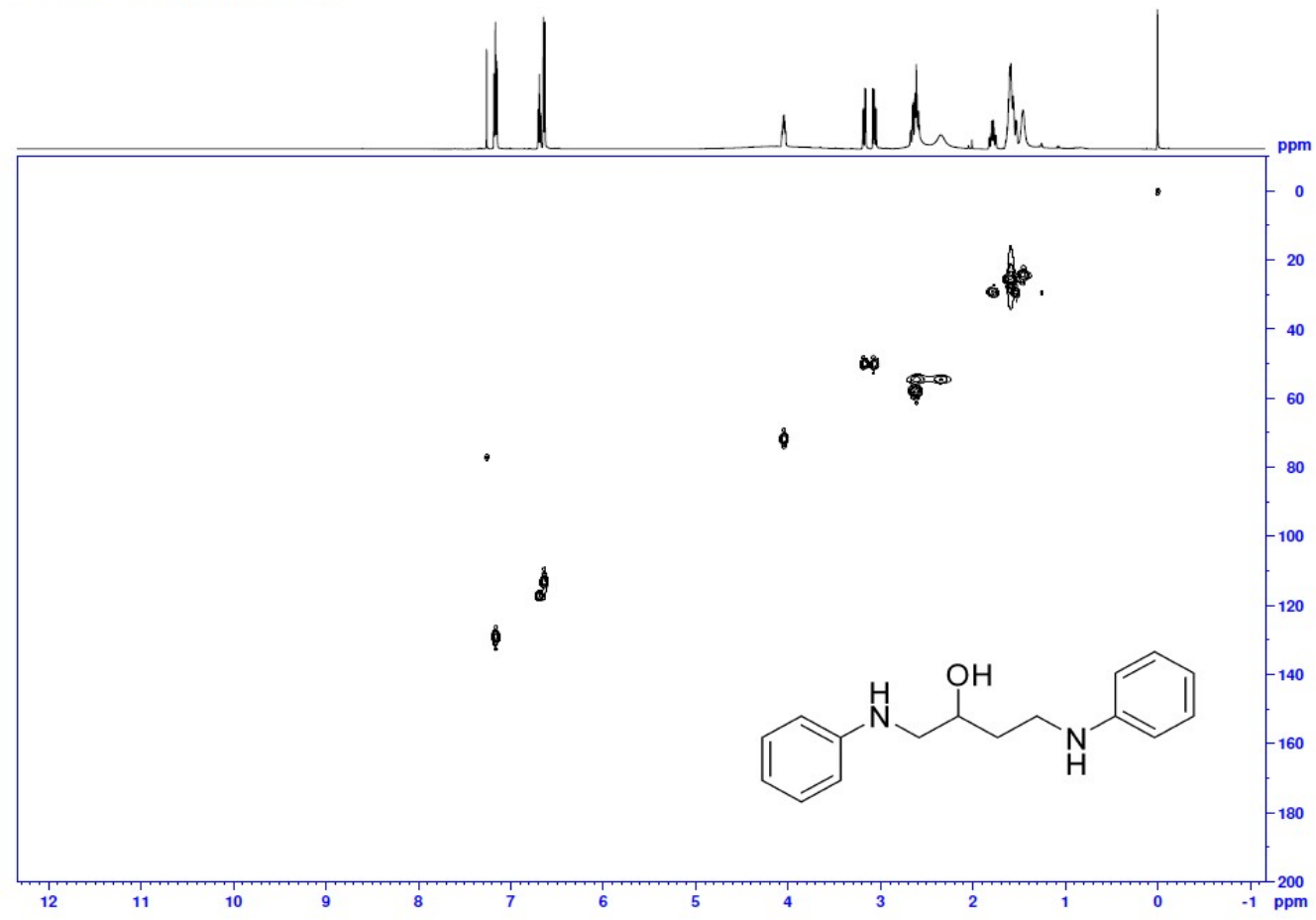


Chiral HPLC of Racemic standard.

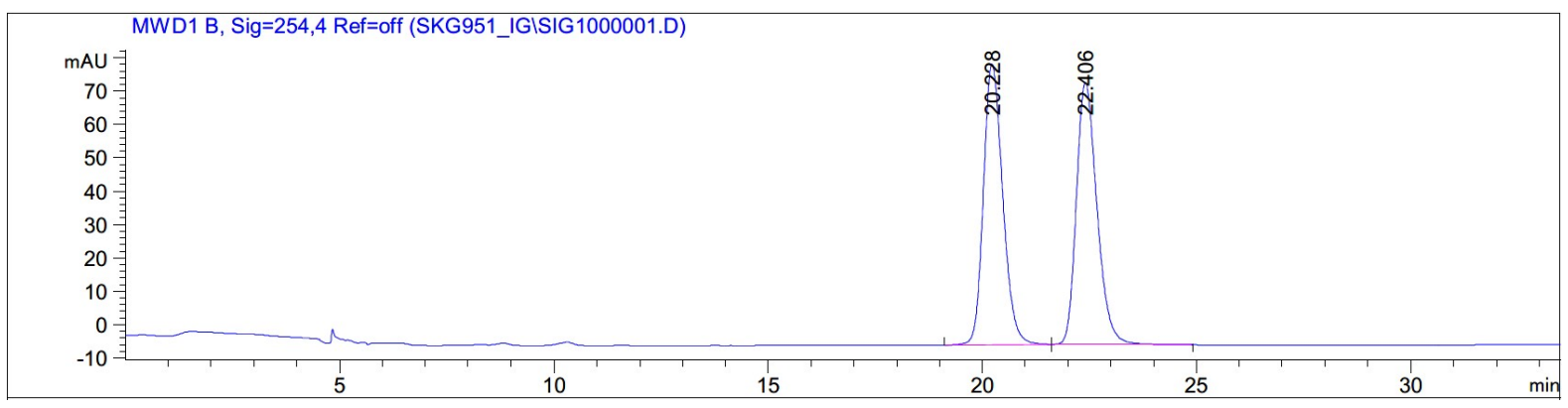

Signal 1: MWD1 B, Sig=254, 4 Ref=off

\begin{tabular}{|c|c|c|c|c|c|c|}
\hline $\begin{array}{c}\text { Peak } \\
\quad \#\end{array}$ & $\begin{array}{c}\text { RetTime } \\
\text { [min] }\end{array}$ & Type & $\begin{array}{l}\text { Width } \\
\text { [min] }\end{array}$ & $\begin{array}{c}\text { Area } \\
{\left[\mathrm{mAU}{ }^{*} \mathrm{~s}\right]}\end{array}$ & $\begin{array}{l}\text { Height } \\
{[\mathrm{mAU}]}\end{array}$ & $\begin{array}{c}\text { Area } \\
\frac{\circ}{0}\end{array}$ \\
\hline & & & & --------- & ----- & \\
\hline 1 & 20.228 & BB & 0.4830 & 2629.23340 & 84.04671 & 0912 \\
\hline 2 & 22.406 & $\mathrm{BB}$ & 0.5161 & 2619.66431 & 78.35108 & 49.9088 \\
\hline
\end{tabular}

Chiral HPLC of ATH product. $96 \%$ ee.

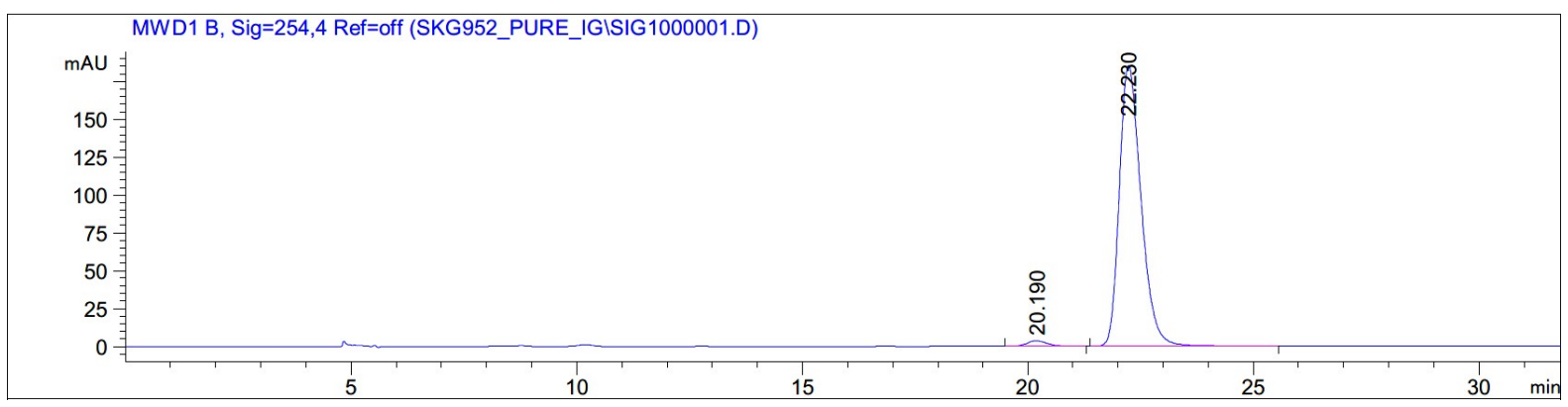

Signal 1: MWD1 B, Sig=254,4 Ref=off

\begin{tabular}{|c|c|c|c|c|c|c|}
\hline $\begin{array}{c}\text { eak } \\
\text { \# }\end{array}$ & $\begin{array}{l}\text { RetTime } \\
\text { [min] }\end{array}$ & Type & $\begin{array}{c}\text { Width } \\
\text { [min] }\end{array}$ & $\begin{array}{c}\text { Area } \\
{\left[\mathrm{mAU}{ }^{*} \mathrm{~s}\right]}\end{array}$ & $\begin{array}{l}\text { Height } \\
\text { [mAU] }\end{array}$ & $\begin{array}{c}\text { Area } \\
\frac{\circ}{0}\end{array}$ \\
\hline & & & & -- & --0 & \\
\hline 1 & 20 . & BB & & 112. & 46 & 868 \\
\hline 2 & 2.230 & BB & 0 . & 6209.47559 & 185.45047 & 98.2132 \\
\hline
\end{tabular}




\section{(R)-1,4-bis(benzyl(methyl)amino)butan-2-ol 60.}<smiles>CN(CC[C@H](O)CN(C)Cc1ccccc1)Cc1ccccc1</smiles>

This compound is novel and was prepared following general procedure $\mathbf{E}$ using ethyl $(R)-4-$ (benzyl(methyl)amino)-3-hydroxy-4-oxobutanoate 7 (0.100 g, $0.377 \mathrm{mmol}, 1.0 \mathrm{eq}), \mathrm{LiAlH}_{4}$ (2M in THF) (0.47 mL, $0.944 \mathrm{mmol}, 1.2 \mathrm{eq})$ in THF $(6 \mathrm{~mL})$ to generate crude material which was further purified by column chromatography $\left(10 \% \mathrm{MeOH}\right.$ in $\left.\mathrm{CHCl}_{3}\right)$ to afford $\mathbf{6 0}$ as a yellow oil (72.3 mg, $0.272 \mathrm{mmol}, 72 \%)$.

TLC: $\mathrm{R}_{\mathrm{f}}$ ca 0.2 (9:1, $\left.\mathrm{CHCl}_{3}: \mathrm{MeOH}\right), \mathrm{UV}$ active, $\mathrm{KMnO}_{4}$ reactive and PMA-reactive; HRMS (ESI) m/z: [M+H] $]^{+}$Calcd for $\mathrm{C}_{20} \mathrm{H}_{29} \mathrm{~N}_{2} \mathrm{O} 313.2274$; Found 313.2270 (error 1.5 ppm); $U_{\max } 3358,2941,2790,1452,1364,1074,735,696 \mathrm{~cm}^{-1}$;

Enantiomeric excess determined by HPLC analysis (Chiralpak IG, $250 \mathrm{~mm} \times 4.6 \mathrm{~mm}$ column, hexane: $\mathrm{PrOH} 93: 7,0.8 \mathrm{~mL} / \mathrm{min}, \lambda=210 \mathrm{~nm}, \mathrm{~T}=25^{\circ} \mathrm{C}$ ), $R$-enantiomer $10.5 \mathrm{~min}, S$ enantiomer $11.4 \min .93 \%$ ee $(R),[\alpha]_{\mathrm{D}}^{22}=-20\left(\mathrm{c}=0.062, \mathrm{CHCl}_{3}\right)$;

${ }^{1} \mathrm{H}$ NMR (500 MHz, $\left.\mathrm{CDCl}_{3}\right) \delta$ 7.32-7.22 (10H, m, ArH), 3.89-3.84 (1H, m, CHOH), 3.56$3.43\left(4 \mathrm{H}, \mathrm{m}, \mathrm{CH}_{2} \mathrm{Ph}\right), 2.68-2.63\left(1 \mathrm{H}, \mathrm{m}, \mathrm{CHCH}_{\mathrm{a}} \mathrm{H}_{\mathrm{b}} \mathrm{CH}_{2}\right), 2.55-2.51\left(1 \mathrm{H}, \mathrm{m}, \mathrm{CHCH}_{\mathrm{a}} \underline{\mathrm{H}}_{\mathrm{b}} \mathrm{CH}_{2}\right)$, $2.43\left(1 \mathrm{H}, \mathrm{dd}, J=12.4,7.8 \mathrm{~Hz}, \mathrm{CHCH}_{\underline{\mathrm{a}}} \mathrm{H}_{\mathrm{b} H} \mathrm{NH}, 2.33\left(1 \mathrm{H}, \mathrm{dd}, J=12.4,5.3 \mathrm{~Hz}, \mathrm{CHCH}_{\underline{a}} \underline{\mathrm{H}} \mathrm{NH}\right)\right.$, $2.22\left(3 \mathrm{H}, \mathrm{s}, \mathrm{NCH}_{3}\right), 2.19\left(3 \mathrm{H}, \mathrm{s}, \mathrm{NCH}_{3}\right), 1.67-1.60\left(2 \mathrm{H}, \mathrm{m}, \mathrm{CHCH}_{\mathrm{a}} \mathrm{H}_{\mathrm{b}} \underline{\mathrm{H}}_{2}\right)$;

${ }^{13} \mathrm{C}$ NMR $\left(126 \mathrm{MHz}, \mathrm{CDCl}_{3}\right) \delta 139.0,138.5,129.3,129.2,128.5,128.4,127.3,127.1,69.3$, 63.5, 62.9, 62.8, 55.8, 42.7, 42.1, 31.4;

$m / z(\mathrm{ESI}) 313.2\left[(\mathrm{M}+\mathrm{H})^{+}, 100 \%\right]$. 


\section{1,4-Bis(benzyl(methyl)amino)butan-2-ol 60.}

${ }^{1} \mathrm{H}$ NMR (500 MHz, $\left.\mathrm{CDCl}_{3}\right)$.

May11-2021

Chemist Shweta Gediya

PROTON.w CDCl3 /opt/topspin3.2 SKG 44

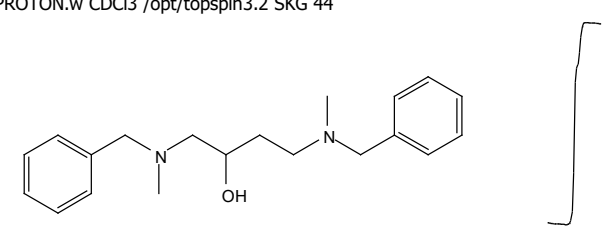

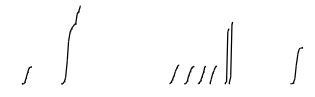

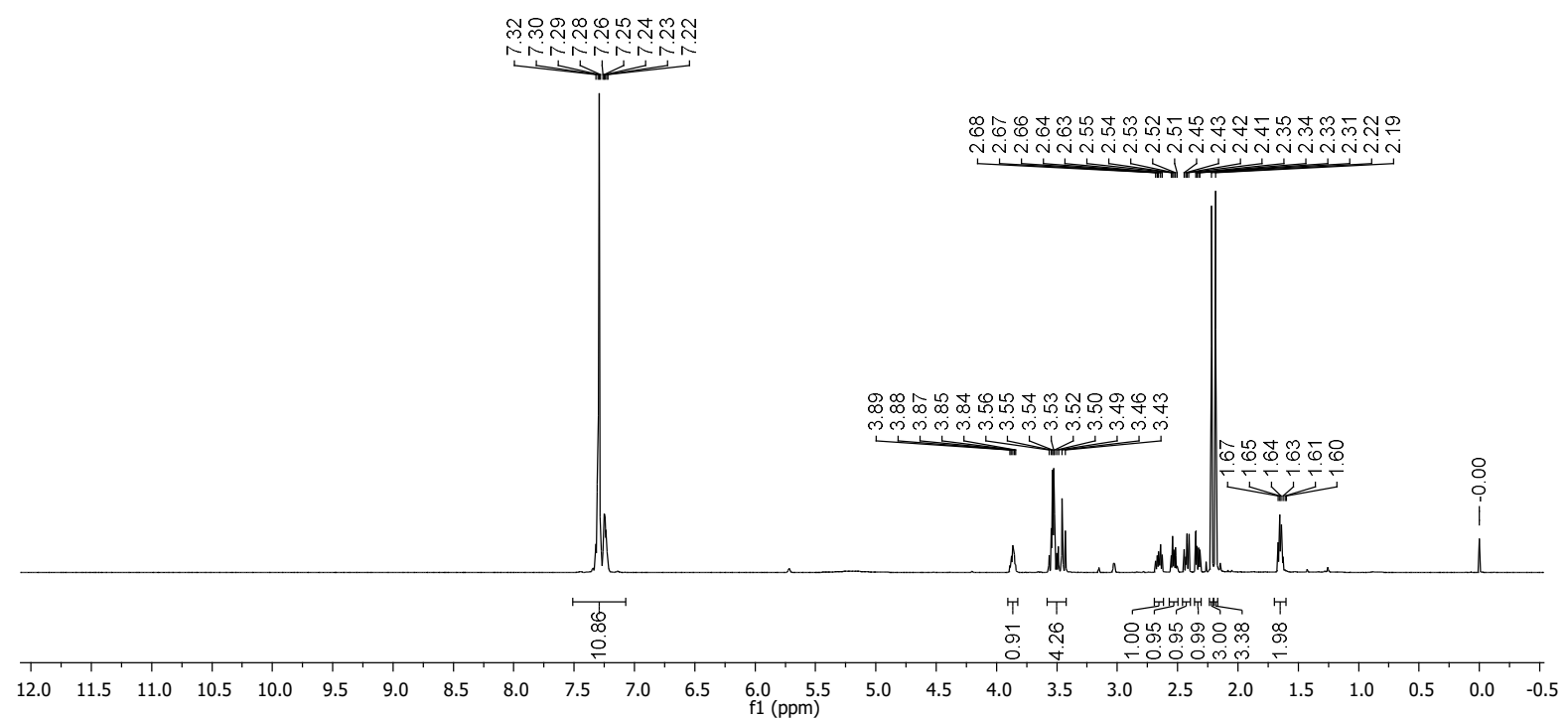

${ }^{13} \mathrm{C} \mathrm{NMR}\left(126 \mathrm{MHz}, \mathrm{CDCl}_{3}\right)$.<smiles>CN(CCC(O)CN(C)Cc1ccccc1)Cc1ccccc1</smiles>

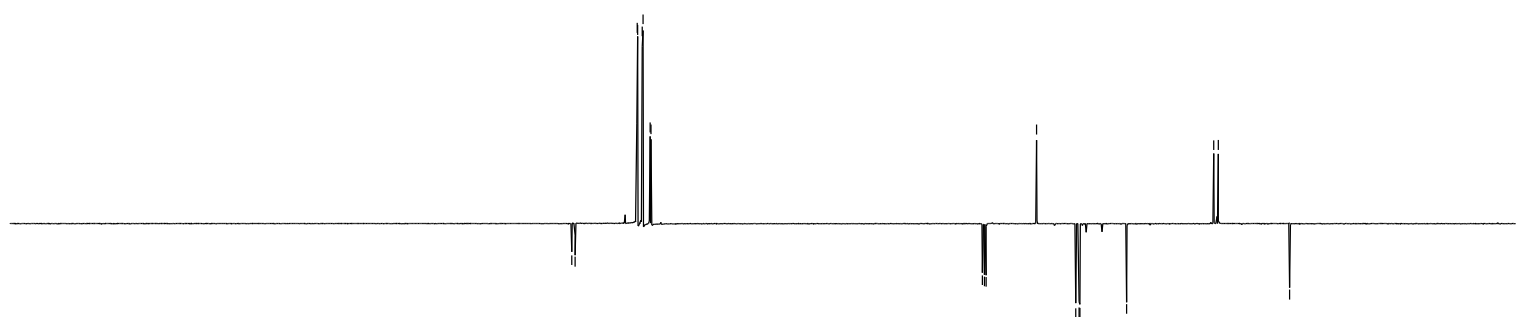

$\begin{array}{lllllllllllllllllllllllll}220 & 210 & 200 & 190 & 180 & 170 & 160 & 150 & 140 & 130 & 120 & \begin{array}{l}110 \\ 110\end{array} & 100 & 90 & 80 & 70 & 60 & 50 & 40 & 30 & 20 & 10 & 0\end{array}$ 
COSY.

Chemist Shweta Gediya

COSY.w CDCl3 /opt/topspin3.2 SKG 44

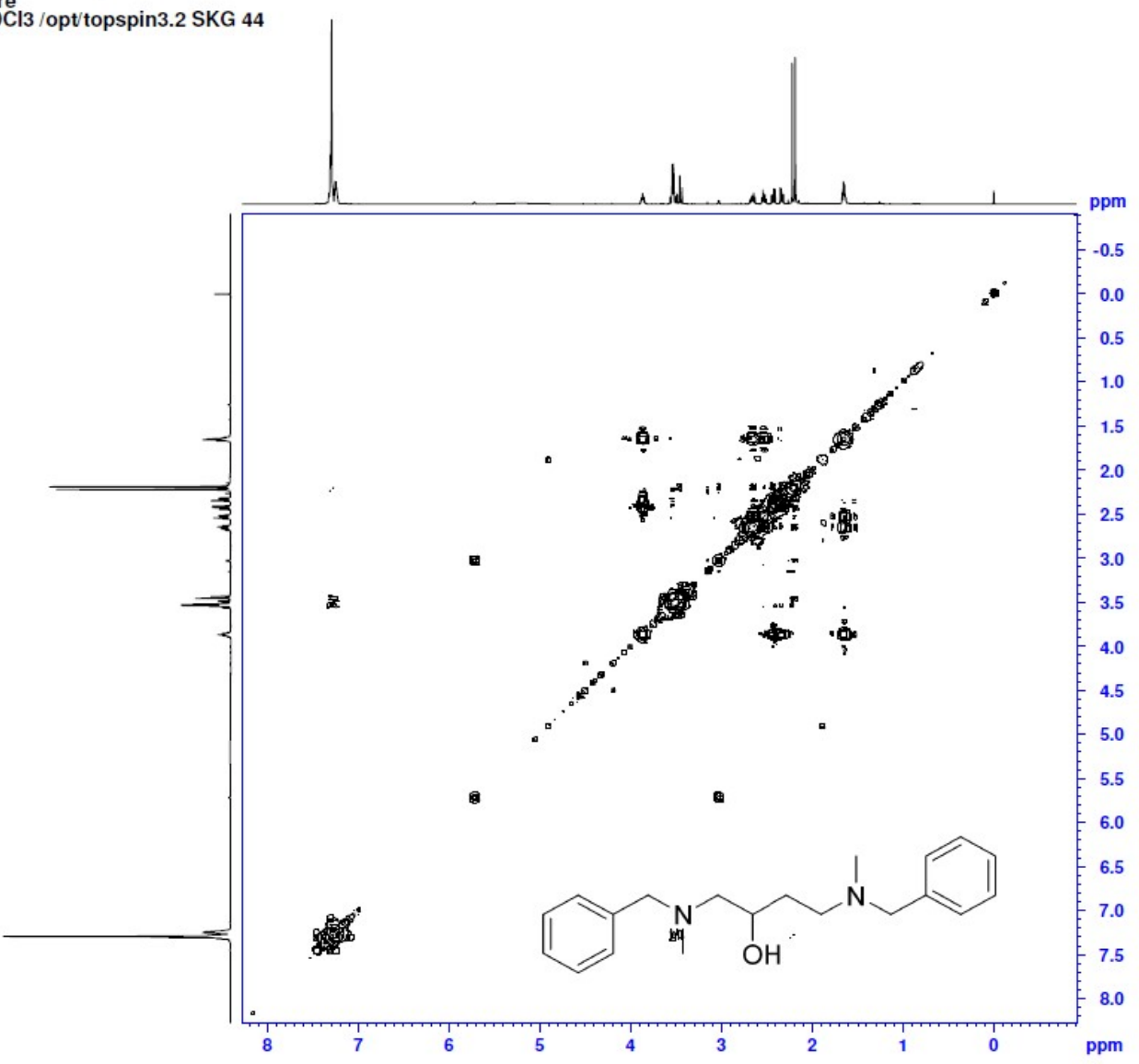

HSQC.

Chemist Shweta Gediya

SKG947 pure 1 HSQC.w CDCl3 /opttopspin3.2 SKG 44

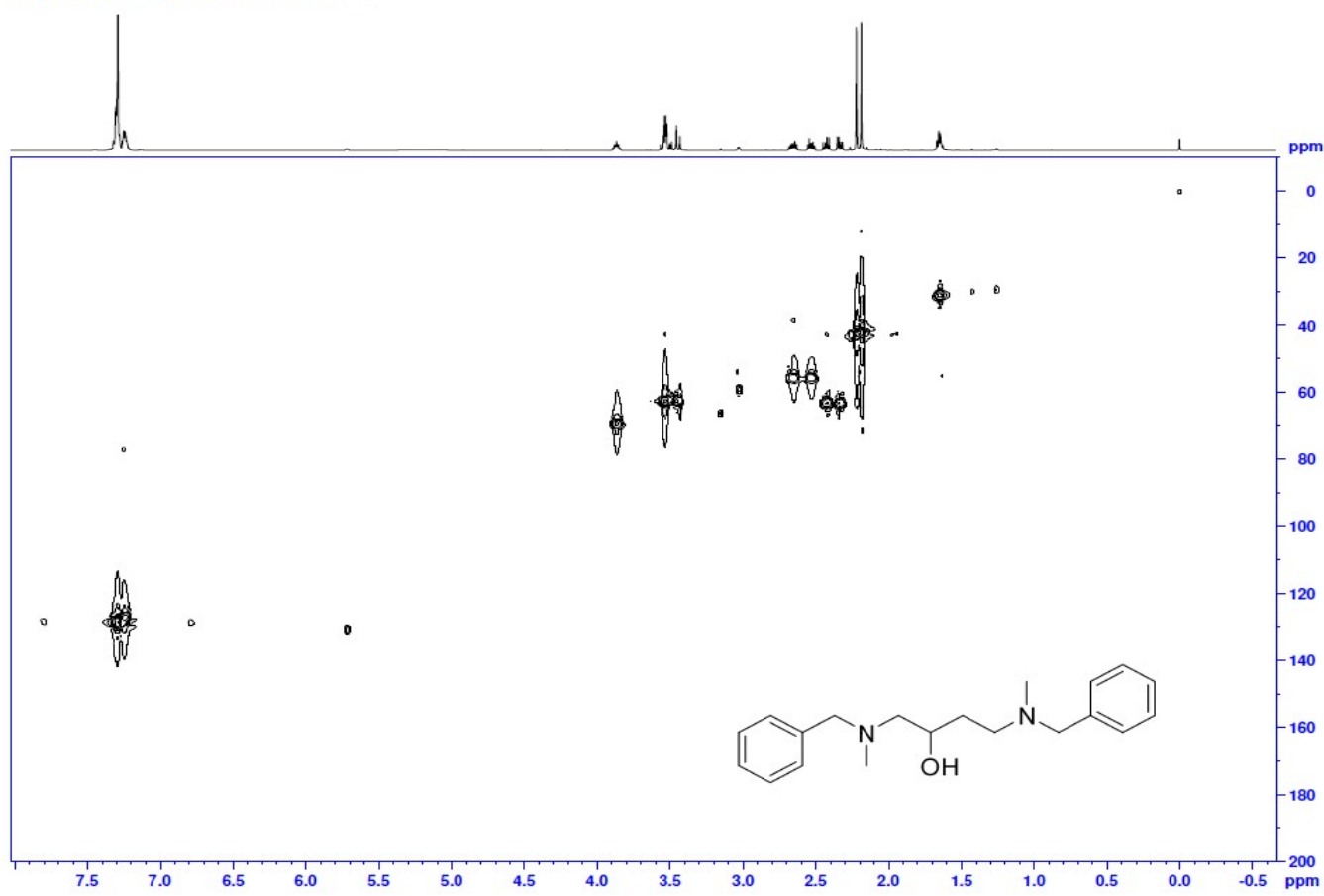




\section{Chiral HPLC of racemic standard.}

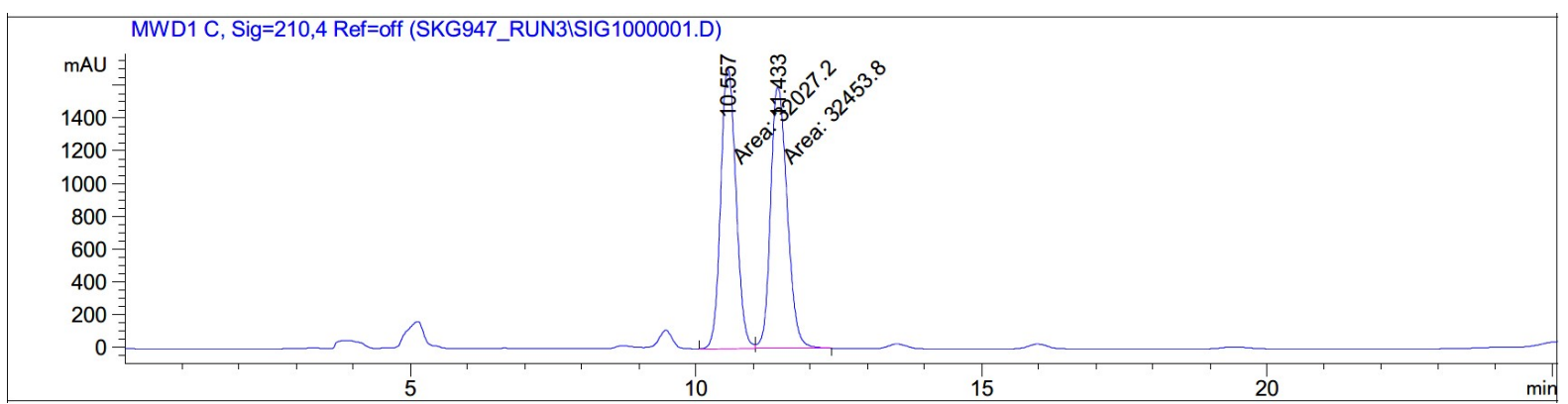

Signal 2: MWD1 C, Sig=210,4 Ref=off

\begin{tabular}{|c|c|c|c|c|c|c|}
\hline $\begin{array}{c}\text { Peak } \\
\quad \#\end{array}$ & $\begin{array}{c}\text { RetTime } \\
\text { [min] }\end{array}$ & Type & $\begin{array}{l}\text { Width } \\
{[\mathrm{min}]}\end{array}$ & $\begin{array}{c}\text { Area } \\
{\left[\mathrm{mAU}{ }^{*} \mathrm{~s}\right]}\end{array}$ & $\begin{array}{l}\text { Height } \\
{[\mathrm{mAU}]}\end{array}$ & $\begin{array}{c}\text { Area } \\
\frac{\circ}{0}\end{array}$ \\
\hline- & & & & --- & ---------- & --- \\
\hline 1 & 10.557 & $\mathrm{MF}$ & 0.3109 & $3.20272 \mathrm{e} 4$ & 1716.63123 & 49.6692 \\
\hline 2 & 11.433 & FM & 0.3379 & $3.24538 \mathrm{e} 4$ & 1600.55615 & 50.3308 \\
\hline
\end{tabular}

Chiral HPLC of ATH product. 93\% ee.

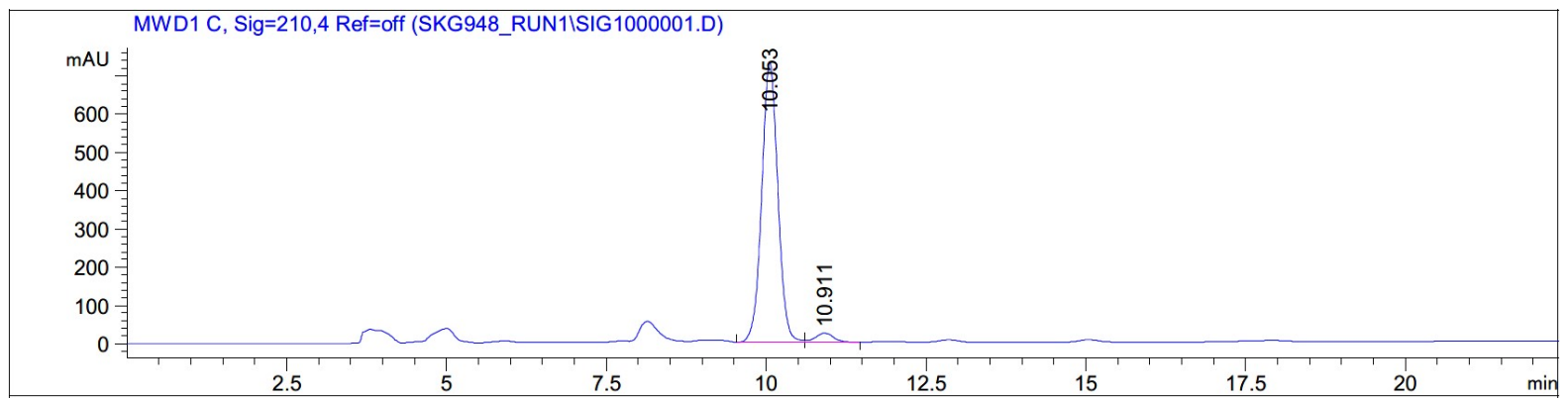

Signal 2: MWD1 C, Sig=210,4 Ref=off

\begin{tabular}{|c|c|c|c|c|c|c|}
\hline $\begin{array}{c}\text { eak } \\
\#\end{array}$ & $\begin{array}{c}\text { RetTime } \\
\text { [min] }\end{array}$ & Type & $\begin{array}{l}\text { Width } \\
\text { [min] }\end{array}$ & $\begin{array}{c}\text { Area } \\
{\left[\mathrm{mAU}^{\star} \mathrm{s}\right]}\end{array}$ & $\begin{array}{l}\text { Height } \\
{[\mathrm{mAU}]}\end{array}$ & $\begin{array}{c}\text { Area } \\
\quad \%\end{array}$ \\
\hline & & & & & & \\
\hline 1 & & $\mathrm{~V}$ & & 1.2 & 313 & 53 \\
\hline 2 & & VV & & 465.43066 & 3.49374 & 947 \\
\hline
\end{tabular}


(R)-N-(2,6-Diisopropylphenyl)-2,4-dihydroxybutanamide 61.<smiles>CC(C)c1cccc(C(C)C)c1NC(=O)[C@H](O)CCO</smiles>

This compound is novel and was prepared following general procedure D using ethyl $(R)-4$ ((2,6-diisopropylphenyl)amino)-3-hydroxy-4-oxobutanoate 33 (100 mg, $0.318 \mathrm{mmol}, 1.0$ eq), $\mathrm{NaBH}_{4}(76 \mathrm{mg}, 3.18 \mathrm{mmol}, 10 \mathrm{eq})$ in methanol $(5 \mathrm{~mL})$ and water $(1 \mathrm{~mL})$ to generate crude material which was further purified by column chromatography $\left(10 \% \mathrm{MeOH}\right.$ in $\left.\mathrm{CHCl}_{3}\right)$ to afford 61 as a white solid (82.0 mg, $0.293 \mathrm{mmol}, 92 \%)$.

TLC: $\mathrm{R}_{\mathrm{f}}$ ca 0.2 (9:1, $\left.\mathrm{CHCl}_{3}: \mathrm{MeOH}\right)$, light $\mathrm{UV}$ active, $\mathrm{KMnO}_{4}$ reactive and PMA-reactive; HRMS (ESI) m/z: $[\mathrm{M}+\mathrm{H}]^{+}$Calcd for $\mathrm{C}_{16} \mathrm{H}_{25} \mathrm{NO}_{3} \mathrm{Na}$ 302.1727; Found 302.1728 (error -0.5 ppm);

$U_{\max } 3500,3328,2961,1636,1513,1114,1052,807 \mathrm{~cm}^{-1}$;

Enantiomeric excess determined by HPLC analysis (Chiralpak IC, $250 \mathrm{~mm} \times 4.6 \mathrm{~mm}$ column, hexane: $\mathrm{PPOH} 85: 15,0.6 \mathrm{~mL} / \mathrm{min}, \lambda=210 \mathrm{~nm}, \mathrm{~T}=25^{\circ} \mathrm{C}$ ), $S$-enantiomer $9.9 \mathrm{~min}, R$ enantiomer $12.3 \mathrm{~min} .98 \%$ ee $(R),[\alpha]_{\mathrm{D}}^{22}=+126\left(\mathrm{c}=0.1, \mathrm{CHCl}_{3}\right)$;

${ }^{1} \mathrm{H}$ NMR $\left(500 \mathrm{MHz}, \mathrm{CDCl}_{3}\right) \delta 8.24(1 \mathrm{H}, \mathrm{s}, \mathrm{CONH}), 7.30(1 \mathrm{H}, \mathrm{t}, J=7.7 \mathrm{~Hz}, \mathrm{ArH}), 7.18(2 \mathrm{H}$, d, $J=7.7 \mathrm{~Hz}, \operatorname{ArH}), 4.64(1 \mathrm{H}, \mathrm{s}, \mathrm{CHO} \underline{\mathrm{H}}), 4.48-4.46(1 \mathrm{H}, \mathrm{m}, \underline{\mathrm{C}} \underline{\mathrm{HOH}}), 3.95-3.87$ (2H, m, $\left.\mathrm{C}_{2} \mathrm{OH}\right), 3.05-3.01\left(3 \mathrm{H}, \mathrm{m}, \mathrm{CH}\right.$ of isopropyl group $\left.+\mathrm{CH}_{2} \mathrm{O} \underline{\mathrm{H}}\right), 2.23-2.17\left(1 \mathrm{H}, \mathrm{m}, \mathrm{C}_{\underline{a}} \mathrm{H}_{\mathrm{b}}\right)$, 2.03-1.96 (1H, m, $\left.\mathrm{CH}_{\mathrm{a}} \underline{\mathrm{H}} \mathrm{b}\right), 1.19\left(12 \mathrm{H}, \mathrm{d}, J=6.8 \mathrm{~Hz}, \mathrm{CH}_{3}\right.$ of isopropyl group);

${ }^{13} \mathrm{C} \mathrm{NMR}\left(126 \mathrm{MHz}, \mathrm{CDCl}_{3}\right) \delta 173.4,146.2,130.5,128.6,123.6,73.1,61.8,35.8,28.9,23.8$, 23.7;

$m / z(\mathrm{ESI}) 302.2\left[(\mathrm{M}+\mathrm{Na})^{+}, 100 \%\right]$. 
N-(2,6-diisopropylphenyl)-2,4-dihydroxybutanamide 61.

${ }^{1} \mathrm{H}$ NMR $\left(500 \mathrm{MHz}, \mathrm{CDCl}_{3}\right)$.

May05-2021

Chemist Shweta Gediya

SKG924

PROTON.w CDCl3 /opt/topspin3.2 SKG 28

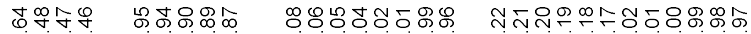

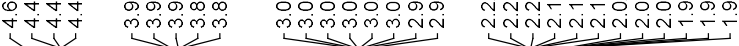

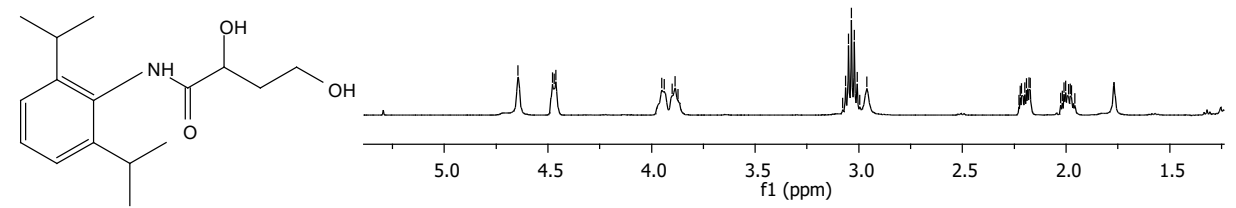

,

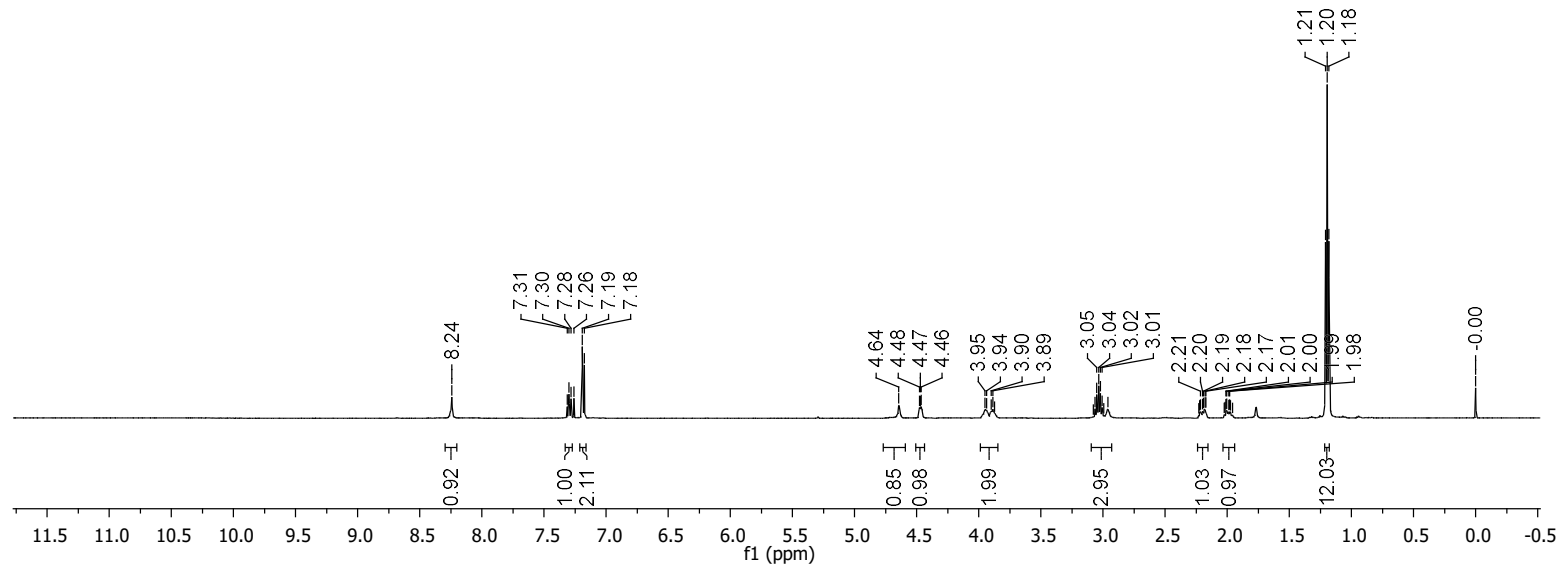

${ }^{13} \mathrm{C} \mathrm{NMR}\left(126 \mathrm{MHz}, \mathrm{CDCl}_{3}\right)$.

May05-2021

Chemist Shweta Gediya

SKG924

C13APT.w CDCl3 /opt/topspin3.2 SKG 28

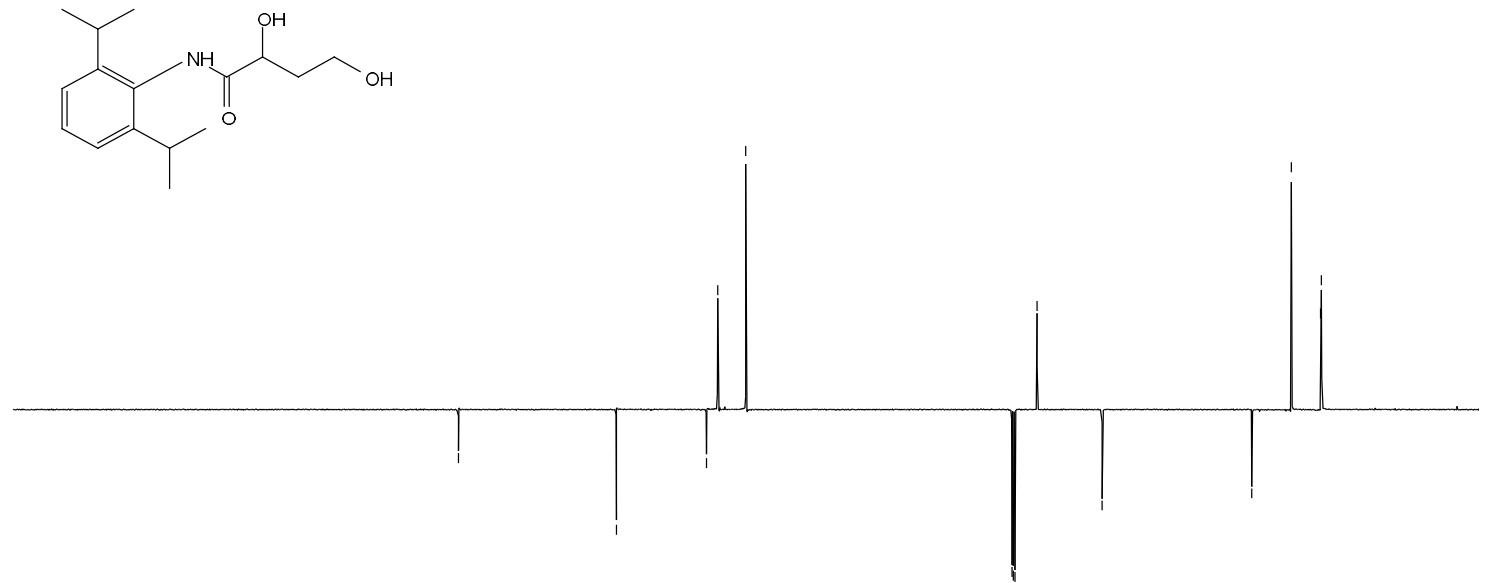

$\begin{array}{rlllllllllllllllllllllllllllllllllllll}250 & 240 & 230 & 220 & 210 & 200 & 190 & 180 & 170 & 160 & 150 & 140 & 130 & 120 & 110 & 100 & 90 & 80 & 70 & 60 & 50 & 40 & 30 & 20 & 10 & 0\end{array}$ 
COSY.

Chemist Shweta Gediya

COSY.w CDCl3 /opt/topspin3.2 SKG 28

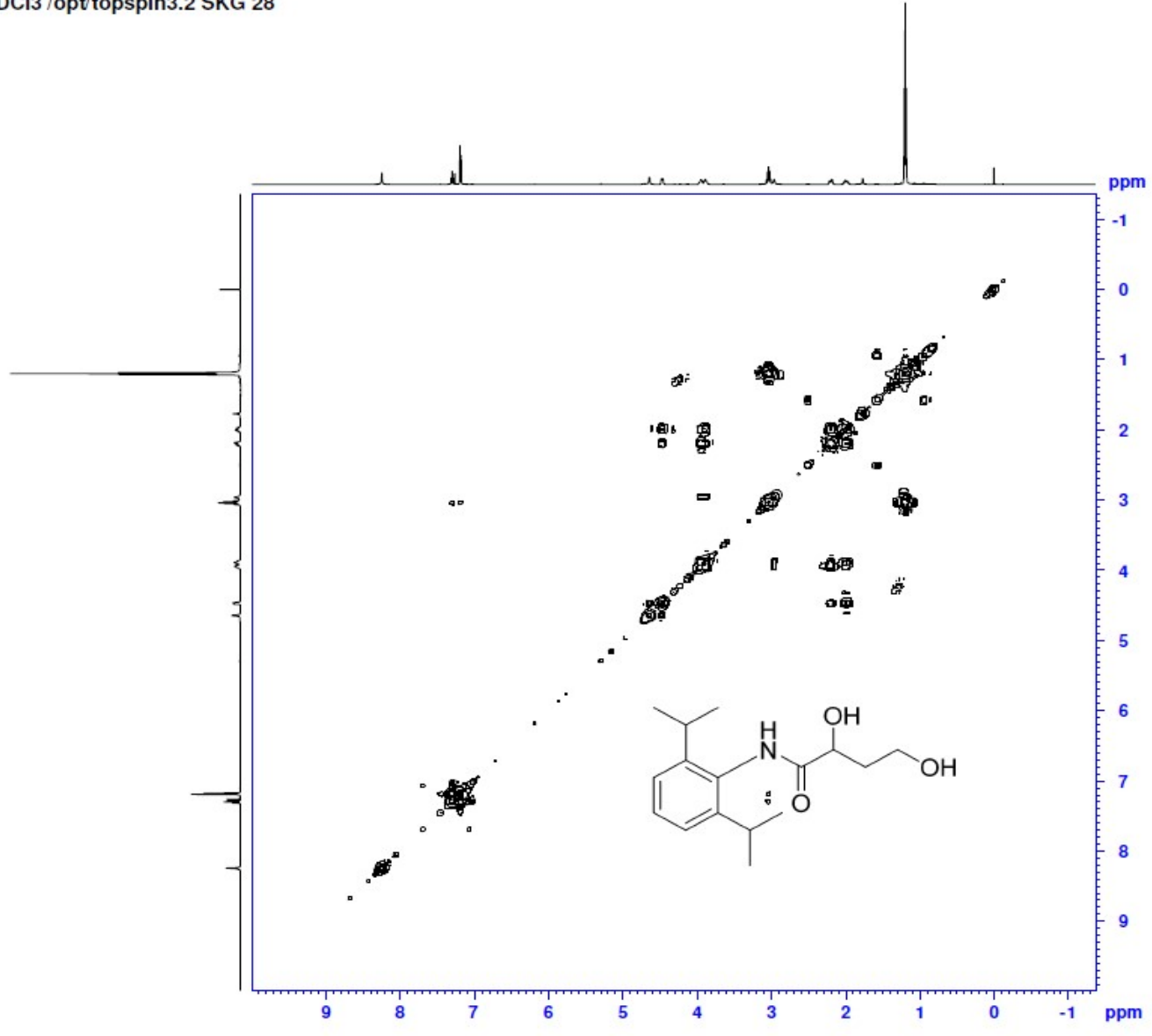

HSQC.

Chemist Shweta Gediya

HSGC.w CDCl3 /opttopspin3.2 SKG 28

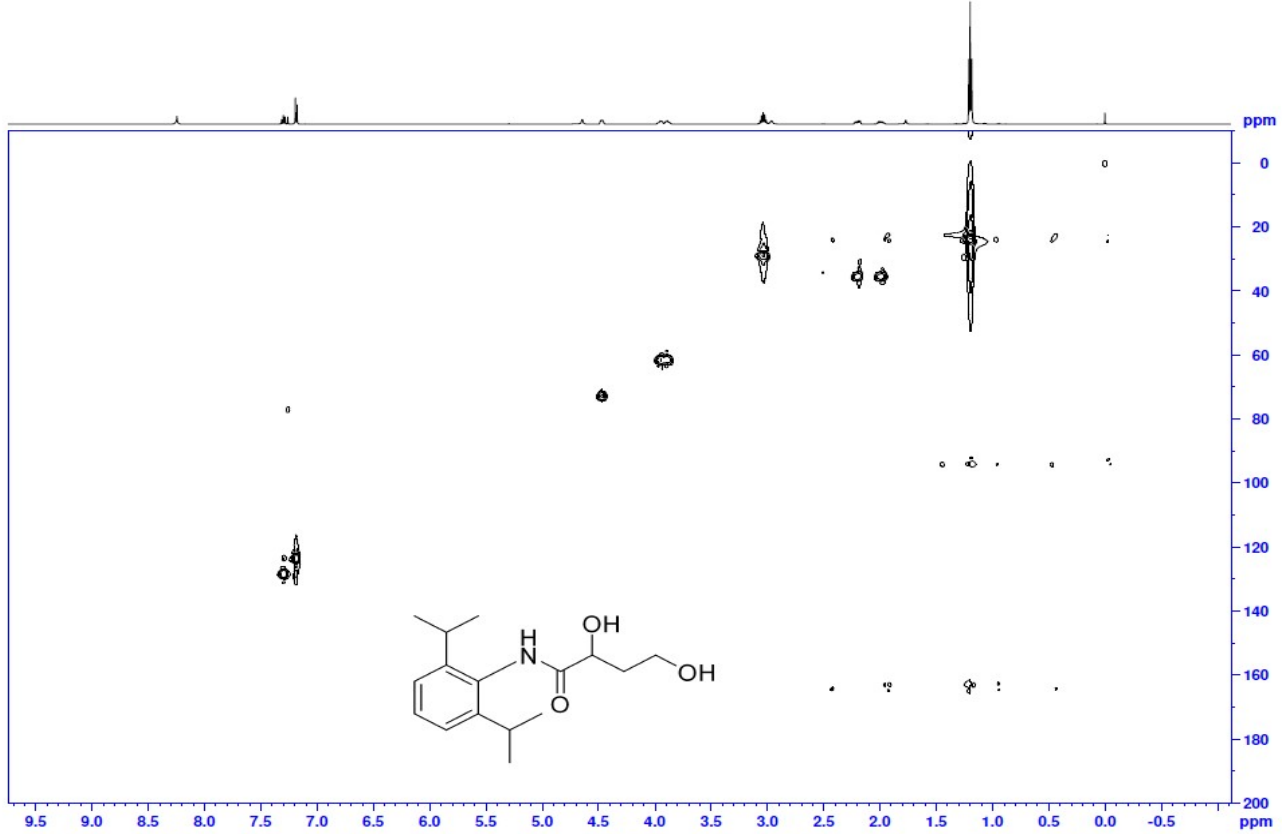




\section{Chiral HPLC of racemic standard.}

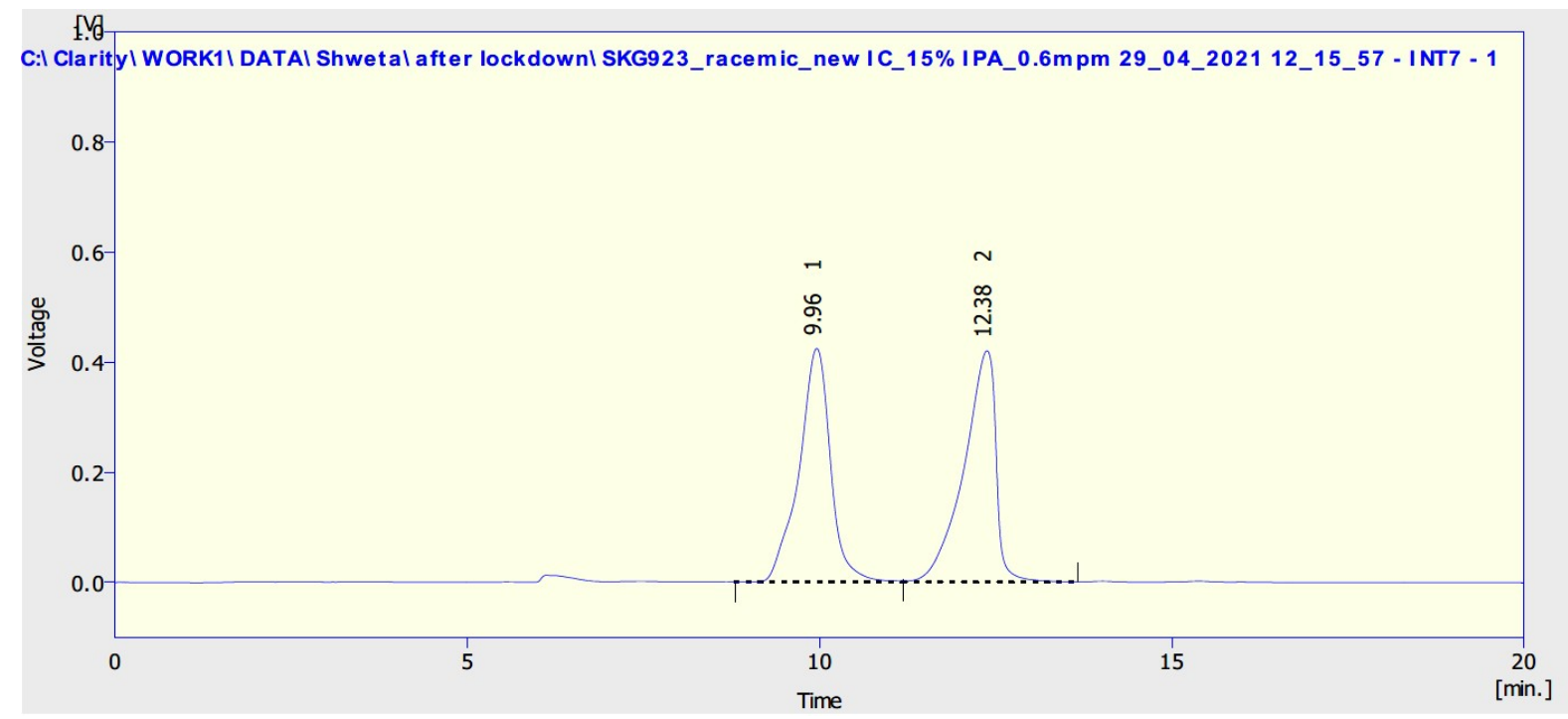

\begin{tabular}{|c|c|c|c|c|c|c|c|}
\hline & $\begin{array}{c}\text { Reten. Time } \\
{[\mathrm{min}]}\end{array}$ & $\begin{array}{c}\text { Area } \\
{[\mathrm{mV} . \mathrm{s}]}\end{array}$ & $\begin{array}{c}\text { Height } \\
{[\mathrm{mV}]}\end{array}$ & $\begin{array}{c}\text { Area } \\
{[\%]}\end{array}$ & $\begin{array}{c}\text { Height } \\
{[\%]}\end{array}$ & $\begin{array}{c}\text { W05 } \\
{[\mathrm{min}]}\end{array}$ & $\begin{array}{c}\text { Compound } \\
\text { Name }\end{array}$ \\
\hline 1 & 9.960 & 13191.670 & 424.082 & 49.8 & 50.3 & 0.44 \\
\hline 2 & 12.377 & 13309.834 & 419.669 & 50.2 & 49.7 & 0.46 & \\
\hdashline & Total & 26501.504 & 843.751 & 100.0 & 100.0 & & \\
\hline
\end{tabular}

\section{Chiral HPLC of ATH products. $98 \%$ ee.}

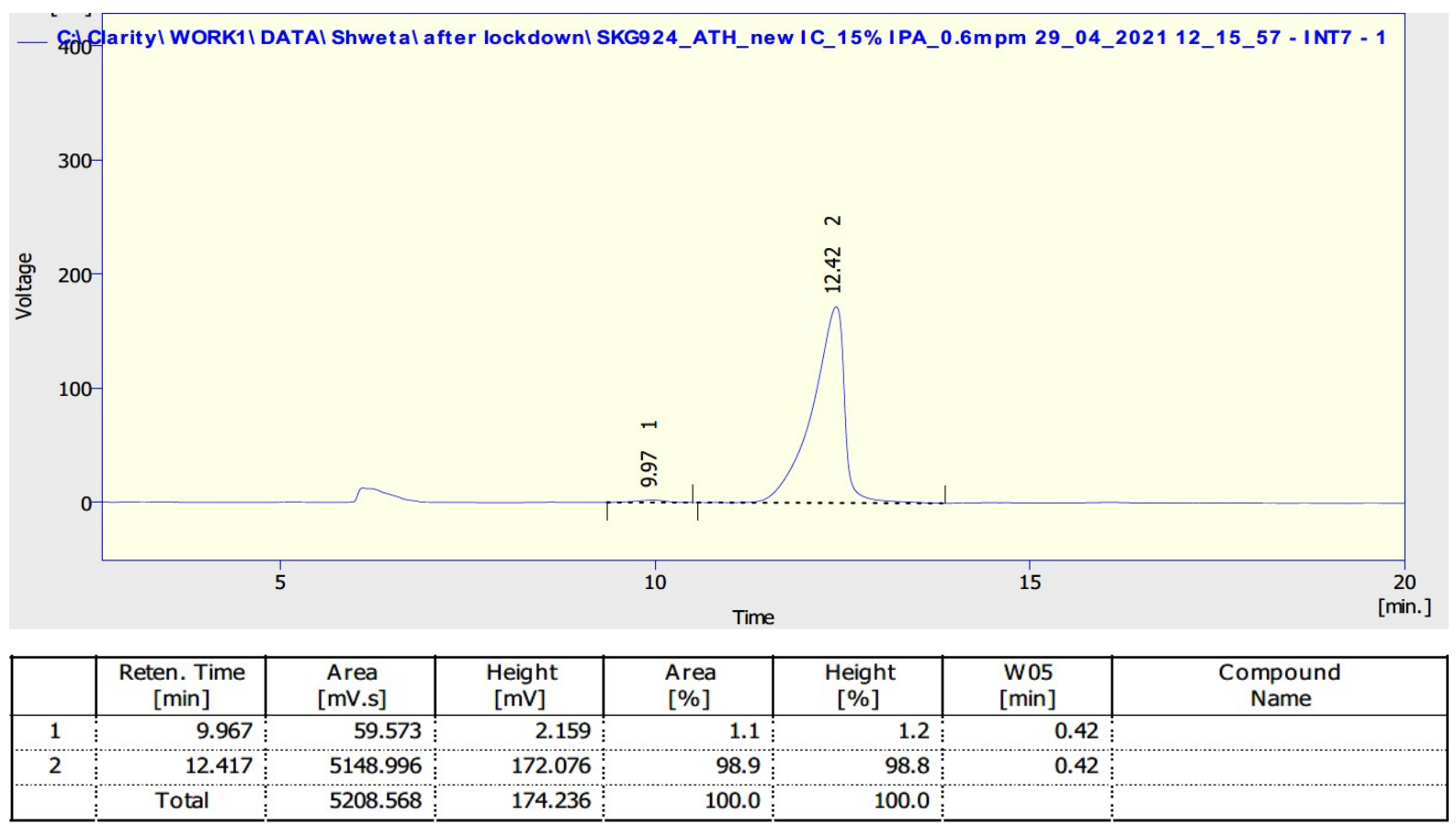


(R)-4-((2,6-Diisopropylphenyl)amino)butane-1,3-diol.<smiles>CC(C)c1cccc(C(C)C)c1NCC(O)CCO</smiles>

This compound is novel and was prepared following general procedure $\mathbf{E}$ using ethyl $(R)-4-$ ((2,6-diisopropylphenyl)amino)-3-hydroxy-4-oxobutanoate 33 (250 mg, $0.784 \mathrm{mmol}, 1.0 \mathrm{eq})$, $\mathrm{LiAlH}_{4}(2 \mathrm{M}$ in THF) $(0.979 \mathrm{~mL}, 1.95 \mathrm{mmol}, 1.2 \mathrm{eq})$ in THF $(10 \mathrm{~mL})$ to generate crude material which was further purified by column chromatography $(80 \%$ EtOAc in pet ether) to afford the product as a clear liquid (125 $\mathrm{mg}, 0.472 \mathrm{mmol}, 60 \%)$. We were unable to achieve a HPLC separation for this product, therefore cannot confirm whether the ee has been reduced. However by analogy with other examples, this would not be expected.

TLC: $\mathrm{R}_{\mathrm{f}}$ ca 0.2 (2:8, Hexane: EtOAc), light UV active, $\mathrm{KMnO}_{4}$ reactive and PMA-reactive; HRMS (ESI) m/z: [M+Na] ${ }^{+}$Calcd for $\mathrm{C}_{16} \mathrm{H}_{28} \mathrm{NO}_{2}$ 266.2115; Found 266.2124 (error -3.6 ppm);

$U_{\max } 3342,2961,2868,1457,1446,1098,1054,948,756 \mathrm{~cm}^{-1}$;

${ }^{1} \mathrm{H}$ NMR $\left(500 \mathrm{MHz}, \mathrm{CDCl}_{3}\right) \delta$ 7.12-7.06 (3H, m, ArH), 4.12-4.08 (1H, m, CㅂOH), 3.94-3.86 $\left(2 \mathrm{H}, \mathrm{m}, \mathrm{CH}_{2} \mathrm{C}_{2} \mathrm{OH}\right), 3.32-3.24(2 \mathrm{H}, \mathrm{m}, \mathrm{CH}$ of isopropyl group), 2.97 (1H, dd, $J=12.1,3.4$ $\left.\mathrm{Hz}, \mathrm{C}_{\underline{a}} \mathrm{H}_{b} \mathrm{CH}\right), 2.86\left(1 \mathrm{H}, \mathrm{dd}, J=12.1,8.3 \mathrm{~Hz}, \mathrm{CH}_{a} \underline{\mathrm{H}_{b}} \mathrm{CH}\right), 1.84-1.70\left(2 \mathrm{H}, \mathrm{m}, \mathrm{C}_{2} \mathrm{CH}_{2} \mathrm{OH}\right)$, $1.24\left(12 \mathrm{H}, \mathrm{d}, J=6.9 \mathrm{~Hz}, \mathrm{CH}_{3}\right.$ of isopropyl group);

${ }^{13} \mathrm{C} \mathrm{NMR}\left(126 \mathrm{MHz}, \mathrm{CDCl}_{3}\right) \delta 142.9,142.5,124.5,123.8,71.0,61.2,57.7,36.4,27.7,24.4$, 24.3;

$m / z(\mathrm{ESI}) 266.2\left[(\mathrm{M}+\mathrm{H})^{+}, 100 \%\right]$. 
4-((2,6-Diisopropylphenyl)amino)butane-1,3-diol.

${ }^{1} \mathrm{H}$ NMR $\left(500 \mathrm{MHz}, \mathrm{CDCl}_{3}\right)$.

May11-2021

Chemist Shweta Gediya

PROTON.w CDCl3 /opt/topspin3.2 SKG 45
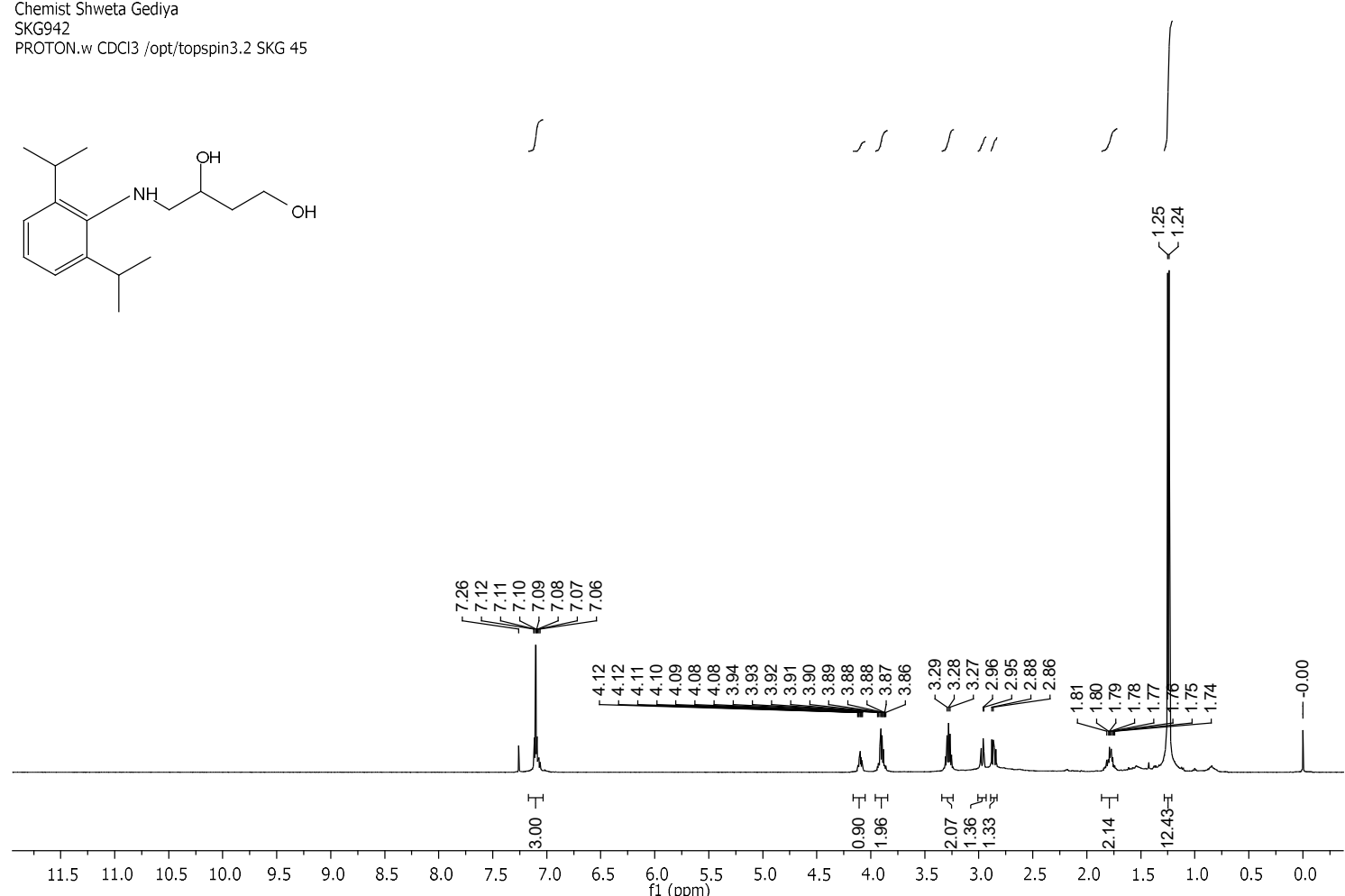

${ }^{13} \mathrm{C}$ NMR (126 MHz, $\left.\mathrm{CDCl}_{3}\right)$.

May11-2021

Chemist Shweta Gediya

SKG942

C13APT.w CDCl3 /opt/topspin3.2 SKG 45
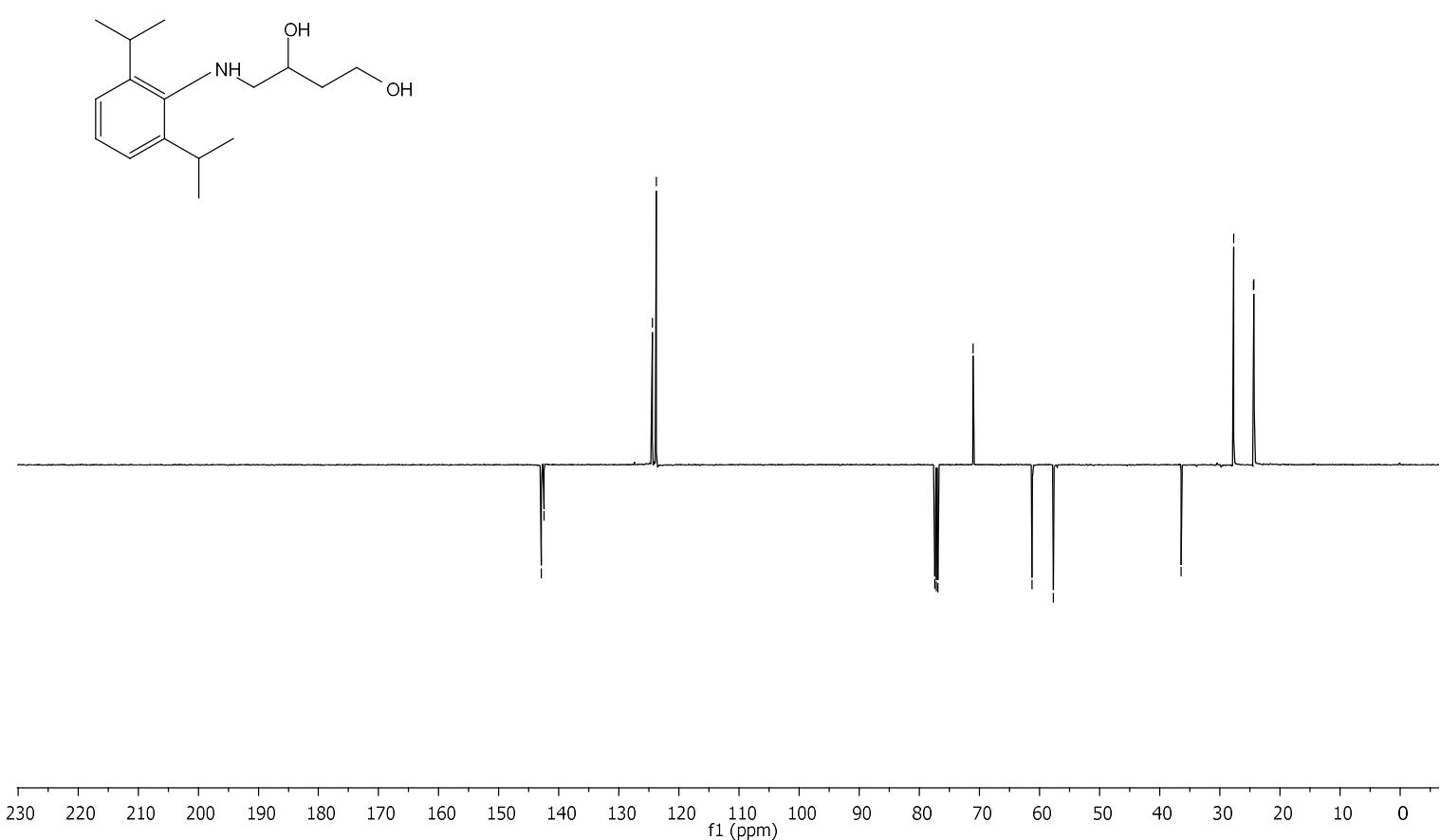
COSY.

Chemist Shweta Gediya

COSY.w CDCl3 /opt/topspin3.2 SKG 45

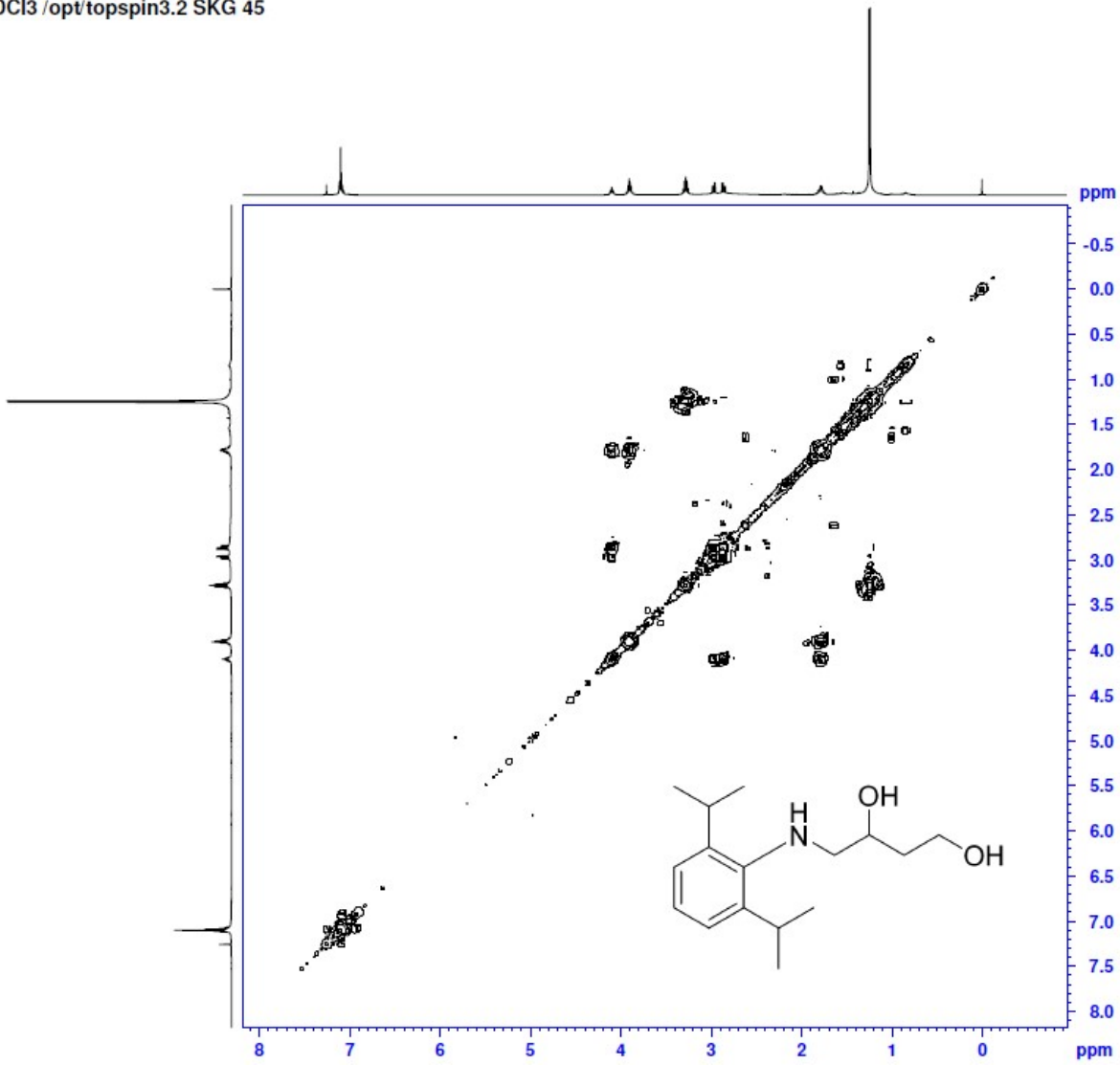

HSQC.

Chemist Shweta Gediya

HSQ94.w CDCl3/opt/topspin 3.2 SKG 45

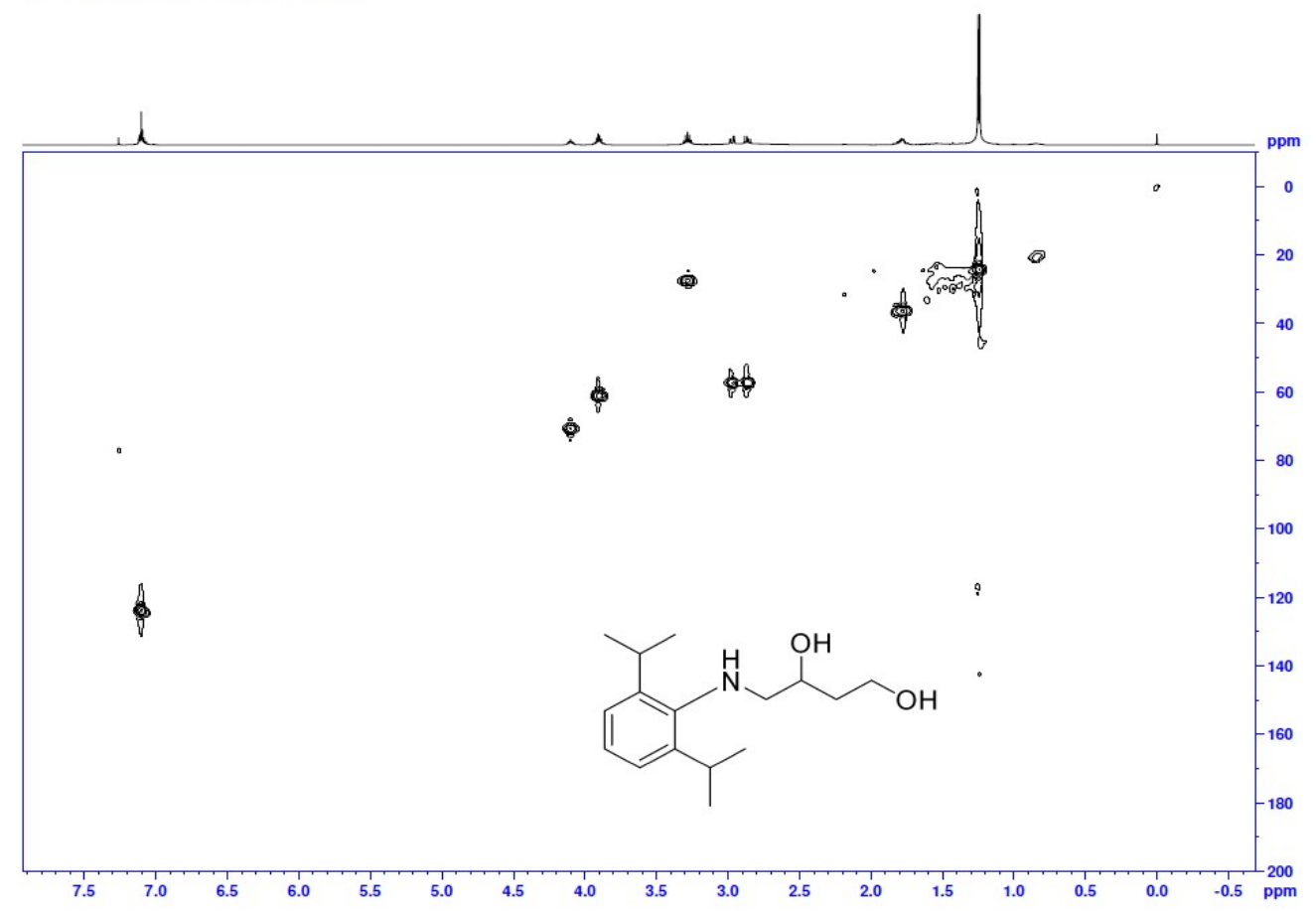




\section{$\underline{\text { X-ray data. }}$}

\section{(R)-N-(4-Chlorophenyl)-2-hydroxy-4-oxo-4-(piperidin-1-yl)butanamide}

\section{(local code skg3) CCDC 2100851. (R)-25.}

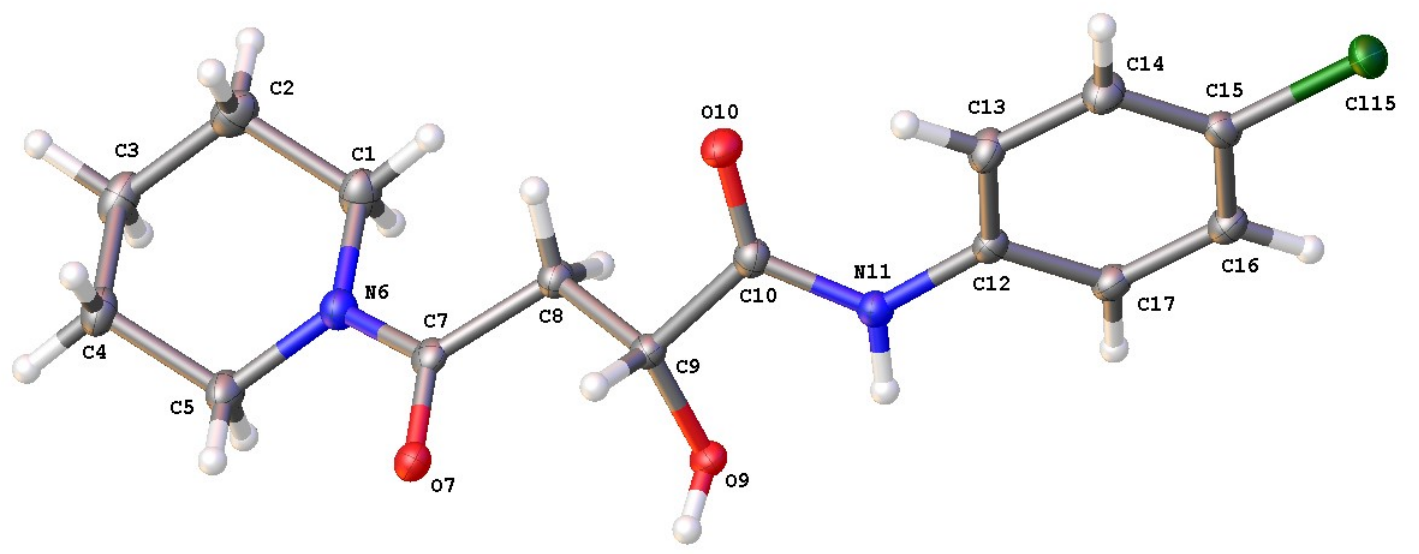

solid state structure of skg3 with atoms labels and thermal ellipsoids drawn at 50\% probability level (and no labels below)

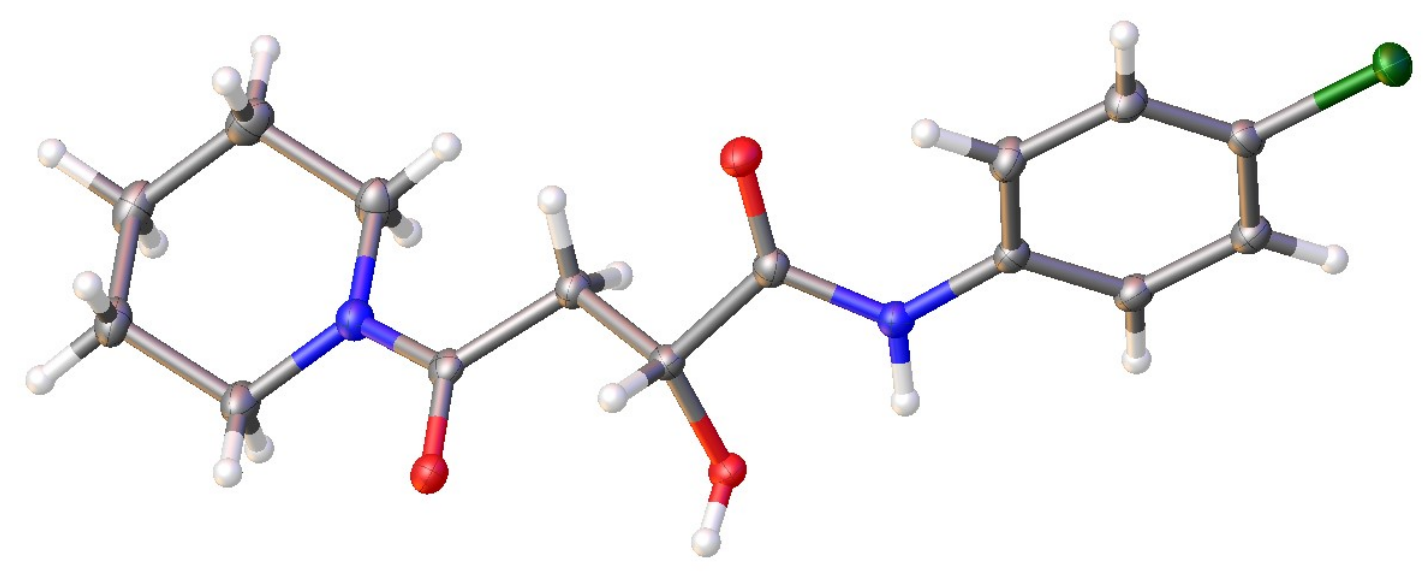

\section{Crystal structure determination of $(R)-25$ [skg3]}

The asymmetric unit contains the alcohol. There are four molecules in the unit cell. The $\mathrm{OH}$ and amide $\mathrm{NH}$ were located in a difference map but refined with restraints. They form short contacts tabulated below

Specified hydrogen bonds (with esds except fixed and riding $\mathrm{H}$ )
D-H H...A
D...A $<$ (DHA)
$0.84 \quad 1.85 \quad 2.6894(17) 172.8 \quad$ O9-H9A...O7_\$1 
$0.882 .152 .6059(18) \quad 111.6 \mathrm{~N} 11-\mathrm{H} 11 \ldots \mathrm{O} 9$ (this is an internal $\mathrm{H}$ bond along the $\mathrm{C}=\mathrm{O}$ dipole)

$0.88 \quad 2.44 \quad 3.2376(19) \quad 150.9 \quad$ N11-H11...O10_\$2

Symmetry operators used to generate symmetry equivalent atoms discussed in above contacts were

$\$ 10.5+X, 1.5-Y, 1-Z$

$\$ 21+\mathrm{X},+\mathrm{Y},+\mathrm{Z}$

The Flack parameter and associated Hooft y parameter are small with a small error so you can be confident in the assignment of the handedness of the crystal chosen

Flack x: -0.009(5) Shelx 2018

Hooft y: -0.013(4) Olex 2

Bulk property advised to confirm handedness of whole sample.

\section{Experimental}

Single crystals of $\mathrm{C}_{15} \mathrm{H}_{19} \mathrm{ClN}_{2} \mathrm{O}_{3}$ [skg3] were grown from methanol. A suitable crystal was selected and mounted on a glass fibre with Fomblin oil and placed on a Rigaku Oxford Diffraction Synergy-S diffractometer with a dual source equipped with a Hybrid pixel array detector. The crystal was kept at 100(2) K during data collection. Using Olex2 [1], the structure was solved with the ShelXT [2] structure solution program using Intrinsic Phasing and refined with the ShelXL [3] refinement package using Least Squares minimisation.

1. Dolomanov, O. V.; Bourhis, L. J.; Gildea, R. J.; Howard, J. A. K.; Puschmann, H. J. Appl. Cryst. 2009 42, 339-341.

2. Sheldrick, G.M. Acta Cryst. 2015, A71, 3-8.

3. Sheldrick, G.M. Acta Cryst. 2015, C71, 3-8.

Crystal Data for $\mathrm{C}_{15} \mathrm{H}_{19} \mathrm{ClN}_{2} \mathrm{O}_{3}(M=310.77 \mathrm{~g} / \mathrm{mol})$ : orthorhombic, space group $\mathrm{P} 2{ }_{1}{ }_{1} 2_{1}$ (no. 19), $a=5.47611(6) \AA, b=9.16092(9) \AA, c=29.7735(3) \AA, V=1493.62(2) \AA^{3}, Z=4, T=$ $100(2) \mathrm{K}, \mu(\mathrm{Cu} \mathrm{K \alpha})=2.372 \mathrm{~mm}^{-1}$, Dcalc $=1.382 \mathrm{~g} / \mathrm{cm}^{3}, 21962$ reflections measured $\left(5.936^{\circ}\right.$ $\left.\leq 2 \Theta \leq 159.448^{\circ}\right), 3194$ unique $\left(R_{\text {int }}=0.0420, \mathrm{R}_{\text {sigma }}=0.0221\right)$ which were used in all calculations. The final $R_{1}$ was 0.0240 (I $\left.>2 \sigma(\mathrm{I})\right)$ and $w R_{2}$ was 0.0623 (all data). 
Table 1 Crystal data and structure refinement for skg3.

Identification code

Empirical formula

Formula weight

Temperature/K

Crystal system

Space group

$\mathrm{a} / \AA$

$\mathrm{b} / \AA$

$\mathrm{c} / \AA \AA$

$\alpha /^{\circ}$

$\beta /{ }^{\circ}$

$\gamma /{ }^{\circ}$

Volume $/ \AA^{3}$

Z

$\rho_{\text {calc }} \mathrm{g} / \mathrm{cm}^{3}$

$\mu / \mathrm{mm}^{-1}$

$\mathrm{F}(000)$

Crystal size $/ \mathrm{mm}^{3}$

Radiation skg3

$\mathrm{C}_{15} \mathrm{H}_{19} \mathrm{ClN}_{2} \mathrm{O}_{3}$

310.77

100(2)

orthorhombic

$\mathrm{P} 2{ }_{1}{ }_{1} 2_{1}$

$5.47611(6)$

9.16092(9)

29.7735(3)

90

90

90

1493.62(2)

4

1.382

2.372

656.0

$0.2 \times 0.06 \times 0.03$

$\mathrm{Cu} \operatorname{K} \alpha(\lambda=1.54184)$

$2 \Theta$ range for data collection/ ${ }^{\circ} 5.936$ to 159.448

Index ranges

$-6 \leq \mathrm{h} \leq 6,-11 \leq \mathrm{k} \leq 11,-37 \leq 1 \leq 37$

Reflections collected 21962

Independent reflections $3194\left[\mathrm{R}_{\text {int }}=0.0420, \mathrm{R}_{\text {sigma }}=0.0221\right]$

Data/restraints/parameters

$3194 / 0 / 191$

Goodness-of-fit on $\mathrm{F}^{2}$ 1.055

Final $R$ indexes $[\mathrm{I}>=2 \sigma(\mathrm{I})] \quad \mathrm{R}_{1}=0.0240, \mathrm{wR}_{2}=0.0620$

Final $\mathrm{R}$ indexes [all data] $\quad \mathrm{R}_{1}=0.0246, \mathrm{wR}_{2}=0.0623$

Largest diff. peak/hole / e $\AA^{-3} 0.17 /-0.18$

Flack parameter $-0.009(5)$ 
(R)-Ethyl-4-((2,6-diisopropylphenyl)amino)-3-hydroxy-4-oxobutanoate 33.

(local code skg7) CCDC 2100850. (R)-33.

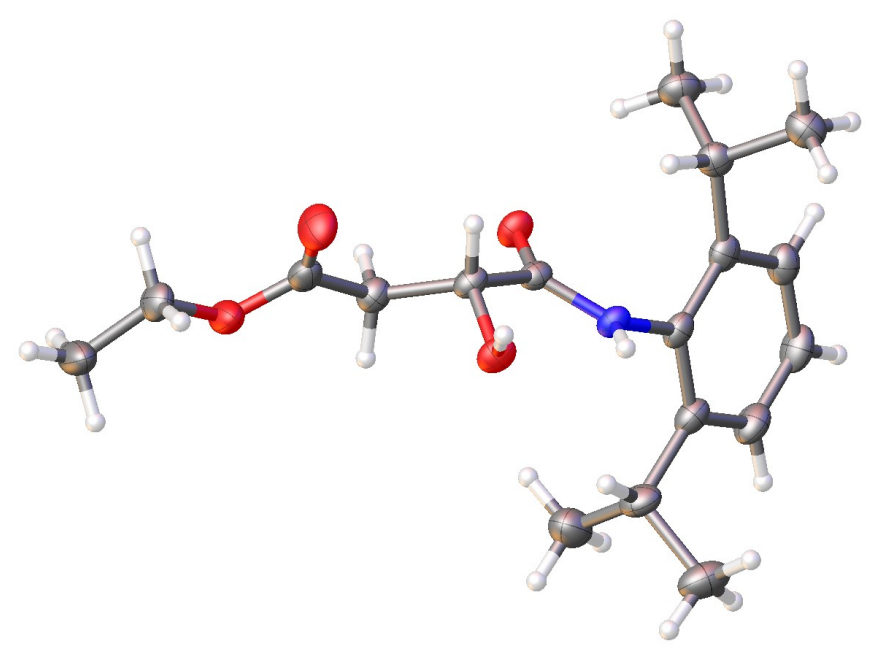

solid state structure of skg7 with thermal ellipsoids drawn at 50\% probability level

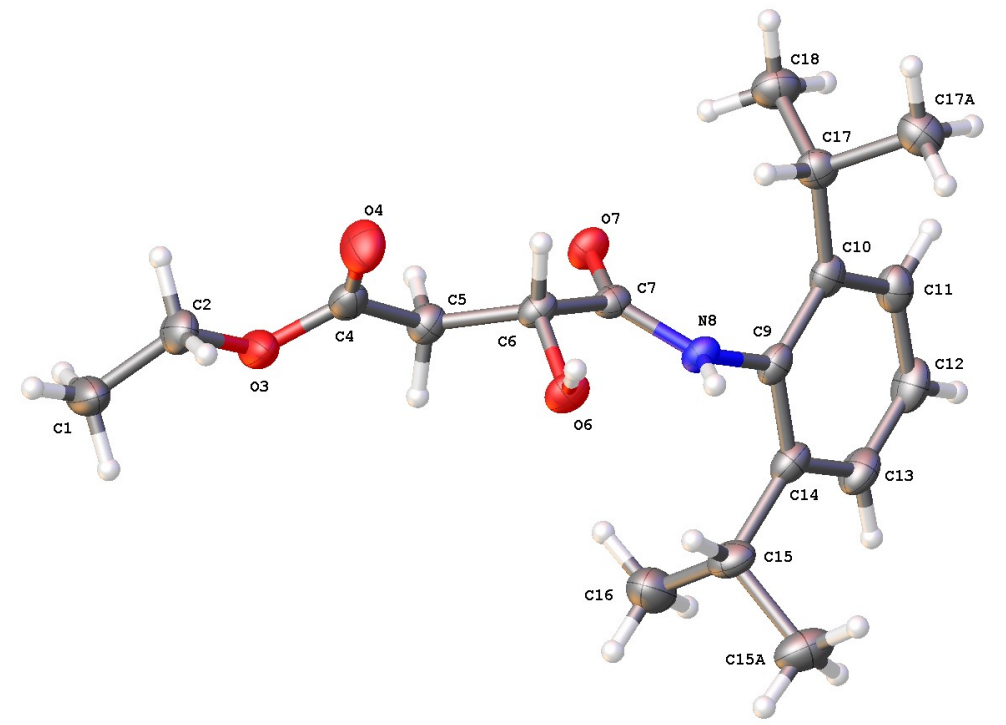

solid state structure of skg7 with atom labels and thermal ellipsoids drawn at 50\% probability level

\section{Crystal structure determination of $(R)-33$ [skg7]}

The asymmetric unit contains the compound. There are four molecules in the unit cell.

The ethyl group of the ester was modelled as disordered over two positions. The occupancy of the of the components was linked to a free variable which refined to 80.20 . The minor component was refined isotropically.

The $\mathrm{NH}$ and $\mathrm{OH}$ were located in a difference map and refined with restraints.

The $\mathrm{OH}$ forms an $\mathrm{H}$ bond tabulated below. 
Specified hydrogen bonds (with esds except fixed and riding $\mathrm{H}$ )
D-H H...A D...A $<($ DHA $)$
$0.84 \quad 1.84 \quad 2.6744(19) \quad 169.7 \quad$ O6-H6...O7_\$1

Symmetry operator used to define symmetry related equivalent atoms in above contact we $\$ 1$ $1+\mathrm{X},+\mathrm{Y},+\mathrm{Z}$

The NH has no traditional acceptor but points into the center of the pi system of a neighbouring ring

The Flack parameter (and associated Hooft y parameter) as a measure of the confidence you can have in the assignment of the handedness of the crystal chosen was

Flack x: 0.02(10) Shelx 2018

Hooft y: 0.01(8) Olex2

This is reasonably small (error a little large but expected for only having an oxygen as the heaviest atom) so you can be reasonably confident in the assignment of the crystal chosen.

Advised to have some determination of the handedness of the bulk sample.

\section{Experimental}

Single crystals of $\mathrm{C}_{18} \mathrm{H}_{27} \mathrm{NO}_{4}$ [skg7] were grown from methanol.

A suitable crystal was selected and mounted on a Mitegen head with Fomblin oil and placed on a Rigaku Oxford Diffraction Synergy-S diffractometer with a dual source equipped with a Hybrid pixel array detector.

The crystal was kept at 100(2) K during data collection. Using Olex2 [1], the structure was solved with the SHELXT [2] structure solution

program using Intrinsic Phasing and refined with the SHELXL [3] refinement package using Least Squares minimisation.

1. Dolomanov, O. V.; Bourhis, L. J.; Gildea, R. J.; Howard, J. A. K.; Puschmann, H. J. Appl. Cryst. 2009 42, 339-341.

2. Sheldrick, G.M. Acta Cryst. 2015, A71, 3-8.

3. Sheldrick, G.M. Acta Cryst. 2015, C71, 3-8.

Crystal Data for $\mathrm{C}_{18} \mathrm{H}_{27} \mathrm{NO}_{4}(M=321.40 \mathrm{~g} / \mathrm{mol})$ : orthorhombic, space group $\mathrm{P} 2{ }_{1}{ }_{12}{ }_{1}$ (no. 19), $a=5.43973(9) \AA, b=12.9815(3) \AA, c=25.7849(4) \AA, V=1820.83(6) \AA^{3}, Z=4, T=$ $100(2) \mathrm{K}, \mu(\mathrm{Cu} \mathrm{K \alpha})=0.665 \mathrm{~mm}^{-1}$, Dcalc $=1.172 \mathrm{~g} / \mathrm{cm}^{3}, 15329$ reflections measured $\left(6.856^{\circ}\right.$ 
$\left.\leq 2 \Theta \leq 159.622^{\circ}\right), 3847$ unique $\left(R_{\text {int }}=0.0393, \mathrm{R}_{\text {sigma }}=0.0341\right)$ which were used in all calculations. The final $R_{1}$ was 0.0364 (I $\left.>2 \sigma(\mathrm{I})\right)$ and $w R_{2}$ was 0.0952 (all data).

\section{Table 1 Crystal data and structure refinement for skg7}

Identification code

Empirical formula

Formula weight

Temperature/K

Crystal system

Space group

$\mathrm{a} / \AA$

$\mathrm{b} / \AA$

$\mathrm{c} / \AA$

$\alpha /{ }^{\circ}$

$\beta /{ }^{\circ}$

$\gamma /{ }^{\circ}$

Volume $/ \AA^{3}$

Z

$\rho_{\text {calcmg }} / \mathrm{mm}^{3}$

$\mu / \mathrm{mm}^{-1}$

$\mathrm{F}(000)$

Crystal size $/ \mathrm{mm}^{3}$

$2 \Theta$ range for data collection

Index ranges

Reflections collected

Independent reflections

Data/restraints/parameters

Goodness-of-fit on $\mathrm{F}^{2}$

Final R indexes $[\mathrm{I}>=2 \sigma(\mathrm{I})]$

Final $\mathrm{R}$ indexes [all data]

Largest diff. peak/hole / e $\AA^{-3}$

Flack parameter skg7

$\mathrm{C}_{18} \mathrm{H}_{27} \mathrm{NO}_{4}$

321.40

100(2)

orthorhombic

$\mathrm{P} 2{ }_{1}{ }_{1} 2_{1}$

5.43973(9)

$12.9815(3)$

25.7849(4)

90

90

90

1820.83(6)

4

1.172

0.665

696.0

$0.12 \times 0.06 \times 0.03$ colourless block

6.856 to $159.622^{\circ}$

$-6 \leq \mathrm{h} \leq 3,-16 \leq \mathrm{k} \leq 16,-32 \leq 1 \leq 32$

15329

$3847[\mathrm{R}$ (int) $=0.0393$ ]

$3847 / 0 / 224$

1.057

$\mathrm{R}_{1}=0.0364, \mathrm{wR}_{2}=0.0935$

$\mathrm{R}_{1}=0.0395, \mathrm{wR}_{2}=0.0952$

$0.28 /-0.24$

$0.02(10)$ 